\title{
Publications of the
}

National Bureau of Standards

July 1, 1957, to June 30, 1960

Includes Titles of Papers Published in Outside Journals

1950 to 1959

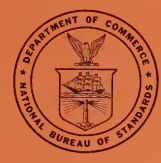

United States Department of Commerce

National Bureau of Standards

Miscellaneous Publication 240 


\section{THE NATIONAL BUREAU OF STANDARDS}

\section{Functions and Activities}

The functions of the National Bureau of Standards are set forth in the Act of Congress, March 3, 1901, as amended by Congress in Public Law 619, 1950 . These include the development and maintenance of the national standards of measurement and the provision of means and methods for making measurements consistent with these standards; the determination of physical constants and properties of materials; the development of methods and instruments for testing materials, devices, and structures; advisory services to government agencies on scientific and technical problems; invention and development of devices to serve special needs of the Government; and the development of standard practices, codes, and specifications. The work includes basic and applied research, development, engineering, instrumentation, testing, evaluation, calibration services, and various consultation and information services. Research projects are also performed for other government agencies when the work relates to and supplements the basic program of the Bureau or when the Bureau's unique competence is required. The scope of activities is suggested by the listing of divisions and sections on the inside of the back cover.

\section{Publications}

The results of the Bureau's work take the form of either actual equipment and devices or published papers. These papers appear either in the Bureau's own series of publications or in the journals of professional and scientific societies. The Bureau itself publishes three periodicals available from the Government Printing Office: The Journal of Research, published in four separate sections, presents complete scientific and technical papers; the Technical News Bulletin presents summary and preliminary reports on work in progress; and Basic Radio Propagation Predictions provides data for determining the best frequencies to use for radio communications throughout the world. There are also five series of nonperiodical publications: Monographs, Applied Mathematics Series, Handbooks, Miscellaneous Publications, and Technical Notes.

Information on the Bureau's publications can be found in NBS Circular 460 , Publications of the National Bureau of Standards (\$1.25) and its Supplement $(\$ 1.50)$, available from the Superintendent of Documents, U.S. Government Printing Office, Washington 25, D.C. 


\section{Publications of the}

\section{National Bureau of Standards \\ July 1, 1957, to June 30,1960}

Includes Titles of Papers Published in Outside Journals 1950 to 1959

(With Subject and Author Indexes)

Betty L. Arnold

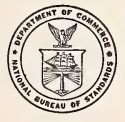

National Bureau of Standards Miscellaneous Publication 240 To Accompany National Bureau of Standards C460, and Supplement-1901 to June 30, 1957 Issued April 3, 1961 



\section{CONTENTS}

Page

Foreword.

General information

1. 1. Purchase procedures _.....................

1. 2. Announcements of Bureau publications

1. 3. Catalogs of publications

1. 4. Mailing lists

1. 5. Depository libraries in the United States

1. 6. Field Offices of the U.S. Department of Commerce

2. Periodicals of the National Bureau of Standards

2. 1. Journal of Research

Section A. Physics and Chemistry

Section B. Mathematics and Mathematical Physics.

Section C. Engineering and Instrumentation

Section D. Radio Propagation

2. 2. Technical News Bulletin

2. 3. Basic Radio Propagation Predictions
3. Titles and abstracts of NBS publications_... 11

3. 1. Research papers $\ldots$

3. 2. Papers from Section A._._...... 34

3. 3. Papers from Section B.

3. 4. Papers from Section $\mathrm{C}_{-}$

3. 5. Papers from Section D_._._._. 48

3. 6. Circulars

3. 7. Monographs _._. 59

3. 8. Handbooks_._.

3. 9. Miscellaneous publications _._._. 62

3. 10. Building materials and structures reports _._. 63

3. 11. Applied mathematics series__._._. 64

3. 12. Technical notes.... 65

4. Titles of papers published in outside journals 1950 to 1959

5. Price list of available publications

6. Author index 167

7. Subject index 


\section{FOREWORD}

The functions of the National Bureau of Standards are set forth in the Act of Congress, March 3, 1901, as amended by Congress in Public Law 619, 1950. These include the development and maintenance of the national standards of measurement and the provision of means and methods for making measurements consistent with these standards; the determination of physical constants and properties of materials; the development of methods and instruments for testing materials, devices, and structures; advisory services to Government agencies on scientific and technical problems, invention and development of devices to serve special needs of the Government; and the development of standard practices, codes, and specifications. The work includes basic and applied research, development, engineering, instrumentation, testing, evaluation, calibration services, and various consultation and information services. The program is carried out in the following technical divisions and offices: Electricity, metrology, heat, radiation physics, analytical and inorganic chemistry, mechanics, organic and fibrous materials, metallurgy, mineral products, building research, applied mathematics, data processing systems, atomic physics, instrumentation, physical chemistry, weights and measures, cryogenic engineering, ionosphere research and propagation, radio propagation engineering, radio standards, radio systems, upper atmosphere and space physics.

The results of the Bureau's work take the form of either actual equipment and devices or published papers, which appear either in the Bureau's own series of publications or in the journals of professional and scientific societies. The Bureau publishes three periodicals, available from the Government Printing Office: The Journal of Research, the Technical News Bulletin, and Basic Radio Propagation Predictions. There are also five series of nonperiodical publications: Monographs, Handbooks, Applied Mathematics Series, Miscellaneous Publications, and Technical Notes.

This publication provides a complete list, with brief abstracts, of Bureau publications issued during the period July 1, 1957 to June 30,1960 . It also includes a list of those papers by Bureau authors that have appeared in nonNational Bureau of Standards Journals from 1950 through 1959. Circular 460 (for the period 1901 to June 30, 1957) and its Supplement (July 1, 1947 to June $30,1957)$ remain in effect.

\section{A. V. Astin, Director.}

\section{Supplementary List of Publications of the National Bureau of Standards July 1, 1957, to June 30, 1960}

This Supplement lists the publications of the National Bureau of Standards from July 1 , 1957 , to June 30,1960 . A brief abstract for each publication is given. It also includes a list of those papers by Bureau authors that have appeared in non-National Bureau of Standards Journals from 1950 through 1959 . Subject and author indexes are included, and general publication information is furnished. This catalog and previous lists (see page 2) give reference to the publications printed by the Bureau during the period 1901 to June 30, 1957. Over 8,000 papers have been issued.

Reprints of papers published in outside journals are available only from the authors themselves or from the outside journal. 



\section{GENERAL INFORMATION}

\subsection{PURCHASE PROGEDURES}

The publications of the Bureau are distributed principally by the Superintendent of Documents, United States Government Printing Office, Washington 25, D.C., who sells them, as long as copies are available, at the prices given in this Circular.

How To Make Remittances. Remittances for publications for which individual sales or subscription prices are shown should be mailed to Superintendent of Documents, U.S. Government Printing Office, Washington 25, D.C., by coupon, postal money express order, or check. Publications cannot be mailed in advance of receipt of remittance. Currency may be sent at sender's risk. Foreign remittances should be made either by international money order or draft on an American bank.

Foreign money, defaced or smooth coins, will not be accepted. Do not send postage stamps.

The letter symbol with each publication number and the full title of the publication must be given when ordering. If 100 copies or more of any publication are ordered, a discount of 25 percent is allowed.

For the convenience of the general public, coupons in the denomination of 5 cents that are good until used in exchange for Government publications sold by the Superintendent of Documents, may be purchased from his office. Address order to Superintendent of Documents, U.S. Government Printing Office, Washington 25, D.C.

Persons who make frequent purchases from the Superintendent of Documents may find a deposit account convenient. Deposits of $\$ 10$ or more are accepted against which orders may be placed without making individual remittances or first obtaining quotations. Order blanks are furnished for this purpose. After the handling of the order has been completed, it will be returned showing the publications supplied, explanations regarding those not sent, the amount of charge, and the balance on deposit.

No charge is made for postage on documents forwarded to points in United States and possessions, or to Canada, Cuba, Mexico, Newfoundland (including Labrador), and certain Central and South American countries. To other countries the regular rate of postage is charged, and reinittances must cover such postage. In computing foreign postage, add one-fourth of the price of the publication.

\subsection{ANNOUNGEMENTS OF BUREAU PUBLICATIONS}

There are several official sources of information as to new publications of the National Bureau of Standards. The following announcements are issued regularly by governmental agencies. In addition, many of the technical journals carry notices of new Bureau publications of interest in their respective fields.

Technical News Bulletin. Issued monthly by the National Bureau of Standards. Announces all new publications by members of the staff, including those appearing in outside journals. Available from the Superintendent of Documents, U.S. Government Printing Office, Washington 25, D.C. Annual subscription, $\$ 1.50 ; \$ 2.25$ foreign. Single copies, 15 cents each.

Monthly Catalog of United States Government Publications. Issued monthly by the Superintendent of Documents, U.S. Government Printing Office, Washington 25, D.C. Annual subscription, with consolidated annual index, $\$ 3$; $\$ 4.50$ foreign.

Price Lists. Publication lists prepared by the Superintendent of Documents on special subjects. These will be furnished free, on application, if the subject concerning which information is desired is stated.

List of Selected Government Publications. Issued semimonthly by the Superintendent of Documents. Each list is arranged alphabetically by subjects, with annotations and prices. May be obtained free from that office.

Business Service Check List. Weekly announcements of publications of the Department of Commerce. Lists titles and prices of National Bureau of Standards publications, as well as those of other offices of the Department of Commerce. Available from the Superintendent of Documents for $\$ 1.50$ a year domestic; $\$ 3.50$ foreign.

\subsection{Catalogs OF BUREAU PUblications}

Previous catalogs and this Circular give a complete list of the titles and brief abstracts of the Bureau's publications up to June 30,1960 . Where the price is given the publication is available from the Superintendent of Documents, U.S. Government Printing Office, Washington 25, D.C., or they may be available for consultation in libraries maintaining sets of Bureau Circulars. 
Cireular C24, 7th edition: Publications of the Bureau of Standards 1901 to 1925.271 pages, including brief abstracts and subject index...

Circular C24 Supplement: Supplementary list of publications of the Bureau of Standards, July 1, 1925 , to December 31, 1931. 214 pages, including brief abstracts and subject index . . . .

Circular 24 Supplement: Supplementary list of publications of the National Bureau of Standards, January 1, 1932, to December 31, 1941. 386 pages, including brief abstracts. The subject and author indexes cover the period 1901 to December 31,1941

Circular 460: Publications of the National Bureau of Standards 1901 to June 30, 1947. 375 pages, including subject and author indexes. Brief abstracts are included for the period January 1, 1942 , to June 30,1947

Supplementary List of Publications of the National Bureau of Standards, July 1, 1947, to June 30 , 1957. 373 pages, including subject and author indexes

- Out of print.
Miscellaneous Publication 240: Publications of the National Bureau of Standards July 1, 1957, to June 30,1960 . Includes Titles of Papers Published in Outside Journals 1950 to 1959.391 pages, including subject and author indexes. . . . .

\subsection{MAILING LISTS}

Names of individuals are not placed on the Bureau's mailing lists. The principal distribution is by the Superintendent of Documents on a sales basis. A limited edition of each paper is printed for Bureau administrative needs and for official distribution to cooperating laboratories, technical organizations, Government agencies, and to leading public and educational institutions libraries. In addition, the Superintendent of Documents distributes copies of selected Bureau publications to the Government depository libraries listed below.

\subsection{DEPOSITORY LIBRARIES IN THE UNITED STATES}

The Superintendent of Documents, United States Government Printing Office, is authorized by law to furnish Government publications to designated depository libraries. The libraries listed below are now receiving certain selected publication series of the National Bureau of Standards for general reference use. Whether or not a given library has a copy of any particular publication can only be determined by inquiry at the library.

ALABAMA

Auburn: Alabama Polytechnic Institute Library.

Birmingham:

Howard College Library.

Birmingham Southern College, M. Paul Phillips Library.

Public Library.

Florence: State Teachers College Library.

Jacksonville: State Teachers College Library.

Mobile (Spring Hill): The Thomas Bryne Memorial, Spring Hill College.

Montgomery:

Department of Archives and History Library.

State and Supreme Court Library.

Tuskegee Institute: Hollis Burke Frissell Library

University: University of Alabama Library.

\section{ALASK A}

College: University of Alaska Library.

Juneau: Alaska Historical Library and Museum.

\section{ARIZONA}

Flagstaff Arizona State Teachers College Library.

Phoenix:

Department of Library and Archives.

Phoenix Public Library.

Tempe: Matthews Library.

Tueson: University of Arizona Library.

\section{ARKANSAS}

Clarksville: College of the Ozarks Library.

College Heights: Arkansas Agricultural and Mechanical College Library.

Conway: Hendrix College Library.

Fayetteville: University of Arkansas Library.

Little Rock: Little Rock Public Library.

Magnolia: Southern State College, J. M. Peace Library.

Russellville: Arkansas Polytechnic College Library.

State College: Arkansas State College Library.

\section{CALIFORNIA}

Bakersfield: Kern County Free Library.

Berkeley: University of California Library.
Claremont: Pomona College, Honnold Library.

Davis: University of California Library.

Eureka: Eureka Free Library.

Fresno: Fresno County Free Library.

Long Beach: Public Library.

Los Angeles:

University of Southern California Library.

Los Angeles Public Library.

Los Angeles State College Library.

Loyola University Library.

Occidental College Library.

University of California at Los Angeles Library.

Northridge: San Fernando Valley State College Library. Oakland: Oakland Public Library.

Pasadena: California Institute of Technology Library. Redding: Shasta County Free Library.

Redlands: University of Redlands Library.

Richmond: Richmond Public Library.

Riverside: Riverside Public Library.

Sacramento:

California State Library.

City Free Library.

San Diego: San Diego Public Library.

San Francisco:

Mechanics Mercantile Library.

San Francisco Public Library.

San Francisco State College, Social Science and Business Library

Santa Barbara: Santa Barbara Public Library.

Stanford Univ.: Stanford University Library.

Stockton: Stockton Free Public Library.

\section{COLORADO}

Boulder: University of Colorado Library.

Colorado Springs: Colorado College, Coburn Library. Denver:

Colorado State Library.

Public Library.

Regis College Library.

U.S. Air Force Academy.

University of Denver, Mary Reed Library.

Fort Collins: Colorado State University Library.

Golden: Colorado School of Mines Library.

Gunnison: Western State College Library.

Pueblo: McClelland Public Library. 


\section{CONNECTICUT}

Bridgeport: Bridgeport Public Library.

Hartford:

Connecticut State Library.

Hartford Public Library.

Trinity College Library.

Middletown: Wesleyan University, Olin Library.

New Haven: Yale University Library.

New London:

Connecticut College, Palmer Library.

U.S. Coast Guard Academy Library.

Storrs: University of Connecticut Library.

Waterbury: Silas Bronson Library.

\section{DELAWARE}

Newark: University of Delaware Library.

Wilmington: Wilmington Institute Free Library.

\section{DISTRICT OF COLUMBIA}

Washington:

Department of Agriculture Library.

Department of Commerce Library.

Department of Health, Education, and Welfare Library.

Department of Interior Central Library.

Department of Justice Main Library.

Department of State Library.

National War College Library.

Navy Department Library.

Post Office Department Library.

Public Library.

Treasury Department Library.

\section{FLORIDA}

Coral Gables: University of Miami Library.

De Land: Sampson Library of John B. Stetson University.

Gainesville: University of Florida Library.

Jacksonville: Jacksonville Public Library.

Lakeland: Lakeland Public Library.

Miami: Miami Public Library.

Tallahassee:

Florida Agricultural and Mechanical College Library. Florida State Library.

Florida State University Library.

Tampa: University of Tampa Library.

Winter Park: Rollins College, Mills Memorial Library.

\section{GEORGIA}

Athens: University of Georgia General Library.

Atlanta:

Atlanta Public Library.

Emory University: Asa Griggs Candler Library.

Georgia State Library.

Augusta: Library of Richmond County Academy and Junior College of Augusta.

Collegeboro: Georgia Teachers College Library.

Dahlonega: North Georgia College Library.

Milledgeville: Georgia State College for Women Library.

Savannah: Savannah Public Library.

Valdosta: Valdosta State College Library.

Honolulu:

\section{HA WAII}

Library of Hawaii.

University of Hawaii Library.

\section{IDAHO}

Boise:

Boise Public Library.

Idaho State Law Library.

Caldwell: Strahorn Memorial Library.

Moscow: University of Idaho Library.

Pocatello: Idaho State College Library.

Rexberg: Ricks College Library.

\section{ILLINOIS}

Carbondale: Southern Illinois University Library.

Carlinville: Blackburn College Library.
Chicago:

Amundsen Junior College Library.

Chicago Public Library.

Chicago Teachers College Library.

John Crerar Library.

Newberry Library.

University of Chicago Libraries.

University of Illinois Library.

Decatur: Decatur Public Library.

Elsah: Prinicipia College, Marshall Brooks Library.

Evanston: Northwestern University Library.

Freeport: Freeport Public Library.

Galesburg: Galesburg Public Library.

Jacksonville: MacMurray College Library.

Joliet: Public Library.

Kankakee: Olivet Nazarene College Library.

Lisle: St. Procopius College Library.

Lockport: Lewis College of Science and Technology Library.

Monmouth: Monmouth College Library.

Normal: Illinois State Normal University Library.

Peoria: Peoria Public Library.

Rock Island: Rock Island Publie Library.

Rockford: Public Library.

Springfield: Illinois State Library.

Urbana: University of Illinois Library.

\section{INDIANA}

Bloomington: Indiana University Library.

Crawfordsville: Wabash College Library.

Evansville: Evansville Public Library.

Fort Wayne: Public Library.

Gary: Gary Public Library.

Greencastle: De Pauw University Library.

Hanover: Hanover College Library.

Indianapolis:

Indiana State Library.

Indianapolis Public Library.

Lafayette: Purdue University Library.

Muncie: Public Library.

Notre Dame: University of Notre Dame Library.

Richmond: Morrison-Reeves Library.

Terre Haute: Indiana State Teachers College Library.

Valparaiso: Valparaiso University Library.

\section{IOWA}

Ames: Iowa State College Library

Cedar Falls: Iowa State Teacheis College Library.

Council Bluffs: Free Public Library.

Des Moines:

Iowa State Traveling Library.

Public Library.

Dubuque: Carnegie Stout Free Public Library.

Fairfield: Fairfield Free Public Library.

Grinnell: Grinnell College Library.

Iowa City: State University of Iowa Library.

Lamoni: Graceland College Library.

Mount Vernon: Cornell College Library.

Sioux City: Public Library.

\section{KANSAS}

Baldwin City: Baker University Library.

Emporia: William Allen White Library, Kansas State Teachers College.

Hays: Forsyth Library of Fort Hays Kansas State College. Highland: Highland Junior College Library.

Lawrence: University of Kansas Library.

Manhattan: Kansas State College Library.

Pittsburg: Porter Library, Kansas State Teachers College.

Salina: Kansas Wesleyan University Library.

Topeka:

Kansas State Historical Society Library.

Kansas State Library.

Wichita: University of Wichita Library.

\section{KENTUCKY}

Ashland: Ashland Public Library.

Barbourville: Union College, Abigail E. Weeks Library. 
Bowling Green: Western Kentucky State Teachers College Library.

Danville: Centre College Library.

Frankfort: State Law Library.

Lexington: University of Kentucky Library.

Louisville:

Louisville Free Public Library.

University of Louisville Library.

Morehead: Johnson Camden Library, Morehead State College.

Murray: Murray State Teachers College Library.

Pikeville: Pikeville College Library.

\section{Baton Rouge:}

\section{LOUISIANA}

Louisiana State University Law Library.

Louisiana State University Library.

Southern University Library.

Lafayette: Southwestern Louisiana Institute Library

Lake Charles: McNeese State College Library.

Natchitoches: Northwestern State College of Louisiana.

New Orleans:

Law Library of Lousiana.

Loyola University Library.

New Orleans Public Library.

Tulane University, Howard-Tilton Memorial Library.

Ruston: Lousiana Polytechnic Institute Library.

Shreveport: Shreve Memorial Library.

\section{MAINE}

Augusta: Maine State Library.

Bangor: Bangor Public Library.

Brunswick: Bowdoin College Library.

Lewiston: Bates College Library.

Orono: University of Maine Library.

Portland: Portland Public Library.

Waterville: Colby College Library.

Annapolis:

MARYLAND

Maryland State Library.

U.S. Naval Academy Library.

Baltimore:

Enoch Pratt Free Library.

Johns Hopkins University Library.

Morgan State College Library.

Peabody Institute Library.

Chestertown: Washington College, George Avery Bunting Library.

College Park: University of Maryland Library.

Gaithersburg: Montgomery County Department of Public Libraries.

Westminster: Western Maryland College Library.

\section{MASSACHUSETTS}

Amherst:

Amherst College Library.

University of Massachusetts, Goodell Library.

Boston: Boston Athenaeum Library.

Boston Public Library.

State Library of Massachusetts.

Brookline: Public Library.

Cambridge:

Harvard College Library, Serials Division.

Massachusetts Institute of Technology Library.

Lowell: Lowell Textile Institute Library.

Lynn: Lynn Public Library.

New Bedford: Public Library.

Tufts College: Tufts College Library.

Wellesley: Wellesley College Library.

Williamstown: Williams College Library.

Worcester:

American Antiquarian Society Library.

Free Public Library.

\section{MICHIGAN}

Ann Arbor: General Library of University of Michigan

Battle Creek: Willard Library.

Benton Harbor: Benton Harbor Public Library.

Bloomfield Hills: Cranbrook Institute of Science Library.
Dearborn: Henry Ford Community College Library.

Detroit:

Detroit Public Library.

University of Detroit Library.

Wayne County Public Library.

Wayne State University Library.

East Lansing: Michigan State College of Agriculture and Applied Science Library.

Flint: Robert N. Mandeville High School Library.

Grand Rapids: Grand Rapids Public Library.

Houghton: Michigan College of Mining and Technology Library.

Kalamazoo: Public Library.

Lansing: Michigan State Library.

Mount Pleasant: Central Michigan College Library.

Muskegon: Hackley Public Library.

Port Huron: Port Huron Public Library.

Saginaw: Hoyt Public Library.

\section{MINNESOTA}

Collegeville: St. Johns University Library.

Duluth: Duluth Public Library.

Minneapolis:

Public Library.

University of Minnesota Library.

Moorhead: State Teachers College Library.

Northfield:

Carleton College Library.

St. Olaf College Library.

Saint Peter: Gustavus Adolphus College Library.

St. Paul:

Minnesota Historical Society Library.

Minnesota State Law Library.

St. Paul Public Library.

Stillwater: Stillwater Public Library.

\section{MISSISSIPPI}

Cleveland: W. B. Roberts Memorial Library, Delta State Teachers College.

Columbus: J. C. Fant Memorial Library of Mississippi State College for Women.

Hattiesburg: Mississippi Southern College Library.

Jackson:

Mississippi Library Commission.

Mississippi State Library.

State College: Mississippi State College, Mitchell Memorial Library.

University: University of Mississippi Library.

\section{MISSOURI}

Cape Girardeau: Kent Library, Southeast Missouri State College.

Columbia: University of Missouri Library.

Fulton: Westminster College Library.

Hannibal: Free Public Library.

Jefferson City:

Lincoln University Library.

Missouri Supreme Court Library.

Kansas City:

Kansas City Public Library.

Rockhurst College Library.

University of Kansas City Library.

Liberty: William Jewell College Library.

Rolla: School of Mines \& Metallurgy Library.

St. Joseph: St. Joseph Public Library.

St. Louis:

St. Louis Public Library.

St. Louis University Library.

Washington University Library.

Springfield: Drury College Library.

Warrensburg: Central Missouri State College Library.

\section{MONTANA}

Bozeman: Montana State College Library. Butte: Montana School of Mines Library.

Helena:

Helena Public Library

Historical Society of Montana Library.

Missoula: State University of Montana Library. 


\section{NEBRASKA}

Blair: Dana College Library.

Crete: Whitin Library of Doane College.

Fremont: Midland College Library.

Lincoln:

Nebraska State Library.

University of Nebraska Library.

Omaha:

Municipal University of Omaha Library.

Omaha Public Library.

Scottsbluff: Scottsbluff Public Library.

\section{NEVADA}

Carson City: Nevada State Library.

Hawthorne: Mineral County Public Library.

Reno: University of Nevada Library.

\section{NEW HAMPSHIRE}

Concord: New Hampshire State Library.

Dover: Dover Public Library.

Durham: University of New Hampshire Library

Hanover: Dartmouth College Library.

Laconia: Laconia Public Library.

Manchester: City Library.

\section{NEW JERSEY}

Atlantic City: Free Public Library.

Bayonne: Free Public Library.

Camden: Camden Free Public Library.

Convent Station: Santa Maria Library of College of St. Elizabeth.

Elizabeth: Public Library.

Jersey City: Free Public Library.

Madison: Rose Memorial Library.

New Brunswick:

Free Public Library.

Rutgers University Library.

Newark: Public Library.

Princeton: Princeton University Library.

Rutherford: Fairleigh Dickinson College Library.

South Orange: Seton Hall University Library.

Trenton:

Division of State Library, Archives and HistoryDepartment of Education.

Free Public Library.

\section{NEW MEXICO}

Albuquerque: University of New Mexico.

Hobbs: Hobbs Public Library.

Las Vegas: New Mexico Highlands University, Rodgers Library.

Santa Fe: New Mexico State Law Library.

Silver City: New Mexico State Teachers College Library.

State College: New Mexico College of Agriculture and Mechanic Arts Library.

\section{NEW YORK}

Albany: New York State Library.

Brooklyn:

Brooklyn College Library.

Brooklyn Public Library.

Pratt Institute Library.

Buffalo:

Buffalo and Erie County Public Library.

Grosvenor Library.

Canton: St. Lawrence University Library.

Elmira: Elmira College Library.

Farmingdale: Long Island Agricultural and Technical Institute Library.

Flushing: Queens College Library.

Glens Falls: Crandall Library.

Hamilton: Colgate University Library.

Ithaca:

Albert R. Mann Library, New York State Colleges of Agriculture and Home Economics.

Cornell University Library.
Jamaica:

Queens Borough Public Library.

St. Johns University Library.

New York City:

College of the City of New York Library.

Columbia University Library.

Cooper Union Library.

Fordham University Library.

New York Law Institute Library.

New York Public Library (Astor Branch).

New York Public Library (Lenox Branch).

New York State Maritime College Library.

New York University General Library.

Newburgh: Newburgh Free Library.

Potsdam: Clarkson College of Technology Library.

Poughkeepsie: Vassar College Library.

Rochester:

Rochester Public Library, Edgerton Branch.

University of Rochester Library.

St. Bonaventure: St. Bonaventure College Library.

Schenectady: Union College Library.

Staten Island: Wagner College Library, Grymes Hill.

Syracuse: Syracuse University Library.

Troy: Troy Public Library.

Utica: Utica Public Library.

West Point: U.S. Military Academy.

Yonkers: Yonkers Public Library.

\section{NORTH CAROLINA}

Chapel Hill: University of North Carolina Library.

Charlotte: Queens College Library.

Cullowhee: Western Carolina College Library.

Davidson: Library of Davidson College.

Durham: Duke University Library.

Greensboro: Agricultural and Technical College Library.

Greenville: East Carolina College Library.

Pembroke: Pembroke State College Library.

Raleigh:

D. H. Hill Library of North Carolina State College. North Carolina State Library.

Salisbury: Catawba College Library.

Wilson: Atlantic Christian College, Clarence L. Hardy Library.

Winston-Salem:

Public Library of Winston-Salem and Forsyth County. Wake Forest College Library.

\section{NORTH DAKOTA}

Bismarck:

State Historical Library.

State Law Library.

Fargo: North Dakota Agricultural College and Experiment Station Library.

Grand Forks: University of North Dakota Library.

Minot: State Teachers College Library.

Valley City: State Teachers College Library.

\section{OHIO}

Akron: Akron Public Library.

Alliance: Mt. Union College Library.

Ashland: Ashland College Library.

Athens: Ohio University Library.

Bluffton: Musselman Library of Bluffton College.

Bowling Green: Bowling Green State University Library.

Bucyrus: Bucyrus Public Library.

Cincinnati:

Public Library.

University of Cincinnati Library.

Cleveland:

Adelbert College Library of Western Reserve University.

Cleveland Public Library.

Columbus:

Columbus Public Library.

Ohio State Library.

Ohio State University Library.

Dayton: Dayton Public Library. 
Delaware: Charles Slocum Library of Ohio Wesleyan University.

Gambier: Kenyon College Library.

Granville: Denison University Library.

Hiram: Hiram College Library.

Marietta: Marietta College Library.

Oberlin: Oberlin College Library.

Oxford: Miami University Library.

Portsmouth: Free Public Library.

Springfield: Warder Public Library.

Steubenville: Carnegie Library.

Toledo: Toledo Public Library.

Van Wert: Brumback Library of Van Wert County.

Youngstown: Youngstown Public Library.

\section{OKLAHOMA}

Ada: East Central State Teachers College Library.

Alva: Northwestern State Teachers College Library.

Durant: Southeastern Teachers College Library.

Edmond: Central State College Library.

Enid: Carnegie Public Library.

Langston: Langston University Library.

Norman: University of Oklahoma Library.

Oklahoma City: Oklahoma State Library.

Shawnee: Oklahoma Baptist University Library.

Stillwater: Oklahoma State University Library.

Tahlequah: Northeastern State Teachers College Library.

Tulsa: University of Tulsa Library.

Weatherford: Southwestern State College Library.

\section{OREGON}

Ashland: Southern Oregon College of Education Library.

Corvallis: Oregon State College Library.

Eugene: University of Oregon Library.

Forest Grove: Pacific University Library.

La Grande: Eastern Oregon College of Education Library.

Portland:

Library Association of Portland.

Reed College Library.

Salem: Oregon State Library.

\section{PENNSYLVANIA}

Allentown: Muhlenberg College Library.

Bethlehem: Lehigh University Library.

Bradford: Carnegie Public Library.

Carlisle: Dickinson College Library.

Erie: Erie Public Library.

Harı isburg: Pennsylvania State Library.

Haverford: Haverford College Library.

Huntingdon: Juniata College Library.

Lancaster: Franklin and Marshall College, Fackenthal Library.

Meadville: Allegheny College Library.

Philadelphia:

Free Library of Philadelphia.

Philadelphia Commercial Museum Library.

Temple University, Sullivan Memorial Library.

University of Pennsylvania Library.

Pittsburgh:

Allegheny Regional Branch Library.

Carnegie Library of Pittsburgh.

University of Pittsburgh Library.

Reading: Reading Public Library.

Seranton: Seranton Public Library.

State College: Pennsylvania State University Library.

Swarthmore: Swarthmore College Library.

Warren: Warren Library Association.

Washington: Memorial Library of Washington and Jefferson College.

Wilkes-Barre: Kings College Library.

Williamsport: James V. Brown Library.

\section{PUERTO RICO}

Mayaguez: University of Puerto Rico, College of Agriculture and Mechanical Arts Library.

Rio Piedras: University of Puerto Rico General Library.

\section{RHODE ISLAND}

Kingston: University of Rhode Island Library.

Providence:

Brown University Library.

Providence Public Library.

Rhode Island State Library.

Westerly: Westerly Public Library.

\section{SOUTH CAROLINA}

Charleston: Charleston College Library.

Clemson: Clemson College Library.

Columbia:

South Carolina State Library.

University of South Carolina Library.

Orangeburg: State Agricultural and Mechanical College.

Rockhill: Carnegie Library of Winthrop College.

\section{SOUTH DAKOTA}

Brookings: Lincoln Memorial Library of South Dakota State College.

Mitchell: Dakota Wesleyan University Library.

Pierre: South Dakota Free Library Commission.

Sioux Falls: Carnegie Free Public Library.

Spearfish: Black Hills Teachers College Library.

Vermillion: University of South Dakota Library.

Yankton: Yankton College Library.

\section{TENNESSEE}

Chattanooga: Chattanooga Public Library.

Clarksville: Austin Peay State College Library.

Johnson City: East Tennessee State College Library.

Knoxville: University of Tennessee Library.

Martin: University of Tennessee Library, Martin Branch.

Memphis: Cossitt Library.

Murfreesboro: Middle Tennessee State College Library.

Nashville:

Joint University Libraries.

Nashville Public Library.

State Library Division, Tennessee State Library and Archives.

Sewanee: University of the South Library.

\section{TEXAS}

Abilene: Hardin Simmons University Library. Austin:

Texas State Library.

University of Texas Library.

Beaumont: Lamar State College of Technology Library.

Canyon: West Texas State Teachers College Library.

College Station: Agriculture and Mechanical College of

Texas Library.

Commerce: East Texas State Teachers College Library.

Dallas:

Dallas Public Library.

Southern Methodist University Library.

Denton: North Texas State Teachers Library.

El Paso: El Paso Public Library.

Fort Worth:

Fort Worth Public Library.

Texas Christian University Library.

Galveston: Rosenberg Library.

Houston:

Houston Public Library.

University of Houston Library.

Huntsville: Sam Houston State Teachers College, Estill Library.

Kingsville: Texas College of Arts and Industries Library.

Lubbock: Texas Technological College Library.

Marshall: Bishop College Library.

San Antonio: Public Library (La Villita Annex).

San Marcos: Southwest Texas State Teachers College Library.

Waco: Baylor University Library. 
UTAH

Ephraim: Snow College Library.

Logan: Utah State Library of Agriculture and Applied Science Library.

Ogden: Carnegie Free Library.

Provo: Brigham Young University Library.

Salt Lake City:

University of Utah Library.

Utah State Library.

\section{VERMONT}

Burlington: University of Vermont Library.

Johnson: Johnson Teachers College Library.

Middlebury: Middlebury College, Egbert Starr Library.

IIontpelier: Vermont State Library.

Northfield: Norwich University Library.

\section{VIRGINIA}

Blacksburg: Virginia Polytechnic Institute Library.

Bridgewater: Bridgewater College Library.

Emory: Emory and Henry College Library.

Fredericksburg: Mary Washington College Library.

Hampden Sydney: Hampden Sydney College Library.

Lexington:

Virginia Military Institute Library.

Washington and Lee University Library.

Norfolk: Norfolk Public Library.

Petersburg: Virginia State College Library.

Richmond: Virginia State Library.

Salem: Bittle Memorial Library of Roanoke College.

University: University of Virginia Library.

Univ. of Richmond, P.O.: University of Richmond Library.

Williamsburg: William and Mary College Library.

\section{WASHINGTON}

Everett: Everett Public Library.

Olympia: Washington State Library.

Pullman: State College of Washington Library.

Seattle:

Seattle Public Library.

University of Washington Library.
Spokane: Spokane Public Library.

Tacoma:

College of Puget Sound Library.

Tacoma Public Library.

Walla Walla: Whitman College Library

\section{WEST VIRGINIA}

Athens: Concord College Library.

Charleston:

Department of Archives and History, State Library.

Kanawha County Public Library.

Elkins: Davis and Eikins College Library.

Fairmont: Fairmont State College Library.

Huntington: Marshall College Library.

Institute: West Virginia State Collegé Library.

Morgantown: West Virginia University Library.

Salem: Salem College Library.

\section{WISCONSIN}

Appleton: Lawrence College Library.

Beloit: Beloit College Library.

Eau Claire: Wisconsin State College Library.

La Crosse: Public Library.

Madison:

State Historical Society Library.

University of Wisconsin Library.

Wisconsin State Library.

Milwaukee:

Law Library of Milwaukee County.

Milwaukee Public Library.

Oshkosh: Oshkosh State College Library.

Racine: Racine Public Library.

Stevens Point: Wisconsin State College Jibrary.

Superior:

Superior Public Library.

Curran Library, Wisconsin State College.

\section{WYOMING}

Casper: Natrona County Public Library.

Cheyenne: Wyoming State Library.

Laramie: University of Wyoming Library.

\subsection{FIELD OFFICES OF THE U.S. DEPARTMENT OF COMMERGE}

Department of Commerce Field Offices are maintained in the cities listed below. Their purpose is to provide ready access, at the local level, to the Department's reports, publications, statistical statements, surveys, as well as to the specialized and experienced staff in charge. Each Field Office serves as an official sales agent of the Superintendent of Documents, U.S. Government Printing Office, making a vailable for purchase locally a wide range of Government publications. The reference library maintained by each Field Office contains many Government and private publications, periodicals, directories, reports, and other reference material.

ALBUQUERQUE, N. MEX. Rm. 321, Post Office Bldg.

ATLANTA 3, GA.

604 Volunteer Bldg.

66 Luckie St., N.W.

BOSTON 9, MASS.

Rm. 232, Post Office \& Courthouse

BUFFALO 3, N.Y.

504 Federal Bldg.

117 Ellicott St.

CHARLESTON 4, S.C.

Area 2, Sergeant Jasper Bldg.

West End Broad St.

CHEYENNE, WYO.

207 Majestic BIdg.

16th St. \& Capitol Ave.
CHICAGO 6, ILL.

Rm. 1302

226 West Jackson Blvd.

CINCINNATI 2, OHIO

915 Fifth Third Bank Bldg.

36 E. Fourth St.

CLEVELAND 1, OHIO

4th Floor

Federal Reserve Bank bidy.

E. 6th St. \& Superior Ave.

DALLAS 1, TEX.

Rm. 3-104 Merchandise Mat

500 South Ervay St.

DENVER 2, COLO.

142 New Custom House

19th \& Stout Street
DETROIT 26, MICH. 438 Federal Bldg.

GREENSBORO, N.C.

Rm. 407, Post Office Bldg

HOUSTON 2, TEX.

610 Scanlan Bldg.

405 Main St.

JACKSONVILLE 1, FLA. 425 Federal Bldg.

311 West Monroe St.

KANSAS CITY 6, MO.

Room 2011

911 Walnut St.

LOS ANGELES 15, CALIF

Rm. 450, Western Pacific Bldg.

1031 S. Broadway

For local telephone listing, consult section devoted to U.S. Government. 
MEMPHIS 3, TENN.

212 Falls Bldg.

22 N. Front St.

MIAMI 32, FLA.

316 Post Office Bldg.

300 N.E. First Ave.

MINNEAPOLIS 1, MINN.

319 Metropolitan Bldg.

2nd Ave. South \& 3rd St.

NEW ORLEANS 12, LA.

1508 Masonic Temple Bldg. 333 St. Charles Ave.

NEW YORK 1, N.Y.

61st Fl. Empire State Bldg. 350 Fifth Ave.

PHILADELPHIA 7, PA.

Jefferson Bldg.

1015 Chestnut St.
PHOENIX, ARIZ.

419 Ellis Bldg.

137 N. 2nd Ave.

PITTSBURGH 22, PA.

817 Fulton Bldg.

107 Sixth Street

PORTLAND 4, OREG.

217 Old U.S. Courthouse 520 S.W. Morrison St.

RENO, NEV.

1479 Wells Ave.

RICHMOND 19, VA.

Rm. 309, Parcel Post Bldg.

11th and Main Streets

ST. LOUIS 1, MO.

910 New Federal Bldg.

1114 Market St.
SALT LAKE CITY 1, UTAH

Room 105

222 S. W. Temple St.

SAN FRANCISCO 11, CALIF. Rm. 419 Customhouse

555 Battery St.

SAVANNAH, GA.

235 U.S. Courthouse and Post Office Bldg.

125-29 Bull St.

SEATTLE 4, WASH.

809 Federal Office Bldg.

909 First Ave. 


\section{PERIODICALS OF THE NATIONAL BUREAU OF STANDARDS}

\subsection{JOURNAL OF RESEARCH}

The Journal of Research reports research and development in physics, mathematics, chemistry, and engineering. Comprehensive scientific papers give complete details of the work, including laboratory data, experimental procedures, and theoretical and mathematical analyses. The Journal also presents review articles by recognized authorities and compilations of information on subjects closely related to the Bureau's technical program. The Journal of Research is issued in four separate sections as follows:

\section{A. PHYSICS AND CHEMISTRY}

This section presents papers of interest primarily to scientists working in these fields. It covers a broad range of physical and chemical research, with major emphasis on standards of physical measurement, fundamental constants, and properties of matter. Issued six times a year.

\section{B. MATHEMATICS AND MATHEMATICAL PHYS- ICS}

This section presents studies and compilations designed mainly for the mathematician and theoretical physicist. Topics in mathematical statistics, theory of experiment design, numerical analysis, theoretical physics and chemistry, logical design and programing of computers and computer systems are covered, together with short numerical tables. Issued quarterly.

\section{ENGINEERING AND INSTRUMENTATION}

This section reports results of interest chiefly to the engineer and the applied scientist. It includes many of the new developments in instrumentation resulting from the Bureau's work in physical measurement, data processing, and development of test methods. It also covers some of the work in acoustics, applied mechanies, building research, and cryogenic engineering. Issued quarterly.

\section{RADIO PROPAGATION}

This section reports research in radio propagation, communications, and upper atmospheric physics. Topics covered include propagation in ionized media, scattering by turbulence, effect of irregular terrain on propagation, diffraction and scattering by solid obstacles, propagation through time-varying media, surface waves, and antennas. Issued six times a year.

\subsection{TECHNICAL NEWS BULLETIN}

This monthly publication summarizes the current research development, and test activities of the Bureau. The articles are brief, with emphasis on the results of research and their significance, chosen for their importance to other scientists, engineers, and to industry. Résumés of longer research reports, important national and international conferences on fundamental science in which the Bureau has represented the Nation, and a bibliography of all publications by members of the staff as published are included. The Bulletin is designed to give a succinct account of the current work of the Bureau.

\subsection{BASIC RADIO PROPAGATION PREDICTIONS}

This is a monthly publication for those concerned with radio communication in determining the best sky-wave frequencies over any path at any time of day for average conditions for the month of prediction, which are made three months in advance. Charts of extraordinary-wave critical frequency for the F2 layer and of maximum usable frequency for a transmission distance of $4,000 \mathrm{~km}$, of highest frequency of sporadic- $\mathrm{E}$ in excess of $15 \mathrm{MC}$ are included. In addition, there are various maps, charts, diagrams, and nomograms needed to make practical application of the world-contour charts, together with examples of their use.

\begin{tabular}{|c|c|c|}
\hline ANNUAL SUBSCRIPTION & Domestic 1 & Forefgn ${ }^{2}$ \\
\hline 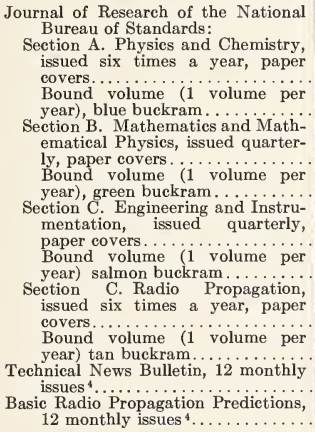 & $\begin{array}{l}\$ 4.00 \\
\left(^{3}\right) \\
2.25 \\
\left(^{3}\right) \\
2.25 \\
\left(^{3}\right)\end{array}$ & $\begin{array}{l}\$ 4.75 \\
\left(^{3}\right) \\
2.75 \\
\left(^{3}\right)\end{array}$ \\
\hline
\end{tabular}

Note. - Send order, with remittance, to Superintendent of Documents, U.S. Government Printing Office, Washington 25, D.C.

1 United States and its possessions, Canada, Cuba, Mexico, Newfoundland (including Labrador), and certain Central and South American countries.

2 Foreign price includes the cost of the publication and postage

a Prices of the bound volumes vary. The Superintendent of Documents will furnish prices on request.

4 These two monthly periodicals may be obtained on a 1-, 2-, 3-year subscription basis (although no reduction in rates can be made). 



\section{TITLES AND ABSTRAGTS OF NBS PUBLICATIONS}

The prices of the publications are shown in "Price list of available publications" on page 161 . The publication may be purchased from the Superintendent of Documents, U.S. Government Printing Office, Washington 25, D.C. (The only exception is the Technical Note Series, see page 165.) If the price is omitted, the publication is out of print, but may be consulted in libraries maintaining reference sets of Bureau publications. Abstracts of publications issued prior to July 1, 1957, are included in Circulars 24, 460, and 460 supplement.

The letter-symbol designations given below are used in this Circular for the publication series indicated, and these letters should be included with the serial number in all requests and references to Bureau publications:

J, Journal of Research

A. Physics and Chemistry

B. Mathematics and Mathematical Physics

C. Engineering and Instrumentation

D. Radio Propagation

C, Circulars.

\subsection{RESEARCH PAPERS}

Research Papers are reprints of individual articles that appeared in Volumes 1-62 of the monthly Journal of Research. They were made available in this form primarily to serve the needs of research workers and technical groups. In July 1959 the Bureau began publishing the Journal in four separate sections (page 34) and Research Papers were discontinued.

\section{RESEARCH PAPERS FROM JOURNAL OF RESEARCH OF THE} NATIONAL BUREAU OF STANDARDS, VOLUME 59, JULYDECEMB R 1957

RP2768. Thermal length changes of some refractory castables, S. J. Schneider and L. E. Mong

The changes in length exhibited by a variety of refractory castables during complete heating and cooling cycles were determined. The tests consisted of determinations on castable specimens that had been heat treated at either $110^{\circ}, 1,050^{\circ}$, or $1,300^{\circ} \mathrm{C}$. A detailed description of the apparatus is given, especially that of the dilatometer assemblies. These assemblies incorporated differential transformers to give a record of length changes as they occurred. The data suggested that the same general length-change characteristics were exhibited by castables that had received the same curing and heat treatment. Length-change curves of all three groups illustrated silica inversions. Curves of the $110^{\circ} \mathrm{C}$ group indicated contractions associated with cement reactions, namely, the loss of water of hydration and the conversion of amorphous alumina to $\gamma$ alumina. $8 \mathrm{p}$.

RP2769. Precise intercomparison of acids by differential potentiometric titration with hydrogen electrodes, R. G. Bates and E. Wichers

The purity of two preparations of benzoic acid, three of oxalic acid, and three of potassium hydrogen phthalate has been compared by a precise determination of the equivalence of each substance with the same solid reference base, sodium carbonate, the only requirements for which were that its composition be uniform and remain constant throughout the series of measurements. The inflection point in each titration was determined by a differential
Mono., Monographs.

H, Handbooks.

MP, Miscellaneous Publications.

BMS, Building Materials and Structures Reports.

AMS, Applied Mathematics Series.

TN, Technical Note Series (use PB number).

potentiometric method utilizing two hydrogen electrodes. The procedure, which gives a degree of precision greater than has previously been reported for acidimetric determinations, is described in detail. When about 0.06 equivalent of acid was used in each titration, the reproducibility attainable was better than 0.002 percent. The failure of the inflection point and the equivalence point to coincide exactly has been considered in evaluating the reliability of the procedure.

The purity of one of the samples of benzoic acid, as determined independently from accurate measurements of the freezing point, was 99.997 mole percent. Single crystals of benzoic acid grown from a melt of this material were selected as a reference acid and were regarded to have a purity of 100.000 percent. In terms of this reference, a preparation of potassium hydrogen phthalate consisting of optically perfect crystals was found to have a purity of 100.000 percent. The method is adaptable to a variety of acids and bases and, hence, provides an additional criterion of purity for these substances. $9 \mathrm{p}$.

RP2770. Mixed-path ground-wave propagation: 2. Larger distances, J. R. Wait and J. Householder

The theoretical results given in part 1 (NBS Research Paper 2687) for ground wave propagation over a mixed path on a flat earth are generalized to a spherical earth. The problem is formulated in terms of the mutual impedance between two vertical dipoles which are located on either side of the boundary of separation. Extensive numerical results are given in graphical form for a mixed land-sea path at frequencies of $10,20,50,100$, and 200 kilocycles per second. 8 p.

RP2771. A spectroscopic study of oils used in oil-extended rubber, F. J. Linnig and J. E. Stewart

Knowledge of the composition of oils used in oil-extended rubber is useful in specif ying oil types and in understanding the properties of the oil-rubber master batches. In this work, elucidation of structure has been attempted by a comparison of infrared spectra between 2 and 15 microns of two series of oil fractions obtained by basically different methods. Fractions of a number of different oils or oil distillates were studied. The spectra were compared with reference to the method of separation and in some cases to the physical and chemical properties of the fractions. There are marked similarities between certain of the 
fractions separated by the two methods. However, the study indicates a variability of structural types from one oil to another. This is especially true for the more polar fractions. It is suggested that this varability, especially with respect to polar groups and other structures leading to active hydrogens, could account for the observed variability in the aging properties of master batches containing different oils or oil fractions.

Spectra were also obtained of a few typical fractions in the ultraviolet range between 210 and 340 millimicrons and in the far infrared between 15 and 40 microns. $14 \mathrm{p}$.

RP2772. Infrared spectra of sugar acetates in solution, H. S. Isbell, F. A. Smith, E. C. Creitz, H. L. Frush, J. D. Moyer, and J. E. Stewart

Infrared absorption spectra are reported for a group of structurally and configurationally related carbohydrate derivatives. The conformation of the pyranose ring and other structural features of the compounds are discussed in relation to the spectra. Most of the absorption that is characteristic of the cyclic carbohydrate structure occurs in the region of 8.4 to $15 \mu$. Although the spectra are complex and difficult to interpret, they show features from which a number of provisional conclusions have been drawn.

It was found that fully acetylated monosaccharides with an axial glycosidic acetate group give a moderately strong absorption band at $8.63 \pm 0.04 \mu$, and at most a weak band at $8.87 \pm 0.06 \mu$; whereas compounds having an equatorial glycosidic acetate group absorb weakly at $8.63 \mu$, and give a moderately strong band at $8.87 \mu$. The spectra for the acetates having the gulo-, ido-, or taloconfigurations appear to indicate the presence of the $\mathrm{C}^{\prime} 2$, or less common chair conformation, or a mixture of conformations.

Acetylated methyl glycosides with the methoxyl group in the axial position give characteristic absorption at $8.33 \pm 0.02 \mu$ and at $8.80 \pm 0.05 \mu$; none of the compounds with the methoxyl group in the equatorial position shows absorption in these regions. Absorption at $8.80 \mu$ is enhanced further by the presence of axial acetyl groups on the ring. On the basis of this generalization, an acetylated methyl glycoside with the $\beta$-gulo structure gives evidence for the presence of the $\mathrm{C}^{\prime} 2$ conformation.

Overlapping bands in the region of 8.0 to $8.2 \mu$ arise from acetyl groups of diverse character, with the result that spectra in this region show characteristic features for some compounds. Thus the pyranose acetates of the pentoses and of rhamnose give a sharp, well-defined band at $8.0 \mu$, but the acetates of the hexoses and higher sugars give a poorly defined band that seems to include an overlapping component with a maximum at higher wavelength. This characteristic may serve to distinguish acetates that do, or do not, have a primary acetyl group $38 \mathrm{p}$.

\section{RP2773. A problem in self-heating of a spherical body, S. M. Genensky}

An analytic steady-state solution is developed for a spherical body in which heat is generated according to a first-order unimolecular-reaction law, and lost at the surface in accordance with Newton's law of cooling. The temperature within the sphere depends chiefly on the radial distance from the center, but also on the ambient temperature, the surface heat-loss coefficient, and the material properties, which are assumed constant. $3 \mathrm{p}$.

RP2774. Properties of arsenic sulfide glass, F. W. Glaze, D. H. Blackburn, J. S. Osmalov, D. Hubbard, and M. H. Black

Samples of arsenic sulfide glass were investigated to determine their chemical and physical properties. Among the properties reported are the hygroseopicity, chemical durability, hydrogen electrode function, expansivity, elastic moduli, modulus of rupture, hardness, transmittance, and indices of refraction.
The main interest in arsenic sulfide glass, for the present, is for its transmittance in the infrared. It is transparent to infrared energy out to approximately 13 microns. Although its physical properties are inferior to those of ordinary silicate glasses, they are superior to those of some of the available materials used in infrared optics. $10 \mathrm{p}$.

RP2775. Thermal conductivity of beryllium oxide from $40^{\circ}$ to $750^{\circ}$ C, D. A. Ditmars and D. C. Ginnings

The thermal conductivity of beryllium oxide has been measured by an absolute method from $40^{\circ}$ to $750^{\circ} \mathrm{C}$. The apparatus employed steady-state longitudinal heat flow along a rod of high-fired beryllium oxide, surrounded by a tube with matching temperature gradient to minimize radial heat loss. The estimated accuracy of the measurements is about 3 percent. However, the values of thermal conductivity of the ideal beryllium oxide crystal are probably considerably higher than the values given because of the lower density ( 87 percent theoretical) of the sample used. $7 \mathrm{p}$.

RP2776. A self-balancing direct-current bridge for accurate bolometric power measurements, G. F. Engen

Until recently, the most accurate microwave power measurements of the bolometric type have required the use of a manual d-c bridge. A self-balancing d-c bridge has been developed that preserves the inherent accuracy of the manual bridge, extends the dynamic range of operation, and greatly simplifies the operating procedure. A general description of the equipment and operating techniques is given, followed by a comprehensive survey of the sources of error accompanying the method and the accuracy achieved. $5 \mathrm{p}$.

RP2777. Some observations on hydrated monocalcium aluminate and monostrontium aluminate, E. T. Carlson

Monocalcium aluminate hydrate, probably having the composition $\mathrm{CaO} \cdot \mathrm{Al}_{2} \mathrm{O}_{3} \cdot 10 \mathrm{H}_{2} \mathrm{O}$, was prepared by precipitation from calcium aluminate solutions at $1^{\circ} \mathrm{C}$, and by hydration of pastes of monocalcium aluminate or aluminous cement. It was obtained as hexagonal prisms, very weakly birefringent, with mean index 1.471. Three molecules of water were expelled by drying over $\mathrm{CaCl}_{2}$, without apparent change in crystal structure; however, the structure was destroyed by heating to $175^{\circ} \mathrm{C}$. Monostrontium aluminate hydrate was obtained by analogous methods in the form of minute needles or prisms with mean index 1.478. The maximum degree of hydration was not successfully determined, as the compound underwent decomposition to $3 \mathrm{SrO} . \mathrm{Al}_{2} \mathrm{O}_{3} \cdot 6 \mathrm{H}_{2} \mathrm{O}$ and gibbsite. $\mathrm{X}$-ray diffraction powder patterns indicate a close similarity in structure between the monocalcium and monostrontium aluminate hydrates. $5 \mathrm{p}$.

RP2778. Turbidity and viscosity measurements on some cationic detergents in water and in sodium chloride solutions, L. M. Kushner, W. D. Hubbard, and R. A. Parker

Light-scattering and viscosity measurements were made on solutions of three cationic detergents in distilled water and in various solutions of sodium chloride. The detergents were dodecylamine hydrochloride, dodecyltrimethylammonium chloride, and tetradecyltrimethylammonium chloride. The first of these was investigated at $30^{\circ} \mathrm{C}$ and the others at $23^{\circ} \mathrm{C}$. The micellar weight and the intrinsic viscosity of the micelles of each detergent were calculated, and the dependence of these quantities on the concentration of sodium chloride is discussed. $7 \mathrm{p}$. 
RP2779. Relative stress-optical coefficients of some National Bureau of Standards optical glasses, R. M. Waxler and A. Napolitano

The relative stress-optical coefficients of 27 optical glasses made at the National Bureau of Standards have been determined. The glasses were loaded in compression, and the amount of birefringence was measured with a polarimeter, using a quarter-wave plate compensator. The stress-optical coefficients determined for the crown glasses ranged between +2.95 and +1.82 brewsters. For the flint glasses, a regular variation of the stressoptical coefficient with weight percent of lead oxide was found with values ranging from +3.18 to -1.16 brewsters. $5 \mathrm{p}$.

RP2780. Physicochemical studies of the destructive alkali-aggregate reaction in concrete, $R$. G. Pike and D. Hubbard

In a further study of the alkali-aggregate reaction in high-alkali cements, 11 reactive and nonreactive experimental aggregates were examined by several procedures. On comparing the results of each method with the standard expansion-bar test, it appears that each property studied has some bearing on the expansion characteristics of each aggregate. For example (1) The chemical durability as determined by the interferometer procedure was poor for the aggregates that showed excessive expansion, but not all aggregates that are shown to be chemically reactive by this procedure will cause expansion, (2) all aggregates are sufficiently hygroscopic to attract the water necessary for the reaction if other properties are also favorable, (3) surface electrical-resistance measurements confirm the hygroscopicity determinations; however, free ions in glasses may have considerably more effect on the surface resistance of certain glasses than do their hygroscopic properties, and (4) the uneven distribution of migratable ions between the aggregate surface and the outer phase offers a mechanism for the osmotic swelling of individual aggregate grains, with accompanying expansion in concrete members. $6 \mathrm{p}$.

RP2781. Insulated loop antenna immersed in a conducting medium, J. R. Wait

A solution is given for the fields of a circular loop in a conducting medium. The loop is assumed to have a uniform current, and it is enclosed by a spherical insulating cavity. The impedance of the loop is also considered. It is shown that the power radiated from the loop varies approximately as the reciprocal of the radius of the cavity for a specified loop current. Furthermore, if the cavity is electrically small, relative to the external medium, the radiation field is not significantly affected by the presence of the cavity. $5 \mathrm{p}$.

RP2782. Noncrystal ionic model for silica glass, L. W. Tilton

A model was constructed of pentagonal rings of symmetrical tetrahedra so connected that all five silicons lie in one plane and all angles $\mathrm{Si}-\mathrm{O}-\mathrm{Si}$ approximate $180^{\circ}$. From an initial tetrahedron the rings extend in six directions to include its six edges, and in the resulting threedimensionable network each ring forms a common interface between dodecahedral cages which necessarily have fivefold symmetry. Such symmetry is incompatible with formation of a crystal lattice and thus the structure can be extended in three dimensions only to a very limited degree and with unavoidable stress and distortional strain. A stressed network cluster of such dodecahedral cages is here called a viTroN.

If the scale in this model for silica glass is taken as $1.6 \mathrm{~A}$ from silicon to oxygen, the model is found to be in accord with other radial distances computed from diffraction data and the density is correct within 10 percent. The apertures of the pentagonal interfaces are of suitable size to explain data on the diffusion of noble gases through silica.
Shared penta faces and intermittent oxygen bridges connect neighboring vitrons and constitute an interstitial tissue of relatively weakened structure that accounts for the observed low tensile strengths of silica glass and provide channels for viseous flow.

The proposed cages would be less pliable at low temperatures and so the interstitial tissues between vitrons should expand in volume on cooling. This "negative" expansion would counter the normal expansion and account for the known extremely small (net) expansivity of silica glass. The accompanying changes in strength and volume of the glass at its weakest places, the interstitial tissues, can account for a number of known "anomalies" in thermal behavior of silica glass with respect to its volume, compressibility, elastic moduli, and viscous flow.

The vitron concept reconciles the crystallite and the network theories of glass by proposing nuclei that cannot grow extensively and a distribution of localized stresses that may constitute an acceptable degree of randomness and provide effective modulations in continuity. This concept of definite but limited mieroregularity in structure suggests that other noncrystal symmetries should be studied for possible interest in other fields such as liquids and high polymers. $16 \mathrm{p}$.

RP2783. A computation of cyclic cubic units, H. Cohn and S. Gorn

The paper is a report on a tabulation of units performed on the EDVAC at the U.S. Army Proving Grounds in Aberdeen, Maryland. The algebraic number fields involved were 45 cyclic cubic fields of discriminant $l^{2}$ where $l$ is one of the primes of form $3 m+1$ from 7 through 499. The object of the search was the discovery of units through a specific method, an algorithm based on Minkowski's geometric number theory but particularly amenable to a stored-program computer. In the computation, combined use was made of integral arithmetic and decimal arithmetic but with careful error analysis. $14 \mathrm{p}$.

RP2784. Estimation of the frequencies of thin elastic plates with free edges, T. Kato, H. Fujita, Y. Nakata, and M. Newman

A variational method is proposed for calculating the frequencies of thin elastic plates with free edges, with rigorous error estimates. As a numerical example, the fundamental frequency of a square plate with the Poisson ratio 0.225 is calculated with a satisfactory result, the possible relative error being less than $1 / 2000$. Generalization to more complicated boundary conditions is straightforward. $18 \mathrm{p}$.

RP2785. A thermochemical study of the reaction of calcium hydroxide, silica gel, and water, E. S. Newman

The heat of reaction of calcium hydroxide, silica gel, and water was measured by a heat-of-solution method. The reaction was carried out at $25^{\circ} \mathrm{C}$ in pastes having a water solid ratio of about 1.0 . The heat of reaction, $-\Delta \mathrm{H}$ at $25^{\circ} \mathrm{C}$, for the reaction

$$
\mathrm{Ca}(\mathrm{OH})_{2}+\mathrm{SiO}_{2} \cdot n \mathrm{H}_{2} \mathrm{O}=\mathrm{CaO} \cdot \mathrm{SiO}_{2} \cdot n \mathrm{H}_{2} \mathrm{O}
$$

was found to be about 10.3 kilocalories. The heat of the reaction

$$
\begin{aligned}
& \mathrm{CaO} \cdot \mathrm{SiO}_{2} \cdot n \mathrm{H}_{2} \mathrm{O}+m \mathrm{Ca}(\mathrm{OH})_{2} \\
& =(1+m) \mathrm{CaO} \cdot \mathrm{SiO}_{2} \cdot(n+m) \mathrm{H}_{2} \mathrm{O}
\end{aligned}
$$

is about 1.6 kilocalories per mole of added $\mathrm{Ca}(\mathrm{OH})_{2}$. About half of the total measured heat of reaction represents the heat of wetting of the reaction products. Differential thermal analyses and drying experiments indicated that $3 \mathrm{CaO} \cdot 2 \mathrm{SiO}_{2} \cdot 2 \mathrm{H}_{2} \mathrm{O}$ was formed in some of the pastes. $10 \mathrm{p}$. 
RP2786. On some expansions for Bessel integral functions, F. Oberhettinger

Investigations by van der Pol and Humbert concerning the Bessel integral function of order zero are extended to Bessel functions of other kinds and to functions related to Bessel functions. $5 \mathrm{p}$.

RP2787. Table of the first moment of ranked extremes, J. Lieblein and H. E. Salzer

Let a sample of $n$ independent random values from the extreme-value distribution, with c.d.f. $\Phi(y)=\exp \left(-e^{-y}\right)$, be arranged in decreasing order and denoted by $y_{1}, y_{2}$, .., $y_{m}, \ldots, y_{n}$. The table gives the expected values for all these order statistics for sample size not exceeding 25 . For the larger samples, up to $n=100$, the expected values are given only for the first 26 largest values. $4 \mathrm{p}$.

RP2788. An approximate expression for gamma-ray degradation spectra, U. Fano and A. T. Nelms

The equation for gamma-ray degradation spectra is reduced to a form suitable for a simple approximation. The approximation is valid in a wavelength range between two Compton wavelengths larger than the wavelength of the source and the region where photoelectric absorption becomes very intense; this range is wide for low $Z$ materials. Input data are provided for application to various materials and source energies. The results of sample calculations are shown and compared with those obtained by standard numerical calculation. $4 \mathrm{p}$.

RP2789. Some infrared bands of deuterium sulfide, H. C. Allen, Jr., E. K. Plyler, and L. R. Blaine

Two regions of the $D^{2} \mathrm{~S}$ spectrum have been observed, 5 and $3.6 \mu$. An analysis of the absorption in the $3.6 \mu$ region attributes it to two overlapping bands with excited states $\left(v^{1}, v^{2}, v^{3}\right)=(1,1,0)$ and $(0,1,1)$. The band center of the $(0,1,1)$ band is found to be some $50 \mathrm{~cm}^{-1}$ lower than the previously accepted value while the $(1,1,0)$ band center is found some $60 \mathrm{~cm}^{-1}$ higher than the previous determination. The absorption near $5 \mu$ seems to arise from the $(0,0,1)$ band centered near $1906 \mathrm{~cm}^{-1}$. Overlapping atmospheric absorption makes a detailed analysis impossible at this time. This band center is almost $100 \mathrm{~cm}^{-1}$ lower than previously reported. The excited state constants for the two bands near $3.6 \mu$ are

$$
\begin{array}{cccc}
(1,1,0) \nu_{0}=2742.77 & A^{*}=5.478 & B^{*}=4.512 & C^{*}=2.395 \\
(0,1,1) \nu_{0}=2754.44 & A^{*}=5.531 & B^{*}=4.521 & C^{*}=2.405 . \\
& 4 \mathrm{p} . & &
\end{array}
$$

RP2790. Some effects of low temperatures and notch depth on the mechanical behavior of an annealed commercially pure titanium, G. W. Geil and N. L. Carwile

Unnotched and notched specimens $\left(60^{\circ}\right.$ notch angle, 0.05 -inch root radius and various notch depths) of an annealed commercially pure titanium were slowly strained to fracture in tension at $+150^{\circ}$ or $+100^{\circ}$ to $-196^{\circ} \mathrm{C}$, to reveal the combined effects of temperature and noteh geometry on the tensile behavior of the metal. Impact tests were made on Charpy V-notch specimens at $+300^{\circ}$ to $-196^{\circ} \mathrm{C}$ for a determination of the impact notchtoughness of the titanium. True stress-true strain relations were determined for the titanium in tension and a study was made of the effects of test temperature, stress system, and interstitial content on the mechanism of deformation and work-hardening characteristics of the metal. $12 \mathrm{p}$.

RP2791. Determination and use of the sag point as a reference point in the heating of glasses, $\mathrm{S}$. Spinner, G. W. Cleek, and E. H. Hamilton

Glasses, when heated, undergo a gradual continuous change from solids to liquids over a rather wide temper- ature range. As a consequence, it has been found necessary to define certain temperature reference points in this transition range so that different glasses may be compared with each other. These points are chosen to correspond to established stages in the annealing or forming process. A new reference point, designated as the sag point, is described. The experimental determination of this point is rapid and simple. Also, it gives useful information concerning the annealing temperatures and ease of formation of glasses of widely varying compositions.

The sag point is defined as the temperature at which a glass fiber 0.5 to 0.8 millimeter in diameter, horizontally supported at 1/2-inch intervals, will sag under its own weight in $25 \pm 5$ minutes. $5 \mathrm{p}$.

RP2792. Formulas for inverse osculatory interpolation in the complex plane, H. E. Salzer

Improved formulas for inverse osculatory interpolation in the complex plane are obtained by inversion of Hermite's formula and the use of appropriate grid point configurations. They cover the cases for $n=2(1) 7$, where $n$ is the number of points required in direct osculatory interpolation. The formulas provide an improved means for inverse interpolation in the complex plane where the first derivative is either tabulated alongside the function or is easily obtained. $6 \mathrm{p}$.

RP2793. An x-ray study of textural stresses in two-phase alloys, C. J. Newton and H. C. Vacher

Internal or residual stresses of the textural stress class are discussed, with special attention to that type resulting from a difference in thermal expansion of two phases present in an alloy. X-ray stress measurements were made on various brasses and steels, and the results were found to be in reasonable agreement with the quantitative theory of F. Lászlo. $5 \mathrm{p}$.

RP2794. Dissociation of 4-chloro-4'-aminodiphenylsulfone, E. E. Sager and F. C. Byers

The ultraviolet absorption spectra of 4-chloro-4'-aminodiphenylsulfone has been determined. The dissociation may be considered as the acidic dissociation of the ammonium ion which is formed with the addition to the base of hydrogen ion from an acid such as hydrochloric acid. Spectrophotometric measurements of several concentrations of 4-chloro- $4^{\prime}$-a minodiphenylsulfone at various known hydrogen-ion concentrations were made at $25^{\circ} \mathrm{C}$, from which the values $p K_{e}$, based upon the concentrations, were determined. From these, the value of $p K_{a}$ was derived. At $25^{\circ} \mathrm{C}, p K_{a}$ is 1.38 , which corresponds to a value of 0.042 mole per liter for $K_{a}$. The activity coefficient terms may be expressed simply as a linear function of the ionic strength. Spectrophotometric measurements were also made at $15^{\circ}, 20^{\circ}, 25^{\circ}, 30^{\circ}$, and $35^{\circ} \mathrm{C}$ on certain solutions in order to calculate the heat of dissociation. It was found to be 21,000 joules $\mathrm{deg}^{-1} \mathrm{~mole}^{-1}$. The basic dissociation constant, $K_{b}$, is $2.4 \times 10^{-13}$, or $p K_{b}$ is 12.62 . $4 \mathrm{p}$.

RP2795. Speed of sound in water by a direct method, M. Greenspan and C. E. Tschiegg

The speed of sound in distilled water was measured over the temperature range $0^{\circ}$ to $100^{\circ} \mathrm{C}$ with an accuracy of 1 part in 30,000 . The results are given as a fifth-degree polynomial and in tables. The water was contained in a cylindrical tank of fixed length, terminated at each end by a plane transducer, and the end-to-end time of flight of a pulse of sound was determined from a measurement of the pulse-repetition frequency required to set the successive echoes into time coincidence. $6 \mathrm{p}$.

RP2796. Pattern of a flush-mounted microwave antenna, J. R. Wait

The numerical results for the far zone radiation from an axial slot on a circular cylinder of perfect conductivity and infinite length are discussed. It is shown that the results 
or large diameter cylinders ean be expressed in a universal orm that is suitable for pattern calculations for arrays of ilots on a gently curved surface. The work is compared vith a related diffraction problem considered by Fock. ip.

RP2797. Standards for $p \mathrm{H}$ measurements from $60^{\circ}$ to $95^{\circ} \mathrm{C}, \mathrm{V}$. E. Bower and R. G. Bates

The NBS standard $p \mathrm{H}$ seale is defined in terms of several eference solutions whose $p \mathrm{H}$ values $\left(p \mathrm{H}_{0}\right)$ have heretofore seen assigned only in the range $0^{\circ}$ to $60^{\circ} \mathrm{C}$. Recent neasurements of the standard potential of the silverilver-chloride electrode at temperatures above $60^{\circ}$ now jermit this scale to be extended to $95^{\circ} \mathrm{C}$.

Electromotive-force measurements of cells with hydrozen and silver-silver-chloride electrodes and containing ouffer-chloride solutions are reported in this paper for the range $60^{\circ}$ to $95^{\circ} \mathrm{C}$. The assignment of $p \mathrm{H}$, values to the following five reference solutions is described: $0.05-m$ potassium tetroxalate; potassium hydrogen tartrate (saturated at $25^{\circ} \mathrm{C}$ ) ; $0.05-m$ potassium hydrogen phthalate; $0.025-m$ potassium dihydrogen phosphate; $0.025-m$ disodium hydrogen phosphate; and $0.01-m$ borax. 4 p.

RP2798. A numerical study of Dedekind's cubic class number formula, H. Cohn

In this paper an analytic formula of Dedekind is used to compute class numbers for a sample of pure cubic fields, employing the National Bureau of Standards electronic computer, the SEAC. The computation is one of major magnitude, and it illustrates the usefulness of combining integral and decimal arithmetic. The class numbers obtained are of much greater magnitude than those of pure cubic fields previously studied by means of hand computer methods. $7 \mathrm{p}$.

RP2799. Impact properties of slack-quenched alloy steels, M. R. Meyerson and S. J. Rosenberg

A method was developed for slack quenching impact specimens so as to produce uniform and accurately controlled hardnesses and microstructures at any location in the cross section. Steels of the $81 x x$ type, without and with boron, and varying in hardenability, were studied to evaluate the effect of slack quenching upon the impact properties.

Slack quenching was shown to be detrimental to the impact properties of the steels. The injurious effects were found to be dependent on the carbon and alloy content of the steels, the degree of slack quenching, and the amount of tempering.

When any two steels were slack quenched to the same hardness, the higher carbon lower alloy steel had impact properties inferior to those of the lower carbon higher alloy steel. Tempering usually improved the properties of both and reduced the differences between them.

The higher the hardness to which any steel was slack quenched, the lower were its impact properties. When tempered to the same hardness, however, the steel originally slack quenched to the higher hardness had the better impact properties. $16 \mathrm{p}$.

RP2800. Synthesis of $\mathrm{C}^{14}$-labeled L-sorbose and L-ascorbic acid, H. L. Frush and H. S. Isbell

A method is presented for preparing $\mathrm{C}^{14}$-labeled L-ascorbic acid (vitamin C) from labeled barium D-gluconate or D-glucose. The process is adapted from the commercial synthesis of vitamin $\mathrm{C}$ through sorbitol, L-sorbose and potassium di-O-isopropylidene-2-keto-L-gulonate. In the cyanohydrin synthesis of labeled D-glucose, barium D-gluconate is used as an intermediate. For preparing labeled L-ascorbic acid, the gluconate is converted to the lactone. This is efficiently reduced, by means of sodium borohydride, directly to sorbitol, obviating the intermediate preparation of D-glucose.

If labeled D-glucose is more readily available than the corresponding gluconic acid (as for instance D-glucose-6-C or randomly labeled D-glucose), the sugar is reduced to sorbitol by the sodium borohydride method; the yield is nearly quantitative.

In a preparation of L-ascorbic acid-6-C14 from barium D-gluconate-1-C ${ }^{14}$ the yield was 41.5 percent, based on the gluconate, or 67.8 percent, based on the intermediate L-sorbose. A mixture of Methyl Cellosolve, ethylene dichloride, and hydrogen chloride was found to be effective in converting potassium di- $O$-isopropylidene-2-keto-I-gulonate to L-ascorbic acid. $4 \mathrm{p}$.

RP2801. A determination of the gamma-ray emission of radium, F. H. Attix and V. H. Ritz

Measurements of the $\gamma$-ray emission of radium (roentgens per milligram-hour at 1 centimeter) have been made by means of a graphite cavity ionization chamber. An auxiliary experimental examination of the stopping-power correction for the non-air-equivalence of the cavity wall is also described. The resulting average value for the $\gamma-\mathrm{r}$ ay emission of radium (enclosed in an 0.5 -millimeter platinum capsule) is $8.26 \pm 0.05$ roentgens per milligram-hour at 1 centimeter, which is based upon the conclusion that the stopping-power data of Bakker and Segre are consistent with the results of the auxiliary experiment. $13 \mathrm{p}$.

RP2802. Radiation from slots on dielectric-clad and corrugated cylinders, J. R. Wait and A. M. Conda

An approximate formula is derived for the radiation pattern of an axially slotted cylinder with a thin dielectric coating. The accuracy of the formula is shown to be sufficient for practical purposes. Using a similar method, the pattern function for a slot on a corrugated cylinder is derived. Extensive numerical results are presented for both dielectric-clad and corrugated cylinders. $10 \mathrm{p}$.

RP2803. Infrared emission spectrum of methane at 3.3 microns, E. K. Plyler and L. R. Blaine

The emission spectrum of methane from the flame of a Bunsen burner has been observed in the region from 2,820 to $3,180 \mathrm{~cm}^{-1}$. Many lines have been measured in the $P_{-}, Q_{-}$, and $R$-branches. The $P$-branch was observed to $P-28$ and the $R$-branch to $R-17$.

The spectrum was observed from the inner cone of the flame and with a rich fuel mixture the $P-12$ line was most intense. This intensity distribution indicated a temperature of about $1,200^{\circ} \mathrm{K}$ for the heated gas. $2 \mathrm{p}$.

RP2804. Term analysis of the first spectrum of rhenium (Re I), P. F. A. Klinkenberg, W. F. Meggers, R. Velasco, and M. A. Catalán

With the aid of new data on wavelengths, intensities, and the Zeeman effect, the structural analysis of the first spectrum of rhenium (Re I) has been extended to include 2,764 lines that are explained as transitions among 282 atomic energy levels. This analysis accounts for 90 percent of the total observed intensity, although only 64 percent of the total number of lines has been classified. From the Zeeman effect, magnetic splitting factors have been derived for 75 percent of the levels, and nearly 40 percent of these have been grouped into designated spectral terms ascribed to specific configurations of electrons. The normal state of neutral Re atoms is $5 d^{5} 6 s^{26} \mathrm{~S}_{21 / 3}$ and the ionization limit is approximately 63530 kaysers or 7.87 electron volts. $30 \mathrm{p}$.

RP2805. An apparatus for measurement of thermal conductivity of solids at low temperatures, R. L. Powell, W. M. Rogers, and D. O. Coffin

A description is given of an apparatus used for determining the thermal conductivities of solids in the temperature range $4^{\circ} \mathrm{K}$ to $300^{\circ} \mathrm{K}$. The apparatus is especially suited to the determination of thermal conductivity over a large temperature interval, enabling coverage of the 
temperatures between the normal boiling points of liquefied gases. Illustrative results are given for an insulator, polytetrafluoroethylene, and for a high-conductivity commercial coalesced copper. The thermal conductivity of the insulator increases monotonically from 0.56 milliwatt per centimeter per degree $\mathrm{K}$ at $5^{\circ} \mathrm{K}$, to 2.32 at $80^{\circ} \mathrm{K}$. The thermal conductivity of the copper has a maximum of 24.9 watts per centimeter per degree $\mathrm{K}$ at $21^{\circ} \mathrm{K}$, and a value of 8.15 at $5^{\circ} \mathrm{K}$. The methods of data analysis and estimation of errors are given. $7 \mathrm{p}$.

$\mathrm{RP} 2806$. Some factors affecting the surface area of hydrated portland cement as determined by water-vapor and nitrogen adsorption, L. A. Tomes, C. M. Hunt, and R. L. Blaine

Brunauer-Emmett-Teller (BET) surface areas of hydrated portland cement have been calculated from watervapor and nitrogen-adsorption data. The adsorption and desorption of water vapor caused measurable decreases in specific surface areas by both water-vapor and nitrogen adsorption. Changes were also produced by wetting and drying. The adsorption and desorption of nitrogen at the boiling point of liquid nitrogen did not produce similar effects.

The specific surface and nonevaporable-water content of a hydrated cement are also somewhat dependent on the initial drying treatment. The effect of measuring watervapor surface and nonevaporable water with specimens dried for different periods of time is also considered. $8 \mathrm{p}$

RP2807. Excitation of surface waves on conducting, stratified, dielectric-clad, and corrugated surfaces, J. R. Wait

An expression for the field of an electric dipole located over a flat surface with a specified surface impedance $Z$ is derived from the formal integral solution by a modified saddle-pcint method. Using the value of $Z$ appropriate for a homogeneous conducting ground, the general expressions reduce to those given by Norton. In this case the phase of $Z$ lies between 0 and $45^{\circ}$. When the phase exceeds $45^{\circ}$, as it may for a stratified ground, the radiated wave of the dipole becomes partially trapped to the interface. This effect is most pronounced for an inductive surface where the phase of $Z$ is $90^{\circ}$, in which case the energy of the wave is confined within a small distance from the surface. Such inductive surfaces are a metallic plane with a thin dielectric film or a corrugated surface.

This unifying treatment provides a link between the surface waves of Zenneck, Sommerfeld, Norton, and Goubau, and indicates that the phase angle of $Z$ controls the extent to which these waves may exist for a dipole excitation. $13 \mathrm{p}$.

\section{RP2808. Cunife wire magnets of small size, I. L. Cooter} and R. E. Mundy

A ductile permanent-magnet alloy, having a composition of 60 percent of copper, 20 percent of nickel, and 20 percent of iron, was cold-drawn from wire, 25 mils in diameter to 5 mils in diameter. Demagnetization curves were obtained for several sizes of the wire in the cold-drawn state. These wires were then given a heat treatment and again demagnetization curves were obtained. Values of coercivity, retentivity, and maximum energy produet are compared for various wire diameters. Although the magnetic properties were adversely affected by the cold-working, they were substantially improved by the heat treatment. $4 \mathrm{p}$.

RP2809. Interpretation of mass spectra of condensates from urban atmospheres, E. R. Weaver, E. E. Hughes, S. M. Gunther, S. Schuhmann, N. T. Redfearn, and R. Gorden, Jr.

A method of interpreting the mass spectra of the very complex mixtures of substances that condense from an urban atmosphere at the temperature of liquid oxygen is described. The number of individual chemical compounds in such a mixture is greatly in excess of the number of masses recorded, and it is accordingly impossible to identify all the compounds present. However, the significant sources of pollutants are much less numerous, and if the produets from each source are of substantially constant composition it is possible to obtain a representative pattern of each and to proceed thereafter to make a fairly good estimate of the character and amount of pollutants in terms of their sources. The greatest simplification is made by treating the gases associated with motor traffic as a unit. $22 \mathrm{p}$.

RP2810. Infrared emission spectrum of silicon carbide heating elements, J. E. Stewart and J. C. Richmond

A method for determining the spectral emittance of a silicon carbide heating element (Globar) was devised and data were obtained over the wavelength range 1.25 to $15.25 \mu$ for four different temperatures. The spectral emittance at all four temperatures, $482^{\circ}, 649^{\circ}, 816^{\circ}$, and $982^{\circ} \mathrm{C}\left(900^{\circ}, 1,200^{\circ}, 1,500^{\circ}\right.$, and $\left.1,800^{\circ} \mathrm{F}\right)$, was found to be approximately constant except for a gradual rise in emittance from 1.25 to about $4 \mu$ and two minimums in the curves at longer wavelengths. The first of these minimums, which occurred at about $9 \mu$, was ascribed to minor amounts of $\mathrm{SiO}_{2}$ present on the surface of the silicon carbide heating element, whereas the second at about $12.5 \mu$ corresponds to one of the strong Raman lines for SiC. 5 p.

RP2811. Increased chemical reactivitv of the surface compared with that in the bulk volume of BrittonRobinson universal buffers, R. G. Pike and D. Hubbard

A direct comparison of the chemical reactivity at the surface with that in the bulk volume of buffer solutions over the range $p \mathrm{H} 7$ to $p \mathbf{H} 11.8$ was made, using Corning 015 glass as the indicator. By observing interferometrically the surface alterations of optically flat specimens brought about by exposures to aqueous buffer solutions under controlled conditions of time, temperature, and $p \mathrm{H}$ it was possible to evaluate, at least semiquantitatively, the difference between the chemical reactivity of the surface and the bulk volume of the solutions. For the buffer at $p \mathrm{H} 7$ the surface reactivity appeared to correspond to a hydrogen ion activity of $p \mathrm{H} 9.4$. The apparent concentration differential between the surface and bulk volume falls off sharply for buffers of increased $p \mathbf{H}$, amounting to

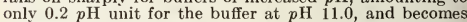
nondetectable at $p \mathrm{H}$ 11.8. Unfortunately the chemical durability characteristics of the glass were such that no differentiation could be made between the hydrogen ion activity of the surface and the bulk volume in solutions more acid than $p H$ 7. $4 \mathrm{p}$.

RP2812. Photometric determination of tungsten in steel and titanium alloys with dithiol, L. A. Machlan and J. L. Hague

A method is described for the direct photometric determination of tungsten in steels. The sample, 0.1 to $0.2 \mathrm{~g}$ in weight, is dissolved in aqua regia, and a sulfuricphosphoric-perchloric acid mixture is added. The solution is evaporated to fumes of sulfuric acid and diluted to volume with diluted sulfuric acid $(1+3)$. An aliquot portion of the solution is treated with dithiol in diluted sulfuric acid solution $(1+3)$ containing sulfur di xide, and molybdenum and certain other potential interferences are removed by extraction with chloroform. Copper and other elements forming insoluble dithiolates are removed by filtration. The tungsten-dithiol complex is then formed, after the addition of hydrochloric acid, stannous chloride, and dithiol, and extracted with butyl acetate. The absorbancy of the tungsten complex is determined at approximately $635 \mathrm{~m} \mu$. An accuracy of 0.005 percent of tungsten, or better, is indicated in the range 0.05 to 0.50 
percent of tungsten, and of about 0.001 percent for amounts of less than 0.05 percent of tungsten. The application of the method to titanium alloys is described in an appendix section. $6 \mathrm{p}$.

RP2813. On the most general form of the compatibility equations and the conditions of integrability of strain rate and strain, E. H. Brown

The most general forms of the equations of compatibility are derived by a very simple analysis in terms of the material strain rate tensor and the rate of change of the Riemann-Christoffel curvature tensor during the defcrmations. It is shown that recent statements on the significance of the compatibility equations, essentially that their satisfaction is equivalent to the condition that the space be locally Euclidean, are misinterpretations based on restricted forms of the equations. In addition, a new form of the conditions of integrability of strain is derived in terms of the rate of change of the curvature tensor. $6 \mathrm{p}$.

RP2814. An apparatus for measuring the piezoresistivity of semiconductors, R. F. Potter and W. J. McKean

A detailed description is given of an apparatus and procedure designed to measure the piezoresistive effect in semiconductors over an extended temperature range. A tensile force up to 1 kilogram can be applied to the sample by means of a calibrated beam balance. The apparatus liss been used for measurements on indium antimonide over the range $78^{\circ} \mathrm{K}$ to $300^{\circ} \mathrm{K}$, and tensile stresses of the order of $5 \times 10^{7}$ dynes per square centimeter can be applied to samples that are cut in a special manner. $4 \mathrm{p}$.

TITLE PAGE AND CONTENTS TO VOLUME $59.4 \mathrm{p}$.

RESEARCH PAPERS FROM JOURNAL OF RESEARCH OF THE NATIONAL BUREAU OF STANDARDS, VOLUME 60 , JANU. ARY-JUNE 1958

RP2815. The condition of certain matrices, III, J. Todd

The condition-numbers of certain matrices associated with various discretizations of the two-dimensional Laplacian operator are estimated. The condition-number gives an estimate of the error obtained by solving the corresponding systems of simultaneous linear equations. 7 p.

RP2816. Infrared study of some structural changes in natural rubber during vulcanization, F. J. Linnig and J. E. Stewart

A knowledge of the structure of vulcanized rubber is essential to the interpretation of vulcanization and oxidation studies and the physical properties of the material.

In the present work an infrared study has been made of structures resulting from a number of different methods of vulcanization. Sulfur vulcanizates show the presence of a shifted double bond, originally observed by Sheppard and Sutherland. The presence of conjugated double bonds is also indicated. Accelerators such as tetramethylthiuram disulfide and zinc dibutyl dithiocarbamate increase the rate of the double-bond shift and reduce the amount of conjugated double bonds. Neither the doublebond shift nor conjugation is observed as a result of vulcanization with tetramethylthiuram disulfide alone, hydrogen sulfide and sulfur dioxide (Peachey process), a peroxide, or gamma rays. These result in a possible decrease in carbonyl structures, and in the case of the last three, possible increased absorption due to $\mathrm{OH}$ and ionized carboxyl groups.

Apparently, the double-bond shift and conjugation are primarily phenomena related to the use of elemental sulfur. The other vulcanization systems studied evidently involve different mechanisms. An implication of the present work is that there may be a relationship between the reported ease of oxidation of sulfur vulcanizates, accelerated vulcanizates, and sulfurless vulcanizates (tetra- methylthiuram disulfide alone), which decreases in the order named, and the probable amount of conjugation in the compound, which decreases in the same order. $13 \mathrm{p}$.

\section{RP2817. Crystal structure of barium hydrogen ortho-} phosphate, G. Burley

The structure of anhydrous $\mathrm{BaHPO}_{4}$ was determined from three Fourier projections. The unit cell is orthorhombic with the space group $\mathrm{Pn} 2_{1} \mathrm{a}-$ No. 33 . The cell dimensions are $a=14.12, b=17.15$, and $c=4.59 \mathrm{~A}$, and it contains twelve formula units. Each $\mathrm{Ba}$ atom has a coordination polyhedron of ten oxygen atoms, being bonded to four phosphate tetrahedra by edge sharing and to two more by corner sharing of oxygen atoms. The phosphate groups are in separate tetrahedra, linked by hydrogen bonding to form continuous chains. $5 \mathrm{p}$.

\section{RP2818. A study of sampling of flame gases, C. Halpern and $\mathrm{F}$. W. Ruegg}

A study of sampling of hot combustion gases by means of water-cooled probes of internal diameter of 0.027 to $0.070 \mathrm{~cm}$ has been made. Effect of conditions of sampling on concentrations of $\mathrm{CO}, \mathrm{CO}_{2}, \mathrm{H}_{2}$, and $\mathrm{H}_{2} \mathrm{O}$ was the primary interest in this study. The probes were unable to quench reactions completely and were unable to preserve the original composition of the gas, but small probes were more effective than larger probes. Sample composition was unaffected by sampling velocity, except that at the higher rates of sampling it appears that gradients of composition and temperature caused the effectiveness of quenching to decrease. Probe material and configuration had little effect on sample composition. $9 \mathrm{p}$.

RP2819. Pyrolysis of polyamides, S. Straus and L. A. Wall

Thermal decompositions of various nylon samples having different molecular weights and composition were investigated by several procedures: (1) The rate of volatilization at temperatures between $310^{\circ}$ and $380^{\circ} \mathrm{C}$; (2) the analysis of the volatile products by mass spectrometry and (3) a direct measurement of volatilizing material obtained by carrying out the pyrolysis within the ionization chamber of a mass spectrometer.

Activation energies based on the rates of volatilization for the various samples varied from 15 to 42 kilocalories. The rate behavior, i.e., the observation of maxima in the rate-versus-conversion plots, is close to that given by theory for random decomposition. The different activation energies appear to be the result of a hydrolytic mechanism which is sensitive to trace polymerization catalysts. Increases in rates were obtained when sulfuric and phosphoric acids were added to nylon. The fluctuations found in activation energies, and the production of $\mathrm{CO}_{2}$ indicates that a hydrolytic decomposition mechanism may be contributing to the over-all process. It appears evident that, compared to a pure hydrocarbon chain, the polyamides are much more susceptible to thermal decomposition. $7 \mathrm{p}$.

RP2820. Some thermodynamic properties of the systems polybutadiene-benzene and polyisobutenebenzene, R. S. Jessup

Experimental data are reported on densities at $26.9^{\circ} \mathrm{C}$ of solutions of polyisobutene in benzene covering the composition range from 0 to 10 percent by weight of polyisobutene, and on relative vapor pressures of solutions of polybutadiene and of polyisobutene in benzene covering the range from 0 to 50 percent of benzene. Values of apparent specific volume of polyisobutene in benzene were calculated from the densities of solvent and solutions. The results of the vapor pressure measurements were compared with the Flory-Huggins equation. The deviations from this equation for the polyisobutene-benzene system are much greater than for the polybutadienebenzene system. The following equations represent the 
activities of the solvent for the polybutadiene-benzene and polyisobutene-benzene systems, respectivaly.

$$
\begin{aligned}
& \ln a_{1}=\ln v_{1}+v_{2}+0.27 v_{2}^{2}+0.06 v_{2}^{3}, \\
& \ln a_{1}=\ln v_{1}+\left(1-\frac{1}{m}\right) v_{2}+0.50 v_{2}^{2}+0.388 v_{2}^{3}+0.184 v_{2}^{12},
\end{aligned}
$$

where $a_{1}$ represents activity of the solvent, $v_{1}$ and $v_{2}$ are volume fractions of solvent and polymer, respectively, and $m$ is the ratio of molal volumes of polymer and solvent. $7 \mathrm{p}$.

RP2821. Infrared measurements with a small grating from 100 to 300 microns, E. K. Plyler and L. R. Blaine

Good resolution has been obtained with a small grating in the region from 100 to 300 microns. A high-pressure mercury lamp, having a quartz inner envelope, was used as a source and a Golay cell as a detector. The radiation was reflected from three filtering plates before passing through the entrance slit. This filtering served to reduce the stray radiation to about 5 percent. $3 \mathrm{p}$.

\section{RP2822. A low-frequency annular-slot antenna, J. R. Wait}

The radiation characteristics of an annular slot cut in an ideally conducting ground plane are discussed. The voltage impressed between the concentric edges is assumed to be constant around the slot. The annular slot is backed by a hemispherical cavity which has imperfectly conducting walls. For a specified voltage, the power radiated in the upper half-space and the power absorbed by the hemispherical cavitv are calculated. It is indicated that the power absorbed can be reduced greatly by lining the walls of the cavity with a wire mesh. A flush-mounted antenna of this type at low frequencies may have certain practical advantages over the more conventional monopole. $6 \mathrm{p}$.

RP2823. Relation between the absorption spectra and the chemical constitution of dyes: XXIX. Interaction of direct azo dyes in aqueous solution, M. N. Inscoe, J. H. Gould, M. E. Corning, and W. R. Brode

Interaction of dyes in aqueous solution results in differences in the spectrum of the mixture when compared with the sum of the spectra of the individual dyes. A general survey, chiefly with azo dyes, was made to determine the effect of dye structure on this interaction.

The effect of other factors on the interaction of direct azo dyes was investigated. The addition of alcohol or non-ionic detergent prevents this interaction, while the presence of inorganic salts or an increase in dye concentration appears to increase the extent of interaction. The effect of acids and bases is dependent upon the nature of the dyes in the mixture.

The results obtained are consistent with the assumption that the forces involved in the interaction of direct azo dyes in mixtures are the same as those causing the aggregation of individual dyes and those binding these dyes to fibers. $19 \mathrm{p}$.

RP2824. Energy spectra of cascade electrons and photons, C. A. Olson and L. V. Spencer

The equations for the energy spectra of electrons and photons in a cascade shower are written in a form suitable for numerical applications with accurate eross sections. Trial calculations were carried out to check the feasibility of a step-by-step numerical integration procedure similar to that used successfully at low energies in noncaseade problems. Numerical results were obtained for lead by using a monoenergetic source of electrons at $360 m c^{2}$ and extreme relativistic cross-sectional forms, which assume complete screening. The method was designed to permit the determination of cascade-shower spectra, spatial distributions, and directional distributions that are exact in the sense that the main limiting factor is the accuracy with which the cross sections are known. The numerical results of the trial calculations were found to agree with previously published results based on more restrictive methods of analysis. $12 \mathrm{p}$.

RP2825. Observer differences in color-mixture functions studied by means of a pair of metameric grays, K. L. Kelly

The Granville metameric gray panels have served to characterize the color vision of observers in an approximate but useful way. Thus, the 39 observers studied, and the four color-mixture functions were classed into five groups according to their indicated amounts of ocular pigmentstion. These panels were also used to measure the correlation of lens and macular pigmentations with observer age, sex, and eye and hair colors. Of the three sets of color-mixture functions intended to refer to $2^{\circ}$ field observation, the 1931 Commission Internationale de L'Eclairage standard observer agrees best. These data thus afford no basis for supplanting the 1931 Commission Internationale de L'Eclairage standard observer with either the Judd " $i$ " or the 1955 Stiles' $2^{\circ}$ color-mixture functions. The 1955 Stiles' $10^{\circ}$ functions, however, agree fairly well. $7 \mathrm{p}$.

RP2826. Precise evaluation of surface area with indirectly calculated dead space, W. V. Loebenstein

The determination of surface area from nitrogen adsorption data obtained at low temperature by volumetric methods is subject to several errors. One error that becomes especially serious when the surface area is small is associated with the calibration of the dead space with helium. This error is large because the volume of adsorbed gas is calculated as a small difference between two comparatively large quantities.

A statistical method of successive approximations has been developed for calculating the dead space. The latter is obtained as that quantity necessary to yield the best agreement with the known isotherm equation. This procedure fixes the value of $V_{m}$ (directly proportional to the surface area). The procedure also provides an estimate of the experimental error associated with the over-all determination. $4 \mathrm{p}$.

RP2827. Sample calculations of gamma-ray penetration into shelters: Contributions of sky shine and roof contamination, M. J. Berger and J. C. Lamkin

An approximate method is presented for calculating the penetration of gamma radiation in shelters. Sample calculations, for an assumed source energy of 1 million electron volts, are given for the following problems: (1) Dose rate inside houses and underground shelters whose roofs are covered with radioactive fallout, and (2) dose rate in open holes due to reflected radiation (sky shine) from fallout contamination on the surrounding ground. A detailed examination is made of the dependence of the dose rate in a shelter on the shape of the shelter, and on the position of the detector within the shelter. The estimated accuracy of the calculations is \pm 30 percent. $8 \mathrm{p}$.

RP2828. Heat content of sodium borohydride and of potassium borohydride from $0^{\circ}$ to $400^{\circ} \mathrm{C}$, T. B. Douglas and A. W. Harman

The heat content of sodium borohydride, $\mathrm{NaBH}_{4}$, and of potassium borohydride, $\mathrm{KBH}_{4}$, was measured, relative to that at $0^{\circ} \mathrm{C}$, at 50-degree intervals from $0^{\circ}$ to $400^{\circ} \mathrm{C}$. The so-called "drop" method was used with a precision Bunsen ice calorimeter and a silver core furnace. Preliminary tests showed that spontaneous decomposition of these compounds is very slow at temperatures up to $400^{\circ} \mathrm{C}$. Measurements were made on two samples of each compound, those of sodium borohydride having been obtained 
from different sources. Smoothed values of the heatcontent function, heat capacity, entropy, and the Gibbs free-energy function were derived from the data. These values were increased by 0.5 percent to correct for the presence of an estimated 1 percent of the corresponding metaborate in each sample. The heat capacity of sodium borohydride increases monotonically with temperature in the range investigated; that of potassium borohydride passes through a point of inflection, which suggests that there may be a broad second-order transition in the neighborhood of room temperature. $8 \mathrm{p}$.

RP2829. Infrared spectra of crystalline polyphenyls, J. E. Stewart and M. Hellmann

Infrared absorption spectra of sixteen selected polyphenyls ranging from biphenyl to $m$-octaphenyl are presented for the 5- to 38-micron region. The observed vibration bands are discussed in terms of a pseudo-symmetry approximation in which the individual rings are assumed to vibrate separately. Indications of interactions between rings are seen in the $\mathrm{C}-\mathrm{H}$ out-of-plane bending vibrations of ortho- and meta-substituted rings. Evidence is given for the existence of two rotational isomers of $m$ quatraphenyl with a free energy difference of about 250 calories per mole. $12 \mathrm{p}$.

RP2830. On the application of steam-driven water jets fór propulsion purposes, J. M. Burgers and A. Ghaffari

Calculations have been made concerning the momentum that can be given to a jet of water by mixing it with a jet of high-speed steam. Apart from an application of the equations of momentum and enthalpy, this raises questions concerning the speed of condensation and the acceleration of the water. $5 \mathrm{p}$.

RP2831. Mas 3 spectra and relative sensitivities of some polyphenyls, P. Bradt and F. L. Mohler

Mass spectra have been measured by evaporating polyphenyls from a tube furnace into the ionization chamber of a $60^{\circ}$ mass spectrometer operated at constant ion accelerating voltage. Mass spectra of pure tetraphenyl, hexaphenyl, and octaphenyl are reported. A synthetic mixture of terphenyl, tetraphenyl, pentaphenyl, hexaphenyl, and octaphenyl in known proportions was evaporated as the temperature was increased step by step from $125^{\circ}$ to $290^{\circ} \mathrm{C}$ and time integrals of the five molecule ion currents were recorded over the time required to completely evaporate the sample. The relative sensitivity increases rapidly with increasing molecular weight. $3 \mathrm{p}$.

RP2832. Infrared spectra of thermally degraded poly (vinyl chloride), R. R. Stromberg, S. Straus, and B. G. Achhammer

The changes in chemical structure occurring in poly (vinyl chloride) as a result of heating in a vacuum in the range $100^{\circ}$ to $400^{\circ} \mathrm{C}$ were studied using infrared spectrophotometry. The principal changes occurring in the residue during pyrolysis in a vacuum were the formation of unsaturated structures and a change from an aliphatic spectrum to one showing aromatic absorption. The data are used to support a previously proposed mechanism of decomposition for poly (vinyl chloride). $6 \mathrm{p}$.

RP2833. Current and potential relations for the cathodic protection of steel in salt water, W. J. Schwerdtfeger

A laboratory investigation was made pertaining to the cathodic protection of steel specimens that were exposed for 60 days to both stagnant and aerated city water, to which was added 3 percent by weight of sodium chloride.

Major consideration was given to the significance of potential as a criterion for protection. Optimum protection was achieved when specimens were controlled at
-0.77 volt with reference to the saturated calomel half cell. Although a good degree of protection was obtained at controlled potentials more noble than -0.77 volt, that is, at the potentials associated with the breaks in cathodic polarization curves, this lesser degree of protection could not be obtained at lower mean current densities. $7 \mathrm{p}$.

RP2834. Joule-Thomson process in the liquefaction of helium, E. H. Brown and J. W. Dean

A dimensionless, normalized, correlating function is introduced for the specific enthalpy of helium. Using this function, the consistency of various enthalpy data is determined. These data are then used to obtain curves of helium liquefaction yield in the region of variable specific heat in terms of heat-exchanger efficiency. $8 \mathrm{p}$.

RP2835. Changes in the properties of an asphalt during the blowing operation, L. R. Kleinschmidt and H. R. Snoke

The physical characteristics and component distribution of an asphalt flux and of asphalt products taken at six two-hour intervals during the conversion, by air blowing, of the flux to a coating-grade asphalt, were determined. The softening point and asphaltene content of the asphalt products increased linearly with time during the blowing operation. There was essentially no change in percentage, refractive index, and viscosity of the water-white oils during blowing. The percentage and refractive index of the dark oils decreased progressively during the blowing operation. $4 \mathrm{p}$.

RP2836. Creep of annealed nickel, copper, and two nickel-copper alloys, W. D. Jenkins and C. R. Johnson

Creep tests were made in tension under constant loads at temperatures of $300^{\circ}, 700^{\circ}$, and $900^{\circ} \mathrm{F}$ on initially annealed specimens of nickel, copper, and 70-percentnickel-30-percent-copper and 30 -percent-nickel-70-percentcopper alloys. Tests at $1,200^{\circ} \mathrm{F}$ were also made on the nickel and the two alloys, but not on the annealed copper as the resistance to creep of copper is relatively low at this temperature. The investigation included a study of the influence of rate of loading on the creep stress and of prior thermal-mechanical history on the creep behavior of the alloys at several selected temperatures. Contour and hardness surveys and metallographic examinations were also carried out on some of the fractured specimens to ascertain the effect of creep on the necking characteristies, hardness, and structures of the metals. $19 \mathrm{p}$.

RP2837. Stress-strain relation of pure-gum rubber vulcanizates in compression and tension, L. A. Wood

The stress-strain curve in tension for a typical puregum rubber vulcanizate after a given period of creep, according to Martin, Roth, and Stiehler, can be represented by an empirical equation

$$
F=M\left(L^{-1}-L^{-2}\right) \exp A\left(L-L^{-1}\right)
$$

where $F$ is the stress based on the original-cross sectional area, and $L$ is the ratio of stressed to unstressed length, $M$ is the slope of the stress-strain curve at $L=1$ and $A$ normally has a value close to 0.38 . The present paper shows by an examination of data published by Sheppard and Clapson, Treloar, and Rivlin and Saunders that the equation is also valid in the region of compression for values of $L$ as small as 0.5 (50 percent compression). The features of the empirical equation are discussed, and comparisons are made with the equation predicted by the statistical theory of rubber elasticity and the equation derived by assuming Hooke's law for the stressed cross section. The consequences of the validity of the empirical equation in terms of the Mooney-Rivlin presentation of the strain energy function are pointed out. The equation 
predicted by the statistical theory represents observed data very well in the compression region from $L=0.5$ to $L=1.0$. The Mooney equation is approximately valid from $L=1.5$ to $L=3.5$. Neither of these equations is satisfactory in the important intermediate region from $L=1.0$ to $L=1.5$. The empirical equation represents the observed data over all three of these regions. It is concluded that Young's modulus $M$ can best be obtained from the intercept of a plot of $\log F /\left(L^{-1}-L^{-2}\right)$ against $\left(L-L^{-1}\right)$. For $0.75<L<2.00$ it is thoroughly satisfactory to determine $M$ as the intercept of a plot of $F^{\prime}\left(L^{-1}-L^{-2}\right)$ against $(L-1) .7 \mathrm{p}$.

RP2838. Tables for diagonalizing second-order matrices, R. E. Trees and C. D. Coleman

Sets of tables are given to facilitate the evaluation of the eigenvalues and eigenvectors of second-order matrices. $14 \mathrm{p}$.

RP2839. Effects of capillary shape on flow characteristics and degradation of polymer solutions, H. S. White and $\mathrm{H}$. V. Belcher

Flow data were obtained using a tapered capillary, a chamfered capillary, and a uniform-bore capillary in a McKee worker consistometer. With polyisobutene-cetane solutions, degradation occurred at lower rates of flow and persisted for a greater number of passes with flow entering the smaller end compared to flow entering the larger end of the tapered capillary. When degradation occurred in both directions at high rates of flow the pressure was larger for flow entering the larger end though after degradation ceased the pressure (used to overcome viscous resistance) was less for flow in this direction, compared to flow entering the smaller end in each case. Errors which might arise in the use of single pass instruments with nonuniform capillaries are pointed out. 5 p.

RP 2840. Ortho-para catalysis in liquid-hydrogen production, D. H. Weitzel, W. V. Loebenstein, J. W. Draper, and O. E. Park

A series of selected or specially prepared eatalysts were studied for their ability to accelerate the ortho to para conversion of hydrogen. The results of this study are presented, and the performance of various catalysts are compared with that of chromic oxide on alumina pellets. An outstanding eatalyst, unsupported hydrous ferric oxide granules, was selected for further study and used in the liquefiers of the National Bureau of Standards Cryogenic Engineering Laboratory. One and one-half liters of this catalyst has now been used to convert more than 100,000 liters of liquid hydrogen to 90 to 95 percent para at an average rate of about 235 liters of liquid per hour There is to date no evidence of decrease in efficiency with continued use. $7 \mathrm{p}$.

RP 2841. Degradation of cellulose in a vacuum with ultraviolet light, J. H. Flynn, W. K. Wilson, and W. L. Morrow

Dried, purified, cotton cellulose sheets were irradiated in a vacuum at $40^{\circ} \mathrm{C}$ with light of $2537 \mathrm{~A}$ wavelength. Hydrogen, carbon monoxide, and carbon dioxide were evolved, the degree of polymerization (D. P.) decreased, and $\mathrm{CHO}$ and $\mathrm{COOH}$ groups were produced. The evolution of hydrogen has not been reported previously. The rate of hydrogen evolution followed a parabolic rate law indicating inhibition by a product. The rate of evolution of earbon monoxide plus earbon dioxide inereased slightly during irradiation. The initial quantum yield was $10^{-2}$ for hydrogen and $10^{-3}$ for carbon monoxide and carbon dioxide. A mechanism is proposed in which alcohol groups are photolyzed to carbonyl with the liberation of hydrogen. $5 \mathrm{p}$.

RP 2842. Cavity ionization as a function of wall material, F. H. Attix, L. DeLa Vergne, and V. H. Ritz

A study has been made of the ionization within a flat cavity chamber under irradiation by $\mathrm{X}$ - and gamma rays in the energy region 38 to 1,250 kilovolts effective (kev). Chamber walls were made of carbon, aluminum, copper tin, and lead, and the wall separation was varied from 0.5 to 10 millimeters. Results are compared with cavity theory. $9 \mathrm{p}$.

\section{RP 2843. Fragmentation of waterdrops in the zone behind an air shock, O. G. Engel}

Observations made on the fragmentation of two waterdrop sizes, after collision with air shocks that were moving at three different supersonic velocities, are reported. The possible mechanisms of various aspects of the fragmentation process are discussed. The experimental observations indicate that high-speed-rain-erosion damage should not be observed on spheres having a diameter as large as 4 feet and moving with a Mach number in the range of 1.3 to 1.7 in rain that has a drop diameter of 1.4 millimeter. Waterdrops of this size should be reduced to mist in the zone of separation between the detached shock and the surface of the sphere according to the results that are reported. A means to extend this protection to spheres of smaller diameter or to rain of larger size is pointed out. The need for further experimental observation of the time required for the fragmentation of waterdrops using shocks moving at higher Mach numbers is indicated to verify and extend the information. $36 \mathrm{p}$.

\section{RP 2844. Transient radio-frequency ground waves over the surface of a finitely conducting plane earth, J. R. Johler}

The complete transient signal is reconstructed after propagation via the ground-wave mode over a finitely conducting plane earth in which the displacement currents are neglected. The theory is illustrated by computations that have been made on formulas derived by the method of the inverse Laplace transformation. The results of this analysis indicate that current sources with sinusoidal form in the time domain could be used to simulate sferics. The methods employed in this analysis can be also used to reconstruct propagated signals of pulsed radio-navigation systems. $5 \mathrm{p}$.

\section{RP 2845. Measurement of current with a Pellat-type} electrodynamometer, R. L. Driscoll

The value of an electric current has been determined in absolute measure by means of an electrodynamometer, and simultaneously by standard cells and standard resistors as currently maintained. The electrodynamometer used was of the Pellat type, and featured a fused silica balance beam and single layer helical coils.

The relation of the NBS ampere to the absolute ampere, from this determination, may be expressed as

\section{NBS ampere $=1.000013$ absolute amperes.}

The uncertainty in this result from all known sources is estimated to be eight parts per million. $10 \mathrm{p}$.

RP2846. Measurement of current with the National Bureau of Standards current balance, R. L. Driscoll and R. D. Cutkosky

Prior to the adjustment of the electrical units in 1948, the value of a eurrent had been determined in absolute units by means of a current balance and simultaneously measured in NBS amperes by comparison with standard resistors and standard cells. This work was reported in RP1449 Similar measurements made recently with an electrodynamometer indicate a possible change in the values of the standards. The present paper reports a repetition of the work described in RP1449. The purpose of this remeasurement was to determine whether or not the standards had changed. Only minor changes were made in the equipment in order that factors which might have introduced small systematic errors in the results would remain unchanged. 
According to the work deseribed in this paper, 1 NBS ampere $=1.000008$ absolute amperes. Recent work with the Pellat electrodynamometer gave the result 1 NBS ampere $=1.000013$ absolute amperes. The weighted mean of these two values is

\section{NBS ampere- $1.000010 \pm 0.000005$ absolute amperes}

The results given above for the current balance differ by $6 \mathrm{ppm}$ from those obtained in 1942 . This indicates, in view of the uncertainties of measurement, that any change in the ampere as maintained by standard resistors and standard cells does not exceed a few parts in a million. $9 \mathrm{p}$.

RP2847. Radial distribution of the center of gravity of random points on a unit circle, F. Scheid

This paper deseribes some Monte Carlo computations carried out on the Standards Electronic Automatic Computer (SEAC) which are related to the problem of random walks. 2 p.

RP2848. An adiabatic calorimeter for the range $30^{\circ}$ to $500^{\circ}$ C, E. D. West and D. C. Ginnings

An adiabatic calorimeter accurate to 0.1 percent and suitable for heat capacity measurements of solids and liquids over the temperature range $30^{\circ}$ to $500^{\circ} \mathrm{C}$ is described. Factors affecting the design and accuracy are discussed. Automatic controls permit one-man operation of the apparatus. Measurements of the heat eapacity of $\mathrm{Al}_{2} \mathrm{O}$ agree to 0.1 percent with earlier measurements made with other calorimeters at the National Bureau of Standards. $8 \mathrm{p}$.

RP2849. An evaluation of the luminous-transmittance requirements for, railroad-signal glassware in terms of standard source A of the International Commission on Illumination, F. C. Breckenridge

As an extension of Research Paper 1688, an estimate has been made of the transmittance for illuminant $A$ of signal glassware having the minimum transmittance acceptable under the specifications of the Signal Section of the Association of American Railroads, which are formulated in terms of tests at $2,360^{\circ} \mathrm{K} .4 \mathrm{p}$.

RP2850. Random notes on matrices, K. Goldberg

Three matrix problems are considered in this paper Bessel functions as limits of determinants, finding all optimal strategies of a matrix game with nonzero value, and conditions for matrices to have equal principal minors. $6 \mathrm{p}$.

RP2851. Range finders using projected images, R, E. Stephens

Two optical range finders of six-inch base length have been designed and constructed for making measurement to arbitrary points on a model. One measures distances from 2.5 feet to 10 feet, the other from 9.7 feet to 25 feet. The accuracy of all distances is plus or minus one-quarter inch or better. Because of unusual requirements of use these range finders operate by projecting two images of a bright cross upon the target, which may be fused into a single image by adjustment of the range knob. $7 \mathrm{p}$.

RP2852. Effect of rib flexibility on the vibration modes of a delta-wing aircraft, W. D. Kroll

A systematic study was made to determine whether decreasing the number of ribs or making the ribs more flexible would have any appreciable effect on the vibration modes and frequencies of a delta-wing aircraft. The modes and frequencies were computed for the basic wing and for the following modifications of the basic wing: (1) One rib outboard of the fuselage removed, (2) two ribs removed, (3) stiffness of ribs outboard of fuselage reduced one-half, and (4) rib stiffness reduced nine-tenths. The results indicated that the frequencies and mode shapes of the modified wings differed little from those of the basic wing and, therefore, that changes similar to these modifications would not appreciably affect the vibration characteristics of delta wings. $7 \mathrm{p}$.

RP2853. Thermal degradation of cellulosic materials, S. L. Madorsky, V. E. Hart, and S. Straus

Fortisan, cellulose triacetate, and $\mathrm{NO}_{2}$-oxidized cellulose were pyrolyzed in a vacuum in the temperature range $180^{\circ}$ to $465^{\circ} \mathrm{C}$. Cotton cellulose and cellulose triacetate were also pyrolyzed in nitrogen at atmospheric pressure. The tar yields were in the decreasing order from: Cotton, Fortisan, cellulose triacetate, and oxidized cellulose. The other volatiles consisted mainly of acetic acid, carbon dioxide, and carbon monoxide, from the triacetate; and water, carbon dioxide, and carbon monoxide from the other celluloses. In all cases there was a carbonaceaus residue (volatilization end point), the amount depending on the nature of the cellulose and the temperature of pyrolysis. When pyrolyzed in nitrogen at atmospheric pressure, cotton cellulose and cellulose triacetate yielded less tar than when pyrolyzed in a vacuum. The tar from cellulose triacetate consisted of a compound whose infrared spectrum resembled that of the original triacetate. Cotton cellulose, Fortisan, and cellulose triacetate do not differ much in their initial rates and activation energies of thermal degradation. Oxidized cellulose has very high initial rates of thermal degradation. $7 \mathrm{p}$.

RP2854. Role of vitrons in alkali silicate binary glasses, L. W. Tilton

Applications of vitron theory to analyses of properties of $\mathrm{R}_{2} \mathrm{O}$ silicate binary glasses indicate that changes in slope on the property-composition curves may occur where there are integral numbers of cations per dodecahedral cavity or eage of the network. From geometrical considerations it is found that 1 or 2 oxygens and 6 sodiums can be accommodated inside a cage, and similarly not more than 1 oxygen and 4 potassiums can be inclosed in a cage.

Data on chemical attack and on solubility losses in water suggest for alkali silicate glasses a maximal average inclosure of oxygen inside the cavities at 16.7-mole percent for $\mathrm{K}_{2} \mathrm{O}, 23.1$ percent for $\mathrm{Na}_{2} \mathrm{O}$, and probably 28.6 percent for $\mathrm{Li}_{2} \mathrm{O}$. These compositions correspond to $1,1.5$, and 2 oxygens per cage. These compositions mark the begining deterioration of the net, possibly because of nonbridging oxygen. Changes in rate of volatilization occur at 28.6-, 37.5-, and 50.0-percent $\mathrm{R}_{2} \mathrm{O}$ for potash, soda, and lithia silicate glasses, repectively. These critical compositions are considered those for which the cavities in the glasses are completely saturated with the modifier oxides, $\mathrm{R}_{2} \mathrm{O}$, and with additional cations, $R$.

Detailed analyses of curves of molar volume versus composition show that the critical points for oxide saturation are evident and that changes in slope may occur at other critical compositions corresponding to integral numbers of cations per cage. It is found that the percentage of 16.7-percent $\mathrm{Na}_{2} \mathrm{O}$ at high temperatures and the saturation point of 37.5 percent at low temperatures are critical in curves for specific (electrical) resistance. The "deep-well" point at 16.7 -percent $\mathrm{Na}_{2} \mathrm{O}$ eritically separates soda silicate glasses that are very readily devitrified from those that contain more oxide modifier and are more stable. $14 \mathrm{p}$.

RP2855. Elastic problem for a ring of uniform force in an infinite body, W. H. Pell

Kelvin has given an integral representation for the displacements in an infinite elastic body produced by a body force acting in an arbitrary portion of this body. $\mathrm{By}$ a limit procedure, solutions have been found for point and line singularities acting in the interior of such a body. A similiar method is used in this paper to obtain the 
displacements produced by a uniform force applied along, and normal to, a circle lying in the interior of an infinite elastic body. $9 \mathrm{p}$.

\section{RP2856. Description and analysis of the second spectrum of molybdenum, Mo II, C. C. Kiess}

Wavelengths and estimated intensities are presented for 3,800 lines of Mo II in the spectral range from $6100 \mathrm{~A}$ in the red to $1550 \mathrm{~A}$ in the ultraviolet. For approximately 970 of these lines, Zeeman patterns have been measured. Analysis of these spectral data shows that about 70 percent of the lines can be accounted for as transitions between levels of the even terms in the electron configurations $4 d^{5}$ and $4 d^{4} 5 s$, and levels of the odd terms in the configuration $4 d^{4} 5 p$. No series have been found from which an ionization potential can be derived. $48 \mathrm{p}$.

\section{RP2857. Forces on cylinders and plates in an oscillating fluid, G. H. Keulegan and L. H. Carpenter}

The inertia and drag coefficients of cylinders and plates in simple sinusoidal currents are investigated. The midsection of a rectangular basin with standing waves surging in it is selected as the locale of currents. The cylinders and plates are fixed horizontally and below the water surface. The average values of the inertia and drag coefficients over a wave cycle show variations when the intensity of the current and the size of the cylinders or plates are changed. These variations, however, can be correlated with the period parameter $U_{\mathrm{m}} T / D$, where $U_{\mathrm{m}}$ is the maximum intensity of the sinusoidal current, $T$ is the period of the wave and $D$ is the diameter of the cylinder or the width of the plate. For the cylinders $U_{\mathrm{m}} T / D$ equaling 15 is a critical condition yielding the lowest value of the inertia coefficient and the largest value of the drag coefficient. For the plates the higher values of the drag coefficient are associated with the smaller values of $U_{\mathrm{m}} T / D$ and the higher values of the mass coefficient with the larger values of $U_{m} T / D$. The variation of the coefficients with the phase of the wave is examined and the bearing of this on the formula for the forces is discussed. The flow patterns around the cylinders and plates are examined photographically, and a suggestion is advanced as to the physical meaning of the parameter $U_{\mathrm{m}} T / D .18 \mathrm{p}$.

RP2858. Reaction of portland cement with carbon dioxide C. M. Hunt, V. Dantzler, L. A. Tomes, and R. L. Blaine

The reaction of portland cement with carbon dioxide was found to be greatly influenced by the moisture environment of the cement. Reaction with carbon dioxide and water also affected the subsequent hydration of the cement. $6 \mathrm{p}$.

RP2859. Average energy of sulfur-35 beta decay, H. H Seliger, W. B. Mann, and L. M. Cavallo

The rate of energy emission in an approximately 400 millicurie source of sulfur-35 was measured by means of a "radiation-balance" microcalorimeter. This value and the disintegration rate of an accurate dilution of the sample measured by means of $4 \pi \beta$-gas-proportional counting yield a mean energy for sulfur-35 beta decay of 50.4 $\left({ }_{-1.2}^{+0.7}\right)$ kev. The half-life has been determined to be $87.16 \pm 0.1$ days. $4 \mathrm{p}$.

RP2860. Effect of structure on the thermal decomposition of polymers, L. A. Wall and R. E. Florin

During recent years a considerable number of theoretical and experimental studies have been published on the kinetics and mechanism of the thermal decomposition of polymers. The treatment of depolymerization as a freeradical chain reaction involving the four basic steps of initiation, propagation, transfer, and termination seems adequate for a fairly complete understanding of the process in a large number of cases. Rate data now available are discussed from this point of view. It is shown that most of the characteristics of the thermal decomposition rate curves are a result of their basic structure and not of trace impurities. The magnitude of the rates and activation energies are, however, sensitive to trace structures or impurities. It is shown that intermolecular transfer will account for the rate behavior in a large number of polymers. In a relatively few other cases intramolecular transfer is indicated to be of more importance. $8 \mathrm{p}$.

RP2861. Comparison of theoretical and empirical relations between the shear modulus and torsional resonance frequencies for bars of rectangular cross section, S. Spinner and R. C. Valore, Jr.

The relations between the modulus of elasticity in shear and the fundamental torsional resonance frequency, mass, and dimensions for bars of rectangular cross section have been evaluated experimentally. The empirical relation was found to be less than the theoretical approximation given by Pickett, by an amount increasing to about $13 / 4$ percent as the cross-sectional width to depth ratio of the bars approached 10 .

In addition to the fundamental torsional resonance frequency, the first overtone of the specimens was also determined. The overtone was found not to be an exact multiple of the fundamental; it increased more than 5 percent over double the value of the fundamental as the width to depth ratio increased to 10 . $6 \mathrm{p}$.

RP2862. Specific volume and degree of crystallinity of semicrystalline poly(chlorotrifluoroethylene), and estimated specific volumes of the pure amorphous and crystalline phases, J. D. Hoffman and J. J. Weeks

The specific volume of poly(chlorotrifluoroethylene) has been measured from $-40^{\circ}$ to $+260^{\circ} \mathrm{C}$. Both quenched and well-crystallized specimens prepared by reproducible procedures were studied. Well-defined glass transitions were found close to $52^{\circ} \mathrm{C}$ in both specimens. Hence the glass temperature of this polymer is essentially independent of the degree of crystallinity. The quasi-equilibrium melting point of the particular type of crystallized specimen used in the investigation was $216^{\circ} \mathrm{C}$. The equilibrium melting temperature is undoubtedly somewhat higher, and probably lies between $220^{\circ}$ and $225^{\circ} \mathrm{C}$.

The degree of crystallinity of the crystallized and quenched specimens was calculated at $T_{\text {from specific- }}$ volume measurements alone, using a straightforward thermodynamic procedure. The method avoids a long "unguided" extrapolation of the liquid data to low temperatures, and does not involve the inexact approximation that the volume-temperature derivative of the glassy and crystalline states are the same. It has the further advantage of not requiring a pure crystal density from another source, such as a unit-cell determination from X-ray data. It was found that the quenched sample was 39 percent crystalline and the well-crystallized one 82 percent. A simple extension of the theory permits the degree of crystallinity to be computed as a function of temperature. The results are compared with those obtained in earlier investigations.

The specific volumes and volume-temperature derivatives of the pure supercooled liquid, glassy, and crystalline phases are estimated over a wide range of temperature. Certain quantities related to the free volume of the glassy state are discussed.

The methods outlined may be of utility in analyzing specific-volume-temperature data on other semicrystalline polymers where the rapid onset of crystallization interferes with a direct study of the supercooled liquid and glassy states, and where independent data on the properties of the pure crystalline phase are not available. $15 \mathrm{p}$.

RP2863. Heat and ultraviolet aging of poly(vinyl chloride), C. F. Bersch, M. R. Harvey, and B. G Achhammer

Four poly (vinyl chloride) polymers preparod with different initiators were exposed to ultraviolet radiant energy 
and to heat, in a vacuum and in air. The gaseous products evolved were analyzed by mass spectrometry. Changes in chemical structure were followed by infrared spectrophotometry. Benzene was among the products evolved in most cases, and acetone was produced during exposure to heat in air. Catalyst fragments or other incorporated impurities affected initiation at very mild conditions. Susceptibility to degradation increased with increasing oxygen content and unsaturation of the untreated polymer. Pyrolysis studies indicated a two stage degradation: (1) dehydrochlorination, and (2) decomposition of the resultant polyene chain. Color formation was attributed to both oxidation and conjugated unsaturation because exposure in air following exposure in a vacuum, and vice versa, caused bleaching of the degraded polymer. $8 \mathrm{p}$.

RP2864. Determination of water vapor from the change in electrical resistance of a hydroscopic film, E. R. Weaver, E. E. Hughes, and A. W. Diniak

The electrical conductivity of a thin film of such a material as phosphoric acid changes over a wide range with changes in the concentration of water in the atmosphere with which it is in contact. By adjusting the pressures of a sample of gas of known composition and one of unknown composition, they can be made to have the same concentration of water (more accurately the same fugacity) shown by the production of equal resistances of the detecting film. Modifications of the apparatus, procedures, and calculations described in an earlier publication are presented and numerous applications of the method are discussed. The method has the merits of simplicity, speed, and great sensitivity. Only small samples are needed, and few substances interfere. $20 \mathrm{p}$.

RP2865. Improved bridge method for the measurement of core losses in ferromagnetic materials at high flux densities, W. P. Harris and I. L. Cooter

Accurate core-loss measurements at high flux densities can be made by bridge methods if the power dissipated in the primary circuit at harmonic frequencies is measured and subtracted from the apparent power dissipated in the ferromagnetic material at fundamental frequency. The determination of this harmonic power term is inconvenient, and must be done with greater accuracy than that required in the final result. An amplifier having negative output resistance was devised and is used in a manner that automatically allows accurate compensation for the harmonic power dissipation. $8 \mathrm{p}$.

RP2866. Stress-strain relationships in yarns subjected to rapid impact loading: 5 . Wave propagation in long textile yarns impacted transversely, J. C. Smith, F. L. McCrackin, and H. F. Schiefer

The behavior of an infinitely long flexible filament after transverse impact is treated theoretically. The filament is assumed to have a tension-strain curve that is always concave downward, and to have no short-time creep or stressrelaxation effects. Under most conditions the impact initiates a variable strain that propagates down the filament between an "elastic wave" front and a "plastic wave" front. A transverse wave, shaped like an inverted $V$, then travels in the constant-strain region behind the plastic-wave front. Under special conditions the transverse-wave front may propagate faster than the plasticwave front, but the shape of the transverse wave remains the same. The theory for both cases is worked out in detail, and some illustrative examples are given. $18 \mathrm{p}$.

RP2867. Measurement of flame speeds by a nozzle burner method, C. Halpern

The literature records a great many measurements, using stationary flames on burners, of the speed with which flame moves through combustible mixtures of gases.
Despite the fact that the method itself seems reasonably simple, the results obtained by various investigators often are not in good agreement. One of the phases of a program of research on combustion has been a study of some of the reasons for the differences among recorded values of flame speed measured by the burner method. The primary objective of this task has been to develop the precautions that should be observed in applying the method, rather than to evolve numerical values of flame speed. This paper describes progress that has been made since the apparatus was described originally in 1951, and presents values of flame speeds of methane-air mixtures obtained since then, together with comparisons of these values with those obtained by two flame theories. $12 \mathrm{p}$.

RP2868. Growth of preferentially oriented aluminum single crystals, T. H. Orem

Monocrystalline aluminum specimens having both a preferential orientation and a specified cross-sectional shape are very difficult to produce. Such specimens must be grown in a vertical furnace, a condition which, while permitting the choice of cross-sectional shape in the specimen, makes it extremely difficult to obtain specimens of preferred orientation. It is fairly easy to grow preferentially oriented monocrystalline aluminum specimens when a horizontal furnace is used, but the use of such a furnace limits the choice of cross-sectional shapes in the finished crystal. Crystals of circular cross-sectional shape are practically impossible to grow in a horizontal furnace.

The method described herein can be used to grow monocrystalline aluminum crystals with any desired orientation or cross-sectional shape. $3 \mathrm{p}$.

RP2869. Marginal performance of corrected ophthalmic lenses, F. E. Washer and W. R. Darling

The measured values of the departures of the marginal meridional and cylindrical powers from the corresponding measured values of the axial powers for 311 corrected ophthalmic lenses are reported. The spherical refractive powers of the lenses studied range from +7.00 to -20.00 diopters with cylindrical powers of 0.00 to 4.00 diopters. The probable errors of measurement are discussed. A set of tolerance values is suggested, and the degree of compliance with these suggested tolerances is shown. $12 \mathrm{p}$.

RP2870. Enthalpy and heat capacity from $0^{\circ}$ to $900^{\circ} \mathrm{C}$ of three nickel-chromium-iron alloys of different carbon contents, T. B. Douglas and A. W. Harman

The enthalpy relative to $0^{\circ} \mathrm{C}$ of three alloys was measured at nine temperatures from $100^{\circ}$ to $900^{\circ} \mathrm{C}$ by a precise "drop" method. The alloys contained approximately 76 percent of nickel, 15 percent of chromium, and 8 percent of iron, with carbon contents of $0.02,0.07$, and 0.11 percent, respectively. The results are almost independent of the several variations in prior heat treatment investigated, and the heat-capacity-temperature curves of the three alloys are almost coincident, but there is a marked shift in each curve within the interval $500^{\circ}$ to $600^{\circ} \mathrm{C}$, $6 \mathrm{p}$.

RP2871. Relative strengths of forty aromatic earboxylic acids in benzene at $25^{\circ}$ C., M. M. Davis and H. B. Hetzer

The relative strengths of benzoic acid, 31 of its monosubstituted derivatives, 7 disubstituted derivatives, and one trisubstituted derivative have been measured in terms of the equilibrium constants $\left(K^{\prime \prime}\right)$ for association with the reference base 1,3-diphenylguanidine in benzene at $25^{\circ} \mathrm{C}$. The measurements were performed spectrophotometrically, using the indicator acid bromophthalein magenta $\mathrm{E}$ (tetrabiomophenolphthalein ethyl ester) as the reference acid, the equilibrium constant $K^{\prime}$ for association 
of the indicator with diphenylguanidine being known from previous work. Equilibrium constants for $p$-orsellinic (2,6-dihydroxy- $p$-toluic) acid and 2,4,6-trinitrobenzoic acid could not be measured, as these acids appear to associate completely with diphenylguanidine under the conditions of the experiments.

Plotting $\log K^{\prime \prime}$ values against the corresponding $p K$ values for aqueous solutions of the acids gives an essentially linear relationship in the case of acids with meta- and parasubstituents only, but the line for para-substituted acids has a slightly different slope from the line for $m$-substituted acids. Similar comparisons were made with published data on relative strengths in alcohols or dioxane-water mixtures. ortho-Substituted acids show marked solvent effects. Explanations of the solvent effects have been suggested, and various theoretical implications and possible applications of the results are discussed. The theoretical discussion includes evaluation of constants of the Hammett equation. $24 \mathrm{p}$.

RP2872. Shape of the liguidus surface as a criterion of stable glass formation, E. H. Hamilton and G. W. Cleek

The slope of the liquidus curve or surface at a point in a phase diagram representing a specific chemical composition has been found to be a reliable indication of the glass-forming tendency of that composition. The observed effect is interpreted in terms of the structure of the glass. $4 \mathrm{p}$.

RP2873. Twinned epitaxy of copper on copper, T. H. Orem

A study of the X-ray diffraction patterns of copper electrodeposited on copper monocrystals having surfaces parallel to the cubic, dodecahedral, and octahedral planes shows an interesting relationship between electrodeposit and base crystal. At the current density used for electrodeposition, the electrodeposit on the surface parallel to the dodecahedral plane was a monocrystalline continuation of the orientation of the base crystal in every respect. The electrodeposit on the surface parallel to the octahedral plane was a highly preferentially oriented polycrystalline electrodeposit made of microcrystals, some of which continued the orientation of the base crystal, whereas others were twinned with respect to the surface plane of the base crystal. In the case of electrodeposition on the surface parallel to the cubic plane, the electrodeposit was also a highly preferentially oriented polycrystalline electrodeposit made up of microcrystals, some of which continued the orientation of the base crystal, whereas others bore a twin relationship to the close-packed planes of the base erystal. $12 \mathrm{p}$.

RP2874. Dielectric constant of deuterium oxide, C. G. Malmberg

An equal ratio-arm, capacitance-conductance bridge op erated at frequencies below 100 kilocycles per second was used to measure the dielectric constant of deuterium oxide with an accuracy of 0.1 percent or better in the range $4^{\circ}$ to $100^{\circ} \mathrm{C}$. A value of 77.94 was found for the dielectric constant at $25^{\circ} \mathrm{C}$. The data fit the relation

$$
\epsilon=87.48_{2}-(0.40509) t+\left(9.638 \times 10^{-4}\right) t^{2}-\left(1.333 \times 10^{-6}\right) t^{3},
$$

with a maximum deviation of less than 0.01 unit in dielectric constant. These values were obtained by using a sample having a deuterium oxide content of 99.38 mole percent. Deviation of these values from those for pure deuterium oxide, as a result of isotopic contamination, is estimated to be less than 0.005 unit. Some aspects of the temperature dependence of the dielectric constant and of that of a macroscopic polarization are discussed. $4 \mathrm{p}$.

RP2875. Additional abseissas and weights for Gaussian quadratures of high order: values for $n=64$, 80 , and $96, P$. Davis and $P$. Rabinowitz

Abscissas and weights for Gaussian quadratures of orders $n=64,80$, and 96 are given to twenty decimal places. 2 p.
RP2876. Mass spectra of aromatic hydrocarbons filtered from smoky air, F. L. Mohler, P. Bradt, and V. H. Dibeler

Public health service chemists collected particulate matter from smoky air and separated aliphatic and aromatic hydrocarbons from this material. These samples have been analyzed by two methods. In one method the sample is slowly evaporated from a tube furnace directly into the mass spectrometer and spectra recorded as the temperature is increased step by step. In the other method the sample is completely vaporized in a heated reservoir and the vapor passed through a leak into the mass spectrometer. Mass spectra of the aliphatic compounds are not reported in detail. Mass spectra of the aromatic fractions evaporated from the tube furnace show sharply defined fractionation with molecule ions of fused-ring aromatics predominant. Compounds containing three to seven fused rings are tentatively identified. The mass spectrum from the reservoir mass spectrometer was recorded at ionizing voltages of $70 \mathrm{v}$ and at a low voltage, which gives predominantly molecule ions. The fused-ring aromatics include compounds that are carcinogenic. $4 \mathrm{p}$.

TITLE PAGE AND CONTENTS TO VOLUME 60. $6 \mathrm{p}$.

RESEARCH PAPERS FROM JOURNAL OF RESEARCH OF THF NATIONAL BUREAU OF STANDARDS, VOLUME 61, JULYDECEMBER 1958

RP2877. The system lime-alumina-water at $1^{\circ} \mathrm{C}, \mathrm{E} . \mathrm{T}$. Carlson

A study was made of phase equilibria in the system $\mathrm{CaO}-\mathrm{Al}_{2} \mathrm{O}_{3}-\mathrm{H}_{2} \mathrm{O}$ at $1^{\circ} \mathrm{C}$. The stable phases are believed to be gibbsite, tetracalcium aluminate hydrate, and $\mathrm{Ca}(\mathrm{OH})_{2}$, but no solubility curve was established for gibbsite because of extreme slowness of reaction. The compound $3 \mathrm{CaO} \cdot \mathrm{Al}_{2} \mathrm{O}_{3} \cdot 6 \mathrm{H}_{2} \mathrm{O}$ appears to be metastable, being very slowly transformed to one or the other of the hexagonal hydrates. Metastable equilibrium curves were established for $\mathrm{CaO} \cdot \mathrm{Al}_{2} \mathrm{O}_{3} \cdot 10 \mathrm{H}_{2} \mathrm{O}$ and $2 \mathrm{CaO} \cdot \mathrm{Al}_{2} \mathrm{O}_{3} \cdot 8 \mathrm{H}_{2} \mathrm{O}$. The concentration at their intersection (a metastable invariant point) is about $0.10 \mathrm{~g}$ of $\mathrm{Al}_{2} \mathrm{O}_{3}$ and $0.38 \mathrm{~g}$ of $\mathrm{CaO}$ per liter. The dicalcium compound can readily be dried to a $6 \mathrm{H}_{2} \mathrm{O}$ stage, giving a characteristic $\mathrm{X}$-ray pattern. During the long storage period required to approach equilibrium, most of the reaction mixtures picked up a little $\mathrm{CO}_{2}$, apparently by absorption through the polyethylene containers, with formation of a carboaluminate, probably $3 \mathrm{CaO} \cdot \mathrm{Al}_{2} \mathrm{O}_{3} \cdot \mathrm{CaCO}_{3} \cdot 11 \mathrm{H}_{2} \mathrm{O} .11 \mathrm{p}$.

RP2878. Heat content of zirconium and of five compositions of zirconium hydride from $0^{\circ}$ to $900^{\circ} \mathrm{C}$, T. B. Douglas and A. C. Victor

Using a precise Bunsen ice calorimeter, a "silver core" furnace, and a "drop" method, the heat content (enthalpy) of zirconium metal and five samples of zirconium hydride (24 to 52 atomic percent hydrogen) was measured over the range $0^{\circ}$ to $900^{\circ} \mathrm{C}$. Thermal hysteresis of the hydrides was investigated in several cases, and corrections were applied for the impurities in the samples. At each temperature up to $550^{\circ} \mathrm{C}$ the heat contents of the hydrides relative to $0^{\circ} \mathrm{C}$ were found to vary linearly with composition, a fact in accord with the phase diagram of the system. The effective heat capacity of each hydride sample reached its maximum value between $550^{\circ}$ and $600^{\circ} \mathrm{C}$, a behavior that can be interpreted as due to the endothermic formation of the high-temperature beta phase. $11 \mathrm{p}$.

\section{RP2879. Uniform transient error, E. L. R. Corliss}

The economy of transient error is discussed. Equations describing error in power-level measurements of transients can be used to compute the design of analyzers so as to distribute transient error in a way compatible with experimental requirements. In addition, consideration of a limiting power-diserimination factor provides a measure of the largest number of bandpass filters that can be over- 
lapped on adjacent channels to yield meaningful information about a rapidly changing signal. A scanning filter can be compared with a set of bandpass filters by calculating the effective number of overlapped bandpass filters providing the same resolution as the scanning filter. The results are applicable to autocorrelation analyzers. Restrietions on rapid signal analysis also are considered for the special case in which power-level discrimination is limited by noise. $6 \mathrm{p}$.

RP2880. Optical T-bench method of measuring longitudinal spherical aberration, F. E. Washer

A method of measuring longitudinal spherical and chromatic aberration by visual means is described. The method employs an especially constructed optical T-bench equipped with nodal slide and an angle-measuring telescope. The underlying theory of the method is presented, together with a brief description of the apparatus used. The results of measurement on a few typical lenses and an analysis of the sources of error are included. $9 \mathrm{p}$.

RP2881. Mass spectrometric study of the rate of thermal decomposition of hydrazoic acid, J. L. Franklin, V. H. Dibeler, and P. P. Morris, Jr.

Hydrazoic acid vapor at low pressures $(0.15 \mathrm{~mm} \mathrm{Hg}$ or less), has been decomposed thermally and the rate of reaction studied mass spectrometrically at $265^{\circ}$ to $325^{\circ} \mathrm{C}$. Nitrogen and ammonia are the only reaction products, and no intermediates were observed. The disappearance of $\mathrm{HN}_{3}$ is first order and the reaction occurs on the walls of the glass reaction vessel. The experimental activation energy is 31 kilocalories per mole. The activation energy for the reaction $\mathrm{HN}_{3} \rightarrow \mathrm{NH}+\mathrm{N}_{2}$ must be at least 39 kilocalories per mole. $6 \mathrm{p}$.

RP2882. Erosion damage to solids caused by high-speed collision with rain, O. G. Engel.

The stresses that are produced when a liquid drop collides with the planar surface of a solid are discussed. These are a result of the impact pressure and the consequent radial flow of the drop. It is concluded that a rainerosion resistant material may be either soft and rubbery or hard and rigid. If it is soft and rubbery it mitigates the applied stresses but it must be able to withstand the mitigated stresses. If it is hard and rigid it does not mitigate the applied stresses and it must be able to withstand the unmitigated stresses. $6 \mathrm{p}$.

RP2883. Emission spectrum of carbon monoxide from 2.3 to 2.5 microns, E. K. Plyler, H. C. Allen, Jr., and E. D. Tidewell

The 2-0, 3-1, 4-2, 5-3, and 6-4 emission bands of carbon monoxide have been measured in the region from 4000 to $4360 \mathrm{~cm}^{-1}$ with a grating spectrometer. A 10,000 line per inch grating used in the second order resolved lines separated by $0.08 \mathrm{~cm}^{-1}$. Several sets of measurements were made on the $2-0$ band and the molecular constants were calculated. The constants in $\mathrm{cm}^{-1}$ are $B_{0}=1.922511 \pm$ $0.000025 D_{0}=6.13 \pm 0.02 \times 10^{-6}$. Using these values and the $B_{0}$ from 1-0 band in conjunction with the microwave constant a new determination of the speed of light is obtained, $c=299,794 \pm 3$ kilometers per second. $4 \mathrm{p}$.

RP2884. Further studies of the influence of a ridge on the low-frequency ground wave, J. R. Wait and A. Murphy

Computations are presented in graphical form for the perturbation of a plane wave by a semicylindrical boss on an otherwise flat ground plane of perfect conductivity. The height of the ridge is comparable to the wavelength. This is an extension of earlier work on the semielliptical boss. $4 \mathrm{p}$.
RP2885. Infrared studies on polymorphs of silicon dioxide and germanium dioxide, E. R. Lippincott, A. Van Valkenburg, C. E. Weir, and E. N.' Bunting

The infrared spectra of coesite, low-temperature tridymite, low-temperature cristobalite-low-temperature quartz, vitreous siliea, hexagonal $\mathrm{GeO}_{2}$, tetragonal $\mathrm{GeO}_{2}$, and vitreous germania are reported from 4,000 to $300 \mathrm{~cm}^{-1}$. Wherever possible an assignment of frequencies has been made on the basis of the selection rules for the crystal symmetry. Three characteristic group frequencies near $1,100,800$, and $480 \mathrm{~cm}^{-1}$ are common to all the polymorphs of $\mathrm{SiO}_{2}$. These frequencies respectively correspond to a stretching mode involving displacements associated primarily with the oxygen atoms, a stretching mode involving displacements associated primarily with the silicon atoms, and $\mathrm{a}$ Si-O bending mode. The presence of these group frequencies in coesite indicates that the coordination of silicon in coesite is tetrahedral and that its high density is associated with the packing of tetrahedral units at an angle approximating 120 degrees. The tetragonal and hexagonal $\mathrm{GeO}_{2}$ polymorphs show a marked difference in spectra due in part to the change from sixfold to fourfold coordination. The assignment of observed frequencies in hexagonal $\mathrm{GeO}_{2}$ is consistent with that made for lowtemperature quartz if allowance is made for the heavier mass of the Ge atom. $10 \mathrm{p}$.

RP2886. Carbon-14 carboxy-labeled polysaccharides, J. D. Moyer and $\mathrm{H}$. S. Isbell

Procedures are given for the preparation of $\mathrm{C}^{14}$ carboxydextran and $\mathrm{C}^{14}$ carboxyinulin. These materials have $\mathrm{C}^{14}$-labeled carboxyl groups in place of the reducing end groups present in the parent polysaccharides. The substances can be prepared cheaply and should find many applications as tracers in biological and chemical research. 3 p.

RP2887. Heat of formation of sodium calcium aluminate, E. S. Newman

The compounds $3 \mathrm{CaO} \cdot \mathrm{Al}_{2} \mathrm{O}_{3}, \mathrm{Na}_{2} \mathrm{SO}_{4} \mathrm{~V}$ (Thenardite), $\mathrm{Na}_{2} \mathrm{O} \cdot 8 \mathrm{CaO} \cdot 3 \mathrm{Al}_{2} \mathrm{O}_{3}$, and $\mathrm{CaSO}_{4}$, anhydrite, were prepared, and their heats of solution in $\mathrm{HCl}, 26.61 \mathrm{H}_{2} \mathrm{O}$ were determined. The heat of solution of $\mathrm{Na}_{2} \mathrm{SO}_{4} \mathrm{~V}$ in water to form $\mathrm{Na}_{2} \mathrm{SO}_{4}, 1000 \mathrm{H}_{2} \mathrm{O}$ was also measured. From these and other data in the literature the heats of formation of $\mathrm{Na}_{2} \mathrm{O} \cdot 8 \mathrm{CaO} \cdot 3 \mathrm{Al}_{2} \mathrm{O}_{3}$ and $\mathrm{NaSO}_{4} \mathrm{~V}$ were calculated to be -2567 and $-330.92 \mathrm{kcal} / \mathrm{mole}$, respectively. $2 \mathrm{p}$.

RP2888. Thermal degradation of polyacrylonitrile, polybutadiene, and copolymers of butadiene with acrylonitrile and styrene, S. Straus and S. L. Madorsky

Polybutadiene and a copolymer consisting of $\mathbf{7 6 . 5}$ percent butadiene and 23.5 percent of styrene were investigated as to the rates of their thermal degradation. The rates for polybutadiene indicated an activation energy of 60 kilocalories per mole. The copolymer had very high initial rates of degradation, followed by a rapid drop, so that it was not possible to obtain a reliable activation energy.

Polyacrylonitrile and a copolymer consisting of 31 percent of acrylonitrile and 69 percent of butadiene were investigated with regard to the nature and distribution of volatile products, as well as to the rates of their thermal degradation. The rates were very high initially, but dropped rapidly so that it was not possible to determine accurately the activation energy. The acrylonitrilebutadiene copolymer showed a decomposition pattern similar to that of polybutadiene. Here, too, the activation energy could not be deduced accurately from the rate curves. Comparative thermal stability in terms of temperature, at which half of the polymer sample is evaporated in 35 minutes of heating, is as follows: $407^{\circ} \mathrm{C}$ for poly- 
butadiene, $374^{\circ} \mathrm{C}$ for SBR, $364^{\circ} \mathrm{C}$ for polystyrene, $360^{\circ} \mathrm{C}$ for $\mathrm{NBR}$, and $316^{\circ} \mathrm{C}$ for polyacrylonitrile. $5 \mathrm{p}$.

RP2889. On the motion of two cylinders in an ideal fluid, L. H. Carpenter

The complex potential of two cylinders moving in an infinite liquid is determined by the method of image doublets, and the solution is expressed as an infinite series in rectangular coordinates. Approximate solutions in finite form are given for various cases. A method for generalizing the solution for the case of more than two cylinders is indicated. Applications to the flow induced by a cylinder moving in the presence of plane boundaries are given and the stream lines are illustrated in certain cases. 5 p.

RP2890. Properties of sodium titanium silicate glasses, E. H. Hamilton and Given W. Cleek

The glass-forming area of the $\mathrm{Na}_{2} \mathrm{O}-\mathrm{TiO}_{2}-\mathrm{SiO}_{2}$ system was surveyed. Glasses were made with refractive indices from 1.5184 to 1.8005 , nu values from 51.5 to 23.2 , and densities from 2.42 to 3.00 . The glasses have higher dispersions and have considerably lower densities than the $\mathrm{PbO}$ glasses with comparable refractive indices. $6 \mathrm{p}$.

RP2891. Wavelengths from thorium-halide lamps, W. F. Meggers and R. W. Stanley

The present system of international secondary standards of wavelength for spectroscopic measurements is based on interferometric determinations of wavelengths emitted at atmospheric pressure by an electric are between iron electrodes. Because of the poor quality and uneven distribution of these iron standards they are not suitable for accurate measurement of wavelengths in the spectra of heavier elements, most of which are more complex and consist of much sharper lines that the standards. Quartztube lamps containing a small quantity of a thorium halide, when excited by microwaves, emit thousands of uniformly sharp and evenly distributed lines whose wavelengths, or positions in a spectrum, can be determined with about one-tenth the error of locating iron-arc lines. Preliminary values of 222 vacuum wavelengths emitted by a thoriumiodide lamp have been measured relative to 5462.2705 and 4047.7144 A emitted by a similar lamp containing mercury198. Fabry-Perot interferometers with plate separations of 25,40 , or 50 millimeters were used with a stigmatic grating spectrograph in making these measurements. The thorium waveleng ths range from 3288.7356 to $6991.5839 \mathrm{~A}$ in vacuum and from 3287.7885 to $6989.6562 \mathrm{~A}$ in standard air. The accuracy in relative value of 27 classified thorium lines is tested by means of the combination principle, which indicates that the average error is less than 1 part in 20 million. 9 p.

RP2892. An electrical-analog method for transient heatflow analysis, A. F. Robertson and D. Gross

An electronic instrument is described which permits solution of transient heat-flow problems by use of direct analogy to electrical networks. The instrument is of the so-called "fast-time" type, simulating the transient involved in times of the order of $10^{-6}$ that of the thermal prototype. A photoformer type of input-signal generation is used and this, together with a variable-frequency master oscillator, permits flexibility of input-signal waveform and time scale.

Some applications of the instrument to the field of fire research are described. The discussion of errors includes consideration of those resulting from the lumping of circuit elements. $11 \mathrm{p}$.

RP2893. Effect of erystal field and spin-orbit coupling on magnetic susceptibility of systems with $f$ electron configuration, C. M. Herzfeld and D. B. Levine

The effects of a crystal field of octahedral symmetry and of spin-orbit coupling on an ion with $f^{2}$ electron con- figuration are considered. Only fourth-degree terms in the crystal-field potential are taken into account. The eigenvalues and eigenvectors of the resulting $33 \times 33$ secular determinant are determined numerically on the 704 electronic computer at the National Bureau of Standards for eight values of $D / \zeta$, where $D$ is a crystal-field parameter, and $\zeta$ the spin-orbit coupling constant of the ion. Perturbations by an external magnetic field are computed using second-order perturbation theory. All eigenvalues are tabulated for $D / \zeta=-10^{-4},-2 \times 10^{-4},-5 \times 10^{-4},-10^{-3}$ $-2 \times 10^{-3},-5 \times 10^{-3},-10^{-2},-10^{-1}$. They can be adapted directly to any finite nonzero positive value of $\zeta$ and, by interpolation, for values of $D / \zeta$ not tabulated. The magnetic susceptibility of a powder is calculated and displayed as a function of temperature and $D / \zeta$, for $\zeta=\infty$, $10^{3} \mathrm{~cm}^{-1}$, and $5 \times 10^{2} \mathrm{~cm}^{-1}$. The relation of the results of the calculations to measurements on compounds of $U^{+4}$ is discussed. $5 \mathrm{p}$.

RP2894. Vibration-rotation bands of ammonia: 1. The combination bands $\nu_{2}+\left(\nu_{1}, \nu_{3}\right)$, W. S. Benedict, E. K. Plyler, and E. D. Tidwell

A general discussion is given of methods used in the analysis of $\mathrm{NH}_{3}$ vibration-rotation spectra, including the derivation of molecular constants and the determination of line strengths and line widths. Results are given for the region 2.15 to $2.48 \mu\left(4,060\right.$ to $\left.4,700 \mathrm{~cm}^{-1}\right)$, in which more than 800 lines have been measured. These have been analyzed into the inversion-doubled perpendicular combination band $\nu_{2}+\nu_{3}\left(\nu_{0}=4416.908\right.$ and $4434.610 \mathrm{~cm}^{-1}$. band strength $=19.7 \mathrm{~cm}^{-2} \mathrm{~atm}^{-1}$ ) and the inversiondoubled parallel combination band $\nu_{1}+\nu_{2} \quad\left(\nu_{0}=4293.716\right.$ and $4320.060 \mathrm{~cm}^{-1}$; band strength $=2.9 \mathrm{~cm}^{-2} \mathrm{~atm}^{-1}$ ). Complete energy levels for these bands have been found up to $J=12$ and 10 , respectively, permitting determinations of molecular constants, which include numerous higherorder effects involving the interaction of rotation, vibration and inversion. The line widths range from a maximum of $0.57 \mathrm{~cm}^{-1} \mathrm{~atm}^{-1}$, when $K=J$ to a minimum of $<0.2 \mathrm{~cm}^{-1}$ atm $^{-1}$, when $K \ll J . \quad 25 \mathrm{p}$.

RP2895. Evaluation of tensile, compressive, torsional, transverse, and impact tests and correlation of results for brittle cermets, M. J. Kerper, L. E. Mong, M. B. Stiefel, and S. F. Holley

Static tests were studied for the determination of mechanical properties of brittle materials. Specimens of brittle materials, represented by cermets having five different compositions, were subjected to tensile, compressive, torsional, transverse, and impact tests. The designs of specimens and apparatus suitability of the tests to the materials, refinements in test procedures, and the variability of results and their correlation were studied. The elastic properties were obtained from tensile, compressive, and transverse tests, and the modulus of rigidity calculated from the results of these tests agreed with that from the torsional test. Tensile strength was obtained from the tensile, torsional, and transverse tests on specimens of comparable sizes in accordance with a limiting tensile strain. Shear strengths were obtained in the compressive tests. The correlation of impact values with mechanical properties was unsatisfactory. $21 \mathrm{p}$.

RP2896. Redetermination of mass spectra of deuteromethanes, F. L. Mohler, V. H. Dibeler, and E. Quinn

Mass spectra of the four deuteromethanes have been remeasured using samples of improved isotopic purity. The relative abundances of fragment ions involving loss of $\mathrm{H}$ or $\mathrm{D}$ atoms from the molecules $\mathrm{CH}_{3} \mathrm{D}, \mathrm{CH}_{3} \mathrm{D}_{2}$, and $\mathrm{CHD}_{3}$ are not proportional to the a priori probabilities of removing $\mathbf{H}$ or $\mathrm{D}$ atoms but have been expressed in terms of the a priori probability times a weighting factor. The weighting factor for removing one $\mathrm{H}$ or $\mathrm{D}$ atom from these molecules can be roughly expressed as positive and negative powers of a single constant $1.19 \pm 0.015$ and the same 
power law holds for the relative abundances of $\mathrm{H}^{+}$and $\mathrm{D}^{+}$. Weighting factors for removing two or more hydrogenic atoms are not consistent with this power law. 2 p.

RP2897. Effect of strain-temperature history on the tensile behavior of titanium and a titanium alloy, G. W. Geil and N. L. Carwile

This study was made to determine the effect of prestraining specimens in tension under uniaxial and multiaxial stresses at a selected temperature on their tensile behavior at a different temperature. Unnotched and notched cylindrical specimens of initially annealed, commercially pure titanium and initially annealed 4-percentaluminum, 4-percent-manganese, titanium alloy were extended at selected temperatures to various true strains and then extended to fracture at another temperature. The ductility retained by the prestrained specimens decreased with increase in the prestrain, whereas the strength indices generally were nearly independent of the amount of the prestraining. However, strength indices obtained on some of the deep-notched specimens of the alloy at $-196^{\circ} \mathrm{C}$ were decreased by prestraining at $+25^{\circ} \mathrm{C}$ as a direct result of the very low retained ductility of the prestrained specimens. $14 \mathrm{p}$.

RP2898. Propagation of very-low-frequency pulses to great distances, J. R. Wait

A theoretical study is presented for the propagation of electromagnetic pulses at very low frequencies to large distances. The space between the earth and the ionosphere is represented as a wave guide with sharply bounded and concentric spherical boundaries. The concept of phase and group velocity and its application to the present problem is discussed in some detail. The influence of the propagation medium on the shape of the envelope of a quasi-monochromatic pulse is also considered. Using an alternative approach, the response of an impulsive source is also calculated and is shown to be a damped oscillatory function of time with a quasi-half-period varying in a predictable manner with distance of travel in agreement with the observations of Norinder and Hepburn. $17 \mathrm{p}$.

RP2899. Transmission and reflection of electromagnetic waves in the presence of stratified media, J. R. Wait

A general analysis is presented for the electromagnetic response of a plane stratified medium consisting of any number of parallel homogeneous layers. The solution is first developed for plane-wave incidence and then generalized to both cylindrical and spherical-wave incidence. Numerical results for interesting special cases are presented and discussed. The application of the results to surfacewave propagation over a stratified ground is considered in some detail. $28 \mathrm{p}$.

RP2900. Pseudoternary system calcium oxide-monocalcium aluminate $\left(\mathrm{CaO} \cdot \mathrm{Al}_{2} \mathrm{O}_{3}\right)$-dicalcium ferrite $\left(2 \mathrm{CaO} \cdot \mathrm{Fe}_{2} \mathrm{O}_{3}\right)$, T. F. Newkirk and R. D. Thwaite

The system $\mathrm{CaO}-\mathrm{CaO} \cdot \mathrm{Al}_{2} \mathrm{O}_{3}-2 \mathrm{CaO} \cdot \mathrm{Fe}_{2} \mathrm{O}_{3}$ is of importance to portland cement chemistry because it provides information on the composition of the iron-bearing phase in cement clinker.

Iron-bearing solid solutions in the system were found to exist as a continuous narrow band extending from $2 \mathrm{CaO} \cdot \mathrm{Fe}_{2} \mathrm{O}_{3}$ to $6.45 \mathrm{CaO} \cdot 2.31 \mathrm{Al}_{2} \mathrm{O}_{3} \cdot \mathrm{F}_{2} \mathrm{O}_{3}$. Additional tie lines were established to show the composition of the iron-bearing solid solution phase (Fss) and liquid phases during the course of crystallization of mixtures within the system. Isotherms have been located for the regions of principal interest. Compatibility relations differ somewhat from those reported by previous investigators. The composition of the iron-bearing phase in equilibrium with $\mathrm{CaO}$ and $3 \mathrm{CaO} \cdot \mathrm{Al}_{2} \mathrm{O}_{3}$ at the ternary invariant point proved to be lower in $\mathrm{Fe}_{2} \mathrm{O}_{3}$ than that reported by Swayze. Other results are in general agreement with the work of Swayze and of Yamauchi. $13 \mathrm{p}$.

RP2901. Heats of formation of diborane and pentaborane, E. J. Prosen, W. H. Johnson, and F. Y. Pergiel

The heats of formation of diborane and pentaborane have been determined by measurements of the heats of decomposition into amorphous boron and hydrogen in a calorimeter. The heats of formation at $25^{\circ} \mathrm{C}$, from amorphous boron and hydrogen, are $6.73+0.52 \mathrm{kcal} / \mathrm{mole}$ for diborane (gas) and $12.99 \pm 0.39$ for pentaborane (gas). 4 p.

RP2902. Temperature of the inversion in cristobalite, R. F. Walker, S. Zerfoss, S. F. Hollev, and L. J. Gross

The inversion temperature of cristobalite prepared from pure precipitated silica gel was compared with that of less pure cristobalite, including material derived from quartz. All materials showed a variability in their inversion temperature which was dependent on their thermal history. However, evidence suggested that a sufficiently extended heat treatment at high temperatures would in each case produce a uniform product with invariant inversion temperatures attributable to the low-high and high-low inversions. It is proposed that the variability of the inversion temperature can be rationalized in terms of three rate processes dependent on the temperature of heattreatment, viz., nucleation, ordering, and crystal growth. The effect of all other factors known to influence the inversion temperature is either to accelerate or retard the rate of one or more of these processes. $11 \mathrm{p}$.

RP2903. Infrared emission spectra of flames under high resolution, E. K. Plyler and E. D. Tidwell

The spectrum of a hydrogen-oxygen flame and an oxyacetylene flame has been measured in the near infrared region with a high-resolution grating spectrometer. In the region from 3.7 to $4.1 \mu$ many lines are observed which have not been classified. They may arise from transitions belonging to the $\nu_{2}$ or $\nu_{3}$ bands of water vapor. The intensity of the first overtone band of $\mathrm{CO}$ was increased when a high-frequency discharge was passed through the flame and the infrared CN band was increased in intensity when $\mathrm{N}_{2} \mathrm{O}$ was mixed with the acetylene. The results are shown in five figures of the observed spectra, $6 \mathrm{p}$.

RP2904. Improved description of hafnium spectra, C. H. Corliss and W. F. Meggers

Earlier descriptions of atomic hafnium spectra, from conventional ares or sparks, have been handicapped by the absence of pure samples and by the presence of a strong background of molecular spectra. Recent availability of highly purified hafnium metal, and the development of a new light source, removed these handicaps. This improved description of hafnium spectra was made by employing electrodeless metal-halide lamps excited, at relatively low pressure and temperature, by microwaves. Lamps of hafnium iodide, hafnium bromide, and hafnium chloride were compared to recognize the spectra of halogens or their compounds and thus arrive at the atomic hafnium lines common to different lamps. The number of lines now ascribed to hafnium spectra exceeds 6,200 as compared with about 2,400 heretofore. The wavelengths range from 1284.88 to $12043.08 \mathrm{~A}$, and estimated relative intensities in different light sources (including ares, sparks, and electrodeless lamps) indicate which lines belong to HfI, HfII, HfII, and Hfrv. The splitting of spectral lines in magnetic fields (Zeeman effect) has also been improved by using magnetic fields of higher intensity, and greater spectrographic resolving power than before. The number of lines for which Zeeman patterns have been observed has been increased from 280 to 1,030 , and the types of complex 
patterns invariably confirm the assignments of lines to Hif or HfII, $56 \mathrm{p}$.

RP2905. Dielectric constant of hydrogen-bonded liquids, F. Buckley

A simple extension is presented of Onsager's electrostatic theory of dielectric polarization to take account of the "excess" polarization due to hydrogen bonding. Dielectric data for a number of liquid mixtures are analyzed and the deviations from ideal behavior expressed in terms of an excess polarization. A method is developed for calculating the excess polarization and certain related molecular parameters. Tentative values of these parameters have been determined for some particularly simple structures. $18 \mathrm{p}$.

RP2906. On the diffraction and reflection of waves and pulses by wedges and corners, F. Oberhettinger

Various problems arising in the theory of the excitation of a perfectly reflecting wedge or corner by a plane, cylindrical, or spherical wave, are dealt with. The incident wave is represented by a line source (acoustic or electromagnetic) parallel to the edge. The spherical wave is emitted by an acoustic point source or by a Hertz dipole with its axis parallel to the edge. The case of an incident plane wave field is obtained as the limiting case (for large distances of the source from the edge) of the cylindrical or spherical wave excitation. $23 \mathrm{p}$.

RP2907. A liquid-helium cold cell for use with an X-ray diffractometer, I. A. Black, L. H. Bolz, F. P. Brooks, F. A. Mauer, and H. S. Peiser

A liquid-helium cold cell for use with the General Electire X-ray goniometer has been designed and construeted. It is used to obtain X-ray diffraction patterns of polycrystalline solids deposited from the gaseous phase on a helium-cooled surface. This work is part of the program for studying the stabilization of free radicals. $5 \mathrm{p}$.

RP2908. The second spectrum of ruthenium (Ru II), A. G. Shenstone and W. F. Meggers

Wavelengths and estimated intensities of 2,227 spectral lines characteristic of singly ionized ruthenium atoms are presented. The wavelengths range from 1054.684 to $6371.29 \mathrm{~A}$. The Zeeman effect has been investigated for 488 lines ranging from 2323 to $4010 \mathrm{~A}$. Analysis of these basic data of $R u$ II has resulted in the classification of 1,633 lines as transitions between 68-even, and 140-odd energy levels. All but three of these levels have been grouped into designated spectral terms and assigned to electron configurations. The low-even terms arise from $4 d^{7}, 4 d^{6} 5 s$, and $4 d^{6} 5 s^{2}$. All the terms from the $4 d^{7}$ configuration have been found, and $4 d^{7} a^{4} \mathrm{~F}$ represents the ground state of Ru II. From two members of the $4 d^{6}$ $n s^{6} \mathrm{D}$ series a limit, $135200 \mathrm{~cm}^{-1}$, has been calculated which indicates a principal ionization potential (I. P.) of 16.76 electron volts for $\mathrm{Ru}^{+}$ions. $39 \mathrm{p}$.

RP2909. Self-ignition temperatures of materials from kinetic-reaction data, D. Gross and A. F Robertson

Results of experimental determinations of the kinetic constants of the self-heating reaction are presented for wood fiberboard, cotton linters, sugar pine, cork, crepe rubber, GRS rubber, natural, synthetic, and blended foam rubber (with and without additive), various oils (raw linseed, cottonseed, rapeseed, sperm, olive, castor, and neatsfoot) applied to cotton gauze in a ratio of 1 part of oil to 6 parts of cotton by weight, ammonium perchlorate, and nitrocellulose plastic. Under the assumption that self-heating follows a first-order reaction, these constants were used to calculate the critical radii of spherical piles for each of four surface temperatures likely to be experienced in long-period storage. Calculated self-ignition temperatures of piles of 1/6-inch-diameter to 22 -inch-diameter spheres of wood fiberboard and 1/8-inchdiameter to 2-inch-diameter spheres of cotton linters were in reasonable agreement with previous measurements by N. D. Mitchell (National Fire Protection Association Quarterly 45: 162, 1951). 5 p.

RP2910. Some studies of atmospheric transmittance on Mauna Loa, R. Stair and R. G. Johnston

Studies are described of the spectral intensity of solar radiation and its transmission through the atmosphere above a new 11,140-foot-altitude station on Mauna Loa, Hawaii, during May and June 1957. The spectral measurements covered the region from 300 millimicrons to 2.5 microns for different air masses from about 3.0 to 1.0 . The amounts of ozone and of water vapor which were present in the upper atmosphere were calculated from spectral data obtained on several different days. $7 \mathrm{p}$.

RP2911. Central notations for the revised ISCC-NBS color-name blocks, K. L. Kelly

Nickerson and Newhall published, in 1941, the central notations of the original ISCC-NBS (Inter-Society Color Council-National Bureau of Standards) color-name blocks which were used in the preparation of the soil and rock color-name charts. In 1955, the ISCC-NBS colorname blocks were revised to accord more closely with usage in the textile and other fields (NBS Circular 553). The central notations of these revised color-name blocks have been computed and are given in the present paper in tabular form. A color chart showing the central colors of the 267 ISCC-NBS color-name blocks would serve for rapid determination of the ISCC-NBS color designation, especially in field work where speed and ease of operation are more important than high accuracy. $5 \mathrm{p}$.

\section{RP2912. Viscosity of $n$-hexadecane, R. C. Hardy}

Attempts to prepare high-purity $n$-hexadecane from commercial cetane by simple laboratory procedures were unsuccessful. Fractional distillation at reduced pressure, of material previously treated with silica gel, produced a few small fractions of about 99.5-mole-pereent purity. The kinematic viscosities of the original material and of the fraction of highest purity, 99.63-mole percent, were found to be the same, 4.4635 centistokes at $20^{\circ}$. All other fractions had lower viscosities. The viscosity of NBS standard sample of $n$-hexadecane, 99.94-mole percent, was found to be 4.4642 centistokes or 3.4540 centipoises at $20^{\circ} \mathrm{C}$. $n$-Hexadecane is not likely to be suitable for use as a second calibration standard for viscometry until a simple, easily defined routine of purification is developed. $4 \mathrm{p}$.

RP2913. Revision of the phase equilibrium diagram of the binary system calcia-titania, showing the compound $\mathrm{Ca}_{4} \mathrm{Ti}_{3} \mathrm{O}_{10}$, R. S. Roth

The compound $\mathrm{Ca}_{4} \mathrm{Ti}_{3} \mathrm{O}_{10}$ has been found to be a stable phase in the system $\mathrm{CaO}_{-\mathrm{TiO}}$. It melts incongruently at about $1755 \pm 10^{\circ} \mathrm{C}$, presumably to $\mathrm{CaTiO}_{3}$ plus liquid. This compound is shown in a revision of the phase diagram for the system $\mathrm{CaO}-\mathrm{TiO}_{2} .4 \mathrm{p}$.

RP2914. Term analysis of the second spectrum of rhenium (Re II), W. F. Meggers, M. A. Catalán, and M. Sales

Wavelengths and estimated intensities of approximately 2,000 spectral lines emitted by singly ionized rhenium atoms were available for this analysis. Observed Zeeman patterns for 220 Re II lines facilitated finding and interpreting the first energy levels. The analysis continued until 49 low-even, and 85 high-odd levels were found. Transitions between these groups of levels account for 1,014 Re II lines including about 70 percent of the tota observed intensity. The ground state of $\mathrm{Re}^{+}$is described by the spectral term $5 d^{6}\left(a^{6} \mathrm{~S}\right) 6 s a^{7} \mathrm{~S}_{3} .21 \mathrm{p}$. 
RP2915. Density formula for alkali silicate glasses from annealing to glass-processing temperatures, L. W. Tilton

An additive formula is given for the computation of specific volumes of molten and compacted alkali silicate binary glasses with modifiers up to 50 -mole percent and from annealing temperatures $\left(400^{\circ}\right.$ or $\left.500^{\circ} \mathrm{C}\right)$ to $1,400^{\circ} \mathrm{C}$. The effective partial volumes, $v_{\mathcal{S}}$, for the silica are postulated as

$$
\left(1 / v_{s}\right)=2.198+r_{m} C_{S}(1723-t),
$$

where $r_{m}$ is the mole fraction of nonsilica and $C_{S}$ is a constant to be evaluated from glass-density data. This is based on the idea that silica networks can contract in volume as temperatures are lowered provided, and in proportion as, modifier ions are present in the glass. The effective partial volumes of $\mathrm{R}_{2} \mathrm{O}$, the nonsilica, are assumed as linear functions of temperature but not of the fraction of silica present.

Computed and observed densities agree within approximately 1 percent. The effective volumes of nonsilica are somewhat smaller than published estimates for the oxides themselves, as should be expected because of interpenetrations. $5 \mathrm{p}$.

RP2916. Thermodynamic properties of gases at high temperature: 1 . Chemical equilibrium among molecules, atoms, and atomic ions considered as clusters, H. W. Woolley

The equilibrium thermodynamic properties of gaseous mixtures at high temperatures are treated by an extension of the cluster theory of Ursell, omitting the assumption of additivity of pair energies. An effective partition function is introduced, which is a convenient function in expressing the increase of the partition function for the entire gas due to the joining together of the parts of the cluster. The law of mass action and the dependence of second and third virial coefficients upon the cluster integrals for pairs and triples of molecules in a mixture have been obtained therefrom. Extension to a partially ionized gas is made by incorporating Mayer's cluster-based extension of the Debye-Hückel theory. 22 p.

RP2917. Parallel testing interferometer, J. B. Saunders

The conventional methods of testing the parallelism of opaque bodies, such as gage blocks, by interferometry require wringing of the body to an optical flat. This operation disturbs the temperature equilibrium, necessitating long periods between tests, especially for long blocks. It often injures the surfaces of both the optical flat and the test body. Also, if the body is a standard gage block, repeated wringings during use ultimately change the dimension. This paper describes an interferometer for measuring the parallelism of gage blocks and other bodies of any reasonable length without the necessity of the wringing operation. Two forms of this instrument are used-one for testing long blocks and another for testing short blocks. Either form can be constructed for testing blocks of any length, but two forms are found to be more practical. $8 \mathrm{p}$.

RP2918. Correction for instrumental drift in flame photometry, B. W. Mulligan and A. F. Haught

The application of the principles of experimental design to correct for drift in instrumental measurements, specifically in flame photometry, is described. Based on the procedure proposed by Youden, samples and reference standards may be measured in combinations of pairs in a manner such that errors caused by drift can be minimized and the actual amount of drift can be determined. Where the drift is dependent on the magnitude of the quantity measured, grouping by magnitude prior to final measurement is necessary. Application of the procedure to flame photometric determination of alkali elements, under conditions of severe drift, resulted in improving coefficients of variation from an original range of 2.0 to 3.5 to a range of 0.6 to 1.2 . $3 \mathrm{p}$.

RP2919. Mechanism of stress-corrosion cracking in the AZ31B magnesium alloy, H. L. Logan

The mechanism of stress-corrosion cracking of annealed AZ31B magnesium alloy in an aqueous $\mathrm{NaClK}_{2} \mathrm{CrO}_{4}$ solution was investigated. Cracking was predominantly an electrochemical process and was shown to be dependent on the rate of strain in the specimen following loading in tension. It is postulated that eracks develop if the protective film on the metal surface is ruptured (over narrow segments of the specimen) at a greater rate than it is repaired in the corroding medium. This would expose film-free metal that is anodic to the filmed metal. $6 \mathrm{p}$.

RP2920. Evaluation of lens distortion by visual and photographic methods, F. E. Washer, W. P. Tayman, and W. R. Darling

The evaluation of lens distortion by photographic and visual methods is discussed. Measurements made on a single lens using the two methods are reported. The precision of measurement of each method is determined which shows that the observed differences must be attributed to systematic error. Various sources of systematic error are considered. Uncompensated differential plate tipping is identified as the most probable cause of the observed differences. A method of correction is developed. It is concluded that when work is done with extreme care and with due account taken of various insidious sources of error, it is possible to achieve comparable results with either method. $7 \mathrm{p}$.

TITLE PAGE AND CONTENTS TO VOLUME $61.6 \mathrm{p}$.

RESEAROH PAPERS FROM JOURNAL OF RESEARCH OF THE NATIONAL BUREAU OF STANDARDS, VOLUME 62 , JANUARY-JUNE 1959

RP2921. Heat of mixing of polybutadiene and benzene, R. S. Jessup

Measurements have been made of the heat of mixing of polybutadiene and benzene at $26.9^{\circ} \mathrm{C}$ by means of a Bunsen-type calorimeter, in which diphenyl ether is the calorimetric medium. The results of these measurements can be represented by the empirical equation

$$
\Delta H^{M}=12.0 v_{1} v_{2},
$$

where $\Delta H^{M}$ is heat of mixing in joules per $\mathrm{cm}^{3}$, and $v_{1}$ and $v_{2}$ are volume fractions of solvent and polymer, respectively The data have been combined with previously reported data on the activity of the solvent in this system to obtain values of $\Delta \bar{S}_{1}$, the partial molal entropy of dilution. The curve of $\Delta \bar{S}_{1} / R v_{2}^{2}$ versus $v_{2}$ for this system lies above the calculated curve of $\Delta \bar{S}_{1}^{*} / R v_{2}^{2}$, where $\Delta \bar{S}_{1}^{*}$ is the "configuartional entropy" of dilution, and also lies above the corresponding experimental curves for several other polymersolvent systems. 5 p.

RP2922. Infrared high-resolution grating spectrometer, E. K. Plyler and L. R. Blaine

An infrared grating spectrometer which can be used as a single- or double-pass instrument has been built and set in operation. The collimating mirror has a focal length of 235 centimeters and the instrument has the highest resolution from 1,600 to $3,500 \mathrm{~cm}^{-1}$. In order to have flexibility in scanning the spectra at different speeds, a special drive mechanism has been built which is made of spur gears and worm gears. The speeds range from 2.5 to 200 minutes of time per degree of rotation. Examples of the spectra resolved by the instrument are given. Lines separated by $0.05 \mathrm{~cm}^{-1}$ are completely resolved and two lines separated by $0.025 \mathrm{~cm}^{-1}$ are partially resolved. $3 \mathrm{p}$. 
RP2923. Determination of titanium, zirconium, niobium, and tantalum in steels: separations by anionexchange, J. L. Hague and L. A. Machlan

A procedure is described for the determination of titanium, zirconium, niobium, and tantalum in steel. Thest elements are concentrated by selective precipitation with cupferron from a hydrochloric acid solution of the sample, and then ignited to the oxides. The oxides are fused, and dissolved in a hydrochloric-hydrofluoric acid mixture. Three separate eluates, containing titanium and zirconium, niobium, and tantalum, result from elution with mixtures containing ammonium chloride, hydrochloric acid, and hydrofluoric acid from a column of Dowex-1 anion-exchange resin. Boric acid is added to the eluates to complex the hydrofluoric acid, and the elements are precipitated with cupferron. Titanium is determined by the hydrogenperoxide method, and zirconium by the phosphategravimetric method. Niobium and tantalum are determined by the hydroquinone- and pyrogallol-photometric methods, or by weighing the oxides. $9 \mathrm{p}$.

RP2924. Preparation of new solution standards of radium, W. B. Mann, L. L. Stockmann, W. J. Youden, A. Schwebel, P. A. Mullen, and S. B. Garfinkel

New radium-solution standards have been prepared in the ranges of 10 micrograms and also $10^{-8}$ and $10^{-11}$ gram of radium element. These have been compared with the National Bureau of Standard's 1940 and 1947 series of radium-solution standards and, as a result of these comparisons, it has been found that the $194010^{-9}$ and $10^{-11}$. gram solution standards contained some 2 to 3 percent more radium element than certified. It has been shown that this difference probably arose in the dilution of the 1940 standards. $6 \mathrm{p}$.

RP2925. Phase equilibrium relations in the binary system lead oxide-niobium pentoxide, R. S. Roth

The phase equilibrium diagram for the binary system lead oxide-niobium pentoxide has been constructed from observations of fusion characteristics and X-ray diffraction data. The system contains six binary compounds with $\mathrm{PbO}: \mathrm{Nb}_{2} \mathrm{O}_{5}$ ratios of $3: 1,5: 2,2: 1,3: 2,1: 1$, and $1: 2$. The compound $\mathrm{PbO} \cdot \mathrm{Nb}_{2} \mathrm{O}_{5}$ was found to melt congruently at $1,343^{\circ} \mathrm{C}$ and have a stable, reversible phase transformation temperature from the low-temperature rhombohedral form to the high-temperature tetragonal form at $1,150^{\circ} \mathrm{C}$. The $5: 2,2: 1$, and $1: 2$ compounds melt congruently at $1,220^{\circ}, 1,233^{\circ}$, and $1,337^{\circ} \mathrm{C}$, respectively; the $3: 1$ and $3: 2$ compounds melt incongruently at $985^{\circ}$ and $1,233^{\circ} \mathrm{C}$, respectively. $12 \mathrm{p}$.

RP2926. Mass spectra of some deuterostyrenes, E. I. Quinn and F. L. Mohler

Mass spectra of alpha, beta, and para deuterostyrene; beta, beta dideuterostyrene; and alpha, beta, beta trideuterostyrene have been measured. The spectra are compared with the intensity distribution computed on the assumption of a random distribution of deuterium in $\mathrm{C}_{8} \mathrm{H}_{8}$. This gives a fair approximation to the observed spectra. The spectra of doubly charged styrenes are computed on the basis of observed half integer peaks in $\mathrm{C}_{8} \mathrm{H}_{8}$ and $\mathrm{C}_{8} \mathrm{H}_{7} \mathrm{D}$. The metastable transition peaks of the deuterostyrenes have been recorded. It is suggested that ionized styrene molecules may form an octatetraene ring which dissociates with a random distribution of deuterium atoms but the observed spectra are not completely random and there may be other modes of fragmentation. $4 \mathrm{p}$.

RP2927. Heat of reaction of diborane with water and the heat of formation of boric oxide, E. J. Prosen, W. H. Johnson, and F. Y. Pergiel

The heat of reaction of gaseous diborane with water to torm gaseous hydrogen and a solution of boric acid $\left(\mathrm{H}_{3} \mathrm{BO}_{3}+1,000 \mathrm{H}_{2} \mathrm{O}\right)$ has been measured by passing gase- ous diborane through two successive bubblers in a calorimeter. The heat of the reaction obtained was:

$$
\begin{gathered}
\Delta \mathrm{H}_{298.15}=-466.34 \pm 2.23 \mathrm{kj} / \mathrm{mole} \\
\text { mole }) .
\end{gathered}
$$

Combination of this value with values previously reported for the heat of decomposition of diborane and the heat of solution of boric oxide yields the following value for the heat of formation of erystalline boric oxide from amorphous boron and gaseous oxygen:

$$
\Delta \mathrm{Hf}_{298 \cdot 15}=-1280.89 \pm \begin{aligned}
& \pm .14 \mathrm{kj} / \mathrm{mole} \\
& \mathrm{kcal} / \mathrm{mole}) .
\end{aligned}
$$

RP2928. Heat of formation of titanium tetrachloride, W. H. Johnson, R. A. Nelson, and E. J. Prosen

The heat of formation of gaseous titanium tetrachloride has been measured by the reaction of metallic titanium with gaseous chlorine in a calorimeter. The value for the heat of formation obtained in this investigation corresponds to the reaction:

$$
\mathrm{Ti}_{(c)}+2 \mathrm{Cl}_{2(s)}=\mathrm{TiCl}_{4(s)},
$$

$\Delta \mathrm{Hf}^{\circ}\left(25^{\circ} \mathrm{C}\right)=-763.2 \pm 2.9 \mathrm{kj} / \mathrm{mole}(-182.4 \pm 0.7 \mathrm{kcl} /$ mole).

Earlier data are discussed briefly. 4 p.

RP2929. Determination of niobium and tantalum in titanium-base alloys, J. L. Hague and L. A. Machlan

A procedure for the determination of niobium and tantalum in titanium-base alloys is described. The sample is dissolved in a mixture of hydrochloric and hydrofluoric acid, and oxidized with a minimum of nitric acid. The resulting solution is transferred to a column containing a strong quaternary amine anion-exchange resin (Dowex-1). Titanium and other alloying elements, except niobium and tantalum, are removed by elution with an ammonium chloride-hydrochloric-hydrofluoric acid solution. Niobium is removed by elution with an ammonium chloridehydrofluoric acid solution. Tantalum is collected in a separate eluate of slightly acid ammonium chloride-ammonium fluoride solution. Boric acid is added to complex the fluoride, the earths acids precipitated with cupferron, ignited to the pentoxides, and weighed. $5 \mathrm{p}$.

RP2930. Properties of zinc borosilicate glasses, E. H. Hamilton, R. M. Waxler, and J. M. Nivert, Jr.

The glass-forming region of the system $\mathrm{ZnO}-\mathrm{B}_{2} \mathrm{O}_{3}-\mathrm{SiO}_{2}$ was surveyed. Glasses were made with compositions within the following limits in mole percent: 50 to $62.5 \mathrm{ZnO}$, 20 to $43.5 \mathrm{~B}_{2} \mathrm{O}_{3}$, and 0 to $20 \mathrm{SiO}_{2}$. They can be melted and poured below $1,400^{\circ} \mathrm{C}$. The glasses have low coefficients of thermal expansion, high values of elastic moduli and Poisson's ratio, and refractive indices from 1.6409 to 1.6798. 4 p.

RP2931. Design and performance of a block-type osmometer, D. McIntyre, G. C. Doderer, and J. H. O'Mara

A block-type osmometer has been designed and constructed utilizing several new features which enhance the precision of the osmotic-pressure measurement and allow simpler manipulative techniques. The details of the construction as well as a discussion of the design and performance of this osmometer are included in the paper. $4 \mathrm{p}$.

RP2932. Heat of formation of titanium tetrabromide, R. A. Nelson, W. H. Johnson, and E. J. Prosen

The heat of formation of solid titanium tetrabromide has been measured by the reaction of titanium metal with 
gaseous bromine in a calorimeter. The following value for the heat of reaction was obtained:

$$
\mathrm{Ti}(\mathrm{c})+2 \mathrm{Br}_{2}(\mathrm{~g})=\mathrm{TiBr}_{4}(\mathrm{c})
$$

$\Delta \mathrm{Hf}^{\circ}\left(25^{\circ} \mathrm{C}\right)=$

$$
-678.16 \pm 4.60 \mathrm{kj} / \mathrm{mole}(-162.08 \pm 1.10 \mathrm{keal} / \mathrm{mole}) \text {. }
$$

Combination of this value with the heat of vaporization of bromine yields the standard heat of formation of $\mathrm{TiBr}_{4}(\mathrm{c})$ from $\mathrm{Ti}(\mathrm{c})$ and $\mathrm{Br}_{2}(\mathrm{liq})$ as $\Delta \mathrm{Hf}^{\circ}\left(25^{\circ} \mathrm{C}\right)=$ $-616.72 \pm 4.60 \mathrm{kj} / \mathrm{mole}(-147.40 \pm 1.10 \mathrm{kcal} / \mathrm{mole}) .3 \mathrm{p}$.

RP2933. Adsorption of polyesters on glass, silica, and alumina, R. R. Stromberg, A. R. Quasius, S. D. Toner, and M. S. Parker

The adsorption of several saturated linear polyesters has been studied under a variety of conditions. The study also included di- $n$-butyl sebacate as a model low molecular weight ester and poly(methyl methacrylate). The adsorbents were type $\mathrm{E}$ glass powder, silica, and alumina. The glass powder and silica were nonporous materials; the alumina contained a large internal pore structure. The rate of adsorption of the polyesters on glass was very rapid and the adsorpt on isotherm was irreversible. Adsorption from a poor solvent was favored over adsorption from a good solvent. A large number of sites are available on the adsorbent for bond formation and the adsorbed polymer most probably extends into the solution in a coiled configuration. Metallic ions, in addition to silicon, appear to be involved in an electrostatic bond between the polymer and the glass. The Langmuir equation is obeyed at high-equilibrium concentrations, although there is noneonformity at the low concentrations. $7 \mathrm{p}$.

RP2934. Theory of the effect of drag on the orbital inclination of an earth satellite, J. P. Vinti

The rotation of the earth's atmosphere with the earth gives rise to a change in the inclination of the orbit of an earth satellite. In this paper it is assumed that the drag is in the direction of the air velocity relative to the satellite and that its magnitude diminishes so rapidly with altitude that it is appreciable only near perigee in an elliptic orbit. Without further assumptions about the properties of the drag function, results are then deduced for the secular changes in inclination up to, but not including, the final ballistic stage of motion. $10 \mathrm{p}$.

\section{RP2935. On a theorem of M. Riesz, M. Pearl}

The structure of the set of skew-symmetric, orthogonal matrices of order 4 with elements in the real field is studied. The first and second regular representations of the quaternions are used to represent these matrices and known properties of the quaternions are exploited to obtain characterizations of the skew-symmetric matrices. In the second part of this paper, the underlying structure of the geometry is changed from the Euclidean to the Lorentzian. It is then shown that there are no skew-symmetric, orthogonal (in the Lorentzian sense) matrices of order $4.6 \mathrm{p}$.

RP2936. Refinement of the crystal structure of triclinic magnesium pyroborate, S. Block, G. Burley, A. Perloff, and R. D. Mason, Jr.

The triclinic modification of $\mathrm{Mg}_{2} \mathrm{~B}_{2} \mathrm{O}_{5}$ was classified as a member of an isostructural series which also includes $\mathrm{Mn}_{2} \mathrm{~B}_{2} \mathrm{O}_{5}, \mathrm{Fe}_{2} \mathrm{~B}_{2} \mathrm{O}_{5}$, and $\mathrm{Co}_{2} \mathrm{~B}_{2} \mathrm{O}_{5}$. The erystal strueture was refined by the use of successive Fourier projections and a least-squares refinement program on an electronic computer. The structure of $\mathrm{Mg}_{2} \mathrm{~B}_{2} \mathrm{O}_{5}$ consists of $\mathrm{B}_{2} \mathrm{O}_{5}-4$ groups and $\mathrm{Mg}^{+2}$ ions. Each magnesium atom is surrounded octahedrally by six oxygen atoms, forming an extended sheet of octahedra. The pyroborate groups share corners with octahedra in the sheets and thus link them. Each boron atom is in planar triangular coordination with three oxygen atoms. The pyroborate group has two such triangles with one shared oxygen. The angle between the planes formed by the triangles is $16^{\circ}$. The average boron-to-oxygen distance is $1.38 \mathrm{~A}$. $6 \mathrm{p}$

RP2937. Heat transfer in laminar flow through a tube, M. Abramowitz, W. F. Cahill, and C. Wade, Jr.

The problem of heat transfer due to laminar flow of a viscous fluid in a channel is studied under the assumption that there is a parabolic distribution of velocity. The effect of axial temperature changes are considered and the solution is based on the simpler situation where axial effects are discussed. The solution, obtained by the method of least squares, is represented in terms of a set of nonorthogonal characteristic functions. These functions and the corresponding characteristic values are determined by numerical integration employing the Runge-Kutta procedure. Finally, asymptotic developments are obtained which are useful in the limiting cases. $5 \mathrm{p}$.

RP2938. Fluorination of haloaromatic compounds, R. E. Florin, W. J. Pummer, and L. A. Wall

The reactions of $\mathrm{BrF}_{3}, \mathrm{CLF}_{3}$, and $\mathrm{IF}_{5}$ with $\mathrm{C}_{6} \mathrm{Cl}_{6}, \mathrm{C}_{6} \mathrm{Br}_{6}$, $\mathrm{C}_{6} \mathrm{Cl}_{5}-\mathrm{CF}_{3}$, and other haloaromatic compounds are described. These reactions are not readily controlled, and explosions frequently occurred, particularly when $\mathrm{BrF}_{3}$ and $\mathrm{ClF}_{3}$ were used. Dehalogenation of the reaction products led to certain aromatic fluorocarbons, for example, $\mathrm{C}_{6} \mathrm{ClF}_{5}$ and $\mathrm{C}_{6} \mathrm{ClF}_{4}-\mathrm{CF}_{3}$. Completely fluorinated aromatic compounds were not easily obtained, and therefore the process is not recommended for the production of these species. 6 p.

RP2939. Synthesis of some disubstituted 3,4,5,6-tetrafluorobenzenes, W. J. Pummer, R. E. Florin, and L. A. Wall

The preparation of 1,2,3,4-tetrafluorobenzene from 2-chloroheptafluorotoluene in a two-step synthesis is described. A new method for the hydrolysis of the trifluoromethyl group was developed using alumina and steam at high temperatures. The products obtained from the hydrolysis of 2-chloroheptafluorotoluene were 2-chloro-3,4,5,6-tetrafluorobenzoyl fluoride and 2,3,4,5tetrafluorochlorobenzene, the decarboxylated product. The latter compound was converted to 1,2,3,4-tetrafluorobenzene by reduction with hydrogen and palladium at $280^{\circ} \mathrm{C}$ and atmospheric pressure. Some of the new compounds prepared, in addition to those mentioned previously, include 2-chloro-3,4,5,6-tetrafluorobenzoic acid, 2-chloro-3,4,5,6-tetrafluoroiodobenzene, and 1,2-diiodo3,4,5,6-tetrafluorobenzene. $5 \mathrm{p}$.

RP2940. Reactions of aromatic fluorocarbons with hydrogen, R. E. Florin, W. J. Pummer, and L. A. Wall

Various palladium and platinum catalysts were used to effect the following hydrogenation reactions at relatively high conversions and yields:

$$
\begin{gathered}
\mathrm{C}_{6} \mathrm{~F}_{4} \mathrm{ClH} \underset{280^{\circ}}{\stackrel{\mathrm{Pd}}{\longrightarrow}} \mathrm{C}_{6} \mathrm{~F}_{4} \mathrm{H}_{2} \\
\mathrm{C}_{6} \mathrm{~F}_{4} \mathrm{ClC} \mathrm{F}_{3} \underset{280^{\circ}}{\stackrel{\mathrm{Pd}}{\longrightarrow}} \mathrm{C}_{6} \mathrm{~F}_{4} \mathrm{HCF}_{3} \\
\mathrm{C}_{6} \mathrm{~F}_{6} \underset{300^{\circ}}{\stackrel{\mathrm{Pt}}{\longrightarrow}} \mathrm{C}_{6} \mathrm{~F}_{5} \mathrm{H}, \mathrm{C}_{6} \mathrm{~F}_{4} \mathrm{H}_{2}
\end{gathered}
$$

Nickel could be used for some of the reactions, but with much greater difficulty. The products of these reactions can be iodinated and brominated, and hence are a starting 
point for the synthesis of many new derivatives of aromatic fluorocarbons. $4 \mathrm{p}$.

RP2941. Exponential integral $\int_{1}^{\infty} e^{-x t} t^{-n} d t$ for large values of $n$, W. Gautschi

An asymptotic expansion is given which is well suited for numerical computation when $n$ is large and $x$ arbitrary positive. $3 \mathrm{p}$.

RP2942. Effect of oxygen on the bonding of gold to fused silica, D. G. Moore and H. R. Thornton

Gold pellets were melted on plaques of polished fused silica in vacuum and then heated for 15 minutes at $1,100^{\circ} \mathrm{C}$ under different oxygen pressures. The force required at room temperature to shear the solidified pellets from the silica surface was found to vary from zero for gold melted in vacuum to 725 pounds per square inch for gold melted under an oxygen pressure of 150 millimeters of mercury. In all cases where bond developed, fracture occurred in the silica rather than at the gold-silica interface. Silica plaques with pellets bonded to them by treatment in oxygen showed no measurable strain when examined with a polarograph, suggesting that the gold had yielded as the specimen cooled.

Tests using $\mathrm{Au} \mathrm{u}^{198}$ as a tracer indicated that gold diffused into the silica lattice at high oxygen pressure but that no diffusion occurred during vacuum heating. Observations made on molten droplets showed no correlation between the contact angles in air and vacuum and the apparent shear strengths. A possible explanation is included to account for this lack of agreement with theory. $9 \mathrm{p}$.

RP2943. Interferometer for large surfaces, J. B. Saunders and F. L. Gross

An interferometer is described that permits the testing of large areas, such as layout plates. The extension to large areas is obtained by causing a collimated beam of light to reflect from the specimen at a large angle of incidence. The resultant fringe pattern is a contour map of the surface relative to an arbitrarily chosen plane and the contour interval is a function of wavelength and angle of incidence. $3 \mathrm{p}$.

RP2944. Glass formation in polymers: II. The system rubber-sulfur, G. M. Martin and L. Mandelkern

Volume versus temperature relations encompassing a wide range in temperature and composition have been determined for a series of natural rubber-sulfur vulcanizates and mixtures. The characteristic temperature of glass formation is deduced from these studies. The glass temperature is found to increase continuously from $-69^{\circ}$ to $+90^{\circ} \mathrm{C}$ in the vulcanizates as the amount of bound sulfur is increased and to be invariant with the composition in the mixtures. A qualitative interpretation of these results is given in terms of the free-volume theory of glass formation. $6 \mathrm{p}$.

RP2945. Elastic deformations in strips with holes loaded through pins, M. Chi and L. K. Irwin

Bickley's solution for the generalized plane stress in a plate loaded through a pin is extended to give the deformation at any point in a plate loaded through one or two pins which are on the same load line. Analytical expressions are derived that are known to apply to a finite strip with a width $\geq 14 a$ and distance between holes $\geq 8 a$, where $a$ is the radius of the hole. Numerical examples are developed and presented in the form of graphs for typical cases. $5 \mathrm{p}$.

RP2946. Absolute light-seattering photometer: I. Design and operation, D. MeIntyre and G. C. Doderer

A new light-scattering photometer has been designed and built for determining the absolute scattering from polymer solutions. The instrument is also capable of performing as a research instrument for making measurements at very low and very high angles, and at very low and very high intensities of scattered light. The instrument scans the angular scattering either manually or automatically while measuring continuously the ratio of the scattered light to the incident light. $7 \mathrm{p}$.

RP2947. Resolution of the dissociation constants of $d, l$ malic acid from $0^{\circ}$ to $50^{\circ} \mathrm{C}$., M. Eden and R. G. Bates

A method for the precise determination of the dissociation constants for the two overlapping dissociation steps of a weak dibasic acid from the same set of experimental data is described. The procedure, based on an earlier suggestion of Speakman (J. Chem. Soc. 1940, 855), is applied to the resolution of the thermodynamic dissociation constants of $d, l$-malic acid at intervals of five degrees from $0^{\circ}$ to $50^{\circ} \mathrm{C}$. The constants were derived from measurements of the electromotive force of cells of the type

$$
\begin{aligned}
& \text { Pt; } \mathrm{H}_{2}(\mathrm{~g}) \text {, malate buffer solution, } \\
& \qquad \mathrm{KCl}(m=0.008), \mathrm{AgCl} ; \mathrm{Ag} .
\end{aligned}
$$

The 39 buffer solutions were prepared from potassium hydrogen malate and from mixtures of potassium hydrogen malate with perchloric acid or potassium hydroxide, and each contained potassium chloride. The first association constant, $K_{1}$, is $3.48 \times 10^{-4}$ at $25^{\circ} \mathrm{C}$. It is given as a function of absolute temperature $(T)$ by

$$
-\log K_{1}=\frac{1358.85}{T}-5.1382+0.013550 T \text {. }
$$

The second constant, $K_{2}$, is $7.99 \times 10^{-6}$ at $25^{\circ} \mathrm{C}$. It is given by

$$
-\log K_{2}=\frac{1658.53}{T^{\prime}}-6.2364+0.019353 T .
$$

The thermodynamic quantities $\Delta G^{\circ}, \Delta H^{\circ}, \Delta S^{\circ}$, and $\Delta C_{p}^{\circ}$ related to each dissociation step were computed and compared with the corresponding constants for other acids closely related structurally to malic acid. $10 \mathrm{p}$.

RP2948. Capacity requirement of a mail sorting device, B. K. Bender and A. J. Goldman

A mathematical model of a sorting device suggested by $\mathrm{S}$. Henig is considered. The relevant parameters are $r$ (the number of destinations for the mail) and $k$ (the number of letters entering the device during each cycle of operation). It is shown that the device will never jam if its capacity is at least $(r-1)(k-1)+k$ letters; this is the lowest possible capacity requirement, and for realistic values of $r$ and $k$ is significantly less than the previously known estimate $r^{2} k$. $3 \mathrm{p}$.

RP2949. Effect of internal radiant heat transfer on temperature distribution, thermal stress, and deflection in box beams, S. Goodman, S. B. Russell, and C. E. Noble

Thirteen box beams were analyzed to determine the effect of internal radiant heat transfer on temperature distribution, thermal stress, and deflection. A rough experimental check of temperature distribution and beam deflection was made for one case. For maximum beam temperatures above $700^{\circ}$ to $900^{\circ} \mathrm{F}$, change in emittance of the interior surfaces of the beam had an appreciable effect on the cover (but not the web) temperatures and to an even greater extent, on the beam deflection. At maximum beam temperatures of $1,200^{\circ} \mathrm{F}$, an increase in interior surface emittance appreciably relieved the maximum thermal stress. $7 \mathrm{p}$. 
RP2950. Mean absolute value and standard deviation of the phase of a constant vector plus a Rayleighdistributed vector, J. R. Johler and L. C. Walters

The mean absolute value of the phase and the standard deviation of the phase of a constant vector plus a Rayleighdistributed vector are determined by an evaluation of the first and second moment integrals of the probability distribution for various values of average relative intensity of the random Rayleigh-distributed component. The results of a quadrature evaluation of the integrals are tabulated over a wide range of values of average relative intensity $\left(k^{2}=0.010\right.$ to 1,000$)$. $4 \mathrm{p}$.

RP2951, Simple rotating molecular still, G. S. Ross and L. J. Frolen

A simple molecular still has been designed to purify materials which have a low vapor pressure and which are thermally unstable. The apparatus contains no ground glass joints and the distillation is accomplished in a completely closed system. The efficiency of the still was found to be better than that for either the pot-type or the falling film-type molecular stills generally used. $1 \mathrm{p}$.

RP2952. Scintillation counter method of intercomparing neutron source strengths by means of a manganous sulfate bath, E. R. Mosburg, Jr.

It has proved feasible to use a scintillation counter to measure the relative activity of a large manganous sulfate bath, thereby determining the neutron-emission rates of neutron sources relative to the national standard neutron source of the National Burcau of Standards. A scintillation counter allows the calibration of sources weaker by a factor of seven than those it was previously possible to calibrate by means of a Geiger-Muller counter. The use of $\mathrm{Cs}^{137}$ as a reference pulse height to which the scintillation counter system is adjusted by means of a two channel pulse height analyzer makes possible the reliable reproduction of results from day to day. By using the scintillation counter the ratio of the national reference standard neutron source (NBS-II) to the national standard neutron source (NBS-I) has been redetermined and is found to be $0.939_{3} \pm 0.001_{7}$. $3 \mathrm{p}$.

RP2953. Phase equilibria in the subsystem barium disilicate-dibarium trisilicate, $R$. S. Roth and E. M. Levin

A revised phase equilibrium diagrain for the subsystem $\mathrm{BaO} \cdot 2 \mathrm{SiO}_{2}-2 \mathrm{BaO} \cdot 3 \mathrm{SiO}_{2}$ is presented. The previously published diagram showing a complete solid solution series was not verified. Instead, two new compounds were identified by X-ray diffraction powder pattern techniques, and two eutectics were located by the quenching method. The compound $3 \mathrm{BaO} \cdot 5 \mathrm{SiO}_{2}$ melts incongruently at $1,423^{\circ}$ $\mathrm{C}$ to form $5 \mathrm{BaO} \cdot 8 \mathrm{SiO}_{2}$ and liquid. The latter compound melts congruently at $1,446^{\circ} \mathrm{C}$. Barium disilicate was found to have a quenchable and slowly reversible phase transformation at about $1,350^{\circ} \mathrm{C}$. The X-ray diffraction powder pattern for $2 \mathrm{BaO} \cdot 3 \mathrm{SiO}_{2}$ was indexed for the first time on the basis of a monoclinic unit cell. $8 \mathrm{p}$.

RP2954. Separation and determination of phosphate, silicate, and arsenate, W. S. Clabaugh and A. Jackson

A method was developed for quantitatively separating and determining phosphate, silicate, and arsenate when they occur together in solution. All three, at a $p \mathrm{H}$ of 1.8 , form heteropoly molybdate complexes when heated just to boiling. Only the phosphate complex is extracted into ether from a solution that contains one volume of concentrated hydrochloric acid plus nine volumes of solution. After removing the phosphate, only the silicate complex is extracted into butanol from a solution that contains two volumes of concentrated hydrochloric acid plus nine vol- umes of solution. The arsenate complex is then extracted into methy isobutyl ketone after readjusting the acidity back to $1+9$ by adding silica-free ammonium hydroxide. The various complexes are reduced by adding stannous chloride to the organic solvent and determined spectrophotometrically. The effect of various salts on the complex formation and the extraction is also reported. $5 \mathrm{p}$.

RP2955. Heat of formation of potassium calcium silicate, E. S. Newman

The heats of solution of $2 \mathrm{CaO} \cdot \mathrm{SiO}_{2}(\mathrm{c}, \beta), \mathrm{CaSO}_{4}(\mathrm{C}$, anhydrite), $\mathrm{K}_{2} \mathrm{SO}_{4}$ (C. II) and six members of a series of substitutional solid solutions of $\mathrm{K}_{2} \mathrm{O}$ in dicalcium silicate were determined in a nitric-hydrofluoric acid mixture by means of an isothermal-jacket calorimeter. From these data and heats of formation taken from the literature, the heat of formation of $\mathrm{K}_{2} \mathrm{O} \cdot 23 \mathrm{CaO} \cdot 12 \mathrm{SiO}_{2}$ (c) was calculated to be $-6,420$ kilocalories per mole at $25^{\circ} \mathrm{C}$. $5 \mathrm{p}$.

RP2956. Heat of formation of boron trichloride, W. H. Johnson, R. G. Miller, and E. J. Prosen

The heat of formation of gaseous boron trichloride has been determined by the direct reaction of gaseous chlorine with amorphous boron in a calorimeter.

$$
\begin{gathered}
\mathrm{B}(\text { amorph })+3 / 2 \mathrm{Cl}_{2} \text { (gas) }=\mathrm{BCl}_{3} \text { (gas) } \\
\Delta \mathrm{Hf}^{\circ}\left(25^{\circ} \mathrm{C}\right)=-407.98 \pm 1.34 \mathrm{kj} / \mathrm{mole} \\
(-97.51 \pm 0.32 \mathrm{kcal} / \mathrm{mole}) .
\end{gathered}
$$

By utilizing the values previously reported for the heats of formation of boric acid, diborane, and pentaborane, the heat of hydrolysis of boron trichloride and the heats of reaction of diborane and pentaborane with chlorine have been obtained. By the use of an estimated value for the heat of sublimation of boron, the average bond energy of the $\mathrm{B}-\mathrm{Cl}$ bond in boron trichloride is found to be 105.2 kcal at $0^{\circ} \mathrm{K}$. The data on the heats of formation of diborane, boric oxide, boric acid, and boron trichloride now form a consistent set of values. 5 p.

RP2957. Thermal degradation of polymers at low rates, S. L. Madorsky

In measuring the rates of thermal degradation of polymers at constant temperatures in the range of $200^{\circ}$ to $500^{\circ} \mathrm{C}$, one encounters a distortion of the initial parts of the rate curves during the time required to heat the specimen to the pyrolysis temperature. This distortion has been largely overcome by using a new apparatus, based on an electronic balance, in which the degradation of the specimen is carried out at very low rates. The apparatus is equipped with an electronic thermostat that controls the temperature to within $\pm 0.1^{\circ} \mathrm{C}$, and also with an automatic recorder that measures both the temperature and the loss of weight of the specimen. Pyrolysis experiments were carried out in a vacuum in this apparatus on pure specimens of polystyrene, poly( $\alpha$-methylstyrene), polymethylene, high-molecular-weight polyethylene, and low-molecular-weight polyethylene. The initial rates of degradation, in percent of sample per minute, varied from 0.008 to 0.095 . Except for the initial parts of the rate curves, in some cases up to about 30 percent loss of weight, the rate curves at low rates resemble closely those previously obtained at high rates of degradation. $10 \mathrm{p}$.

RP2958. Pits in metals caused by collision with liquid drops and soft metal spheres, O. G. Engel

An equation is developed to give pit depth as a function of collision velocity for pits formed in soft to medium-hard metal plates as a result of collision with liquid drops. The rear face of the target plate must be a free surface. The plate thickness must not be less than 1.5 to 2.0 times the drop diameter nor greater than 4 to 5 times the drop diameter. It is shown that, under the same conditions on 
the target plate, the equation is also applicable to pits formed in soft to medium hard metal plates as a result of collision with spheres of the same metal that flow radially as a result of the collision. Pit-depth-versus-velocity data obtained in other laboratories were used to test the equation. Metals used as targets were copper, 1100-0 aluminum, 2024-0 aluminum, lead, steel, soft iron, and zinc. Mercury was used as the drop liquid against copper, aluminum, lead, and steel. Water was used as the drop liquid against copper, aluminum, and lead. Spheres of copper, aluminum, lead, soft iron, and zinc were used against targets of the same materials, respectively. The equation can be used to calculate the dynamic compressive yield strength of soft to medium-hard metals. $18 \mathrm{p}$.

\section{RP2959. Earth currents near a monopole antenna with} symmetrical top loading, J. R. Wait

Expressions for the fields are developed for a vertical ground-based monopole with a cone or disk located at the top of the antenna to simulate umbrella top loading. The current distribution on the structure is assumed. Using spherical-wave functions, the magnetic-field distribution on the ground plane near the base of the antenna is computed and illustrated by graphs. For the case where the antenna is electrically small, the currents flowing on the cone or disk are shown to contribute only slightly to the total field. $9 \mathrm{p}$.

RP2960. Infrared absorption spectra of some cyclic acetals of sugars, R. S. Tipson, H. S. Isbell, and J. E. Stewart

The infrared absorption spectra of twenty-eight 1-methoxyethylidene and isopropylidene acetals of sugars have been recorded and analyzed. The formulas of the compounds were grouped according to structure, and the absorption bands for each group were considered in relation to the bands found for the other structurally related compounds. No bands were found for the unequivocal detection of the 1,3-dioxolane ring attached respectively to an aldopentofuranose or a ketopentofuranose, an aldopentopyranose, an aldohexofuranose, an aldohexopyranose or a ketohexopyranose, a uronic acid salt or lactone, a sugar alcohol, or a sugar keto-acid salt. Regardless of the fine structure of the dioxolane compounds, certain strong bands were observed. but the bands are not easily recognized from an inspection of one or two spectra and are not eharacteristic of these compounds only. $26 \mathrm{p}$.

RP2961. Earth currents near a top-loaded monopole antenna with special regard to electrically small L- and T-antennas, H. L. Knudsen

An investigation has been made of the ground currents near a top-loaded monopole with nonazimuthal symmetry. Formulas have been developed for the surface current density produced by an inclined, straight wire over a horizontal ground plane for an arbitrary current distribution on the antenna. Working formulas have been developed and numerical calculations of the surface current density on the ground plane have been carried out for the case of a small antenna with a linear current distribution. These results have been used for the calculation of the contribution to the surface current density due to the top loading in the case of an L-antenna and in the case of a T-antenna. In each case both the absolute value of the surface eurrent density arising from the top loading and the relative value of its $\phi$-component have been plotted, as it may be expected that this component under certain circumstances may be important in calculating the ground losses in the case of a system of radial ground wires. $14 \mathrm{p}$.

TITLE PAGE AND CONTENTS TO VOLUME $62.5 \mathrm{p}$.
3.2 PAPERS FROM THE JOURNAL OF RESEARCH OF THE NATIONAL BUREAU OF STANDARDS, SECTIONA. PHYSICS AND CHEMISTRY, VOLUME 63A, JULY-DECEMBER 1959

July-August 1959

Description and analysis of the first spectrum of iodine, C. C. Kiess and C. H. Corliss

An extensive survey of the spectra of iodine has led to a list of more than 900 lines emitted by neutral atoms in

the region from $23070 \mathrm{~A}$ in the infrared to $1195 \mathrm{~A}$ in the extreme ultraviolet. Wavelengths between $12304 \mathrm{~A}$ and 2061 A were derived from measurements of spectrograms obtained with gratings of high dispersion. Wavelengths of lines outside these limits are the computed values for lines observed on photometric tracings of the infrared, inaccessible to photography, and in the ultraviolet with a vacuum-grating spectrograph. For many of the lines Zeeman patterns were obtained in a magnetic field of about 37,000 oersteds. With these data many of the lines have been classified as combinations between odd levels from the electron configurations $5 s^{2} 5 p^{4} n p$ and $5 s^{2} 5 p^{4} n f$ and even levels from the configurations $5 s^{2} n s$ and $5 s^{2} 5 p^{4} n d$. Among these levels several sets have been recognized as forming Rydberg sequences that are in close agreement in placing the ground state $5 p^{6}{ }^{2} \mathrm{P}_{\text {ir }}$ of I I at $84,340 \mathrm{~cm}^{-1}$ below the ground state $5 p^{4}{ }^{3} \mathrm{P}^{2}$ of I II. This gives 10.45 electronvolts for the ionization potential of the neutral iodine atom. A strong infrared line at $13148.8 \mathrm{~A}$ is explained as a magnetic dipole transition between the levels of the ground term $5 p^{5}{ }^{2} \mathrm{P}^{\circ}$. $18 \mathrm{p}$.

$\mathrm{CH}$ in the solar spectrum, C. E. Moore and H. P. Broida

$\mathrm{CH}$ lines in the solar spectrum have been identified by direct comparison of measured laboratory and solar wavelengths and intensities. The comparisons of individual rotational lines are included in a series of tables arranged according to electronic and vibrational transitions. $35 \mathrm{p}$.

Infrared studies in the 1 - to 15 -micron region to 30,000 atmospheres, C. E. Weir, E. R. Lippincott, A. Van Valkenburg, and E. N. Bunting

A pressure cell was constructed using a pair of type II diamonds for study of infrared spectra of solids in the 1- to 15-micron region. Using commercial infrared equipment, spectra can be studied routinely to calculated pressures as high as 30,000 atmospheres. Under pressure, bands generally shift to higher frequencies and decrease in intensity. The magnitude of both changes depends on the mode of vibration. Occasionally major changes in spectra occur. In calcite the carbon-oxygen symmetric stretching, mode $\nu_{1}$, becomes active at elevated pressures while the doubly degenerate $\nu_{3}$, stretching, and $\nu_{4}$, bending, frequencies split. From the shift in frequency of $\nu_{1}$ with pressure the "compressibility", $\left[\left(-1 / \mathrm{R}_{0}\right)(\mathrm{dR} / \mathrm{dp})\right]$, of the $\mathrm{C}-\mathrm{O}$ bond length, $R$, is calculated to be $2.8 \times 10^{-7} / \mathrm{atmos}-$ phere. Major spectral changes are not observed in the same pressure ranges in other carbonates having the calcite or aragonite structures. The results for calcite may be explained by a shift of the $\mathrm{CO} \overline{3}$ ion from the trigonal axis under pressure. $8 \mathrm{p}$.

Phosphinoborine compounds: mass spectra and pyrolysis, L. A. Wall, S. Straus, R. E. Florin, F. L. Mohler, and P. Bradt

The mass spectra of tetramethylphosphinoborine trimer, $\left[\mathrm{P}\left(\mathrm{CH}_{3}\right)_{2} \mathrm{~B}\left(\mathrm{CH}_{3}\right)_{2}\right]_{3}$ (I) and a compound, $\mathrm{P}_{5}\left(\mathrm{CH}_{3}\right)_{9} \mathrm{~B}_{5} \mathrm{H}_{9}$, (II) prepared from dimethylphosphinoborine were observed and the compounds were pyrolyzed at 300 to $500^{\circ} \mathrm{C}$. Most peaks in the spectrum of (I) came from the $\mathrm{P}-\mathrm{B}$. $\mathrm{B}-\mathrm{C}$, and $\mathrm{P}-\mathrm{C}$ cleavages. The mass spectrum of (II) was much more complicated with evidence for methyl group redistribution. 
The pyrolysis of both compounds indicates a very complicated mechanism with many unidentifiable compounds. Trends in the formation of volatile products indicate that both compounds are completely decomposed in $4 \mathrm{hr}$ at $450^{\circ} \mathrm{C}$. Compound (I) produces trimethylboron, which disappears rapidly above $400^{\circ} \mathrm{C}$. Neither (I) nor (II) formed ethane or elemental phosphorus. $4 \mathrm{p}$.

Experimental and theoretical study of kinetics of bulk crystallization in poly(chlorotrifluoroethylene), J. D. Hoffman, J. J. Weeks, and W. M. Murphey

The rate of isothermal bulk crystallization of poly(chlorotrifluoroethylene), $T_{m}=221^{\circ} \mathrm{C}$, was measured from $170^{\circ}$ to $200^{\circ} \mathrm{C}$. The intrinsic bulk crystallization, which accurately followed an $n=2$ law, was shown to be a result of the injection of primary nuclei sporadically in time, with one-dimensional growth of centers derived from these nuclei. The crystallites are exceedingly small. The one-dimensional growth process was isolated by uncleating specimens with seed crystals, and its temperature-dependence determined between $191^{\circ}$ and $205^{\circ} \mathrm{C}$. The seed crystal isotherms followed an $n=1$ law. The temperature coefficients of the rate of nucleation and the rate of growth were both strongly negative.

A theory of homogeneous nucleation that takes into account the segmental character of the polymer chains is developed in some detail. A cylindrical nucleus is assumed. In the temperature range near the melting point, region $A$, where the radius and length of the nucleus are unrestricted, the rate of nucleation is shown to be proportional to $\exp \left(-\alpha / T^{3} \Delta T^{2}\right)$. The nucleation rate is proportional to $\exp \left(-\beta / T^{2} \Delta T\right)$ in region $B$, which extends from somewhat below the melting point to considerably lower temperatures; the length of the nucleus has a constant value $l_{0}$ in this region, but the radius is unrestricted. (In the above expressions, $\alpha$ and $\beta$ are constants). Finally, at sufficiently low temperatures, region $\mathrm{C}$ is entered. Under certain circumstances, the rate of nucleation in region $\mathrm{C}$ will be extremely rapid, and correspond to a "nucleative collapse" of the supercooled liquid state. A calculation of the one-dimensional growth rate shows that it is proportional to $\exp \left(-\gamma / T^{2} \Delta T\right)$ where $\beta=\gamma$.

A careful analysis of the experimental data obtained between $170^{\circ}$ and $200^{\circ} \mathrm{C}$ clearly showed that both the rate of nucleation and the rate of growth were proportional to $\exp \left(-\beta / T^{2} \Delta T\right)$, and not $\exp \left(-\alpha / T^{3} \Delta T^{2}\right)$. The primary nucleation event was thus of type $B$ in this interval. A detailed analysis of the data is given, and surface free energies and the dimensions of the nuclei quoted. Quenching experiments, where the polymer was crystallized well below $170^{\circ} \mathrm{C}$, gave a firm indication of the existence of region $\mathrm{C}$.

An experimental study was made of the extremely slow crystallization process that prevailed when the degree of crystallinity became high. The onset of this stage of the crystallization was interpreted as being the result of a massive degree of impingement. This interpretation is justified by the calculations of Lauritzen, who has given a theory of impingements that predicts a pseudoequilibrium degree of crystallinity.

As indicated above, the growth process originating at homogeneous nuclei is not of a three-dimensional or spherulitic character in the region of study. Such stray spherulites as do appear in this region are shown to originate at heterogeneities. The possibility that the intrinsic growth process may become three-dimensional at crystallization temperatures sufficiently near $T_{m}$ is discussed. $32 \mathrm{p}$.

$$
\text { September-October } 1959
$$

Reflection of fast neutrons from water, M. J. Berger and J. W. Cooper

The backscattering of fast $(0.3,1,3,6,9$, and $14 \mathrm{Mev})$ neutrons from a semi-infinite water medium has been calculated by the Monte Carlo method. The informa- tion obtained includes the joint angular and spectral distribution of the reflected neutrons, the dependence of the number albedo and energy albedo on the source energy and obliquity, and the contributions to the albedo of successive orders of scattering. The spectra were calculated down to epithermal energies $(\sim 0.5 \mathrm{ev})$. The results for each case are based on the analysis of 3,000 neutron histories, generated with the use of an IBM-704 computer. In the random sampling, elastic scattering from hydrogen and oxygen, inelastic scattering from oxygen, and absorption due to $n-\alpha$ and $n-p$ processes were taken into account. The cross sections for some of these interactions are not well known. Parallel calculations with different assumptions about the cross sections were made in order to estimate how sensitively the albedo depends on the cross sections. The paper includes a selfcontained description of the Monte Carlo method, its application to the calculation of radiation diffusion and in particular to the neutron albedo problem. Emphasis is placed on the technique of correlated sampling which makes possible an accurate estimate of albedo differences resulting from different assumptions about the cross sections. The random sampling computations were supplemented by analytical calculations of the single-scattering albedo. This was useful for an understanding of the Monte Carlo results because a considerable fraction of the reflected neutrons return after only one collision. $44 \mathrm{p}$.

Some vibrational-rotational bands of deuterated methanes, H. C. Allen, Jr., and E. K. Plyler

A parallel band at $2,200 \mathrm{~cm}^{-1}$ and a perpendicular band at $2,780 \mathrm{~cm}^{-1}$ of $\mathrm{CH}_{3} \mathrm{D}$ have been observed under high resolution and analysed. The analysis of the perpendicular band revealed the presence of l-type doubling in the doubly degenerate excited state. From the analysis of the parallel band it is found that $B_{0}=3.880 \mathrm{~cm}^{-1}$. A hybrid band of $\mathrm{CD}_{3} \mathrm{H}$ has been observed near $2,600 \mathrm{~cm}^{-1}$. Both active components, $A$ and $E$ are observed and analysed. The ground state $B_{0}$ value found from this analysis is in good agreement with previous determinations. $8 \mathrm{p}$.

Precise coulometric titration of acids and bases, J. K. Taylor and S. W. Smith

Apparatus and techniques are described for the titration of acids and bases by constant-current coulometry. The precision and accuracy of the method are indicated by titrations of benzoic acid, potassium acid phthalate, adipic acid, hydrochloric acid, and sodium carbonate. Standard deviations of \pm 0.003 percent have been obtained which indicate that the reliability of the method is equal to or exceeds that of classical analytical procedures. $7 \mathrm{p}$.

Heat of formation of titanium tetraiodide, W. H. Johnson,

A. A. Gilliland, and E. J. Prosen

The heat of formation of titanium tetraiodide was determined relative to that of titanium tetrabromide by comparison of their heats of hydrolysis in dilute sulfuric acid. The difference in the heats of formation may be expressed by the equation:

$$
\begin{gathered}
\mathrm{TiI}_{4}(\mathrm{c})+2 \mathrm{Br}_{2}(\mathrm{liq})=\mathrm{TiBr}_{4}(\mathrm{c})+2 \mathrm{I}_{2}(\mathrm{c}), \\
\Delta H\left(25^{\circ} \mathrm{C}\right)=-230.91 \pm 0.75 \mathrm{kj} / \mathrm{mole} \\
(-55.19 \pm 0.18 \mathrm{kcal} / \mathrm{mole}) .
\end{gathered}
$$

By taking the heat of formation of $\mathrm{TiBr}_{4}(\mathrm{c})$ as -616.72 $\pm 4.60 \mathrm{kj} / \mathrm{mole}$, the heat of formation of $\mathrm{TiI}_{4}(\mathrm{c})$ is caleulated to be $-385.81 \pm 4.64 \mathrm{kj} / \mathrm{mole}(-91.21 \pm 1.11$ $\mathrm{kcal} / \mathrm{mole})$. The heats of hydrolysis of $\mathrm{TiBr}_{4}$ and $\mathrm{TiCl}_{4}$ were similarly measured; the value obtained for the difference $(186.77 \pm 1.34 \mathrm{kj} / \mathrm{mole})$ is in good agreement with the difference between the directly determined heats of formation $(187.11 \pm 5.35 \mathrm{kj} / \mathrm{mole}) .6 \mathrm{p}$. 
Reactions of pentafluorohalobenzenes, W. J. Plummer and L. A. Wall

Both pentafluorobromobenzene and pentafluoroiodobenzene are reactive intermediates and can be used to introduce the perfluorophenyl $\left(\mathrm{C}_{5} \mathrm{~F}_{6} \rightarrow\right)$ group int 5 a variety of new compounds. The chief methods used are the Grignard coupling or addition reaction as well as the Ullmann condensation reaction. Some of the new compounds prepared were pentafluorobenzonitrile, perfluorodiphenyl, pentafluorophenyl- $\alpha$-ethanol, and pentafluorostyrene. 3 p.

Tritium-labeled compounds I. Radioassay of tritiumlabeled compounds in "infinitely thick" films with a windowless, gas-flow, proportional counter, H. S. Isbell, H. L. Frush, and R. A. Peterson

A simple, sensitive, and reliable techniaue has been devised for the radioassay of nonvolatile, water-soluble tritium compounds. The substance to be analyzed is dissolved in an aquenus solution of a thickening agent, preferably sedium $O$-(carboxymethvl)cellulose or sodium alginate. The solution is placed in a shallow planchet, and after evaporation of the water, the resulting flm, which is "infinitely thick" to tritium beta particles, is counted with a $2 \pi$, windowless, gas-flow, proportional counter. By means of an empirical factor, determined with a substance of known radioactivitv, the counts are converted to microcuries. In a film having a thickness of $0.7 \mathrm{mg} / \mathrm{cm}^{2}$, the counting efficiency is about 4 percent; the standard deviation from the mean, obtained in a series of routine measurements, was less than 2 percent. An assay can readily be made with tritium-containing material having 0.01 microcurie of radioactivity. The method, which is applicable to nonvolatile, water-soluble solids, solutions, or liquids, is suitable for routine analyses. 5 p.

Tritium-labeled compounds II. General-purpose apparatus, and procedures for the preparation, analysis, and use of tritium oxide and tritium-labeled lithium borohydride, H. S. Isbell and J. D. Moyer

A general-purpose manifold is described, which is useful for numerous procedures involving tritium gas and tritiumlabeled materials.

Methods are given in detail for (a) converting tritium gas to tritium oxide, (b) preparing tritium-labeled lithium borohydride, and (c) conducting a variety of reactions in a closed system. Auxiliary equipment is shown, including water traps, reaction flasks, and apparatus for preparing solutions and making filtrations in closed systems. Methods are presented for assaying tritium-labeled water by (a) dissolving it in a phosphoric anhydride-sulfuric acid solution and counting in a windowless, gas-flow, proportional counter, or (b) converting it to hydrogen- $t$ and assaying it in an ionization chamber. The use of tritiumlabeled lithium borohydride is illustrated by the preparation of D-galactitol-1- $t$ from D-galactose. Subsequent papers will describe the use of this apparatus in the synthesis of tritium-labeled carbohydrates. The detailed description of techniques and apparatus should be helpful to others who are interested in using tritium. $7 \mathrm{p}$.

Preparation of trichloride and tetrachloride of molybdenum, D. E. Couch and A. Brenner

Molybdenum trichloride was successfully prepared in quantity by the reduction of molybdenum pentachloride with hydrogen. The most satisfactory yields were obtained with a 4 to 5 mole excess of hydrogen at pressures of 100 psi or higher and at a temperature of $125^{\circ} \mathrm{C}$.

Molybdenum tetrachloride was prepared by direct reaction of molybdenum trichloride with molybdenum pentachloride in a sealed tube or steel bomb maintained at $250^{\circ} \mathrm{C}$. X-ray patterns of the various chlorides were obtained. $4 \mathrm{p}$.
Infrared spectra of asphalts, B. D. Beitchman

A procedure for preparing thin films of airblown asphalts and a spectroseopic study of such films throughout the infrared region of 2.5 to 15 microns are described. The relationship of transmittance of several absorption bands with the durability of the asphalt is discussed. Exposure to ultraviolet radiation produced changes in transmittance at four wavelengths which, in a series of asphalts studied, showed statistically significant correlations with durability. 5 p.

November-December 1959

Multiple ionization of rare gases by electron impact, M. Krauss, R. M. Reese, and V. H. Dibeler

Electron impact studies of multiple ionization processes in helium, neon, argon, and xenon appear to support theoretical conclusions that the threshold probability for $n$-fold ionization is proportional to the $n$th power of the electron energy in excess of the threshold energy. The probability law applies, for the cases studied, over a considerable energy range that, for all but $\mathrm{He}^{2+}$, includes the possible onset of more than one mode of ionization. The presence of a Boltzmann spread in the energy of the electron beam or specific focusing effects due to ion source geometry are found to affect only the foot of the probability curve. By the use of certain assumptions, an estimate is also made of the departure from a ${ }^{3} \mathrm{P}$ ionization probability curve resulting from onset of ionization to the ${ }^{1} \mathrm{D}$ and is states. $4 \mathrm{p}$.

Light scattering by commercial sugar solutions, C. J. Rieger and F. G. Carpenter

Using a direct measure of seattered light, it was found that commercial sugar solutions scatter light predominantly in a forward direction. The scattering at angles less than $30^{\circ}$ was as much as one hundred times that at right angles to the incident beam.

It was found that the light scattering by commercial sugar solutions is inversely dependent on wavelength to a power of between 2 and 3 , and that severe multiple scattering occurs when the turbidity of the solution is larger than $2 \times 10^{-1} \mathrm{~cm}^{-1}$ at $436 \mathrm{~m} \mu$. The scattering of commereial sugar solutions is compared with that of highly purified sucrose.

A method is discussed that will enable a good approximation of the turbidity of commercial sugar solutions to be made from a single forward scattering measurement at an angle of about $20^{\circ}$ with respect to the incident light beam. A correction for scattered light in transmission measurements of these solutions is also introduced. $7 \mathrm{p}$.

Analysis of the first spectrum of ruthenium (Ru I), K. G. Kessler

The analysis of the first spectrum of ruthenium has been extended with the aid of digital computers. A total of 105 even and 206 odd levels are listed with observed Land $^{\circ} g$-factors for 54 even and 148 odd levels. A complete list of approximately 3,400 classified lines in the range 2013 to $11484 \mathrm{~A}$ is presented. The ionization limit calculated from a two member series is $59410 \mathrm{~cm}^{-1}$ or $7.364 \mathrm{v} .39 \mathrm{p}$.

Supplementary Zeeman data for the first spectrum of ruthenium (Ru I), J. R. MeNally, Jr. and K. G. Kessler

Zeeman data are listed for 207 lines of Ru I between 2400 and $5400 \mathrm{~A}$, all of which have been classified. The spectrograms were made at the Massachusetts Institute of Technology and were analyzed there and at the National Bureau of Standards. 2 p.

Low even configurations in the first spectrum of ruthenium (Ru I), Part 2, R. E. Trees

A published calculation for the $4 d^{8}, 4 d^{7} 5 s$, and $4 d^{6} 5 s^{2}$ configurations of Ru I (R, E, Trees, J. Opt. Soc. Am, 49. $838(1959))$ is repeated in steps. Displacements produced 
by configuration interaction are evaluated, and departures of term positions from familiar expectations in the absence of configuration interaction are explained. The weaker perturbations produced by second-order effects of the spin-orbit interaction are then determined. It is shown that the neglect of these effects in published hand computations has obscured the remarkably good agreement between theory and observation that is obtainable in spectra of the second long period. The eigenvectors are based on "third-order eigenfunctions" which describe the levels simply, and show the degree of $L S$-coupling in a more quantitative manner. $6 \mathrm{p}$.

Thermal degradation of polymers at high temperatures, S. L. Madorsky and S. Straus

Work on thermal degradation of polymers has previously been carried out at temperatures up to about $500^{\circ} \mathrm{C}$. In the present work the range has been extended to $850^{\circ} \mathrm{C}$. Polystyrene was pyrolyzed in a vacuum and also in helium at atmospheric pressure at $362^{\circ}$ and at $850^{\circ} \mathrm{C}$. Analysis of the volatile products indicates that higher temperatures and higher pressures cause a greater fragmentation of the volatile products. Samples of poly (vinylidene fluoride), polyacrylonitrile, and polytrivinylbenzene, were pyrolyzed in a vacuum at temperatures from $350^{\circ}$ to $800^{\circ} \mathrm{C}$. The more volatile products were analyzed qualitatively and quantitatively in a mass spectrometer. The less volatile products were tested for their average molecular weight by a microcryoscopic method.

Rates of thermal degradation were also determined for the last three polymers. The activation energies in the temperature range $218^{\circ}$ to $440^{\circ} \mathrm{C}$. were found to be 48 , 31 , and $73 \mathrm{kcal} / \mathrm{mole}$, respectively, for poly (vinylidene fluoride), polyacrylonitrile, and polytrivinylbenzene. $8 \mathrm{p}$.

Influence of impurities on the pyrolysis of polyamides, S. Straus and L. A. Wall

A study has been made of the effect of drying and purification of polycaprolactam (nylon-6) on its thermal decomposition. The conclusions drawn from previous work, that a hydrolytic mechanism is at least partially operating due to the presence of water and acid polymerization catalysts, are confirmed. Purification of the material decreases the rate of degradation and the production of $\mathrm{CO}_{2}$, and increases the preexponential factor and the activation energy. The hydrolytic decomposition, which competes with the thermal free-radical degradation process, would be expected to produce carboxylic acids and amines, the former decomposing further to form $\mathrm{CO}_{2}$. The preexponential factor is increased by sample purification from $10^{7}$ to $10^{10}$ and the activation energy from 34 to $43 \mathrm{kcal} / \mathrm{mole}$. The decomposition behavior of the purest sample is a nearly perfect example of the random decomposition of linear polymer chains. All other samples of polyamides thus far studied also appear to decompose by random or nearly random mechanisms. 5 p.

A preliminary list of levels and $g$-values for the first spectrum of thorium (Th I), R. Zalubas

The present state of the analysis of the first spectrum of thorium (Th I) is discussed briefly. Even and odd levels are listed in tables 1 and 2 . The low even levels form terms arising from the configurations $6 d^{2} 7 s^{2}$ and $6 d^{3} 7 s$. The Th I standard wavelengths that fit into the known level arrays are presented in table $3.4 \mathrm{p}$.

\section{$\mathrm{OH}$ in the solar spectrum, C. E. Moore and H. P. Broida}

Revised identifications of $\mathrm{OH}$ lines in the solar spectrum have been made from the detailed laboratory analyses of the $\mathrm{A}^{2} \Sigma^{+}-\mathrm{X}^{2}$ II bands. In the $(0,0),(1,1)$, and $(2,2)$ bands a total of 175 solar lines are ascribed to $\mathrm{OH}$ unblended; 124 have $\mathrm{OH}$ as a partial contributor. Laboratory intensities along the branches of the separate bands have been used as a guide in making the solar identifications. 17 p.
Use of chebychev polynomials in thin film computations, K. D. Mielenz

From Herpin's expression for the $m$ th power of a multilayer matrix, very simple closed formulas are derived for the matrices and optical constants of any multilayer with a periodic structure.

According to Epstein's theorem, any symmetrical multilayer is equivalent to a fictitious monolayer. A simple expression for the equivalent index and thickness of this monolayer is deduced for the case of a periodic and symmetrical sequence of equally thick films.

As compared to any other method of numerical computation, the suggested formulation provides a considerable saving of time and work. In a numerical example, this saving amounts to about 80 percent. $4 \mathrm{p}$.

TITLE PAGE AND CONTENTS TO VOL. 63A. 3 p.

PAPERS FROM THE JOURNAL OF RESEARCH OF THE NA TIONAL BUREAU OF STANDARDS, SECTION A. PHYSICS AND CHEMISTRY, VOLUME 64A, JANUARY-JUNE 1960

January-February 1960

The "1958 $\mathrm{He}^{4}$ scale of temperatures." Part 1. Introduction, F. G. Brickwedde and Part 2. Tables for the 1958 temperature scale, H. van Dijk, M. Durieux, J. R. Clement, and J. K. Logan

The generally used practical scale of temperatures between $1^{\circ}$ and $5.2^{\circ} \mathrm{K}$ is the $\mathrm{He}^{4}$ vapor pressure scale based on an accepted vapor pressure equation or table. In Sèvres (near Paris), October 1958, the International Committee on Weights and Measures recommended for international use the "1958 $\mathrm{He}^{4}$ Scale" based on a vapor pressure table arrived at through international cooperation and agreement. This table resulted from a consideration of all reliable $\mathrm{He}^{4}$ vapor pressure data obtained using gas thermometers, and paramagnetic susceptibility and carbon resistor thermometers. The theoretical vapor pressure equation from statistical thermodynamics was used with thermodynamic data on liquid $\mathrm{He}^{4}$ and the vapor equation of state to insure satisfactory agreement of the vapor pressure table with reliable thermodynamic data. $17 \mathrm{p}$.

Energy levels and spectrum of neutral helium $\left({ }^{4} \mathrm{He} \mathrm{I}\right)$, W. C. Martin

A table of energy levels based on the most accurate observations now available is given for the neutral ${ }^{4} \mathrm{He}$ atom. The wavelengths contained in a revised list of ${ }^{4} \mathrm{He}$ I lines from $320 \mathrm{~A}$ to $21132 \mathrm{~A}$ are also based on the best measurements, over half of them having been calculated from the wavenumber separations of appropriate levels. Several previously disturbing features of the ${ }^{4} \mathrm{He}$ term scheme are obviated by the revised level values. In a discussion of the experimental results for this spectrum some comparisons with theory are made. $10 \mathrm{p}$.

Vibration-rotation structure in absorption bands for the calibration of spectrometers from 2 to 16 microns, E. K. Plyler, A. Danti, L. R. Blaine, and E. D. Tidwell

Suitable bands of common gases have been tabulated and remeasured wherever necessary from 2 to 16 microns to obtain an accuracy of about $0.03 \mathrm{~cm}^{-1}$ throughout the region and to provide good calibrating points at frequent intervals. Some 600 rotation-vibration lines are illustrated in 20 spectrograms and wavenumbers are listed in companion tables with considerable intercomparison with worthy data obtained in other laboratories. The absorption bands were remeasured or calibrated by using either a precisely graduated grating circle or standard atomic lines with the fringe system formed by a Fabry-Perot interferometer. Characteristic features of the individual bands are discussed briefly and references to other publications are given. The substances used for calibration include $\mathrm{H}_{2} \mathrm{O}, \mathrm{CO}_{2}, \mathrm{CO}, \mathrm{HCl}, \mathrm{HBr}, \mathrm{NH}_{3}, \mathrm{C}_{2} \mathrm{H}_{2}, \mathrm{CH}_{4}, \mathrm{~N}_{2} \mathrm{O}$, and polystyrene film. $20 \mathrm{p}$. 
Combustion calorimetry with fluorine: constant pressure flame calorimetry, G. T. Armstrong and R. S. Jessup

Instruments and methods have been developed and are described for the measurement of heats of reaction between fluorine and other gaseous materials. Verification of the amount of reaction of hydrogenous materials is possible. The estimated accuracy of measurements is about 0.3 percent. Lack of certainty of the magnitude of corrections to be applied for hydrogen fluoride nonideality is an important factor. The heat of formation of hydrogen fluoride is found to be $-64.4 \pm 0.25 \mathrm{kcal} / \mathrm{mole}$ on the basis of the reaction of fluorine with ammonia. $11 \mathrm{p}$.

Pits in metals caused by collision with liquid drops and rigid steel spheres, O. G. Engel

A pit-depth-versus-velocity equation developed earlier was tested further with experimental data obtained using target plates of electrolytic tough pitch copper, 1100-O aluminum, and 2024-O aluminum, the static strength properties of which were measured by testing tensile specimens. The projectiles used to produce the pits were mercury drops, waterdrops, and steel spheres. It was found that the numerical constants in the equation for projectiles that flow during and as a result of the collision are different from those for projectiles that do not flow (hardened steel spheres). Curves calculated using the equation were found to be in acceptable agreement with experimental pit-depth-versus-velocity data for collisions of the indicated projectiles with target plates of the three metals used with the exception of the case of steel-sphere impingement against $2024-\mathrm{O}$ aluminum alloy. In this case work-hardening of the target metal seems to foster a mode of pit formation that was not considered in the development of the pit-depth-versus-velocity equation. $12 \mathrm{p}$.

Theory of formation of polymer erystals with folded chains in dilute solution, J. I. Lauritzen, Jr., and J. D. Hoffman

A detailed interpretation of the kineties of homogeneous nueleation and growth of crystals of a linear homopolymer from dilute solution is given. The probability of forming both nuclei with folded chains, and conventional bundlelike nuclei, from dilute solution is analyzed. It is predicted that at sufficiently high dilution, critical nuclei of length $\mathbf{I}_{p}^{*}$ will be formed from single polymer molecules by sharp folding of the chain backbone. The step height of the nucleus is given approximately by $\mathrm{l}_{\mathrm{p}}^{*}=4 \sigma_{e} / \Delta \mathrm{f}$. Here $\sigma_{e}$ is the free energy required to form a unit area of the loopcontaining end surfaces, and $\Delta \mathrm{f}$ is the free energy difference per unit volume of crystal between the crystalline and solution states. The quantity $\Delta \mathbf{f}$ is approximately proportional to the degree of supercooling $\Delta \mathrm{T}$. The growth of these nuclei is then analyzed. After growth, the resulting crystal is flat and platelike, the loops formed by the chain folds being on the upper and lower surfaces. Kinetic factors determine that the distance between the flat surfaces in the grown erystal will vary over only a narrow range about a value that is in the vicinity of $I^{*}=4 \sigma_{e} / \Delta \mathrm{f}$. (Neglecting effects due to edge free energies, the theoretical upper and lower limits are $1 *=4 \sigma_{e} / \Delta \mathrm{f}$ and $I^{*}=2 \sigma_{e} / \Delta \mathrm{f}$, respectively.) In some cases the predicted temperature dependence of the step height of the grown crystal, $\mathrm{I}^{*}=$ const. $\Delta \mathrm{T}$, may be modified by the existence of a constant term resulting from the presence of an edge free energy $\epsilon_{p}$. A grown loop-type crystal is predicted to be stable in comparison with a bundlelike erystal of the same shape and volume in a sufficiently dilute solution. The logarithm of the nucleation rate is approximately proportional to $1 /(\boldsymbol{\Delta} \mathbf{T})^{2}$ near the melting point. The cxponent $n$ in the free growth rate law is predicted under various assumptions. To the extent that comparison is possible, the predictions given agree with the experimental results obtained by Keller and O'Connor and others on single crystals of unbranched polyethylene grown from dilute solution.

A survey is given of homogeneous nucleation in bulk polymers, where the conventional bundlelike nucleus con- taining segments from many different molecules is valid, and the essential results compared with those calculated for the dilute solution case.

The theory given for loop nuclei is both general and precise enough at the critical points to suggest that, on crystallization from sufficiently dilute solution, crystals of a definite step height are commonly to be expected for other crystallizable linear polymers than polyethylene, provided loop formation is sterically possible. $30 \mathrm{p}$.

Studies of beryllium chromite and other beryllia compounds with $\mathrm{R}_{2} \mathrm{O}_{3}$ oxides, C. E. Weir and A. Van Valkenburg

Reactions between $\mathrm{BeO}$ and $\mathrm{R}_{2} \mathrm{O}_{3}$ oxides at high temperatures were studied. Compound formation was observed between $\mathrm{BeO}$ and the following oxides: $\mathrm{B}_{2} \mathrm{O}_{3}, \mathrm{Al}_{2} \mathrm{O}_{3}, \mathrm{Ga}_{2} \mathrm{O}_{3}$ $\mathrm{Y}_{2} \mathrm{O}_{3}$, $\mathrm{La}_{2} \mathrm{O}_{3}$, and $\mathrm{Cr}_{2} \mathrm{O}_{3}$. No reaction was observed with $\mathrm{Sc}_{2} \mathrm{O}_{3}$, $\mathrm{In}_{2} \mathrm{O}_{3}$, and $\mathrm{Fe}_{2} \mathrm{O}_{3}$. Detailed studies were made of $\mathrm{BeO} \cdot \mathrm{Cr}_{2} \mathrm{O}_{3}$ which is isostructural with $\mathrm{BeO} \cdot \mathrm{Al}_{2} \mathrm{O}_{3}$. $\mathrm{BeO}$. $\mathrm{Cr}_{2} \mathrm{O}_{3}$ is a semiconductor. Optical and $\mathrm{X}$-ray data are given for all reaction products. $4 \mathrm{p}$.

Uranium-platinum system, J. J. Park and D. P. Fickle

The phase diagram of the uranium-platinum system was constructed from data obtained by thermal analysis, metallographic examination, and X-ray diffraction. The system is characterized by four intermetallic compounds: $\mathrm{U} \mathrm{Pt}$, formed peritectoidally at $961^{\circ} \mathrm{C}$; $\mathrm{UPt}_{2}$, formed peritectically at $1,370^{\circ} \mathrm{C} ; \mathrm{UPt}_{3}$, melting congruently at $1,700^{\circ}$ $\mathrm{C}$; and $\mathrm{UPt}_{5}$, formed peritectically at $1,460^{\circ} \mathrm{C}$. One eutectic occurs at $1,005^{\circ} \mathrm{C}$ and $12 \mathrm{a} / \mathrm{o}$ platinum, and a second at $1,345^{\circ} \mathrm{C}$ and $87.5 \mathrm{a} / \mathrm{o}$ platinum. The maximum solubilities are $4.5 \mathrm{a} / \mathrm{o}$ uranium in platinum and $5 \mathrm{a} / \mathrm{o}$ platinum in gamma-uranium. Platinum lowers the gamma-beta uranium transformation to $705^{\circ} \mathrm{C}$ and the betaalpha transformation to $589^{\circ} \mathrm{C}$. $11 \mathrm{p}$.

Nitriding phenomena in titanium and the $6 \mathrm{~A} 1-4 \mathrm{~V}$ titanium alloy, J. R. Cuthill, W. D. Hayes, and R. E. Seebold

Nitriding unalloyed titanium in purified nitrogen at $1,800^{\circ} \mathrm{F}$ produced a uniformly thick case that consisted of five distinct zones. The same treatment applied to a $6 \mathrm{~A} 1-4 \mathrm{~V}$ titanium alloy resulted in a thinner nitride case that consisted of three zones and elongated nitride grains that penetrated into the core at approximately 45 degrees to the specimen surface. The aluminum appears to be responsible for the formation of the elongated grains. These grains, in turn, appear to be responsible for the nitriding having a more adverse effect on the toughness of the alloy than of the unalloyed titanium, as indicated by preliminary impact test results. The nitride case on the titanium appears to increase in thickness with increase in nitriding time without limit. The nitride case exhibits a hardness equivalent to about 77 Rockwell $\mathrm{C}$ at the surface down to almost 50 Rockwell $\mathrm{C}$ at the interface. $7 \mathrm{p}$.

Stability of silver and pyrex in perchloric acid-silver perchlorate solutions and in conductivity water, D. N Craig, C. A. Law, and W. J. Hamer

The stability of mint silver, purified mint silver, and Pyrex fritted erucibles in aqueous solutions of perchloric acid, in aqueous solutions of perchloric acid containing silver perchlorate, and in conductivity water at room temperature was determined. The stability of the silver in various states of subdivision was studied. The corrosion current-density for mint silver in sheet form is $1.1 \times 10^{-8} \mathrm{amp} \mathrm{cm}^{-2}$ for 20 percent aqueous solutions of

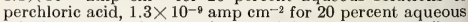
solutions of perchloric acid containing 0.5 percent silver perchlorate, and within the limits of detection is zero for conductivity water. Pyrex has high stability at $25^{\circ} \mathrm{C}$ in 20 percent aqueous solutions of perchloric acid and in 20 percent aqueous solutions of perchloric acid containing 0.5 percent silver perchlorate. Finely-divided silver contained in Pyrex erucibles was found to be highly stable 
when the crucibles were filled repeatedly with aqueous solutions of perchloric acid containing silver perchlorate, rinsed with conductivity water, and dried at $105^{\circ} \mathrm{C}$. These observations are important in the determination of the Faraday by the anodic dissolution of silver in aqueous solutions of perchloric acid which is now underway at the National Bureau of Standards. $7 \mathrm{p}$.

Note on the preparation of sodium amalgam in the form of pellets, H. S. Isbell, H. L. Frush, and N. B. Holt

A procedure is described for the preparation of sodium amalgam in the form of pellets. $2 \mathrm{p}$.

\section{March-April 1960}

A carbon-14 beta-ray standard, benzoic acid-7-C ${ }^{14}$ in toluene, for liquid scintillation counters, W. F. Marlow and R. W. Medlock

A earbon-14 beta-ray standard for use in liquid scintillation counting has been prepared and standardized. The sample consists of benzoic acid-7- $\mathrm{C}^{14}$ dissolved in toluene. Samples of the solution were oxidized quantitatively in a Paar oxygen bomb, and the radioactivity of the earbon dioxide was compared with the radioactivity of carbon dioxide prepared quantitatively from the Bureau's sodium carbonate-C ${ }^{14}$ standard. 4 p.

A comparison of experimental and theoretical relations between Young's modulus and the flexural and longitudinal resonance frequencies of uniform bars, $\mathrm{S}$. Spinner, T. W. Reichard, and W. E. Tefft

The relations from which Young's modulus may be computed from mechanical flexural and longitudinal resouance frequencies have been established by an empirical method using two sets of steel bars. Both sets contained rectangular and cylindrical specimens. For longitudinal vibration of evlindrical specimens, the agreement between the empirical curves and Bancroft's corresponding theoretical relation was within experimental error if Poisson's ratio for both sets is taken to be 0.292 . For flexural vibrations, the agreement between the empirical curve and the corresponding theoretical relation developed by Pickett is also within experimental error for about the same value of Poisson's ratio for the rectangular specimens of both sets; but for clylindrical specimens, the empirical values are somewhat lower than those predicted by the theory. $9 \mathrm{p}$.

Determination of copolymer composition by combustion analysis for carbon and hydrogen, L. A. Wood, I. Madorsky, and R. A. Paulson

A detailed description is given of the method of combustion analysis for carbon and hydrogen to determine the composition of a copolymer from its carbon-hydrogen ratio. The apparatus and procedures have been developed at the National Bureau of Standards over a period of years. The method has been applied chiefly to determine what fraction of a styrene-butadiene copolymer is derived from styrene. Minor ingredients are removed by extraction, with the exception of the bound mercaptan residue for which correction must be made. The amount of bound mercaptan residue is determined from measurements of the sulfur content of the copolymer by the Carius method. Measurements are made of the oxygen content and the ash content, although these do not enter into the calculations. The standard deviation of a measurement of carbon-hydrogen ratio is approximately 0.0010 and is independent of st vrene content. This corresponds to a standard deviation of about 0.036 percent bound styrene for polymers of low styrene content and 0.018 percent for polymers of high styrene content. The accuracy of the method is demonstrated by the fact that observations of carbon-hydrogen ratio for four out of five samples of polybutadiene differed by less than one standard deviation from the theoretical value. $6 \mathrm{p}$
Some effects of aging on the surface area of portland cement paste, C. M. Hunt, L. A. Tomes, and R. L. Blaine

A hardened cement paste cured at room temperature, from which part of the evaporable water has been removed by vacuum drying, has been studied. The surface area has been shown to decrease with time depending upon the the amount of evaporable water left in the paste. This change is the opposite of that usually observed during hydration and probably represents some collodial growth phenomena analogous to aging observed in other collodial gels. Both water vapor and nitrogen adsorption measurements have been used to show the effects of aging in cement paste.

Wet or dry paste is shown to undergo less change than paste of intermediate evaporable water content, so that if surface area after storage is plotted as a function of evaporable water content, a curve with a minimum is obtained. With increasing storage temperature there is some indication that this minimum might shift towards lower water content.

Aging is shown to occur during the initial drying of a cement paste, so that even the initial surface area of a cement paste depends upon the manner in which the paste has been dried. $7 \mathrm{p}$.

\section{Conformations of the pyranoid sugars. I. Classification of} conformers, H. S. Isbell and R. S. Tipson

An improved system is presented for indicating the principal conformaticns of pyranoid sugars and derivatives, by attaching two symbols to the systematic name. The first symbol shows the kind of pyranoid ring; for example, $\mathrm{B}=\mathrm{a}$ boat, $\mathrm{C}=$ the chair, and $\mathrm{S}=\mathrm{a}$ skew form. (The three boat and six skew rings are distinguished by subscript numerals referring to exoplanar ring-atoms.) The second symbol differentiates between two conformations that have the same type of ring, by describing as $\mathrm{A}$ or $\mathrm{E}$ the axial or equatorial character of the reference group at a selected ring-atom. If the anomeric group is not quasi, the $\alpha$ anomeric group is used as the reference group. For sugars and derivatives having a quasi anomeric group, and for relatives lacking an anomeric group, the $\mathrm{A}$ or $\mathrm{E}$ classification is based on the reference group at the low est numbered, nonquasi, asymmetric ring-atom. $6 \mathrm{p}$.

Tritium-labeled compounds III. Aldoses-1-t, H. S. Isbell, H. L. Frush, N. B. Holt, and J. D. Moyer

In a new method for preparing aldoses labeled with tritium at carbon 1 , the aldonic lactone is reduced with lithium borohydride- $t$ in pyridine solution. The method is suitable for preparing aldoses-1-t having high specific activities. The crude product contains a susbtantial proportion of the corresponding alditol-1- $t$, but the pure aldose-1- $t$ is readily separated by fractional recrystallization or paper chromotography. By means of an isotopedilution technique, yields were determined for the following aldoses- $1-t$ and the corresponding alditols-1-t: D-arabinose$1-t$, D-xylose-1- $t$, D-ribose-1-t, D-glucose-1-t, D-galactose-1- $t$, D-mannose-1-t-, L-rhamnose-1- $t$, maltose-1- $t$, and lactose$1-t$.

These labeled materials were also prepared by reducing the corresponding lactones with sodium amalgam in tritiated water. Although this latter method is not suitable for preparing labeled aldoses of high specific activity, the products are more readily purified than those obtained by reducing the lactones with lithium borohydride-t.

D-Glucose-1-t, obtained by each of these reduction methods, was oxidized with bromine, and the resulting D-gluconic acid was found to be nonradioactive. Hence, in the samples oxidized, tritium was present only at $\mathbf{C} 1$.

An apparatus used for reclaiming tritiated water by freeze-drying is depicted: it incorporates an efficient device for trapping entrained solids or liquids. $4 \mathrm{p}$. 
Determination of aluminum in precipitation hardening stainless steel and high temperature alloys, L. A. Machlan, J. L. Hague, and E. J. Meros.

A procedure is described for the determination of aluminum in high temperature alloys. Aluminum is selectively precipitated with 8-hydroxyquinoline from an ammoniacal solution of the alloy containing citrate and cyanide as complexing agents. The precipitate is ignited under oxalic acid, the oxides fused, and dissolved in acid. A caustic precipitation is made, an aliquot of the filtrate treated with hydrogen peroxide, and the aluminum precipitated with 8-hydroxyquinoline. The aluminum hydroxyquinolate is filtered on a fritted-glass crucible, dried, and weighed. $4 \mathrm{p}$.

\section{Mny-Juse 1960}

Phase shift effects in Fabry-Perot interferometry, C. J. Koester

A method is demonstrated for utilizing in Fabry-Perot interferometry the data on reflection phase shift dispersion obtained from fringes of equal chromatic order. Unknown wavelengths can be calculated from the Fabry-Perot patterns obtained with a large etalon spacing, even without prior knowledge of the phase shift of the reflecting surfaces. When the theoretical phase shift as a function of wavelength is known approximately, then the correct orders of interference can be determined for both the Fabry-Perot fringes and fringes of equal chromatic order. From the wavelengths of the latter the phase shift dispersion can be measured to an accuracy of about $10 \mathrm{~A}$. The method is especially useful for reflectors with large dispersion of phase shift, such as multilayers. Results in the visible spectrum are reported for aluminum films and a pair of dielectric 15-layer broadband reflectors. $10 \mathrm{p}$.

Infrared absorption spectrum of methane from $247^{\circ}$ to $3200 \mathrm{~cm}^{-1}$, E. K. Plyler, E. D. Tidwell, and L. R. Blaine

The absorption spectrum of methane has been measured from 2470 to $3200 \mathrm{~cm}^{-1}$ with a high-resolution infrared spectrometer. Pressures from 0.01 to $4.5 \mathrm{~cm}$ of the gas were used in order to observe both intense and weak lines. A total of 2,460 lines were measured in the entire region. The $\nu_{3}$ band at $3018 \mathrm{~cm}^{-1}$ was measured at very slow scanning rates and the lines of the $P$ and $R$ branches were resolved into several components. The observed spectrum is shown in five figures. $12 \mathrm{p}$.

Elastic constants of synthetic single erystal corundum at room temperature, J. B. Wachtman, Jr., W. E. Tefft, D. G. Lam, Jr., and R. P. Stinchfield

The six elastic constants (and six elastic compliances) of corundum were determined in the kilocycle per second frequency range by an accurate resonance method. The results were checked in the megacycle per second range with a less accurate, pulse velocity method. The elastic moduli for polycrystalline alumina calculated from the single crystal compliances determined by the resonance method are in good agreement with experimental values obtained on high density polyerystalline alumina. The variation of Young's modulus and of the shear modulus with orientation was ealeulated from the compliances and the results are shown graphically. The results of the present work do not agree well with previous work on single crystal sapphire. The specification of orientation and the theory used to calculate the elastic constants are given in detail to support the contention that the results of the present work are correct. $15 \mathrm{p}$.

Radial distribution study of vitreous barium borate, A. Bienenstock, A. S. Posner, and S. Block

X-ray diffraction, radial distribution studies of a 20 percent barium oxide, 80 percent $\mathrm{B}_{2} \mathrm{O}_{3}$ glass have been performed using both the atomic and electronic distribution functions. From these distributions, the average bariumbarium distance has been determined as $6.76 \mathrm{~A}$. This distance is in good agreement with that predicted by Levin and Block on the basis of a structural interpretation of immiscibility data. $5 \mathrm{p}$.

Separation and determination of small quantities of aluminum in steel, B. B. Bendigo and R. K. Bell

A method is described for determining small amounts of aluminum ( 0.01 to 0.3 percent) in stainless and carbon steels. A perchloric-sulfuric acid solution of the steel is electrolyzed in a mercury cathode cell to remove most of the iron, and an extraction with chloroform is made to remove elements such as aluminum, residual iron, and titanium as cupferrates from a solution buffered at $p \mathrm{H}$ 3.5. These elements are converted from cupferrates to perchlorates; all except aluminum are then extracted as cupferrates with chloroform from $4 \mathrm{~N}$ hydrochloric acid. Aluminum in the acid solution is determined photometrically with aluminon (ammonium aurintricarboxylate) at a wave length of approximately 540 millimicrons. An accuracy of \pm 0.005 percent aluminum is indicated. $4 \mathrm{p}$.

Conformations of the pyranoid sugars. II. infrared absorption spectra of some aldopyranosides, R. S. Tipson and H. S. Isbell

The conformations of twenty-four aldopyranosides have been studied by analysis of their infrared absorption spectra. The most stable conformations of twelve of the glycosides had previously been assigned by Reeves from a study of their instability factors; these conformations were assumed to apply to the crystalline state, for which the spectra had been recorded. The compounds were classified into (a) configurationally and (b) structurally related groups, and the spectra were intercompared. The analysis revealed groups of absorption bands which showed a concerted shift on change of anomeric disposition.

With these groups of absorption bands thus identified, intercomparison with nine of the remaining spectra afforded evidence that the anomeric group (1) is axial in methyl D-glycero- $\alpha$-L-gluco-heptopyranoside, methyl Dglycero- $\alpha$-L-manno-heptopyranoside, and methyl $\mathrm{D}$-glycero$\alpha$-D-gulo-heptopyranoside; (2) is equatorial in methyl 6 deoxy- $\beta$-L-mannopyranoside, methyl D-glycero- $\beta$-D-guloheptopyranoside, and cyclohexyl D-glycero- $\beta$-D-gulo-heptopyranoside; and (3) either is quasi or occurs as different (or mixed) axial and equatorial forms in methyl $\alpha$-D-lyxopranoside, methyl $\beta$-D-lyxopranoside, and (possibly) $\alpha$-D-methylgulopyranoside.

Three of the glycosides were available as their crystalline complexes with calcium chloride. The spectra of these complexes were also examined, and the effect of co-crystallization with calcium chloride is pointed out. $25 \mathrm{p}$.

3.3. PAPERS FROM THE JOURNAL OF RESEARCH OF THE NATIONAL BUREAU OF STANDARDS, SECTION B. MATHEMATICS AND MATHEMATICAL PHYSICS, VOLUME 63B, JULY-DECEMBER 1959

July-September 1950

Relations between summation methods and integral transformations, W. Greub

Relations between the Lototsky method of summation and those of Borel, Euler, and Knopp are obtained by associating an integral transformation with the series transformation. $13 \mathrm{p}$.

On a modification of Watson's lemma, F. Oberhettinger

The method of steepest descents is extended to the case when a saddle point and a pole of arbitrary order are involved. An application to a problem in diffraction theory is demonstrated. $3 \mathrm{p}$.

Principal submatrices of a full-rowed non-negative matrix, K. Goldberg

It is shown that one of the principal submatrices of an incidence matrix without zero rows is a permutation 
matrix. From this an inspection method is deseribed for determining whether a non-negative matrix is nilpotent. Application is made to graph theory. $2 \mathrm{p}$.

Zeros of certain polynomials, A. J. Goldman

Let $P$ be a real parameter. It is proved that all roots of $z^{n+1}-z^{n}+P=0$ lie in the open unit disk, if and only if $0<P<2 \sin \pi /(4 n+2) .2$ p.

Tables of transport integrals: a supplement, W. M. Rogers, W. J. Hall, and R. L. Powell

Tables of values of the transport integrals, defined by

$$
J_{n}(x) \equiv \int_{0}^{x} \frac{e^{z} z^{n} d z}{\left(e^{2}-1\right)^{2}},
$$

were previously published in NBS Circular 595 for values of $n$ from 2 through 17 . In this paper, values are given for the integrals where $n$ is 18 and 20 and where $x$ ranges from 0.2 to 50.0 in steps of $0.28 \mathrm{p}$.

Lens design: a new approach, O. N. Stavroudis

This paper describes a new method of defining the total aberrations of an optical system and its application to lens design. A system of partial differential equations defining optical image formation is written in the form of 4 by 4 matrices and is applied to the derivation of lens aberrations. It is shown that the form of the matrices facilitates the computation of these aberrations and that equations of condition on the lens parameters can be derived. The use of this method is illustrated through its application to a simple, well-known problem. $12 \mathrm{p}$.

Analytical integration of the differential equation for water storage, V. M. Yevdjevich

The integration of the storage differential equation at present is usually done mostly by graphical or numerical procedures. An approach to the analytical integration of that equation is the subject of this paper. A new method of fitting the given background curves by mathematically tractable expressions is introduced. The storage-outflow discharge relation is expressed in the form of a power function. A general differential equation for water storage $y^{\prime}+c P y^{2}-c y^{k}=0$ is derived, with $c$ and $k$ constants for the given reservoir, outflow shape and type of flow, and $P$ being the inflow hydrograph. The analytical solutions of this equation for $P=0, P=$ constant, and certain $P=f(t)$ are given for the integrable cases (tables 1 to 3 , eqs (12) to (29)). The application of the results obtained is discussed at the end of the paper. $10 \mathrm{p}$.

Compressible turbulent boundary layers with heat transfer and pressure gradient in flow direction, A. Walz

The best-known theoretical works on boundary-layer problems, especially in the case of compressible flow without or with heat transfer, are related to the laminar boundary layer, although the turbulent boundary layer is, in practice, of ten more interesting. The laminar boundary layer is more easily accessible to theoretical treatment because clearly defined relations exist between the viscosity $\mu$ and the shear stresses $\pi$. In the turbulent case, empirical relations must be introduced. Therefore, attempts to get exact solutions are not worthwhile, while efforts to obtain approximate solutions, based for instance on the von Karman-Pohlhausen principle (Z. Angew. Math. u. Mech. 1, 233, and 252, 1921) of utilizing integral conditions, appear to be appropriate to this problem. In the last few years the accuracy of such approximate solutions for the incompressible case was noticeably improved by the application of a new energy integral condition in connection with a new empirical law for the dissipation in turbulent boundary layers, stated by $\mathrm{J}$. Rotta (Ingr-Arch. 20, 195, 1952) and E. Truckenbrodt
(Ingr-Arch. 20, 212, 1952). The empirical laws for dissipation and for turbulent wall friction, which are needed in the present approximate theory, are formulated on the basis of available measurements for incompressible flow . Generalization to the compressible flow with heat transfer is made from physical considerations. Calculated results agree satisfactorily with available experimental data. Some possibilities for improving as well as for simplifying the approximation theory are outlined. $18 \mathrm{p}$.

October-December 1959

Applications of a theorem on partitioned matrices, E. V. Haynsworth

A reduction formula for partitioned matrices is applied to block-stochastic matrices and other types of partitioned matrices in order to reduce the computation in finding the eigenvalues. Such matrices occur frequently in physics and chemistry, and the reduction formulas given here have been applied successfully in practical problems. In addition, some results of $\mathrm{A}$. Brauer on stochastic matrices and of J. Williamson in partitioned matrices are generalized. 6 p.

Capacity requirement of a mail sorting device: II, A. J. Goldman

The combinatorial analysis of a mathematical model of a sorting device suggested by S. Henig is completed. The relevant parameters are $r$, the number of destinations for the mail, and $k$, the number of letters entering the device during each cycle of operation. The capacity of the device, if it is never to jam, should be between $r k$ and $r k-$ $(r-1)$ inclusive; arguments indicating that the latter value is preferable are given. $4 \mathrm{p}$.

Analy tic comparison of suggested configurations for automatic mail sorting equipment, B. K. Bender and A. J. Goldman

Analytic methods are developed to aid in determining the equipment configuration which achieves sorting of outgoing mail at a given (required) rate at least cost. The techniques are applied to a specific numerical problem; several of the suggested configurations are quickly eliminated, and a "hybrid" of two of the proposed configurations is found which comes within four percent of optimum (if a certain pair of parameters is chosen correctly). $22 \mathrm{p}$.

New method of solution for unretarded satellite orbits, J. P. Vinti.

An axially symmetric solution of Laplace's equation in oblate spheroidal coordinates is found, which may be used as the gravitational potential about an oblate planet. This potential, which makes the Hamilton-Jacobi equation for a satellite orbit separable, has an expansion in zonal harmonics in which the amplitudes of the zeroth and second harmonics can be adjusted to agree exactly with the values for any axially symmetric planet and a fourth harmonic which then agrees approximately with the latest value for that of the earth. The net result is therefore a reduction of the problem of satellite motion to quadratures, with use of a potential field that is much closer to the empirically accepted one for the earth than any heretofore used as the starting point of a calculation. It may thus be possible to do the gravitational theory of a satellite orbit very accurately without use of perturbation theory. The method can take into account a first harmonic in the potential, in case observations are reduced to a center which does not coincide with the center of mass of the planet. $12 \mathrm{p}$.

\section{Effect of sudden water release on the reservoir free outflow} hydrograph, V. M. Yevdjevich

The free outflow hydrograph is studied in the case of a sudden water release from a reservoir. The outflow hydrograph is called free when it is not affected by the 
tailwater levels. Both the effect of the steep negative wave, created by sudden water release, and the effect of the flow resistance are analyzed through the use of a fictitious inflow hydrograph. The water accelerated by the steep negative wave movement, whether or not the flow resistance is taken into account, gives this fictitious hydrograph. The procedures are given for the computation of the wave celerities and the new water velocities along the reservoir, for the computation of wavefront heights and the maximum outflow discharges, and for the determination of the fictitious and total inflow hydrograph. The examples are given for the pyramidal reservoirs of rectangular and parabolic cross sections. A general procedure for the determination of the reservoir free outflow hydrograph in an approximate form is derived. $13 \mathrm{p}$.

Uniform asymptotic expansions for Weber parabolic cylinder functions of large orders, F. W. J. Olver

Expansions of solutions of the differential equation

$$
\frac{d^{2} w}{d t^{2}}=\mu^{4}\left(t^{2}-1\right) w
$$

are sought for large values of $|\mu|$, which are uniformly valid with respect to arg $\mu$ and unrestrieted values of the complex variable $t$. Two types of expansion are found. Those of the first type are in terms of elementary functions and are valid outside the neighborhoods of the points $t= \pm 1$, the turning points of the differential equation. The second are in terms of Airy functions and hold in unbounded regions containing one of the turning points.

The special forms of the expansions when the variables are real are considered in detail, and asymptotic expansions for the zeros of solutions of the differential equation are found by reversion. Numercial examples are included. $39 \mathrm{p}$.

TITLE PAGE AND CONTENTS TO VOL. 63B. $3 \mathrm{p}$.

PAPERS FROM THE JOURNAL OF RESEARCH OF THE NA TIONAL BUREAU OF STANDARDS, SECTION B. MATHEMATICS AND MATHEMATICAL PHYSICS, VOLUME 64BJANUARY-JUNE 1960

Janusry-March 1960

Theory of diffraction in microwave interferometry, D. M Kerns and E. S. Dayhoff

Microwave Michelson and Fabry-Perot interferometers are respectively considered as instances of: (1) A "reflection system", consisting of a radiating-receiving system and a reflecting object (e.g., a finite mirror); and (2) a "transmission system", consisting of a radiating system and a receiving system with an object (e.g., a FabryPerot etalon) interposed. The basic theoretical objective is the calculation of the amplitude and phase of the (timeharmonic) received signal in the systems considered. The electromagnetic field in space transmission paths is represented in terms of continuous angular spectra of vectorial plane waves, and the elements of the systems are described by means of suitable tensor scattering matrices (having both diserete and continuous indices). Needed seattering matrices are considered known; relationships to experiinentally determinable data are outlined. The general case of either the reflection or transmission system is soluble formally in terms of a series of integrals stemming from the Liouville-Neumann series solution of certain integral equations. Formulas are obtained for models of the Michelson and Fabry-Perot instruments with arbitrary radiating and receiving characteristics. The theory and various features of the instruments considered, including Fresnel-region (or quasi-optical) behavior, are illustrated by means of examples obtained by choosing relatively simple and rather hypothetical analytical expressions for the radiating and receiving characteristics. $13 \mathrm{p}$.

Some solutions for electromagnetic problems involving spheroidal, spherical, and cylindrical bodies, J. R. Wait

Solutions are presented for the low-frequency electromagnetic response to an oscillating magnetic dipole by conducting bodies of simple shape. The quasi-stationary approximation is employed throughout, which is valid when the relevant dimensions of the problem are all small compared to the free-space wavelength. This amounts to matching solutions of the wave equation within the bodies to solutions of Laplace's equation outside. The results have application to geophysical prospecting. $18 \mathrm{p}$.

Kantorovich's inequality, M. Newman

An elementary proof with a generalization of an inequality of Kantorovich is given. 2 p.

A si mmetric continuous poker model, A. J. Goldman and J. J. Stone

Beginning with von Neumann, mathematicians concerned with the rational analysis of conflict situations have realized that investigation of accurate or simplified versions of common card games leads to techniques and insights applicable to situations of military or economic interest. In the present paper, a symmetric poker model one stage more complicated than the original von Neumann game is solved. There are two bet levels, $a, b$, and an ante of 1 unit $(a>b>1)$; no raises are permitted. The game has a unique optimal strategy, which forbids bluffing on a low hand. The limiting case $b=1$ is shown to vield the von Neumann model. $6 \mathrm{p}$.

Moebius function on the lattice of dense subgraphs, R. E.

Nettleton and M. S. Green

The Moebius function $f_{k}$ on the lattice of $k$-dense subgraphs of a connected graph, defined in a previous paper, is calculated for graphs $G$ containing isthmuses and articulators. $f_{1}$ evaluated for the null graph $\phi$ is shown to vanish if $G$ contains an isthmus, while for any integer $q$ there exist graphs containing articulators for which $f_{1}(\phi)=q$. The "lattice of path sets" joining a pair of points and the lattice of graphs "associated with $G$ and a subgraph $G^{\prime \prime \prime}$ are defined and the Moebius functions on these lattices are shown in certain cases to be related to $f_{1} .7 \mathrm{p}$.

The minimum of a certain linear form, K. Goldberg

The positive minimum of the integral linear form $L\left(x_{1}, \ldots, x_{n}\right)=a_{1} x_{1}+\ldots+a_{n} x_{n}$ is found subject to the conditions $a_{i}>0$ and $L\left(x_{1}, \ldots, x_{n}\right) \geq 2 a_{n} x_{i}$ for $i=1,2$, n. 2 p.

Space of $k$-commutative matrices, M. Marcus and N. A. Khan

Let $[A, X]=A X-X A$ and $[A, X]_{k}=\left[A,[A, X]_{k-1}\right]$. Those matrices $X$ which " $k$-commute" with a fixed matrix $A$ are investigated. In particular, the dimension of the null space of the linear transformation $T(X)=[A, X]_{k}$ when $A$ is nonderogatory is determined. $4 \mathrm{p}$.

Selected bibliography of statistical literature, 1930 to

1957: I. Correlation and regression theory, L. S. Deming

This is the first in a series of bibliographies dealing with various specific subjects in the field of statistics. References and titles of important contributions in correlation and regression theory have been taken from technical journals published throughout the world since 1930. $14 \mathrm{p}$.

Selected bibliography of statistical literature, 1930 to 1957: II. Time series, L. S. Deming

This is the second in a series of bibliographies dealing with various specific subjects in the field of statistics. References and titles of important contributions to the study of time series have been taken from a wide variety of technical journals published in the many languages and countries which have been actively engaged in statistical analysis. 8 p. 
Non-self-adjoint boundary value problems in ordinary differential equations, W. Greub and W. C. Rheinboldt

It is shown that the theory of non-self-adjoint linear ordinary differential equations can be simplified and unified if, instead of specifying linear boundary conditions in the conventional way, one merely specifies the linear subspace determined by the boundary conditions. If this is done, the corresponding linear space of the adjoint problem is the orthogonal complement of the original space with respect to the scalar product defined by the right-hand side of Green's boundary formula. 8 p.

Criteria for the existence and equioscillation of best Tchebycheff approximations, J. R. Rice

Let $F(a, x)=\Sigma a_{i} \varphi_{i}(x)$ where $\varphi_{i}(x)$ are continuous in $[0,1]$ and the $a_{1}$ are real parameters. The following theorem answers the principal questions of a general nature in the theory of Tchebycheff approximations: Theorem. Let $\{\varphi,(x)\}$ form a Tehebycheff set and let $f(x)$ be an arbitrary function continuous on $[0,1]$. Then (A) $f(x)$ possesses a best approximation, (B) a necessary and sufficient condition that $F\left(a^{*}, x\right)$ be a best approximation to $f(x)$ is that $\max \left|F\left(a^{*}, x\right)-f(x)\right|$ alternates at least $n$ times in $[0,1]$, (C) the best approximation to $f(x)$ is unique. A. Haar [Math. Ann. 78, 43-56 (1928)] posed and answered the following question: What conditions on $F$ are necessary and sufficient for theorem $\mathrm{C}$ to be valid? The condition he found is that $\left\{\varphi_{i}(x)\right\}$ must form a Tchebycheff set. This paper poses and answers the following three questions: (1) What conditions on $F$ are necessary and sufficient for theorem A to be valid? (2) What conditions on $F$ are necessary and sufficient for theorem B to be valid? (3) What conditions on $F$ are necessary and sufficient for both theorems $\mathrm{A}$ and $\mathrm{B}$ to be valid?

This paper does not tacitly assume that the $a_{i}$ may assume all values. $3 \mathrm{p}$.

Note on the solution of Riccati's differential equation, H. H. Howe

Three recurrence formulas are developed, giving the solution of a particular case of Riccati's equation in power series valid in the neighborhoods of $0, \infty$, and an arbitrary point, respectively. The first two were programed for computation on the SEAC. 4 p.

On a generalization of the index notation for absolute tensors of arbitrary order, E. H. Brown

A generalized vector index notation is introduced which facilitates study of properties of tensors regardless of order and variance of components. This generality is obtained by replacing the set of $m$ indices, each of which vary in a single domain of $n$ integers, by one vector index which varies over the integral lattice points of an $m$-dimensional domain. The notation also suggests an elegant treatment of order transformations of tensors, $5 \mathrm{p}$.

Upper and lower bounds for the center of flexure, L. E. Payne

There is disagreement in the literature as to how the center of flexure of an isotropic elastic beam should be defined. In either of the two most widely accepted definitions, upper and lower bounds for the coordinates of the center of flexure are obtained. $7 \mathrm{p}$.

Half-round inductive obstacles in rectangular waveguide, D. M. Kerns

Formulas are derived for the accurate calculation of the lowest-mode, lumped-element representation of perfectly conducting half-round inductive obstacles in rectangular waveguide. These obstacles consist of either one or two opposed semicircular cylindrical indentations extending across the narrow sides of the waveguide. They seem especially suitable for use as precise calculable standards of reflection or impedance in waveguide. Schwinger's integral equation approach is used to obtain stationary expressions for the desired parameters as functionals of the surface currents on the obstacles. Upper bounds are obtained for one of the two parameters. Explicit formulas are derived for the values of the parameters under the assumption of $n$-term Fourier sine-series expansions for the obstacle currents. Rapid convergence is indicated by numerical evaluations for $n=1,2$, and 3 . In the process of obtaining expressions suitable for numerical calculation, an expansion (believed to be new) of the Green's function of the problem is obtained and the sums of certain infinite series of Bessel's functions occurring in this expansion are expressed in terms of definie integrals. A brief numerical table of these sums, sufficient for the evaluation of the $n=1$ approximation, is included. $18 \mathrm{p}$.

3.4. PAPERS FROM THE JOURNAL OF RESEARCH OF THE NATIONAL BUREAU OF STANDARDS, SECTION C, ENGINEERING AND INSTRUMENTATION, VOLUME 63C, JULYDECEMBER 1959

July-September 1959

Creep of cold-drawn nickel, W. D. Jenkins and C. R. Johnson

Creep tests were made in tension under constant loads at temperatures of $300^{\circ}, 700^{\circ}, 900^{\circ}$, and $1,200^{\circ} \mathrm{F}$ on specimens of nickel initially cold-drawn to 40 -percent reduction in area. None of the equations for defining the strain-time relationship proposed by other investigators was found to be suitable to express or predict the creep-test results of this investigation with high accuracy because these equations inadequately describe the changes in structure accompanying the creep processes. However, conformance to the parabolic strain-time law was obtained over limited ranges of stresses and strains. The effects of cold-drawing on the creep properties are discussed. Certain etching techniques were employed that gave evidence of the presence and movement of dislocations in the structure. $18 \mathrm{p}$.

Friction and endurance of prelubricated and unlubricated ball bearings at high speeds and extreme temperatures, H. S. White

Endurance tests were made with 20-millimeter bore prelubricated ball bearings, at 10,000 revolutions per minute, using polyester felt rings saturated with di(2-ethylhexy) sebacate containing appropriate additives, at ambient temperatures ranging from $80^{\circ}$ to $400^{\circ} \mathrm{F}$. Tests were made with greases for comparative purposes. Results indicate that endurance with these oil-soaked pads is comparable with the high-temperature performance of MIL-G-3278 grease at $250^{\circ} \mathrm{F}$ and MIL-L-3545 grease at $300^{\circ} \mathrm{F}$. Some endurance tests were made with special bearings (20-millimeter bore) made of high-speed tool steel, prelubricated at $450^{\circ} \mathrm{F}$ ambient temperature, and unlubricated at $650^{\circ} \mathrm{F}$ ambient temperature $(5,000$ revolutions per minute at $650^{\circ} \mathrm{F}$ ). Experiments were made with greased bearings and with felt-pad oiled bearings in gyros and small high-speed direct-current motors at high temperatures. With oiled bearings and with unlubricated bearings, wearing or breaking of separators (ball cages) was the most common cause of failure. Compared to low-temperature greases MIL-G-3278 and MIL-G-7421 with oiled bearings starting friction was low and running friction was about one-tenth that of greased bearings at $-75^{\circ} \mathrm{F}$ ambient temperature. $11 \mathrm{p}$.

Effect of light and water on the degradation of asphalt, L. R. Kleinschmidt and H. R. Snoke

Coatings prepared from two blown petroleum asphalts in a thickness range of 0.002 to 0.04 inches were exposed to accelerated test conditions and outdoors. When exposed to light only, a surface film, insoluble in common asphalt solvents, was formed. The formation of this surface film was accompanied by a gain in weight of the coatings, apparently due to an oxygen pickup. This sur- 
face film retarded further degradation of the maltenes during the exposures made to light only. When the coatings were immersed in water after exposure, of sprayed with water during exposure, or exposed outdoors, they lost weight. These decreases in weight were found to be in part due to the extraction of water-soluble, light-degraded material. Their magnitudes were dependent upon the asphalt exposed, the thickness of the exposed coatings, and the exposure conditions. The relationship between the losses in weight and water-soluble material, when considered in conjunction with the oxygen content of the asphalts and the water-soluble materials, indicated that volatile degradation products were also formed.

When the surface skin formed by the action of light was partially removed by washing with water, percentage decreases were noted in the water-white oils, dark oils, and asphaltic resins. Since these decreases were unequal in magnitude and since the losses in weight were dependent on the thickness of the coating, it was concluded that lightdegradable components of the asphalt had migrated to the surface to replace degraded material that had been washed away. $6 \mathrm{p}$.

\section{Current and potential relations for the cathodic protection} of steel in a high resistivity environment, W. J. Schwerdtfeger

In order to evaluate potential and current criteria for the cathodic protection of bare low-carbon steel in a highresistivity environment, specimens were exposed in the laboratory for a period of two months to a soil having a resistivity of about 20,000 ohm-centimeter.

Previous work in low-resistivity environments by the author and by other investigators has shown that corrosion can be reduced to a negligible degree by polarizing a steel structure to -0.85 volt (protective potential) with reference to a copper-copper sulfate electrode. In such studies by the author, cathodic polarization curves have also been shown to be useful in indicating the current density required for cathodic protection.

In the present study the above criteria were again evaluated. In addition to protecting the steel at the protective potential (free of $I R$ drop), the effect on protection of including $I R$ drop caused by the protective current was also noted. Also, cathodic polarization curves were obtained on a recorder in conjunction with a bridge circuit to eliminate the $I R$ drop.

The results show that the best degree of protection was achieved on the specimen controlled at -0.77 volt (without $I R$ ) with reference to a saturated calomel half-cell. This is approximately equivalent to the protective potential -0.85 volt with reference to the copper-copper sulfate electrode. Applied current indicated by the break (change-in-slope) in the cathodic polarization curve agreed reasonably well with the actual current necessary to maintain polarization at -0.77 volt (free of $I R$ ).

The current required for protection was about three times the magnitude of the corrosion current; therefore, the corrosion reaction was either under anodic control (unlike previous studies) or an equivalent type of control which was caused by high resistance at anodic areas. $9 \mathrm{p}$.

A tilting air-lubricated piston gage for pressures below one-half inch of mercury, U. O. Hutton

A description is given of a tilting dead-weight piston gage constructed at the National Bureau of Standards for ranges of differential pressure up to about 0.5 inch of mercury. A resolution of better than 1 part in a hundred thousand of full scale has been obtained by use of the toolmaker's sine bar method of angle measurement. The scale is a linear function of the sine. The instrument can be calibrated from basic measurements of length and weight, is rugged, and may be constructed in almost any laboratory mechanical shop. Sources of possible errors in reading are discussed in detail. Comparative tests with certain other gages or manometers are cited wherein linearity was found to be within 1 part in 10,000 and agreement within 2.5 parts in 10,000 . The uses of the gage are briefly discussed. $11 \mathrm{p}$.
Compact multi-anvil wedge-type high pressure apparatus, E. C. Lloyd, U. O. Hutton, and D. P. Johnson

Apparatus for generating high pressures by application of force by an anvil against each face of a solid polyhedron is described. The equipment constructed utilizes a tetrahedron of pyrophyllite and four tungsten carbide anvils as described earlier by H. T. Hall. External force is applied to only one of the anvils, and wedge reaction forces act on the other anvils, permitting the equipment to be used in a conventional hydraulic press. Examples of results of use of the equipment are given. Success of the design has been shown by the repeated generation of pressures in excess of 100,000 atmospheres indicating that large existing presses might be used to generate pressures of this order in volumes of several cubic inches. For higher pressures a two-stage multi-anvil apparatus is proposed in which the second-stage anvils are embedded in a large pyrophyllite tetrahedron to obtain necessary supporting forces. $6 \mathrm{p}$.

\section{A coulometric-titration coulometer, S. W. Smith and J. K. Taylor}

A highly precise coulometer is described which permits time integration of currents totaling 100 coulombs or more with a precision of about 1 part in 100,000. The current to be integrated oxidizes hydroquinone in an electrolysis cell, producing quinone and acid. The quinone is then reduced by constant-current coulometric titration, the end point being indicated by hydrogen-ion concentration measurements. $4 \mathrm{p}$.

Electron beam magnetometer, L. Marton, L. B. Leder, J. W. Coleman, and D. C. Schubert

A theoretical investigation of the electron optics of an electron beam deflection method for detecting small magnetic fields is presented. It is shown that remarkably high sensitivity can be reached. A laboratory model of such a magnetometer was constructed, and it was demonstrated that the theoretical estimate of sensitivity, $3 \times 10^{-5}$ ampere per oersted, could be attained in practice. A discussion of the possible improvements which could extend the sensitivity of the device is also given. $7 \mathrm{p}$.

A refined $\mathrm{X}$-band microwave microcalorimeter, G. F. Engen

The microcalorimetric method for evaluation of the efficiency and substitution error of a bolometer mount proposed by Macpherson and Kerns has been the object of further study and refinement at the Boulder Laboratories of the National Bureau of Standards, and an improved instrument based on this technique has been recently placed in operation.

The new microcalorimeter design features are: (1) Greatly improved ambient temperature control, permitting higher sensitivity and resolution; (2) improved d-c instrumentation; (3) improved mechanical construction giving better repeatability; (4) relocation of the thermopile so that it no longer is attached directly to the bolometer mount, thus providing flexibility in the choice of termination; and (5) a more comprehensive treatment of the calorimetric substitution or equivalence error. These features permit the determination of the effective efficiency of a bolometer mount to an accuracy of better than 0.2 percent. $6 \mathrm{p}$.

Ootober-December 1950

Conical coaxial capacitors and their advantages, M. C. Selby

Adjustable capacitors having electrodes in the form of coaxial cones or frustums have been used on rare occasions in the past, but their potential superiority to other types of capacitors for some important applications has been overlooked. The advantage of this geometry over cylindrical or disk forms is that the practical capacitance range is several times larger. An example cites the capacitance ranges for the same mechanical and percentage 
accuracy of a disk, cylindrical and conical type to be 10 , 40 , and 168 to 1 , respectively. An approximate equation was derived for this conical capacitor and close agreement is shown between computed and measured values of capacitance versus electrode displacement. Multiple cone and specially shaped electrodes are suggested to obtain large values of capacitance with an appreciable saving of space and further increased range of capacitance. $3 \mathrm{p}$.

A photoelectric followup and recording system, and its application to remote observations of the beam in high-precision balances, H. A. Bowman and L. B. Macurdy

A device is described which permits a significant increase in the frequency of the oscillations of the beam of a balance, thereby shortening the interval during which upsetting ambient conditions can act. The device is a servo-driven photoelectric followup system responding to the positionmodulated light signal reflected from a mirror attached to the balance $12 \mathrm{ft}$ away from the photocell. Reproducibility in observing the position of the light beam is 0.001 in, or less. $6 \mathrm{p}$.

A stroboscopic vibration analyzer, S. Edelman, R. Brooks, S. Saito, E. Jones, and E. R. Smith

An instrument for studying the amplitude and phase relations which exist in complex periodic processes is described in this paper. The process treated here is vibration of complicated bodies. The motion at each point of interest is detected by a vibration pickup. The signal from each pickup is heterodyned with a common signal that differs from the vibration frequency by a small amount to produce a set of signals at the difference frequency. The desired relations are exhibited at the difference frequency which is low enough to give a slow motion effect. The output circuitry allows scope for ingenuity to choose a presentation suited to a particular problem, $7 \mathrm{p}$.

Evaluation of lens distortion by the inverse nodal slide, F. E. Washer and W. R. Darling

The evaluation of lens distortion by means of the inverse nodal slide method is discussed. The sources of error inherent in the method are investigated. Emphasis is given to the effect of asymmetric use of apertures. Measurements are reported on the same lens for which distortion values were measured by the direct nodal slide and photographic methods. A comparison of results obtained by the three different methods is given. It is concluded that accurate values of the distortion of a lens can be obtained by the inverse nodal slide method and that the precision of measurement is comparable to that of the two other methods mentioned when proper attention to sources of error is given. $8 \mathrm{p}$

Evaluation of lens distortion by the modified goniometric method, F, E. Washer and W. R. Darling

This is the third of a series of papers dealing with factors affecting the accuracy of measurement of lens distortion by various visual and photographic processes. This paper presents the results of a study of the visual modified goniometric method which employs a viewing theodolite and accurate linear scale. Sources of error are investigated and discussed. Measurements are reported on the same lens that was used in the analysis of two visual and one photographic distortion measuring processes. A comparison of results by the four different methods is given. It is concluded that accurate values of lens distortion can be obtained by the modified goniometric method provided care is taken to use centered apertures and to preserve known angular relationships between telescope pointings and successive locations. $8 \mathrm{p}$.

Proposed criteria for defining load failure of beams, floors, and roof constructions during fire tests, J. V. Ryan and A. F. Robertson

A brief account is presented of procedures used in development of criteria for defining the point at which fire test specimens fail to sustain load during test. It is proposed that both a deflection of $L^{2} / 800 d$ and an hourly rate of deflection of $L^{2} / 150 \mathrm{~d}$ be taken as an indication of load failure. In these formulas, $L$ is the span between supports of the member or element found to be critical under fire exposure, and $d$ is the distance between upper and lower extreme fibers of the particular structural component or assembly. $4 \mathrm{p}$.

Conductive flooring for hospital operating rooms, T. $H$. Boone, F. L. Hermach, E. H. MacArthur, and R. C. MeAuliff

Characteristics and performance of available types of conductive flooring materials were investigated in the laboratory. The study showed that the electrodes and instruments used to measure the floor greatly affected the measured resistance, but that the method specified by the National Fire Protection Association for measuring the electrical resistance reasonably simulated the conditions under which a floor functions in reducing electrostatic hazards. The physical, chemical, and serviceability characteristics of conductive floorings investigated showed results comparable with those of nonconductive flooring of the same type. Consequently, with some limitations, an architect may base his choice of a conductive flooring material on his knowledge of the behavior of similar nonconductive materials. $16 \mathrm{p}$.

Measurement of the aging of rubber vulcanizates, J.

Mandel, F. L. Roth, M. N. Steel, and R. D. Stiehler

A study of aging data in the literature and of measurements made at the National Bureau of Standards indicates that ultimate elongation is the best of the tensile properties for characterizing the deterioration of rubber vulcanizates during storage at various temperatures. Ultimate elongation (strain at failure) decreases during aging for all types of rubber vulcanizates; whereas tensile strength and modulus may increase, decrease, or remain essentially unchanged.

This study includes measurements of ultimate elongation of a nitrile rubber vulcanizate after various periods of storage at temperatures of $23^{\circ}, 34^{\circ}, 45^{\circ}, 57^{\circ}, 70^{\circ}, 85^{\circ}$, and $100^{\circ} \mathrm{C}$. It also includes a study of the published data on ultimate elongation obtained in an interlaboratory test conducted by Subcommittee 15 of ASTM Committee D-11, involving vulcanizates of five different rubbers stored at $25^{\circ}, 70^{\circ}, 100^{\circ}$, and $121^{\circ} \mathrm{C}$.

The change in ultimate elongation over prolonged periods of storage cannot be expressed by a simple mathematical equation. However, during most of the useful storage life of a rubber vulcanizate, the elongation decreases approximately linearly with the square root of time. The data indicate that for some vulcanizates an estimate of storage life at room temperature can be made from measurements of ultimate elongation at two or more elevated temperatures. $5 \mathrm{p}$.

$$
\text { TITLE PAGE AND CONTENTS TO VOL. 63C. } 3 \text { p. }
$$

PAPERS FROM THE JOURNAL OF RESEARCH OF THE NA. TIONAL BUREAU OF STANDARDS, SECTION C, ENGINEERING AND INSTRUMENTATION, VOLUME 64C, JANUARYJUNE 1960

Power loss and operating temperature of tires, R. D. Stiehler, M. N. Steel, G. G. Richey, J. Mandel, and R. H. Hobbs

The power loss of pneumatic tires was measured under steady-state conditions by means of two dynamometers, one of which measured the total power input and the other power output. A steel wheel was used to measure the power loss in the equipment and in windage. The power required to flex the tire (input power minus output power minus equipment and windage losses), was not affected by the tractive effort (output power). The coefficient of rolling resistance, a dimensionless quantity, was calculated by means of the equation: $R=(P / S L)$, where $P$ is power loss of tire, $S$ is speed, and $L$ is load. This coefficient increased at an increasing rate with the slip angle (angle 
between plane of tire and direction of travel) and was approximately doubled at an angle of 2 degrees.

The change in $R$ with speed varied and appeared to depend on construction of the tire. Both nylon and steel wire truck tires exhibited a decrease in $R$ with speed, rayon truck tires showed either no change or a linear increase in $R$ with speed, and for passenger car tires $R$ increased at an increasing rate with speed, particularly for a rayon tire. $R$ increased with load for all but the steel wire tire. The change in $R$ with inflation pressure was studied only for rayon truck tires. $R$ increased linearly with the reciprocal of the pressure but the rate was dependent on the speed and load conditions.

The type of rubber had a pronounced effect on $R$ and combinations of natural and styrenebutadiene rubber caused $R$ to be larger than expected from the values of $R$ for tires made from a single rubber. Varying the type of carbon black in the treads of truck tires had no effect on $R$, but SAF black in passenger car tires eaused $R$ to be larger than that when HAF black was used. Because of constructional differences, no conclusions on effect of cord could be drawn. However, $R$ for a steel wire truck tire was the lowest observed, and the values for rayon tires were lower than those for nylon tires except at high speeds.

The temperature rise of the air in the inner tube was found to be related to the power loss by the relation: $\Delta T / P=G+H /(S L)^{0.8}$ where $G$ and $H$ are parameters dependent on the thermal resistance of the rubber compounds and of the interface between tire and air or roadway, respectively. The ratio $\Delta T / P$ remained essentially unchanged by ehanges in inflation pressure and did not appear to be affected by the type of cord. $11 \mathrm{p}$.

Effects of antioxidants on asphalt durability, B. D. Beitehman

The mechanism by which asphalt degrades during weathering can be studied profitably by observing the action of materials which inhibit this degradation.

A number of antioxidants which are believed to function either by their ability to inhibit chain reactions or to decompose peroxides were studied for their effectiveness in retarding weight loss and promoting durability of roofing asphalts. Inhibitors of chain reactions in some cases inhibited weight loss without extending durability. Compounds which decompose peroxides were observed to be capable of extending the iurability of an asphalt and also of retarding weight loss. Phenothiazine, which is believed to function primarily as a peroxide decomposer, proved to be the most outstanding of the inhibitors tested.

A study made in one asphalt of the dependence of phenothiazine activity on concentration indicated that a concentration of 2 percent was optimum. In several other asphalts, 2 percent phenothiazine retarded weight loss to varying extents without significantly altering the durability of the asphalt

Synergistic activity was shown by the use of an inhibiting mixture containing phenothiazine and $N$-phenyl-2naphthylamine.

The results of this study support the hypothesis of a free radical process being involved to a considerable degree in asphaltic degradation during weathering. $5 \mathrm{p}$.

Temperature stratification in a nonventing liquid helium Dewar, L. E. Scott, R. F. Robbins, D. B. Mann, and B. W. Birmingham

The presence of a large temperature gradient in Dewars used for transporting helium is undesirable because it may be accompanied by unnecessarily high internal pressures when the contents are sealed. In a study of the problems, such gradients were observed in experiments conducted with a 39.7-liter stainless steel Dewar. A method is shown for calculating the pressure rise in the absence of temperature gradients and the results are compared with the observed pressure rise. In some cases curves representing calculated and observed pressure rise intersect. A possible explanation of this situation is given. The destratifying effect of a concentrated heat input and of copper rods is shown. $5 \mathrm{p}$.
Expansion engines for hydrogen liquefiers, E. H. Brown

Criteria are developed for the practicability of expansion engines and expansion turbines in large hydrogen liquefiers. Some additional data on pertinent properties such as the sonic velocity of normal hydrogen are included. The analysis suggests that use of expansion turbines in hydrogen liquefiers having a production capacity of less than 5,000 liters per hour ordinarily would not be justified. For greater capacities, however, use of modern materials and design should make expansion turbine performance even more favorable than in liquefiers for denser fluids. $12 \mathrm{p}$.

A statistical chain-ratio method for estimating relative volumes of mail to given destinations, N. C. Severo and A. E. Newman

A sampling method, called the chain ratio method, is applied in estimating the distribution of mail by destination. Variances and coefficients of variation for the estimators are given. The details and results of three applications of this sampling method to outgoing first-class letter mail are given. These studies were conducted by the National Bureau of Standards in San Francisco, Los Angeles, and Baltimore. $11 \mathrm{p}$.

Standard free-air chamber for the measurement of low energy X-rays (20 to 100 kilovolts-constant-potential), V. H. Ritz

A description of the new National Bureau of Standards "low" energy free-air chamber is given. The standard chamber is designed to measure the exposure dose in roentgens for X-ray beams generated at potentials from 20 to 100 kilovolts-constant-potential (kvep) with flltrations ranging from 2 millimeters of beryllium to 2 millimeters of beryllium plus 4 millimeters of aluminum. The chamber has been compared with the National Bureau of Standards "medium" energy standard at 60,75 , and 100 kvep with filtrations of 3,3 , and 4 millimeters of aluminum, respectively. The two standard chambers agreed to within 0.3 percent. $5 \mathrm{p}$.

Transmittance of materials in the far infrared, E. K. Plyler and L. R. Blaine

The transmittance of several crystalline materials with thicknesses of about $5 \mathrm{~mm}$ has been measured from 17 to $55 \mu$. The crystals are sodium chloride, potassium chloride, potassium bromide, thallium bromide-iodide, cesium bromide, and cesium iodide. Also the transmittance of a polyethylene film containing carbon black has been measured to $100 \mu$ and an example of its use as a transmission filter for the far infrared is given. $2 \mathrm{p}$.

Equipment and method for photoelectric determination of image contrast suitable for using square wave targets, F. W. Rosberry

Conventional measurements of the resolving power of lenses employ measuring photographs of test charts containing an array of accurately spaced parallel lines. This method has limited precision because of the variability of photographic emulsions and is time-consuming in operation. This paper extends previous work by others in obviating these differences by using a direct photoelectric scanning of a line-pattern image formed by the lens under test. Square wave high contrast resolving power targets with two different line pattern arrangements were used as test objects. The image was moved across a stationary slit and photomultiplier tube. The output was recorded as relative transmission. $8 \mathrm{p}$.

Formation of silver sulfide in the photographic image during fixation, C. I. Pope

A photographic silver image is made permanent (fixed) after development by bathing it in a solution containing thiosulfate which forms a soluble thiosulfate complex with 
the residual silver halide. Some of the silver in the image is sulfided by the thiosulfate during fixation. The purpose of this study was to determine the amount of sulfiding of the silver in the image during fixation of film and paper. The amount of the silver reacting depends on the type of the light-sensitive layer. Also, during bleaching, the residual thiosulfate in the film or paper reacts with silver in a potassium dichromate-sulfuric acid bleach bath to form silver sulfide. For one paper, it was shown that the amount of silver sulfide which was formed in the bleach bath increased with the increase of the concentration of the residual thiosulfate in the paper. A procedure was developed for the reduction of silver sulfide in an emulsion layer to silver so that the silver sulfide may be determined in terms of the optical density of the silver deposit. The use of hypo eliminators was investigated and a test procedure was found for testing the effectiveness of hypo eliminators. A small amount of potassium iodide added to the fixing bath was found effective in preventing most of the sulfiding of the silver image during fixation. 9 p.

\section{Capacitor calibration by step-up methods, T. L. Zapf}

Step-calibration methods are used in many physical laboratories for the extension of measurements to quan tities far removed from the magnitude of greatest accuracy at which absolute determinations are made. The excellent precision of repetitive substitution procedures is exploited by step-up or step-down methods to extend measurements to higher or lower magnitudes without serious degradation of accuracy. The application of step-up techniques to the calibration of variable air capacitors is described in this paper as a practical example of the method. $5 \mathrm{p}$.

April-June 1960

Measurement of cobalt-60 and cesium-137 gamma rays with a free-air chamber, H. O. Wyckoff

Design data for free-air chambers measuring cobalt-60 and cesium-137 gamma rays in roentgens are presented. It has been shown that the Jaffé-Zanstra method of obtaining the saturation current is adequate for air pressures of about 4 to 12 atmospheres. Also, radiation measurements of the gamma rays from cobalt-60 and cesium-137 made by a cavity chamber and a free-air chamber agree to within the experimental errors. $11 \mathrm{p}$.

Apparatus for the measurement of the normal spectral emissivity in the infrared, A. G. Maki, R. Stair, and R. G. Johnston

Apparatus and methods are described for measuring in the infrared the normal spectral emissivity of metals and coatings or oxides which tightly adhere to metals. Examples of the use of this apparatus are given in measurements of the emissivity of platinum and of oxidized Inconel within the spectral region of 1.5 to 15 microns. Measured values were reproduced to better than 5 percent. 4 p.

Electrostatic deflection plates for cathode-ray tubes.

I. Design of single-bend deflection plates with parallel entrance sections.

II. Deflection defocusing distortion of single-bend deflection plates with parallel entrance sections, L. Frenkel

In section I, a plate design system is offered which allows rapid and accurate determination of mechanical plate parameters to achieve given electrical plate characteristics. The design is suitable for single-bend plates with parallel entrance sections. The design curves were calculated under the conventional assumptions of small deflection theory, but corrections for the entrance and exit fields are included. The method of calculating the curves and corrections is indicated in an appendix.

Section II deals with deflection defocusing of such plates, a convenient formula for calculating the defocusing distortions of single-bend deflection plates is derived and compared with experiment. This type of distortion is proportional to the square of the deflection angle and is shown to be sensitive to plate design. In general, long deflection plates give lower distortions while the most "economical" plates yield larger distortions. Post deflection is shown to lead to increased distortions. $11 \mathrm{p}$.

The functional synthesis of linear plots, J. P. Vinti and R. F. Dressler

In practical engineering or experimental work one often encounters a function $F$ of many variables, $F$ ( $x$ 's, $y^{\prime}$ s, $z^{\prime}$ s), represented only by the families of curves obtained by plotting $F$ against each of the $x$ 's on Cartesian graph paper, against each of the $y$ 's on semilog paper, and against each of the $z$ 's on double-log paper. It frequently happens that these curves are all approximately straight lines over a limited range of interest. On the assumption that they are all true straight lines, the present note shows how to synthesize all the graphical representations, for any number of parameters, into the most general formula possible, expressing $F$ as the product of a multilinear function of the $x$ 's and the exponential of a constant-free multilinear function of the $y$ 's and of the $\log z$ 's, the coefficients in both multilinear functions being independent of the $x$ 's, $y$ 's, and $z^{\prime}$ s. 5 p.

Radiation field from a rectangular source, J. H. Hubbell, R. L. Bach, and J. C. Lamkin

Many radiation shielding problems involve calculations of the response of an isotropic detector to radiation of arbitrary angular distribution from uniform rectangular sources. In calculations of this type the family of integrals $\int s\left(\cos \theta d S / r^{2}\right) P_{l}(\cos \theta)$ and the integral $\int s\left(d S / r^{2}\right) \exp (-\mu t /$ $\cos \theta)$ are frequently encountered, where $\theta$ is obliquity with respect to an axis perpendicular to the plane containing the rectangular radiant surface, $S, r$ is the distance from an element of source area, $d S$, to the detector, $\mu$ is the attenuation coefficient, and $t$ is the barrier thickness. Solutions of the first type of integral facilitate use of Legendre expansion representations of radiation directional distributions, and may also have application in other radiant surface studies, such as illumination and heat exchange engineering. The second integral relates to exponentially attenuated radiation from a plane isotropic rectangular source separated from the detector by a layer of material of thickness $t$. Formulas, expansions, and numerical results are presented. $18 \mathrm{p}$.

Microwave attenuation measurements with accuracies from 0.0001 to 0.06 decibel over a range of 0.01 to 50 decibels, G. F. Engen and R. W. Beatty

The application of certain power stabilization and measurement techniques to the problem of attenuation measurement has yielded a measurement system with a stability and resolution of the order of 0.0001 decibel. A practical application for this technique was recently provided in the calibration of a rotary vane type of variable microwave attenuator.

In order to take complete advantage of this increased stability it was necessary to apply refined techniques to the evaluation and reduction of mismateh error.

This proved to be by far the most exacting practical application of the eited techniques encountered to date, but the results of this calibration showed excellent agreement with the mathematically predicted values used in marking the attenuator dial. $7 \mathrm{p}$.

Effect of oleophobic films on metal fatigue, H. E. Frankel, J. A. Bennett, and W. L. Holshouser

The fatigue strengths of a low-alloy steel, a magnesium alloy, and a copper-beryllium alloy were increased by coating the specimens with certain polar organic compounds. Also the dispersion of the results was much less for coated specimens of these materials than for clean ones. Similar tests showed no effect with titanium or 
6061 aluminum alloy, and only a slight improvement for 17-7 PH stainless steel.

The full beneficial effect of the coatings was found only with compounds having a carbon chain of at least twelve, and this effect was not significantly reduced when the coated specimens were tested in water. Organic solvents (benzene and xylene) had a deleterious effect on the fatigue life of materials that were improved by oleophobic coatings.

It is suggested that the effect of the coatings is principally due to their ability to present a barrier to water and oxygen molecules. $4 \mathrm{p}$.

Ratio-recording spectroradiometer, H. K. Hammond III, W. L. Holford, and M. L. Kuder

A ratio-recording spectroradiometer has been constructed primarily for determining the relative spectral irradiance from fluorescent lamps in the visible spectrum. Radiant flux from a test source and a comparison source is transmitted or reflected by separate diffusers. The irradiance from each diffuser is sampled alternately of the order of 100 times a second by a double prism monochromator with cam-linearized wavelength drive from 360 to $760 \mathrm{~m} \mu$. A 14-stage multiplier phototube with $\mathrm{S}-20$ response is used with an electronic gate and integrator circuits to compare the spectral irradiances from the two sources. When the phototube outputs are unequal, a servo unit adjusts apertures in each beam to equalize them. The measured parameter is the amount of adjustment required for equalization at each wavelength. The apertures are adjusted by a cam coupled to a pen which records the ratio on a three-cycle logarithmic strip chart. The instrument requires about 8 minutes to record the spectrum at $10 \mathrm{~m} \mu$ per inch. An expanded scale of $1 \mathrm{~m} \mu$ per inch is used for evaluating the energy in the spectral lines. The speed of wavelength scan may be made inversely proportional to the unbalance signal, if desired, to provide ample time to record spectral lines accurately. Symmetry of beam treatment is demonstrated by interchanging test and comparison sources and recording the inverse ratio. $7 \mathrm{p}$.

An intermittent-action camera with absolute time calibration, G. Hefley, R. H. Doherty, and E. L. Berger

A detailed description is presented of a film-recording system in which a randomly occurring event and its absolute time are recorded simultaneously. The system consists of a 16-millimeter framing camera capable of intermittent operation at a maximum rate of 140 frames per second (fps) and a clock capable of reading out time with an absolute accuracy of plus or minus 1 millisecond (msec). 7 p.

3.5 PAPERS FROM THE JOURNAL OF RESEARCH OF THE NATIONAL BUREAU OF STANDARDS, SECTION D. RADIO PROPAGATION VOLUME 63D, JULY-DECEMBER 1959

\section{July-Ausuat 1959}

Preliminary results of the National Bureau of Standards radio and ionospheric observations during the international geophysical year, D. M. Gates

A review is given of the activities of the National Bureau of Standards during the International Geophysical Year. The equipment used on each project is described and preliminary results of the observations are given. The following areas of research are discussed: (1) World Warning Agency, (2) Ionospheric Vertical Sounding Stations, (3) VHF Propagation, (4) VHF Equatorial Forward Scatter, (5) Radio Noise Network, (6) Radio Satellite Observations, (7) Airglow Observations, and (8) World Data Center for Airglow and Ionosphere. $14 \mathrm{p}$.

Origin of [OI] 5577 in the airglow and the aurora, F. E. Roach, J. W. McCaulley, and E. Marovich

The distribution of 5577 zenith intensities at stations in the subauroral zone is found to be unimodal with no discontinuity at the visual threshold. This is interpreted as evidence that 5577 airglow and 5577 aurora may have a common origin. $4 \mathrm{p}$.

Comparison of absolute intensities of [OI] 5577 in the auroral and subauroral zones, F. E. Roach, J. W. McCaulley, and C. M. Purdy

The distribution of 5577 zenith intensities is compared for Fritz Peak, Colo., in the subauroral zone and Thule, Greenland, near the geomagnetic pole. The absolute intensity is bright enough to permit visual detection at the Colorado station during 2 percent of the time and at the Greenland station, 27 percent of the time. The distribution curves have a general similarity, suggesting a phenomenological similarity in the excitation mechanisms at the two stations. $2 \mathrm{p}$.

Origin of "very-low-frequency emissions," R. M. Gallet and R. A. Helliwell

Selective traveling-wave amplification in the outer ionosphere is postulated to explain very-low-frequency emissions, a class of very low-frequency (1 to 30 kilocycles per second) natural noise. By analogy with the mechanism of traveling wave tubes, low-level ambient noise in the outer ionosphere is amplified in streams of incoming ionized solar particles at frequencies for which the stream and wave velocities are equal. Required velocities are in the range 0.01 to $0.1 c$ (where $c$ is the velocity of light). Streams with densities of the order of one electron per cubic centimeter would provide sufficient energy. Phenomena which can be explained qualitatively by the theory are the hiss, quasi-constant tones, dawn chorus and related transients, and very long trains of whistler echoes. A quantitative example shows how the theory can reproduce the general form of certain cliaracteristic discrete spectra "hooks" of emissions, and how this leads to definite values of particle velocity and a law for the distribution of electron density in the outer ionosphere. $7 \mathrm{p}$.

\section{Climatology of ground-based radio ducts, B. R. Bean}

An atmospheric duct is defined as occurring when geometrical optics indicate that a radio ray leaving the transmitter and passing upwards through the atmosphere is sufficiently refracted that it is traveling parallel to the earth's surface. Maximum observed incidence of ducts was determined as 13 percent in the tropics, 10 percent in the arctic and 5 percent in the temperate zone by analysis of 3 to 5 years of radiosonde data for a tropical, temperate, and arctic location. Annual maximums are observed in the winter for the arctic and summer for the tropics. The arctic ducts arise from ground-based temperature inversions with the ground temperature less than $-25^{\circ} \mathrm{C}$ while the tropical ducts are observed to occur with slight temperature and humidity lapse when the surface temperature is $30^{\circ} \mathrm{C}$ and greater. $6 \mathrm{p}$.

Power requirements and choice of an optimum frequency for a worldwide standard-frequency broadcasting station, A. D. Watt and R. W. Plush

Calculations are presented for the expected transmission characteristies and atmospheric noise levels in the 8- to 100 -ke band. When these are combined with carrier-tonoise requirements for a given precision of frequency comparison, it is indicated that a minimum radiated power in the order of 10 to $100 \mathrm{kw}$ for frequencies in the vicinity of $20 \mathrm{kc}$ will be required to provide worldwide coverage. Minimum observation times of 15 to 30 min appear to be required for these transmitter powers in order to obtain a precision of frequency comparison of 1 part in $10^{9}$ for typical transmission paths. Carrier-to-noise requirements and the factors determining this ratio are considered for typical receiving systems. $10 \mathrm{p}$. 
Measurements of phase stability over a low-level tropospheric path, M. C. Thompson, Jr., and H. B. Janes

A knowledge of the statistics of atmosphere-induced variations in the phase of the received signal (i.e., variations in electrical path length) is essential in evaluating the reliability of any system using radio waves for measuring distance and/or velocity. This paper describes an analysis of phase variations measured at 9,400 megacycles per second over a 9.4-mile path near Boulder, Colorado, during a 40-hour period in September 1958. The power spectral density of these variations is shown to be approximately proportional to $f^{-2.8}$ over a wide range of frequencies throughout the period of recording. The long-term phase variations are closely correlated with atmospheric refractivity measurements made at the path terminals. $7 \mathrm{p}$.

\section{System loss in radio wave propagation, K. A. Norton}

A summary is presented of the ways in which the concept of system loss and the closely related concepts of transmission loss, basic transmission loss, propagation loss, and path antenna gain may be used for precise, yet simple, descriptions of some of the characteristics of radio wave propagation which are important in the design of radio systems. Definitions of various terms associated with the concept of system loss are given which introduce a greater flexibility into its use without any loss in precision. It is shown that the use of these added terms and concepts makes feasible the extension of the use of this method of description to any portion of the radio spectrum. A more general formula for the system loss is given which may be used for antennas with an arbitrarily small separation. Using this formula it is shown that the system loss between small electric or magnetic dipoles separated by a distance $d \ll \lambda$ can be made arbitrarily small even though the individual antennas have large circuit losses. Formulas are developed for the percentage of time that a desired signal is free of interference, and these are used to demonstrate methods for the efficient use of the spectrum. In particular, contrary to general belief, it is shown that efficiency is promoted by the use of high power and high antennas and, in the case of a broadcast service, sufficiently small separations so that there is appreciable mutual interference. An analysis is made of the variance of the path antenna gain in ionospheric scatter propagation. Methods are given for the calculation of the transmission loss for the ground wave and tropospheric scatter modes of propagation through a turbulent model atmosphere with an exponential gradient. Examples of such calculations are given which cover a wide range of frequencies and antenna heights. Finally, examples are given of the expected range of various tropospheric point-to-point scatter systems such as an FM multichannel teletype system, a television relay or an FM broadcast relay. $21 \mathrm{p}$.

Mode expansion in the low-frequency range for propagation through a curved stratified atmosphere, H. Bremmer

This expansion is particularly useful when considering ionospheric propagation at low frequencies. The complex problem dealing with two media, viz., a homogeneous earth and a surrounding stratified atmosphere, leads to intractable expressions. However, as the influence of the earth may be accounted for by an approximate boundary condition at the earth's surface, the problem is then reduced to that of the outer medium only. The coefficients of the mode expansion for this simplified problem will be derived while taking into account the earth's curvature; however, the latter proves to be negligible under very general conditions. The expansion to be derived is wanted in particular when studying the influence of a gradual transition in the electron density with height at the lower edge of the ionosphere. $11 \mathrm{p}$.

Transmission and reflection by a parallel wire grid, M. T. Decker

A comparison is made at $\mathrm{X}$-band frequencies of the theoretical and measured transmission and reflection co- efficients for a parallel wire grid. The methods used are applicable to the measurements of these factors for various building materials. $4 \mathrm{p}$.

Synoptic variation of the radio refractive index, B. R. Bean and L. P. Riggs

The synoptic variation of the atmospheric radio refractive index, evaluated from standard weather observations, is examined during an outbreak of polar continental air. It is found that the reduced-to-sea-level value of the refractive index is a more sensitive synoptic parameter than the station value. The reduced value is quite sensitive to the humidity and density structure of the storm under study while the great station elevation dependence of the station value tends to mask synoptic changes. The reduced value changes systematically with the approach and passage of the polar front. The present system shows a consistent increase of the reduced value in the warm sector of the wave and a marked decrease behind the cold front. $7 \mathrm{p}$.

Low-frequency propagation paths in arctic areas, A. D.

Watt, E. L. Maxwell, and E. H. Whelan

The very low ground conductivities encountered in arctic areas, and the particular ionospheric conditions prevailing at high latitudes, can lead to rather unusual radiation and propagation conditions. In order to determine the magnitude of these effects, field intensities from transmitters located in the Labrador and Greenland areas were measured both on the surface of the earth and during several aircraft flights over this area. The many factors involved in lf propagation are considered and calculated field intensities compared with experimental values. Under conditions where the initial portion of the propagation path is across icecap or permafrost, the attenuation observed is very great, and when the propagation path extends out over sea water, the field intensity recovery taking place after the coastline is crossed is very marked. Estimates of skywave field intensity appear to agree with the observed results provided the radiated field pattern is suitably modified by the antenna cutback factor which accounts for the presence of a finitely conducting curved earth. These vertical patterns based on work by Wait, along with the field intensity flight data, indicate that the siting of low-frequency stations several miles or more inland in arctic regions may cause a great increase in total transmission path loss. $14 \mathrm{p}$.

Stratification in the lower ionosphere, C. Ellyett and J. M. Watts

A survey of the evidence for stratification in the ionosphere below $100 \mathrm{~km}$ is given, covering radio and optical observations, and rocket measurements. The conclusion is reached that one stratum at about $85 \mathrm{~km}$ is observed consistently, and that other fine structure exists but has no long time constancy of height or pattern. There is no series of preferred heights below $100 \mathrm{~km}$. The authors consider explanations which may account for the observations, and advocate the testing of radio methods of exploration in conjunction with rocket measurements in order to develop the most practicable means of obtaining accurate electron density versus height profiles on a synoptic basis. $18 \mathrm{p}$.

Effect of small irregularities on the constitutive relations for the ionosphere, K. G. Budden

Irregularities in the ionosphere which are small compared with one wavelength may modify the constitutive relations, and hence, may affect the refractive indices for electromagnetic waves. The modifications are in some ways similar to those which would be introduced into the Appleton-Hartree formula by a Lorentz force. The theory is given first for the case when the irregularities extend only in one dimension, and it is found that even in a loss-free medium the refractive index now has an imaginary part 
which might be associated with loss of energy from the wave by scattering. The theory for three-dimensional irregularities is then discussed but is more difficult, and a method of successive approximations is used. The results indicate that small irregularities may play an important part in the propagation of very-low-frequency radio waves in the ionosphere. In particular, they may explain why "whistlers" are observed only on comparatively rare occasions. $15 \mathrm{p}$.

Ionospheric investigations using the sweep-frequency pulse technique at oblique incidence, V. Agy and K. Davies

This paper describes the present state of obliqueincidence investigations of the ionosphere, using the sweepfrequency pulse technique, with special reference to the work carried out at the National Bureau of Standards. After a short review of the published literature, obliqueincidence sweeps are presented showing the diurnal and seasonal variations on two east-west paths of lengths 1,150 kilometers and 2,370 kilometers. The diserepancies between observed and calculated maximum usable frequencies are presented for both paths and then various phenomena of interest are shown. Finally, the above phenomena are discussed in the light of existing knowledge and theory and, in particular, it is shown that the discrepancies between observed and calculated maximum usable frequencies are unlikely to be caused by magnetoionic deviation of the ray. $24 \mathrm{p}$.

Fields in electrically short ground systems: an experimental study, A. N. Smith and T. E. Devaney

An experimental study of magnetic field distribution in a simplified radial ground system on poorly conducting soil under an electrically short, toploaded monopole is described. It is shown that the distribution is that expected from the theory of J. R. Wait in those portions of the radial system satisfying the assumptions of the theory, and that the theory may still be successfully applied for $H$-field power loss computations even when this is not fully the case. The particular model system studied exhibits a condition suggesting damped standing waves on the radials in the area where the radial spacing exceeds that required by the theory. $6 \mathrm{p}$.

Diffraction of electromagnetic waves by smooth obstacles for grazing angles, J. R. Wait and A. M. Conda

The diffraction of electromagnetic waves by a convex cylindrical surface is considered. Attention is confined primarily to the region near the light-shadow boundary. The complex-integral representation for the field is utilized to obtain a correction to the Kirchhoff theory. Numerical results are presented which illustrate the influence of surface curvature and polarization on the diffraction pattern. Good agreement with the experimental results of Bachynski and Neugebauer is obtained. The effect of finite conductivity is also considered. $17 \mathrm{p}$.

Very-low-frequeney radiation spectra of lightning discharges, W. L. Taylor and A. G. Jean

Spectral analyses are given of the groundwave portion of 33 sferic waveforms recorded from cloud-to-ground lightning discharges which occurred at distances ranging between about 150 and 600 kilometers from Boulder, Colo. Frequencies of peak energy lie between 5 and 20 kilocycles per second, which agree favorably with other published results. The average value of energy calculated from the groundwave pulses was found to be 26,600 joules, which is lower than values derived from other experiments. Various parameters, such as the peak amplitude and duration of the first half-cycle, are related to the radiated energy of the stroke. $6 \mathrm{p}$.
Radio-wave scattering by tropospheric irregularities, A. D. Wheelon

The subject of radio-waye scattering by turbulent irregularities in the tropospheric index of refraction is reviewed. Descriptions of the turbulent medium are considered first, together with a status report on physical theories for the spectrum of irregularities. Phase and amplitude scintillations induced on electromagnetic waves propagated along line-of-sight paths are discussed next. The complementary problems of signal statistics and electromagnetic-propagation calculations are summarized and compared with available data. Both the geometrical optics and wave theory approaches are discussed. The theory of propagation beyond the horizon by scattering from the same irregularities is then described. A current review of the predictions for received power, signal fluctuations, and antenna effects is given. The paper is primarily a review of the essential and auxiliary predictions of scatter theory, but also contains a considerable amount of unpublished research by the author. $29 \mathrm{p}$.

Study at 1,046 megacycles per second of the reflection coefficient of irregular terrain at grazing angles, R. E. MeGavin and L. J. Maloney

An experimental determination of the reflection coefficient over rough terrain is reported. The reflected signal received over rough terrain is considered to be made up of two components, one that is a specular component and the other a Rayleigh-distributed component. Where one terminal is low, the Rayleigh component is considered to be small with respect to the specular component but increases in relative magnitude as the height of the lower terminal increases. A terminal height is reached where the specular component is no longer significant, and the reflected energy is essentially Rayleighdistributed. A terminal height is quickly reached above which the mean value of the reflected energy is relatively constant, of a low value, and independent of the grazing angle. $14 \mathrm{p}$.

Synoptic study of the vertical distribution of the radio refractive index, B. R. Bean, L. P. Riggs, and J. D. Horn

An analysis of the vertical structure of an intense outbreak of continental polar air is presented in terms of the radio refractive index of the atmosphere. Employed for the first time is a reduced index analogous to potential temperature. The reduced value more clearly shows the refractive index structure than the classical methods used heretofore. This new unit is a measure of both atmospheric density and humidity and shows, on a single cross section, the airmass structure and the dynamic mixing of air around the frontal interface. $6 \mathrm{p}$.

November-Deeember 1959

Radio-refractive-index climate near the ground, B. R. Bean and J. D. Horn

The radio refractive index of air is a function of atmospheric pressure, temperature, and humidity and is found to vary in a systematic fashion with climate. It was found that the surface value of the refractive index may be estimated four to five times more accurately from charts of reduced-to-sea-level values than from similar sized charts of surface index. Worldwide maps of 5-year means of this reduced value are presented for the months of February and August, for the minimum monthly mean value of the year and for the range of monthly mean values, Year-to-year variation of monthly means is also considered. Applications of these data to the prediction of radio field strengths indicate a possible 30 -decibel difference in median level of identically equipped tropospheric communications systems due to climate alone. $13 \mathrm{p}$. 
Path antenna gain in an exponential atmosphere, W. J. Hartman and R. E. Wilkerson

The problem of determining path antenna gain is treated here in greater detail than previously. The method used here takes into account for the first time the ex ponential decrease of the gradient of refractive index with height, and a scattering cross section inversely proportional to the fifth power of the scattering angle. Results are given for all combinations of beamwidths and path geometry, assuming that symmetrical beams are used on both ends of the path and that atmospheric turbulence is isotropic. The result appears as a function of both of the beamwidths, in addition to other parameters, and thus the loss in gain cannot be determined independently for the transmitting and receiving antennas. The values of the loss in gain are generally lower than the previous estimates for which a comparison is possible. $14 \mathrm{p}$.

Effect of atmospheric horizontal inhomogeneity upon ray tracing, B. R. Bean and B. A. Cahoon

The tracing of radio rays is normally carried out under the assumption that the refractive index varies only in the vertical direction. Although this assumption appears to be quite reasonable in the average or climatic sense, it is seldom satisfied under actual conditions and is strongly violated by horizontal airmass changes occurring near frontal and land-sea interfaces. This latter case is investigated by tracing rays through two instances of observed marked horizontal variation of the refractive index. The bending for these ray paths was then compared with values obtained under the normal assumption of horizontal homogeneity.

Although at 1 kilometer and above these horizontal changes appear to have little effect, rays emitted at low elevation angles are sensitive to extreme horizontal variations of the atmosphere near the surface, such as those associated with ducting conditions. However, since it appears that such conditions occur less than 15 percent of the time at most locations, the majority of ray-path calculations may be carried out under the normal assumption of horizontal stratification of the refractive index. $6 \mathrm{p}$.

On the correlation of solar noise fluctuations in harmonically related bands, L. R. O. Storey

A method is proposed for the study of the solar corona, by observing a delayed correlation between rapid fluctuations of enhanced solar radio emission in harmonically related frequency bands. The correlation is expected in those types of emission that are produced by nonlinear plasma oscillations. The delay of the fundamental frequency with respect to the harmonic would be brought about by dispersive group retardation in the corona. The method appears to be most suitable for use with type II bursts, though it might also be applied to other types of nonthermal solar emission. $4 \mathrm{p}$.

A monochromatic low-latitude aurora, F. E. Roach and E. Marovich

A monochromatic (6300 A) auroral arc occurred over Colorado on September $29 / 30,1957$. It seems to have been a continuation of a similar arc observed at Haute Provence, France, on the same night. Its intensity decreased during the night from about 7,000 rayleighs to 2,000 rayleighs compared with a normal zenith intensity of 100 to 200 rayleighs. It was relatively fixed (geographically) during the night, south of and apparently independent of a visual aurora that was active to the north from 0100 to 0400 m.s.t. The magnetic dipole lines of force from the arc extend out into space between the two Van Allen radiation belts. It is speculated that the arc may be associated with one of the belts. $5 \mathrm{p}$.
Pattern synthesis for slotted-cylinder antennas, J. R. Wait and J. Householder

The radiation from a cylinder excited by an array of axial slots is discussed. A procedure for synthesizing a given radiation pattern is developed with particular attention being paid to a Tchebyscheff-type pattern. Specifying the side lobe level and the width of the main beam, the required source distributions are computed for a number of cases. The effect of using a finite number of slot elements to approximate the continuous source distribution is also considered.

Central Radio Propagation Laboratory exponential reference atmosphere, B. R. Bean and G. D. Thayer

The background and development of an exponential model of atmospherie radio refractivity, the "C.R.P.L. Exponential Reference Atmosphere" is outlined. A set of ray tracings for the model is presented in the form of tables of refraction variables for the complete range of observed values of surface refractive index. A detailed analysis of the accuracy of the ray tracing and tabulation methods is made for these tables. The variables are presented as numbers between one and ten multiplied by the appropriate power of ten, thus maintaining a maximum number of significant figures. The tables may be used for the solution of practical refraction problems involving elevation angle errors, range errors, and similar quantities. 3 p.

Excitation mechanisms of the oxygen 5577 emission in the upper atmosphere, E. Tandberg-Hanssen and F. E. Roach

Possible excitation mechanisms for the green 5577 emission are considered in the light of recent data on the dynamics of the upper atmosphere. Photochemical reactions as affected by mass motions as well as excitation directly due to the mass motions are analyzed. It is concluded that either or both mechanisms could probably account for the observed emission. $6 \mathrm{p}$.

A method for measuring local electron density from an artificial satellite, L. R. O. Storey

A method is proposed for measuring the electron density at known points in the outer ionosphere, by the use of vlf receiving equipment in an artificial satellite, in conjunction with a vlf transmitter on the ground. The transmitter would radiate continuous waves, which would be propagated through the ionosphere in the "whistler" mode. The basis of the method is a measurement of the local wave admittance of the medium, by comparison of the signals received on an electric dipole and on a loop.

A further proposal is made for an integrated vlf satellite experiment, in which several different types of observation would be made simultaneously. $16 \mathrm{p}$.

TITLE PAGE AND CONTENTS TO VOL. 63D. $4 \mathrm{p}$.

PAPERS FROM THE JOURNAL OF RESEARCH OF THE NA. TIONAL BUREAU OF STANDARDS, SECTION D. RADIO PROPAGATION, VOLUME 64D, JANUARY-JUNE 1960

\section{January-February 1960}

Effect of antenna size on gain, bandwidth, and efficiency, R. F. Harrington

A theoretical analysis is made of the effect of antenna size on parameters such as gain, bandwidth, and efficiency. Both near-zone and far-zone directive gains are considered. It is found that the maximum gain obtainable from a broadband antenna is approximately equal to that of the uniformly illuminated aperture. If higher gain is desired, the antenna must necessarily be a narrow-band device. In fact, the input impedance becomes frequency sensitive so rapidly that, for large antennas, no significant increase in gain over that of the uniformly illuminated aperture is 
possible. Also, if the antenna is lossy, the efficiency falls rapidly as the gain is increased over that of the uniformly illuminated aperture. $12 \mathrm{p}$.

Surface-wave resonance effect in a reactive cylindrical structure excited by an axial line source, A. L. Cullen

It is shown that a purely reactive cylinder excited by a neighboring line source can, under suitable conditions, give rise to a radiation pattern closely approximating the function $\cos n \theta$.

In a numerical example, a cylinder of three transverse electro-magnetic (T.E.M.) wavelengths circumference has a surface reactance chosen to emphasize the term $\cos 6 \theta$ in the Fourier series of the resultant radiation pattern. It is shown that only 1.1 percent of the total power delivered to the line source is radiated in unwanted modes.

It is also shown that the position of the line source does not affect this result to first order provided that $k(b-a) \ll 1$, where $b-a$ is the distance of the line source from the cylindrical surface. $7 \mathrm{p}$.

Basic experimental studies of the magnetic field from electromagnetic sources immersed in a semi-infinite conducting medium, M, B. Kraichman

Using electromagnetic sources, consisting of various dipoles and loops immersed in a concentrated sodium chloride solution, measurements were made verifying the magnetic field propagation equations in air, derived previously by several authors. The receiver was farther away from the source than a wavelength in the conducting medium, but much closer than a wavelength in air.

An expression is derived giving the value of the magnetic field in air due to a rectangular loop with a horizontal axis by assuming the loop to consist of two electric dipoles corresponding to the horizontal members. Experimental data verifying this expression are presented.

Also, using submerged electric dipoles, measurements were made of the magnetic field in air which show that the field is determined solely by the current in the horizontal radiating wires of the dipoles. $5 \mathrm{p}$.

A very-low-frequency antenna for investigating the ionosphere with horizontally polarized radio waves, $R$. S. Macmillan, W. V. T. Rusch, and R. M. Golden

The advantages of a horizontal half-wave resonant antenna for very-low-frequency propagation experiments lie in its relatively simple and inexpensive corstruction and in its radiation pattern which is maximum in the vertical direction. The radiation fields of this type of antenna located at the surface of a conducting earth consist of: 1, A horizontally polarized space-wave field radiated in the perpendicular bisector plane of the antenna; and 2, a vertically polarized groundwave field radiated along the axis of the antenna. This vertically polarized field is zero at right angles to the antenna. These fields have been experimentally verified.

The use of a 50-kilocycle horizontal half-wave antenna for vertical-incidence ionospheric sounding experiments is described. The radiation pattern of this antenna is well suited for ionospheric soundings since a receiver located in the groundwave null receives only the reflected skywave signal.

Ground-resistivity measurements made at a number of locations in Central and Southern California were correlated with the geology of the terrain. This correlation showed that the ground resistivity is highest (a condition necessary for optimum antenna efficiency) in areas where the underlying rock formations are relatively unfractured. The amount of annual rainfall and other climatic conditions have little or no effect on the resistivity.

Finally, a unique antenna system is presented which employs resonant loading circuits to convert a section of an existing power line into a horizontal half-wave verylow-frequency transmitting antenna. $9 \mathrm{p}$
Effects of high-altitude nuclear explosions on radio noise, C. A. Samson

High-altitude nuclear explosions over Johnston Island in August 1958 appear to have had a rather pronounced effect on the radio noise recorded at Kekaha, Hawaii. Graphs are presented showing the hour-to-hour variation of the noise during August at eight frequencies from 13 $\mathrm{kc}$ to $20 \mathrm{Mc}$. All frequencies seem to have been affected, and the drop in received noise power amounted to as much as $32 \mathrm{db}$ in the hour following the first explosion. The period of time over which abnormal noise conditions were observed suggests that high-altitude nuclear explosions may have a rather persistent effect on radio communications at certain frequencies. $4 \mathrm{p}$.

Measured frequency spectra of very-low-frequency atmospherics, T. Obayashi

New spectroscopes recording continuously the amplitude-frequency spectra of vlf atmospherics have been developed. Two receivers cover the frequency ranges 1 to $10 \mathrm{kc}$ and 5 to $70 \mathrm{kc}$ sweeping the respective bands repeatedly, and their outputs are displayed on intensity modulated cathode-ray tubes which are photographed on slowly moving film.

Observations have been carried out since June 1958 , and it appears that the results provide an excellent experimental basis for comparisons with the mode theory of vlf ionospheric propagation. It is found that the frequency spectrum of distant atmospherics indicates a pronounced absorption near 3 to $5 \mathrm{kc}$, a broad intensity maximum around 10 to $20 \mathrm{kc}$, and a general decrease towards higher frequencies with undulating peaks. The selective absorption bands appearing in the spectrum are variable according to the time of day and seasons. These changes may be interpreted loosely as an ionospheric effect which is associated with the cutoff frequency of the waveguide bounded by the earth and the ionosphere. The solar flare effect on vlf atmospherics propagation is also revealed, which indicates a sudden shift of the spectrum to higher frequencies owing to the increase of ionization and the lowering of a reflecting height of the ionosphere. $8 \mathrm{p}$.

Determination of the amplitude-probability distribution of atmospheric radio noise from statistical moments, W. Q. Crichlow, C. J. Roubique, A. D. Spaulding, and W. M. Beery

During the International Geophysical Year, the National Bureau of Standards established a network of atmospheric noise recording stations throughout the world. The $\mathrm{ARN}-2$ noise recorder at these stations measures three statistical moments of the noise: average power, average voltage, and average logarithm of the voltage. An empirically-derived graphical method of obtaining an amplitude-probability distribution from these three moments, and its development, is presented. Possible errors, and their magnitudes, are discussed. 8 p.

Measurements of coastal deviation of high-frequency radio waves, C. W. McLeish

The angular deviation of the phase front of a wave propagated across a fresh water shoreline has been measured over the frequency range from 3 to $20 \mathrm{Mc}$. The deviation is found to be roughly half that which theoretically would be obtained if the same sites were adjacent to infinitely conducting surfaces. $3 \mathrm{p}$.

An exact earth-flattening procedure in propagation around a sphere, B. Y.-C. Koo, and M. Katzin

By a refinement of the procedure used in the usual earth-Hattening approximation, the problem of propagation around a spherical earth is reduced to an exact 
equation of the same form. Thereby the earth-flattening procedure becomes applicable to arbitrarily large heights and distances. It is also found that existing solutions of the approximate equations can be re-evaluated to yield the exact solutions for slightly different refractive index distributions. $4 \mathrm{p}$.

Limit of spatial resolution of refractometer cavities, W. J. Hartman

Filter factors that determine an upper limit for the wave numbers for which refractometer measurements can be used to calculate the spectrum of refractivity are derived in this paper based on the assumption that refractometers measure a weighted average of the refractive index in a volume of air surrounding the center of the refractometer cavity. Two models are used assuming the weighting function has spherical symmetry around the center and one model is used assuming the function has cylindrical symmetry. All models result in a simple mathematical form which should be easy to use in further theoretical developments. $8 \mathrm{p}$.

\section{Conference on arctic communication, R. C. Kirby and C. G. Little}

News and commentary on arctic communication conference. $8 \mathrm{p}$.

Tropospheric scatter propagation and atmospheric circulations, W. F. Moler and D. B. Holden

Transhorizon vhf and uhf fields exhibit deep fades or large signal enhancements of several hours' duration, as the propagation mechanism alternates between partial reflection and scattering caused by turbulent dielectric fluctuations in the atmosphere. Such alternations occur when strong refractive layers develop below $3,000 \mathrm{ft}$. Surface wind streamline analyses show that mesoscale centers of convergence or divergence cause local redistribution of refractve layering, tending to produce the change from one mechanism to the other.

Current scattering theory and the empirical findings of others are examined to determine the gross meteorological factors that influence changes in scattered fields. The two variables in the turbulent scattering coefficients, the scattering angle and the intensity of dielectric fluctuations at high wave numbers, are found to be dependent upon the refractive layering and the thermal stability of the airmass. It has been shown elsewhere that refractivity and stability are principally functions of the vertical velocity in the atmosphere. It is shown here that the direction and relative magnitude of the vertical velocity can be inferred from the upper-tropospheric wind velocity divergence. Received scattered signals are found to be well correlated with computed velocity divergence.

It is suggested that the variations of scattered signal level or range can be predicted in a routine manner by regular meteorological personnel using ordinarily available meteorological data. $13 \mathrm{p}$.

Layered earth propagation in the vicinity of Point Barrow, Alaska, G. M. Stanley

The relative field strength of a vertically polarized low-frequency radio signal was measured as a function of distance over several radial paths in the vicinity of Point Barrow, Alaska. The attenuation of the recorded signal was very much less than predicted by the theory of propagation of a ground wave signal over a plane, homogeneous, infinitely conducting earth. The analysis of these data in terms of a plane, layered, finitely conducting earth appears to resolve the anomaly. $3 \mathrm{p}$.$$
\text { March-April } 1960
$$

Optimum frequencies for outer space communication, G. W. Haydon

Frequency dependence of radio propagation and other technical factors which influence outer space communica- tions are examined to provide a basis for the selection of frequencies for communication between earth and a space vehicle or for communication between space vehicles. $5 \mathrm{p}$.

The joint use of the ordinary and extraordinary virtual height curves in determining ionospheric layer profiles, L. R. O. Storey

An extension of Budden's matrix method for determining ionospheric layer profiles is described. When analyzing vertical incidence ionograms by the matrix method, it is usual to interpret the virtual height curve for the ordinary mode only. Errors then arise from the presence in the lower ionosphere of low-density ionization for which the plasma frequency is less than the lowest frequency observed. In the proposed extension of the method, such errors are reduced by making use of the extraordinary virtual height curve as well as of the ordinary. $14 \mathrm{p}$.

Measured statistical characteristics and narrow-band teletype message errors on a single-sideband 600-mile-long ultrahigh-frequency tropospheric radio link, E. F. Florman and R. W. Plush

Measurements of a 417 megacycles per second unmodulated radio carrier over a 600 -mile tropospheric path indicated that the variations of the received carrier envelope amplitude with time over 30 -minute periods roughly approximated a Rayleigh distribution in the majority of the tests. Cumulative time distributions of the carrier fade durations were obtained over a range of carrier envelope power levels and were found to resemble corresponding distributions for narrow band thermal noise. The fade rate of the carrier envelope, at the median power level, was less than 0.2 cycle per second; this comparatively low fade rate is thought to be due mainly to the narrow $\left(1^{\circ}\right)$ antenna beam widths that were used and the consequent low order of multipath propagation of the radio waves. The half hour median power levels of the received carrier varied over a range of approximately \pm 8 decibels. The effective low-pass bandwidth of the carrier envelope spectrum was found to vary from 0.06 to 0.17 cycle per second. The measured median transmission loss was approximately 183 decibels which is within 3.5 decibels of the calculated value for the summer afternoon hours covered by the tests.

With antennas spaced (normal to the path) at each end by 150 wavelengths it was found that parallel-path, divergent-path, or convergent-path types of transmission gave cross-correlation coefficients of the carrier envelopes which ranged from 0.08 to 0.20 . For the same antenna spacing but using crossed-path type of transmission, the cross-correlation coefficient was 0.57 . Diversity measurements of single-path crossed polarization type of transmission indicated that the cross-correlation coefficient of the carrier envelopes was very close to unity.

Nondiversity narrow-band FSK error measurements indicated that an 18-decibel signal-to-noise power ratio over an effective bandwidth of 290 cycles per second (at the limiter-discriminator input) for a fading signal resulted in 0.8 percent binary errors and 4.0 percent teletype character errors. Extrapolation of these results indicates that a signal-to-noise ratio of 27 decibels is required to reduce the teletype character error rate to 0.1 percent in the same effective bandwidth. $9 \mathrm{p}$.

Impedance of a corner-reflector antenna as a function of the diameter and length of the driven element, A. C. Wilson

Impedance measurements have been made for a monopole in a corner reflector over an image plane as a function of the monopole length, diameter, and position within the corner-reflector structure. The results are presented as a family of curves which should be useful in the design of the driven element for a corner-reflector antenna of the size described in this paper and for other corner-reflector antennas with similar parameters. $3 \mathrm{p}$. 
The electric field at the ground plane near a top-loaded monopole antenna with special regard to electrically small L- and T-antennas, H. L. Knudsen and T. Larsen

The present article deals with the calculation of the electric field strength at the ground plane near electrically small top-loaded antennas having a known current distribution, with special reference to $L$ - and T-antennas. The formulas and numerical values obtained here for this component may be used in calculating the contribution to the ground losses around an antenna of the above-mentioned type due to the vertical component of the earth current.

An exact expression involving an integral has been obtained for the electric field strength at the ground plane due to the current in a linear antenna having an arbitrary inclination. If the length and the height of the antenna is small compared to the wavelength, and if the current distribution on the antenna can be expressed by a finite number of terms of a power series, it is theoretically possible to obtain a closed expression for the field at the ground plane. However, only in special eases does this expression become sufficiently simple to be of practical value for numerical calculations.

Working formulas have been obtained and numerical calculations carried out for the near zone field of an electrically small vertical or horizontal antenna with a linear current distribution. Based on these results, a calculation has been made of the electric field strength at the ground plane near electrically small L- and $T$-antennas. Also the relative contribution to this component due to the top loading has been calculated. $13 \mathrm{p}$.

Terrestrial propagation of very-low-frequency radio waves. A Theoretical Investigation, J. R. Wait

A self-contained treatment of the waveguide mode theory of the propagation of very-low-frequency radio waves is presented. The model of a flat earth with a sharply bounded and homogeneous ionosphere is treated for both vertical and horizontal dipole excitation. The properties of the modes are discussed in considerable detail.

The influence of earth curvature is also considered by reformulating the problem using spherical wave functions of complex order. The modes in such a curved guide are investigated and despite the initial complexity of the general solution, many interesting and limiting cases may be treated in simple fashion to yield useful and convenient formulas for calculation.

Other factors considered are the influence of the earth's magnetic field, antipodal effects, resonator type oscllations, and the influence of stratification at the lower edge of the ionosphere. $52 \mathrm{p}$.

Aurora of October 22/23, 1958, at Rapid City, South Dakota, F. E. Roach and E. Marovich

During the night of October 22/23, 1958, auroral activity at Rapid City, South Dakota, included (a) a visible aurora in the northern part of the sky and; (b) a "monochromatic" (6300 A) are through the zenith with an azimuth $12^{\circ}$ from east-west (geomagnetic). The intensity changes of the arc were independent of the changes in the visible aurora. It moved slowly southward during the night corresponding to a linear speed of about 8 meters per second if its height is 300 kilometers. It is suggested that it is a member of a family of monochromatic ares which have until recently escaped detection because their red color makes them invisible even though intrinsically intense. $5 \mathrm{p}$.

$$
\text { May-June } 1960
$$

A theory of radar scattering by the moon, T. B. A. Senior and $\mathbf{K}$. M. Siegel

A theory is described in which the moon is regarded as a "quasi-smooth" scatterer at radar frequencies. A scattered pulse is then composed of a number of individual returns each of which is provided by a single scattering area. In this manner it is possible to account for all the major features of the pulse, and the evidence in favor of the theory is presented. From a study of the measured power received at different frequencies, it is shown that the scattering area nearest to the earth is the source of a specular return, and it is then possible to obtain information about the material of which the area is composed. The electromagnetic constants are derived and their significance discussed. $13 \mathrm{p}$.

A theory of wavelength dependence in ultrahigh frequency transhorizon propagation based on meteorological considerations, R. Bolgiano, Jr.

Recent radio data indicate that the wavelength dependence of ultrahigh frequency transhorizon propagation varies widely in ime. This is in contradiction with theoretical explanations previously set forth. Each attempt to account for the underlying effects of ever-present atmospheric motions has, in the past, pointed toward a unique form of the dependence. Extensive discussions have resulted as to the validity and relative merits of the various forms, but at no time has a variable wavelength dependence been proposed.

Since scatter propagation theory has predicted so satisfactorily the broad aspects of the radio signals, it is retained as the basis for further analysis. A new model is developed for the structure of refractive index fluctuations induced by turbulence. Grounded on a theory of homogeneous turbulence in a stably stratified atmosphere, which has been developed concurrently by the author, this new model provides an explanation for the observed distribution of wavelength dependence. It suggests that at times when the dynamic stability of the air within the scattering volume is neutral the received power should be nearly independent of radio wavelength. On the other hand, when the atmosphere is dynamically stable the signal strength should be proportional to the square, or higher power, of the wavelength.

These predictions have been tested by comparing the results of a scaled-frequency experiment with simultaneous meteorological data gathered along the path. Richardson's number for the 1- to 3-kilometer layer, within which the principal scattering volume lies, has been employed as an index of dynamic stability, though it falls short of ideal in some respects. The 0.8 value of correlation found between Richardson's number and the wavelength dependence is highly suggestive that a relation of the nature predicted does, in fact, exist. $7 \mathrm{p}$.

A preliminary study of radiometeorological effects on beyond-horizon propagation, F. Ikegami

A study was made of American and Japanese radiometeorological data in order to suggest the dominant factors in propagation beyond the horizon. The diurnal variability of radio field strengths seems to be sensitive to the crossover height of rays tangent to the radio horizon and disappears for erossover heights greater than about 500 meters. High hourly median field strengths were observed in Japan corresponding to the existence of a marked refractive index discontinuity layer in a common volume of two antenna beams. The results of these experiments suggest that laminar structures of the atmosphere play an important role in beyond-horizon radio propagation. $8 \mathrm{p}$.

The trade-wind inversion as a transoceanic duct, $M$.

Katzin, H. Pezzner, B. Y.-C. Koo, J. V. Larson, and J. C. Katzin

Radiosonde data for stations in the South Atlantic trade-wind belt are analyzed to determine the potentialities of the trade-wind inversion as an elevated duct for transoceanic radio transmission. These were supplemented by refractometer soundings made by an aircraft during the latter part of 1958 . These records indicate that a duct is present in the majority of the cases. Since it is known that the radiosonde underestimates ducting because of its slow response, it is concluded that a duct is present practically all the time. On the basis of the data analyzed, an experiment with two aircraft is suggested to test the propagation potentialities of this mechanism. A fre- 
quency of around 200 megacylces per second appears to be a good choice for an initial experiment. $7 \mathrm{p}$.

An aulysis of propagation measurements made at 418 megacylces per second well beyond the radio horizon (a digest), H. B. Janes, J. C. Stroud, and M. T. Decker

During an 18-month period in 1952 and 1953, transmission loss measurements at 418 megacycles were made over a 134-mile path between Cedar Rapids, Iowa, and Quincy, Illinois. Continuous recordings made simultaneously at several receiving antenna heights from 30 to 665 feet yielded information on diurnal and seasonal variations in both the hourly median basic transmission loss and in height gain. These data are compared to predictions made using the method developed by Rice, Longley, and Norton and are found to be in good agreement, particularly at the lower antenna heights. An analysis of the correlation of short-term signal level variations observed at horizontally and vertically spaced antennas is described. 3 p.

On the calculation of the departures of radio wave bending from normal, B. R. Bean and E. J. Dutton

The calculation of nonnormal tropospheric bending of radio waves is treated in terms of a reduced-to-sea-level value of the refractive index. This method emphasizes departures of bending from the average bending for the United States and consists of visualizing ray bending as consisting of two parts; an "average" component and a "departure-from-average" component. The "average" component comprises most of the bending and is obtained accurately from refraction tabulations while the component due to departures is easily obtained by graphical means. $5 \mathrm{p}$.

On the mode theory of very-low-frequency propagation in the presence of a transverse magnetic field, D. D. Crombie

The effect of a purely transverse horizontal magnetic field on the propagation of very-low-frequency (vlf) waves is considered. It is shown that the magnetic field introduces nonreciprocity, and that for propagation along the magnetic equator, the rate of attenuation is less for westto-east propagation than for east-to-west propagation. 3 p.

On the theory of reflection of low- and very-low-radiofrequency waves from the ionosphere, J. R. Johler and L. C. Walters

The regiorous application of the magneto-ionic theory to the calculation of reflection coefficients for a sharply bounded ionosphere model is carried out. The paper is illustrated with computations applicable to the $D$-region or the E-region of the ionosphere. The quasi-longitudinal approximation is derived from this theory and the range of validity of this approximation is illustrated. The restrictions imposed by the use of a sharply bounded model ionosphere are discussed. $18 \mathrm{p}$.

Focusing, defocusing, and refraction in a circularly stratified atmosphere, K. Toman

Focusing, defocusing, astronomical refraction and path length of rays as a function of the departure angle $\Delta$ of the ray at the source is described for cases with the source outside, inside, or on the boundary of a circular stratification. Relative to zero elevation angle symmetrical and centrosymmetrical distributions are found. $2 \mathrm{p}$.

Response of a loaded electric dipole in an imperfectly conducting cylinder of finite length, C. W. Harrison, Jr., and R. W. P. King

Analytical relationships are developed which permit calculation of the power in the load impedance of an electric probe, symmetrically located within an imper- fectly condueting cylinder of small radius compared to the wavelength, in terms of the electric field incident upon the cylinder. $5 \mathrm{p}$.

Impedance characteristics of a uniform current loop having a spherical core, S. Adachi

The radiation impedance is derived by the electromotive force method in a convenient form as the sum of the selfradiation impedance of a loop in the free space and an additional term due to the reaction between the loop and the sphere which is proportional to the well-known expansion coefficient of a magnetic-type scattered wave from a sphere in an incident plane wave. The first antiresonance frequency has been given in the form of a universal curve for a very small uniform current loop with core of an arbitrary composition of $\mu_{s}$ and $\epsilon_{s}$, subject to the condition that the refraction coefficient $N=\sqrt{\mu_{s} \epsilon_{s}}$ is extremely large. Some numerical calculations show that high $-\mu$ core is desirable for a comparatively lower frequency region, and high $-\epsilon$ core is rather desirable in an antiresonance region. $5 \mathrm{p}$.

\subsection{CIRGULARS}

Circulars are compilations of information on various subjects related to the Bureau's scientific and technical activities. They not only include the results of Bureau studies but give data of general interest from other sources. The Circular series was discontinued in June 1959. After this date material that would formerly have been published in the Circular series has been largely directed to the Journal of Research and the new Monograph series (page 59). See "Price List of Available Publications," page 161.

C440 Supplement. Viscosities of sucrose solutions at various temperatures: Tables of recalculated values, J. F. Swindells, C. F. Snyder, R. C. Hardy, and P. E. Golden

The tables of viscosities of sucrose solutions appearing in NBS Circular 440 were calculated, assuming the value of the viscosity of water at $20^{\circ} \mathrm{C}$ to be 1.0050 centipoises. Subsequently, a redetermination of the absolute viscosity of water at $20^{\circ} \mathrm{C}$ established the value 1.0020 centipoises. In addition, more precise values have been obtained for the calibration constants of the viscometers used in the original measurements of suerose solutions upon which the tables were largely based. Incorporating these more precise values, the above tables have been recalculated. July $31,1958.7 \mathrm{p}$.

\section{C467, Vol. III. Atomic energy levels, C. E. Moore}

The compilation of Atomic Energy Levels as derived from the analyses of optical spectra, exclusive of hyperfine structure, has continued. Volume I of Circular 467, issued in 1949, contained data for 206 spectra of the first 23 elements in the periodic table, hydrogen through vanadium. The energy levels are listed by terms starting with the ground state zero. A uniform style of notation is used, and for complex spectra arrays of observed terms are included for comparison with similarly arranged arrays of predicted terms, which are given in the Introduction for each isoelectronic sequence. For each spectrum a brief discussion of the analysis is given, together with a detailed bibliography. Limits and ionization potentials are given when known. Similarly, the configurations of the individual terms are included together with $g$-values derived from the observed Zeeman patterns.

Volume II issued in 1952, is similarly arranged. It includes data for 152 spectra of the 18 elements chromium through niobium. 
Volume III was issued in 1958, continuing the program to heavier elements. This volume contains similar data for 124 spectra of 32 elements, molybdenum through lanthanium and hafnium through actinum.

This completes the periodic table for the two groups of elements known as rare earth. The atomic spectra of these elements will be covered in Volume IV of Circular 467 , which is now being started.

These volumes contain much unpublished material and are useful to workers in many fields of science. This program has stimulated work on spectrum analysis not only in the laboratory but also among theoretical workers. Circular 467, Volume I, June 15, 1949 . 309 p. Circular 467, Volume II, August 15, 1952. 227 p. Circular 467, Volume III, May 1, 1958.245 p.

C539, Vol. 7. Standard X-ray diffraction powder patterns. Data for 53 substances, H. E. Swanson, N. T. Gilfrich, and M. I. Cook

Fifty-three standard X-ray diffraction powder patterns are presented. Forty-six are to replace sixty-two patterns already represented in the X-ray Powder Data File, and seven are for substances not previously represented. The X-ray Powder Data File is a compilation of diffraction patterns from all sources and is used for the identification of unknown crystalline materials by matching spacing and intensity measurements. In this Circular, comparison is made of all powder diffraction data available for each of the substances reported. The patterns were made with a Geiger counter X-ray diffractometer, using samples of high purity. The $d$-values were assigned Miller indices determined by comparison with calculated interplaner spacings and from space group considerations. The densities and lattice constants were calculated, and the refractive indices were measured whenever possible.

Included are X-ray data for the following fifty-three substances: $\mathrm{AlCl}_{3} \cdot 6 \mathrm{H}_{2} \mathrm{O}, \quad \mathrm{NH}_{4} \mathrm{NO}_{3}, \quad\left(\mathrm{NH}_{4}\right)_{2} \mathrm{C}_{2} \mathrm{O}_{4} \cdot \mathrm{H}_{2} \mathrm{O}$, $\mathrm{NH}_{4} \mathrm{ClO}_{4}, \mathrm{BaMoO}_{4}, \mathrm{BaS}, \mathrm{BaWO}_{4}, \mathrm{CdCO}_{3}, \mathrm{CdSe}, \mathrm{CaCrO}_{4}$, $\mathrm{Ca}\left(\mathrm{NO}_{3}\right)_{2}, \mathrm{CaS}, \mathrm{Cs}_{2} \mathrm{SO}_{4}, \mathrm{AuSb}_{2}, \mathrm{AuSn}, \mathrm{LaF}_{3}, \mathrm{LaOCl}$, $\mathrm{PbMoO}_{4}, \mathrm{PbWO}_{4}, \mathrm{LiIO}_{3}, \mathrm{LiNO}_{3}, \mathrm{MgCO}_{3}, \mathrm{MgSO}_{4} \cdot 7 \mathrm{H}_{2} \mathrm{O}$, $\mathrm{MgS}, \mathrm{MnCO}_{3}, \mathrm{Hg}_{2} \mathrm{Br}_{2}, \quad \mathrm{HgSe}, \mathrm{NiSO}_{4} \cdot 6 \mathrm{H}_{2} \mathrm{O}, \mathrm{KBrO}_{3}$, $\mathrm{KCNO}, \mathrm{K}_{2} \mathrm{TiF}_{6}, \mathrm{KIO}_{4}, \mathrm{KMnO}_{4}, \mathrm{RbBr}, \mathrm{AgClO}_{3}, \mathrm{Ag}_{2} \mathrm{MoO}_{4}$, $\mathrm{Ag}_{2} \mathrm{SO}_{4}, \mathrm{NaIO}_{3}, \mathrm{NaIO}_{4}, \mathrm{NaClO}_{4}, \mathrm{SrMoO}_{4}, \mathrm{SrS}, \mathrm{SrWO}$, $\mathrm{NH}_{3} \mathrm{SO}_{3}, \mathrm{TeO}_{2}, \mathrm{TIBr}, \mathrm{Tl}_{3} \mathrm{PO}_{4}, \mathrm{TIPO}_{4}, \mathrm{SnTe}, \mathrm{CO}\left(\mathrm{NH}_{2}\right)_{2}$, $\mathrm{Zn}_{2} \mathrm{SiO}_{4}, \mathrm{ZnSO}_{4}$, and $\mathrm{Zr}\left(\mathrm{SO}_{4}\right)_{2} \cdot 4 \mathrm{H}_{2} \mathrm{O}$. September $27,1957$. $70 \mathrm{p}$.

C539, Vol. 8. Standard X-ray diffraction powder patterns. Data for 61 substances, H. E. Swanson, N. T. Gilfrich, M. I. Cook, R. Stinchfield, and P. C. Parks

Sixty-one standard X-ray diffraction powder patterns are presented. Thirty-three are to replace thirty-nine patterns already represented in the X-ray Powder Data File, and twenty-eight are for substances not previously represented. The X-ray Powder Data File is a compilation of diffraction patterns from all sources and is used for the identification of unknown crystalline materials by matching spacing and intensity measurements. In this Circular, comparison is made of all powder diffraction data available for each of the substances reported. The patterns were made with a Geiger counter X-ray diffractometer, using samples of high purity. The $d$-values were assigned Miller indices determined by comparison with calculated interplanar spacings and from space group considerations. The densities and lattice constants were calculated, and the refractive indices were measured whenever possible.

Included are $\mathrm{X}$-ray data for the following sixty-one substances: $\mathrm{Al}_{2} \mathrm{O}_{3} \cdot 6 \mathrm{CaO} \cdot 3 \mathrm{SO}_{3} \cdot 31 \mathrm{H}_{2} \mathrm{O},\left(\mathrm{NH}_{4}\right)_{2} \mathrm{SeBr}_{6},\left(\mathrm{NH}_{4}\right)_{2}-$ $\mathrm{TeBr}_{6},\left(\mathrm{NH}_{4}\right)_{2} \mathrm{IrCl}_{6},\left(\mathrm{NH}_{4}\right)_{2} \mathrm{PdCl}_{6},\left(\mathrm{NH}_{4}\right)_{2} \mathrm{TeCl}_{6}, \mathrm{NH}_{4} \mathrm{VO}_{3}$, $\left(\mathrm{NH}_{4}\right)_{3} \mathrm{PO}_{4}\left(\mathrm{MoO}_{3}\right)_{12} \cdot 4 \mathrm{H}_{2} \mathrm{O}, \mathrm{Be}_{2} \mathrm{SiO}_{4}, \mathrm{BiOBr}, \mathrm{CaBr}_{2} \cdot 6 \mathrm{H}_{2} \mathrm{O}$, $\mathrm{Ca}\left(\mathrm{HCO}_{2}\right)_{2}, \mathrm{CeF}_{3}, \mathrm{CsBrO}_{3}, \mathrm{Cs}_{2} \mathrm{PtBr}_{6}, \mathrm{Cs}_{2} \mathrm{SeBr}_{6}, \mathrm{CsClO}_{3}$, $\mathrm{CsCr}\left(\mathrm{SO}_{4}\right)_{2} \cdot 12 \mathrm{H}_{2} \mathrm{O}, \quad \mathrm{CsBF}_{4}, \quad \mathrm{CsGa}\left(\mathrm{SO}_{4}\right)_{2} \cdot 12 \mathrm{H}_{2} \mathrm{O}, \quad \mathrm{Er}_{2} \mathrm{O}_{3}$, $\mathrm{GaPO}_{4}\left(\alpha\right.$-quartz type), $\mathrm{GeO}_{2}$ (tetragonal), In $\mathrm{PO}_{4}, \mathrm{~Pb}-$ $\left(\mathrm{HCO}_{2}\right)_{2}, \quad \mathrm{~Pb}_{3} \mathrm{O}_{4}$ (minimum), $\mathrm{Pb}_{5}\left(\mathrm{PO}_{4}\right)_{3} \mathrm{OH}$ (lead hydroxyapatite), $\mathrm{LiClO}_{4} \cdot 3 \mathrm{H}_{2} \mathrm{O}, \mathrm{NdF}_{3}, \mathrm{NdOCl}, \mathrm{NiSiF}_{6} \cdot 6 \mathrm{H}_{2} \mathrm{O}$, $\mathrm{NbSi}_{2}, \mathrm{~K}_{2} \mathrm{PtBr}_{6}, \mathrm{~K}_{2} \mathrm{SeBr}_{6}, \mathrm{KReO}_{4}, \mathrm{~K}_{3} \mathrm{PO}_{4}\left(\mathrm{MoO}_{3}\right)_{12} \cdot 4 \mathrm{H}_{2} \mathrm{O}$,
$\mathrm{KCNS}, \mathrm{RbBrO}, \mathrm{Rb}_{2} \mathrm{TeBr}_{6}, \mathrm{RbClO}_{3}, \mathrm{Rb}_{2} \mathrm{TeCl}_{6}, \mathrm{Rb}_{2} \mathrm{SO}_{4}$, $\mathrm{ScPO}_{4}, \mathrm{AgI}$ (iodyrite), $\mathrm{AgReO}_{4}, \mathrm{Na}_{2} \mathrm{CO}_{3} \cdot \mathrm{H}_{2} \mathrm{O}$ (thermonatrite), $\mathrm{Sr}\left(\mathrm{HCO}_{2}\right)_{2}, \quad \mathrm{Sr}\left(\mathrm{CHO}_{2}\right)_{2} \cdot 2 \mathrm{H}_{2} \mathrm{O}, \quad \mathrm{SrI}_{2} \cdot 6 \mathrm{H}_{2} \mathrm{O}, \quad \mathrm{TaSi}_{2}$, $\mathrm{TlBrO}_{3}, \mathrm{TlClO}_{3}, \mathrm{TlIO}_{3}, \mathrm{TICNS}, \mathrm{Ti}_{5} \mathrm{Si}_{3}, \mathrm{WS}_{2}, \mathrm{~V}_{2} \mathrm{O}_{5}, \mathrm{YPO}_{4}$ (xenotime), $\mathrm{ZnCO}_{3}$ (smithsonite), $\mathrm{ZnSiF}_{6} \cdot 6 \mathrm{H}_{2} \mathrm{O}$, and $\mathrm{ZnSO}_{4} \cdot 7 \mathrm{H}_{2} \mathrm{O}$ (goslarite). April 1, 1959. $76 \mathrm{p}$.

C539, Vol. 9. Standard X-ray diffraction powder patterns. Data for 43 substances, H. E. Swanson, M. I. Cook, T. Isaacs, and E. H. Evans

Forty-three standard X-ray diffraction powder patterns are presented. Thirty-one are to replace forty-one patterns already given in the X-ray Powder Data File, and twelve are for substances not previously included. The X-ray Powder Data File is a compilation of diffraction patterns from all sources and is used for the identification of unknown crystalline materials by matching spacing and intensity measurements. In this Circular, comparison is made of all powder diffraction data available for each of the substances reported. The patterns were made with a Geiger counter X-ray diffractometer, using samples of high purity. The $d$-values were assigned Miller indices determined by comparison with calculated interplanar spacings and from space group considerations. The densities and lattice constants were calculated, and the refractive indices were measured whenever possible.

Included are X-ray data for the following forty-three substances: $\alpha-\mathrm{Al}_{2} \mathrm{O}_{3}$ (corundum), $\mathrm{NH}_{4} \mathrm{~N}_{3},\left(\mathrm{NH}_{4}\right) \mathrm{HCO}_{3}$ (teschemacherite), $\left(\mathrm{NH}_{4}\right)_{2} \mathrm{PtBr}_{6}, \mathrm{NH}_{4} \mathrm{ReO}_{4}, \quad\left(\mathrm{NH}_{4}\right)_{2} \mathrm{SO}_{4}$ (mascagnite), $\mathrm{BeAl}_{2} \mathrm{O}_{4}$ (chrysoberyl), $\mathrm{Be}_{3} \mathrm{Al}_{2}\left(\mathrm{SiO}_{3}\right)_{6}$ (beryl), $\mathrm{BiOI}, \mathrm{CdBr}, \mathrm{CdCl}_{2} 12 \mathrm{CaO} \cdot 7 \mathrm{Al}_{2} \mathrm{O}_{3}, \mathrm{Ca}_{3} \mathrm{Fe}_{2} \mathrm{Si}_{3} \mathrm{O}_{12}$ (andradite), $\mathrm{Cs}_{2} \mathrm{TeBr}_{6}, \mathrm{CsNO}_{3}, \beta-\mathrm{CrPO}_{4}, \mathrm{CoAl}_{2} \mathrm{O}_{4}, \mathrm{CoO}, \mathrm{Co}_{3} \mathrm{O}_{4}$, $\mathrm{Dy}_{2} \mathrm{O}_{3}, \mathrm{ErPO}_{4}, \mathrm{Ho}_{2} \mathrm{O}_{3}, \mathrm{MgCr}_{2} \mathrm{O}_{4}$ (picrochromite), $\mathrm{MnAl}_{2} \mathrm{O}_{4}$ (galaxite), $\mathrm{MnFe}_{2} \mathrm{O}_{4}$ (jacobsite), $\mathrm{Mn}_{2} \mathrm{O}_{3}$ (partridgeite), $\mathrm{HgO}$ (montroydite), $\mathrm{Nd}\left[\left(\mathrm{C}_{2} \mathrm{H}_{5}\right) \mathrm{SO}_{4}\right]_{3} \cdot 9 \mathrm{H}_{2} \mathrm{O}, \mathrm{NiAl}_{2} \mathrm{O}_{4}, \mathrm{Ni}_{2}$ $\mathrm{GeO}_{4}, \mathrm{KBH}_{4}, \mathrm{~K}_{3} \mathrm{Co}\left(\mathrm{NO}_{2}\right)_{6}, \mathrm{~K}_{3} \mathrm{ZrF}_{7}, \mathrm{PrOCl}, \gamma-\mathrm{AgI}, \mathrm{AgIO}$, $\mathrm{NaBH}_{4}, \mathrm{SrZrO}_{4}, \mathrm{~S}, \mathrm{TeO}_{2}$ (tellurite), $\mathrm{Tm}_{2} \mathrm{O}_{3}, \mathrm{TiO}_{1 \cdot 515}$, and $\mathrm{ZnI}_{2}$. February 25, 1960 . $64 \mathrm{p}$.

C552, 3d ed. Standard materials. Issued by the $\mathrm{Na}$ tional Bureau of Standards

A descriptive listing of the various Standard Materials issued by the National Bureau of Standards is given. A schedule of fees and weights, as well as directions for ordering, is included. Summarized tables of analyses are presented, to indicate the type of standards of composition presently available. Announcements of new standards will be made in scientific and trade journals, and the current status of the various standards will be indicated by an insert sheet issued with this Circular. April 8, 1959. 27 p. (Supersedes C552, 2d ed., April 15, 1957).

C563, 1st Supplement. Periodicals and serials received in the Library of the National Bureau of Standards, August 1958, N. J. Hopper and H. W. Reinhart

This supplement lists new periodical titles added to the collection of the National Bureau of Standards Library from April 1955 to August 1958. It also includes changes in title, titles that have ceased publication, and titles that are no longer received.

A list of Russian periodicals currently received, and also of translations of Russian periodicals, is included at the end of this publication.

The footnote references are the same as those used in the original list. December $30,1958.10 \mathrm{p}$.

\section{C577 Supplement. Energy loss and range of electrons and positrons, A. T. Nelms}

Tabulations of the mean energy loss due to ionization and excitation which include the density effect and the range derived from this quantity are given for electrons and positrons in several materials. July $30,1958.31 \mathrm{p}$. 
C583 Supplement. X-ray attenuation coefficients from 10 kev to $100 \mathrm{Mev}, \mathrm{R}$. T. McGinnies

A revision is given of the $\mathrm{X}$-ray attenuation coefficients presented in National Bureau of Standards Circular 583. Table 4 of that publication is eliminated, and a new table is given for each material for photon energies less than $100 \mathrm{kev}$. The uncertainties in the estimates of attenuation coefficients at low energies are from 3 to 5 percent, which is the same as was previously given at higher energies. The cross sections for scattering are unchanged. Two values are listed for the photoelectric cross section, one calculated from the Sauter-Stobbe formulas and the other derived from new experimental evidence. The procedures for smoothing experimental data are described and are generally the same as were used in Circular 583. In addition to the systematic coverage of the region from 10 kev to $100 \mathrm{Mev}$, some data are included for a number of elements based on experimental measurements below 10 kev and above $100 \mathrm{Mev}$. A comparison is made between calculated and experimental total attenuation coefficients at energies above $10 \mathrm{Mev}$. October $30,1959.10 \mathrm{p}$.

C584. Simulator for use in development of jet engine controls, E. S. Sherrard

A method of simulation and cost estimates of simulator components are given for a simulator to be used in the development of turbojet engine control systems for aircraft. The simulator employs typical d-c analog computer components. It is capable of representing a twin-spool, variable-nozzle, afterburning turbojet engine, as well as the engine's controller. The simulator is of moderate accuracy, flexibility, and cost. It is intended to be used in the determination of the stability and performance of the engine control system. September 16, 1957. 17 p.

\section{C585. The measurement of thickness, G. Keinath}

The numerous methods for the measurement of thickness in laboratory or shop are treated in seven groups according to physical operating principles: Mechanical - weight/dimension relationships, acoustics, vibration, displacement with various conversions; chemical-stripping, spectrochemical analysis; electrical-dielectric breakdown, resistance, electrochemical, capacitance, thermoelectricity; magnetic-attractive force, reluctance, saturation, inductance, eddy currents; optical-microscopy (also electron microscopy), interferometry, diffraction, shadow; X-rayabsorption, diffraction backscatter, spectrometry; radioactive radiation - absorption, backscatter, tracers. Ranges, accuracies, advantages, and limitations are discussed. A bibliography of references, a limited list of suppliers, and a detailed index of the gages, methods, applications, and trade names covered are appended. January 20, 1958. $79 \mathrm{p}$.

\section{C586. Electric hygrometers, A. Wexler}

This Circular is a review of the art of measuring the moisture content of air by the methods of electric hygrometry. The basis of these methods is the change in electrical resistance of a hygroscopic material with change in humidity. September 3,1957. $21 \mathrm{p}$.

C587. Electroforming of waveguide components for the millimeter-wavelength range, A. A. Feldmann

The technique of electroforming has become very important in the production of precision waveguide components. In the millimeter-wavelength range it represents almost the sole method of construction for precision components. Given machine-shop facilities, normal laboratory facilities, and a few auxiliary items, electroforming of the best quality can be done without much difficulty. This is of particular importance to research workers in university or Government laboratories. Simple techniques are outlined that permit the production of highquality millimeter components with a minimum of equip- ment, time, or specialized knowledge of electrodeposition. November 15,1957 . $13 \mathrm{p}$.

C588, Determination and correlation of flow capacilies of pneumatic components, D. H. Tsai and M. M. Slawsky

Some of the problems of measurement and correlation of flow capacities of pneumatic components are discussed. A dimensionless "area factor," defined as the ratio of the "effective area" of the component to some reference area (equation 7), is introduced. The physical significance of the area factor and its experimental determination are discussed in some detail. Sample data are also included to show that this area factor provides a valid and convenient basis for comparing the flow capacities of components, regardless of their size and design, and over a wide range of test conditions. October 15, 1957. $7 \mathrm{p}$.

C589. Tables of dielectric dispersion data for pure liquids and dilute solutions, F. Buckley and A. A. Maryott

Primary dielectric dispersion data and characteristic dispersion parameters are tabulated for almost 200 substances in the liquid state and for dilute aqueous and nonaqueous solutions with more than 150 solutes. There are 6 tables and 1 section of graphs. There are 4 tables for pure liquids, 2 containing summaries of the derived dispersion parameters and 2 containing the primary data. The section on graphs supplements the tables for pure liquids and contains reproductions of pertinent data that are available only in the form of graphs. November 1 , 1958. $95 \mathrm{p}$.

\section{C590. Methods of testing thermocouples and thermo- couple materials, Wm. F. Roeser and S. T. Lon- berger}

Various methods used for testing thermocouples and thermocouple materials and the precautions that must be observed in order to attain various degrees of accuracy are described. In particular, the methods that have been developed and used at the National Bureau of Standards are outlined in detail, and some guidance is given to the reader in the selection of the method best adapted to a given set of conditions.

Consideration is given primarily to the calibration of platinum versus platinum-rhodium, copper-constantan, Chromel-Alumel, and iron-constantan thermocouples. February 6, 1958, 21 p. (Supersedes RP768).

C591, Sect. 1. System design of digital computers at the National Bureau of Standards: methods for highspeed additions and multiplication

1. A logic for high-speed addition, A. Weinberger and J. L. Smith

A method is described whereby increased speed of operation in a binary digital adder is obtained by organizing the electronic elements for the carry-generating network into a more efficient system. This network is so organized that certain special auxiliary functions of the digits in the two numbers to be added are formed during the early phases of the addition cycle. These auxiliary functions are used during subsequent phases of the cycle to generate the carry digits in large groups simultaneously instead of generating each carry individually and sequentially as is the case in conventional adder designs.

When associated with a suitably fast storage device, this type of adder permits the design of arithmetic processing units that operate up to 150 times faster than earlier National Bureau of Standards computers, using the same basic types of electronic elements.

\section{Shortcut multiplication for binary digital computers} J. L. Smith and A. Weinberger

A method for increasing the basic multiplication speed of a computer is described in which certain time-consuming 
but non-essential steps are omitted. Such steps are ordinarily included when the elementary definition of binary multiplication as a simple sequence of repeated addition and single-position shift operations is applied in a straightforward fashion. It is shown that most of these elementary steps can be omitted for any continuous string of similar digits that occurs in the multiplier number. Fullest advantage of this can be taken if provision is made to perform various multiposition shifts as rapidly as possible. Implementing the method requires the continual sensing of several of the multiplier digits in advance in order to discover which of the elementary steps are non-essential and can be omitted. Speed factors of up to 3 over conventional methods can generally be obtained. February 14, 1958. $22 \mathrm{p}$.

\section{C592. Nickel and its alloys, J. G. Thompson}

A review is presented of available information about the occurrence, recovery and refining, properties, and uses of high-purity and commercial forms of nickel, and about the properties and industrial applications of its important alloys. The Circular is a revision of National Bureau of Standards C485 issued in 1950 . February 5, 1958. 87 p. (Supersedes C485).

C593. The federal basis for weights and measures. A historical review of Federal legislative effort, statutes, and administrative action in the field of weights and measures in the United States, R. W. Smith

A review is presented, for the period 1776-1956, very largely in chronological form, of congressional efforts and accomplishments in the general weights and measures area, with particular emphasis on units and standards.

Certain important and closely related administrative actions initiated by Hassler and Mendenhall in the nineteenth century are cited. Current Federal statutes having weights and measures significance are discussed briefly. In its entirety the Circular presents a connected and reasonably comprehensive story of the Federal contribution to the legislative basis for weights and measures administration in the United States. June 5, 1958, 23 p.

C594. Preparation, maintenance, and application of standards of radioactivity, W. B. Mann and H. H. Seliger

The methods available for the preparation and maintenance of primary and secondary standards of radioactivity are reviewed, and the applications of such standards to problems in physies are discussed. June 11, 1958. $47 \mathrm{p}$.

C595. Tables of transport integrals $J_{n}(x) \equiv \int_{0}^{x} \frac{e^{z} z^{n} d z}{\left(e^{z}-1\right)^{2}}$,

W. M. Rogers and R. L. Powell

The transport integrals, $J_{n}(x)$, defined by

$$
J_{n}(x) \equiv \int_{0}^{x} \frac{e^{z} z^{n} d z}{\left(e^{z}-1\right)^{2}}
$$

are utilized often in the development of theories describing the thermal conductivity, electrical conductivity, thermal electromotive force, specific heat, and other similar transport properties of solids. The tables include values to six significant figures of the integral for the index integer $n$ ranging from 2 through 17 , and for the limit of integration $x$ ranging from 0.1 , by 0.1 intervals, to the limiting upper value, that ranges from 25 for $n=2$ to 40 for $n=17$. The three series utilized to represent the integral in different ranges are derived. The limiting values in the upper range and the asymptotic series expansions in the lower range are also given. Auxiliary tables include the values of the Riemann Zeta numbers and Bernoulli numbers utilized in the calculations. July $3,1958.46 \mathrm{p}$.
C596. Single-phase transfer of liquefied gases, R. B. Jacobs

The problems encountered in the single-phase transfer of liquefied gases are discussed in detail. A general system of equations and the empirical information required for the design of long-distance transfer systems are presented.

A closed-form solution of the mathematical equations for incompressible flows is obtained and discussed. Information required for numerical computations involving helium, hydrogen, nitrogen, and oxygen is presented in graphical form. The numerical solution for three general problems involving hydrogen and oxygen are obtained. December 1, 1958. $42 \mathrm{p}$.

\section{C597. Energy spectrum resulting from electron slowing down, R. T. MeGinnies}

The process of electron slowing down is described qualitatively and the resulting energy spectrum defined in terms of differential track length. A method developed by Spencer and Fano for the calculation of the electron spectra has been applied extensively by means of an automatic computer. The accumulation of secondary knockon electrons is included in the calculations and bremsstrahlung losses are neglected. Tabulations of differential track length are given for aluminum, copper, tin, lead, air, water, bone, muscle, and polyethylene $\left(\mathrm{C}_{2} \mathrm{H}_{4}\right)$, with up to 17 source energies each. Source energies range from 10.46 million electron volts to 6.438 kilo electron volts. A tabulation of the probability for the production of knock-on secondary electrons is also given. These data are an abstract of a much larger tabulation, printed by the computing machine, which is available on loan from the library of the National Bureau of Standards. February 20, 1959. $16 \mathrm{p}$.

C598. Techniques for accurate measurement of antenna gain, H. V. Cottony

Comparison of published results of experimental antenna measurements, particularly gains, reveals apparent discrepancies of the order of one or more decibels. Experimental work at the National Bureau of Standards on scaled model antennas for long-range VHF communication via ionospheric seatter revealed some sources of difficulties and led to the adoption of special precautions resulting in significantly more consistent and, it is believed, more accurate results. The procedures are based on the comparison method but include, in addition to the standard antenna, the use of a third antenna here designated as the reference antenna. To obtain more accurate measurements it was found essential to correct for the standing wave pattern in the field set up, presumably, by reflections from the irregularities in the terrain. Special features of instrumentation, including methods for minimizing and measuring matching losses, are described. The accuracy of the techniques has been verified by measuring the gain of an antenna, the value of which could be accurately calculated. December $1958.10 \mathrm{p}$.

C599. On the theory of fading properties of a fluctuating signal imposed on a constant signal, H. Bremmer

This paper deals with a theoretical investigation of the fading properties of a signal composed of a fluctuating contribution, and another steady contribution with fixed amplitude and phase. It is assumed that the central limit theorem may be applied to two proper quantities describing the fading signal as a quasi-monochromatic function of the time. The results are applicable to any autocorrelation function for the fluctuating contribution.

The first part of the paper (sections 1 to 16 ) is mainly restricted to the idealized case in which any two components of the fluctuating part of the complete signal that are in quadrature with respect to their phase do have identical statistical properties; the fluctuating part is then termed a "random" signal. This idealized case is shown to constitute but an approximation if applied to the fluctuating field due to first order scattering in a turbulent 
atmosphere. Therefore, in the second part of the paper (sections 17 to 26) the theory has been extended to fluctuating contributions (then termed "quasi-random" contributions) not satisfying the above condition of isotropy. All results then depend on two complex correlation functions $a(\tau)$ and $b(\tau)$ instead of on the single function $a(\tau)$ governing the simplified theory. In contrast to $a(\tau)$, the function $b(\tau)$ does not exclusively depend on the energy spectrum of the fluctuating contribution.

The fading properties investigated for the composed signal are the distribution functions of both the amplitude and phase, as well as the average number of crossings of each of them (per unit time interval) through any given level. The complicated general formulas reduce to simple expressions in the two extreme cases of (a) absence of the steady signal (e.g., tropospheric scatter propagation to distances far beyond the transmitter's horizon), and (b) predominance of the steady signal (e.g., line of sight propagation to distances well within the horizon). The first limiting case leads, when neglecting the influence of $b(\tau)$ (then being very small), to the well-known Rayleigh distribution for the amplitude, to a homogeneous distribution for the phase, and to a fading rapidity (defined as the average number of crossings per unit time interval through the level most frequently passed) which is for the amplitude faster than for the phase by a factor of 3.04 . The second limiting ease amounts to normal distributions for both amplitude and phase, and to fading rapidities of these quantities which are only identical insofar as the different behaviour of the in-phase and in-quadrature component (with respect to the steady signal) does have no numerical importance. On the other hand, measurements of the difference in the amplitude-fading rapidity and the phase-fading rapidity will reveal the effect of the asymmetry with respect to the two mentioned components of the scattered signal. May 25, 1959. $32 \mathrm{p}$.

C600. Calibration of liquid-in-glass thermometers, J. F. Swindells

This Circular contains information of general interest to both manufacturers and users of liquid-in-glass thermometers, as well as those who wish to calibrate thermometers or submit them to the National Bureau of Standards for calibration. Important elements of thermometer design are discussed, and eligibility requirements for certificates or reports of tests are given. Factors affecting the use of common types of liquid-in-glass thermometers are included together with tables of tolerances and resonably attainable accuracies. The calculation of corrections for the temperature of the emergent stem is given in detail for various types of thermometers and conditions of use. The Circular also describes the techniques and equipment used in the calibration procedures and provides instructions for applicants requesting thermometer calibration services. January 8, 1959. 21 p. (Supersedes C8).

C601. Recorder survey: recording surfaces and marking methods, G. Keinath

This Circular surveys the characteristics and comparative advantages of continuous traces, dotted traces, and printed characters, as produced by inking, incision, impression, indentation, deposition, heat, light, electric discharge, electron beam, magnetism, chemical action, or fluid streamlines. Descriptive and reference material is included on three physical components of the recording system-the reservoir of material or energy, the marking point or matrix positioned by the measuring element, and the chart surface which preserves the record. September 1 , 1959. $41 \mathrm{p}$.

C602. Testing of glass volumetric apparatus, J. C. Hughes

This Circular contains specifications and tolerances for glass volumetric apparatus of precision grade. Detailed information is given as to dimensions, graduations, inscriptions, and tolerances for burets, pipets, flasks, eylindrical graduates, and certain kinds of special apparatus.
A deseription of test methods used and the reports furnished and directions for submitting apparatus are included. April 1, 1959. 14 p. (Supersedes C434).

C603. Stark broadening functions for the hydrogen lines, A. B. Underhill and J. H. Waddell

Numerical values of the intensities of the Stark components of the hydrogen lines and the Stark broadening functions, $S(\alpha)$, are presented for all series members up to and including $n=18$. The $S(\alpha)$ functions are tabulated for lines of the Balmer, Lyman, Paschen, and Brackett series of hydrogen. The data can be used to compute self-consistent profiles of the hydrogen lines in stellar spectra. May 1, 1959. $94 \mathrm{p}$.

\subsection{MONOGRAPHS}

Monographs are usually contributions to the technical literature which are too lengthy for publication in the Journal of Research. They often provide extensive compilations of information on subjects related to the Bureau's technical program. Until July 1959 most of this type of material was published in the Cireular series (page 55). See "Price List of Available Publications," page 161 .

\section{Mono. 1. Energy dissipation by fast electrons, L. V. Spencer}

Tabulations are given of the energy dissipated by fast electrons at different distances from monoenergetic electron sources, for plane perpendicular and point isotropic sources. A summary of the theoretical methods and data utilized, and a table of spatial moments are also included. September 10, 1959 . $70 \mathrm{p}$.

Mono. 2. Temperature-induced stresses in solids of elementary shape, L. H. Adams and R. M. Waxler

In general, a solid subjected to non-uniform temperature change develops internal stresses. These are determined by, (1) the temperature distribution within the solid, and (2) certain physical constants of the material. Although the necessary fundamental relations are known, the computation of stresses in even simple solids has heretofore been a tedious operation. For two varieties of heating, the equations determining stress have now been put in convenient form for practical use, and tables of certain temperature functions are offered as a means of quickly determining stresses in a slab, in a cylinder, or in a sphere subjected to either of two modes of heating. The temperature-distribution tables independently will provide a useful means for the ready estimation of temperature gradients. June 21, 1960. $27 \mathrm{p}$.

Mono. 3, Vol. I. Table of wavenumbers, $2000 \mathrm{~A}$ to $7000 \mathrm{~A}$, and Vol. II. Table of wavenumbers, $7000 \mathrm{~A}$ to $1000 \mu$, C. D. Coleman, W. R. Bozman, and W. F. Meggers

A two-volume table for converting wavelengths in standard air to wavenumbers in vacuum was computed by using the equation $\sigma_{\mathrm{yac}}=\mathbf{1} / \lambda\left(\mathrm{n}_{\mathrm{nir}}\right)$, where $n$ was computed from Edlén's 1953 equation for the refractive index of air. Wavenumbers are given to the nearest 0.001 $\mathrm{K}\left(\mathrm{cm}^{-1}\right)$ for wavelengths from 2000 to $7000 \mathrm{~A}$ in Volume I, and $7000 \mathrm{~A}$ to $1000 \mu$ in Volume II. Proportional tables are given for linear interpolation between entries of $\lambda$. Also included are the vacuum increase in wavelength, $\lambda(n-1)$; and the refractivity of standard air in the form $(n-1) 1000$. Mono. 3, Vol. I, May 2, 1960. 500 p. Mono. 3, Vol. II, May 16, 1960. 534 p. 
Mono. 4. CRPL exponential reference atmosphere, B. R. Bean and G. D. Thayer

The background and development of an exponential model of atmospheric radio refractivity, the CRPL Exponential Reference Atmosphere, is outlined. A set of ray tracings for the model is presented in the form of tables of refraction variables for the complete range of observed values of surface refractive index. A detailed analysis of the accuracy of the ray tracing and tabulation methods is made for these tables. The variables are presented as numbers between 1 and 10 multiplied by the appropriate power of 10 , thus maintaining a maximum number of significant figures. The tables may be used for the solution of practical refraction problems involving elevation angle errors, range errors, and similar quantities. October 29,1959 . $67 \mathrm{p}$.

Mono. 5. Preservation of documents by lamination, W. K. Wilson and B. W. Forshee

The chemical stability and physical properties of cellulose acetate film used to preserve and restore old or damaged documents by lamination were investigated, Pretreatment of documents with alkaline media before lamination is desirable if the paper contains an appreciable amount of acid. The lamination process does not degrade cellulose acetate film to a measurable extent. Acid-free papers are not degraded during lamination, but papers containing acid are degraded in proportion to the amount of acid present. Tissue added to the laminate increases the tensile strength and internal tear resistance, but decreases edge-tear resistance as compared to that of film alone extending bevond the paper.

The loss of plasticizer with time from a cellulose acetate laminating film does not impair the properties of the laminate. Composition and performance specifications for a cellulose acetate laminating film suitable for archival use are presented. The properties of polyethylene and polyethylene terephthalate films of interest in connection with their possible use for the protection of documents are discussed. October 30,1959. 20 p.

\section{Mono. 6. Properties of high-temperature ceramics and cermets. Elasticity and density at room temperature, S. M. Lang}

In order to provide some of the basic data necessary for the effective utilization of ceramics and cermets in various high-temperature applications, a specimen "bank" of such materials, mainly commercially fabricated, was established for the measurement of physical properties and constants. This Monograph describes: (1) The materials and some of their fabrication data; (2) bulk densities; (3) theoretical densities; and (4) the dynamic room-temperature elastic constants. Data are given for 46 sets of specimens, representing 20 different materials; these include oxides, carbides, borides, cermets, and an intermetallic compound. A statistical evaluation was used for analyzing the data.

Results of the room-temperature measurements show that: (1) Significant variations are common both in the specimens of one group and from group to group of specimens prepared of the same material; (2) the largest variations occur for specimens formed by hot-pressing, although average values are higher for hot-pressed specimens; and (3) measurements of the dynamic elastic constants by the sonic method are more sensitive as indicators of homogeneity and group uniformity than bulk-density measurements. March 1, $1960.45 \mathrm{p}$.

\section{Mono. 7. Precise measurement of heat of combustion with a bomb calorimeter, R. S. Jessup}

This Monograph gives detailed descriptions of apparatus and methods which are used at the National Bureau of Standards for precise determinations of heats of combustion of liquid hydrocarbon fuels. Numerical examples are given of methods of calculating results of measurements from observed data. The technique of making and filling glass bulbs to contain samples of volatile liquid fuels is described.

The accuracy of the methods described is about 0.1 percent. This is intermediate between the accuracy of 0.01 or 0.02 percent attained in certain measurements on pure compounds, and the accuracy of several tenths of one percent obtainable with published standard procedures for measurements on fuels. February 26, 1960. 23 p.

Mono. 8. Mercury barometers and manometers, W. G. Brombacher, D. P. Johnson, and J. L. Cross

The various designs of mercury barometers and manometers are briefly described, with a more extended discussion of the various design elements which may affect the achievable accuracy. Sources of error in measuring pressures are described in considerable detail, particularly for portaable instruments, including scale, temperature, gravity, capillarity, vacuum errors and return gas column. Methods of minimizing those errors and of making the corrections, including extensive tables, are presented. Standard conditions are defined and the pertinent properties of mercury given. The paper contains 65 literature references. May 20,1960, $59 \mathrm{p}$.

Mono. 9. A method for the dynamic determination of the elastic, dielectric, and piezoelectric constants of quartz, S. A. Basri

Several dynamic determinations have been made of the constants of quartz. Most of these determinations do not take into account the piezoelectric effect; those that do, suffer from certain deficiencies which are discussed in this paper.

Taking into account the piezoelectric effect, expressions for the frequency of longitudinal vibration of rectangular bars and thickness shear vibration of infinite plates are derived and applied to the determination of the constants of quartz. On the basis of present theoretical knowledge, it is suggested that the best procedure is to measure the frequency of vibration of 2 particular cuts for rectangular bars, and 7 cuts for plates, and to measure the capacitance at zero frequency of a rectangular bar. These 10 measurements provide the data for determining the 6 elastic, 2 dielectric, and 2 piezoelectric constants of quartz uniquely.

For accurate measurements, it will first be necessary to determine the linear coefficients of expansion of quartz to higher accuracy than is available at present. June 1, 1960. $22 \mathrm{p}$.

Mono. 10. The "1958 $\mathrm{He}^{4}$ seale of temperatures". Part

1. Introduction, F. G. Brickwedde and Part

2. Tables for the 1958 temperature scale, H. van Dijk, M. Durieux, J. R. Clement, and J. K. Logan

The generally used practical scale of temperatures between $1^{\circ}$ and $5.2^{\circ} \mathrm{K}$ is the $\mathrm{He}^{4}$ vapor pressure scale based on an accepted vapor pressure equation or table. In Sèvres (near Paris), October 1958, the International Committee on Weights and Measures recommended for international use the "1958 He Scale" based on a vapor pressure table arrived at through international cooperation and ageement. This table resulted from a consideration of all reliable $\mathrm{He}^{4}$ vapor pressure data obtained using gas themometers, and paramagnetic susceptibility and carbon resistor thermometers The theoretical vapor pressure equation from statistical thermodynamics was used with thermodynamic data on liquid $\mathrm{He}^{t}$ and the vapor equation of state to insure satisfactory agreement of the vapor pressure table with reliable thermodynamic data. (Reprinted from the Journal of Research of the NBS-A Physics and Chemistry, Vol. 64A, No. 1, January-February 1960). June 17, 1960. $17 \mathrm{p}$.

Mono. 11. Conductive flooring for hospital operating rooms, T. H. Boone, F. L. Hermach, E. H. MacArthur, and R. C. MeAuliff

Characteristics and performance of available types of conductive flooring materials were investigated in the 
laboratory. The study showed that the electrodes and instruments used to measure the floor greatly affected the measured resistance, but that the method specified by the National Fire Protection Association for measuring the electrical resistance reasonably simulated the conditions uuder which a floor functions in reducing electrostatic hazards. The physical, chemical and serviceability characteristics of conductive floorings investigated showed results comparable with those of nonconductive flooring of the same type. Consequently, with some limitations, an architect may base his choice of a conductive flooring material on his knowledge of the behavior of similar nonconductive materials. (Reprinted from the Journal of Research of the NBS-C, Engineering and Instrumentation, Vol. 63C, No. 2, October-December 1959). March 21, $1960.16 \mathrm{p}$.

Mono. 12. Stabilization of free radicals at low temperatures

This volume is the final report of the National Bureau of Standards Free Radicals Research Program. The three-year program which ran from September 1956 to October 1959 was devoted to the study of the properties of reactive molecular fragments trapped in inert matrices at low temperatures. The first paper describes the organization and operations of the Free Radicals Research Program. The next seven papers summarize the technical accomplishments of the NBS Program, as viewed against the status of the entire field of radical trapping. The ninth paper is a summary of the Fourth International Symposium on Free Radical Stabilization which was held in Washington, D.C., August 31 through September 2, 1959. The final paper is a complete cross-referenced bibliography of papers published as a result of the research completed during the three-year period of the Program. August 1, $1960.110 \mathrm{p}$.

Mono. 13. Mechanical properties of structural materials at low temperatures, a compilation from the literature, R. M. MeClintock and H. P. Gibbons

The tensile strength, yield strength, tensile elongation, and impact energy of about two hundred materials, metallic and nonmetallic, are given graphically as functions of temperature between $4^{\circ}$ and $300^{\circ}$ Kelvin. June 1,1960 . $180 \mathrm{p}$.

Mono. 14. Bibliography on molecular and crystal structure models, D. K. Smith

A bibliography on molecular and crystal structure models is presented. The references are classified into those discussing models in general, static models, dynamic models, or construction devices. The static models are further classified in molecular (Fisher-Hirschfelder-Taylor type), closed packing, open molecular, open crystal structure, open with parallel rods, polyhedral, and miscellaneous models. A short annotation is given which describes the model types and indicates the more significant articles pertaining to them. May 20, $1960.7 \mathrm{p}$.

Mono. 15. Calibration of line standards of length and measuring tapes at the National Bureau of Standards, L. V. Judson

The methods used at the National Bureau of Standards in calibrating line standards of length and measuring tapes submitted for standardization are outlined. The equipment used is described briefly. There is a discussion of some considerations that should be given as to whether or not a standard should be submitted to the Bureau. Instructions are given for submitting items to the Bureau for calibration. The appendix contains useful information on the use of steel tapes. May 20, 1960.

11 p. (Supersedes C572).
Mono. 16. Vibration-rotation structure in absorption bands for the ealibration of spectrometers from 2 to 16 microns, E. K. Plyler, A. Danti, L. R. Blaine, and E. D. Tidwell

Suitable bands of common gases have been tabulated and remeasured wherever necessary from 2 to 16 microns to obtain an accuracy of about $0.03 \mathrm{em}^{-1}$ throughout the region and to provide good calibrating points at frequent intervals. Some 600 rotation-vibration lines are illustrated in 20 spectrograms and wavenumbers are listed in companion tables with considerable intercomparison with worthy data obtained in other laboratories. The absorption bands were remeasured or calibrated by using either a precisely graduated grating circle or standard atomic lines with the fringe system formed by a Fabry-Perot interferometer. Characteristic features of the individual bands are discussed briefly and references to other publications are given. The substances used for calibration include $\mathrm{H}_{2} \mathrm{O}, \mathrm{CO}_{2}, \mathrm{CO}, \mathrm{HCl}, \mathrm{HBr}, \mathrm{NH}_{3}, \mathrm{C}_{2} \mathrm{H}_{2}, \mathrm{CH}_{4}, \mathrm{~N}_{2} \mathrm{O}$, and polystyrene film. (Reprinted from the Journal of Research of the NBS-A, Physics and Chemistry, Vol. 64A, No. 1, January-February 1960.) June 21, 1960. $20 \mathrm{p}$.

\section{Mono. 17. New description of thorium spectra, R. Zalubas}

Wavelengths and estimated intensities in electrodeless lamp and spark sources are presented for 15121 lines of Th I, Th II, Th III, and Th IV in the spectral range from 2000 to $11550 \mathrm{~A}$. Previously published interferometric values are in this table rounded off to three decimal places and followed by the letter i. June 21, $1960.103 \mathrm{p}$.

\subsection{HANDBOOKS}

These are recommended codes of engineering and industrial practice, including safety codes, developed in cooperation with the national organizations and others concerned. In many cases the recommended requirements are given official status through their incorporation in local ordinances by State and municipal regulatory bodies. See "Price List of Available Publications," page 161.

H28 (1957) - Part II. Screw-thread standards for Federal services, 1957. Amends in part H28 (1944) (and in part its 1950 Supplement).

Part II includes standards for pipe threads, Dryseal pipe threads, hose coupling and fire hose coupling threads, gas cylinder valve outlet, inlet, and safety device threads, and hose connections for welding and cutting equipment. Parts I and II supersede all of Handbook H28 (1944) and the 1950 Supplement thereto excepting sections IX and X and appendix 5. November 16, 1959. 119 p.

\section{H63. Protection against neutron radiation up to 30 million electron volts}

Information for protection of personnel working near reactors, accelerators and radioactive neutron sources is included in this report. The physical problems of measuring neutron radiation and absorbed neutron dose are discussed as well as the current status of information on the biological effects of neutrons. Rules are recommended for safe operation of neutron sources and for protection of personnel. The recommendations of this Handbook take into consideration the January 8,1957 , statement of the National Committee on Radiation Protection and Measurement lowering the maximum permissible levels for radiation workers. November $22,1957.88$ p. 
H64. Design of free-air ionization chambers, H. O. Wyckoff and F. H. Attix

The material in this Handbook is a collection of the data required for designing free-air ionization chambers. These chambers are principally used for the calibration of the smaller clinical and field instruments. Until recently, they were used in a few of the large national standards laboratories; with the increase in radiation therapy, more of the national laboratories and many industries have become interested in providing such calibration facilities.

The handbook contains information for the design of primary standards to measure radiation in roentgens from the majority of the sources now used in medical radiology. For a given design, it also includes data on the magnitude of the corrections required to obtain a determination of the number of roentgens in 50 to $500 \mathrm{kv}$ X-ray beams from the ionization measurements. Sample computations and an estimate of the possible errors in such determinations are included. Some of this data is based on information obtained during intercomparisons of the free-air chambers of the national standards laboratories of various countries. December 13, 1957. $16 \mathrm{p}$.

H65. Safe handling of bodies containing radioactive isotopes (A guide for surgeons, pathologists, and funeral directors)

This is one of a series of reports prepared by the $\mathrm{Na}$ tional Committee on Radiation Protection and Measurements. The present Handbook is a revision of Handbook 56, Safe Handling of Cadavers Containing Radioactive Isotopes. This revision was prepared in order to include information on additional isotopes used in therapy and encountered in the handling of bodies, and to conform with the lowered maximum permissible radiation levels recently recommended both nationally and internationally. July 10, 1958. 20 p. (Supersedes H56).

\section{H66. Safe design and use of industrial beta-ray sources}

This Handbook was prepared to serve as a guide toward the safe design, manufacture, installation, use, maintenance, and disposal of beta-ray sealed sources for industrial applications. In addition, it may be of some help to the reader in complying with the radiation protection regulations issued by the U.S. Atomic Energy Commission and other Federal and State agencies. However, as these governmental regulations may be changed from time to time, they may differ in detail from the recommendations that are given. The Handbook was prepared by Subcommittee 8 on Sealed Beta-Ray Sources of the ASA-Z54 Sectional Committee on Protection for the Industrial Use of Radiation. May 28, 1958. 28 p.

H67. Checking prepackaged commodities. A manual for weights and measures officials, M. W. Jensen

A manual for State and local weights and measures officials, describing a method for controlling various types of prepackaged commodities. March 20, 1959. $27 \mathrm{p}$.

H68. Tabulation of data on receiving tubes, C. P. Marsden, W. J. Keery, and J. K. Moffitt

A tabulation of Receiving-Type Electron Tubes with some characteristics of each type has been prepared in the form of two major listings, a Numerical Listing in which the tubes are arranged by type number, and a Characteristic Listing in which the tubes are arranged by tube type and further ordered on the basis of one or two important parameters. The tabulation is accompanied by a listing of similar tube types and basing diagrams for the listed tubes. November $1,1959.110 \mathrm{p}$.

H69. Maximum permissible body burdens and maximum permissible concentrations of radionuclides in air and in water for occupational exposure

This Handbook represents a complete revision of Handbook 52, originally published in 1953 . It was prepared jointly by the NCRP and the ICRP. All of the material in the earlier handbook was reviewed and revised in light of the basic changes in our permissible dose concepts introduced in January 1957 and April 1958. Maximum permissible body burdens and maximum permissible concentrations of radionuclides in air and in water are given for approximately 300 radionuclides as compared with about 100 in the original report. As a result of the review, some of the values have been revised up or down and some have remained the same as in the earlier report. The more detailed discussion of some of the derivations and the bibliography have been published in the ICRP Committee 3 report on X-rays Up to $3 \mathrm{Mev}$ and Alpha and Gamma Rays From Sealed Sources, 1958 revision. (Pergamon Press, New York, N.Y., April 1960). June 5, 1959. 95 p. (Supersedes H52).

\section{H71. Specifications for dry cells and batteries}

This seventh edition of American Standard Specification for Dry Cells and Batteries, American Standard C18, includes new tests, up-to-date data, and covers new types of dry cells. An alarm test with results on batteries suitable for alarm circuits is a new feature of this seventh edition of the specification. Performance data on flashlight batteries, radio batteries, hearing-aid batteries, etc., have been brought up to date. Batteries for use with transistors are included in this specification for the first time. December 29, 1959.20 p. (Supersedes C559).

\subsection{MISCELLANEOUS PUBLICATIONS}

As the name implies, this series includes material which, because of its character or because of its size, does not fit into any of the other regular publication series. Some of these are charts, administrative pamphlets, Annual Reports, Weights and Measures Conference Reports, and other subjects appropriate to the Miscellaneous series. See "Price List of Available Publications", page 161 .

\section{M221. Hydraulic research in the United States, 1957, H. K. Middleton}

Research and development projects being conducted in 1957 in hydraulic and hydrologic laboratories of universities and Federal agencies throughout the United States and Canada are briefly described. A list of the contributing laboratories is given. The status of continuing projects covered by previous issues of the publication is reported upon, as well as on new projects in progress, the results of completed work are given. References to publications relating to the projects and an extensive subject index are included. July 24, 1957. 220 p.

\section{M222. Report of the 42d National Conference on Weights and Measures, 1957}

A report of the proceedings of the forty-second National Conference on Weights and Measures, held in Washington, D.C., June $3,4,5,6$, and 7,1957 , and attended by state, county, and city weights and measures officials. $133 \mathrm{p}$.

\section{M223. Annual report 1957 National Bureau of Standards}

This is a summarized illustrated report of the research and development activities of the Bureau in the fields of physics, chemistry, engineering, and mathematics, during the fiscal year ending June 30,1957 . Brief accounts are included relating to projects completed by or in progress in the Bureau's scientific and technical divisions, including electricity and electronies, optics and metrology, heat, atomic and radiation physics, chemistry, mechanics, organic and fibrous materials, metallurgy, mineral products, building technology, applied mathematics, data processing systems, cryogenic engineering, radio propagation, radio standards, and basic instrumentation. 
A statement of the Bureau's testing, ealibration, and Standard Samples activities by Divisions is also included, as well as a review of its technical services and cooperation, both national and international. $143 \mathrm{p}$.

M224. Hydraulic research in the United States, 1958, H. K. Middleton

Research and development projects being conducted in 1958 in hydraulic and hydrologic laboratories of universities and Federal agencies throughout the United States and Canada are briefly described. A list of the contributing laboratories is given. The status of continuing projects covered by previous issues of the publication is reported upon, as well as on new projects in progress, the results of completed work are given. References to publications relating to the projects and an extensive subject index are included. August 15, $1958.167 \mathrm{p}$.

M225. Report of the 43d National Conference on Weights and Measures, 1958

A report of the proceedings of the forty-third National Conference on Weights and Measures, held in Washington, D.C., June $9,10,11,12$, and 13,1958 , and attended by state, county, and city weights and measures officials. $146 \mathrm{p}$.

M226. Research highlights of the National Bureau of Standards, Annual report 1958

This is a summarized illustrated report of the research and development activities of the Bureau in the fields of physics, chemistry, engineering, and mathematics, during the fiscal year ending June 30,1958 . Brief accounts are included relating to projects completed by or in progress in the Bureau's scientific and technical divisions, including electricity and electronies, optics and metrology, heat, atomic and radiation physies, chemistry, mechanics, organic and fibrous materials, metallurgy, mineral projects, building technology, applied mathematies, data processing systems, cryogenic engineering, radio propagation, radio standards, and basic instrumentation.

A statement of the Bureau's testing, calibration, and Standard Samples activities by Divisions is also included, as well as a review of its technical services and cooperation, both national and international. $138 \mathrm{p}$.

M227. Hydraulic research in the United States, 1959, H. K. Middleton

Research and development projects being conducted in 1959 in hydraulic and hydrologic laboratories of universities and Federal agencies throughout the United States and Canada are briefly described. A list of the contributing laboratories is given. The status of continuing projects covered by previous issues of the publication is reported upon, as well as on new projects in progress, the results of completed work is given. References to publications relating to the projects and an extensive subject index are included. October $1,1959.188 \mathrm{p}$.

M228. Report of the 44th National Conference on Weights and Measures, 1959

A report of the proceedings of the forty-fourth National Conference on Weights and Measures, held in Washington, D.C., June $8,9,10,11$, and 12,1959 , and attended by state, county, and city weights and measures officials. $144 \mathrm{p}$.

M229. Research highlights of the National Bureau of Standards, Annual report 1959

This is a summarized illustrated report of the research and development activities of the Bureau in the fields of physics, chemistry, engineering, and mathematies, during the fiscal year ending June 30,1959 . Brief accounts are included relating to projects completed by or in progress in the Bureau's scientific and technical divisions, including electricity and electronics, optics and metrology, heat, atomic and radiation physics, chemistry, mechanies, organic and fibrous materials, metallurgy, mineral products, building technology, applied mathematics, data processing systems, cryogenic engineering, radio propagation, radio standards, and basic instrumentation.

A statement of the Bureau's testing, calibration, and Standard Samples activities by Division is also included, as well as a review of its technical services and cooperation, both national and international. $169 \mathrm{p}$.

\subsection{BUILDING MATERIALS AND STRUC- TURES REPORTS}

This series reports the results of research in building technology work at the National Bureau of Standards. The objective is to provide government, the interested professions, and the building industry with technical data useful in the preparation of building codes, standards, and engineering design criteria. Subjects of primary interest are the properties of building materials, structures, equipment, and facilities. Effective July 1959, this series was discontinued. Papers on building technology are now published in the Journal of Research (usually Section C. Engineering and Instrumentation), or the Monograph series (page 59). See "Price List of Available Publications", page 161 .

BMS140, 3d ed. Building construction and maintenance, E. R. Meggers

Selected references on building materials, equipment, good construction practices for new construction, and the modernization and maintenance of buildings. January $15,1959.30 \mathrm{p}$.

BMS144, 2d Supplement. Sound insulation of wall, floor, and door constructions, R. V. Waterhouse, R. D. Berendt, and R. K. Cook

Sound insulation data are presented for building structures measured at the National Bureau of Standards in the period July 1955 to December 1956 . These figures constitute the second supplement to the data published in Building Materials and Structures Report 144. The accuracy of the figures is discussed. Details are also given of a new average figure, called the Energy Average, for the over-all sound insulation of a panel, and why it is preferable to the Decibel Average, which it is designed to supersede. December 1, 1958. $13 \mathrm{p}$.

BMS151. Thermal resistance of airspaces and fibrous insulations bounded by reflective surfaces, H. E. Robinson, L. A. Cosgrove, and F. J. Powell

Observed insulating values are presented for 28 test panels, of which 23 were insulated with reflective membranes used alone or in combination with fibrous insulations. The panels were tested at five different orientations corresponding to use as walls, ceilings, or floors. The observed insulating values of panels are compared with calculated values based on a method and data developed in previous work at the National Bureau of Standards, and summarized in an appendix to this report. Observed values for panels agreed within 10 percent, in most cases, with calculated values. Departures of observed from calculated values are attributed to such factors as nonuniformity of airspace thickness, moisture condensation on reflective surfaces, air circulation between tandem airspaces, and effects due to local high conductance at edges of insulations or radiation from framing members. November $14,1957.22 \mathrm{p}$. 
BMS152. Wind pressures in various areas of the United States, G. N. Brekke

A procedure is described for developing a wind-pressure map that shows minimum resultant wind pressures (30 feet above oround) for design purposes throughout the continental United States. This map was used in the 1955 revision of the American Standards Association's standard A58.1, American Standard Building Code Requirements for Minimum Design Loads in Buildings and Other Structures. It is based on records of annual fastestsingle-mile wind speeds at $155 \mathrm{U}$. S. Weather Bureau stations. In computing resultant wind pressures from these data, allowance was made for gusts and for building shape. Values for 15 of the stations were also adjusted for altitude or for unusual local wind conditions. A table of design wind pressures at various heights from less than 30 to more than 1,200 feet above ground is derived for use with the map. The principal sources of strong winds affecting building constructions are briefly reviewed. April 24, 1959. 8 p.

\subsection{APPLIED MATHEMATICS SERIES}

The Applied Mathematies Series contains mathematical tables, manuals and studies of special interest to physicists, engineers, chemists, biologists, mathematicians, computers and others engaged in scientific and technical work. Some of the volumes are reissues, to meet a continuing demand, of the Mathematical Tables prepared by the Project for the Computation of Mathematical Tables conducted by the Federal Works Agency, Work Projects Administration for the City of New York, under the scientific sponsorship of and made available through the National Bureau of Standards. The Mathematical Tables series (M'T) as originally issued is out of print; the list, by title, is given in NBS Circular 460 .

When the Applied Mathematics Division was established at the National Bureau of Standards in July 1947, the Mathematical Tables Project became identified with the unit of this Division known as the Computation Laboratory. See "Price List of Available Publications", page 161.

AMS49. Further contributions to the solution of simultaneous linear equations and the determination of eigenvalues

Three related but independently presented papers describe some recently developed techniques for the solution of certain problems of numerical analysis: Kernel polynomials in linear algebra and their numerical applications, by E. L. Stiefel; The quotient-difference algorithm, by P. Henrici; Solution of eigenvalue problems with LR-transformation, by $\mathrm{H}$. Rutishauser. The techniques developed are useful in solving simultaneous linear equations, in finding the eigenvalues of a matrix, in the construction of orthogonal polynomials, in the factorization of polynomials, in expanding into continued fractional functions defined by power series of Laplace integrals, and in many other problems. January $15,1958.81 \mathrm{p}$.

AMS50. Tables of the bivariate normal distribution function and related functions

This volume is a compilation and extension of various tables of Karl Pearson, Evelyn Fix, Jerzy Neyman and $\mathrm{H}$. H. Germond relating to the bivariate normal frequency function $g(x, y, z)=\left(1 / 2 \pi \sqrt{1-r^{2}}\right) \quad \exp \left[-\frac{1}{2}\left(x^{2}+y^{2}-2 r x y\right) /\right.$ $\left.\left(1-r^{2}\right)\right]$. Table I gives values of $L(h, k, r)=\int_{h}^{\infty} d x \int_{k}^{\infty}$ $g(x, y, r) d y$ for $h, k=0(.1) 4, r=0(.05) 0.95(.01) 1,6 D$. Table II gives values of $L(h, k,-r)$ for $h, k=0(.1) u_{n}$ where $u_{n} \leq 4$ and $L<1 / 2.10^{-7}, r=0(.05) .95(.01) 1,7 D$. The major portion of tables I and II have been compiled from Karl Pearson's "Tables for Statisticians and Biometricians," Part II. Table III tabulates $V(h, \lambda h)=\int_{0}^{\Lambda} d x \int_{0}^{\lambda x} z(x) z(y) d y$ where $z(t)=(1 / 2 \pi) \exp \left(-\frac{1}{2} t^{2}\right)$ for $\lambda=0.1(.1) 1, h=0(.01) 4(.02) 4.6$ (.1) 5.6,, $7 D$ Table IV gives $V(\lambda h, h)$ for $\lambda=0.1(.1) 1$, $h=0(.01) 4(.02) 5.6, \infty, 7 D$. Finally table $V$ gives values of $(1 / 2 \pi) \sin ^{-1} r$ for $r=0(.01) 1,8 D$. The Introduction is composed of two main parts, one dealing with the statistical applications of these tables, the other relating to interpolation and the computation of the tables. June 15, 1959. $258 \mathrm{p}$.

AMS51. Tables of the exponential integral for complex
arguments

The function, $E_{1}(z)=\int_{z}^{\infty}\left(e^{-u} / u\right) d u, z=x+i y$, is tabulated to six decimal places, for $0 \leq x \leq 4,0 \leq y \leq 10$, with intervals between the arguments so chosen that quadratic interpolation will yield the fullest attainable accuracy over most of the region. Also tabulated is $E_{1}(z)$ to ten decimal places over the region $0 \leq-x, y \leq 3.1$ at intervals of 0.1 , To facilitate interpolation the function $E_{1}(z)+\log _{e} z$ is tabulated for $0 \leq x, y \leq 1$ to six decimal places and for $0 \leq-x, y \leq 1$ to ten decimal places. For the region $4 \leq x \leq 10,0 \leq y \leq 10$ the auxiliary function $e^{x} E_{1}(z)$ is tabulated to six decimal places since interpolation for the same number of significant figures is easier for this function than for $E_{1}(z)$. Finally, a short table of $e^{z} E_{1}(z)$ over the region $0 \leq+x, y \leq 20$ is given to six decimal places over varying intervals. May 15, 1958. $634 \mathrm{p}$.

\section{AMS52. Integrals of airy functions}

Part I gives tables of integrals of the Airy function

where

$$
f(x)=\int_{0}^{x} A i(-t) d t ; \quad F(x)=\int_{0}^{x} f(t) d t
$$

$$
A i(x)=\frac{1}{\pi} \int_{0}^{x} \cos \frac{t^{3}}{3}+x t d t
$$

with second central differences for $x=-2(.01) 5$ to 8 decimal places and 7 decimal places, respectively. Part 2 gives tables of the modified Airy integral

$$
A_{0}(x)=\int_{0}^{\infty} e^{-x t-(t 3 / 3)} d t
$$

and its derivative $A^{\prime}{ }_{0}(x)$ with second central differences for $x=0(.01) 1(.02) 5(.05) 11,1 / x=.01(.01) .1$, to 8 decimal places. In addition, a skeleton table is given of $G(x)=$ $\int_{0}^{x} A_{0}(t) d t$ for $x=.5,1(1) 11$, to 8 decimal places. May $15,1958.28 \mathrm{p}$.

AMS53. Table of natural logarithms for arguments between five and ten to sixteen decimal places

The table gives 16-place values of the natural (to the bases) logarithms of the decimal numbers from 5 to 10 at intervals of 0.0001 . This volume is a companion volume to AMS31. The table was originally issued in the MT series as volume IV in a four-volume table of natural logarithms MT12. March 28, 1958. 506 p. (Supersedes MT12).

AMS54. Fractional factorial experiment designs for factors at three levels, W. S. Connor and M. Zelen

This publication contains a collection of fractional factorial designs of the series $\left(1 / 3^{v}\right) \times 3^{n}$ for $p=1,2,3,4,5$, and for $n$ ranging from 4 to 10 factors. The experiment 
plans are constructed so that the treatment combinations are grouped into blocks, thus allowing the experimenter to take advantage of any homogenous grouping of the experimental material. May 1,1959 . $37 \mathrm{p}$.

AMS56. Tables of osculatory interpolation coefficients, H. E. Salzer

The tables give the coefficients $A_{i}(p)$ and $B_{i}(p)$ where

$$
f\left(x_{0}+p h\right)=\sum_{\left[i=\frac{n-1}{2}\right]}^{\left[\frac{n}{2}\right]}\left\{A_{i}(p) f_{i}+B_{i}(p) h f_{i}^{\prime}\right\}
$$

for $n=2(1) 5$ and $p=-\left[\frac{n-1}{2}\right]$ (1.0) $\left[\frac{n}{2}\right]$, exact values or nine decimal places. These coefficients facilitate interpolation when both a function and its first derivative are tabulated. May 8, 1959. $25 \mathrm{p}$.

\section{AMS57. Basic theorems in matrix theory, M. Marcus}

This publication is a survey of the basic identities and inequalities of matrix theory, and is intended as a handy reference for research workers and students. Included are results dealing with elementary properties, canonical forms, invariance, congruence, commutativity, orthogonalization, eigenvalues, determinants, sub-matrices, rank, determinant and rank inequalities, numerical methods for inversion and eigenvalues, and condition numbers. No attempt has been made to provide proofs for the theorems listed; and all statements are made directly in terms of matrices rather than linear transformations. References are given after each result, unless the result is found in most textbooks on matrices. January 22, $1960.27 \mathrm{p}$.

\section{TECHNICAL NOTE SERIES}

This series was initiated in 1959 to supplement the Bureau's regular publications program. Technical Notes provide a maans for making available scientific data that are of transient or limited interest. They are available for sale by the Office of Technical Services, U.S. Department of Commerce, Washington 25, D.C. (Order by PB number only). Sea "Price List of Available Publications", page 161 .

TN1 (PB151360). A double-pulse total-absorption fast neutron spectrometer, L. J. Nicastro and R. S. Caswell

A fast neutron spectrometer utilizing the double-pulse total-absorption technique has been developed for use in the energy range 1-20 Mev. The total absorption spectrometer uses a hydrogenous scintillator, in which a fast neutron may lose all its energy, together with a detector for slow neutrons. Scintillation detection of the disintegration caused by absorption of a slow neutron in an element of high reaction cross section serves to identify those neutrons which lost essentially all their energy in the hydrogenous scintillator.

Various organic liquid scintillators loaded with natural methyl borate, and a plastic scintillator with a boron-10 or lithium- 6 slow neutron scintillation detector nearby were all tested with monoenergetic neutrons obtained from the $\mathrm{D}(\mathrm{d}, \mathrm{n}) \mathrm{He}^{3}$ and $\mathrm{T}(\mathrm{d}, \mathrm{n}) \mathrm{He}^{4}$ reactions. The best experimental results were obtained with a plastic scintillator and a $\mathrm{Li}^{6} \mathrm{I}(\mathrm{Eu})$ crystal. Representative data for this spectrometer are presented. For comparison purposes, the results of Monte Carlo calculations, made by Leiss for a phenylcylohexane liquid scintillator loaded with methyl borate ( $\mathrm{B}^{10}$ enriched), are also included. April 24, 1959. $25 \mathrm{p}$.
TN2 (PB151361). World maps of F2 critical frequencies and maximum usable frequeney factors, D. H. Zacharisen

This publication reports six months of contour maps and charts for use in predieting F2-layer maximum usable frequencies. Prediction maps for each even hour of Greenwich Mean Time and charts in which time is continuous along the abscissa are given for the months of January, March, June, July, September and December.

The four parameters used for predicting MUFs are foF 2 and the $4000 \mathrm{~km}$ MUF factor for a twelve-month running average Zurich sunspot number of 50 , and the rates of change of foF 2 and $4000 \mathrm{~km}$ MUF factor with sunspot number. The first three parameters use a map presentation with GMT constant over the surface of the map. The fourth parameter uses a chart presentation in which the ordinate is geomagnetic latitude and the abscissa is local time. April 1959. $244 \mathrm{p}$.

TN3 (PB151362). Detwinning quartz crystals, F. P.

Attempts were made to detwin quartz by cooling through the inversion temperature while a d.c. potential was applied and later with RF potential across the plate. BT, AT and $X$ cut plates were used. Air was largely removed to improve the insulation but this was not wholly satisfactory because of the difficulty of adequately degassing the sample. Some samples yielded untwinned plates, others did not. It appeared that different samples of quartz behaved differently in respect to ease of changing the twin pattern. The method used did not appear commercially useful because of the erratic behavior of different pieces of quartz. April 1959, 5 p.

TN4 (PB151363). The vapor pressures of some hydrocarbons in the liquid and solid state at low temperatures, W. T. Ziegler

This paper presents a brief review of the literature pertaining to the vapor pressure of 11 hydrocarbons at low temperatures. These hydrocarbons are methane, ethane, propane, $n$-butane, isobutane (2-methyl propane), $n$-pentane, ethylene, propylene, 1-butene, acetylene and cyclopropane. All of these except cyclopropane are encountered in the purification of hydrogen from refinery sources. Vapor pressure data are given for most of these hydrocarbons over the pressure range 0.001 to $1,500 \mathrm{~mm}$ $\mathrm{Hg}$. These data have been used to estimate the triplepoint pressures of the hydrocarbons where these pressures were not found in the literature. Some data for the vapor pressures of solid methaue, ethane, n-butane, ethylene, acetylene and cyclopropane are included. May 1959. $17 \mathrm{p}$.

TN5 (PB151364). Tables of median hourly values of the solar zenith angle $(x)$ for 35 locations, R. E. McDuffie

This report consists of tables of hourly values of cosine $x$ from sunrise to sunset for the 15 th day of each month appropriate to 35 vertical incidence ionospheric stations associated with the National Bureau of Standards. February 1959. $38 \mathrm{p}$.

TN6 (PB151365). An analysis of propagation measurements made at $418 \mathrm{Mc}$ well beyond the radio horizon, H. B. Janes, J. C. Stroud, and M. T. Decker

This report presents the result of an analysis of transmission loss measurements made at $418 \mathrm{Mc}$ over the 134mile path from Cedar Rapids, Iowa to Quincy, Ill., during 1952 and 1953 . The data consisted chiefly of continuous simultaneous recordings of signal level at several receiving antenna heights, ranging from 30 to 665 feet above ground. These data are reduced to tabulations of hourly median values of basic transmission loss and fading 
range. These values, as well as the hourly difference in transmission loss observed at two heights (height-gain) are also shown plotted in seatter diagrams versus time of day for each of the 13 two-week recording periods. The medians for each recording period of all hourly values of median basic transmission loss, fading range and heightgain are plotted versus time of year to show any seasonal variation in these statisties. A formula developed at NBS for predicting the median basic transmission loss in tropospheric scatter propagation is shown to be in good agreement with the data. The results of a study of the correlation of short-term signal variations observed at horizontally and vertically spaced antennas are given. May 1959. $84 \mathrm{p}$.

TN7 (PB151366). Low- and very low-radiofrequeney tables of ground wave parameters for the spherical earth theory: the roots of Riccati's differential equation, J. R. Johler, L. C. Walters, C. M. Lilley

The roots of Riccati's differential equation are tabulated in detail throughout the low- and very low-radiofrequency part of the spectrum. The zeroes and certain other parameters used in the calculation of the amplitude and phase of the ground wave by the rigorous series of residues are also tabulated. February 1, 1959. 86 p. (Supplementary numerical data for C573).

TN8 (PB151367). Thermodynamie properties of helium at low temperatures and high pressures, D. B. Mann and R. B. Stewart

The most comprehensive investigations of the thermodynamic properties of normal helium I and II were published over a period of the last 30 years by W. H. Keesom. A culmination of most of this work was published by Keesom in 1942. Since that time, numerous investigators have found several diserepancies in this original work in the temperature range below $20^{\circ} \mathrm{K}$. Recent interest in the compressed liquid region, an area not covered extensively by Keesom's work has resulted in new experimental data. These investigations and corrections to Keesom's work have been published, not as additions to the original Keesom work, but as separate and sometimes isolated articles of very narrow interest and not in readily usable form. Also the new data have little value until their relation to existing data is established.

Methods of property diagram construction are also explained, and the results are presented in the form of temperature-entropy and enthalpy-entropy diagrams. PresBures to 100 atmospheres, temperatures from $0^{\circ} \mathrm{K}$ to $20^{\circ}$ $\mathrm{K}$ and specific volumes from 5 liters $/ \mathrm{kgm}$. to 800 liters/ kgm. are presented. May $1959.39 \mathrm{p}$.

TN9 (PB151368). Frequency dependence of VHF ionospheric scattering, J. C. Blair

Results of frequency dependence measurements in VHF scatter propagation are given. Data included were taken from continuous recordings of transmissions over the 1300 $\mathrm{Km}$ path from Long Branch, Illinois to Boulder, Colorado. Frequencies of $30,40,50,74$ and $108 \mathrm{Mc} / \mathrm{s}$ were recorded simultaneously. Data were included for the period September 1957 to June 30, 1958.

One principal result is that over the full range of frequencies observed, received power closely follows the exponential relationship $P_{r} \frac{1}{f^{n}}$. The value of $n$ varied diurnally from 5 to 8 , expressed for constant-aperture receiving antenna.

Among other results reported are the relationship of short-term signal amplitude distributions to relative influence 0 ? meteoric activity as well as the fading rates observed, and the effect on the signal of SID's.

The results presented herein are presented prior to completion of actual calibrating measurements on the antenna systems and are subject to some review after such measurements are made. April 1959. 52 p.
TN10 (PB151369). Calculated behavior of a fast neutron spectrometer based on the total absorption principle, J. E. Leiss

Performance calculations for a total absorption fast neutron spectrometer are presented. The spectrometer detecting element is a boron-10-loaded liquid scintillator. By making the scintillator a thin disk, only those neutrons whose first collision is a large-energy-loss hydrogen collision have appreciable chance of remaining in the spectrometer long enough to be captured. The expected energy resolution and efficiency of this type of spectrometer are determined, and are comparable to other types of fast neutron spectrometers. April 14, 1959. $36 \mathrm{p}$.

TN11 (PB151370). Penetration of gamma rays from isotropic sources through aluminum and concrete, M. J. Berger and L. V. Spencer

Semi-analytical expressions, with numerically specified parameters, are given which represent the gamma ray dose distribution in infinite aluminum or concrete media, for sources that are monoenergetic (with energies between 10.22 Mev and 0.0341 Mev), isotropic, and have the form of an infinite plane, point, disk or spherical surface. May 11, $1959.13 \mathrm{p}$.

TN12 (PB151371). Transmission loss in radio propagation: II, K. A. Norton

In an earlier report with this title the concept of transmission loss was defined and its advantages explained. In this report a survey will be made of the transmission losses expected for a wide range of conditions, i.e., for distances from 10 to 10,000 statute miles; for radio frequencies from 10 ke to $100,000 \mathrm{Me}$; for vertical or horizontal polarization; for ground waves, ionospheric waves, and tropospheric waves; over sea water or over land which may be either rough or smooth; and for various geographical and climatological regions. June $1959,151 \mathrm{p}$.

TN13 (PB151372). Technical considerations leading to an optimum allocation of radio frequencies in the band 25 to $60 \mathrm{MC}$, K. A. Norton

The object of this study is to outline very briefly the technical considerations leading to an optimum allocation of radio frequencies in the band 25 to $60 \mathrm{Mc}$. This report will indicate what technical information is now available and where it may be obtained, as well as what additional information should be obtained in the future. June 1959. $96 \mathrm{p}$.

TN14 (PB151373). Analysis of ionospheric vertical soundings for electron density profile data. I. Facilities for convenient manual reduction of ionograms, J. W. Wright and R. B. Norton

Facilities for manual reduction of ionospheric vertical soundings to true heights are prepared using the "10 point" ratios of Ventrice and Schmerling. A study of the effects of the earth's magnetic field on true height calculations permits selection of five sets of these ratios assuring equal accuracy of application for any part of the world. The facilities available are in the form of transparent overlays and matching special graph paper. July 1959.13 p.

TN15 (PB151374). Prediction of the cumulative distribution with time of ground wave and tropospheric wave transmission loss. Part I-The prediction formula, P. L. Rice, A. G. Longley, and K. A. Norton

This report describes a method for predicting the cumulative distribution with time of transmission loss at fre- 
quencies above 10 megacycles per second over paths of arbitrary length. The method makes use of available information about terrain profiles and surface meteorological data, and is based on the CRPL radio standard atmospheres, in which the radio refractive index decreases linearly with height for the first kilometer above ground, and then decreases exponentially with height. Discussion of the theoretical basis for this formula and a demonstration of its accuracy by comparison with experimental data are reserved for later parts of this report. July $1959.81 \mathrm{p}$.

TN16 (PB151375). Some applications of statistical sampling methods to outgoing letter mail characteristics, N. C. Severo, A. E. Newman, S. M. Young, and M. Zelen

This paper presents applications of statistical sampling procedures especially devised to procure information about the characteristics of outgoing letter mail. The results of four separate studies carried out in the Washington, D.C., San Francisco, and Los Angeles post offices are herein summarized. The techniques used in the various studies were developed so that the required information would be of predetermined reliability and could be gathered without the use of a large staff and without interrupting the flow of mail. The four studies presented concern: (1) letter size and color characteristics, (2) ratio of hand canceled mail to machine canceled mail, (3) top and bottom clearance space of an addressed envelope, (4) proportions of long and short letters. July $1959.129 \mathrm{p}$.

TN17. (PB151376). Canceled.

TN18 (PB151377). Radio noise data for the International Geophysical Year July 1, 1957December 31, 1958, W. Q. Crichlow, C. A. Samson, R. T. Disney, and M. A. Jenkins

Radio noise data were obtained during the IGY from fifteen stations in a world-wide network equipped with the ARN-2 noise recorder developed by the National Bureau of Standards. Tables are presented which give the median values of recorded noise for each month at all hours of the day, and also the seasonal average of the noise by four-hour time blocks. July 27, 1959. 235 p.

TN18-2 (PB151377-2). Quarterly radio noise dataMarch, April, May 1959, W. Q. Crichlow, C. A. Samson, R. T. Disney, and M. A. Jenkins

Radio noise power, voltage, and logarithm of the voltage are being recorded at a network of field stations throughout the world. The data recorded at these locations during the season March, April, May 1959 are presented. The tabulation shows month-hour median and decile values of the power and the median values of the other two parameters as deviations below the power. The month-hour median values for all three parameters are averaged over a three-month, four-hour period to give seasonal time-block values corresponding to the time-block values presented in CCIR Report No. 65. March 14, 1960. $61 \mathrm{p}$.

TN19 (PB151378). Analysis of ionospheric vertical soundings for electron density profile data. II. Extrapolation of observed electron density profiles above $h_{\max } F 2$, J. W. Wright

A tentative model of the $F$ region above $h_{\max }$ is provided to permit extrapolation of electron density profiles into this region. The model corresponds to a simple "Chapman" curve, and may be fitted to true height data (obtained in part I) by a simple graphical process. June 1959. $8 \mathrm{p}$.
TN20 (PB151379). A preliminary analysis of amplitude scintillations of radio stars observed at Boulder, Colorado, R. S. Lawrence and J. L. Jespersen

Variations in the apparent flux from the radio source Cygnus-A are recorded at 53 and $108 \mathrm{mc} / \mathrm{s}$ using a twoelement phase-sweeping interferometer located at Boulder. An ionospheric sounder operating at Ellsworth, Nebraska, provides simultaneous vertical-incidence measurements of the ionosphere along the path. Amplitude scintillations observed at Boulder over a six-month period are compared with the ionograms taken at Ellsworth. Positive correlation is found between amplitude scintillations and spread $\mathrm{F}$, while a small negative correlation is found with sporadic E. The present result appears to be significant but a full year's data are being accumulated to disclose seasonal or diurnal effects. Detailed analysis of the scintillations indicates that the probability distribution of the amplitude can be represented by the Rice probability distribution function. The scintillation rate for $108 \mathrm{mc} / \mathrm{s}$ seems to be more rapid than for $53 \mathrm{mc} / \mathrm{s}$. July 1959 . $19 \mathrm{p}$.

TN21 (PB151380). Variations of gamma cassiopeiae, S. R. Pottasch

The variations of gamma Cassiopeiae between 1890 and 1950 are summarized and discussed. The variations of visual magnitude, color temperature, radius and electron density of the shell, and the spectral variations are depicted. August 1959. 29 p.

TN22 (PB151381). Precise time synchronization of widely separated clocks, A. H. Morgan

This paper describes known precise methods of setting a group of widely separated clocks to precisely the same time and keeping them in close agreement indefinitely; most of the proposed methods are now available. An estimate of the accuracies of each method are given. Also, there is some discussion of high frequency radio propagation theory pertinent to two of the methods and a few sets of measurements of the propagation delay time of high frequency signals from WWV to WWVH are given. Several graphs and tables are included to simplify some of the calculations. July 1959.65 p.

TN23 (PB151382). Design of single frequency filters, F. F. Fulton, Jr.

Efficient procedures are shown for designing filters formed by a number of identical resonant eircuits loosely coupled together, and which are required to accept one narrow band of frequencies and reject another narrow band somewhat removed in frequency, without any special requirements on the shape of the attenuation curve in between these regions. The design is based on using a large number of sections. August 20, 1959. 10 p.

TN24 (PB151383), Radio wave propagation in an inhomogeneous atmosphere, J. R. Wait

A self-contained treatment of the theory of radio waves in an inhomogeneous atmosphere is given. The refractive index is assumed to vary with height above the earth in a monotonic fashion. Variation according to an exponential law is used for illustration of general principles. For this case, rigorous series formulas are developed for the distance to the horizon for an elevated point in the atmosphere. September 10, 1959. $20 \mathrm{p}$.

TN25 (PB151384). Communication theory aspects of television bandwidth conservation, W. C. Coombs

New concepts of communication bandwidth utilization and conservation are in prospect through difference signal modulation systems in which only relative changes information is transmitted in lieu of absolute amplitudes. By 
these systems, the changes data function is made one of time as well as of amplitude, so that better advantage can be taken of redundancies in the video signal.

Advantageous conversions of information rate are made feasible by reason of a tremendous disparity. existing between the extremely high information capacity of conventional television systems and the relatively very low perception capability of a human observer channel. Information conversions more consistent with perception capabilities of the human channel are achieved in difference signal modulation without usual great expansion of bandwidth that would be required to reproduce every possible absolute amplitude of each datum in the whole video picture mosaic directly.

Conversion to binary digital form accrues the further advantages of binary systems, including greater immunity to noise, greater adaptability to discrete data storage media, greater ease of scrambling for security, and greater amenability of encoding to optimum parametric controls for most advantageous transmission. August 1959. 26 p.

TN26 (PB151385). Survey of Central Radio Propagation Laboratory research in tropospheric propagation 1948-1956, J. W. Herbstreit and P. L. Rice

This report summarizes and abstracts publications concerned with the National Bureau of Standards tropospheric propagation research program dating back to the formation of the Central Radio Propagation Laboratory. Some technical papers are reproduced here as supplements to this report and excerpts from some of the longer technical reports are also included. September 1959. $211 \mathrm{p}$.

TN27 (PB151386), Distribution of mail by destination at the San Francisco, Los Angeles, and Baltimore Post Offices, N. C. Severo and A. E. Newman

This report presents the results of the application of the sampling method presented in a paper by Norman C. Severo and Arthur E. Newman entitled "A statistical chain-ratio method for estimating relative volumes of mail to given destinations", NBS Journal of Research $64 C$, No. 1, 37, January-March 1960 . These studies were made at the San Francisco, Los Angeles, and Baltimore Post Offices. December 1959. $55 \mathrm{p}$.

TN28 (PB151387). A history of vertical-incidence ionosphere sounding at the National Bureau of Standards, S. C. Gladden

This is an attempt to treat in chronological order the development of vertical incidence ionosphere sounding at the National Bureau of Standards through 1957. Of necessity many phases of the radio work of the Bureau have been omitted or treated lightly as not being germane to the principal purpose of this history. With the exception of early workers in the field, names of individuals are to be found only in the references.

Source material includes the monthly, quarterly, and annual reports of Section 6, Division 1' (the old Radio Section), and of the Central Radio Propagation Laboratory; reports of the Interservice Radio Propagation Laboratory; the declassified reports of the International Radio Propagation Conference; the National Bureau of Standards Journal of Research; the NBS Technical News Bulletin; published and unpublished reports of NBS; periodical literature, such as the Proceedings of the Institute of Radio Engineers, the Physical Review, Nature, Journal of Geophysical Research (formerly Terrestrial Magnetism and Atmospheric Electricity), and Science. September 1959. $134 \mathrm{p}$.

TN29 (PB151388). Photographic dosimetry at total exposure levels below $20 \mathrm{mr}$, M. Ehrlich and W. L. MeLaughlin

Assemblies of commercial photographic material sandwiched between two plastic scintillators can be used to measure high-energy $\mathrm{X}$ - or gamma-ray exposures down to $1 \mathrm{mr}$ and less. The energy dependence of the assemblies' response is much less than that of a conventional photographic dosimeter, in some instances allowing an exposure interpretation with an accuracy of \pm 25 percent over the energy range from about $0.1 \mathrm{Mev}$ to $1.25 \mathrm{Mev}$. However, low-intensity reciprocity failure limits the range of applicability of the system.

Conventional photographic dosimeters, not incorporating scintillators, are usually preferable for routine personnel dosimetry. By extending the monitoring period, it may be possible to avoid personnel dosimetry below $20 \mathrm{mr}$ entirely. However, by doing this, one introduces additional difficulties because of instabilities in the photographic image. In some instances, an increase in effective emulsion thickness, achieved by using stacks of identical films, may lead to an increase in emulsion sensitivity sufficient to extend the useful range of a film badge well below $20 \mathrm{mr}$, without the use of a scintillator. October 1959. $23 \mathrm{p}$.

\section{TN30 (PB151389). Aerodynamic phenomena in stellar atmospheres - A bibliography}

A bibliography and short abstract of papers appearing in the astronomical literature between 1920 and 1960 on the subject of aerodynamic phenomena in stellar atmospheres. Prepared collaboratively by P. Ledoux, Institut d'Astrophysique, Liege; B. E. J. Pagel, Royal Observatory, Herstmonceux; J. Tuominen, University of Helsinki; C. Pecker, Institut d'Astrophysique, Paris; R. Lust, Max Planck Institut, Munich; G. Righini and G. Kalamai, Observatorio Astrofisico di Arcetri, Firenze; S. Miyamoto, Kwasan Observatory, Kyoto; S. A. Kaplan, University of Lwow, and S. B. Pickelner, University of Moscow and Simeis Observatory; C. A. Whitney, Smithsonian Astrophysical Observatory, Cambridge; and L. L. House and R. N. Thomas, National Bureau of Standards, Boulder, in an attempt to provide a working bibliography for the Fourth Symposium on Stellar Atmospheres, to be held during August 1960 at Varenna, Italy, under the sponsorship of the International Astronomical Union and International Union of Theoretical and Applied Mechanics. September $1959.89 \mathrm{p}$.

TN31 (PB151390). An atlas of oblique-incidence ionograms, W. Agy, K. Davies, and R. Salaman

This atlas is intended to serve a twofold purpose: first, to provide a compilation of records, of a type becoming standard in the field of ionospheric research, for those workers who are not now familiar with them, and second, to present records which are characteristic of the specific paths used by the National Bureau of Standards for consideration by those using other paths. November 1959. $135 \mathrm{p}$.

\section{TN32. (PB151391), Canceled.}

TN33 (PB151392). Distribution of incoming lettermail at the Baltimore, Md. City Post Office, B. M. Levin and A. E. Newman

Lettermail at the Baltimore, Md. post office was sampled and studied for the purpose of estimating the distribution characteristics of letters for local delivery. Separate studies were conducted for first- and third-class mail and the results are reported both separately and combined. The study demonstrated an effective method of sampling incoming lettermail. It also yielded data needed for the design of efficient procedures for sorting mail using machinery now being developed. October 1959 . $114 \mathrm{p}$.

TN34 (PB151393). Resistance diode bridge circuit for temperature control, L. H. Bennett and $\mathrm{V}$. M. Johnson

The conventional ac bridge gives irregular performance including loss of temperature control when the temperature error exceeds a certain critical value. The present 
note describes a simple method of achieving stable temperature control over a large range of temperatures. October $1959.4 \mathrm{p}$.

TN35 (PB151394). Service area of an airborne television station, M. T. Decker

As a step in the evaluation of a proposed airborne television network, the service to be expected from an airborne station has been calculated for a wide variety of operating conditions. The use of basic transmission loss to describe the radio propagation effects enables the results to be used when equipment parameters are changed as system requirements and economic considerations dictate. October 1959 . $43 \mathrm{p}$.

TN36 (PB151395). A multiplet table of astrophysical interest (Revised Edition). Part I. Table of multiplets and Part II. Finding list of all lines in the table of multiplets, C. E. Moore

The leading lines in 143 atomic spectra of 85 chemical elements are listed in related groups called multiplets. For each spectrum the multiplets are arranged in order of decreasing wavelength. Estimated intensities, excitation energies, and multiplet designations are given for the individual lines, and each multiplet is assigned a number. An extensive bibliography covers the source material used for the compilation.

The table is presented in two parts:

Part I includes the multiplets, with the spectra of each element being given in order of increasing ionization, and the elements in order of increasing atomic number.

Part II is a Finding List in which all the lines in Part I are entered in order of increasing wavelength, with their multiplet numbers.

The range of the Table is from $2951 \mathrm{~A}$ to $13164 \mathrm{~A}$. A supplementary table of "Forbidden Lines" extends from 2972 A to 12645 A. November 1959. 256 p.

TN37 (PB151396). Application of RF micropotentiometers for calibration of signal generators to $1000 \mathrm{Mc}, \mathrm{L}$. F. Behrent

With the RF Micropotentiometer, signal generator output voltage can be calibrated to $1000 \mathrm{Mc}$ simply and accurately if the procedures outlined in this paperare carefully followed. The sources of error are discussed and methods for minimizing or eliminating them are described. Topics discussed include: rf shielding, selection of a suitable rf detector, impedance matching and the proper selection of voltage reference planes. January $1960.14 \mathrm{p}$.

TN38 (PB151397). Design and construction of a liquid hydrogen temperature refrigeration system, D. B. Chelton, J. W. Dean, and B. W. Birmingham

Maintaining a liquid hydrogen bubble chamber at $27^{\circ} \mathrm{K}$ has been achieved with an automatically controlled closed circuit hydrogen reirigeration system of 300 watts capacity. The system is sufficiently flexible to be used on other experimental apparatus requiring refrigeration at liquid hydrogen temperatures. Several control systems are discussed. Experimental evidence is compared to predicted performance for design operating conditions.

General design charts are developed that enable heat exchanger lengths and associated operating parameters to be determined for the pertinent heat exchanger configuration when employed in liquid hydrogen refrigerators of other capacities. January 12, 1960. $37 \mathrm{p}$.

TN39 (PB151398). Helium refrigeration and liquefaction using a liquid hydrogen refrigerator for precooling, D. B. Chelton, J. W. Dean, T. R. Strobridge, B. W. Birmingham, and D. B. Mann

Consideration is given to the use of a hydrogen refrigerator to assist in the production of temperatures below those obtained with hydrogen. A hydrogen refrigerator is used to maintain a precooling evaporator at or near $21^{\circ} \mathrm{K}$ in a helium gas circuit. The helium circuit may be arranged to produce liquid for external use or to produce refrigeration between $21^{\circ} \mathrm{K}$ and $4.2^{\circ} \mathrm{K}$ in a closed system.

Charts have been developed that show the requirements of the composite helium-hydrogen system and the effect of heat exchanger performance. The relative quantities of refrigeration (hydrogen and helium) at various temperature levels have been determined. January 27,1960 . $31 \mathrm{p}$.

TN40-1 (PB151399-1). Mean electron density variations of the quiet ionosphere, IMarch 1959, J. W. Wright and L. A. Fine

The CRPL has initiated a program for large-scale computation of electron density profiles from ionospheric vertical soundings. Scaling is performed at field stations permitting computation of hourly profiles at the central laboratory. These profiles are combined to form hourly mean quiet profiles for each station and month. The results of this program for one month are illustrated graphically. This report is the first of a series illustrating the electron density variations in the mean quiet ionosphere between latitudes $15^{\circ} \mathrm{N}$ and $50^{\circ} \mathrm{N}$ along the $75^{\circ} \mathrm{W}$ meridian. February 1960 . $50 \mathrm{p}$.

TN40-2 (PB151399-2). Mean electron density variations of the quiet inosphere, 2April 1959 , J. W. Wright and L. A. Fine

The CRPL has initiated a program for large-scale computation of electron density profiles from ionospheric vertical soundings. Scaling is performed at field stations permitting computation of hourly profiles at the central laboratory. These profiles are combined to form hourly mean quiet profiles for each station and month. The results of this program for the month of April are illustrated graphically. This report is the second of a series illustrating the electron density variations in the mean quiet ionosphere between latitudes $15^{\circ} \mathrm{N}$ and $50^{\circ} \mathrm{N}$ along the $75^{\circ} \mathrm{W}$ meridian. February $1960.50 \mathrm{p}$

TN41 (PB151400). Expendable modules as bases for disposal-at-failure maintenance, $\mathrm{R}$. O. Stone, P. Meissner, and K. M. Schwarz

The maintenance of future electronic equipment will more than likely exceed the capabilities of existing maintenance activities if present maintenance procedures are continued. Design trends such as miniaturization, printed circuitry, encapsulation, and modular construction all point toward disposal-at-failure maintenance. Disposalat-failure modular design would help to solve some of the future maintenance problems and would also be compatible with future design trends.

It has been suspected that disposal-at-failure maintenance would be too costly to be practical. This report compares the costs to procure, support and maintain an equipment designed with expendable modules to the costs to procure, support, and maintain a similar equipment designed with repairable modules. It was found that the total costs in either case were approximately equal. Since the cost factor is not important in the decision between expendable assembly or repairable assembly maintenance, other factors, such as improved reliability of equipment, smaller size, lighter weight, and improved maintenance, all advantages that may be gained through the use of expendable design, should be carefully studied. These advantages may be so important as to completely determine a decision in favor of expendability.

Modules of various electrical sizes (from 1 to 12 tubes) have been compared from the standpoint of total over-all procurement cost, in order to determine an optimum 
module size. On the basis of calculations made, it has been concluded that the optimum module which would lead to lowest over-all procurement cost and at the same time be of aid in the solution of logistic and maintenance problems would be one containing from 4 to 8 tubes. This is true for both disposal-at-failure and repairable modules. February $1960.79 \mathrm{p}$.

TN42 (PB151401). Analog-digital conversion equipment for electrocardiographic data, L. Taback

A corrected orthogonal 3-lead system has been used to record electrocardiograms directly from patients at Veterans Hospitals, using three FM channels of magnetic tape. A pilot facility has been designed and assembled by NBS to permit a medical technician to inspect these on an oscilloscope and select a significant cardiac cycle. This is automatically sampled at millisecond intervals and the numerical values are stored in digital form on magnetic tape acceptable to an electronic computer. Upon writing various programs for the digital computer, the cardiac researcher will have a flexible tool for objective analysis of large quantities of biological data by a variety of possible criteria. April 1960.40 p.

TN43 (PB151402). A summary of VHF and UHF tropospheric transmission loss data and their long-term variability, D. A. Williamson, V. L. Fuller, A. G. Longley and P. L. Rice

Cumulative distributions of hourly median basic transmission loss are presented for 135 beyond-line-of-sight radio paths in the United States. In order to allow for seasonal trends of transmission loss, the year is divided into a summer period, May through October, and a winter period, November through April.

The long-term variability of observed hourly medians is compared with predicted variability based on empirical curves by Rice, Longley and Norton. March 1960. 174 $\mathrm{p}$

TN44 (PB151403). Tables for the statistical prediction of radio ray bending and elevation angle error using surface values of the refractive index, B. R. Bean, B. A. Cahoon and G. D. Thayer

Radio ray bending, $\mathrm{T}$, and elevation angle error, $\mathrm{E}$, have been calculated for a wide range of meteorological conditions at 13 climatically diverse U.S. radiosonde stations. The parameters in the observed linear regression equations of $\mathrm{T}$ and $\mathrm{E}$ upon the surface value of the refractive index are given for heights of 0.1 to 70 kilometers and initial elevation angles of the ray from 0 to 900 milliradians. March 16, $1960.22 \mathrm{p}$.

\section{TN45 (PB151404). Operating instructions for ARN-2 auxiliary log-linear noise recorder, R. T. Disney and C. A. Samson}

Auxiliary equipment has been added to the noise power recorder, ARN-2, to measure the average voltage of the noise envelope and the average logarithm of the noise envelope. These two additional parameters of the noise give enough information to determine the average characteristics of the noise. The operation of the equipment is discussed and standard operating procedures are outlined. January 1960 . $30 \mathrm{p}$.

TN46 (PB151405). Experimental plating of gun bores to retard erosion, V. A. Lamb and J. P. Young

Methods for plating the bores of gun barrels are described, including details of fixture design, solution composition, and operating conditions. Extensive firing tests were performed, which show that chromium plate increases the life of barrels about 2- to 5-fold, depending on the type and caliber of barrel. Optimum thickness of plate ranges from 0.0015 inch in the smallest calibers to 0.015 inch in cannon.

Certain modifications of the barrels enhance the improvement provided by the chromium plate. Choking of the bore at the muzzle produces a marked improvement in accuracy life of caliber 0.30 and 0.50 barrels. It is less effective in barrels of larger caliber. Specially shaped lands, designed to reduce the concentration of engraving stresses at land corners, significantly improve the performance of $20 \mathrm{~mm}$ barrels. Other modifications, such as hardening of the basis steel by nitriding and increasing the length of the land "run-up", result in moderate improvements in some types of barrels. Physical properties of the chromium plates tested were varied. Ordinary "hard" chromium yields the best performance in most types of barrels.

Chromium plating has been adopted as standard production practice for caliber $0.30,0.50$, and $20 \mathrm{~mm}$ barrels, and for several calibers of cannon. May 1960. $99 \mathrm{p}$.

TN47 (PB151406). An improved hydrogen atom beam furnace, R. Klein and J. Pararas

An improvement in the construction of the tungsten furnace for use in a hydrogen atom-beam apparatus has been effected by fabricating the furnace from a tungsten rod. This avoids the possibility of leakage of molecular hydrogen which may occur when the tungsten tube is made from rolled tungsten sheet, the technique used heretofore. The fabrication of the seamless furnace and the method of mounting are described. May 1960. $7 \mathrm{p}$.

TN48 (PB151407). Report on the IGY oblique-incidence sporadic-E and F-scatter program, J. W. Finney and E. K. Smith

The IGY Oblique-Incidence Sporadic-E Measurements program was instigated to test the longitude effect in temperate zone sporadic $\mathrm{E}$. To accomplish this two $\mathrm{CW}$ 50 Mc circuits, both approximately 800 miles in length, were installed, one between the Philippines and Okinawa in the Far East, and the other between Panama and Cuba in the Caribbean. In addition to sporadic $\mathrm{E}$ a very peculiar evening signal was observed during the equinoxes on the far eastern circuit which we have referred to as the "evening signal anomaly" or the "Far Eastern Anomaly". Sporadic E, as expected, was three to five times more frequent in the Far East than in the Caribbean, the factor depending upon what transmission loss level is taken. The evening signal anomaly appears to be of F-region origin and is at least 100 times more frequent over the Far Eastern circuit. March $1960.94 \mathrm{p}$.

TN49 (PB151408). Dynamic measurements of the magnetoelastic properties of ferrites, V. E. Bottom

The relations between the mechanical and magnetic properties of a ferrite are derived for the small signal or reversible condition using thermodynamic principles. The equation of motion of a ferrite ring driven in its fundamental mode is set up and solved leading to the equivalent electrical circuit of the magnetostriction resonator. From mechanical measurements of the density and dimensions of the ring and electrical and frequency measurements of the resonator, the elastic modulus, permeability, magnetostriction coefficients and loss factors in the ferrite can be determined. Apparatus is proposed for performing the above measurements. $1960.43 \mathrm{p}$.

TN50 (PB151409). Magnetic drum directory and programming system for codesorting letter mail, P. C. Tosini

This report is an analysis and extension of the Rabinow Engineering Company's proposal for a magnetic drum file-directory and special purpose computer to be used in 
a test coded-address ("codesorting") letter-mail sorting system scheduled for late 1960 installation at the Washington, D.C. Post Office. The report is composed of 6 major sections that successively examine the major considerations involved. A short introduction is presented first. Then the general problem of coding the fields composing addresses is discussed. This area is analyzed in terms of facilitating both the human coding process and the computer coded address-to-bin-number translation. Addresses are classified into two types: "standard" and "non-standard". When coded, their fields must be unique and yet be sufficiently flexible to specify a variety of addresses. They must furthermore adequately specify some more common forms of misaddressed mail that is presently correctly sorted manually. A slightly amended form of Coding Plan E-1 is then presented and analyzed in terms of the conditions specified for coded addresses in general.

After the presentation of the coding area, the local mail sort translation program of the Rabinow proposal is discussed in detail. It is then extended to include the outgoing mail translation program and non-standard and special address forms. The information and instruction characters necessitated by the programs are listed in detail and the effects of the file-directory source document preparation determined. A coding keyboard is then suggested.

The programming is then reexamined and it is determined that all addresses can be treated as special cases of the standard address forms at an increased cost in memory space and with a saving in the special instruction symbols required. Finally, alternative methods of file-directory information storage are explored and evaluated in terms of memory space requirements, table look-up and directory access times, and conceptual simplicity. June 1960 . 54 p.

TN51 (PB161552). Isotopic abundance ratios reported for reference samples stocked by the National Bureau of Standards, F. L. Mohler

The National Bureau of Standards maintains a stock of reference samples of isotopic abundance and this paper gives results that have been reported on measurements of these samples. Some information has been received on 24 of the 32 samples. The paper includes a list of the reference samples and tables of the results reported with notes on these results. May $1960.14 \mathrm{p}$.

TN52 (PB161553). Field strength calculations for E.L.F. radio waves, J. R. Wait and N. F. Carter

The mode theory of propagation of electromagnetic waves at extremely low frequencies $(1.0$ to $3000 \mathrm{c} / \mathrm{s})$ is briefly reviewed in this paper. Starting with the representation of the field as a sum of modes, approximate formulas are presented for the attenuation and phase constants. At the shorter distances, where the range is comparable to the wavelength, the spherical-earth mode series is best transformed to a series involving cylindrical wave functions. This latter form is used to evaluate the near field behavior of the various field components. The effect of the earth's magnetic field is also evaluated using a quasi-longitudinal approximation which is particularly appropriate for propagation in the magnetic meridian. In general it is indicated that if the gyro frequency is comparable or greater than the effective value of the collision frequency, the presence of the earth's magnetic field may be important for E.L.F. In this case the attenuation may be increased somewhat. The influence of a purely transverse magnetic field is also considered.

This technical note is actually a numerical supplement to the paper "Mode Theory and the Propagation of ELF Radio Waves," by J. R. Wait, J. Research N.B.S., 64D, July-Aug. 1960; however, for sake of completeness the relevant theory is reviewed. March $1960.35 \mathrm{p}$.
TN53 (PB161554). Carrier frequency dependence of the basic transmission loss in tropospheric forward scatter propagation, K. A. Norton

A further interpretation is given of certain Lincoln Laboratory data obtained in an experiment using scaled antennas as presented in a recent letter to the Proceedings of the IRE from Bolgiano. This paper has four objectives: first, to clarify the significance of these data from the standpoint of the engineer developing long-range tropospheric scatter systems; second, to apply a further statistical analysis to these data; third, to consider their significance as regards the theory of radio propagation through a turbulent atmosphere; and fourth, to describe a suitable method for the measurement of the meteorological parameters entering the theory. May 12, 1960. $64 \mathrm{p}$.

TN54 (PB161555). Determination of a general index of effort in sorting mail by conventional methods, S. Henig

The conventional method of sorting letters in stages is described. It is shown, through reference to available statistics, that about 90 percent of letters originating within the areas served by several large post offices will be involved in no more than five stages to be completely sorted to a carrier, firm or section of boxes. A method is presented for the accounting of each stage's individual letter readings which are defined as the units of measurement for the index of sorting effort. It is found that the upper bound average number of readings per letter for three large post offices varies from 3.16 to 2.98 and that these bounds are applicable to 96.2 to 92.4 percent of the local originating first class letters. It is anticipated that the values of such indexes for all large post offices will be consistently close to the range already determined. June 1960. 9 p.

TN55 (PB161556). Investigation of bearing creep of two forged aluminum alloys, L. Mordfin, N. Halsey, and P. J. Granum

Fourteen bearing specimens of 7075-T6 and 2014-T6 forged aluminum alloys were tested in creep at $400^{\circ} \mathrm{F}$. The 2014-T6 specimens had greater ereep resistance and less ductility than the 7075-T6 specimens.

For each material the bearing ereep resistance varied directly with edge distance and bolt tightness. Prior exposure of the 2014-T6 alloy to elevated temperature reduced its bearing creep resistance.

The validity of a previously proposed theory of creep in bolted joints was examined using the test data together with results from an earlier study. The theory was found to hold for 7075-T6 but not for 2014-T6. August 1960. $31 \mathrm{p}$.

TN56 (PB161557). A bibliography of the physical equilibria and related properties of some cryogenic systems, T. M. Flynn

A bibliography of approximately 700 references is presented on the physical equilibria and related properties of several important cryogenic systems. The systems considered are the pure components and mixtures of: Hydrogen, Helium, Nitrogen, Carbon Dioxide, Carbon Monoxide, Methane, Ethane, and Propane. May 1960.123 p.

TN57 (PB161558). Variable capacitor calibration with an inductive voltage divider bridge, T. L. Zapf

The use of an inductive voltage divider bridge for the calibration of three-terminal and two-terminal variable air capacitors is discussed. May 1960 . $6 \mathrm{p}$. 
TN58 (PB161559), A survey and bibliography of recent research in the propagation of VLF radio waves, J. R. Wait

This report presents a general, but brief, survey of the field. Attention is confined primarily to terrestrial propagation, and thus solar and exospheric phenomena are generally excluded although certain germane references are given in the bibliography.

First a brief description of recent advances of ground wave propagation is given. This is followed by sections on ray and mode concepts of ionospheric propagation. The research dealing with the waveforms of atmospherics is also considered. Finally some recent applications of VLF propagation are described. While the emphasis is on the theoretical approaches used, reference to corroborating experimental work is included. It is hoped that the shortcomings in a brief article of this kind are partially compensated by the inclusion of an extensive bibliography on the subject arranged under subject classification. While attention is confined primarily to the triennium (1957-59) a number of basic references prior to 1957 are included.

The VLF band is here defined as the decade 3 to $30 \mathrm{kc} / \mathrm{s}$ whereas the ELF band covers the range $1.0 \mathrm{c} / \mathrm{s}$ to $3 \mathrm{kc} / \mathrm{s}$. May $1960.44 \mathrm{p}$. 


\section{TITLES OF PAPERS PUBLISHED IN OUTSIDE JOURNALS 1950 TO 1959}

On pages 75 to 160 are listed papers by the Bureau staff that have appeared in outside journals during the period 1950 to 1959 . The listing is alphabetical by title under year of publication. Each paper is preceded by a number that serves to key the paper to the index. These papers are not for sale by the Government, usually they may be obtained directly from the author or from the publisher of the appropriate journal. 


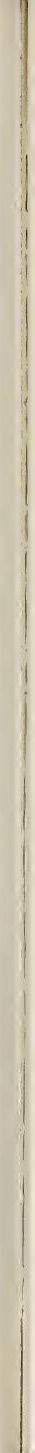


1. A class of mean value functions, E. F.

Beckenbach. Am. Math. Monthly 57, No. 1, $1(1950)$.

2. A comparison of meteor activity with occurrence of sporadic E reflections, V. C. Pineo. Science $112,50(1950)$.

3. A new method for determining the value of the Faraday, D. N. Craig and J. I. Hoffman. Phys, Rev. 80, 487 (1950).

4. A new technique for cutting very thin sections and its application to the electron microscopy of fibers, S. B. Newman. ASTM Bull. 163, 57 (1950).

5. A note on the four by four Latin squares,

W. J. Youden. Biometrics 6, 289 (1950).

6. A recording microwave refractometer, G.

Birnbaum. Rev. Sci. Instr. 21, 169 (1950).

7. A reduction of area gage for use at low

temperatures, G. W. Geil and N. L. Carwile. ASTM Bull. 163, 75 (1950).

8. A remote-control method of opening ampoules of active materials, J. E. Sherwood. Rev. Sci. Instr. 21, 570 (1950).

9. A resistance-temperature relation for low temperature thermometry, H. J. Hoge. Rev. Sci. Instr. 21, 815 (1950).

10. A source of error in radio phase measuring systems, R. Bateman, E. F. Florman, and A. Tait. Proc. IRE 38, 612 (1950).

11. A sulfate susceptibility test for portland cements, W. C. Taylor and R. H. Bogue. Portland Cement Assoc. Fellowship 58, 223 (1950).

12. A survey of ceramics for nuclear reactors,

R. F. Geller. Nucleonics 7, No, 4, 3 (1950).

13. A thickness gage for ceramic coatings, C. C. Gordon and J. C. Richmond. J. Am. Ceram. Soc. 33, 295 (1950).

14. A versatile pneumatic instrument based on critical flow, W. A. Wildhack, Rev. Sci. Instr. 21, No. 1, 25 (1950).

15. A VHF match meter, P. G. Sulzer. Television Eng. 1, No. 7, 4 (1950).

16. Acceptance sampling by variables, with special reference to the case in which quality is measured by average of dispersion, J. H. Curtiss. Acceptance Sampling: A symposium given at the 105th annual meeting. Am. Statistical Assoc. (Washington, D. C.) (1950).

17. Unas signed.

18. Adjustment of high-precision frequency and time standards, J. M. Shaull. Proc. IRE 38, $6(1950)$

19. Aging of Karakul and seal fur skins, E. T. Steiner and E. R. Hosterman. J. Am. Leather Chemists Assoc. 45, 579 (1950).

20. American Dental Association Specification No. 9 for dental silicate cement, First revision, effective July 1, 1950, G. C. Paffenbarger, A. C. Swaney, I. C. Schoonover, and G. Dickson. J. Am. Dental Assoc. 40, 186 (1950).

21. Amerikanische Kunststoffentwicklung im Jahre 1949, G. M. Kline. Kunststoffe (Munchen, Germany) 40, No. 2, 59 (1950).
22. An analysis of some anomalous properties of equiphase contours, G. A. Hufford. Proc. IRE 38, 614 (1950)

23. An elementary introduction to the calculus of variations, M. R. Hestenes. Mathematics 23, No. 5, 249 (1950).

24. An instrument for mechanically differentiating curves, A. H. Scott. Rev. Sci. Instr. 21, 397 (1950).

25. Analysis of natural gas, M. Shepherd. Anal. Chem. 22, 885 (1950).

26. Apparatus for determining freezing points at saturation pressure from time-temperature freezing and melting experiments, A. R. Glasgow, Jr., N. C. Krouskop, and F. D. Rossini. Anal. Chem. 22, 1521 (1950).

27. Application of photoelectric multiplier tubes to the sensitive measurement of absorption or of changes of relative light intensities, O. Oldenberg and H. P. Broida. J. Opt. Soc. Am. 40, 381 (1950).

28. Atomic definition of primary standards, R. D. Huntoon and U. Fano. Nature (London, England) 166,167 (1950).

29. Attenuation due to rainfall, H. E. Bussey. FM-TV Radio Commun. 10, No. 2, 12 (1950).

30. Background eradication in thick-layered emulsions, M. Wiener and H. Lagoda. Rev. Sci. Instr. 21, No. 1, 39 (1950).

31. Calibration of audiometers, E. L. R. Corliss and W. F. Snyder. J. Acoust. Soc. Am. 22,837 (1950).

32. Calibration of precision airplane mapping cameras, F. E. Washer and F. A. Case. Photogrammetric Eng. 16, 502 (1950).

33. Cascade-connected attenuators, R. W. Beatty. Proc. IRE 38, 1190 (1950).

34. Characteristics of the Institute for Numerical Analysis Computer, H. D. Huskey. Math. Tables and Other Aids to Computation 4, No. 30, 103 (1950).

35. Chemical spectroscopy. 1950 Edgar Marburg Lecture before the American Society for Testing Materials, W. R. Brode. Am. Soc. Testing Materials Proc. 50, 513 (1950).

36. Coefficients for polar complex interpolation, H. E. Salzer. J. Math. and Phys. 24, No. 2, 96 (1950).

37. Color phase contrast microscopy: Requirements and applications, C. P. Saylor, A. T. Brice, and F. Zernike. J. Opt. Soc. Am. 40, $329(1950)$

38. Color television, N. Smith. Sci. Am. 183, No. 6, 13 (1950).

39. Comparative tests in a single laboratory, W. J. Youden. ASTM Bull. 166, 48 (1950).

40. Comparison of variously derived solar indexes, H. S. Moore and M. Stein. J. Geophys. Research 55, 423 (1950).

41. Coupling chart for solenoid coils, P. G. Sulzer. Television Eng. 1, No. 6, 20 (1950).

42. Coverage of standard frequency station

WWVH, E. L. Hall. Television Eng. 1, No. 8, 16; No. 10, 20 (1950).

43. Degradation of high polymers, R. Simha.

J. Polymer Sci. Letter to Editor 5, 515 (1950). 
44. Depolymerization as a chain reaction, $\mathbf{R}$. Simha, L. A. Wall, and P. J. Blatz. J. Polymer Sci. 5, 615 (1950).

45. Determination of attenuation from impedance measurements, R. W. Beatty. Proc. IRE 38, 897 (1950).

46. Determination of reverberant sound absorption coefficient from acoustic impedance measurement, A. London. J. Acoust. Soc. Am. 22, 263 (1950).

47. Device for filtering and precise dispensing of solutions in closed systems, S. Rothman. Anal. Chem. 22, 367 (1950).

48. Dispersion in $\mathrm{NH}_{3}$ in the microwave region, G. Birnbaum. Phys. Rev. 77, 144 (1950).

49. Dissociation constant of aqueous ammonia at $0^{\circ}$ to $50^{\circ}$ from EMF studies of the ammonium salt of a weak acid, R. G. Bates and G. D. Pinching. J. Am. Chem. Soc. 72, 1393 (1950)

50. Effect of fuel-immersion on laminated plastics, W. A. Crouse, M. Carickhoff, and M. A. Fisher, Trans, Am, Soc, Mech. Engrs. 72, 175 (1950).

51. Effects of acid treatment on acid and abrasion resistance of porcelain enamels, W. N. Harrison, J. C. Richmond, and J. R. Crandall. J. Am. Ceram. Soc. 33, 314 (1950).

52. E1 sistema I. C. I. para la especificacion del color, D. B. Judd. Anales de la Real Sociedad Espanola de Fisica y Quimica (Madrid, Spain) 46, 123 (1950).

53. Electron optical properties of spacecharged clouds, L. Marton and D. L. Reverdin. J. Appl. Phys. 21, 842 (1950).

54. Electrons vs, photons: A comparison of microscopes, L. Marton. J. Opt. Soc. Am. 40,269 (1950).

55. Evaluation of adhesives for acoustical tile, F. W. Reinhart, B. D. Loos, and N. J. DeLollis. ASTM Bull. 169, 57 (1950).

56. Exploration of electrostatic and magnetic fields, L. Marton. Sci. Monthly 71, 3 (1950).

57. Factors affecting the water vapor permeability of leather, J. R. Kanagy and R. A. Vickers. J. Am. Leather Chemists Assoc. $45,211(1950)$.

58. Flow behavior of concentrated GR-S rubber solutions, A. B. Bestul and H. V. Belcher.

J. Colloid Sci. 5, 303 (1950).

59. For wear prevention, S. A. McKee, J. F. Swindells, H. S. White, and W. Mountjoy. SAE J. 58, 47 (1950).

60. Forbidden lines in the spectra of impure mercury 198, K. G. Kessler. Phys. Rev. $77,559(1950)$

61. Formulas for numerical integration of first and second order differential equations in the complex plane, H. E. Salzer. J. Math. and Phys, 29, No. 3, 207 (1950).

62. Frequency and amplitude stability of the cathode-coupled oscillator, P. G. Sulzer. Proc, IRE 38, 540 (1950).

63. Galactic radio waves, G. Reber. Leaflet Astron. Soc, Pacific No. 295, (1950).

64. Generalization of a theorem of Osgood to the case of continuous approximation. A. M. Ostrowski. Proc. Am. Math. Soc. 1, No. 5, $648(1950)$.
65. Gibbs ${ }^{\prime}$ phenomenon for Hausdorff means, O. Szasz. Trans. Am. Math. Soc. 69,440 (1950).

66. High-speed motion pictures in textile research. E. K. Fischer and J. C. Burnett. Textile Research J. 20, 259 (1950).

67. Higher-order approximations in ionospheric wave-propagation, J. Feinstein. J. Geophys. Research 55, 161 (1950).

68. How statistics improves physical, chemical, and engineering measurements, $W$. J. Youden. Revised text of a lecture, presented earlier by Dr. Youden, has been issued in mimeographed form by (and copies are available from) the Committee on Experimental Design, Agricultural Research Administration, U. S. Department of Agriculture, Washington, D. C.

69. How the U. S. Weather Bureau uses Fax \& Telemetering: How FM expedites weather data distribution, C. A. Kettering and G. F. Montgomery. FM- TV Radio Commun, 10, No. 10, $17(1950)$

70. Identification and microdetermination of nickel, H. I. Feinstein. Anal. Chem, 22, $723(1950)$

71. Index for rating diagnostic tests, $W, J$, Youden. Cancer 3, No. 1, 32 (1950).

72. Inequalities concerning ultraspherical polynomials and Bessel functions, O. Szasz. Proc. Am. Math. Soc. 1, No. 2, 256 (1950).

73. Influence of strain rate and temperature on the creep of cold drawn ingot iron, W. D. Jenkins and T. G. Digges. Trans. Am. Soc. Metals 42, 1128 (1950).

74. Influence of temperature on the adsorption of water vapor by collagen and leather, J. R. Kanagy. J. Am. Leather Chemists Assn. $45,12(1950)$.

75. Infrared absorption spectra of twelve substituted benzene derivatives from 15 to 40 microns, E. K. Plyler. Faraday Society Discussion No. 9, (University Press, Aberdeen, England) (1950).

76. Instrumentation in perspective, W. A. Wildhack. Science 112, 515 (1950).

77. Interdisciplinary approach to science personmel, M. E. Stevens. Personnel Administration 12, No. 3, $27(1950)$.

78. Intermediate-frequency gain stabilization 'with inverse feedback, G. F. Montgomery. Proc. IRE 38, 662 (1950).

79. International exchange of scientific information, W. R. Brode. Chem, \& Eng. News 28,4332 (1950).

80. Ionosphere observations at $50 \mathrm{kc}, \mathrm{J}, \mathrm{N}$. Brown and J. M. Watts, J. Geophys. Research 55, 179 (1950).

81. Is otope effect in the superconductivity of mercury, E. Maxwell. Phys. Rev. 78, 477 (1950).

82. Lubrication of plain journal bearings, $E$. N. Bair, Sr, , S. A. McKee and H. S. White. The Virginia Tech. Eng. 23, 26 (1950).

83. Lunar stratification of the F2 layer at Huancayo, Peru. T. N. Gautier, R. W. Knecht, and A. G. McNish. Proc, General Assembly, Intern. Sci. Radio Union (Zurich, Switzerland) Sept. 11-22, 1950, VII, Pt. 2, $216(1950)$. 
83A. Matching loads on a magic tee, A. C. MacPherson and D. M. Kerns. Electronics 23, No. 9 (1950).

84. Matrix inversion by a Monte Carlo method, G. E. Forsythe and R. A. Leibler. Math. Tables and Other Aids to Computation 4, No. $31,127(1950)$.

85. Measurement of high temperatures in gas streams, A. I. Dahl. Bull. A \& M College of Texas 5th series 6, $30(1950)$ and Petrol Refiner 29, No. 3, 115 (1950).

86. Measurement of surface roughness of electrodeposited and electropolished surfaces by means of the microinterferometer, A. G. Strang and F. Ogburn. 37th Annual Proc. Am. Electroplaters' Soc. 125 (1950).

87. Metric differential geometry, E. F. Beckenbach, Mathematics 23, No. 3, 143 (1950).

88. Microwave attenuation statistics estimated from rainfall and water vapor statistics, H. E. Bussey. Proc. IRE 38, 781 (1950).

89. Microwave frequency dividers, H. Lyons. J. Appl. Phys. 21, 59 (1950).

90. Microwave power stabilizer, I. K. Munson, Rev. Sci. Instr. 21, 622 (1950).

91. Modification of electron microscope for electron optical shadow method, J. A. Simpson and A. Van Bronkhorst. Rev. Sci. Instr. 21, 669 (1950).

92. Modified resonant circuits match impedances, P. G. Sulzer. Tele-Tech. 9, No. 11, $41(1950)$.

93. Monostable multivibrator design, G. F. Montgomery. Tele-Tech. 9, No. 10, 31 (1950).

94. Natural and synthetic rubbers, N. Bekkedahl. Anal. Chem. 22, 253 (1950).

95. Note on the focusing of electron beams in certain magnetic fields, P. A. Sturrock. Proc. Phys. Soc. (London, England) 63, 954 (1950).

96. Note on Vincent's theorem, A. M. Ostrowski. Annals of Math. 52, 702 (1950).

97. Notes on modern numerical analysis. I. Solution of differential equations by recurrence relations, J. Todd. Math. Tables and Other Aids to Computation 4, No. 29, 39 (1950).

98. Notes on numerical analysis, 2. Note on the condition of matrices. O. Taussky, Math. Tables and Other Aids to Computation 4, No. $30,111(1950)$.

99. Notes on the care and use of electrical instruments, F. D. Weaver. Instruments 23, 1236 (1950).

100. Numerical evaluation of the Fermi betadistribution function, I. Feister. Phys, Rev. 78,375 (1950).

101. Observations of second sound radiation by the thermal Pitot tube, J. R. Pellam. Phys. Rev. 78, 818 (1950).

102. On a summation method of O. Perron, O. Szasz. Math. Z. (Berlin, Germany) 52, 631 (1950).

103. On the Gibbs' phenomenon for Euler means, O. Szasz. Acta Scientiarum Mathematicarum (Szeged, Hungary) 12, Pt. B, 107 (1950).

104. On the relative extrema of Bessel function, O. Szasz. Bolletino della Unione Mathematica Italiana (Bologna, Italy) (Firenze) III, 225 (1950).
105. On the relative extrema of the Laguerre orthogonal functions, J. Todd. Bollettino della Unione Matematica Italiana (Bologna, Italy) Serie 3, Anno 5, No. 2, 122 (1950).

106. On the relative extrema of ultraspherical polynomials, O. Szasz. Bollettino della Unione Matematica Italiana (Bologna, Italy) Serie 3, Anno 5, No. 2, 125 (1950).

107. Petroleum hydrocarbons, B. J. Mair. Ind. \& Eng. Chem. 42, 1355 (1950).

108. Piezoelectric constants of alpha- and beta-quartz at various temperatures, R. K. Cook and P. G. Weissler. Phys. Rev. 80, 712 (1950).

109. Plastics: Organic, F. W. Reinhart and G. M. Kline. Medical Physics, Vol. II, 728 (1950).

110. Precision thermostat for high temperatures, W. R. Eubank. Rev. Sci. Instr. 21, 845 (1950).

111. Predicted lines of $\mathrm{Fe} I$ in the arc and in the sun, C. C. Kiess and C. E. Moore. Astron. J. 55, 173 (1950).

112. Preparing cuprammonium solvent and cellulose solutions, H. F. Launer and W. K. Wilson. Anal. Chem. 22, 375 (1950).

113. Present status of standards for shoe last sizes, R. B. Hobbs. J. Nat. Assoc. Chiropodists No. 10, 30 (1950).

114. Review of "Proceedings of the Berkeley Symposium on Mathematical Statistics and Probability, "C. Eisenhart. Psychometrica 15,448 (1950).

115. Propagation of sound in rarefied helium, M. Greenspan. J. Acoust. Soc. Am. 22, $568,(1950)$.

116. Pyrolysis of hydrocarbon polymers, S. L. Madorsky. Science 111, 360 (1950).

117. Radio frequency mass spectrometer, W. H. Bennett. J. Appl. Phys. 21, 2 (1950).

118. Radio noise of ionospheric origin, H. V. Cottony. Science 111, 41 (1950).

119. Rapid extraction of resins from chilte and guayule rubbers, J. W. Wood and R. J. Fanning. Rubber Age 68, No. 2, 195 (1950).

120. Rate of shrinkage of tendon collagen: Further effects of tannage and liquid environment on the activation constants of shrinkage, C. E. Weir and T. J. Carter. J. Am. Leather Chemists Assoc. 45, 421 (1950).

121. Recombination and attachment in the Fregion during the eclipse of May 20, 1947, J. Savitt. J. Geophys, Research 55, 385 (1950).

122. Recording microwave refractometer, G. Birnbaum. Rev. Sci. Instr. 21, 2 (1950).

123. Reflected ray suppression, H. E. Bussey. Proc. IRE 38, 1453 (1950).

124. Refractive index of silver chloride for visible and infrared radiant energy, E. K. Plyler, L. W. Tilton and R. E. Stephens. J. Opt. Soc. Am. 40, 540 (1950).

125. Refractory castables, preparation and some properties, R. A. Heindl and Z. A. Post. J. Am. Ceram. Soc. 33, 230 (1950). 126. Unassigned. 
127. Report of the Committee on Atomic Weights of the American Chemical Society, E. Wichers, J. Am. Chem. Soc. 72, 1431 (1950).

128. Report on the methods used in the 1949 AGA-ASME orifice meter tests, F. M. Patridge. Instruments 23, 189 (1950).

129. Research on roofings, H. R. Snoke. Building Research Advisory Board Conf. Report. Research Corporation Conf. (Building Research Advisory Board, Div, of Engr. and Ind., Natl. Res. Council, Washington, D. C. (1950).

130. Safe floors and floor finishes, P. A. Sigler. Soap and Sanit. Chemicals 26, No. 9, 121 (1950).

131. Saturation backscattering of positive electrons, H. H. Seliger. Phys, Rev. 78, 491 (1950).

132. Some economic aspects of the bone char process in sugar refining, V. R. Deitz and J. M. Brown. Intern. Sugar J. (London, England) 52, 147 (1950).

133. Some statistical designs for experiments, G. Warren. Proc. 12th Annual Porcelain Enamel Inst. Forum (University of Illinois, Urbana, Ilinois) p. 76 (1950).

134. Specification of color rendering properties of fluorescent lamps, C. W. Jerome and D. B. Judd. Preprint No. 4, Nat1. Tech. Conf, of the Illum. Eng. Soc., Pasadena, California (Aug. 21-24, 1950).

135. Stable electronic voltage regulator, P. G. Sulzer. Electronics 23, No. 12, 162 (1950).

136. Standards help in the selection of sheets and pillowcases, W. D. Appel. Hospitals 24, 82 (1950).

137. Statistics and planning tests at elevated temperatures, W. J. Youden. Proceedings of the Society for Experimental Stress Analysis XI, No. 2, 219 (1950).

138. Statistics in analytical chemistry, W. J. Youden. Ann. New York Acad. of Sci. 52, Art. 6,815 (1950).

139. Stress-strain and elongation graphs for alclad aluminum-alloy 24 S-T 86 sheet, J. A. Miller. Natl. Advisory Comm. for Aeronaut., Washington, D. C. TN 2094 (1950).

140. Stroboscopic mapping of time-variable fields, L. Marton and D. L. Reverdin. J. Appl. Phys, 21, 617 (1950).

141. Studies on galvanic couples. I. Measurement of electromotive force and internal resistance of cells during current flow, H. D. Holler. J. Electrochem. Soc. 97, 271 (1950).

142. Studies on galvanic couples. II. Some potential-current relations in galvanic corrosion, H. D. Holler. J. Electrochem. Soc. 97, 277 (1950).

143. Studies on galvanic couples. III. Polarization and cathodic protection, H. D. Holler. J. Electrochem. Soc. 97, 453 (1950).

144. Study of radio reflections from meteor trails in research on the upper atmosphere, A. G. McNish. Air Force Cambridge Research Center: Geophysical Research Paper No. 7 (Proc. of the Conf. on Ionospheric Res, , June 1949), p. 105 (1950).
145. Summation of slowly convergent series, O. Szasz. J. Math. and Phys. 28, No. 4, $272(1950)$.

146. Tables of integrals of Struve functions, M. Abramowitz. J. Math. and Phys. 29, 49 (1950).

147. Errata in "Tables relating to Hankel integrals of order zero", by L. Schwarz, published in Luftfahrtforschung, 20, No. 12, 341 (1943), and translated by J. Lotsof, Cornell Aeronautics Laboratory, May 1946. Math. Tables and Other Aids to Computation IV, No. 30, 100 (1950).

148. The atomic clock, a universal standard of frequency and time, H. Lyons. The American Scholar 19, 159 (1950).

149. The condition of a certain matrix, J. Todd. Proc. Cambridge Phil. Soc. 46, Pt. 1,116 (1950).

150. The disintegration scheme of $\mathrm{I}^{131}$, I. Feister and L. F. Curtiss. Phys, Rev. 78, 179 (1950).

151. The influence of the ground on the calibration and use of VHF field-intensity meters, F. M. Greene. Proc. IRE 38, No. 6 (1950).

152. The Institute for Numerical Analysis. An unsigned item prepared by J. H. Curtiss and published in the University of California Faculty Bulletin (Berkeley, California) 19, No. 7, 67 (1950).

153. The preparation of high-purity hydrogen deuteride, A. Fookson, P. Pomerantz, and E. H. Rich. Science 112,748 (1950).

154. The present status of color television; a report by the Senate Advisory Committee on Color Television, N. Smith, E. U. Condon, S. L. Bailey, W. L. Everitt and D. G. Fink. Proc. IRE 38, 980 (1950).

155. The selection of a limited number from many possible conditioning treatments for alloys to achieve best coverage and statistical evaluation, J. M. Cameron and W. J. Youden. Am. Soc. Testing Materials Proc. 50, 951 (1950).

156. Theoretical analysis of fractionating process of adsorption, B. J. Mair, J. W. Westhaver, and F. D. Rossini. Ind. \& Eng. Chem. 42,1279 (1950).

157. Thermal rayleigh disk in liquid helium II, J. R. Pellam and P. M. Morse. Phys. Rev. 78,474 (1950).

158. Those government researchers, H. N. Eaton. Plumbing and Heating Business 13, No. $4,58(1950)$.

159. Transmission of reverberant sound through double walls, A. London. J. Acoust. Soc. Am. 22, 270 (1950).

160. Treatment of leather with Castiloa and Hevea rubbers, R. Oehler, T. J. Kilduff, and S. Dahl. J. Am. Leather Chemists Assoc. 45,378 (1950).

161. Tritanopia with abnormally heavy ocular pigmentation, D. B. Judd, L. Plaza, and D. Farnsworth. J. Opt. Soc. Am. 40, 833 (1950).

162. Tungsten helical spring balance, S. L. Madorsky, Rev. Sci. Inst. 21, 393 (1950). 
163. Units of radioactivity, L. F. Curtiss, R. D. Evans, W. Johnson, and G. T. Seaborg. Rev. Sci. Instr. 21, 94 (1950).

164. Use of admixtures as integral waterproofing and dampproofing materials, B. E. Foster. $\mathrm{J}$. Am. Concrete Inst. 22, (Proc, 47), 46 (1950).

165. Use of statistics to determine precision of test methods, W. J. Youden and J. M. Cameron. Am. Soc. Testing Materials Spec. Tech. Publ. No. 103, 27 (1950).

166. Viscosities of very dilute polymer solutions, S. Rothman, R. Simha, and S. G. Weissberg. J. Polymer Sci. 5, 141 (1950). 167. Volume changes in small concrete cylinders during freezing and thawing, R. C. Valore, Jr., Title No. 46-26 J. Am. Concrete Inst. 21,417 (1950).
168. VTM circuits, M. C. Selby. Electronics 23, No. 5, $110(1950)$.

169. Wide-band FM adapter reduces interference, P. G. Sulzer. Radio-Electronics 21, 24 (1950).

170. Wide range decade frequency generator, J. M. Shaull. Tele-Tech 9, No. 11, 36 (1950).

171. Wide-range R-C oscillator, P. G. Sul zer. Electronics 23, No, 9, 88 (1950).

172. X-ray calibration of radiation survey meters, pocket chambers and dosimeters, F. H. Day, Ra-Det (Tech. Info. Div. ORE, AEC, Oak Ridge, Tenn.) 3, No. 4, 3 (1950). 173. Zirconia and alumina shapes, L. E. Mong and J. J. Donoghue. Am. Ceramic Soc. Bull. 29, 405 (1950). 
174. A bar magnet velocity meter, T. A. Perls and E. Buchmann. Rev. Sci. Instr. 22, 475 (1951).

175. A ceramic accelerometer of wide frequency range, $\mathrm{L}$. T. Fleming. Instruments 24 , No. 8,105 (1951).

176. A circuit for simultaneously recording the range, amplitude, and duration of radar-type reflections, V. C. Pineo and R. C. Peck. Rev. Sci. Instr. 22, 112 (1951).

177. A guide to tables on punched cards, G. $\mathrm{Blanch}$ and E. C. Yowell. Math. Tables and Other Aids to Computation 5, No. 36, 185 (1951).

178. A high temperature, broad-band Stark cell for microwave spectroscopy, L. J. Rueger, H. Lyons, and R. G. Nuckolls, Rey. Sci. Instr. 22, 428 (1951).

179. A logarithmic slidewire for a self-balancing potentiometer, M. Greenspan and M. C. Thompson, Jr, Rev, Sci, Instr, 22, 799 (1951).

180. A new method for the measurement of the isotopic abundance of solids, H. Somer and J. A. Hipple. Phys. Rev. 83, 229 (1951). 181. A note on a bridged-T network, P. G. Sulzer. Proc. IRE 39, 819 (1951).

182. A proposed training program, W. S. Bussey. Scale J. 37, No. 7, 4 (1951).

183. A reflectionless waveguide termination, R. E. Grantham. Rev. Sci. Instr. 22, 828, (1951).

184. A simple type $\mathrm{X}(\mathrm{t})$ - $\mathrm{Y}$ recorder, $\mathrm{T}$. A. Perls and W. A. Wildhack. Rev. Sci. Instr. 22, No. 7, 541 (1951).

185. A "simpson's rule" for the numerical evaluation of Weiner's integrals in function space. R. H. Cameron. Duke Math. J. 18, No, 1 ; 111 (1951).

186. A source of error in radio phase measuring systems (Discussion), R. Bateman, E. F. Florman, A. Tait, and E. Rechtin. Proc. IRE 39, 436 (1951).

187. A survey of audio-frequency power amplifier circuits, P. G. Sulzer. Audio Eng. 35, No, 5, 15 (1951).

188. Accomplishments at the International Conference in Bournemouth, England, Part III, W. D. Appel. (Technical Committee on Textiles International Organization for Standardization (ISO) Meeting). Am. Dyestuff Reptr. 40, No. 23, 735 (1951).

189. Agenda for Commission on Symbols, Units, and Nomenclature of the International Union of Pure and Applied Physics, July 1951, F. G. Brickwedde. Chem. Eng. News 29, No. 7, 588; Phys. Today 4, 33; Science 113, 213 (1951).

190. Air replaces sand in "no-fines" concrete, R. C. Valore, Jr., and W. C. Green. J. Am. Concrete Inst. 22, No. 10, 833 (1951).

191. An incremental delay pulse gerierator, G. ts. Montgomery, Electronics 42, No. 2, 218 (1951).

192. An iterative method for finding characteristic vectors of a symmetric matrix, $W$. Karush. Pacific J. Math. 1, No, 2, 233 (1951).
193. Analysis of ionic recombination including ion production during measurement, W. B. Kunkel. Phys, Rev. 84, 218 (1951).

194. Analysis of solids with the mass spectrometer, J. G. Gorman, E. J. Jones, and J. A. Hipple. Anal. Chem. 23, 438; Phys. Rev. 82, 768 (1951).

195. Applications of the theory of quadratic forms in Hilbert space to the calculus of variations, M. R. Hestenes, Pacific J. Math. 1, No. 4, 525 (1951).

196. Atomic clock and frequency stabilization on microwave spectral lines, C. H. Townes. J. Appl. Phys. 22, 1365 (1951).

197. Atomic spectra for the astrophysicist, C. E. Moore. Science 113, No. 2946, 669 (1951).

198. Basic standards in science, E. U. Condon. J. Franklin Inst. 251, No. 1, 103 (1951).

199. Boron needs well-made steel plus intelligent heat treatment (Part of a panel discussion on "Saving precious alloys by intelligent use of alternatives".), T. G. Digges. Metal Progr. 59,823 (1951).

200. Building technology at the National Bureau of Standards, L. D. C. Nobel. Informes construc. (Madrid, Spain) 4, No. 36, 1 (1951). 201. Ceguera para el color y teorias de la visión cromatica, D. B. Judd. Anales real soc. españ. fis, y quim. (Madrid, Spain). 47,35 (1951).

202. Certain Fourier transforms of distributions, E. Lukacs and O. Szasz, Can. J. Math. 3, No. 2, 140 (1951).

203. Change of free energy with pressure of the reaction nepheline + albite $=2$ jadeite, H. S. Yoder and C. E. Weir. Am. J. Sci. 249, 683, (1951).

204. Checking money-value graduations on a computing scale, W. S. Bussey. Scale J. 38 , No, 2, 6 (1951).

205. Classes of matrices and quadratic fields, O. Taussky. Pacific J. Math. 1, 127 (1951). 206. Combination bands of ethylene, E. K. Plyler. J. Chem. Phys, 19, 658 (1951). 207. Combustion in Bunsen flames, F, R. Caldwell, H. P. Broida, and J. J. Dover. Ind. Eng. Chem. 43, 2731 (1951).

208. Comparative strengths of some adhesiveadherend systems, N. J. DeLollis, N. Rucker, and J. E. Weir. Trans, Am. Soc. Mech. Engr8. 73, 183 (1951).

209. Comparing outputs from precision time standards, J. M. Shaull and C. M. Kortman. Electronics 24, No. 4, 102 (1951).

210. Constant-amplitude oscillator. N. C. Hekimian. Electronics 24, 164 (1951).

211. Correction factors for the balancing effect in one leg of a U-type manometer, H. S. Bean. Gas 27, 48 (1951).

212. Correction factors for the balancing effect of a gas in one leg of a manometer, H. S. Bean and F. C. Morey. Instruments 24, 528; Oil and Gas J. 50, No. 11, 107 (1951).

213. Corrosion factors in design, F. M. Reinhart. Product Engr. 22, No. 7, 101 (1951). 
214. Coulomb wave functions expressed in terms of Bessel-Clifford functions and Bessel functions, M. Abramowitz. J. Math. and Phys. 29, 303 (1951).

215. Crystal chemistry symposium, F. Ordway. Ceram. Age 58, No. 5, 28 (1951).

216. Design details of a linear magnetic UHF oscillator attenuator, F. Reggia. FM-TV

Radio Commun. 11, No. 10, 16 (1951).

217. Deterioration of silicate cements in the tropics, T. E. Fischer and I. C. Schoonover. U. S. Armed Forces Med. J. 2, 907 (1951).

218. Determination of dissociation constants in a mixture of two weak acids by electromotive force measurements, R. G. Bates. J. Am. Chem. Soc. 73, 2259 (1951).

219. Determination of grease in leather, E. W. Zimmerman and E. F. Pangborn. J. Am. Leather Chemists Assoc. 46, 342 (1951).

220. Determination of the extreme values of the spectrum of a bounded self-adjoint operator, W. Karush. Proc. Am. Math. Soc. 2, No. 6, 980 (1951).

221. Developing seals for abrasive-fluid mixtures, J. Rabinow. Machine Design 23, 128 (1951).

222. Diagonal tension in reinforced concrete beams, A. P. Clark. J. Am. Concrete Inst. 23, 145 (1951).

223. Direct resinous filling materials: temperature rise during polymerization, $\mathrm{R}$. B. Wolcott, G. C. Paffenbarger, and I. C.

Schoonover. J. Am. Dental Assoc. 42, 253 (1951).

224. Directional distribution of 1040 -kev radiation from a high voltage $X-r$ ay tube, N. Goldste in, U. Fano, and H. O. Wyckoff. J. Appl. Phys, 22, 417 (1951).

225. Dispersion of F2-layer critical frequencies, M. L. Phillips and H. S. Moore. Proc. IRE 39,717 (1त51).

226. Early work in applied optics at the

National Bureau of Standards, E. C. Critten-

den. J. Opt. Soc. Am. 41, 367 (1951).

227. Effect of melamine resin on chemical tests of paper, W. K. Wilson, J. L. Harvey, and A. A. Padgett. Tappi 34, 410 (1951).

228. Effective earth's radius for radiowave propagation beyond the horizon, W. Miller. J. Appl. Phys. 22, No. 1, 55 (1951).

229. Effects of ionosphere disturbances on low frequency propagation, J. M. Watts and J. N. Brown. J. Geophys. Research 56, No. 3, 403, (1951).

230. Electron-optical exploration of space charge in a cut-off magnetron, D. L. Reverdin. J. Appl. Phys. 22, 257 (1951).

231. Elimination of randomization in certain statistical decision procedures and zero-sum two-person games, A. Dvoretzky, A. Wald, and J. Wolfowitz. Ann. Math. Stat. 22, 1 (1951). 232. Emission spectrographic standards, B. F. Scribner and C. H. Corliss. Anal. Chem. 23, 1548 (1951).

233. Evaluation of automatic computing machine 8, F. L. Alt. Product Engr. 22, No. 11, 146 (1951).

234. Evaluation of coaxial slotted-line impedance measurements, H. E. Sorrows, W. E. Ryan and R. C. Ellenwood. Proc. IRE 39, 162 (1951).
235. Fifth-order aberration in an optical system, R. K. Anderson. Proc. Computation Seminar (IBM) p. 130 (IBM Corp. , 590 Madison Ave., New York, N. Y.) (1951).

236. Fifty years of scientific standardization, E. C. Crittenden. Standardization 22, No. 11, 349 (1951).

237. Film-forming properties of cellulose acetate propionates. Effect of solvents, diluents, and plasticizers. A. G. Roberts and S. G.

Weissberg. Ind. Eng. Chem. 43, 2088 (1951). 238. Fixed capacitors of electronic circuits, P.S. Schmidt. Elec. Mfg. 47, No. 5, 100 (1951). 239. Fixture unit ratings as used in plumbing system design, H. N. Eaton and J. L. French. Housing and Home Finance Agency Housing Research Paper No. 15 (1951).

240. Fluctuations in the refractive index of the atmosphere at microwave frequencies, G. Birnbaum. Phys. Rev. 82, 110 (1951).

241. Formulas for calculating the error function of a complex variable, H. E. Salzer. Math. Tables and Other Aids to Computation 5, 67 (1951).

242. Formulas for finding the arguments for which a function has a given derivative, H. E. Salzer. Math. Tables and Other Aids to Computation 5, No. 36, 213 (1951).

243. Gated-beam mixer, S. Rubin and G. E. Boggs. Electronics 24, 196 (1951).

244. Gauss to Gerling on relaxation, G. E. Forsythe. Math. Tables and Other Aids to Computation 5, No. 36, 255 (1951).

245. Grease consistometer is modified to operate at constant shear rates, higher viscosities, H. W. White. Petroleum Processing 6, 285 (1951).

246. Heat capacity of crystalline dextrose between $25^{\circ}$ and $95^{\circ} \mathrm{C}$. T. B. Douglas, A. F. Ball, and J. L. Torgesen. J. Am. Chem. Soc. 73,1360 (1951).

247. Heat delivery in a compressible flow and applications to hot-wire anemometry, C. M. Tchen. Natl. Advisory Comm. Aeronaut. Tech. Note 2436 (1951).

248. Hydrogen-oxygen reaction at the second explosion limit, H. P. Broida. J. Chem. Phys. 19,790 (1951).

249. Identities and inequalities concerning orthogonal polynomials and Bessel functions, O. Szasz. J. D'Analyse Mathematique (Jerusalem, Israel) 1, 116 (1951).

250. Influence of the sun upon the ionosphere, N. Smith. (Part of a Conference on the sun in the service of man.) Proc. Am. Acad. Arts Sci. 79, 181 (1951).

$250 \mathrm{~A}$. Information theory, C. H. Page. J. Wash. Acad. Sci. 41, 245 (1951).

251. Interlaboratory comparison of thermal conductivity determinations with guarded hot plates, H. E. Robinson and T. W. Watson. Am. Soc. Testing Materials Spec. Tech. Publ. No. 119 (1951).

252. List of geomagnetic observatories and the saurus of values, H. F. Johnston. J. Geophys. Research 56, 431 (1951).

253. Locating sources of variability in a proсевs, W. J. Youden. Ind. Eng. Chem. 43, 2059 (1951).

254. Magnetic susceptibility of manganese solenide, R. Lindsay. Phys. Rev, 84, 569 (1951). 
255. Materials, W. R. Brode, J. Franklin Inst. 25, 81 (1951).

256. Measuring power factor of low-loss dielectrics, J. L. Dalke and R. C. Powell. Electronics 24, No. 8, 224 (1951).

257. Measuring time and frequency in Hawaii, V. E. Heaton. Tele-Tech. 10, No. 3, 36 (1951).

258. Microwave components: Precision casting vs. electroforming, A. A. Feldmann. Materials and Methods 34, No. 1, 70 (1951).

259. Microwave measurements of the dielectric properties of gases, G. Birnbaum, S. J. Kryder and H. Lyons, J. Appl. Phys. 22, No. 1 , 95 (1951).

260. Microwave spectra of deutero-ammonias, H. Lyons, L. J. Rueger, R. G. Nuckolls and M. Kessler. Phys, Rev, 81, 630 (1951).

261. Modified locked-oscillator frequency dividers, P. G. Sulzer. Proc, IRE 39, 1535 (1951).

262. Mold growth and the $\mathrm{pH}$ of tartrate buffer solutions, R. G. Bates. Anal. Chem. 23, 813 (1951).

263. Motion in the solar atmosphere as deduced from radio measurements, G. Reber. Science 113 , No. 2934,312 (1951).

264. Natural and synthetic rubbers, N. Bekkedahl. Anal. Chem. 23, 243 (1951).

265. Net heat of combustion of AN-F- 58 aircraft fuels, S. Rothberg and R. S. Jessup. Ind. Eng. Chem. 43, 981 (1951).

266. New light on self-ignition, N. D. Mitchell. Natl. Fire Prot. Quart. 45, No. 2, 165 (1951).

267. New matrix transformations for obtaining characteristric vectors, W. Feller and G. E. Forsythe. Quart. Appl. Math. 8, No. 4, 325 (1951).

268. New resolving power test chart, F. E. Washer and F. W. Rosberry. J. Opt. Soc. Am. 41, 597 (1951)

269. New tools for research, W. A. Wildhack. Science 114, No. 2962, 3 (1951).

270. Note on an infinite integral, A. M. Ostrowski. Duke Math. J. 18, No. 2, 355 (1951).

271. Notes on numerical analysis, 4. Relaxation and step-by-step methods, L. Fox. Math. Tables and Other Aids to Computation 5, 92 (1951).

272. Notes on numerical analysis, 5. Tablemaking for large arguments. The exponential integral, L. Fox and J. C. P. Miller. Math. Tables and Other Aids to Computation 5, 163 (1951).

273. NRL Aleutian radio eclipse expedition, J. Hagen, F. T. Haddock, and G. Reber. Sky and Telescope 10, No. 5, 111 (1951).

274. Numerical methods associated with Laplace's equation, W. E. Milne. Proc. of a Second Symposium on Large-Scale Digital Calculating Machinery, Annals of the Harvard Computation Laboratory (Cambridge, Massachusetts), XXVI, 152 (1951).

275. On a Tauberian theorem for Abel summability, O. Szasz. Pacific J. Math. 1, 117 (1951).

276. On positive harmonic functions of ultraspherical polynomials, W. Seidel and O. Szasz. J. London Math. Soc, (London, England), 26, 36 (1951).
277. On singular pertubation problems in theory of non-linear vibrations, W. Wasow. Actes du Colloque International des Vibrations Non Lineaires, Ile de Porquerolles, France. Publications Scientifiques et Techniques du Ministere de l'Air No. 281 (1951).

278. On some connections between probability theory and differential and integral equations, M. Kac. Second Berkeley Symposium on Mathematical Statistics and Probability held by the University of California from July 31 to August $12,1950,189$ (1951).

279. On some trigonometric transforms. O. Szasz. Pacific J. Math. 1, No. 2, 291 (1951). 280. On subharmonic, harmonic, and linear functions of two variables, E. F. Beckenbach. Revista Universidad Nacional del Tucuman, Ser. A, Matematica y Fisica Teorica (Tucuman, Argentina) 8, No. 1-2, 7, Publication No. 602 (1951).

281. On the duration of random walks. W. Wasow. Anals Math. Stat. 22, No. 2, 199 (1951).

282. On the inversion of matrices by random walks, W. Wasow. Math. Tables and Other Aids to Computation VI, No. 38, 78 (1951). 283. On the relative extrema of the Hermite orthogonal functions, O. Szasz. J. Indian Math. Soc. (Madras, India) 15, Pt. B, 129 (1951).

284. On the variation of the determinant of a positive definite matrix, A. M. Ostrowski and O. Taussky. Koninkl. Nederl. Akademie Van Wetenschappen (Amsterdam, Holland). Proc. Series. A, 54, No. 5; Indag. Math. 13, No. 5 (1951).

285. Optical rotation of polarized light by chemical compounds, W. R. Brode. J. Opt. Soc. Am. 41, 987 (1951).

286. Pertubation characteristic functions and their application to electron optics, P. A. Sturrock. Proc. Roy. Soc. (London, England) A, 210, 269 (1951).

287. Physics. Measure for measure: Some problems and paradoxes of precision. F. B. Silsbee. J. Wash. Acad. Sci. 41, 213 (1951). 288. Preservation of the Declaration of Independence and the Constitution of the United States, G. M. Kline. ASTM Bull. No. 178, 32 (1951).

289. Problems of drainage and venting, R. S.

Wyly. Master Plumber and Heating Contractor 21 , No. 3, 22 (1951).

290. Proposals for the mechanical resolution of German syntax patterns, V. A. Oswald, Jr., and S. L. Fletcher, Jr. Modern Language Forum 36, No. 3-4, 1 (1951).

291. Pyrolysis of mixtures of ethane and ethaned , L. A. Wall and W. J. Moore. J. Am. Chem. Soc. 73,2840 (1951).

292. Quantitative determination of natural rubber hydrocarbon by refractive index measurements, R. J. Fanning and N. Bekkedahl. Anal. Chem. 23, 1653 (1951).

293. R-branch heads of some $\mathrm{CO}_{2}$ infrared bands in the $\mathrm{CO}+\mathrm{O}_{2}$ flame spectrum. W. S. Benedict, R. C. Herman, and S. Silverman. J. Chem. Phys. 19, 1325 (1951). 
294. R-F solenoid design chart, P. G. Sulzer. Tele-Tech 10, No. 5, 45 (1951).

295. Radio wave generation by multi-stream charge interaction, J. Feinstein and H. K. Sen. Phys. Rev. 83, No. 2, 405 (1951).

296. Radix table for trigonometric functions and their inverses to high accuracy, H. E.

Salzer. Math. Tables and Other Aids to Computation V, 9 (1951).

297. Recent developments in the Ramberg vacuum tube accelerometer, S. Levy. Proc. Soc. Exptl. Stress Anal., 9, No. 1, 151 (1951). 298. Recurrent determinants of Legendre and of ultraspherical polynomials, E. F. Beckenbach, W. Seidel and O. Szasz. Duke Math. J. $18,1,1$ (1951).

299. Relations among certain ranges of vector measures, A. Dvoretzky, A. Wald and J.

Wolfowitz. Pacific J. Math. 1, 59 (1951).

300. Relative back-scattering of electrons and positrons, W. Miller. Phys. Rev. 82 , No. 3, 452 (1951).

301. Unassigned.

302. Resin bonding of hardwood fibers in offset papers, B. W. Scribner, M. B. Shaw, M. J. O'Leary and J. K. Missimer. Tappi 34,481 (1951).

303. Resin bonding of offset papers containing mineral fillers, M. J. O'Leary, B. W. Scribner and J. K. Missimer. Tappi 34, 506 (1951). 304. Rotational temperatures of $\mathrm{OH}$ in methaneair flames, H. P. Broida. J. Chem. Phys. 19,1383 (1951).

305. Second order determinants of Legendre polynomials, G. E. Forsythe. Duke Math. J. 18, No. 2, 361 (1951).

306. Selecting engineering adhesives, F, W. Reinhart. Product Engr. 22, 123 (1951).

307. Some aspects of depolymerization kinetics, R. Simha and L. A. Wall. J. Polymer Sci. 6, No. 1, 39 (1951).

308. Some factors influencing the road wear of tires, R. D. Stiehler, M. N. Steel, and J. Mandel. Trans. Inst. Rubber Ind. (Cambridge, England) 27, No. 6, 298 (1951).

309. Some problems in ultramicrotomy, S. B. Newman. Bull. Intern. Assoc. Med. Museums 32,100 (1951).

310. Some problems on random walks in space, A. Dvoretzky and P. Erdos. Second Berkeley Symposium on Mathematical Statistics and Probability held by the University of California from July 31 to Aug. 12, 1950, 353 (1951).

311. Special lubricants tests, H. S. White and D. Zei. Mech. Eng. 73, 657 (1951).

312. Specific heat of beryllium between $0^{\circ}$ and $900^{\circ}$ C., D. C. Ginnings, T. B. Douglas and A. F. Ball. J. Am. Chem. Soc. 73, 1236 (1951).

313. Specifications for electrodeposited coatings, F. Ogburn. Elec. Mfg. 48, 119 (1951). 314. Spectrophotometric evidence for the absence of free aldehyde groups in periodateoxidized cellulose, J. W. Rowen, F. H. Forziati and R. E. Reeves. J. Am. Chem. Soc. 73,4484 (1951).

315. Spurious resolution of photographic lenses, R. N. Hotchkiss, F. E. Washer, and F. W. Rosberry. J. Opt. Soc. Am. 41, 600 (1951).
316. Standards for electrical measurements F. B. Silsbee. Elec. Eng. 70, No. 3, 202 (1951).

317. Suppressing microwaves by zonal screens, H. E. Bussey, Tele-Tech, 10, No, 12, 45 (1951).

318. Synthetic detergents, L. M. Kushner and J. I. Hoffman. Sci. Am. 185, No. 4, 26 (1951). 319. Systematic ionospheric winds, C. D. Salzberg and R. Greenstone. J. Geophys. Research 56 , No. 4, 521 (1951).

320. Table of the integral, $\int^{z} e^{-\mu^{3}} d u$. M.

Abramowitz. J. Math. and Phys. 30, No. 3 162 (1951)

321. Tauberian theorems for summability

$\left(R_{1}\right)$, O. Szasz. Am. J. Math. 73, No. 4, 779 (1951).

322. Technetium in the sun, C. E. Moore. Science 114, 59 (1951).

323. Technical aspects of industrial and office quieting, A. London. Proc. Second Annual Nat. Noise Abatement Symp., Armour Research Foundation (Chicago, Illinois) 2, 75 (1951).

324. Technique for locating sources of variability in a process, W. J. Youden. Ind. Eng. Chem. 45, No. 9, 2059 (1951).

325. Textile Forschung and Prufung am National Bureau of Standards, H. F. Schiefer. Sonderdruck aus Melliand Textilberichte (Heidelberg, Germany) 32, No. 125, 188 (1951).

326. The cascade-binary counter with feedback, G. F. Montgomery. J. Appl. Phys, 22, 780 (1951).

327. The chemistry of portland cement, R. H, Bogue. Editorial Dossat, S. A. (Madrid, Spain) (1951).

328. The construction and operation of thoria resistor-type furnaces, S. M. Lang and R. F, Geller. J. Am. Ceram. Soc. 34, 193 (1951). 329. The convergence of Seidel iterants of nearly symmetric matrices, P. Stein. Math. Tables and Other Aids to Computation V, 236 (1951).

330. The determination of eigenvectors and eigenvalues of symmetric matrices, E. C.

Yowell. Proc. Computation Seminar, IBM, p. 112 (1951).

331. The determination of freezing point of engine antifreezes, R. E. Mallonee and F. L. Howard. ASTM Bull. No. 172, 43 (1951). 332. The determination of nitrogen in leather by the Kjeldahl method, S. Dahl and R. Oehler. J. Am. Leather Chemists Assoc. 46, 317 (1951).

333. The emission spectra of first-stage combustion in an engine, H. P. Broida, W. J. Levedahl and F. L. Howard. J. Chem. Phys. 19,797 (1951).

334. The estimation of parameters in certain stochastic processes, H. B. Mann. Sankhya (Calcutta, India) 11, Pt. 2, 97 (1951).

335. The fisherian revolution in methods of an experimentation, W. J. Youden, J. Am. Stat. Assoc. 46,47 (1951).

336. The gated-beam mixer, S. Rubin and G. Boggs. Electronics 24, No, 10, 196 (1951). 337. The Institute for Numerical Analysis of the National Bureau of Standards, J. H. Curtiss. Monthly Research Rept. of the Office of Naval Research, Dept. Navy P. 8 (Issue of May 1951); Abbreviated version in Am. Math. No. 58, 372, 
338. The interpretation of radar waves from meteor trails, J. Feinstein. J. Geophys. Research 56, No. 1, 37 (1951).

339. The National Bureau of Standards and its international relations, W. R. Brode. Foreign Commerce Weekly 42, No. 11, 3 (1951).

340. The rating of water-current meters, $R$. $H$. Dickman. Water Power (Westminister, England) 3,330 (1951).

341. The relation between the absorption spectra and the chemical constitution of dyes. XXII.

Cis-trans isomerism in thioindigo dyes, G. M. Wyman and W. R. Brode. J. Am. Chem. Soc. 73,1487 (1951).

342. The relation between the absorption spectra and the chemical constitution of dyes. XXIV. Absorption spectra of some thioindigo dyes in sulfuric acid, W. R. Brode and G. M. Wyman. J. Am. Chem. Soc. 73, 4267 (1951).

343. The sea-level latitude variation of fast cosmic-ray neutrons, P. S. Gill and L. F. Curtiss. Phys. Rev. 84, 591 (1951).

344. The tensile forces in tightened bolts, A. H. Stang. Product Engr. 22, No. 2, 118 (1951). 345. The thermal hydrogen-oxygen reaction at relatively low temperatures, H. P. Broida and O. Oldenberg. J. Chem. Phys. 19, 196 (1951).

346. The use of components of variance in preparing schedules for sampling of baled wool, J. M. Cameron. Biometrics 7, 83 (1951).

347. The variation with frequency of the signal range of FM and television broadcasting stations, K. A. Norton and E. W. Allen. J. Brit. Inst. Radio Engr. 11, 93 (1951).
348. The wave-frequency dependence of the duration of radar-type echoes from meteor trails, V. C. Pineo and T. N. Gautier. Science 114,460 (1951).

349. Transmittance of cesium bromide crystals, E. K. Plyler and F. P. Phelps. J. Opt. Soc. Am. 41, 209 (1951).

350. Treadwear of tires. Influence of type of rubber and black. J. Mandel, M. N. Steel and R. D. Stiehler. Inḍ. Eng. Chem. 43, 2901 (1951).

351. Tropospheric propagation beyond the horizon, J. Feinstein. J. Appl. Phys. 22, 1292 (1951).

352. Tuning systems employing feedback amplifiers, P. G. Sulzer. Electronics 24, 252 (1951).

353. Two consequences of the transposition theorem on linear inequalities, T, S. Motzkin. Econometrica 19, No. 2, 184 (1951).

354. Two-stage autoignition of some hydrocarbons, W. J. Levedahl and F. L. Howard. Ind. Eng. Chem. 43, 2805 (1951).

355. Using mathematics for design, (How industry can make better use of mathematics.) An interview with J. H. Curtiss. Product Eng. 22,156 (1951).

356. Volume-temperature relationships for the room temperature transition in Teflon, F. A. Quinn, Jr., D. E. Roberts and R. N. Work. J. Appl. Phys, 22, 1084 (1951).

357. Wide-range resonators for VHF and UFH, G. F. Montgomery and P. G. Sulzer. Electronics 42, No. 5, 200 (1951). 
358. A broad-band coaxial stark cell for microwave spectroscopy, L. J. Ruegar and R. G. Nuckolls. Rev. Sci. Instr. 23, 635 (1952).

359. A collision path from the earth to the moon in the restricted problem of three bodies, A. Goldstein and C. E. Froberg. Kgl. Fysiograf. Sallskap. I. Lund Forh (Lund, Sweden) 22, No. 14, 1 (1952).

360. A combination crossed-field and time-offlight mass spectrometer, J. A. Hipple and H. A. Sommer. Phys, Rev. 85, 714 (1952). 361. A comparison of CW field intensity and backscatter delay, W. L. Hartsfield and R. Silberstein. Proc. IRE 40, 1700 (1952).

362. A comparison of methods for evaluating trends in time series of tropospheric radio field strength data, P. L. Rice. IRE Trans. Ant. Prop. AP-3, 144 (1952).

363. A hydride bath for the electrodeposition of aluminum, D. E. Couch and A. Brenner. J. Electrochem. Soc. 99, No. 6, 234 (1952). 364. A low voltage regulator, S, Rubin. Television Eng. 3, No. 3, 8 (1952).

365. A meter for timing the flow of very small volumes of a gas, J. C. Westmoreland. Natl. Advisory Comm. Aeronaut. RM 52109 (1952).

366. A method for the determination of converging factors, applied to the asymptotic expansions for the parabolic cylinder functions, J. C. P. Miller. Proc. Cambridge Phil. Soc. (London, England) 48, Pt. 2, 243 (1952).

367. A model for epitaxy in metal-rock salt pairs, O. G. Engel. J. Chem. Phys. 20, No. 7, 1174 (1952).

368. A new infrared band system of FeO, A. M. Bass and W. S. Benedict. Astrophys. J. $116,652(1952)$.

369. A new kind of radio propagation at very high frequencies observable over long distances, D. K. Bailey, R. Bateman, L. V. Berkner, H. G. Booker, G. F. Montgomery, E. M. Purcell, M. W. Salisbury and J. B. Wiesner. Phys. Rev. 86, No. 2, 141 (1952). 370. A note on a selective $\mathrm{R}-\mathrm{C}$ bridge, P. G. Sulzer. Proc. IRE 40, 338 (1952).

371. A note on the polarization of low frequency ionosphere echoes, J. M. Watts. J. Geophys. Research 57, No. 2, 287 (1952).

372. A numerical solution of the helium wave equation with the SEAC, J. H. Wegstein. Proc. Assoc. Computing Machinery (Toronto, Canada, ) p. 34 (Assoc. for Computing Machinery, New York, N. Y.) (1952).

373. A remark on M. M. Day's characterization of inner-product spaces and a conjecture of L. M. Blumenthal, I. J. Schoenberg. Proc. Am. Math. Soc. 3, 961 (1952).

374. A shutter-illumination device for the study of phototropic dyes, J. H. Gould and W. R. Brode. J. Opt. Soc. Am. 42, No. 6, 380 (1952).

375. Unassigned.

376. A simplified method for measuring the attenuation of balanced transmission lines, R. C. Powell. Proc. Nat1. Electronics Conf, 1951 (Nat1. Electronics Conf., Inc., Chicago, Ilinois) 7, 287 (1952).
377. A source of error in the measurement of radiated harmonics, F. M. Greene. Proc. IRE 40, 486 (1952).

378. A statistical approach to the problem of multiple radio interference to $F M$ and television service, K. A. Norton, H. Staras, and M. Blum, IRE Trans. Ant. Prop. AP-1, 43 (1952).

379. A suggested program for industry-sponsored shoe research, R. B. Hobbs, Leather and Shoes 123, 8 (1952).

380. A theorem on convex cones, with applications to linear inequalities, J. W. Gaddum. Proc. Am. Math. Soc. 3, No. 6, 957 (1952).

381. A variable heart pump permitting independent control of rate, output, and ejection velocity, H. P. Broida, E. D. Freis, and J. C. Rose. Science 115, No. 2996, 603 (1952).

382. Abrasion resistance measurements on cotton fabrics made with the blade type abradant and the Schiefer abrasion testing machine, H. F. Schiefer. Proc. Spring Meeting, Textile Qual. Control Assoc. p. 65 (1952).

383. Absence of spontaneous emission of neutrons from samarium, S. D. Chatterjee and R. N. Olcott. Phys, Rev. 85, 147 (1952). 384. Adiabatic demagnetization and the temperature scale below $1^{\circ} \mathrm{K}, \mathrm{D}$. de Klerk. Science 116,335 (1952).

385. Aging changes in clinical thermometers, L. C. Liberatore and R. E. Wilson. J. Am. Ceram. Soc. 35, 203 (1952).

386. Alternative derivations of Fox's escalator formulae for latent roots, G. E. Forsythe. Quart. J. Mech. and Appl. Math. (London, England) 5, Pt. 2, 191 (1952).

387. Amelung glasses compared with some modern commercial glasses, D. Hubbard, L. B. Jenkins, and E. M. Krumrine. Sci. Monthly LXXV, No. 6, 327 (1952).

388. An alkaline solution of potassium chromate as a transmittancy standard in the ultraviolet, G. W. Haupt. Condensation of this paper appeared in J. Opt. Soc. Am. 42, 441 (1952). 389. An alternative "predictor-corrector" process, T. H. Southard and E. C. Yowell. Math. Tables and Other Aids to Computation VI, No. 40,253 (1952).

390. An elementary note on powers of quaternions, H. E. Salzer. Amer. Math. Mo. 59, No. 5,298 (1952).

391. An essential property of the Fourier transforms of distribution functions, E. Lukacs. Proc. Am. Math. Soc. 3, No. 3, 508 (1952). 392. An experimental rapid access memory using diodes and capacitors, A. W. Holt. Proc. Assoc. Computing Machinery, p. 133 (Assoc. for Computing Machinery, New York, N. Y.) (1952).

393. An extension of Gauss' transformation for improving the condition of systems of linear equations, G. E. Forsythe and T. S. Motzkin. Math. Tables and Other Aids to Computation 6 , No. 37, 9 (1952).

394. An integral equation approach to the problem of wave propagation over an irregular surface, G. A. Hufford. Quart. Appl. Math. 9, No. 4, 391 (1952). 
395. An optical model for nucleon-nuclei scattering, R. E. LeLevier and D. S. Saxon. Phys. Rev. 87, 40 (1952).

396. An U-H-F moon relay, P. G. Sulzer, G. F. Montgomery, and I. H. Gerks. Proc. IRE 40, 361 (1952).

397. Analysis of goniophotometric reflection curves, I. Nimeroff. Condensation, J. Opt. Soc. Am. 42, 579 (1952).

398. Automatic counting machine, H. M. Joseph and C. Stansbury. Elect. Mfg. 49, No. 3, 100 (1952).

399. Automotive research at the National Bureau of Standards, F. L. Howard. NADA Mag. 24, No. 3, 118 (1952).

400. Average radio-ray refraction in the lower atmosphere, M. Schulkin. Proc. IRE 40, 554 (1952).

401. Bergmans integraloperator erster Art und Riemannsche Funktion, P. Henrici. Z. Angew. Math. u Phys. (Basel, Switzerland) 3, 228 (1952).

402. Bonding of plastic teeth to heat cured denture base resins, I. C. Schoonover, T. E. Fischer, A. F. Serio, and W. T. Sweeney. J. Am. Dental Assoc. 44, 285 (1952).

403. Boolean geometry. 1. L. M. Blumenthal.

Rend. Circ. Mat. Palermo II, No. 1, 1 (1952). 404. Boundary value problems for multiply connected domains, F. L. Alt. Proc. Assoc. Computing Machinery, Jointly Sponsored by the Assoc. for Computing Machinery and the MelIon Institute, Pittsburgh, Pennsylvania, May 2-3, 1952, p. 193 (Assoc. for Computing Machinery, New York, N. Y.) (1952).

405. Bounds for the greatest latent root of a positive matrix, A. M. Ostrowski. J. London Math. Soc. (London, England) 27, Pt. 2, No. 106,253 (1952).

406. Unassigned.

407. Calibration of commercial field-strength meters, C. C. Cook. Tele-Tech. 11, No. 10, 44 (1952).

408. Carbon black differentiation by electrical resistance of vulcanizates, J. E. McKinney and F. L. Roth. Ind. Eng. Chem. 44, 159 (1952).

409. Unassigned.

410. Chart speeds design of feedback amplifiers, N. C. Hekimian. Electronics 25, No. 9,153 (1952).

411. Chebyshev polynomials in the solution of large-scale linear systems, C. Lanczos.

Proc. Assoc. Computing Machinery, Toronto, Canada, p. 124 (Assoc. for Computing Machinery, New York, N. Y.).(1952).

412. Classes of matrices and quadratic fields

(II), O. Taussky. J. London Math. Soc.

(London, England) 27, Pt. 2, No. 106, 237 (1952).

413. Computer aids to code checking, I. C. Diehm. Proc. Assoc. Computing Machinery, Toronto, Canada, p. 19 (Assoc. for Computing Machinery, New York, N. Y.) (1952).

414. Condition for radiation from a solar plasma, J. Feinstein. Phys. Rev. 85, 145 (1952).

415. Constant-level device for liquids, D. E. Couch and A. Brenner. Anal. Chem. 24, 922 (1952).
416. Construction and use of subroutines for the SEAC, J. Levin. Proc. Assoc. Computing Machinery, Jointly Sponsored by the Assoc. for Computing Machinery and the Mellon Institute, Pittsburgh, Pennsylvania, May 2-3, 1952. p. 173 (Assoc. for Computing Machinery, New York, N. Y.) (1952).

417. Convergence of a method of solving linear problems, W. Karush. Proc. Am. Math. Soc. 3, No. 6, 839 (1952).

418. Cosmic radio noise intensities in the VHF band, H. V. Cottony and J. R. Johler. Proc. IRE 40,1053 (1952).

419. Cracking in masonry caused by expansion of mortar, J. W. McBurney. Proc. Am. Soc. Testing Materials 52, 1228 (1952).

420. Criteria for the characterization of kaolinite, halloysite, and a related mineral in clays and soils, L. Bramao, J. G. Cady, S. Hendricks, and M. Swerdlow. Soil Sci. 73, No. 4,273 (1952).

421. Crystallographic investigation of tricalcium phosphate hydrate, A. S. Posner and S. R. Stephenson. J. Dental Research 31, No. 3, 371 (1952).

422. Density, expansivity, and viscosity of molten alkali silicates, L. Shartsis, S. Spinner, and W. Capps. J. Am. Ceram. Soc. 35, No. 6, 155 (1952).

423. Dental amalgam: The effect of mechanical condensation of some physical properties, G.

Ryge, G. Dickson, I. C. Schoonover. J. Am. Dental Assoc. 45, 269 (1952).

424. Design features of a magnetic drum memory for the National Bureau of Standards Western Automatic Computer (SWAC), R. Thorensen. Proc. of the Symp. on Electronic Computers held by the Western Sect. of the Elect. Computers Prof. Group of the IRE, April 30, May 1-2, 1952 (University of California, Los Angeles, California) II, 1-II:9 (1952).

425. Determination of critical depth in spatially variable flow, G. H. Keulegan. 2nd Proc. Midwestern Conf. on Fluid Méchanics, March 17-19, 1952, Ohio State University, Engineering and Experiment Station Bul. 149, p. 67 (1952).

426. Determination of nitrogen and sulfur in steel, J. J. Furey, W. R. Sayre, and J. L. Hague. Anal. Chem. 24, 199 (1952).

427: Development of VHF field-intensity standards, F. M. Greene and M. Solow. Proc. IRE 40, No. 5, 573 (1952).

428. Diaphragm error and testing rate in the paper bursting test, F. T. Carson and V. Worthington. Tappi 35, No. 12, 539 (1952). 429. Diathermy generators, E. L. Hall. Arch. Phys. Med. 33, 28 (1952).

430. Diathermy rules and the physician, E. L. Hall. Arch. Phys. Therapy 33, 163 (1952). 431. Dielectric constant of water vapor, G. Birnbaum and S. D. Chatterjee. J. Appl. Phys. 23, 220 (1952).

432. Distribution of electrical conduction currents in the vicinity of thunderstorms, R. E. Holzer and D. S. Saxon. J. Geophys. Research 57, No. 2, 207 (1952). 
433. Editorial on Roentgen ray protection design, L. S. Taylor. Am. J. Roentgenol., Radium Therapy Nuclear Med. 68, 972 (1952). 434. Effects of biaxial stretch-forming, B. M. Axilrod, M. A. Sherman, V. Cohen, and I. Wolock. Modern Plastics 30, 117 (1952).

435. Effects of humidity during fabrication of polyester laminates, J. E. Wier, D. C. Pons, and B. M. Axilrod. SPE J. 8, No. 9, 8 (1952).

436. Effects of moderate biaxial stretch-forming on tensile and crazing properties of acrylic plastic glazing, B. M. Axilrod, M. A. Sherman, V. Cohen, and I. Wolock. Natl. Advisory Comm. Aeronaut. Tech. Note 2779 (1952).

437. Electrical noise from instrument cables subjected to shock and vibration, T. A. Perls. J. Appl. Phys. 23, No. 6, 674 (1952).

438. Electron interferometer, L. Marton.

Phys. Rev. 85, No. 6, 1057 (1952).

439. Electron microscopy of soil clays and related materials, E. B. Kinter, A. M. Wintermyer, and M. Swerdlow. Public Roads J. Highway Research 27, No. 5, 89 (1952).

440. Electron-tube curve generator, M. L. Kuder. Electronics 25, No. 3, 118 (1952).

441. Electronic computers aiding management control, H. D. Huskey and V. R. Huskey. J. Accountancy 93, No. 1, 69 (1952).

442. Emission spectra on autoignited heptaneair mixtures, W. J. Levedahl and H. P. Broida. Anal. Chem. 24, 1776 (1952).

443. Emission spectroscopy, W. F. Meggers. Anal. Chem. 24, No. 1, 23 (1952).

444. Experimental production of nodular graphite in cast iron, A. I. Krynitsky and $\mathrm{H}$. Stern. Foundry 80 , No. 3, 106; Pt. 2, 80 , No. 4,98 (1952).

445. Experimental statistics, R. J. Hader and W. J. Youden, Anal. Chem. 24, 120 (1952). 446. Factors affecting the properties of rayon fabrics, L. H. Jameson, B. L. Whittier, and H. F. Schiefer. Textile Research J. 22, No. 9, 599 (1952).

447. Fast coincidences with Cerenkov counters, Z. Bay, M. R. Cleland, and F. McLernon, Phys. Rev. 87, 901 (1952).

448. Fifty years of electrochemical theory, W. J. Hamer. J. Electrochem. Soc. 99, No. $12,331 \mathrm{C}(1952)$.

449. Fifty years of electrodeposition, W. Blum. J. Electrochem. Soc. 99, No. 2, 31C (1952). 450. Filters for the infrared region, E. K. Plyler and J. J. Ball. J. Opt. Soc. Am, 42, 269 (1952).

451. Fluid exchange at the margins of dental restorations, R. J. Nelsen, R. B. Wolcott, and G. C. Paffenbarger. J. Am. Dental Assoc. 44,288 (1952).

452. Fluorescence of teeth: A means of investigating their structure, G. Dickson, A. F. Forziati, M. E. Lawson, Jr. , and I. C. Schoonover. J. Am. Dental Assoc. 45, 661 (1952).

453. Formulas for numerical differentiation in the complex plane, H. E. Salzer. J. Math. and Phys. 31, No. 3, 155 (1952).

454. Foundry crucible service life, R. A. Heind1. Am. Foundryman 22, 40 (1952).
455. Frequency-deviation meter plots drift, N. C. Hekimian. Electronics 25, No. 6, 134 (1952).

456. Frequency standards, W. D. George. Cathode Press (Machlett Laboratories, Inc., Stamford, Connecticut) 9, No. 2, 2 (1952).

457. Gapless coverage in air-to-ground communications at frequencies above $50 \mathrm{Mc}, \mathrm{K}$. A. Norton and P. L. Rice, Proc. IRE 40, 470 (1952).

458. Genuine cooperation between manufacturers and weights and measures officials,

W. S. Bussey. Scale J. 38, No. 8, 7 (1952).

459. Ground-to-air cochannel interference at 2900 Mc, P. L. Rice, W. V. Mansfield, and J. W. Herbstreit. IRE Trans, Ant, Prop. AP- 6, 1 (1952).

460. Growth and infrared transmission of cesium iodide crystals, E. K. Plyler and F. P. Phelps, J. Opt. Soc. Am. 42, 432 (1952).

461. Hazards from static electricity, F. L. Hermach. Military Engr. 144, 287 (1952). 462. Heat capacity of potassium and three potassium-sodium alloys between $0^{\circ}$ and $800^{\circ}$, the triple point and heat of fusion of potassium, T. B. Douglas, A. F. Ball, D. C. Ginnings, and W. D. Davis, J. Am. Chem. Soc. 74, 2472 (1952).

463. High-power square-pulse generator, W. E. Williams, Jr. Electronics 25, No. 10, 144 (1952).

464. Impact strength of some thermosetting plastics at low temperatures, J. J. Lamb, D. A. George, H. A. Baker, and L. E. Sieffert. ASTM Bull. No. 181, 67 (1952).

465. Improved photogrid techniques for determination of strain over short gage lengths, J. A. Miller. Proc. Soc. Exptl. Stress Anal. Semiann. Rept. 10, 29 (1952).

466. Improvement in gain stability of the superheterodyne mixer through the application of negative feedback, G. E. Boggs. Proc. IRE 40 , No. 2, 202 (1952).

467. Influence coefficients of tapered cantilever beams computed on SEAC, S. Levy. ASME Paper No. 52-A-25 (1952).

468. Influence of splitting on the strength of chrome-tanned steer hides, J. R. Kanagy, W. Leser, E. B. Randall, T. Carter, and C. Mann. J. Am. Leather Chemists Assoc. 47, 329 (1952).

469. Instantaneous power spectra, C. H. Page. J. Appl. Phys. 23, 103 (1952).

470. Internal reflection in the troposphere and propagation beyond the horizon, T. J. Carroll. IRE Trans. Ant. Prop. AP-2, 9 (1952).

471. International co-operation on standardization of textile testing methods, W. D. Appel. J. Home Econ. 44, No. 10, 773 (1952). 472. Interpretation of high-frequency C-W field-intensity records with the aid of simultaneous pulse data, R. Silberstein. Proc. IRE 40,974 (1952).

473. Interpretation of tests for resistance to abrasion of textiles, H. F. Schiefer and C. W. Werntz. Textile Research J. 22, 1 (1952). 474. Kinetics of chain depolymerization, $R$. Simha and L. A. Wall. J. Phys. Chem. 56, No. 6, 707 (1952). 
475. Kinetics of $\mathrm{OH}$ radicals from flame emission spectra. IV. A study of the hydrogen-oxygen flame, H. P. Broida and K. E. Shuler. J. Chem. Phys. 20, 168 (1952). 476. Kinetics of $\mathrm{OH}$ radicals from flame emission spectra. V. A study of acetyleneoxygen flame, H. P. Broida and K. E. Shuler. J. Chem. Phys. 20, 1383 (1952).

477. Manufacture of paper from glass fibers, M. J. O'Leary, B. W. Scribner, J. K. Missimer, and J. J. Erving. Tappi 35, No. 7,289 (1952).

478. Mass spectrometry, V. H. Dibeler and J. A. Hipple. Anal. Chem. 24, 27 (1952).

479. Materiales para pisos, P. A. Sigler and T. H. Boone. Ingenieria Internacional Construccion (McGraw-Hill Book Co., Inc. , New York, N. Y.) 40, No. 7, 50 (1952).

480. Measurement of dynamic properties of rubber, R. S. Marvin. Ind. Eng. Chem. 44, 696 (1952).

481. Measurement of the effect of irregular terrain on VHF and UHF directive antenna patterns, R. S. Kirby, J. M. Taff, and H. S. Moore. IRE Trans. Ant. Prop. AP-3, 167 (1952).

482. Mechanical testing of solid materials, W. Ramberg. Appl. Mech. Rev. 5, 241 (1952). 483. Military field X-ray equipment, H. I. Amory and S. W. Smith. Radiology 59, No. 6, 879 (1952).

484. Minimizing anomalies in reflectance measurements with the Beckman quartz spectrophotometer, H. K. Hammond, III, and I. Nimeroff. J. Opt. Soc. Am. 42, 367 (1952). 485. National Bureau of Standards and state weights and measures administration, W. E. Bussey. Scale J. 38, No. 6, 9 (1952).

486. National Bureau of Standards Semi-

Centennial 1901-1951, L. S. Taylor. Am. J. Roentgenol., Radium Therapy Nuclear Med. 67, 112 (1952).

487. Natural and synthetic rubbers, N. Bekkedahl. Anal. Chem. 24, 279 (1952).

488. New frontiers in business management control are being established by electronic computers, H. D. Huskey and V. R. Huskey. J. of Accountancy, 93, 69 (1952).

489. New techniques for electronic miniaturization, R. L. Henry, R, K-F. Scal, and G. Shapiro. Proc. IRE (Sydney, Australia) 13, No. 3, 75 (1952).

490. Note on the displacement pressure method for measuring the affinity of liquids for solids, N. J. Delollis. J. Phys. Chem. 56, No. 2, 193 (1952).

491. Nuclear-resonance magnetic field control, H. A. Thomas, Electronics 25, No, 1, 114 (1952).

492. Object-color changes from daylight to incande scent filament illumination, H. Helson, D. B. Judd, and M. H. Warren, Hlum. Eng. 47,221 (1952).

493. Oblique incidence propagation at $300 \mathrm{kc}$ using the pulse technique, J. M. Watts. J. Geophys. Research 57, 487 (1952).

494. Oil flow in plain journal bearings, S. A. McKee. Trans. Am. Soc. Mech. Engrs. 74, 841 (1952).
495. Unassigned.

496. On analytic characteristic functions, E. Lukacs and O. Szasz. Pacific J. Math. 2, No. 4, 615 (1952).

497. On integration of parabolic equations by difference methods, F. John. Comm. Pure and Appl. Math. 5, No. 2, 155 (1952).

498. On lineal entire functions of $n$ complex variables, T. S. Motzkin and I. J. Schoenberg. Proc. Am. Math. Soc. 3, No. 4, 517 (1952).

499. On representations of finite groups. T. S. Motzkin and O. Taussky. Koninkl. Nederl. Akademie Van Wetenschappen Proc. (Amsterdam, Holland) Series A, 55, No. 5; Indag. Math. (Amsterdam, Holland) 14, No. 5, 511 (1952).

500. On the Gibbs phenomenon for a class of linear transforms, O. Szasz. Acad. Serve. Sci. Publ. Inst. Math. 4, 135 (1952).

501. On the nature of the decay of a meteor trail, J. Feinstein. Proc. Phys. Soc. (London, England) 65, Pt. 9, 741 (1952).

502. On the numerical solution of equations involving differential operators with constant coefficients, G. Blanch. Math. Tables and Other Aids to Computation 6, No, 40, 219 (1952).

503. On the relation between the conductance and the noise power spectrum of certain electronic streams, J. J. Freeman. J. Appl. Phys, 23, No. 11, 1223 (1952).

504. On the rounding off of difference tables for linear interpolation, A. M. Ostrowski. Math. Tables and Other Aids to Computation 6, No. 40,212 (1952).

505. Optical spectrophotometric analysis of hydrogen-deuterium mixtures in presence of air. H. P. Broida and G. H. Morgan. Anal. Chem. 24, 799 (1952).

506. Optical systems for increasing the available intensity from flames, E. K. Plyler and H. J. Kostkowski. J. Opt. Soc. Am. 42, 360 (1952). 507. Pairs of matrices with property L, T. S. Motzkin and O. Tausgky. Trans. Am. Math. Soc. 73 , No. 1,108 (1952).

508. Partial reflections in tropospheric propagatien, J. Feinstein. IRE Trans. Ant. Prop. AP- 3, 101 (1952).

509. Penetration and diffusion of X-rays: Calculation of spatial distributions by semiasymptotic methods, L. V. Spencer. Phys. Rev. 88, No. 4, 793 (1952).

510. Physical and physico-chemical constants of leather and collagen, C. E. Weir. J. Soc. Leather Trades' Chemists 36, 155 (1952).

511. Physical properties of electrodeposited metals, I: Nickel, 3: The effect of plating variables on the structure and properties of electrodeposited nickel, V. Zentner, A. Brenner, and C. W. Jennings, Plating 39, 865 (1952). 512. Plastics, G. M. Kline and R. B. Seymour. Ind. Eng. Chem. 44, 2339 (1952).

513. Polarization measurements of low-frequency echoes, E. L. Kilpatrick. J. Geophys. Research 57, No. 2, 221 (1952). 
514. Polyhedral flames with hydrogen and hydrocarbons, H. P. Broida and W. R. Kane. J. Chem. Phys. 20, No. 6, 1042 (1952).

515. Polymer degradation studies by absorption and mass spectrometry, B. G. Achhammer. Anal. Chem. 24, No. 12, 1925 (1952).

516. Precise measurements in the infrared spectrum of carbon monoxide, E. K. Plyler, W. S. Benedict, and S. Silverman. J. Chem. Phys, 20, 175 (1952).

517. Preparation of small quantities of germanium tetramethyl, J. H. Lengel and V. H. Dibeler. J. Am. Chem. Soc. 74, 2683 (1952). 518. Programming for finding the characteristic values of Mathieu's differential equation and the spheroidal wave equation, G. Blanch. Proc. of the Elect. Computer Symp. held by the Western Section of the Elect. Computers Prof. Group of the IRE, April 30, May 1-2, 1952 (University of California, Los Angeles, California) XIV:1 (1952).

519. Proof testing rubber tires, R. D. Stiehler. Ordnance 36 , No. 192,1018 (1952).

520. Properties of polymethyl alpha-chloroacrylate, M. C. Slone, J. J. Lamb, and F. W. Reinhart. Modern Plastics 29, No. 10, 109 (1952).

521. Properties of self-curing denture base resins, H. J. Caul, J. W. Sanford, and A. F. Serio. J. Am. Dental Assoc. 44, 295 (1952). 522. Properties of the $\mathrm{Cr}^{+++}$ion in the paramagnetic alums at low temperatures, R. P. Hudson. Phys. Rev. 88, No. 3, 570 (1952). 523. Quantitative determination of copper in natural rubber, L. T. Milliken. Rubber Age 71 , No. 1, 64 (1952).

524. Radio measurement of winds aloft, $R$. Greenstone. Aeronaut. Dig. 64, No. 2, 56 (1952).

525. Random flight with multiple partial correlations, C. M. Tchen. J. Chem. Phys. 20, No. 2, 214 (1952).

526. Rates of thermal degradation of polystyrene and polyethelene in a vacuum, S. L. Madorsky. J. Polymer Sci. 9, No. 2, 133 (1952).

527. Refractive indices of cesium bromide, W. S. Rodney and R. J. Spindler. J. Opt. Soc. Am. 42, 431 (1952).

528. Relations among certain specification properties of building brick and effects of differences in raw materials and methods of forming, J. W. McBurney, J. C. Richmond, and M. A. Copeland. J. Am. Ceram. Soc. 35, No. 12, 309 (1952).

529. Remarks on some modular identities, $M$. Newman. Trans. Am. Math. Soc. 73 , No. 2, 313 (1952).

530. Unassigned.

531. Report of the committee on atomic weights of the American Chemical Society, E. Wichers. J. Am. Chem. Soc. 74, 2447 (1952).

532. Panel discussion--Report on the NBS optical image evaluation symposium, F. E. Washer, D. P. Feder, and R. V. Shack. Reprinted from Photogrammetric Eng. 18, 575 (1952).
533. Resistivity, dielectric constant, and power factor of leather, C. E. Weir. J. Am. Leather Chemists Assoc. 47, 711 (1952).

534. Rotational temperatures of $\mathrm{OH}$ in several flames, H. P. Broida and G. T. Lalos. J. Chem. Phys. 20, No. 9, 1466 (1952).

535. Sampling of side upper leather II, C. Mann, J. Mandel, M. N. Steel, and J. R. Kanagy. J. Am. Leather Chemists Assoc. 47,352 (1952).

536. Scattering of electromagnetic energy in a randomly inhomogeneous atmosphere, $\mathrm{H}$. Staras. J. Appl. Phys. 23, No. 10, 1152 (1952).

537. Scientific measurement and technological progress, H. Odishaw. Ind. Lab. 3, No. 9, 8 (1952).

538. Secondary electrons: average energy loss per ionization, U. Fano. Chapter in Symp. on Radiobiology, The Basic Aspects of Radiation Effects on Living Systems (John Wiley \& Sons, Inc., New York, N. Y.) (1952).

539. Series-resonant high-voltage supply, P. G. Sulzer. Electronics 25, No, 9, 157 (1952).

540. Service range for air-to-ground and airto-air communications at frequencies above 50 Mc., R. S. Kirby, J. W. Herbstreit, and K. A. Norton. Proc. IRE 40, 525 (1952).

541. Service tests of boys' shoes, R. B. Hobbs. Am. Shoemaking Pt. 1. 222, No. 6, 15; Pt. 2. 222, No. 7, 17 (1952).

542. Sigma phase in high-purity 18-10, S. J. Rosenberg and C. R. Irish. Metal Progr. 61, No. 5, 92 (1952).

543. Similarity properties of the two-fluid model of superconductivity, E. Maxwell. Phys, Rev. 87, No. 6, 1126 (1952).

544. Solar "enhanced radiation" and plasma oscillations, H. K. Sen. Phys. Rev. 88, No. 4,816 (1952).

545. Some general precepts for programmers, E. C. Yowell. Proc. of the Symp. on Elect. Computers held by the Western Section of the Elect. Computers Prof. Group at the IRE, April 30, May 1-2, 1952 (University of California, Los Angeles, California) X:1-X:6 (1952).

546. Some methods for evaluating trends in time series of tropospheric radio field strength data, P. L. Rice. IRE Trans. Ant. Prop. AP- 3, 144 (1952).

547. Some observations on the evaporation of water from cellulose, H. Bogaty, K. S. Campbell, and W. D. Appel. Textile Research J. 22, 75 (1952).

548. Some relations among the blocks of symmetrical group divisible designs, W. S. Connor. Annals of Math. Stat. 23, No. 4, 602 (1952).

549. Sources and identifying symbols of Government specifications, S. F. Booth. Metal Progr. 62, No. 4, 109 (1952).

550. Spectral lines as frequency standards, H. Lyons. Ann. N. Y. Acad. Sci. 55, Art. 5, 831 (1952).

551. Spectroscopic analysis of deuterium in hydrogen-deuterium mixtures, H. P. Broida and J. W. Moyer. J. Opt. Soc. Am. 42, 37 (1952).

552. Stable-output oscillator, S, Rubin. Electronics 25, No. 10, 154 (1952). 
553. Statistical aspects of analytical determinations, W. J. Youden. The Analyst 77, No. 921,874 (1952).

554. Sterochemical research, W. R. Brode. Record Chem. Progr. Summer Issue 45 (1952). 555. Stress and strain at onset of crazing of polymethyl methacrylate at various temperatures, M. A. Sherman and B. M. Axilrod. Nat1. Advisory Comm. Aeronaut. Tech. Note 2778 (1952).

556. Stress studies of bulkhead intersections for welded tankers, W. R. Campbell, L. K. Irwin, and R. C. Duncan. Welding J. 31, No. 2, 68 (1952).

557. Study of degradation of polystyrene by means of mass spectrometry, B. G. Achhammer, M. J. Reiney, L. A. Wall, and F. W. Reinhart. J. Polymer Sci. 8, No. 5, 555 (1952).

558. Study of the variation of the physical and chemical properties of chrome-tanned leather and the selection of a sampling location, J. R. Kanagy, C. Mann, and J. Mandel. J. Soc. Leather Trades' Chemists (London, England) 36,231 (1952).

559. Sufficient conditions for the convergence of Newton's method in complex Banach spaces, M. I. Stein. Proc. Am. Math. Soc. 3, No. 6, 858 (1952).

560. Superconductivity of the isotopes of tin, E. Maxwell. Phys. Rev. 86, No. 2, 235 (1952).

561. Sweep-frequency oblique-incidence ionosphere measurements over a $1,150 \mathrm{~km}$ path, P. G. Sulzer and E. E. Ferguson. Proc. IRE 40,1124 (1952).

562. Systems of equations, matrices, and determinants, O. Taussky and J. Todd. Mathematics Mag. 26, No. 1, Sept. p. 9 and 26, No. 2, Dec. p. 71 (1952).

563. Tables for constructing and for computing the operating characteristics of single sample plans, J. M. Cameron. Ind. Qual. Control 9, No. 1, Pt. 1, 37 (1952).

564. Temperature prediction in electronic design, P. J. Selgin and B. K. Hawes. Elec. Mfg. 50, No. 4, 116 (1952).

565. Testing wholesale meters, W. S. Bussey. Scale J. 38 , No. 4,7 (1952).

566. The admittance of a diode with a retarding field, J. J. Freeman. J. Appl. Phys. 23, 743 (1952).

567. The backscattering of positrons and electrons, H. H. Seliger. Phys, Rev. 88, 408 (1952).

568. The construction and operation of a high pressure diffusion cloud chamber, J. M. Wyckoff. Phys. Rev. 87, 185 (1952). 569. The decay of the Cd15 isomers, R. W. Hayward, Phys, Rev, 87, 202 (1952). 570. The determination of impedance with a double-slug transformer, R. C. Ellenwood and E. H. Hurlburt. Proc. IRE 40, 1690 (1952).

571. The differences in the relationship between ionospheric critical frequencies and sunspot number for different sunspot cycles, S. M. Ostrow and M. Pokempner. J. Geophys, Research 57 , No. 4, 473 (1952).
572. The effect of molding processes on some properties of denture resins, A. H. Grunewald. G. C. Paffenbarger, and G. Dickson. J. Am. Dental Assoc. 44, 269 (1952).

573. The effect of recent mass measurements on the faraday, H. Sommer, S. N. Craig, J. I. Hoffman, and J. A. Hipple. Phys. Rev. 85, 740 (1952).

574. The effect of sporadic-E on television reception, E. K. Smith. IRE Trans. Ant. Prop. AP-2, 54 (1952).

575. The electromagnetic field of rotating uniformly magnetized sphere, A. Banos, Jr. and R. K. Golden. J. Appl. Phys. 23, No. 12, 1294 (1952).

576. The emission spectra of nitrogen bornbarded by high energy protons, R. J. Shalek, T. W. Bonner, and L. M. Branscomb. Phys. Rev. 85, 739 (1952).

577. The expansion theorem for pseudo-analytic functions, S. Agmon and L. Bers. Proc. Am. Math. Soc. 3, No. 5, 757 (1952).

578. The infrared emission spectra of $\mathrm{OH}, \mathrm{CO}$, and $\mathrm{CO}_{2}$ from $3 \mu$ to $5.5 \mu$, E. K. Plyler and J. J. Ball. J. Chem. Phys. 20, 1178 (1952). 579. The interpretation of chemical data, W. J. Youden. Conf. Papers of the 5 th Annual Convention of the Am. Soc. for Quality Control, May 23, 1951 (Rochester Institute of Technology, Rochester, N. Y.) 8, No. 6, 90 (1952). 580. The ionization potential of the $\mathrm{CF}_{3}$ radical and some fluorocarbon bond energies obtained by electron impact, V. H. Dibeler, R. M. Reese, and F. L. Mohler. J. Chem. Phys. 20, 761 (1952).

581. The isotope effect in superconductivity, E. Maxwell. Phys. Today 5, No. 12, 14 (1952); Phys. Rev. 5, 13 (1952).

582. The kinorama as an evaluator in aviation lighting, F. C. Breckenridge. nlum. Eng. 47, 561 (1952).

583. The limiting polarization of magnetoionic waves, J. Feinstein. IRE Trans. Ant. Prop. AP-3, 19 (1952).

584. The magnetic threshold field curve of a superconductor, R. P. Hudson. Phys. Rev. 85 , No. 2, 382 (1952).

585. The microwave measurement of variations in atmospheric refractive index, G. Birnbaum, H. E. Bussey, and R. R. Larson. IRE Trans. Ant. Prop. AP-3, 74 (1952).

586. The nature of gradient reflections, J. Feinstein. IRE Trans. Ant. Prop. AP-4, 2 (1952).

587. The necessity for uniformity of test procedures, M. W. Jensen. Scale J. 39, No. 3, 7 (1952).

588. The operating characteristic of the average chart, E. P. King. Ind. Qual. Control 9, No. 3,30 (1952).

589. The oxidation of cellulose by ozone in small concentrations, H. Bogaty, K. S. Campbell, and W. D. Appel. Textile Research J. 22,81 (1952).

590. The present status of temperature scales, H. F. Stimson. Science 116, 339 (1952).

591. The probability distribution of the phase of the resultant vector sum of a constant vector plus a Rayleigh distributed vector, K. A. Norton, E. L. Schultz, and H. Yarbrough. J. Appl. Phys. 23, No. 1, 137 (1952). 
592. The refractories industry in some European countries, R. A. Heindl. The Refractories Inst. Tech. Bull. No. 90, p. 1 (1952).

593. The relation between the absorption spectra and the chemical constitution of dyes, $\mathrm{XXV}$. Phototropism and cis-trans isomerism in aromatic azo compounds. W. R. Brode, J. H. Gould, and G. M. Wyman. J. Am. Chem. Soc. 74, No. 18. 4641 (1952).

594. The reliability of measured values. Part I. Fundamental concepts, C. Eisenhart. Photogrammetric Eng. 18, No. 3, 542 (1952). 595. The role of partial reflections in tropospheric propagation beyond the horizon, J. Feinstein. IRE Trans. Ant. Prop. AP-2, 2 (1952).

596. The stochastic independence of symmetric and homogeneous linear and quadratic statistics, E. Lukacs. Ann. Math. Stat. 23, No. 3, 442 (1952).

597. The sums of dihedral and trihedral angles in a tetrahedron, J. W. Gaddum. Am. Math. Monthly 59, No. 6, 370 (1952).

598. The use of sub-routines on SEAC for numerical integrations of differential equations and for Gaussian quadrature, P. Rabinowitz. Proc. Assoc. for Computing Machinery, Toronto, Canada. p. 88 (Assoc. for Computing Machinery, New York, N. Y.) (1952).

599. The vapor pressure of $\mathrm{He}^{3}-\mathrm{He}^{4}$ mixtures, R. A. Nelson and W. Band. Phys. Rev. 88, No. 6, 1431 (1952).

600 . The variation of intensity of fast cosmicray neutrons with altitude, L. F. Curtiss and P. S. Gill. Phys, Rev. 85, No. 2, 309 (1952). 601 . The variation of the physical and chemical properties of split and unsplit chrome-tanned leathers, E. B. Randall, T. J. Carter, T. J. Kilduff, C. Mann, and J. R. Kanagy. J. Am. Leather Chemists Assoc. 47, 404 (1952).

602. Thermal batteries, R. B. Goodrick and R. C. Evans. J. Electrochem. Soc. 99, No. 8, 207 (1952).

603. Thermal Rayleigh disk measurements in liquid helium II. , J. R. Pellam and W. B. Hanson. Phys. Rev. 85, No. 2, 216 (1952). 604. Thermodynamic properties of gaseous difluorodichloromethane (freon-12), J. F. Masi. J. Am. Chem. Soc. 74, 4738 (1952). 605. Total collision cross sections of negative atomic iodine ions in nitrogen and argon, F. L. McCrackin. Phys. Rev. 86, No. 1, 135 (1952). 606. Transmission loss of space waves propagated over irregular terrain, K. A. Norton. IRE Trans. Ant. Prop. AP-3 (1952).

607. Treatment of leather with polyisobutylene, R. Oehler, S. Dahl, and T. J. Kilduff. J. Am. Leather Chemists Assoc. 47, 642 (1952).
608. Two existence theorems for systems of linear inequalities, L. M. Blumenthal. Pacific J. Math. 2, No. 4, 523 (1952).

09. Two explicit formulae for the distribution function of the sums of $\mathrm{n}$ uniformly distributed independent variables, A. M. Ostrowski. Archiv Der Mathematik (Basel, Switzerland) 3, No. 6, 3 (1952).

610. Ueber den Einfluss der Gewebekonstruktion auf den Scheuerwiderstand, H. F. Schiefer and C. W. Werntz. Textil-Praxis (Stuttgart, Germany) 57, 1 (1952).

611. Units and dimensions in physics, C. H. Page. Am. J. Phys. 20, 1 (1952).

612. Use of continued fractions in high-speed computing, D. Teichroew. Math. Tables and Other Aids to Computation 6, No. 39, 127 (1952). 613. Use of random numbers, F. Proschan. Ind. Qual. Control 9, No. 1, 32 (1952).

614. Use of subroutines on SWAC, R. S. Lipkis. Proc. Assoc. for Computing Machinery, p. 231 (1952).

615. Using arc-discharge tubes in regulated low-voltage supplies, G. E. Boggs. FM-TV Radio Communication Mag. 12, No. 10, 25 (1952).

616. Vapor pressure of benzene above $100^{\circ} \mathrm{C}$., P. Bender, G. T. Furukawa and J. R. Hyndman. Ind. Eng. Chem. 44, 387 (1952).

617. Variational calculation of scattering cross sections, E. Gerjuoy and D. S. Saxon. Phys. Rev. 85, No. 5, 939 (1952).

618. Variations of physical and chemical properties within and between vegetable retanned cow and steer sides, J. R. Kanagy, E. B. Randall, T. J. Carter, R. A. Kinmonth, and C. Mann. J. Am, Leather Chemists Assoc. 47, 726 (1952).

619. Versatile core boxes, R. A. Harwell and J. H. Schaum. Foundry 80, No. 5, 154 (1952). 620. Very high frequency tropospheric recording measurements of plane and circular polarized waves in the Great Lakes area, J. S. Hill, G. V. Waldo, and H. Staras. Abstract published in IRE Trans. Ant. Prop. AP-1, 42 (Feb. 1952); entire paper published as FCC Memo. 62838 .

621 . Viscosities of 0.25 to 90 percent GR-S rubber solutions, A. B. Bestul, H. V. Belcher, F. A. Quinn, Jr., and C. B. Bryant. J. Phys. Chem. 56, 432 (1952).

622. What use is spectroscopy? W. F. Meggers. Appl. Spectroscopy 6, No. 4, 6 (1952).

623. World-wide standard frequency broadcast reception, E. L. Hall. Tele-Tech. 11, No. 6, 46 (1952); Pt. 2. 11, No. 7, 64 (1952).

624. Zeros of $I_{n+1}(x) J_{n}(x)+J_{n+1}(x) I_{n}(x), G$. Blanch. Math. " Computation 6, No. 37, 58 (1952). 
625. A comparative survey of spectrophotometers in the $210-760 \mathrm{~m} \mu$ region, W. R. Brode, J. H. Gould, G. M. Wyman, and J. E. Whitney. J. Opt. Soc. Am. 43, 862 (1953).

626. A dual-amplitude axial-load fatigue machine, A. E. McPherson. Proc. Soc. Exptl. Stress Anal. 10, 143 (1953).

627. A high-power pulse generator, W. E.

Williams, Jr. Radio-Electronic Eng. Section, Radio and TV News 49, 32 (1953).

628. A method of preparing uniform films of bituminous materials, S. H. Greenfield. ASTM Bull. No. 193, 50 (1953).

629. A note on wave propagation through an inhomogeneous medium, G. A. Hufford. J. Appl. Phys. 24, 268 (1953).

630. A Neumann series for the product of two Whittaker functions, P. Henrici. Proc. Am. Math. Soc. 4, 331 (1953).

631. A numerical analyst's fifteen-foot shelf, G. E. Forsythe. Math. Tables and Other Aids to Computation VII, 211 (1953).

632. A probe tube method for the transfer of threshold standards between audiometer earphones, E. L. R. Corliss and M. D. Burkhard. J. Acoust. Soc. Am. 25, 990 (1953).

633. A relative damping criterion for linear systems, J. F. Koenig. Applications and Industry, No. 9, 291 (1953); also Trans. Am. Inst. Elec. Engrs. 72, Pt. 2, 291 (1953).

634. A reliable locked-oscillator pulse timer, P. G. Sulzer. Tele-Tech 12, No. 4, 68 (1953). 635. A study of some of the meterological effects on radio propagation at $96.3 \mathrm{mc}$ between Richmond, Va., and Washington, D. C. , D. L. Randall. Bull. Am. Meterol. Soc. 35, 56 (1953).

636. A table of Wilson quotients and the third Wils on prime, K. Goldberg. J. London Math. Soc. 28, pt. II, 252 (1953).

637. A variable length re-entrant cavity for dielectric measurements from 100 to $400 \mathrm{Mc}$, J. H. Beardsley. Rev. Sci. Instr. 24, 180 (1953).

638. A VHF and microwave matching termination, R. C. Ellenwood and W. E. Ryan. Proc. IRE 41, 104 (1953).

639. Absorption in the low-frequency wing of the $\mathrm{NH}_{3}$ inversion spectrum, G. Birnbaum and A. A. Maryott. J. Chem. Phys. 21, 1774 (1953).

640. Accurate radio-frequency microvoltages, M. C. Selby. Elec. Eng. 72, 515 (1953); Am. Inst. Elec. Engrs. Trans. p. 1 (May 1953 issue); Electronics 6, 158 (1953).

641. Addendum to a guide to tables on punched cards, G. Blanch and E. C. Yowell. Math. Tables and Other Aids to Computation VII, 1 (1953).

642. Advancements in dielectrics, 1952, R. C. Powell and J. L. Dalke. Proc. IRE 41, 452 (1953).

643. Alpha-gamma angular correlation in ionium $\left(\mathrm{TH}^{230}\right)$, G. M. Temmer and J. M. Wyckoff. Phys, Rev, 92, 913 (1953).

644. American Dental Association specification N. 12 for denture base resin (second revision), A. C. Swaney, G. C. Paffenbarger, H. J. Caul, and W. T. Sweeney. J. Am. Dental Assoc, 46, 54 (1953).
645. Amide nitrogen of collagen and hide powder, J. M. Cassel and E. McKenna. J. Am. Leather Chemists Assoc. 48, 142 (1953). 646. An accurate time-modulated pulse circuit, C. E. Tschiegg and M. Greenspan. Rev. Sci. Instr. 24, 183 (1953).

647. An automatic controller for radiofrequency induction-heating unit, J. J. Theron. Rev. Sci. Instr. 24, 281 (1953).

648. An eleven megacycle interferometer for low pressure gases, M. Greenspan and M. C. Thompson, Jr. J. Acoust. Soc. Am. 25, 92 (1953).

649. An estimate of the density and motion of solar material from observed characteristics of solar radio outbursts, H. K. Sen. Australian J. Phys. 6, 67 (1953).

650. An improved cuvette densitometer for cardiac output determination by the dye-dilution method, S. R. Gilford, D. E. Gregg, O. W. Shadle, T. B. Ferguson, and L. A. Marzetta. Rev. Sci. Instr. 24, 696 (1953).

651. An ionosphere recorder for low frequencies, J. C. Blair, J. N. Brown, and J. M. Watts. J. Geophys. Research 58, 99 (1953). 652. Analyzing straight-line data, F. S. Acton. J. Chem. Education 30, 128 (1953).

653. Anelasticity of quartz, R. K. Cook and R. G. Breckenridge. Phys. Rev. 92, No. 6, 1419 (1953).

654. Application of viscosity methods to the study of branching in polysaccharides, $M$. Wales, P. A. Marshall, S. Rothman, and S. G. Weissberg. Ann. N. Y. Acad. Sci. 57, 353 (1953).

655. Applied optics at NBS, I. C. Gardner. Phys. Today 6, No. 9, 8 (1953).

656. Asymptotic solution of the differential equation of hydrodynamic stability in a domain containing a transition point, W. Wasow. Ann. Math. 58, 222 (1953).

657. Beta-ray spectrometers, R. W. Hayward. Chapter in vol. 5 of Advances in Electronics (Academic Press Inc., New York, N. Y.) (1953). 658. Biaxial stretch-forming of acrylics, I. Wolock, B. M. Axilrod, and M. A. Sherman. Modern Plastics 31, 128 (1953).

659. Calculation of peaked angular distributions from legendre polynomial expansions and an application to the multiple scattering of charged particles, L. V. Spencer. Phys. Rev. 90, 146 (1953).

660. Cemented piezoelectric accelerometers, E. Jones. Rev. Sci. Instr. 24, 1151 (1953). 661. Change in the inversion spectrum of $\mathrm{ND}_{3}$ from resonant to nonresonant absorption, G. Birnbaum and A. A. Maryott. Phys. Rev. 92, 270 (1953).

662. Changes of sign of sums of random variables, P. Erdos and G. A. Hunt. Pacific J. Math. 3, 673 (1953).

663. Characteristics of the magnetic attenuator at UHF, F. Reggia and R. W. Beatty. Proc. IRE 41, 93 (1953).

664. Chart for TE model piston attenuator, C. M. Allred. Electronics 26, 142 (1953); Electronics Buyer's Guide Is sue 26 , No. $6 \mathrm{~A}$, R8 (1953). 
665. Chemical analyses, surface area, and thermal reactions of natural graphite, and refractoriness of the ashes, L. Mackles, R. A. Heindl, and L. E. Mong. J. Am. Ceram. Soc. 36,266 (1953).

666. Chemical analys is of GR-S by complete solution procedures. Gross constituents in GR-S containing soap, F. J. Linnig, J. M. Peterson, D. M. Edwards, and W. L. Acherman. Anal. Chem. 25, 1511 (1953).

667. Chemical analysis of GR-S by complete solution procedures. Titration of mineral and organic acids in toluene-ethanol solution, F. J. Linnig and A. Schneider. Anal. Chem. 25, 1515 (1953).

668. Chemical methods for separating and determining metals contained in platiniferous materials, R. Gilchrist. Anal. Chem. 25, 1617 (1953).

669. Closure to discussion by Prof. Maxwell of "Stress and Strain at the Onset of Crazing of Polymethyl Methacrylate at Various Temperatures" by M. A. Sherman and B. M. Axilrod, B. M. Axilrod. ASTM Bull. No. 194, p. 61 (1953).

670. Colorimetric determination of tetrachlorohydroquinone, S. Dahl. Anal. Chem. 25, 1724 (1953).

671. Completely continuous normal operators with property L, I. Kaplansky. Pacific J. Math. 3, 721 (1953).

672. Computational experience in solving linear programs, A. J. Hoffman, M. Mannos, D. Sokolowsky, and N. A. Wiegmann. J. Soc. Ind. Appl. Math. 1, 17 (1953).

673. Computer memory uses conventional C-R tubes, A. W. Holt and W. W. Davis, Electronics 26, 178 (1953).

674. Confidence and tolerance intervals for the normal distribution, F. Proschan. J. Am. Stat. As soc. 48, 550 (1953).

675. Continental maps of four ionospheric disturbances, R. S. Lawrence. J. Geophys. Research 58, 219 (1953).

676. Control charts may be all right, but--, F. Proschan. Ind. Qual. Cont. 9, 56 (1953). 677. Data concerning atomic spectra, W. F. Meggers and C. E. Moore. J. Opt. Soc. Am. 43,415 (1953).

678. Decay constant in vibrating systems, $R$. D. Laughlin. Radio-Electronic Eng. Sect., Radio and Television News 49, 11 (1953). 679. Deformation twinning in charpy $V$-notch specimens of ingot iron. G. W. Geil and N. L. Carmete. Welding J. Research Suppl. 32, 273- F (1953).

680. Degradation and range straggling of highenergy radiations, U. Fano. Phys. Rev. 92, 328 (1953).

681. Degradation des polymeres par les rayons et les neutrons, L. A. Wall and M. Magat. J. Chim. Phys. 50, 308 (1953).

682. Degradation of polyisobutylenes on shearing in solution, A. B. Bestul and H. V. Belcher. J. Appl. Phys. 24, 1011 (1953).

683. Designing electroformed parts, W. H. Metzger and V. A. Lamb. Machine Design 25, 124 (1953).
684. Determination of dynamic stress-strain curves from strain waves in long bars, W. R. Campbell. Proc. Soc. Exptl. Stress Anal. Semiann. Rep. 10, 113 (1953).

685. Determination of phosphate by differential spectrophotometry, A. Gee and V. R. Deitz. Anal. Chem. 25, 1320 (1953).

686. Development of turbulence-measuring equipment, L. S. Kovasznay. Nat. Advisory Comm. Aeronaut. Tech. Note 2839 (1953). 687. Dilute solution thermodynamic behavior of flexible chain molecules: polymethyl methacrylate, T. G. Fox and L. Mandelkern. J. Chem. Phys. 21, 187 (1953).

688. Direct and indirect filling resins: a review of some physical and chemical properties, G. C. Paffenbarger, R. J. Nelsen, and W. T. Sweeney. J. Am. Dental Assoc. 47, 516 (1953).

689. Direct filling resins: dimensional changes resulting from polymerization shrinkage and water sorption, D. L. Smith and I. C. Schoonover. J. Am. Dental Assoc. 46, 540. (1953). 690. Direct-indicating recording instruments, S. R. Gilford. Elec. Mfg. 52, 114 (1953).

691. Durability of boys' shoes, R. B. Hobbs. J. Home Economics 45, 32 (1953).

692. Dynamic compliance, dynamic modulus, and equivalent Voight and Maxwell models for polyisobutylene, H. Leaderman and R. S. Marvin. J. Appl. Phys. 24, 812 (1953).

693. Effect of light on coated groundwood papers, W. K. Wilson and J. L. Harvey. Tappi 36, 459 (1953).

694. Effect of straining rate on the compressive strength and elastic properties of concrete, D. Watstein. J. Am. Concrete Inst. 24, No. 49-52, 729 (1953).

695. Effect of tensile strain at low temperatures on deformation twinning in ingot iron, G. W. Geil and N. L. Carwile. J. Metals 5, 213 (1953); Trans. Am. Inst. Mining Met. Engrs. 197,213 (1953).

696. Effect of variation of circuit parameters on the excitation of spectra by capacitor discharges, C. H. Corliss. Spectrochim. Acta 5,378 (1953).

697. Effectiveness of vacuum investing in the elimination of surface defects in gold castings, H. W. Lyon, G. Dickson, and I. C. Schoonover. J. Am. Dental Assoc. 46, 197 (1953). 698. Effects of atomic radiation on polymers, L. A. Wall and M. Magat. Modern Plastics 30, 111 (1953).

699. Effects of high degrees of biaxial stretchforming on crazing and other properties of acrylic plastic glazing, I. Wolock, B. M. Axilrod, and M. A. Sherman. Natl. Advisory Comm. Aeronaut, Research Memo, RM53Dl4 (1953).

700. Effects of self-absorption of rotational "temperatures" of $\mathrm{OH}$ in flames, H. P. Broida. J. Chem. Phys. 21, 1165 (1953).

701. Effects of vibrational state on rotational intensity distribution in $\mathrm{OH}, \mathrm{A}$. M. Bass and H. P. Broida. J. Chem. Phys. 21, 173 (1953). 
702. Electrical indicating instruments used in early Edison central stations, H. B. Brooks. J. Franklin Inst. 256, 401 (1953).

703. Electrical properties of indium antimonide, R. G. Breckenridge, W. R. Hosler, and W. Oshinsky. Phys, Rev, 91, 243 (1953).

704. Electrical properties of titanium dioxide semiconductors, R. G. Breckenridge and W. R. Hosler. Phys. Rev. 91, 793 (1953).

705. Electrodeless metal-halide lamps, C. H. Corliss, W. R. Bozman, and F. O. Westfall. J. Opt. Soc. Am. 43, 398 (1953).

706. Electron beam interferometer, L. Marton, J. A, Simpson, and J. A. Suddeth. Phys, Rev. 90,490 (1953).

707. Electron interferometry, L. Marton. Science 118, 470 (1953).

708. Elements of a mathematical theory of probability, J. H. Curtiss. Mathematics Mag. 26, 233 (1953).

709. Eleven-Mev thick target bremsstrahlung, J. W. Motz, W. Miller, and H. O. Wyckoff. Phys. Rev. 89, 968 (1953).

710. Endurance of helical springs related to properties of the wire, H. C. Burnett and C. L. Staugaitis. Metal Progr. 64, 77 (1953).

711. Energy levels in $\mathrm{F}^{18}$ from alpha-particle reactions in nitrogen, N. P. Heydenburg and G. M. Temmer. Phys. Rev. 92, 89 (1953).

712. Engineering management in a government laboratory, J. G. Reid. Proc. Natl. Elec. Conf. (Am. Inst. Elec. Engrs.) 8, 734 (1953).

713. Estimating the standard deviation of a normal population, E. P. King. Ind. Quality Control 10,30 (1953).

714. Evaluation of a new cuvette densitometer for determination of cardiac output, $O$. W. Shadle, T. B. Ferguson, D. E. Gregg, and S. R. Gilford. Circulation Research 1, No.13, 200 (1953).

715. Evaluation of adhesion of organic coatings by ultracentrifugal and other methods, A. Malloy, W. Soller, and A. G. Roberts. Paint, Oil, and Chem. Rev, 116, No. 18, 14 and 116 , No. 19,26 (1953)

716. Evaluation of the integral $\int_{0}^{\infty o} e^{-u^{2}-(x / u)} d u$, M. Abramowitz, J. Math. Phys. 32, 188 (1953).

717. Extension of the second spectrum of zir-

conium into the infrared, C. C. Kiess, J. Opt.

Soc. Am. 43, 1024 (1953).

718. Factoren, die die Eigenschaften von

Reyongeweben beeinflussen, L. H. Jameson.

Textil-Praxis (Stuttgard, Germany) 8, 769

(1953).

719. Feedback amplifiers with stabilized output impedances, N. C. Hekimian. Tele-Tech 12, 103 (1953).

720. Filter radiometry and some of its applications, R. Stair. J. Opt. Soc. Am, 43, 971 (1953)

721. Fluorescense of teeth reveals structure, G. Dickson, A. F. Forziati, and M. E. Lawson, Jr. N. Y. J. Dentistry 23, 299 (1953).

722. FM data reduction from magnetic tape recorders, L. Costrell. Rev. Sci. Instr. 24, 76 (1953).

723. Frequency-deviation meter plots drift, N. C. Hekimian. Radio and Television News (Radio-Electronic Engineering Section) 50, No. 4, 32 (1953).
724. Gamma-ray attenuation, U. Fano. Part I, Nucleonics 11, 8 (1953); Part II, Nucleonics 11,55 (1953).

725. Gamma-ray measurements by the magnetic analysis of Compton electrons, J. W. Motz, W. Miller, H. O. Wyckoff, H. F. Gibson and F. S. Kirn. Rev. Sci. Instr. 24, 929 (1953).

726. Geometrical characterization of nuclear states and the theory of angular correlations, U. Fano. Phys. Rev. 90, 577 (1953).

727. Geographical and height distribution of the gradient of refractive index, B. R. Bean. Proc. IRE 41,549 (1953).

728. Unassigned.

729. Heat capacity standards for the range $14^{\circ}$ to $122^{\circ} \mathrm{K}$, D. C. Ginnings and G. T. Furukawa. J. Am. Chem. Soc. 75, 522 (1953).

730. High-frequency scatter sounding experiments at the National Bureau of Standards, R. Silberstein. Science 118, 759 (1953).

731. History of optical glass production in the United States, F. W. Glaze. Am. Ceram. Soc. Bull. 32, 242 (1953).

732. Hydraulic turbine contra-angle handpiece, R. J. Nelsen, C. E. Pelander, and J. W. Kumpula. J. Am. Dental Assoc. 47, 324 (1953).

733. Hyperfine structure formulas for LS coupling, R. E. Trees. Phys. Rev. 92, 308 (1953).

734. Impact properties of yarns made from different fibers, H. F. Schiefer, W. D. Appel, J. F. Krasny, and G. G. Richey. Textile Res, J. 23,489 (1953).

735. Improvement in response of 4- $\pi$ gammaionization chambers, C. C. Smith and H. H. Seliger. Rev. Sci. Instr. 24, 474 (1953).

736. Improvements in flame photometric determination of sodium in portland cement, J. J. Diamond and L. Bean. Anal. Chem. 25, 1825 (1953).

737. Improvements in the NBS primary standard of frequency. Philco Techrep. Div. Bul. (Philadelphia, Pa.) 3, No. 1, 11 (1953).

738. Influence coefficients of tapered cantilever beans computed on SEAC, S. Levy. Am. Soc. Testing Materials 20, 1 (1953).

739. Influence of the ground on the calibration and use of VHF field-intensity meters, F. M. Greene. Proc. IRE 14, 58 (1953).

740. Influence of variations in rotors, dies, and rate of shear on Mooney viscosity, G. E. Decker and F. L. Roth. India Rubber World 128, 339 (1953).

741. Information processing in social and industrial research, J. L. McPherson, S. N. Alexander, H. B. Horton, and E. Glaser. Sci. Monthly 76, 100 (1953).

742. Infrared measurements with a cesium iodide prism, N. Acquista and E. K. Plyler. J. Opt. Soc. Am. 43, 977 (1953).

743. Infrared multiplets and the solar spectrum, C. E. Moore. J. Opt. Soc. Am. 43, No. 11, 1014 (1953).

744. Infrared spectrometry with a cesium iodide prism, E. K. Plyler and N. Acquista. J. Opt. Soc. Am. 43, 212 (1953). 
745. Intrinsic viscosity-molecular weight relationships for dextran, M. Wales, P. A. Marshall, and S. G. Weissberg. J. Polymer Sci. 10, 229 (1953).

746. Investigation of structural failures of welded ships, M. L. Williams and G. A. Ellinger. Welding J. 32, 498-s (1953). 747. Ionization and dissociation of methyl siloxanes by electron impact, V. H. Dibeler, F. L. Mohler, and R. M. Reese. J. Chem. Phys. 21, 180 (1953).

748. Is omorphous substitution in enamel apatite, A. S. Posner and S. R. Stephenson. J. Am. Dental Assoc. 46, 257 (1953).

749. Junction transactor circuit applications, P. G. Sulzer. Electronics 26, No. 8, 170 (1953).

750. Large reductions of VHF transmission loss and fading by the presence of a mountain obstacle in beyond-line-of-sight paths, F. H.

Dickson, J. J. Egli, J. W. Herbstreit, and G. S. Wickiser. Proc. IRE 41, 967 (1953).

751. Lead storage batteries--essential to modern transportation, W. J. Hamer. Capital Chemist 3, 7 (1953).

752. Looking ahead in mechanics, W. Ramberg. J. Wash. Acad. Sci. 43, 241 (1953).

753. Looking into the nucleus, F. Bitter. J.

Opt. Soc. Am. 43, 233 (1953).

754. Lossy cable attenuator pads, R. W.

Beatty and C. E. Huber. Rev. Sci. Instr. 24, 1002 (1953).

755. Low-distortion transistor audio os cillator, P. G. Sulzer. Electronics 26, 171 (1953).

756. Magnetic properties below $1^{\circ} \mathrm{K}$, D. de Klerk. Phys. Today 6, No. 2, 4 (1953).

757. Making one measurement do the work of two, W. J. Youden and W. S. Connor. Chem. Eng. Progr. 49, 549 (1953).

758. Mass spectrum of gallium vapor, $S$. Antkiw and V. H. Dibeler. J. Chem. Phys. 21,1890 (1953).

759. Mathematical services useful in industry, A. S. Cahn, Jr. Proceedings of Symposium on Industrial Applications of Automatic Computing Equipment held by the Midwest Research Institute, Kansas City, Mo., January 8, 1953, p. 19 (1953).

760. Measurement of color, gloss, and haze, H. K. Hammond, III, and G. W. Ingle. Symposium on Plastics Testing, ASTM Spec. Tech. Publ. No. 132 , p. 25 (1953).

761. Measurement of treadwear of commercial tires, R. D. Stiehler, G. G. Richey, and J. Mandel. Rubber Age 73, 201 (1953).

762. Measuring the stiffness of paper, R. B.

Hobbs and V. Worthington. Modern Packaging 26, 127 (1953).

763. Metodi probabilistici per la soluzione numerica di alcuni problemi di analisi, W. Wasow. Rend. Mat. App. V, XI, 336-346 (Roma 1952); also is sued separately as Publicazioni dell' Istituto per le Applicazioni del Calcolo N. 354 (Roma, Italy) (1953).

764. Metric methods in integral and differential geometry, J. W. Gaddum. Am. J. Math. 75, 30 (1953).

765. Microstructure of dental amalgam, D. L.

Smith, G. W. Ferguson, and I. C. Schoonover.

J. Am. Dental Assoc. 47, 305 (1953).
1953

766. Microwave absorption spectrum of $\mathrm{ND}_{3}$, R. G. Nuckolls, L. J. Rueger, and H. Lyons. Phys. Rev. 89, 1101 (1953).

767. Millimeter wavelength dispersion of water vapor, G. Birnbaum. J. Chem. Phys. 21, 57 (1953).

768. Minimax theorems, K. Fan. Proc. Natl. Acad. Sci. 39, 42 (1953).

769. Mismatch errors in microwave power measurements, R. W. Beatty and A. C. Mac Pherson. Proc. IRE 41, 1112 (1953).

770. Modes of vibration of a suspended chain, D. S. Saxon and A. S. Cahn, Jr. Quart. J. Mech. and Appl. Math. 6, Pt. 3, 273 (1953).

771. National Bureau of Standards aircraft lighting, research, development and testing, U. S. Naval Aviation Electronics Digest, p. 25 (December 1953). (This article is unclassified by action of NavAer 2545 of 23 Mar 1954, although the Digest itself is classified.)

772. Natural and synthetic rubbers, N. Bekkedahl. Anal. Chem. 25, 54 (1953).

773. Noise comparator for microwaves, J. J. Freeman. Radio and TV News (Radio-Elec. Eng. Sect.) 49, No. 3, 11 (1953).

774. Normal fluid concentration in liquid helium II below $1^{\circ} \mathrm{K}, \mathrm{D}$. de Klerk, R. P. Hudson, and J. R. Pellam. Phys. Rev. 89, 662 (1953). 775. Notation for atomic spectra, W. F. Meggers and C. E. Moore. J. Opt. Soc. Am. 43, 422 (1953).

776. Note on the indentation of heavy leather, R. B. Hobbs. J. Am. Leather Chemists Assoc. 68,349 (1953).

777. Note on the rmal stabilization of austenite iron-carbon-nickel alloys, S. J. Rosenberg. Acta Met. 1, 376 (1953).

778. Nuclear magnetic resonance of aligned radioactive nuclei, N. Bloembergen and G. M. Temmer. Phys. Rev. 89, 883 (1953).

779. On a computation of the capacity of a cube, P. Davis and W. F. Cahill. Math. Tables and Other Aids to Computation VII, 272 (1953).

780. On absolute measurement, N. E. Dorsey and C. Eisenhart. Sci. Monthly 77, 103 (1953). 781. On over and under relaxation in the theory of the cyclic single step iteration, A. M. Ostrowski. Math.Tables and Other Aids to Computation 7, No. 43, 152 (1953).

782. On polya frequency functions. III, The positivity of translation determinants with an application to the interpolation problem by spline curves, I. J. Schoenberg and A. Whitney. Trans. Am. Math. Soc. 74, 246 (1953).

783. On smoothing operations and their generating functions, I. J. Schoenberg. Bull. Am. Math. Soc. 59, 199 (1953).

784. On some procedures for the rejection of suspected data, E. P. King. J. Am. Stat. Assoc. 48,531 (1953).

785. On the approximation of linear elliptic differential equations by difference equations with positive coefficients, T. S. Motzkin and W. Wasow. J. Math. and Phys. 31, 253 (1953). 786. On the derivative of a polynomial and Chebyshev approximation, T. S. Motzkin and J. L. Walsh. Proc. Am. Math. Soc. 4, 76 (1953). 
787. On the determination of critical micelle concentrations by a bubble pressure method, L. M. Kushner and W. D. Hubbard, J. Phys. Chem. 57, 898 (1953).

788. On the exact evaluation of the variances and covariances of order statistics in samples from the extreme-value distribution, J. Lieblein. Ann. Math. Stat. 24, No. 2, 282 (1953). 789. On the solution of the differential equation occurring in the problem of heat convection in laminar flow through a tube, M. Abramowitz. J. Math. and Phys. 32, 184 (1953).

790 . On the zeros of polynomials and the degree of linear systems, J. F. Koenig. J. Appl. Phys. 24, 476 (1953).

791. Pairs of matrices with property L, T. S. Motzkin and O. Taussky. Proc. Natl. Acad. Sci. 39, 961 (1953).

792. Pairs of normal matrices with property L, N. A. Wiegmann. Proc. Am. Math. Soc. 4,35 (1953).

793. Penetration and diffusion of hard X-rays: Polarization effects, L. V. Spencer and C. Wolff. Phys. Rev. 90, 510 (1953).

794. Permeability and some other properties of a variety of refractory materials, G. B. Massengale, L. E. Mong and R. A. Heindl. J. Am. Ceram. Soc. 36, 222 (1953).

795. Physical measurement and medicine, A. V. Astin. Med. Ann. Dist. Columbia 22,639 (1953).

796. Plastic electrets, H. H. Wieder and S. Kaufman. J. Appl. Phys. 24, 156 (1953).

797. Plastic electrets and their applications, H. H. Wieder and S. Kaufman. Elec. Eng. 72,511 (1953).

798. Poisson's ratio of aircraft sheet materials for large strains, S. Goodman and S. B. Russell. Proc. First Midwestern Conf. on Solid Mechanics (University of nlinois, Urbana, Illinois) p. 1 (1953).

799. Precision transistor oscillator, P. G. Sulzer. Radio and TV News (Radio-Elec. Eng. Sect.) 49 , No. 5, 18 (1953).

800. Preparation of bituminous materials for spinning, L. R, Kleinschmidt. ASTM Bull. No. 193,53 (1953).

801. Preparation of metallic iron of high purity, G. A. Moore. J. Metals 5, No. 11, 1443

(1953); Trans. Am. Inst. Mining and Metallurgical Engrs. (Mining Branch) 197, 1443 (1953). 802. Processing of two-dimensional patterns by scanning techniques, L. S. Kovasznay and H. M. Joseph. Science 118, 475 (1953).

803. Products volatilized from a channel and a furnace black on heating to $1,000^{\circ} \mathrm{C}, \mathrm{S}$. L. Madorsky, V. E. Hart, and S. Straus. Rubber Age 84, 238 (1953).

804. Propagation of errors in spectrophotometric colorimetry, I. Nimeroff. J. Opt. Soc. Am. 43, 531 (1953).

805. Pulp and paper, R. B. Hobbs and W. K. Wilson. Chem. Eng. News 31, 40 (1953). 806. Radiant glass heating panels, P. R. Achenbach. Heating and Ventilating 50, 83 (1953). 807. R. F. micropotentiometers, M. C. Selby. (Radio-Electronic Eng. Sect.) Radio \& TV News 50 , No. 4,5 (1953).
808. Radio wave scattering in tropospheric propagation, J. W. Herbstreit, K. A. Norton, P. L. Rice and G. E. Schafer. Convention Record of the IRE, Pt. 2, Antennas and communications, p. 85 (1953).

809. Radiophotoluminescence dosimetry system of the U. S. Navy, J. H. Schulman, W. Shurcliff, R. J. Ginther, and F. H. Attix. Nucleonics 11,52 (1953).

810. Radix table for obtaining hyperbolic and inverse hyperbolic functions to many places, H. E. Salzer. J. Math, and Phys. 32, 197 (1953).

811. Rates and activation energies of thermal degradation of styrene and acrylate polymers in a vacuum, S. L. Madorsky. J. Polymer Sci. 11,491 (1953).

812. Refractive index of potassium bromide for infrared radiant energy, R. E. Stephens, E. K. Plyler, W. S. Rodney, and R. J. Spindler. J. Opt. Soc. Am. 43, 110 (1953).

813. Rejection of outlying observations, F. Proschan. Am. J. Phys, 21, 520 (1953).

814. Removal of manganese prior to calcium and magnesium precipitations, B. L. Ingram and L. Bean. Anal. Chem. 25, 1217 (1953). 815. Representation of gas properties in terms of molecular clusters, H. W. Woolley. J. Chem. Phys. 21, 236 (1953).

816. Rotational and vibrational "temperatures" of $\mathrm{CH}$ in flames at atmospheric pressure, H. P. Broida. J. Chem. Phys. 21, 340 (1953).

817. Rotational intensity distributions of $\mathrm{OH}$ and $O D$ in electrodeless discharge through water vapor, H. P. Broida and W. R. Kane. Phys. Rev. 89, 1053 (1953).

818. Rotational "temperature" of $\mathrm{OH}$ in diluted flames, W. R. Kane and H. P. Broida. J. Chem. Phys, 21, 347 (1953).

819. Scientific teamwork in a Computation Laboratory, J. H. Curtiss. Teamwork in research, edited by G. P. Bush and L. H. Hattery, Chapter 17 (American University Press) (1953).

820. SEAC, S. Greenwald, R. D. Haueter, and S. N. Alexander. Proc. IRE 41, 1300 (1953). 821. Second sound propagation in liquid helium II, J. R. Pellam. Phys. Today 6, 4 (1953). 822 . Second sound velocity measurements below $1^{\circ} \mathrm{K}$, D. de Klerk, R. P. Hudson, and J. R. Pellam. Phys. Rev. 89, 326 (1953).

823. Semiconducting intermetallic compounds, R. G. Breckenridge. Phys. Rev. 90, 488 (1953).

824. Sequential decision problems for processes with continuous time parameter. Testing hypotheses, A. Dvoretsky, J. Kiefer, and J. Wolfowitz. Ann. Math. Stat. 24, No. 2, 254 (1953).

825. Sets of three measurements, W. J. Youden. Sci. Monthly 77, 143 (1953).

826. Signal generator system for low output levels, J. W. Herbstreit. Electronics 26, No. 1,218 (1953).

827. Silicate cement: method of mixing in a closed container to prevent effects of exposure to atmosphere, A. H. Grunewald, G. Dickson, G. C. Paffenbarger, and I. C. Schoonover. J. Am. Dental Assoc. 46, 184 (1953). 
828. Singular perturbation methods for nonlinear oscillations, W. Wasow. Proceedings of a Symposium on Nonlinear Circuit Analysis, held by the Polytechnic Institute of Brooklyn, N. Y. P. 75 (1953).

829. Slide rule computes radio refractive index of air, S. Weintraub. Electronics 26, No. 1, 182 (1953).

830. Solderless electronic assembly with cellular units, P. J. Selgin. Elec. Mfg. 52, 139 (1953).

831. Solving linear algebraic equations can be interesting, G. E. Forsythe. Bull. Am. Math. Soc. 59, 299 (1953).

832. Sorption of dextran to collodion membranes, S. Rothman, A. Schwebel, and S. G. Weissberg. J. Polymer Sci. 11, 381 (1953).

833. Specification of color-rendering properties of fluorescent lamps, C. W. Jerome and D. B. Judd. Ilum. Eng. 48, 259 (1953).

834. Spectral energy distribution of the International Commission on flumination light sources $A, B$, and C, R. Davis, K. S. Gibson, and G. W. Haupt. Condensation of this paper appeared in J. Opt. Soc. Am. 43, 172 (1953). 835. Standard samples, E. Wichers. Science 117,7 (1953).

836. Standards of temperature, R. E. Wilson. Phys. Today 6, 10 (1953).

837. Steric hindrance to coplanarity in o-fluorobenzidines, A. J. Bilbo and G. M. Wyman. J. Am. Chem. Soc. 75, 5312 (1953).

838. Stress and strain at onset of crazing of polymethyl methacrylate at various temperatures, M. A. Sherman and B. M. Axilrod. ASTM Bull. 191, 65 (1953).

839. Stress-solvent crazing of acrylics, B. M. Axilrod and M. A. Sherman. Modern Plastics 30,130 (1953).

840. Structural analys is and influence coefficients for delta wings, S. Levy. J. Aeronaut. Sci, 20, No. 7, 449 (1953).

841. Studies on the polar amino acid content of collagen and related material, J. M. Cassel, E. McKenna, and A. Glime. J. Am. Leather Chemists Assn. 48, 277 (1953).

842. Study of the burst test as applied to military upper leathers, E. B. Randall, C. Mann, J. R. Kanagy, and J. Mandel. J. Am. Leather Chemists Assoc. 48, 84 (1953).

843. Survey of experimental heat capacities of ten technically important gases, J. F. Masi. Trans. Am. Soc. Mech. Engrs,, p. 1067 (1953).

844. Symposium on Recent Developments in the Evaluation of Natural Rubber-Introduction, N. Bekkedahl, ASTM Spec, Tech, Pub. No. 136 , p. 1 (1953).

845. Synthetic textiles, W. D. Appel. Americana Annual 1, 697 (1953).

846. Synthetically bonded molding sand, M. T. Zemantowsky. Am. Foundryman 23, 57 (1953). 847. Systematic calculations of gamma-ray penetration, H. Goldstein, J. E. Wilkins, Jr., and L. V. Spencer. Phys. Rev. 89, 1150 (1953). 848. Teflon insert makes needle valve vacuum tight, L. J. Rueger. Rev. Sci. Instr. 24, 551 (1953).
849. Temperature coefficients of non-Newtonian viscosity at fixed shearing stress and at fixed rate of shear, A. B. Bestul and H. V. Belcher. J. Appl. Phys. 24, 696 (1953).

850. Tests for colorfastness of textiles under consideration in the International Organization for Standardization, W. D. Appel. Am. Dyestuff Reptr. 42, 871 (1953).

851. The action of boron in enhancing the hardenability of steel, T. G. Digges. SAE J. 61, 24 (1953).

852. The chain block design, W. J. Youden and

W. S. Connor. Biometrics 9, 127 (1953).

853. The coefficients of certain infinite products, M. Newman. Proc. Am. Math. Soc. 4, 435 (1953).

854. The color stability of direct filling resins, H. J. Caul and I. C. Schoonover. J. Am. Dental Assoc. 47, 448 (1953).

855. The constants in the equation for atmospheric refractive index at radio frequencies, E. K. Smith and S. Weintraub. Proc. IRE 41, 1035 (1953).

856. The convergence of numerical iteration, H. A. Antosiewicz and J. M. Hammersley. Am. Math. Monthly 60, 604 (1953).

857. The double description method, T. S. Motzkin, H. Raiffa, G. L. Thompson, R. M. Thrall. Contributions to the theory of games II, Edited by H. W. Kuhn and A. W. Tucker, Annals of Math. Study 28, Chapter 3 (Princeton, N. J.) (1953).

858. The effect of illuminance of a lens interposed between the source and the illuminated surface, R. E. Stephens. J. Opt. Soc. Am. 43,704 (1953).

859. The effect of sporadic-E on television reception, E. K. Smith. J. Radio-Electronics 24, No. 6, 54 (1953).

860. The emission spectrum of $\mathrm{OH}$ from 1.4 to 1. $7 \mu$, W. S. Benedict, E. K. Plyler, and C. J. Humphreys. J. Chem. Phys. 21, 398 (1953).

861. The freezing of water in pipes and vessels, R. S. Dill. Heating and Ventilating 50, 96 (1953).

862. The graduation of precise circles, B. L.

Page. Surveying and Mapping 13, 149 (1953). 863. The half-life of $I^{131}$, H. H. Seliger, L. Cavallo, and S. V. Culpepper. Phys. Rev. 90, 443 (1953).

864. The identification of solar lines, C. E. Moore. Chapter 4 of The Sun, Volume I of the Solar System, p. 186 (Edited by G. P. Kuiper, The University of Chicago Press, Chicago, Dlinois) (1953).

865. The limiting thickness of an electrolyzed gas film capable of sustaining a given negative pressure, L. J. Briggs. J. Chem. Phys. 21, 779 (1953).

866. The luminous reaction between carbon monoxide and atomic oxygen, H. P. Broida and A. G. Gaydon. Trans, Faraday Soc. 49, 1190 (1953).

867. The measurement of gas temperatures by immersion-type instruments, E. F. Fiock and A. I. Dahl. J. Am. Rocket Soc. 23, 155 (1953). 
868. The mechanism of formation of $\mathrm{OH}, \mathrm{CH}$, and $\mathrm{HCO}$ in flame spectra, using deuterium as tracer, H. P. Broida and A. G. Gaydon. Proc. Roy. Soc. (London, England) 218, 60 (1953). 869. The melting transition of polymethylene, L. Mandelkern, M. Hellmann, D. W. Brown, D. E. Roberts, and F. A. Quinn, Jr. J. Am. Chem. Soc. 75, 4093 (1953).

870. The monthly refractive gradient for the United States and its application to predicting the geographical and annual trend of VHF transmission loss, B. R. Bean and F. M. Meaney. Proc. Conf, Radio Meteorol. Supplement III-2, (The University of Texas, Austin, Texas) (1953). 871. Unassigned.

872. The NBS primary frequency standard, $J$. H. Shoaf. Radio and TV News 49, No. 1, 10 (1953).

873. Unassigned.

874. The non-Maxwellian distribution in a shock front and the anomaly of the chromospheric temperature, H. K. Sen. Phys. Rev. 92, 861 (1953).

875. The nuclear moments of technetium-99, K. G. Kessler and R. E. Trees. Phys. Rev. 92,303 (1953).

876. The number of farthest points, T. S. Motzkin, E. G. Straus, and F. A. Valentine. Pacific J. Math. 3, 221 (1953).

877. The piston gage as a precise measuring instrument, D. P. Johns on and D. H. Newhall. Trans. Am. Soc. Mech. Engrs. 75, 301 (1953). 878. Unassigned.

879. The preparation of two fluorinated p-dihalobenzenes, M. Hellmann and A. J. Bilbo, J. Am. Chem. Soc. 75, 4590 (1953).

880. The principles of experimental design, W. J. Youden. Appeared as a chapter in "Research Operations in Industry," (Papers delivered at the Third Annual Conference on Industrial Research, June 1953), P. 317 (Kings Crown Press, Columbia University, New York, N. Y.) (1953).

881 . The relation between the absorption spectra and the chemical constitution of dyes. XXVI. Effect of solvent and of temperature on the cistrans isomerization of azo dyes, W. R. Brode, J. H. Gould, and G. M. Wyman. J. Am. Chem. Soc. 75,1856 (1953).

882. The sieve problem for all-purpose computers, D. H. Lehmer. Math.Tables and Other Aids to Computation 7, 6 (1953).

883. The stiffness of paper, F. T. Carson and V. Worthington. Tappi 36, 36 (1953).

884. The structure of turbulence in fully developed pipe flow, J. Laufer. Nat. Advisory Comm. Aeronaut, Tech. Note 2954 (1953). 885. The SWAC-design features and operating experience, H. D. Huskey, R. Thorensen, B. F. Ambrosio, and E. C. Yowell. Proc. IRE 41,1294 (1953).

886. The testing of electrical instruments, F. L. Hermach. Proc. Instr. Soc. Am. 8, 18 (1953).

887. The use of zonal constants in the calculation of beam flux, T. H. Projector. nlum. Eng. 48, 189 (1953).

888. The $\mathrm{V}_{9}$ fundamental of $\mathrm{C}_{2} \mathrm{~F}_{6}, \mathrm{E}$. K. Plyler and D. E. Mann. J. Chem. 'Phys. 21, 1116 (1953).
889. The vapor pressures of the deuteromethanes, G. T. Armstrong, F. G. Brickwedde, and R. B. Scott. J. Chem. Phys. 21, 1297 (1953).

890. The variation of the spectrum of a normal matrix, A. J. Hoffman and H. Wielandt. Duke Math. J. 20, 37 (1953).

891. The vibration-rotation spectrum of $D_{2} O$, W. S. Benedict, N. Gailar, and E. K. Plyler. J. Chem. Phys. 21, 1301 (1953).

892. The vibration-rotation spectrum of HDO, W. S. Benedict, N. Gailar, and E. K. Plyler. J. Chem. Phys. 21, 1302 (1953).

893. The vibrational spectrum of chlorotrifluoroethylene, D. E. Mann, N. Acquista, and E. K. Plyler. J. Chem. Phys. 21, 1949 (1953). 894. The year 1952 in review, G. M. Kline. Modern Plastics 30, 111 (1953).

$895_{3}$ Thermal Rayleigh disk measurements in $\mathrm{He}^{3}-\mathrm{He}^{4}$ mixtures, B. Weinstock and J. R. Pellam. Phys, Rev. 89, 521 (1953).

896. Thermoelectric power in germanium single crystals, H. P. R. Frederikse. Phys, Rev, 91, 491 (1953).

897. Thermoelectric power of germanium below room temperature, H. P. R. Frederikse. Phys. Rev. 92, 248 (1953).

898. Thickness and compressibility of fabrics, H. F. Schiefer. ASTM Bull. No. 192, TP138, 48 (1953).

899. Transistor band spotter, P. G. Sulzer. Radio and Television News, Radio-Electronic Engineering Section, 49, No. 6, 52 (1953). 900. Transistor frequency standard, P. G. Sulzer. Electronics 36, 206 (1953).

901. Transmission loss in radio propagation, K. A. Norton. Proc. IRE 41, 146 (1953).

902. UHF magnetic attenuator, F. Reggia. Radio-Electronic Eng. Sect. Radio and TV News 49 , No. 4, 12 (1953).

903. Underearth crystal resonators, T. A. Pendleton. Proc. IRE 41, 1612 (1953).

904. Versatile goniometer for projection photometry, T. H. Projector. Ilum. Eng. 48, 192 (1953).

905. Unassigned.

906. Vibration- rotation spectrum of HDO, E. K. Plyler, W. S. Benedict and N. Gailar. J. Chem. Phys, 21, 1302 (1953).

907. Viscosity and electrical resistivity of molten alkali borates, L. Shartsis, W. Capps; and S. Spinner. J. Am. Ceram. Soc. 36, 319 (1953).

908. Voltage breakdown tester, W. E. Williams, Jr. Rev. Sci. Instr. 24, 458 (1953).

909. Vulcanization characteristics of natural rubber, R. D. Stiehler and F. L. Roth. Symp. on Recent Developments in the Evaluation of Natural Rubber, 1952; ASTM Spec. Tech. Pub. No. 136, p. 50 (1953); India Rubber World 127, 783 (1953) Rubber Chem. Tech. 26, 593 (1953). 910. Wave propagation in an anisotropic, inhomogeneous medium, J. Feinstein. J. Geophys. Research 58, 223 (1953).

911. Wide-range calibrator for vibration pickups, W. A. Yates and M. Davidson. Electronics 26, 183 (1953).

912. Zeros of the derivative of Bessel functions of fractional order, H. E. Salzer. Math. Tables and Other Aids to Computation VII, 69 (1953). 
913. A basic method of determining the dynamic characteristics of accelerometers by rotation, W. A. Wildhack and R. O. Smith. J. Instr. Soc. Am. 1, No. 12, paper No. 54 (1954).

914. A broadband, low-level, error-voltage detector, M. L. Kuder. Rev. Sci. Instr. 25, No. 5, 464 (1954).

915. A comparison of amplitude and angle modulation for narrow-band communication of binarycoded messages in fluctuation noise, G. F. Montgomery. Proc. IRE 42, 447 (1954).

916. A comparison of X-ray standards, H. O. Wyckoff, G. H. Aston, and E. E. Smith. British J. Radiol. 27, No. 318, 325 (1954).

917. A flex tension test for leather, T. J. Carter and J. R. Kanagy. J. Am. Leather Chemists Assoc. 49, 23 (1954).

918. A graphical interpretation of a three-components theory of chromatic adaptation in terms of the CIE chromaticity diagram, G. Wyszecki. J. Opt. Soc. Am. 44, 787 (1954).

919. A high vacuum seal-off valve, R. J. Richards. Rev. Sci. Instr. 25, 520 (1954).

920. A method for the study of the circulation in the dog using a mechanical left ventricle, J. C. Rose, H. P. Broida, C. A. Hufnagel, J. F. Gillespie, P. J. Rabile, and E. D. Freis. J. Appl. Physiol. 7, 580 (1954).

921. A multiple-purpose orthonormalizing code and its uses, P. Davis and P. Rabinowitz. J. Assoc. Computing Machinery 1, No. 4, 183 (1954). 922. A new method of analyzing extreme-value data, J. Lieblein. Natl. Advisory Comm. Aeronaut. Tech. Note 3053 (1954).

923. A note on partially balanced designs, M. Zelen. Ann. Math. Stat. 25, No. 3, 599 (1954).

924. A note on sweep-frequency backscatter observations, R. Silberstein. J. Geophys. Research 59, 138 (1954).

925. A note on the oxyquinolate determination of magnesium oxide in cement, L. Bean and N. J. Tucker. ASTM Bull. (TP224) No, 201, 62 (1954).

926. A plan for studying the accuracy and precision of an analytical procedure, F. J. Linnig, J. Mandel, and J. M. Peterson. Anal. Chem. 26, No. 7, 1102 (1954).

927. A pound of gas? H. S. Bean. Gas 30 , No. 12, 31 (1954).

928. A property of the normal distribution, E. Lukacs and E. P. King. Ann. Math. Stat. 25, No. 2, 389 (1954).

929. A pulsed magnetic extractor for removing the electron beam from a betatron, R. S. Foote and B. Petree, Rev. Sci. Instr. 25, 694 (1954). 930. A regular rhombohedral lattice sampling of Munsell renotation space, G. Wyszecki. J. Opt. Soc. Am. 44, 725 (1954).

931. A remark on the smoothing problem, H. A. Antosiewicz and A. J. Hoffman. Management Sci. 1, No. 1, 92 (1954).

932. A representation for solutions of analytic systems of linear differential equations, H. A. Antosiewicz and M. Abramowitz, J. Wash. Acad. Sci. 44, No, 12, 382 (1954).

933. A revised analysis of the solar spectrum from 2990 to $2635 \mathrm{~A}, \mathrm{~N}$. L. Wilson, R. Tousey, J. D. Purcell, F. S. Johnson, and C. E. Moore. Astrophys. J. 119, No. 3, 590 (1954).
934. A similarity principle in the backscattering of $\gamma$-rays, U. Fano. Radiation Research 1, No. 5,495 (1954).

935. A stable voltage controlled logarithmic attenuator, G. E. Boggs. Proc. IRE 42, 696 (1954).

936. A Stokes-parameter technique for the treatment of polarization in quantum mechanics, U. Fano. Phys, Rev, 93, 121 (1954).

937. A study of some operations involved in cement analysis, L. Bean and E. J. Hackney, ASTM Bull. No. 197, 43 (1954).

938. A technique for sweep frequency polarization measurements at low frequencies, E. L. Kilpatrick. J. Geophys, Research 59, 345 (1954).

939. A time-proportional electronic thermostat, F. A. Ransom. Cont. Eng. 1, 85 (1954). 940. Acceptance sampling of electroplated articles, J. M. Cameron and F. Ogburn, Plating 41, No. 1, 43 (1954).

941. Acoustic impedance of a right circular cylindrical enclosure, F. Biagi and R. K. Cook. J. Acoust. Soc. Am. 26, No. 4, 506 (1954). 942. Adiabatic apparatus for the study of selfheating of poorly conducting materials, W. H. Raskin and $A$. F. Robertson. Rev. Sci. Instr. 25,541 (1954).

943. Advancements in dielectrics, 1953, R. C. Powell and J. L. Dalke. Proc. IRE 42, 705 (1954).

944. American dielectric standards, R. C. Powell. Elec. J. 153, 1779 (1954).

945. American standard building code requirements for masonry, J. W. McBurney. Mag. of Standards 25, No. 11, 358 (1954).

946. An acoustic method for the measurement of vibration amplitudes, W. Koidan. J. Acoust. Soc. Am, 26, 428 (1954).

947. An electron interferometer, L. Marton, J. A. Simpson, and J. A. Suddeth. Rev. Sci. Instr. 25, No. 11, 1099 (1954).

948. An embedding theorem for balanced incomplete block designs, M. Hall, Jr., and W. S. Connor. Can. J. Math. 6, No. 1, 35 (1954).

949. Unassigned.

950. An improved optical test for spherical aberration, J. B. Saunders. Letter to Editor. J. Opt. Soc. Am. 44, No. 8, 664 (1954).

951. An integrating and differentiating bar-magnet velocity meter for use in ballistocardiography, T. A. Perls and C. W. Kissinger. Rev. Sci. Instr. 25, No. 10, 983 (1954).

952. An isoperimetric inequality for closed curves convex in even-dimensional Euclidean spaces, I. J. Schoenberg. Acta Math. (Uppsala, Sweden) 91, 143 (1954).

953. Analysis for some partially balanced incomplete block designs having a missing block, M. Zelen. Biometrics 10, No. 2, 273 (1954).

954. Analysis of brittle behavior in ship plates, M. L. Williams. ASTM Spec. Tech. Publ. No. 158,11 (1954).

955. Application of variational methods to intermediate and high energy scattering, E. Gerjuoy and D. S. Saxon. Phys. Rev. 94, 478 (1954). 
956. Approximations in linear viscoelasticity theory: Delta-function approximations, $\mathrm{H}$. Leaderman. J. Appl. Phys. 25, 294 (1954).

957. Asymptotic lower bounds for the frequencies of certain polygonal membranes, G. E. Forsythe. Pacific J. Math. 4, No. 2, 467 (1954).

958. Atoms and ions in the sun, C. E. Moore. Science 119, No. 3093, 449 (1954).

959. Backscattering of the cobalt- 60 gamma rays from infinite media, E. V. Hayward and J. H. Hubbell. J. Appl. Phys. 25, 506 (1954). 960. Becker value of manila rope by photoelectric reflectometry, S. B. Newman, H. K. Hammond, III, and H. F. Riddell. ASTM Bull. No. 199,84 (1954).

961. Betatron X-rays: How much concrete for shielding? F. S. Kirn and R. J. Kennedy. Nucleonics 12, 44 (1954).

962. Bias supplied for direct-coupled circuits, J. Reaves, Electronics 27, No. 8, 172 (1954).

963. Building and using dielectric amplifiers, A. Silverstein. Electronics 27, 150 (1954).

964. Cellular concretes. Part 1. Composition and methods of preparation, R. C. Valore, Jr. J. Am. Concrete Inst. 25, 773 (1954). Part 2. Physical properties. J. Am. Concrete Inst. 25, 817 (1954).

965. Certain fourier transforms of distribution: II, E. Lukacs and O. Szasz. Can. J. Math. 6, 186 (1954).

966. Chain block designs with two-way elimination of heterogeneity, J. Mandel. Biometrics 10,251 (1954).

967. Characteristic roots of quaternion matrices, O. Taussky. Archiv Der Mathematik (Wurzburg, Germany) 5, 99 (1954).

968. Characteristics of turbulence in a boundary layer with zero pressure gradient, P. S. Klebanoff. Nat1. Advisory Comm. Aeronaut. Tech. Note 3178 (1954).

969. Chemical education in Switzerland, R. G. Bates. The Capital Chemist 4, No. 5, 150 (1954).

970. Color and turbidity of sugar products, B. W. Liggett and V. R. Deitz. Advances in Carbohydrate Chem. 9, 247 (1954).

971. Combined translational and relaxational dispersion of sound in gases; M. Greenspan. J. Acoust. Soc. Am. 26, 70 (1954).

972. Comparative $100 \mathrm{mc}$ measurements at distances far beyond the radio horizon, A. P. Barsis. IRE Conv. Record, March 22-25, 1954, [2], Pt. 1, p. 98 (1954).

973. Comparison of sodium hydroxide and sulfuric acid methods for analys is of wool admixed with cotton and viscose rayon, E. Horowitz and J. Mandel. Am. Dyestuff Reprt. 43, No. 19, 613 (1954).

974. Comparison of theories and experiments for the hydraulic dam-break wave, R. F. Dressler. Proc. Intern. Union Geodesy and Geophys. (Rome, Italy) 3, 319 (1954).

975. Complex biorthogonality for certain sets of polynomials, P. Davis and H. Pollak. Duke Math. J. 21, No. 4, 653 (1954).

976. Composition of apparent shearing forces during shear degradation of polymers, A. B. Bestul. J. Appl. Phys. 25, 1069 (1954).
977. Continuous measurement of atmospherica ozone by an automatic photoelectric method, R. Stair, T. C. Bagg, and R. G. Johnston. Proc. Joint Army-Navy Air Force Conf. on Elastomer Research and Devel., January 12-13, 1954 (Washington, D. C.), Natl. Acad. Sci.-Natl. Research Council Publ. 370, p. 75 (1954).

978. Contractibility and convexity, H. W. Kuhn. Proc. Am. Math. Soc. 5, No. 5, 777 (1954).

979. Corrosion of aluminum alloys by exhaust gases, F. M. Reinhart. Corrosion 10, No. 12, 421 (1954).

980. Coulomb wave functions in the transition region, M. Abramowitz and H. A. Antosiewicz. Phys. Rev. 96, No. 1, 75 (1954).

981. Critical field phenomena in an isotropic paramagnetic crystal, E. Ambler and R. P. Hudson, Phys. Rev. 96, No. 4, 907 (1954).

982. Cross sections for charge transfer collisions of low energy ions in $\mathrm{N}_{2}$ and $\mathrm{O}_{2}, \mathrm{R}, \mathrm{F}$. Potter. J. Chem. Phys. 22, 974 (1954).

983. Crystallization in butadiene-styrene copolymers, L. A. Wood. J. Appl. Phys. 25, No. 7,851 (1954).

984. Current problems in refining with bone char, V. R. Deitz. Sugar J. 49, 44 (1954).

985. Depolymerization of polymethylene and polyethylene, L. A. Wall, S. L. Madorsky, D. W. Brown, S. Straus, and R. Simha. J. Am. Chem. Soc. 76, 3430 (1954).

986. Deterioration during storage of alginate hydrocolloidal dental impression material, K. R. Pfeiffer, J. L. Harvey, and G. M. Brauer. U. S. Armed Forces Med. J. 5, No. 9, 315 (1954).

987. Determination of inorganic constituents in sucrose solutions, A. Gee, L. P. Domingues, and V. R. Deitz. Anal. Chem, 26, 1487 (1954). 988. Determination of loads in the presence of thermal stresses, S. Levy. J. Aeronaut. Sci. 21, No, 10, 659 (1954).

989. Determination of viscosity of exhaust-gas mixtures at elevated temperatures, J. C. Westmoreland. Natl. Advisory Comm. Aeronaut. Tech. Note 3180 (1954).

990. Determination of water content in Freon12 circulating in a refrigeration system, A. W. Diniak, E. E. Hughes, and M. Fujii. Refrig. Eng. 62, No. 2, 56 (1954).

991. Die bestimmung thermodynamischer aciditatskonstanten, R. G. Bates and G. Schwarzenbach. Hevl. Chim. Acta (Basel, Switzerland) 37,1069 (1954).

992. Dielectric measurements up to $500^{\circ} \mathrm{C}$, symposium on temperature stability of electrical insulating materials, A. H. Scott, P. Ehrlich, and J. F. Richardson. Proc. Am. Soc. Testing Materials Spec. Tech. Publ. No, 161 (1954).

993. Differential cross-section measurement for 1-Mev bremsstrahlung, J. W. Motz and W. Miller. Phys. Rev. 96, 544L (1954).

994. Dimethylphosphinoborine trimer: mass spectra and thermal decomposition, R. E. Florin, L. A. Wall, F. L. Mohler, and E. Quinn. J. Am. Chem. Soc. 76, 3344 (1954). 995. Dose measurements in water using scintillation and ionization detectors, B. Zendle and H. W. Koch. Radiation Research 1, No. 6, 568A (1954). 
996. Drain casting of a high-grade magnesium oxide support used in an experimental vacuum tube, W. G. Haliday and J. R. Nall. Rev. Sci. Instr. 25, No. 12, 1225 (1954).

997. Effect of chemical combination of the characteristic energy loss of electrons, L. B. Leder and L. Marton. Phys. Rev. 95, 1345 (1954).

998. Effect of exposure to soils on the properties of asbestos-cement pipes, M. Romanoff and I. A. Denison. Corrosion 10, 169 (1954). 999. Effect of molecular weight on the crazing and tensile properties of polymethyl methacrylate, I. Wolock, M. A. Sherman, and B. M. Axilrod. Natl. Advisory Comm. Aeronaut. Research Memo 54AO4 (1954).

1000. Effect of sample preparation on precision in the hide substance determination, S. Dahl. J. Am. Leather Chemists Assoc. 49, 515 (1954).

1001. Effects of internal motion in the microwave spectrum of methylamine, D. R. Lide, Jr. J. Chem. Phys. 22, 1613 (1954).

1002. Effects of multiaxial stretching on crazing and other properties of transparent plastics, I. Wolock and D. A. George. Natl. Advisory Comm. Aeronaut. Research Memo 54F22 (1954).

1003. Effects of plastic and steel surfaces on clotting time of human blood, J. C. Rose and H. P. Broida, Proc, Soc. Exptl. Biol, Med. J. 86,384 (1954).

1004. Effects of resin coating methods and other variables on physical properties of glass-fabric reinforced polyesters, J. E. Wier, D. C. Pons, and B. M. Axilrod. Natl. Advisory Comm. Aeronaut. Research Memo 54G26 (1954).

1005. Einschliessung von Eigenwerten hermitescher Matrizen nach dem Abschnittsverfahren, H. Wielandt. Arch. Math. (Tübinger, Germany) 5, 108 (1954).

1006. Elastic scattering of photons, E. G. Fuller and E. V. Hayward. Phys. Rev. 94, 732 (1954).

1007. Electrical and optical properties of intermetallic compounds. I. Indium antimonide, R. G. Breckenridge, R. F. Blunt, W. R. Hosler, H. P. R. Frederikse, J. H. Becker, and W. Oshinsky. Phys. Rev. 96, 571 (1954); II. Gallium antimonide, R. F. Blunt, W. R. Hosler, and H. P. R. Frederikse. Phys. Rev. 96, 576 (1954); III. Aluminum antimonide, R. F. Blunt, H. P. R. Frederikse, J. H. Becker, and W. R. Hosler. Phys. Rev. 96, 578 (1954). 1008. Electrical measurements in the selection of bolt materials for service underground, W. J. Schwerdtfeger. Corrosion 10, 355 (1954). 1009. Electroforming, V. A. Lamb and W. H. Metzger. Tool Engr. 33, 55 (1954).

1010. Electroless plating comes of age, A. Brenner. Metal Finishing 52, No. 11, 68; No. 12, 61 (1954).

1011. Electron microscopy, M. Swerdlow. Anal. Chem. 26, 178 (1954).

1012. Electrothermic instruments for the measurement of alternating current and voltage, F. L. Hermach. Proc. Symp. Natl. Phys, Lab., Precisions of Electrical Measurement, Teddington, England, (H. M. Station Office, London, England) (1954).
1013. Emission spectroscopy, W. F. Meggers. Anal. Chem. 26, 54 (1954).

1014. Energy loss of electrons in passage through thin films, L. Marton and L. B. Leder. Phys. Rev. 94, 203 (1954).

1015. Energy spectrum resulting from electron slowing down, L. V. Spencer and U. Fano. Phys. Rev. 93, 1172 (1954).

1016. Energy transfer in hot gases, E. K. Plyler and W. S. Benedict. Reprint of "Highresolution spectra of hydro-carbon flames in the infrared" from Symp. at Washington, D. C. (1954).

1017. Estimation of correlation coefficients from scatter diagrams, G. R. Sugar. J. Appl. Phys. 25, 354 (1954).

1018. Evaluation of Coulomb wave functions along the transition line, M. Abramowitz and P. Rabinowitz. Phys. Rev. 96, No. 1, 77 (1954).

1019. Experiments in the solution of differential equations by Monte Carlo methods, J.

Todd. J. Wash. Acad. Sci. 44, No. 12, 377 (1954).

1020. Extending the range of the NBS stiffness tester, V. Worthington. Tappi 37, 384 (1954). 1021. Film badge dosimetry: how much fading occurs? W. L. McLaughlin and M. Ehrlich. Nucleonics 12, No. 10, 34 (1954).

1022. Gain stable mixers and amplifiers with current feedback, G. E. Boggs. Proc. IRE 42 , No. 7, 1145 (1954).

1023. Generalized commutators of matrices and permutations of factors in a product of three matrices, O. Taussky. Studies in Math. and Mechanics, p. 67 (Academic Press, Inc., New York, N. Y.) (1954).

1024. Geographic and temporal distribution of polar blackouts, V. Agy. J. Geophys. Research 59, No. 4, 499 (1954).

1025. Giauque-Kelving proposal, F. G. Brickwedde. Phys. Today 7, 4 (1954).

1026. Glass fiber papers, R. B. Hobbs. Sixth Annual Report of Tech. Assoc. Graphic Arts, p. 104 (1954).

1027. Heat content of lead from $0^{\circ}$ to $900^{\circ}$, and the heat of fusion, T. B. Douglas and J. L. Dever. J. Am. Chem. Soc. 76, 4824 (1954).

1028. Heats of wetting of collagen, leather, and other organic and fibrous materials, J. R.

Kanagy. J. Am. Leather Chemists Assoc. 49, 646 (1954).

1029. High-power pulser aids cathode studies, L. A. Marzetta, Electronics 27, 178 (1954). 1030. High-speed machine computation of ideal gas thermodynamic functions. I. The isotopic water molecules, A. S. Friedman and L. Haar. J. Chem. Phys. 22, 2051 (1954).

1031. High-speed stroboscope for accelerometer calibration, P. G. Sulzer, E. R. Smith, and S. Edelman. Rev. Sci. Instr. 25, No. 8, 837 (1954).

1032. High-temperature liquids, R. E. Flor in and T. W. Mears. Conf. on Reactor Heat Transfer, Report ENL 2446, 89, Brookhaven National Laboratories (Brookhaven, Long Island, N. Y.) (1954).

1033. Improved dip coater, A. G. Roberts and R. S. Pizer. Anal. Chem. 26, 790 (1954). 
1034. Improved stainless steel needle valve, A. R. Glasgow, Jr., and G. S. Ross. Anal. Chem. 26, 2003 (1954).

1035. Index of refraction of fused-quartz glass for ultraviolet, visible, and infrared wavelengths, W. S. Rodney and R. J. Spindler. J. Opt. Soc. Am. 44, No. 9, 677 (1954).

1036. Index to the literature on spectrochemical analysis. Part III-1946-1950 (226 pgs),

B. F. Scribner and W. F. Meggers. Am. Soc. Testing Materials Spec. Tech. Pub. 41-C (1954).

1037. Inelastic collisions and the Moliere theory of multiple scattering, U. Fano. Phys. Rev. 93, 117 (1954).

1038. Infrared absorption of liquid water from 2 to 42 microns, E. K. Plyler and N. Acquista. J. Opt. Soc. Am. 44, 505 (1954).

1039. Infrared photoconductivity due to neutral impurities in germanium, E. Burstein, J. W. Davisson, E. E. Bell, W. J. Turner, and $\mathrm{H}$. G. Lipson. Phys. Rev. 93, 65 (1954).

1040. Infrared spectrum of hydrogen sulfide, II. The $5100 \mathrm{~cm}^{-1}$ region, E. K. Plyler and H. C. Allen, Jr. J. Chem, Phys. 22, 1104 (1954).

1041. Instrumental drift, W. J. Youden. Science 120,627 (1954).

1042. Instrumentation--Revolution in industry, science, and warfare, W. A. Wildhack. Science 120, 15A (1954).

1043. Instruments for magnetic high frequency measurements, P. H. Haas, R. D. Harrington, R. C. Powell, and A. L. R asmussen. Wright Air Development Center Tech. Report 54-238 (1954).

1044. Intensity distributions in nitrogen bands excited in auroras and by high-energy protons and hydrogen atoms, L. M. Branscomb, R. J. Shalek, and T. W. Bonner. Trans. Am. Geophys. Union 35, 107 (1954).

1045. Introductory remarks on the dosimetry of ionizing radiations, U. Fano. Radiation Research 1, 3 (1954).

1046. Invariance of insidedness in projective transformations of the Maxwell triangle, G. Wyszecki. J. Opt. Soc. Am. 44, 524 (1954). 1047. Kinetics of crystallization in polymers, I. Bulk polymers, L. Mandelkern, F. A. Quinn, and P. J. Flory, Jr. J. Appl. Phys. 25,830 (1954).

1048. Laboratory standard microphones, R. K. Cook. Acustica (Zurich, Switzerland) 4, 101 (1954).

1049. Lead resistance errors in watthourmeter phantom load test, F. L. Hermach and T. L. Zapf. Elec. World 141, 113 (1954). 1050. Linear functional equations and interpolation series, P. Davis. Pacific J. Math. 4, No. 4, 503 (1954).

1051. Linear programming in bid evaluation, L. Gainen, D. J. Honig, and E. D. Stanley. Logistics Research Quart. 1, 48 (1954).

1052. Liquid level indicator for condensed gases at low temperature, W. E. Williams, Jr. and E. Maxwell. Rev. Sci. Instr. 25, No. 2, 111 (1954).

1053. Lithium fluoride; Heat content from $0^{\circ}$ to $900^{\circ}$, the melting point and heat of fusion, T. B. Douglas and J. L. Dever. J. Am. Chem. Soc. 76,4826 (1954).
1054. Location of the auroral absorption zone, V. Agy. J. Geophys, Research 59, 267 (1954). 1055. Low temperature physics, F. G. Brickwedde. J. Am. Chem. Soc. 76, 2030 (1954). 1056. Low-pass duplexing system for highfrequency pulse transmitters, W. L. Hartsfield and R. Silberstein. Tele-Tech, 13, 76 (1954).

1057. Magnetic and thermal properties of chromic methylamine alum below $1^{\circ} \mathrm{K}, \mathrm{R}, \mathrm{P}$. Hudson and C. K. McLane. Phys. Rev. 95, No. 4,932 (1954).

1058. Masonry cracking and damage caused by moisture expansion of clay tile, J. W. McBurney. Am. Soc. Testing Materials Proc. 54, 1219 (1954).

1059. Masonry requirements up to date, J. W. McBurney. Standards 25, No. 11, 358 (1954). 1060. Mass spectrometry, V. H. Dibeler.

Anal. Chem. 26, 58 (1954).

1061. Mathematical foundations and computational methods for a digital logic machine, R. S. Ledley. J. Operations Research Soc. Am. 2, No. 3, 249 (1954).

1062. Maximum usable frequencies and lowest usable frequencies for the path Washington to Resolute Bay, S. M. Ostrow. Letter to Editor, J. Geophys. Research 59, 3 (1954).

1063. Measurement and theoretical study of electrical conductivity and Hall effect in oxide cathodes, R. Forman. Phys. Rev. 96, No. 6, 1479 (1954).

1064. Measurement of burning velocities, E. F. Fiock. Section K, vol. 9, Princeton University Series on High Speed Aerodynamics and Jet Propulsion (Princeton, N. J.) (1954). 1065. Measurement of creep of acrylic glazing plastics exposed to temperature gradients, M. C. Slone and F. W. Reinhart. SPE J. 10, 29 (1954).

1066. Measurement of distortion produced in guarded electrostatic fields by grounded external plates, W. Miller and R. J. Kennedy. Rev. Sci. Instr. 25, 1031 (1954).

1067. Measurements of chromium potassium alum below $1^{\circ} \mathrm{K}, \mathrm{E}$. Ambler and R. P. Hudson. Phys. Rev. 95, 1143 (1954).

1068. Measuring circuit for radiometers, R. P. Teele. J. Opt. Soc. Am. 44, No. 11, 860 (1954).

1069. Mechanical properties of direct filling resins, W. T. Sweeney, W. D. Sheehan, and E. L. Yost. J. Am. Dental Assoc. 49, 513 (1954).

1070. Mechanical stops for use with helical potentiometers, P. D. Lowell, W. Hakkarinen, and L. M. Allison. Rev. Sci. Instr. 25, No. 4, 398 (1954).

1071. Message error in diversity frequencyshift reception, G. F. Montgomery. Proc. IRE 42, No. 7, 1184 (1954).

1072. Mesures dans l'infrareuge avec un prisme en iodure de cesium, E. K. Plyler. Extrait dur Journal de Physiquet et le Radium, (Paris, France) 15, 519 (1954).

1073. Meteorological instrumentation at the National Bureau of Standards, W. K. Gautier. Weatherwise 7, No. 4, 86 (1954).

1074. Microwave absorption by the nonpolar gas $\mathrm{CO}_{2}$, G. Birnbaum, A. A. Maryott, and P. F. Wacker. J. Chem. Phys. 22, 1782 (1954). 
1075. Microwave spectrum of benzonitrils, D. R. Lide, Jr. J. Chem. Phys, 22, 1577 (1954).

1076. Montecarlo calculations of $\gamma$-ray backscattering and transmission, M. J. Berger. Radiation Research 1, No. 5, 489 (1954).

1077. Motion of small particles in skew shape suspended in a viscous liquid, C. M. Tchen. J. Appl. Phys. 25, 463 (1954).

1078. Motion of the storm-D region, V. Agy. Nature 173, 445 (1954).

1079. Multiple scattering of relativistic electrons, L. V. Spencer and C. H. Blanchard. Phys. Rev. 93, 114 (1954).

1080. Nature of adhesion, F. W. Reinhart. J. Chem. Educ. 31, 128 (1954).

1081. New techniques for evaluating natural corundum ores, H. F. Car1, H. W. Jaffe, and A. Hockman. Mining Eng. 6, No. 4, 402 (1954).

1082. Note on the adjustment of Mooney viscometer die closure, G. E. Decker. ASTM Bull. No. 195, (TP13) (1954).

1083. Note on the Bragg-Gray cavity principle for measuring energy dissipation, U. Fano. Radiation Research 1, 237 (1954).

1084. Note on the circle theorem of hydrodynamics, E. Levin. Quart. Appl. Math. 12, No. 3, 315 (1954).

1085. Note on the strength of picric acid, R. G. Bates and G. Schwarzenbach. Experientia 10, 482 (1954).

1086. Nuclear elastic scattering of photons, E. V. Hayward and E. G. Fuller. Phys. Rev. 95, 1106 (1954).

1087. Nucleonics, C. L. Gordon. Anal. Chem. 26, 176 (1954).

1088. Obituary notice on L. F. Richardson, J. Todd. Math. Tables and Other Aids to Computation 8, 242 (1954).

1089. Observations in abrasion testing, J. R. Giles. Proc. Porcelain Enamel Inst. Forum 16,136 (1954).

1090. Observations on field exposures of enameled ranges, J. R. Crandall. Proc, of the Porcelain Enamel Inst. Forum 16, 27 (1954). 1091. On a theorem of Ostrowski and Taussky, R. Bellman and A. J. Hoffman. Archiv Der Mathematik 5, 123 (1954).

1092. On modified divided differences I, G. Blanch. Math. Tables and Other Aids to Computation 8, No. 45, 1 (1954).

1093. On representations and extension of bounded linear functionals defined on classes of analytic functions, P. Davis and J. L. Walsh. Trans. Am. Math. Soc. 76, No. 2, 190 (1954).

1094. On rotational viscous flow through a tube, P. Henrici. J. Appl. Math. and Phys. (Basel, Switzerland), V, No. 6, 511 (1954). 1095. On strongly continuous stochastic processes, E. Lukacs. Sankhya 13, 219 (1954). 1096. On the estimation of quadrature errors for analytic functions, P. Davis and P. Rabinowitz. Math. Tables and Other Aids to Computation 8 , No. 48,193 (1954).

1097. On the linear iteration procedures for symmetric matrices, A. M. Ostrowski. Rend. Mat. App. \{V\} VIII, No. 3-4, 140 (1954).

1098. On the practical evaluation of integrals, M. Abramowitz, J. Soc. Indust. App. Math. 2 , 20 (1954).
1099. On the solution of the caterer problem, J. W. Gaddum, A. J. Hoffman, and D.

Sokolowsky. Logistics Research Quart, 1, 223 (1954).

1100. On the spectrum of a one parametric family of matrices, A. M. Ostrowski. J. Reine Angew Math. (Berlin, Germany) 193, 143 (1954). 1101 . On two problems in abstract algebra connected with Horner's rule, A. M. Ostrowski. Studies in Math. and Mechanics, p. 40 (Academic Press, Inc., New York, N. Y.) (1954).

1102. Optimum grouping in one-criterior variance components analysis, E. P. King. J. Am. Stat. Assoc. 49, 637 (1954).

1103. Paper production on the upswing, R. B. Hobbs and W. K. Wilson. Chem. Eng. News 32, 40 (1954).

1104. Phosphorescence of atoms and molecules of solid nitrogen at $4.2^{\circ} \mathrm{K}, \mathrm{H}$. P. Broida and J. R. Pellam. Phys, Rev, 95, No. 3, 845 (1954).

1105. Photodetachment of the hydrogen negative ion, L. M. Branscomb and W. L. Fite. Phys. Rev. 93, 651 (1954).

1106. Photodisintegration of helium 4, E. G. Fuller. Phys. Rev. 96, 1306 (1954).

1107. Physical chemistry of synthetic rubbers, L. A. Wood. Chapter X in the book "Synthetic Rubber" p. 316 (John Wiley and Sons, Inc. , New York, N. Y.) (1954).

1108. Plastic deformation of ceramic-oxide single crystals, J. B. Wachtman, Jr., and L. H. Maxwell. J. Am. Ceram. Soc. 37, 291 (1954).

1109. Plastics literature in Government reports, J. Kanegis and I. Wolock. Am. Chem. Soc. Advances in Chem. Ser. No. 10, 151 (1954).

1110. Plate separation requirements for standard free-air ionization chambers, F. H. Attix and L. DelaVergne. Radiology 63, No. 6, 853 (1954). 1111. Precision quartz resonator frequency standards, J. H. Shaaf and J. M. Shaull. Proc. IRE 42, No. 8, 1300 (1954).

1112. Predictions of the present sunspot cycle, A. G. McNish and J. V. Lincoln. Trans. Am. Geophys. Union 35, 709 (1954).

1113. Preferred circuits manual with engineering notes, J. H. Muncy. Bu. Aeronaut. Report 3492 (1954).

1114. Pressure calibration of condenser microphones above $10,000 \mathrm{cps}, \mathrm{B}$. Simmons and F. Biagi. J. Acoust. Soc. Am. 26, 693 (1954).

1115. Pressure-volume temperature relations of liquid normal deuterium, A. S. Friedman, M. Trzeciak, and H. L. Johnston. J. Am. Chem. Soc. 76, 1552 (1954).

1116. Probability limits for the average chart when process standards are unspecified, E. P. King. Ind. Qual. Control 10, 62 (1954).

1117. Procedure used to improve the quality of ionospheric data, S. C. Gladden. Trans. Am. Geophysical Union 35, 398 (1954).

1118. Programs for computing hypergeometric series, W. F. Cahill. Math. Tables and Other Aids to Computation 8, 36 (1954).

1119. Prolonged space-wave fadeouts at 1, 046 Mc observed in Cheyenne Mountain propagation program, B. R. Bean. Proc. IRE 42, No. 5, 848 (1954). 
1120. Propagation constant in rectangular wave guide of finite conductivity, D. M. Kerns and R. W. Hedberg. J. Appl. Phys. 25, No. 12, 1550 (1954).

1121. Properties of fibers, W. D. Appel.

Smithsonian Physical Tables, p. 241 (Smithsonian Institution, Washington, D. C. ) (1954).

1122. Properties of plastic films, M. C. Slone and F. W. Reinhart. Modern Plastics 31, 203 (1954).

1123. Pulse-height measurements of recoils from $\mathrm{B}^{10}(\mathrm{n}, \alpha) \mathrm{Li}^{7}, \mathrm{~J}$. A. DeJuren and $\mathrm{H}$. Rosenwasser. Phys. Rev, 93, 831 (1954). 1124. Radioactivity of In 117 and Sbl17, C. L. McGinnis. Bull. Am. Phys. Soc. 29, No. 1, 43 A (1954).

1125. Radiology biology, U. Fano. Chapter I in the book, Principles of radiological physics (McGraw-Hill Publishing Co., New York, N. Y.) I, 1 (1954).

1126. Recent trends in glass analysis, F. W. Glaze. Am. Ceram. Soc. Bull. 33, 45 (1954).

1127. Regular and irregular Coulomb wave functions expressed in terms of Bessel-Clifford functions, M. Abramowitz. J. Math. Phys. 33, 111 (1954).

1128. Relation between net heat of combustion and aniline-gravity product of aircraft fuels, R. S, Jessup and J. A. Cogliano. ASTM Bull. No. 201, (T P217), 55 (1954).

1129. Report of the Committee on Atomic Weights of the American Chemical Society, E. Wichers. J. Am. Chem. Soc. 76, 2033 (1954).

1130. Research and standards for the plastics industry, G. M. Kline. Modern Plastics 31, No. 12,127 (1954).

1131. Review of some methods of flow measurement, W. A. Wildhack. Science 120, No. 3110 , 191 (1954).

1132. Rheology of polyisobutylene. I. Theory and experimental procedures, H. Leaderman, J. Polymer Sci. 13, 371 (1954); ii. Low molecular weight polymers, H. Leaderman, R. G. Smith, and R. W. Jones, J. Polymer Sci. 14, 47 (1954); and IV. Calculation of the retardation time function and dynamic response from creep data, H. Leaderman, Proc. Second Internat. Congress on Rheology, edited by V. G. W. Harrison (Butterworth's Scientific Publications, London, England) (1954).

1133. Role of nickel dip in the enameling of sheet steel, D. G. Moore, J. W. Pitts, and W. N. Harrison. J. Am. Ceram. Soc. 37, No. 8,363 (1954).

1134. Scale and reading errors of electrical indicators, F. D. Weaver. Instr, and Automation 27, No. 11, 1812 (1954).

1135. Scattered radiation contribution to depth dose data for 35-Mev and 90-Mev synchrotron $\mathrm{X}$-rays, J. W. Boag and B. Zendle. Phys, Rev. 95, 608A (1954).

1136. Scintillation spectrometers for measuring total energy of X-ray photons, R. S. Foote and H. W. Koch. Rev. Sci. Instr. 25, 746 (1954). 1137. Second sound attenuation in liquid helium II, W. B. Hanson and J. R. Pellam, Phys. Rev. 95, 321 (1954).
1138. Smithsonian Physical Tables, Vol. 120, Pub. 4169 prepared by W. E. Forsythe. Table 664 "Elements in the Sun's Atmosphere", C. E. Moore. (Table 620 "Solar Wave Lengths" and Tables 710, 711 "Binding Energies of Electrons' are from papers listed above.) (Smithsonian Institution, Washington, D. C.) (1954).

1139. Smooth patterns of production, A. J. Hoffman and W. Jacobs. Management Science 1, 86 (1954).

1140. Some applications of extreme-value theory, E. J. Gumbel and J. Lieblein. Am. Stat. 8, 14 (1954).

1141. Some aspects of the charge and discharge processes in lead-acid storage batteries, D. N. Craig and W. J. Hamer. Appl. and Ind. No. 11, 22 (1954).

1142. Some implications of Liapunov's conditions for stability, H. A. Antosiewicz and P. Davis. J. Rational Mechanics and Analysis 3, No. 4, 447 (1954).

1143. Some information theory aspects of propagation through time varying media, J. Feinstein. IRE Conv. Record, Mar. 22, 1954, [2] pt. 1, Antennas and Propagation, p. 87.

1144. Some ions of high kinetic energy in mass spectra of polyatomic molecules, F. L. Mohler, V. H. Dibeler, and R. M. Reese. J. Chem. Phys. 22, 394 (1954).

1145. Some remarks on commutators of matrices, K. Fan. Arch. Math. (Tubinger, Germany), 5,102 (1954).

1146. Some results of sweep-frequency investigation in the low frequency band, J. M. Watts and J. N. Brown. J. Geophys. Research 59, 71 (1954).

1147. Some stochastic problems in wave propagation, Part I, J. Feinstein. IRE Trans, Ant. Prop. AP-2, 23 (1954).

1148. Some theorems for partially balanced designs, W. S. Connor and W. H. Clatworthy. Ann. Math. Stat. 25, 100 (1954).

1149. Space charge wave amplification in a shock front and the fine structure of solar radio noise, H. K. Sen. Australian J. Phys. 7, 30 (1954).

1150: Spatial distribution of energy dissipation by high-energy X-rays, H. Brysk. Phys, Rev. 96 , No. 2, 419 (1954).

1151. State control of protection against ionizing radiation, L. S. Taylor. Am. J.

Roentgenol., Radium Therapy Nuclear Med. 71, 691 (1954); Acta Radiol. (Stockholm, Sweden) Suppl. 116, 21 (1954).

1152. Structure of some iridium-osmium alloys, H. C. Vacher, C. J. Bechtoldt, and E. Maxwell. J. Metals 6, No. 1, 80 (1954); Trans. Am. Inst. Mining Engrs. 200, 80 (1954).

1153. Studies of oxidation behavior of enameling iron during firing, A. G. Eubanks, J. C. Richmond, and D. G. Moore. Finish 11, No. 5, 50 (1954).

1154. Subharmonic crystal oscillators, M. C. Thompson, Jr., C. E. Tschiegg, and M. Greenspan. Rev. Sci. Instr. 25, 8 (1954). 1155. Summary of symposium on color of transparent and translucent products, D. B. Judd. ASTM Bull. No. 202, p. 72 (1954). 
1156. Surface tension, density, viscosity, and electrical resistivity of molten binary alkalineearth borates, L. Shartsis and H. F. Shermer. J. Am. Ceram. Soc. 37, No. 11, 544 (1954). 1157. Survey of adhesion and types of bonds involved, F. W. Reinhart. Chapter in a book "Adhesion and Adhesives, Fundamentals and Practice," p. 9 (John Wiley \& Sons, Inc., New York, N. Y.) (1954).

1158. Sweep frequency backscatter--some observations and deductions, R. Silberstein. IRE Trans. Ant. Prop. AP-2, No. 2, 56 (1954). 1159. Swelling of collagen and modified collagen, J. M. Cassel and E. McKenna. J. Am. Leather Chemists Assoc. 49, 553 (1954).

1160. Tables 142 to 144 , the rmal expansion, P. Hidnert and H. S. Krider. 9th Edition of the Smithsonian Phys. Tables (Smithsonian Institution, Washington, D. C. ) (1954).

1161. Tables of expected values of $1 / \mathrm{X}$ for positive Bernoulli and Poisson variables, E. Grab and I. R. Savage. J. Am. Stat. Assoc. 49,169 (1954).

1162. Temperature dependence of the microwave absorption of $\mathrm{NH}_{3}, \mathrm{G}$. Birnbaum and A. A. Maryott. J. Chem. Phys. 22, No. 8, 1457 (1954).

1163. Temperature measurements in highvelocity streams of hot gas, E. F. Fiock and A. I. Dahl. Proc. First Iowa Thermodynamics Symp., p. 190, State University of Iowa (Iowa City, Iowa)(1954).

1164. Temperature measurements in the Mooney viscometer, G. E. Decker and R. D. Stiehler. ASTM Bull. No. 195, (TP7) (1954).

1165. Temperatures developed in rotating dental cutting instruments, D. C. Hudson and W. T. Sweeney. J. Am. Dent. Assoc. 48, 127 (1954).

1166. Terminology for describing the performance of analytical and other precise balances, L. B. Maccurdy, H. K. Alber, A. A. Benedetti-Pichler, H. Carmichael, A. H. Corwin, R. M. Fowler, E. W. D. Huffman, P. L. Kirk, and T. W. Lashof. Anal. Chem. 26, 1190 (1954).

1167. Test methods, specifications and standards for plastics, G. M. Kline. Am. Chem. Soc. Advances in Chem. Ser. No. 10, 145 (1954).

1168. Testing a quick-weighing balance, T. W. Lashof and L. B. Macurdy. Anal. Chem. 26, 707 (1954).

1169. Testing of photographic lenses at the National Bureau of Standards, F. E. Washer. Photographic Eng. 5, No. 1, 37 (1954). 1170. The albedo of various materials for 1 Mev photons, E. V. Hayward and J. H. Hubbell. Phys. Rev. 93, 955 (1954).

1171. The attenuation of gamma rays at oblique incidence, F. S. Kirn, R. J. Kennedy, and H. O. Wyckoff. Radiology 63, 94 (1954). 1172. The calcium silicate hydrates, R. H. Bogue. Proc. International Congress of Industrial Chem,, Portland Cement Assoc. Fellowship Paper No. 69 (1954).

1173. The compressibility of liquid normal hydrogen from the boiling point to the central point at pressures up to 100 atmospheres, $H$. L. Johnston, W. E. Keller, and A. S. Friedman. J. Am. Chem. Soc. 76, 1482 (1954).
1174. The concentration dependence of the sedimentation constants of flexible macromolecules, M. Wales and K. E. Van Holde. J. Polymer Sci. 14, 81 (1954).

1175. The condition of certain matrices, II, J. Todd. Arch. Math. (Tubenger, Germany) 5,249 (1954).

1176. The decomposition of CF4 in flames, D. E. Mann, H. P. Broida, and H. P. Squires. J. Chem. Phys. 22, 348 (1954).

1177. The development of some infrared transmitting glasses, G. W. Cleek and E. H. Hamilton. Proc. of the Conference of Infrared Optical Materials, Filter and Films; Eng. Research and Dev. Laboratories; The Eng. Ctr. Ft. Belvoir, Va., p. 1 (1954).

1178. The disintegration of $\mathrm{Ba}^{113}$, R. W. Hayward, D. D. Hoppes, and H. Ernst. Phys. Rev. $93,916(1954)$.

1179. The disintegration of $\mathrm{Nb}^{91}$ and $\mathrm{Nb} 92$, R. W. Hayward, D. D. Hoppes, and H. Ernst. Bull. Am. Phys. Soc. 29, No. 7, 16A (1954). 1180. The distribution of linear energy transfer, or "ion density" for fast neutrons in water, J. W. Boag. Radiation Research 1, No. 4, 323 (1954).

1181. The drying twist in plant fibers, S. B. Newman and H. F. Riddell. Textile Research J. 24,113 (1954).

1182. The dynamic bulk viscosity of polyisobutylene, R. S. Marvin, R. Aldrich, and H. S. Sack. J. Appl. Phys. 25, No. 10, 1213 (1954). 1183. The dynamic mechanical properties of polyisobutylene, R. S. Marvin. Proc. Second Intern. Congr. Rheol., Edited by V. G. W. Harrison (Butterworth's Scientific Publications), (London, England) (1954).

1184. The effect of preheating on thermal expansion of silica-gypsum investments, G. F. Glasson, W. T. Sweeney, and I. C. Schoonover. J. Am. Dental Assoc. 48, 433 (1954).

1185. The Herzberg bands of $\mathrm{O}_{2}$ in an oxygen afterglow and in the night sky spectrum, H. P. Broida and A. G. Gaydon. Proc. Roy. Soc., Series[A] 222, 181 (1954).

1186. The influence of heat treating variables on the martensite transformation in SAE 1050 steel, M. R. Meyerson and S. J. Rosenberg. Trans. Am. Soc. Metals 45, 1225 (1954).

1187. The infrared spectrum of hydrogen sulfide in the $5100 \mathrm{~cm}^{-1}$ region, H. C. Allen, Jr., and E. K. Plyler. J. Chem. Phys. 22, 1104 (1954).

1188. The International Geophysical Year: A progress report, J. Kaplan and A. H. Shapley. News Report Natl. Research Council 4, No. 6, 1 (1954).

1189. The measurement of ionizing radiations for biological purposes, L. Marinelli and L. S. Taylor. Biological Effects of Radiation (McGrawHill Book Co., Inc., New York, N. Y.) (1954). 1190. The need for a new type of frequency and time standard, W. D. George. Proc. IRE 42 , 1349 (1954).

1191. The nightglow: a review of observational results, F. E. Roach. Proc. Intern. Union Geodesy and Geophys. (Rome, Italy) (1954). $1191 \mathrm{~A}$. The physical properties of terpenes. III. The vapor pressures of alpha pinene and beta pinene, J. E. Hawkins and $G_{3}$ T. Armstrong. 
1192. The preparation of some aryl silanes, M. Maienthal, M. Hellmann, C. P. Haber, L. A. Hymo, S. Carpenter, and A. J. Carr. J. Am. Chem. Soc. 76, 6392 (1954).

1193. Unassigned.

1194. The relation of occlusal surfaces to the stability of artificial dentures, R. S. Ledley. J. Am. Dental Assoc. 48, 508 (1954).

1195. The relative basicities of tribenzylamine and tribenzylamine oxide in benzene and water. Preparation and properties of tribenzylamine oxide, M. M. Davis and H. B. Hetzer. J. Am. Chem. Soc. 76, 4247 (1954).

1196. The relaxation method for linear inequalities, S. Agmon. Can. J. Math. 6, 382 (1954). 1197. The relaxation method for linear inequalities, T. S. Motzkin and I. J. Schoenberg. Can. J. Math. 6, 393 (1954).

1198. The representation of integers by binary quadratic rational forms, K. Goldberg, $M$. Newman, E. G. Straus, and J. D. Swift. Arch. Math. (Tübinger, Germany) 5, 12 (1954).

1199. The response of earphones in ears and couplers, M. D. Burkhard and E. L. R. Corliss. J. Acoust. Soc. Am. 26, No. 5, 679 (1954).

1200. The standardization of beta-emitting nuclides, H. H. Seliger and A. Schwebel. Nucleonics 12, No. 7, 54 (1954).

1201. The temperature-independent para-

magnetism of ammonium hexabromoosmate, IV, R. B. Johannesen and A. R. Lindberg. J. Am. Chem. Soc. 76, 5349 (1954).

1202. The theory of the three-crystal electron interferometer, J. A. Simpson. Rev. Sci. Instr. 25, No. 11, 1105 (1954).

1203. The thermal insulating value of airspaces, H. E. Robinson and F. J. Powlitch. Housing and Home Finance Agency (Washington, D. C.) Housing Research Paper No. 32 (1954).

1204. The transmission of monoenergetic positrons and electrons, H. H. Seliger. Phys. Rev. 95,610 A (1954).

1205. The vibrational spectra of n-dimethylaminodiborane, D. E. Mann. J. Chem. Phys. 22, 70 (1954).

1206. The vibrational spectrum of bromotrifluoroethylene, D. E. Mann, N. Acquista, and E. K. Plyler. J. Chem. Phys. 22, 1199 (1954). 1207. The viscosity, thermal conductivity, and Prandtl number for air, $\mathrm{O}_{2}, \mathrm{~N}_{2}, \mathrm{NO}, \mathrm{H}_{2}, \mathrm{CO}$, $\mathrm{CO}_{2}, \mathrm{H}_{2} \mathrm{O}, \mathrm{He}$ and $\mathrm{A}$, J. Hilsenrath and ${ }^{2} \mathrm{Y}$. S. Touloukian, Trans. Am. Soc, Mech. Engrs. 76 , No. 6, 967 (1954).

1208. The Zeeman effect in the first spectrum of hafnium, W. F. Meggers. J. Opt. Soc. Am. 44,348 (1954).

1209. Thermal degradation of polystyrene and polyethylene, S. L. Madorsky. J. Polymer Sci. 13, 185 (1954).

1210. Unassigned.
1211. Thick target bremsstrahlung spectra for 1.00-, 1.25-, and 1.40- Mev electrons, W. Miller, J. W. Motz, and C. Cialella. Phys. Rev. 96, No. 5, 1344 (1954).

1212. Threshold field properties of some superconductors, E. Maxwell and O. S. Lutes. Phys. Rev. 95, 333 (1954).

1213. Total-absorption X-ray spectrometry, application to betatron experiments, H. W. Koch and R. S. Foote. Nucleonics 12, 51 (1954). 1214. Transmission of reverberant sound through walls, R. V. Waterhouse. Acustica 4,290 (1954).

1215. Transport processes as functions of the Heisenberg and Obukoff theories of turbulence, C. M. Tchen. Phys, Rev. 93, 4 (1954).

1216. Transverse strength testing machine for denture resins, W. T. Sweeney, H. J. Caul, and W. Gneug. J. Am. Dental Assoc. 49, 174 (1954).

1217. Triathanolamin als puffersubstanz, R. G. Bates and G. Schwarzenbach. Chim. Acta 37, 1439 (1954).

1218. Turbulent processes as observed in boundary layer and pipe, G. B. Schubauer. J. Appl. Phys. 25, 188 (1954).

1219. Two early papers on the relation between extreme values and tensile strength, J. Lieblein. Biometrika (London, England) 41, 559 (1954).

1220. Use of deuterium to investigate extent of branching in polystyrene, L. A. Wall and D. W. Brown. J. Polymer Sci. 14, No. 78, 513 (1954).

1221. Ueber die Funktionen von Gegenbauer, P. Henrici. Arch. Math. (Tubinger, Germany) 5,92 (1954).

1222. Values of physical constants of different rubbers, L. A. Wood. Section of Smithsonian Physical Tables, 9th Ed. Edited by W. E. Forsyth (Smithsonian Institution) Washington, D. C.) (1954).

1223. Variational principles for the acoustic field, E. Gerjuoy and D. S. Saxon. Phys. Rev. 94 , No. 6, 1445 (1954).

1224. Vibrational spectra of trifluorethylene and trifluoroethylene-d, D. E. Mann, N. Acquista, and E. K. Plyler. J. Chem. Phys. 22,1586 (1954).

1225. Viscometric and turbidimetric measurements on dilute aqueous solutions of a nonionic detergent, L. M. Kushner and W. D. Hubbard. J. Phys. Chem. 58, 1163 (1954).

1226. Water demand rates for electric drinkingwater coolers of the bubbler type, P. R. Achenbach and C. W. Phillips. Refrig. Eng. , 62, No. 12,37 (1954).

1227. What is the heat pump? P. R. Achenbach. Parents Mag. 29, 56 (1954).

1228. Zeeman effect in spark lines of ruthenium, W. F. Meggers and G. R. Harrison. J. Opt. Soc. Am. 43, 816 (1954). 
1229. A cavity ionization theory including the effects of energetic secondary electron, L. V. Spencer and F. H. Attix. Radiology 64, No. 1, 113 (1955).

1230. A comparison of X-ray standards, H. O. Wyckoff and G. H. Aston. Acta Radiol. Suppl. 117,17 (1955).

1231. A comparison theorem for eigenvalues of normal matrices, K. Fan. Pacific J. Math. 5, Suppl. 2, 911 (1955).

1232. A determinantal inequality, K. Fan and J. Todd. J. London Math. Soc. 30, 58 (1955). 1233. A dynamic spectrum analyzer for Sacramento Peak, D. H. Menzel, J. W. Warwick, and $R$. S. Lawrence. Harvard College Observatory (Cambridge, Massachusetts), Scientific Report 20 (1955).

1234. An extremum property of sums of eigenvalues, $H$. Wielandt. Proc. Am. Math. Soc. 6, No. 1, 106 (1955).

1235. A graphical method for single-point determination of intrinsic viscosity, V. E. Hart. J. Polymer Sci. 17, 215 (1955).

1236. A microwave microcalorimeter, A. C. MacPherson and D. M. Kerns. Rev. Sci. Instr. 14, 27 (1955).

1237. A note on group matrices, O. Taussky. Proc. Am. Math. Soc. 6, 984 (1955).

1238. A note on the estimation of sodium carbonate in photographic developers, C. I. Pope. J. Soc. Motion Picture Television Engr8. 64, 420 (1955).

1239. A note on the preparation of solid ozone and atomic oxygen, H. P. Broida and J. R. Pellam. J. Chem. Phys. 23, 409 (1955). 1240. A pilot plant study of the process for treating heavy leather with polyisobutylene and other polymers, R. Oehler, J. H. Davis, and R. A. Kinmouth. J. Am. Leather Chemists Assoc. 50, 16 (1955).

1241. A proposed index of current noise for composition resistors, G. T. Conrad, Jr. Proc. Electronic Components Symp. 118. (Sponsored by Am. Inst. Elec. Engr. New York, N. Y.) (1955).

1242. A review of observational results in airglow photometry, F. E. Roach. Annales Geophys. (Paris, France) II, No. 2, 214 (1955).

1243. A review of pressure drop across columns of bone char, F. G. Carpenter. Intern. Sugar J. 57,12 (1955).

1244. A revision of American Dental Association specification No. 3 for dental impression compound, J. W. Stanford, G. C. Paffenbarger, and W. T. Sweeney. J. Am. Dental Assoc. 51, 56 (1955).

1245. A semi-micro dilution viscometer, V. E. Hart. J. Polymer Sci. 17, 207 (1955).

1246. A theorem on alternatives for pairs of matrices, H. A. Antosiewicz. Pacific J. Math. 5, Suppl. 1, 641 (1955).

1247. A theory of cavity ionization, F. H.

Attix and L. V. Spencer. Radiol. Research 3, 239 (1955).

1248. A tree from the viewpoint of lightning, F. M. Defandorf. J. Wash. Acad. Sci. 45, 11 (1955).

1249. Abrasive jet method for measuring abrasion resistance of organic coatings, A. G. Roberts, W. A. Crouse, and R. S. Pizer. ASTM Bull. No. 208, 36 (1955).
1250. Absorption by concrete of X-rays and gamma-rays, B. E. Foster. BuDocks Technical Digest No. 55 and 56 (May 1955).

1251. Accelerated aging of record papers compared with normal aging, W. K. Wilson, J. L. Harvey, J. Mandel, and T. L. Worksman. Tappi 38, No. 9, 543 (1955).

1252. Activities of the National Bureau of Standards Cryogenic Engineering Laboratory, R. B. Scott. Conf, de physique des basses temperatures, September 2-8, 1955, 368 (Union Internationale de Physique pure et appliquee Institut Internationale du Froid) (Paris, France) (1955).

1253. Additional theorems for general Legendre and Gegenbauer functions, P. Henrici. J. Rational Mech. Anal. 4, No. 6, 983 (1955). 1254. Aerodynamical mechanisms producing electronic density fluctuations in turbulent ionized layers, R. M. Gallet. Proc. IRE 43, 1240 (1955).

1255. Alignment of cerium ${ }^{141}$ and neodynium 147 E. Ambler, R. P. Hudson, and G. M. Temmer. Phys. Rev. 97, 1212 (1955).

1256. Alignment of three rare-earth isotopes, E. Ambler, R. P. Hudson, and G. M. Temmer. Bull. Intern. Inst. Refrig., Commun. 143, 255 (1955).

1257. American Dental Association specification No. 13 for self-curing repair resins, J. W. Stanford, C. L. Burns, and G. C. Paffenbarger. J. Am. Dental Assoc. 51, 425 (1955).

1258. Amplitude, scale, and spectrum of refractive index inhomogeneities in the first 125 meters of the atmosphere, G. Birnbaum and H. E. Bussey. Proc. IRE 43, 1412 (1955). 1259. An algebraic proof of the isoperimetric inequality for polygons, K. Fan, O. Taussky, and J. Todd. J. Wash. Acad. Sci. 45, No. 11 , 334 (1955).

1260. An alternative proof of a theorem on unimodular groups, M. Newman. Proc. Am.

Math. Soc. 6, 998 (1955).

1261. An evaluation of electronic data processing equipment, S. N. Alexander. Proc. Automatic Data Processing Conf. (Harvard Graduate School of Business Administration, Cambridge, Massachusetts) (1955).

1262. An identity for the coefficients of certain modular forms, M. Newman. J. London Math. Soc. 30,488 (1955).

1263. Analysis of fluorinated polyphenyls by mass spectrometer, P. Bradt and F. L. Mohler. Anal. Chem. 27, 875 (1955).

1264. Analytic study of war games, H. A. Antosiewicz. Naval Research Logistics, Office of Naval Research (Washington, D.C.) 2, No. 3, 181 (1955).

1265. Angular distribution of multiple-scattered gamma radiation from a plane isotropic source, M. J. Berger. J. Appl. Phys. 26, No. 12, 1504 (1955).

1266. Application of photoelasticity to the design of tensile specimens for ceramic metal materials, M. B. Stiefel. Bull. Am. Ceram. Soc. 34 , No. 5, 133 (1955).

1267. Application of two methods of numerical analysis to the computation of the reflected radiation of a point source, P. Henrici. J. Wash. Acad. Sci. 45, 38 (1955). 
1268. Atomic flames: Spectra "temperatures" and products, R. E. Ferguson and H. P. Broida. Fifth Symposium (International) on Combustion (Rheinhold Pub. Corp., New York, N. Y.) p. 754 (1955).

1269. Atomic spectra, C. E. Moore. Chapter 19 of Fundamental Formulas of Physics, D. H. Menzel, Ed. p. 451 (Prentice-Hall, Inc., New York, N. Y.) (1955).

1270. Unassigned.

1271. Aurora and airglow, C. T. Elvey and F. E. Roach. Sci. American 193, No. 3, 140 (1955).

1272. Automatic computation of nerve excitation, K. S. Cole, H. A. Antosiewicz, and P. Rabinowitz. J. Soc. Indust. Appl. Math. 3, 153 (1955).

1273. Automatic cut-off valve for ion-exchange columns, C. A. Hewitt. Anal. Chem. 27, 865 (1955).

1274. Automatic instrument for electron scattering measurements, L. Marton, J. A. Simpson, and T. F. McCraw. Rev. Sci. Instr. 26, No. 9, 855 (1955).

1275. Begrundung fur die Beschaftigung mit numerischer Analysis, Yahres, J. Todd. Bericht de DMV (Tubingen, Germany) 58, No. 1, 11 (1955). (Translation of "Motivation for working in numerical analysis").

1276. Unassigned.

1277. Breaking velocities, strain energies, and theory neglecting wave propagation, Part II, F. L. McCrackin, H. F. Schiefer, J. C. Smith, and W. K. Stone. Textile Research J. 25,529 (1955).

1278. Bremsstrahlung differential cross-section measurements for $0.5-$ and $1.0-$ Mev electrons, J. W. Motz. Phys, Rev. 100, No. 6, 1560 (1955).

1279. Building research in the U. S. before 1947, D. E. Parsons. Proc. Conference on Building Research, October 21-23, 1953. Bull. 1 of Div, of Bldg. Research, Natl. Research Council, Ottawa, Canada, p. 1 (1955).

1280. Can we legislate ourselves into radiation safety? L. S. Taylor. Nucleonics 13, No. 3, 17 (1955).

1281. Capacity of plumbing stacks, R. S.

Wyly and H. N. Eaton. The Plumber and J. of Heating (London, England) 76, No. 912, 26 (1955).

1282. Cathode film studies by the drainage method, A. Brenner and G. Wranglen. Sartrych ur Svensk Kemish Tidskrift (Sweden) 67, 81 (1955).

1283. Cavity techniques for permeability measurements in the VHF region, R. D. Harrington. 6th Electronic Components Conf. Proc. May 27-29, 1955 (Los Angeles, Calif.), p. 27 (1955).

1284. Cement, R. H. Bogue. Encyclopedia Americana 6, 188; Portland Cement Association Fellowship Paper No. 68 (1955).

1285. Ceramic coatings for nuclear reactors (a progress report), J. C. Richmond, H. G. Lefort, C. N. Williams, and W. N. Harrison. J. Am. Ceram. Soc. 38, No. 2, 72 (1955).
1286. Chaines de Markoff dans les ensenbles abstraits et applications aux processus avec. regions absorbantes et au probleme des boucles (formerly, Time-discrete stochastic processes in arbitrary sets, with applications to processes with absorbing regions and to the problems of loops in Markoff chains), D. van Dantzig. Annales Inst. H. Poincare (Paris, France) 14, 145 (1955).

1287. Characteristic energy losses of electrons in solids, L. Marton, L. B. Leder, and H. Mendlowitz. Advances in Electronics and Electron Phys. 7, 183 (1955).

1288. Unas signed.

1289. Color and chemical constitution, W. R. Brode. Am. Scientist 43, No. 2, 259 (1955). 1290. Comparison of methods for measuring the tensile and tear properties of plastic films, F. W. Reinhart and J. Mandel. ASTM Bull. No. 209, 50 (1955).

1291. Components for mechanized production, B. L. Davis. Radio-Electronic Eng. 24, No. 3, 12 (1955).

1292. Compressibility of binary alkali borate and silicate glasses at high pressures, C. E. Weir and L. Shartsis. J. Am. Ceram. Soc. 38, No. 9, 299 (1955).

1293. Computation of vibration modes and frequencies on SEAC, W. F. Cahill and S. Levy. J. Aeronaut. Sci. 22, No. 12, 837 (1955). 1294. Continuous analys is of ortho-parahydrogen mixtures, D. H. Weitzel and L. E. White. Rev. Sci. Instr. 26, 290 (1955).

1295. Unassigned.

1296. Contribution to the study of transport phenomena in gases at high densities, A. Michels, J. A. M. Cox, A. Botzen, and A. S. Friedman. J. Appl. Phys. 26, 843 (1955). 1297. Corrosion research at the National Bureau of Standards, J. G. Thompson. Corrosion Technology (London, England)2, 102 (1955).

1298. Crazing of acrylic resins, W. T. Sweeney, G. M. Brauer, and I. C. Schoonover. J. Am. Dental Research 34, 306 (1955).

1299. Creep and creep rupture characteristics of some riveted and spot-welded lap joints of aireraft materials, L. Mordfin. Natl. Advisory Comm. Aeronaut. Tech. Note 3412 (1955).

1300. Cross-field error in unsymmetrical range finders, R. E. Stephens. J. Opt. Soc. Am. 45, No. 3,146 (1955).

1301. Cryogenics, F. G. Brickwedde, R. P. Hudson, and E. Ambler. Ann. Rev. Phys. Chem. 6, 25 (1955).

1302. Crystal-stabilized pulse-pair generator, M. C. Thompson, Jr. Rev. Sci. Instr. 26, 617 (1955).

1303. Cumulative fatigue damage of axially loaded Alclad 75S-T6 and Alclad 24S-T3 aluminum-alloy sheet, I. Smith, D. M. Howard, and F. C. Smith. Natl. Advisory Comm. Aeronaut. Tech. Note 3923 (1955).

1304. Cyclic strain testing machine for leather, T. J. Kilduff and J. R. Kanagy. J. Am. Leather Chemists Assoc. 50, No. 10, 489 (1955). 
1305. Dangers of radiation, R. J. Nelsen and S. W. Smith. J. Am. Dental Assoc. 50, No. 5,581 (1955).

1306. Dental cements, G. C. Paffenbarger, W. T. Sweeney and P. J. Schoubee. Intern. Dental J. 5, 584 (1955).

1307. Dental materials specifications and certification annual for 1954, G. C. Paffenbarger and W. T. Sweeney. Am. Dental Assoc. Publication (1955).

1308. Determination of aldehyde in cellulose: A review of methods, W. K. Wilson. Tappi 38, 274 (1955).

1309. Determination of carbon monoxide in studies of air pollution, M. Shepherd, S. Schuhmann, and M. Kilday. Anal. Chem. 27, 380 (1955).

1310. Determination of glucose by means of sodium chlorite, H. F. Launer, W. K. Wilson, and J. H. Flynn. Indian Pulp and Paper 9, 399 (1955).

1311. Determination of sulfur in rubber vulcanizates, E. W. Zimmerman, V. E. Hart, and E. Horowitz. Anal. Chem. 27, No. 10, 1606 (1955).

1312. Direct observation of nuclear absorption and elastic scattering of X-rays, H. W. Koch. Proc. 1954 Glasgow Conference on Nuclear and Meson Physics (Pergamon Press, New York, N. Y.) (1955).

1313. Discrete analogs of inequalities of Wirtinger, K. Fan, O. Taussky, and J. Todd. Monatsh. Mathematik (Vienna, Austria) Bd. $59 / 2$ (1955).

1314. Discrete approximations to elliptic differential equations, W. Wasow. Zeit. angew Math. Phys. (Basil, Switzerland) 6, Pt. 2, 81

(1955). Disintegration of $5.8^{\mathrm{d}} \mathrm{Sb}^{120}$, C. L. McGinnis, Bul. Am. Phys. Soc. 30, No. 1, 33A (1955).

1316. Disintegration of selenium-73, R. W. Hayward and D. D. Hoppes. Bull. Am. Phys, Soc. 30 , No. 1, 33A (1955).

1317. Distribution of electron scattering by gold, L. Marton, J. A. Simpson, and T. F. McCraw. Phys. Rev. 99, No. 2, 495 (1955).

1318. Distribution of phase diagrams of interest to ceramists, E. Levin. Bull. Am. Ceram. Soc. 34, No. 12, 399 (1955).

1319. Dose to walls in radiographic rooms, S. W. Smith and R. J. Kennedy. Radiology 64, $114 \mathrm{~A}$ (1955).

1320. Double scattering of electrons with magnetic interaction, $H$. Mendlowitz and $K$. M. Case. Phys. Rev. 97, 33 (1955).

1321. Dry cell standards broadened--now includes mercury-, air-, and flat-type cells, J. P. Schrodt and W. J. Hamer. Mag. of Standards 26, No. 5, 132 (1955).

1322. Dynamics in the inlet system of a fourstroke single-cylinder engine, C. F. Taylor, J. C. Livengood, and D. H. Tsai. Trans. Am. Soc. Mech. Engrs. 77, No. 7, 1133 (1955). 1323. Eddy-current mutual-inductance transducers with high-conductivity reference plates, H. M. Joseph and N. Newman. Commun. and Electronics (published by Am. Inst. Elec. Engr 8.) Paper 55-4, No. 17, p. 1 (1955).
1324. Education in radiation protection, L. S. Taylor. Janeway Lecture, 1954, Am. J.

Roentgenol. Radium Theraphy Nuclear Med. 73, 193 (1955).

1325. Effect of an anodic (HAE) coating on the fatigue strength of magnesium alloy specimens, J. A. Bennett. Am. Soc. Testing Materials Proc. 55, 1015 (1955).

1326. Effect of dis sociation on thermodynamic properties of pure diatomic gases, H. W. Woolley. Natl. Advisory Comm. Aeronaut. Tech. Note 3270 (1955).

1327. Effect of light on coated groundwood papers, W. K. Wilson and J. L. Harvey. Indian Pulp and Paper 9, 444 (1955).

1328. Effect of temperature and degree of polymerization on the sorption of water by polymethyl methacrylate, G. M. Brauer and W. T. Sweeney. Modern Plastics 32, No. 7, 138 (1955).

1329. Effect of the oxygen content of the furnace atmosphere on the adherence of vitreous coatings to iron, A. G. Eubanks and D. G. Moore. J. Am. Ceram. Soc. 38, No. 7, 226 (1955). 1330. Electrical and optical properties of intermetallic compounds. IV. Magnesium stannide, R. F. Blunt, H.P.R. Frederikse, and W. R. Hosler. Phys. Rev. 100, No. 2, 663 (1955). 1331. Electrodeposition of some of the less common metals from nonaqueous media, A. Brenner. Record of Chem. Progr. 16, No. 4, 241 (1955). 1332. Electrolytic coatings on magnesium base alloys from alkaline chromate solutions, $F$. Ogburn, H. I. Salmon, and M. L. Kronenberg. Plating 42, 271 (1955).

1333. Electrolytic preparation of molybdenum from fused salts, IV. Preparation of reduced molybdenum chlorides from molybdenite concentrate, S. Senderoff and R. J. Labrie. J. Electrochem. Soc. 102, 77 (1955).

1334. Electron affinity of atomic oxygen, L. M. Branscomb and S. J. Smith. Phys. Rev. 98, No. 4, 1127 (1955).

1335. Energy losses of electrons and their influence on resolving power, L. Marton, L. B. Leder, H. Mendlowitz, J. A. Simpson, and C. Marton. Proc. of the Toulouse Conference, Centre National de la Recherche Scientifique (Paris, France) p. 175 (1955).

1336. European experience with electronic computers, S. N. Alexander. First Proc., Electronics in Management (American University, Washington, D. C.) (1955).

1337. Evaluation of the Boor-Quartermaster snag tester for coated fabrics and plastic films, F. W. Reinhart, L. Boor, C. Brown, and J. J. Lamb. ASTM Bull. No. 210, 50 (1955).

1338. Experimental cross section for photodetachment of electrons from $\mathrm{H}-$ and $\mathrm{D}-$, L. M. Branscomb and S. J. Smith. Phys. Rev. 98, No. 4, 1028 (1955).

1339. Experimental evidence for the existence of abnormal OH rotational "temperatures" in low pressure flames, H. P. Broida and H. J. Kostkowski. J. Chem. Phys. 23, 754 (1955). 1340. Experimental temperature measurements in flames and hot gases, H. P. Broida. Temperature, Its Measurement and Control in Science and Industry (Reinhold Pub. Co., New York, N. Y.) 2, 265 (1955). 
1341. Extension of the Sen-White paper on atmospheric oscillations, M. L. White. J. Geophys. Research 60, 531 (1955).

1342. Factors affecting radio propagation in the TV and FM bands, W. F. Utlaut. Tele-Tech. 14, No. 6, 98 (1955).

1343. Factors influencing the behavior of polymers exposed to high-energy radiation, L. A. Wall. J. Polymer Sci. 17, 141 (1955).

1344. Farm milk tank testing, M. W. Jensen. Proc, 11th Annual Dairy Technology Conf. (Univ, of Md., College Park, Md.) p. 6 (Nov. 1955); Proc. Milk Industry Foundation Convention (Dairy Industries Foundation, Washington, D. C. ) p. 41 (Oct. 1955).

1345. Fatigue and static tents of flush riveted joints, D. M. Howard and F. C. Smith. Product Eng. Handbook No. 3, Section G, p. G2 (McGraw-Hill Book Co., Inc., New York, N. Y. ) (1955)

1346. Flame photometric determination of strontium in portland cement, J. J. Diamond. Anal. Chem. 27, 913 (1955).

1347. Flames, H. P. Broida and H. J. Morowitz. Sci. Monthly 80, No. 1, 3 (1955).

1348. Fluorescence and average lifetime of excited $\mathrm{OH}\left({ }^{2} \mathbf{\Sigma}^{+}\right)$in flames, H. P. Broida and T. Carrington. J. Chem. Phys, 23, 2202 (1955).

1349. Frequency multipliers and converters for measurement and control, J. M. Shaull. TeleTech. 14, No. 4, section 1,86 (1955).

1350. Fully convex normed linear spaces, K. Fan and I. Glicksburg. Proc. Natl. Acad. Sci. 41, No. 11, 947 (1955).

1351. Further infrared measurements with a cesium iodide prism molecular spectroscopy, E. K. Plyler. The Institute of Petroleum (London, England) (1955).

1352. Galvanomagnetic effects in InSb, W. R. Hosler and H. P. R. Frederikse, Phys, Rev. 98,1532 A (1955).

1353. Gas tube stabilized power supplies, S. Rubin and D. Scott. Tele-Tech 14, No. 7, 70 (1955).

1354. Geometric factors in electrical measurements relating to corrosion and its prevention, W. J. Schwerdtfeger and I. A. Denison. Corrosion 2,25 (1955).

1355. Heat units and temperature scales for calorimetry, H. F. Stimson. Am. J. Phys. 23, 614 (1955).

1356. High precision automatic frequency comparator and recorder, J. M. Shaull. Tele-Tech 14 , No. 1, 58 (1955).

1357. High-speed computation of ideal gas the rmodynamic functions. II. The diatomic free radicals of the isotopic hydrides of oxygen and sulphur, L. Haar and A. S. Friedman. J. Chem. Phys. 23, No. 5, 869 (1955).

1358. High-stability bridge-balancing oscillator, P. G. Sulzer. Proc, IRE 43, 701 (1955).

1359. High-stability frequency standards, P. G. Sulzer. Tele-Tech 14, No. 6, 108 (1955). 1360. How to solve a linear programming problem, A. J. Hoffman. Proc, of Second Symp. Linear Programming, held jointly by NBS and the USAF, 397 (Dept. of Defense) (1955).
1361. Hysteresis effect in multiplier phototube noise, C. A. Ziegler and H. H. Seliger. J. Appl. Phys. 26, No. 10, 1225 (1955).

1362. Improved Hepp-Scatchard osmometer, F. R. McCann and S. Rothman. J. Polymer Sci. 18, 151 (1955).

1363. Indentation pressure of a smooth circular punch, E. Levin. Quart. Appl. Math. 13, No. 2, 133 (1955).

1364. Information theory aspects of propagation through time-varying media, J. Feinstein.

J. Appl. Phys. 26, 219 (1955).

1365. Infrared absorption spectrum of n-dimethyl-amino-diborane, J. E. Stewart. J. Chem. Phys. 23, No. 11, 2204 (1955).

1366. Infrared flame spectra, E. K. Plyler. Microchim. Acta 2-3, 421 (1955).

1367. Infrared measurements, past and present, E. K. Plyler. J. Franklin Inst. 259. No. 1, 17 (1955).

1368. Inner bremsstrahlung from $v^{49}$, R. W. Hayward and D. D. Hoppes. Bull. Am. Phys. Soc. 30, No. 3, 59 A (1955).

1369. Instrument for measuring the marginal power of spectacle lenses, F. E. Washer. J. Opt. Soc. Am. 45, No. 9, 719 (1955).

1370. Instrumentation and automation, W. A. Wildhack. Science 122, 1146 (1955).

1371. Instrumentation and the future of mankind, W. A. Wildhack. Instr. Soc. Am. J. 2, No.9, 430 (1955).

1372. Instrumentation research at the National Bureau of Standards, W. A. Wildhack. Ann. N. Y. Acad, Sci, 60,809 (1955).

1373. Inter ference patterns in reverberant sound fields, R. V. Waterhouse. J. Acoust. Soc. Am. 27, 247 (1955).

1374. Internal rotation in methyltrifluoromethyl acetylene, D. R. Lide, Jr. and D.

Kivelson. J. Chem. Phys, 23, 2191 (1955).

1375. International methods for testing colorfastness of textiles, W. D. Appel. Mag. of Standards 26, No. 2, 44 (1955).

1376. International plastic committee considers standard tests, G. M. Kline. Mag. of Standards 26, No. 10, 305 (1955).

1377. Investigation of homogeneity of six proposed NBS-GMC zinc-base spectrographic standards, R. E. Michaelis. General Motors Spectrographic Committee Special Publication (1955).

1378. Investigations of the properties of corrugated diaphragms, W. A. Wildhack, R. F. Dressler, and E. C. Lloyd. Am. Soc. Mech. Engrs. preprint 55-A-181 (1955).

1379. Ion-atom interchange in air, R. F. Potter. J. Chem. Phys. 23, No. 12, 2462 (1955). 1380. Ionization chambers for radiation data during dental X-ray exposure, D. C. Hudson and J. W. Kumpula. U. S. Armed Forces Med. J. 6, No. 8, 1131 (1955).

1381. Isotope analysis using dimethylmercury, V. H. Dibeler. Anal. Chem. 27, 1958 (1955).

1382. Kinetics of crystallization in polymers.

II. Polymer-diluent mixtures, L. Mandelkern. J. Appl. Phys. 26, 443 (1955).

1383. Kleine Bemerkung zur asymptotischen Entwicklung des Fehlerintegrals, P. Henrici. Z. angew. Math. u Phys. (Basil, Switzerland) 6,145 (1955). 
1384. Rebuttal to comments on "Large reduction of VHF transmission loss and fading by the presence of a mountain obstacle in beyond-lineof-sight paths, "by F. H. Dickson, J. J. Egli, J. W. Herbstreit and G. S. Wickizer, (Proc. IRE 41, 967 (1953)), by J. H. Crysdale, by J. W. Herbstreit, F. H. Dickson, J. J. Egli and G. S. Wickizer. Proc. IRE 43, 627 (1955). 1385. Least pth power polynomials on a real finite point set, T. S. Motzkin and J. L. Walsh. Am. Math. Soc. 78, No. 1, 67 (1955).

1386. Light scattering and micelle structure in . the system sodium dodecyl sulfate-sodium chloride-water, L. M. Kushner and W. D. Hubbard. J. Colloid Sci. 10, No, 4, 428 (1955). 1387. Light waves and length standards, I. C. Gardner. J. Opt. Soc. Am. 45, No. 9, 685 (1955).

1388. Limitations on rapid signal analysis, E. L. R. Corliss. J. Wash. Acad. Sci. 45, No. 11,359 (1955).

1389. Lindeck potentiometer, D. W. Oliver. Rev. Sci. Instr. 26, No. 11, 1078 (1955).

1390. Lithium: heat content from $0^{\circ}$ to $900^{\circ}$, triple point and heat of fusion, and thermodynamic properties of the solid and liquid, T. B. Douglas, L. F. Epstein, J. L. Dever, and W. H. Howland. J. Am. Chem. Soc. 77, 2144 (1955).

1391. Low-capacitance power supply, J. Reaves. Radio-Electronic Eng. 24, No. 6, 16 (1955).

1392. Low temperature scales from $90^{\circ}$ to $5^{\circ} \mathrm{K}$, R. B. Scott. Am. Inst. Phys. Temperatures, its Measurement and Control in Science and Industry 2, 179 (Reinhold Publ. Corp. , New York York, N. Y.) (1955).

1393. Low-temperature thermal conductivity of a free-machining copper, R. L. Powell and D. O. Coffin. Rev. Sci. Instr. 26, 516 (1955).

1394. Macromolecular chemistry, S. L. Madorsky. Science 121,81 (1955).

1395. Magnetic cooling, E. Ambler and R. P. Hudson. Published in Reports on Progress in Physics, Proc. British Phys. Soc. (London, England) 18, 251 (1955).

1396. Magnetic susceptibilities of some U(IV) compounds, C. A. Hutchison, Jr., and C. M. Herzfeld. J. Chem. Phys. 23, 1650 (1955). 1397. Manganese thermal neutron activation cross section, J. A. DeJuren and J. Chin.

Phys, Rev. 99, No. 1, 191 (1955).

1398. Unassigned.

1399. Measurement of correlation coefficients in reverberant sound fields, R. K. Cook, R. V. Waterhouse, R. D. Berendt, S. Edelman, and M. C. Thompson, Jr. J. Acoust. Soc. Am. 27 , No. 6, 1072 (1955).

1400. Measurement of the effect of two-dimensional and three-dimensional roughness elements on boundary-layer transition, P. S. Klebanoff, G. B. Schubauer, and K. D. Tidstrom. J. Aeronaut. Sci. 22, 11 (1955).

1401. Measurements of correlation, height gain, and path antenna gain at $1046 \mathrm{Mc}$ on spaced antennas far beyond the radio horizon, A. F.

Barghausen, M. T. Decker and L. J. Maloney. IRE Conv. Record 3, Pt. 1, 78 (1955).
1402. Measurement of the phase of radio waves received over transmission paths with electrical lengths varying as a result of atmospheric turbulence, J. W. Herbstreit and M. C. Thompson, Jr. URSI Symp. on Electromagnetic Wave Theory, June 1955 (Ann Arbor, Mich.); Proc. IRE 43, 1391 (1955).

1403. Measurements on the saturation vapour pressure of liquid helium in the range $1.4^{\circ}$ $4.2^{\circ} \mathrm{K}$, E. Ambler and R. P. Hudson. Bull. Intern. Inst. Refrig., Commun. 147, 605 (1955). 1404. Mechanical impedance of the forehead and mastoid, E. L. R. Corliss and W. Koidan. J. Acoust. Soc. Am. 27, No. 6, 1164 (1955). 1405. Mechanism of hygroscopic expansion of dental casting investments, H. W. Lyon, G. Dickson, and I. C. Schoonover. J. Dental Research 34, 44 (1955).

1406. Mechanisms of alkali-aggregate reaction, R. G. Pike, D. Hubbard, and H. Insley. J. Am. Concrete Inst. 27, No. 1, 13 (1955).

1407. Method for restoring original appearance of impregnated leather, J. H. Davis and R. Oehler. J. Am. Leather Chemists Assoc. 50, 38 (1955).

1408. Mircowave absorption in compressed oxygen, A. A. Maryott and G. Birnbaum. Phys, Rev. 99, 1886 (1955).

1409. Mooney viscometer cure characteristics of Hevea rubber, G. E. Decker and J. Mandel. ASTM Bull. No. 209, 47 (1955).

1410. Motivation for working in numerical analysis, J. Todd. Commun. on Pure and Appl. Math. 8, No. 1, 97 (1955).

1411. Multistage autoignition of engine fuels, W. J. Levedahl. Fifth Symposium (International) on Combustion (Reinhold Pub. Corp., New York, N. Y. ) p. 372 (1955).

1412. National Bureau of Standards preferred circuits program, J. H. Muncy. Elec. Eng. 74 , No. 12,1088 (1955).

1412A. Natural and synthetic rubbers. Biennial review of physical and chemical test methods, N. Bekkedahl and M. Tryon. Anal. Chem. 27, 589 (1955).

1413. Negative oxygen ions in the upper atmosphere: The affinity and radiative attachment coefficient of atomic oxygen, L. M. Branscomb and S. J. Smith. Trans. Am. Geophys. Union $36,5,755$ (1955).

1414. New method of recording the sound transmission loss of walls as a continuous function of frequency, R. V. Waterhouse and R. K. Cook. J. Acoust. Soc. Am. 27, No. 5, 967 (1955). 1415. New techniques for fabrication of airborne electronic equipment, R. K-F. Scal. Proc. IRE 43 , No. 1,4 (1955).

1416. Nightglow heights: a reinterpretation of old data, F. E. Roach and A. B. Meinel. Astrophys. J. 3, 554 (1955).

1416A. Noise in composition resistors, G. T. Conrad, Jr. Proc. Natl. Electronics Conf. Electronics Conf. Yearbook 10, 1 (1955).

1417. Noise measurements of composition resistors. I. The method and equipment. II. Characteristics and comparison of resistors, G. T. Conrad, Jr. IRE Trans, on Component Parts CP4, 61 (1955). 
1418. Nonlinear theory of space-charge wave in moving, interacting electron beams with application to solar radio noise, H. K. Sen. Phys, Rev. 97, 849 (1955).

1419. Nonlinear viscoelastic behavior of rubber in shear, H. Leaderman. J. Polymer Sci. 16, No. 82, 261 (1955).

1420. Nuclear moments of $\mathrm{Ac}^{227}$, M. Fred, F. S. Tomkins, and W. F. Meggers. Phys. Rev. 98, 1514 (1955).

1421. Observations of distant meteor-trail echoes followed by ground scatter, W. L. Hartsfield. J. Geophys. Research 60, 53 (1955).

1422. Oil-soaked felt-pad lubrication of ball bearings at high speed and high temperature, H. S. White, J. F. Swindells, and H. V. Belcher. Lubrication Eng. 11, 182 (1955).

1423. On a problem in the theory of mechanical quadratures, P. Davis. Pacific J. Math. 5, Suppl. 1, 669 (1955).

1424. On certain series expansions involving Whittaker functions and Jacobi polynomials, P. Henrici. Pacific J. Math. 5, Suppl. 1, 725 (1955).

1425. On eigenvalues of sums of normal matrices, H. Wielandt. Pacific J. Math 5, 633 (1955).

1426. On generating functions of the Jacobi polynomials, P. Henrici. Pacific J. Math. 5, Supp1. 2, 923 (1955).

1427. On helical springs of finite thickness, P. Henrici. Quart. Appl. Math. 13, No. 1, 106 (1955).

1428. On moments of order statistics from the Weibull distribution, J. Lieblein. Ann. Math. Stat. 26, No. 2, 330 (1955).

1429. On non-linear differential equations of the second order with integrable forcing term, H. A. Antosiewicz, J. London Math. Soc. 30,65 (1955).

1430. On power spectra and the minimum detectable signal in measurement systems, J. J. Freeman. J. Appl. Phys. 26, 236 (1955). 1431. On the dependence of the constants in the Curie-Weiss law on exchange, C. M. Herzfeld. J. Chem. Phys, 23, 398 (1955).

1432. On the design of two-dimensional nozzles by the method of characteristics, B. Chaix and P. Henrici. J. Aeronaut. Sci. 22, 140 (1955). 1433. On the kinetics of elementary gas phase reactions at high temperatures, K. E. Shuler. Fifth Symposium (International) on Combustion (Reinhold Pub. Corp., New York, N. Y.) p. 56 (1955).

1434. On the origin of the electronically excited $\mathrm{C}_{2}$ radical in hydrocarbon flames, R. E. Ferguson. J. Chem. Phys. 23, 2085 (1955). 1435. On the vibration of a square clamped plate, M. Abramowitz and W. F. Cahill. J. Assoc. Computing Machinery 2, No. 3, 162 (1955).

1436. Optical properties of cesium iodide, W. S. Rodney. J. Opt. Soc. Am. 45, No. 11, 987 (1955).

1437. Oxidation studies of some natural graphites, R. A. Heindl and N. F. Mohler. J. Am. Ceram. Soc. 38 , No. 3, 89 (1955).
1438. Oxygen chemisorption on carbon adsorbents, W. V. Loebenstein and V. R. Deitz. J. Phys. Chem. 59, 481 (1955).

1439. Oxygen quenching and wavelength shifters in liquid scintillator $\mathrm{s}, \mathrm{C}$. A. Ziegler, H. H. Seliger, and I. Jaffe. Phys. Rev. 99, No. 2, 663 (1955); Bull. Am. Phys. Soc. 30, No. 3, 63 A (1955).

1440. Pairs of matrices with property L. II, T. S. Motzkin and O. Taussky. Trans. Am. Math. Soc. 80, No. 2, 387 (1955).

1441. Paper milk containers, M. W. Jensen. Scale J. 41, No. 12, 5 (1955).

1442. Partially replicated latin squares, W. J. Youden and J. S. Hunter. Biometrics 11, 399 (1955).

1443. Permissible exposure to ionizing radiation, L. S. Taylor. Proc, of Intern. Conf, on Peaceful Uses of Atomic Energy (Geneva, Switzerland) (1955).

1444. Photoeffects in intermetallic compounds, H. P. R. Frederikse and R. F. Blunt. Proc. IRE 43, 1828 (1955).

1445. Physical characteristics of titanium carbide type cermets at elevated temperatures, F. P. Knudsen, R. F. Geller, and R. E. Moreland. J. Am. Ceram. Soc. 38, No. 9, 312 (1955).

1445A. Physical mathematics, C. H. Page. (D. Van Nostrand Co., Inc. New York, N. Y.) (1955).

1446. Physiological monitor for anesthesia, S. R. Gilford and H. P. Broida. Electronics 28, No. 10, 130 (1955).

1447. Plating standards and specifications, F. Ogburn. Electroplating Eng. Handbook, Chapter 7 (Reinhold Pub. Corp. New York, N. Y.) (1955).

1448. Polarization energy for $3 \mathrm{~d}$ and $4 \mathrm{p}$ electrons, R. E. Trees, Phys, Rev, 97, 686 (1955).

1449. Pores in collagen and leather, R. R. Stromberg and M. Swerdlow. J. Am. Leather Chemists Assoc. 50, No. 7, 336 (1955).

1450. Portable potentiometer and thermostated containers for standard cells, F. L. Hermach and A. W. Spinks. Rev. Sci. Instr. 26, 770 (1955).

1451, Potassium iodide fast neutron detector, B. Brown. Rev. Sci. Instr. 26, 970 (1955). 1452. Practical test methods for the Porcelain Enameling Industry, J. R. Giles. Proc. Porcelain Enamel Inst. Forum 17, 55 (1955).

1453. Precision 5-10 kv high-voltage supply, L. Costrell. Nucleonics 13, No. 3, 63 (1955). 1454. Preferred orientation in stark rubber studied with an automatic integrating pole-figure goniometer, C. J. Newton, L. Mandelkern, and D. E. Roberts. J. Appl. Phys. 26, No. 12, 1521 (1955).

1455. Preparation and maintenance of radioactive standards by the National Bureau of Standards, W. B. Mann. Acta Radiol. Suppl. 117, 50 (1955).

1456. Pressure drop in pneumatic components and systems, M. M. Slawsky, M. Lutsky, and A. E. Schneidlin. Product Eng. Handbook No. 3, Section J, p. J2 (McGraw-Hill Book Co., Inc., New York, N. Y.) (1955). 
1457. Problems arising in engineering investigations, W. J. Youden. Proc. 1st Annual Statistical Engineering Symposium, held by the Chemical Corps Engineering Agency at Army Chemical Center, Md., 60 (1955).

1458. Proceedings of the Second Symposium in Linear Programming, held jointly by the National Bureau of Standards and the United States Air Force (January, 1955).

1459. Progress in metal cleaning and finishing, A. Brenner. Metal Progr. 68, No. 3, 113 (1955)

1459 A. Progress report by Committee of Optical Society of America on uniform color scales, D. B. Judd, J. Opt. Soc. Am. 45, 673 (1955). 1460. Properties of $\mathrm{Mg}_{2} \mathrm{Sn}, \mathrm{R}$. F. Blunt, H. P. R. Frederikse, and W. R. Hosler. Phys. Rev. 98,1533 A (1955).

1461. Properties of pore conductors, R. Forman. J. Appl. Phys. 26, No. 9, 1187 (1955). 1462. Protection in the use of dental roentgenographic equipment, S. W. Smith. J. Am. Dental Assoc. 51, 12 (1955).

1463. Protection of low-strategic alloys with a chromium boride-nickel cermet coating, D. G. Moore and J. R. Cuthill. Am. Ceram. Soc. Bull. 34, 11 (1955).

1464. Pyrolysis of $\alpha$ and $\beta$ deuterostyrene polymers, L. A. Wall, D. W. Brown, and V. E. Hart. J. Polymer Sci. 15, No. 79, 157 (1955).

1465. Pyrophosphate formation upon ignition of precipitated basic calcium phosphates, A. Gee and V. R. Deitz. J. Am. Chem. Soc. 77, 2961 (1955).

1466. Pulp and paper (Annual Review) R. B. Hobbs and W. K. Wilson. Chem. Eng. News 33,114 (1955).

1467. Radiation protection in therapeutic radiology, H. O. Wyckoff. Am. J. Roentgenol.

Radium Therapy Nuclear Med. 73, 655 (1955). 1468. Radio observations (al-CM) of dense dark nebulae, B. J. Bok, R. S. Lawrence and T. K. Menon. Publs. Astron. Soc. Pacific 67, No. 395,108 (1955).

1469. Radio transmission at VHF by scattering and other processes in the lower ionosphere, D. K. Bailey, R. Bateman, and R. C. Kirby. Proc. IRE 43, No. 10, 1181 (1955).

1470. Radio transmission loss vs distance and antenna height at $100 \mathrm{Mc}, \mathrm{P}$. L. Rice and F. T. Daniel. IRE Trans. Ant. Prop. AP-3, 59 (1955). 1471. Radioactivity of In 117 and Sb117, C. L. McGinnis. Phys. Rev. 97, 93 (1955).

1472. Rate of thermal diffusion of polymer molecules in solution, J. D. Hoffman and $B . H$. Zimm. J. Polymer Sci. 15, 405 (1955).

1473. Reaction of sodium chlorite with some aldoses and modified celluloses, W. K. Wilson and A. A. Padgett. Tappi 38, 292 (1955).

1474. Reagent chemicals, E. Wichers. American Chemical Society Specifications, 1955. Prepared by the Comm. on Analytical Reagents, published by Am. Chem. Soc. (1955).

1475. Reference samples of isotopic abundance, F. L. Mohler. Science 122, No. 3164,334 (1955).

1476. Relationships of dental materials to clinical subjects, R. J. Nelson. J. Dental Education 19, 62 (1955).
1477. Report on International Standards Organization Technical Committee 61, G. M. Kline. Mag. of Standards 26, No. 2, 47 (1955).

1478. Report on the Second revision of Rowland's table of solar wavelengths. Proc. Rydberg Centennial Conf., Lunds Universitets (Lund, Sweden), Arsskrift N. F. and 2, 50, No. 21,104 (1955).

1479. Residual lattice strains in sectioned bars of plastically deformed iron, C. J. Newton and H. C. Vacher. J. Metals 7, No. 11, 1193 (1955).

1480. Résume of dental research, G. C. Paffenbarger. J. Am. College Dentists 22, 21 (1955).

1481. Safety color codes, H. J. Keegan.

Inter-Society Color Council News Letter No. 121,10 (1955).

1482. Scattering at oblique incidence from ionospheric irregularities; summary of an informal talk, D. K. Bailey. In the physics of the ionosphere; report of the Physical Society Conference on the physics of the ionosphere, held at the Cavendish Laboratory, Cambridge, September 1954) p. 99 (The Physical Society, London, England) (1955).

1483. Science in radiology, L. S. Taylor.

Radiology 64, 429 (1955).

1484. Scintillation crystal-TV device X-rays objects in motion, J. S. Pruitt. Nucleonics 13 , No. 8, 26 (1955).

1485. Significance of the results of some physical tests on upper leather, J. R. Kanagy. J. Am. Leather Chemists Assoc. 50, No. 3, 112 (1955).

1486. Silver electrode, C. L. Gordon. Anal. Chem. 27, No. 9, 1508 (1955).

1487. Simple humidity lag apparatus, S. Hasegawa, S. B. Garfinkel, and A. Wexler. Rev. Sci. Instr. 26, No. 12, 1196 (1955).

1488. Small grating spectrometer for the far infrared region, E. K. Plyler and N. Acquista. J. Chem. Phys. 23, 752 (1955).

1489. Solar Hafilaments and geomagnetic disturbances, H. I. Leighton and D. E. Billings. J. Atmospheric and Terrest. Phys, 7, 349 (1955).

1490. Solar radio astronomy at the National Bureau of Standards. In Proc. 7 th Western Amateur Astronomers' Convention (Yosemite, California) 13, (1955).

1491. Some aspects of the large scale liquefaction of hydrogen, B. W. Birmingham. Proc. Instr. Soc. Am. Paper 55-2-1, 10, Pt. 2, 1 (1955).

1492. Some developments in vibration measurement, S. Edelman, E. Jones, and E. R. Smith. J. Acoust. Soc. Am. 27, 728 (1955).

1493. Some factors affecting the dimensional stability of the $\mathrm{Ag}-\mathrm{Sn}(\mathrm{Cu}-\mathrm{Zn})$ amalgams, J. A. Mitchell, I. C. Schoonover, G. Dickson, and H. C. Vacher. J. Dental Research 34, No. 2, 273 (1955).

1494. Some factors influencing the cutting characteristics of rotating dental cutting instruments, D. C. Hudson, J. L. Hartley, R. B. Moore, and W. T. Sweeney. J. Am. Dental Assoc. 50, 377 (1955). 
1495. Some fading characteristics of regular VHF ionospheric propagation, G. R. Sugar. Proc. IRE 43, No. 10, 1432 (1955).

1496. Some inequalities concerning positivedefinite Hermitian matrices, K. Fan. Proc. Cambridge Phil. Soc. 51, 414 (1955).

1497. Some metric inequalities in the space of matrices, K. Fan and A. J. Hoffman. Proc. Am. Math. Soc. 6, No. 1, 111 (1955).

1498. Some results of a sweep-frequency propagation experiment over an 1150-KM east-west path, B. Wieder. J. Geophys. Research 60 , 395 (1955).

1499. Some tropospheric scatter propagation measurements near the radio horizon, H. B. Janes and P. I. Wells. Proc. IRE 43, 1336 (1955).

1500. Sorption of macromolecules to solid surfaces: sorption of dextran to cellulose nitrate membranes in the presence of serum albumin and surface-active agents, S. Rothman, J. Mandel, F. R. McCann, and S. G. Weissberg. J. Colloid Sci. 10, No. 4, 338 (1955).

1501. Sources of transport coefficients and correlations of thermodynamic and transport data, J. Hilsenrath. Second AGARD Combustion Colloquium, Liege, Belgium (1955).

1502. Specific heats of collagen and leather,

J. R. Kanagy. J. Am. Leather Chemists Assoc. 50 , No. 10,444 (1955).

1503. Spectroscopic determination of the dissociation energy of the $\mathrm{OH}$ radical, G. A. Hornbeck. Fifth Symp. (International) on Combustion (Reinhold Pub. Corp., New York, N. Y.) 790 (1955). 1504. Spectroscopy, W. F. Meggers. Encyclopedia Brittanica 21, 180 (1955).

1505. Standard natural rubber, R. D. Stiehler and F. L. Roth. Rubber Age 78,77 (1955).

1506. Standard test for photoflash cells, H. J. DeWane, Mag. of Standards 26, No. 5, 135 (1955).

1507. Standards at the National Bureau of Standards, W. J. Hamer. Yale Sci. Mag. 30, No. 2, 7 (1955).

1508. Standards for electronics, A. V. Astin. Proc. National Electronics Conference (Chicago, Il1.) 11,1 (1955).

1509. Standards for plastics, G. M. Kline.

Chap. in Plastics in Building 95, Advances in Chemistry Series 10, 145 (1955).

1510. Stark effect, W. F. Meggers. Encyclopedia Brittanica 21,334 (1955).

1511. Statistical principles of testing. J. Mandel. Ind. Quality Control 12, 18 (1955).

1512. Statistics--A tool in testing rubber, J. Mandel and R. D. Stiehler. ASTM Bull. No. 204, 44 (1955).

1513. Stress corrosion cracking of a gold wed-

ding ring, F. M. Reinhart. Corrosion 11, 503t (1955).

1514. Stress-strain relationships in yarns subjected to rapid impact loading, H. F. Schiefer, J. C. Smith, F. L. McCrackin, and W. K. Stone. Am. Soc. Testing Materials Spec. Tech. Publ. 176, "Symposium on Impact Testing", 126 (1955).
1515. Stress-strain relationships in yarns subjected to rapid impact loading. Part I. Equipment, testing procedure, and typical results, W. K. Stone, H. F. Schiefer, and G. Fox. Textile Research J. 25, 520 (1955).

1516. Stress-strain relationships in yarns subjected to rapid impact loading. III. Effect of wave propagation, J. C. Smith, F. L. Mc Crackin, and H. F. Schiefer. Textile Research J. 25, No. 8, 701 (1955).

1517. Structure theorems for modular subgroups, M. Newman. Duke Math. J. 22, No. 1,25 (1955).

1518. Studies of crystal growth, and electrical and optical properties of gray tin, J. H. Becker. Phys. Rev. 98, 1192A (1955).

1519. Superconducting transitions in tin whiskers, O. S. Lutes and E. Maxwell. Phys. Rev. 97 , No. 6, 1718 (1955).

1520. Sweep-frequency pulse-transmission measurements over a $2400-\mathrm{KM}$ path, P. G. Sulzer. J. Geophys, Research 60, 483 (1955). 1521. Synthesis and properties of fluorinated polyphenyls, M. Hellmann, A. J. Bilbo, and W. J. Pummer. J. Am. Chem. Soc. 77,3650 (1955).

1522. Synthetic textiles, W. D. Appel.

Encyclopedia Americana, p. 732 (1955).

1523. Table of characteristic values of Mathieu's equation for large values of the parameter, G. Blanch and I. Rhodes. J. Wash. Acad. Sci. 45,166 (1955).

1524. Teaching pay in the Soviet Union, L. S. Taylor. Letter to Editor Science Teacher 22, No. 6, 264 (1955).

1525. Tektites and the lost planet, R. Stair. Smithsonian Institution Annual Report No. 4194, 217 (Smithsonian Institution, Washington, D.C.) (1955).

1526. Temperature control during mixing of rubber compounds, F. L. Roth, G. E. Decker, and R. D. Stiehler. Rubber World 132, 482 (1955).

1527. Temperature dependence of mechanical shear degradation, P. Goodman and A. B. Bestul. J. Polymer Sci. 18, No. 88,235 (1955).

1528. Temperatures in atomic explosions, F. G. Brickwedde. Temperature, Its Measurement and Control in Science and Industry (Reinhold Pub. Co., New York, N. Y.) 2, 395 (1955). 1529. Textiles at the National Bureau of Standards, W. D. Appel. Proc. Am. Assoc. Textile Chemists Colorists 44 , No. 23, 795 (1955). 1530. The behavior of long helical springs under fluctuating load, C. L. Staugaitis and H. C. Burnett. ASTM Bull. 55, 918 (1955).

1531. The effect of an anodic (HAE) coating on the fatigue strength of magnesium alloy specimens, J. A. Bennett. ASTM Bull. 55, 1015 (1955).

1532. The generation of Coulomb wave functions by means of recurrence relations, I. A. Stegun and M. Abramowitz. Phys. Rev. 98, 1851 (1955).

1533. The heat of formation of tetrafluoromethane, R. S. Jessup and R. E. McCoskey and R. A. Nelson. J. Am. Chem. Soc. 77, 244 (1955). 
1534. The height of the nightglow by the van Rhijn method, F. E. Roach and A. B. Meinel. Astrophys. J. 122, No. 3, 530 (1955).

1535. The helium vapor-pressure scale of temperature, R. P. Hudson. Temperature, Its Measurement and Control in Science and Industry (Reinhold Pub. Co., New York, N. Y.) 2, 185 (1955).

1536. The interconnection of two digital computers, M. E. Stevens. Am. Inst. Elec. Engrs. Fall General Meeting, CP55-736 (Chicago, 표.) (1955).

1537. The ionosphere, T. N. Gautier. Sci. American 193, No. 3, 126 (1955).

1538. The mechanisms of material transport during sintering, R. F. Walker. J. Am. Ceram. Soc. 38, No. 6, 187 (1955).

1539. The National Bureau of Standards' 1000lb balance, H. H. Russell. The Scale J. 41, No. 11,6 (1955).

1540. Unassigned.

1541. The probability distribution of the amplitude of a constant vector plus a Rayleigh-distributed vector, K. A. Norton, L. E. Vogler, W. V. Mansfield, and P. J. Short. Proc. IRE 43,1354 (1955).

1542. The rate of fading in propagation through a turbulent atmosphere, K. A. Norton, P. L. Rice, H. B. Janes, and A. P. Barsis. Proc. IRE 43, 1341 (1955).

1543. The relation between the absorption spectra and the chemical constitution of dyes. XXVIII. The hydration of Azo dyes in organic solvents, W. R. Brode, I. L. Seldin, P. E. Spoerri, and G. M. Wyman. J. Am. Chem. Soc. 77,2762 (1955).

1544. The scatter propagation is sue, K. A. Norton and J. B. Wiesner. Introduction to the special issue of Proc. IRE. Proc. IRE 43, 1181 (1955).

1545. The spectral radiant energy from the sun through varying degrees of smog at Los Angeles, R. Stair. Proc. 3d National Air Pollution Symposium (Los Angeles, California) (1955). 1546. The spectral-transmissive properties of plastics for use in eye protection, R. Stair. Am. Standards Assoc. Special Is sue (New York, N. Y.) (1955).

1547. The spectral-transmissive properties of the tektites, R. Stair. Geochim, et Cosmochim. (Pergamon Press, New York, N. Y.) 7, 43 (1955).

1548. The theory of electronpenetration, L. V. Spencer. Phys. Rev. 98, 1597 (1955).

1549. Unassigned.

1550. The use of angular distance in estimating transmission loss and fading range for propagation through a turbulent atmosphere over irregular terrain, K. A. Norton, P. L. Rice, and L. E. Vogler. Proc. IRE 43, 1488 (1955). 1551. The use of cesium iodide prisms to 50 microns, E. K. Plyler and N. Acquista. N. 3 del Supplemento al Vol. 2, Serie X, II Nuovo Cimento, p. 629 (Bologna, Italy) (1955).

1552. Unassigned.

1553. Unassigned.

1554. Theory of dielectric relaxation for a single-axis rotator in a crystalline field, II. , J. D. Hoffman. J. Chem. Phys. 23, No. 7, 1331 (1955).
1555. Thermal and gravitational excitation of atmospheric oscillations, H. K. Sen and M. L. White. J. Geophys. Research 60, No. 4, 483 (1955).

1556. Thermal decomposition of polymers, L. A. Wall. Chem. Corps Report, Report of Symposium VII, Incendiary Gels and Their Instrumentation, p. 103 (1955).

1557. Thermal degradation of polymers as a function of molecular structure and branching, S. L. Madorsky and S. Straus. Consiglio Nazionale Delle Ricerche (Rome, Italy) 25, 3 (1955).

1558. Thermodynamics of crystallization in high polymers: natural rubber, D. E. Roberts and L. Mandelkern. J. Am. Chem. Soc. 77, 781 (1955).

1559. Thermoelectric power of indium antimonide, H. P. R. Frederikse and E. V. Mielczarek. Phys. Rev. 99, 1889 (1955).

1560. Transmission of positrons and electrons, H. H. Seliger. Phys. Rev. 100, No. 4, 1029 (1955).

1561. Transmittance of infrared energy by glasses, F. W. Glaze. Am. Ceram. Soc. Bull. 34, No. 9, 291 (1955).

1562. Treatment of liquid $\mathrm{He}^{3}$ based on the "cell" model, H. N. V. Temperley. Phys. Rev. 97, 835 (1955).

1563. Uber pH-Werte nichtwasseriger Losungen, R. G. Bates and G. Schwarzenbach. Helv. Chim. Acta (Basil, Switzerland) 38, 699 (1955).

1564. Ultrasonic switch aids diversity reception, G. F. Montgomery. Electronics 28, No. 11, 1169 (1955).

1565. Ultraviolet spectrophotometry of low pressure explosions, G. J. Minkoff, A. J. Everett, and H. P. Broida. Fifth (International) Symposium on Combustion (Reinhold Pub. Corp., New York, N. Y.) p. 779 (1955).

1566. Understressing as a means of eliminating the damaging effect of fatigue stressing, H. E. Frankel and J. A. Bennett. ASTM Bull. 55, 891 (1955).

1567. Unimodular integral circulants, O. Taussky. Math. Zeitschr (Berlin, Germany) 63,286 (1955).

1568. Unsolved problems in review, A. T.

McPherson. ASTM Bull. No. 210 , p. 12 (1955).

1569. Use of partially oriented solid samples and unpolarized radiant energy in the analysis of infrared absorption spectra, J. E. Stewart. J. Chem. Phys. 23, No. 5, 986 (1955).

1570. Valve for cold fluids, R. J. Richards and R. B. Jacobs. Rev. Sci. Instr. 26, No. 7, 730 (1955).

1571. Variability of wool content in part wool blankets, E. Horowitz and W. S. Connor. ASTM Bull. No. 208, 42 (1955).

1572. Variation in distortion with magnification, A. A. Magill. J. Opt. Soc. Am. 45, No. 3, 148 (1955).

1573. Variations in preferred orientation and modulus of elasticity in cold drawn music spring wire, H. C. Burnett and C. J. Newton. Wire and Wire Products 30, No. 11, 1375 (1955). 
1574. Velocity of light from the molecular constants of carbon monoxide, E. K. Plyler, L. R. Blaine, and W. S. Connor. J. Opt. Soc. Am. 45, No. 2, 102 (1955).

1575. Vibrational energy of deuterium cyanide, H. C. Allen, Jr., E. D. Tidwell, and E. K. Plyler. J. Chem. Phys. 23, No. 7, 1356 (1955). 1576. Vibrational spectrum of 1-fluoro-1-chloroethylene, E. K. Plyler, D. E. Mann, and N. Acquista. J. Chem. Phys. 23, 2122 (1955). 1577. Vibrational spectrum of fluorotrichloroethylene, D. E. Mann and E. K. Plyler. J. Chem. Phys. 23, No. 11, 1989 (1955).

1578. Weatherability of porcelain enamels, D. G. Moore. Proc. of Conf. Architectural Porcelain Enamel, Auspices of Architectural Division-Porcelain Enamel Institute, p. 39 (1955).
1579. Weathering of porcelain enamel, D. G. Moore. Proc. Porcela in Enamel Inst. 17, 136 (1955).

1580. Weights and measures approval seals, M. W. Jensen. Scale J. 41 , No. 5, 2 (1955). 1581. World unification of screw threads, I. H. Fullmer. Mag. of Standards 26, No. 10, 306 (1955).

1582. X-ray attenuation in lead, aluminum, and concrete in the range 275 to 525 kilovolts, W. Miller and R. J. Kennedy. Radiology 65, No. 6,920 (1955).

1583. X-ray diffraction studies of tooth structures, A. S. Posner. Norelco Reptr. 2, 26 (1955).

1584. Zeeman effect. W. F. Meggers. Encyclopedia Brittanica 23, 940 (1955). 
1584A. A bend-test method of determining the stress required to cause creep in tension, $\mathrm{J}$. B. Wachtman, Jr., and L. H. Maxwell. ASTM Bull. 211, 38 (1956).

1585. A brief look at the history of automation, A. V. Astin. Signal 10, No. 4, 44 (1956). 1586. A comparison of the annual mean solar and lunar atmospheric tides in barometric pressure, as regards to their worldwide distribution of amplitude and phase, S. Chapman. J. Atmospheric and Terrest. Phys. 8, 1 (1956). 1587. A configuration of taillights and brakelights, F. C. Breckenridge. Reports of Night Visibility Committee, Highway Research Board Bul. 146, p. 56 (1956).

1588. A direct approach to the problem of stability in the numerical solution of partial differential equations, J. Todd. Comm. Pure Appl. Math. 9, No. 3, 597 (1956).

1589. A frequency standard at low temperatures, W. D. George. Proc. 10th Ann. Symp. on Frequency Control. Signal Corps Engineering Laboratories, p. 197 (May 1956).

1590. A function generator for two independent variables, L. Taback. Control Eng. 3, No. 11, 129 (1956).

1591. A high energy electron-nuclear scattering, H. Mendlowitz. Phys. Rev. 102, No. 2, 527 (1956).

1592. A method for determining rate equation for reactions in which the concentration of the reactant is unknown, J. H. Flynn. J. Phys. Chem. 60, 1332 (1956).

1593. A method for measuring surface flammability of materials using a radiant energy source, A. F. Robertson, D. Gross, and J. Loftus. Am. Soc. Testing Materials Proc. 56, 1437 (1956).

1594. A new technique for the measurement of microwave standing-wave ratios; A. C. MacPherson and D. M. Kerns. Proc. IRE 44, 1024 (1956).

1595. A note on the normal distribution, S. Geisser. Ann. Math. Stat. 27, 858 (1956). 1596. A one-microsecond adder using onemegacycle circuitry, A. Weinberger and J. L. Smith. IRE Trans. Electronic Computers EC-5, No. 2, 65 (1956).

1597. A paper chromatographic analysis for collagen and collagen derivatives, J. M. Cassel. J. Am. Leather Chemists Assoc. $51,223(1956)$.

1598. A photometric unit for the airglow and aurora, D. M. Hunten, F. E. Roach, and J. W. Chamberlain. J. Atmospheric and Terrest. Phys. 8, 345 (1956).

1599. A radioisotope study of the nickel dip, J. C. Richmond and H. B. Kirkpatrick. J. Am. Ceram. Soc. 39, No. 2, 39 (1956).

1600 . A study of magnetic maps of the sun, $M$. B. Wood. Astrophys. J. 124, 447 (1956). 1601. A study of the impact properties of borontreated steels, S. J. Rosenberg and J. D. Grimsley. J. Iron and Steel Inst. 182, 278 (1956).

1602. A study of thermistor materials for use as temperature-sensing elements in the highvelocity exhaust gases of jet-type engines, G. F. Blackburn, P. D. Freeze, and F. R. Caldwell. Wright Air Devel. Ctr. Tech. Rept. (Ohio) p. 54, Suppl. 1 (1956).
1603. A subroutine for computations with rational numbers, P. Henrici. J. Assoc. Computing Machinery 3, No. 1, 6 (1956).

1604. A survey of the application of ceramics as vacuum tube envelopes, C. Marsden. Third Annual Meeting Tube Techniques Conf. (Advisory Group on Electron Tubes, New York, N. Y.) (1956).

1605. A table of the coefficients of the powers of $\eta(\gamma)$, M. Newman. Konink1. Ned. Akad. Wetenschap, Proc. 59, 2 (1956); Indag. Math. 18, 2 (1956).

1606. A theory of cavity ionization, L. V. Spencer. Cancer Yearbook (Yearbook Publishers, Chicago, IIl.) p. 499 (1956).

1607. Abundance of free atoms in solid nitrogen condensed at $4.2^{\circ} \mathrm{K}$ from a gas discharge, $\mathrm{H}$. P. Broida and O. S. Lutes. J. Chem. Phys. 24, No. 2, 484 (1956).

1608. Accelerating effect of aminos on polymerization of methyl methacrylate, G. M. Brauer, R. M. Davenport, and W. C. Hansen. Modern Plastics 34, No. 3, 153 (1956).

1609. Adsorption of gases and vapors on glass spheres. II. Two dimensional condensation of oxygen, J. L. Shereshefsky and C. E. Weir. J. Phys. Chem. 60, 1162 (1956).

1610. Algebraic equations satisfied by roots of natural numbers, E. G. Straus and O. Taussky. Pacific J. Math. 6, 97 (1956).

1611. Alignment of three odd-A rare-earth nuclei, E. Ambler, R. P. Hudson, and G. M. Temmer. Phys. Rev. 101, No. 1, 196 (1956).

1612. Alkaline solutions for $\mathrm{pH}$ control, R. G. Bates and V. E. Bower. Anal. Chem. 28, 1322 (1956).

1613. Alloys of gallium with powdered metals as possible replacement for dental amalgam, D. L. Smith, and H. J. Caul. J. Am. Dental Assoc. 53,315 (1956).

1614. American Dental Association specifications for dental materials, G. C. Paffenbarger and W. T. Sweeney. J. Am. Dental Assoc. 2d Edition (1956).

1615. An A-C bridge for the audio-frequency range, B. L. Dunfee. Commun. and Electronics, Am. Inst. Elec. Engrs. No. 24, 123 (1956). 1616. An analog computer for radioactive fallout prediction, H. K. Skramstad and J. H. Wright. Proc. Natl. Simulation Council (1956).

1617. Unas signed.

1618. An application of the Monte Carlo method to a problem in gamma-ray diffusion, M. J. Berger. Symp. Monte Carlo Methods (John Wiley \& Sons, Inc., New York, N. Y.) (1956).

1619. An eight decade logarithmic ohmmeter, S. B. Garfinkel. J. Instr. Soc. Am. 3, No. 2, 54 (1956).

1620. An elasticity and dielectric loss of quartz, R. K. Cook and J. H. Wasilik. J. Appl. Phys. 27, No. 7, 836 (1956).

1621. An intercomparison of the roentgen standards of Great Britain and USA, G. H. Aston and F. H. Attix. Acta Radiol. 46, 747 (1956).

1622. An investigation of slot radiators in rectangular metal plates, D. G. Frood and J. R. Wait. Proc. Inst. Elec. Engrs. (London, England) 103, Pt. B. No. 7, 103 (1956). 
1623. Unassigned.

1624. Analysis of atomic spectra with electronic digital computers, K. G. Kessler, S. B. Prusch, and I. A. Stegun. J. Opt. Soc. Am. 46, No. 12, 1043 (1956).

1625. Analysis of the (0.1) and (1.2) bands of the $2 \triangle-2 \pi$ system of $C H, N$. H. Kiess and H. P. Broida. Astrophys. J. 123, No. 1, 166 (1956).

1626. Analytical mass spectrometry, V. H.

Dibeler. Org. Anal. 3, 387 (1956).

1627. Annual review of pulp and paper industry,

R. B. Hobbs and W. T. Jones. Ind. Eng. Chem. 48, 11 (1956).

1628. Apparatus for etching fine points under controlled conditions, R. A. Schrack and R. C. Placious. Rev. Sci. Instr. 27, No. 6, 412 (1956).

1629. Apparatus for the direct determination of the dynamic bulk modulus, S. Edelman, J. E. McKinney, and R. S. Marvin. J. Appl. Phys. 27,425 (1956).

1630. Application of low Mu triodes to series regulator circuits, S. Rubin. Trans. of RETMA Symp. (1956).

1631. Applications of standards of radioactivity, H. H. Seliger. Intern. J. Appl. Radiation and Isotopes 1, 215 (1956).

1632. Approximate theory on the stability of interfacial waves between two streams, C. M. Tchen. J. Appl. Phys. 27, No. 12, 1533 (1956). 1633. Arc-lengths along the lines of force of a magnetic dipole, S. Chapman and M. Suguira. J. Geophys. Research 61, 485 (1956).

1634. Atomic radiation and polymers, L. A. Wall. J. Soc. Plastics Engr. 12, No. 3, 17 (1956).

1635. Atomic theory of electromagnetic interactions in dense materials, U. Fano. Phys. Rev. 103, No. 5, 1202 (1956).

$1635 \mathrm{~A}$. Attenuation of $86-$ and $176-\mathrm{Mev}$ synchrotron X-rays in concrete and lead, W. Miller and R. J. Kennedy. Radiol. Research 4, 360 (1956). 1636. Australite (meteoric) glass, D. Hubbard, E. M. Krumrine, and R. Stair. Trans. Am. Geophys. Union 37, No. 6, 767 (1956).

1637. Unassigned.

1638. Automatic computations with power series, P. Henrici. J. Assoc. Computing Machinery 3, No. 1, 10 (1956).

1639. Basis for standards for radiation protection, L. S. Taylor. Proc. Meeting Society for Non-Destructive Testing, J. Wash. Acad. Sci. 46, No. 3, 69 (1956).

1640. Behaviour of "pure gum" rubber vulcanizates in tension, G. M. Martin, F. L. Roth, and R. D. Stiehler. Trans. Inst. Rubber Ind. 32, No. 6, 189 (1956).

1641. Best approximation polynomials of given degree, J. L. Walsh. Proc. 6th Symp. Appl. Math. (Am. Math. Soc., Santa Monica, Calif. , 1953) p. 213 (1956).

1642. Boron steels, T. G. Digges. Symp. Production, Properties, and Applications of Alloy and Special Steels, Natl. Metallurgical Lab. , India (1956).

1643. Bremsstrahlung polarization measurements for 1.0-Mev electrons, J. W. Motz. Phys. Rev. 104, No. 3, 557 (1956).
1644. Calibration of the portable Swedish freeair chamber equipment at the U. S. National Bureau of Standards, H. O. Wyckoff and R. Thoraeus. Acta Radiol. 46, 741 (1956).

1645. Calorimetric comubstion in a bomb of compounds containing carbon, hydrogen, oxygen, and nitrogen, E. J. Prosen. Chapter 6 in the book Experimental Thermochemistry (Interscience Publ. Inc., New York, N. Y.) p. 129 (1956).

1646. Chemiluminescence as a tool in the study of liquid flow boundaries, A. S. Friedman. J. Appl. Phys. 27, 417 (1956).

1647. Chromium for gun bores, J. P. Young.

Am. Rifleman 104, 39 (1956).

1648. Chromium plating of gun bores, V. A. Lamb and J. P. Young. Ann. Proc. Am.

Electroplaters' Soc., 260 (1956).

1649. Clamp for handling radioactive liquids, A. Schwebel. Nucleonics 14, No. 9, 112 (1956). 1650. Clues to ionospheric conditions in the southern auroral zone, A. H. Shapley. In Antarctica in the International Geophys. Year: based on a Symp. on the Antarctic, Cosponsored by the U. S. Natl. Comm. for the IGY of the Natl. Acad. Sci. , Natl. Sci. Foundation and Am. Geophys. Union (Wa shington, D. C.) p. 86 (1956).

1651. Color rendition in fluorescent sources of illumination, H. Helson, D. B. Judd, and M. Wilson. Hlum. Eng. 51, 329 (1956).

1652. Commutator for periodically changing the direction and magnitude of direct currents, F. Ogburn and H. I. Salmon. Plating 43, 343 (1956).

1653. Commutators of $\mathrm{A}$ and $\mathrm{A} * \mathrm{~T}$. Kato and $\mathrm{O}$. Taussky. J. Wash. Acad. Sci. 46, 38 (1956).

1654. Commuting bilinear transformations and matrices, O. Taussky and J. Todd. J. Wash. Acad. Sci. 46, 373 (1956).

1655. Comparison of the characteristic energy losses of electrons with the fine structure of the $\mathrm{X}$-ray absorption spectra, L. B. Leder, H. Mendlowitz, and L. Marton. Phys. Rev. 101, No. 5, 1460 (1956).

1656. Compilation of phase diagrams of interest to ceramists, E. M. Levin, H. F. McMurdie, and F. P. Hall. Book, Phase of Ceramists (Am. Ceram. Soc.) (1956).

1657. Compressibility factor, density, specific heat, enthalpy, entropy, free energy function, viscosity, and thermal conductivity of steam, L. Fano, J. H. Hubbell, and C. W. Beckett. Natl. Advisory Comm. Aeronaut. Tech. Note 3273 (1956).

1658. Compressibility of binary alkaline-earth borate glasses at high pressures, C. E. Weir and L. Shartsis. J. Am. Ceram. Soc. 39, No. 9, 319 (1956).

1659. Conference on quantum interactions of the free electron, L. Marton. Rev. Mod. Phys. 28, No. 3, $172(1956)$.

1660. Confidence intervals for a proportion, E. L. Crow. Biometrika (London, England) 43, Pts. 3 and 5, 423 (1956).

$1660 \mathrm{~A}$. Contributions to Dictionary of Physics and Electronics, C. H. Page. (D. Van Nostrand Co. Inc. , New York, N. Y.) (1956).

1661. Contributions to the theory of rank order statistics--the two sample case, I. R. Savage. Ann. Math. Stat. 27, 3 (1956). 
1662. Convenient microwave harmonic generator, R. B. Riley. Rev. Sci. Instr. 27, 174 (1956). 1663. Correlation in VHF propagation over irregular terrain, R. S. Kirby and F. M. Capps. IRE Trans. on Ant. Prop. AP-4, No. 1, 77 (1956).

1664. Corrosion, observing 3d year anniversary of "Corrosion Technology," J. G. Thompson. Corrosion Tech. 3, No. 4, 105 (1956).

1665. Cracking of reinforced concrete flexural members, A. P. Clark. J. Am. Concrete Inst. 27, 851 (1956).

1666. Cryoscopic determination of purity of highly reactive substances, A. R. Glasgow, Jr., and M. Tenenbaum. Anal. Chem. 28, 1907 (1956). 1667. Crystal structure of anhydrous indium phosphate and thallic phosphate by $\mathrm{X}$-ray diffraction, R. C. L. Mooney. Acta Cryst. 9, No. 95-204, Pt. 2, 113 (1956).

1668. Currents excited on a conducting surface of large radius of curvature, J. R. Wait.

IRE Trans. Microwave Theory and Tech. MTT-4, 143 (1956).

1669. Dental materials and instruments 1951-55, G. C. Paffenbarger. Intern. Dental J. 6, No. 3, 299 (1956).

1670. Design of experiments in operations research, W. J. Youden. Operations Research for Management II, 282 (1956).

1671. Determinanten mit ueberwiegender Hauptdiagonale und die absolute Konvergenz von linearen Iterationsprozessen, A. M. Ostrowski. Comment, Math. Helv. 30, 175 (1956).

1672. Determination of resin content of glass fiber polyester laminates containing a calcium carbonate filler, S. D. Toner. Anal. Chem. 28, 1109 (1956).

1673. Determination of the monomer content of polymethyl methacrylate, H. J. Caul, L. A. Wall, and N. Acquista. J. Am. Dental Assoc. 53,56 (1956).

1674. Development and evaluation of ceramic coatings for thermocouples, F. H. Sawada, C. J. Carter, P. D. Freeze, and G. F. Blackburn. Wright Air Devel. Ctr. Tech. Report 56-135 (1956).

1675. Development of automatic computers, F. L. Alt. Appeared in "Electronics in Management", L. H. Hattery and G. P. Bush, editors (The University Press of Washington, D. C.) p. 15 (1956).

1676. Differential cross-section measurements of thin-target bremsstrahlung produced by $2.7-$ to 9.7-Mev electrons, N. Starfelt and H. W. Koch. Phys. Rev. 102, 1598 (1956).

1677. Dilworth's theorem on partially ordered sets, G. B. Dantzig and A. J. Hoffman. Ann. Math. Studies No. 38, 207 (1956).

1678. Discussion of the paper "Heavy steelaggregate concrete", by E. I. Fiensenheiser and $B$. A. Wasil, discussed by B. E. Foster. J. Am. Concrete Inst. 28, No. 6, 1143 (1956).

1679. Discussion of the paper "Wave action in diffusers for exhaust-pipe systems, with special reference to the scavenging of two-stroke engines", by F. J. Wallace and G. Boxer, discussed by D. H. Tsai. Proc. Inst. Mech. Engrs. 170, 1152 (1956).
1680. Disintegration of selenium-73, R. W. Hayward and D. D. Hoppes. Phys. Rev. 101, No. 1, 93 (1956).

1681. Distribution of electrons scattered by solids, J. A. Simpson, T. F. McCraw, and L. Marton. Phys. Rev. 104, No. 1, 64 (1956). 1682. Documentation in instrumentation, W. A. Wildhack, J. Stern, and J. Smith. Studies in Coordinate Indexing Vol. 111, Documentation, Inc. (1956).

1683. Durability of deaired brick, J. W. McBurney and P. V. Johnson. J. Am. Ceram. Soc. 39, No. 5, $159(1956)$.

1684. Dynamic and static tests for mechanical properties of fired plastic refractories and other more resilient materials, L. E. Mong and W. L. Pendergast. J. Am. Ceram. Soc. 39, No. 9, 293 (1956).

1685. Dynamics of complete right ventricular failure in dogs maintained with an extracorporeal left ventricle, J. C. Rose, E. J. Lazaro, and H. P. Broida. J. Am. Heart Assoc. 4, No. 2, 173 (1956).

1686. Effect of dissolved air on the speed of sound in water, M. Greenspan and C. E. Tschiegg. J. Acoust. Soc. Am. 28, 501 (1956).

1687. Effect of size on the inlet-system dynamics in four-stroke, single-cylinder engines, $D$. $H$. Tsai. Trans. Am. Soc. Mech. Engrs. 78, No. 1, 197 (1956).

1688. Effect of the ground screen on the field radiated from a monopole, J. R. Wait. IRE Trans. on Ant. Prop. AP- 4, 179 (1956).

1689. Effect of 20-year marine exposure on some aluminum alloys, F. M. Reinhart and G. A. Ellinger. Am. Soc. Testing Materials Spec. Tech. Publ. 175, 47 (1956).

1690 . Effects of multiaxial stretching on crazing and other properties of transparent plastics, I. Wolock and D. A. George. SPE J. 12, 20 (1956).

1691. Elastic and plastic behavior of the ferrite lattice in a low-alloy steel, H. C. Vacher, R. Liss, and R. W. Mebs. Acta Met. 4, No. 5, 532 (1956).

1692. Elastic moduli of glasses at elevated temparatures by a dynamic method, S. Spinner. J. Am. Ceram. Soc. 39, No. 3, 113 (1956). 1693. Elastic moduli of indium antimonide, R. F. Potter. Phys. Rev. 103, No. 1, 47 (1956).

1694. Elastic scattering of electrons and positrons by point nuclei, J. A. Doggett and L. V. Spencer. Phys. Rev. 103, No. 6, 1597 (1956).

1695. Electrical conduction in magnesium stannide at low temperatures, H. P. R. Frederikse, W. R. Hosler, and D. E. Roberts. Phys. Rev. 101, No. 6, 1653 (1956).

1696. Electrodeposition of metals from organic solutions, Part I. General Survey, A. Brenner. J. Electrochem. Soc. 103, No. 12, 652 (1956). 1697. Electrodeposition of metals from organic solutions, Part II. Further studies on the electrodeposition of aluminum from a hydride bath, J. H. Connor and A. Brenner. J. Electrochem. Soc. 103, No. 12, 657 (1956).

1698. Electron affinity of atomic sulfur and empirical affinities of the light elements, L. M. Branscomb and S. J. Smith. J. Chem. Phys. 25, No. 3, 598 (1956). 
1699. Electron characteristic energy losses in metals and compounds, L. B. Leder. Phys.

Rev. 103, No. 6, 1721 (1956).

1700. Electron injection in betatrons, M. Danos.

Bul. Am. Phys. Soc. Ser. II, No. 1, 344, Abstract (1956).

1701. Electron interference and phase effects. Electron interference phenomena, L. Marton. Proc. Intern. Conf. Electron Microscopy, July 1954 (Royal Microscopical Society, London, England) p. 272 (1956).

1702. Electron interference experiments, J. A.

Simpson, Rev. Mod. Phys. 28, No. 3, 254 (1956).

1703. Electron microscopy, M. Swerdlow, A. J. Dalton, and L. S. Birks. Anal. Chem. 28, No. 4, Pt. II, 597 (1956).

1704. Electron optical image of an atomic beam,

L. Marton, D. C. Schubert, and S. R. Mielczarek. J. Appl. Phys. 27, No. 4, 419 (1956).

1705. Emission spectroscopy, W. F. Meggers.

Anal. Chem. 28, 616 (1956).

1706. Entoptic color-perceptions of the macular pigment by observers of normal and colordefective vision according to the three-components theory, D. B. Judd. Proc. 1953

Colloquium on Optical Problems in Vision (Madrid, Spain) (1956).

1707. Error bounds for the eigenvalues of symmetric integral equations, $H$. Wielandt. Proc. 6th Symp. Appl. Math. (Am. Math. Soc., Santa Monica, California, 1953) p. 261 (1956).

1708. Excitation and separation of pure highorder modes in large high- $Q$ cavities, C. T. Zahn and W. G. Schweitzer, Jr. , J. Appl. Phys. 27, No. 8, 929 (1956).

1709. Experimental proof for the existence of nonthermal rotational distributions of $\mathrm{OH}$ $\left({ }^{2} \Sigma^{+}\right)$in flames, H. P. Broida and H. J. Kostkowski. J. Chem. Phys. 25, No. 4, 676 (1956).

1710. Extension of Munsell renotation system to very dark colors, D. B. Judd and G. Wyszecki. J. Opt. Soc. Am. 46, 281 (1956).

1711. Farm milk tank testing, M. W. Jensen.

Milk Products J. p. 17 (1956).

1712. First report of the special committee on world-wide ionospheric soundings, A. H. Shapley. Intern. Sci. Radio Union (URSI) Inform. Bull. 99, 48 (1956).

1713. Fluorescent sunlamps in laboratory aging tests for plastics, F. W. Reinhart and M. K. Mutchler. ASTM Bull. No. 212, 45 (1956).

1714. Unassigned.

1715. Future office and factory automation, Interpreter (Insurance Accounting and

Statistical Association), 15, No. 9, 1 (1956).

1716. Galvanomagnetic effects in $\mathrm{n}$-type InSb at $4.2^{\circ} \mathrm{K}, \mathrm{H}$. P. R. Frederikse and W. R. Hosler. Can. J. Phys. 34, 1377 (1956).

1717. Galvanometer efficiency as a design parameter, F. K. Harris. Commun, and Electronics, Am. Inst. Elec. Engrs. 24, 176 (1956).

1718. Gamma radiation from $\mathrm{Ag}^{105}, \mathrm{R}$. W. Hayward and D. D. Hoppes. Bull, Am. Phys. Soc. Series II, No. 1, 42 (1956).

1719. Generalization of a theorem of Konig, A. J. Hoffman. J. Wash. Acad. Sci. 46, 211 (1956).

1720. Generalizations of identities for the coefficients of certain modular forms, M. Newman. J. London Math. Soc. 31, 205 (1956).
1721. Generalized tables of corrections to thermodynamic properties for nonpolar gases, W. H. Woolley and W. S. Benedict. Natl. Advisory Comm. Aeronaut. Tech. Note 3272 (1956).

1722. Generation and te sting of pseudo-random numbers, O. Taussky and J. Todd. Proc. Symp. Monte Carlo Methods (Gainesville, Fla., 1954) p. 15 (1956).

1723. Geological application of extreme-value methods to interpretation of cobbles and boulders in gravel deposits, W. C. Krumbein and J. Lieblein. Trans. Am. Geophys. Union 37, No. 3, 313 (1956).

1724. Gloss meter comparisons, I. Nimeroff, H. K. Hammond, III, J. C. Richmond, and J. R. Crandall. J. Am. Ceram. Soc. 39, No. 3, 103 (1956).

1725. Government specifications, S. F. Booth. Plating 43, No, 4, 490 (1956).

1726. Gravitational and thermal oscillations in the earth's upper atmosphere, M. L. White. J. Geophys. Research 61, 489 (1956).

1727. Heat flow in a fluid with eddying flow, $W$. H. Durfee. J. Aeronaut. Sci. 23, 188 (1956).

1728. High frequency induced electroluminescence in zinc sulfide, G. G. Harman and R. L. Raybold. Phys. Rev. 104, No. 6, 1498 (1956).

1729. High-gain antennas for VHF scatter propagation, H. V. Cottony. Trans, IRE on Commun. Systems CS- 4, 56 (1956).

1730. High speed flip-flops for the millimicrosecond region, Z. Bay and N. T. Grisamore. IRE Trans. on Elec. Computers EC-5, 3 (1956).

1731. High vacuum gimbals, R. A. Schrack.

Rev. Sci. Instr. 27, No. 3, 172 (1956).

1732. Hindered rotation in symmetric-asymmetric molecules, D. C. Burkhard. Trans. Faraday Soc. 52, No. 397, Pt. 1, 1 (1956).

1733. How the National Bureau of Standards contributes to building codes, J. P. Thompson. Building Officials Conference of America, Inc. , News 4, No. 12, 1 (1956).

1734. In vitro production of experimental enamel caries, A. F. Forziati, F. L. Losee, W. W. Pigman, W. H. Jennings, and M. E. Lawson, Jr. J. Am. Dental Assoc. 53, 655 (1956).

1735. Incomplete equilibrium and temperature measurement, C. M. Herzfeld. J. Wash. Acad. Sci. 46, 269 (1956).

1736. Indirect transitions in indium antimonide, R. F. Potter. Phys. Rev. 103, 861 (1956).

1737. Influence of copper ions on the adherence of vitreous coatings to stainless steel, D. G. Moore and A. G. Eubanks. J. Am. Ceram. Soc. 39 , No. 10,357 (1956).

1738. Influence of exposure conditions on the accelerated durability testing of asphalt, L. R. Kleinschmidt. ASTM Bull. No. 213, p. 69 (1956).

1739. Infrared spectra of hydrogen cyanide and deuterium cyanide, H. C. Allen, Jr., E. D. Tidwell, and E. K. Plyler. J. Chem. Phys. 25, No. 2, 302 (1956).

1740. Infrared spectrum of hydrogen sulfide, H. C. Allen, Jr., and E. K. Plyler. J. Chem. Phys. 25, No. 6, 1132 (1956). 
1741. Infrared spectrum of hydrogen sulfide from 2200-2800 $\mathrm{cm}^{-1}$, H. C. Allen, Jr., P. C. Cross, E. K. Plyler, and L. R. Blaine, J. Chem. Phys. 24, No. 1, 35 (1956).

1742. Insulating concretes, R. C. Valore, Jr. J. Am. Concrete Inst. 28, 5 (1956).

1743. Integral boundary points of convex polyhedra, A. J. Hoffman and J. B. Kruskal. Ann. Math. Studies No. 38, 223 (1956).

1744. Interaction of stretching vibrations and inversion in ammonia, W. S. Benedict and E. K. Plyler. J. Chem. Phys. 24, No. 4, 904 (1956).

1745. Interfacial waves in viscoelastic media, C. M. Tchen. J. Appl. Phys. 27, No. 5, 431 (1956).

1746. International comparisons of radioactivity standards, A. V. Astin, E. C. Bullard, and W. B. Lewis. Science 123, 895 (1956).

1747. Interpretation of spectra of atoms and molecules in solid nitrogen condensed at $4.2^{\circ} \mathrm{K}$, C. M. Herzfeld and H. P. Broida. Phys. Rev. 101,606 (1956).

1748. Introduction, L. Marton. To July 1956 is sue Rev. Mod. Phys, containing all papers of the Intern. Conf. "Quantum Interactions of the Free Electron" 28, No. 3, 171 (1956).

1749. Ionization and dissociation of $\mathrm{NF}_{3}$ by electron impact, R. M. Reese and V. H. Dibeler. J. Chem. Phys. 24, 1175 (1956). 1750. Ionospheric effects produced by solar flare radiation, V. Agy. Phys. Rev. 102, 917 (1956).

1751. Iron catalyst for production of liquid para-hydrogen, D. H. Weitzel and O. E. Park. Rev. Sci. Instr. 27, No. 1, 57 (1956). 1752. Jerkmeter for ballistocardiography, T. A. Perls and C. W. Kissinger. Rev. Sci. Instr. 27, No. 1, 51 (1956).

1753. Kinetics of capillary shear degradation in concentrated polymer solutions, A. B.

Bestul. J. Chem. Phys. 24, No. 6, 1196 (1956).

1754. Laboratory and field exposure studies of leather fungicides, S. Dahl and A. M. Kaplan. J. Am. Leather Chemists Assoc. 51, No. 3, 119 (1956).

1755. Legislative control of radiation, L. S.

Taylor. Radiology 66, No. 2, 246 (1956).

1756. Les Tektites - Ces objets de verre tombes du ciel proviennent-ils d'une planete perdue? R. Stair. Sciences et Evenir (Paris, France) No. 116, 441 (1956).

1757. Linear programming, A. J. Hoffman.

Appl. Mech. Rev. 9, 185 (1956).

1758. Lines of the chemical elements in astronomical spectra. P. W. Merrill; Appendix A-- Partial Grotrian diagrams of astrophysical interest, C. E. Moore and P. W. Merrill. Carnegie Inst. Washington, D. C. , Publ. 610, 103 (1956).

1759. Liquid-s cintillator temperature effects, H. H. Seliger and C. A. Ziegler. Nucleonics 14, No. 4,49 (1956).

1760. Longitudinal impact tests of long bars with a slingshot machine, W. Ramberg and L. K. Irwin. Am. Soc. Testing Materials Spec. Tech. Publ. No. 176, Symp. Impact Testing, June 1955 (1956).

1761. Low frequency radiation from a horizontal antenna over a spherical earth, J. R. Wait. Can. J. Phys, 34, 586 (1956).
1762. Machine attacks on problems whose variables are permutations, C. B. Tompkins.

Proc. 6th Symp. Appl. Math. (Am. Math. Soc., Santa Monica, Calif., Aug. 1953) (1956).

1763. Machines for retrieving and correlating recorded information, S. N. Alexander. Proc. Conf. on Practical Utilization of Practical Knowledge (Western Reserve Univ. Press, Cleveland, Ohio, 1956).

1764. Mass spectrometry, V. H. Dibeler.

Anal. Chem. 28, 610 (1956).

1765. Unassigned.

1766. Unassigned.

1767. Meaning and standardization of $\mathrm{pH}$ measurements, R. G. Bates. ASTM Symp. on $\mathrm{pH}$ Measurements, Am. Soc. Testing Materials Spec. Tech. Pub. No. 190 (1956).

1768. Measurement of the resistance-strain relation and Poisson's ratio for copper wires, T. E. Wells, Paper No. 56-8-3, Proc. Instr. Soc. Am. 11, No. 9, 377 (1956).

1769. Measurement of $\mathrm{W}_{\text {air }}$ for sulfur- 35 betarays, Z. Bay, W. B. Mann, H. H. Seliger, and $\mathrm{H}$. O. Wyckoff. Radiol. Research Abstract 5, No. 4, 9 (1956).

1770. Measurements of the phase of signals received over transmission paths with electrical lengths as a result of atmospheric turbulence, J. W. Herbstreit and M. C. Thompson, Jr. IRE Trans, on Ant. Prop. AP-4, 352 (1956).

1771. Measurements on sound absorbers for jet-engine test cells, R. V. Waterhouse, R. K. Cook, and R. D. Berendt. J. Acoust. Soc. Am. 28, No. 4, 688 (1956).

1772. Mechanical tests on specimens from large aluminum-alloy forgings, J. A. Miller and A. L. Albert. Natl. Advisory Comm. Aeronaut. Tech. Note 3729 (1956).

1773. Mechanism of autoignition in benzene-air mixtures, A. Beckers and W. J. Levedahl. Ind. Eng. Chem. 48, 411 (1956).

1774. Melting of copolymers, P. J. Flory and L. Mandelkern. J. Polymer Sci. 21, No. 98, 345 (1956).

1775. Melting-point and phase-equilibrium determinations, S. M. Lang. High Temp. Technol. p. 409 (J. Wiley \& Sons, New York, N. Y., and Chapman and Hall, London, England.) (1956).

1776. Methods for measuring the " $Q$ " of large reactors, C. Peterson, B. L. Dunfee, and F. L. Hermach. Am. Inst. Elec. Engrs. Trans. Paper No. 56-87 (1956).

1777. Metrology and production, A. V. Astin. Ordnance 40, No. 216, 973 (1956).

1778. Microwave absorption in compressed gases; saturated hydrocarbons, A. A. Maryott and G. Birnbaum. J. Chem. Phys. 24, 1022 (1956).

1779. Millimicrosecond coincidence circuits, Z. Bay. Nucleonics 14, No. 4, 56 (1956).

1780. Unassigned.

1781. Multiple reflections between the earth and the ionosphere in VLF propagation, J. R. Wait and A. Murphy. Geofisica Pura e Applicata 35, 61 (1956).

1782. Near infrared spectrum of nitrous oxide, E. K. Plyler, E. D. Tidwell, and H. C. Allen, Jr. J. Chem. Phys. 24, No. 1, 95 (1956). 
1783. New materials for magnetic cooling and thermometry below $1^{\circ} \mathrm{K}, \mathrm{E}$. Ambler and R, P. Hudson. Phys, Rev, 102, 916 (1956).

1784. New paper products in competition with textiles, R. B. Hobbs. Am. Dyestuff Reptr. 45,479 (1956).

1785. Unassigned.

1786. Notes on post-treatment for HAE coatings,

H. I. Salmon and F. Ogburn. Plating 43, 1251 (1956).

1787. Nuclear elastic scattering of photons, E. G. Fuller and E. V. Hayward. Phys. Rev. 101, No. 2, 692 (1956).

1788. Numerical analysis, Review of, J. Todd.

Proc. Symp. Applied Mathematics Vol. VI,

Science 124, No. 3233, 1211 (1956).

1789. Numerical experiments in potential

theory using orthonormal functions, P. Davis and P. Rabinowitz. J. Wash. Acad. Sci. 46, $12(1956)$.

1790. Oblique-incidence measurements of the heights at which ionospheric scattering of the VHF radio waves occurs, V. Pineo. J. Geophys. Research 61, 165 (1956).

1791. On a generalization of the normal basis in abelian algebraic number fields, M. Newman and $O$. Taussky. Commun. on Pure and Appl. Math. (New York University, New York, N. Y.) 9, 85 (1956).

1792. On asymptotic series for functions occurring in the theory of diffraction of waves by wedges, F. Oberhettinger. J. Math, and Phys. 34, No. 4, 245 (1956).

1793. On systems of distinct representatives, A. J. Hoffman and H. W. Kuhn. Ann. Math. Studies No. 38, 199 (1956).

1794. On systems of linear inequalities, K. Fan. Ann. Math. Studies 38, 99 (1956).

1795. On the asymptotic transformation of certain distributions into the normal distribution, W. Wasow. Proc. 6th Symp. Appl. Math. (Am. Math. Soc. Santa Monica, Calif., 1953) p. 251 (1956).

1796. On the conductance of slots, J. R. Wait.

IRE Trans. on Ant. Prop. AP-4, 124 (1956).

1797. On the existence of identities for the coefficients of certain modular forms, $M$. Newman. J. London Math. Soc. 31, 350 (1956). 1798. On the Lerch zeta functions, F. Oberhettinger. Pacific J. Research 6, No. 1, 117 (1956).

1799. On the propagation of electromagnetic and acoustic pulses. Part I: Diffraction of pulses by wedges, F. Oberhettinger. Z. Phys. $146,423(1956)$.

1800. On the waveform of a radio atmosphere at short ranges, J. R. Wait. Proc. IRE 44, 1052 (1956).

1801. Outline of characteristics of an imageforming system, R. V. Shack. J. Opt. Soc. Am. 9, 755 (1956).

1802. Oxidative degradation of styrene and deuterostyrene polymers, L. A. Wall, M. R. Harvey, and M. Tryon. J. Phys. Chem. 60, 1306 (1956).

1803. Parameters of the 15-Mev X-rays

scattered by $\mathrm{C}^{12}$, E. V. Hayward and E. G.

Fuller. Physica 22, 1138 (1956).

1804. Unassigned.

1805. Phase generator for tropospheric research, R. W. Hubbard and M. C. Thompson, Jr.

Electronics 29, No. 10, 220 (1956).
1806. Photoconductivity and photovoltaic effect in intermetallic compounds, H. P. R. Frederikse and R. F. Blunt. Photoconductivity Conf. Atlantic City, N. J. (John Wiley \& Sons, Inc., New York, N. Y.) p. 414 (1956).

1807. Photoemission from silver into sodium chloride, thallium chloride, and thallium bromide, W. J. Turner, Phys. Rev. 101, No. 6, 1653 (1956).

1808. Physical standards for the electronics industry, A. V. Astin. Signal 10, No. 5, 45 (1956).

1809. Pitfalls in computation, I. A. Stegun and M. Abramowitz. J. Soc. Indust. Appl. Math. 4, 207 (1956).

1810. Plastic springs, F. W. Reinhart and S. B. Newman. Product Eng. 27, 183 (1956).

1811. Plastics, G. M. Kline. Grolier Encyclopedia (1956 ed); J. Eng. Mech. Div. Proc. Am. Soc. Civil Engrs. 84, Paper 1071, EM 4 (1956).

1812. Point-to-point radio relaying via the scatter mode of tropospheric propagation, K. A. Norton. IRE Trans, on Commun. Systems CS-4, No. 1, 39 (1956).

1813. Polymer structure research at the National Bureau of Standards, N. Bekkedahl. Revue General de Caoutchouc (Paris, France) 33, 1129 (1956).

1814. Power supplies for 60-cycle tests of electrical instruments and meters, F. L. Hermach. Instr. Soc. Am. Proc. 11, Paper No. 56-21-3 (1956).

1815. Precise coulometric titrations, J. K. Taylor and S. W. Smith. Science 124, No. 3228 , 940 (1956).

1816. Preparation of pure hydroxyapatite crystals, A. Perloff and A. S. Posner. Science 124, No. 3222, 583 (1956).

1817. Prevention of microbiological deterioration of leather, S. Dahl. J. Am. Leather Chemists Assoc. 51, No. 3, 104 (1956).

1818. Problems of logical design, A. L. Leiner. Proc. Convention on Digital Techniques, Inst. Elec. Engrs, (London, England.) (1956).

1819. Processing denture base resins: heatcuring type, J. W. Stanford and G. C. Paffenbarger. J. Am. Dental Assoc. 53, 72 (1956). 1820. Programming of scientific computers, J. H. Wegstein and S. N. Alexander. Control Eng. 3, No. 5, 87 (1956).

1821. Unassigned.

1822. Progress report on automatic data processing in Government, 1956, S. N. Alexander. University of Michigan (Ann Arbor, Michigan) (1956).

1823. Propagation of sound in five monoatomic gases, M. Greenspan. J. Acoust. Soc. Am. 28, No. 4, 644 (1956).

1824. Properties of concrete at high rates of loading, D. Watstein. Am. Soc. Testing Materials Spec. Tech. Publ. 176, Symp. Impact Testing, June 1955 (1956).

1825. Properties of fluorine compounds, the vibrational spectrum of tetrabromoethylene, D. E. Mann, J. H. Meal, and E. K. Plyler. J. Chem. Phys. 24, No. 5, 1018 (1956).

1826. Discussion of paper, Properties of portland cement pastes cured at elevated temperatures and pressures, C. M. Hunt. J. Am. Concrete Inst. 28, Pt. 2, No. 6, 1934 (1956). 
1827. Pulse generator and high-speed memory circuit, Z. Bay and N. T. Grisamore. IRE Trans. on Elec. Computers EC-5, No. 4, 213 (1956).

1828. Punched-cell batteries with polyethylene glycol electrolytes, R. E. Wood. J. Electrochem. Soc. 103, 417 (1956).

1829. Quantum interactions of the free electron, H. Mendlowitz. Phys. Today 9, No. 8, 27 (1956).

1830. Radiation patterns of circumferential slots on moderately large conducting cylinder 8 , J. R. Wait and J. Kates. Inst. Elec. Engrs. Mono. No. $167 \mathrm{R}, 1$ (1956).

1831. Radiation protection for the general practitioner, L. S. Taylor. Southern Med. J. of the Southern Medical As soc. 49, 8 (1956).

1832. Radiation regulations, L. S. Taylor. Hospitals 30, No. 13, 23 (1956).

1833. Radiation resistance of dipoles in an interface between two dielectrics, J. R. Wait. Can. J. Phys. 34, 24 (1956).

1834. Radiative electron capture in cesium131, D. D. Hoppes and R. W. Hayward. Phys. Rev. 104, No. 2, 368 (1956).

1835. Radiative orbital electron capture in vanadium-49, R. W. Hayward and D. D. Hoppes. Phys. Rev. 104, No. 1, 183 (1956). 1836. Radio observations of interstellar neutral hydrogen clouds, R. S. Lawrence. Astrophys. J. 123, No. 1, 30 (1956).

1837. Reaction of nitrous acid with collagen, J. M. Cassel. J. Am. Leather Chemists Assoc. 51, 406 (1956).

1838. Reciprocity law for X-rays, Part 1: Validity for high-intensity exposures in the negative region, M. Ehrlich and W. L. McLaughlin. J. Opt. Soc. Am. 46, No. 10, 797 (1956).

1839. Reciprocity law for X-rays, Part 2: Failure in the reversal region, M. Ehrlich. J. Opt. Soc. Am. 46, No. 10, 801 (1956). 1840. Record papers and their preservation, W. K. Wils on. Capital Chemist 6, 46 (1956).

1841. Reduction of adjacent-channel interference from on-off keyed carriers, A. D. Watt, R. M. Coon, and V. J. Zurich, IRE Trans. on Commun. Systems CS 4,41 (1956).

1842. Refraction and dispersion of thallium bromide iodide, W. S. Rodney and I. H. Malitson, J. Opt. Soc. Am, 46, No. 11, 956 (1956).

1843. Reinforced plastic springs, F. W. Reinhart and S. B. Newman. SPE J. 12, 8 (1956).

1844. Relation between refractive index and elastic moduli of a borosilicate glass after heat treatment, S. Spinner. J. Am. Ceram. Soc. 39, No. 11, 390 (1956).

1845. Relationships between aurora and sporadic-E echoes at Barrow, Alaska, R. W. Knecht. J. Geophys. Research 61, 59 (1956).

1846. Relationship between residual monomer and some properties of self-curing dental resins, H. J. Caul, W. T. Sweeney, and G. C. Paffenbarger. J. Am. Dental Assoc. 53, 60 (1956).
1847. Relaxation of vibrational nonequilibrium distributions: I. Collisional relaxation of a system of harmonic oscillators, R. J. Rubin and K. E. Shuler. J. Chem. Phys. 25, 59 (1956); II. The effect of the collisional transition probabilities on the relaxation behavior, J. Chem. Phys, 25, 68 (1956).

1848. Report on comparative $100 \mathrm{Mc}$ measurements for three transmitting antenna heights, A. P. Barsis and R. E. McGavin. IRE Trans. on Ant. Prop. AP-4, 168 (1956).

1849. Report on Joint Commission for Spectroscopy Meeting, C. E. Moore. J. Opt. Soc. Am. 46,1004 (1956).

1850. Report on reducing sugars. Effect of clarification with neutral lead acetate before determining reducing sugars in molasses, $E$. J. McDonald. J. Assoc. Offic. Agr. Chemists $39,676(1956)$.

1851. Report on revision of atmospheric radio noise data, W. Q. Crichlow and R. T. Disney. CCIR Report No. 65, VIII Plenary Assembly, Warsaw (1956) (International Radio Consultative Committee, Secretariat, Geneva, Switzerland) (1956).

1852. Report on sugars and sugar products, C. F. Snyder. J. Assoc. Offic. Agr. Chemists 39,676 (1956).

1853. Research on effects of prestraining and notch sharpniess on the notch strength of materials, G. W. Geil and N. L. Carwile. Wright Air Devel. Ctr. Tech. Rept. 56-402 (1956).

1854. Resin cements and posteriortype directfilling resins, P. J. Schoubee, G. C. Paffen barger, and W. T. Sweeney. J. Am. Dental Assoc. 52, 584 (1956).

1855. Resistencia a la tension de los plasticos, F. W. Reinhart. Revista de Plasticos 7, No. 41,236 (1956).

1856. Re sponse function of $\mathrm{NaI}$ (T1) scintillation counters, M. J. Berger and J. A. Doggett. Rev. Sci. Instr. 27, No. 5, 269 (1956).

1857. Ricerca e normalizzazione per I'Industria delle materie plastische, G. M. Kline. Poliplasti (E. T.A.S. Editrice, Milano, Italy) 2, 2529, 62 (1956).

1858. Role of oxygen in the quenching of liquid scintillators, H. H. Seliger, C. A. Ziegler, and I. Jaffe. Phys. Rev. 101, No. 3, 998 (1956).

1859. Rotation-vibration spectra of deuterated water vapor, E. K. Plyler, W. S. Benedict, and N. Gailar. J. Chem. Phys, 24, 1139 (1956). 1860. Unassigned.

1861. Safety color codes, H. J. Keegan. Mag. of Standards 27, 21 (1956); J. Opt. Soc. Am. 46,74 (1956).

1862. Screw thread standardization, A. T. McPherson. Standards Engrs. Soc. Proc, for 1956 (Proc. of the 5th Annual Natl. Meeting (Washington, D. C.) (1956).

1863. Search for the microwave spectrum of perchlorylfluoride, D. R. Lide, Jr., and D. E. Mann. J. Chem. Phys. 25, 595 (1956). 1864. Sensitivity and life data on Bourdon tubes, H. L. Mason. Trans. Am. Soc. Mech. Engrs. 30, No. 1, 65 (1956). 
1865. Sequential flow cooling of electronic equipment, P. Meissner. Elec. Mfg. 58, No. 6, 120 (1956).

1866. Shielding of a transient electromagnetic dipole field by a conductive sheet,. J. R. Wait. Can. J. Phys. 34, 890 (1956).

1867. Slit function effects in the direct measurement of absorption line half-widths and intensities, H. J. Kostkowski and A. M. Bass. J. Opt. Soc. Am. 46, No. 12, 1060 (1956).

1868. Sodium borohydride reduction of aldonic lactones, H. L. Frush and H. S. Isbell. J. Am. Chem. Soc. 78 , No. 12, 2844 (1956). 1869. Solvability and consistency for linear equations and inequalities, H. W. Kuhn. Am. Math. Mo. 63, 217 (1956).

1870. Some activities of the NBS in the field of fire research, A. F. Robertson. Natl. Acad. Sci. and Natl. Research Council Publ. No. 475, (1956).

1871. Some algebraic number theory estimates based on the Dedekind eta-function, H. Cohn. Am. J. Math. 78, 791 (1956).

1872. Some aspects of tropospheric radio wave propagation, A. P. Barsis, IRE Trans, on Broadcast Transmission Systems-6, 1 (1956). 1873. Some computational problems in algebraic number theory, O. Taussky. Proc. 6th Symp. App. Math. of Am. Math. Soc. (Santa Monica, Calif., 1953) p. 187 (1956).

1874. Some meteorological effects on scattered radio waves, B. R. Bean. IRE Trans, on Commun. CS -4 , No, 1, 32 (1956).

1875. Some Monte Carlo experiments in computing multiple integrals, P. Davis and P. Rabinowitz. Math. Tables and Other Aids to Computation 10, 1 (1956).

1876. Some physical properties of gallium copper-tin alloys, D. L. Smith, H. J. Caul, and W. T. Sweeney. J. Am. Dental Assoc. 53, 677 (1956).

1877. Some physical properties of high-density thorium oxide, S. M. Lang and F. P. Knudsen. J. Am. Ceram. Soc. 39, No. 12, 415 (1956). 1878. Sources of error in various methods of airplane camera calibration, F. E. Washer. Photogrammetric Eng. 22, No. 4, 727 (1956). 1879. Sources of transport coefficients and correlations of thermodynamic and transport data, J. Hilsenrath. Agard Selected Combustion Problems, II Fundamentals and Aeronautical Applications. (Buttersworth Sci. Pub., London, England) p. 199 (1956).

1880. Specifications, standards and codes, governmental aspects, R. S. Dill. Symp. Am. Soc. Heating and Air Conditioning Engrs. Problems of Standards and Codes, p. 16 (1956). 1881. Spectra emitted from solid nitrogen condensed at $4.2^{\circ} \mathrm{K}$ from a gas discharge, A. M. Bass and H. P. Broida. Phys. Rev. 101, No. 6, 1740 (1956).

1882. Spectral absorption method for determining population "temperatures" in hot gases, H. J. Kostkowski and H. P. Broida. J. Opt. Soc. Am. 46 , No. $4,246(1956)$.

1883. Spectroscopic observations on pentaborane-air flames and explosions, W. G. Berl, E. L. Gayhart, H. L. Olsen, H. P. Broida, and K. E. Shuler. J. Chem. Phys. 25, No. 4, 797 (1956).
1884. Spectrum of frequency-shift radio phototransmissions, A. D. Watt. IRE Trans, on Commun. Systems CS-4, 27 (1956).

1885. Spectrum of the $\mathrm{C}_{3}$ molecule between $3600 \mathrm{~A}$ and $4200 \mathrm{~A}, \mathrm{~N}$. H. Kiess and H. P. Broida. Can. J. Phys. 34, 1471 (1956).

1886. Specular-glos measurement of ceramic materials, I. Nimeroff, H. K. Hammond, III. J. C. Richmond, and J. R. Crandall. J. Am. Ceram. Soc. 39, No. 3, 103 (1956).

1887. Spin-spin absorption in chromic methylammonium alum and manganous chloride tetrahydrate at very low temperatures, E. Ambler and R. P. Hudson. Physica XXII, 866 (1956). 1888. Spin-spin absorption in manganous chloride tetrahydrate, E. Ambler and R. P. Hudson. Phys. Rev. 104, 1506 (1956).

1889. Spin-spin relaxation at very low temperatures in chromic methylammonium alum, E. Ambler and R. P. Hudson. Phys. Rev. 104, 1500 (1956).

1890. Stability of oscillations of superposed fluids, C. M. Tchen. J. Appl. Phys. 27, No. 7,760 (1956).

1891. Stabilization film processing for $X$ - and $\gamma$-ray dosimetry, S. Levinos and M. Ehrlich. Nucleonics 14, No. 7, 72 (1956).

1892. Stable carbon isotope analysis of optical spectroscopy, R. E. Ferguson and H. P. Broida. Anal. Chem. 28, 1436 (1956).

1893. Stable systems of differential equations with integrable forcing term, H. A. Antosiewicz. J. London Math. Soc. 31, 208 (1956).

1894. Unassigned.

1895. Standard electrode potential of the silver, silver chloride electrode, R. G. Bates, E. A. Guggenheim, H. S. Harned, D. J. Ives, G. J. Janz, C. B. Monk, R. A. Robinson, R. H. Stokes, and W. F. J. Wynne-Jones. J. Chem. Phys. 25, 361 (1956).

1896. Statistical methods in chemistry, J. Mandel and F. J. Linnig. Anal. Chem. 28, 770 (1956).

1897. Steric effects in Azo- and indigo dyes, W. R. Brode. Recent Advances in Chem. Colouring Matters (London, England) SP-4, 1 (1956).

1898. Stress-corrosion cracking of type 304 austenitic stainless steel, H. L. Logan and R. J. Sherman, Jr. Welding J. Research Suppl. 35 , No. 8,389 (1956).

1899. Stress-strain relationships in yarns subjected to rapid impact loading, H. F. Schiefer, J. C. Smith, F. L. McCrackin, and W. K. Stone. Am. Soc. Testing Materials Spec. Tech. Publ. 185. Symp, on Speed of Testing on NonMetallic Materials, p. 47 (1956).

1900. Stress-strain relationship in yarns tested at rates of straining up to a million percent per minute, H. F. Schiefer, J. C. Smith, F. L. McCrackin, W. K. Stone, and K. M. Towne. Physics of Wool and Other Viscoelastic Fibers, D, 148 (1956) (Proc. International Wool Textiles Research Conference, August 20-25, 1955, (Sydney, Australia) (1956).

1901. Structural rearrangements in silicate glasses during annealing, L. W. Tilton. J. Soc. Glass Technol. (British) 40, T338 (1956). 
1902. Structure of a magnetohydrodynamic shock wave in a plasma of infinite conductivity, H. K. Sen. Phys. Rev. 102, 5 (1956).

1903. Student research on ASTM problems,

A. T. McPherson. ASTM Bull. 217, 31 (1956).

1904. Studies of dose distributions in water for betatron X-rays to $37 \mathrm{Mev}, \mathrm{B}$. Zendle, H. W. Koch, J. McElhinney, and J. W. Boag. Radiol. Research 5, No. 22, 107 (1956).

1905. Studies on leather by means of a sonic technique, J. R. Kanagy and M. Robinson. J. Am. Leather Chemists Assoc. 51, 174 (1956).

1906. Sulfinic acid derivatives as accelerators in the polymerization of methyl methacrylate, G. M. Brauer and F. R. Burns. J. Polymer Sci. 19, No. 92, 311 (1956).

1907. Surface currents excited by an infinite slot on half-planes and ribbons, J. R. Wait and M. O'Grady. IRE Trans. on Ant. Prop. AP-4, No. 1, 47 (1956).

1908. Survey of geomagnetic eclipse phenomena, S. Chapman. Special Suppl. J. Atmospheric and Terrest. Phys. 6, 221 (1956).

1909. Synthesis of maltose-1- $\mathrm{C}^{14}$, maltobionodeltalactone-1- $\mathrm{C}^{14}$ and lithium maltobionate-1$\mathrm{C}^{14}$ from 3-(-D-glucopyranosyl)-D-arabinose, H. S. Isbell and R. Schaffer. J. Am. Chem. Soc. 78 , No. 9, 1887 (1956).

1910. Systems of distinct representatives and linear programming, A. J. Hoffman and H. W. Kuhn. Am. Math. Mo. 63, 455 (1956).

1911. Techniques and theory of fast coincidence experiments, Z. Bay. IRE Trans. on Nuclear Science NS-3, No. 4, 12 (1956).

1912. Tektites and the lost planet, R. Stair. Sci. Monthly 83, 1 (1956).

1912 A. Tektites, meteoric glass, R. Stair. Discovery (Norwich, England) 17, No. 10, 408 (1956).

1913. Temper brittleness of boron-treated steel, S. J. Rosenberg. Wright Air Devel. Ctr. Tech. Rept. 56-396 (1956).

1914. Temperature coefficient of the speed of sound in water near the turning point, $M$. Greenspan, C. E. Tschiegg, and F. Breckenridge. J. Acoust. Soc. Am. 28, 500 (1956). 1915. Temperature effects in gas-free liquid scintillators, H. H. Seliger and C. A. Ziegler. IRE Trans. on Nuclear Science NS-3, 4 (1956).

1916. Temperature inversions of anhydrous gallium orthophosphate, A. Perloff. J. Am. Ceram. Soc. 39, No. 3, 83 (1956).

1917. Temperature studies of air in a truck tire, G. G. Richey, R. H. Hobbs, and R. D. Stiehler. Rubber Age 79, 273 (1956).

1918. Unassigned.

1919. The absolute photometry of the Gegenschein. F. E. Roach and M. H. Rees. Symp. on the Airglow and the Aurora, September 1955, Belfast (Pergamon Press, London, England) p. 142 (1956).

1920. The adhesion of electroplated coatings to titanium, C. Stanley and A. Brenner. Ann. Proc. Am. Electroplaters' Soc. p. 123 (1956). 1921. The assignment problem, T. S. Motzkin. Proc. 6th Symp. Appl. Math. of Am. Math. Soc. (Santa Monica, Calif. 1953) p. 109 (1956). 1922. The Boltzman equation from the statistical mechanical point of view. M. S. Green. J. Chem. Phys. 25, 836 (1956).
1923. The crystal structure of aluminum phosphate and gallium phosphate, low cristobalite type, R. C. L. Mooney. Acta Cryst. 9, 728 (1956).

1924. The crystallization of flexible polymer molecules, L. Mandelkern. Chem. Rev. 56, No. 5, 903 (1956).

1925. The distinction between initiation and propagation of a fatigue crack, J. A. Bennett. Intern. Conf. on Fatigue of Metals, Inst. Mech. Engr 5. (London, England) (1956).

1926. The effect of particle size on the bulk density and strength properties of uranium dioxide specimens, M. D. Burdick and H. S. Parker. J. Am. Ceram. Soc. 39, No. 5, 181 (1956).

1927. The effects of weathering on certain mortars exposed in brick masonry with and without caps and flashings, J. W. McBurney. Am. Soc. Testing Materials Proc. 56, 1273 (1956).

1928. The electrical resistance to the earth of a live tree, F. M. Defandorf. Am. Inst. Elec. Engrs. Trans. Paper No. 56, 627, p. 1 (1956).

1929. The engineer at the National Bureau of Standards, A. V. Astin. Am. Eng. 26, No. 9, 18 (1956).

1930. The flame photometric determination of manganese in cement, J. J, Diamond. Anal. Chem. 28, 328 (1956).

1931. The formal power series for $\log \mathrm{e}^{\mathrm{x}} \mathrm{e}^{\mathrm{y}}, \mathrm{K}$. Goldberg. Duke Math. J. 23, 13 (1956).

1932. The infrared spectrum and rotational constants of carbon disulfide, H. C. Allen, Jr., E. K. Plyler, and L. R. Blaine. J. Am. Chem. Soc. 78,4843 (1956).

1933. The infrared spectrum and structure of perchlorlyfluoride, D. R. Lide, Jr., and D. E. Mann. J. Chem. Phys. 25, 1128 (1956).

1934. The infrared spectrum of acetylene- $d_{i}$, H. C. Allen, Jr., E. D. Tidwell, and E. K. Plyler. J. Am. Chem. Soc. 78, 3034 (1956). 1935. The integration of concepts in the terminology of measurement and control, H. L'. Mason. Trans. Am. Soc. Mech. Engrs. 30, No. 1,1 (1956).

1936. The ionosphere IGY program in the Arctic, A. H. Shapley. Proc. Arctic Conf. (Stockholm, Sweden, May 22-25, 1956) Int. Sci. Radio Union Info. Bul. 98, 29 (1956).

1937. The logical design of a one-microsecond parallel adder using one-megacycle circuitry, A. Weinberger and J. L. Smith. Am. Inst. Elec. Engrs. Proc. Western Joint Computer Conf. T-85 (1956).

1938. The melting behavior of polybutadienes, L. Mandelkern, M. Tryon, and F. A. Quinn, Jr. J. Polymer Sci. 19, 77 (1956).

1939. The National Bureau of Standards and its services to industry, R. D. Stiehler. Industries et Sciences. (L'Association des Ingenieurs Techniciens de Charleroi. Rhode-Staint-Geneselex-Bruxelles, Belgium), 30, No. 4, 17 (1956).

1940. The nature, cause and effect of porosity in electrodeposits, II. Radiographic detection of porosity in electrodeposits, F. Ogburn and M. Hilkert. Ann. Proc. Am. Electroplaters' Soc. 43,256 (1956).

1941. The nickel dip: a radioisotope study of metallic deposits in porcelain enameling, J. C. Richmond and H. B. Kirkpatrick. Natl. Advisory Comm. Aeronaut. Tech. Note 3577 (1956). 
1942. The normalizer of certain modular subgroups, M. Newman. Can. J. Math. 8, No. 1, 29 (1956).

1943. The number of absolute points of a correlation, A. J. Hoffman, M. Newman, E. G. Straus, and O. Taussky. Pacific J. Math. 6, 83 (1956).

1944. The preparation and maintenance of standards of radioactivity, W. B. Mann. J. Appl. Radiation and Isotopes 1, 3 (1956).

1945. The propagation characteristics of the frequency band $152-162 \mathrm{Mc}$ which is available for marine radio communications, $H$. $T$. Dougherty. Appendix F to report of Spec. Committee 19 of Radio Tech. Commission for Marine Services "Study of a reliable short range radio telephone system" (Washington, D. C. ) (1956).

1946. The radiation pattern of an antenna mounted on a surface of large radius of curvature, J. R. Wait, Proc. IRE 44, 694 (1956).

1947. The radiation patterns and conductances of slots cut on rectangular metal plates, J. R. Wait and D. G. Frood. Proc. IRE 44, 1469 (1956).

1948. The reactions of ammonia and hydrazine with oxygen atoms and hydrogen atoms in atomic flames, G. E. Moore, K. E. Shuler, S. Silverman, and R. C. Herman. J. Phys, Chem. 60,813 (1956).

1949. The rotation-vibration spectra of deuterated water vapor, W. S. Benedict, N. Gailar, and E. K. Plyler. J. Chem. Phys, 24, No. 6, 1139 (1956).

1950. The structure of anhydrous scandium phosphate, R. C. L. Mooney. Acta Cryst. 9, 677 (1956).

1951. The synthesis and infrared absorption spectra of some m-polyphenyls, R. L. Alexander, Jr. J. Organic Chem. 21, 1464 (1956).

1952. The thermal conductivity of argon for temperatures between $0^{\circ}$ and $75^{\circ} \mathrm{C}$ and at pressures up to 2500 atmospheres, A. Michels, A. Botzen, A. S. Friedman, and V. J. Sengers. Physica 22, 121 (1956).

1953. The uncertainty of measurements, J. Mandel. Chem. Corps Engineering Command, Proc. 2d Annual Statistical Engineering Symp. (Edgewood, Maryland) p. 57 (1956).

1954. The use of an AC bridge to measure core loss at high induction, I. L. Cooter and W. P. Harris. Commun, and Electronics. Am. Inst. Elec. Engrs. No. 24, 252 (1956).

1955. The use of scintillation spectrometers in the study of continuous gamma- and X-ray spectra between 0.01 and $20 \mathrm{Mev}, \mathrm{N}$. Starfelt. Kungl. Fysiografiska Sallskapets I. Lund Forhandlinger Bd 26, Nr 5 (University of Lund, Lund, Sweden) (1956).

1956. The Washington area scientists-forteachers program, J. K. Taylor. J. Chem. Educ. 33, 461 (1956).

1957. Theoretical electromotive forces for cells containing a single solid or molten chloride electrolyte, W. J. Hamer, M. S. Malmberg, and B. Rubin. J. Electrochem. Soc. 103, 8 (1956).

1958. Thermal and photochemical processes in polystyrene in the glassy state, L. A. Wall and M. Tryon. Nature 178, 101 (1956).
1959. Unassigned.

1690. Thermal decomposition of manganous and ferrous carbonates, H. E. Kissinger, H. F. McMurdie, and B. Simpson. J. Am. Ceram. Soc. 39 , No. 5, 168 (1956).

1961. Thermal degradation of unvulcanized and vulcanized rubber in a vacuum, S. Straus and S. L. Madorsky. Ind, Eng. Chem. 48, 1212 (1956).

1962. Thermal stresses and deformation in beams, S. Levy. Aeronaut. Eng. Rev. 15, 10 (1956).

1963. Thermochemistry and thermodynamics of substances, C. W. Beckett, M. S. Green and H. W. Woolley. Ann. Rev. Phys. Chem. 7, 287 (1956).

1964. Thermodynamic properties of gaseous nitrogen, H. W. Woolley. Natl. Advisory Comm. Aeronaut. Tech. Note 3271 (1956).

1965. Thermodynamics of crystallization in high polymers: gutta percha, L. Mandelkern, F. A. Quinn, Jr., and D. E. Roberts. J. Am. Chem. Soc. 78,926 (1956).

1966. Three ways to increase efficiency of liquid scintillators, C. A. Ziegler, H. H. Seliger, and I. Jaffe. Nucleonics 14, No. 5, 84 (1956).

1967. Transient fields of a vertical dipole over a homogeneous curved ground, J. R. Wait. Can. J. Phys. 34, 27 (1956).

1968. Transients in signal analysis, E. L. R.

Corliss, J. Wash. Acad. Sci. 46, 305 (1956).

1969. Unimodular matrices of order two which commute, K. Goldberg. J. Wash. Acad. Sci. 46,337 (1956).

1970. Use of the sensitivity criterion for the comparison of the Bekk and Sheffield smoothness testers, T. W. Lashoff, J. Mandel, and V. Worthington. Tappi 39, No. 7, 532 (1956).

1971. VHF propagation by ionospheric scatteringsurvey of experimental results, R. C. Kirby. IRE Trans, on Commun. Systems CS-4, No. 1, 17 (1956).

1972. VHF propagation measurements in the Rocky Mountain region, R. S. Kirby, H. T. Dougherty, P. L. McQuate. IRE Trans. on Vehicular Commun. VC-6, 13 (1956).

1973. Vibrational spectrum of tetrabromoethylene, D. E. Mann, J. H. Meal, and E. K. Plyler. J. Chem. Phys. 24, 1018 (1956). 1974. Vistas in astronomy, C. E. Moore. Chapter 13, Vol. 2, Atomic Spectra - Their role in astrophysics, p. 1209 (Pergamon Press, New York, N. Y.) (1956).

1975. Volume temperature relations of amorphous polymers over an extended temperature range, G. M. Martin, S. S. Rogers, and L. Mandelkern. J. Polymer Sci. 20, 579 (1956). 1976. Weights and measures administration in the U. S., W. S. Bussey and M. W. Jensen. Supplement to "The Monthly Review" of the Inst. of Weights and Measures Admin. (April 1956).

1977. Why screw threads must be everybody's business, I. H. Fullmer. Proc. 7th Natl. Conf. on Standards, p. 45, Am. Stds. Assoc. (1956). 1978. Zirconia reactions in binary oxide systems, R. S. Roth. J. Am. Ceram. Soc. 39, No. 5, 196 (1956). 
1979. A collaborative study on the cinchonine method for determination of lignosulfonates in vegetable extract blends, S. Dahl and J. Mandel. J. Am. Leather Chemists Assoc. 52, 184 (1957).

1980. A determinantal inequality of H. P.

Robertson, M. Marcus. J. Wash. Acad. Sci. 47,264 (1957).

1981. A discussion of "Design of corrugated diaphragms" by J. A. Haringx, R. F. Dressler. Trans. Am. Soc. Mech. Eng. 79, 61 (1957). 1982. A field emitter point projection X-ray microscope, L. Marton, R. A. Schrack, and R. C. Placious. X-ray Microscopy and Microradiography (Academic Press Inc., New York, N. Y.) p. 287 (1957).

1983. A function to aid in the fitting of kinetic data to a rate equation, J. H. Flynn. J. Phys. Chem. 61, 110 (1957).

1984. A large liquid hydrogen bubble chamber, D. B. Chelton, D. B. Mann, and R. A. Byrns. Proc. 1956 Cryogenic Engineering Conference, Paper H-2, p. 325. Edited by K. D. Timmerhaus, Chemical Engineering Dept., University of Colorado (Boulder, Colorado) (1957).

1985. A matrix minimization problem, A. J. Goldman. J. Wash. Acad. Sci. 47, No. 12, 405 (1957).

1986. A mechanical refrigeration process for the no-loss storage of liquid hydrogen, B. W. Birmingham. Refrig. Eng. 65, No. 42-44, 86 (1957).

1987. A method for measuring the mucosal surface contours of impressions, casts, and dentures, N. W. Rupp, G. Dickson, M. E. Lawson, Jr., and W. T. Sweeney. J. Am. Dential Assoc. 54,24 (1957).

1988. A new arrangement for ortho-para conversion of liquid hydrogen in the large CEL-NBS liquefier, V. J. Johnson. Proc. 1956 Cryogenic Engineering Conference, Paper A-4, p. 19. Edited by K. D. Timmerhaus, Chemical Engineering Dept., University of Colorado (Boulder, Colorado) (1957).

1989. A new point of view in the measurement of light and color, D. B. Judd. Anales real soc. espan. fis. y quim, 53A, No. 1-2, 43 (1957). 1990. A note on symmetric functions of eigenvalues, M. Marcus and R. Thompson. Duke Math. J. 24, 43 (1957).

1991. A note on the climatic variation of absolute humidity, B. R. Bean and B. A. Cahoon. Bull. Am. Meteorol. Soc. 38, No. 7, 395 (1957).

1992. A note on the propagation of the transient ground wave, J. R. Wait. Symp. on the Prop. of V. L. F. Radio Waves, Sponsored by the NBS Boulder Laboratories and IRE Prof. Group on Ant. and Prop., January 23-25, 1957 (Boulder, Colorado), Appendix D, V. 4, 67; Can. J. Phys. 35,1146 (1957).

1993. A panoramic X-ray dental machine, D. C. Hudson, J. W. Kumpula, G. Dickson, U. S.

Armed Forces Med. J. 8, No. 1, 46 (1957). 1994. A photographic personnel dosimeter for $\mathrm{X}$-radiation in the range from $30 \mathrm{kev}$ to beyond $1 \mathrm{Mev}, \mathrm{M}$. Ehrlich. Radiology 68, No. 4, 549 (1957).

1995. A progress report on SEAC, M. R. Fox.

Signal 11, No. 7, 18 (1957).
1996. A proposed specification for dental chromium-cobalt casting alloys, D. F. Taylor and W. T. Sweeney. J. Am. Dental Assoc. 54, 44 (1957).

1997. A pulse method for determining dynamic stress-strain relations, W. Ramberg and L. K. Irwin. Trans. IX ${ }^{\mathrm{e}}$ Congres International de Mechanique Appliquee (Brussels, Belgium) 8, 480 (1957).

1998. A reproducible and stable silver-silver oxide electrode, W. J. Hamer and D. N. Craig. J. Electrochem. Soc. 104, 206 (1957).

1999. A service for the Nation's technology, A. V. Astin. Mag. of Standards 28, No. 7, 196 (1957).

2000. A simple technic for making porcelain jacket crowns, H. Sacchi and G. C. Paffenbarger. J. Am. Dental Assoc. 54, 366 (1957).

2001. A simplified method of locating the point of symmetry, F. E. Washer. Photogrammetric Eng. XXIII, No. 1, 75 (1957).

2002. A technological challenge to the textile industry, R. B. Hobbs. Can. Textile J. 74, 53 (1957).

2003. A theory of the production of VLF noise (so-called drawn chorus) by traveling wave amplification in the exosphere of the earth, R. M. Gallet and R. A. Helliwell. Symp. on the Prop. of VLF Radio Waves, Sponsored by the NBS Boulder Laboratories and IRE Prof. Group on Ant. Prop. , January 23-25, 1957 (Boulder, Colorado), Paper No. 20, V2 (1957).

2004. Unassigned.

2005. Abrasion resistance, R. D. Stiehler.

Encyclopedia of Chemistry (Reinhold Publ.

Corp., New York, N. Y.) p. 1 (1957).

2006. Absence of interference effects in the $\beta$ decay of polarized $\mathrm{CO}^{56}$ and $C O^{58}$ nuclei, $\mathrm{E}$.

Ambler, R. W. Hayward, D. D. Hoppes, and R. P. Hudson. Phys. Rev. 108, 503 (1957).

2007. Ab solute intensities of the 721 and $742 \mathrm{~cm}^{-1}$

bands of $\mathrm{CO}_{2}, \mathrm{H}$. J. Kostkowski and L. D. Kap-

lan. J. Chem. Phys. 26, 1252 (1957).

2008. Absolute measurement of $W_{\text {air }}$ for sulfur-

35 beta rays, Z. Bay, H. H. Seliger, and H. O. Wyckoff. Radiation Research 7, No. 6, 558 (1957).

2009. Absolute temperature below $1^{\circ} \mathrm{K}$ : chromic methylammonium alum as a thermometric substance, E. Ambler and R. P. Hudson, J. Chem. Phys. 27, 378 (1957).

2010. Absorption of sound by patches of absorbent materials, R. K. Cook. J. Acoust. Soc. Am. 29, 329 (1957).

2011. Adsorption of $\mathrm{C}^{14}$ dextran to human blood platelets and red blood cells in vitro, S. Rothman, E. Adelson, A. Schwebel, and R. D. Langdell. Vox Sanguinis 2, 104 (1957).

2012. An adjustable sliding termination for rectangular waveguide, $R$. W. Beatty. IRE Trans. on Microwave Theory and Tech, MTT-3, 192 (1957).

2013. An exact solution for a cylindrical cavity containing a gyromagnetic material, H. E. Bussey and L. A. Steinert. Proc. IRE 45, Pt. 1, 693 (1957). 
2014. An experimental study of the strength and fatigue of glass at very low temperatures, R. H. Kropschot and R. P. Mikesell. Proc. 1956 Cryogenic Engineering Conference, Paper D-5, p. 136. Edited by K. D. Timmerhaus, Chemical Engineering Dept., University of Colorado (Boulder, Colorado) (1957).

2015. An experimental test of parity conservation in beta decay, C. S. Wu, E. Ambler, R. W. Hayward, D. D. Hoppes, and R. P. Hudson. Phys, Rev. 105, 1413 (1957).

2016. An inclusion theorem for modular groups, M. Newman. Proc. Am. Math. Soc. 8, 125 (1957).

2017. An intercomparison of the French and U. S. roentgen ray standards, A. Allisy, L. DeLaVergne, and H. O. Wyckoff. Acta Radiol. 48 , No. 6, 486 (1957).

2018. An isotopic tracer study of carbon formation in hydrocarbon flames, R. E. Ferguson. Combustion and Flame 1, No. 4, 431 (1957). 2019. An observation of audio-frequency electromagnetic noise during a period of solar disturbance, J. M. Watts. J. Geophys, Research 62 , 199 (1957).

2020. Analytical procedures: natural and synthetic rubbers, M. Tryon. Anal. Chem. 29, 714 (1957)

2021. Angular correlation of radiations with parallel angular moments, U. Fano. Il Nuovo Cimento (Roma, Italy) Serie X 5, 1358 (1957). 2022. Anisotropic relaxation peak in the internal friction of crystalline quartz, J. H. Wasilik. Phys, Rev, 105, 1174 (1957).

2023. Unassigned.

2024. Annual review of pulp and paper industry, R. B. Hobbs and W. K. Wilson. Ind. Eng. Chem. 49, 42A (1957).

2025. Another milestone in screw thread standardization, I. H. Fullmer. Fasteners 12 , No. 4,3 (1957).

2026. Unassigned.

2027. Application of configuration interaction to the $\mathrm{H}_{3}$ complex, B. J. Ransil. J. Chem. Phys, 26, No. 4, 97 (1957).

2028. Aspects of gamma ray penetration theory relevant to shielding problems, USNRDL Revs. and Lectures 1, No. 29, 25 (1957).

2029. Asphalt research at the National Bureau of Standards, H. R. Snoke. ASTM Bull. No. 220 , p. 41 (1957).

2030. Asymptotic behavior of tests on the mean of a logarithmic-normal distribution with known variances, N. C. Severo. Ann. Math. Stat. 28,1044 (1957).

2031. Atmospheric research, International Geophysical Year, R. J. Slutz. Americana Annual, p. 60. (An Encyclopedia of the Events of 1956, Americana Corp., New York, N. Y.) (1957).

2032. Attenuation of 14. I- Mev neutrons in water, R. S. Caswell, R. F. Gabbard, D. W. Padgett, and W. P. Doering. Nuclear Sci. and Engr. 2, 143 (1957).

2033. Audio-frequency electromagnetic hiss recorded at Boulder in 1956, J. M. Watts. Symp. on Prop. of V. L. F. Radio Waves, Sponsored by the NBS Boulder Laboratories and IRE Prof. Group on Ant. and Prop., January 23-25, 1957 (Boulder, Colorado), Paper No. 17, V. 2; Geofis. pura e appl. (Milano, Italy) 37, 169 (1957).
2034. Automatic lens design methods, D. P. Feder. J. Opt. Soc. Am. 47, No, 10,902 (1957).

2035. Automatic temperature regulation and recording in precision adiabatic calorimetry, E. D. West and D. C. Ginnings. Rev. Sci. Instr. 28, 1070 (1957).

2036. Automatic timer simplifies small-current measurements, L. Costrell and F. H. Attix. Nucleonics 15, No. 2, 83 (1957).

2037. Basic standards for science and industry, R. D. Huntoon. Electronic Design 5, No. 16, 38 (1957); Proc. Conf. Instrumentation and Control in the Process Industries, February 6-7, 1957 (Armour Research Foundation, Illinois Institute of Technology, Chicago, nlinois ) (1957).

2038. Breathing oxygen storage dewars, W. A. Wilson. Proc. 1956 Cryogenic Engineering Conference, Paper B-1, p. 54. Edited by K. D. Timmerhaus, Chemical Engineering Dept., University of Colorado (Boulder, Colorado) (1957).

2039. Bureau of Standards comments on Loran communications, E. K. Smith. IRE Trans, on Aeronaut, Navigational Elec. ANE-4, 84 (1957). 2040. Calcification in lysine deficiency, R. C. Likins, L. A. Bavetta, and A. S. Posner. Arch. of Biochem, and Biophys. 70, 401 (1957). 2041. Calculation of an optical merit function and its derivatives with respect to the system parameters, D. P. Feder, J. Opt. Soc. Am. 47 , No. 10,913 (1957).

2042. Calculation of cryoscopic data, C. P. Saylor. Anal. Chim. Acta 17, 36 (1957).

2043. Calculation of energy dissipation by gamma radiation near the interface between two media, M. J. Berger. J. Appl. Phys. 28, No. 12, 1502 (1957).

2044. Calculations of ionospheric reflection coefficients at very low radio frequencies, J. R. Wait and L. B. Perry. J. Geophys. Research 62, No. 1, 43 (1957).

2045. Calibration of airplane cameras, F. E. Washer. Photogrammetric Eng. 23, No. 5, 890 (1957).

2046. Calibration of shock and vibration pickups, W. Ramberg. Noise Control 3, No. 5, 23 (1957).

2047. Calorimetric determination of the power in a $1400 \mathrm{kev}$ X-ray beam, J. McElhinney, B. Zendle, and S. R. Domen, Radiol, Research 6. No. 1,40 (1957).

2047A. Capillary viscometry, A. B. Bestul. J. Soc. Cosmetic Chemists 8, No, 6, 345 (1957)

2048. Catalysis of the ortho-parahydrogen conversion, D. H. Weitzel, J. W. Draper, O. E. Park, K. D. Timmerhaus, and C. C. Van Valin. Proc. 1956 Cryogenic Engineering Conference, Paper A-4, p. 12. Edited by K. D. Timmerhaus, Chemical Engineering Dept., University of Colorado (Boulder, Colorado) (1957).

2049. Central Radio Propagation Laboratory, F. H. Brown. Signal 12, No. 4, 9 (1957). 2050. Changes in agar-agar type duplicating material and agar-agar on heating and storage, P. M. Margetis and W. C. Hansen. J. Am. Dental Assoc. 54, 737 (1957). 
2051. Characteristics and present requirements of ferrites, R. D. Harrington. Proc. 13th Ann. Meeting Metal Powder Assoc., April 30-May 1, 1957 (Chicago, Dlinois) p. 177 (1957).

2052. Characteristics of atmospheric noise from 1 to $100 \mathrm{Kc} / \mathrm{s}$, A. D. Watt and E. L. Maxwell. Symp. on the Prop. of V. L. F. Radio Waves, Sponsored by the NBS Boulder Laboratories and IRE Prof. Group on Ant, and Prop. , January 23-25, 1957 (Boulder, Colorado), Paper No. 35, V. 3; Proc. IRE 45, 787 (1957). 2053. Characteristics of metal-clad laminates, D. S. Hoynes. Elec. Mfg. 59, No. 4, 104 (1957).

2054. Characteristics of some insulations for liquid oxygen transfer lines, D. A. Van Gundy and R. B. Jacobs. Proc, 1956 Cryogenic Engineering Conference, Paper E-1, p. 156. Edited by K. D. Timmerhaus, Chemical Engineering Dept., University of Colorado (Boulder, Colorado) (1957).

2055. Characteristics of ultrasonic vibrations, W. T. Sweeney. J. Am. Dental Assoc. 55, 819 (1957).

2056. Clamp-on microammeter measures a-c current, G. F. Montgomery and C. Stansbury. Electronics 30 , No. 12, 152 (1957).

2057. Classes of positive definite unimodular circulants, M. Newman and O. Taussky. Can. J. Math. 9, 71 (1957).

2058. Comments on a recent paper by Deresiewicz on plane waves in a thermoelastic solid, M. Greenspan. J. Acoust. Soc. Am. 29, 966 (1957).

2059. Comments on the attenuation versus frequency characteristics, J. R. Wait. Symp. on the Prop. of V. L.F. Radio Waves, Sponsored by the NBS Boulder Laboratories and IRE Prof. Group on Ant. and Prop., January 23-25, 1957 (Boulder, Colorado), Appendix A, V. 4, 43; Proc. IRE 45, 768 (1957).

2060. Commutativity in finite matrices, $O$. Taussky. Am. Math. Mo. 64, 229 (1957). 2061. Comparative characteristics of mediumpriced fully automatic computers for statistical applications, M. E. Stevens and S. N. Alexander. Proc. of First Conf. on the Design of Experiments in Army Research, Development and Testing, Office of Ordnance Research (Durham, N. C.) OORR 57-1 (June 1957).

2062. Comparison of cryoscopic determinations of purity of benzene by thermometric and calorimetric procedures, A. R. Glasgow, Jr., G. S. Ross, A. T. Horton, D. Enagonio, H. D. Dixon, C. P. Saylor, G. T. Furukawa, M. L. Reilly, and J. M. Henning. Anal. Chim. Acta 17, 54 (1957); Proc. I. U. P. A. C., International Symp. on Purity Control by Thermal Analysis (Amsterdam, The Netherlands) (1957).

2063. Comparison of mechanical properties of flag sheets, molded shapes, and postformed shapes of cotton fabric-phenolic laminates, F. W. Reinhart, C. L. Good, P. S. Turner, and I. Wolock. Nat1. Advisory Comm. for Aeronaut. Tech. Note 3825 (1957).
2064. Complete night of vertical-incidence ionosphere soundings covering frequency range from $50 \mathrm{Kc} / \mathrm{s}$ to $25 \mathrm{Mc} / \mathrm{s}, \mathrm{J}$. M. Watts. J. Geophys. Research 62, 484 (1957).

2065. Complete sequences and approximations in normed linear spaces, P. Davis and K. Fan. Duke Math. J. 24, 183 (1957).

2066. Computation of effective intensity of flashing lights, C. A. Douglas. Ilum. Eng. 52, No. 12, 641 (1957).

2067. Condensation interfaces in two-phase flows, P. Chiarulli and R. F. Dressler. J. Appl. Phys. 28, 990 (1957).

2068. Congruences for the coefficients of modular forms and some new congruences for the partition function, M. Newman. Can. J. Math. 9, 549 (1957).

2069. Construction and application of a class of modular functions, M. Newman. Proc. London Math. Soc. 7, 334 (1957).

2070. Conversion of payroll processes at the National Bureau of Standards from manual to punched cards, P. R. McClennon. Federal Accountant 7, No. 2, 64 (1957).

2071. Creep behavior of structural joints of aircraft materials under constant loads and temperatures, L. Mordfin and A. C. Legate. Natl. Advisory Comm. Aeronaut. Tech. Note 3842 (1957).

2072. Creep of crystalline nonmetals, J. B. Wachtman, Jr. Am. Soc. Metals Symp., Oct. 6, 1956, Creep and Recovery p. 344 (1957). 2073. Cryogenic characteristics of wire resistance strain gages, R. M. McClintock. Proc. 1956 Cryogenic Engineering Conference, Paper E-4, p. 172. Edited by K. D. Timmerhaus, Chemical Engineering Dept., University of Colorado (Boulder, Colorado) (1957).

2074. Crystal mount and techniques for measuring high frequency induced electroluminescence, G. G. Harman. Rev. Sci. Instr. 28, No. 2, 127 (1957).

2075. Current situation with regard to permissible radiation exposure levels, L. S. Taylor. Radiology 69, No. 1, 6 (1957).

2076. Cutting characteristics of dental burs as shown by high speed photomicrography, J. L. Hartley, D. C. Hudson, W. T. Sweeney, and W. P. Richardson. U. S. Armed Forces Med. J. 8,209 (1957).

2077. Density expansions of distribution functions. I. Virial expansion for finite closed systems; canonical ensemble, I. Oppenheim and P. Mazur. Physica 23, 197 (1957).

2078. Density expansions of distribution functions. II. Density expansions in the grand canonical ensemble, I. Oppenheim and P. Mazur. Physica 23, 216 (1957).

2079. Dental Research, G. C. Paffenbarger. Pub, in Book "Developments in Dentistry During the Last 25 Years," Med. Digest of India (Bombay, India), p. 351 (1957).

2080. Description of color, D. B. Judd. Proc. Perkin Centennial (Ogden Printing Co., New York, N. Y.) (1957).

2081. Description of states in quantum mechanics by density matrix and operator techniques, U. Fano. Rev. Modern Phys. 29, No. 1, 74 (1957). 
2082. Design and performance of a portable infiltration meter, C. W. Coblentz, and P. R. Achenbach. Heating, Piping, Air Conditioning 29 , No. 7, 155 (1957); Trans. Am. Soc. Heating and Air Conditioning Engrs., Paper 477 (1957).

2083. Design of experiments in research and development, W. J. Youden. Proc. of Conference on Design of Experiments in Army Research, Development, and Testing, Office of Ordnance Research Report 57-1 (Durham, N. C. ) p. 9 (1957).

2084. Development of craze and impact resistance in glazing plastics by multiaxial stretching, G. M. Kline, I. Wolock, B. M. Axilrod, M. A. Sherman, D. A. George, and V. Cohen. Natl. Advisory Comm. Aeronaut. Tech. Report 1290 (1957).

2085. Development of methods for evaluation of rotating dental diamond abrasive instruments, J. L. Hartley, D. C. Hudson, W. T. Sweeney, and G. Dickson. J. Am. Dental Assoc. 54, 637 (1957).

2086. Diffraction of a spherical wave pulse by a half plane screen, J. R. Wait. Can. J. Phys. 35, No. 5, 693 (1957).

2087. Diffusion of particles with memory, P. L. Bender. Proc. Natl. Acad. Sci. 43, No. 5, 412 (1957).

2088. Dimensions, units, and standards, A. G. McNish. Phys. Today 10, No. 4, 19 (1957). 2089. Dipole moment of perchloryl fluoride, A. A. Maryott and S. J. Kryder.J.Chem. Phys. 27, No. 5, 1221 (1957).

2090. Direct-coupled power amplifier for cryostat heating control, R. D. Goodwin and J. R. Prucell. Rev. Sci. Instr. 26, 581 (1957). 2091. Disaster monitoring with amateur photographic film and with dental X-ray film, $M$. Ehrlich. Radiology 68, No. 2, 251 (1957).

2092. Discussion of paper "Pressure-flow characteristics of pneumatic valves" by F. D. Ezekiel and J. L. Shearer, D. H. Tsai. ASME Trans. 79, 1589 (1957).

2093. Distillation of hydrogen-deuterium mixtures, T. M. Flynn, D. H. Weitzel, K. D. Timmerhaus, P. C. Vander Arend, and J. W. Draper. Proc. 1956 Cryogenic Engineering Conference, Paper A-6, p. 39. Edited by K. D. Timmerhaus, Chemical Engineering Dept., University of Colorado (Boulder, Colorado) (1957).

2094. Does rationalization change units? F. B. Silsbee. Elec. Eng. 76, 4 (1957).

2095. Early history and development of building codes, J. P. Thompson. BOCA News (Published by the Building Officials Conference of America, Inc.) 6, No. 1, 2 (1957).

2096. Effect of crystal orientation on fatiguecrack initiation in polycrystalline aluminum alloys, J. G. Weinberg and J. A. Bennett. Natl. Advisory Comm. Aeronaut. Tech. Note 3990 (1957).

2097. Effect of illumination on the oxidation of copper single crystals in water, J. Kruger. J. Appl. Phys. 28, No. 10, 1212 (1957).

2098. Effect of microwave signals incident upon different regions of a dc hydrogen glow, B. J. Udelson. J. Appl. Phys. Letter to Editor 28, No. 3, 380 (1957).
2099. Effect of new lower permissible exposure levels on use of radioactive isotopes, L. S. Taylor. Proc. United Nations Educational, Scientific and Cultural Organization Conf., Paris, France (Sept. 1957); Impact 8, 209 (1957).

2100. Effect of super-refractive layers on tropospheric signal characteristics in the Pacific coast region, A. P. Barsis and F. M. Capps. IRE Wescon Conv. Record 1, Pt. I, 116 (1957).

2101. Effective intensity of flashing lights, T. H. Projector. Illum. Eng. 52, No. 12, 630 (1957).

2102. Effects of boundary and inhomogeneities on the penetration of gamma radiation, $M$. J. Berger. USNRDL Revs, and Lectures 1, No. 29,47 (1957).

2103. Elastic distortion error in the dead weight piston gage, D. P. Johnson, J. L. Cross, J. P. Hill, and H. A. Bowman. Ind. Eng. Chem. 49, 2046 (1957).

2104. Elastic waves in anisotropic media, J. L. Synge. J. Math. Phys. 35, 323 (1957).

2105. Electrical properties of samarium doped barium titanate, G. G. Harman. Phys. Rev. 106, No. 6, 1358 (1957).

2106. Electrodeposition of metals from organic solutions. III. Preparation and electrolysis of titanium and zirconium compounds in nonaqueous media, W. E. Reid, Jr., J. M. Bish, and A. Brenner. J. Electrochem. Soc. 104, No. 1, 21 (1957).

2107. Electrodeposition of metals from organic solutions. IV. Electrodeposition of beryllium and beryllium alloys, G. B. Wood and A. Brenner. J. Electrochem. Soc. 104, No. 1, 29 (1957).

2108. Electrodeposition of metals from organic solutions. V. Electrodeposition of magnesium alloys, J. H. Connor, W. E. Reid, Jr., and G. B. Wood. J. Electrochem. Soc. 104, 38 (1957).

2109. Electron scattering phenomena, L. Marton. J. Sci. Ind. Research 16a, No. 6, 221 (1957).

2110. Emissivities of metallic surfaces at $76^{\circ} \mathrm{K}, \mathrm{M}$. M. Fulk and M. M. Reynolds. J. Appl. Phys. 28, 1464 (1957).

2111. Energy and angular distribution of X-rays scattered in lead, J. H. Hubbell and E. V. Hayward and W. F. Titus. Phys. Rev. 108, No. 6, 1361 (1957).

2112. Evaluation of analytical methods by the sensitivity criterion, J. Mandel and R. D. Stiehler. Anal. Chem. 29, 17A (1957). 2113. Evaluation of width of cracks in concrete at the surface of reinforcing steel by means of tensile bond specimens, D. Watstein, and R. G. Mathey. Proc. RILEM Symp. on Bond and Crack Formation of Reinforced Concrete (Stockholm, Sweden) (1957).

2114. Excess noise in microwave crystal diodes used as rectifiers and harmonic generators, J. M. Richardson and J. J. Faris. IRE Trans. on Microwave Theory and Tech. MTT-5, No. 3, 208 (1957). 
2115. Existence theorems and extreme solutions for inequalities concerning convex functions or linear transforms, K. Fan. Math. Z. (Berlin, Germany) 68, 205 (1957).

2116. Experiences with incomplete block designs: examples, W. S. Connor. Proc. Symp. on the Design of Industrial Experiments, North Carolina State College (John Wiley \& Sons, New York, N. Y.) p. 193 (1957).

2117. Experimental equipment for communication utilizing meteor bursts, R. G. Ochs and R. J. Carpenter. IRE WESCON Conv. Session 46: Ionospheric Prop., August 20-23, 1957 , San Francisco, California, IRE WESCON Conv. Record, Pt. 1, 283 (1957).

2118. Unassigned.

2119. Experiments in processing pictorial information with a digital computer, $R$. A. Kirsch, L. Cahn, L. C. Ray, and G. H. Urban. 1957 Eastern Joint Computer Conf. Proc. (Wa shington, D. C.) (1957).

2120. Experiments with many factors, $M$. Zelen. Proc. 3d Annual Statistical Engineering Symp. (Chemical Center, Md.) p. 1 (1957).

2121. Field-strength variations recorded on a VHF ionospheric scatter circuit during the solar event of February 23, 1956, H. I. Leighton. J. Geophys. Research Letter to Editor 62, 483 (1957).

2122. Finding chemical records by digital computers, L. C. Ray and R. A. Kirsch. Science 126, No. 3278,814 (1957).

2123. Fire extinguishment by means of dry powder, C. S. McCamy, H. Shoub, and T. G. Lee, Proc. 6th Symp. (International) on Combustion (Reinhold Pub. Corp., New York, N. Y.) p. 795 (1957).

2124. Fire research at the National Bureau of Standards, A. F. Robertson. Proc. Fire Research, lst Correlation Conf. 1956, Natl. Academy of Sci. Publ. No. 475, p. 40 (1957). 2125. Fitting a straight line to certain types of cumulative data, J. Mandel. J. Am. Stat. Assoc. 52, 552 (1957).

2126. Flexibility of thin porcelain enameled sheet steel, A. G. Eubanks, J. R. Crandall, J. C. Richmond. Bull. Am. Ceram. Soc. 36, No, 2, 59 (1957).

2127. Forecasting of disturbed HF communication conditions, R. C. Moore. J. Atmospheric and Terrest. Phys. Special Suppl. 1957 Proc. of the Polar Atmosphere Symp., July 2-8, 1956 (Oslo, Norway) Pt. II, 147 (1957).

2128. Formal procedures for connecting terminals with a minimum total wire length, $\mathrm{H}$. Loberman and A. Weinberger. J. Assoc. Computing Machinery 4, No. 4, 428 (1957). 2129. Frozen free radicals, C. M. Herzfeld and A. M. Bass. Sci. American 3, 91 (1957). 2130. Frozen free radicals: The problem and the program at the National Bureau of Standards, L. T. Milliken. Capital Chemist 7, No. 8, 244 (1957).

2131. Fungicidal effectiveness of compounds applied to leather, S. Dahl and A. M. Kaplan. J. Am. Leather Chemists Assoc. 52, 536 (1957).

2132. Further experiments on $\beta$-decay of polarized nuclei, E. Ambler, R. W. Hayward, D. D. Hoppes, R. P. Hudson, and C. S. Wu. Phys. Rev. 106, No. 6, 1361 (1957).
2133. Further studies of an electron thickness gage: circuitry operating characteristics, and calibration, F. P. Brodell and A. Brenner. Plating 44, 591 (1957).

2134. Gaging and testing farm milk tanks, M. W. Jensen. Proc. Natl. Conf. Milk (Bulk) Handling (Michigan State University, Lansing, Michigan) p. 62 (1957).

2135. Galvanomagnetic effects in n-type indium antimonide, H, P. R. Frederikse and W. R. Hosler. Phys, Rev, 108, 1136 (1957).

2136. Galvanomagnetic effects in p-type indium antimonide, H. P. R. Frederikse and W. R. Hosler. Phys. Rev. 108, 1146 (1957).

2137. Games with random payoff matrices, A. J. Goldman. Summary Rept. 3d Conf. on Games, ONR Logistics Project (Princeton University, Princeton, N. J.) (1957).

2138. Gamma irradiation of polymethyl methacrylate and polystyrene, L. A. Wall and D. W. Brown. J. Phys. Chem. 61, 129 (1957).

2139. Gamma-ray spectroscopy, H. W. Koch. Chapter 15, Trace Analysis, Edited by Yoe \& Koch (John Wiley \& Sons, Inc., New York, N. Y.) P. 413 (1957).

2140. Generation of Bessel Functions on high speed computers, I. A. Stegun and $M$. Abramowitz. Math. Tables and Other Aids to Computation, 11, 255 (1957).

2141. Geographic distribution of geophysical stations on the polar cap, A. H. Shapley. J. Atmospheric and Terrest. Phys. Special Suppl. 1957, Proc. of the Polar Atmosphere Symp., July 2-8, 1956 (Oslo, Norway) Pt. II, 108 (1957).

2142. Glass formation in polymers: I. The glass transitions of the poly-(n-alkyl methacrylates), S. S. Rogers and L. Mandelkern. J. Phys. Chem. 61, 985 (1957).

2143. Gloss measurement--past, present, and future, H. K. Hammond, III. Am. Paint J. 41 , No. 37,94 (1957).

2144. Government and industry use of common standards, A. T. McPherson. Proc, of the Eighth Natl. Conf. on Standards (Am. Std. Assoc.), p. 26, (1957).

2145. Graphical solution of the single-degree-offreedom vibration problem with arbitrary damping and restoring forces, W. H. Pell. J. Appl. Mech. 24, No. 2, 311 (1957).

2146. Head regulator for cutoff valve, L. Bean. Anal. Chem. 29, No. 6, 98 (1957).

2147. Heat transfer through foams and powders, M. M. Fulk, R. J. Devereaux, and J. E.

Schrodt. Proc. 1956 Cryogenic Engineering Conference, Paper E-3, p. 166. Edited by K. D. Timmerhaus, Chemical Engineering Dept., University of Colorado (Boulder, Colorado) (1957).

2148. Heats of combustion of some peroxides and the heats of formation of acetate, propionate, and butyrate radicals, I. Jaffe, E. J. Prosen, M. Szwarc. J. Chem. Phys. 27, 416 (1957). 2149. Heats of wetting of modified collagen and other materials, J. R. Kanagy and J. M. Cassel. J. Am. Leather Chemists Assoc. 52, 248 (1957).

2150. Henry Norris Russell, C. E. Moore. The Observatory 77, No. 897, 67 (1957). 
2151. Hexafluorobenzene from the pyrolysis of tribromofluoromethane, M. Hellman, E.

Peters, W. J. Pummer, and L. A. Wall. J.

Am. Chem. Soc. 79, 5654 (1957).

2152. High-dispersion spectra of mars, C. C.

Kiess, C. H. Corliss, H. K. Kiess, E. L. R.

Corliss. Astrophys. J. 126, No. 3, 579 (1957).

2153. High-order harmonics for X-band

oscillator stabilization, M. C. Thompson, Jr., and

J. V. Cateora. Rev. Sci. Instr. 28, 656 (1957).

2154. High pressure gas handling equipment for

autoclaves in chemical research, R. D. Goodwin.

Ind. Engr. Chem. 49, 861 (1957).

2155. High-repetition-rate mercury pulser,

L. Costrell. Nucleonics 15, No. 11, 112 (1957).

2156. Hydrogen liquefaction by a dual pressure process, D. B. Chelton, J. Macinko, and J.

Dean. Refrig. Eng. 65, No. 8, 39 (1957).

2157. Increased rate of liquefaction of the

National Bureau of Standards helium liquefier,

D. B. Lawson. Rev. Sci. Instr. 28, 204 (1957).

2158. Inelastic scattering of 20-kev electrons in metal vapors, L. B. Leder. Phys. Rev. 107, No. 6, 1569 (1957).

2159. Influence of temperature on the adsorption of chrome $\left(\mathrm{Cr}_{2} \mathrm{O}_{3}\right)$ by calf skin squares, J. R. Kanagy. J. Am. Leather Chemists Assoc. 52, 142 (1957).

2160. Infrared absorption spectra of urea, thiourea, and some thiourea-alkali halide complexes, J. E. Stewart. J. Chem. Phys. 26, 248 (1957).

2161. Infrared and raman spectra of transfluorodichloroethylene, E. K. Plyler and D. E. Mann. J. Chem. Phys. 26, 773 (1957).

2162. Infrared spectrum of carbonyl sulfide, H. C. Allen, Jr., E. K. Plyler, and L. R.

Blaine. J. Chem. Phys. 26, No. 2, 400 (1957).

2163. Intermittent communications with a

fluctuating signal, G. F. Montgomery. Proc. IRE 45, No. 12, 1684 (1957).

2164. International textile work shows fast progress, W. D. Appel. Mag. of Standards 28, 38 (1957).

2165. Introduction to the VLF papers, J. R. Wait.

Proc. IRE 45, 739 (1957).

2166. Investigations of the properties of corrugated diaphragms, W. A. Wildhack, R. F. Dressler, and E. C. Lloyd. Trans. Am. Soc.

Mech. Engrs, , 79, 56 (1957).

2167. Invited discussion on the Haringx diaphragm theory, R. F. Dressler. Trans. Am. Soc. Mech. Engrs. 79, No. 1, 61 (1957).

2168 . Ionization and dissociation of hexafluorobenzene by electron impact, V. H. Dibeler, R. M. Reese, and F. L. Mohler. J. Chem. Phys. 26, No. 2, 304 (1957).

2169. Ionization and dissociation of oxygen difluoride by electron impact, V. H. Dibeler, R. M. Reese, and J. L. Franklin. J. Chem. Phys. 27, 1296 (1957).

2170. Ionization and dissociation of perchlorylfluoride by electron impact, V. H. Dibeler, R. M. Reese, and D. E. Mann. J. Chem. Phys. 27, 176 (1957).

2171. Ionospheric forward scatter, K. L.

Bowles. Annals of the International Geophysical Year, 3, Pt. 4, p. 346 (1957).
2172. Ionospheric reflection coefficients at VLF, A. G. Jean, L. J. Lange, and J. R. Wait. Symp. on the Prop. of V. L. F. Radio Waves, Sponsored by the NBS Boulder Laboratories and IRE Prof. Group on Ant. and Prop., January 23-25, 1957 (Boulder, Colorado), Paper No. 31, V. 3 .

2173. Ionospheric vertical soundings, J. W. Wright, R. W. Knecht, and K. Davies. Annals of the International Geophysical Year, 3, Pt. 1, 1 (Pergamon Press, New York, N. Y.) (IGY Instruction Manual: The Ionosphere, Pt. 1) (1957).

2174. Kinetics of the reaction between nickel and chlorine above $1,100^{\circ} \mathrm{K}$, J. D. McKinley, $\mathrm{Jr}$, , and K. E. Shuler. Proc. International Meeting of Chemistry (Paris, France), Section de Chimie Minerale, p. 637 (1957).

2175. Lattice energies of ionic cubic crystals, C. E. Weir. Phys. Rev. 108, No. 1, 19 (1957). 2176. Light scattering measurements of a fractionated nonionic detergent, L. M. Kushner, W. D. Hubbard, and A. Doan. J. Phys. Chem. 61,371 (1957).

2177. Long distance transfer of liquefied gases, R. B. Jacobs. Proc. 1956 Cryogenic Engineering Conference, Paper G-7, p. 303. Edited by K. D. Timmerhaus, Chemical Engineering Dept. , University of Colorado (Boulder, Colorado) (1957).

2178. Low-cost microvolt potentiometer, W. H. Wood. Rev. Sci. Instr. 28, No. 3, 202 (1957).

2179. Low frequency standard transmission, W. D. George. Proc. 11th Annual Symp. on Freq. Control, May 7-9, 1957 (Fort Monmonth, N. J.) p. 574 (1957).

2180. Low temperature physics, E. Ambler. Science, 126, 1096 (1957).

2181. Low-temperature thermal conductivity of some commercial coppers, R. L. Powell,

H. M. Roder, and W. M. Rogers. J. Appl. Phys. 28, 1282 (1957).

2182. Luminescence de $L^{\prime}$ azote solide $\left(4,2^{\circ} \mathrm{K}\right)$ contenant des atomes ou radicaux libres, $H$. P. Broida. Effet de la dilution par L'argon $\left({ }^{1}\right)$, Le J. de Physique et le radium (Paris, France) 18,593 (1957).

2183. Luminous reaction between carbon monoxide and atomic nitrogen, H. P. Broida and D. F. Heath. J. Chem. Phys. 26, 1352 (1957).

2184. Materials for small, oil-free bearings, H. S. White. Materials and Methods 45 , No. 4, 135 (1957).

2185. Unassigned.

2186. Mathematics--a note on values of a quadratic form, M. Marcus. J. Wash. Acad. Sci. 47, No. 4, 97 (1957).

2187. Maximum permissible radiation exposures to man, L. S. Taylor. Radiation Research 6, No. 4, 513 (1957); Radiology 68, No. 2, 260 (1957).

2188. Measured statistical characteristics of VLF atmospheric radio noise, A. D. Watt and and E. L. Maxwell. Proc. IRE 45, 55 (1957). 2189. Measurement of a service area for television broadcasting, R. S. Kirby. IRE

Trans. Broadcast Transmission Systems BTS-7, 23 (1957). 
2190. Measurement of atmospheric noise, W. Q. Crichlow, G. Foldes, F. J. Hewitt, F. Horner, H. Shinkawa, and A. W. Sullivan. Recommendation No. 1 and Annex, Proc. XIth Gen. Assembly International Sci. Radio Union, Boulder, Colorado, August 22-September 5, 1957, XI, Pt. 4, 99, 1957. U. R.S. I. (Intern. Sci. Radio Union) Info. Bull. No. 105, 10

(September-October 1957).

2191. Measurement of voltage ratio at audio frequencies, W. C. Sze. Commun. Electronic 32,444 (1957).

2192. Measuring with the electron, L. Marton. J. Sci. Ind. Research 16A, No. 10, 429 (1957). 2193. Mechanical properties of some engineering materials, R. H. Kropschot, R. M. McClintock, and D. A. Van Gundy. Proc. 1956 Cryogenic Engineering Conference, Paper C-2, p. 93. Edited by K. D. Timmerhaus, Chemical Engineering Dept., University of Colorado (Boulder, Colorado) (1957).

2194. Mechanism of high energy radiation effects in polymers, R. Simha and L. A. Wall. J. Chem. Phys. 61, 425 (1957).

2195. Mechanism of transition at subsonic speeds, G. B. Schubauer. Proc. Symp. on Boundary Layer Research, Intern. Union for Theo, and Appl. Research, August 26-29, 1957, (Freiburg, Germany) p. 85 (1957).

2196. Unassigned.

2197. Medida del color en la industria, D. B. Judd. Boletin Tberomericano de Cultura

Tecnica (Madrid, Spain) 1, 13 (1957).

2198. Micromethylation of polysaccharides, H. S. Isbell, H. L. Frush, B. H. Bruckner, G. N. Kowkabany, and G. Wampler. Anal. Chem. 29, 1523 (1957).

2199. Microstructure of the human tooth, A. The dentinoenamel junction, F. L. Losee, W. H. Jennings, and M. E. Lawson, Jr. J. Dental Research 36, No. 6, 911 (1957).

2200. Microwave measurements of the properties of a dc hydrogen discharge, B. J. Udelson, J. E. Creedon, and J. C. French. J. Appl. Phys. 28, No. 6, 717 (1957).

2201. Microwave power measurements employing electron beam techniques, H. A. Thomas. Proc. IRE 45, No. 2, 205 (1957).

2202. Microwave spectra of molecules exhibiting internal rotation. I. Propylene, D. R. Lide, Jr., and D. E. Mann. J. Chem. Phys. 27, 868 (1957); II. Methylallene, J. Chem. Phys. 27, 874 (1957).

2203. Mode calculations for V. L. F. ionospheric propagation, H. H. Howe and J. R. Wait. Symp. on the Prop. of V. L.F. Radio Waves, Sponsored by the NBS Boulder Laboratories and IRE Prof. Group on Ant. and Prop., January 23-25, 1957 (Boulder, Colorado), Paper No. 36, V. 3; Further comments, V. 4, 91 .

2204. Modern batteries, W. J. Hamer. IRE Trans. Component Parts CP-4, No. 3, 86 (1957).

2205. Molecules in the solar spectrum, H. P. Broida and C. E. Moore. 7th Intern. Astrophys. Symp. Proc. Memoirs of Royal Soc. (Liege, Belgium) Series 4, Vol. 18, 217 (1957).

2206. Multichannel pulse height analyzers, $H$. W. Koch and R. W. Johnston, Nat1. Acad. Sci. Natl. Research Council Publ. 467 (1957).
2207. Multi-factor experiments for evaluating reliability, M. Zelen. Proc, of Joint Military Industry Guided Missile Reliability Symp. (Point Mugu, California) p. 2-17 (1957).

2208. National Bureau of Standards equatorial region V.H.F. scatter research program for the I. G. Y., K. L. Bowles and R. S. Cohen. QST 41, 11 (1957).

2209. National physical standards and design of experiment, W. J. Youden. Bull.International Statistical Inst. 35, 191 (1957).

2210. Negative ions, L. M. Branscomb. Chapter in Advances in Electronics and Electron Physics (Academic Press Inc., New York, N. Y.) (1957).

2211. Neutron-insensitive gamma-ray dosimeter, R. S. Caswell. Radiology 68,101 (1957).

2212. New federal standard on colors, H. J. Keegan. J. Opt. Soc. Am. 47, 330 (1957).

2213. New standard wind load requirements, J. P. Thompson. Eng. News-Record 158, No. 6,33 (1957).

2214. New ultra high strength steel, S. J.

Rosenberg and C. R. Irish. Materials and Methods 45, 145 (1957).

2215. Noise control requirements in building codes, R. V. Waterhouse, Chapter 40, Handbook of Noise Control (McGraw Hill, New York, N. Y.) (1957).

2216. Noise investigation at V. L. F. by the National Bureau of Standards, W. Q. Crichlow. Symp. on the Prop. of V. L.F. Radio Waves, Sponsored by the NBS Boulder Laboratories and IRE Prof. Group on Ant. and Prop., January 23-25, 1957 (Boulder, Colorado), Paper No. 42, V. 3; Proc. IRE 45, 778 (1957).

2217. Nonresonant absorption of symmetric top molecules: Shape of the nonresonant spectra, G. Birnbaum. J. Chem. Phys. 27, 360 (1957). 2218. Note on bounds for certain determinants, E. V. Haynsworth. Duke Math J. 24, No. 3, 313 (1957).

2219. Note on L.F. portable antennas operating over ice and snow covered terrain, J. R. Wait. Symp. on the Prop. of V. L. F. Radio Waves, Sponsored by the NBS Boulder Laboratories and IRE Prof. Group on Ant. and Prop., January 23-25, 1957 (Boulder, Colorado), Appendix C, V. 4,62 .

2220. Note on the dielectric properties of magnesium-strontium titanates, S. Marzullo and E. N. Bunting. J. Am. Chem. Soc. 40, No. 8,285 (1957).

2221. $\gamma_{3}$ band of methane, H. C. Allen, Jr. and E. K. Plyler. J. Chem. Phys. Letters 26, No. 4,972 (1957).

2222. On matrix classes corresponding to an ideal and its inverse, O. Taussky. Ilinois J. Math. 1, 108 (1957).

2223. On prediction of system performance from information on component performance, J. R. Rosenblatt. Proc. IRE Western Joint Computer Conf. (Los Angeles, California), p. 85 (1957). 2224. On small precision bottles of quartz and low-loss glass, S. Ruthberg, and E. I. Klein. Rev. Sci. Instr. 28, No. 3, 205 (1957). 
2225. On some aspects of prediction in the study of complex systems, J. R. Rosenblatt. Proc. of the NYU-RCA Working Conf. on Theory of Reliability, (Ardsley-on-Hudson, N. Y.) p. 24 (1957).

2226. On standard methods of measurement in architectural acoustics, R. V. Waterhouse. J. Acoust. Soc. Am. 29, 544 (1957).

2227. On subdeterminants of doubly stochastic matrices, M. Marcus. Illinois J. Math. 1, 583 (1957).

2228. On the atmospheric dynamo theory, M. L. White. J. Geophys. Research 62, 329 (1957). 2229. On the Hilbert matrix T. Kato. Proc. Am. Math. Soc, 8, 73 (1957).

2230. On the identity relationship for fractional replicates of the $2^{\text {n }}$ series, R. C. Burton and W. S. Connor. Ann. Math. Stat. 28, 3 (1957). 2231. On the independence of tests of randomness and other hypotheses, I. R. Savage. J. Am. Stat. Assoc. 52, 53 (1957).

2232. On the low rates of equilibration in dialysis experiments with ionic surface-active agents, L. M. Kushner and R. A. Parker. J. Phys. Chem. 61, 822 (1957).

2233. On the measurement of the conductivity of a fluid contained in a cylindrical vessel, J. R. Wait. Can. J. Technol. 34, 410 (1957).

2234. On the mode theory of VLF ionospheric propagation, J. R. Wait. Geofis, pura e appl. (Milan, Italy) 37, 103 (1957).

2235 . On the relaxation of vibrational nonequilibrium distributions, III: The effect of radiative transitions on the relaxation behavior, R. J. Rubin and K. E. Shuler. J. Chem. Phys. 26, No. 1, 137 (1957).

2236. On the representation of a certain integral involving Bessel functions by hypergeometric series, P. Henrici, J. Math. Phys. 36, No. 2, 151 (1957).

2237. On the specification of transient nonequilibrium systems, K. E. Shuler. 6th Intern. Symp. on Combustion (Reinhold Publ. Corp. , New York, N. Y.) p. 371 (1957).

2238. On the theory of reflection from a wire grid parallel to an interface between homogeneous media, J. R. Wait. Appl. Sci. Research Section B, 6, 259 (1957).

2239. Unassigned.

2240. Organizing a network of computers to meet deadlines, A. L. Leiner, W. A. Notz, J. L. Smith, and A. Weinberger. 1957 Eastern Joint Computer Conf. Proc. (Washington, D. C.) p. 115 (1957).

2241. Oxygen and water vapor absorption of radio waves in the atmosphere, $B$. $R$. Bean and R. Abbott. Geofis. pura e appl. (Milan, Italy) 37, 127 (1957).

2242. Pairs of matrices of order two which generate free groups, K. Goldberg and M. Newman. Illinois J. Math. 1, No. 3, 446 (1957). $2242 \mathrm{~A}$. $\mathrm{pH}$ and the modern analyst, R. G. Bates, Anal. Chem. 29, 15A (1957).

2243. Performance of pumps with liquefied gases, K. B. Martin, R. B. Jacobs, and R. J. Hardy. Proc. 1956 Cryogenic Engineering Conference, Paper G-6, p. 295. Edited by K. D. Timmerhaus, Chemical Engineering Dept., University of Colorado (Boulder, Colorado) (1957).
2244. Performance of three-millimeter harmonic generators and crystal detectors, J. M. Richardson and R. B. Riley, IRE Trans. on Microwave Theory and Tech. MTT-5, 131 (1957).

2245. Photodetachment of atmospheric negative ions, L. M. Branscomb. Conf. on Chem. Aeronomy (Pergamon Press, London, England) (1957).

2246. Photodetachment studies of negative ions, L. M. Branscomb, S. J. Smith, D. S. Burch, and S. Geltman. Third Intern. Conf, on Ionization Phenomena in Gases. Societa Italiana di Fiscia (Venice, Italy) (1957).

2247. Photometric observations of the airglow, F. E. Roach. Annals Intern. Geophys. Year 4 Pt. 1, 115 (1957).

2248. Photon self-absorption and scattering by the $15.1-\mathrm{Mev}$ level in $\mathrm{C}^{12}$, E. V. Hayward and

E. G. Fuller. Phys. Rev. 106, 991 (1957).

2249. Photoneutron yields in the rare earth region, B. Petree, M. S. Weiss, and E. G. Fuller. Bull. Am. Phys. Soc. Ser. II, No. 2, 16 (1957).

2250. Photoproduction of neutral mesons from carbon near threshold, J. E. Leiss. Am. Phys. Soc. Ser. II, No. 2, 6 (1957).

2251. Physics--the elasticity of rubber, L. A. Wood. J. Wash. Acad. Sci. 47, No. 9, 281 (1957).

2252. Piezoresistance of indium antimonide, $R$. F. Potter. Phys. Rev, 108, No. 3, 652 (1957).

2253. Plastic deformation of ceramic-oxide single crystals, II., J. B. Wachtman, Jr., and L. H. Maxwell. J. Am. Ceram. Soc. 40, No. 11, 377 (1957).

2254. Plastics, F. W. Reinhart, The Encyclopedia of Chemistry (Reinhold Publ, Corp., New York, N. Y.) p. 756 (1957).

2255. Plastics for aircraft. Part II. Transparent glazing materials, G. M. Kline, I. Wolock, and I. G. Callomon. Army-NavyCommerce Bull. ANC-17 (1957).

2256. Polar blackout occurence patterns, V. Agy. J. Atmospheric and Terrest. Phys. Special Suppl. 1957, Proc. of the Polar Atmospheric Symp. July 2-8, 1956 (Oslo, Norway) Pt. II, 129 (1957).

2257. Polarization of sferics, A. G. Jean, L. J. Lange, and J. R. Wait. Symp. on the Prop. of V. L. F. Radio Waves, Sponsored by the NBS Boulder Laboratories and IRE Prof. Group on Ant. and Prop., January 23-25, 1957 (Boulder, Colorado) Abstracts, Paper No. 32, V. 3 (1957). 2258. Polarographic determination of trace quantities of iodide, S. W. Smith and J. K. Taylor. Anal. Chem. 29, 301 (1957).

2259. Practical suggestions for reducing radiation exposure in diagnostic examinations,

L. S. Taylor. Am. J. Roentgenol. Radium Therapy Nuclear Med. 78, No. 6, 983 (1957). 2260. Precise intercomparison of acids by differential potentiometric titration with the hydrogen electrode, R. G. Bates and E. Wichers. Actas Do Congress (Lisboa, Portugal) 1, 3 (1957). 2261. Precision measurement, I. C. Gardner. Ordnance XII, 222 (1957).

2262. Preparation of carbon-14-labeled cyanide, J. D. Moyer and H. S. Isbell. Anal. Chem. 29, 393 (1957). 
2263. Present status of research on the physical constants at the (United States) National Burtau of Standards, R. D. Huntoon and A. G. McNish. N. 1 del supplemento Vol. 6, Serie X, II Nuovo Cimento (Bologna, Italy,) p. 146 (1957).

2264. Prism effect, camera tipping, and tangential distortion, F. E. Washer. Photogrammetric Engr. 23, No. 4, 721 (1957).

2265. Propagation of a pulse across a coast line, J. R. Wait. Proc. IRE 45, 1550 (1957).

2266. Propagation of errors in tristimulus colorimetry, I. Nimeroff. J. Opt. Soc. Am. 47,697 (1957).

2267. Propagation of the radiofrequency ground wave transient sinusoidal over a finitely conducting plane earth, J. R. Johler. Symp. on the Prop. of V. L. F. Radio Waves, Sponsored by the NBS Boulder Laboratories and IRE Prof. Group on Ant. and Prop. , January 23-25, 1957 (Boulder, Colorado), Paper No. 16, V. 2; Geofis. pura e appl. (Milano, Italy), 37, 116 (1957).

2268. Properties of materials at low temperatures, R. J. Corruccini. Chem. Eng. Progr. 53, Pt. I, No. 6, 262; Pt. II, No. 7, 343; Pt. III, No. 8 , 397 (1957).

2269. Properties of Struve functions, R. K. Cook. J. Wash. Acad. Sci. 47, 365 (1957).

2270. Proposal for standard frequency broadcast at very low frequency, W. D. George. Symp. on the Prop. of V. L. F. Radio Waves, Sponsored by the NBS Boulder Laboratories and IRE Prof.

Group on Ant. and Prop. , Ja nua ry 23-25, 1957

(Boulder, Colorado), Paper No. 41, V. 3 (1957).

2271. Proposed nomenclature for linear

viscoelastic behavior, $H$. Leaderman. Trans.

Soc. Rheology I, 312 (1957).

2272. Proposed specification for plastic teeth,

W. T. Sweeney, R. L. Myerson, E. E. Rose, and J. O. Semmelman. J. Prosthetic Dentistry 7,420 (1957).

2273. Protective coating adhesion measurement using an electronic averaging device for the adherometer, A. G. Roberts and R. S. Pizer. ASTM Bull. No. 221, 53 (1957).

2274. Pulse sky wave phenomena observed at $100 \mathrm{Kc}$, R. H. Doherty. Symp. on the Prop. of V. L. F. Radio Waves, Sponsored by the NBS Boulder Laboratories and IRE Prof. Group on Ant, and Prop., January 23-25, 1957 (Boulder, Colorado), Abstract, Paper No. 44, V. 3 (1957). 2275. Purification of mercury and its physical properties, C. L. Gordon and E. Wichers. Ann. N. Y. Acad. Sci. 65, No. 5, 369 (1957).

2276. Quantitative chromatographic procedure for determining dextrose in sugar mixtures, $E$. J. McDonald. Anal. Chem. 29, 32 (1957).

2277. Radiation exposure and the use of radioisotopes, L. S. Taylor. Impact of Sci. on Soc. (Paris, France) 8, No. 4 (1957).

2278. Raman spectrum of triethylborane, E. J. Blau and B. W. Mulligan. J. Chem. Phys. 26, 1085 (1957).

2279. Range straggling of high-energy electrons in carbon, J. E. Leiss, S. Penner, and C. S. Robinson. Phys. Rev. 107, 1544 (1957).

2280. Reaction of dimeric 5-aldo-1,2-0-isopropylidene-D-xylo-pentofuranose with cyanide and the preparation of calcium 1,2-0-isopropylidene-Lidofururonate-6-C $C^{14}$ dihydrate, R. Sch affer and H. S. Isbell. J. Am. Chem. Soc. 79, 3867

(1957).
2281. Relation between roughness of interface and adherence of porcelain enamel to steel, J. C. Richmond, D. G. Moore, H. B. Kirkpatrick, and W. N. Harrison. Inst, Vitreous Enamellers Ltd. Bull. (Published by J. Am. Ceramic Soc.) 7, No. 5, 69 (1957).

2282. Unassigned.

2283. Reliability starts with circuit design, J. H. Muncy. Aviation Age 27, 1 (1957).

2284. Report of the subcommission on the question: "What are the most readily measured characteristics of terrestrial radio noise from which the interference to different types of communications systems can be determined?" prepared by W. Q. Crichlow. Proc. of the XIIth General Assembly, Intern. Sci. Radio Union, Boulder, Colorado, 1957, XI, Pt. 4, 9 (1957).

2285. Representation of nonlinear functions of two input variables on analog equipment, D. A. Elliott. Trans. Am. Soc. Mech. Engrs. 79, 489 (1957).

2286. Reproducibility of a pen-and-chart type of recording instrument under dynamic conditions, D. H. I'sai and E. W. Hogue. ASME Paper 57A209 (1957).

2287. Results of Bureau of Standards 45-year corrosion study, M. Romanoff. Petroleum Engr. 29, No. 3 and 4, 615 (1957).

2288. Results of ionospheric drift measurements in the United States, V. Agy. J. Atmospheric and Terrest. Phys. Special Suppl. 1957, Proc. of the Polar Atmosphere Symp., July 2-8, 1956 (Oslo, Norway), Pt. II, 23 (1957).

2289. Results of laboratory tests of high duty and super duty fireclay plastic refractories, R. A. Heindl and W. L. Pendergast. Bull. Am. Ceram. Soc. 36 , No. 1, 6 (1957).

2290. Review of 1956 dental research: dental materials and instrumentation, G. C. Paffenbarger and J. W. Stanford. J. Am. Dental Assoc. 54, 465 (1957).

2291. Unassigned.

2292. Rotational lines of $\mathrm{CH}, \mathrm{OH}$, and $\mathrm{CN}$ in the solar spectrum, C. E. Moore and H. P. Broida. 7th Intern. Astrophys. Symp. Proc. Memoirs Royal Sci.(Liege, Belgium) Series 4, 18, 252 (1957).

2293. Scientists' and public's responsibility, A. V. Astin. Phys. Today 10, No. 11, 23 (1957). 2294. Second report of the special committee on world-wide ionospheric soundings of the URSI/ AGI Committee, May 1957, A. H. Shapley, Chairman, Boulder, Colorado, National Bureau of Standards, Central Radio Propagation Laboratory (1957).

2295. Secondary chromatic aberration, R. E.

Stephens. J. Opt. Soc. Am. 47, No. 12, 1135 (1957).

2296. Sedimentation equilibrium of flexible cha in molecules, L. Mandelkern, L. C. Williams, and S. G. Weissberg. J. Phys. Chem. 61, 271 (1957).

2297. Sharp line absorption in silica near 3400 $\mathrm{cm}^{-1}$, A. M. Bass and H. P. Broida. J. Opt. Soc. Am. 47, 163 (1957).

2298. Shellac, C. C. Hartman. Offic. Dig. Federation Paint Varnish Production Clubs 29, No. $393,1028(1957)$. 
2299. Silicate cements: How to select and use them, G. C. Paffenbarger. Trans. Am. Dental Soc. of Europe (John Wright and Sons Ltd. , Bristol, England) (1957).

2300. Simple technique for diplexing 10,000 Mc and video signals on coaxial cables. M. C.

Thomspon, Jr., and D. M. Waters, Rev. Sci. Instr. 28, 206 (1957).

2301. Sing-around ul $t$ rasonic velocimeter for liquids, M. Greenspan and C. E. Tschiegg. Rev. Sci. Instr. 28, No. 11, 897 (1957).

2302. Some examples of the use of high-speed computers in statistics, J. M. Cameron. Proc. Conf. Designs of Experiments in Army Research, Development, and Testing, Off. Ord. Research Rept. 57-1, 129 (1957).

2303. Some extreme value results for indefinite Hermitian matrices, M. Marcus, B. N. Moyles, and R. Westwick. Illinois J. Math. 1, 449 (1957). 2304. Some implications of slant-Es, E. K. Smith and R. Knecht. J. Atmospheric and Terrest. Terrest. Phys. Spec. Suppl. 1957, Proc. Polar Atmospheric Symp. July 1956 (Oslo, Norway) Pt. II, 195 (1957).

2305. Some theorems about pr (n), M. Newman. Can. J. Math. 9, 68 (1957).

2306. Specific heats of liquid metals and liquid salts, T. B. Douglas. Trans. Am. Soc. Testing Materials 79, 23 (1957).

2307. Spectra of afterglows and discharges from nitrogen-oxygen mixtures, U. H. Kurzweg, A. M. Bass, and H. P. Broida. J. Mol. Spectroscopy 1,184 (1957).

2308. Spectrophotometry and aerial photoreconnaissance, H. J. Keegan and J. C. Schleter. Abstract J. Opt. Soc. Am. 47, 1050 (1957). 2309. Spectroscopic studies of solids condensed at $4.2^{\circ} \mathrm{K}$ from electric discharge through nitrogen, oxygen, hydrogen, water, and ammonia, H. P. Broida. Proc. Conf. on Chem. Aeronomy. The Threshold of Space (Pergamon Press, London, England) (1957).

2310. Spectroscopic study of electronic flame temperatures and energy distributions, $H$. P. Broida and K. E. Shuler. J. Chem. Phys. 27, No. 4, 933 (1957).

2311. Spectroscopic survey of energy distributions of $\mathrm{OH}, \mathrm{C}_{2}$ and $\mathrm{CH}$ radicals in low-pressure acetylene-oxygen flames, H. P. Broida and D. F. Heath. J. Chem. Phys. 26, 223 (1957). 2312. Spectrum analysis of sferics, W. L. Taylor. Symp. on the Prop. of V. L. F. Radio Waves, Sponsored by the NBS Boulder Laboratories and the IRE Prof. Group on Ant, and Prop., January 23-25, 1957 (Boulder, Colorado), Paper No. 33, V. 3 (1957).

2313. Spectrum of the $\mathrm{C}_{3}$ molecule between $3600 \AA$ and $4200 \AA, N$. H. Kiess and H. P. Broida. Mem. Soc. Roy. Sci. (Liege, Belgium) 18,544 (1957).

2314. Sphere-gap volt-time curves-standards for steep front surge measurements, J. H. Park and H. N. Cones. Am. Inst. Elec. Engrs. Conf. Paper No. CP57-215 (1957).

2315. Stability of quartz resonators at very low temperatures, F. P. Phelps. Proc. 11th Ann. Symp. on Frequency Control, May 7-9, 1957 (Ft. Monmouth, N. J.) p. 256 (1957).

2316. Stabilization of free radicals at low temperatures, H. P. Broida. Annal N. Y. Acad. Sci. 67, Article 9, 530 (1957).
2317. Statistical results and their shortcomings concerning the ionosphere within the auroral zone, R. W. Knecht. J. Atmospheric and Terrest. Phys. Special Suppl. 1957, Proc. of the Polar Atmosphere Symp. July 2-8, 1956 (Os1o, Norway) Pt. II, 109 (1957).

2318. Strength and fatigue of glas at very low temperatures, R. H. Kropschot and R. P. Mikesell. J. Appl. Phys. 28, 610 (1957).

2319. Structural analysis of clinical dextrans by periodate oxidation and isotope dilution techniques, J. D. Moyer and H. S. Isbell. Anal. Chem. 29, 1862 (1957).

2320. Structural interpretation of immiscibility in oxide systems: 1 . Analysis and calculation of immiscibility, E. M. Levin and S. Block, J. Am. Ceram. Soc. 40, No. 3, 95 (1957).

2320A. Structural interpretation of immiscibility in oxide systems: 2 . Coordination principles applied to immiscibility, M. Levin and S. Block. J. Am. Ceram. Soc. 40, No. 4, 113 (1957). 2321. Structure of 5-aldo-1,2-0-is opropylideneD-xylo-pentofuranose, R. Schaffer and H. S. Isbe11. J. Am. Chem. Soc. 79, 3864 (1957). 2322. Structure of the methylamine molecule, I. Microwave spectrum of $\mathrm{CD}_{3} \mathrm{ND}_{2}, \mathrm{D}$. R. Lide, Jr. J. Chem. Phys. 27, 343 (1957).

2323. Structure of the methylamine molecule. II. Theory of internal motions and application to $\mathrm{CD}_{3} \mathrm{NO}_{2}, \mathrm{D}$. Kivels on and D. R. Lide, Jr. J. Chem. Phys. 27, 353 (1957).

2324. Studies in nonequilibrium rate processes. I. The relaxation of a system of harmonic oscillators, E. W. Montroll and K. E. Shuler. J. Chem. Phys. 26, 454 (1957).

2325. Studies in nonequilibrium rate processes. II. The relaxation vibrational nonequilibrium in distributions in chemical reactions and shock waves, K. E. Shuler. J. Phys. Chem. 61, 849 (1957).

2326. Studies of transequatorial ionospheric propagation by the scatter-sound method, by Villard-Stein-Yeh. Comments by R. Silberstein. J. Geophys. Research 62, 645 (1957).

2327. Studies on electroless nickel plating, C. H. de Minjer and A. Brenner. Plating 44, 1297 (1957)

2328. Study of accuracy in chemical analysis using linear calibration curves, J. Mandel and F. J. Linnig. Anal. Chem. 29, 743 (1957). 2329. Superconductivity of microscopic tin filaments, O. S. Lutes. Phys. Rev. 105, No. 5, 1451 (1957).

2330. Suppression of bubbling in boiling refrigerants, G. J. Minkoff, F. I. Scherber, and A. K. Stober. Nature 180, 1413 (1957).

2331. Sur 1'utilisation des observations meterorologiques courantes en propagation radioelectrique, B. R. Bean. L'Onde Electrique (Paris, France) 37, No. 362, 411 (1957).

2332. Survey of computer development and applications, S. N. Alexander. Proc. Conf. on Sci. and Tech. for the Deans of Engineering. Purdue Univ. (Lafayette, Indiana) (1957).

2333. Survey of hazards of handling liquid oxygen, C. S. McCamy. Ind. Eng. Chem. 49, 81A (1957). 2334. Symbolic designations for electrical connections, A. Weinberger and $\mathrm{H}$. Loberman. J. Assoc. Computing Machinery 4, No. 4, 420 (1957). 2335. Systems of inequalities involving convex functions, K. Fan, I. Glicksburg, and A. J. Hoffman. Proc. Am. Math. Soc. 8, 617 (1957). 
2336. Tables $4 f-1$ through $4 f-6$ on thermal expansion. P. Hidnert and H. S. Krider. Am. Inst. Phys. Handbook (McGraw-Hill Pub. Co. Inc., New York, N. Y.) (1957).

2337. Tables of spectra, C. E. Moore. Trans. Intern. Astron. Union 9, 212 (1957).

$2337 \mathrm{~A}$. Tacan coverage and channel requirements, M. T. Decker. IRE Trans. Aeronaut. Navigational Electronics ANE-4, No. 3, 135 (1957).

2338. Technique for monitoring triatiated-water vapor in air, C. A. Ziegler and A. Schwebel. Nucleonics 15, No. 1, 64 (1957).

2339. Techniques for electroforming of precision waveguide components in the millimeter wavelengths, A. A. Feldmann. Rev. Sci. Instr. 28, 295 (1957).

2340. Temperature dependence of distribution functions in quantum statistical mechanics, I. Oppenheim and J. Ross. Phys. Rev. 107, No. 1, 28 (1957).

2341. Tension testing of plastics, F. W. Reinhart. Am. Soc. Testing Materials Symp. on Testing of Nonmetallic Materials No. 194 (1957). 2342. The analysis of convariance for incomplete block design, M. Zelen. Biometrics 13, No. 3 , 309 (1957).

2343. The analysis of incomplete block designs, M. Zelen. J. Am. Stat. Assoc. 52, 204 (1957). 2344. The attenuation of Rayleigh waves with depth in a medium with two surface layers, R. Stoneley and U. Hochstrasser. Monthly Notices of Royal Astron. Soc. Geophys. (London, England Supplement 7, 279 (1957).

2345. The attenuation versus frequency characteristics of VLF radio waves, J. R. Wait. Proc. IRE 45,768 (1957).

2346. The aurora in middle and low latitudes,

S. Chapman. Nature (Mac Millan and Co.,

London, England) 179, No. 4549, 7 (1957).

2347. The clamp-type alternating-current microammeter, G. F. Montgomery and C. Stansbury. Am. Inst. Elec. Engrs. Paper No. 57114 (1957). 2348. The condenser microphone as a displacement detector calibrator, W. Koidan. J. Acoust. Soc. Am. 29, No. 7, 813 (1957).

2349. The effect of heat treatment of the susceptibility of sand cast aluminum alloy 220 to stress corrosion cracking, F. M. Reinhart.

Corrosion 13, 17 (1957).

2350. The effect of prism on the location of the principal point, F. E. Washer. Photogrammetric Eng. 23, No. 13, 520 (1957).

2351. The effect of receiver bandwidth on amplitude distribution of VLF atmospheric noise, F. F. Fulton. Symp. on the Prop. of V. L. F. . Radio Waves, Sponsored by the NBS Boulder Laboratories and IRE Prof. Group on Ant. and Prop., January 23-25, 1957 (Boulder, Colorado), Paper No. 37, V. 3 (1957).

2352. The effective electrical constants of soil at low frequencies, J. R. Wait. Proc. IRE 45, 1411 (1957).

2353. The effects of fungicides on deterioration of leather, S. Dahl. J. Am. Leather Chemists Assoc. 52, 611 (1957).

2354. The effects of rates of straining on some tensile properties of polyester film, C. F. Bersch, F. L. McCrackin, and K. F. Plitt. Modern Plastics 35, 171 (1957).
2355. The electronic structure and magnetic properties of uranyl-like ions, II. Plytonyl, J. C. Eisenstein and M. H. L. Pryce. Proc. Roy. Soc. (London, England) 238, 31 (1957). 2356. The emission spectrum of OH from 2.8 to $4.1 \mu, \mathrm{H}$. C. Allen, Jr., E. K. Plyler, and L. R. Blaine. Spectrochem. Acta 9, 126 (1957). 2357. The flash photolysis of chlorine monoxide, S. H. C. Edgecombe, R. G. W. Norrish, and B. A. Thrush. Proc. Roy. Soc. (London, England) 243, 24 (1957).

2358. The galvanic corrosion theory for adherence of porcelain enamel ground coats to steel, D. G. Moore, J. W. Pitts, J. C. Richmond, and W. N. Harrison. Inst. Vitreous Enamellers Ltd. Bull. (Published by J. Am. Ceram. Soc.) 7, No. 4 (1957). 2359. The gassing of dry cells, E. M. Otto and W. G. Eicke, Jr. J. Electrochem. Soc. 104, 199 (1957).

2360. The geometrical optics of VLF sky wave propagation, J. R. Wait and A. Murphy. Symp. on the Prop. of V. L. F. Radio Waves, Sponsored by the NBS Boulder Laboratories and IRE Prof. Group on Ant. and Prop., January 23-25, 1957 (Boulder, Colorado), Paper No. 5, V. 1; Proc. IRE 45, 754 (1957).

2361. The Government's bookstore, L. T.

Milliken. Capital Chemist 7, No. 1, 10

(1957).

2362. The heat capacity of aluminum oxide in

the range $300^{\circ}$ to $700^{\circ} \mathrm{K}, \mathrm{E}$. D. West and D. C.

Ginnings. J. Phys. Chem. 61, 1573 (1957).

2363. The impact absorbing capacity of textile yarns, J. C. Smith, F. L. McCrackin, and H. F. Schiefer. ASTM Bull. No. 220, 52 (1957).

2364. The impedance of a wire grid parallel to a dielectric interface, J. R. Wait. IRE Trans. on Microwave Theory and Tech. MTT-5, 99 (1957).

2365. Unassigned.

2366. The International Commission on Radiological units and measurements, L. S. Taylor. International Associations (Pergamon Press, London, England) 9, 594 (1957).

2367. The ionic character and elastic moduli of zinc blend lattices, R. F. Potter. Phys. Chem. of Solids 3, 223 (1957).

2368. The ionic dissociation of $2,4-, 2,6-, 3$, 4-dichlorobenzoic acids in water, M. M. Davis and H. B. Hetzer. J. Phys. Chem, 61, 123 (1957).

2369. The ionic dissociation of 2, 6-dimethylbenzoic acid in water, M. M. Davis and H. B. Hetzer, J. Phys. Chem. 61, 125 (1957).

2370. The isotropic length of polymer networks, D. E. Roberts, L. Mandelkern, and P. J. Flory. J. Am. Chem. Soc. 79, 1515 (1957). 2371. The long distance horizontal radiation pattern of a high-frequency antenna, R. Silverstein. IRE Trans. on Ant. Prop. AP-5, 397, (1957).

2372. The microwave spectrum and structure of sulfurylfluoride, D. R. Lide, Jr., D. E. Mann, and R. M. Fristrom. J. Chem. Phys. 25, 734 (1957).

2373. The mode theory of V. L. F. ionospheric propagation for finite ground conductivity, J. R. Wait. Symp. on Prop. of V. L. F. Radio Waves, Sponsored by the NBS Boulder Laboratories and IRE Prof. Group on Ant. and Prop., January 23-25, 1957 (Boulder, Colorado), Paper No. 6, V. 1; Proc. IRE 45, 760 (1957). 
2374. The new safety code for portable metal ladders, R. L. Lloyd. Mag. of Standards 28, No. 4, 104 (1957).

2375. The number of representations of a quadratic form as a sum of four squares, G. Pall and O. Taussky. Proc. Roy. Irish Acad. Sect. A, No. 3,23 (1957).

2376. The philosophy underlying radiation protection, L. S. Taylor. Am. J. Roentgenol. 2377. The precise measurement of the infrared spectra of molecules of the atmosphere, E. K. Plyler and E. D. Tidwell. Memoires Royal Sci. Soc. Liege, quatrieme serie, Inst. Astrophys. (Liege, Belgium) 28, 426 (1957).

2378. The principle of randomization in the design of experiments, C. Eisenhart. Proc. Conf. on Design of Experiments, Off. Ord. Research Report 57-1, 15-16 (1957).

2379. The probability of a saddle point, A. J. Goldman. Am. Math. Monthly (Math. Notes) 64, 729 (1957).

2380. The role of electronic computers in air traffic control, R. D. Elbourn. IRE Conv. Record 5, Pt. 8, 114 (1957).

2381. The spectrum of the electron density fluctuations in the ionosphere, R. M. Gallet. J. Atmospheric and Terrest. Phys. Special Suppl. 1957, Proc, of the Polar Atmosphere Symp., July 2-8, 1956 (Oslo, Norway) Pt. II, 165 (1957).

2382. The strontium oxide content of portland cements, L. Bean. ASTM Bull. No. 224, 42 (1957).

2383. The structure of bone mineral, A. S. Posner. Clinical Orthopedics No. 9, p. 5 (1957). 2384. The tensile properties of selected steels as a function of temperature, E. P. Klier. Welding Research Council Bull. 35 (1957).

2385. The torsion of a hollow square, J. L. Synge and W. F. Cahill. Quart. Appl. Math. 15, No. 3, 217 (1957).

2386. The transient behavior of the electromagnetic ground wave on a spherical earth, J. R. Wait. IRE Trans. Ant. Prop. AP-5, No, 2, 198 (1957).

2387. The transmission of Rayleigh waves across an ocean floor with two surface layers, R. Stoneley. Bull. Seismological Soc. Am. 47, 7 (1957). 2388. The use of surface weather observation to predict the total atmospheric bending of radio rays at small elevation angles, B. R. Bean and B. A. Cahoon. Proc. IRE 45, 1545 (1957). 2389. The utility of meteor burst for intermittent radio communication, G. F. Montgomery and G. R. Sugar. Proc. IRE 45, No. 12, 1684 (1957). 2390. The waveguide mode theory of VLF ionospheric propagation, J. R. Wait and H. H. Howe. Proc. IRE 45, 95 (1957).

2391. The "wave-guide"mode" theory of the propagation, K. G. Budden. Symp. on the Prop. of V. L. F. Radio Waves, Sponsored by the NBS Boulder Laboratories and IRE Prof. Group on Ant. and Prop. January 23-25, 1957 (Boulder, Colorado), Appendix B, V. 4, 50; Proc. IRE 45,772 (1957); Comments V. 4, 96 (1957). 2392. Theory of the forbidden transition of nitrogen atoms trapped in solids, C. M. Herzfeld. Phys. Rev. 107, 1239 (1957).
2393. Thermal conductivities of copper and copper alloys, R. L. Powell, W. M. Rogers, and H. M. Roder. Proc. 1956 Cryogenic Engineering Conference, Paper E-3, p. 166. Edited by K. D. Timmerhaus, Chemical Engineering Dept., University of Colorado (Boulder, Colorado) (1957).

2394. Thermodynamic properties at high tem. peratures: Ideal gas thermal functions to 25,000 deg. $\mathrm{K}$ for diatomic molecules, oxygen, nitrogen, nitricocide, and their molecule ions, C. W. Beckett and L. Haar. Proc. Conf. on Thermodynamic and Transport Properties of Fluids, July 10-12, 1957 (London, England) p. 27 (1957). 2395. Tough problems solved with tricky tooling, F. P. Brown and C. E. Pelander. Machinery 64 , No. 1,149 (1957).

2396. Transistor beta tester, G. F. Montgomery. Electronics 30, No. 5, 198 (1957).

2397. Transmission of air-borne noise through walls and floors, R. K. Cook and P. Chrzanowski. Handbook of Noise Control (McGraw-Hill Book Co. Inc., New York, N. Y.) Chapter 20 (1957). 2398. Transmission of ultrasonic vibrations in metals, R. W. Mebs and D. B. Ballard. WrightAir Devel. Ctr. Tech. Rept. 56-457 (1957).

2399. Two resistance-type hot-pressing furnaces for laboratory use, R. F. Walker. Rev. Sci. Instr. 28, No. 7, 563 (1957).

2400. Uniqueness theory for asymptotic expansions in general'regions, P. Davis. Pacific J. Math. 7, 849 (1957).

2401. Unit-cell data of the lead niobate $\mathrm{PbNb}_{2} \mathrm{O}_{6}$, R. S. Roth. Acta Cryst. 10, Pt. 6, 437 (1957). 2402. U.S.A. National Committee report, URSI subcommission 6.3 , antennas and waveguides, and annotated bibliography, H. V. Cottony, R. S. Elliott, E. C. Jordan, V. H. Rumsey, K. M. Siege1, J. R. Wait, and O. C. Woodyard. Proc. 12th Gen. Assembly, Intern. Sci.

Radio Union, August-September 1957 (Boulder, Colorado)11, Pt. 6, 121 (1957).

2403. Use of carbon-14 and tritium for the study and characterization of cellulose and other polysaccharides, H. S. Isbell, H. L. Frush, and J. D. Moyer. Tappi 40, No. 9, 739 (1957).

2404. Use of computers in statistics, J. M. Cameron. Proc. 4th Annual High-Speed Computer Conf. (Louisiana State University, Baton Rouge, La.) p. 67 (1957).

2405. Utilizzazione delle osservazioni meteorologiche correnti nella propagation radio-electrica, B. R. Bean. Post e telecomun. 25, 1120 (1957). 2406. Vacuum-insulated transfer tube, R. B. Jacobs and R. J. Richards. Rev. Sci. Instr. 28, 291 (1957).

2407. Variation of elastic constants and static strains with hydrostatic pressure: a method for calculation from ultrasonic measurements, $R$. K. Cook. J. Acoust. Soc. Am. 29, 445 (1957). 2408. Very low temperature data: Properties of paramagnetic salts, R. P. Hudson. Chapter 4-b, Am. Inst. Phys. Handbook (McGraw-Hill Pub. Co. Inc., New York, N. Y.) p. 14 (1957).

2409. Vibrational spectrum and force constants of diboron tetrachloride, D. E. Mann and L. Fano. J. Chem. Phys. 26, 1665 (1957).

2410. Vibrational structure in the ionization efficiency curves of hydrogen and deuterium molecules, M. Krauss and A. Kropf. J. Chem. Phys. 26, 1776 (1957). 
2411. Vibration-rotation bands of ammonia II-the molecular dimensions and harmonic frequencies of ammonia and deuterated ammonia, W. S. Benedict and E. K. Plyler. Can. J. Phys. 35, 1235 (1957).
2412. Visual auroral observations, S. Chapman (with D. S. Kimball, J. Paton, O. Schneider, and I. L. Thomsen). Annals. Intern. Geophys. Year 4, Pt. 1, 41 (1957).

2413. Water-soluble degradation products of asphalt, A. Schriescheim and S. H. Greenfeld. ASTM Bull. 220, 43 (1957). 
2414. A-C zero locator, L, Costrell. Electronics 31, 98 (1958).

2415. A compact microwave refractometer for use in small aircraft, M. C. Thompson, Jr., and $M$. J. Vetter. Rev. Sci. Instr. 29, No. 12, 1093 (1958).

2416. A comparison of insulating materials. J. E. Schrodt, M. M. Fulk, W. A. Reynolds, and W. S. Flanery. Proc, 1957 Cryogenic Engineering Conference, Abstract, Paper G-7, p. 416. Edited by K. D. Timmerhaus. Chemical Engineering Department, University of Colorado (Boulder, Colorado) (1958).

2417. A delegate reports on small tools, F. P. Brown. Mag. of Standards 29, No. 8, 235 (1958).

2418. A dispersion function of paramagnetic relaxation, P. H. Fang. Physica (Amsterdam, Holland) 24,970 (1958).

2419. A dry, static calorimeter for $r f$ power measurement, P. A. Huds on and C. M. Allred. IRE Trans. Instrumentation I-7, No, 3-4, 292 (1958).

2420. A facility for the evaluation of resistance strain gages at elevated temperatures, R. L. Bloss. Am. Soc. Testing Materials Spec. Tech. Publ. No. 230, 57 (1958).

2421. A feedback amplifier with negative output resistance for magnetic measurements, $W$. $P$. Harris and I. L. Cooter. IRE Natl. Conv. Record, Pt. 5, p. 217 (1958).

2422. A free precession determination of the proton gyromagnetic ratio, P. L. Bender and R. L. Driscoll. IRE Trans. Instrumentation I-7, No. 3-4, 176 (1958).

2423. A long-distance pulse-propagation experiment on 20.1 megacycles, R. Silberstein. J. Geophys. Research 63, No. 3, 445 (1958).

2424. A magnetic specimen mount for fractography, D. B. Ballard and J. A. Bennett. Metal Prog. 74, No. 5, 114 (1958).

2425. A method for measuring the directivity of directional couplers, G. E. Schafer and R. W. Beatty. IRE Trans. Microwave Theory and Tech. MTT-6, No. 4, 419 (1958).

2426. A method of controlled slack quenching of impact bars, M. R. Meyerson and S. J. Rosenberg. Rev. de Metallurgie 55, No. 4, 317 (1958). 2427. A method of speeding up iterations with superlinear convergence, A. M. Ostrowski. J. Math. Mech. 7, 117 (1958).

2428. A modulator for microwave mixers, G. E. Schafer. IRE Trans. Microwave Theory and Tech. MTT-6, No. 3, 333 (1958).

2429. A note on confidence intervals in regression problems, J, Mandel. Annals Math. Stat. 49, No. 3, 903 (1958).

2430. A novel rocket hoist, J. A. Dickinson. Elevator World 6, No. 2, 13 (1958).

2431. A phenomenological theory of induced electrical polarization, J. R. Wait. Can. J. Phys. 36, 1634 (1958).

2432. A pretied tie for communication line wires, C. R. Ballard and R. R. Bouche. Conf. Paper No. CP 58-266 presented at the Annual Meeting of Am. Inst. Elec. Engrs. (1958).

2433. A property of additively closed families of distributions, E. L. Crow. Annals Math. Stat. 29, No. 3, 892 (1958).
2434. A proposed standard for facilities for scientific conferences, A. T. McPherson. Mag. of Standards 29, No. 7, 202 (1958).

2435. A recording torsion testing machine for wire, H. C. Burnett. ASTM Bull. No. 227 (1958).

2436. A review of the state of the art and future trends in electrochemical battery system. I. The more common systems, W. J. Hamer. Proc. Seminar on Advanced Energy Sources and Conversion Techniques, ASTIA, No. AD209301, p. 41 ; Dept. Comm. OTS No. PB151461. 2437. A study of earth currents near a VLF monopole antenna with a radial wire ground system, J. R. Wait. Proc. IRE 46, No. 8, 1 (1958).

2438. A study of VLF field strength data! both old and new, J. R. Wait. Geofisica Pura e Applicata (Milano, Italy) 41, 73 (1958).

2439. A survey of Lyapunov's second method, $H$. A. Antosiewicz. "Contributions to the theory of nonlinear os cillations, "Vol. IV, ed. by S. Lefshetz, p. 141 (Annals of Mathematics Studies No. 141 (Princeton University Press, Princeton, New Jersey) (1958).

2440. A survey of some recent advances in polymex physics, and their relation to dielectric properties, A. J. Curtis. Insulation p. 15 (1958).

2441. A system of names for binary numbers, J. Stern. Science 128, 594 (1958).

2442. A toric bending specimen for investigation of geometrical factors in fatigue, J. A. Bennett J. G. Weinberg. ASTM Bull. No. 234, p. 53 (1958).

2443. A unique thermal conductivity gas analyzer, J. R. Purcell, J. W. Draper, and D. H. Weitzel. Proc. 1957 Cryogenic Engineering Conference, Paper D-4, p. 191. Edited by K. D. Timmerhaus. Chemical Engineering Department, University of Colorado (Boulder, Colorado) (1958).

2444. Absolute measurement of $W$ for polonium-210 alpha particles, Z. Bay andr $H$. H. Seliger. Radiation Research 9, No. 1, 90 (1958). 2445. Absorption spectra of solids condensed at low temperatures from electric discharges, A. M. Bass and H. P. Broida. J. Mol. Spectroscopy 2,42 (1958).

2446. AC-DC transfer instruments for current and voltage measurements, F. L. Hermach. IRE Trans. Instrumentation I-7, No. 3-4, 235 (1958). 2447. Accelerating convergence of iterative processes, J. H. Wegstein. Communications, Assoc. Computing Machinery 1, No. 6, (1958).

2448. Accuracy, F. W, Reinhart. Juniata College Bull. 55, No. 4, 2 (1958).

2449. Acrylic resins in prosthetic dentistry, W. T. Sweeney. Dental Clin. of North America 593 (1958).

2450. Age to indium resonance for D-D neutrons in water, V. Spiegel, Jr., D. W. Oliver, and R. S. Caswell. Nuclear Sci. and Eng. 4, No. 4, $546(1958)$.

2451. American Dental Association specifications for dental materials (third ed.), G. C. Paffenbarger, J. W. Stanford, and W. T. Sweeney (1958). 
2452. Amplitude stabilization of a microwave signal source, G. F. Engen. IRE Trans. Microwave Theory and Tech. MTT-6, No. 2, 202 (1958).

2453. An analog-digital simulation for the design and improvement of man-machine systems, H. K. Skramstad, A. A. Ernst and J. P. Nigro. 1957 Eastern Joint Computer Conf. Proc. (Inst. Radio Engrs., New York, N. Y.) (1958).

2454. An analysis of dielectric loss in two urea addition compounds, J. I. Lauritzen, Jr. J. Chem. Phys. 28, No. 1, 118 (1958).

2455. An equation for calculating the number of chain scissions in the photochemical degradation of solid polymers, J. H. Flynn. J. Polymer Sci. 27, 83 (1958).

2456. An extension to the mode theory of VLF ionospheric propagation, J. R. Wait. J. Geophys. Research 63, No. 1, 125 (1958).

2457. An impact test for leather based on an alternating punching force, T. J. Carter. J. Am. Leather Chemists Assoc. 53, 250 (1958).

2458. An investigation of the perturbations impassed upon radio waves penetrating the ionosphere, R. S. Lawrence. Proc. IRE 46, 1 (1958).

2459. Unassigned.

2460. Analys is of photocross sections, A. S. Penfold and J. E. Leiss. Physics Dept. Rept. (University of Illinois, Champaign, Ill.) (1958).

2461. Angular distribution in neutral meson decay, J. E. Leiss and R. A. Schrack. Phys. Rev. 109, 1326 (1958).

2462. Atmospheric research, D. M. Gates.

Americana Annual, p. 62 (1958).

2463. Atomic and molecular negative ions in stellar atmospheres, L. M. Branscomb, and B. E. F. Pagel. Monthly Notices Roy. Astron. Soc. 118, No. 3, 258 (1958).

2464. Atomic beam sources and standard of length, K. G. Kessler, R. L. Barger, and W. G. Schweitzer, Jr. IRE Trans. Instrumentation I-7, No. 3-4, 181 (1958).

2465. Autographic stress-strain recorders, R. R. Bouche and D. R. Tate. ASTM Bull. No. 228, 33 (1958).

2466. Automatic coding principles, J. H. Wegstein. Proc. Symp. on Advanced Programming Methods for Digital Computers, Washington, D. C. , June 1956 (Office of Naval Research, Department of the Navy, Washington, D. C.), p. 3 (1958).

2467. Automation and future management information systems, S. N. Alexander. Proc. 4th Military-Industry Packaging and Handling Symp., Navy Department, September 30-October 2, 1958 , p. 23, (Washington, D. C.) (1958).

2468. Automation in the laboratory, B. F.

Scribner. Capital Chemist 8, 38 (1958).

2469. Basic research in Europe, D. M. Gates. Science 128, No. 3318, 227 (1958).

2470. Basic standardization, S. F. Booth. Report of the 1st Annual Standardization Symp. Army Chem. Corps Engr. Command, March 24-25, 1958 (Army Chem. Center, Maryland) p. $90(1958)$.

2471. Behavior of young children under conditions simulating entrapment in refrigerators, K. Bain, M. L. Faegre, and R. S. Wyly. Pediatrics

22, No. 4, Pt. I, 628 (1958).
2472. Beta-alumina-type structure in the system lanthana-alumina, R. S. Roth and S. Hasko. J. Am. Ceram. Soc. 41, No. 4, 146 (1958).

2473. Beta-gamma correlations from polarized manganese-52, E. Ambler, R. W. Haywa rd, D. D. Hoppes, and R. P. Hudson. Phys. Rev. 110 , No. 3, 787 (1958); Proc. Intern. Congr. on Nuclear Phys, (Paris, France, July 1958) and Proc. Kamerlingh Onnes Conf. on Low Temp. Phys., Leiden, The Netherlands, Supplement of Physica (June 1958).

2474. Bonding agents for high-temperature strain gages, D. G. Moore. Am. Soc. Testing Materials, Spec. Tech. Publ. No. 230, 106 (1958).

2475. Bose-Einstein condensation and the lambda transition in liquid helium, M. S. Green. Phys. Rev. Letters 1, 409 (1958).

2476. Branched-chain higher sugars, R. Schaffer and H. S. Isbell. J. Am. Chem. Soc. 80, 756 (1958).

2477. Bremsstrahlung cross-section measurement for 50-kev electrons, J. W. Motz and R. C. Placious. Phys. Rev. 109, 235 (1958).

2478. Brief history of the National Committee on Radiation Protection and Measurements, L. S. Taylor. Health Phys. J. 1, 3 (1958).

2479. Bright rims in diffuse nebulae, S. R. Pottasch, Rev. Mod. Phys. 30, No. 3, 1053 (1958).

2480. Broad and narrow beam attenuation of Ir ${ }^{192}$ gamma rays in concrete, steel, and lead, V. H. Ritz. Nondestructive Testing 16, No. 3, 269 (1958).

2481. Calculated calibration points for negative ion appearance potentials, L. M. Branscomb. J. Chem. Phys. 29, No. 2, 452 (1958)

2482. Calculation of gaseous heat conduction in dewars, R. J. Corrruccini. Proc. 1957 Cryogenic Engineering Conference, Paper G-1, p. 353. Edited by K. D. Timmerhaus. Chemical Engineering Dept., University of Colorado (Boulder, Colorado) (1958).

2483. Calibration of thermocouples at low temperatures, M. D. Bunch and R. L. Powell.

Proc. 1957 Cryogenic Engineering Conference, Paper E-6, p. 269. Edited by K. D. Timmerhaus. Chemical Engineering Dept., University of Colorado (Boulder, Colorado) (1958).

2484. Career opportunities in the Government, W. R. Brode and G. E. Hilbert. Chem. Eng. News 36, Pt. II, 70 (1958).

2485. Charge-storage pulse-height analyzers for use with pulsed accelerators, L. Costrell and R. E. Brueckmann. Nuclear Instr. (NorthHolland Publishing Co., Amsterdam, Holland) 3, 350 (1958).

2486. Chemiluminescence in the system atomic sodium plus atomic hydrogen, J. D. McKinley, Jr., and J. C. Polanyi. Can. J. Chem. 36, 107 (1958).

2486A. Classification and nomenclature for standards of measurement, A. G. McNish. IRE Trans. Instrumentation I- 7, No. 3-4, 371 (1958).

2487. Colorimetry from precision spectrophotometry, H. J. Keegan. J. Opt. Soc, Am. 48,281 (1958). 
2488. Collection of ions produced by alpha particles in air, Z. Bay and H. H. Seliger. Bull. Am. Phys. Soc. II, 3, No. 3, Session Y-9 (1958). 2489. Comments on capacities of plumbing drains, R. S. Wyly. Yearbook of the Am. Soc. Sanit. Engr. 36, 243 (1958).

2490. Commission III on ionospheric radio, world-wide soundings committee: Status report by A. H. Shapley, Chairman, Intern. Sci. Radio Union (U. R.S. I. ) Info. Bull. No. 108, 13 (1958). 2491. Communication via meteor bursts, G. F. Montgomery. Radio-Electronics 29, 88 (1958). 2492. Comparison of first, second, and third approximations in Bacher and Goudsmit's theory of atomic spectra, R. E. Trees, J. Opt. Soc. Am. 48, 293 (1958).

2493. Comparison of phase difference and Doppler shift measurements for studying ionospheric fine structure using earth satellites, M. C. Thompson, Jr., and D. M. Walters. Letter Proc. IRE 46, No. 12, 1960 (1958).

2494. Complementary use of nuclear magnetic resonance and infrared absorption studies in organic phosphorus chemistry, H. Finegold. Ann. N. Y. Acad. Sci. 70, 875 (1958).

2495. Compound semiconductors, H. P. R. Frederikse, J. Metals 10, 346 (1958).

2496. "Computers," F. L. Alt. An item in The Encyclopedia of Chemistry Supplement, p. 91 (Reinhold Publishing Corp., New York, N. Y.) (1958).

2496A. Conditions at the ionization and shock fronts in collisions of gas clouds--Bright rims in diffuse nebulae, Part VI, S. R. Pottasch. Rev. Modern Phys. 30, No. 3, 1053 (1958). 2497. Conference on photonuclear reactions, J. E. Leiss. Phys. Today 11, No. 9, 18 (1958).

2498. Congruences for the coefficients of modular forms and for the coefficients of $j(\tau), M$. Newman. Proc. Am. Math. Soc. 9, 609 (1958). 2499. Continuous phase difference measurements of earth satellites, J. W. Herbstreit and M. C. Thompson, Jr. Proc. IRE 46, No. 8, 1535 (1958).

2500. Contributions to Handbook of Physics,

Edited by E. U. Condon and H. Odishaw (McGrawHill Book Co., Inc., New York, N. Y.) (1958):

Arithmetic, F. L. Alt, Chapter 1, Pt. 1, p. $1-4$.

Color vision and colorimetry, D. B. Judd, Chapter 4, Pt. 6, p. 6-64.

Control mechanisms, H. K. Skramstad and G.

L. Landsman, Chapter 9, Pt. 2, p. 2-69.

Electrolytic conductivity and electrode processes, W. J. Hamer and R. E. Wood, Chapter 9, Pt. 4, p. 4-138.

Electronic circuits, C. H. Page, Chapter 4, Pt. 4 , p. 4-47.

Elements of probability, C. Eisenhart and $\mathbf{M}$.

Zelen, Chapter 12, Pt. 1, p. 1-134.

Geometry, A. J. Hoffman, Chapter 8, Pt. 1, p. 1-97.

Integral equations, M. Abramowitz, Chapter 6, Pt. 1, p. 1-90.

Neutron physics, C. O. Muehlhause, Chapter 7, Pt. 9, p. 9-125.

Nuclear electromagnetic radiations, R. W. Hayward, Chapter 6, Pt. 9, p. 9-106.
Statistical design of experiments, W. J. Youden, Chapter 13, Pt. 1, p. 1-165.

Thermometry and pyrometry, R. E. Wilson and R. D. Arnold, Chapter 3, Pt. 4, p. 5-30.

2501. Control of factors affecting the reproducibility of mechanical properties of refractory semi-dry press specimens, L. E. Mong and D. M. Adelman. J. Am. Ceram. Soc. 41, No. 7, 267 (1958).

2502. Correlation correction to the activation energy for diffusion in crystalline solids, J. R. Manning. Phys. Rev, Letters 1, No. 10, 501 (1958).

2503. Corrosion research at the National Bureau of Standards, J. Kruger. J, Ind, Eng. Chem. $50,55 \mathrm{~A}(1958)$.

2504. Covariances of least-squares estimates when residuals are correlated, M. M. Siddiqui. Ann. Math. Stat. 29, 1251 (1958).

2505. Creep and static strengths of large bolted joints of forged aluminum alloys under various temperature conditions, L. Mordfin, G. E.

Greene, N. Halsey, R. H. Harwell, Jr., and R. L. Bloss. Inst. of Aeronaut. Sci. Preprint No. 779 (1958).

2505A. 1958 critique of VHF ionospheric scatter communication. (Survey Paper), R. C. Kirby. Record of Natl. Symp. on Extended Range and Space Communications, October 6-7, 1958 (Washington, D. C.), p. 90 (1958).

2506. Cryostat for electron bombardment and electron diffraction work, E. M. i Horl and L.

Marton. Rev. Sci. Instr. 29, No. 10, 859 (1958). 2507. Crystallization kinetics in polymeric systems, L. Mandelkern. Chapter in the book "Growth and Perfection of Crystals," p. 467 (John Wiley and Sons, New York, N. Y.) (1958). 2508. Crystallographic data for yohimbine hydrochloride, G. Burley and G. M. Brown. Anal. Chem. 30, 154 (1958).

2509. Crystallography of cold drawn music wire, H. C. Burnett and C. J. Newton. Wire and Wire Products 33, No. 1, 66 (1958).

2510. Unassigned.

2511. Degradation of collagen by heat, J. M. Cassel. J. Am. Leather Chemists Assoc. 53, 507 (1958).

2512. Demands on teachers in a technological society, F. W. Brown. Colorado School J. 14 (1958).

2513. Design and construction of a blackbody and its use in the calibration of a grating spectroradiometer, G. T. Lalos, R. J. Corruccini, and H. P. Broida. Rev. Sci. Instr. 29, 505 (1958).

2514. Design data for ortho-parahydrogen converters. D. H. Weitzel, C. C. Van Valin and J. W. Draper. Proc. 1957 Cryogenic Engineering Conference, Paper B-2, p. 73. Edited by K. D. Timmerhaus. Chemical Engineering Dept. , University of Colorado (Boulder, Colorado) (1958).

2515. Design of simple DC resistance thermometer bridges for wide-range temperature control, R. D. Goodwin. Paper E-5, p. 254.

Edited by K. D. Timmerhaus. Chemical Engineering Dept., University of Colorado (Boulder, Colorado) (1958). 
2516. Design of simple resistance thermometer bridges for wide-range control of low temperatures, R. D. Goodwin. Rev. Sci. Instr. 29, 497 (1958).

2517. Determination of reducing sugars and reducing end groups in polysaccharides by reaction with carbon-14-labeled cyanide, J. D. Moyer and $\mathrm{H}$. S. Isbell. Anal. Chem. 30, No. 12, 1975 (1958).

2518. Determination of some compressive properties of human enamel and dentin, J. W. Stanford, G. C. Paffenbarger, J. W. Kumpula, and W. T. Sweeney. J. Am. Dental Assoc. 57, 487 (1958).

2519. Developments in instruments. W. T. Sweeney. Intern. Dental J. 8, No. 2, 238 (1958). 2520. Dielectric properties of titania or tin oxide containing varying proportions of rare-earth oxides, S. Marzullo and E. N. Bunting. J. Am. Ceram. Soc. 41, No. 1, 40 (1958).

2521. Differential thermal analysis above $1200^{\circ}$ C, T. F. Newkirk. J. Am. Ceram. Soc. 41, 409 (1958).

2522. Digital reduction of spectrophotometric data to Munsell renotations, H. J. Keegan, W. C. Rheinboldt, J. C. Schleter, J. P. Menard, and D. B. Judd. J. Opt. Soc. Am. 48, 863 (1958).

2523. Dimensional stability of denture base resins, W. E. Mowery, C. L. Burns, G. Dickson, and W. T. Sweeney. J. Am. Dental Assoc. 57,345 (1958).

2524. Direct nitrogen calibration of dead space in gas absorption systems, W. V. Loebenstein. J. Chem. Phys. 29, No. 1, 236 (1958).

2525. Discussion of the paper - "Heat treatment affects properties of enamel-steel composites," by J. H. Healey and L. K. Breeze - J. C. Richmond. J. Am. Ceram. Soc. 41, 386 (1958), 2526. Distillation of hydrogen isotopes, T. M. Flynn, K. D. Timmerhaus, and D. H. Weitzel. Colorado Engr. 55, 12 (1958).

2527. Distribution of a serial correlation coefficient near the ends of the range, M. M. Siddiqui. Ann. Math. Stat. 29, 852 (1958). 2528. Effect of calcium treatment on solubility and calcium uptake of synthetic hydroxyapatite and rat molar enamel, R. C. Likins, A. S. Posner, and A. C. Steere. J. Am. Dental Assoc. 57, 335 (1958).

2529. Effect of degree in orientation and crystal size on the scattering of $20-\mathrm{kev}$ electrons by aluminum, L. Marton, J. A. Simpson, J. A. Suddeth, M. D. Wagner, and H. Watanabe. Phys. Rev. 110, No. 5, 1057 (1958).

2530. Effect of suspension position on apparent values of internal friction determined by Forster's method, J. B. Wachtman, Jr., and W. E. Tefft. Rev. Sci. Instr. 29, No. 6, 517 (1958). 2531. Unassigned.

2532. Effect of transverse atmospheric drag on satellite orbits, W. A. Wildhack. Science 128, 309 (1958).

2533. Effects of molding pressure on strength properties of glass-fiber reinforced plastics, S. D. Toner, I. Wolock, and F. W. Reinhart. SPE J. 14, 40 (1958).
2534. Elastic properties of pure-gum rubber vulcanizates, L. A. Wood. Proc. Joint A rmyNavy-Air Force Conf. on Elastomer Research and Development 2, 5th meeting (October 1958) p. 363 (Materials Laboratory, Wright Air Development Center, Wright-Patterson Air Force Base, Ohio) (1958).

2535. Elastic scattering of photons by tantalum, E. G. Fuller and E. V. Hayward. Phys. Rev. Letters 1, No. 12, 465 (1958).

2536. Elasticity, strength, and other related properties of some refractory castables, S. J. Schneider and L. E. Mong. J. Am. Ceram. Soc. 41, No. 1, 27 (1958).

2537. Electrical contact resistance of copper, copper functions at low temperatures, R. L. Powell and A. A. Aboud. Rev. Sci. Instr. 29, 248 (1958).

2538. Electrochemical calorimetry, J. M. Sherfey and A. Brenner. J. Electrochem. Soc. 105, 665 (1958).

2539. Electroluminescence from the surface layer of $\mathrm{BaTiO}_{3}, \mathrm{SrTiO}_{3}$ and associated materials, G. G. Harman. Phys. Rev. 111, No. 1,27 (1958).

2540. Unassigned.

2541. Electron absorption spectrometer using an improved velocity analyzer, L. Marton and J. A. Simpson. Rev. Sci. Instr. 29, No. 7, 567 (1958).

2542. Electron impact studies of sulfur dioxide and sulfuryl fluoride, R. M. Reese, V. H.

Dibeler, and J. L. Franklin. J. Chem. Phys. 29 , No. 4,880 (1958).

2543. Electronic structure of some diatomic hydrides, M. Krauss. J. Chem. Phys. 28, 1021 (1958).

2544. Emission spectra from mixtures of atomic nitrogen and organic substances, N. H. Kiess and H. P. Broida. 7th Symp. on Combustion (Butterworths, London, England) p. 207 (1958).

2545. Emission spectroscopy, B. F. Scribner. Anal. Chem. 30, 596 (1958).

2546. Energy release from discharged monatomic gasses trapped at $4^{\circ} \mathrm{K}, \mathrm{G}$. J. Minkoff and F. I. Scherber. J. Chem. Phys. 28, No. 5, 992 (1958).

2547. Epoxy resins as cryogenic structural adhesives, R. M. McClintock and M. J. Hiza. Proc. 1957 Cryogenic Engineering Conference, Paper F1-3, p. 305. Edited by K. D. Timmerhaus. Chemical Engineering Dept., University of Colorado (Boulder, Colorado) (1958); Modern Plastics 35, 172 (1958).

2548. Essential similarity: a counter-example, A. J. Goldman. Am. Math. Monthly (Math. Notes) 65, 564 (1958).

2549. Étalons de temps atomiques et moléculaires au National Bureau of Standards, R. C. Mockler. Procès-Verbaux des Séances, Rapport et Annexes, IRE Session (1957), Comite Consultatif pour la Définition de la Seconde, p. 38. (Comité International des Poids et Mesures, 2, serie, tome 26B) Paris, France, Gauthier-Villars (1958). 
2550. Etalons et mesures de frequence et d'intervalle de temps aux Etats - Unis d'Amerique de 1954 á 1957, W. D. George. Proces Verbaux des Séances, Rapport et Annexes, IRE Session (1957), Comité Consultatif pour la Définition de la Seconde, p. 34. (Comité International des Poids et Mesures, Procès-Verbaux des Séances. 2. serie, tome 26-B) Paris, France, GauthierVillars (1958).

2551. Evaluation of bremsstrahlung cross sections at the high-frequency limit, U. Fano, H. W. Kocli, and J. W. Motz. Phys. Rev, 112, No. 5, 1679 (1958).

2551A. Evaporation of active species trapped in a solid condensed from discharged nitrogen,

H. P. Broida and M. Peyron. J. Chem. Phys. 28, 725 (1958).

2552. Exact solution for a gyromagnetic sample and measurements on a ferrite, H. E. Bussey and L. A. Steinert. IRE Trans. Microwave Theory and Tech. MTT-6, 72 (1958).

2553. Excitation of surface waves on plane and curved impedance boundaries, J. R. Wait.

Congres International sur la Propagation des

Ondes Radio-Electriques, October 6-11, 1958

(Liege, Belgium). Resume des communications, p. 26 (1958).

2554. Excitation of the oxygen red lines in twilight, J. W. Chamberlain. Astrophys. J. 127, No. 1, 54 (1958).

2555. Experimental evaluation of the oxygen microwave absorption as a possible atomic frequency standard, J. M. Richardson. J. Appl. Phys. 29, No. 2, 137 (1958).

2556. Experimental photodetachment cross section and the ionospheric detachment rate for $0(1)$, S. J. Smith, D. S. Burch, and L. M. Branscomb. Ann. Geophys. Tome 14, No. 2, 225 (1958).

2557. Experiments on the beta-decay of polarized nuclei, E. Ambler, R. W. Hayward, D. D. Hoppes, and R. P. Hudson. Proc. Kamerlingh Onnes Conference, Leiden, Holland, 1958, Physica (Supplement) 24, S64 (1958).

2558. Expression in terms of molecular distribution functions for the entropy density in an infinite system, R. E. Nettleton and M. S. Green. J. Chem. Phys. 29, 1365 (1958). 2559. Extension of the Munsell renotation system, J. C. Schleter, D. B. Judd, and H. J. Keegan. J. Opt. Soc. Am. 48, 863 (1958).

2560. Extreme useful range of VHF transmission by scattering from the lower ionosphere, R. C. Kirby. IRE Natl. Conv. Record 6, Pt, 1, 112 (1958).

2561. Fabrication techniques for ceramic $\mathrm{X}$-band cavity resonators, M. C. Thompson, Jr., F. E. Freethey, and D. M. Waters. Rev. Sci. Instr. 29, No. 10,865 (1958).

2562. Fifty years of signal lighting, F. C. Breckenridge. Illum. Eng. 53, 311 (1958).

2563. Fire research in the United States, A. F. Robertson. Fire Research and Fire Prevention Conf., 1958 Bulletin No. 2, Division Building Research, Nat1. Research Council Pub. NRC 5011 , p. 51 (Ottawa, Canada) (1958).

2564. First meeting on radio climatology, B. R. Bean. Proc. IRE Letter 46, 1425 (1958).
2565. Flame spread properties of building

finish materials, D. Cross and J. Loftus. ASTM

Bull. No. 230 (1958).

2566. Flexural cracks in reinforced concrete beams, M. Chi and A. F. Kirstein. J. Am. Concrete Inst. No. 54 (1958).

2567. Fluoridation, A. S. Posner. Encyclopedia of Chemistry, Supplement, p. 128

(Reinhold Publishing Co., New York, N. Y.) (1958).

2568. Fractional factorial experiments of $2^{\mathrm{m}_{3} \mathrm{n}}$ series, W. S. Conner. Proc. 14th Annual Clinic of the Rochester Society for Quality Control, February 18, 1958 (Rochester, New York), p. 59 (1958).

2569. Fracture phenomena and molecular weight in polymethyl methacrylate, S. B. Newmin and I. Wolock. J. Appl. Phys. 29, No. 1, 49 (1958). 2570. Free radical stabilization in condensed phases, S. Golden. J. Chem. Phys. 29, 61 (1958).

2571. Free radical statistics, J. L. Jackson and E. W. Montroll. J. Chem. Phys. 28, 1101 (1958).

2572. Free radicals research symposium, J. W. Moyer. Phys. Today 11, 14 (1958).

2573. Further identities and congruences for the coefficients of modular forms, M. Newman. Can. J. Math. 10, 577 (1958).

2574. Gains of finite-size corner-reflector antennas, H. V. Cottony and A. C. Wilson. IRE Trans. Ant. Prop. AP-6, No. 4, 366 (1958). 2575. Glass dewars for optical studies at low temperatures, L. J. Schoen, L. E. Kuentzel, and H. P. Broida. Rev. Sci. Instr. 29, No. 7, 633 (1958).

2576. Glass transition temperatures of copolvmers, L. A. Wood. J. Polymer Sci. 28, No. 117,319 (1958).

2577. Handmade bolts support the Capitol dome, L. K. Irwin. Fasteners 13, No. 3, 5 (1958). 2578. Harmonic generation with ideal rectifiers, C. H. Page. Proc, IRE 46, No. 10, 1738 (1958).

2579. Heat transfer in laminar flow, M. Abramowitz. Abstracts of Short Communications and Scientific Programme, International Congress of Mathematicians (Edinburgh, Scotland) 135 (1958).

2580. Heat transfer measurements on refrigerated-food trailers, H. D. Johns on, J. C. W inter, C. W. Phillips, J. W. Grimes, and P. R. Achenbach. U. S. Dept. of Agriculture, Agricultural Marketing Service (AMS-250) (June 1958).

2581. Heat transfer to boiling liquid nitrogen and hydrogen flowing axially through narrow annular passages, R. J. Richards, R. F. Robbins, R. B. Jacobs, and D. C. Holten. Proc. 1957 Cryogenic Engineering Conference, Paper G-3, p. 375 . Edited by K. D. Timmerhaus, Chemical Engineering Department, University of Colorado (Boulder, Colorado) (1958).

2582. Heats of adsorption from charge-transfer complex theory, R. J. Brodd. J. Phys. Chem. 62,54 (1958). 
2583. High-frequency impedance standards at the National Bureau of Standards, R. C. Powell, R. M. Jickling, and A. E. Hess. IRE Trans. Instrumentation I-7, No. 3-4, 270 (1958).

2584. High-frequency magnetic permeability measurements using toroidal coils, R. D. Harrington and R. C. Powell. Proc. IRE 46, 784 (1958).

2585. High-frequency standards of the Electronic Calibration Center, National Bureau of Standards, Boulder Laboratories, Boulder, Colorado, M. C. Selby. IRE Trans. Instrumentation I-7, No. 3-4, 262 (1958).

2586. High input impedance transistor amplifier,

G. F. Montgomery. Electronic Design 6, No. 16,48 (1958).

2587. High-speed computations in the kinetics of free-radical degradation, I. Random initiation. R. Simha, L. A. Wall, and J. Bram. J. Chem. Phys. 29, No, 4, 894 (1958).

2588. High-vacuum temperature gradient furnace, L. Marton and J. A. Suddeth. Rev. Sci. Instr. 29 , No. 5, 440 (1958).

2589. History of the International Commission on Radiological Protection, L. S. Taylor.

Health Physics J. 1, 2 (1958).

2590. Hydrogen liquefaction cycles, J. Macinko, D. B. Chelton, and J. Dean, Proc, 1957 Cryogenic Engineering Conference, Paper A-1, p. 1. Edited by K. D. Timmerhaus, Chemical

Engineering Dept., University of Colorado (Boulder, Colorado) (1958).

2591. Ignition of Kel-F and teflon, L. Greenspan. Rev. Sci. Instr. 29, 172 (1958).

2592. Immiscibility and surface tension of some simple borates, A. B. Bestul, L. Shartsis, and H. F. Shermer. J. Am. Ceram. Soc. 41, 507 (1958).

2593. Importance of the Faraday to elemental constants and electricity standards, R. D.

Huntoon and A. G. MeNish. Nature 181, 1194 (1958).

2594. Impregnation of sole leather with polymers, B. H. Fouquet and J. R. Kanagy. J. Am.

Leather Chemists Assoc. 53, No. 6, 336 (1958).

2595. Improved dimensional stability in laminated map paper, G. L. McLeod and T. L. Worksman. Tech. Assoc. Pulp Paper Ind. 41, No. 9, 484 (1958).

2596. Improved electrical differentiation of retarding potential measurements, L. B. Leder and J. A. Simpson. Rev. Sci. Instr. 29, No. 7, 571 (1958).

2597. Improvements in liquid scintillators, $\mathrm{H}$. H. Seliger. Proc. Northwestern Technical Institute Symp. on Liquid Scintillation Counting, p. 115 (Pergamon Press, New York, N. Y.) (1958).

2598. Influence of lower permissible levels on atomic operations in the United States, L. S. Taylor. Proc. 2d Intern. Conf. on the Peaceful Uses of Atomic Energy, United Nations (Geneva, Switzerland) 23, 211 (1958).

2599. Infrared absorption of oxygen discharge products and ozone at $4^{\circ} \mathrm{K}, \mathrm{K}$. B. Harvey and A. M. Bass. J. Mol. Spectroscopy 2, 405 (1958).

2600. Infrared phase-contrast reflectometer,

W. S. Rodney and E. Djurle. J. Opt. Soc. Am. 48 , No. 6, 388 (1958).
2601. Input-output, key or bottleneck, R. D. Elbourn. Proc. Computer Applications Symp. , October 29-30, 1958 (Armour Research Foundation, Chicago, Illinois), p. 69 (1958).

2602. Instrumentation--where we stand, A. V. Astin. ASTM Bull. No, 232, 7 (1958),

2603. Internal barrier in ethane, D. R. Lide, Jr. , J. Chem. Phys. Note 29, 1426 (1958). 2604. Ionization and dissociation of hydrazoic acid and methyl azide by electron impact, J. L. Franklin, V. H. Dibeler, R. M. Reese, and M. Krauss. J. Am Chem. Soc. 80, 298 (1958). 2605. Isolation of the diffusion layer at an electrode and the determination of concentration polarization, T. Yannakopoulos and A. Brenner. J. Electrochem. Soc. 105, No. 9, 521 (1958).

2606. Isotope effect in oxidation of D-mannitol2-Cl4 by acetabacter suboxydans, H. L. Frush and L. J. Tregoning. Science 128, No. 3324, 597 (1958).

2607. Kinetic spectroscopy in the far ultraviolet: the flash photolysis of ethyl compounds, B. A. Thrush. Proc. Roy. Soc. 243, 555 (1958). 2608. Kinetics of the high temperature heterogeneous reaction of chlorine and nickel between 1200 and $1700^{\circ} \mathrm{K}$, J. D. McKinley, Jr. , and K.

E. Shuler. J. Chem. Phys. 28, No. 6, 1207 (1958).

2609. La medida de la luz y el color, D. B. Judd. Anales Real Soc, Espan. Fis, y Quim. (Madrid, Spain)53A, 43 (1958).

2610. Laboratory tests with turboprop lubricants, H. S. White. Am. Soc. Lubrication Engrs. Trans. 1, 51 (1958).

2610A. Liquid helium cryostat with an integral super-conducting resonator, E. Maxwell and F. A. Schmidt. Suppl, au Bull, de l'Institut Intern. du Froid, Commission 1, Delft, Holland, Annexe 1958-1, p. 95 (1958).

2611. Limitations in the photographic dosimetry of X- and gamma-rays, W. L. McLaughlin and M. Ehrlich. Radiation Research 9, No, 1, 148 (1958).

2612. Limiting resolution of electron spectrometers, L. Marton. Rev. Sci. Instr. 29, No. 5, 438 (1958).

2613. Liquid-flowmeter calibration techniques, M. R. Shafer and F. W. Ruegg. Trans. Am. Soc. Mech. Engrs. 80, No. 7, 1369 (1958).

2614. Liquid scintillation counting, $H . H$.

Seliger. Proc. Symp. on Measurements and Standards of Radioactivity, Natl. Acad. Sci., Natl, Research Council, Nuclear Science Series No. 24, 47 (1958).

2615. Low and medium frequency radio propagation (abstract), K. A. Norton. Congres Intern. sur la Propagation des Ondes Radio-Electriques, October 6-11, 1958 (Liege, Belgique) Resume des communications, p. 20 (1958).

2616. Low-angle $\mathrm{X}$-ray diffraction of fibrous polyethylene, L. Mandelkern, C. R. Worthington, and A. S. Posner. Science 127, 1052 (1958). 2617. Unassigned.

2618. Low temperature distillation of hydrogen isotopes, K. D. Timmerhaus, D. H. Weitzel, and T. M. Flynn. Chem. Eng. Progr. 54, No. 6,35 (1958).

2619. Low-temperature microcell for infrared study of condensed gases, J. E. Stewart. Anal. Chem. 20, 2073 (1958). 
2620. Low-temperature properties of plastic foams, R. M. McClintock. Soc. of Plastics Engrs. J. 14, No. 11, 36 (1958).

2621. Unassigned.

2622. Luminescence of solid nitrogen $\left(4.2^{\circ} \mathrm{K}\right)$ containing atoms of free radicals. Effect of traces of oxygen, hydrogen and water vapor, H. P. Broida and M. Peyron. J. Phys. Radium 19 , No. 4,480 (1958).

2623. Mass spectrometric study of the decomposition of hydrazoic acid by the electric discharge, J. L. Franklin, J. T. Herron, P. Bradt, and V. H. Dibeler. J. Am. Chem. Soc. 80,6188 (1958).

2624. Mass spectrometry, V. H. Dibeler and R. M. Reese. Anal. Chem. 30, 604 (1958).

2625. Materials of construction: their properties and methods of test, A. T. McPherson, J. Am. Inst. Arch. 29, No. 3, 143 (1958).

2626. $50 \mathrm{Mc}$ oblique transmission experiment near the magnetic equator, $R$. S. Cohen and K. L. Bowles, IRE Trans. Ant. Prop. AP-6, 316 (1958).

2627. Measurement of light and color, D. B. Judd. Illum. Eng. 53, 61 (1958).

2628. Measurement of the gamma-ray dose near the interface between two media, W. F. Titus. Nuclear Sci. and Eng. 3, No. 5, 609 (1958).

2629. Mechanical failures of metals in service, J. A. Bennett and W. L. Holshouser. Eng. Materials Handb, 36, 2 (1958).

2630. Mechanical properties of high polymers,

H. Leaderman. Ann, Rev. Phys, Chem, 9, 179 (1958).

2631. Mechanisms of polymer formation and decomposition, R. Simha and L. A. Wall. Catalysis Series 6, Chapter 3, p. 191, edited by P. H. Emmett (Reinhold Publ. Co., New York, N. Y.) (1958).

2632. Mechanized computation of thermodynamic tables at the National Bureau of Standards - The calculation of the equilibrium composition and thermodynamic properties of dissociated and ionized gaseous systems, J. Hilsenrath and J. H. Wegstein. Proc. Joint Conf. on Thermodynamic and Transport Properties of Fluids, London, England, July 10-12, 1958 (Institution of Mechanical Engineers, London, England), p. 79 (1958).

2633. Mechanized conversion of colorimetric data to Munsell renotations, W. C. Rheinboldt and J. P. Menard. J. Opt. Soc. Am. 48, 864 (1958).

2634. Unassigned.

2635. Microbiological fractionation of the hydrogen isotopes, P. E. Cloud, Jr., V. H. Dibeler, I. Friedman, and F. D. Sisler. Science 127, 3311 (1958).

2636. Microwave double-sweep method for analysis of time-dependent cavity characteristics, S. Ruthberg. Rev. Sci. Instr. 29, No. 11, 999 (1958).

2637. Microwave spectra of molecules exhibiting internal rotation. III. Trimethylamine, D. R. Lide, Jr., and D. E. Mann. J. Chem. Phys. 27,572 (1958).

2638. Microwave spectra of molecules exhibiting internal rotation. IV. Isobutyane, tertiary butyl fluoride and trimethylphospine, D. R. Lide, Jr., and D. E. Mann. J. Chem. Phys. 29, 914 (1958).
2639. Miscellaneous observations on the alkaliaggregate reaction and the ionic charge on hydrated cement, R. G. Pike and D. Hubbard. Natl. Acad. Sci., Natl. Research Council, Publ. Highway Research Board Bull. 171, p. 16 (1958). 2639A. Modulation studies of VHF ionospheric scattering, J. W. Koch. Record of Natl. Symp. on Extended Range and Space Communications, October 6-7, 1958 (Washington, D. C.), p. 114 (1958).

2640. Movements of airglow cells, F. E. Roach, E. Tandberg-Hanssen, and L. R. Megill. J. Atmospheric and Terrest. Phys. 13, 122 (1958).

2641. Multipurpose standard for microchemical analysis, W. H. Smith. Anal. Chem. 30, 149 (1958).

2642. Mutarotation, hydrolysis, and rearrangement reactions of glycosylamines, H. S. Isbell and H. L. Frush. J. Organic Chem. 23, 1309 (1958).

2643. Narrow hyperfine absorption lines of $\mathrm{C}_{\mathrm{s}} 133$ in various buffer gases, $E$. C. Beaty and P. L. Bender. Phys. Rev. 112, No. 2, 450 (1958).

2644. NBS alpha standards, coincidence counting, and radiative electron capture, R. W. Hayward. Proc. Symp. on Measurements and Standards of Radioactivity, Natl. Acad. Sci., Natl. Research Council, Nuclear Science Series No. 24,12 (1958).

2645. Near infrared solar radiation measurements by balloon to an altitude of 100,000 ft., D. M. Gates, D. G. Murcray, C. C. Shaw, and R. J. Herbold. J. Opt. Soc. Am. 48, No. 12,1010 (1958).

2646. Net heat of combustion of aviation gasoline and its correlation with other properties, G. T. Armstrong, R. S. Jessup, and T. W. Mears. Chem. \& Eng. Data Series 3, 20 (1958).

2647. New apparatus at the NBS for absolute capacitance measurement, M. C. McGregor, J. F. Hersh, R. D. Cutkowsky, F. K. Harris, and $F$. R. Kotter. IRE Trans. Instrumentation I- 7, No. 3-4, 253 (1958).

2648. Nonresonant absorption of symmetric top molecules. Collision cross section, G. Birnbaum and A. A. Maryott. J. Chem. Phys. 29, 1422 (1958).

2649. Note on accelerating components in particle spectrometers, H. Mendlowitz. Rev. Sci. Instr. 29, No. 8, 701 (1958).

2650. Note on circular disks containing the eigenvalues of a matrix, K. Fan. Duke Math. J. 25, 441 (1958).

2651. Note on the determination of molecular structure from spectroscopic data, V. W. Laurie. J. Chem. Phys. 28, 704 (1958).

2652. Unassigned.

2653. Nuclear matter distributions from coherent neutral pion production, J. E. Leiss and R. A. Schrack. Rev. Modern Phys, 30, No. 2, 456 (1958).

2654. Numerical experiments in potential theory using the Nehari estimates, U. Hochstrasser. Math. Tables and Other Aids to Computation 12, 26 (1958).

2655. Observation of vertical-incidence scatter from the ionosphere at $41 \mathrm{Mc} / \mathrm{sec}$., K. L. Bowles. Phys. Rev. Letters 1, No. 12, 454 (1958).

2656. Off-path propagation at VHF, V. C. Pineo. Proc. IRE 46, 922 (1958).

2657. On a determinantal inequality, M. Marcus. Am. Math. Monthly (Math. Notes) 65, 266 (1958). 
2658. On doubly stochastic transforms of a vector, M. Marcus. Oxford Quart. J. Math. 9, 74 (1958).

2659. On Gauss' speeding up device in the theory of single step iteration, A. M. Ostrowski. Math. Tables and Other Aids to Computation 12, No. 62, $116(1958)$.

2660. On-off temperature control for electrically heated filaments, R. E. Little and J. D. McKinley, Jr., Rev. Sci. Instr. 29, No. 12, 1143 (1958).

$2660 \mathrm{~A}$. On prediction of system behavior, J. R. Rosenblatt. Proc. of the New York UniversityIndustry Conf. on Reliability Theory (New York, N. Y.), p. 39 (June 1958).

2661. On the approximate daytime constancy of the absorption of radio waves in the lower ionosphere, S. Chapman and K. Davies. J. Atmospheric and Terrest. Phys. 13, 86 (1958).

2662. On the behavior in the large of the integral curves of a non-linear differential equation, A. Ghaffari. Abstracts of Short Communications and Scientific Programme, Intern. Congress of Mathematicians (Edinburgh, Scotland), p. 50 (1958).

2663. On the bounds of a one-parametric family of matrices, A. M. Ostrowski. J. Reine Angew. Math. (Berlin, Germany) 200,190 (1958).

2664. On the calculation of properties of gases at elevated temperatures, 1. Amdur and J. Ross. Combustion and Flames 2, 412 (1958).

2665. On the calculations of transverse current loss in buried wire ground systems, J. R. Wait. Appl. Sci. Research 7, Sec. B, 81 (1958).

2666. On the derivative of Bessel functions with respect to the order, F. Oberhettinger. J. Math. Phys. 37, 75 (1958).

2667. On the electroviscous effect in dilute aqueous solutions on ionic detergents, R. A. Parker and S. P. Wasik. J. Phys. Chem. 62, 967 (1958).

2668. On the interpretation of hydrodynamic data for dilute protein solutions, L. Mandelkern and H. A. Scheraga. J. Phys. Chem. 62, 370 (1958).

2669. On the long-range correlation model of the photonuclear effect, M. Danos. Nuclear Phys. 5,23 (1958).

2670. On the measurement of ground conductivity at VLF, J. R. Wait and A. M. Conda. IRE Trans, Ant. Prop. AP-6, No. 3, 273 (1958).

2671. On the perturbation of the vibrational equilibrium distribution reactant molecules by chemical reactions, K. E. Shuler. 7th Symp. on Combustion (Butterworths, London, England), p. 87 (1958).

2672. On the quality of gray tin crystals and their rate of growth, J. H. Becker. J. Appl. Phys. 29, No. 7, 1110 (1958).

2673. On the theory of propagation of electromagnetic waves along a curved surface, J. R. Wait. Can. J. Phys. 36, 9 (1958).

2674. On the transmission error function for meteor-burst communication, G. F. Montgomery. Proc. IRE 46, 1423 (1958).

2675. On the use of the early Balmer lines to extend the photospheric model, R. G. Athay and R. N. Thomas. Astrophys. J. 127, No. 1, 96 (1958).
2676. Operation of bearings and pumps at low temperatures, K. B. Martin, R. B. Jacobs, and R. J. Hardy. Proc. 1957 Cryogenic Engineering Conference, Paper D-6, p. 209. Edited by K. D. Timmerhaus. Chemical Engineering Dept., University of Colorado (Boulder, Colorado) (1958).

2677. Optical detection of narrow $\mathrm{Rb}^{87}$ hyperfine absorption lines, P. L. Bender, E. C. Beaty, and A. R. Chi. Phys. Rev. Letters 1, No. 9, 331 (1958).

2678. Oscillatory galvanomagnetic effects in n-type indium arsenide, H. P. R. Frederikse and W. R. Hosler. Phys. Rev. 110, 880 (1958). 2679. Output of a sound source in a reverberation chamber and other reflecting environments, R. V. Waterhouse. J. Acoust. Soc. Am. 30 , 4 (1958).

2680. Oxidative degradation of a series of deuterated styrene polymers, M. Tryon and L. A. Wall. J. Phys, Chem. 62, No. 6, 697 (1958). 2681. Oxygen red lines in the airglow, I. Twilight and night excitation processes, J. W. Chamberlain. Astrophys. J. 127, No. 1, 54 (1958).

2682. Pattern of an antenna on a curved lossy surface, J. R. Wait and A. M. Conda. IRE Trans. Ant. Prop. AP-6, No. 4, 348 (1958). 2683. Photodetachment cross section and the electron affinity of atomic oxygen, L. M. Branscomb, D. S. Burch, S. J. Smith, and S. Geltman. Phys. Rev. 111, No. 2, 504 (1958). 2684. Photodetachment of $\mathrm{O}_{2-}^{-}$, D. S. Burch, S. J. Smith, and L. M. Branscomb. Phys. Rev. 112, 171 (1958).

2685. Photometer for measurement of the effective intensity of condenser-discharge lights, C. A. Douglas. Illum. Eng. 53, 205 (1958). 2686. Photoneutron yields in the rare-earth region, E. G. Fuller, B. Petree, and M. S. Weiss. Phys. Rev. 112, No. 2, 554 (1958).

2687. Physical properties of chromium cobalt alloys, D. F. Taylor, W. A. Leibfritz, and A. G. Adler. J. Am. Dental Assoc. 56, 343 (1958).

2688. Physical properties of plastic teeth, W. T. Sweeney, E. L. Yost, and J. G. Fee. J. Am. Dental Assoc. 56, 883 (1958).

2689. Physics--The electrostatic field and the symmetry of snowflakes, R. A. Schrack. J. Wash. Acad. Sci. 48, No. 9, 273 (1958).

2690. Piezoelectric effect in indium antimonide,

J. H. Wasilik and R. B. Flippen. Phys. Rev. Letters 1, No. 7, 233 (1958).

2691. Pilot plant studies of the low temperature distillation of hydrogen isotopes, T. M. Flynn, K. D. Timmerhaus, and D. H. Weitzel. Proc. 1958 Cryogenic Engineering Conference, Paper H-1, p. 464 . Edited by K. D. Timmerhaus, Chemical Engineering Dept., University of Colorado (Boulder, Colorado) (1958); Chem. Eng. Progr. 54, No. 6, 35 (1958).

2692. PILOT, the NBS multicomputer system, A. L. Leiner, W. A. Notz, J. L. Smith, and A. Weinberger. Proc. Eastern Joint Computer Conf. (Philadelphia, Pennsylvania) p. 164 (1958). 2693. Plastic phosphor matrix for fast neutron detection, B. Brown and E. C. Hooper, Jr. Nucleonics 16, 96 (1958).

2694. Polarization near the high-frequency limit of 500-kev bremsstrahlung, J. W. Motz and R.

C. Placious. Phys. Rev. 112, No. 4, 1039 (1958). 
2695. Possible movements of 5577 airglow, $F$. E. Roach, E. Tandberg-Hanssen, and L. R. Megill. J. Atmospheric and Terrest. Phys. $13,122(1958)$

2696. Precision measurement and race for technological supremacy, A. V. Astin. Mag. of Standards 29, No. 2, 36 (1958).

2697. Preface to Proc. 3d Symp. on cosimical gas dynamics, J. M. Burgers and R. N. Thomas. Smithsonian Astrophysical Observatory, June 1957 (Cambridge, Massachusetts), Rev. Modern Phys. 30, 908 (1958).

2698. Pressure-sensitive diaphragm-type null detector, D. White and J. Hilsenrath. Rev, Sci. Instr. 29, No. 7, 648 (1958).

2699. Pressures developed in cement pastes and mortars by the alkali-aggregate reaction, R. G. Pike. Natl. Acad. Sci., Natl. Research Council Pub, Highway Research Board Bul, 171, p. 34 (1958).

2700. Probing the ionosphere, A. H. Shapley and R. J. Slutz. In American Geophysical Union. Geophysics and the IGY, Geophysical Monograph No. 2, 45 (1958).

2701. Production of a square temperature wave in a filament operating at low temperatures, $R$. Klein and J. A. Simpson. Rev. Sci. Instr. 29, No. 9, $770(1958)$.

2702 Products of cool flame oxidation of propane2-C 13, R. E. Ferguson and C. R. Yokley. 7 th Symp. on Combustion (Butterworths, London, England), p. 113 (1958).

2703. Properties of some experimental map papers containing synthetic fibers, G. L. McLeod. Tappi 41, No. 8, 430 (1958).

2704. Proposed recommendation on ionospheric vertical soundings after the I. G. Y., A. H. Shapley. (Memorandum No. 18), Intern. Sci. Radio Union (U. R. S. I.) Inform. Bull. No. 111, 42 (1958).

2705. Protection of molybdenum from oxidation at elevated temperatures, D. E. Couch, H. Shapiro, J. K. Taylor, and A. Brenner. J. Electrochem. Soc. 105, No. 8, 450 (1958). 2706. Proton gyromagnetic ratio, R. L. Driscoll and P. L. Bender. Phys. Rev. Letters 1, No. 11, 413 (1958).

2707. Pyrolysis of poly-alpha-methylstyrene, D. W. Brown and L. A. Wall. J. Phys. Chem, 62, 848 (1958).

2708. Quantum mechanical effects on the surface tension of simple liquids, I. Oppenheim. Il Nuovo Cimento Supplemento al IX, No. 1, 180 (1958).

2709. Radiation exposure as a reasonable calculated risk, L. S. Taylor. Proc. UNESCO Conf. (Paris, France), September 1957; Impact 8, 209 (1957); Health Phys. J. 1, 62 (1958).

2710. Radiation monitoring over long-distance telephone lines and direct field lines, L. Costrell. IRE Trans. Nuclear Sci. NS-5, No, 3, 21 (1958).

2711. Radioactivity of $\mathrm{In}^{120} \mathrm{Sb}^{120}$, C. L. McGinnis. Phys, Rev. 109, 888 (1958).

2712. Radioactivity standardization in the United States, W. B. Mann and H. H. Seliger. Proc. 2d Intern. Conf, on the Peaceful Uses of Atomic Energy, United Nations (Geneva, Switzerland) 21, 90 (1958).

2713. Radio propagation research at the National Bureau of Standards, F. W. Brown. Am. J. Phys. 26, 628 (1958).
2714. Radium standards, internal gas counting and the United States radioactivity standards program, W. B. Mann. Proc. Symp. on Measurements and Standards of Radioactivity, Natl. Acad. Sci., Natl. Research Council, Nuclear Science Series, No. 24, 17 (1958). 2715. Rapid impact loading of textile yarns, J. C. Smith. Proc. Symp. on High-speed Testing, Plas-Tech. Equipment Corp., (Interscience Publ. Inc., New York, N. Y.) (1958).

2716. Rapport sur l'etalonnage de deux lampes etalons secondaires a ruban de tungstene par quatre laboratories nationaux, H. J. Kostkowski. Proces-Verbaux Comite Consultatif de Thermometrie, aupres du Comite International de Poids et Mesures (Paris, France) 5, 133 (1958).

2717. Reaction of carbon monoxide with graphite carbon at $450^{\circ} \mathrm{C}$, V. R. Deitz and E. J. Prosen. Nature 118, 109 (1958).

2718. Reactions of hexafluorobenzene, W. J. Pummer and L. A. Wall. Science 127, 643 (1958), 2719. Recent deveopments in NBS spectrographic standard samples of iron-base alloys, R. E. Michaelis. Appl. Spectros copy 12, 114 (1958). 2720. Recent developments in the field of microwave power measurements at the National Bureau of Standards, G. F. Engen. IRE Trans. Instrumentation I-7, No. $3-4,304$ (1958).

2721. Recent theoretical studies of the propagation of long waves (abstract), J. R. Wait. Congres Intern. sur la Propagation des Ondes Radio-Electriques, October 6-11, 1958 (Liege, Belgium). Resume des communications p. 27 (1958).

2722. Recently developed microwave impedance standards and methods of measurement, R. W. Beatty and D. M. Kernş. IRE Trans. Instrumentation I-7, No, 3-4, 319 (1958).

2723. Reduction of adjacent-channel interference components from frequency-shift-keyed carriers, A. D. Watt, V. J. Zurich, and R. M. Coon. IRE Trans. Commun. Systems CS-6, 39 (1958). 2724. Refinement of the hydroxyapatite structure, A. S. Posner, A. Perloff, and A. F. Diorio. Acta Cryst. 11, 308 (1958).

2725. Unassigned.

2726. Refractive index of arsenic trisulfide, W. S. Rosney, I. H. Malitson, and T. A. King. J. Opt. Soc. Am. 48, No. 9, 633 (1958).

2727. Refractive index of synthetic sapphire, I. H. Malitson, V. F. Murphy, Jr., and W. S. Rodney. J. Opt. Soc. Am. 48, No. 1, 72 (1958). 2728. Regulated supply offsets line changes, L. Costrell. Electronics 31, 100 (1958).

2729. Relation between actual and artificial weathering, F. W. Reinhart. Am, Soc. Testing Materials Spec. Tech. Publ. No. 236, 57 (1958).

2730. Removal of nitrogen from hydrogen with silica gel at low temperatures, V. J. Johnson. Proc. 1958 Cryogenic Engineering Conference, Paper A-2, p. 11. Edited by K. D. Timmerhaus, Chemical Engineering Dept., University of Colorado (Boulder, Coloradó) (1958).

2731. Report on atomic weights for 1956-57, E. Wichers. J. Am. Chem. Soc. 80, 4121 (1958). 2732. Report on reducing sugars, E. J. McDonald. J. Assoc. Official Agri. Chem. 41, No, 3, 623 (1958).

2733. Report on the determination of small amounts of radium in solution, J. E. Harding, A. Schwebel, and L. L. Stockman. J. Assoc. Official Agri. Chem. 41, No. 2, 311 (1958). 
2734. Research at NBS Boulder Laboratories on quartz crystal resonators and oscillators at low temperatures, F. P. Phelps and A. H. Morgan. Proc. 12th Annual Symp. on Frequency Control, May 6-8, 1958, U. S. Army Signal Research and Development Laboratory (Ft, Monmouth, New Jersey), p. 162 (1958).

2735. Research in normal threshold of hearing, J. P. Albrite, R. E. Shutts, M. B. Whitlock, Jr., R. K. Cook, E. L. R. Corliss, and M. D. Burkhard. A. M. A. Arch. Otolaryngol. 68, 194 (1958).

2736. Research in plating at the National Bureau of Standards, A. Brenner. Plating Management 3, No. 5, 14 (1958).

2737. Research is everybody's business, A, V. Astin. Tappi 41, No. 5, 14A (1958).

2738. Resins in prosthetic dentistry, W. T, Sweeney. Chapter in the Dental Clinics of North America, p. 593 (W. B. Saunders Co., Philadelphia, Pennsylvania) (1958).

2739. Resistance measurement of ceramic-type strain-gage cements, J. W. Pitts, E. Buzzard, and D. G. Moore. Am. Soc. Testing Materials Spec. Tech. Publ. No. 230, 67 (1958).

2740. Response functions of total-absorption spectrometers, H. W. Koch and J. M. Wyckoff. Reprint IRE Trans. Nuclear Sci. NS-5, No. 3, 127 (1958).

2741. Response of a large sodium iodide scintillation detector to high energy X-rays, J. H. Hubbell. Rev. Sci. Instr. 29, No. 1, 65 (1958).

2742. Reverberation chamber study of the sound power output of subsonic air jets, R. V. Waterhouse and R. D. Berendt. J. Acoust. Soc. Am. 30,114 (1958).

2743. Reversible contractile processes in fibrous macromolecules, L. Mandelkern, D. E. Roberts, and A. F. Diorio. J. Am. Chem. Soc. 80,500 (1958).

2744. Review of analytical distillation, R. T. Leslie and E. C. Kuehner. Anal. Chem. 30, 629 (1958).

2745. Revision of Commercial Standard CS115-44 for porcelain enameled hot water tanks, J. C. Richmond. Proc. Porcelain Enamel Inst. 20, 51 (1958).

2746. RF permeameter techniques for testing ferrite cores, A. L. Rasmussen and A. E. Hess. Elec. Mfgr. 61, 86 (1958).

2747. RF voltmeter calibrating consoles, M. C. Selby, L. F. Behrent, and F. X. Ries. IRE Natl. Conv. Record 6, Pt. 5, 251 (1958).

2748. Scientific developments and methods in practice (Dentistry in 1967), G. C. Paffenbarger and R. L. Bowen. J. Am. Dental Assoc. 56, 785 (1958).

2749. Scintillation counting in experiments on parity conservation, R. W. Hayward and D. D. Hoppes, Proc. 6th Scin. Counter Symp. IRE Trans. Nuclear Science NS-5, 161 (1958).

2750. Scintillation counting of beta emitters, $H$. H. Seliger. Proc. Intern. Conf, on Padioisotopes in Scientific Research UNESCO (1958).

2751. Separation of hydrogen isotopes by multicomponent distillation, T. M. Flynn, K. D. Timmerhaus, D. H. Weitzel, and J. W. Draper. Proc. 1957 Cryogenic Engineering Conference, Paper A-6, p. 58. Edited by K. D. Timmerhaus. Chemical Engineering Dept., University of Colorado (Boulder, Colorado) (1958).
2752. Separation of isomeric polyphenyls by adsorption chromatography, M. Hellmann, R. L. Alexander, Jr., C. F. Coyle. Anal. Chem. $30,1206(1958)$.

2753. Separation of the products of cool flame oxidation of propane, C. R. Yokley and R. E. Ferguson. Combustion and Flame 2, No, 2, 117 (1958).

2754. Shape of liquid immiscibility volume in the system barium oxide-boric oxide-silica, E. M. Levin and G. W. Cleek, J. Am. Ceram. Soc. 41 , No. 5, 175 (1958).

2755. Shape of the high-energy end of the electron bremsstrahlung spectrum, E. G. Fuller, E. V. Hayward, and H. W. Koch. Phys. Rev. 109, 630 (1958).

2756. Silicate cement: How to select and use them, G. C. Paffenbarger and J. W. Stanford. Dental Practioner 8, No. 12, 387 (1958).

2757. Simplified approach to spin in Dirac theory, H. Mendlowitz. Am. J. Phys. 26, No. 1, 17 (1958).

2758. Single path phase measuring system for three-centimeter radio waves, M. C. Thompson, Jr., and M. J. Vetter. Rev, Sci. Instr. 29,148 (1958).

2759. Solar cycle influence on the lower ionosphere on the VHF forward scatter, C. D. Ellyett and H. Leighton. Proc. IRE 46, No. 10, 1711 (1958).

2760. Some characteristics of VLF propagation using atmospheric waveforms, W. L. Taylor and L. J. Lange. In Recent Advances in Atmospheric Electricity; Proc, 2d Conf, on atmospheric Electricity, Portsmouth, New Hampshire, May 20-23, 1958 (Pergamon Press, New York, N. Y.), p. 609 (1958).

2761. Some mechanical properties of Mylar and Dacron polyester strands at low temperatures, R. P. Mikesell and R. P. Reed. Rev. Sci. Instr. 29, No. 8, 734 (1958).

2762. Some properties of diamond, E. N. Bunting and A. Van Valkenburg. Am. Mineralogist $43,102(1958)$.

2763. Some properties of glasses in the system varium oxide-boric oxide-silica, E. H. Hamiltion, G. W. Cleek, and O. H. Grauer. J. Am. Ceram. Soc. 41, No. 6, 209 (1958).

2764. Some properties of lightning impulses which produce whistlers, R. A. Helliwell, A. G. Jean, and W. L. Taylor. Proc. IRE Letter $46,1760(1958)$.

2765. Some properties of polymer networks formed from oriented chains of natural rubber, D. E. Roberts and L. Mandelkern. J. Am. Chem. Soc. 80,1289 (1958).

2766. Some studies of the upper atmosphere in the auroral zone, S. Matsushita. Ann. Geophys. 14,483 (1958).

2767. Source function in a non-equilibrium atmosphere. II. Depth dependence of source function for resonance and strong subordinate lines, J. T. Jeffries and R. N. Thomas. Astrophys. J. 127, No. 3, 667 (1958).

2768. Source of the non-migratable ionic charges developed by portland and high-alumina cements during hydration, R. G. Pike and D. Hubbard. Natl. Acad. Sci., Natl. Research Council,

Proc. Highway Research Board, 37, 256 (1958). 2769. Unassigned. 
2770. Spectral band-pass determinations by a dynamic approach, I. Nimeroff and J. Laufer. J. Opt. Soc. Am. 48, 864 (1958).

2771. Spectroscopic study of electronic flame temperatures and energy distributions, H. P. Broida and K. E. Shuler. Rev. French Inst. Petro, and Ann. Combus. Liquid 13, 620 (1958). 2772. Splitting of the giant resonance for deformed nuclei, E. G. Fuller and M. S. Weiss. Phys. Rev. 112, No. 2, 560 (1958).

2773. Sporadic E observed on VHF oblique incidence circuits, E. K. Smith. North Atlantic Treaty Organization. Sporadic E Ionization, Ionospheric Research Meeting, AGARD Avionics Panel, September 1958 (Cambridge, England), AGARDograph 34, p. 129 (1958).

2774. Stabilized free radicals, J. L. Jackson. J. Wash. Acad. Sci. 48, No. 6, 181 (1958).

2775. Stable nitrogen isotope analysis by optical spectroscopy, H. P. Broida and M. W. Chapman. Anal. Chem. 30, No. 12, 2049 (1958).

2776. Standards and measurements for electronics, A. V. Astin. IRE Trans. Instrumentation I- 7, No. 3-4, 134 (1958).

2777. Unassigned.

2777A. Standards - good and bad, A. T.

McPherson. Boletime de Metrologia No. 4, 7 , (1958).

2778. Statistical mechanical theory of transport processes, I. Oppenheim. Proc. Midwestern Conf. on Theoretical Phys. Washington University (St. Louis, Mo.) p. 142 (1958).

2779. Statistical methods in chemistry, J. Mandel and F. J. Linnig. Anal. Chem. 30, 739 (1958).

2780. Statistical treatment of analytical data, J. Mandel. Proc. Conf. on Chemical Control Problems, Natl. Plant Food Instr., p. 1 (October 1958).

2781. Status of absolute measurements at the National Bureau of Standards, R. L. Driscoll, J. L. Thomas, and R. D. Cutkosky. ProcesVerbaux, C.I. P. M., p. 36 (1958).

2782. Strains in beams having diagonal cracks, D. Watstein and R. G. Mathey. J. Am. Concrete Inst. 30 , No. 6, Title No. 55-46 (1958); Proc. Am. Concrete Inst. 55, 717 (1958-59). 2783. Stress-strain relationship in yarns subjected to rapid impact loading. V. Wave propagation in long textile yarns impacted transversly, J. C. Smith, F. L. McCrackin, and H. F. Schiefer. Textile Research J. 28, No. 4, 288 (1958).

2784. Stroboscopic interferometer for vibration measurement, E. R. Smith, S. Edelman, E. Jones, and V. A. Schmidt. J. Acoust. Soc. Am. 30, 867 (1958).

2785. Structural interpretation of immiscibility in oxide systems: 3 . Effect of alkalis and alumina in ternary systems, E. M. Levin and S. Block. J. Am. Ceram. Soc. 41, No. 2, 49 (1958).

2786. Studies in nonequilibrium rate processes. III. The vibrational relaxation of a system of anharmonic oscillators, N. W. Bazley, E. W. Montroll, R. J. Rubin, and K. E. Shuler. Errata in J. Chem. Phys. 29, No. 5, 1185 (1958).
2787. Studies in nonequilibrium rate processes. IV. The rotational and vibrational relaxation of a system of rotating oscillators, R. C. Herman and K. E. Shuler. J. Chem. Phys. 29, 366 (1958).

2788. Studies of leather fungicides, S. Dahl and A. M. Kaplan. J. Am. Leather Chemists Assoc. 53, 103 (1958).

2789. Studies of stress-corrosion cracking of Austenitic stainless steel, H. L. Logan. Research Suppl. Welding J. 37, 4635 (1958).

2790. Suppression of bubbling in boiling refrigerants as an aid in optical measurements, G. J. Minkoff and F. I. Scherber. J. Opt. Soc. Am. 48, 358 (1958).

2791. Sur les limites du pouvoir de resolution des spectrometres corpusculaires, L. Marton. Bull. acad. roy. de Belg., p. 159 (March 1958). 2792. Surface erosion of filled plastics, S. B. Newman, S. D. Toner and Achhammer, B. G. Modern Plastics 36, 135 (1958).

2793. Surface resistivity of cotton, wool, and nylon fabrics at different temperatures and relative humidities, H. H. Walker. Textile Research J. 28, No. 12, 1047 (1958).

2794. Surface tension measurements with a strain-gauge-type testing machine, S. B. Newman and W. M. Lee. Rev. Sci. Instr. 29, 785 (1958).

2795. Synthetic cuspidine, A. Van Valkenberg and G. F. Rynders. Am. Mineralogist 43, Nos. 11 and 12, 1195 (1958).

2796. Tables of line strengths for rotational transitions of asymmetric rotor molecules, R. H. Schwendeman and V. W. Laurie.

(Pergamon Press, London, England) (1958). 2797. Technical aspects of large scale liquid helium liquefaction and transportation, D. B. Mann, B. W. Birmingham, and P. C. Vander Arend. Proc, 1957 Cryogenic Engineering Conference, Paper D-4, p. 191. Edited by K. D. Timmerhaus. Chemical Engineering Dept. , University of Colorado (Boulder, Colorado) (1958).

2798. Temperature dependence of the characteristic energy loss of electrons in aluminum, L. B. Leder and L. Marton. Phys. Rev. 112, No. 2, 341 (1958).

2799. Temperature du point du zinc et comparison de deux determinations du point du soufre dans un appareil ferme, J. L. Riddle. Proces-Verbaux Comite Consultatif de Thermometrie, auxpres du Comite Intern. des Poids et Mesures (Paris, France) 5, 111 (1958).

2800. Temperature microcell for infrared study of condensed gases, J. E. Stewart. Anal. Chem. 30, 2073 (1958).

2801. Tension testing of rubber rings, F. L. Roth and R. D. Stiehler. ASTM Bull. No. 233, 38 (1958).

2802. Test for dimensional changes in woven fabrics in high temperature laundering, W. D. Appel. Am. Dyestuff Reptr. 47, No. 7, 213 (1958).

2803. The addition of hydrogen atoms to solid olefins at $-195^{\circ}$, R. Klein and M. D. Scheer. J. Am. Chem. Soc. 80, 1007 (1958).

$2803 \mathrm{~A}$. The algebra of electronics, C. H. Page (D. Van Nostrand Co. Inc., New York, N. Y.) (1958). 
2804. The ammonia maser as an atomic frequency and time standard, R. C. Mockler, J. Barnes, R. Beehler, H. Salazar, and L. Fey. IRE Trans. Instrumentation I-7, No. 3-4, 201 (1958).

2805. The application of the theory of stochastic processes to chemical kinetics, E. W. Montroll and K. E. Shuler. Adv, in Chem. Phys. 1, 361 (Interscience Pub. Inc., New York, N. Y.) (1958).

2806. The atmospheric bending of radio waves

(Abstract), B. R. Bean. Congres International sur la Propagation des Ondes Radio-Electriques, October 6-11, 1958 (Liege, Belgium). Resume des Communications p. 3 (1958).

2807. The characteristic size of airglow cells, F. E. Roach, E. Tandberg-Hanssen, and L. R. Megill. J. Atmospheric and Terrest. Phys. 13, 113 (1958).

2808. The coefficients in an allocation problem, R. J. Aumann and J. B. Kruskal. Naval Research Logistics Quart. 5, 111 (1958).

2809. The definition and measurement of the time constant and response time of thermal converters, F. L. Hermach. Trans. Am. Inst. Elec. Engrs. 77, Pt. I, 277 (1958); Communications and Electronics, No. 37, 277 (1958).

2810. The effect of echo on the operation of high frequency communication circuits, D. K. Bailey. IRE Trans. Ant. Prop. AP-6, No. 4, 325 (1958). 2810A. The elasticity of rubber, L. A. Wood. Rubber Chem, and Technol. 31, No. 5, 959 (1958).

2811. The electrolytic preparation of molybdenum from fused salts. V. Electrorefining studies in the presence of tin, iron, copper, silicon, and nickel, D. E. Couch and S. Senderoff. Trans. Metallurgical Soc. of Am. Inst. Metallurgical Engrs. p. 320 (1958).

2812. The electron affinity of atomic oxygen, L. M. Branscomb. Nature 182, 248 (1958). 2813. The energy dissociation of the $\mathrm{C}-\mathrm{H}$ bond in the formyl radical, R. Klein and L. J. Schoen. J. Chem. Phys. Comments and Errata 29, 953 (1958).

2814. The evaluation of matrix inversion programs, M. Newman and J. Todd. J. Soc. Indust. Appl. Math. 6, 466 (1958).

2815. The Haystaq system: past, present and future, H. R. Koller, E. Marden, and H. Pfeffer. Preprinted in Proc. Intern. Conf. on Scientific Info. November 11-16, 1958 (American Documentation Inst., Washington, D. C.) Area 5, p. 317 (1958).

2816. Unassigned.

2817. The height of nightglow 5577, F. E. Roach, L. R. Megill, M. H. Rees, and E. Marovich. J. Atmospheric and Terrest. Phys. 12, Nos. 2/3, 171 (1958).

2818. The helium-vapor pressure scale of temperatures, F. G. Brickwedde. Phys. Today 11,23 (1958).

2819 . The impact testing of various alloys at low temperatures, R. P. Mikesell and R. P. Reed. Proc. 1957 Cryogenic Engineering Conference, Paper F-4, p. 316. Edited by K. D. Timmerhaus. Chemical Engineering Dept., University of Colorado (Boulder, Colorado) (1958).
2820. The infrared spectrum of tertiary butyl fluoride, D. E. Mann, N. Acquista, and D. R. Lide, Jr. J. Mol. Spectros copy 2, 575 (1958). 2821. The interpretation of night-time low- frequency ionograms, J. M. Watts. J. Geophys. Research 63, 717 (1958).

2822. The Kosters double-image prism, J,

B. Saunders. Appendic C of "Concepts of Classical Optics", p. 393 (W. H. Freeman and Co., San Francisco, California) (1958).

2823. The mean deviation of the Poisson distribution, E. L. Crow. Biometrika 45, 556 (1958). 2824. The need for more knowledge of materials in civil engineering, A. T. McPherson. Conf. Basic Research in Civil Engr. September 10-11, 1958. Am. Soc. Civil Engrs. Natl. Sci.

Foundation, George Washington University

(Wa shington, D. C. ) p. 20 (1958).

2825. The night airglow, F. E. Roach.

American Geophysical Union. Geophysics and the IGY, Geophysical Monograph No. 2, 97, (1958).

2826. The patterns of a slot-array antenna on a finite and imperfect ground plane, J. R. Wait and A. M. Conda. L'Onde Electrique 38, No. 376 bis (Proc. Intern. Congress Ultra High Frequency Circuits and Antennas, October 21-26, 1957, Paris, France) p. 7 (1958).

2827. The program of training and graduate instruction in dental materials at the National Bureau of Standards, W. T. Sweeney. J. Dental Educ. 22, 217 (1958).

2828. The reaction of hydrogen atoms with solid olefins at $-195^{\circ}, \mathrm{R}$. Klein and M. D. Scheer. J. Phys. Chem. 62, 1011 (1958).

2829. The reaction of metal oxides with o-ethyoxybenzoic acid and other chelating

agents, E. M. Brauer, E. E. White, Jr. and M. G. Moshanos. J. Dental Research 37, 547 (1958).

2830. The sky and eye, F. E. Roach and P. M. Jammick. Sky and Teles cope. 17, 164 (1958). 2831. The specific volume and degree of crystallinity of poly(chlorotribluoroethylene), J. D. Hoffman and J. J. Weeks. J. Polymer Sci. 28, No. 117, 472 (1958).

2832. The Stokes flow problem for a class of axially symmetric bodies, L. E. Payne and W. H. Pell. Abstract, Notices Am. Math. Soc. 5,468 (1958).

2833. The structure of ethylene from infrared spectra, H. C. Allen, Jr. and E. K. Plyler. J. Am. Chem. Soc. 80, 2673 (1958).

2833A. The thermal E. M. F. of several thermometric alloys, R. L. Powell and M. D. Bunch. Supplement au Bulletin de 1'Institut Intern, du Froid (Delft, Holland), Comm. 1, 129 (1958). 2834. The thermodynamic driving force in nucleation and growth processes, J. D. Hoffman. Chem. Phys. Note 29, No. 5, 1192 (1958).

2835. The uniqueness of the triangular association scheme, W. S. Connor. Ann. Math. Stat. 29,262 (1958).

2836. The use of group divisible designs for asymmetrical factorial arrangements, M. Zelen. Ann. Math. Stat. 29, 22 (1958).

2837. The use of nickel-aluminum alloy coatings for the protection of molybdenum from oxidation, D. E. Couch, H. Shapiro, and A. Brenner. Electrochem. Soc. 105, No. 8, 485 (1958). 
2838. The use of sweep-frequency backscatter data for determining oblique-incidence ionospheric characteristics, R. Silberstein. J. Geophys. Research 63, 335 (1958).

2839. The zirconium-hydrogen system: Some thermodynamic properties from a heat content study, T. B. Douglas, J. Am. Chem. Soc. 80 , 5040 (1958).

2840. Theory of the stark effect of the NO molecule, M. Mizushima. Phys. Rev. 109, No. 5, 1557 (1958).

2841. Theory of threshold energy dependence of photodetachment of diatomic molecular negative ions, S. Geltman. Phys, Rev. 112, No. 1, 176 (1958).

2841A. Thermal and electrical conductivity of aluminum and aluminum alloys, W. J. Hall, H. M. Roder, and R. L. Powell. In LowTemperature Physics and Chemistry; 1957 Proc. 5 th International Conf., University of Wisconsin (Madison, Wisconsin), p. 389 (1958).

2841B. Thermal and electrical conductivity of pure copper, H. M. Roder, R. L. Powell, and W. J. Hall. In Low-Temperature Physics and Chemistry; 1957 Proc. 5th Intern. Conf., University of Wisconsin (Madison, Wisconsin), p. 364 (1958).

2842. Thermal conductivities of common commercial aluminum alloys, W. J. Hall, R. L. Powell, and H. M. Roder. Proc. 1957 Cryogenic Engineering Conference, Paper G-6, p. 408. Edited by K. D. Timmerhaus, Chemical Engineering Dept., University of Colorado (Boulder, Colorado) (1958).

2843. Thermal degradation of polymers at temperatures up to about $800-900^{\circ} \mathrm{C}$, S. L. Madorsky. Proc. Joint Army-Navy-Air Force Conf. on Elastomer Research and Development, 2, 5th meeting (October 1958), p. 511 (Materials Laboratory, Wright Air Development Center, Wright-Patterson Air Froce Base, Ohio) (1958). 2844. Thermal diffusion in ionized gases, $S$. Chapman, Proc. Phys. Soc. 72, 353 (1958). 2844A. Thermal emf of some thermometric alloys, M. D. Bunch, R. L. Powell, and R. J. Corruccini, In Low-Temperature Physics and Chemistry; 1957 Proc. 5th Intern. Conf., University of Wiscons in (Madison, Wisconsin), p. 484 (1958).

2845. Thermoconductivity of tin-indium alloys in the normal state, C. A. Shiffman. Proc. Phys. Soc. (London, England) 71, No. 460, 597 (1958).

2846. Thermodynamics of crystallization in high polymers: Poly(ethylene), F. A. Quinn, Jr. and L. Mandelkern. J. Am. Chem. Soc. 80,3178 (1958).

2847. Thermometric study of the frozen products from the nitrogen microwave discharge, B. J. Fontana. J. Appl. Phys. 29, 1668 (1958).

2848. Time-temperature behavior of rubber from measurements of indentation hardness, F. L. Roth. Proc. Joint Army-Navy-Air Force Conf. on Elastomer Research and Development 2, 5th meeting (October 1958), p. 399 (Materials Laboratory, Wright Air Development Center, WrightPatterson Air Force Base, Ohio) (1958).

2849. To the edge of space, A. H. Shapley. Sci, Teacher 25, No. 2, 69 (1958).
2850. Topological proofs for certain theorems on matrices with non-negative elements, Ky Fan. Monatsch. Math. 62, 219 (1958).

2851. Transmission los s curves for propagation at very low radio frequencies, J. R. Wait. IRE Trans, Commun. Systems CS- 6, No. 2, 58 (1958).

2852. Transmittance and reflectance of cesium iodide in the far infrared region, E. K. Plyler and N. Acquista. J. Opt. Soc. Am. 48, No.9, 668 (1958).

2852A. Transport properties of dilute alloys of copper, R. L. Powell, W. J. Hall, and H. M. Roder. Physica 24, S176 (1958).

2853. Trapped radicals, H. P. Broida, Endeavor 17, 208 (1958).

2854. Tropospheric effects on 6-MC pulses, R. Silberstein. Proc. IRE Letter 46, No, 1, 1968 (1958).

2855. Two forms of mathematical induction, A. Schach. Math. Mag. 32, No, 2, 83 (1958). $2855 \mathrm{~A}$. United States planning for the large scale liquefaction and transportation of helium, D. B. Mann and B. W. Birmingham. Physica 24, S144 (1958).

2856. Unscrambling of gamma-ray scintillation spectrometer pulse-height distributions, J. H. Hubbell and N. E. Schofield. IRE Trans. Nuclear Sci. NS-5, No. 3, 156 (1958).

2857. Unsteady nonlinear waves in sloping channels, R. F. Dressler. Abstracts of Short Communications and Scientific Programme, Intern. Congress of Mathematicians (Edinburg, Scotland) 138 (1958); Proc. Roy Soc. [A], 247, 186 (1958).

2858. Using an electronic computer to reduce weathering test data, A. Potter. Proc. Porcelain Enamel Inst. 20, 73 (1958).

2859. Vapor phase or tho-para conversion in the large CEL-NBS hydrogen liquefier, W. A. Wilson and D. H. Weitzel. Proc. 1957 Cryogenic Engineering Conference, Paper B-3, p. 85. Edited by K. D. Timmerhaus. Chemical Engineering Dept., University of Colorado (Boulder, Colorado) (1958).

2860. Vertical distribution of light from gasfilled candlepower standards, L. E. Barbrow and S. W. Wilson. Ilum. Eng. 53, 645 (1958). 2861. Vibration-rotation bands of ammonia, III. The region 3.2-4.3 microns, E. K. Plyler, W. S. Benedict, and E. D. Tidwell. J. Chem. Phys, 29, 829 (1958).

2862. Vibrational energy exchange between diatomic molecules and a surface, R. C. Herman and R. J. Rubin. J. Chem. Phys. 29, 591 (1958). 2863. Viscoelastic phenomena in amorphous high polymeric systems, H. Leaderman. Chapter 1 , page 1, Vol. II. Rheology-theory and application (Academic Press Inc., New York, N. Y.) (1958). 2864. Viscosity density, and electrical resistivity of molten alkaline-earth borate glasses with three mole percent of potassium oxide, L. W. Coughanour, L. Shartsis, and H. F. Shermer. J. Am. Ceram. Soc, 41, 324 (1958). 2865. Wall effects on rotational population of $\mathrm{OH}^{2} \Sigma^{+}$in a microwave discharge, T. Carrington and H. P. Broida. J. Mol. Spectros copy 2, No. 3,273 (1958). 
2866. Wax batteries, R. E. Wood. Proc. 12th Annual Battery Research and Develop. Conf. Sponsored by Power Sources Div. U. S. Army Signal Corp. Research \& Devel, Lab. (Ft. Mammouth, New Jersey), p. 62 (1958).

2867. Unassigned.

2868. Wind-induced vibration of telephone and distribution conductors, R. R. Bouche, A. A. Lee, C. R. Ballard, and F. J. On. Am. Inst. Elec. Engrs. Middle Eastern District Meeting Conf. Paper (April 1958).

2869. Wireless microphone uses F-M modulation, G. F. Montgomery. Electronics 31, No. 1, 54 (1958).
2870. World-wide soundings committee: Memorandum 17--Future Work, A. H. Shapley. URSI. (Intern. Sci. Radio Union) Info. Bul. No. 110, 55 (July-August 1958). 2871. WWV standard frequency transmissions, W. D. George. Proc. IRE 46, 910 (1958). 2872. WWV standard frequency transmissions, D. M. Kerns, Proc. IRE Letter 46, 1881 (1958).

2873. Zinc phosphate and silicate cements, G. C. Paffenbarger and J. W. Stanford. Chapter in Dental Clin. North America, 561 (W. B. Saunders Co., Philadelphia, Pa.) (1958). 
2874. A model of the chromosphere from radio and optical data, R. G. Athay. Paris Symp. Radio Astronomy (IAU Symp. No. 9 and URSI Symp. No. 1), paper 16, p. 98 (1959).

2875. A model of the electron corona with reference to radio observations, G. Newkirk, Jr. Paris Symp. Radio Astronomy (IAU Symp. No. 9 and URSI Symp. No. 1), paper 27, p. 149 (1959).

2876. A new method of checking the consistency of precedence matrices, R. B. Marimont. J. Assoc. Computing Mach. 6, No. 2, 164 (1959). 2877. A note on the computation of $x^{2}, M$. G. Natrella. Am. Stat. 13, No. 1, 20 (1959). 2878. A radio-astronomical test of the ballistic theory of light emission, L. R. O. Storey and R. S. Lawrence. The Observatory Correspondence to Editor 79, No. 911, 150 (1959). 2879. A re-examination to the crystal structure of $\alpha$ and $\beta$ nitrogen, L. H. Bolz, M. E. Boyd, F. A. Mauer, and H. S. Peiser. Acta Cryst. 12,237 (1959).

2880. A relationship between the lower ionosphere and the [OI] 5577 nightglow emission, J. W. McCaulley and W. S. Hough, J. Geophys. Research 64, No. 12, 2307 (1959).

2881. A statistician and the post office: A case history in operations research, N. C. Severo. Trans. 1959 Conf. Admin. Appl. Div. of Am. Soc. Quality Control (Milwaukee, Wis.) (1959). 2882. A study of limb flares and associated events, C. S. Warwick and M. B. Wood. Astrophys. J. 128, No. 3, 801 (1959).

2883. A study of 17-7 PH stainless steel, N. L. Carwile and S. J. Rosenberg, WADC Technical Report 58-653 (June 1959). Available from the Office of Technical Services, Department of Commerce, Washington 25, D. C.

2884. A study of the morphology of ionospheric storms, S. Matsushita. J. Geophys. Research 64,305 (1959).

2885. A survey of the present knowledge of sporadic-E ionization, J. A. Thomas and E. K. Smith. J. Atmospheric and Terrest. Phys. 13, 295 (1959).

2886. Abelian groups of unimodular matrices, E. C. Dade. Illinois J. Math. 3, 11 (1959). 2887. Absorption spectrum of vibrationally excited $\mathrm{N}_{2}$ in active nitrogen, $\mathrm{K}$. Dressler. J. Chem. Phys. Letter to Editor 30, 1621 (1959).

2888. Adsorption, diffusion, and evaporation of carbon monoxide on tungsten, R. Klein. J. Chem. Phys. 31, No. 5, 1306 (1959).

2889. Aerodynamic heating and fatigue, W. D. Kroll. NASA Memorandum 6-4-59W (1959). 2890. Aldehyde, carboxyl, pentosan, and degree of polymerization data on the ICCA "standard" pulps, W. L. Morrow. Tech. Assoc. Pulp Paper Ind. 42, No. 3, 169 (1959).

2891. American Dental Association contribution to dental science: Physical research, G. C. Paffenbarger and W. Souder. J. Am. Dental Assoc. 58, 97 (1959).

2892. An evaluation of various thermoplastic materials for use as iodine vapor barriers, $S$. D. Toner. SPE J. 15, 48 (1959).

2893. An improved 8-hydroxyquinoline method for the determination of magnesium oxide in portland cement, H. A. Berman. ASTM Bull. No. 237,51 (1959).
2894. An infrared study of hydrogen bonding in solid $\mathrm{H}_{2} \mathrm{O}$ and $\mathrm{H}_{2} \mathrm{O}-\mathrm{H}_{2} \mathrm{O}_{2}$ mixtures, K. B. Harvey and $P$. Giguere. J. Mol. Spectroscopy 3, 36 (1959).

2895. Analysis of millimicrosecond pulses using a charge-storage type pulse-height analyzer, L. Costrell and R. E. Brueckmann. Proc. 2d Symp. on Advances in Fast Pulse Techniques for Nuclear Counting, February 12-13, 1959 (University of California, Berkeley, California) (1959).

2896. Analysis of vibrational relaxation data in shock wave experiments, K. E. Shuler. J. Chem. Phys. Note 30, 1631 (1959).

2897. Antennas and waveguides, and annotated bibliography, H. V. Cottony, R. S. Elliott, E. C. Jordan, V. H. Rumsey, K. M. Siegel, J. R. Wait, and O. C. Woodyard. U.S.A. National Committee report, International Sci. Radio Union Subcommission 6.3. Inst. Radio Engrs. Trans, on Ant. Prop. AP-7, 87 (1959).

2898. Application of the theory of stochastic processes to chemical kinetics, E. W. Montroll and K. F. Shuler. Chapter in book, Advances in Chemical Physics (Interscience Publishers, New York, N. Y.) Vol. I, p. 361 (1959).

2899. Artificial auroras resulting from the 1958 Johnston Island nuclear explosions, J. M. Malville. J. Geophys. Research 64, No. 12, 2267 (1959).

2900. Atomic electron affinities, H. Johnson and F. Rohrlich. Nature No. 4656,183 (1959).

2901. Basic physics of atoms and molecules, U. Fano and L. Fano. (John Wiley and Sons, Inc., New York, N. Y.) 414 pages (1959).

2902. Beta-gamma correlations from polarized nuclei, E. Ambler, R. W. Hayward, D. D. Hoppes, and R. P. Hudson. Proc. Congres International de Physique Nucleaire, 1958, Interactions Faibles, Chapter III, p. 831 (Paris, France) (1959).

2903. Branched-chain higher sugars. I. A 9-aldo4-C-formyl-nonose derivative, R. Schaffer and H. S. Isbell. J. Am. Chem. Soc. 81, 2178 (1959).

2904. Branched-chain higher sugars. II. A diethylidene-oxtose, R. Schaffer and H. S. Isbell. J. Am. Chem. Soc. 81, 2838 (1959).

2905. Bremsstrahlung and the photoelectric effect as inverse processes, K. W. McVoy and U. Fano. Phys. Rev. 116, No. 5, 1168 (1959). 2906. Can the scales of atomic weights and nuclidic masses be unified? E. Wichers. Phys. Today 12, No. 1, 28 (1959).

2907. Cavity resonator dielectric measurements on rod samples, H. E. Bussey. Insulation p. 26 (1959).

2908. Characteristics of deposits, W. H. Metzger, Jr. Am, Soc. Testing Materials Symp. on Electroless Nickel Plating 265, 13 (1959).

2909. Characterization of the high-speed impact behavior of textile yarns, J. C. Smith, F. L. McCrackin, and H. F. Schiefer. Conference Issue J. Textile Inst. (Trans.) 50, No. 1, T55 (1959).

2910. Chemical structure and stability relationships in polymers, B. G. Achhammer, M. Tryon, and G. M. Kline. Kunststoffe combined with German Plastics Digest 49, No. 11, 600 (1959); Modern Plastics 37, No. 4, 131 (1959). 
2910A. Coatings for space vehicles, J. C. Richmond. Proc. Ist Symp. Surface Effects on Space and Craft Materials, Sponsored by USAF Air Research and Development Command and Lockheed Aircraft Corp., May 12-13, 1959 (Palo Alto, Calif.), p. 182 (John Wiley and Sons, New York, N. Y.) (1959).

2911. Comments on the structure of $1,1,1$-trifluoro-2-butyne, V. W. Laurie. J. Chem. Phys. Letter 30, 1101 (1959).

2912. Comparison of the ionization produced in air by alpha particles near 5-Mev and by beta particles, Z. Bay and P. A. Newman. Bull. Am. Phys. Soc. II: 4, No. 4, 217 (1959).

2913. Computer defines color, H. J. Keegan. Chem. Eng. News 37, No. 10, 37 (1959).

2914. Conductivity of plasmas to microwaves, P. H. Fang. Phys. Rev. 113, No. 1, 13 (1959). 2915. Confidence intervals for the expectation of a Poisson variable, E. L. Crow and R. S. Gardner. Biometrika 46, 441 (1959).

2916. Constant temperature liquid helium bath and reproducibility of resistance thermometers, H. H. Plumb and M. H. Edlow. Rev. Sci. Instr. 30, 376 (1959).

2917. Contribution of the annihilation radiation to the gamma-ray flux in lead, M. J. Berger J. H. Hubbell, and I. Reingold. Phys. Rev. 113 , No. 3, 857 (1959).

2918. Convexity of the field of a linear transformation, A. J. Goldman and M. Marcus. Can. Math. Bull. 2, 15 (1959).

2919. Correlation effects in impurity diffusion, J. R. Manning. Phys. Rev. 116, No. 4, 819 (1959).

2920. Cosmic examples of heat conduction in very rare rotating or expanding gases, S. Chapman. Extrait des Ann. de Geophys. Tome 15, No. 4, 434 (1959).

2921. Creep behavior of transparent plastics at

elevated temperatures, F. L. McCrackin and C. F. Bersch. SPE Tech Paper 5, 94 (1959). 2922. Cryogenic engineering, R. B. Scott. (D. Van Nostrand Co., New York, N. Y.) (1959). 2923. Crystallization kinetics in polymeric systems, L. Mandelkern. SPE J. 15, 63 (1959).

2924. Degradation of cellulose acetate films, W. K. Wilson and B. W. Forshee. SPE J. 15, No. 2, 146 (1959).

2925. Dense subgraphs and connectivitv, R. E. Nettleton, K. Goldberg, and M. S. Green. Can. J. Math. 11, 262 (1959).

2926. Departures from the Saha equation under varying conditions of Lyman continuous opacity, S. R. Pottasch and R. N. Thomas. Astrophys. J. 130, No. 3, 941 (1959).

2927. Departures of hydrogen from L. T. E. in a stellar atmosphere and the consequent structure of the solar chromosphere, S. R. Pottasch. Communications de l'Observatoire Royal de Belgique No. 157, entitled The empirical determination of the stellar photospheric structure, Paper 11, 67 (1959).

2928. Determination of acetylene in air in concentrations from ten parts per billion to ten parts per million, E. E. Hughes and R. Gorden Jr. Anal. Chem. 31, 94 (1959).
2929. Diffusion of particles in turbulent flow, C. M. Tchen. Advances in Geophysics 6: "Atmospheric diffusion and air pollution", p. 165, Proc. Symp. Oxford, England, August 1958 (Academic Press Inc., New York, N. Y.) (1959).

2930. Distribution of matter with temperature in the emission corona, D. E. Billings.

Astrophys. J. 130, No. 3, 961 (1959).

2931. Diurnal change of ionospheric heights deduced from phase velocity, J. R. Wait. Proc. IRE 47, No. 5, 98 (1959).

2932. Downcoming radio waves, measurement of characteristics, J. R. Wait. Elec. and Radio Engr. 36, No. 3, 106 (1959).

2933. Effect of fluorides on infrared transmittance of certain silicate glasses, G. W. Cleek and T. S. Scuderi. J. Am. Ceram. Soc. 42 , No. 12,599 (1959).

2934. Electrical discharge induced luminescence of solids at low temperatures, L. J. Schoen and R. Rebbert. J. Mol. Spectroscopy 3, No. 4, 417 (1959).

2935. Electrical measurements - in the core curriculum, F. R. Kotter. Proc. 3d Natl. Conf. on Analog and Digital Instrumentation, April 20-21, 1959, Philadelphia, Pennsylvania (Sponsored by Recording and Controlling Instrumentation Committee of AIEE). Am. Inst. Elec. Engrs. p. 15 (1959).

2936. Electrolysis of organic solvents with reference to the electrodeposition of metals, A. Brenner. J. Electrochem. Soc. 106, 148 (1959).

2937. Electron characteristic energy losses in some intermetallic compounds, B. Gauthe.

Phys. Rev. 114, No. 5, 1265 (1959).

2938. Electron impact studies of aromatic hydrocarbons. I. Benzene, napthalene, anthracene, and phenanthrene, M. E. Wacks and V. H. Dibeler. J. Chem. Phys. 31, No. 6, 1557 (1959).

2939. Electron impact studies of hydrazine and the methyl-substituted hydrazines, V. H.

Dibeler, J. L. Franklin, and R. M. Reese, J. Am. Chem. Soc. 81,68 (1959).

2940. Electron spin resonance spectra from gamma-irradiated solid nitrogen, L. A. Wall, D. W. Brown, and R. E. Florin. J. Chem. Phys. 30, No. 2, 602 (1959).

2941. Electronic quenching of $\left.\mathrm{OH}^{2} \Sigma^{+}\right)$in flames and its significance in the interpretation of rotational relaxation, T. Carrington. J. Chem. Phys. 30, 1087 (1959).

2942. Energetic species trapped at $4.2^{\circ} \mathrm{K}$ from gaseous discharges, G. J. Minkoff, F. I.

Scherber, and J. S. Gallagher. J. Chem. Phys. 30,753 (1959).

2943. Etude aux infrarouges de certains solides condenses a partir de decharges en phase gazeuse, K. B. Harvey and H. W. Brown. J. chim. phys. 56, No. 2392, 745 (1959).

2944. Etude spectroscopique des produits de la decharge electrique dan L'Azote condenses a l'etat solide a tres basse temperatures, prevues en faveur de 1'existence d'Azote triatomique dan le solide, M. Peyron, E. M. Horl, H. W. Brown, and H. P. Broida. J. chim. phys. 56, No. 2392, 736 (1959). 
2945. Unassigned.

2946. Experiments with the cell $\mathrm{Bi}, \mathrm{Bi}_{2} \mathrm{O}_{3}$, $\mathrm{ZnCl}_{2} / \mathrm{ZnCl}_{2} / \mathrm{ZnCl}_{2}, \mathrm{Zn}$ between 450 and $510^{\circ}$, R. E. Wood. J. Phys. Chem. 63, 525 (1959). 2947. Exposure standards and radiation protection regulations, L. S. Taylor. Section 3, p. 2 Radiation Hygiene Handbook, edited by Hansen Blatz (McGraw-Hill Book Co., Inc., New York, N. Y.) (1959).

2948. Factors affecting the accuracy of distortion measurements made on the nodal slide optical bench, F. E. Washer and W. R. Darling. J. Opt. Soc. Am. 49, No. 6, 517 (1959). 2949. Fading rate recorder for propagation research, J. W. Koch, W. B. Harding, and R. J. Jansen. Electronics 32, No. 51, 78 (1959). 2950. False negative permanent strains observed with resistance wire strain gages, C. J. Newton. ASTM Bull. 235, 42 (1959).

2951. Fatigue properties of high strength steels, H. E. Frankel, J. A. Bennett, and W. A. Pennington. Trans. Am. Soc. Metals 52 (1959). 2952. Fifty years of signal lighting, F. C. Breckenridge. Brochure is sued by the Illum. Eng. Soc. 21 pages (1959).

2953. Flow conversion kinetics of ortho and parahydrogen, D. H. Weitzel, J. H. Blake, and M. Konecnik. Proc. 1958 Cryogenic Engineering Conference, Paper E-2, p. 286. Edited by K. D. Timmerhaus, Chemical Engineering Dept., University of Colorado (Boulder, Colorado) (1959).

2954. Formation of ozone from atomic oxygen at low temperatures, R. A. Ruehrwein and J. S. Hashman. J. Chem. Phys, 30, No. 3, 823 (1959).

2955. Future developments of electronic data processing systems, S. N. Alexander. Proc. 8th Annual Symp. Federal Govt. Accountants Assoc., November 17-18, 1958, The Federal Accountant VIII, No. 4, 28 (1959).

2956. Gamma radiation from $\mathrm{Zn} 63$, R. W. Hayward, E. F. Pessoa, D. D. Hoppes, and R. Van Lieshout. Il Nuovo Cimento 11, 53 (1959). 2957. General relations between fluxes from collimated point and plane sources of radiation, M. J. Berger and L. V. Spencer. Phys, Rev. 113 , No. 2, 408 (1959).

2958. Geomagnetic disturbance and velocity of slow-drift solar radio bursts, M. B. Wood and C. S. Warwick. Nature 184, 1471 (1959).

2959. Geomagnetic effects of high-altitude nuclear explosions, A. G. McNish. J. Geophys. Research 64, No. 12, 2253 (1959).

2960. Green coronal line intensity and geomagnetism, C. S. Warwick. J. Geophys. Research 64, No. 5, 527 (1959).

2961. High-frequency limit of bremsstrahlung in the Sauter approximation, U. Fano. Phys, Rev. 116 , No. 5, 1156 (1959).

2962. IGY instruction manual, Pt. 1: World days and communications, A. H. Shapley. Ann. Intern. Geophys. Year Vol. 7, Pt. 1, p. 1 (Pergamon Press, New York, N. Y.) (1959). 2963. IGY observations of F-layer scatter in the far east, R. Bateman, J. W. Finney, E. K. Smith, L. H. Tveten, and J. M. Watts. J. Geophys. Research 64, 403 (1959).

2964. Improvements in liquid scintillators, $H$. H. Seliger and B. Agranoff. Anal. Chem. 31, No. 9, 1607 (1959).
2965. Index to the literature on spectrochemical analysis, Part IV, 1950-1955, B. F. Scribner and W. F. Meggers. Am. Soc. for Testing Materials Publ. No. 41D (1959).

2966. Initial and remanent permeability spectra of Yttrium iron garnet, R. D. Harrington and A. L. Rasmussen. Proc. IRE 47, 98 (1959). 2967. Interference of antioxidant in the determination of low polymer in SBR synthetic rubber, L. T. Milliken and F. J. Linnig. J. Polymer Sci. XLI, No. 138, 544 (1959).

2968. Interference of orbital and spin currents on bremsstrahlung and photoelectric effect, U. Fano, K. W. McVoy, and J. R. Albers. Phys. Rev. 116, No. 5, 1159 (1959).

2969. Interplanetary space and the earth's outermost atmosphere, S. Chapman. Proc. Roy. Soc. A, 253, 462 (1959).

2970. Irreducible tensorial sets, U. Fano and G. Racah. Academic Press Inc., New York, N. Y. (1959).

2971. Kinetics of $\mathrm{D}$ atom reactions with $\mathrm{H}_{2}, \mathrm{R}$. Klein, J. R. McNesby, M. D. Scheer, and L. J. Schoen. J. Chem. Phys. 30, No. 1, 58 (1959).

2972. Kinetics of nitrogen atom recombination, J. T. Herron, J. L. Franklin, P. Bradt, and V. H. Dibeler. J. Chem. Phys. Letters 29 , No. 1, 230 (1958); J. Chem. Phys. 30, 879 (1959). 2973. La recherche sur les radicaux libres au National Bureau of Standards, H. P. Broida. J. chim. phys. 56, No. 2392, 813 (1959).

2974. Leonard Euler's integral: A historical profile of the gamma function, P. Davis. Am. Math. Mo. 66, 849 (1959).

2975. Light emission from solid nitrogen during and after electron bombardment, E. M. Horl. J. Mol. Spectroscopy 3, No. 4, 425 (1959).

2976. Line shape and $f$ value in the OH $25+$.

$2 \pi$ transition, T. Carrington. J. Chem. Phys. 31, No. 5, 1243 (1959).

2977. Local standards laboratories, A. T. McPherson. Instr. and Automation 32, 92 (1959).

2978. Low-energy limit of the photonuclear pseudodeuteron effect, M. Danos. Bull. Am. Phys. Soc. Serv. II, 4, 102 (1959).

2979. Low-temperature X-ray studies on Rice's blue material, L. H. Bolz, F. A. Mauer, and H. S. Peiser. J. Chem. Phys. 30, No. 1, 349 (1959).

2980. Magnetic losses at low temperatures, E.

H. Brown and J. R. Brennand Jr. Proc. 1958 Cryogenic Engineering Conference, Paper A-5, p. 65. Edited by K. D. Timmerhaus, Chemical Engineering Dept., University of Colorado (Boulder, Colorado) (1959); J. Appl. Phys. 30, No. 1, 112 (1959).

2980A. Magnetic properties of polycrystalline materials, D. M. Grimes, R. D. Harrington, and A. L. Rasmussen. J. Phys. Chem. Solids 12,28 (1959).

2981. Magnitoye okhlazhdenye, E. Ambler and R. P. Hudson. Uzpekhi Physicheskhikh Nauk 47,445 (1959).

2982. Mass spectrometric study of the reactions of some hydrocarbons with active nitrogen, J. T. Herron, J. L. Franklin, and P. Bradt. Can. J. Chem. 37, 579 (1959). 
2983. Measurement of the flow of liquefied gases with sharp-edged orifices, R. J. Richards, R. B. Jacobs, and W. J. Pestalozzi. Proc. 1958 Cryogenic Engineering Conference, Paper E-1, p. 272. Edited by K. D. Timmerhaus, Chemical Engineering Dept., University of Colorado (Boulder, Colorado) (1959).

2984. Measurement systems for high-level dosimetry, E. J. Hart, H. W. Koch, B. Petree, J. H. Schulman, S. I. Taimuty, and H. O. Wyckoff. 2d United Nations Intern. Conf. on the Peaceful Uses of Atomic Energy, Vol. 21, Health and Safety: Dosimetry and Standards, P/1927 USA, p. 188 (Pergamon Press, London, England) (1959).

2985. Mechanical properties of insulating plastic foams at low temperatures, R. M. McClintock. Proc. 1958 Cryogenic Engineering Conference, Paper B-6, p. 132. Edited by K. D. Timmerhaus, Chemical Engineering Department, University of Colorado (Boulder, Colorado) (1959).

2986. Mechanism of contraction in the muscle fibre - ATP system, L. Mandelkern, A. S. Posner, A. F. Diorio, and K. Laki. Proc. Natl. Acad. Sci. 45, 814 (1959).

2987. Mechanistic a spects of hearing, E. L. R. Corliss. J. Acoust. Soc. Am. Abstract 31, 841 (1959).

2988. Mechanized computation of thermodynamic tables at the National Bureau of Standards: The calculation of the equilibrium composition and thermodynamic properties of dissociation and ionized gaseous systems, J. Hilsenrath, M. Klein, and D. Y. Sumida. Proc. Symp. Thermodynamic and Transport Properties of Gases, Liquids, and Solids, (Reprinted from Thermodynamic and Transport Properties of Gases, Liquids, and Solids), Am. Soc. Mech. Engrs. p. 416 (1959).

2989. Men and electrons, L. Marton. J. Wash. Acad. Sci. 49, No. 4, 97 (1959).

2990. Methods of experimental physics, H. P. R. Frederikse, V. A. Johnson, and W. W. Scanlon. Chapter 7.6, Thermomelectric Effects. Edited by K. Lark-Horovitz and V. A. Johnson (Academic Press Inc., New York, N. Y.) p. 114 (1959).

2991. Microwave spectra of molecules exhibiting internal rotation, V. Barrier height in ethylchloride and ethyl bromide, D. R. Lide, Jr. J. Chem. Phys. 30, 37 (1959).

2992. Microwave spectrum of methyl germane, V. W. Laurie, J. Chem. Phys. 30, 1210 (1959) 2993. Mismatch errors in cascade-connected variable attenuators, G. E. Schafer and A. Y. Rumfelt. IRE Trans. on Microwave Theory and Tech. MTT-7, No. 4, 447 (1959).

2994. Molecular complexes and their spectra. IX. Infrared absorption by iodine in its pyridine complexes and in benzene, E. K. Plyler and R. S. Mulliken. J. Am. Chem. Soc, 81, 823 (1959). 2995. Multi-factor experiments, M. Zelen and W. S. Connor. Ind. Quality Control 15, No. 9, 1 (1959).

2996. Multiple ionization of sodium vapor by electron impact, V. H. Dibeler and R. M. Reese. J. Chem. Phys. 31, 282 (1959).

2997. Natural and synthetic rubbers: Review of test methods, M. Tryon and F. J. Linnig. Anal. Chem. Appl. Revs. 31, Pt. II, No. 4, 767 (1959).
2998. New standards for science and industry, A. T. McPherson. Am. Soc. Quality Control, Proc. 4th Annual Quality Control Symp.,

(Southern Methodist Univ., Dallas, Texas), p. 103 (1959).

2999. Note on measur ement of sine-wave response of lenses, R. E. Stephens. J. Opt. Soc. Am. 49, No. 4, 413 (1959).

3000. Nuclear magnetic resonance in semiconductors, O. Kraus. Phys, and Chem, of Solids (Pergamon Press, London, England) 8 , 504 (1959).

3001. OH emission in the earth's atmosphere, D. M. Gates. Proc. 2d Natl. Infrared Information Symp. 4, No. 1, 145 (1959).

3002 . On models of the atmospheric radio refractive index, B. R. Bean and G. D. Thayer. Proc. IRE 47, No. 5, 740 (1959).

3003. On the convergence of the Rayleigh quotient iteration for the computation of the characteristic roots and vectors, II, A. M. Ostrowski. Arch. Ratl. Mech. Anal. 2, No. 5, 423 (1959).

3004 . On the convergence of the Rayleigh quotient iteration for the computation of characteristic roots and vectors, VI. (Usual Rayleigh quotient for nonlinear elementary divisors), A. M. Ostrowski. Arch. Ratl. Mech. Anal. 4, No. 2, 153 (1959).

3005. On the diffraction of electromagnetic pulses by curved conducting surfaces, J. R. Wait and A. M. Conda. Can. J. Phys. 37, 1384 (1959).

3006. On the electromagnetic response of an imperfectly conducting thin dyke, J. R. Wait. Geophysics XXIV, No. 1, 167 (1959).

3007. On the hydrogen evolution reactions, $R$. J. Brodd. J. Electrochem. Soc. 106, No. 1, 74 (1959).

3008. On the minimum of the permanent of a doubly stochastic matrix, M. Marcus and $M$. Newman. Duke Math. J. 26, 61 (1959).

3009 . On the numerical integration of periodic analytic functions, P. Davis. Chapter in the Book On Numerical Approximation, Edited by R. E. Langer, (University of Wisconsin Press, Madison Wisconsin), p. 45 (1959).

3010 . On the theory of reflection from a wire grid parallel to an interface between homogeneous media (II), J. R. Wait. Appl. Sci. Research Section B, 7, No. 5, 355 (1959).

3011. Optimum antenna height for ionospheric scatter propagation, R. G. Merrill. IRE Natl. Conv. Record Pt. I, p. 10 (1959).

3012. Photodetachment cross section of the negative hydrogen ion, S. J. Smith and D. S. Burch. Phys. Rev. Letters 2, No. 4, 1645 (1959).

3013. Photolysis of oxygen in solution in liquid nitrogen and in liquid carbon monoxide at $1849 \mathrm{~A}$, J. R. McNesby. J. Chem. Phys. 31, No. 1, 283 (1959).

3014. Photon scattering and self absorption in lead and bismuth at $7 \mathrm{Mev}$, E. G. Fuller and E. Hayward. Proc. Congres International de Physique Nucleaire, 1958, Nuclear Reaction in Nuclear Forces, Pt. 2, Chapter I, p. 62 (Paris, France) (1959).

3015. Pile oscillator measurements of eta, C. O. Muehlhause, Nuclear Sci. and Engr. 5, No. 4, 225 (1959). 
3016. PILOT, a new multiple computer system, A. L. Leiner, W. A. Notz, J. L. Smith, and A. Weinberger. J. Assoc. Computing Mach. 6, No. 3, 313 (1959).

3017. Poisoning and reactivation of orthoparahydrogen conversion catalyst, R. N. Keeler and K. D. Timmerhaus. Proc. 1958 Cryogenic Engineering Conference, Paper E-3, p. 296. Edited by K. D. Timmerhaus, Chemical

Engineering Dept., University of Colorado (Boulder, Colorado) (1959).

3018. Polymorphism in barium disilicate, $R$. S. Roth and E. Levin. Am. Mineralogist 44, No. 3-4, 452 (1959).

3019. Prediction of pressure drop in two-phase single-component fluid flow, M. R. Hatch, R. B. Jacobs, R. J. Richards, R. N. Boggs, and G. R. Phelps. Proc. 1958 Cryogenic Engineering Conference, Paper F-4, p. 357 . Edited by K. D. Timmerhaus, Chemical Engineering Dept. University of Colorado (Boulder, Colorado) (1959).

3020. Prediction of sunspot numbers for cycle 20, W. B. Chadwick. Nature 184, 1787 (1959). 3021. Present status and future prospects of emission spectroscopy, W. F. Meggers. Rev. Universelles des Mines (Liege, Belgique), No. 5 , p. 7 (1959).

3022. Program of the International Commission on Radiological Units and Measurements, L. S. Taylor, L. H. Gray, and H. O. Wyckoff. 2d United Nations Intern. Conf. on the Peaceful Uses of Atomic Energy, Vol. 21, Health and Safety: Dosimetry and Standards, P/2243 WHO, p. 81 (Pergamon Press, London, England) (1959). 3023. Propagation and production of electromagnetic waves in a plasma, R. M. Gallet. Il Nuovo Cimento Supplemento 13, No. 1, 234 (1959). 3024. Propagation of a ground waye pluse around a finitely conducting spherical earth from a damped sinusoidal source current, J. R. Johler, and L. C. Walters. IRE Trans. Ant. Prop. AP-7, No. 1, 1 (1959).

3025. Quantum-mechanical calculation of the probability of an exchange reaction for constrained linear encounters, J. Mazur and R. J. Rubin. J. Chem. Phys. 31, No. 5, 1395 (1959).

3026. Rack for standard resistors, P. H.

Lowrie, Jr. Rev. Sci. Instr. Letter to

Editor 30, No. 4, 291 (1959).

3027. Radiation attenuation data, H. O. Wyckoff. Section 8, p. 1, Radiation Hygiene Handbook, edited by Hanson Blatz (McGraw-Hill book Co. , Inc., New York, N. Y.) (1959).

3028. Radiation from a small loop immersed in a semi-infinite conducting medium, J. R. Wait.

Can. J. Phys. 37, 672 (1959).

3029. Unassigned.

3030. Reactions en chaine de radicaux geles, J. L. Jackson. J. chim. phys. 56, No. 2392, 771 (1959).

3031. Recent advances in cryogenic engineering,

R. B. Jacobs. ARS J. 29, No. 4, 245 (1959).

3032. Recent experimental evidence favouring the $\mathrm{pK}_{1}(\mathrm{p})$ correlation function for describing the turbulence of refractivity in the troposphere and stratosphere, K. A. Norton. J. Atmosspheric and Terrrest. Phys. 15, 206 (1959).
3033. Reception of space diversity transmitters, J. W. Koch. Wireless World (England) 65, No. 10,512 (1959).

3034. Reflectors for a microwave Fabry-Perot interferometer, W. Culshaw. IRE Trans. Microwave Theory and Tech. MTT-7, No. 2, 221 (1959).

3035. Relative measurement of the photodetachment cross section for $\mathrm{H}^{-}, \mathrm{S}$. J. Smith and D. S. Burch. Phys. Rev. 116, No. 5, 1125 (1959). 3036. Report of the Committee on the American Standards Association, F. E. Washer. Photogrammetric Engr. XXV, No. 2, 309 (1959).

3037. Rheology of polyisobutylene. III. Elastic recovery, non-Newtonian flow, and molecular weight distribution, H. Leaderman, R. G. Smith, and L. C. Williams. J. Polymer Sci. 36,233 (1959).

3038 . Rotational relaxation in nitrogen, oxygen, and air, M. Greenspan. J. Acoust. Soc. Am. 31,155 (1959).

3039. Sampling of leather, J. Mandel and C. W. Mann. J. Sci. and Ind. Research 18A, No. 12,575 (1959).

3040. Sauter theory of the photoelectric effect, U. Fano, K. W. McVoy, and J. R. Albers. Phys. Rev. 116, No. 5, 1147 (1959).

3041. Science fairs, A. T. McPherson. ASTM Bull. No. 237, 29 (1959).

3042. Unassigned.

3043. Selection of glasses for three color achromats, R. E. Stephens. J. Opt. Soc. Am. 49, No. 4, 398 (1959).

3044. Semiautomatic Townsend balance system, S. B. Garfinkel. Rev. Sci. Instr. 30, No. 6, 439 (1959).

3045. Unas signed.

3046. Some color demonstrations I have shown, D. B. Judd. J. Opt. Soc. Am. 49, 322 (1959). 3047. Some elementary inequalities relating to a gamma and incomplete gamma function, W. Gautschi. J. Math. Phys. 39, 77 (1959). 3048. Some evidence for structural anomalies in pure cristobailite, R. F. Walker, S. J. Schneider, and R. S. Roth. J. Am. Ceram. Soc. 42 , No. 12,642 (1959).

3049. Some methods for reducing heat leak through support members in liquefied gas storage vessels, R. W. Arnett, L. O. Mullen, and K. A. Warren. Proc. 1958 Cryogenic Engineering Conference, Paper G-1, p. 410. Edited by K. D. Timmerhaus, Chemical Engineering Dept. , University of Colorado (Boulder, Colorado) (1959).

3049A. Some methods used at the National Bureau of Standards for measuring thermal emittance at high temperatures, J. C. Richmond. Proc, 1st Symp. Surface Effects on Space and Craft Materials. Sponsored by USAF Air Research and Development Command and Lockheed Aircraft Corp., May 12-13, 1959 (Palo Alto, Calif.), p. 182 (John Wiley and Sons, New York, N. Y.) (1959). 
3050. Some properties of flameproof fabrics, M. W. Sandholzer. Am. Dyestuff Reptr. 48, No. 2, 37 (1959).

3051. Some radiological applications of gammaray transport theory, M. J. Berger and L. V. Spencer. Radiation Research 10, No. 5, 552 (1959).

3052. Spectra emitted from solid nitrogen condensed at very low temperatures from a gas discharge, M. Peyron and H. P. Broida. J. Chem. Phys. 30, No. 1, 139 (1959).

3053. Spectral emittance of ceramic-coated and uncoated specimens of Inconel and stainless steel, J. C. Richmond and J. E. Stewart. J. Am. Ceram. Soc. 42, No. 12, 633 (1959). 3054. Spectroscopic evidence for triatomic nitrogen in solids at very low temperatures, M. Peyron, E. M. Horl, H. W. Brown, and H. P. Broida. J. Chem. Phys. 30, 1304 (1959). 3054A. Standard frequency transmission and time signals, W. D. George. Proc. 2d all-IRIG Symp., prepared by Secretariat, Inter-Range Instrumentation Group, October 1958 (White Sands Missile Range, New Mexico), IRIG Document No. 107-58, 141 (1959).

3055. Standards for neutron flux measurement and neutron dosimetry, R. S. Caswell, J. Chin, and E. R. Mosburg, Jr. 2d United Nations Intern. Conf. on the Peaceful Uses of Atomic Energy, Vol. 21, Health and Safety: Dosimetry and Standards, P/752, USA, p. 92 (Pergamon Press, London, England) (1959).

3055A. Status of ASTM methods and standards for appearance evaluation. I. Nimeroff. Am. Soc. Testing Materials Spec. Tech. Publ. 258, 3 (1959).

3056. Structural changes in irradiated plastics films, C. F. Bersch, R. R. Stromberg, and B. G. Achhammer. SPE Tech. Papers 5, 5 (1959).

3057. Studies of borate minerals. VI: Veatchite, J. R. Clark, M. E. Mrose, A. Perloff, and G. Burley. Am. Mineralogist 44, No. 11-12, 1141 (1959).

3058. Study of the setting of plaster, K. D. Jorgesen and A. S. Posner. J. Dental Research 38, 491 (1959).

3059. Surface tension of alkaline-earth borates containing 3 mole percent of potassium oxide, L. Shartsis, A. B. Bestul, and H. Shermer. J. Am. Ceram. Soc. 42, 242 (1959).

3060. Systematic research, A. V. Astin. Ordnance 43 , No. 233, 741 (1959).

3061. Tables of the speed of sound in water, $M$. Greenspan and C. E. Tschiegg. J. Acoust. Soc. Am. 31, No. 1, 75 (1959).

3062. Temperature dependence of the breakdown field of barium titanate, P. H. Fang and W. S. Brower. Phys. Rev. 113, 456 (1959). 3063. Testing and operation of ball bearings submerged in liquefied gases, K. B. Martin and R. B. Jacobs. Proc. 1958 Cryogenic Engineering Conference, Paper H-2, p. 476. Edited by K. D. Timmerhaus, Chemical Engineering Dept., University of Colorado (Boulder, Colorado) (1959); ASLE Trans. 2, No. 1, 101 (1959).
3064. The ampere, F. B. Silsbee. Proc. IRE 47 , No. 5, 643 (1959).

3065. The ASTM crude natural rubber subcommittee, N. Bekkedahl and R. G. Seaman. Rubber World 189, 693 (1959).

3066. The basis of our measuring system, A. G. McNish. Proc. IRE 47, No. 5, 636 (1959). 3067. The calculation of the field in a homogeneous conductor with a wavy interface, J. R. Wait. Proc. IRE 47, No. 6, 1155 (1959).

3068. The day-to-day coordination of IGY observations, A. H. Shapley. Proc. IRE 47, 323 (1959).

3069. The design of impact-resisting fabric structures, J. C. Smith and H. F. Schiefer. Chapter 13 on Textile Engineering, Encyclopedia of Man-Made Textiles (Textile Book Publishers, Division of Interscience Publishers, New York, N. Y.) (1959).

3070. The diode reactance modulator, G. F. Montgomery. Elec. Eng. 77, 615 (1958); AIEE Trans. on Commun. and Electronics 78, No. 40, 980 (1959).

3071. The earth and its environment, S. Chap-

man. Proc. IRE 47, No. 2, 137 (1959).

3072. The effect of a ceramic coating on the creep behavior of some high temperature alloys, J. R. Cuthill, J. C. Richmond, and N. J. Tighe. Bull. Am. Ceram. Soc. 39, 4 (1959).

3073. The effect of multipath distortion on the choice of operating frequencies for high-frequency communication circuits, D. K. Bailey. IRE Trans. Ant. Prop. AP-7, No. 4, 397 (1959). 3074. The effect of sporadic $E$ on VHF transmission in North America, R. M. Davis, E. K. Smith, and C. D. Ellyett. IRE Spec. Govt. Is sue 47 , No. 5, 762 (1959).

3075. The effect of the earth's magnetic field on m. u. f. calculations, K. Davies. J. Atmospheric and Terrest. Phys. 16, 187 (1959). 3076. The electromotive force method of determining ionization constants: Ionization of bisulfate ion, W. J. Hamer. Chapter in the Book The Structure of Electrolytic Solutions (J. Wiley and Sons, Inc., New York, N. Y.) p. 236 (1959). 3077. The emission of He I $\lambda$ 10830, during the great flare of August 26, 1958, E. TandbergHanssen, W. Curtis, and K. Watson. Astrophys. J. 129, No. 1, 238 (1959).

3078. The evaluation of small color differences: I. Visual observations, J. C. Richmond and W. N. Harrison. Bull. Am. Ceram. Soc. 38, 292 (1959).

3078A. The evolution of certified reference materials, J. I. Hoffman. Anal. Chem. 31, No. 12, 1934 (1959).

3079. The heat capacity of sulfur from $25^{\circ}$ to $250^{\circ}$, the heats and temperatures of transition and fusion, E. D. West. J. Am. Chem. Soc. 81,29 (1959).

3080 . The inner solar corona during June 1959 , G. A. Newkirk, G. W. Curtis, D. K. Watson, R. Manning, and J. Shelby. Nature 184, 1308 (1959).

3081. The internal energy of highly ionized gases, J. Hilsenrath, M. S. Green, and C. W. Beckett. IXth Intern. Astronautical Congr. Proc. Amsterdam, Holland, 1958. Springer Verlag (Vienna, Austria) p. 120 (1959). 
3082. The magnetic interaction of $\mathrm{H}_{3}, \mathrm{~V}$. Griffing, J. L. Jackson, and B. J. Ransil. J. Chem. Phys. 30, 1066 (1959).

3083. The melting of crystalline polymers, L. Mandelkern. Rubber Chem. and Technol.

XXXII, No. 5, 1392 (1959).

3084. The night airglow, F. E. Roach. Proc. IRE 47, 267 (1959).

3085. The nova outburst. I. The nova outburst; II. The radiative cooling of an expanding shell, S. R. Pottasch. III. The ionization of hydrogen gas by an exciting star, J. Jefferies and S. R. Pottasch. IV. The intensity of $\mathrm{H} \alpha$; and V. The temperature and radius of the central exciting star and observation of elements other than hydrogen, S. R. Pottasch. Ann. Astrophys. J. $22,297,310,318,394$, and 412 (1959). 3086. The origin of the current nomenclature for the ionospheric layers, R. Silberstein. J. Atmospheric and Terrest. Phys. 13, 382 (1959). 3087. The peek-a-boo system, W. A. Wildhack and J. Steril. Chapter 6, Punched Cards, p. 125 (Reinhold Publ. Co., New York, N. Y.) (1959). 3088. The plasma jet as a spectroscopic source, J. Margoshes and B. F. Scribner. Spectrochim Acta XIV, No. 2, 138 (1959).

3089. The power output of a sound source in a reflecting environment, $R$. V. Waterhouse. Dissertation (Catholic University of America, Washington, D. C.) (1959).

3090. The present experimental status of rareearth spectra, W. F. Meggers. Opt. i Spekt 6, 429 (1959).

3091. The riometer--a device for the continuous measurement of ionospheric absorption, C. G. Little and $\mathrm{H}$. Leinbach. Proc. IRE 47, No. 2, 315 (1959).

3092. The spectral emittance of uncoated and ceramic-coated Inconel and type 321 stainless steel, J. C. Richmond and J. E. Stewart. NASA Memo 4-9-59W (1959).

3093. The stability of austenitic stainless steels at low temperatures as determined by magnetic measurements, R. P. Reed and R. P. Mikesell. Proc. 1958 Cryogenic Engineering Conference, Paper B-2, p. 84, Edited by K. D. Timmerhaus, Chemical Engineering Dept, , University of Colorado (Boulder, Colorado) (1959).

3094. The system magnesium oxide-germanium dioxide, C. R. Robbins and E. M. Levin. Am. J. Sci. 257,63 (1959).

3095. The tensile and impact strength of annealed and welded 5086 aluminum down to $20^{\circ} \mathrm{K}$, R. P. Mikesell and R. P. Reed. Proc. 1958 Cryogenic Engineering Conference, Paper B-3, p.101. Edited by K. D. Timmerhaus, Chemical Engineering Dept, University of Colorado (Boulder, Colorado) (1959). 3096. The theory of extreme values, J. Mandel. ASTM Bull. No. 236, 29 (1959); Tech. Eng. News 40, No. 7, 28 (1959).

3097. The very low-frequency emissions generated in the earth's exosphere, R. M. Gallet. Proc. IRE 47, 211 (1959).

3098. The weighted compounding of two independent significance tests, M. Zelen and L. S. Joel. Ann. Math. Stat. 30, No. 4, 885 (1959).
3099. Thermal decomposition of poly(vinyl chloride), R. R. Stromberg, S. Straus, and B. G. Achhammer. J. Polymer Sci. 35, No. 129, 355 (1959).

3100. Thermal degradation of polyethylene oxide and polypropylene oxide, S. L. Madorsky and S. Straus. J. Polymer Sci. 36, 183 (1959).

3100A. Unassigned.

3101. Total photoelectric cross sections of copper, molybdenum, silver, tantalum, and gold at $662 \mathrm{kev}$, W. F. Titus. Phys. Rev. 115, No. 2, 351 (1959).

3102. Transmission of power in radio propagation, J. R. Wait. Elec, and Radio Engr. 36, No. 4, 146 (1959).

3103. Trucks and trailers, C. W. Phillips. Vol. 1, Chapter 32, Am. Soc. Refrig. Engrs., Data Book, p. 32 (1959).

3104. Turbulent motion, G. B. Schubauer and C. M. Tchen. Section B. Vol. V, Princeton Series, High Speed Aerodynamics and Jet Propulsion, "Turbulent Flows and Heat Transfer", p. 75 (Princeton University Press, Princeton, N. J.) (1959).

3105. $U_{235}$ fission product decay spectra at various times after fission, A. Nelms and J. Cooper. Health Physics 1, 427 (1959).

3106. Un nouveau critere d'univalence des transformations dans un $\mathrm{R}^{\mathrm{n}}$, A. M. Ostrowski. Compt. Rend. Acad. Sci. 248, 348 (1959).

3107. Use of a direct current amplifier and recorder to balance a Mueller resistance bridge, G. T. Armstrong, P. K. Wong, and L. A. Krieger. Rev. Sci. Instr. 30, 339 (1959). 3108. Unassigned.

3109. Value of the Rydberg constant, W. C. Martin. Phys. Rev. 116, No. 3, 654 (1959).

3110. Variations of surface tension with body and surface concentrations, A. B. Bestul. J. Am. Ceram. Soc. 42, 236 (1959).

3111. Volcanoes on the moon, M. W. Windsor. Nature 184, 1556 (1959).

3112. Weighted restricted partitions, M. Newman. Acta Arith. V, 371 (1959).

3113. Wide-range cryostat temperature control, R. D. Goodwin. Proc. 1958 Cryogenic Engineering Conference, Paper H-3, p. 487. Edited by K. D. Timmerhaus, Chemical Engineering Dept., University of Colorado (Boulder, Colorado) (1959).

3114. Young's modulus of various refractory materials as a function of temperature, J. B. Wachtman, Jr., and D. G. Lam, Jr. J. Am. Ceram. Soc. 42, 254 (1959).

3115. Zeeman-split absorption lines as very narrow pass filters, K. G. Kessler and W. G. Schweitzer, Jr. J. Opt. Soc. Am, 49, 199 (1959). 


\section{PRICE LIST OF AVAILABLE PUBLICATIONS}

The following is a list of National Bureau of Standards publications issued from 1901 to date that may be purchased from the Superintendent of Documents, U.S. Government Printing Office, Washington 25, D.C., at the prices given. See "How To Purchase NBS Publications," page 1.

The tabulation brings up to date information given in Circular 460 and its printed Supplement, and should be referred to instead of Circular 460 and Supplement to determine if a given publication is still available.

National Bureau of Standards publications that are out of print are available for reference use in many of the leading scientific, technical, educational, and Government depository libraries.

\begin{tabular}{|c|c|c|c|c|c|}
\hline \multicolumn{6}{|c|}{ RESEARCH PAPERS } \\
\hline \multicolumn{2}{|l|}{ No. } & \multirow[t]{2}{*}{ Price } & \multicolumn{2}{|r|}{ No. } & \multirow{2}{*}{$\frac{\text { Price }}{\$ 0.20}$} \\
\hline 768 Superseded by C5 & & & \multicolumn{2}{|c|}{2279} & \\
\hline \multicolumn{6}{|c|}{ CIRCULARS } \\
\hline No. & Price & No. & Price & No. & Price \\
\hline $\begin{array}{l}8 \text { Superseded by C600 } \\
31 \text { (4th edition) } \\
47 \text { Superseded by } \mathrm{M} 1 \\
74 \\
142\end{array}$ & $\begin{array}{r}\$ 0.30 \\
1.25 \\
1.25 \\
.40\end{array}$ & $\begin{array}{l}461 \\
462 \\
465 \\
466 \text { Supersede } \\
467 \text { Volume I }\end{array}$ & \begin{tabular}{l|r} 
& $\$ 3.75$ \\
& 1.25 \\
by C559 & .30 \\
& 4.75
\end{tabular} & $\begin{array}{l}509 \text { Supplement } \\
510 \\
510 \text { Supplement No. } \\
510 \text { Supplement No.2 } \\
512\end{array}$ & $\begin{array}{r}\$ 0.50 \\
4.00 \\
3.25 \\
.35 \\
.20\end{array}$ \\
\hline $\begin{array}{l}328 \text { Superseded by } \mathrm{C} 5 \\
332 \text { Superseded by } \mathrm{C} 5 \\
376 \\
383 \text { Superseded by C4 } \\
389\end{array}$ & $24 \cdot 10$ & $\begin{array}{l}467 \text { Volume II } \\
467 \text { Volume II } \\
468 \\
469 \\
470\end{array}$ & $\begin{array}{r}4.00 \\
2.50 \\
.40 \\
.15 \\
.30\end{array}$ & $\begin{array}{l}514 \\
515 \\
516 \\
518\end{array}$ & $\begin{array}{r}.40 \\
.15 \\
.10 \\
.65\end{array}$ \\
\hline $\begin{array}{l}398 \text { Superseded by C5 } \\
402 \\
419 \\
422 \\
427\end{array}$ & $\begin{array}{r}52 \\
.05 \\
.10 \\
.15 \\
.10\end{array}$ & $\begin{array}{l}472 \\
474 \text { Supersede } \\
475 \\
477\end{array}$ & 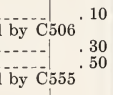 & $\begin{array}{l}519 \\
521 \\
523 \\
524\end{array}$ & $\begin{array}{l}\text { 2. } 00 \\
2.00 \\
1.50 \\
2.00 \\
2.25\end{array}$ \\
\hline $\begin{array}{l}428 \text { Superseded by C5 } \\
429 \\
430 \\
4334 \text { Superseded by C6 }\end{array}$ & $\begin{array}{r}33 \\
.20 \\
.10 \\
1.50 \\
02\end{array}$ & $\begin{array}{l}478 \text { Supersede } \\
484 \\
485\end{array}$ & by C509 & $\begin{array}{l}526 \\
528 \\
531 \\
532 \\
533 \ldots\end{array}$ & $\begin{array}{r}2.25 \\
\text { 1. } 50 \\
.35 \\
.15 \\
1.75\end{array}$ \\
\hline $\begin{array}{l}435 \text { Superseded by C4 } \\
440 \\
440 \text { Supplement } \\
441 \\
442\end{array}$ & $\begin{array}{r}66 \\
4.25 \\
.05 \\
.05 \\
.05\end{array}$ & $\begin{array}{l}488 \text { Section } 1 . \\
488 \text { Section } 2 \\
490 \\
492\end{array}$ & $\begin{array}{l}.55 \\
.70 \\
.25 \\
.15 \\
.10\end{array}$ & $\begin{array}{l}535 \\
536 \\
537 \\
538\end{array}$ & $\begin{array}{l}.35 \\
.20 \\
.20 \\
.15 \\
.45\end{array}$ \\
\hline $\begin{array}{l}443 \\
447 \\
450 \\
453\end{array}$ & $\begin{array}{r}.05 \\
3.50 \\
.10 \\
79.10\end{array}$ & $\begin{array}{l}495 \text { Supersede } \\
18 \\
497 \\
498 \\
501\end{array}$ & \begin{tabular}{|r|r|} 
by Mono. \\
\\
-15 \\
-5
\end{tabular} & $\begin{array}{l}539 \text { Volume }{ }^{2} \ldots \ldots \\
539 \text { Volume } 6 \ldots \ldots \\
539 \text { Volume } 7 \ldots \ldots \\
539 \text { Volume } 8 \ldots \\
539 \text { Volume } 9 . \ldots\end{array}$ & $\begin{array}{l}.45 \\
.40 \\
.40 \\
.45 \\
.40\end{array}$ \\
\hline $\begin{array}{l}454 \\
455 \\
459 \\
460 \\
460 \text { Supplement } 1 \text { - }\end{array}$ & $\begin{array}{r}.15 \\
.15 \\
.05 \\
\text { 1. } 25 \\
\text { 1. } 50\end{array}$ & $\begin{array}{l}503 \\
505 \\
506 \text { Supersede } \\
508 \text { Supersede } \\
509\end{array}$ & \begin{tabular}{l|l} 
& .05 \\
by $\mathrm{C} 576$ & .15 \\
by $\mathrm{C} 561$
\end{tabular} & $\begin{array}{l}540 \ldots \\
541 \\
542 \\
543 \\
545\end{array}$ & $\begin{array}{r}1.25 \\
.20 \\
.55 \\
.15 \\
.50\end{array}$ \\
\hline
\end{tabular}


CIRCULARS

\begin{tabular}{|c|c|c|c|c|c|}
\hline No. & Price & No. & Price & No. & Price \\
\hline 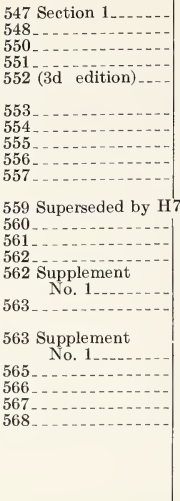 & $\begin{array}{r}\$ 0.35 \\
.40 \\
.35 \\
2.00 \\
.35 \\
2.00 \\
.30 \\
.10 \\
.50 \\
.30 \\
\\
.15 \\
.55 \\
.50 \\
.25 \\
.20 \\
\\
.10 \\
.30 \\
1.00 \\
.20\end{array}$ & $\begin{array}{l}569 \\
571 \\
572 \text { Superseded by } \\
\text { Mono. } 15 \\
573 \\
574 \\
575 \\
576 \\
577 \\
577 \text { Supplement } \\
578 \\
579 \\
580 \\
581 \\
582 \\
583 \text { Supplement } \\
584 \\
585 \\
586 \\
587 \\
588 \\
589 \\
590 \\
591 \text { Section } 1 \\
592\end{array}$ & $\begin{array}{r}.25 \\
.50 \\
.20 \\
.35 \\
.20 \\
.15 \\
.15 \\
.30 \\
.30 \\
.15 \\
3.00 \\
.15 \\
1.50 \\
3.25 \\
.15 \\
.20 \\
.50 \\
.20 \\
.15 \\
.10 \\
.50 \\
.20 \\
.20 \\
.60 \\
.30\end{array}$ & $\begin{array}{l}594 \ldots \\
595 \\
596 \\
597 \\
598 \\
599 \\
600 \\
601 \\
602 \ldots \\
603 \ldots\end{array}$ & $\begin{array}{r}\$ 0.35 \\
.40 \\
.30 \\
.20 \\
.15 \\
\\
.25 \\
.20 \\
.30 \\
.20 \\
.65\end{array}$ \\
\hline \multicolumn{6}{|c|}{ MONOGRAPHS } \\
\hline No. & Price & No. & Price & No. & Price \\
\hline 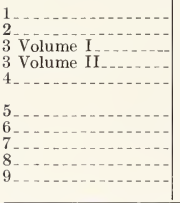 & $\begin{array}{r}\text { s0. } 45 \\
.25 \\
6.00 \\
6.00 \\
.45 \\
.20 \\
.20 \\
.25 \\
.40 \\
.15\end{array}$ & $\begin{array}{l}10 \ldots \\
11 \\
12\end{array} 1$ & $\begin{array}{r}\$ 0.20 \\
.20 \\
\text { 1. } 50 \\
\text { 1. } 50 \\
.15\end{array}$ & $\begin{array}{l}15 \ldots \\
16 \\
17\end{array}$ & $\begin{array}{r}\$ 0.15 \\
.20 \\
.65\end{array}$ \\
\hline
\end{tabular}


BUILDING MATERIALS AND STRUCTURES REPORTS

\begin{tabular}{|c|c|c|c|c|c|}
\hline No. & Price & No. & Price & No. & Price \\
\hline $\begin{array}{r}4 \\
10 \\
11 \\
17 \text { Superseded by } \\
\text { seded by BMS1 } \\
17 \text { supplem ent } 2 \text { S } \\
19 \text { seded by B BIS1 } \\
25 \\
39 \\
52 \\
56 \\
58 \\
64 \\
66 \\
67 \\
71 \\
73\end{array}$ & $\begin{array}{r}\text { s0. } 10 \\
.25 \\
15 \\
10 \\
10 \\
\text { MIS1 } \\
\text { uper- } \\
44 \\
\text { uper- } \\
44116 \\
.25 \\
.10 \\
.15 \\
.10 \\
.15 \\
.10 \\
.40 \\
.20 \\
.30 \\
.10 \\
.20 \\
.35 \\
.20 \\
.25 \\
.25 \\
30\end{array}$ & $\begin{array}{l}102 \ldots 5 \\
106 \\
110 \\
112 \\
114 \\
116 \\
117 \\
118 \\
121 \\
122 \\
124 \\
125 \\
126 \\
127 \\
128 \\
129 \\
130 \\
131 \\
132 \\
133 \\
135 \\
136\end{array}$ & $\begin{array}{r}80.15 \\
1.50 \\
.15 \\
.20 \\
.15 \\
.25 \\
.20 \\
.25 \\
.25 \\
.35 \\
.20 \\
.15 \\
.30 \\
.20 \\
.15 \\
.20 \\
.10 \\
.15 \\
.15 \\
.25 \\
.25 \\
.15 \\
.15 \\
.25\end{array}$ & $\begin{array}{l}140 \text { (3d edition) } \\
141 \\
142 \\
143 \\
144 \\
144 \text { Supplement } \\
144 \text { Supplement } 2 \\
146 \\
147 \\
148 \\
149 \\
150 \\
151 \\
152\end{array}$ & $\begin{array}{r}\$ 0.30 \\
.20 \\
.25 \\
.35 \\
.40 \\
.05 \\
.10 \\
.20 \\
.15 \\
.20 \\
.15 \\
.20 \\
.15 \\
.20 \\
.15\end{array}$ \\
\hline
\end{tabular}

1 Supplement No. 1, Dec. 20, 1940.

2 Supplement No. 2, Sept. 15,1947

APPLIED MATHEMATICS SERIES

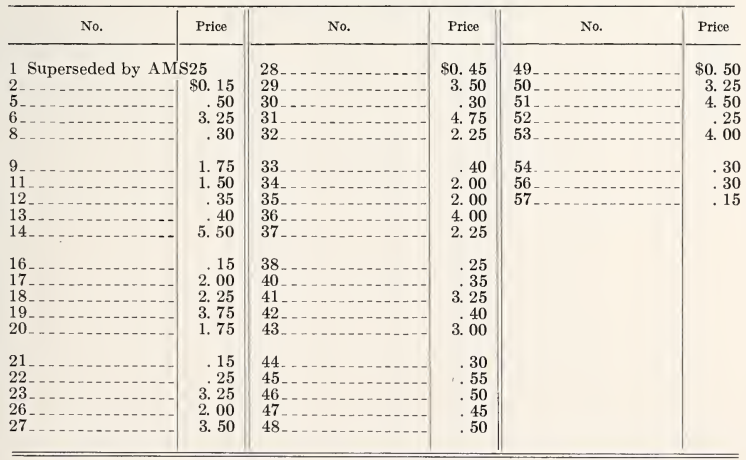


HANDBOOKS

\begin{tabular}{|c|c|c|c|c|c|}
\hline No. & Price & No. & Price & No. & Price \\
\hline 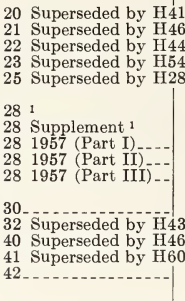 & $\begin{array}{r}1.25 \\
.75 \\
.60 \\
2.25\end{array}$ & $\begin{array}{l}44 \text { (2d edition) } \\
\text { looseleaf. } \\
47 \text { Superseded by H62 } \\
48 \\
49 \\
50\end{array}$ & $\begin{array}{r}\$ 1.00 \\
2 \quad .15 \\
.15 \\
.20 \\
9 \quad .20 \\
9.15 \\
.25 \\
.25 \\
\\
\\
.15 \\
.20 \\
.35 \\
.40\end{array}$ & $\begin{array}{l}64 \ldots \\
65 \\
66 \\
67 \\
68 \\
69 \\
71\end{array}$ & $\begin{array}{r}\$ 0.20 \\
.15 \\
.20 \\
.35 \\
1.00 \\
.35 \\
.25\end{array}$ \\
\hline
\end{tabular}

1 H28 and its 1950 supplement are superseded by H28 (1957), pts. I, II, and III.

MISCELLANEOUS PUBLICATIONS

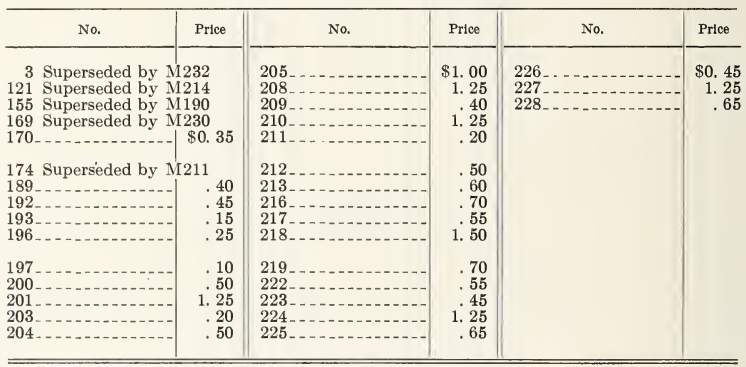

BUILDING AND HOUSING PUBLICATION

\begin{tabular}{|c|c|}
\hline No. & Price \\
\hline $13 \ldots$ & $\$ 1.50$ \\
\hline
\end{tabular}


The following is a list of the National Bureau of Standards Technical Note Series. This series is available only by purchase from the U.S. Department of Commerce, Office of Technical Services, Washington 25, D.C., at the prices indicated. The "PB" number in parenthesis is to be used when ordering this series.

\begin{tabular}{|c|c|c|c|}
\hline No. & Price & No. & Price \\
\hline $\begin{array}{l}\text { TN1 (PB151360) } \\
\text { TN2 (PB151361) } \\
\text { TN3 (PB151362) } \\
\text { TN4 (PB151363) } \ldots \\
\text { TN5 (PB151364) }\end{array}$ & $\begin{array}{r}\$ 0.75 \\
\text { 3. } 50 \\
.50 \\
.75 \\
\text { 1. } 25\end{array}$ & $\begin{array}{l}\text { TN31 (PB151390) } \\
\text { TN33 (PB151392) } \\
\text { TN34 (PB151393) } \\
\text { TN35 (PB151394) } \\
\text { TN36 (PB151395) }\end{array}$ & $\begin{array}{r}\$ 2.25 \\
2.50 \\
.50 \\
.75 \\
4.00\end{array}$ \\
\hline $\begin{array}{l}\text { TN6 (PB151365) } \\
\text { TN7 (PB151366) } \\
\text { TN8 (PB151367) } \\
\text { TN9 (PB151368) } \\
\text { TN10 (PB151369) }\end{array}$ & $\begin{array}{l}\text { 2. } 25 \\
\text { 2. } 25 \\
\text { 1. } 25 \\
\text {. } 75 \\
\text { 1. } 00\end{array}$ & $\begin{array}{l}\text { TN37 (PB151396) } \\
\text { TN38 (PB151397) } \\
\text { TN39 (PB151398) } \\
\text { TN40-1 (PB151399-1) } \\
\text { TN40-2 (PB151399-2) }\end{array}$ & $\begin{array}{r}.50 \\
.75 \\
.50 \\
\text { 1. } 25 \\
\text { 1. } 25\end{array}$ \\
\hline $\begin{array}{l}\text { TN11 }(\mathrm{PB} 151370) \\
\text { TN12 }(\mathrm{PB} 151371) \\
\text { TN13 (PB151372) } \\
\text { TN14 (PB151373) } \\
\text { TN15 (PB151374) }\end{array}$ & $\begin{array}{r}.50 \\
\text { 3. } 00 \\
\text { 2. } 50 \\
.50 \\
\text { 1. } 50\end{array}$ & $\begin{array}{l}\text { TN41 }(\text { PB151400) } \\
\text { TN42 (PB151401) } \\
\text { TN43 (PB151402) } \\
\text { TN44 (PB151403) } \\
\text { TN45 (PB151404) }\end{array}$ & $\begin{array}{l}\text { 2. } 25 \\
\text { 1. } 25 \\
\text { 2. } 25 \\
.50 \\
.50\end{array}$ \\
\hline $\begin{array}{l}\text { TN16 (PB151375) } \\
\text { TN18 (PB151377) } \\
\text { TN18-2 (PB151377-2) } \\
\text { TN19 (PB151368) } \\
\text { TN20 (PB151379) }\end{array}$ & $\begin{array}{l}\text { 2. } 75 \\
\text { 2. } 50 \\
\text { 4. } 00 \\
\text {. } 50 \\
\text { 1. } 00\end{array}$ & $\begin{array}{l}\text { TN46 (PB151405) } \\
\text { TN47 (PB151406) } \\
\text { TN48 (PB151407) } \\
\text { TN49 (PB151408) } \\
\text { TN50 (PB151409) }\end{array}$ & $\begin{array}{l}\text { 2. } 50 \\
\text { 2. } 50 \\
\text { 2. } 50 \\
\text { 1. } 00 \\
\text { 1. } 75\end{array}$ \\
\hline $\begin{array}{l}\text { TN21 }(\mathrm{PB} 151380) \\
\text { TN22 (PB151381) } \\
\text { TN23 (PB151382) } \\
\text { TN24 (PB151383) } \\
\text { TN25 (PB151384) }\end{array}$ & $\begin{array}{r}.75 \\
\text { 1. } 50 \\
.50 \\
\text { 1. } 00 \\
.50\end{array}$ & $\begin{array}{l}\text { TN51 (PB161552) } \\
\text { TN52 (PB161553) } \\
\text { TN53 (PB161554) } \\
\text { TN54 (PB161555) } \\
\text { TN55 (PB161556) }\end{array}$ & $\begin{array}{r}.50 \\
.50 \\
\text { 1. } 00 \\
.50 \\
\text { 1. } 00\end{array}$ \\
\hline $\begin{array}{l}\text { TN26 (PB161385) } \\
\text { TN27 (PB151386) } \\
\text { TN28 (PB151387) } \\
\text { TN29 (PB151388) } \\
\text { TN30 (PB151389) }\end{array}$ & $\begin{array}{r}\text { 1. } 00 \\
\text { 1. } 50 \\
\text { 2. } 00 \\
\text {. } 50 \\
\text { 1. } 25\end{array}$ & $\begin{array}{l}\text { TN56 (PB151557) } \\
\text { TN57 (PB161558) } \\
\text { TN58 (PB161559) } \ldots\end{array}$ & $\begin{array}{r}1.75 \\
.50 \\
.75\end{array}$ \\
\hline
\end{tabular}


Errata to Accompany National Bureau of Standards

Miscellaneous Publication 240, Publications

of the NBS, July 1, 1957 to June 30, 1960

HOW TO USE AUTHOR AND SUBJECT INDEXES

(Beginning on page 167)

Natjonal Bureau of Standards publications are 1dentified in the author and subject indexes by the series designation for papers published by the NBS (pages 11-72) and by assigned number for papers published in outside journals (pages 73-160). The following tables will assist in locaing publication listings referred to in the indexes:

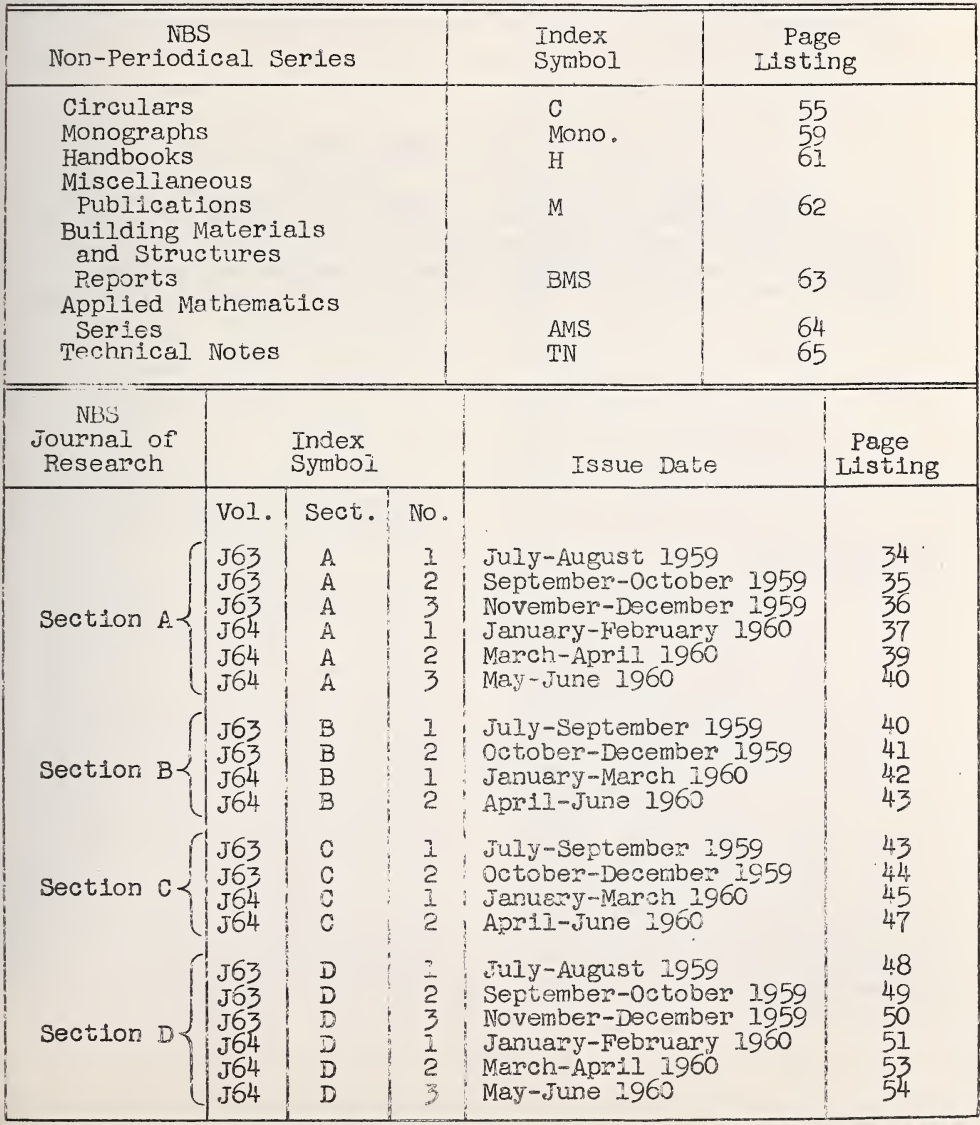




\begin{tabular}{|c|c|c|c|}
\hline Periodical & $\begin{array}{c}\text { Index } \\
\text { Symbol }\end{array}$ & Issue Date & $\begin{array}{c}\text { Page } \\
\text { Listing }\end{array}$ \\
\hline $\begin{array}{c}\text { Research } \\
\text { Papers* }\end{array}$ & RP & July 1957 to June 1959 & 11 \\
\hline
\end{tabular}

*This numbering series for Journal of Research papers was discontinued when the four-section Journal was established in July 1959.

Papers in Outside Journals

Author-index citations to papers published in outside journals (non-NBS) give the journal designation followed by the assigned number corresponding to the paper listing beginning on page 75. Subject-index citations to outside papers are designated by the assigned number only (page 75).

May 22, 1961 


\section{AUTHORS INDEX}

$\mathbf{A}$

Abbott, R., Bean, B. R., Oxygen and water vapor absorption of radio waves in the atmosphere. Geofis. pura e appl. (Milan, Italy) 37, 127 (1957). 2241.

Aboud, A. A., Powell, R. L., Electrical contact resistance of copper, copper functions at low temperatures. Rev. Sei. Instr. 29, 248 (1958). 2537.

Abramowitz, $M$., Coulomb wave functions expressed in terms of Bessel-Cliff ord and Bessel functions. J. Math. and Phys. 29, 303 (1951). 214.

Evaluation of the integral $\int_{0}^{\infty} e^{-u^{2}-(x / u)} d u$. J. Math. and Phys. 32, 188 (1953). 716.

Heat transfer in laminar flow. Abstracts of Short Communications and Scientific Programme, Internat tional Congress of Mathematicians (Edinburgh, Seotland) p. 135 (1958). 2579

Integral equations. Handbook of Physics, Edited by E. U. Condon and $\mathrm{H}$. Odishaw, Chapter 6, Pt. 1, p. 1-90 (McGraw-Hill Book Co., Inc., New York, N.Y.) (1958), 2500 .

On the practical evaluation of integrals. J. Soc. Indust. Appl. Math. 2, 20 (1954). 1098.

On the solution of the differential equation occuring in the problem of heat convection in laminar flow through a tube. J. Math. and Phys. 32, 184 (1953) 789.

Regular and irregular Coulomb wave functions expressed in terms of Bessel-Clifford functions. J Math. and Phys. 33, 111 (1954). 1127.

Table of the integral, $\int_{0}^{z} e^{->3} d u$. J. Math. and Phys. 30, No. 3, 162 (1951). 320.

Tables of interrals of Struve functions. J. Math. and Phys. 29, 49 (1950). 146.

Abramowitz, M., Antosiewicz, H. A., A representation for solutions of analytic systems of linear differential equations. J. Wash. Acad. Sci. 44, No. 12, 382 (1954). 932 .

Coulomb wave functions in the transition region. Phys. Rev. 96, No. 1, 75 (1954). 980.

Abramowitz, $M$., Cahill, $W$. $F$., On the vibration of a square clamped plate. J." Assoc. Computing Machinery 2, No. 3, 162 (1955). 1435.

Abramowitz, M., Cahill, W. F., Wade, C., Jr., Heat transfer in laminar flow through a tube. J 62, 101 (1959) RP2937.

Abramouitz, M., Rabinowitz, $P$., Evaluation of coulomb wave functions along the transition line. Phys Rev. 96, No. 1, $77(1954)$. 1018.

Abramowitz, M., Stegun, I. A., Generation of Bessel functions on high speed computers. Math. Tables and Other Aids to Computation 11, 255 (1957). 2140. Pitfalls in computation. J. Soc. Indust. Appl. Math. 4, 207 (1956). 1809 .

The generation of Coulomb wave functions by means of recurrence relations. Phys. Rev. 98, 1851 (1955). 1532.

Achenbach, P. R., Radiant glass heating panels. Heating and Ventilating 50, 83(1953). 806.

What is the heat pump? Parents Mag. 29, 56 (1954). 1227.

Achenbach, P. R., Coblentz, C. W., Design and performance of a portable infiltration meter. Heating, Piping, Air Conditioning 29, No. 7, 155 (1957); Trans.
Am. Soc. Heating and Air Conditioning Engrs., Paper 477 (1957). 2082.

Achenbach, P. R., Johnson, H. D., Winter, J. C., Phillips, C. W., Grimes, J. W., Heat transfer measurements on refrigerated-food trailers. U.S. Dept. of Agriculture, Agricultural Marketing Service (AMS-250), (June, 1958). 2580.

Achenbach, P. R., Phillips, C. W., Water demand rates for electric drinking-water coolers of the bubbler type. Refrig. Eng. (62, No. 12, 37 (1954). 1226.

Acherman, W. L., Lining, F. J., Peterson, J. M., Edwards, D. M., Chemical analysis of GR-S by complete solution procedures. Gross constituents in GR-S containing soap. Anal. Chem. 25, 1511 (1953). 666.

Achhammer, B. G., Polymer degradation studies by absorption and mass spectrometry. Anal. Chem. 24, No. 12, 1925. (1952) 515 .

Achhammer, B. G., Bersch, C. F., Stromberg, R. R., Structural changes in irradiated plastics films. SPE Tech. Paper 5, 5 (1959). 3056.

Achhammer, B. G., Harvey, M. R., Bersch, C. F., Heat and ultraviolet aging of poly(vinyl chloride).' J 60 , 481 (1958) RP2863.

Achhammer, B. G., Newman, S. B., Toner, S. D., Surface erosion of filled plastics. Modern Plastics 36, 135 (1958). 2792.

Achhammer, B. G., Reiney, M. J., Wall, L. A., Reinhart, $F$. $W$., Study of degradation of polystrene by means of mass spectrometry. J. Polymer Sci. 8, No. 5 , $555(1952)$. 557

Achhammer, B. G., Stromberg, R.R., Straus, S., Thermal decomposition of poly(vinyl chloride). J. Polymer Sci. 35, No. 129, 355 (1959). 3099.

Infrared spectra of thermally degraded poly (vinylchloride). J 60, 147 (1958) RP2832.

Achhammer, B. G., Tryon, M., Kline, G. M., Chemical structure and stability relationships in polymers. Kunststoffe combined with German Plastics Digest 49, No. 11, 600 (1959); Modern Plasties 37, No. 4, 131 (1959). 2910.

Acquista, N., Caul, H. J., Wall, L. A., Determination of the monomer content of polymethyl methacrylate. J. Am. Dental Assoc. 53, 56 (1956). 1673.

Acquista, N., Mann, D. E., Lide, D. R., Jr., The infrared spectrum of tertiary butyl fluoride. J. Mol. Spectroscopy 2,575 (1958), 2820.

Acquista, N., Mann, D. E., Plyler, E. K., The vibrational spectrum of bromotrifluoroethylene. J. Chem. Phys. 22, 1199 (1954). 1206.

The vibrational spectrum of chlorotrifluoroethylene. J. Chem. Phys. 21, 1949 (1953). 893.

Vibrational spectra of trifluoroethylene and trifluoroethylene-d. J. Chem. Phys, 22, 1586 (1954). 1224.

Acquista, N., Plyler, E. K., Infrared absorption of liquid water from 2 to 42 microns. J. Opt. Soc. Am. 44, 505 (1954). 1038.

Infrared measurements with a cesium iodide prism. J. Opt. Soc. Am. 43, 977 (1953). 742.

Infrared spectrometry with a cesium iodide prism. J. Opt. Soc. Am. 43, 212 (1953). 744.

Small grating spectrometer for the far infrared region. J. Chem. Phys. 23, 752 (1955). 1488.

The use of cesium iodide prisms to 50 mierons. $\mathrm{N}$. 3 del Supplemento al Vol. 2, Serie x, Il Nuovo Cimento, p. 629 (Bologna, Italy) (1955). 1551. Transmittance and reflectance of cesium iodide in the far infrared region. J. Opt. Soc. Am. 48, No. 9, 668 (1958). 2852. 
Acquista, N., Plyler, E. K., Mann, D. E., Vibrational spectrum of 1-fluoro-1-chloroethylene. J. Chem. Phys. 23, 2122 (1955). 1576.

Acton, F. S., Analvzing straight-line data. J. Chem. Education 30, 128 (1953). 652 .

Adachi, S., Impedance characteristics of a uniform current loop having a spherical core. J 64D3. 295 (1960).

Adams, L. H., Waxler, $R$. M., Temperature-induced stresses in solids of elementary shape. (1960) Mono. 2.

Adelman, D. M., Mong, L. Ex, Control of factors affeeting the reproducibility of mechanical properties of refractory semi-dry press specimens. J. Am. Ceram. Soe. 41, No. 7, $267(1958) .2501$.

Adelson, E., Rothman, S., Schwebel, A., Langdell, R. D., Adsorption of $\mathrm{C}^{14}$ dextran to human blood platelets and red blood cells in vitro. Vox Sanguinix 2, 104 (1957). 2011.

Adler, A. G., Taylor, D. F., Leihfritz, W. A., Physical properties of chromium cobalt alloys. J. Am. Dental Assoe. 56. 343 (1958), 2687.

Agmon, S., The relaxation method for linear inequalities. Can. J. Math. 6, 382 (1954). 1196

Agmon, S., Bers, L., The expansion theorem for pseudoanalytic functions. Proc. Am. Math. Soc. 3, No. 5, 757 (1952). 577.

Agranoff, B., Seliger, H. H., Improvements in liquid scintillators. Anal. Chem. 31, No. 9, 1607 (1959). 2964.

Agy, $V$. Geographic and temporal distrihution of polar blackouts. J. Geophys. Research 59, 499 (1954). 1024. Ionospheric effects produced by solar flare radiation. Phys. Rev, 102, 917 (1956). 1750.

Location of the auroral absorption zone. J. Geophys. Research 59, 267 (1954). 1054.

Motion of the storm-D region. Nature 173, 445 (1954). 1078 .

Polar blackout occurence patterns. J. Atmospheric and Terres. Phys. Special Suppl. 1957, Proc. of the Polar Atmosphere Symp. (Oslo, Norway) July 2-8 1956, Pt. II, 129 (1957). 2256.

Results of ionospheric drift measurements in the United States. J. Atmospheric and Terrest. Phys. Special Suppl. 1957, Proc. of the Polar Atmosphere Symp. (Oslo, Norway) July 2-8, 1956, Pt. II, 23 (1957). 2288.

A gy, $V$. Davies, $K$. Ionospheric investigations using the sweep-frequency pulse technique at oblique incidence. J 63D2, 151 (1959)

Agy, V., Davies, K., Salaman, R., An atlas of obliqueincidence ionograms. (1959) TN31 (PB151390).

Alber, $H . K .$, Macurdy, L. B., Benedetti-Pichler, A. A. Carmichael, H., Corwin, A. H., Fowler, R. M., Huffman, E. W. D., Kirk, P. L., Lashof, T. W., Terminology for describing the performance of analytical and other precise balances. Anal. Chem. 26, 1190 (1954). 1166.

Albers, J. R., Fano, U., McVoy, K. W., Interference of orbital and spin currents on bremsstrahlung and photoelectric effect. Phys. Rev. 116, No. 5, 1159 (1959), 2968.

Sauter theory of the photoelectric effect. Phys. Rev. 116, No, 5, 1147 (1959), 3040.

Albert, A. L., Miller, J.A., Mechanical tests on specimens from large aluminum-alloy forgings. Natl. Advisory Comm. Aeronaut. Tech. Note 3729 (1956). 1772.

Albrite, J. P., Shutts, R. E., Whitlock, M. B., Jr., Cook, R. K., Corliss, E. L. R., Burkhard, M. D. Research in normal threshold of hearing. A.M.A. Arch. Otolaryngol. 68, 194 (1958). 2735.

Aldrich, R., Marvin, R. S., Sack, H. S., The dynamic bulk viscosity of polyisobutylene. J. Appl. Phys. 25, No. 10, 1213 (1954), 1182

Alexander, $R$. L., Jr., The syntheses and infrared absorption spectra of some $m$-polyphenyls. J. Organic Chem. 21, 1464 (1956). 1951.

Alexander, R. L., Jr., Hellmann, M., Coyle, C. F., Separation of isomeric polyphenyls by adsorption chromatography. Anal. Chem. 30, 1206 (1958). 2752.
Alexander, S. N., An evaluation of electronic data processing equipment. Proc. Automatic Data Processing Cong. (Harvard Graduate School of Business Administration, Cambridge, Mass.) (1955). 1261.

Automation and future management information systems. Proc. 4th Military-Industry Packaging and Handling Symp. Navy Department, September 30 -October 2, 1958 , p. 23, Washington, D. C. (1958). 2467.

European experience with electronic computers. First Proc., Electronics in Management (American University, Washington D. C.) (1955). 1336.

Future developments of electronic data processing systems. Proc. 8th Annual Symp. Federal Govt. Accountants Assoc., November 17-18, 1958, The Federal Accountant VIII, No. 4, 28 (1959). 2955.

Machines for retrieving and correlating recorded information. Proc. Conf. on Practical Utilization of Practical Knowledge, (Western Reserve Univ. Press, Cleveland, Ohio, (1956). 1763.

Progress report on automatic data processing in Government. University of Michigan, Ann Arbor, Michigan (1956). 1822.

Survey of computer development and applications. Proc. Conf, on Sci. and Tech. for the Dean of Engineering, Purdue Univ. (Lafayette, Indiana) (1957). 2332.

Alexander, S. N., Greenwald, S., Haueter, R. D., SEAC. Proc. IRE 41, 1300 (1953). 820.

Alexander, S. N., McPherson, J. L., Horton, H. B., Glaser $E$., Information processing in social and industrial research. Sei. Monthly $\mathbf{7 6}, 100$ (1953). 741.

Alexander, S. N., Stevens, M. E., Comparative characteristics of medium-priced fully automatic computers for statistical applications. Proc. of First Conf. on the Design of Experiments in Army Research, Development and Testing. Office of Ordnance Research (Durham, N.C.) OORR 57-1 (June 1957). 2061

Alexander, S. N., Wegstein, J. H., Programming of scientific computers. Control Eng. 3, No. 5, 87 (1956). 1820.

Allen, $E, W$., Norton, $K . A$., The variation with frequency of the signal range of FM and television broadeasting stations. J. Brit. Inst. Radio Engr. 11, 93 (1951). 347.

Allen, $H . C$. Jr. Cross, P. C. Plyler, E. K., Blaine, L. R., Infrared spectrum of hydrogen sulfide from 2200$2800 \mathrm{~cm}^{-1}$. J. Chem. Phys. 24, No. 1, 35 (1956). 1741.

Allen, H. C., Jr., Plyler, E. K., Infrared spectrum of hydrogen sulfide, II. The $5100 \mathrm{~cm}^{-1}$ region. J. Chem. Phys. 22, 1104 (1954). 1040.

Infrared spectrum of hydrogen sulfide. J. Chem. Phys. 25, No. 6, 1132 (1956), 1740.

$\nu_{3}$ band of methane, J. Chem. Phys. 26, No. 4, 972 (1957). 2221.

Some vibrational-rotational bands of deuterated methanes. J 63A2, 145 (1959).

The infrared spectrum of hydrogen sulfide in the $5100 \mathrm{~cm}^{-1}$ region. J. Chem. Phys. 22, 1104 (1954). 1187.

The structure of ethylene from infrared spectra. J Am. Chem. Soc. 80, 2673 (1958). 2833.

Allen, H. C., Jr., Plyler, E. K., Blaine, L. R., Infrared spectrum of carbonyl sulfide. J. Chem. Phys. 26, No. 2,400 (1957). 2162.

Some infrared bands of deuterium sulfide. J 59, 211 (1957) RP2789.

The emission spectrum of $\mathrm{OH}$ from 2.8 to $4.1 \mu$. Spectrochem. Acta 9, 126 (1957) 2356.

The infrared spectrum and rotational constants of carbon disulfide. J. Am. Chem. Soc. 78, 4843 (1956). 1932.

Allen, H. C., Jr., Plyler, E. K., Tidwell, E. D., Emission spectrum of carbon monoxide from 2.3 to 2.5 microns. J 61, 53 (1958) RP2883.

Near infrared speetrum of nitrous oxide. J. Chem. Phys. 24, No. 1, 95 (1956). 1782 
Allen, H. C., Jr., Tidwell, E. D., Plyler, E. K., Infrared spectra of hydrogen cyanide and deuterium cyanide. J. Chem. Phys. 25, No. 2, 302 (1956). 1739.

The infrared spectrum of acetylene-d $d_{1}$. J. Am. Chem. Soc. 78, 3034 (1956). 1934.

Vibrational energy of deuterium cyanide, J. Chem. Phys. 23, No. 7, 1356 (1955), 1575.

Allison, L. M., Lowell, P. D., Hakkarinen, W., Mechanical stops for use with helical potentiometers. Rev. Sci. Instr. 25. No. 4. 398 (1954). 1070.

Allisy, A., DeLaVergne, L., and Wyckoff, H. O., An intercomparison of the French and U.S, roentgen ray standards, Acta Radiol. 48, No. 6, 486 (1957). 2017.

Allred, C. M., Chart for ' $\mathrm{TE}_{11}$ model piston attenuator. Electronics 26, 142, (1953); Electronics Buyer's Guide Issue 26, No. 6A, R8 (1953). 664 .

Allred, C. M., Hudson, P. A., A dry, static calorimeter for $\mathrm{RF}$ power measurement. IRE Trans. Instrumentation I- 7 , No. 3-4, 292 (1958). 2419.

Alt, F. L., Arithmetic. Handbook of Physics, Edited by E. U. Condon and H. Odishaw, Chapter 1, Pt. 1, p. 1-4 (McGraw-Hill Book Co., Inc., New York, N.Y.) (1958). 2500.

Boundary value problems for multiply connected domains. Proc. Assoc. Computing Machinery, Jointly Sponsored by the Assoc. for Computing Machinery and the Mellon Institute, Pittsburgh, Pennsylvania, May 2-3, 1952, p. 193 (Assoc. for Computing Machinery, New York, N.Y.) (1952). 404.

Computers. An item in The Encyclopedia of Chemistry Supplement, p. 91 (Reinhold Publ. Corp., New York, N.Y.) (1958). 2496.

Development of automatic computers. Appeared in "Electronics in Management," L. H. Hattery and G. P. Bush editors (The University Press of Washington, D.C.), p. 15 (1956). 1675.

Evaluation of automatic computing machines. Product Engr. 22, No. 11, 146 (1951). 233.

Ambler, E., Low temperature physics. Science 126, 1096 (1957). 2180.

Ambler, E., Brickwedde, F. G., Hudson, R. P., Cryogenics. Ann. Rev. Phys, Chem. 6, 25 (1955). 1301.

Ambler, E., Hayward, R. W., Hoppes, D. D., Hudson, R. P., Absence of interference effects in the $\beta$ decay of polarized $\mathrm{CO}^{50}$ and $\mathrm{CO}^{58}$ nuclei. Phys. Rev. 108, 503 (1957). 2006.

Beta-gamma correlations from polarized manganese52. Phys. Rev, 110, No. 3, 787 (1958); Proc. Intern. Congr. on Nuclear Phys. (Paris, France, July 1958) and Proc. Kamerlingh Conf. on Low Temp. Phys. Leiden, The Netherlands, Supplement of Physica. (June 1958). 2473.

Beta-gamma correlations from polarized nuclei. Proc. Congres Intern. de Physique Nucleaire, 1958, Interactions Faibles, Chapter III, p. 831 (Paris, France) (1959). 2902.

Experiments on the beta-decay of polarized nuclei. Proc. Kamerlingh Onnes Conference, Leiden, Holland, 1958; Physica (Supplement), 24, S64 (1958). 2557.

Ambler, E., Hayward, R. W., Hoppes, D. D., Hudson, $R$.'P., and $W u, C$.S., Further experiments on $\beta$ decay of polarized nuclei. Phys. Rev, 106, No. 6, 1361 (1957). 2132.

Ambler, E., Hudson, R. P., Absolute temperature below $1^{\circ} \mathrm{K}$ : chromic methylammonium alum as a thermometric substance. J. Chem. Phys. 27, 378 (1957). 2009.

Critical field phenomena in an isotropic paramagnetic crystal. Phys, Rev. 96, No. 4, 907 (1954). 981. Magnetic cooling. Published in Reports on Progress in Physics, Proc. British Phys. Soc. (London, England) 18, 251 (1955). 1395.

Magnitoye okhlazhdenye. Uzpekhi Physicheskhikh Nauk 47, 445 (1959). 2981.

Measurements of chromium potassium alum below $1^{\circ} \mathrm{K}$. Phys. Rev. 95, 1143 (1954). 1067.

Measurements on the saturation vapour pressure of liquid helium in the range $1.4^{\circ}-4.2^{\circ} \mathrm{K}$. Bull. Intern. Inst. Refrig., Commun, 147, 605 (1955). 1403.

New materials for magnetic cooling and thermometry below $1^{\circ} \mathrm{K}$. Phys, Rev. 102, 916 (1956). 1783.

Spin-spin absorption in chromic methylammonium alum and manganous chloride tetrahydrate at very low temperatures. Physica XXII, 866 (1956), 1887. Spin-spin absorption in manganous chloride tetrahydrate. Phys, Rev, 104, 1506 (1956), 1888.

Spin-spin relaxation at very low temperatures in chromic methylammonium alum. Phys. Rev. 104, 1500 (1956). 1889

Ambler, E., Hudson, R. P., Temmer, G. M., Alignment ofcerium $^{141}$ and neodynium ${ }^{147}$. Phys. Rev, 97, 1212 (1955). 1255.

Alignment of three odd-A rare-earth nuclei. Phys. Rev. 101, No. 1, 196 (1956). 1611.

Alignment of three rare-earth isotopes. Bull. Intern. Inst. Refrig., Commun. 142, 255 (1955). 1256.

Ambler, E., Wu, C. S., Hayward, R. W., Hoppes, D. D., Hudson, R. P., An experimental test of parity conservation in beta decay. Phys. Rev, 105, 1413 (1957). 2015.

Ambrosio, B. F., Huskey, H. D., Thorensen, R., Yowell, $E$. C., The SWAC-design features and operating experience. Proc. IRE 41, 1294 (1953). 885.

Amdur, I., Ross, J., On the calculation of properties of gases at elevated temperatures. Combustion and Flames 2, $412(1958) .2664$.

Amory, H. I., Smith, S. W., Military field X-ray equipment. Radiology 59, No. 6, 879 (1952). 483.

Anderson, $R$. K., Fifth-order aberration in an optical system. Proc. Computation Seminar (IBM) p. 130 (1951). 235.

Antkiw S., Dibeler, V. H., Mass spectrum of gallium vapor. J. Chem. Phys, 21, 1890 (1953). 758.

Antosiewicz, H. A., A survey of Lyapunov's second method. "Contributions to the theory of nonlinear oscillations." Vol. IV, ed. by S. Lefshetz, p. 141 (Annals of Mathematics Studies No. 141 (Princeton University Press, Princeton, New Jersey) (1958). 2439.

A theorem on alternatives for pairs of matrices. Pacific J. Math. 5, Suppl. 1, 641 (1955). 1246.

Analytic study of war games. Naval Reserve Logistics, Office of Naval Research (Washington, D.C.), 2, No. 3, 181 (1955), 1264.

On non-linear differential equations of the second order with integrable forcing term. J. London Math. Soc. 30, 65 (1955). 1429.

Stable systems of differential equations with integrable forcing term. J. London Math. Soc. 31, 208 (1956). 1893.

Antosiewicz, H. A., Abramowitz, M., A representation for solutions of analytic systems of linear differential equations. J. Wash. Acad. Sci. 44, No. 12, 382 (1954). 932 .

Coulomb wave functions in the transition region. Phys. Rev. 96, No. 1, 75 (1954). 980.

Antosiewicz, H. A., Cole, K. S., Rabinowitz, P., Automatic computation of nerve excitation. J. Soc. Indust. Appl. Math. 3, 153 (1955). 1272.

Antosiewicz, H. A., Davis, P., Some implications of Liapunov's conditions for stability. J. Rational Mechanics and Analysis 3, No. 4, 447 (1954): 1142. Antosiewicz, H. A., Hammersley, J. M., The convergence of numerical iteration. Am. Math. Mo. 60, 604 (1953). 856.

Antosiewicz, H. A., Hoffman, A. J., A remark on the smoothing problem. Management Sci. 1, No. 1, $92(1954)$. 931.

Appel, $W$. D., Accomplishments at the International Conference in Bournemouth, England, Part III. (Technical Committee on Textiles International Organization for Standardization (ISO) Meeting). Am. Dyestuff Reptr. 40, No. 23, 735 (1951). 188. International co-operation on standardization of textile testing methods. J. Home Econ. 44, No. 10, 773 (1952). 471 . 
International methods for testing colorfastness of textiles. Mag. of Standards 26, No. 2, 44 (1955). 1375.

International textile work shows fast progress. Mag. of Standards 28, 38 (1957). 2164.

Properties of fibers. Smithsonian Physical Tables, p. 241 (Smithsonian Institution, Washington, D.C.) (1954). 1121.

Standards help in the selection of sheets and pillowcases. Hospitals 24, 82 (1950). 136.

Synthetic textiles. Americana Annual 1, 697 (1953). 845; Encyclopedia Americana, p. 732 (1955). 1522. Test for dimensional changes in woven fabrics in high temperature laundering. Am. Dyestuff Reptr. 47, No. 7,213 (1958), 2802 .

Tests for colorfastness of textiles under consideration in the International Organization for Standardization. Am. Dyestuff Reptr. 42, 871 (1953). 850.

Textiles at the National Bureau of Standards. Proc. Am. Assoc. Textile Chemists Colorists 44, No, 23, 795 (1955). 1529.

Appel, W. D., Bogaty, H., Campbell, K. S., Some observations on the evaporation of water from cellulose. Textile Research J. 22, 75 (1952), 547.

The oxidation of cellulose by ozone in small concentration. Textile Research J. 22, 81 (1952). 589.

Appel, W. D., Schiefer, H. F., Krasny, J. F., Richey, G. G., Impact properties of yarns made from different fibers. Textile Research J. 23, 489 (1953). 734.

Armstrong, G. T., Brickwedde, F. G., Scott, R. B., The vapor pressures of the deuteromethanes. J. Chem. Phys. 21, 1297 (1953). 889.

Armstronq, G. T., Hawkins, J. E., The physical properties of terpenes. III. The vapor pressures of alpha pinene and beta pinene. J. Am. Chem. Soc. 76, 3756 (1954). $1191 \mathrm{~A}$.

Armstrong, G. T., Jessup, R. S., Combustion calorimetry with fluorine: constant pressure flame calorimetry. J 64A 1, 49 (1960).

Armstrong, G. T., Jessup, R. S., Mears, T. W., Net heat of combustion of aviation gasoline and its correlation with other properties. Chem. \& Eng. Data Series 3. 20 (1958). 2646.

Armstrong, G. T., Wong, P. K., Krieger, L. A., Use of a direct current amplifier and recorder to balance a Mueller resistance bridge. Rev. Sci. Instr. 30, 339 (1959). 3107.

Arnett, R. W., Mullen, L. O., Warren, K. A., Some methods for reducing heat lead through support members in liquefied gas storage vessels. Proc. 1958 Cryogenic Engineering Conference, Paper G-1, p. 410 . Edited by K. D. Timmerhaus, Chemical Engineering Dept., University of Colorado (Boulder, Colorado) (1959). 3049 .

Arnold, R. D., Wilson, R. E., Thermometry and pyrometry. Handbook of Physies, Edited by E. U. Condon and $\mathrm{H}$. Odishaw, Chapter 3, Pt. 4, p. 5-30 (McGraw-Hill Book Co., Inc., New York, N.Y.) (1958). 2500 .

Astin, A. V., A brief look at the history of automation. Signal 10, No. 4, 44 (1956). 1585.

A service for the Nation's technology. Mag. of Standards 28, No. 7, 196 (1957). 1999.

Instrumentation-where we stand. ASTM Bull. No. 232, 7 (1958). 2602.

Metrology and production. Ordnance 40, No. 216, $973(1956) .1777$.

Physical measurement and medicine. Med. Ann. District of Columbia 22, 639 (1953). 795.

Physical standards for the electronics industry. Signal 10, No. 5, 45 (1956), 1808.

Precise measurement and race for technological supremacy. Mag. of Standards 29, No. 2, 36 (1958). 2696.

Research is everybody's business. Tappi 41, No. 5, $14 \mathrm{~A}(1958) .2737$.

Scientists' and public's responsibility. Phys. Today 10, No. 11, 23 (1957). 2293.

Standards and measurements for electronics. IRE
Trans. Instrumentation I- 7 , No. 3-4, 134 (1958). 2776.

Standards for electronics. Proc. National Electronics Conference (Chicago, Ill.) 11, 1 (1955). 1508. Systematic research. Ordnance 43, No. 233, 741 (1959). 3060.

The engineer at the National Bureau of Standards. Am. Engr. 26, No. 9, 18 (1956). 1929.

Astin, A. V., Bullard, E. C., Lewis, W. B., International comparisons of radioactivity standards. Science 123, 895 (1956). 1746.

Aston, G. H., Attix, F. H., An intercomparison of the roentgen standards of Great Britain and USA. Acta Radiol. 46, 747 (1956). 1621.

Aston, G. H., Wyckoff, H. O., A comparison of X-ray standards. Acta Radiol. Suppl. 117, 17 (1955). 1230.

Aston, G.H., Wyckoff, H. O., Smith, E. E., A comparison of X-ray standards. British J. Radiol. 27, No. 318,325 (1954). 916.

Athay, $R$ G., A model of the chromosphere from radio and optical data. Paris Symp. Radio Astronomy (IAU Symp. No. 9 and URSI Symp. No. 1), paper 16, p. $98(1959)$. 2874 .

Athay, R. G., Thomas, R. N., On the use of the early Balmer lines to extend the photospheric model. Astrophys. J. 127, No. 1, 96 (1958). 2675.

Attix, F. H., Aston, G. H, An intercomparison of the roentgen standards of Great Britain and USA. Acta Radiol. 46, 747 (1956). 1621.

Attix, F. H., Costrell, L., Automatic timer simplifier small-current measurements. Nucleonics 15, No. 2, $83(1957) .2036$

Attix, F. H., DeLaVergne, L., Plate separation requirements for standard free-air ionization chambers. Radiology 63, No. 6, 853 (1954). 1110.

Attix, F. H., DeLaVergne, L., Ritz, V. H., Cavity ionization as a function of wall material. J 60, 235 (1958) RP2S42.

Attix, F. H., Ritz, V. H., A determination of the gammaray emission of radium. J 59, 293 (1957) RP2801. Attix, F. H., Schulman, J. H., Shurcliff, W., Ginther, R. J., Radiophotoluminescence dosimetry system of the U.S. Navy. Nucleonics 11, 52 (1953). 809.

Attix, $F, H$. Spencer, $L$. $V$. A cavity ionization theory including the effects of energetic secondary electron. Radiology 64, No. 1, 113 (1955). 1229.

A thenry of cavity ionization. Radiol. Research 3, 239 (1955). 1247.

Attix, F. H., Wyckoff, H. O., Design of free-air ionization chambers. (1957) H64.

Aumann, R. J., Kruskal, J. B., The coefficient in an allocation problem. Naval Research Logistics Quart. 5, 111 (1958). 2808.

Axilrod, B. M., Closure to discussion by Prof. Maxwell of "Stress and Strain at the Onset of Crazing of Polymethyl Methacrylate at Various Temperatures" by M. A. Sherman and B. M. Axilrod. ASTM Bull. No. 194, 61 (1953). 669.

Axilrod, B. M., Kline, G. M., Wolock, I., Sherman, M. A., George, D. A., Cohen, V., Development of craze and impact resistance in glazing plastics by multiaxial stretching. Natl. Advisory Comm. Aeronaut. Tech. Report 1290 (1957). 2084.

Axilrod, B. M., Sherman, $M$. A., Stress and strain at onset of crazing of polymethyl methacrylate at various temperatures. Natl. Advisory Comm. Aeronaut. Tech. Note 2778, (1952), 555.

Stress and strain at onset of crazing of polvmethyl methacrylate at various temperatures. ASTM Bull. No. 191,65 (1953). 838.

Stress-solvent crazing of acrylies. Modern Plastics 30, 130 (1953). 339.

Axilrod, B. M., Sherman, M. A., Cohen, V., Wolock, I., Effects of biaxial stretch-forming. Modern Plastics 30, 117 (1952). 434.

Effects of moderate biaxial stretch-forming on tensile and crazing properties of acrylic plastic glazing. 
Natl. Advisory Comm. Aeronaut. Tech. Note. 2779 (1952). 436

Axilrod, B. M., Wier, J. E., Pons, D. C., Effects of humidity during fabrication of polyester laminates. SPE J. \&, No. 9, 8 (1952). 435.

Effects of resin coating methods and other variables on physical properities of glass-fabric reinforced polyesters. Natl. Advisory Comm. Aeronaut. Research Memo. 54G26 (1954). 1004.

Axilrod, B. M., Wolock, I., Sherman, M. A., Biaxial stretch-forming of acrylics. Modern Plastics 31, 128 (1953). 658 .

Effects of high degrees of biaxial stretch-forming on crazing and other properties of acrylic plastic glazing. Natl. Advisory Comm. Aeronaut. Research Memo. 53D14 (1953). 699.

Effect of molecular weight on the crazing and tensile properities of polymethyl methacrylate. Natl. Advisory Comm, Aeronaut. Research Memo. 54A04 (1954). 999.

\section{B}

Bach, R. L., Hubbell, J, H., Lamkin, J. C., Radiation field from a rectangular source. J 64C2, 121 (1960).

Bagg, T. C., Stair, R., Johnston, R. G., Continuous measurement of atmospheric ozone by an automatic photoelectric method. Proc. Joint Army-Navy-Air Force Conf. on Elastomer Research and Devel., January 12-13, 1954 (Washington, D.C.), Natl. Acad. Sci.-Natl. Research Council Publ. 370, p. 75 (1954). 977 .

Bailey, $D$. K., The effect of multipath distortion on the choice of operating frequencies for high-frequency communication circuits. IRE Trans. Ant. Prop. AP-7, No. 4, 397 (1959). 3073.

The effect of echo on the operation of high-frequency communication circuits. IRE Trans. Ant. Prop. AP-6, No. 4, 325 (1958). 2810.

Bailey, D. K., Bateman, R., Berkner, L. V., Booker, $H$ G., Montgomery G. F., Purcell, E. M., Salisbury, $M$. W., Wiesner, J. B., A new kind of radio propagation at very high frequencies observable over long distances. Phys. Rev. 86, No. 2, 141 (1952). 369.

Bailey, D. K., Bateman, R., Kirby, R. C., Radio transmission at VHF by scattering and other processes in the lower ionosphere. Proc. IRE 43, No. 10, 1181 (1955). 1469.

Bailey, S. L., Smith, N., Condon, E. U., Everitt, W. L., Fink, D. G., The present status of color television; a report by the Senate Advisory Committee on Color Television. Proc. IRE 38, 980 (1950). 154.

Bain, K., Faegre, M. L., Wyly, R. S., Behavior of young children under conditions simulating entrapment in refrigerators. Pediatrics 22, No, 4, Pt. I, 628 (1958). 2471.

Bair, E. N., Sr., McKee, S. A., White, H. S., Lubrication of plain journal bearings. The Virginia Tech. Eng. 23, 26 (1950). 82.

Bcker, H. A., Lamb., J. J., George, D. A., Sieffert, L. E., Impact strength of some thermosetting plastics at low temperatures. ASTM Bull. No. 181, 67 (1952). 464.

Ball, A. F., Douglas, T. B., Ginnings, D. C., Davis, W. D., Heat capacity of potassium and three potassiumsodium alloys between $0^{\circ}$ and $800^{\circ}$, the triple point and heat of fusion of potassium. J. Am. Chem. Soc. 74, 2472 (1952). 462.

Ball, A. F., Douglas, T. B., Torgesen, J. L., Heat capacity of crystalline dextrose between $25^{\circ}$ and $95^{\circ} \mathrm{C}$. J. Am. Chem. Soc. 73, 1360 (1951). 246.

Ball, A. F., Ginnings, D. C., Douglas, T. B., Specific heat of beryllium between $0^{\circ}$ and $900^{\circ} \mathrm{C}$. J. Am. Chem. Soc. 73, 1236 (1951). 312.

Ball, J. J., Plyler, E. K., Filters for the infrared region. J. Opt. Soc. Am. 42, 269 (1952). 450.

The infrared emission spectra of $\mathrm{OH}, \mathrm{CO}$, and $\mathrm{CO}_{2}$ from $3 \mu$ to $5.5 \mu$. J. Chem. Phys. 20, 1178 (1952). 578.
Ballard, C. R., Bouche, R. R., A pretied tie for communication line wires. Conf. Paper No. CP58-266 presented at the Annual Meeting of Am. Inst. Elee. Engrs. (1958). 2432.

Ballard, C. R., Bouche. R. R., Lee, A. A., On, F. J., Windinduced vibration of telephone and distribution conductors. Am. Inst. Elec. Engrs. Middle Eastern District Meeting Conf. Paper (April 1958). 2868.

Ballard, D. B., Bennett, J. A., A magnetic specimen mount for fractography. Metal Prog. 74, No. 5, 114 (1958), 2424

Ballard, D. B., Mebs, R. W., Transmission of ultrasonic vibrations in metals. Wright Air Devel. Ctr. Tech. Rept. 56-457 (1957). 2398.

Band, W., Nelson, R. A., The vapor pressure of $\mathrm{He}^{3} \mathrm{He}^{4}$ mixtures. Phys, Rev, 88, No. 6, 1431 (1952). 599.

Banos, A., Jr., Golden, R., K., The electromagnetic field of rotating uniformly magnetized sphere. J. Appl. Phys. 23, No. 12, 1294 (1952). 575.

Barbrow, L. E., Wilson, S. W. Vertical distribution of light from gas-filled candlepower standards. Illum. Eng. 53, 645 (1958). 2860.

Barger, R. L., Kessler, K. G., Schweitzer, W. G., Jr., Atomic beam sources and standard of length. IRE Trans. Instrumentation 1-7, No. 3-4, 181 (1958). 2464.

Barghausen, A. F., Decker, M. T., Maloney, L. J., Measurements of correlation, height gain, and path antenna gain at $1046 \mathrm{Mc}$ on spaced antennas far beyond the radio horizon. IRE Conv. Record 3, Pt. 1, 78 (1955). 1401.

Barnes, J., Mockler, R. C., Beehler, R., Salazar, H., Fey, L., The ammonia maser as an atomic frequency and time standard. IRE Trans. Instrumentation I-7, No. 3-4, 201 (1958), 2804.

Barsis, A. P., Comparative $100 \mathrm{mc}$ measurements at distances far beyond the radio horizon. IRE Conv. Record, March 22-25, 1954, [2], Pt. 1, p. 98 (1954). 972 .

Some aspects of tropospheric radio wave propagation. IRE Trans. Broadeast Transmission Systems BTS-6, 1 (1956). 1872.

Barsis, A. P., Capps, F. M., Effect of super-refractive layers on tropospheric signal characteristics in the Pacific coast region. IRE Wescon Conv. Record 1, Pt. I, 116 (1957). 2100.

Barsis, A. P., McGavin, R. E., Report on on comparative $100 \mathrm{Mc}$ measurements for three transmitting antenna heights. IRE Trans. Ant. Prop., AP-4, 168 (1956), 1848.

Barsis, A. P., Norton, K. A., Rice, P. L., Janes, H. B., The rate of fading in propagation through a turbulent atmosphere. Proc. IRE 43, 1341 (1955). 1542.

Basri, S. A., A method for the dynamic determination of the elastic, dielectric, and piezoelectric constants of quartz. (1960) Mono, 9.

Bass, A. M., Benedict, W. S., A new infrared band system of FeO. Astrophys. J. 116, 652 (1952). 368.

Bass, A. M., Broida, H. P., Absorption spectra of solids condensed at low temperatures from electric discharges. J. Mol. Spectroscopy 2, 42 (1958). 2445. Effects of vibrational state on rotational intensity in distribution in OH. J. Chem. Phys. 21, 173 (1953). 701 .

Sharp line absorption in silica near $3400 \mathrm{~cm}^{-1}$. J. Opt. Soc. Am. 47, 163 (1957). 2297.

Spectra emitted from solid nitrogen condensed at $4.2^{\circ}$ $\mathrm{K}$ from a gas discharge. Phys. Rev. 101, No. 6, 1740 (1956). 1881.

Bass, A. M., Harvey, K. B., Infrared absorption of oxygen discharge products and ozone at $4^{\circ} \mathrm{K}$. J. Mol. Spectroscopy 2, 405 (1958). 2599.

Bass, A. M., Herzfeld, C. M., Frozen free radicals. Sci. American 3, 91, (1957). 2129.

Bass, A. M., Kostkowski, H. J., Slit function effects in the direct measurement of absorption line half-widths and intensities. J. Opt. Soc. Am. 46, No. 12, 1060 (1956). 1867. 
Bass, A. M., Kurzweg, U. H., Broida, H. P., Spectra of afterglows and discharges from nitrogen-oxygen mixtures. J. Mol. Spectroscopy 1, 184 (1957). 2307.

Bateman, R., Bailey, D. K., Berkner, L. V., Booker, H. G., Montgomery, G. F., Purcell, E. M., Salisbury, M. W., Wiesner, J. B., A new kind of radio propagation at very high frequencies observable over long distances. Phys. Rev, 86, No, 2, 141 (1952), 369.

Bateman, R., Bailey, D. K., Kirby, C., Radio transmission at $\mathrm{VHF}$ by scattering and other processes in the lower ionosphere. Proc. IRE 43, No. 10, 1181 (1955). 1469 .

Bateman, R., Finney, J. W., Smith, E. K., Tveten, L. H., Watts, J. M., IGY observations of F-layer scatter in the far east. J. Geophys. Research 64, 403 (1959). 2963.

Bateman, R., Florman, E. F., Tait, A., A source of error in radio phase measuring systems. Proc. IRE 38, $612(1950) .10$.

Bateman, R., Florman, E. F., Tait, A., Rechtin, E., A source of error in radio phase measuring systems. (Discussion), Proc. IRE 39, 436 (1951). 186.

Bates, $R$. G., Chemical education in Switzerland. Capital Chemist 4, No. 5, 150 (1954). 969.

Determination of dissociation constants in a mixture of two weak acids by electromotive force measurements. J. Am. Chem. Soc. 73, 2259 (1951). 218. Meaning and standardization of $\mathrm{pH}$ measurements. ASTM Symp. on $\mathrm{pH}$ Measurements, Am. Soc. Testing Materials Spec. Tech. Publ. No. 190 (1956). 1767.

Mold growth and the $\mathrm{pH}$ of tartrate buffer solutions. Anal. Chem. 23, 813 (1951). 262.

$\mathrm{pH}$ and the modern analyst. Anal. Chem. 29, 15A (1957). 2242A.

Bates, R. G., Bower, $V$. E., Alkaline solutions for $\mathrm{pH}$ control. Anal. Chem. 28, 1322 (1956). 1612.

Standards for $\mathrm{pH}$ measurements from $60^{\circ}$ to $95^{\circ} \mathrm{C}$. J 59, 261 (1957) RP2797.

Bates, R. G., Eden, $M$, Resolution of the dissociation constants of d, 1 -malic acid from $0^{\circ}$ to $50^{\circ} \mathrm{C}$. J 62 , 161 (1959) RP2947.

Bates, R. G., Guggenheim, E. A., Harned, H. S., Ives, D. J., Janz, G.J., Monk, C. B., Robinson, R. A., Sokes, R. H., Wynne-Jones, W. F. J., Standard electrode potential of the silver, silver chloride electrode. J. Chem. Phys. 25, 361 (1956). 1895.

Bates, R. G., Pinching, G. D., Dissociation constant of aqueous ammonia at $0^{\circ}$ to $50^{\circ}$ from EMF studies of the ammonium salt of a weak acid. J. Am. Chem. Soc. 72, $1393(1950), 49$.

Note on the strength of picric acid. Experientia 10, 482 (1954), 1085.

Triathanolamin als puffersubstanz. Helv. chim. acta (Basil, Switzerland) 37, 1439 (1954). 1217.

Uber pH-Werte nichtwässeriger Lösungen. Helv. chim. acta (Basil, Switzerland) 38, 699 (1955). 1563.

Bates, R. G., Schwarzenbach, $G$., Die bestimmung thermodynamischer aciditatskonstanten. Helv, chim. acta (Basil, Switzerland) 37, 1069 (1954). 991.

Bates, R. G. Wichers, E., Precise intercomparison of acids by differential potentiometric titration with hydrogen electrodes. J 59, 9 (1957) RP2769.

Precise intercomparison of acids by differential potentiometric titration with the hydrogen electrode. Actas do Congress (Lisboa, Portugal) 1, 3 (1957). 2260 .

Bavetta, L. A., Likins, R. C., Posner, A. S., Calcification in lysine deficiency. Arch. of Biochem. and Biophys. 70, 401 (1957). 2040.

Bay, Z., Millimicrosecond coincidence circuits. Nucleonics 14, No. 4, 56, (1956). 1779.

Techniques and theory of fast coincidence experiments. IRE Trans. Nuclear Science NS-3, No, 4, 12 (1956). 1911.

Bay, Z., Cleland, M.R., McLernon, F., Fast coincidences with Cerenkov counters. Phys. Rev. 87, 901 (1952). 447.
Bay, Z. Grisamore, $N$. T., High speed flip-flops for the millimicrosecond region. IRE Trans. Elec. Computers $\mathbf{E C}-5,3$ (1956). 1730.

Pulse generator and high-speed memory circuit. IRE Trans. Elec. Computers EC-5, No. 4, 213 (1956). 1827.

Bay, Z., Mann, W. B., Seliger, H. H., Wyckoff, H. O., Measurement of $\mathrm{W}_{\mathrm{B} \text { ir }}$ for sulfur-35 betarays. Radiol. Research 5, No. 4, Abstract 9 (1956). 1769.

Bay, Z., Newman, P. A., Comparison of the ionization produced in air by alpha particles near 5-Mev and by beta particles. Bull. Am. Phys. Soc. 11:4, No. 4, 217 (1959). 2912

Bay, Z., Seliger, H. H., Absolute measurement of $\mathrm{W}_{\text {ait }}$ for polonium-210 alpha particles. Radiation Research 9, No. 1, 90 (1958). 2444.

Collection of ions produced by alpha particles in air. Bull. Am. Phys. Soc. II, 3, No. 3, Session Y-9 (1958), 2488.

Bay, Z., Seliger, H. H., Wyckoff, $H$. O., Absolute measurement of $W_{\mathrm{air}}$ for sulfur-35 beta rays. Radiation Research 7, No. 6, 558 (1957). 2008.

Bazley, N. W., Montroll, E. W., Rubin, R. J., Shuler, $K$. E., Studies in nonequilibrium rate processes, III. The vibrational relaxation of a system of anharmonic oscillators. Errata in J. Chem. Phys. 28, 700 (1958), 2786

Bean, B. R., Climatology of ground-based radio ducts. J 63D 1, 29 (1959).

First meeting on radio climatology. Proc. IRE Letter 46, 1426 (1958). 2564.

Geographical and height distribution of the gradient of refractive index. Proc. IRE 41, 549 (1953). 727. Prolonged space-wave fadeouts at $1,046 \mathrm{Mc}$ observed in Cheyenne Mountain propogation program. Proc. IRE 42, No, 5, 848 (1954). 1119.

Some meteorological effects on scattered radio waves. IRE Trans. Commun. Systems CS-4, No. 1, 32 (1956). 1874.

Sur l'utilisation des observations meteorologiques courantes on propogation radioelectrique. L'Onde Electrique (Paris, France) 37, No. 362, 411 (1957). 2331. The atmospheric bending of radio waves. (Abstract) Congres International sur la Propagation des Ondes Radio-Electriques, October 6-11, 1958 (Liege, Belgium), Resume des Communications p. 3 (1958). 2806.

Utilizzazione delle osservazioni metearologiche correnti nella propagation radioelectrica. Post e tele comun. 25, 1120 (1957). 2405

Bean, B. R., Abbott, R., Oxygen and water vapor absorption of radio waves in the atmosphere. Genfis. pura e appl (Milan, Italy) 37, 127 (1957). 2241.

Bean, B. R., Cahoon, B. A., A note on the climatic variation of absolute humidity. Bull. Am. Meteorol. Soc. 38, No. 7, 395 (1957). 1991.

Effect of atmospheric horizontal inhomogeneity upon ray tracing. J 63D 3, 287 (1959).

The use of surface weather observation to predict the tntal atmospheric bending of radio rays at small elevation angles. Proc. IRE 45, 1545 (1957). 2388.

Bean, B. R. Cahoon, B. A., Thayer, G. D. Tables for the statistical prediction of radio ray bending and elevation angle error using surface values of the refractive index. (1960) TN44 (PB151403).

Bean, B. R., Dutton, E. J., On the calculation of the departures of radio wave bending from normal. J 64D 3, 259 (1960).

Bean, B. R., Horn, $J, T$. Radio-refractive-index climate near the ground. J 63D 3,259 (1959).

Bean, B. R., Meaney, $F$. M., The monthly refractive gradient for the United States and its application to predicting the geographical and annual trend of VHF transmission loss. Proc. Conf. Radio Meteorol. (The University of Texas, Austin, Texas) Supplement III-2 (1953). 870.

Bean, B. R., Riggs, L. P., Svnoptic variation of the radio refractive index. $J$ 63D 1,91 (1959). 
Bean, B. R., Riggs, L., P., Horn, J. D., Synoptic study of the vertical distribution of the radio refractive index. J 63D2, 249, (1959).

Bean, B. R.. Thayer, G. D., CRPL exponential reference atmosphere. (1959) Mono. 4.

Central Radio Propagation Laboratory exponential reference atmosphere. J 63D3, 315 (1959).

On models of the atmospheric radio refractive index. Proc. IRE 47, No. 5, 740 (1959). 3002.

Bean, H. S., A pound of gas? Gas 30, No. 12, 31 (1954). 927.

Correction factors for the balancing effect in one leg of a U-type manometer. Gas 27, 48 (1951). 211.

Bean, H. S., Morey, F. C., Correction factors for the balancing effect of a gas in one leg of a manometer. Instruments 24, 528; Oil and Gas J. 50, 11, 107 (1951). 212.

Bean, $L$., Head regulator for cutoff valve. Anal. Chem. 29, No. 6, 987 (1957). 2146.

The strontium oxide content of portland cements. ASTM Bull. No. 224, 42 (1957). 2382.

Bean, L., Diamond, J. J., Improvements in flame photometeric determination of sodium in portland cement. Anal. Chem. 25, 1825 (1953). 736.

Bean, L., Hackney, E. J., A study of some operations involved in cement analysis. ASTM Bull. No. 197, 43 (1954). 937.

Bean, L., Ingram, B. L., Removal of manganese prior to calcium and magnesium precipitations. Anal. Chem. 25, 1217 (1953). 814.

Bean, L., Tucker, N.J., A note on the oxyquinolate determination of magnesium oxide in cement. ASTM Bull. No. 201 (TP244), 62 (1954). 925.

Beardsley, J. H., A variable length re-entrant cavity for dielectric measurements from 100 to $400 \mathrm{Mc}$. Rev. Sci. Instr. 24, 180 (1953). 637.

Beaty, E. C., Bender, P. L., Narrow hyperfine absorption lines of $\mathrm{C}_{\mathrm{s}}{ }^{133}$ in various buffer gases. Phys. Rev. 112, No. 2, 450 (1958). 2643.

Beaty, E. C., Bender, P. L., Chi, A. R., Optical detection of narrow $\mathrm{Rb}^{87}$ hyperfine absorption lines. Phys. Rev. Letters 1, No. 9, 331 (1958), 2677.

Beatty, R. W., An adjustable sliding termination for rectangular waveguide. IRE Trans. Microwave Theory and Tech. MTT-3, 192 (1957). 2012.

Cascade-connected attenuators. Proc. IRE 38, 1190 (1950). 33.

Determination of attenuation from impedance measurements. Proc. IRE 38, 897 (1950). 45.

Beatty, $R . W .$, Engen $G, F$. Microwave attenuation measurements with accuracies from 0.0001 to 0.06 decible over a range of 0.01 to 50 decibles. J $64 \mathbf{C} 2$, 139 (1960).

Beatty, R. W., Huber, C. E., Lossy cable attenuator pads. Rev. Sci. Instr. 24, 1002 (1953). 754

Beatty, R. W., Kerns, D. M., Recently developed microwave impedance standards and methods of measurement. IRE Trans. Instrumentation I- $\gamma$, No. 3-4, 319 (1958). 2722.

Beatty, R. W., MacPherson, A. C., Mismatch errors in microwave power measurements. Proc. IRE 41, 1112 (1953). 769

Beatty, R. W., Reggia, F., Characteristics of the magnetic attenuator at UHF. Proc. IRE 41, 93 (1953). 663.

Beatty, R. W., Schafer, G. E., A method for measuring the directivity of directional couplers. IRE Trans. Microwave Theory and Tech. MTT-6, No. 4, 419 (1958). 2425.

Bechtoldt, C. J., Vacher, H. C., Maxwell, E., Structure of some iridium-osmium alloys. J. Metals 6, No. 1 , 80 (1954); Trans. Am. Inst. Mining Engrs. 200, 80 (1954). 1152.

Beckenbach, $E, F$., A class of mean value functions. Am. Math. Mo. 57, No. 1, 1 (1950). 1.

Metric differential geometry. Mathematies 23, No. $3,143(1950)$. 87.

On subharmonic, harmonic and linear functions of two variables. Revista Universidad Nacional del
Tucuman, Ser. A, Matematica y Fisica Teorica Tucuman, Argentina 8, No. 1-2, 7, Publ. No. 602 (1951). 280 .

Beckenbach, E. F., Seidel, W., Szasz, O., Recurrent determinants of Legendre and of ultraspherical polynomials. Duke Math. J. 18, 1 (1951). 298.

Becker, J. H., On the quality of gray tin crystals and their rate of growth. J. Appl. Phys. 29, No. 7, 1110 (1958). 2672.

Studies of crystal growth and electrical and optical properties of gray tin. Phys. Rev. 98, 1192A (1955). 1518 .

Becker, J. H., Blunt, R. F., Frederikse, H. P. R., Hosler, $W^{2}$. R., Electrical and optical properties of intermetallic compounds, III. Aluminum antimonide. Phys. Rev. 96, 578 (1954). 1007.

Becker, J. H., Breckenridge, R. G., Blunt, R. F., Hosler, W. R., Frederikse, H. P. R., Becker, J. H., Oshinsky, $W$., Electrical and optical properties of intermetallic compounds. I. Indium antimonide. Phys. Rev. 96, 571 (1954). 1007.

Beckers, A., Levedahl, W. J., Mechanism of autoignition in benzene-air mixtures. Ind. Eng. Chem. 48, 411 (1956). 1773.

Beckett, C. W., Fano, L., Hubbell, J. H., Compressibility factor, density, specific heat, enthalpy, entropy, free energy function, viscosity, and thermal conductivity of steam. Natl. Advisory Comm. Aeronaut. Tech. Note 3273 (1956). 1657.

Beckett, C. W., Green, M. S., Woolley, H. W., Thermochemistry and thermodynamics of substances. Ann. Rev. Phys. Chem, y, 287 (1956). 1963.

Beckett, C. W., Haar, L., Thermodynamic properties at high temperatures: Ideal gas thermal functions to 25,000 deg. $\mathrm{K}$ for diatomic molecules, oxygen, nitrogen, nitricocide, and their molecule ions. Proc. Conf. on Thermodynamic and Transport Properties of Fluids, July 10-12, 1957 (London, England) p. 27 (1957). 2394 .

Beckett, C. W., Hilsenrath, J., Green, M. S., The internal energy of highly ionized gases. IXth' Intern. Astronautical Congr. Proc. Amsterdam, Holland, 1958. Springer Verlag (Vienna, Austria), p. 120 (1959). 3081 .

Beehler, R., Mockler, R. C., Barnes, J., Salazar, H., Fey, $L$., The ammonia maser as an atomic frequency and time standard. IRE Trans. Instrumentation I- $\mathbf{\gamma}$, No. 3-4, 201 (1958). 2804.

Beery, W. M., Crichlow, W. Q., Roubique, C. J., Spaulding, A. D., Determination of the amplitude-probability distribution of atmospheric radio noise from statistical moments. J 64D 1, 49 (1960).

Behrent, L. F., Application of RF micropotentiometers for calibration of signal generators to $1000 \mathrm{Mc}$ (1960). TN37 (PB151396).

Behrent, L. F., Selby, M. C., Ries, F. X., RF voltmeter calibrating consoles. IRE Natl. Conv. Record 6, Pt. 5, 251 (1958). 2747.

Beitchman, B. D., Effects of antioxidants on asphalt durability. J 64C1, 13 (1960). Infrared spectra of asphalts. J 63A2, 189 (1959).

Bekkedahl, N., Natural and synthetic rubbers. Anal. Chem. 22, 253 (1950). 94; 23, 243 (1951). 264. Anal. Chem, 24, 279 (1952). 487; Anal. Chem. 25, 54 (1953). 772.

Polymer structure research at the National Bureau of Standards. Revue General de Caoutchoue (Paris, France) 33, 1129 (1956). 1813. Symposium on recent developments in the evaluation of natural rubber-Introduction. Am. Soc. Testing Materials Spec. Tech. Publ. No. 136, p. 1 (1953). 844.

Bekkedahl, N., Fanning, R. J., Quantitative determination of natural rubber hydrocarbon by refractive index measurements. Anal. Chem. 23, 1653 (1951). 292.

Bekkedahl, N., Seaman, R. G., The ASTM crude natural rubber subcommittee. Rubber World 189, 693 (1959). 3065 . 
Bekkedahl, N., Tryon, $M$., Natural and synthetic rubber. Biennial review of physical and chemical test methods. Anal. Chem. 27, 589 (1955). 1412A.

Belcher, H. V., Bestul, A. B., Degradation of polyisobutylenes on shearing in solution. J. Appl. Phys. 24, 1011 (1953). 682.

Flow behavior of concentrated GR-S rubber solutions. J. Colloid Sci. 5, 303 (1950). 58.

Temperature coefficients non-Newtonian viscosity at fixed shearing stress and at fixed rate of shear. J. Appl. Phys. 24, 696 (1953). 849

Belcher, H. V., Bestul, A. B., Ouinn, F. A., Jr., Bryant, C. B., Viscosities of 0.25 to 90 percent GR-S rubber solutions. J. Phys. Chem. 56, 432 (1952). 621.

Belcher, H. V., White H. S., Effects of capillary shape on flow characteristics and degradation of polymer solutions. J 60, 215 (1958) RP2839.

Belcher, H. V., White, H. S., Swindells, J. F., Oil-soaked felt-pad lubrication of ball bearings at high speed and high temperature. Lubrication Eng. 11, 182 (1955), 1422

Bell, E. E., Burstein, E., Davisson, J. W., Turner, W. J., Lipson, H. G., Infrared photoconductivity due to neutral impurities in germanium. Phys. Rev. 93, 65 (1954). 1039 .

Bell, R. K., Bendigo, B. B., Separation and determination of small quantities of aluminum in steel. J $64 \mathbf{A} 3$, $235(1960)$.

Bellman, R., Hoffman, A. J., On a theorem of Ostrowski and Taussky. Archiv der Mathematic 5, 123 (1954). 1091

Bender, B. K., Goldman, A. J., Analytic comparison of suggested configurations for automatic mail sorting equipment. J 63B2, 83 (1959).

Capacity requirement of a mail sorting device. J 62, 171 (1959) RP2948

Bender, C. P., Furukawa, G. T., Hyndman, J. R., Vapor pressure of benzene above $100^{\circ} \mathrm{C}$. Ind. Eng. Chem. 44, 387 (1952). 616 .

Bender, $P$. L. Diffusion of particles with memory. Proc. Natl. Acad. Sci. 43, No. 5, 412 (1957). 2087.

Bender, P. L., Beaty, E. C., Narrow hyperfine absorption lines of $\mathrm{C}_{8}{ }^{133}$ in various buffer gases. Phys. Rev. 112, No. 2, 450 (1958). 2643.

Bender, P. L., Beaty, E. C., Chi, A. R., Optical detection of narrow $\mathrm{Rb}^{87}$ hyperfine absorption lines. Phys. Rev. Letters 1, No, 9, 331 (1958). 2677.

Bender, P. L., Driscoll, R. L., A free precession determination of the proton gyromagnetic ratio. IRE Trans. Instrumentation I-7, No. 3-4, 176 (1958). 2422.

Proton gyromagnetic ratio. Phys. Rev. Letters 1 No. 11,413 (1958). 2706.

Bendigo, B. B., Bell, R. K., Separation and determination of small quantities of aluminum in steel. J $\mathbf{6 4 A 3}$, 235 (1960).

Benedetti-Pichler, A. A., Macurdy, L. B., Alber, H. K., Carmichael, H., Corwin, A. H., Fowler, R. M., Huffman, E. W. D., Kirk, $P$. I., Lashof, T. W., Terminology for describing the performance of analytical and other precise balances. Anal. Chem. 26, 1190 (1954). 1166.

Benedict, $W$. S., Bass, A. M., A new infrared band system of FeO. Astrophys. J.116, 652 (1952). 368.

Benedict, W. S., Gailar, N., Plyler, E. K., The rotationvibration spectra of deuterated water vapor. J. Chem. Phys. 24, No. 6, 1139 (1956). 1949.

The vibration-rotation spectrum of $\mathrm{D}_{2} \mathrm{O}$. J. Chem. Phys. 21, 1301 (1953). 891.

The vibration-rotation spectrum of HDO. J. Chem. Phys. 21, 1302 (1953). 892.

Benedict, W. S., Herman, R. C., Silverman, S., R-branch heads of some $\mathrm{CO}_{2}$ infrared bands in the $\mathrm{CO}+\mathrm{O}_{2}$ flame spectrum. J. Chem. Phys. 19, 1325 (1951). 293.

Benedict, W. S., Plyler, E. K., Energy transfer in hot gases. Reprint of "High-resolution spectra of hydrocarbon flames in the infrared" from Symp. at Washington, D.C. (1954). 1016.
Interaction of stretching vibrations and inversion in ammonia. J, Chem. Phys. 24, No. 4, 904 (1956). 1744

Vibration-rotation bands of ammonia. II. The molecular dimensions and harmonic frequencies of ammonia and deuterated ammonia. Can. J. Phys. 35, 1235 (1957). 2411.

Rotation-vibration spectra of deuterated water vapor. J. Chem. Phys. 24, 1139 (1956). 1859.

Benedict, W. S., Plyler, E. K., Humphreys, C. J., The emission spectrum of $\mathrm{OH}$ from 1.4 to $1.7 \mu$. J. Chem. Phys. 21, 398 (1953). 860.

Benedict, W. S., Plyler, E. K., Silverman, J., Precise measurements in the infrared spectrum of carbon monoxide. J. Chem. Phys. 20, 175 (1952). 516.

Benedict, W. S., Plyler, E. K., Tidwell, E. D., Vibrationrotation bands of ammonia: $I$. The combination bands $\nu_{2}-\left(\nu_{1}, \nu_{3}\right)$. J 61, 123 (1958) RP2894.

Vibration-rotation bands of ammonia, III. The region 3.2-4.3 microns. J. Chem. Phys. 29, 829 (1958). 2861.

Benedict, W. S., Woolley, W. H., Generalized tables of corrections to thermodynamic properties for nonpolar gases. Natl. Advisory Comm. Aeronaut. Tech. Note 3272 (1956), 1721 .

Bennett, J.A., Effect of an anodic (HAE) coating on the fatigue strength of magnesium alloy specimens. Am. Soc. Testing Materials Proc. 55, 1015 (1955). 1325. The distinction between initiation and propagation of a fatigue crack. Intern. Conf. on Fatigue of Metals, Inst. Mech. Engrs. (London, England) (1956). 1925. The effect of an anodic (HAE) coating on the fatigue strength of magnesium alloy specimens. ASTM Bull. No. 55, 1015 (1955). 1531.

Bennett, J. A., Ballard, D. B., A magnetic specimen mount for fractography. Metal Progr. 74, No. 5, 114 (1958). 2424 .

Bennett, J. A., Frankel, H. E., Understressing as a means of eliminating the damaging effect of fatigue stressing. ASTM Bull. 55, 891 (1955) 1566.

Bennett, J. A., Frankel, H. E., Holshouser, W. L., Effect of oleophobic films on metal fatigue. J 64C2, 147 (1960).

Bennett, J. A., Frankel, H. E., Pennington, W. A., Fatigue properties of high strength steels. Trans. Am. Soc. Metals 52 (1959). 2951.

Bennett, J. A., Holshouser, $W$. L., Mechanical failures of metals in service. Eng. Materials Handbook 36, 2 (1958). 2629.

Bennett, J. A., Weinberg, J. G., A torie bending specimen for investigation of geometrical factors in fatigue. ASTM Bull. No. 234, 53 (1958). 2442.

Effect of crystal orientation of fatique-crack initiation in polycrvstalline aluminum alloys. Natl. Advisory Comm. Aeronaut. Tech. Note 3990 (1957). 2096.

Bennett, L. H., Johnson, $V$. M., Resistance diode bridge circuit for temperature control. (1959) TN34 (PB151393). Bennett, $W . H$., Radio frequency mass spectrometer. J. Appl. Phys. 21, 2 (1950). 117.

Berendt, R. D., Cook, R. K., Waterhouse, R. V., Edelman, $S$, Thompson, $M$. C., Jr., Measurement of correlation coefficients in reverberant sound fields. J. Acoust. Soc. Am. 27, No. 6, 1072 (1955). 1399.

Berendt, R. D., Waterhouse, R. V., Reverberation chamber study" of the sound power output of subsonic air jets. J. Acoust. Soc. Am, 30, 114 (1958). 2742.

Berendt, R. D., Waterhouse, R. V., Cook, R. K., Measurements on sound absorbers for jet-engine test cells. J. Acoust. Soc. Am. 28, No. 4, 688 (1956). 1771. Sound insulation of wall, floor, and door constructions. (1958) BMS144, 2d Suppl.

Berger, E. L., Hefley, G., Doherty, R. H., An intermittentaction camera with absolute time calibration. J 64C2, 159 (1960).

Berger, $M . J$. An application of the Monte Carlo method to a problem in gamma-ray diffusion. Symp. Monte Carlo Methods (John Wiley \& Sons, Inc., New York, N.Y.) (1956). 1618.

Angular distribution of multiple-scattered gamma 
radiation from a plane isotropic source. J. Appl. Phys, 26, No. 12, 1504 (1955). 1265.

Calculation of energy dissipation by gamma radiation near the interface between two media. J. Appl. Phys. 28, No. 12, 1502 (1957). 2043.

Effects of boundary and inhomogeneities on the penetration of gamma radiation. USNRDL Revs. and Lectures 1, No. 29, 47 (1957), 2102.

Monte Carlo calculations of gamma-ray backscattering and transmission. Radiation Research 1, No. 5, 489 (1954). 1076.

Eerger, M.J., Cooper, J. W., Reflection of fast neutrons from water. J 63A2, 101 (1959).

Berger, M.J., Doggett, J. A., Response function of NaI (Tl) scintillation counters. Rev. Sci. Instr. 27, No. 5, 269 (1956). 1856.

Berger, M. J., Hubbell, J. H., Reingold, I., Contribution of the annihilation radiation to the gamma-ray flux in lead. Phys. Rev. 113, No. 3, 857 (1959). 2917.

Berger, M. J., Lamkin, J. C., Sample calculations of gamma-ray penetration into shelters: contributions of sky shine and roof contamination. J 60, 109 (1958) $\mathrm{RP} 2827$.

Berger, M.J., Spencer, L. V., General relations between fluxes from collimated point and plane sources of radiation. Phys. Rev. 113, No. 2, 408 (1959). 2957. Penetration of gamma rays from isotropic sources through aluminum and concrete. (1959) TN11 (PB151370).

Some radiological applications of gamma-ray transport theory. Radiation Research 10, No. 5, 552 (1959). 3051

Berkner, L. V., Dailey, D. K., Bateman, R., Booker, H. G., Montgomery, G. F., Purcell, E. M., Salisbury, $M$. W., Wiesner, J.B., A new kind of radio propagation at very high frequencies observable over long distances. Phys. Rev. 86, No. 2, 141 (1952). 369 .

Berl, W. G., Gayhart, E. L., Olsen, H. L., Broida, H. P., Schuler, K. E., Spectroscopic observations on pentaborane-air flames and explosions. J. Chem. Phys. 25, No. 4, 797 (1956). 1883.

Berman, H. A., An improved 8-hydroxyquinoline method for the determination of magnesium oxide in portland cement. ASTM Bull. No. 237, 51 (1959). 2893.

Bers, L., Agmon, S., The expansion theorem for pseudoanalytic functions. Proc. Am. Math. Soc. 3, No. 5, 757 (1952). 577 .

Bersch, C. F., Harvey, M. R., Achhammer, B. G., Heat and ultraviolet aging of poly(vinyl chloride). J 60, 481 (1958) RP2863.

Bersch, C. F., McCrackin, F. L., Creep behavior of transparent plastics at elevated temperatures. SPE Tech. Paper 5, 94 (1959). 2921.

Bersch, C. F., McCrackin, F. L., Plitt, K. F., The effects of rates of straining on some tensile properties of polyester film. Modern Plastics 35, 171 (1957). 2354.

Bersch, C. F., Stromberg, R. R., Achhammer, B. G., Structural changes in irradiated plastics films. SPE Tech. Paper 5, 5 (1959). 3056.

Bestul, A. B., Capillary viscometry. J. Soc. Cosmetic Chemists 8, No. 6, 345 (1957). 2047A.

Composition of apparent shearing forces during shear degradation of polymers. J. Appl. Phys. 25, 1069 (1954). 976.

Kinetics of capillary shear degradation in concentrated polymer solutions. J. Chem. Phys. 24, No. 6, 1196 (1956). 1753.

Variations of surface tension with body and surface concentrations. J. Am. Ceram. Soc. 42, 236 (1959). 3110 .

Bestul, A. B., Belcher, H. V., Degradation of polyisobutylenes on shearing in solution. J. Appl. Phys. 24, 1011 (1953). 682.

Flow behavior of concentrated GR-S rubber solutions. J. Colloid Sci. 5, 303 (1950). 58 .

Temperature coefficients of non-Newtonian viscosity at fixed shearing stress and at fixed rate of shear. J. Appl. Phys. 24, 696 (1953). 849.
Bestul, A. B., Belcher, H. V., Quinn, F. A., Jr., Bryant, C. $B$., Viscosities of 0.25 to 90 percent GR-S rubber solutions. J. Phys. Chem. 56, 432 (1952). 621.

Bestul, A. B., Goodman, P., Temperature dependence of mechanical shear degradation. J. Polymer Sci. 18, No. 88, 235 (1955). 1527.

Bestul, A. B., Shartsis, L., Shermer, H. F., Immiscibility and surface tension of some simple borates. J. Am. Ceram. Soc. 41, 507 (1958). 2592.

Surface tension of alkaline-earth borates containing 3 mole percent of potassium oxide. J. Am. Ceram. Soc. 42, 242 (1959). 3059.

Biagi, $F$. Cook, R. K., Acoustic impedance of a right circular cylindrical enclosure. J. Acoust. Soc. Am. 26, No. 4, 506 (1954). 941.

Biagi, F., Simmons, B., Pressure calibration of condenser microphones above $10,000 \mathrm{cps}$. J. Acoust. Soc. Am. 26, 693 (1954). 1114 .

Bienenstock, A., Posner, A. S., Block, S., Radial distribution study of vitreous barium borate. J 64A3, 229 (1960).

Bilbo, A. J., Hellmann, M., The preparation of two fluorinated $p$-dihalobenzenes. J. Am. Chem. Soc. 75, 4590 (1953). 879.

Bilbo, A. J., Hellmann, M., Pummer, W. J., Synthesis and properties of fluorinated polyphenyls. J. Am. Chem. Soc. 77,3650 (1955). 1521.

Bilbo, A. J., Wyman, G. M., Steric hinderance to coplanarity in 0 -fluorobenzidines. J. Am. Chem. Soc. 75, 5312 (1953). 837.

Billings, D. E., Distribution of matter with temperature in the emission corona. Astrophys. J. 130, No. 3, 961 (1959). 2930.

Billings, D. E., Leighton, H. I., Solar $\mathrm{H} \alpha$ filaments and geomagnetic disturbances. J. Atmospheric and Terrest. Phys. 7,349 (1955). 1489.

Birks, L. S., Swerdlow, M., Dalton, A. J., Electron microscopy. Anal. Chem. 28, No. 4, Pt. II, 597 (1956). 1703 .

Birmingham, B. W., A mechanical refrigeration process for the no-loss storage of liquid hydrogen. Refrig. Eng. 65, No. 42-44, 86 (1957). 1986.

Some aspects of the large scale liquefaction of hydrogen. Proc. Instr. Soc. Am. Paper 55-2-1, 10, Pt. 2, 1 (1955). 1491.

Birmingham, B. W., Chelton, D. B., Dean, J. W., Design and construction of a liquid hydrogen temperature refrigeration system. (1960) TN38 (PB151397).

Birmingham, B. W., Chelton, D. B., Dean, J. W., Strobridge, T. R., Mann, D. B., Helium refrigeration and liquefaction using a liquid hydrogen refrigerator for precooling. (1960) TN39 (PB151398).

Birmingham, B. W., Mann, D. B., United States planning for the large scale liquefaction and transportation of helium. Physica 24, S144 (1958). 2855A.

Birmingham, B. W., Mann, D. B., Vander Arend, P. C., Technical aspects of large scale liquid helium liquefaction and transportation. Proc. 1957 Cryogenic Engineering Conference, Paper D-4, p. 191, Edited by K. D. Timmerhaus, Chemical Engineering Dept., University of Colorado (Boulder, Colorado) (1958). 2797.

Birmingham, B. W., Scott, L. E., Robbins, R. F., Mann, $D . B$., Temperature stratification in a nonventing liquid helium dewar. J 64C1, 19 (1960).

Birnbaum, G., A recording microwave refractometer. Rev. Sci. Instr. 21, 169 (1950). 6.

Dispersion is $\mathrm{NH}_{3}$ in the microwave region. Phys. Rev. 7 \%, 144 (1950). 48.

Fluctuations in the refractive index of the atmosphere at microwave frequencies. Phys. Rev. 82, 119 (1951). 240.

Millimeter wavelength dispersion of water vapor. J. Chem. Phys. 21, 57 (1953). 767.

Nonresonant absorption of symmetric top molecules: shape of the nonresonant spectra. J. Chem. Phys. 27, 360 (1957). 2217 .

Recording microwave refractometer. Rev. Sci. Instr. 21, 2 (1950). 122. 
Birnbaum, $G_{\circ}$, Bussey, H. E., Amplitude, scale, and spectrum of refractive index inhomogeneities in the first 125 meters of the atmosphere. Proc. IRE $\mathbf{4 3}$, 1412 (1955). 1258.

Birnbaum, G., Bussey, H. E., Larson, R. R., The microwave measurement of variations in atmospheric refractive index. IRE Trans. Ant. Prop. AP-3, 74 (1952). 585 .

Birnbaum, G., Chatterjee, S. D., Dielectric constant of water vapor. J. Appl. Phys, 23, 220 (1952). 431.

Birnbaum, G., Kryder, S. J., Lyons, $H$., Microwave measurements of the dielectric properties of gases. J. Appl. Phys, 22, No. 1, 95 (1951). 259.

Birnbaum, G., Maryott, A. A., Absorption in the lowfrequency wing of the $\mathrm{NH}_{3}$ inversion spectrum. J. Chem. Phys. 21, 1774 (1953). 639.

Change in the inversion spectrum of $\mathrm{ND}_{3}$ from resnnant to nonresonant absorption. Phys. Rev. 92, 270 (1953). 661.

Microwave absorption in compressed oxygen. Phys. Rev. 99, 1886 (1955). 1408.

Microwave absorption in compressed gases; saturated hydrocarbons. J. Chem. Phys. 24, 1022 (1956). 1778 .

Nonresonant absorption of symmetric top molecules. Collision crosssection. J. Chem. Phys. 29, 1422 (1958). 2648.

Temperature dependence of the microwave absorption of $\mathrm{NH}_{3}$. J. Chem. Phys. 22, No. 8, 1457 (1954). 1162 .

Birnbaum, G., Maryott, A. A., Wacker, P. F., Microwave absorption by the nonpolar gas $\mathrm{CO}_{2}$. J. Chem. Phys. 22, 1782 (1954). 1074.

Bish, J. M., Reid, W. E., Jr., Brenner, A., Electrodeposition of metals from organic solutions. III. Preparation and electroylsis of titanium and zireonium compounds in nonaqueous media. J. Electrochem. Soc. 104, No. 1, 21 (1957). 2106.

Bitter, $F$. Looking into the nucleus. J. Opt. Soc. Am. 43,233 (1953). 753 .

Black, I. A., Bolz, L. H., Brooles, F. P., Mauer, F. A., Peiser, $H$. S., A liquid-helium cold 'cell for use with an X-ray diffractometer. J 61, 367 (1958) RP2907.

Black, M. H., Glaze, F. W., Blackburn, D. H., Osmalov, $J$. S., Hubbard, D., Properties of arsenic sulfide glass. J 59, 83 (1957) RPि2774.

Blackburn, D. H., Glaze, F. W., Osmalov, J. S., Hubbard, D., Black, $M$. H., Properties of arsenic sulfide glass. J 599, 83 (1957) Rि2774.

Blackburn, G. F., Freeze, P. D., Caldwell, F. R., A study of thermistor materials for use as temperature-sensing elements in the high-velocity exhaust gases of jet-type engines. Wright Air Devel. Ctr. Tech. Rept. (Ohio) p. 54 Suppl. 1 (1956). 1602.

Blackburn, G. F., Sawada, F. H., Carter, C. J., Freeze. $P$. D., Development and evaluation of ceramic coatings for thermocnuples. Wright Air Devel. Ctr. Tech. (Ohio) Rept. 56-135 (1956). 1674.

Blaine, L. R., Allen, H. C., Jr., Cross, P. C., Plyler, E. K., Infrared spectrum of hydrogen sulfide from 2200 $2800 \mathrm{~cm}^{-1}$. J. Chem. Phys. 24, No. 1, 35 (1956). 1741 .

Blaine, L. R., Allen, H. C., Jr., Plyler, E. K., Infrared spectrum of carbonyl sulfide. J. Chem.' Phys. 26, No. 2, 400 (1957). 2162.

Snme infrarer bands of deuterium sulfide. J 59, 211 (1957) RP2789.

The emission spectrum of $\mathrm{OH}$ from 2.8 to $4.1 \mu$. Spectrochem. Acta 9, 126 (1957). 2356.

The infrared spectrum and rotational constants of carbon disulfide. J. Am. Chem. Soc. 78, 4843 (1956). 1932 .

Blaine, L. R., Plyler, E. K., Infrared emission spectrum of methane at 3.3 microns. J 59, 317 (1957) RP2803. Infrared high-resolution grating spectrometer. J. 62, 7 (1959) RP2922.

Infrared measurements with a small grating from 100 to 300 microns. J 60, 55 (1958) RP2821.
Transmittance of materials in the far infrared. $J$ 64C1, 55 (1960).

Blaine, L. R., Plyler, E. K., Connor, W. S., Velocity of light from the molecular constants of carbon monoxide. J. Opt. Soc. Am. 45, No. 2, 102 (1955). 1574.

Blaine, L. R., Plyler, E. K., Danti, A., Tidwell, E. D., Vibration-rotation structure in absorption bands for the calibration of spectrometers from 2 to 16 microns. J 64A 1, 29 (1960); (1960) Mono. 16.

Blaine, L. R., Plyler, E. K., Tidwell, E. D., Infrared absorption spectrum of methane from 2470 to 3200 $\mathrm{cm}^{-1}$. J 64A3, 201 (1960).

Blaine, R. L., Hunt, C. M., Dantzler, V., Tomes, L. A., Reaction of portland cement with carbon dioxide. J 60, 441 (1958) RP2858.

Blaine, R. L., Hunt, C. M., Tomes, L. A., Some effects of aging on the surface area of portland cement paste. J 64A2, 163 (1960).

Blaine, R. L., Tomes, L. A., Hunt, C. M., Some factors affecting the surface area of hydrated portland cement as determined by water-vapor and nitrogen adsorption. J 59, 357 (1957) RP2806.

Blair, J. C., Frequency dependence of VHF ionospheric scattering. (1959) TN9 (PB151368).

Blair, J. C., Brown, J. N., Watts, J. M., An ionosphere recorder for low frequencies. J. Geophys. Research 58, 99 (1953). 651.

Blake, J. H., Weitzel, D. H., Konecnik, M., Flow conversion, kineties of ortho and parahydrogen. Prnc., 1958 Cryogenic Engineering Conference, Paper E-2, p. 286. Edited by K. D. Timmerhaus, Chemical Engineering Dept., University of Colorado (Boulder, Colorado) (1959). 2953.

Blanch, G., On modified divided differences, I. Math. Tables and Other Aids to Computation 8, No. 45, 1 (1954). 1092.

On the numerical solution of equations involving differential operators with constant coefficients. Math Tables and Other Aids to Computation, 6, No. 40, 219 (1952). 502.

Programming for finding the characteristic values of Mathieu's differential equation and the spheroidal wave equation. Proc. of the Elec. Computer Symp. held by the Western Section of the Elec. Computers Prof. Group of the IRE, April 30, May 1-2, 1952. (University of California at Los Angeles, California) XIV:1 (1952). 518.

Zeros of $I_{n+1}(x) J_{n}(x)+J_{n+1}(x) I_{n}(x)$. Math, Tabies and Other Aids to Computation 6, No. 37, 58 (1952). 624.

Blanch, G., Rhodes, I., Table of characteristic values of Mathieu's equation for large values of the parameter. J. Wash. Acad. Sci 45, 166 (1955). 1523.

Blanch, G., Yowell, E. C., A guide to tables on punched cards. Math. Tables and Other Aids to Computation 5, No. 36, 185 (1951). 177.

Addendum to a guide to punched cards. Math. Tables and Other Aids to Computation VII, 1(1953). 641 .

Blanchard, C. H., Spencer, L. V., Multiple scattering of relativistic electrons. Phys. Rev. 93, 114 (1954). 1079.

Blatz, P. J., Simha, R., Wall, L. A., Depolymerization as a chain reaction. J. Polymer Sci. 5, 615 (1950). 44.

Blau, E. J., Mulligan, B. W., Raman spectrum of triethylborane. J. Chem. Phys. 26, 1085 (1957). 2278.

Block, S., Bienenstock, A., Posner, A. S., Radial distribution study of vitreous barium borate. J 64A3, $229(1960)$.

Block, S., Burley, G., Perloff, A., Mason, R. D., Jr., Refinement of the erystal structure of triclinic magnesium pyroborate. J 62, 95 (1959) RP2936. Block, S., Levin, E. M., Structural interpretation of
immiscibility in oxide systems: 1 . Analysis and calculation of immiscibility. J. Am. Ceram. Soc. 40, No. 3, 95 (1957). 2320 .

Structural interpretation of immiscibility in oxide 
systems: 2. Coordination principles applied to immiscibility. J. Am. Ceram. Soc. 40, No. 4, 113 (1957). $2320 \mathrm{~A}$.

Structural interpretation of immiscibility in oxide systems: 3. Effect of alkalis and alumina in ternary systems. J. Am. Ceram. Soc. 41, No. 2, 49 (1958). 2785.

Bloembergen, N., Temmer, G. M., Nuclear magnetic resonance of aligned radioactive nuclei. Phys. Rev. 89,883 (1953). 778 .

Bloss, R. L., A facility for the evaluation of resistance strain gages at elevated temperatures. Am. Soc. Testing Materials Spec. Tech. Publ. No. 230, 57 (1958). 2420.

Bloss, R. L., Mordfin, L., Greene, G. E., Halsey, N., Harwell, $R$. H., Jr., Creep and static strengths of large bolted joints of forged aluminum alloys under various temperature conditions. Inst. of Aeronaut. Sci. Preprint No. 779 (1958). 2505.

Blum, W., Fifty years of electrodeposition. J. Electrochem. Soc. 99, No. 2, 31C (1952), 449.

Blum, M., Norton, $K$. A., Staras, H., A statistical approach to the problem of multiple radio interference to FM and television service. IRE Trans. Ant. Prop. A P-1, 43 (1952). 378.

Blumenthal, L. M., Boolean geometry. 1. Rendiconti del Circolo Mathematico di Palermo II, No. 1, 1 (1952). 403.

Two existance theorems for systems of linear inequalities. Pacific J. Math. 2, No. 4, 523 (1952). 608.

Blunt, R. F., Breckenridge, R. G., Hosler, W. R., Frederikse, H. P. R., Becker, J. H., Oshinsky, W., Electrical and optical properties of intermetallic compounds, I. Indium antimonide. Phys, Rev, 96, 571 (1954). 1007.

Blunt, R. F., Frederiske, $H, P, R$., Photoconductivity and photovoltaic effect in intermetallic compounds. Photoconductivity Conf., Atlantic City, N.J. (John Wiley and Sons, Inc., New York, N.Y.) p. 414 (1956). 1806.

Photoeffects in intermetallic compounds. Proc. IRE 43, 1828 (1955). 1444.

Blunt, R. F., Frederikse, H. P. R., Becker, J. H., Hosler, $W$. $R$., Electrical and optical propertits of intermetallic compounds, III. Aluminum antimonide. Phys. Rev. 96, 578 (1954). 1007.

Blunt, R. F., Frederiske, H. P. R., Hosler, W. R., Electrical and optical properities of intermetallic compounds, IV. Magnesium stannide. Phys. Rev. 100, No. 2, 663 (1955). 1330.

Properities of $\mathrm{Mg}_{2} \mathrm{Sn}$. Phys. Rev, 98, 1533A (1955). 1460 .

Blunt, R. F., Hosler, W. R., Frederikse, H. P. R., Electrical and optical properties of intermetallic compounds, II. Gallium antimonide. Phys. Rev. 96, 576 (1954). 1007.

Boag, J. W., The distribution of linear energy transfer, or "ion density" for fast neutrons in water. Radiation Research 1, No. 4, 323 (1954). 1180.

Boag, J. W., Zendle, B., Scattered radiation contribution to depth dose data for $35-\mathrm{Mev}$ and $90-\mathrm{Mev}$ synchrotron X-rays. Phys. Rev. 95, 608A (1954). 1135.

Boag, J. W., Zendle, B., Koch, H. W., McElhinney, J., Studies of dose distributions in water for betatron $\mathrm{X}$-rays to $37 \mathrm{Mev}$. Radiol. Research 5, No. 2, 107 (1956). 1904.

Bogaty, H., Campbell, K. S., Appel, W. D., Some observations on the evaporation of water from cellulose. Textile Research J. 22, 75 (1952). 547.

The oxidation of cellulose by ozone in small concentration. Textile Research J. 22, 81 (1952). 589.

Boggs, G. E., A stable voltage controlled logarithmic attenuator. Proc. IRE 42, 696 (1954). 935.

Gain stable mixers and amplifiers with current feedback. Proc. IRE 42, No. 7, 1145 (1954). 1022. Improvement in gain stability of the superheterodyne mixer through the application of negative feedback. Proc. IRE 40, No. 2, 202 (1952). 466.

Using arc-discharge tubes in regulated low-voltage supplies. Radio Communication Mag. 12, No. 10 25 (1952). 615 .

Boggs, G. E., Rubin, S., Gated-beam mixer. Electronics 24, 196 (1951). 243.

Bogue, $R$. H., Cement. Encyclopedia Americana 6, 188; Portland Cement Association Fellowship Paper No. 68 (1955). 1284.

The calcium silicate hydrates. Proc. International Congress of Industrial Chem., Portland Cement Assoc. Fellowship Paper No. 69 (1954). 1172.

The chemistry of portland cement. Editorial Dossat, S. A. (Madrid, Spain) (1951). 327.

Bogue, R. H., Taylor, $W$. C., A sulfate susceptibility test test for portland cements. Portland Cement Assoc. Fellowship 58, 223 (1950). 11.

Boggs, R. N., Hatch, M. R., Jacobs, R. N., Richards, R. J., Phelps, G. R. Prediction of pressure drop in two-phase single-component fluid flow. Proc. 1958 Cryogenic Engineering Conference, Paper F-4, p. 357 . Edited by K. D. Timmerhaus, Chemical Engineering Dept., University of Colorado (Boulder, Colorado) (1959). 3019 .

Bok, B. J., Lawrence, $R$, S., Menon, T. K., Radio observations (al-CM) of dense dark nebulae. Publ. Astron. Soc. Pacific 67, No. 395, 108, (1955). 1468.

Bolgiano, R., Jr., A theory of wavelength dependence in ultrahigh frequency transhorizon propagation based on meteorological considerations. $J$ 64D3, 231 (1960).

Bolz, L. H., Black, I. A., Brooks, F. P., Mauer, F. A. Peiser, H. S., A liquid-helium cold cell for use with an X-ray diffractometer. J 61, 367 (1958) RP2907.

Bolz, L. H., Boyd, M. E., Mauer, F. A., Peiser, H. S., A re-examination of the crystal structure of $\alpha$ and $\beta$ nitrogen. Acta Cryst. 12, 237 (1959). 2879.

Bolz, L. H., Mauer, F. A., Peiser, H. S., Low-temperature X-ray studies on Rice's blue material. J. Chem. Phys. 30, No. 1, 349 (1959). 2979.

Bonner, T. W., Branscomb, L. M., Shalek, R. J., Intensity distributions in nitrogen bands excited in auroras and by high-energy protons and hydrogen atoms. Trans. Am. Geophys. Union 35, 107 (1954). 1044.

Bonner, T. W., Shalek, R. J., Branscomb, L. M., The emission spectra of nitrogen bombarded by high energy protons. Phys. Rev. 85, 739 (1952). 576.

Booker, H. G., Bailey, D. K., Bateman, R., Berkner, L. V., Montgomery, G. F., Purcell, E. M., Salisbury, M. W., Wiesner, J. B., A new kind of radio propagation at very high frequencies observable over long distances. Phys., Rev. 86, No. 2, 141 (1952). 369.

Boone, T. H., Hermach, F. L., MacArthur, E. H., McAuliff, $R$. C., Conductive flooring for hospital operating rooms. J 63C2, 125 (1959); (1960) Mono. 11.

Boone, T. H., Sigler, $P$. A., Materiales para pisos Ingenieria Internacional Construccion. (McGraw-Hill Book. Co. Inc., New York, N.Y.) 40, No. 7, 50 (1952). 479.

Boor, L., Reinhart, F. W., Brown, C., Lamb, J.J., Evaluation of the Boor-Quartermaster snag tester for coated fabrics and plastic films. ASTM. Bull. No. 210, 50 (1955). 1337.

Booth, S. F., Basic standardization. Report of the 1st Annual Standardization Symp. Army Chem. Corps. Engr. Command, March 24-25, 1958, (Army Chem. Center, Maryland) p. 90 (1958), 2470. Government specifications. Plating 43, No, 4, 490493 (1956). 1725.

Sources and identifying symbols of Government Specifications. Metal Progr. 62, No. 4, 109 (1952). 549.

Bottom, V. E., Dynamic measurements of the magnetoelastic properties of ferrites. (1960) TN49 (PB151408).

Botzen, A., Michels, A., Cox, J. A. M., Friedman, A. S., Contribution to the study of transport phenomena in gases at high densities. J. Appl. Phys. 26, 843 (1955). 1296 . 
Botzen, A., Michels, A., Friedman, A. S., Sengers, V. J., The thermal conductivity of argon for temperatures between $0^{\circ} \mathrm{C}$ and $75^{\circ} \mathrm{C}$ and at pressures up to 2500 atmospheres. Physica 22, 121 (1956). 1952.

Bouche, R. R., Ballard, C. R., A pretied tie for communication line wires. Conf. Paper No. CP 58-266 presented at the Annual Meeting of Am. Inst. Elec. Engrs. (1958). 2432.

Bouche, R. R., Lee, A. A., Ballard, C. R., On, F.J., Windinduced vibration of telephone and distribution conductors. Am. Inst. Elec. Engrs. Middle Eastern District Meeting Conf. Paper (April 1958). 2868.

Bouche, R. R., Tate, D. R., Autographic stress-strain recorders. ASTM Bull. No. 228, 33 (1958). 2465.

Bowen, R. I., Paffenbarger, G. C., Scientific developments and methods in practice (Dentistry in 1967). J. Am. Dental Assoc. 56, 785 (1958). 2748.

Bower, V. E., Bates, R. G., Alkaline solutions for $\mathrm{pH}$ control. Anal. Chem. 28, 1322 (1956). 1612. Standards for $\mathrm{pH}$ measurements from $60^{\circ}$ to $95^{\circ} \mathrm{C}$. J 59, 261 (1957) RP2797.

Bowles, K. L., Ionospheric forward scatter. Annals. of the Intern. Geophys. Year 3, Pt. 4, 346 (1957). 2171.

Observation on vertical-incidence scatter from the ionosphere at $41 \mathrm{Mc} / \mathrm{sec}$. Phys. Rev. Letters 1, 454 (1958). 2655.

Bowles, K. L., Cohen, R. S., 50 Mc oblique transmission experiment near the magnetic equator. IRE Trans. Ant. Prop. AP-6, 316 (1958). 2626.

National Bureau of Standards equatorial region V.H.F. scatter research program for the I.G.Y. QST 41, 11 (1957). 2208.

Bowman, H. A., Johnson, D. P., Cross, J. L., Hill, J. P. Elastic distortion error in the dead weight piston gage. Ind. Eng. Chem. 49, 2046 (1957), 2103.

Bowman, H. A., Macurdy, L. B., A photoelectric followup and recording system, and its application to remote observations of the beam in high precision balances. J 63C2, 91 (1959).

Boyd, M. E., Bolz, L. H., Mauer, F. A., Peiser, H. S., A re-examination of the crystal structure of $\alpha$ and $\beta$ nitrogen. Acta Cryst. 12, 237 (1959). 2879.

Bozman, W. $R$, Coleman, C. D., Meggers, W. F., Table of wavenumbers, $2000 \mathrm{~A}$ to $7000 \mathrm{~A}$. (1960) Mono. 3, Vol. I.

Table of wavenumbers, $7000 \mathrm{~A}$ to $1000 \mu$.

(1960)

Mono. 3, Vol. II

Bozman, W. R., Corliss, C. H., Westfall, F. O., Electrodeless metalhalide lamps. J. Opt, Soc. Am. 43, 398 (1953). 705 .

Bradt, P., Franklin, J. L., Herron, J. $T$. Dibeler, $V . H$. Mass spectrometric study of the decomposition of hydrazoic acid by the electric discharge. J. Am. Chem. Soc. 80, 6188 (1958). 2623.

Bradt, P., Herron, J. T., Franklin, J. L., Mass spectrometric study of the reactions of some hydrocarbons with active nitrogen. Can. J. Chem. 37, 579 (1959). 2982 .

Bradt, P., Herron, J. T., Franklin, J. L., Dibeler, V. H., Kinetics of nitrogen atom recombination. J. Chem. Phys. Letters 29, No. 1, 230 (1958); J. Chem. Phys. 30, 879 (1959). 2972.

Bradt, P., Mohler, F. L., Analysis of fluorinated polyphenvls by mass spectrometer. Anal. Chem. 27,875 (1955). 1263

Mass spectra and relative sensitivities of some polyphenyls. J 60, 143 (1958) RP2831.

Bradt, P., Mohler, F. L., Dibeler, V. H., Mass spectra of aromatic hydrocarbons filtered from smoky air. J 60 , 615 (1958) RP2876.

Bradt, P., Wall, L. A., Straus, S., Florin, R. E., Mohler, F. L., Phosphinoborine compounds: mass spectra and pyrolysis. J 63A1, 63 (1959).

Bram, J., Simha, R., Wall, L. A., High-speed computations in the kinetics of free-radical degradation, I. Random initiation. J. Chem. Phys. 29, No. 4, 894 (1958). 2587.
Bramao, L., Cady, J. G., Hendricks, S., Swerdlow, M. Criteria for the characteriz ation of kaolinite halloysite, and a related mineral in clays and soils. Soil Sci. $\mathbf{7 3}$, No. 4, 273 (1952). 420 .

Branscomb, L. M., Calculated calibration points for negative ion appearance potentials. J. Chem. Phys. 20, No. 2, 452 (1958). 2481.

Negative ions. Chapter, Advances in electronics and electron physics (Academic Press, Inc., New York, N. Y.) (1957). 2210

Photodetachment of atmospheric negative ions. Conf. on Chem. Aeronomy (Pergamon Press, London, England) (1957). 2245.

The electron affinity of atomic oxygen. Nature 182, 248 (1958). 2812.

Branscomb, L. M., Burch, D. S., Smith, S. J., Photodetachment of $\mathrm{O}_{2}-$. Phys. Rev. 112, 171 (1958). 2684

Branscomb, L. M., Burch, D. S., Smith, S. J., Geltman, S., Photodetachment cross section and the electron affinity of atomic oxygen. Phys. Rev. 111, No. 2, $504(1958) .2683$.

Branscomb, L. M., Fite, W. L., Photodetachment of the hydrogen negative ion. Phys. Rev. 93, 651 (1954). 1105 .

Branscomb, L. M., Pagel, B. E. F., Atomic and molecular negative ions in stellar atmospheres. Monthly Notices Roy. Astron. Soc. 118, No. 3, 258 (1958). 2463.

Branscomb, L. M., Shalek, R. J., Bonner, T. W., Intensity distributions in nitrogen bands excited in auroras and by high-energy protons and hydrogen atoms. Trans. Am. Geophys. Union 35, 107 (1954). 1044.

The emission spectra of nitrogen bombarded by high energy protons. Phys. Rev. 85, 729 (1952). 576.

Branscomb, L. $M .$, Smith, S. J., Electron affinity of atomic oxygen. Phys. Rev. 98, No. 4, 1127 (1955). 1334 .

Electron affinity of atomic sulfur and empirical affinities of the light elements. J. Chem. Phys. 25, No. 3, 598 (1956). 1698.

Experimental cross section for photodetachment of electrons from $\mathrm{H}$ - and D-. Phys. Rev. 98, No. 4, 1028 1955). 1338.

Negative oxygen ions in the upper atmosphere: The affinity and radiative attachment coefficient of atomic oxygen. Trans. Am. Geophys. Union 36, No. 5, 755 (1955). 1413

Branscomb, L. M., Smith, S. J., Burch, D. S., Experimental photodetachment cross section and the ionospheric detachment rate for $\mathrm{O}_{2}^{-}$. Ann. Geophys. Tome 14, No. 2, 225 (1958). 2556.

Branscomb, L. M. Smith, S. J., Burch, D. S., Geltman, S., Photodetachment studies of negative ions. Third Intern. Conf. on Ionization Phenomena in Gases, Societa Italiana di Fiscia. (Venice, Italy) (1957). 2246.

Brauer, E. M., White, E. E., Jr., Moshanos, M. G., The reaction of metal oxides with 0 -ethyoxybenzoic acid and other chelating agents. J. Dental Research 37, 547 (1958). 2829.

Brauer, G. M.,Burns, $F$. R., Sulfinic acid derivatives as accelorators in the polymerization of methyl methacrylate. J. Polymer Sci. 19, No. 92, 311 (1956). 1906.

Brauer, G. M. Davenport, R. M., Hansen, W. C., Accelerating effect of aminos on polymerization of methyl methacrylate. Modern Plastics 34, No. 3, 153 (1956) 1608.

Brauer, G. M., Pfeiffer, K. R., Harvey, J. L., Deterioration during storage of alginate hydrocolloidal dental impression material. U.S. Armed Forces Med. J. 5, No. 9,315 (1954). 986 .

Braver, G. M., Sweeney, W. T., Effect of temperature and degree of polymerization on the sorption of water by polymethyl methacrylate. Modern Plastics 32, No. 7, 138 (1955). 1328.

Brauer, G. M., Sweeney, W. T. Schoonover, I. C., Crazing of acrylic resins. J. Am. Dental Research 34, 306 (1955). 1298. 
Breckenridge, F., Greenspan, M., Tschiegg, C. E., Temperature coefficient of the speed of sound in water near the turning point. J. Acoust. Soc. Am. 28, 500 (1956). 1914.

Breckenridge, $\dot{F}$. C., A configuration of taillights and brakelights. Reports of Night Visibility Committee, Highway Research Board Bull. 146, 56 (1956). 1587 .

An evaluation of the luminous-transmittance requirements for railroad-signal glassware in terms of standard source $\mathrm{A}$ of the International Commission on Illumination. J 60, 317 (1958) RP2849.

Fifty years of signal lighting. Illum. Eng. 53, 311 (1958). 2562; Brochure issued by the Illum. Eng. Soc. 21 pages (1959). 2952.

The Kinorama as an evaluator in aviation lighting. Illum. Eng. 47, 561 (1952). 582.

Breckenridge, R. G., Semiconducting intermetallic compounds. Phys. Rev. 90, 488 (1953). 823.

Breckenridge, R. G., Blunt, R. F., Hosler, W.R., Frederikse, $H$. P. R., Becker, J. H., Oshinsky, $W$., Electrical and optical properities of intermetallic compounds. I. Indium antimonide. Phys. Rev. 96, 571 (1954). 1007.

Breckenridge, R. G., Cook, R. K., An elasticity of quartz. Phys. Rev, 92, No. 6, 1419 (1953). 653.

Breckenridge, R. G., Hosler, W. R., Electrical properties of titanium dioxide semiconductors. Phys. Rev. 91, 793 (1953), 704 .

Breckenridge, R. G., Hosler, W. R., Cshinsky, W., Electrical properties of indium antimonide. Phys. Rev. 91, 243 (1953). 703.

Brekke, G. N. Wind pressures in various areas of the United States. (1959) BMS152.

Bremmer, $H$., Mode expansion in the low-frequency range for propagation through a curved stratified atmosphere. J 63D 1, 75 (1959)

On the theory of fading properties of a fluctuating signal imposed on a constant siznal. (1959) C599.

Brennand, J. R., Jr., Brown, E. H., Magnetic losses at low temperatures. Proc. 1958 Cryogenic Engineering Conference, Paper A-5, p. 65 . Edited by K. D. Timmerhaus, Chemical Engineering Dept., Üniversity of Colorado (Boulder, Colorado) (1959); J. Appl. Phys. 30, No. 1, 112 (1959). 2980.

Brenner, A., Electrodeposition of metals from organic solutions, Part I. General Survey. J. Electrochem. Soc. 103, No. 12, 652 (1956). 1696.

Electrodeposition of some of the less common metals from nonaqueous media. Record of Chem. Progr. 16, No. 4, 241 (1955). 1331.

Electroless plating comes of age. Metal Finishing 52, No, 11, 68; No. 12, 61 (1954). 1010.

Electrolysis of organic solvents with reference to the electrodeposition of metals. J. Electrochem. Soc. 106, 148 (1959). 2936.

Progress in metal cleaning and finishing. Metal Progr. 68, No. 3, 113 (1955). 1459.

Research in plating at National Bureau of Standards. Plating Management 3, No. 5, 14 (1958). 2736.

Brenner, A., Brodell, F. P., Further studies of an electrnnic thickness gage: circuitry, operating characteristics, and calibration. Plating 44, 591 (1957). 2133.

Brenner, A., Connor, J. H., Electrodeposition of metals for organic solutions, Part II. Further studies on the electrodeposition of aluminum from a hydride bath. J. Electrnchem. Soc. 103, No, 12657 (1956). 1697.

Brenner, A., Couch, D. E., A hydride bath for the electrodeposition of aluminum. J. Electrochem. Soc. 99, No. 6. $234(1952), 363$.

Constant-level device for liquids. Anal. Chem. 24, 922 (1952). 415.

Preparation of trichloride and tetrachloride of molybdenum. J 63A2, 185 (1959).

Brenner, A., Couch, D. E., Shapiro, $H$., The use of nickel-aluminum alloy coatings for the protection of molybdenum from oxidation. J. Electrochem. Soc. 105, No. 8, 485 (1958). 2837.
Brenner, A., Couch, D. E. Shapiro, H., Taylor, J. K., Protection of molybdenum from oxidation at elevated temperatures. J. Electrochem. Soc. 105, No. 8, 450 (1958). 2705.

Brenner, A., de Minjer, C. H., Studies on electroless nickel plating. Plating 44, 1297 (1957). 2327.

Brenner, A., Reid, W. E., Jr., Bish, J. M., Electrodeposition of metals from organic solutions. III. Preparation and electrolysis of titanium and zirconium compounds in nonaqueous media. J. Electrochem. Soc. 104, No. 1, 21 (1957). 2106.

Brenner, A., Sherfey, J. M., Electrochemical calorimetry. J. Electrochem. Soc. 105, 665 (1958). 2538.

Brenner, A., Stanley, C., The adhesion of electroplated coatings to titanium. Ann. Proc. Am. Electroplaters' Soc. p. $123(1956) .1920$.

Brenner, A., Wood, G. B., Electrodeposition of metals from organic solutions, Part IV: Electrodeposition of beryllium and beryllium alloys. J. Electrochem. Soc. 104, No. 1, 29 (1957). 2107.

Brenner, A., Wranglen, G., Cathode film studies by the drainage method. Sartrych ur Svensk Kemish Tidskrift (Sweden) 67, 81 (1955). 1282.

Brenner, A., Yannakopoulos, T., Isolation of the diffusion layer at an electrode and the determination of the concentration polarization. J. Electrochem. Soc. 105, No. 9, 521 (1958). 2605.

Brenner, A., Zentner, $V .$, Jennings, $C$. $W$. ., Physical properties of electrodeposited metals, I: Nickel, 3: The effect of plating variables on the structure and properties of electrodeposited nickel. Plating 39, 865 (1952). 511.

Brice, A. T., Saylor, C. P., Zernike, F., Color phase contrast microscopy: requirements and applications. J. Opt. Soc. Am. 40, 329 (1950). 37.

Brickwedde, F. G., Agenda for Commission on Symbols, Units, and Nomenclature of the International Union of Pure and Applied Physics, July 1951. Chem. Eng. News 29, No. 7, 588; Phys. Today 4, 33; Science 113, 213 (1951). 189.

Giauque-Kelving proposal. Phys. Today 7,4 (1954). 1025.

Low temperature physics, J. Am. Chem. Soc. 76, 2030 (1954). 1055.

Temperatures in atomic explosions. Temperature, Its Measurement and Control in Science and Industry (Rheinhold Pub. Corp., New York, N.Y.) 2, 395 (1955). 1528.

The helium-vapor pressure scale of temperatures. Phys. Today 11, 23 (1958). 2818.

The "1958 $\mathrm{He}^{4}$ scale of temperatures", Part 1. Introduction. J 64A1, 1 (1960); (1960) Mono. 10.

Brickwedde, F. G., Armstrong, G. T., Scott, R. B., The vapor pressures of the deuteromethanes. J. Chem. Phys. 21, 1297 (1953). 889.

Brickwedde, F. G., Hudson, R. P., Ambler, E., Cryogenics. Ann. Rev. Phys. Chem. 6, 25 (1955). 1301.

Briggs, L. J., The limiting thickness of an electrolyzed gas film capable of sustaining a given negative pressure. J. Chem. Phys. 21, 779 (1953). 865.

Brodd, R. J., Heats of adsorptions from charge-transfer complex theory. J. Phys. Chem. 62, 54 (1958). 2582 .

On the hydrogen evolution reactions. J. Electrochem. Soc. 106, No. 1, 74 (1959). 3007.

Brode, W. R., Chemical spectroscopy, 1950 Edgar Marburg Lecture before the American Society for Testing Materials. Am. Soc. Testing Materials Proc. 50, 513 (1950). 35.

Color and chemical constitution. Am. Scientist 43, No. 2, 259 (1955). 1289

International exchange of scientific information. Chem. \& Eng. News 28, 4332 (1950). 79.

Materials. J. Franklin Inst. 25, 81 (1951). 255.

Optical rotation of polarized light by chemical compounds. J. Opt. Soc. Am. 41, 987 (1951). 285.

Steric effects in azo and indigo dyes. Recent 
Advances in Chem. Colouring Matters (London, England) SP-4, 1 (1956). 1897.

Sterochemical research. Record Chem. Progr. Summer Issue 45 (1952), 554.

The National Bureau of Standards and its international relations. Foreign Commerce Weekly 42, No. 11,3 (1951). 339 .

Brode, $W$. R., Gould, J. H., A shutter-illumination device for the study of phototropic dyes. J. Opt. Soc. Am. 42, No. 6, 380 (1952). 374.

Brode, W. R., Gould, J. H., Wyman, G. M., The relation between the absorption spectra and the chemical constitution of dyes. XXV. Phototropism and cistrans isomerism in aromatic azo compounds. J. Am.Chem. Soc. 74, No. 18, 4641 (1952). 593

The relation between the absorption spectra and the chemical constitution of dyes. XXVI. Effect of solvent and of temperature on the cis-trans isomerization of azo dyes. J. Am. Chem. Soc. 75, 1856 (1953). 881 .

Brode, W. R., Gould, J. H., Wyman, G. M., Whitney, J. E., A comparative survey of spectrophotometers in the $210-760 \mathrm{~m} \mu$ region. J. Opt. Soc. Am. 43, 862 (1953). 625

Brode, W. R., Hilbert, G. E., Career opportunities in the Government. Chem. Eng. News 36, Pt. II, 70 (1958). 2484

Brode, W. R., Insco, M. N., Gould, J. H., Corning, M. E., Relation between the absorption spectra and the chemical constitution of dyes: XXIX. Interaction of direct azo dyes in aqueous solution. J 60, 65 (1958) RP2823.

Brode, W. R., Seldin, I. L., Spoerri, P. E., Wyman, G. M., The relation between the absorption spectra and the chemical constitution of dyes. XXVIII. The hydration of azo dyes in organic solvents. J. Am. Chem. Soc. 77, 2762 (1955). 1543.

Brode, W. R., Wyman, G. M. The relation between the absorption spectra and the chemical constitution of dyes. XXII. Cis-trans isomerism in thioindigo dyes. J. Am. Chem. Soc. 73, 1487 (1951). 341.

The relation between the absorption spectra and the chemical constitution of dyes. XXIV. Absorption spectra of some thioindigo dyes in sulfuric acid. J. Am. Chem. Soc. 73, 4267 (1951). 342.

Brodell, $F$. P., Brenner, A., Further studies of an electronic thickness gage: circuitry, operating characteristics, and calibration. Plating 44,591 (1957). 2133.

Broida, H. $P_{\text {., Effects of self-absorption of rotational }}$ "temperatures" of OH in flames. J. Chem. Phys. 21, 1165 (1953). 700 .

Experimental temperature measurements in flames and hot gasses. Temperature, Its Measurement and Control in Science and Industry (Reinhold Publ. Corp., New York, N.Y.) 2, 264 (1955). 1340.

Hydrogen-oxygen reaction at the second explosion limit. J. Chem. Phys, 19, 790 (1951). 248.

La recherche sur les radicaux libres au National Bureau of Standards. J. chim. phys. 56, No. 2392, 813 (1959). 2973.

Luminescence de L'azote solide $\left(4,2^{\circ} \mathrm{K}\right)$ contenant des atomes ou radicaux libres. Effet de la dilution par L'argon (1). Le J. de physique et la radium (Paris, France) 18, 593 (1957). 2182.

Rotational temperatures of $\mathrm{OH}$ in methane-air flames. J. Chem. Phys, 19, 1383 (1951), 304.

Rotational vibrational "temperatures" of $\mathrm{CH}$ in flames at atmospheric pressure. J. Chem. Phys. 21, 340 (1953). 816.

Spectroscopic studies of solids condensed at $4.2^{\circ} \mathrm{K}$ from electric discharge through nitrogen, oxygen, hydrogen, water and ammonia. Proc. Conf. on Chem. Aeronomy. The Threshold of Space (Pergamon Press, London, England) (1957). 2309.

Stabilization of free radicals at low temperatures. Annal. N.Y. Acad. Sci. 67, Article 9, 530 (1957), 2316. Trapped radicals. Endeavor 17, 208 (1958). 2853.

Broida, H. P., Bass, A. M., Absorption spectra of solids condensed at low temperature from electric dis- charges. J. Mol. Spectroscopy 2, 42 (1958). 2445 Effects of vibrational state on rotational intensity distribution in OH. J. Chem. Phys. 21, 173 (1953). 701 .

Sharp line absorption in silica near $3400 \mathrm{~cm}^{-1}$. J. Opt. Soc. Am. 47, 163 (1957). 2297.

Spectra emitted from solid nitrogen condensed at $4.2^{\circ} \mathrm{K}$ from a gas discharge. Phys. Rev, 101, No. 6, 1740 (1956). 1881 .

Broida, H. P., Berl, W. G., Gayhart, E. L., Olsen, H. L., Shuler, $K$. E., Spectroscopic observations on pentaborane-air flames and explosions. J. Chem. Phys. 25, No. 4, 797 (1956). 1883.

Broida, H. P., Caldwell, F. R., Dover, J. J., Combustion in Bunsen flames. Ind. Eng. Chem. 43, 2731 (1951). 207.

Broida, $H$. P., Carrington, $T$., Fluorescence and average lifetime of excited $\left.\mathrm{OH}^{2} \Sigma^{+}\right)$in flames. J. Chem. Phys. 23, 2202 (1955), 1348.

Wall effects on rotational population of $\mathrm{OH}^{2} \Sigma^{+}$in a microwave discharge. J. Mol. Spectroscopy 2 , No. 3, 273 (1958). 2865

Broida, H. P., Chapman, $M$. W., Stable nitrogen isotope analysis by optical spectroscopy. Anal. Chem. 30, No. 12, 2049 (1958). 2775.

Broida, $H$. P., Fergusen, $R$. E., Atomic flames: spectra "temperatures" and products. Fifth Symposium (International) on Combustion (Reinhold Publ. Corp. New York, N.Y.) p. 754 (1955). 1268.

Stable carbon isotope analysis of optical spectroscopy. Anal. Chem. 28, 1436 (1956). 1892.

Broida, H. P., Freis, E. D., Rose, J. C., A variable heart pump permitting independent control of rate, output and ejection velocity. Science 115, No. 2996, 603 (1952). 381 .

Broida, H. P., Gaydon, A. G., The Herzberg bands of $\mathrm{O}_{2}$ in an oxygen afterglow and in the night sky spectrum. Proc. Roy. Soc., Series [A] 222, 181 (:954). 1185.

The luminous reaction between carbon monoxide and atomic oxygen. Trans. Faraday Soc. 49, 1190 (1953). 866.

The mechanism of formation of $\mathrm{OH}, \mathrm{CH}$, and $\mathrm{HCO}$ in flame spectra, using deuterium as tracer. Proc. Roy. Soc. (London, England) 218, 60 (1953). 868.

Broida, H. P., Gilford, S. R., Physiological monitor for anesthesia. Electronics 28, No. 10, 130 (1955). 1446.

Broida, H. P., Heath, D. F., Luminous reaction between carbon monoxide and atomic nitrogen. J. Chem. Phys. 26, 1352 (1957). 283.

Spectroscopic survey of energy distributions of $\mathrm{OH}$, $\mathrm{C}_{2}$ and $\mathrm{CH}$ redicals in low-pressure acetylene-oxygen flames. J. Chem. Phys. 26, 223 (1957). 2316.

Bioida, H. P., Herzfeld, C. M., Interpretation of spectra of atoms and molecules in solid nitrogen condensed at $4.2^{\circ} \mathrm{K}$. Phys. Rev, 101, 606 (1953). 1747.

Broida, H. P., Kane, W. R., Polyhedral flames with hydrogen and hydrocarbons. J. Chem. Phys, 20, No. 6. 1042 (1952). 514 .

Rotational intensity distributions of $\mathrm{OH}$ and $\mathrm{OD}$ in electrodeless discharge through water vapor. Phys. Rev. 89, 1053 (1953). 817.

Rotational "temperature" of $\mathrm{OH}$ in diluted flames. J. Chem. Phys. 21, 347 (1953). 818.

Broida, H. P., Kiess, N. H., Analysis of the $(0.1)$ and (1.2) bands of the ${ }^{2} \Delta^{-2} \pi$ system of $\mathrm{CH}$. Astrophys. J. 123, No. 1,166 (1956). 1625.

Emmission spectra from mixtures of atomic nitrogen and organic substances. 7 th Symp. on Combustion (Butterworths, London, England), p. 207 (1958). 2544.

Spectrum of the $\mathrm{C}_{2}$ molecule between $3600 \AA$ and $4200 \AA$. Can. J. Phys. 34, 1471 (1956). 1885. Spectrum of the $\mathrm{C}_{3}$ molecule between $3600 \AA$ and $4200 \AA$. Mem. Soc. Roy. Sci. (Liege, Belgium) 18, 544 (1957). 2313.

Broida, H. P., Kostkowski, H. J., Experimental evidence for the existence of abnormal OH rotational "tem- 
peratures" in low pressure flames, J. Chem. Phys. 23, 754 (1955). 1339 .

Experimental proof for the existence of nonthermal rotational distribution of $\mathrm{OH}\left({ }^{2} \Sigma^{+}\right)$in flames. $\mathrm{J}$ Chem. Phys. 25, No. 4, 676 (1956). 1709.

Spectral absorption method for determining population "temperatures" in hot gases. J. Opt. Soc. Am. 46. No. 4, 246 (1956), 1882

Broida, H. P., Kurzweg, U. H., Bass, A. M. Spectra of afterglows and discharges from nitrogen-oxygen mixtures J. Mol. Spectroscopy 1, 184 (1957), 2307.

Broida, H. P., Lalos, G. T., Rotational temperature of $\mathrm{OH}$ in several fiames. J. Chem. Phys. 20, No, 9 1466 (1952). 534.

Broida, H. P., Lalos, G. T., Corruccini, R, J., Design and construction of a blackbody and its use in the calibration of a grating spectrometer. Rev. Sci. Instr. 29, 505 (1958), 2513 .

Broida, H. P., Levedahl, W. J., Emission spectra on autoignited heptane-air mixtures. Anal. Chem. 24, 1776 (1952). 442.

Broida, H. P., Levedahl, W. J., Howard, F. L. The emission spectra of first-stage combustion in an engine. J. Chem. Phys. 19, 797 (1951). 333.

Broida, H. P., Lutes, O. S., Abundance of free atoms in solid nitrogen condensed at $4.2^{\circ} \mathrm{K}$ from a gas discharge. J. Chem. Phys. 24, No. 2, 484 (1956). 1607.

Broida, H. P., Mann, D. E., Squires, H. P., The decomposition of $\mathrm{CF}_{4}$ in flames. J. Chem. Phys. 22, 348 (1954). 1176.

Broida, H. P., Minkoff, G. J., Everett, A. J., Ultraviolet spectrophotometry of low pressure explosions. Fifth International Symposium on Combustions (Reinhold Publ. Corp., New York, N.Y.) p. 779 (1955) 1565 .

Broida, H. P., Moore, C. E., $\mathrm{CH}$ in the solar spectrum. J $63 \mathrm{~A} 1,19$ (1959).

Molecules in the solar spectrum. 7th Intern. Astrophys. Symp. Proc. Memoirs of Roy. Soc. (Liege, Belgium) Series 4, Vol. 18, 217 (1957), 2205. $\mathrm{OH}$ in the solar spectrum. J E3A 3, 279 (1959).

Rotational lines of $\mathrm{CH}, \mathrm{OH}$ and $\mathrm{CN}$ in the solar spectrum. 7th Intern. Astrophys. Symp. Proc. Memoirs of Roy. Soc. (Liege, Belgium) Series 4, Vol. 18, 252 (1957). 2292.

Broida, H. P., Morgan, G. H., Optical spectrophotometric analysis of hydrogen-deuterium mixtures in presence of air. Anal. Chem. 24, 799 (1952). 505.

Broida, H. P., Morowitz, H. J., Flames. Sci. Monthly 80 , No, 1,3 (1955). 1347.

Broida, H. P., Moyer, J.W. Spectroscopic analysis of deuterium in hydrogen-deuterium mixtures. $\vec{J}$. Opt. Soc. Am. 42, 37 (1952). 551.

Broida, $H$. P., Oldenberg, O., Application of photoelectric multiplier tubes to the sensitive measurement of absorption or of changes of relative light intensities. J. Opt. Soc. Am. 40, 381 (1950). 27.

The thermal hydrogen-oxygen reaction at relatively low temperatures. J. Chem. Phys, 19, 196 (1951). 345 .

Broida, H. P., Pellam, J.R., A note on the preparation of solid ozone and atomic oxygen. J. Chem. Phys. 23, 409 (1955). 1239.

Phosphorescence of atoms and molecules of solid nitrogen at $4.2^{\circ} \mathrm{K}$. Phys. Rev. 95, No. 3, 845 (1954). 1104.

Broida, H. P., Peyron, $M$., Evaporation of active species trapped in a solid condensed from discharged netrogen. J. Chem. Phys. 28, 725 (1958). 2551A.

Luminescence of solid nitrogen $\left(4.2^{\circ} \mathrm{K}\right)$ containing atoms of free radicals. Effect of traces of oxygen, hydrogen and water vapor. J. Phys. Radium 19, No. 4,480 (1958). 2622 .

Spectra emitted from solid nitrogen condensed at very low temperature from a gas discharge. J. Chem. Phys. 30, No. 1, 139 (1959). 3052.

Broida, H. P., Peyron, M., Horl, E. M., Brown, H. W., Etude spectroscopique des produits de la decharge electrique dan L'Azote condenses a l'etat solide a tres basse temperatures, prevues en faveur de l'existence d'Azote triatomique dan le solide. J. chim. phys. 56, No. 2392, 736 (1959). 2944.

Spectroscopic evidence for triatomic nitrogen in solids at very low temperatures. J. Chem Phys. 30, 1304 (1959). 3054.

Broida, H. P., Rose, J. C., Effects of plastic and steel surfaces on clotting time of human blood. Proc. Soc. Exptl. Biol. Med. J. 86, 384 (1954). 1003.

Broida, H. P., Rose, J. C., Hufnagel, C. A., Gillespie, J. F., Rabile, P. J., Freis, E. D. A method for the study of the circulation in the dog using a mechanical left ventricle. J. Appl. Physiol. 7, 580 (1954). 920.

Broida, H. P., Rose, J. C., Lazaro, E. J., Dynamics of complete right ventricular failure in dogs maintained with an extracorporeal left ventricle. J. Am. Heart Assoc. 4, No. 2, 173 (1956). 1685.

Broida, H. P., Schoen, L. J., and Kuentzel, L. E., Glass dewars for optical studies at low temperatures. Rev. Sci. Instr. 29, No. 7, 633 (1958). 2575.

Broida, H. $P$. Shuler, $K$. $E$., Kinetics of OH radicals from flame emission spectra. IV. A study of the hydrogen-oxygen flame. J. Chem. Phys, 20, 168 (1952). 475 .

Kinetics of $\mathrm{OH}$ radicals from flame emission spectra V. A study of acetylene-oxygen flame. J. Chem. Phys. 20, 1383 (1952). 476.

Spectroscopic study of electronic flame temperatures and energy distributions. J. Chem. Phys. 27, No. 4, 933 (1957). 2310; Rev. French Instr. Petro. and Ann. Combus. Liquid 13, 620 (1958). 2771.

Brombacher, W. G., Johnson, D. P., Cross, J. L., Mercury barometers and manometers. (1960) Mono. 8.

Brooks, F. P., Black, I. A., Bolz, L. H., Mauer, F. A. Peiser, H. S., A liquid-helium cold cell for use with an X-ray diffractometer. J 61, 367 (1958) RP2907.

Brooks, H. B., Electrical indicating instruments used in early Edison central stations. J. Franklin Inst. $\mathbf{2 5 6}$ 401 (1953). 702.

Brooks, R., Edelman, S., Saito, S., Jones, E., Smith, E. R. A stroboscopic vibration analyzer. J 63C2, 97 (1959).

Brower, W. S., Fang, P. H., Temperature dependance of the breakdown field of barium titanate. Phys. Rev. 113, 456 (1959). 3062.

Brown, B., Potassium iodide fast neutron detector. Rev. Sci. Instr. 26, 970 (1955), 1451.

Brown, B., Hooper, E. C., Jr., Plastic phosphor matrix for fast neutron detection. Nucleonics 16, 96 (1958) 2693.

Brown, C., Reinhart, F. W., Boor, L., Lamb, J. J., Evaluation of the Boor-Quartermaster snag tester for coated fabrics and plastic films. ASTM Bull. No. 210, 50 (1955). 1337.

Brown, D. W., Mandlekern, L., Hellmann, M., Roberts D. E., Quinn, F. A., Jr., The melting transition of polymethylene. J. Am. Chem. Soc. 75, 4093 (1953). 869 .

Brown, D. W., Wall, L. A., Gamma irradiation of polymethyl methacrylate and polystrene. J. Phys. Chem. 61, 129 (1957). 2138.

Pyrolysis of poly-alpha-methylstyrene. J. Phys. Chem. 62, 848 (1958). 2707.

Use of deuterium to investigate extent of branching in polystyrene. J. Polymer Sci. 14, No. 78, 513 (1954). 1220.

Brown, D. W. Wall, L. A., Florin, R. E. Electron spin resonance spectra from gamma-irradiated solid nitrogen. J. Chem. Phys. 30, No. 2, 602 (1959). 2940.

Brown, D. W. Wall, L. A., Hart, V. E., Pyrolysis of $\alpha$ and $\beta$ deuterostyrene polymers.'J. Polymer Sci. 15, No.79, 157 (i955). 1464.

Brown, D. W., Wall, L. A., Madorsky, S. L., Straus, S. Simha, R., Depolymerization of polymethylene and polyethylene. J. Am. Chem. Soc. 76, 3430 (1954). 985 .

Brown, E. $H$., Expansion engines for hydrogen liquefiers. J 64C1, 25 (1950).

On a generalization of the index notation for absolute tensors of arbitrary order. J 64B2, 99 (1960). 
On the most general form of the compatibility eouations and the conditions of integrability of strain rate and strain. J 59, 421 (1957) RP2813.

Brown, E. H., Brennand, J. R., Jr., Magnetic losses at low temperatures. Proc. 1958 Cryogenic Engineering Conference, Paper A-5, p. 65. Edited by K. D. Timmerhaus, Chemical Engineering Dept., University of Colorado (Boulder, Colorado) (1959); J. Appl. Phys. 30, No. 1, 112 (959). 2980.

Brown, E. H., Dean, $J$. W., Joule-Thomson process in the liouefaction of helium. J 60, 161 (1958) RP2834.

Brown, F. H., Central Radio Propagation Laboratorv. Signal 12, No. 4, 9 (1957). 2049.

Brown, F. P., A delegate reports on small tools. Mag. of Standards 29, No. 8, 235 (1958). 2417.

Brown, F. P., Pelander, C. E., Tough problems solved with tricky tooling. Machinery 64, No. 1, 149 (1957). 2395.

Brown, $F . W .$, Demands on teachers in a technological society. Colorado School J. 14 ('958). 2512.

Radio propagation research at the National Bureau of Standards. Am. J. Phys. 26, 628 (1958). 2713.

Brown, G. M., Burley, G., Crystallographic data for yohimbine hydrochloride. Anal. Chem. 30, 154 (1958). 2508.

Brown, H. W., Harvey, $K$. B., Etude aux infrarouges de certains solides condenses a partir de decharges en phase gazeuse. J. chim. phys. 56, No. 2392, 745 (1959), 2943.

Brown, H. W., Peyron, M., Horl, E. M., B:oida, H. P., Etude spectroscopicue des producits de la decharge electricue dan L'Azote condenses a l'etat solide a tres basses temperatures, prevues en faveur de l'existence d'Azote triatomicue dan le solide. J. chim. phys. 56, No. 2392, 736 (1959). 2944.

Spectroscopic evidence for triatomic nitrogen in solids at very low temperatures. J. Chem. Phys. 30, 1304 (1959). 3054 .

Brown, J. M., Dietz, V.R., Some economic aspects of the bone char process in sugar refining. Intern. Sugar J. (London, England) 52, 147 (1950). 132.

Brown, J. N., Blair, J. C., Watts, J. M., An ionosphere recorder for low frequencies. J. Geophys. Research 58,99 (1953). 651.

Brown, J. N., Watts, J. M., Effects of ionosphere disturbances on low frecuency propagation. J. Geophys. Research 56, No. 3, 403 (1951). 229.

Ionosphere observations at $50 \mathrm{kc}$. J. Geophys. Research 55, $179(950) .80$.

Some results of sweep-freouency investigation in the low frequency band. J. Geophys. Research 59, 71 (1954). 1146.

Bruckner, B. H., Isbell, H. S., Frush, H. L., Kowkabany, G. N., Wampler, G., Micromethylation of polysaccharides. Anal. Chem. 29, 1523 (1957). 2198.

Brueckmann, R. E., Costrell, L., Analysis of millimicrosecond pulses using a charge-storage type puise-height analyzer. Proc. 2d Symp. on Advances in Fast Pulse Techniques for Nuclear Counting, February 12-13, 1959 (University of California, Berkeley, Calif.) (1959). 2895.

Charge-storage pulse height analyzers for use with pulsed accelerators. Nuclear Instr. (North-Holland Publ. Co., Amsterdam, Holland) 3, 350 (1958). 2485.

Bryant, C. B., Bestul, A. B., Belcher, H. V., Quinn, F. A., $J r . \quad$ Viscosities of 0.25 to 90 percent GR-S rubber solutions. J. Phys. Chem. 56, 432 (1952). 621.

Brysk, H., Spatial distribution of energy dissipation by high-energy X-rays. Phys. Rev. 96, No. 2, 419 (1954). 1150.

Buchmann, E., Perls, T. A., A bar magnet velocity meter. Rev. Sci. Instr. 22, 475 (1951). 174.

Buckley, $F$., Dielectric constant of hydrogen-bonded liquids. J 61, 325 (1958) RP2905.

Buckley, F., Maryott, A. A., Tables of dielectric dispersion data for pure liquids and dilute solutions. (1958) C589.
Budden, $K$. G., Effect of small irregularities on the constitutive relations for the ionosphere. J 63D2, 135 (1959).

The "wave-guide mode" theory of the propagation of very low frecuency radio waves. Symp. on the Prop. of V.L.F. Radio Waves, Sponsored by the NBS Boulder Laboratories and IRE Prof. Group on Ant. and Prop., January 23-25, 1957 (Boulder, Colorado), Appendix B, V. 4, 50; Proc. IRE 45, 772 (1957). 239.

Bullard, E. C., Astin, A. V., Lewis, W. B., International comparisons of radioactivity standards. Science 123, 895 (1956). 1746.

Bunch, M. D., Powell, R. L., Calibration of thermocouples at low temperatures. Proc. 1957 Cryogenic Engineering Conference, Paper E-6, p. 269, Edited by K. D. Timmerhaus, Chemical Engineering Dept., University of Colorado (Boulder, Colorado) (1958). 2483.

The thermal E.M.F. of several thermometric alloys. Suppl. au Bull. Inst. Intern, du Froid (Delft, Holland), Comm. 1, 129 ('958). 2833A.

Bunch, M. D., Powell, R. L., Corruccini, R. J., Thermal emf of some thermometric alloys. In Low-Temperature Physics and Chemistry; 1957 Proc. 5th Intern. Conf., University of Wisconsin (Madison, Wisconsin), p. 484 (1958). $2844 \mathrm{~A}$.

Bunting, E. N., Lippincott, E. R., Van Valkenburg, A., Weir, C. E., Infrared studies on polymorphs of silicon dioxide and germanium dioxide. J 61, 61 (1958) RP2885.

Dielectric properties of titania or tin oxide containing varying proportions of rare-earth oxides. J. Am. Ceram. Soc. 41, No. 1, 40 (1958). 2520.

Note on the dielectric properties of magnesium-strontium titanates. J. Am. Ceram. Soc. 40, No. 8, 285 (!957). 2220 .

Bunting, E. N., Van Valkenburg, A., Some properties of diamond. Am. Mineralogist 43, 102 (1958). 2762.

Bunting, E. N., Weir, C. E., Lippincott, E. R., Van Valkenburg, $A$., Infrared studies in the 1 - to 15-micron region to 30,000 atmospheres. J 63A 1, 55 (1959).

Burch, D. S., Branscomb, L. M., Smith, S. J., Gellman, S. Photodetachment cross section and the electron affinity of atomic oxygen. Phys. Rev. 111, No. 2, 504 (1958). 2683.

Photodetachment studies of negative ions. Third Intern. Conf. on Ionization Phenomena in Gases, Societa Italiana di Fiscia (Venice, Italy) (1957). 2246.

Burch, D. S., Smith, S. J., Photodetachment cross section of the negative hydrogen ion. Phys. Rev. Letters 2, No. 4, 1645 (1959). 3012.

Relative measurement of the photodetachment cross section for $\mathrm{H}^{-}$. Phys. Rev. 116, No. 5, 1125 (1959). 3035 .

Burch, D. S., Smith, S. J., Branscomb, L. M., Experimental photodetachment cross section and the ionospheric detachment rate for $0_{2}^{-}$. Ann. Geophys. Tome 14, No. 2,225 (1958). 2556.

Photodetachment of $0_{\overline{2}}^{-}$. Phys. Rev. 112, 171 (1958). 2684.

Burdick, M. D., Parker, H. S., The effect of particle size on the bulk density and strength properties of uranium dioxide specimens. J. Am. Ceram. Soc. 39, No. 5, 181 (1956). 1926.

Burgers, J. M., Ghaffari, $A_{\text {., }}$ On the application of steamdriven water jets for propulsion purposes. J 60, 137 (1958) RP2830.

Burgers, J. M. Thomas, R. N., Preface to Proc. 3d Symp. on Cosmical Gas Dynamies. Smithsonian Astrophysical Observatory, June 1957 (Cambridge, Massachusetts); Rev. Modern Phys. 30, 908 (1958). 2697.

Burkhard, $D$. C., Hindered rotation in symmetric-asymmetric molecules. Trans. Faraday Soc. 52, No. 397 , Pt. 1, 1 (1956). 1732.

Burkhard, M. D., Albrite, J. P., Shutts, R. E., Whitlock, M. B., Jr., Cook, R. K., Corliss, E. L. R., Research in normal threshold of hearing. A. M. A. Arch. Otolaryngol. 68, 194 (1958). 2735.

Burkhard, M. D., Corliss, E. L. R., A probe tube method for the transfer of threshold standards between 
audiometer earphones. J. Acoust. Soc. Am. 25, $990(1953)$. 632.

The response of earphones in ears and couplers. J Acoust. Soc. Am. 26, No. 5, 679 (1954). 1199.

Burley, G., Crystal structure of barium hydrogen orthophosphate. J 60, 23 (1958) RP2817.

Burley, G., Block, S., Perloff, A., Mason, R. D., Jr., Refinement of the crystal structure of triclinic magnesium pyroborate. J 62, 95 (1959) RP2936.

Burley, G., Brown, G. M., Crystallographic data for yohimbine hydrochloride. Anal. Chem. 30, 154 (1958). 2508.

Burley, G., Clark, J. R., Mrose, M. E., Perloff, A. Studies of borate ninerals. VI: Veatchite. Am. Nineralogist 44, No. $11-12,1141$ (1959). 3057.

Burnett, $H$. C., A recording torsion testing machine for wire. ASTM Bull. No, 227 (1958). 2435.

Burnett, H. C., Newton, C. J., Crystallegraphy of eold drawn music wire. Wire and Wire Products 33, No. 1, 66 (1958). 2509 .

Variations in preferred orientation and modulus of elasticity in cold drawn music spring wire. Wire and Wire Products 30, No. 11, 1375 (1955). 1573.

Burnett, H. C., Staugaitis, C. L., Endurance of helical springs related to properties of the wire. Metal Progr. 64, 77 (1953). 710.

The behavior of long helical springs under fluctuating load. ASTM Bull. No. 55, 918 (1955). 1530.

Burnett, J. C., Fischer, E. K., High-speed motion pictures in textile research. Textile Research J. 20, 259 $(1950) .66$.

Burns, C. L., Mowery, W. E., Dickson, G., Sweeney, W. T. Dimensional stability of denture base resins. J. Am. Dental Assoc. 57, 345 (1958). 2523.

Burns, C. L., Stanford, J. W., Paffenbarger, G. C., American Dental Association specification No. 13 for self-curing repair resins. J. Am. Dental Assoc. 51, $425(1955) .1257$.

Burns, F. R., Brauer, G. M., Sulfinic acid derivatives as accelerators in the polymerization of methyl methacrylate. J. Polymer Sci. 19, No. 92, 311 (1956). 1906

Burstein, E., Davisson, J.W., Bell, E. E., Turner, W. J. Lipson, H. G., Infrared photoconductivity due to neutral impurities in germanium. Phys. Rev. 93, 65 (1954). 1039.

Burton, R. C., Conner, $W$. S., On the identity relationship for fractional replicates of the $2^{n}$ series. Ann. Math. Stat. 28, 3 (1957). 2230.

Bussey, H. E., Attenuation due to rainfall. FM-TV Radio Commun. 10, No. 2, 12 (1950). 29.

Cavity resonator dielectric measurements on rod samples. Insulation p. 26 (1959). 2907.

Microwave attenuation statistics estimated from rainfall and water vapor statistics. Proc. IRE 38 , $781(1950) .88$

Reflected ray suppression. Proc. IRE 38, 1453 (1950). 123 .

Suppressing microwaves by zonal screens. TeleTech. 10, No. 12,45 (1951). 317.

Bussey, H. E., Birnbaum, G., Amplitude, scale, and spectrum of refractive index inhomogeneities in the first 125 meters of the atmosphere. Proc. IRE 43, 1412 (1955). 1258.

Bussey, H. E., Birnbaum, G., Larson, R. R., The microwave measurement of variations in atmospheric refractive index. IRE Trans. Ant. Prop. AP-3, 74 (1952). 585

Bussey, H. E., Steinert, L. A., An exact solution for a cylindrical cavity containing a gyromagenetic material. Proc. IRE 45, Pt. 1, 693 (1957). 2013.

Exact solution for a gyromagnetic sample and measurements on a ferrite. IRE Trans. Mierowave Theory and Tech. MTT=6, 72 (1958). 2552.

Bussey, $W$. S., A proposed training program. Scale J. 37, No. 7, 4 (1951). 182.

Checking money-value graduations on a computing scale. Scale J. 38, No. 2, 6 (1951). 204.
Genuine cooperation between manufacturers and weights and measures officials. Scale J. 38, No. 8, 7 $(1952) .458$.

National Bureau of Standards weights and measures administration. Seale J. 38, No. 6, 9 (.952). 48.5. Testing wholesale meters. Scale J. 38, No, 4, 7 (1952). 565.

Bussey, W. S., Jensen, M. W., Weights and measures administration in the U.S. Supplement to "The Monthly Review" of the Inst. of Weights and Measures Admin. (April 1956). 1976.

Buzzard, E., Pitts, $J, W$., Moore, $D . G$., Resistance measurement of ceramic-type strain-gage cements. Am. Soc. Testing Materials Spec. Tech. Publ. No. 230, 67 (1958). 2739.

Byers, F. C., Sager, E. E., Dissociation of 4-chloro-4' aminodiphenylsulfone. $\mathrm{J} \quad \mathbf{5 9}, 245$ (1957) RP2794.

Byrns, R. A., Chelton, D. B., Mann, D. B., A large liquid hydrogen bubble chamber. Proc. 1956 Cryogenic Engineering Conference, Paper $\mathrm{H}-2$, p. 325, Edited by K. D. Timmerhaus, Chemical Engineering Dept. University of Colorado (Boulder, Colorado) (1957). 1984.

C

Cady, J. G., Bramao, L., Hendricks, S., Swerdlow, M. Criteria for the characterization of kaolinite halloysite, and a related mineral in clays and soils. Soil Sci. 73, No. 4, 273 (1952). 420.

Cahill, $W . F$., Programs for computing hypergeometric series. Math. Tables and other Aids to Computation 8,36 (1954). 1118.

Cahill, W. F., Abramowitz, $M_{*}$, On the vibration of a souare clamped plate. J. Assoc. Computing Machinery 2, No. 3, 162 (1955). 1435.

Cahill, W. F., Abramowitz, M., Wade, C., Jr., Heat transfer in laminar flow through a tube. J 62,101 (1959) RP2937.

Cahill, W. F., Davis, P., On a computation of the capacity of a cube. Math. Tables and Other Aids to Computation VII, 272 (Oct. 1953). 779.

Cahill, W. F., Levy, S., Computation of vibration modes and frequencies on SEAC. J. Aeronaut. Sci. 22, No. 12,837 (1955). 1293.

Cahill, W.F., Synge, J.L., The torsion of a hollow square Quart. Appl. Math. 15, No. 3, 217 (1957). 2385.

$C a h n, A$. S., Jr., Mathematical services useful in industry. Proceedings of Symposium on Industrial Applications of Automatic Computing Equipment held by the Midwest Research Institute, Kansas City, Mo., January 8, 1953, p. 19 (1953). 759.

Cahn, A. S., Jr., Saxon, D. S., Modes of vibration of a suspended chain. Quart. J. Mech. and Appl. Math. 6, Pt. 3, 273 (1953). 770.

Cahn, L., Kirsch, R. A., Ray, L. C., Urban, G. H., Experiments in processing pictorial information with a digital computer. 1957 Eastern Joint Computer Conf. Proc., Washington, D.C. (1957), 2119.

Cahoon, B. A., Bean, B. R., A note on the climatic variation of absolute humidity. Bull. Am. Meteorol. Soc. 38, No. 7, 395 (1957). 1991.

Effect of atmospheric horizontal inhomogeneity, upon ray tracing. J 63D3, 287 (1959).

The use of surface weather observation to predict the total atmospheric bending of radio rays at small elevation angles. Proc. IRE 45, 1545 (1957). 2388.

Cahoon, B. A., Bean, B. R., Thayer, G. D., Tables for the statistical prediction of radio ray bending and elevation angle error using surface values of the refractive index. (960) TN44 (PB151403).

Caldwell, F. R., Blackburn, G. F., Freeze, P. D., A study of thermistor materials for use as temperature-sensing elements in the high-velocity exhaust gases of jet-type engines. Wright Air Devel. Ctr. Tech. Rept. (Ohio) p. 54, Suppl. 1 (1956). 1602.

Caldwell, F. R., Broida, H. P., Dover, J. J., Combustion in Bunsen flames. Ind. Eng. Chem. 43, 2731 (1951). 207. 
Callomon, I. G., Kline, G. M., Wolock, I., Plastics for aircraft. Pt. II. Transparent glazing materials. Army-Navy-Commerce Bull. ANC-17 (1957). 2255.

Cameron, J. M., Some examples of the use of high-speed computers in statistics. Proc. Conf. Designs of Experiments in Army Research, Nevelopment, and Testing, Off. Ord. Researeh Rept. 57-1, 129 (1957). 2302. Tables for constructing and for computing the operating characteristics of single sample plans. Ind. Qual. Control 9, No. 1, Pt. 1, 37 (1952). 563.

The use of components of variance in preparing schedules for sampling of baled wool. Biometries 7 , 83 (1951). 346.

Use of computers in statistics. Proc. Fourth Annual High-Speed Computer Conf. (Louisiana State University, Baton Rouge, La.,) p. 67 (1957). 2404.

Cameron, J. M., Ogburn, F., Acceptance sampling of electroplated articles. Plating 41, No. 1, 43 (1954). 940.

Cameron, J. M., Youden, W.J., The selection of a limited number from many possible conditioning treatments for alloys to achieve best coverage and statistical evaluation. Am. Soc. Testing Materials Proc. 50, 951 (1950). 155.

Use of statistics to determine precision of test methods. Am. Soc. Testing Materials Spec. Tech. Publ. No. 103, $27(1950) .165$.

Cameron, R. H., A "simpson's rule" for the numerical evaluation of Weiner's integrals in function space. Duke Math. J. 18, No. 1, 111 (1951). 185.

Campbell, K. S., Bogaty, H., Appel, W. D., Some observations on the evaporation of water from cellulose. Textile Research J. 22, 75 (1952). 547.

The oxidation of cellulose by ozone in small concentration. Textile Research J. 22, 81 (1952). 589.

Campbell, $W . R$. Determination of dynamic stress-strain curves from strain waves in long bars. Proc. Soc. Exptl. Stress Anal. Semi-ann. Rept. 10, 113 (1953). 684.

Campbell, W. R., Irwin, L. K., Duncan, R. C., Stress studies of bulkhead intersections for welded tankers. Welding J. 31, No. 2, 68 (1952). 556.

Capps, F. M., Barsis, A. P., Effect of super-refractive layers on tropospheric signal characteristics in the Pacific coast region. IRE Wescon Conv. Record 1, Pt. I, $116(-957) .2100$.

Capps, F. M., Kirby, R. S., Correlation in VHF propagation over irregular terrain. IRE Trans. Ant. Prop. AP-4, No. 1, 77 (1956), 1663.

Capps, $W$., Shartsis, L., Spinner, S., Density, expansivity and viscosity of molten alkali silicates. J. Am. Ceram. Soc. 35, No. 6, 155 (1952). 422.

Viscosity and electrical resistivity of molten alkali borates. J. Am. Ceram. Soc. 36, 319 (:953). 907.

Carickhoff, M., Crouse, W. A., Fisher, M. A., Effect of fuel-immersion on laminated plastics. Trans. Am. Soc. Mech. Engrs. 72, 175 (1950). 50.

Carl, H. F., Jaffe, H. W., Hockman, A., New technicues for evaluating natural corundum ores. Min. Eng. 6, No. 4, 402 (1954). 1081.

Carlson, E. T., Some observations on hydrated monocalcium aluminate and monostrontium aluminate. J 59, 107 (1957) RP2777.

The system lime-alumina-water at $1^{\circ} \mathrm{C}$. J 61, 1 (1958) RP2877.

Carmete, J. L., Geil, G. W., Deformation twinning in charpy V-notch specimens of ingot iron. Welding J. Research Suppl. 32, 273-F (1953). 679.

Carmichael, H., Macurdy, L. B., Alber, H. K., BenedettiPichler, A. A., Corwin, A. H., Fowler, R. M., Huffman, E. W. D., Kirk, P. L., Lashof, T. W., Terminology for describing the performance of analytical and other precise balances, Anal. Chem. 26, 1190 (1954). 1166 .

Carpenter, F.G., A review of pressure drop across columns of bone char. Intern. Sugar J. 57, 12 (1955). 1243.

Carpenter, F. G., Rieger, C. J., Light seattering by commercial sugar solutions. J 63A3, 205 (1959).
Carpenter, L. H., On the motion of two cylinders in an ideal fluid. J 61, 83 (1958) RP2889.

Carpenter, L. H., Keulegan, G. H., Forces on eylinders and plates in an oscillating fluid. J 60, 423 (1958) RP2857.

Carpenter, R. J., Ochs, R. G., Experimental equipment for communication utilizing meteor bursts. IRE Wescon Conv. Session 46: Ionospheric Prop. August 20-23, 1957, San Francisco, California, IRE Wescon Conv. Record, Pt. 1, 283 (i957). 2117. Carpenter, S., Maienthal, M., Hellmann, M., Haber, C. P., Hymo, L. A., Carr, A.J., The preparation of some aryl silanes. J. Am. Soc. Chem. 76, 6392 (1954). 1192.

Carr, A. J., Maienthal, M., Hellmann, M., Haber, C. P., Hymo, L. A., Carpenter, S., The preparation of some aryl silanes. J. Am. Chem. Soc. 76, 6392 (1954). 1192.

Carrington, T., Electronic quenching of $\mathrm{OH}\left({ }^{2} \Sigma^{+}\right)$in flames and its significance in the interpretation of rotational relaxation. J. Chem. Phys. 30, 1087 (1959). 2941.

Line shape and $\mathrm{F}$ value in the $\mathrm{OH}^{2} \Sigma^{+}-2 \Pi$ transition. J. Chem. Phys. 31, No. 5, 1243 (1959). 2976.

Carrington, T., Broida, H. P., Fluorescence and average life time of excited $\mathrm{OH}\left({ }^{2} \Sigma^{+}\right)$in flames. J. Chem. Phys. 23, 2202 (1955) 1348

Wall effects on rotational population of $\mathrm{OH}^{2} \Sigma^{+}$in a microwave discharge. J. Mol. Spectroscopy. 2, No. 3,273 (1958). 2865.

Carroll, T. J., Internal reflection in the troposphere and propagation beyond the horizon. IRE Trans. Ant. Prop. AP-2, 9 (1952). 470.

Carson, $F, T$. Worthington, $V$, Diaphragm error and testing rate in the paper bursting test. Tappi 35, No. $12,539(1952)$. 428.

The stiffness of paper. Tappi 36, 36 (1953). 883.

Carter, C. J., Sawada, F. H., Freeze, P. D., Blackburn, $G$. F., Development and evaluation of ceramic coatings for thermocouples. Wright Air Devel. Ctr. Tech. Rept. (Ohio) 65-135 (1956). 1674.

Carter, N. F., Wait, J. R., Field strength calculations for E.L.F. radio waves. (1960) TN52 (PB161553).

Carter, T.J., An impact test for leather based on an alternating punching force. J. Am. Leather Chemists Assoc. 53, 250 (1958). 2457.

Carter, T. J., Kanagy, J. R., A flex tension test for leather. 'J. Am. Leather Chemists Assoc. 49, 23 (1954). 917

Carter, T. J., Kanagy, J. R., Leser, W., Randall, E. B., Mann, C., Influence of splitting on the strength of chrome-tanned steer hides. J. Am Leather Chemists Assoc. 47, 329 (1952). 468.

Carter, T. J., Kanagy, J. R., Randall, E. B., Kinmonth, R. A., Mann, C., Variations of physical and chemical properties within and between vegetable re-tanned cow and steer sides. J. Am. Leather Chemists Assoc. 47, 726 (1952), 618.

Carter, T. J., Randall, E. B., Kilduff, T. J., Mann, C., Kanagy, J.R., The variation of the physical and chemical properties of split and unsplit chrome-tanned leathers. J. Am. Leather Chemists Assoc. 47, 404 (.952). 601 .

Carter, T. J., Weir, C. E., Rate of shrinkage of tendon collagen: Further effects of tannage and licuid environment on the activation constants of shrinkage. J. Am. Leather Chemists Assoc. 45, 421 (1950). 120.

Carwile, N. L., Geil, G. W., A reduction of area gage for use at low temperatures. ASTM Bull. No, 163, 75 (1950). 7. Effect of strain-temperature history on the tensile behavior of titanium and a titanium alloy. J 61, 173 (1958) RP2897.

Effect of tensile strain at low temperatures on deformation twinning in ingot iron. J. Metals 5, 213 (1953); Trans. Am. Inst. Mining Met. Engrs. 197, 213 (1953). 695.

Research on effects of prestraining and notch sharpness on the notch strength of materials. WADC Tech. Rept. 56-402 (1956). 1853. 
Some effects of low temperatures and notch depth on the mechanical behavior of an annealed commercially pure titanium. J 59, 215 (1957) RP2790.

Carwile, N. L., Rosenberg, S. J., A study of 17-7 PH stainless steel. WADC Tech. Rept. 58-653 (1959). 2883.

Case, F. A., Washer, F. E., Calibration of precision airplane mapping cameras. Photogrammetric Eng. 16, $502(1950)$. 32 .

Case, K. M., Mendlowitz, H., Double scattering of electrons with magnetic interaction. Phys. Rev. 97, 33 (1955). 1320.

Cassel, J. M., A paper chromatographic analysis for collagen and collagen derivatives. J. Am. Leather Chemists Assoc. 51, 223 (1956). 1597.

Degradation of collagen by heat. J. Am. Leather Chemists Assoc. 53, 507 (1958). 2511.

Reaction of nitrous acid with collagen. J. Am. Leather Chemists Assoc. 51, 406 (1956). 1837.

Cassel, J. M., Kanagy, J.R., Heats of wetting of modified collagen and other materials. J. Am. Leather Chemists Assoc. 52, 248 (1957). 2149.

Cassel, J. M., McKenna, E., Amide nitrogen of collagen and hide powder. J. Am. Leather Chemists Assoc. 48,142 (1953). 645 .

Swelling of collagen and modified collagen. J. Am. Leather Chemists Assoc. 49, 553 (1954). 1159.

Cassel, J. M., McKenna, E., Glime, A., Studies on the polar amino acid content of collagen and related material. J. Am. Leather Chemists Assoc. 48, 277 (1953). 841.

Caswell, $R$.S., Neutron-insensitive gamma-ray dosimeter. Radiology 68, 101 (1957). 2211.

Caswell, R. S., Gabbard, R. F., Padgett, D. W., Doering, W. $P$., Attenuation of $14.1 \mathrm{Mev}$ neutrons in water. Nuclear Sci, and Eng. 2, 143 (1957), 2032.

Caswell, R. S., Mosburg, E. R., Jr., Chin, J., Standards for neutron flux measurement and neutron dosimetry. 2d United Nations Intern. Conf. on the Peaceful Uses of Atomic Energy. Vol. 21, Health and Safety: Dosimetry and Standards, P/752, USA, p. 92 (Pergamon Press, London, England) (1959). 3055.

Caswell, R. S., Nicastro, L. J., A double-pulse totalabsorption fast neutron spectrometer. (1959) TN1 (PB151360).

Caswell, R. S., Spiegel, $V ., J r$, Oliver, D. W. Age to indium resonance for 'D-D'neutrons in water. Nuclear Sci. and Eng. 4, No. 4, 546 (1958). 2450.

Catalan, $M$. A., Klinkenberg, P. F. A., Meggers, W. F. Velasco, R., Term analysis of the first spectrum of rhenium (Re r.) J 59, 319 (1957) RP2804.

Catalan, $M$. A., Meggers, $W$. $F$., Sales, $M$., Term analysis of the second spectrum of rhenium $(\operatorname{Re} n) . J 61$, 441 (1958) RP2914.

Cateora, J. V. Thompson, M. C., Jr., High-order harmonics for X-band oscillator stabilization. Rev. Sci Instr. 28, 656 (1957). 2153.

Caul, H. J., Sanford, J.W., Serio, A. F., Properties of self-curing denture base resins. J. Am. Dental Assoc. 44, 295 (1952). 521.

Caul, H.J., Schoonover, I. C., The color stability of direct filling resins. J. Am. Dental Assoc. 47, 448 (1953). 854.

Caul, H. J., Smith, D. L., Alloys of gallium with powdered metals as possible replacement for dental amalgam. J. Am. Dental Assoc. 53, 315 (1956). 1613.

Caul, H. J., Smith, D. L., Sweeney, W. T., Some physical properties of gallium copper-tin alloys. J. Am. Dental Assoc. 53, 677 (1956). 1876.

Caul, H. J., Swaney, A. C., Paffenbarger, G. C., Sweeney, $W . T$., American Dental Association specification No. 12 for denture base resin (second revision). J. Am. Dental Assoc. 46, 54 (1953). 644.

Caul, H. J., Sweeney, W. T., Gneug, W., Transverse strength testing machine for denture resinst J. Am. Dental Assoc. 49, 174 (1954). 1216.

Caul, H. J., Sweeney, W. T., Paffenberger, G. C., Relationship between residual monomer and some proper- ties of self-curing dental resins. J. Am. Dental Assoc. $53,60(1956)$. 1846.

Caul, H. J., Wall, L. A., Acquista, N., Determination of the monomer content of polymethyl methacrylate. J. Am. Dental Assoc. 53, 56 (1956). 1673.

Cavallo, L., Seliger, H. H., Culpepper, S. V., The half-life of I131. Phys. Rev. 90, 443 (1953). 863.

Cavallo, $L$. M., Seliger, $H$. $H$., Mann, $W . \dot{B}$., Average energy of sulfur-35 beta decay. J 60, 447 (1958) RP2 859 .

Chadwick, $W$. B., Prediction of sunspot numbers for cycle 20 . Nature 184, 1787 (1959). 3020.

Chaix, B., Henrici, $P$., On the design of two-dimensional nozzles by the method of characteristics. J. Aeronaut. Sci. 22, 140 (1955). 1432.

Chamberlain, $J . W$., Excitation of the oxygen red lines in twilight. Astrophys. J. 127, No. 1, 54 (1958). 2554 .

Oxygen red lines in the airglow, I. Twilight and night excitation processes. Astrophys. J. 127, No. 1, 54 (1958). 2681.

Chamberlain, J. W., Hunten, D. M., Roach, F. E., A photometric unit for the airglow and aurora. J. Atmospheric and Terrest. Phys. 8, 345 (1956). 1598.

Chapman, M. W., Broida, H. P., Stable nitrogen isotope analysis by optical spectroscopy. Anal. Chem. 30, No. 12, 2049 (1958). 2775.

Chapman, S., A comparison of the annual mean solar and lunar atmospheric tides in barometric pressure, as regards their worldwide distribution of amplitude and phase. J. Atmospheric and Terrest. Phys. 8, 1 (1956). 1586 .

Cosmic examples of heat conduction in very rare rotating or expanding gases. Extrait des Ann. de Ceophys. Tome 15, No. 4, 434 (1959). 2920.

Interplanetary space and the earth's outermost atmossphere, Proc. Roy. Soc. [A] 253, 462 (1959). 2969. Survey of geomagnetic eciipse phenomena, Special Suppl. J. Atmospheric and Terrest. Phys. 6, 221 ('956). 1908.

The aurora in middle and low latitudes. Nature (Mac.Millan \& Co., London, England) 179, No. 4549, 7 (1957). 2346.

The earth and its enviroment. Proc. IRE 47, No. 2, 137 (1959). 3071.

Thermal diffusion in ionized gases. Proc. Phys. Soc. 72, 353 (1958). 2844.

Chapman, S., Davies, $K$., On the approximate daytime constancy of the absorption of radio waves in the lower ionosphere. J. Atmospheric and Terrest. Phys. 13, 86 (1958). 2661 .

Chapman, S. (with aid of Kimball, D. S., Paton, J., Schneider, O., and Thomsen, I. L.), Visual auroral observations. Annals Intern. Geophys. Year 4, Pt. 1, 41 (1957), 24'2.

Chapman, S., Suguira, $M$., Arc-lengths along the lines of force of a magnetic dipole. J. Geophys. Research 61, 485 (1956). 1633.

Chatterjee, S. D., Birnbaum, G., Dielectric constant of water vapor. J. Appl. Phys. 23, 220 (1952). 431.

Chatterjee, S. D., Olcott, R. N., Absence of spontaneous emission of neutrons from samarium. Phys. Rev. 85, 147 (1952). 383.

Chelton, D. B., Dean, J. W., Birmingham, B. W., Design and construction of a liquid hydrogen temperature refrigeration system. (1960) TN38 (PB151397).

Chelton, D. B., Dean, J. W., Strobridge, T. R., Birmingham, $B$. W., Mann, D. B., Helium refrigeration and liquefaction using a liquid hydrogen refrigerator for precooling. (1950) TN39 (PB151398)

Chelton, D. B., Macinko, J., Dean, J., Hydrogen liquefaction by a dual pressure process. Refrig. Eng. 65, No. 8,39 (1957). 2156.

Hydrogen liquefaction cycles. Proc. 1957 Cryogenic Engineering Conf., Paper A-1, p. 1. Edited by K. D. Timmerhaus, Chemical Engineering Dept., University of Colorado (Boulder, Colorado) (1958). 2590 . 
Chelton, D. B., Mann, D. B., Byrns, R. A., A large licuid hydrogen bubble chamber. Proc. 1956 Cryogenic Engineering Conf., Paper H-2, p. 325, Edited by K. D. Timmerhaus, Chemical Engineering Dept., University of Colorado (Boulder, Colorado) (1957). 1984.

Chi, A. R., Bender, P. L., Beaty, E. C., Optical detection of narrow $\mathrm{Rb}^{87}$ hyperfine absorption lines. Phys. Rev. Letters 1, No. 9, 331 (1958). 2677.

Chi, M. L., Irwin, K., Elastic deformation in strips with holes loaded through pins. J 62, 147 ( 959$)$ RP2945.

Chi, M., Kirstein, A. F., Flexural cracks in reinforced concrete beams. J. Ảm. Concrete Inst. No. 54 (1958). 2566.

Chiarulli, P., Dressler, R. F., Condensation interfaces in two-phase flows. J. Appl. Phys. 28, 990 (1957). 2067

Chin, J., Caswell, R. S., Mosburg, E. R., Jr., Standards for neutron flux measurement and neutron dosimetry. 2d United Nations Intern. Conf. on the Peaceful Uses of Atomic Energy, Vol. 21, Health and Safety: Dosimetry and Standards, P/752, USA, p. 92 (Pergamon Press, London, England) (1959). 3055.

Chin, J., DeJuren, J. A., Manganese thermal neutron activation cross section. Phys. Rev. 99, No. 1, 191 (1955), 1397.

Chrzanowski, P., Cook, R. K., Transmission of airborne noise through walls and floors. Handbook of Noise Control (McGraw-Hill Book Co., Inc., New York, N.Y.) Chapter 20 (1957). 2397

Cialella, C., Miller, W., Motz, J. W., Thick target bremsstrahlung spectra for 1.00-, 1.25-, and 1.40-Mev electrons. Phys, Rev. 96, No. 5, 1344 (1954). 1211.

Clabaugh, W. S., Jackson, A., Separation and determination of phosphate, silicate, and arsenate. J 62, 201 (1959) RP2954.

Clark, A. P., Cracking of reinforced concrete flexual members. J. Am. Concrete Inst. 27, 851 (1956). 1665 .

Diagonal tension in reinforced concrete beams. J. Am. Concrete Inst. 23, 145 (1951). 222.

Clark, J. R., Mrose, M. E., Perloff, A., Burley, G., Studies of borate minerals. VI: Veatchite. Am. Mineralogist 44, No. 11-12, 1141 ('959). 3057.

Clatworthy, $W$. H., Connor, $W$. S., Some theorems for partially balanced designs. Ann. Math. Stat. 25, $100(1954)$. 1148.

Cleek, G. W. Hamilton, E. $H$. Properties of sodium titanium silicate glasses. J 61, 89 (1958) RP2890. Shape of the liquidus surface as a criterion of stable glass formation. J 60, 593 (1958) RP2872.

The development of some infrared transmitting glasses. Proc. of the Conf. of Infrared Optical Materials, Filter and Films. Eng. Research and Dev. Laboratories, The Eng. Ctr. Ft Belvoir, Va. p. 1 (1954). 1177 .

Cleek, G. W., Hamilton, E. H., Grauer, O. H., Some properties of glasses in the system barium oxide-silica. J. Am. Ceram. Soc. 41, No. 6, 209 (1958). 2763.

Cleek, G. W., Levin, E. M., Shape of liouid immiscibility volume in the system barium oxide-boric oxide-silica. J. Am. Ceram. Soc. 41, No. 5, 175 (1958). 2754.

Cleek, G. W., Scuderi, T. S., Effect of fluorides on infrared transmittance of certain silicate glasses. J. Am Ceram. Soc. 42, No. 12, 599 (959). 2933

Cleek, G. W., Spinner, S., Hamilton, E.H., Determination and use of the sag point as a reference point in the heating of glasses. J 59, 227 (1957) RP2791.

Cleland, $M$. R., Bay, Z., McLernon, $F$. Fast coincidences with Cerenkov counters. Phys. Rev. 87, 901 (1952). 447.

Clement, J. R., van Dijk, H., Durieux, M., Logan, J. K., The "1958 $\mathrm{He}^{4}$ scale of temperatures", Pt. 2, Tables for the 1958 temperature scale. J 64A 1, 1 (1960); (1960) Mono.10.

Cloud, P. E., Jr., Friedman, I., Sisler, F. D., Dibeler, V. H., Microbiological fractionation of the hydrogen isotopes. Science 127, 13 (1958). 2635.
Coblentz, C. $W$., Achenbach, P. R., Design and performance of a portable infiltration meter. Heating, Piping, Air Conditioning 29, No. 7, 155 (1957); Trans. Am. Soc. Heating and Air Conditioning Engrs., Paper 477 (1957). 2082 .

Coffin, D. O., Powell, R. L., Low-temperature thermal conductivity of a free-machining copper. Rev. Sci. Intr. 26, 516 (1955). 1393.

Coffin, D. O., Powell, R. L., Rogers, W. M., An apparatus for measurement of thermal conductivity of solids at low temperatures. J 59, 349 (1957) RP2805.

Cogliano, J. A., Jessup, R. S., Relation between net heat of combustion and aniline-gravity product of aircraft fuels. ASTM Bull. No. 201 (TP217), 55 (1954). 1128.

Cohen, R. S., Bowles, K. L., 50 Me oblique transmission experiment near the magnetic equator. IRE Trans. Ant. Prop. AP-6, 316 (1958). 2626.

National Bureau of Standards equatorial region V.H.F. scatter research program for the I.G.Y. QST 41, 11 (1957). 2208.

Cohen, V., Axilrod, B. M., Sherman, M. A., Wolock, I., Effects of biaxial stretch-forming. Modern Plastics 30, 117 (1952) 434 .

Effects of moderate biaxial stretch-forming on tensile and crazing properties of acrylic plastic glazing. Natl. Advisory Comm. Aeronaut. Tech. Note 2779 (1952). 436.

Cohen, V., Kline, G. M., Wolock, I., Axilrod, B. M., Sherman, M. A., George, D. A., Development of craze and impact resistance in glazing plastics by multiaxial stretching. Natl. Advisory Comm. Aeronaut. Tech. Rept. 1290 (1957). 2084.

Cohn, H., A numerical study of Dedekind's cubic class number formula. J 59, 265 (1957) RP2798.

Some algebraic number theory estimates based on the Dedekind eta-function. Am. J. Math. 78, 791 (1956). 1871 .

Cohn, H., Gorn, S., A computation of cyclic cubic units. J 59, 155 (1957) RP2783.

Cole,.K. S., Antosiewicz. H. A., Rabinowitz, P., Automatic computation of nerve excitation. J. Soc. Indust. Appl. Math. 3, 153 (1955). 1272.

Coleman, C. D., Bozman, W. R., Meggers, W. F., Table of wavenumbers, $2000 \mathrm{~A}$ to $7000 \mathrm{~A}$.' (1960) 'Mono.3, Vol. I.

Table of wavenumbers, $7000 \mathrm{~A}$ to $1000 \mu$. (1960) Mono. 3, Vol. II.

Coleman, C. D., Trees, R. E., Tables for diagonalizing second-order matrices, J 60, 201 (1958) R P2838.

Coleman, J. W., Leder, L. B., Marton, L., Schubert, D. C., Electron beam magnetometer. J 63C 1, 69 (1959).

Conda, $A . M_{\text {., }}$ On the measurement of ground conductivity at VLF. IRE Trans. Ant. Prop. AP-6, No. 3, 273 (1958), 2670.

Conda, A. M., Wait, J.R., Diffraction of electromagnetic waves by smooth obstacles for grazing angles. J 63D2, 181 (1959).

On the diffraction of electromagnetic pulses by curved conducting surfaces. Can. J. Phys. 37, 1384 (1959). 3005 .

On the measurement of ground conductivity at VLF. IRE Trans. Ant. Prop. A P-6, No. 3, 273 (1958). 2670. Pattern of an antenna on a curved lossy surface. IRE Trans. Ant. Prop. A P-6, No. 4, 348 (1958). 2682. Radiation from slots on dielectric-clad and corrugated cylinders. J 59, 307 (1957) RP2802.

The patterns of a slot-array antenna on a finite and imperfect ground plane. L'Onde Electrique 38, No. 376 bis (Proc. Intern. Congress Ultra High Frequency Circuits and Antennas, October 21-26, 1957, Paris, France) p. 7 (1958). 2826.

Condon, E. U., Basic standards in science. J. Franklin Inst. 251, No. 1, 103 (1951). 198.

Condon, E. U., Smith, N. Bailey, S. L., Everitt, W. L., Fink, $D . G_{.}, \quad$ The present status of color television; a report by the Senate Advisory Committee on Color Television. Proc. IRE 38, 980 (1950). 154. 
Cones, H. N., Park, J.H., Sphere-gap volt-time curvesstandards for steep front surge measurements. Am. Inst. Elec. Engrs. Conf. Paper No. CP57-215 (1957). 2314 .

Connor, J. H., Brenner, A., Electrodeposition of metals for organic solutions, Part II. Futher studies on the electrodeposition of aluminum from a hydride bath. J. Electrochem. Soc. 103, No. 12, 657 (1956). 1697.

Connor, J. H., Reid, W. E., Jr., Wood, G. B., Electrodeposition of metal from organic solutions. V. Electrodeposition of magnesium and magnesium alloys. J. Electrochem. Soc. 104, 38 (1957), 2108.

Some relation among the blocks of symmetrical group divisible designs. Ann. Math. Stat. 23, No. 4, 602 (1952), 548 .

The uniqueness of the triangular association scheme. Ann. Math. Stat. 29, 262 (1958). 2835.

Connor, WV. S., Experiences with incomplete block designs: examples. Proc. Symp. on the Design of Industrial Experiments, North Carolina State College, (John Wiley \& Sons, New York, N.Y.) p. 193 (1957). 2116.

Fractional factorial experiments of $2^{\mathrm{m}} 3^{\mathrm{n}}$ series. Proc. 14th Ann. Clinic of the Rochester Society for Quality Control, February 18, 1958, (Rochester, New York), p. 59 (1958), 2568

Connor, W. S., Burton, R. C., On the identity relationship for fractional replicates of the $2^{n}$ series. Ann. Math. Stat. 28, 3 (1957). 2230.

Connor, W. S., Clatworthy, W. H., Some theorems for partially balanced designs. Ann. Math. Stat. 25, 100 (1954). 1148.

Connor, W. S., Hall, M., Jr., An embedding theorem for balanced incomplete block designs. Can. J. Math. 6, No. 1, 35 (1954). 948.

Connor, W. S., Horowitz, E., Variability of wool content in part wool blankets. ASTM Bull. No. 208, 42 (1955). 1571 .

Connor, W. S., Plyler, E. K., Blaine, L. R., Velocity of light from the molecular constants of carbon monoxide. J. Opt. Soc. Am. 45, No. 2, 102 (1955). 1574. The chain block design. Biometries 9, 127 (1953). 852 .

Connor, W. S., Youden, W. J., Making one measurement do the work of two. Chem. Eng. Progr. 49, 549 (1953). 757 .

Connor, W. S., Zelen, $M$., Fractional factorial experiment designs for factors at three levels. (1959) AMS 54.

Multi-factor experiments. Ind. Quality Control 15, No. 9, 1 (March 1959). 2995.

Conrad, G. T., Jr., A proposed index of current noise for composition resistors. Proc. Electronic Components Symp. p. 118 (Sponsored by Am. Inst. Elec. Engrs. New York, N.Y.). (1955). 1241.

Noise in composition resistors. Proc. Natl. Electronics Conf. Electronics Conf. Yearbook 10, 1 (1955). $1416 \mathrm{~A}$.

Noise measurements of composition resistors. I. The method and equipment. II. Characteristics and comparison of resistors. IRE Trans. Component Parts CP4, 61 (1955). 1417.

Cook, C. C., Calibration of commercial field-strength meters. Tele-Tech. 11, No. 10, 44 (1952). 407

Cook, M. I. Swanson, H. E., Gilfrich, N. T. Standard X-ray diffraction powder patterns. (1957) C539, Vol. 7.

Cook, M. I., Swanson, H. E., Gilfrich, N. T., Stinchfield, R., Parks, P. C., Standard X-ray diffraction powder patterns. (1959) C539, Vol. 8.

Cook, M. I., Swanson, H. E., Isaacs, T., Evans, E. H., Standard X-ray diffraction powder patterns, (1960) C539, Vol. 9.

Cook, R. K., Absorption of sound by patches of absorbent materials. J. Acoust. Soc. Am. 29, 329 (1957). 2010. Laboratory standard microphones. Acustica (Zurich, Switzerland) 4, 101 (1954) 1048

Properties of Struve functions. J. Wash. Acad. Sci. 47, 365 (1957). 2269 .
Variation of elastic constants and static strains with hydrostatic pressure: a method for calculation from ultrasonic measurements. J. Acoust. Soc. Am. 29, 445 (1957), 2407.

Cook, R. K., Albrite, J. P., Shutts, R. E., Whitlock, M. B., $J r$, Corliss, E. L. R., Burkhard, $M, D$. Research in normal threshold of hearing. A.M.A. Arch. Otolaryngol. 68, 194 (1958), 2735 .

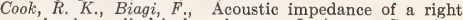
circular cylindrical enclosure. J. Acoust. Soc. Am. 26, No. 4, 506 (1954), 941.

Cook, $R$. $K$, Breckenridge, $R$. G., Anelasticity of quartz. Phys. Rev. 92, No, 6, 1419 (1953). 653.

Cook, R. K., Chrzanowski, $P$., Transmission of airborne noise through walls and floors. Handbook of Noise Control (McGraw-Hill Book Co., Inc., New York, N.Y.) Chapter 20 (1957). 2397.

Cook, R. K., Wasilik, J. H. An elasticity and dielectric loss of quartz. J. Appl. Phys, 27, No. 7, 836 (1956). 1620.

Cook, R. K., Waterhouse, $R, V$, New method of recording the sound transmission loss of walls as a continuous function of frequency. J. A coust. Soc. Am. 27, No. 5, 967 (1955). 1414.

Cook, R. K., Waterhouse, R. V., Berendt, R. D., Measurements on sound absorbers for jet-engine test cells. J. Acoust. Soc. Am. 28, No. 4, 688 (1956). 1771. Sound insulation of wall, floor, and door constructions. (1958) BMS144, 2d Suppl.

Cook, R. K., Waterhouse, R. V., Berendt, R. D., Edelman, S., Thompson, $M$. C., Jr., Measurement of correlation coefficients in reverberant sound fields. J. Acoust Soc. Am. 27, No. 6, 1072 (1955), 1399.

Cook, R. K., Weissler, P. G., Piezoelectric constants of alpha- and beta-quartz at various temperatures. Phys. Rev, 80, 712 (1950), 108.

Coombs, W. C., Communication theory aspects of television bandwidth conservation. (1959) TN25 (PB151384)

Coon, R. M., Watt, A. D., 7 urich, V. J., Reduction of adjacent-channel interference components from frequency-shift-eyed carriers. IRE Trans. Commun. Systems CS-6, 39 (1958). 2723.

Reduction of adjacent-channel interference from onoff keyed carriers. IRE Trans. Commun. Systems CS-4, 41 (1956). 1841.

Cooper, J. W., Berger, $M$. J., Reflection of fast neutrons from water. J 63A2, 101 (1959).

Cooper, J., Nelms, A., $\quad \mathrm{U}_{235}$ fission product decay spectra at various times after fission. Health Physics 1,427 (1959), 3105

Cooter, I. L., Harris, W. P., A feedback amplifier with negative output resistance for magnetic measurements. IRE Natl. Conv. Record, Pt. 5, p. 217 (1958). 2421.

Improved bridge method for the measurement of core losses in ferromagnetic materials at high flux densities. J 60, 509 (1958) RP2865.

The Use of an AC bridge to measure core loss at high induction. Commun. and Electronics. Am. Inst. Elec. Engrs., No. 24, 252 (1956). 1954.

Cooter, I. L., Mundy, R. E., Cunife wire magnets of small size. J 59, 379 (1957) RP2808.

Copeland, M. A., McBurney, J. W., Richmond, J. C., Relations among certain specification properties of building brick and effects of differences in raw materials and methods of forming. $J$. Am. Ceram. Soc. 35, No. 12,309 (1952). 528.

Corliss, C. H., Effect of variation of circuit parameters on the excitation of spectra by capacitor discharges. Spectrochim. Acta 5, 378 (1953). 696.

Corliss, C. H., Bozman, W. R., Westfall, $F$. O., Electrodeless metalhalide lamps. J. Opt. Soc. Am. 43, 398 (1953). 705 .

Corliss, C. H., Kiess, C. C., Description and and analysis of the first spectrum of iodine. J 63A1, 1 (1959.)

Corliss, C. H., Kiess, C. C., Kiess, H. K., Corliss E. L. R., High-dispersion spectra of mars. Astrophys. J. 126, No. 3, 579 (1957). 2152 . 
Corliss, C. H., Meggers, W. F., Improved description of hafnium spectra. J 61, 269 (1958) RP2904.

Corliss, C. H., Scribner, B. F., Emission spectrographic standards. Anal. Chem. 23, 1548 (1951). 232

Corliss, E. L. R., Limitations on rapid signal analysis. J. Wash. Acad. Sci. 45, No. 11, 359 (1955). 1388. Mechanistic aspects of hearing. J. Acoust. Soc. Am. Abstract 31, 841 (1959). 2987.

Transients in signal analysis. J. Wash. Acad. Sei. 46, 305 (1956). 1968.

Uniform transient error. J 61, 25 (1958) RP2879.

Corliss, E. L. R., Albrite, J. P., Shutts, R. E., Whitlock, M. B., Jr., Cook, R. K., Burkhard, M. D., Research in normal threshold of hearing. A. M. A., Arch. Otolaryngol. 68, 194 (1958). 2735.

Corliss, E. L. R., Burkhard, M. D., A probe tube method for the transfer of threshold standards between audiometer earphones. J. Acoust. Soc. Am. 25, 990 (1953). 632 .

The response of earphones in ears and couplers. J Acoust. Soc. Am. 26, No. 5, 679 (1954). 1199.

Corliss, E. L. R., Kiess, C. C., Corliss, C. H., Kiess, H. K. High-dispersion spectra of mars. Astrophys, J. 126, No. 3, 579 (1957). 2152.

Corliss, E. L. R., Koidan, W., Mechanical impedance of the forehead and mastoid, J. Acoust. Soc. Am. 27, No. 6, 1164 (1955). 1409.

Corliss, $E . \dot{L}, R$. Snyder, $W . F$., Calibration of audiometers, J. Acoust. Soc. Am. 22, 837 (1950), 31

Corning, M. E., Inscoe, M. N., Gould, J. H., Brode, W. R., Relation between the absorption spectra and the chemical constitution of dyes: XXIX. Interaction of direct azo dyes in aqueous solution. J 60, 65 (1958) $\mathrm{RP} 2823$

Corruccini, R.J. Calculation of gaseous heat conduction in dewars. Proc. 1957 Cryogenic Eng. Conf. Paper G-1, p. 353, Edited by K. D. Timmerhaus, Chemical Engineering Dept., University of Colorado (Boulder, Colorado) (1958), 2482

Properties of materials at low temperatures. Chem. Eng. Progr. 53, Pt. I, No. 6, 262; Pt. II, No. 7, 343; Pt. III, No. 8, 397 (1957). 2268.

Corruccini, R. J., Bunch, M. D., Powell, R. L., Thermal emf of some thermometric alloys. In Low-Temperature Physics and Chemistry; 1957 Proc. 5th Intern. Conf., University of Wisconsin (Madison, Wisconsin), p. 484 (1958), $2844 \mathrm{~A}$.

Corruccini, R. J., Lalos, G. T., Broida, H. P., Design and construction of a blackbody and its use in the calibration of a grating spectroradiometer. Rev. Sci. Instr. 29, 505 (1958), 2513.

Corwin, A. H., Macurdy, L. B., Alber, H. K., BenedettiPichler, A. A., Carmichael, H., Fowler, R. M., Huffman, E. W. D., Kirk, P. L., Lashof, T. W., Terminology for describing the performance of analytical and other precise balances. Anal. Chem. 26, $1190(1954) \quad 1166$.

Cosgrove, L. A., Robinson, H. E., Powell, F. J., Thermal resistance of airspaces and fibrous insulations bounded by reflective surfaces (1957). BMS151.

Costrell, L., A-C zero locator. Ëlectronics 31, 98 (1958). 2414.

FM data reduction from magnetic tape recorders. Rev. Sci, Instr, 24, 76 (1953) 722

High-repetition-rate mercury pulser. Nucleonics 15, No. 11,112 (1957). 2155.

Precision 5-10 kv high-voltage supply. Nucleonies 13, No. 3,63 (1955). 1453 .

Radiation monitoring over long-distance telephone lines and direct field lines. IRE Trans. Nuclear Sci. NS-5, No. 3, 21 (1958), 2710.

Regulated supply offsets line changes. Electronics 31, 100 (1958). 2728 .

Costrell, L., Attix, F. H., Automatic timer simplifies small-current measurements. Nucleonics 15, No. 2, $83(1957) .2036$

Costrell, L., Brueckmann, R. E., Analysis of millimicrosecond pulses using a charge-storage type pulse-height analyzer. Proe. 2d Symp. on Advances in Fast
Pulse Techniques for Nuclear Counting, February 12-13, 1959 (University of California, Berkeley, California) (1959). 2895.

Charge-storage pulse-height analyzers for use with pulsed accelerators. Nuclear Instr. (North Holland Publ. Co., Amsterdam, Holland) 3, 350 (1958). 2485. Cottony, $H$. V., High-gain antennas for VHF scatter propagation. IRE. Trans. Commun. Systems CS-4, 56 (1956). 1729.

Radio noise of ionospheric origin. Science III, 41 (1950). 118.

Techniques for accurate measurement of antenna gain. (1958) C598.

Cottony, H. V., Elliott, R. S., Jordan, E. C., Rumsey, V. H., Siegel, K. M., Wait, J. R., Woodyard, O. C., Antennas and waveguides, and annotated bibliography. U.S.A. National Committee Report, Intern. Sci. Radio Union Subcommission 6.3. IRE Trans. Ant. Prop. A P-7, 87 (1959). 2897.

U.S.A. National Committee report, URSI Subcommission 6.3 , antennas and waveguides, and annotated bibliography. Proc. 12th Gen. Assembly, International Scientific Radio Union, August-September 1957 (Boulder, Colorado), 11, Pt. 6, 121 (1957). 2402. Cottony, H. V., Johler, J. R., Cosmic radio noise intensities in the VHF band. Proc. IRE 40, 1053 (1952). 418 .

Cottony, H. V., Wilson, A. C., Gains of finite-size cornerreflector antennas. IRE Trans. Ant. Prop. AP-6, No. 4, 366 (1958). 2574.

Couch, D. E., Brenner, A., A hydride bath for the electrodeposition of aluminum. J. Electrochem. Soc. 99, No. 6,234 (1952). 363.

Constant-level device for liquids. Anal. Chem. 24, $922(1952), 415$.

Preparation of trichloride and tetrachloride of molybdenum. J 63A2, 185 (1959).

Couch, D. E., Senderoff, S., The electrolytic preparation of molybdenum from fused salts. V. Electrorefining studies in the presence of tin, iron, copper, silicon, and nickel. Trans. Met. Soc. of AIME, p. 320 (1958). 2811 .

Couch, D. E., Shapiro, H., Brenner, A., The use of nickelaluminum alloy coatings for the protection of molybdenum from oxidation. J. Electrochem. Soc. 105, No. 8, 485 (1958). 2837.

Couch, D. E., Shapiro, H., Taylor, J. K., Brenner, A., Protection of molybdenum from oxidation at elevated temperatures. J. Electrochem. Soc. 105, No. 8, 450 (1958). 2705.

Coughanour, L. W., Shartsis, L., Shermer, H. F. Viscosity density, and electrical resistivity of $m$ olten alkaline-earth borate glasses with three mole percent of potassium oxide. J. Am. Cera n. Soc. 41, 324, (1958). 2864

Cox, J. A. M., Michels, A., Botzen, A., F iedman, A. S., Contribution to the study of transport phenomena in gases at high densities. J. Appl. Phys, 26, 843 (1955). 1296 .

Coyle, C. F., Hellmann, M., Alexander, R. L., Jr. Separation of isomeric polyphenyls by adsorption chromatography. Anal. Chem. 30, 1206 (1958). 2752.

Craig, $D, N$., Hamer, $W . J .$, A reproducible and stable silver-silver oxide electrode. J. Electrochem. Soc. 104, 206 (1957). 1998.

Some aspects of the charge and discharge processes in lead-acid storage batteries. Appl, and Ind., No. 11, 22 (1954). 1141.

Craig, D. N., Hofman, J.I., A new method for determining the value of the Faraday. Phys. Rev. 80, $487(1950) .3$.

Craig, D. N., Law, C. A., Hamer, W. J., Stability of silver and pyrex in perchloric acid-silver perchlorate solutions and in conductivity water. J 64A1, 127 (1960).

Craig, S. N., Sommer, H., Hoffman, J. I., Hipple, J. A., The effect of recent mass measurements on the Faraday. Phys. Rev. 85, 740 (1952). 573. 
Crandall, $J . R$., Observations on field exposures of enameled ranges. Proc. of the Porcelain Enamel Inst. Forum 16, 27 (1954). 1090.

Crandall, J. R., Eubanks, A. G., Richmond, J. C., Flexibility of thin procelain enameled sheet steel. Bull. Am. Ceram. Soc. 36, No. 2, 59 (1957). 2126.

Crandall, J. R., Harrison, W. N., Richmond, J.C., Effects of acid treatment on acid and abrasion resistance of porcelain enamels. J. Am. Ceram. Soc. 33, 314 (1950). 51 .

Crandall, J. R., Nimerof, I., Hammond, H. K. III., Richmond, J. C., Gloss meter comparisons. J. Am. Ceram. Soc. 39, No. 3, 103 (1956). 1724.

Specular-gloss measurement of ceramic materials. J. Am. Ceram. Soc. 39, No. 3, 103 (1956). 1886.

Creedon, J. E., Udelson, B. J. French, J. C., Microwave measurements of the properties of a dc hydrogen discharge. J. Appl. Phys. 28, No. 6, 717 (1957). 2200.

Creitz, E. C., Isbell, H. S., Smith, F. A., Frush, H. L., Moyer, J. D., Stewart, J, E., Infrared spectra of sugar acetates in solution. J 59, 41 (1957) RP2772.

Crichlow, W. Q., Noise investigation at V.L.F. by the National Bureau of Standards. Symp. on the Prop. of V.L.F. Radio Waves, Sponsored by the NBS Boulder Labs. and IRE Prof. Group on Ant. and Prop., January 23-25, 1957 (Boulder, Colo.), Paper No. 42, V. 3; Proc. IRE 45, 778 (1957). 2216.

Report of the subcommission on the question: "What are the most readily measured characteristics of terrestrial radio noise from which the interference to different types of communications systems can be determined?" Proc. XIIth Gen. Assembly. Intern. Sci. Radio Union, Boulder, Colo. 1957, XI, Pt. 4, 9 (1957). 2284.

Crichlow, W. Q., Disney, R. T., Report on revision of atmospheric radio noise data. CCIR Report No.65, VIII Plenary Assembly, Warsaw (1956) (Intern. Radio Consultative Comm., Secretar., Geneva, Switzerland). 1851.

Crichlow, W. Q., Foldes, G., Hewitt, F. J., Horner, F. Shinkawa, H., Sullivan, A. W., Measurement of atmospheric noise. Recommendation No. 1 and Annex, Proc. XIIth Gen. Assembly Intern. Sci. Radio Union, Boulder, Colorado, August 22-September 5, 1957, XI, Pt. 4, 99, 1957. U.R.S.I. (Intern. Sci. Union) Info. Bull. No. 105, 10 (September-October 1957). 2190.

Crichlow, W. Q., Roubique, C. J., Spaulding, A. D., Beery, $W . M$., Determination of the amplitude-probability distribution of atmospheric radio noise from statistical moments. J 64D1, 49 (1960).

Crichlow, W. Q., Samson, C. A., Disney, R. T., Jenkins, M. A., Quarterly radio noise data-March, April, May 1959. (1960) TN18-2 (PB151377-2).

Radio noise data for the International Geophysical Year July 1, 1957-December 31, 1958. (1959) TN18 (PB151377).

Crittenden, E. C., Early work in applied optics at the National Bureau of Standards. J. Opt. Soc. Am. 41, 367 (1951). 226.

Fifty years of scientific standardization. Standardization 22, No. 11, 349 (1951). 236.

Crombie, D. D., On the mode theory of very-low-frequency propagation in the presence of a transverse magnetic field. J 64D 3, 265 (1960).

Cross, J. L., Brombacher, W. G., Johnson, D. P., Mercury barometers and manometers. (1960) Mono.8.

Cross, J. L., Johnson, D. P., Hill, J. P., Bowman, H. A. Elastic distortion error in the dead weight piston gage. Ind. Eng. Chem. 49, 2046 (1957). 2103.

Cross, P. C., Allen, H. C., Jr., Plyler, E. K., Blaine, L. R., Infrared spectrum of hydrogen sulfide from $2200-2800$ $\mathrm{cm}^{-1}$. J. Chem. Phys. 24, No. 1, 35 (1956). 1741.

Crouse, W. A., Carickhoff, M., Fisher, A., Effect of fuelimmersion on laminated plastics. Trans. Am. Soc. Mech. Engrs. 72, 175 (1950). 50.
Crouse, W. A., Roberts, A. G., Pizer, R. S., Abrasive jet method for measuring abrasion resistance of organic coatings. ASTM Bull. No, 208, 36 (1955), 1249

Crow, E. L., A property of additively closed families of distributions. Ann. Math. Stat. 29, No. 3, 892 (1958). 2433.

Confidence intervals for a proportion. Biometrika (London, England) 43, Pts. 3 and 5, $423(1956) .1660$. The mean deviation of the Poisson distribution. Biometrika 45, 556 (1958), 2823.

Crow, E. L., Gardner, R. S., Confidence intervals for the expectation of a Poisson variable. Biometrika $\mathbf{4 6}$, 441 (1959). 2915.

Crysdale, J. H., Herbstreit, J. W., Dickson, F. H., Egli, J. J., Wickizer, G. S., Rebuttal to comments on "Large reduction of VHF transmission loss and fading by the presence of a mountain obstacle in beyond-line-of-sight paths", by F. H. Dickson, J. J. Egli, J. W. Herbstreit, and G. S. Wickizer. (Publ. in Proc. IRE 41, 967 (1953)). Proc. IRE 43, 627 (1955). 1384.

Cullen, A. L., Surface-wave resonance effect in a reactive cylindrical structure excited by an axial line source. J 64D 1, 13 (1960).

Culpepper, S. V., Seliger, H. H., The half-life of $\mathrm{I}^{131}$. Phys. Rev. 90, 443 (1953). 863 .

Culshaw, $W$., Reflectors for a microwave Fabry-Perot interfermeter. IRE Trans. Microwave Theory and Tech. MMT-7, No. 2, 221 (1959). 3034.

Curtis, A.J., A survey of some recent advances in polymer physics, and their relation to dielectric properties. Insulation p. 15 (1958). 2440.

Curtis, G. W., Newkirk, G. A., Watson, D. K., Manning, R., Shelby, J., The inner solar corona during June 1959 . Nature 184, 1308 (1959). 3080.

Curtis, W., Tandberg-Hanssen, E., Watson, K., The emission of $\mathrm{HeI}, \lambda$ 10830, during the great flare of August 26, 1958. Astrophys. J. 129, No. 1, 238 (1959). 3077

Curtiss, J. H. Acceptance sampling by variables, with special reference to the case in which quality is measured by average of dispersion. Acceptance Sampling: A symposium given at the 105th ann. meeting, Am. Stat. Assoc. (Washington, D.C.) (1950). 16.

Elements of a mathematical theory of probability. Math. Mag. 26, 233 (1953). 708.

Interview with; on using mathematies for design. (How industry can make better use of mathematics). Prod. Eng. 22, 156 (1951). 355.

Scientific teamwork in a Computation Laboratory. Teamwork in research, edited by G. P. Bush and L. H. Hattery, Chapter 17 (American University Press) (1953). 819.

The Institute for Numerical Analysis. An unsigned item, University of California Faculty Bull. (Berkeley, Calif.) 19, No. 7, 67 (1950), 152.

The Institute for Numerical Analysis of the National Bureau of Standards. Monthly Research Rept. of the Office of Naval Research, Dept. Navy, p. 8 (Issue of May 1951); Abbreviated version in Am. Math. No. 58, $372(1951), 337$

Curtiss, L. F., Evans, R. D., Johnson, W., Seaborg, G. T., Units of radioactivity. Rev. Sei. Instr. 21, 94 (1950). 163.

Curtiss, L. F., Gill, P. S., The sea-level latitude variation of fast cosmic-ray neutrons. Phys. Rev. 84, 591 (1951). 343.

Curtiss, L. F., Feister, I., The disintegration scheme of I131. Phys. Rev. 78, 179 (1950), 150.

The variation of intensity of fast cosmic-ray neutrons with altitude. Phys. Rev. 85, No. 2, 309 (1952). 600.

Cuthill, J.R., Hayes, W. D., Seebold, R. E., Nitriding phenomena in titanium and the $6 \mathrm{~A} 1-4 \mathrm{~V}$ titanium alloy. J 64A1, 119 (1960).

Cuthill, J. R., Moore, D. G., Protection of low-strategic alloys with a chromium boride-nickel cermet coating. Am. Ceram. Soc. Bull. 34, 11 (1955). 1463.

Cuthill, J. R., Richmond, J. C., Tighe, N. J., The effect of a ceramic coating on the creep behavior of some high temperature alloys. Bull. Am. Ceram. Soc. 39, 4 (1959). 3072. 
Cutkosky, R. D., Driscoll, R. L., Measurement of current with the National Bureau of Standards current balance. J 60, 297 (1958) RP2846.

Cutkosky, R. D., Driscoll, R. L., Thomas, J. L., Status of absolute measurements at the National Bureau of Standards. Procès-Verbaux, C.I.P.M., p. 36, (1958). 2781.

Cutkowsky, J. F., McGregor, M. C., Hersh, J. F., Harris, F. K., Kotter, F. R., New apparatus at the National Bureau of Standards for absolute capacitance measurement. IRE Trans. Instrumentation 1-7, No. 3-4, 253 (1958). 2647.

Dade, E. C., Abelian groups of unimodular matrices. Illinois J. Math. 3, 11 (1959). 2886.

Dahl, A. I., Measurement of high temperatures in gas streams. Bull. A \& M College of Texas 5th series 6, 30 (1950); Petrol. Refiner 29, No. 3, 115 (1950). 85.

Dahl, A. I., Fiock, E. $F$., Temperature measurements in high-velocity streams of hot gas. Proc. First Iowa Thermodynamics Sym. p. 190, State University of Iowa (Iowa City, Iowa) (1954). 1163.

The measurement of gas temperatures by immersiontype instruments. J. Am. Rocket Soc. 23, 155 (1953). 867.

Dahl $S$, Colorimetric determination of tetrachlorohydroquinone. Anal. Chem, 25, 1724 (1953). 670. Effect of sample preparation on precision in the hide substance determination. J. Am. Leather Chemists Assoc. 49, 515 (1954). 1000.

Prevention of microbiological deterioration of leather. J. Am. Leather Chemists Assoc. 51, No. 3, 104 (1956). 1817.

The effects of fungicides on deterioration of leather. J. Am. Leather Chemists Assoc. 52, 611 (1957). 2353.

Dahl, S., Kaplan, A. M., Fungicidal effectiveness of compounds applied to leather. J. Am. Leather Chemists Assoc. 52, 536 (1957). 2131.

Laboratory and field exposure studies of leather fungicides. J. Am. Leather Chemists Assoc. 51, No. 3,119 (1956) 1754 .

Studies of leather fungicides. J. Am. Leather Chemists Assoc. 53, 103 (1958). 2788.

Dahl, $S$., and Mandel, $J$., A collaborative study on the cinchonine method for determination of lignosulfonates in vegetable extract blends. J. Am. Leather Chemists Assoc. 52, 184 (1957). 1979.

Dahl, S., Oehler, R., The determination of nitrogen in leather by the' Kjeldahl method. J. Am. Leather Chemists Assoc. 46, 317 (1951). 332.

Dahl, S., Oehler, R., Kilduff, T.J., Treatment of leather with Castiloa and Hevea rubbers. J. Am. Leather Chemists Assoc. 45, 378 (1950). 160.

Treatment of leather with polyisobutylene, J. Am. Leather Chemists Assoc. 47, 642 (1952). 607.

Dalke, J. L., Powell, R. C., Advancements in dielectrics, 1952. Proc. IRE 41, 452 (1953). 642.

Advancements in dielectrics, 1953. Proc. IRE 42, 705 (1954). 943.

Measuring power of low-loss dielectrics. Electronics 24, No. 8, 224 (1951). 256.

Dalton, A. J., Swerdlow, M., Birks, L. S., Electron microscopy. Anal. Chem. 28, No. 4, Pt. II, 597 (1956) 1703 .

Daniel, F. T., Rice, P. L., Radio transmission loss versus distance and antenna height at $100 \mathrm{Mc}$. IRE Trans. Ant. Prop. A P-3, 59 (1955), 1470.

Danos, $M$., Electron injection in betatrons. Bull. Am. Phys. Soc. Serv. II, No. 1, 344, Abstract (1956). 1700 .

Low-energy limit of the photonuclear pseudodeuteron effect. Bull. Am. Phys. Soc., Serv. II, 4, 102 (1959). 2978 .

On the long-range correlation model of the photonuclear effect. Nuclear Phys. 5, 23 (1958). 2669.

Danti, A., Plyler, E. K., Blaine, L. R., Tidwell, E. D. Vibration-rotation structure in absorption bands for the calibration of spectrometers from 2 to 16 microns; J. 64A 1, 29 (1960), (1960) Mono. 16.

Dantzig, G. B., Hoffman, A. J., Dilworth's theorem on partially ordered sets. Ann. Math. Studies No. 38, 207 (1956). 1677.

Dantzler, V., Hunt, C. M., Tomes, L. A., Blaine, R. L., Reaction of portland cement with carbon dioxide. $J$ 60, 441 (1958) RP2858.

Darling, W. R., Washer, F. E., Evaluation of lens distortion by the inverse nodal slide. J 63C2, 105 (1959). Evaluation of lens distortion by the modified goniometric method. J 63C2, 113 (1959).

Factors affecting the accuracy of distortion measurements made on the nodal slide optical bench. J. Opt. Soc. Am. 49, No. 6, 517 (1959). 2948.

Marginal performance of corrected ophthalmic lenses. J 60, 551 (1958) RP2869.

Darling, W. R., Washer, F. E., Tayman, W. P., Evaluation of lens distortion by visual and photographic methods. J 61, 509 (1958) RP2920.

Daven port, R. M., Brauer, G. M., Hansen, W. C., Accelerating effect of aminos on polymerization of methyl methacrylate. Modern Plasties 34, No. 3, 153 (1956). 1608.

Davidson, M., Yates, W. A., Wide-range calibrator for vibration pickups. Electronics 26, 183 (1953). 911.

Davies, K., The effect of the earth's magnetic field on m.u.f. calculations. J. Atmospheric and Terrest. Phys, 16, 187 (1959). 3075.

Davies, $K$., Agy, V., Ionospheric investigations using the sweep-frequency pulse technique at oblique incidence. J 63D2, 151 (1959).

An atlas of oblique-incidence inograms. (PB151390).)

Davies, $K$., Chapman, $S$., On the approximate daytime constancy of the absorption of radio waves in the lower ionosphere. J. Atmospheric and Terrest. Phys. 13, 86 (1958). 2661.

Davies, K., Wright, J. W., Knecht, R. W., Ionospheric vertical soundings. Ann. of the Intern. Geophys. Year, 3, Pt. 1, 1 (Pergammon Press, New York, N.Y.) (IGY Instruction Manual: The Ionosphere Pt. 1) (1957). 2173.

Davis, B. L., Components for mechanized production. Radio-Electron. Eng. 24, No. 3, 12 (1955). 1291

Davis, J. H., Oehler, R., Method for restoring original appearance of impregnated leather. J. Am. Leather Chemists Assoc. 50, 38 (1955). 1407.

Davis, J.H., Oehler, R., Kinmouth, R. A., A pilot plant study of the process for treating heavv leather with polyisobutylene and other polymers. J. Am. Leather Chemists Assoc. 50, 16 (1955). 1240.

Davis, M. M., Hetzer, H. B., Relative strengths of forty aromatic carboxylic acids in benzene. J 60, 569 (1958) RP2871.

The ionic dissociation of 2, 4-, 2, 6-, 3, 4-dichlorobenzoic acids in water. J. Phys. Chem. 61, 123 (1957). 2368.

The ionic dissocation of 2,6-dimethvlbenzoic acid in water. J. Phys. Chem. 61, 125 (1957). 2369.

The relative basicities of tribenzylamine and tribenzylamine oxide in benzene and water. Preparation and properties of trihenzylamine oxide. J. Am. Chem. Soc. 76, 4247 (1954), 1195.

Davis, P., Leonard Euler's integral: a historical profile of the gamma function. Am. Math. Mo. 66, 849 (1959). 2974.

Linear functional equations and interpolation series. Pacific J. Math. 4, No. 4, 503 (1954). 1050.

On a problem in the theory of mechanical quadratures. Pacific J. Math. 5, Suppl. 1, 669 (1955). 1423. On the numerical integration of periodic analytic functions. Chapter in the book, On Numerical Approrimation, Edited by R. E. Langer, (University of Wisconsin Press, Madison, Wisconsin), p. 45, (1959). 3009 .

Uniqueness theory for asymptotic expansions in general regions. Pacific J. Math. 1, 849 (1957). 2400 . 
Davis, P., Antosiewics, H. A., Some implications of Liapunov's conditions for stability. J. Rational Mechanics and Anal. 3, No. 4, 447 (1954). 1142.

Davis, $P$., Cahill, W. H., On a computation of the capacity of a cube. Math. Tables and Other Aids to Computation VII, 272 (Oct. 1953). 779.

Davis, $P$., Fan, K., Complete sequences and approximations in normed linear spaces. Duke Math. J. 24, 183 (1957). 2065.

Davis, P., Pollak, H., Complex biorthogonality for certain sets of polynomials. Duke Math. J. 21, No. 4, 653 (1954). 975 .

Davis, P., Rabinowitz, P., A multiple-purpose orthonormalizing code and its uses. J. Assoc. Computing Machinery 1, No. 4, 183 (1954). 921.

Additional abscissas and weights for Gaussian quadratures of high order: values for $N=64,80$, and 96. J 60, 613 (1958) RP2875.

Numerical experiments in potential theory using orthonormal functions. J. Wash. Acad. Sci. 46, 12 (1956). 1789.

On the estimation of quadrature errors for analytic functions. Math. Tables and Other Aids to Computation 8, No. 48, 193 (1954). 1096.

Some Monte Carlo experiments in computing multiple integrals. Math. Tables and Other Aids to Computation 10, 1 (1956). 1875.

Davis, $P_{\text {., }}$ Walsh, J. L., On representations and extension of bounded linear functionals defined on classes of analytic functions. Trans. Am. Math. Soc. 76, No. 2, 190 (1954). 1093.

Davis, R., Gibson, K. S., Haupt, G. W., Spectral energy distribution of the International Commission on Illumination light sources A, B, and C. Condensation of this paper appeared in J. Opt. Soc. Am. 43, 172 (1953). 834.

Davis, R. M. Smith, E. K., Ellyett, C. D., The effect of sporadic E on VHF transmission in North America. IRE Spec. Govt. Issue 47, No. 5, 762 (1959). 3074.

Davis, W. D., Douglas, T. B., Ball, A. F., Ginnings D. C., Heat capacity of potassium and three potassiumsodium alloys between $0^{\circ}$ and $800^{\circ}$, the triple point and heat of fusion of potassium. J. Am. Chem. Soc. 74,2472 (1952). 461.

Davis, $W$. W., Holt, $A$. $W$., Computer memory uses conventional C-R tubes. Electronics 26, 178 (1953). 673.

Davisson, J. W., Burstein, E., Bell, E. E., Turner, W. J., Lipson, $H$. G., Infrared photoconductivity due to neutral impurities in germanium. Phys. Rev. 93, 65 (1954). 1039.

Day, F. H., X-ray calibration of radiation survey meters, pocket chambers and dosimeters. Ra-Det (Tech. Info. Div. ORE, AEC, Oak Ridge, Tenn.) 3, No. 4,3 (1950). 172

Dayhoff, E. S., Kerns, D. M., Theory of diffraction in microwave interferometry. J 64B 1, 1 (1960).

Dean, J., Chelton, D. B., Macinko, J., Hydrogen liquefaction by dual pressure process. Refrig. Eng. 65, No. 8,39 (1957). 2156.

Hydrogen liquefaction eycles. Proc. 1957 Cryogenic Eng. Conf. Paper A-1, p.1, Edited by K. D. Timmerhaus, Chem. Eng. Dept., University of Colorado (Boulder, Colorado) (1958). 2590.

Dean, J. W., Brown, E. H., Joule-Thomson process in the liquefaction of helium. J 60, 161 (1958) RP2834.

Dean, J. W., Chelton, D. B., Birmingham, B. W., Design and construction of a liquid hydrogen temperature refrigeration system. (1960) TN38 (PB151397).

Dean, J. W., Chelton, D. B., Strobridge, T. R., Birmingham, B. W., Mann, D. B., Helium refrigeration and liquefaction using a liquid hydrogen refrigerator for precooling. (1960) TN39 (PB151398).

Decker, G. E., Note on the adjustment of Mooney viscometer die closure. ASTM. Bull. No. 195, (TPl3) (1954). 1082.

Decker, G. E., Mandel, J., Mooney viscometer cure characteristics of Hevea rubber. ASTM. Bull. No. 209, 47 (1955). 1409.
Decker, G. E., Roth, F. L., Influence of variations in rotors, dies, and rate of shear on Mooney viscosity. India Rubber World 128, 339 (1953). 740.

Decker, G. E., Roth, F. L., Stiehler, R. D., Temperature control during mixing of rubber compounds. Rubber World 132, 482 (1955). 1526.

Decker, G. E., Stiehler, R. D., Temperature measurements in the Mooney viscometer. ASTM Bull. No. 195, (TP7) (1954). 1164.

Decker, $M$. T. Service area of an airborne television station. (1959) TN35 (PB151394).

Tacan coverage and channel requirements. IRE Trans. Aeronaut. Navigational Electronics. ANE-4, No. 3, 135 (1957), $2337 \mathrm{~A}$.

Transmission and reflection by a parallel wire grid. J 63D 1, 87 (1959)

Decker, M. T., Barghausen, A. F., Maloney, L. J., Measurements of correlation, height gain, and path antenna gain at $1046 \mathrm{Mc}$ on spaced antennas far beyond the radio horizon. IRE Conv. Record $3, \mathrm{Pt} .1,78$ (1955), 1401.

Decker, M. T., Janes, H. B., Stroud, J. C., An analysis of propagation measurements made at 418 megacycles per second well beyond the radio horizon (a digest). J 64D 3,255 (1960).

An analysis of propagation measurements made at $418 \mathrm{Me}$ well beyond the radio horizon. (1959) TN6 (PB151365).

Defandorf, $F . M$., A tree from the viewpoint of lightning. J. Wash. Acad. Sci. 45, 11 (1955). 1248.

The electrical resistance to the earth of a live tree. Am. Inst. Elec. Engrs. Trans. Paper No. 56-627, p. 1 (1956). 1928

Deitz, V. R., Current problems in refining with bone char. Sugar J, 49, 44 (1954). 984.

Deitz, V. R., Brown, J. M., Some economic aspects of the bone char process in sugar refining. Intern. Sugar J. (London, England) 52, 147 (1950). 132.

Deitz, V. R., Gee, A., Determination of phosphate by differential spectrophotometry. Anal. Chem. 25, 1320 (1953). 685 .

Pyrophosphate formation upon ignition of precipitated basic calcium phosphates. J. Am. Chem. Soc. 7r, 2961 (1955). 1465.

Deitz, V. R., Gee, A., Domingues, L. P., Determination of inorganic constituents in sucrose solutions. Anal. Chem. 26, 1487 (1954). 987.

Deitz, V. R., Liggett, B. W., Color and turbidity of sugar products. Advances in Carbohydrate Chem. 9, 247 (1954). 970.

Deitz, V. R., Loebenstein, W. $V$., Oxygen chemiadsorption on carbon adsorbents. J. Phys. Chem. 59, 481 (1955). 1438.

Deitz, V.R., Prosen, E. J., Reaction of carbon monoxide with graphite carbon at $450^{\circ} \mathrm{C}$. Nature 118, 109 $(1958), 2717$.

DeJuren, J. A., Chin, J., Manganese thermal neutron activation cross section. Phys. Rev. 99, No. 1, 191 (1955), 1397.

DeJuren, J. A., Rosenwasser, $H$. , Pulse-height measurements of recoils from $\mathrm{B}^{10}\left(n,{ }^{\alpha}\right) \mathrm{Li}^{7}$. Phys. Rev. 93, 831 (1954). 1123.

de Klerk, D., Adiabatic demagnetization and the temperature scale below $1^{\circ} \mathrm{K}$. Science 116, 335 (1952). 384 .

Magnetic properties below $1^{\circ} \mathrm{K}$. Phys. Today 6, No. 2, 4 (1953). 756.

de Klerk, D., Hudson, R. P., Pellam, J. R., Normal fluid concentration in liquid helium II below $1^{\circ} \mathrm{K}$. Phys. Rev. 89, 662 (1953). 774.

Second sound velocity measurements below $1^{\circ} \mathrm{K}$. Phys. Rev. 89, 326 (1953). 822.

DeLaVergne, L., Allisy, A., Wyckof, H. O., An intercomparison of the French and U.S. roentgen ray standards. Acta Radiol. 48, No. 6, 486 (1957). 2017.

DeLaVergne, L., Attix, F. H., Plate separation requirements for standard free-air ionization chambers. Radiology 63, No 6, 853 (1954). 1110. 
DeLaVergne, L., Attix, F. H., Ritz, V.H., Cavity ionization as a function of wall material. J 60, 235 (1958) RP2842.

DeLollis, $N . J ., \quad$ Note on the displacement pressure method for measuring the affinity of liquids for solids. J. Phys. Chem. 56, No. 2, 193 (1952). 490.

DeLollis, N. J., Reinhart, F. W., Loos, B. D., Evaluation of adhesives for acoustical tile. ASTM Bull. No. $169,57(1950) .55$

DeLollis, N. J., Rucker, N., Weir, J. E., Comparative strengths of some adhesive-adherent systems. Trans. Am. Soc. Mech. Engrs. 73, 183 (1951). 208.

Deming, L. S., Selected bibliography of statistical literature, 1930 to 1957: I. Correlation and regression theory. J 64B 1, 55 (1960).

Selected bibliography of statistical literature, 1930 to 1957: II. Time series. J 64B 1, 69 (1960).

de Minjer, $\dot{G}$. H., Brenner, A., Studies on electroless nickel plating. Plating 44, 1297 (1957). 2327.

Denison, I. A., Romanoff, $M$., Effect of exposure to soils on the properties of asbestos-cement pipes. Corrosion 10, 169 (1954). 998.

Denison, I. A., Schwerdtfeger, $W . J .$, Geometric factors in electrical measurements relating to corrosion and its prevention. Corrosion 2, 25 (1955). 1354.

Devaney, T. E., Smith, A. N., Fields in electrically short ground systems. J 63D2, 175 (1959).

Dever, J. L., Douglas, T. B., Heat content of lead from $0^{\circ}$ to $900^{\circ}$, and the heat of fusion. J. Am. Chem. Soc. 76, 4824 (1954). 1027

Lithium fluoride; heat content from $0^{\circ}$ to $900^{\circ}$, the melting point and heat of fusion. J. Am. Chem. Soc. 76, 4826 (1954). 1053.

Dever, J. L., Douglas, T. B., Epstein, L. F., Howland, $W$. $H$., Lithium ; beat content from $0^{\circ}$ to $900^{\circ}$, triple point and heat of fusion, and thermodynamic properties of the solid and liquid. J. Am. Chem. Soc. 7\%, 2144 (1955). 1390.

Devereaux, R. J., Fulk, M. M., Schrodt, J. E., Heat transfer through foams and powders. Proc. 1956 Cryogenic Eng. Conference, Paper E-3, p. 166. Edited by K. D. Timmerhaus, Chem. Eng. Dept., Univ. Colorado (Boulder, Colorado) (1957). 2147.

DeWane, H.J., Standard test for photoflash cells. Mag. of Standards 26, No. 5, 135 (1955), 1506.

Diamond, J. J., Flame photometric determination of strontium in portland cement. Anal. Chem. 27, 913 (1955). 1346.

The flame photometric determination of manganese in cement. Anal. Chem. 28, 328 (1956). 1930. Improvements in flame photometric determination of sodium in portland cement. Anal. Chem. 25, 1825 (1953), 736.

Dibeler, $V$. $H$., Analytical mass spectrometry. Org. Anal. 3, 387 (1956), 1626.

Isotope analysis using dimethylmercury. Anal. Chem. 27, 1958 (1955). 1381

Mass spectrometry. Anal. Chem. 26, 58 (1954). 1060; Anal. Chem. 28, 610 (1956). 1764.

Dibeler, V. H., Antkiw, S., Mass spectrum of gallium vapor. J.' Chem. Phys, 21, $1890 \quad(1953)$ 758

Dibeler, V. H., Bradt, P., Mohler, F. L., Mass spectra of aromatic hydrocarbons filtered from smoky air. J 60,615 (1958) RP2876.

Dibeler, V. H., Cloud, P. E., Jr., Friedman, I., Sisler, F. D., Microbiological fractionation of the hydrogen isotopes. Science 127, 13 (1958). 2635.

Dibeler, V. H., Franklin, J. L., Herron, J. T., Bradt, P. Mass spectrometric study of the decomposition of hydrazoic acid by the electric discharge, J. Am. Chem. Soc. $80,6188(1958), 2623$.

Dibeler, V. H., Franklin, J. L., Morris, P. P., Jr., Mass spectrometric study of the rate of thermal decomposition of hydrazoic acid. J 61, 41 (1958) RP2881.

Dibeler, V. H., Franklin, J. L., Reese, R. M., Electron impact studies of hydrazine and the methyl-sub stituted hydrazines. J. Am. Chem. Soc. 81, 68 (1959). 2939.
Dibeler, V. H., Franklin, J. L., Reese, R. M., Krauss, M., Ionization and dissociation of hydrazoic acid and methyl azide by electron impact. J. Am. Chem. Soc. 80, 298 (1958). 2604.

Dibeler, V. H., Herron, J. T., Franklin, J. L., Bradt, P., Kineties of nitrogen atom recombination. J. Chem. Phys. Letters 29, No, 1, 230 (1958); J. Chem Phys. $\mathbf{3 0}, 879$ (1959). 2972.

Dibeler, V. H., Hipple, J. A., Mass spectrometry. Anal. Chem. 24, 27 (1952). 478.

Dibeler, $V .{ }^{\prime} H_{.}$, Krauss, $M$. ., Reese, $R$. M., Multiple ionization of rare gases by electron impact. J 63 A 3, 201 (1959).

Dibeler, $V . H$. Lengel, J. $H$., Preparation of small quantities of germanium tetramethyl. J. Am. Chem. Soc. 74, 2683 (1952). 517.

Dibeler, V. H., Mohler, F. L., Cuinn, E., Redetermination of mass spectra of deuteromethanes. J 61, 171 (1958) RP2896.

Dibeler, V. H., Mohler, F. L., Reese, R. M., Ionization and dissociation of methyl siloxanes by electron impact. J. Chem. Phys. 21, 180 (1953). 747.

Some ions of high kinetic energy in mass spectra of polyatomic molecules. J. Chem. Phys. 22, 394 (1954), 1144.

Dibeler, V. H., Reese, R. M., Ionization and dissociation of $\mathrm{NF}_{3}$ by electron impact. J. Chem. Phys. 24, 1175 (1956). 1749.

Mass spectrometry. Anal. Chem. 30, 604 (1958). 2624 .

Multiple ionization of sodium vapor by electron impact. J, Chem. Phys, 31, 282 (1959). 2996.

Dibeler, $V$. H., Reese, R. M., Franklin, J. L., Electron impact studies of sulfur dioxide and sulfuryl fluoride. J. Chem. Phys, 29, No. 4, 880 (1958). 2542.

Ionization and dissociation of oxygen difluoride by electron impact. J. Chem Phys. 27, 1296 (1957). 2169.

Dibeler, V. H., Reese, R. M., Mann, D. E., Ionization and dissociation of perchlorylfuoride by electron impact. J. Chem. Phys. 2\%, 176 (1957). 2170.

Dibeler, $V . H$., Reese, $R$. M. Mohler, $F$. L., Ionization and dissociation of hexafluorobenzene by electron impact. J. Chem. Phys, 26, No. 2, 304 (1957). 2168. The ionization potential of the $\mathrm{CF}_{3}$ radical and some fluorocarbon bond energies obtained by electron impact. J. Chem. Phys, 20, 761 (1952). 580.

Dibeler, V. H., Wacks, $M$. E., Electron impact studies of aromatic hydrocarbons. I. Benzene, napthalene, anthracene, and penanthrene. J. Chem. Phys. 31, No. 6, $1557(1959), 2938$

Dickenson, J.A., A novel rocket hoist. Elevator World 6, No. 2, 13 (1958). 2430.

Dickman, $R$. $H$., The rating of water-current meters. Water Power (Westminister, England) 3, 330 (1951). 340 .

Dickson, F. H., Crysdale, J. H., Herbstreit, J. W., Egli, J. J., Wickizer, G. S., Rebuttal to comments on "Large reduction of VHF transmission loss and fading by the presence of a mountain obstacle in beyondline-of-sight paths", by F. H. Dickson, J. J. Egli, J. W. Herbstreit, and G. S. Wickizer. (Pub. in Proc. IRE 41, 967, (1953)). Proc. IRE 43, 627 (1955). 1384.

Dickson, F. H., Egli, J.J., Herbstreit, J. W., Wickizer, G. S., Large reductions of VHF transmission loss and fading by the presence of a mountain obstacle in beyond-lineof-sight paths. Proc. IRE 41, 967 (1953). 750.

Dickson, G., Forziati, A. F., Lawson, M. E. Jr., Fluorescense of teeth reveals structure. N.Y. J. Dentistry 23, 299 (1953). 721.

Dickson, G., Forziati, A. F., Lawson, M. E., Jr., Schoonover, I. C., Fluorescence of teeth: a means of investigating their structure, J. Am. Dental Assoc. 45, 661 (1952). 452.

Dickson, G., Grunewald, A. H., Paffenbarger, G. C., The effect of molding processes on some properties of denture resins. J. Am. Dental Assoc. 44, 269 (1952). 572. 
Dickson, G., Grunewald, A. H., Paffenbarger, G. C., Schoonover, $I$. C., Silicate cement: method of mixing in a closed container to prevent effects of exposure to atmosphere. J. Am. Dental Assoc. 46, 184 (1953). 827.

Dickson, G., Hartley, J. L., Hudson, D. C., Sweeney W. T., Development of methods for evaluation of rotating dental diamond abrasive instruments. J. Am. Dental Assoc. 54, 637 (1957). 2085.

Dickson, G., Hudson, D. C., Kumpula, J. W., A panoramic X-ray dental machine. U.S. Armed Forces Med. J. 8, No. 1, 46 (1957). 1993.

Dickson, G., Lyon, H. W., Schoonover, I. C., Effectiveness of vacuum investing in the elimination of surface defects in gold castings. J. Am. Dental Assoc. 46, 197 (1953). 697.

Dickson, G., Lyon, H. W., Schoonover, I. C., Mechanism of hygroscopic expansion of dental casting investments. J. Dental Research 34, 44 (1955). 1405.

Dickson, G., Mitchell, J.A., Schoonover, I. C., Vacher, H. C., Some factors affecting the dimensional stability of the $\mathrm{A} g-\mathrm{S} n(\mathrm{C} u-\mathrm{Z} n)$ amalgams. J. Dental Research 34, No, 2, $273(1955) .1493$.

Dickson, G., Mowery, W. E., Burns, C. L., Sweeney, W. T., Dimensional stability of denture base resins. J. Am. Dental Assoc. 57, 345 (1958). 2523.

Dickson, G., Paffenbarger, G. C., Swaney, A. C., Schoonover, I. C. American Dental Assoc. Specification No. 9 for dental silicate cement. J. Am. Dental Assoc. 40, $186(1950) .20$.

Dickson, G., Rupp, N. W., Lawson, M. E., Jr., Sweeney, $W$. T., A method for measuring the mucosal surface contours of impressions, casts, and dentures. J. Am Dental Assoc. 54, 24 (1957). 1987.

Dickson, G., Ryge, G., Schoonover, I. C., Dental amalgam: the effect of mechanical condensation of some physical properties. J. Am. Dental Assoc. 45, 269 (1952). 423.

Diehm, I. C., Computer aids to code checking. Proc. Assoc. Computing Machinery (Toronto, Canada), p. 19 (Assoc. for Computing Machinery, New York, N.Y.) (1952). 413.

Digges, $T \cdot \dot{G}$., $\quad$ Boron needs well-made steel plus intelligent heat treatment. (Part of a panel discussion on "Saving precious alloys by intelligent use of alternatives"). Metal Progr. 59, 823 (1951). 199.

Boron steels. Symp, on Production, Properties, and Applications of Alloy and Special Steels, Natl. Met. Lab. India (1956). 1642.

The action of boron in enhancing the hardenability of steel. SAE J. 61, 24 (1953). 851.

Digges, T. G., Jenkins, W. D., Influence of strain rate and temperature on the creep of cold drawn ingot iron. Trans. Am. Soc. Metals 42, 1128 (1950). 73.

Dill, $R$. S., Specifications, standards and codes, governmental aspects. Symp. Am. Soc. Heating and Air Conditioning Engrs. "Problems of Standards and Codes", p. 16 (1956). 1880.

The freezing of water in pipes and vessels. Heating and Ventilating $\mathbf{5 0}, 96$ (1953). 861.

Diniak, A. W., Hughes, E. E., Fujii, M., Determination of water content in Freon-12 circulating in a refrigeration system. Retrig. Eng. 62, No. 2, 56 (1954). 990.

Diniak, A. W., Weaver, E. R., Hughes, E. E., Determination of water vapor from the change in electrical resistance of a hygroscopic film. J 60, 489 (1958) RP2864.

Diorio, A. F., Mandelkern, L., Posner, A. S., Laki, K. Mechanism of contraction in the muscle ilbre-ATP system. Proc. Natl. Acad. Sci, 45, 814 (1959), 2986.

Diorio, A. F., Mandelkern, L., Roberts, D. E., Reversible contractile processes in fibrous macromolecules. J. Am. Chem. Soc. 80, 500 (1958), 2743.

Diorio, A. F., Perlof, A., Posner, A. S., Refinement of the hydroxyapatite structure. Acta Cryst. 11, 308 (1958). 2724.

Disney, R. T., Crichlow, W. Q., Report on revision of atmospheric radio noise data. CCIR Rept. No. 65, VIII Plenary Assembly, Warsaw (1956) (Intern, Radio
Consultat. Comm., Secretar., Geneva, Switzerland). 1851.

Disney, R. T., Crichlow, W. O., Samson, C. A., Jenkins, $M$. A., Quarterly radio noise data-March, April, May 1959. (1960) TN18-2 (PB151377-2).

Disney, R. T., Crichlow, W Q., Samson, C. A., Jenkins, M.A., Radio noise data for the International Geophysical Year July 1, 1957-December 31, $1958 . \quad$ (1959) TN18 (PB151377).

Disney, R. T., Samson, C. A., Operating instructions for ARN-2 auxiliary log-linear noise recorder. (1960) TN45 (PB151404).

Ditmars, D. A., Ginnings, D. C., Thermal conductivity of beryllium oxide from $40^{\circ}$ to $750^{\circ} \mathrm{C}$. J 59, 93 (1957) RP2775.

Dixon, H. D., Glasgow, A. R. Jr., Ross, G. S., Horton, A. T., Enagonio, D., Saylor, C. P., Furukawa, G. T., Reilly, M. L., Henning, J. M., Comparison of eryoscopic determinations of purity of benzene by thermometric and calorimetric procedures. Anal. Chim. Acta 17, 54 (1957); Proc. of I.U.P.A. Intern. Symp. on Purity Control by Thermal Analysis (Amsterdam, The Netherlands) (April 1957). 2062.

Djurle, E., Rodney, W. S., Infrared phase contrast refractometer. J. Opt. Soc. Am. 48, No. 6, 388 (1958). 2600 .

Doan, A., Kushner, L. M., Hubbard, W. D., Light scattering measurements of a fractionated nonionic detergent. J. Phys. Chem. 61, 371 (1957). 2176.

Doderer, G. C., McIntyre, D., Absolute light-scattering photometer: I. Design and operation. J 62, 153 (1959) RP2946.

Doderer, G. C. McIntyre, D., O'Mara, J. H., Design and performance of a block-type osmometer. J 62, 63 (1959) RP2931.

Doering, W. P., Caswell, R. S., Gabbard, R. F., Padgett, $D$. W., Attenuation of 14.1 Mev neutrons in water. Nuclear Sci. and Engr. 2, 143 (1957). 2032.

Doggett, J. A., Berger, $M$. J., Response function of NaI (Ti) scintillation counters. Rev. Sci. Instr. 27 , No. 5, 269 (1956). 1856.

Doggett, J. A., Spencer, L. V., Elastic scattering of electrons and positrons by point nuclei. Phys. Rev. 103, No. 6, 1597 (1956). 1694.

Doherty, R. H., Pulse sky wave phenomena observed at 100 Kc. Symp. on the Prop. of V.L.F. Radio Waves, Sponsored by the NBS Boulder Laboratories and IRE Prof. Group on Ant. and Prop., January 23-25, 1957 (Boulder, Colo.), Abstract, Paper No. 44, V. 3 (1957). 2274 .

Doherty, R. H., Hefley, G., Berger, E. L., An intermittentaction camera with absolute time calibration. J 64C2, $159(1960)$.

Domen, S. R., McElhinney, J., Zendle, B., Calorimetric determination of the power in a $1400 \mathrm{kev}$ X-ray beam. Radiol. Research 6, No. 1, 40 (1957). 2047.

Domingues, L. P., Gee, A., Deitz, V. R., Determination of inorganic constituents in sucrose solutions. Anal. Chem. 26, 1487 (1954). 987.

Donoghue, J. J., Mong, L. E., Zirconia and alumina shapes. Am. Ceram. Soc. Bull. 29, 405 (1950). 173.

Dorsey, N. E., Eisenhart, $C$, , On absolute measurement. Sci. Monthly 77, 103 (1953). 780.

Dougherty, H. T., The propagation characteristics of the frequency band $152-162 \mathrm{Mc}$ which is available for marine radio communications. Appendix $\mathrm{F}$ to report of Spec. Comm. 19 of Racio Tech. Comm. for Marine Servic «s "Study of a reliable short range radio telephone system. (Washington, D.C.) (1956). 1945.

Dougherty, H. T., Kirby, R. S., McOuate, P. L., VHF propagation measurements in the Rocky Mountain region. IRE Trans. Vehicular Commun. VC-6, 13 (1956). 1972.

Douglas, C. A., Computation of effective intensity of flashing lights. Illum. Eng. 52, No. 12, 641 (1957). 2066.

Photometer for measurement of the effective intensity of condenser-discharge lights. Illum. Eng. 53, 205 (1958). 2685. 
Douglas, T. B., Specific heats of liquid metals and liquid salts. Trans. Am. Soc. Testing Materials 79, 23 (1957). 2306.

The zirconium-hydrogen system: Some thermodynamic prorerties from a heat content study. J. Am. Chem. Soc. 80, 5040 (1958). 2839.

Douglas, T. B., Ball, A. F., Ginnings D. C., Davis, W. D., Heat capacity of potassium and three potassiumsodium alloys retween $0^{\circ}$ and $800^{\circ}$, the triple point and heat of fusion of potassium. J. Am. Chem. Soc. 74, 2472 (1952). 462.

Douglas, T. B., Ball. A. F., Torgesen, J. L., Heat capacity of crystalline dextrose betweer. $25^{\circ}$ and $95^{\circ} \mathrm{C}$. J. Am. Chem. Soc. 73, 1360 (1951). 246.

Douglas, T. B., Dever, J. L., Heat content of lead from $0^{\circ}$ to $900^{\circ}$, and the heat of fusion. J. Am. Chem. Soc. 76, 4824 (1954). 1027.

Lithium fluoride; heat content from $0^{\circ}$ to $900^{\circ}$ the melting point and heat of fusion. J. Am. Chem. Soc. 76, 4826 (1954). 1053.

Douglas, T. B., Epstein, L. F., Dever, J. L., Howland, $W$. $H$., Lithium: heat content from $0^{\circ}$ to $900^{\circ}$, triple point and heat of $\mathrm{f}^{\prime} \mathrm{sion}$, and thermodynamic properties of the solid and liquid. J. Am. Chem. Soc. 77, 2144 (1955). 1390.

Douglas, T. B., Ginnings, D. C., Ball, A. F., Specific heat of beryllium hetween $0^{\circ}$ and $900^{\circ}$ C. J. Am. Chem. Soc. 73, 1236 (1951). 312.

Douglas, T. B., Harman, $A$. $W$., Enthalpy and heat capacity from $0^{\circ}$ to $900^{\circ} \mathrm{C}$ of three nickel-chromiumiron allovs of different carbon contents. J 60, 563 (1958) RP2870.

Douglas, T. B., Harman, A. W., Heat content of sodium borohydride and of rotassium rorohydride from $0^{\circ}$ to $400^{\circ} \mathrm{C}$. J 60, 117 (1958) RP2828.

Douglas, T. B., Victor, A. C., Heat content of zirconium and five compositions of zirconium hydride from $0^{\circ}$ to $900^{\circ} \mathrm{C}$. J 61, 13 (1958) RP2878.

Dover, J. J., Caldwell, $F$. R., Broida, H. P., Combustion in Bunsen flames. Ind. Eng. Chem. 43, 2731 (1951). 207.

Draper, J. W., Flynn, T. M., Timmerhaus, K. D., Weitzel, $D$. $H$., Separation of hydrogen isotopes by multicomnonent distillation, Proc. 1957 Cryogenic Eng. Conf., Paper A-6, p. 58, Edited by K. D. Timmerhai's, Chem. Eng. Dent., Univ. of Colorado, (Boulder, Colo.) (1958). 2751.

Draper, J. W., Flynn, T. M., Weitzel, D. H., Timmerhaus, K. D., Vander Arend, P.C., Distillation of hydrogendeuterium mixtures. Proc. 1956 Cryogenic Eng. Conf., Paper A-6, p. 39, Edited by K. D. Timmerhaus, Chem. Eng. Dept., Univ. Colorado (Boulder, Colo.) (1957). 2093.

Draper, J. W., Purcell, J. R., Weitzel, D. H., A unique thermal conductivity gas analyzer. Proc. 1957 Cryogenic Eng. Conf. Parer D-4, p. 191, Edited by K. D. Timmerhaus, Chem. Eng. Dept., Univ. Colorado (Boulder, Colo.) (1958). 2443.

Draper, J. W., Weitzel, D, H., Loebenstein, W. V., Park, $O$. E., Orthopara catalvsis in liquid-hydrogen production. J 60, 221 (1958) RP2840.

Draper, J. W., Weitzel, D, H., Park, O. E., Timmerhaus, K. D. Van Valin, C. C., Catalysis of the ortho-rarahydrogen conversion. Proc. 1956 Cryogenic Eng. Conf., Paper A-4, p. 12. Edited by K. D. Timmerhavs, Chem. Eng. Dept., Univ. Colorado (Boulder, Colo.) (1957). 2048.

Draper, J. W., Weitzel, D. H., Van Valin, C. C., Design data for ortho-parahydrogen converters. Proc. 1957 Cyrogenic Eng. Conf., Paper B-2, p. 73, Edited by K. D. Timmerhaus, Chem. Eng. Dept., Univ. Colorado (Boulder, Colo.) (1958). 2514,

Dressler, $K$., Atsorption spectrum of vibrationally excited $\mathrm{N}_{2}$ in active nitrogen. J. Chem. Phys. Letter to Editor 30, 1621 (1959). 2887.

Dressler, $R$. F., A discussion of "design of corrugated diaphragms". Trans. Am. Soc. Mech. Eng. 79, 61 (1957), 1981 .

Comparison of theories and experiments for the hydraulic dam-break wave. Proc. Intern. Union Geodesy and Geophys. (Rome, Italy) 3, 319 (1954) 974.

Invited discussion on the Haringx diaphragm theory. Trans Am. Soc. Mech. Engrs. 79, No. 1, 61 (1957). 2167 .

Unsteady nonlinear waves in sloping channels. Abstracts of Short Communications and Scientific Programme, Intern. Cong. of Mathematicians (Edenburgh, Scotland) p. 138 (1958); Proc. Roy Soc. [A] 247, 186 (1958). 2857.

Dressler, $R$. F., Chiarulli, $P$., Condensation interfaces in two-phase flows. J. Appl. Phys. 28, 990 (1957). 2067.

Dressler, $R$. F., Vinti, $J$. P., The functional synthesis of linear plots. J 64C2, 115 (1960).

Dressler, R. F., Wildhack, W. A., Lloyd, E. C., Investigations of the prorerties of corrugated diaphragms. Am. Soc. Mech. Engrs, preprint 55-A-181 (1955). 1378; Trans. ASME 79, 65 (1957). 2166.

Driscoll, R. L., Measurement of current with a Pellattype electrodynamometer. J 60, 287 (1958) RP2845.

Driscoll, $R$. L., Bender, $P$. L., A free precession determination of the proton gvromagnetic ratio. IRE Trans. Instrumentation $\mathbf{I}-7$, No. 3-4, 176 (1958). 2422.

Proton gyromagnetic ratio. Phys. Rev. Letters 1, No. 11,413 (1958). 2706.

Driscoll, R. L. Cutkosky, R. D., Measurement of current with the National Bureau of Standards current balance. J 60, 297 (1958) RP2846.

Driscoll, R. L., Thomas, J. L., Cutkosky, R. D., Status of absolute measurements at the National Bureau of Standards. Precès-Verbaux, C.I.P.M., p. 36 (1958). 2781 .

Duncan, R. C., Campbell, W. R., Irwin, L. K., Stress studies of bulkhead intersections for welded tankers. Welding J. 31, No. 2, 68 (1952). 556.

Dunfee, B. L., An A-C bridge for the audio-frequency range. Commun. and Electron. Am. Inst. Elec. Engrs. No. 24, 123 (1956). 1615.

Dunfee, B. L., Peterson, C., Hermach, F. L., Methods for measuring the "Q" of large reactors. Am. Inst. Elec. Engrs. Trans. Paper No. 56-87 (1956). 1776.

Durfee, $W$. H., Heat flow in a fluid with eddying flow. J. Aeronaut, Sci. 23, 188 (1956). 1727.

Durieux, M., van Dijk, H., Clement, J. R., Logan, J. K. The "1958 $\mathrm{He}^{4}$ scale of temperatures", Part 2. Tables for the 1958 temperature scale. J 64A1, 1 (1960); (1960) Mono. 10.

Dutton, E. J., Bean, B.R., On the calculation of the departires of radio wave bending from normal. $J$ 641 3, 259 (1960).

Dvoretsky, A., Erdos, P., Some problems on random walks in space. 2d Berkely Symp. Math. Stat. and Prob. held by the University of California from July 31 to August 12, 1950, p. 353 (1951). 310.

Dvoretsky, A., Kiofer, J., Wolfowitz, J., Sequential decision problems for processes with continuous time parameter. Testing hypostheses. Ann. Math. Stat, 24, No. 2, 254 (1953). 824.

Dvoretzky, A., Wald, A., Wolfowitz, J., Elimination of randomization in certain statistical decision procedures and zero-sum two-person games. Ann. Math. Stat. 22, 1 (1951). 231.

Relations among certain ranges of vector measures. Pacific J. Math. 1, 59 (1953), 299.

\section{$\mathbf{E}$}

Eaton, H. N., Those government researchers. Plumbing and Heating Business 13, No. 4, 58 (1950). 158.

Eaton, H. N., French, J. L., Fixture unit ratings as used in plumbing system design. Housing and Home Finance Agency Housing Research Paper No. 15 (1951). 239.

Eaton, H. N., Wyly, R. S., Capacity of plumbing stacks. The Plumher and $\mathbf{J}$. of Heating (London, England) 76, No. 912,26 (1955). 1281. 
Edelman, S., Brooks, R., Saito, S., Jones, E., and Smith, E. R., A stroboscopic vibration analyzer. J $63 \mathrm{C} 2$, 97 (1959).

Edelman, S., Cook, R. K., Waterhouse, R. V., Berendt, R. D., Thompson, M. C., Jr., Measurements of correlation coefficients in reverberant sound fields. J. Acoust. Soc. Am. 27, No. 6, 1072 (1955). 1399.

Edelman, S., Jones, E., Smith, E. R., Some developments in vibration measurement. J. Acoust. Soc. Am. 27, $728(1955) .1492$.

Edelman, S., McKinney, J. E., Marvin, R. S., Apparatus for the direct determination of the dynamic bulk modulus, J. Appl. Phys. 27, 425 (1956). 1629.

Edelman, S., Smith, E. R., Jones, E., Schmidt, V. A., Stroboscopic interferometer for vibration measurement. J. Acoust. Soc. Am. 30, 867 (1958). 2784.

Edelman, S., Sulzer, P. G., Smith, E. R., High-speed stroboscope for accelerometer calibration. Rev. Sci. Instr. 25, No. 8, 837 (1954). 1031.

Eden, M., Bates, R. G., Resolution of the dissociation constants of $\mathrm{d}, l$-malic acid from $0^{\circ}$ to $50^{\circ} \mathrm{C}$. J 62, 161 (1959) RP2947.

Edgecombe, S. H. C., Norrish, R. G. W., Thrush, B. A., The flash photolysis of chlorine monoxide. Proc. Roy. Soc. (London, England) 243, 24 (1957). 2357.

Edlow, M. H., Plumb, H. H., Constant temperature liquid helium bath and reproducibility of resistance thermometers. Rev. Sci. Instr. 30, 376 (1959). 2916.

Edwards, D. M., Linnig, F. J., Peterson, J. M., Acherman, W. L., Chemical analysis of GR-S by complete solution procedures. Gross constituents in GR-S containing soap. Anal. Chem. 25, 1511 (1953). 666.

Egli, J. J., Crysdale, J. H., Herbstreit, J. W., Dickson, F. H., Wickizer, G. S., Rebuttal to comments on "Large reduction of VHF transmission loss and fading by the presence of a mountain obstacle in beyondline-of-sight paths," by F. H. Dickson, J. J. Egli, J. W. Herkstreit, and G. S. Wickizer. (Publ. in Proc. IRE 41, 967 (1953)). Proc. IRE 43, 627 (1955). 1384.

Egli, J. J., Dickson, F. H., Herbstreit, J. W., Wickizer, G. S., Large reductions of VHF transmission loss and fading by the presence of a mountain obstacle in beyond-line-of-sight paths. Proc. IRE 41, 967 (1953). 750 .

Ehrlich, M., A photographic personnel dosimeter for Xradiation in the range from $30 \mathrm{kev}$ to beyond $1 \mathrm{Mev}$. Radiology 68, No. 4, 549 (1957). 1994.

Disaster monitoring with amateur photographic film and with dental X-ray film. Radiology 68, No. 2, 251 (1957). 2091.

Reciprocity law for X-rays, Part 2: failure in the reversal region. J. Opt. Soc. Am. 46, No. 10, 801 (1956). 1839.

Ehrlich, M., Levinos, S., Stabilization film processing for $\mathrm{X}$ - and $\gamma$-ray dosimetry. Nucleonics 14, No 7,72 (1956). 1891.

Ehrlich, M., McLaughlin, W. L., Film badge dosimetry: how much fading occurs? "Nucleonics 12, No. 10, 44 (1954). 1021.

Limitations in the photographic dosimetry of $\mathrm{X}$ and gamma-rays. Radiation Research 9, No. 1, 148 (1958). 2611 .

Photographic dosimetry at total exposure levels below $20 \mathrm{mr}$. (1959) TN29 (PB151388).

Reciprocity law for X-rays, Part 1: Validity for highintensity exposures in the negative region. J. Opt. Soc. Am. 46, No. 10, 797 (1956). 1838.

Ehrlich, P., Scott, A. H., Richardson, J. F., Dielectric measurements up to $500^{\circ} \mathrm{C}$, symposium on temperature stability of electrical insulating materials. Am. Soc. Testing Materials Spec. Tech. Publ. No. 161 (1954). 992.

Eike, W. $\dot{G}$. Jr., Otto, E. M., The gassing of dry cells. J. Electrochem. Soc. 104, 199 (1957). 2359.

Eisenhart, C., Review of "Proceedings of the Berkeley Symp. on Mathematical Statistics and Probability". Psychometrica 15, 448 (1950). 114.

The principle of randomization in the design of ex- periments. Proc. Conf. on Design of Experiments, Off. Ord. Research Rept. 57-1, 15-16, (1957). 2378. The reliability of measured values. Part I. Fundamental concepts. Photogrammetric Eng. 18, No. 3, $542(1952)$. 594.

Eisenhart, $C$., Dorsey, $N$. E., On absolute measurement. Sei. Monthly 77, 103 (1953). 780.

Eisenhart, $C_{\text {. }}$ Zelen, $M_{\text {., }}$ Elements of probability. Handbook of Physics, Edited by E. U. Condon and H. Odishaw, Chapter 12, Pt. 1, p. 1-134 (McGraw-Hil Book Co., Inc., New York, N.Y.) (1958). 2500.

Eisenstein, J. C., Pryce, M. H. L., The electronic structure and magnetic properties of uranyl-like ions, II. Plytonyl. Proc. Roy. Soc. (London, England) 238, 31 (1957). 2355.

Elbourn, R. D., Input-output, key or bottleneck. Proc. Computer Applications Symp., October 29-30 (Armour Research Foundation, Chicago, Illinois), p. 69 (1958). 2601.

The role of electronic computers in air traffic control. IRE Conf. Record 5, Pt. 8, 114 (1957). 2380.

Ellenwood, R. C., Hurlburt, E. H., The determination of impedance with a double-slug transformer. Proc. IRE 40, 1690 (1952). 570.

Ellenwood, $R$. C., Ryan, $W$. E., A VHF and microwave matching termination. Proc. IRE 41, 104 (1953). 638.

Ellenwood, R. C., Sorrows, H. E., Ryan, W. E., Evaluation of coaxial slotted-line impedance measurements. Proc. IRE 39, 162 (1951). 234.

Ellinger, G. A., Reinhart, F. M., Effect of 20-year marine exposure on some aluminum alloys. Am. Soc. Testing Materials Spec. Tech. Publ. No. 175, 47 (1956). 1689.

Ellinger, G. A., Williams, M. L., Investigation of structural failures of welded ships. Welding J. 32, 498-S (1953). 746 .

Elliott, D. A., Representation of nonlinear functions of two input variables on analog equipment. Trans. Am. Soc. Mech. Engrs. 79, 489 (1957). 2285.

Elliott, R. S., Cottony, H. V., Jordon, E. C. Rumsey, V. H., Siegel, K. M., Wait, J. R., Woodyard, O. C., Antennas and waveguides, and annotated bibliography. U.S.A. National Committee report, International Sci. Radio Union Subcommission 6.3. IRE Trans. Ant. Prop. AP-7, 87 (1959). 2897.

U.S.A. National Committee report URSI Subcommission 6.3, antennas and waveguides, and annotated bibliography. Proc. 12th Gen. Assembly, Intern. Sci. Radio Union, August-September 1957 (Boulder, Colo.) 11, Pt. 6, 121 (1957). 2402.

Ellyett, C. D., Davis, R. M., Smith, E. K., The effect of sporadic E on VHF transmission in North America. IRE Spec. Govt. Issue 47, No. 5, 762 (1959). 3074.

Ellyett, C. D., Leighton, H. I., Solar cycle influence on the lower ionosphere and on VHF forward scatter. Proc. IRE 46, No. 10, 1711 (1958). 2759.

Ellyett, C. D., Watts, J. M., Stratification in the lower ionosphere. J 63D2, 117 (1959).

Elvey, C. T., Roach, F. E., Aurora and airglow. Sci. Ámerican 193, No. 3, 140 (1955). 1271.

Enagonio, D., Glasgow, A. R., Jr., Ross, G. S., Horton, A. T., Dixon, H. D., Saylor, C. P. Furukawa, G. T., Reilly, M. L., Henning, J. M., Comparison of cryoscopic determinations of purity of benzene by thermometric and calorimetric procedures. Anal. chim. acta 1\%, 54 (1957); Proc. of I.U.P.A. Intern. Symp. on Purity Control by Thermal Analysis (Amsterdam, The Netherlands, April 1957). 2062.

Engel, O. G., A model for epitaxy in metal-rock salt pairs. J. Chem. Phys. 20, No. 7, 1174 (1952). 367. Erosion damage to solids caused by high-speed collision with rain. J 61, 47 (1958) RP2882.

Fragmentation of waterdrops in the zone behind an air shock. J 60, 245 (1958) RP2843.

Pits in metals caused by collision with liquid drops and rigid steel spheres. J 64A1, 61 (1960).

Pits in metals caused by collision with liquid drops and soft metal spheres. J 62, 229 (1959) RP2958. 
Engen, G. F , A refined X-band microwave microcalorimeter. J' 63C1, $77\left(19^{-} 9\right)$.

A self-balancing direct-current bridge for accurate bolometric power measurements. J 59, 101 (1957) RP2776.

Amplitude stabilization of a microwave signal source. IRE Trans. Microwave Theory and Tech. MTT-6, 202 (1958). 2452.

Recent developments in the field of microwave power measurements at the National Bureau of Standards. IRE Trans. Instrumentation I-7, No. 3-4, 304 (1958). 2720.

Engen, G. F., Beatty, R. W., Mierowave attenuation measurements with accuracies from 0.0001 to 0.06 , decibel over a range of 0.01 to 50 decibels. J 64 C2, 139 (1960).

Epstein, L. F., Douglas, T. B., Dever, J. L., Howland, W. $H$., Lithium: heat content from $0^{\circ}$ to $900^{\circ}$, triple point and heat of fusion, and thermodynamic properties of the solid and liquid. J. Am. Chem. Soc. 7\%, 2144 (1955). 1390.

Erdös, P., Dvoretsky, A., Some problems on random walks in space. 2nd Berkeley Symp. on Math. Stat. and Prob. held by the University of California from July 31 to August 12,1950 , p. 353 (1951). 310

Erdös, $P$., Hunt, G. A., Changes of sign of sums of random variables. Pacific J. Math. 3, 673 (1953). 662.

Ernst, A. A., Skramstad, H. K., Nigro, J. P., An analogdigital simulation for the design and improvement of man-machine systems. 1957 Eastern Joint Computer Conf. Proc. (Inst. Radio Engrs., New York, N.Y.) (1958). 2453.

Ernst, H., Hayward, R. W., Hoppes, D. D., The disintegration of $\mathrm{Ba}^{113}$. Phys. Rev. 93, 916 (1954). 1178. The disintegration of $\mathrm{Nb}^{91}$ and $\mathrm{Nb}^{92}$. Bull. Am. Phys. Soc. 29, No. 7, 16A (1954). 1179.

Erving, J. J., O'Leary, M. J., Scribner, B. W., Missimer, $J$. $K$., Manufacture of paper from glass fibers. Tappi 35, No. 7, 289 (1952). 477.

Eubank, $W$. R., Precision thermostat for high temperatures. Rev. Sci. Instr. 21, 845 (1950). 110.

Eubanks, A. G., Crandall, J. R., Richmond, J. C., Flexibility of thin porcelain enameled sheet steel. Bull. Am. Ceram. Soc. 36, No. 2, 59 (1957). 2126.

Eubanks, A. G., Moore, D. G., Effect of the oxygen content of the furnace atmosphere on the adherence of vitreous coatings to iron. J. Am. Ceram. Soc. 38, No. 7, $226(1955) .1329$.

Influence of copper ions on the adherence of vitreous coatings to stainless steel. J. Am. Ceram. Soc. 39, No. 10,357 (1956). 1737.

Eubanks, A. G., Richmond, J. C., Moore, D. G., Studies of oxidation behavior of enameling iron during firing. Finish 11, No. 5, 50 (1954). 1153.

Evans, E. H., Swanson, H. E., Cook, M. I., Isaacs, T., Standard X-ray diffraction powder patterns. (1960) C539, Vol. 9.

Evans, R. C., Goodrick, R. B., Thermal batteries. J. Electrochem. Soc. 99, No. 8, 207 (1952). 602.

Evans, R. D., Curtiss, L. F., Johnson, W., Seaborg, G. T. Units of radioactivity. 'Rev. Sci. Instr. 21, 94 (1950).' 163.

Everett, A. J., Minkof, G. J., Broida, H. P., Ultraviolet spectrophotometry of low pressure explosions. Fifth (Intern.) Symp. on Combustion, p. 779 (Reinhold Publ. Corp., New York, N.Y. 1955). 1565.

Everitt, W. L., Smith, N., Condon, E. U., Bailey, S. L., Fink, D. G., The present status of eolor television; a report by the Senate Advisory Committee on Color Television. Proc. IRE 38, $980(1950) .154$.

Faegre, M. L., Bain, K., Wyly, R. S., Behavior of young children under conditions simulating entrapment of children in refrigerators. Pediatrics 22 , No. 4, Pt. I, 628 (1958). 2471.
Fan, K., A comparison theorem for eigenvalues of normal matrices. Pacific J. Math. 5, Suppl. 2, 911 (1955). 1231.

Existence theorems and extreme solutions for inequalities concerning convex functions or linear transforms. Math. Z. (Berlin, Germany) 68, 205 (1957). 2115. Minimax theorems. Proc. Natl. Acad. Sci. 39, 42 (Jan. 1953). 768.

Note on circular disks containing the eigenvalues of a matrix. Duke Math. J. 25, 441 (1958). 2650. On systems of linear inequalities. Ann. Math. Studies 38, 99 (1956), 1794.

Opological proofs for certain theroems on matrices with non-negative elements. Monatsch. Math. 62, 219 (1958). 2850.

Some inequalities concerning positive-definite $\mathrm{Her}-$ mitian matrices. Proc. Cambridge Phil. Soc. 51, 414 (1955). 1496.

Some remarks on commutators of matrices. Arch. Math. (Tübinger, Germany) 5, 102 (1954). 1145.

Fan, $K$., Davis, P., Complete sequences and approximations in normed linear spaces. Duke Math. J, 24, 183 (1957). 2065.

Fan, ${ }^{\prime}$., Glicksburq, I., Fully convex normed linear spaces. Proc. Natl. Acad. Sci. 41, No. 11, 947 (1955). 1350.

Fan, K., Glicksburg, I., Hoffman, A. J., Systems of inequalities involving convex functions. Proc. Am. Math. Soc. 8, 617 (1957). 2335.

Fan, K., Hofman, A. J., Some metric inequalities in the space of matrices.' Proc. Am. Math. Soc. 6, No. 1,111 (1955). 1497.

Fan, K., Taussky, O., Todd, $J .$, An algebraic proof of the isoperimetric inequality for polygons. J. Wash. Acad. Sci. 45, No. 11, 334 (1955). 1259.

Discrete anologs of inequalities of Wirtinger. Monatsh. Math. Bd. 59/2 (Vienna, Austria) (1955). 1313.

Fan, K., Todd, J., A determinantal inequality. J. London Math. Soc. 30, 58 (1955). 1232,

Fang, P. H., A dispersion function of paramagnetic relaxation. Physica (Amsterdam, Holland) 24, 970 (1958). 2418.

Conductivity of plasmas to microwaves. Phys. Rev. 113, No. 1, 13 (1959). 2914.

Fang, $P$. H., Brower, W. S., Temperature dependence of the breakdown field of barium titanate. Phys. Rev. 113, 456 (1959). 3062.

Fanning, R. J., Bekkedahl, N., Quantitative determination of natural rubber hydrocarbon by refractive index measurements. Anal. Chem. 23, 1653 (1951). 292 .

Fanning, R. J., Wood, J. W., Rapid extraction of resins from chilte and guayule rubbers. Rubber Age 68, No. 2, $195(1950)$. 119.

Fano, L., Hubbell, J. H., Beckett, C. W., Compressibility factor, density, specific heat, enthalpy, entropy, free energy function, viscosity, and thermal conductivity of steam. Natl. Advisory Comm. Aeronaut. Tech. Note. 3273 (1956). 1657.

Fano, L., Mann, D. E., Vibrational spectrum and force constants of diboro tetrachloride. J. Chem. Phys. 26, 1665 (1957). 2409.

Fano, $U$., A similarity principle in the backscattering of gamma-rays. Radiation Reaserch 1, No. 5, 495 (1954). 934 .

A Stokes-parameter technique for the treatment of polarization in quantum mechanies. Phys. Rev. 93,121 (1954). 936.

Angular correlation of radiations with parallel angular momenta. Il Nuovo Cimento (Roma, Italy) Serie X 5, $1358(1957)$. 2021.

Atomic theory of electromagnetic interactions in dense materials. Phys. Rev. 103, No. 5, 1202 (1956). 1635.

Degradation and range straggling of high-energy radiations. Phys, Rev. 9\%, 328 (1953). 680.

Description of states in quantum mechanics by density matrix and operator techniques. Rev. Modern Phys 29. No. 1, 74 (1957). 2081. 
Gamma-ray attenuation. Part I, Nucleonics 11, 8 (1953); Part II, Nucleonics 11, 55 (1953). 724. Geometrical characterization of nuclear states and the theory of angular correlations. Phys. Rev. 90, 577 (1953). 726 .

High-frequency limit of bremsstrahlung in the Sauter approximation. Phys, Rev, 116, No.5, 1156 (1959).2961. Inelastic collisions and the Moliere theory of multirle scattering. Phys. Rev. 93, 117 (i954). 1037. Introductory remarks on the dosimetry of ionizing radiations. Radiation Research 1, 3 (1954). 1045. Tote on the Pragg-Gray cavity princirle for measiring energy dissipation. Radiation Research 1, 237 (1954). 1083 .

Radiology hiology. Chapter I in the Book, Princinles of radiological phrsics (MeGraw-Hill Book Co., New York, X.Y.), I, 1 (1954). 1125.

Secondary electrons: average er.ergy loss rer ionization. Chapter in Symp. on Radio'iology, The Basic Aspects of Radiation Effects on Iiving Srstems (John Wiley \& Sons, Inc., New York, N.Y.) (1952). 538.

Fano, $U$., Fano, $L_{+}$, Basic phrsics of atoms and molecules. (John Wiley and Sons, Inc., New York, N.Y.), 414 pages $(1959) .2901$.

Fano, $U$, Goldstein, $N$., Wyckoff, $H$. O., Directional distribution of 1040 -kev radiation from a high voltage X-ray tube. J. Appl. Phvs. 22, 417 (1951). 224.

Fano, U., Huntoon, R. D., Atomic definition of primarv standards. Nature (London, England) 166, 167 (1950). 28.

Fano, U., Koch, H. W., Motz, J. W., Evaluation of bremsstrahlung cross sections at the high-frequency limit. Phys. Rev. 112, No. 5, 1679 (1958). 2551.

Fano, $U$., McVoy, $K$. W., Bremsstrahlung and the photoelectric effect as inverse processes. Phys. Rev. 116, No. 5, 1168 (1959). 2905.

Fano, $U$., McVoy, $K$. W., Albers, J.R., Interference of orhital and srin currents on bremsstrahlung and photoelectric effect. Phys. Rev. 116, No. 5, 1159 (1959). 2968.

Sauter theory of the photoelectric effect. Phys. Rev. 116. No. 5, 1147 (1959). 3040.

Fano, U., Nelms, A. T., An approximate expression for gamma-ray degradation spectra. J 59, 207 (1957) RP2788.

Fano, U., Racah, G., Irreducihle tensorial sets. Academic Press Inc., New York, N.Y. (1959). 2970.

Fano, U., Spencer, L. V., Energy spectum resulting from electron slowing down. Phys. Rev. 93, 1172 (1954). 1015.

Faris, J.J., Richardson, J. M., Excess noise in microwave crvstal diodes used as rectifiers and harmonic generators. IRE Trans. Microwave Theory and Tech. MTT-5, No. 3, 208 (1957), 2114.

Farnsworth, D., Judd, D. B., Plaza, L., Tritanopia with abnormally heavy ocular pigmentation. J. Opt. Soc. Am. 40, 833 (1950). 161.

Feder, D. P., Automatic lens design methods. J. Opt. Soc. Am. 47, No. 10, 902 (1957). 2034.

Calculation of an optical merit function and its derivatives with resrect to the svstem parameters. J. Opt. Soc. Am. 47, No. 10, 913 (1957). 2041.

Feder, D. P., Washer, F. E., Shark, R. V., Panel discussion-report on the NBS optical image evaluation symposium, reprinted from Photogrammetric Eng. 18, 575 (1952). 532 .

Fee, J. G., Sweeney, W. T., Yost, E. L., Physical properties of plastic teeth. J. Am. Dental Assoc. 56, $883(1958) .2688$.

Feinstein, $H$. I., Indentification and microdetermination of nickel. Anal. Chem. 22, 723 (1950). 70.

Condition for radiation from a solar plasma. Phys. Rev. 85, 145 (1952). 414.

Higher-order approximations in ionospheric wave-propagation. J. Geophys. Research 55, 161 (1950). 67. Information theory aspects of propagation through time-varying media. J. Appl. Phys. 26, 219 (1955). 1364.
On the nature of the decay of a meteor trail. Proc. Phys. Soe. (London, England) 65, Pt. 9, 741 (1952). 501.

Partial reflections in tropospheric propagations. IRE Trans. Ant. Prop. AP-3, 101 (1952). 508.

Some information theory aspects of propagation through time varying media. IRE Conv. Record, Mar. 22, 1954, [2], Pt. 1, Ant. Prop., p. 87. 1143.

Some stochastie problems in wave propagation. Part I. IRE Trans. Ant. Prop. AP-2, 23 (1954). 1147.

The interpretation of radar waves from meteor trails. J. Geophys. Research 56, No. 1, 37 (1951). 338.

The limiting polarization of magneto-ionic waves.

IRE Trans. Ant. Prop. AP-3, 19 (1952). 583.

The nature of gradient reflections. IRE Trans. Ant. Prop. AP-4, 2 (1952). 586.

The role of partial reflections in tropospheric propagation beyond the horizon. IRE Trans. Ant. Prop. A P-2, 2 (1952). 595.

Tropospheric propagation beyond the horizon. J. Appl. Phys. 22, 1292 (1951). 351.

Wave propagation in an anisotropic, inhomogeneous media. J. Geophys. Research 58, 223 (1953). 910.

Feinstein, J., Sen, H. K., Radio wave generation by multi-stream charge interaction. Phys. Rev. 83, No. 2, 405 (1951). 295.

Feister, I., Numerical evaluation of the Fermi betadistribution function. Phys. Rev. 78, 375 (1950). 100.

Feister, I., Curtiss, L. F., The disintegration scheme of ${ }^{131}$. Phys. Rev. 78, 179 (1950). 150.

Feldmann, A. A., Electroforming of waveguide components for the millimeter-wavelength range. (1957) C587.

Microwave components: precision casting vs electroforming. Materials and Methods 34, No. 1, 70 (1951), 258.

Techniques for electroforming of precision waveguide components in the millimeter wavelengths. Rev. Sci. Instr. 28, 295 (1957). 2339.

Feller, W., Forsythe, G. E., New matrix transformations for obtaining characteristic vectors. Quart. Appl. Math. 8, No. 4, 325 (1951). 267.

Ferouson, E. E., Sulzer, $P . G$., Sweep-frequency obliqueincidence ionospheric measurements over a $1,150 \mathrm{~km}$ path. Proc. IRE 40, 1124 (1952). 561.

Ferguson, G. W. Smith, D. L., Schoonover, I. C., Microstructure of dental amalgam. J. Am. Dental Assoc. 47,305 (1953). 765 .

Ferguson, R. E., An isotopic tracer study of carbon formation in hydrocarbon flames. Combustion and Flame 1, No. 4, 431 (1957). 2018.

On the origin of the electronically excited $\mathrm{C}_{2}$ radical in nydrocarbon flames. J. Chem. Phys. 3, 2085 (1955). 1434

Ferguson, R. E., Broida, H. P., Atomic flames: spectra "temperatures" and products. Fifth Symp. (Intern.) on Combustion. (Reinhold Publ. Corp., New York, N.Y.), p. 754 (1955). 1268.

Stable carbon isotope analysis of optical spectroscopy. Anal. Chem. 28, 1436 (1956). 1892.

Ferguson, R. E., Yokley, C. R., Products of cool flame oxidation of propane-2-C13. 7th Symp. on Combustion, p. 113 (Butterworths, London, England) (1958). 2702 .

Separation of the products of cool flame oxidation of propane. Combustion and Flame 2, No. 2, 117 (1958). 2753.

r. uson, T. B., Gilford, S. R., Gregg, D. E., Shadle, O. W., Marzetta, L. A., An improved cuvette densitometer for cardiac output determination by the dye-dilution method. Rev. Sci. Instr. 24, 696 (1953). 650.

Ferguson, T. B., Shadle, O. W., Gregg, D. E., Gilford, S. R., Evaluation of a new cuvette densitometer for determination of cardiac output. Circulation Research 1, No. 3, 200 (1953). 714.

Fey, L., Mockler, R. C., Barnes, J. Beehler, R., Salazar, H., The ammonia maser as an atomic frequency and time 
standard. IRE Trans. Instrumentation I- 7 , No. 3-4, 201 (1958), 2804.

Fickle, D. P., Park, J. J., Uranium-platinum system. J 64A1, 107 (1960).

Fine, L. A., Wright, J. W., Mean electron density variations of the quiet ionnsphere. (1960) TN40-1 (PB151399-1); (1960) TN40-2 (PB151399-2).

Finegold, H., Complementary use of nuclear magnetic resonance and infrared absorption studies in organic phosphorus chemistry. Ann. N.Y. Acad. Sci. 70, 875 (1958). 2494.

Fink, D. G., Smith, N., Condon, E. U., Bailey, S. L., Everitt, W. L., The present status of color television; a report by the Senate Advisory Committee on Color Television. Proc. IRE 38, 980 (1950). 154.

Finneu, J. W., Bateman, R., Smith, E. K., Tveten, L. H., Watts, $J . M$., IGY observations of $F$-layer seatter in the far east. J. Geophys. Research 64, 403 (1959). 2963.

Finney, J. W., Smith, E. K. Jr., Report on the IGY oblique-incidence sporadic- $E$ and $F$-scatter program. (1960) TN48 (PB151407)

Fiock, E. F., Measurement of burning velocities. Section K, Vol. 9, Princeton University Series on High Speed Aerodynamies and Jet Propulsion (Princeton, N.J.) (1954), 1064 .

Fiock, E. F., Dahl, A. I., Temperature measurements in high-velocity streams of hot gas. Proc. First Iowa Thermodynamies Symp., p. 190, State University of Iowa (Iowa City, Iowa) (1954). 1163.

The measurement of gas temperatures by immersiontype instruments. J. Am. Rocket Soc. 23, 155 (1953). 867.

Fischer, E. K., Burnett, J. C., High-speed motion pictures in textile research. Textile Research J. 20, 259 (1950). 66.

Fischer, T. E., Schoonover, I. C., Deterioration of silicate cements in the tropics. U.S. Armed Forces Med. J. 2, 907 (1951). 217.

Fischer, T. E., Schoonover, I. C., Serio, A. F., Sweeney, W. $T$., Bonding of plastic teeth to heat cured denture base resins. J. Am. Dental Assoc. 44, 285 (1952). 402 .

Fisher, M. A., Crouse, W. A., Carickhoff, M., Effect on fuel-immersion on laminated plastics. Trans. Am. Snc. Mech. Engrs. 72, 175 (1950). 50.

Fite, W. L., Branscomb, L. M., Photodetachment of the hydrogen negative ion. Phys. Rev. 93, 651 (1954). 1105 .

Flanery, W. S., Schrodt, J. E., Fulk, M. M., McReynolds, $W$. A., A comparison of insulating materials. Proc. 1957 Cryogenic Eng. Conf., Abstract, Paper G-7, p. 416, Edited by K. D. Timmerhaus, Chem. Eng. Dept., University of Colorado, (Boulder, Colo.) (1958). 2416.

Fleming, L. T., A ceramic accelerometer of wide frequency range. Instruments 24, No. 8, 105 (1951). 175.

Fletcher, S. L., Jr., Oswald, V. A., Jr., Proposals for the mechanical resolution of German syntax patterns. Modern Language Forum 36, No. 3-4, 1 (1951). 290.

Flippen, R. B., Wasilik, J. H., Piezoelectric effect in indum antimonide. Phys. Rev. Letters 1, No. 7, 233 (1958). 2690.

Florin, R. E., Mears, T. W., High-temperature liquids. (1954) Conf. on Reactor Heat Transfer, Report ENL 2446,89 , Brookhaven National Laboratories, (Brookhaven, Long Island, N.Y.). (1954) 1032.

Florin, R. E., Pummer, W. J., Wall, L. A., Fluorination of haloaromatic compounds. J 62, 107 (1959) RP2938.

Reactions of aromatic fluorocarbons with hydrogen. J 62, 119 (1959) RP2940.

Synthesis of some disubstituted $3,4,5,6$-tetrafluorobenzenes. J 62, 113 (1959) RP2939.

Florin, R. E. Wall, L. A., Effect of structure on the thermal decomposition of polymers. J 60,451 (1958) RP2860.
Florin, R. E., Wall, L. A., Brown, D. W., Electron spin resonance spectra from gamma-irradiated solid nitrogen. J. Chem. Phys. 30 No. 2, 602 (1959). 2940. Florin, R. E., Wall, L. A., Mohler, F. L., Quinn, E. Dimethylphosphinoborine trimer: mass spectra and thermal decomposition. J. Am. Chem. Soc. 76, 3344 (1954). 994.

Florin, R. E., Wall, L. A., Straus, S., Mohler, F. L., Bradt, $P$., Phosphinoborine compounds: mass spectra and pyrolysis. J 63A1, 63 (1959).

Florman, E. F., Bateman, R., Tait, $I$., A source of error in radio phase measuring systems. Proc. IRE 38, $612(1950) .10$.

Florman, E.F., Bateman, R., Tait, A., Rechtin, E., A source of error in radio phase measuring systems. (Discussion) Proc. IRE 39, 436 (1951). 186.

Florman, E. F., Plush, R. W., Measured statistical characteristics and narrow-band teletype message errors on a single-sideband 600 -mile-lnng ultrahigh-frequency tropospheric radio link. J 64D2, 125 (1960).

Flory, P.J., Mandelkern, L., Melting of cnpolymers. J. Polymer Sci. 21, No. 98, 345 (1956). 1774.

Flory, P. J., Mandelkern, L., Quinn, F.A., Jr., Kinetics of crystallization in polymers. I. Bulk polymers. J. Appl. Phys. 25, 830 (1954). 1047.

Flory, P.J., Roberts, D. E., Mandelkern, L., The isotropic length of polymer networks. J. Am. Chem. Soc. 79, 1515 (1957). 2370.

Flynn, J. H., A function to aid in the fitting of kinetic data to a rate equation. J. Phys. Chem. 61, 110 (1957). 1983.

A method for determining rate equation for reactions in which the concentration of the reactant is unknown. J. Phys. Chem. 60, 1332 (1956). 1592.

An equation for calculating the number of chain scissions in the photochemical degradation of solid polymers. J. Polymer. Sci. 27, 83 (1958), 2455.

Flynn, J. H., Launer, H. F., Wilson, W. K., Determination of glucose by means of sodium chlorite. Indian Pulp and Paper 9, 399 (1955), 1310.

Flynn, J. H., Wilson, W. K., Morrow, $W$. L., Degradation of cellulnse in a vacuum with ultraviolet light. J 60, 229 (1958) RP2841.

Flynn, T. M., A bibliography of the physical equilibria and related properties of some cryogenic systems. (1960) TN56 (PB161557).

Flynn, T. M., Timmerhaus, K. D., Weitzel, D. H., Distillation of hydrogen isotopes. Colorado Engr. 55, 12 (1958). 2526 .

Low temperature distillation of hydrogen isotopes. Chem. Eng. Prngr. 54, No. 6, 35 (1958). 2618.

Pilot plant studies of the low temperature distillation of hydrogen isntopes. Proc. 1958 Cryogenic Eng. Conf., Paper H-1, p. 464. Edited by K. D. Timmerhaus, Chemical Engineering Dept., Univ, of Colorado (Boulder, Colorado) (1958); Chem. Eng. Progr. 54 , No. 6, 35 (1958). 2691.

Flynn, T. M., Timmerhaus, K. D., Weitzel, D. H., Draper, $J$. $W$." Separation of hydrogen isotopes by multicomponent distillation. Proc. 1957 Cryogenic Eng. Conf., Paper A-6, p. 58, Edited by K. D. Timmerhaus, Chem. Eng. Dept., University of Colorado (Boulder, Colo.) (1958). 2751.

Flynn, T. M., Weitzel, D. H., Timmerhaus, K. D., Vander Arend, P. C., Draper, $\vec{J} . W$, Distillation of hydrogen-deuterium mixtures. Proc. 1956 Cryogenic Eng. Conf., Paper A-6, p. 39, Edited by K. D. Timmerhaus, Chem. Eng. Dept., University of Colorado (Boulder, Colo.) (1957). 2093.

Foldes, G., Crichlow, W. O., Hewitt, F.J., Horner, F., Shinkawa, H., Sullivan, A. W., Measurement of atmospheric noise. Recommendation No. 1 and Annex, Proc. XIIth Gen. Assembly Intern. Sci. Radio Union, Boulder, Colorado, August 22-September 5 , 1957, XI, Pt. 4, 99, 1957. U.R.S.I. (Intern. Sci. Rario Union) Inform. Bull. No. 105, 10 (Sept.-Oct. 1957). 2190. 
Fontana, B. J., Thermometric study of the frozen products from the nitrogen microwave discharge. J. Appl. Phys. 29, 1668 (1958). 2847.

Fookson, A., Pomerantz, P., Rich, E. H., The preparation of high-purity hydrogen deuteride. Science 112, 748 (1950). 153 .

Foote, R. S., Koch, H. W., Scintillation spectrometers for measuring total energy of X-ray photons. Rev. Sci. Instr. 25, 746 (1954). 1136.

Foote, R. S., Koch, H. W., Total-ahsorption X-ray spectrometry, application to betatron experiments. Nucleonics 12, 51 (1954). 1213.

Foote, R. S., Petree, B., A pulsed magnetic extractor for removing the electron beam from a betatron. Rev. Sci. Instr. 25, 694 (1954). 929.

Forman, R., Measurement and theoretical study of electrical conductivity and Hall effect in oxide cathodes. Phys. Rev, 96, No. 6, 1479 (1954). 1063.

Properties of pore conductors. J. Appl. Phys. 26, No. 9,1187 (1955). 1461.

Forshee, B. W., Wilson, W. K., Degradation of cellulose a cetate films. SPE J. 15, No. 2, 146 (1959). 2924. Preservation of documents by lamination. (1959) Mono. 5.

Forsythe, G. E., A numerical analyst's fifteen-foot shelf. Math. Tables and Other Aids to Computation VII, 211 (1953). 631.

Alternative derivations of Fox's escalator formulae for latent roots. Qvart. J. Mech. and Appl. Math. (London, England) 5, Pt. 2, 191 (1952). 386.

Asymptotic lower bounds for the frequencies of certain polygonal membranes. Pacific J. Math. 4, No. 2, 467 (1954). 957.

Gauss to Gerling on relaxation. Math. Tables and Other Aids to Computation 5, No. 36, 255 (1951). 244.

Second order determinants of Legendre polynomials. Duke Math. J. 18, No. 2, 361 (1951). 305.

Solving linear algebraic equations can be interesting. Bull. Am. Math. Soc. 59, 299 (1953). 831.

Forsythe, G. E., Feller, $W$., New matrix transformations for obtaining characteristic vectors. Quart. Appl. Math. 8, No. 4, 325 (1951). 267.

Forsythe, G. E., Leibler, R. A., Matrix inversion by a Monte Carlo method. Math. Tables and Other Aids to Computation 4, No. 31, 127 (1950). 84.

Forsythe, G. E., Motzkin, T. S., An extension of Gauss' transformation for improving the condition of svstems of linear equations. Math. Tables and Other Aids to Computation 6, No. 37, 9 (1952). 393.

Forsythe, W. E., Smithsonian Physical Tables, Vol. 120, Publ. 4169 ; C. E. Moore, Tahle 664 "Elements in the Sun's Atmosphere". (Smithsonian Institution, Washington, D.C.) (1954). 1138.

Forziati, A. F., Dickson, G., Lawson, M. E., Jr., Fluorescense of teeth reveals structure. N.Y. J. Dentistry 23, 299 (1953). 721.

Forziati, A. F., Dickson, G., Lawson, M. E., Jr., Schoonover, I. C., Fluorescence of teeth: a means of investigating their structure. J. Am. Dental Assoc. 45, 661 (1952). 452 .

Forziati, A. F., Losee, F. L., Pigman, W. W., Jennings, $W$. $H$., Lawson, $M, E$., Jr., In vitro production of experimental enamel caries. J. Am. Dental Assoc. 53, 655 (1956). 1734.

Forziati, F. H., Rowen, J. W. Reeves, R. E., Spectrophotometric evidence for the absence of free aldehyde gro'ps in periodate-oxidizied cellulose. J. Am, Chem. Soc. 73, 4484 (1951). 314.

Foster, B. E., Absorption by concrete of X-rays and gamma-ravs. BuDocks Technical Digest No. 55 and 56 (May 1955). 1250.

Discussion of the paper "Heavy steel-aggregate concrete" by E. I. Fiesenheiser and B. A. Wasil. J. Am. Concrete Inst. 28 No. 6, 1143 (1956). 1678. Use of admixtures as integral waterproofing and dampproofing materials. J. Am. Concrete Inst. 22, (Proc. 47) 46 (1950). 164.
Fouquet, B. H., Kanagy, J.R., Impregnation of sole leather with polymers. J. Am. Leather Chemists Assoc. 53, No. 6, 336 (1958), 2594.

Fowler, R. M., Macurdy, L. B., Alber, H. K., BenedettiPichler, A. A., Carmichael, $H$., Corwin, A. H., Hufman, E. W. D., Kirk, P. L., Lashof, T. W.', Terminology for describing the performance of analytical and other precise balances. Anal. Chem. 26, 1190 (1954). 1166.

Fox, G., Stone, W. K., Schiefer, H. F., Stress-strain relationships in yarns subjected to rapid impact loading. Part I. Equipment, testing procedure, and typical results. Textile Research J. 25, 520 (1955). 1515.

Fox, $L$. Notes on numerical analysis. 4. Relaxation and step-by-step methods. Math. Tables and Other Aids to Computation 5, 92 (1951). 271.

Fox, L., Miller, J. C. P., Notes on numerical analysis. 5. Table-making for large arguments. Math. Tables and Other Aids to Computation 5, 163 (1951). 272.

Fox, M. R., A progress report on SEAC. Signal 11, No. 7, 18 (1957). 1995.

Fox, T. G., Mandelkern, L., Dilute solution thermodynamic behavior of flexible chain molecules: polymethyl methacrylate. J. Chem. Phys. 21, 187 (1953). 687.

Frankel, H. E., Bennett, J. A., Understressing as a means of eliminating the damaging effect of fatigue stressing. ASTM Bull. No. 55, 891 (1955). 1566.

Frankel, H. E., Bennett, J. A., Holshouser, W. L., Effect of oleophobic films on metal fatigue. J 64C2, 147 (1960).

Frankel, H. E., Bennett, J. A., Pennington, W. A., Fatigue properties of high strength steels. Trans. Am. Soc. Metals 52, (1959). 2951.

Franklin, J. L., Dibeler, V. H., Morris, P. P., Jr., Mass spectrometric study of the rate of thermal decomposition of hydrazoic acid. J 61, 41 (1958) RP 2881.

Franklin, J. L., Dibeler, V. H., Reese, R. M., Electron impact studies of hydrazine and the methyl-substituted hydrazines. J. Am. Chem. Soc. 81, 68 (1959). 2939.

Ionization and dissociation of oxygen difluoride by electron impact. J. Chem. Phys. 27, 1296 (1957). 2169.

Franklin, J. L., Dibeler, V. H., Reese, R. M., Krauss, M., Ionization and dissociation of hydrazoic and methyl azide by electron impact. J. Am. Chem. Soc. 80, 298 (1958). 2604.

Franklin, J. L., Herron, J. T., Bradt, P., Mass spectrometric study of the reactions of some hydrocarbons with active nitrogen. Can. J. Chem. 37, 579 (1959). 2982.

Franklin, J. L., Herron, J. T., Bradt, P., Dibeler, V. H., Kinetics of nitrogen atom recombination. J. Chem. Phys. Letters 29, No. 1, 230 (1958); J. Chem. Phys. 30, 879 (1959). 2972 .

Mass spectrometric study of the decomposition of hydrazoic acid by the electric discharge. J. Am. Chem. Soc. 80, 6188 (1958), 2623.

Franklin, J. L., Reese, R. M., Dibeler, V. H., Electron impact studies of sulfur dioxide and sulfuryl fluoride. J. Chem. Phys. 29, No. 4, 880 (1958). 2542.

Fred, M., Tomkins, F. S., Meggers, $W$. F. Nuclear moments of Ac ${ }^{227}$. Phys. Rev. 98, 1514 (1955). 1420 .

Frederiske, H. P. R., Compound semiconductors. J. Metals 10, 346 (1958). 2495.

Thermoelectric power in Ge single crystals. Phys. Rev. 91, 491 (1953). 896.

Thermoelectric power of germanium below room temperature. Phys. Rev. 92, 248 (1953). 897.

Frederiske, H. P. R., Blunt, R. F., Photoconductivity and photovoltaic effect in intermetallic compounds. Photoconductivity Conf., Atlantic City, N.J., (John Wiley and Sons, Inc., New York, N. Y.) p. 414 (1956). 1806.

Photoeffects in intermetallic compounds. Proc. IRE 43, 1828 (1955). 1444. 
Frederikse, H.P. R., Blunt, R. F., Becker, J. H., Hosler, $W . R$., Electrical and optical properties of intermetallic compounds, III. Aluminum antimonide. Phys. Rev. 96, 578 (1954). 1007.

Frederikse, H. P. R., Blunt, R. F., Holser, W. R., Electrical and optical properties of intermetallic compounds. II. Gallium antimonide. Phys. Rev. 96, 576 (1954). 1007.

Electrical and optical properties of intermetallic compounds. IV. Magnesium stannide. Phys. Rev. 100, No. 2, 663 (1955). 1330.

Properties of $\mathrm{Mg}_{2}$ Sn. Phys. Rev. 98, 1533A (1955). 1460.

Frederikse, H. P. R., Breckenridge, R. G., Blunt, R. F., Hosler, W. R., Becker, J. H., Oshinsky, W., Electrical and optical properties of intermetallic compounds. I. Indium antimonide. Phys. Rev. 96, 571 (1954). 1007.

Frederikse, $H . P, R$, Hosler, $W . R$., Galvanomagnetic effects in InSb. Phys. Rev. 98, 1532A (1955). 1352.

Galvanomagnetic effects in $n$-type $\mathrm{I} n \mathrm{~S} b$ at $4.2^{\circ} \mathrm{K}$. Can. J. Phys, 34, 1377 (1956). 1716.

Galvanomagnetic effects in $n$-type indium antimonide. Phys. Rev. 108, 1136 (1957). 2135.

Galvanomagnetic effects in $p$-type indium antimonide. Phys. Rev. 108, 1146 (1957). 2136.

Oscillatory galvanomagnetic effects in $n$-type indium arsenide. Phys, Rev, 110, 880 (1958). 2678.

Frederikse, H. P. R., Hosler, W. R., Roberts, D. E., Electrical conduction in magnesium stannide at low temperatures. Phys. Rev. 101, No. 6, 1653 (1956). 1695 .

Frederikse, H. P. R., Johnson, V. A., Scanlon, W. W., Methods of experimental physics. Chapter 7.6, Thermoelectric Effects. Edited by K. Lark-Horovitz and V. A. Johnson (Academic Press Inc., New York, N.Y.) p. 114 (1959) 2990.

Frederikse, H. P. R., Mielczarek, E. V., Thermoelectric power of indium antimonide. Phys. Rev. 99, 1889 (1955) 1559

Freeman, J. J., Noise comparator for microwaves. J. J. Freeman. Radio and TV News (Radio-Elec. Eng. Sect.) 49, No. 3, 11 (1953). 773.

On power spectra and the minimum detectable signal in measurement systems. J. Appl. Phys. 26, 236 (1955). 1430 .

On the relation between the conductance and the noise power spectrum of certain electronic streams. J. Appl. Phys. 23, No. 11, 1223 (1952). 503.

The admittance of a diode with a retarding field. J. Appl. Phys. 23, 743 (1952). 566.

Freethey, F. E., Thompson, M. C., Jr., Waters, D. M. Fabrication techniques for ceramic X-band cavity resonators. Rev. Sci. Instr. 29, No. 10, 865 (1958). 2561 .

Freeze, P. D., Blackburn, G. F., Caldwell, F. R., A study of thermistor materials for use as temperaturesensing elements in the high-velocity exhaust gases of jet-type engines. Wright Air Devel. Ctr. (Óhio) Tech. Rept. p. 54, Suppl. 1 (1956), 1602

Freeze, P. D., Sawada, F. H., Carter, C. J., Blackburn, $G$. F., Development and evaluation of ceramic coatings for thermocouples. Wright Air Devel. Ctr. (Ohio) Tech. Rept. 56-135 (1956). 1674.

Freis, E. D., Broida, H. P., Rose, J. C., A variable heart pump permitting independent control of rate, output and ejection velocity. Science 115, No. 2996, 603 (1952). 381 .

Freis, E. D., Rose, J. C., Broida, H. P., Hufnagel, C. A., Gillespie, J. F., Rabile, $P$, J. A method for the study of the circulation in the dog using a mechanical left ventricle. J. Appl. Physiol. 7, 580 (1954). 920.

French, J. C., Udelson, B.J., Creedon, J. E., Microwave measurements of the properties of a de hydrogen discharge. J. Appl. Phys. 28, No. 6, 717 (1957). 2200 .

French, J, L., Eaton, H. N., Fixture unit ratings as used in plumbing system design. Housing and Home
Finance Agency Housing Research Paper No. 15 (1951). 239.

Frenkel, L., Electrostatic deflection piates for cathoderay tubes. I. Design of single-bend deflection plates with parallel entrance sections. II. Deflection defocusing distortion of single-bend deflection plates with parallel entrance sections. J 64C2, 103 (1960).

Friedman, A. S., Chemiluminescence as a tool in the study of liquid flow boundaries. J. Appl. Phys. 27, 417 (1956). 1646.

Friedman, A. S., Haar, L., High-speed machine computation of ideal gas thermodynamic functions. I. The isotopic water molecules. J. Chem. Phys. 22, 2051 (1954). 1030 .

High-speed machine computation of ideal gas thermodynamic functions. II. The diatomic free radicals of the isotopic hydrides of oxygen and sulphur. J. Chem. Phys. 23, No. 5, 869 (1955). 1357.

Friedman, A. S., Johnston, H. L., Keller, W. E., The compressibility of liquid normal hydrogen from the boiling point to the central point at pressures up to 100 atmospheres. J. Am. Chem. Soc. 76, 1482 (1954). 1173.

Friedman, A. S., Michels, A., Botzen, A., Sengers, V. J., The thermal conductivity of argon for temperatures between $0^{\circ} \mathrm{C}$ and $75^{\circ} \mathrm{C}$ and at pressures up to 2500 atmospheres. Physica 22, 121 (1956). 1952.

Friedman, A. S., Michels, A., Cox, J. A. M., Botzen, A., Contribution to the study of transport phenomena in gases at high densities. J. Appl. Phys. 26, 843 (1955), 1296.

Friedman, I., Cloud, P. E., Jr., Sisler, F. D., Dibeler, V. H., Microbiological fractionation of the hydrogen isotopes. Science 12\%, 13 (1958). 2635.

Friedman, A. S., Trzeciak, M., Johnston, H. L., Pressurevolume temperature relations of liquid normal deuterium. J. Am. Chem. Soc. 76, 1552 (1954). 1115.

Fristrom, R. M., Lide, D. R., Jr., Mann, D. E., The microwave spectrum and structure of sulfuryfluoride. J. Chem. Phys. 25, 734 (1957). 2372.

Froberg, C. E., Goldstein, A., A collision path from the earth to the moon in the restricted problem of three bodies. Kgl. Fysiograf. Sallskap. I. Lund Förh. (Lund, Sweden) 22, No. 14, 1 (1952). 359.

Frolen, L. J., Ross, G. S., Simple rotating molecular still. J 62, 187 (1959) RP2951.

Frood, D. G., Wait, J.R., An investigation of slot radiators in rectangular metal plates. Proc. IEE (London, England) 103, Pt. B, No. 7, 103 (1956). 1622. The radiation patterns and conductances of slots cut on rectangular metal plates. Proc. IRE 44, 1469 (1956). 1947.

Frush, H. L., Isbell, H. S., Mutarotation, hydrolysis, and rearrangement reactions of glycosylamines. J. Organic Chem. 23, 1309 (1958). 2642.

Sodium borohydride reduction of aldonic lactones. J. Am. Chem. Soc. 78, No. 12, 2844 (1956). 1868. Syntheses of $\mathrm{C}^{14}$-labeled L-sorbose and L-ascorbic acid. J 59, 289 (1957) RP2800.

Frush, H. L., Isbell, H. S., Bruckner, B. H., Kowkabany, G. N., Wampler, G., Micromethylation of polysaccharides. Anal. Chem. 29, 1523 (1957). 2198.

Frush, H. L., Isbell, H. S., Holt, N. B., Note on the preparation of sodium amalgam in the form of pellets. J 64A 1, 135 (1960).

Frush, H. L., Isbell, H. S., Holt, N. B., Moyer, J. D., Tritium-labeled compounds III. Aldoses-1-t. J 64A2, 177 (1960).

Frush, H. L., Isbell, H. S., Moyer, J. D., Use of carbon14 and tritium for the study and characterization of cellulose and other polysaccharides. Tappi 40, No, 9, 739 (1957). 2403.

Frush, H. L., Isbell, H. S., Peterson, R. A., Tritiumlabeled eompounds I. Radioassay of tritium-labeled compounds in "infinitely thick" films with a windowless, gas-flow proportional counter. J 63A2, 171 (1959). 
Frush, H. L., Isbell, H. S., Smith, F. A., Creitz, E. C. Moyer, J. D., Stewart, J. E., Infrared spectra of sugar acetates in solution. J 59, 41 (1957) RP2772.

Frush, H. L., Tregoning, L.J., Isotope effect in oxidation of D-mannitol-2-C't by acetobacter suboxydans. Science 128, No. 3324,597 (1958). 2606.

Fujii, M., Diniak, A. W., Hughes, E. E., Determination of water content in Freon-12 circulating in a refrigeration system. Refrig. Eng. 62, No. 2, 56 (1954). 990.

Fujita, H., Kato, T., Nakata, Y., Newman, M., Estimation of the frequencies of thin elastic plates with free edges. J 59, 169 (1957) RP2784.

Fulk, M. M. Devereaus, $R$. J., Schrodt, $J, E$., Heat transfer through foams and powders. Proc. 1956 Cryogenic Engineering Conference, Paper E-3, p. 166, Edited by K. D. Timmerhaus, Chem. Eng. Dept., University of Colorado (Boulder, Colo.) (1957). 2147.

Fulk, M. M., Reynolds, M. M., Emissivities of metallic surfaces at $76^{\circ} \mathrm{K}$. J. Appl. Phys. 28, 1464 (1957). 2110.

Fulk, M. M., Schrodt, J. E., McReynolds, W. A., Flanery, W. S., A comparison of insulating materials. Proc. 1957 Cryogenic Engineering Conference, Abstract, Paper G-7, p. 416, Edited by K. D. Timmerhaus, Chem. Eng. Dept., University of Colorado (Boulder, Colo.) (1958). 2416.

Fuller, E. G., Photodisintegration of helium 4. Phys. Rev. 96, 1306 (1954). 1106.

Fuller, E. G., Hayward, E. I., Elastic scattering of photons. Phys. Rev. 94, 732 (1954). 1006.

Elastic scattering of photons by tantalum. Phys. Rev. Letters 1, No. 12, 465 (1958). 2535.

Nuclear elastic scattering of photons. Phys. Rev. 95, 1106 (1954). 1086; Phys. Rev. 101, No. 2, 692 (1956). 1787.

Parameters of the $15-\mathrm{Mev} \mathrm{X}$-rays seattered by $\mathrm{C}^{12}$. Physica 22, 1138 (1956). 1803.

Photon scattering and self absorption in lead and bismuth at 7 Mev. Proc. Congres Intern. de Physique Nucleaire, 1958, Nuclear Reaction in Nuclear Forces, Pt. 2, Chapter I, p. 62 (Paris, France) (1959). 3014.

Photon self-absorption and scattering by the 15.1Mev level in C12. Phys. Rev. 106, 991 (1957). 2248.

Fuller, E. G., Hayward, E. V., Koch, H. W., Shape of the high-energy end of the electron bremsstrahlung spectrum. Phys. Rev, 109, 630 (1958). 2755.

Fuller, E. G., Petree, B., Weiss, M. S., Photoneutron yields in the rare earth region. Bull. Am. Phys. Soc. Series II, No. 2, 16 (1957). 2249.

Photoneutron yields in the rare earth region. Phys. Rev. 112, No. 2, 554 (1958). 2686.

Fuller, E. G., Weiss, $M$. S., Splitting of the giant resonance for deformed nuclei. Phys. Rev. 112, No. 2, 560 (1958). 2772 .

Fuller, V.L., Williamson, D. A., Longley, A. G., Rice, P. L., A summary of VHF and UHF tropospheric transmission loss data and their long-term variability. (1960) TN43 (PB151402).

Fullmer, I. H., Another milestone in screw thread standardization. Fasteners 12, No. 4, 3 (1957). 2025. Why screw threads must be everybody's business. Proc. 7 th Natl. Conf. on Standards, p. 45, Am. Stds. Assoc. (1956). 1977

World unification of screw threads. Mag. of Stand ards 26, No. 10, 306 (1955). 1581.

Fulton, $F . F$., $J r$., Design of single frequency filters. (1959) TN23 (PB151382).

The effect of receiver bandwidth on amplitude distribution of VLF atmospheric noise. Symp. on the Prop. of VLF Radio Waves, Sponsored by the NBS Boulder Laboratories and IRE Prof. Group on Ant. and Prop., January 23-25, 1957 (Boulder, Colo.), Paper No. 37, V 3 (1957). 2351.

Furey, J. J., Sayre, W. R., Hague, J. L., Determination of nitrogen and sulfur in steel. Anal. Chem. 24, 199 (1952). 426.
Furukawa, G. T., Bender, C. P., Hyndman, J. R., Vapor pressure of benzene above $100^{\circ} \mathrm{C}$. Ind, Chem. 44, 387 (1952). 616.

Furukawa, G. T., Ginnings, D. C., Heat capacity standards for the range $14^{\circ}$ to $122^{\circ} \mathrm{K}$. J. Am. Chem. Soc. 75, $525(1953): 729$

Furukawa, G. T., Glasgow, A. R., Jr., Ross, G. S., Horton, A. T., Enagonio, D., Dixon, H. D., Saylor, C. P., Reilly, M. L., Henning, J. M., Comparison of cryoscopic determinations of purity of benzene by thermometric and calorimetric procedures. Anal. chim. acta 17, 54 (1957); Proc. of I.U.P.A. Intern. Symp. on Purity Control by Thermal Analysis (Amsterdam, The Netherlands) (April 1957). 2062.

Gabbard, R. F., Caswell, R. S., Padgett, D. W., Doering. W. P. Attenuation of $14.1 \mathrm{Mev}$ neutrons in water Nuclear Sci. and Engr. 2, 143 (1957). 2032.

Gaddum, $J . W$. A theorem on convex cones, with applications to linear inequalities. Proc. Am. Math. Soc. 3 . No. 6,957 (1952), 380 .

Metric methods in integral and differential geometry. Am. J. Math. 75, 30 (1953). 764.

The sums of dihedral and trihedral angles in a tetrahedron. Am. Math. Monthly 59, No. 6, 370 (1952). 597.

Gaddum, J. W., Hoffman, A. J., Sokolowsky, D., On the solution of the caterer problem. Logistics Research Quart. 1, 223 (1954). 1099

Gailar, N., Benedict, W. S., Plyler, E. K., The rotationvibration spectra of deuterated water vapor. J. Chem. Phys. 24, No. 6, 1139 (1956). 1949.

The vibration-rotation srectrum of $\mathrm{D}_{2} \mathrm{O}$. J. Chem. Phys. 21, 1301 (1953). 841.

The vihration-rotation spectrum of HDO. J. Chem. Phys, 21, 1302 (1953). 892

Rotation-vihration spectra of deuterated water vapor. J. Chem. Phys. 24, 1139 (1956). 1859.

Gainen, L., Honig, D. J., Stanley, E. D., Linear programming in hid evaluation. Logisties Research Quart. 1, 48 (1954). 1051

Gallagher, J. S., Minkoff, G. J., Scherber, F. I., Energetic species trapped at $4.2^{\circ}$ K from gaseous discharges. J. Chem. Phys, 30, 753 (1959), 2942.

Gallet, $R$. M., Aerodynamical mechanisms producing electronic density fluctuations in turbulent ionized layers. Proc. IRE 43, 1240 (1955). 1254.

The spectrum of the electron density fluctuations in the ionosphere. J. Atmospheric and Terrest. Phys. Special Suppl. 1957, Proc. of the Polar Atmosphere Symp. (Oslo, Norway) July 2-8, 1956, Pt. II, 165 (1957). 2381 .

Propagation and production of electromagnetic waves in a plasma. Il Nuovo Cimento Supplemento 13, No. 1, 234 (1959). 3023.

The very low-frequency emissions generated in the earth's exosnhere. Proc. IRE 47, 211 (1959). 3097.

Gallet, R. M., Helliwell, R. A., A theory of the production of VLF noise (so-called drawn chorus) by traveling wave amplification in the exosphere of the earth Symp on the Prop of VLF Radio Waves, Sponsored by the NBS Boulder Laboratories and IRE Prof, Group on Ant. Prop., January 23-25, 1957 (Boulder, Colorado), Paper No, 20, V 2 (1957). 2003. Origin of "very-low-frequency emissions". J 63D1, $21(1959)$

Gardner, I. C., Applied optics at NBS. Phys. Today 6 , No. 9,8 (1953). 655.

Light waves and length standards. J. Opt. Soc. Am. 45, No. 9, 685 (1955). 1387.

Precision measurement. Ordnance XLI, 222 (1957). 2261 .

Gardner, R. S., Crow, E. L., Confidence intervals for the expectation of a Poisson variable. Biometrika 46, 441 (1959). 2915. 
Garfinkel, S. B., An eight decade logarithmic ohmmeter. J. Instr. Soc. Am. 3, No. 2, 54 (1956). 1619.

Semiautomatic Townsend balance system. Rev. Sci. Instr. 30, No. 6, 439 (1959). 3044.

Garfinkel, S. B., Hasegawa, S., Wexler, A., Simnle humidity lag apparatus. Rev. Sci. Instr. 26, No. 12 , $1196^{\circ}$ (1955). 1487

Garfinkel, S. B., Mann, W. B., Stockmann, L. L., Youden, W. J. Schwebel, A. Mullen, P. A., Preparation of new solution standards of radium. J 62, 21 (1959) RP2924.

Gates, D. M., Atmospheric research. Americana Annual p. $62(1958) .2462$.

Basic research in Europe. Science 128, No. 3318, 227 (1958). 2469.

$\mathrm{OH}$ emission in the earth's atmosphere. Proc. $2 \mathrm{~d}$ Natl. Infrared Information Symp, 4, No. 1, 145 (1959). 3001.

Preliminary resilts of the National Bureau of Standards radio and ionospheric orservations during the International Geophysical Year. J 63D1, 1 (1959).

Gates, D. M., Murcray, D. G., Shaw, C. C., Herbold, R. J., Near infrared solar radiation measmrements by balloon to an altitude of $100,000 \mathrm{ft}$. J. Opt. Soc. Am. 48, No. 12, 1010 (1958). 2645.

Gauischi, W. Exponential integral $\infty^{-z t} t^{-n} d t$ for large values of $n$. J 62, 123 (1959) RP2941.

Gauthe, B., Electron characteristic energy losses in some intermetallic compounds. Phys. Rev. 114, No. 5, 1265 (1959), 2937

Gautier, T. N., The ionosphere. Sei. American 193, No. 3,126 (1955). 1537

Gautier, T. N., Knecht, R. W., McNish, A. G., Lunar stratification of the F2 layer at Huancayo, Peru. Proc. General Assemhly, Intern. Sci. Rario Union, Zurich, Switzerland, Sept. 11-22, 1950, VIII, Pt. 2, 216 (1950). 83.

Gautier, T. N., Pineo, V. C., The wave-frequency dependence of the duration of radar-type echoes from meteor trails. Science 114, 460 (1951). 348.

Gautier, $W$. $K$., Meteorological instrumentation at the National Bureau of Standards. Weatherwise $\boldsymbol{\gamma}$, No. 4, 86 (1954). 1073.

Gautschi, $W$., Some elementary inequalities relating to a gamma and incomplete gamma function. J. Math. and Phys. 39, 77 (1059). 3047 .

Gaydon, A. G., Broida, H. P., The Herzberg bands of $\mathrm{O}_{2}$ in an oxygen afterglow and in the night sky spectrum. Proc. Roy. Soc. Series [A] 22\%, 181 (1954) 1185 .

The luminots reaction between carbon monoxide and atomic oxygen. Trans. Faraday Soc. 49, 1190 (1953). 866

The mechanism of formation of $\mathrm{OH}, \mathrm{CH}$, and $\mathrm{HCO}$ in flame spectra, using deuterium as tracer. Proc. Roy. Soc. (London, England) 218, 60 (1953). 868.

Gayhart, E. L., Berl, W. G., Olsen, H. L., Broida, H. P. Shuler, K. E., Spectroscopic observations on pentaborane-air flames and explosions. J. Chem. Phys. 25, No. 4, 797 (1956). 1883.

Gee, A., Deitz, V. R., Determination of phosphate by differential spectrophotometry. Anal. Chem. 25, 1320 (1953). 685.

Pyrophosphate formation upon ignition of precipitated basic calcium phosphates. J. Am. Chem. Soc. 7 y, $2961(1955)$ 1465.

Gee, A., Domingues, L. P., Detz, V.R., Determination of inorganic constituents in sucrose solutions. Anal Chem. 26, 1487 (1954). 987.

Geil, G. W., Carmete, J. L., Deformation twinning in charpy $V$-notch specimens of ingot iron. Welding J. Research Suppl. 32, 273-F (1953). 679.

Geil, G. W., Caruile, N. L., A reduction of area gage for use at low temperatures. ASTM Bull. No. 163, $75(1950) .7$.

Effect of strain-temperature history on the tensile behavior of titanium alloy. J 61, 173 (1958) RP2897. Effect of tensile strain at low temperatures on deformation twinning in ingot iron. J. Metals 5, 213
(1953); Trans. Am. Inst. Mining Met. Engrs. 197, 213 (19.53) 695.

Research on effects of prestraining and noteh sharpness on the notch strength of materials. Wright Air Devel. Ctr. (Obio) Tech. Rept. 56-402 (1956). 1853.

Some effects of low temperatures and notch depth on the mechanical behavior of an annealed commercially pure titanium. J 59, 215 (1957) RP2790.

Geissner, S., A note on the normal distribution. Ann. Math. Stat. 27, 858 (1956). 1595.

Geller, R. F., A survey of ceramics for nuclear reactors. Nucleonics 7 , No. 4, 3 (1950). 12.

Geller, R. F., Knudsen, F. P., Moreland, R. E., Physical characteristics of titanium carbide type cermets at elevated temperatures. J. Am. Ceram. Soc. 38, No. 9, 312 (1955). 1445.

Geller, R. F., Lang, S. M., The construction and operation of thoria resistor-type furnaces. J. Am. Ceram. Soc. 24, 193 (1951). 328.

Geltman, S., Theory of threshold energy dependence of photodetachment of diatomic molecular negative ions. Phys. Rev. 112, No. 1, 176 (1958). 2841.

Geltman, S., Branscomb, L. M., Burch, D. S., Smith, S. J., Photodetachment eross section and the electron affinity of atomic oxygen. Phys. Rev. 111, No. 2, 504 (1958). 2683.

Geltman, S., Branscomb, L. M., Smith, S. J., Burch, D. S., Photodetachment studies of negative ions. Third Intern. Conf. on Ionization Phenomena in Gases, Societa Italiana di Fiscia (Venice, Italy) (1957). 2246.

Genensky, S. $M$., A problem in self-heating of a spherical body. J 59, 79 (1957) RP2773.

George, D. A., Kline, G. M., Wolock, I., Axilrod, B. M., Sherman, D. A., Cohen, $V$., Development of craze and impact resistance in glazing plastics by multiaxial stretching. Natl. Advisory Comm. Aeronaut. Tech. Report 1290 (1957). 2084.

George, D. A., Lamb, J. J., Baker, H. A., Sieffert, L. E., Impact strength of some thermosetting plastics at low temperatures. ASTM Bull. No. 181, 67 (1952). 464.

George, D. A., Wolock, I., Effects of multiaxial stretching on crazing and other properties of transparent plastics. Natl. Advisory Comm. Aeronaut. Research Memo. 54F22 (1954). 1002.

Effects of multiaxial stretehing on crazing and other properties of transparent plastics. SPE J. 12, 20 (1956). 1690.

George, W. D., A frequency standard at low temperature. Proc. 10th Ann. Symp. on Frequency Control. Signal Corps Engineering Laboratories, p. 197 (1956). 1589. Etalons et mesures de fréquence et d'intervalle de temps aux Etats-Unis d' Amérique de 1954 á 1957. Procès Verbaux des Séances, Rapport et Annexes, IRE Session (1957), Comité Consultatif pour la Définition de la Seconde, p. 34, (Comité International des Poids et Mesures, Procès-Verbaux des Seances, 2 serie, tome 26-B) Paris, France, Gautier-Villars (1958). 2550. Frequency standards. Cathode Press (Machlett Laboratories, Inc., Stamford, Connecticut) 9, No. 2, 2 (1952). 456

Low frequency standard transmission. Proc. 11th Annual Symp. on Frequency Control, May 7-9, 1957 (Fort Monmouth, N.J.) p. 574 (1957). 2179.

Proposal for standard frequency broadcast at very low frequency. Symp. on the Prop. of V.L.F. Radio Waves, Sponsored by the NBS Boulder Laboratories and IRE Prof. Group on Ant, and Prop., January 23-25, 1957 (Boulder, Colorado), Paper No. 41, V. 3. 2270.

Standard frequency transmission and time signals. Proc. 2d all-IRIG symp., prepared by Secretariat, Inter-Range Instrumentation Group, October 1958 (White Sands Missile Range, New Mexico), IRIG Document No. $107-58$, p. 141 (1959). 3054A.

The need for a new type of frequency and time standard. Proc. IRE 42, 1349 (1954). 1190.

WWV standard frequency transmissions. Proc. IRE 46, 910 (1958). 2871. 
Gerjuoy, E., Saxon, D. S., Application of variational methods to intermediate and high energy scattering. Phys. Rev. 94, 478 (1954). 955.

Variational calculation of scattering cross sections. Phys. Rev. 85, No. 5, 939 (1952). 617.

Variational principles for the acoustic field. Phys. Rev. 94, No. 6, 1445 (1954). 1223.

Gerks, I. H., Sulzer, P. G., Montgomery, G. F., A U-H-F moon relay. Proc. IRE 40, 36 (1952). 396.

Ghaffari, A., On the behavior in the large of the integral curves of a nonlinear differential equation. Abstracts of Short Communications and Scientific Programme, Intern. Congress of Mathematicians (Edinburgh, Scotland), p. 50 (1958). 2662.

Ghaffari, A., Burgers, J. M., On the application of steamdriven water jets for propulsion purposes. J 60, 137 (1958) RP2830.

Gibbons, H. P., McCintock, R. M., Mechanical properties of structural materials at low temperatures. A compilation from the literature. (1960) Mono.13.

Gibson, H. F., Motz, J. E., Miller, W., Wyckoff, H. O., Kirm, F. S., Gamma-ray measurements by the magnetic analysis of Compton electrons. Rev. Sci. Instr. 24, 929 (1953). 725.

Gibson, K. S., Davis, R., Haupt, G. W., Spectral energy distribution of the International Commission on Illumination light sources A, B, and C. Condensation of this paper appeared in J. Opt. Soc. Am. 43, 172 (1953). 834.

Giguere, P., Harvey, K. B., An infrared study of hydrogen bonding in solid $\mathrm{H}_{2} \mathrm{O}$ and $\mathrm{H}_{2} \mathrm{O}-\mathrm{H}_{2} \mathrm{O}_{2}$ mixtures. J. Mol. Spectroscopy 3, 36 (1959). 2894.

Gilchrist, R., Chemical methods for separating and determining metals contained in platiniferous materials. Anal. Chem. 25, 1617 (1953). 668.

Giles, J. R., Observations in abrasion testing. Proc. Porcelain Enamel Inst. Forum 16, 136 (1954). 1089. Practical test methods for the Porcelain Enameling Industry. Proc. Porcelain Enamal Inst. Forum 17, 55 (1955). 1452

Gilford, S. R., Direct-indicating recording instruments. Elec. Mfg. 52, 114 (1953). 690.

Gilford, S. R., Broida, H. P., Physiological monitor for anesthesia. Electronics 28, No. 10, 130 (1955). 1446 .

Gilford, S. R., Gregg, D. E., Shadle, O. W., Ferguson, T. B., Marzetta, L. A., An improved cuvette densitometer for cardiac output determination by the dye-dilution method. Rev. Sci. Instr. 24, 696 (1953). 650.

Gilford, S. R., Shadle, O. W., Ferguson, T. B., Gregg, D. E., Evaluation of a new cuvette densitometer for determination of cardiac output. Circulation Research 1, No. 3, 200 (1953). 714.

Gilfrich, N. T., Swanson, H. E., Cook, M. I., Standard X-ray diffraction powder patterns. (1957) C539, Vol. 7.

Gilfrich, N. T., Swanson, H. E., Cook, M. I., Stinchfield, $R$., Parks, $P$. C., Standard X-ray diffraction powder patterns. (1959) C539, Vol. 8.

Gill, P. S., Curtiss, L. F., The sea-level latitude variation of fast cosmic-ray neutrons. Phys. Rev. 84, 591 (1951). 343.

The variation of intensity of fast cosmic-ray neutrons with altitude. Phys. Rev. 85, No. 2, 309 (1952). 600.

Gillespie, J. F., Rose, J. C., Broida, H. P., Hufnagel, C. A., Rabile, P. J., Freis, E. D., A method for the study of the circulation in the dog using a mechanical left ventricle. J. Appl. Physiol. 7, 580 (1954). 920.

Gilliland, A. A., Johnson, W. H., Prosen, E. J., Heat of formation of titanium tetraiodide. $J \mathbf{6 3 A 2}$, 161 (1959).

Ginnings, D. C., Ditmars, D. A., Thermal conductivity of beryllium oxide from $40^{\circ}$ to $750^{\circ} \mathrm{C}$. J $\mathbf{5 9}, 93$ (1957) RP2775.

Ginnings, D. C., Douglas, T. B., Ball, A. F., Specific heat of beryllium between $0^{\circ}$ and $900^{\circ}$ C. J. Am. Chem. Soc. 73, 1236 (1951). 312.

Ginnings, D. C., Douglas, T. B., Ball, A. F., Davis, W. D., Heat capacity of potassium and three potassium- sodium alloys between $0^{\circ}$ and $800^{\circ}$, the triple point and heat of fusion of potassium. J. Am. Chem. Soc. 74, $2472(1952) .462$.

Ginnings, D. C., Furukawa, G. T., Heat capacity standards for the range $14^{\circ}$ to $122^{\circ} \mathrm{K}$. J. Am. Chem. Soc. $75,522(1953)$. 729.

Ginnings, D. C., West, E. D., An adiabatic calorimeter for the range $30^{\circ}$ to $500^{\circ} \mathrm{C}$. J 60, 309 (1958) RP2848 .

Automatic temperature regulation and recording in precision adiabatic calorimetry. Rev. Sci. Inst. 28, 1070 (1957). 2035.

The heat capacity of aluminum oxide in the range $300^{\circ}$ to $700^{\circ}$ K. J. Phys. Chem. 61, 1573 (1957). 2362.

Ginther, R. J., Schulman, J. H., Shurcliff, W., Attix, F. H., Radiophotoluminescence dosimetry system of the U.S. Navy. Nucleonics 11, 52 (1953). 809.

Gladden, S. C., A history of vertical-incidence ionosphere sounding at the National Bureau of Standards. (1959) TN28 (PB151387).

Procedure used to improve the quality of ionospheric data. Trans. Am. Geophys. Union 35, 398 (1954). 1117.

Glaser, E., McPherson, J. L., Alexander, S. N., Horton, $H$. B., Information processing in social and industrial research. Sci. Monthly 76, 100 (1953). 741.

Glasgow, A. R., Jr., Krouskop, N. C., Rossini, F. D., Apparatus for determining freezing points at saturation pressure from time-temperature freezing and melting experiments. Anal. Chem. 22, 1521 (1950). 26.

Glasgow, A. R., Jr., Ross, G. S., Improved stainless steel needle valve. Anal. Chem, 26, 2003 (1954). 1034.

Glasgow, A. R., Jr., Ross, G. S., Horton, A. T., Enagonio, D., Dixon, H. D., Saylor, C. P., Furukawa, G. T. Reilly, M. L., Henning, J. M., Čmparison of eryoscopic determinations of purity of benzene by thermometric and calorimetric procedures. Anal. Chim. Acta 17, 54 (1957); Proc. of I.U.P.A. International Symp. on Purity Control by Thermal Analysis (Amsterdam, The Netherlands) (April 1957). 2062.

Glasgow, A. R., Jr., Tenebaum, M., Cryoscopic determination of purity of highly reactive substances. Anal. Chem. 28, 1907 (1956). 1666.

Glasson, G. F., Sweeney. W. T., Schoonover, I. C., The effect of preheating on thermal expansion of silicagypsum investments. J. Am. Dental Assoc. 48, 433 (1954). 1184.

Glaze, $F . W$. History of optical glass production in the United States. Am. Ceram. Soc. Bull. 32, 242 (1953). 731.

Recent trends in glass analysis. Am. Ceram. Soc. Bull. 33, 45 (1954). 1126.

Transmittance of infrared energy by glasses. Am. Ceram. Soc. Bull. 34, No. 9, 291 (1955). 1561.

Glaze, F.W., Blackburn, D. H., Osmalov, J.S., Hubbard, D., Black, $M$. H., Properties of arsenic sulfide glass. J 59, 83 (1957) RP2774.

Glicksburg, I., Fan, K., Fully convex normed linear spaces. Proc. Nati. Acad. Sci. 41, No. 11, 947 (1955), 1350 .

Glicksburg, I., Fan, K., Hoffman, A. J., System of inequalities involving convex functions. Proc. Am. Math. Soc. 8, 617 (1957). 2335.

Glime, A., Cassel, J., McKenna, E., Studies on the polar amino acid content of collagen and related material. J. Am. Leather Chemists Assoc. 48, 277 (1953). 841.

Gneug, W., Sweeney, $W$. T., Caul, H. J., Transverse strength testing machine for denture resins. J. Am. Dental Assoc. 49, 174 (1954). 1216.

Goldberg, $K$., A table of Wilson quotients and the third Wilson prime. J. London Math. Soc. 28, Pt. II, 252 (1953). 636.

Principal submatrices of a full-rowed non-negative matrix. J 63B 1, 19 (1959).

Random notes on matrices. J 60, 321 (1958) RP2850. The formal power series for $\log e^{x} e^{y}$. Duke Math. J. 23, 13 (1956). 1931. 
The minimum of a certain linear form. J 64B 1 , 49 (1960).

Unimodular matrices of order two which commute. J. Wash. Acad. Sci. 46, 337 (1956). 1969.

Goldberg, K., Nettleton, R. E., Green, M. S., Dense subgraphs and connectivity. Can. J. Math. 11, 262 (1959), 2925.

Goldberg, K., Newman, M., Pairs of matrices of order two which generate free groups. Illinois J. Math. 1, No. 3, 446 (1957). 2242 .

Goldberg, K., Newman, M., Straus, E. G., Swift, J. D., The representation of integers by binary quadratic rational forms. Arch. Math. (Tübenger, Germany) 5, $12(1954), 1198$.

Golden, P. E., Swindells, J. F., Snyder, C. F., Hardy, R. C., Viscosities of sucrose solutions at various temperatures: tables of recalculated values. (1958) C440 Suppl.

Golden, R. K., Banos, A., Jr., The electromagnetic field of rotating uniformly magnetized sphere. J. Appl. Phys, 23, No. 12, 1294 (1952). 575.

Golden, R. M., Macmillan, R. S., Rusch, W. I'. T., A very-low-frequency antenna for investigating the ionosphere with horizontally polarized radio waves. J 64D 1, 27 (1960).

Golden, S., Free radical stabilization in condensed phases. J. Chem. Phys. 29, 61 (1958). 2570.

Goldman, A. J., A matrix minimization problem. J. Wash. Acad. Sci. 47, No. 12, 405 (1957). 1985.

Capacity requirement of a mail sorting device: II. J 63B2, 79 (1959).

Essential similarity: a counter-example. Am. Math. Mo. (Math. Notes) 65, 546 (1958), 2548.

Games with random payoff matrices. Summary Rept. 3d Conf. on Games, ONR Logistics Project (Princeton University, Princeton, N.J.) (1957). 2137.

The probability of a saddle point. Am. Math. Mo. (Math Notes) 64, 729 (1957). 2379.

Zeros of certain polynomials. J 63B 1, 21 (1959).

Goldman, A. J., Bender, B. K., Analytic comparison of suggested configurations for automatic mail sorting equipment. J 63B2, 83 (1959)

Capacity requirement of a mail sorting device. $\quad \mathrm{J} 62$, 171 (1959) RP2948.

Goldman, A.J., Marcus, $M$., Convexity of the field of a linear transformation. Can. Math. Bull. 2, 15 (1959). 2918 .

Goldman, A. J., Stone, J. J., A symmetric continuous poker model. J 64B 1, 35 (1960).

Goldstein, A., Froberg, C. E., A collision path from the earth to the moon in the restricted problem of three bodies, Kgl. Fysiograf. Sallskap. I. Lund Förh (Lund, Sweden) 22, No. 14, 1 (1952), 359.

Goldstein, H., Wilkins, J., Jr., Spencer, L. Y., Systematic calculations of gamma-ray penetration. Phys. Rev. 89,1150 (1953). 847.

Goldstein, N., Fano, U., Wyckoff, $H . O$, Directional distribution of $1040 \mathrm{kev}$ radiation from a high voltage X-ray tube. J. Appl. Phys. 22, 417 (1951). 224.

Good, C. L., Reinhart, F. W., Turner, P. S. Wolock, I., Comparison of mechanical properties of flat sheets, molded shapes and postformed shapes of cotton fabric-phenolic laminates. Natl. Advisory Comm. Aeronaut. Tech. Note 3825 (1957). 2063.

Goodman, P., Bestul, A. B., Temperature dependence of mechanical shear degradation. J. Polymer Sci. 18, No. 88, 235 (1955). 1527.

Goodman, S., Russell, S. B., Poisson's ratio of aircraft sheet materials for large strains. Proc. First Midwestern Conf. on Solid Mechanics (University of Illinois, Urbana, Illinois) p. 1 (1953). 798.

Goodman, S., Russell, S. B., Noble, C. E., Effect of internal radiant heat transfer on temperature distribution, thermal stress, and deflection in box beams. J 62, 175 (1959) RP2949.

Goodrick, R. B., Evans, R. C., Thermal batteries. J Electrochem. Soc. 99, No. 8, 207 (1952). 602

Goodwin, R. D. Design of simple DC resistance thermometer bridges for wide-range temperature control.
Paper E-5, p. 254. Edited by K. D. Timmerhaus, Chemical Engineering Dept., University of Colorado (Boulder, Colorado) (1958). 2515.

Design of simple resistance thermometer bridges for wide-range control of low temperatures. Rev. Sci.

Instr. 29, 497 (1958). 2516.

High pressure gas handling equipment for autoclaves in chemical research. Ind. Engr. Chem. 49, 861 (1957). 2154.

Wide-range crynstat temperature control. Proc. 1958 Cryogenic Engineering Conference, Paper H-3, p. 487. Edited by K. D. Timmerhaus, Chemical Engineering Dept., University of Colorado (Boulder, Colorado) (1959). 3113.

Goodwin, R. D., Purcell, J. R., Direct-coupled power amplifier for cryostat heating control. Rev. Sci. Instr. 26, 581 (1957). 2090.

Gorden, R., Jr., Hughes, E. E., Determination of acetylene in air in concentrations from ten parts per billion to ten parts per million. Anal. Chem. 31, 94 (1959). 2928.

Gorden, R., Jr., Weaver, E. R., Hughes, $E_{.} E_{u}$, Gunther, S. M., Schuhmann, S., Redfearn, N. T., Interpretation of mass spectra of condensates from urban atmospheres. J 59, 383 (1957) RP2809.

Gordon, C. C., Richmond, J. C., A thickness gage for ceramic coatings. J. Am. Ceram. Soc. 33, 295 (1950). 13.

Gordon, C. L., Nucleonics. Anal. Chem. 26, 176 (1954). 1087.

Silver electrode. Anal. Chem. 27, No. 9, 1508 (1955). 1486.

Gordon, C. L., Wichers, $E$., Purification of mercury and its physical properties. Ann. N.Y. Acad. Sci. 65, No. 5, 369 (1957), 2275.

Gorman, J. G., Jones, E. J., Hipple, J. A., Analysis of solids with the mass spectrometer. Anal. Chem. 23, 438; Phvs. Rev. 82, 768 (1951). 194.

Gorn, S., Cohn, H., A computation of cyclic cubic units. J 59, 155 (1957) RP2783.

Gould, J. H., Brode, W. R., A shutter-illumination device for the study of phototropic dyes. J. Opt. Soc. Am. 42, No. 6, 380 (1952). 374.

Gould, J. H., Brode, W. R., Wyman, G. M., The relation between the absorption spectra and the chemical constitution of dyes. XXV. Phototropism and cistrans isomerism in aromatic azo compounds. J. Am. Chem. Soc. 74, No. 18, 4641 (1952). 593.

The relation between the absorption spectra and the chemical constitution of dyes. XXVI. Effect of solvent and of temperature on the cis-trans isomerization of azo dyes. J. Am. Chem. Soc. 75,1856 (1953). 881 .

Gould, J.H., Brode, W. R., Wyman, G. M., Whitney, J. E., A comparative survey of spectrophotometers in the 201-760 $\mathrm{m} \mu$ region. J. Opt. Soc. Am. 43, 862 (1953). 625 .

Gould, J. H., Inscoe, M. N., Corning, M. E., Brode, W. R., Relation between the absorption snectra and the chemical constitution of dyes: XXIX. Interaction of direct azo dyes in aqueous solution. J 60,65 (1958) RP2823.

Grab, $E$., Savage, I. R., Tables of expected values of 1/X' for positive Bernoulli and Poisson variables. J. Am. Stat. Assoc. 49, 169 (1954). 1161.

Grantham, $R$. E., A reflectionless waveguide termination. Rev. Sci. Instr. 22, 828 (1951). 183.

Granum, P. J., Mordfin, L., Halsey, $N$., Investigation of bearing creep of two forged aluminum alloys. (1960) TN55 (PB161556).

Graver, O. H., Hamilton, E. H., Cleek, G. W., Some properties of glasses in the system barium oxidesilica. J. Am. Ceram. Soc. 41, No. 6, 209 (1958). 2763.

Gray, L. H., Taylor, L. S., Wyckoff, H. O., Program of the International Commission on Radiological Units and Measurements, 2d United Nations Intern. Conf. on the Peaceful Uses of Atomic Energy, Vol. 21, Health and Safety: Dosimetry and Standards, 
$\mathrm{P} / 2243$ WHO, p. 81 (Pergamon Press, London, England) (1959). 3022 .

Green, M. S., Bose-Einstein condensation and the lambda transition in liquid helium. Phys. Rev. Letters 1, 409 (1958). 2475.

The Boltzman equation from the statistical mechanical point of view. J. Chem. Phys, 25, 836 (1956). 1922.

Green, M. S., Beckett, C. W., Woolley, H. W., Themochemistry and thermodynamics of substances. Ann. Rev. Phys. Chem. 7, 287 (1956). 1963.

Green, M. S., Hilsenrath, J., Beckett, C. W., The internal energy of highly ionized gases. IXth Intern. Astronautical Congr. Proc. Amsterdam, Holland, 1958. Springer Verlag (Vienna, Austria), p. 120 (1959). 3081 .

Green, M. S., Nettleton, R. E., Expression in terms of molecular distribution functions for the entropy density in an infinite system. J. Chem. Phys. 29, 1365 (1958). 2558.

Moeb us function on the lattice of dense subgraphs. J 64B 1, 41 (1960).

Green, M. S., Nettleton, R. E., Goldberg, K., Dense subgraphs and connectivity. Can. J. Math. 11, 262 (1959). 2925.

Green, W. C. Talore, R. C., Jr., Air replaces sand in "no-fines" concrete. J. Am. Concrete Inst. 22, No. 10,833 (1951). 190.

Greene, F. M., A source of error in the measurement of radiated harmonics. Proc. IRE 40, 486 (1952). 377.

Influence of the ground on the calibration and use of VHF field-intensity meters. Proc. IRE 14, 58 (1953). 739.

The influence of the ground on the calibration and use of VHF field-intensity meters. Proc. IRE 38, No. 6 (1950). 151.

Greene, F. M., Solow, M., Development of VHF field intensity standards. 'Proc. IRE 40, No. 5, 573 (1952). 427.

Greene, G. E., Mordfin, L., Halsey, N., Harwell, R. H., Jr., Bloss, R. L., Creep and static strengths of large bolted joints of forged aluminum alloys under various temperature conditions. Inst. of Aeronaut. Sci. Preprint No. 779 (1958). 2505.

Greenfeld, S. H., A method of preparing uniform films of bituminous materials. ASTM Bull. No. 193, 50 (1953). 628.

Greenfeld, S. H., Schriesheim, A., Water-soluble degradation products of asphalt. 'ASTM Bull. No. 220, 43 (1957). 2413.

Greenspan, L., Ignition of Kel-f and Teflon. Rev. Sei. Instr. 29, 172 (1958). 2591.

Greenspan, M., Combined translational and relaxational dispersion of sound in gases. J. Acoust. Soc. Am. 26, 70 (1954). 971 .

Comments on a recent paper by Deresiewicz on plane waves in a thermoelastic solid. J. Acoust. Soc. Am. 29, 966 (1957). 2058.

Propagation of sound in five monoatomic gases. J. Acoust. Soc. Am. 28, No. 4, 644 (1956). 1823.

Propagation of sound in rarefied helium. J. Acoust. Soc. Am. 22, 568 (1950). 115.

Rotational relaxation in nitrogen oxygen, and air. J. Acoust. Soc. Am. 31, 155 (1959). 3038.

Greenspan, M., Thompson, M. C., Jr., A logarithmic slidewire for a self-balancing potentiometer. Rev. Sci. Instr. 22, 799 (1951). 179.

An eleven megacycle interferometer for low pressure gases. J. Acoust. Soc. Am. 25, 92 (1953). 648.

Greenspan, M., Thompson, M. C., Jr., Tschiegg, C. E. Subharmonic crystal oscillators. Rev. Sci. Instr. 25, $8(1954)$. 1154.

Greenspan, M., Tschiegg, C. E., An accurate time-modulated pulse circuit. Rev. Sci. Instr. 24, 183 (1953). 646.

Effect of dissolved air on the speed of sound in water. J. Acoust. Soc. Am. 28, 501 (1956). 1686.
Sing-around ultrasonic velocimeter for liquids. Rev. Sci. Instr. 28, No. 11, 897 (1957). 2301.

Speed of sound in water by a direct method. J $\mathbf{5 9}$, 249 (1957) RP2795.

Tables of the speed of sound in water. J. Acoust. Soc. Am. 31, No, 1, 75 (1959). 3061.

Greenspan, M., Tschiegg, C. E., Breckenridge, F., Temperature coefficient of the speed of sound in water near the turning point. J. Acoust. Soc. Am. 28, 500 (1956). 1914.

Greenstone, R., Radio measurement of winds aloft. Aeronaut. Dig. 64, No. 2, 56 (1952). 524.

Greenstone, R., Salzberg, C. D., Svstematic ionospheric winds. J. Geophys. Research 566, No. 4, 521 (1951), 319.

Greenwald, S., Haueter, R. D., Alexander, S. N., SEAC, Proc. IRE 41, 1300 (1953). 820.

Gregg, D. E., Gilford, S. R., Shadle, O. W., Ferguson, T. B., Marzetta, L. A., An improved cuvette densitometer for cardiac output determination by the dye-dilution method, Rev. Sci. Instr. 24, 696 (1953). 650.

Gregg, D. E., Shadle, O. W., Ferguson, T. B., Gilford, S. R., Evaluation of a new cuvette densitometer for determination of cardiac output. Circulation Research $\mathbf{1}$, No. 3, 200 (1953). 714.

Grenb, $W$., Relations between summation methods and integral transformations. J 63B 1, 1 (1959).

Greub, W., Rheinboldt, W. C., Non-self-adjoint boundary val"e problems in ordinary differential equations. J 64B 2, 83 (1960).

Griffing, V., Jackson, J. L., Ransil, B. J., The magnetic interaction of $\mathrm{H}_{3}$. J. Chem. Phys. 30, 1066 (1959). 3082.

Grimes, D. M., Harrington, R. D., Rasmussen, A. L., Magnetic prorerties of polycrystalline materials. J. Phys. Chem. Solids 12, 28 (1959). 2980A.

Grimes, J. W., Johnson, H. D., Winter, J. C., Phillips, C. W., Achenbach, P.R., Heat transfer measurements on 'refrigerated-food trailers. U.S. Dept. of Agriculture, Agricultural Marketing Service (AMS-250) (June 1958), 2580.

Grimsley, J. D., Rosenberg, S. J., A study of the impact properties of boron-treated steels. J. Iron and Steel Inst. 182, 278 (1956). 1601.

Grisamore, N. T., Bay, Z., High speed flip-flops for the millimicrosecond region. IRE Trans. Elec. Computers EC-5, 3 (1956). 1730.

Pulse generator and high-speed memory circuit. IRE Trans. Elec. Computers EC-5, No. 4, 213 (1956). 1827.

Gross, D., Loftus, J., Flame spread properties of building finish materials. ASTM Bull. No. 230 (1958). 2565.

Gross, D., Robertson, A. F., An electrical-analog method for transient heat-flow analysis. J 61, 105 (1958) RP2892.

Self-ignition temreratures of materials from kineticreaction data. J 61, 413 (1958) RP2909.

Gross, D., Robertson, A. F., Loftus, J., A method for measuring surface flammatility of materials using a radiant energy source. Am. Soe. Testing Materials Proc. 56, 1437 (1956). 1593.

Gross, F. L., Saunders, J. B., Interferometer for large surfaces. J 62, 137 (1959) RP2943.

Gross, L. J., Walker, R. F., Zerfoss, S., Holley, S. F., Temperature of the inversion in cristobalite. J 61, 251 (1958) RP2902.

Grunewald, A. H., Dickson, G., Paffenbarger, G. C., Schoonover, I. C., Silicate cement: method of mixing in a closed container to prevent effects of exrosure to atmosphere. J. Am. Dental Assoc. 46, 184 (1953). 827.

Grunewald, A. H., Paffenbarger, G. C., Dickson, G., The effect of molding processes on some properties of denture resins. J. Am. Dental Assoc. 44, 269 (1952). 572

Guggenheim, E. A., Bates, R. G., Harned, H. S., Ives, D. J., Monk, C. B., Robinson, R. A., Stokes, R. H., WynneJones, $W . F$. J., Standard electrode potential of the silver, silver chloride electrode. J. Chem. Phys. 25, 361 (1956). 1895. 
Gumbel, E. J., Lieblein, $J$., Some applications of extremevalue theory. Am. Stat. 8, 14 (1954). 1140.

Gunther, S. M., Weaver, E. R., Hughes, E. E., Schuhmann, S., Redfearn, $N$. T., Gorden, R., Jr., Interpretation of mass spectra of condensates from urban atmosphere. J 59, 383 (1957) RP2809.

\section{H}

Haar, L., Beckett, C. W., Thermodynamic properties at high temperatures: Ideal gas thermal functions to 25,000 deg. K for diatomic molecules, oxygen, nitrogen, nitricocide, and their molecule ions. Proc. Conf. on Thermodynamic and Transport Properties of Fluids, July 10-12 1957 (London, England) p. 27 (1957). 2394.

Haar, L., Friedman, A. S., High-speed machine computation of ideal gas thermodynamic functions. I. The isotopic water molecules. J. Chem. Phys. 22, 2051 (1954). 1030.

High-speed computation of ideal gas thermodynamic functions. II. The diatomic free radicals of the isotopic hydrides of oxygen and sulphur. J. Chem. Phys. 23, No. 5, 869 (1955). 1357.

Haas, P. H., Harrington, R. D., Powell, R. C., Rasmussen, A. L., Instruments for magnetic high frequency measurements. Wright Air Derelopment Center Tech. Report 54-238 (1954), 1043.

Haber, C. P., Maienthal, M., Hellmann, M., Hymo, L. A., Carpenter, S., Carr, A.J., The preparation of some aryl silanes. J. Am. Chem. Soc. 76, 6392 (1954). 1192 .

Hackney, E. J., Bean, L., A study of some operations involved in cement analysis. ASTM Bull. No. 197, $43(1954) .937$.

Haddock, F. T., Hagen, J., Reber, G., NRL Aleutian radio eclipse expedition. Sky and Telescope 10, No. 5, 111 (1951), 273.

Hader, R. J., Youden, W. J., Experimental statistics. Anal. Chem. 24, 120 (1952). 445.

Hagen, J., Haddock, F. T., Reber, G., NRL Aleutian radio eclipse expedition. Sky and Telescope 10, No. $5,111(1951) .273$.

Hague, J. L., Furey, J. J., Sayre, W. R., Determination of nitrogen and sulfur in steel. Anal. Chem. 24, 199 (1952). 426.

Hague, J. L., Machlan, L. A., Determination of niobium and tantalum in titanium-base alloys. $\mathrm{J} \mathbf{6 2}, 53$ (1959) RP2929.

Determination of titanium, zirconium, niobium, and tantalum in steels: separations by anion-exchange. J 62, 11 (1959) RP2923.

Photometric determination of tungsten in steel and titanium alloys with dithiol. J 59, 415 (1957) RP2812 .

Hague, J. L., Machlan, L. A., Meros, E. J., Determination of aluminum in precipitation hardening stainless steel and high temperature alloys. J 64A2, 181 (1960).

Haliday, W. G., Nall, J.R., Drain casting of a high-grade magnesium oxide support used in an experimental vacuum tube. Rev, Sci. Instr, 25, No, 12, 1225 (1954), 996.

Hakkarinen, $W$., Lowell, $P$. D., Allison, L. $M$, Mechanical stops for use with helical potentiometers. Rev. Sci. Instr. 25, No. 4, 398 (1954). 1070.

Coverage of standard frequency station WWVH. Television Eng. 1, No. 8, 16; No. 10, 20 (1950), 42 . Diathermy generators. Arch. Phys. Med. 33, 28 (1952). 429 .

Diathermy rules and the physician. Arch. Phys. Therapy 33, 163 (1952). 430.

Hall, $E$. L., World-wide standard frequency broadcast reception. Tele-Tech. 11, No. 7, 64 (1952); Pt. 2. 11, No. 7, 64 (1952). 623

Hall, F. P., Levin, E. M., McMurdie, H. F., Compilation of phase diagrams of interest to ceramists. Book, Phase of Ceramists (Am. Ceram. Soc.) (1956). 1656.

Hall, M., Jr., Connor, W. S., An embedding theorem for balanced incomplete block designs. Can. J. Math. 6, No. $1,35(1954) .948$
Hall, W. J., Powell, R. L., Roder, H. M., Thermal conductivities of common commercial aluminum alloys. Proc. 1957 Cryogenic Engineering Conference, Paper G-6, p. 408 , Edited by K. D. Timmerhaus, Chemical Engineering Dept., University of Colorado (Boulder, Colorado), (1958). 2842.

Transport properties of dilute alloys of copper. Physica 24, S176 (1958), 2852A

Hall, W. J., Roder, H. M., Powell, R. L., Thermal and electrical conductivity of aluminum and aluminum alloys. In Low-Temperature Physies and Chemistry; 1957 Proc. 5th Intern. Conf., University of Wisconsin (Madison, Wisconsin), p. 389 (1958), 2841A. Thermal and electrical conductivity of pure copper. In Low-Temperature Physics and Chemistry; 1957 Proc. 5th Intern. Conf., University of Wisconsin (Madison, Wisconsin), p. 364 (1958), 2841B.

Hall, $W$. J., Rogers, $W . M$., Powell, R. L., Tables of transport integrals: a supplement. $\vec{J}$ 63B 1,23 (1959).

Halpern, C., Measurement of flame speeds by a nozzle burner method. J 60, 535 (1958) RP2867.

Halpern, C., Ruegg, $F$. W., A study of sampling of flame gases. J 60, 29 (1958) RP2818.

Halsey, N., Mordfin, L., Granum, P. J., Investigation of bearing creep of two forged aluminum alloys. (1960) TN55 (PB161556).

Halsey, N., Mordfin, L., Greene, G. W., Harwell, R. H., Jr., Bloss, $R$, L., Creep and static strengths of large bolted joints of forged aluminum alloys under various temperature conditions. Inst. of Aeronaut. Sci. Preprint No. 779 (1958). 2505.

Hamer, $W . J ., \quad$ A review of the state of the art and future trends in electrochemical battery system. I. The more common systems, Proc. Seminar on Advanced Energy Sources and Conversion Techniques. ASTIA, No. AD209301, p. 41; Dept. Comm. OTS No. PB151461. 2436 .

Fifty years of electrochemical theory. J. Electrochem. Soc. 99, No. 12, 331C (1952). 448.

Lead storage batteries-essential to modern transportation. Capital Chemist 3, 7 (1953). 751.

Modern batteries. IRE Trans. Component Parts CP-4, No. 3, 86 (1957). 2204.

Standards at the National Bureau of Standards. Yale Sei. Mag. 30, No. 2, 7 (1955). 1507.

The electromotive force method of determining ionization constants: ionization of bisulfate ion. Chapter in the book "The Structure of Electrolytic Solutions" (J. Wiley \& Sons, Inc., New York, N.Y.) p. 236 (1959), 3076 .

Hamer, $W, J$, Craig $D . N$. A reproducible and stable silver-silver oxide electrode. J. Electrochem. Soc. 104, 206 (1957). 1998.

Some aspects of the charge and discharge processes in lead-acid storage batteries. Appl. and Ind. No. 11, 22 (1954). 1141 .

Hamer, $W . J .$, Craig, D. N., Law, C. A., Stability of silver and pyrex in perchloric acid-silver perchlorate solutions and in conductivity water. J 64A1, 127 (1960).

Hamer, W. J., Malmberg, M. S., Rubin B., Theoretical electromotive forces for cells containing a single solid or molten chloride electrolyte. J. Electrochem. Soc. 103, 8 (1956). 1957.

Hamer, $W . J .$, Schrodt, $J . P ., \quad$ Dry cell standards broadened-now includes mercury-, air-, and flat-type cells. Mag. of Standards 26, No. 5, 132 (1955). 1321.

Hamer, $W, J$ Wood, $R, E$. Electrolytic conductivity and electrode processes. Handbook of Physics, Edited by E. U. Condon and H. Odishaw, Chapter 9 , Pt. 4, p. 4-138 (McGraw-Hill Book Co., Inc., New York, N.Y.) (1958). 2500

Hamilton, E. H. Cleek, G. W. Properties of sodium titanium silicate glasses. J 61, 89 (1958) RP2890. Shape of the liquidus surface as a criterion of stable glass formation. J 60, 593 (1958) RP2872.

The development of some infrared transmitting glasses. Proc. of the Conference of Infrared Optical 
Materials, Filter and Film; Eng. Research and Dev. Laboratories; The Eng. Ctr. Ft Belvoir, Va,, p. 1 (1954), 1177.

Hamilton, E. H., Cleek, G. W., Grauer, O. H., Some properties of glasses in the system barium oxide-silica. J. Am. Ceram. Soc. 41, No. 6, 209 (1958), 2763

Hamilton, E. H., Spinner, S., Cleek, G. W., Determination and use of the sag point as a reference point in the heating of glasses. J 59, 227 (1957) RP2791.

Hamilton, E. H., Waxler, R. M., Nivert, J. M., Jr., Properties of zinc borosilicate glasses. J 62, 59 (1959) RP2930.

Hammersley, J. M., Antowiewicz, H. A., The convergence of numerical iteration. Am. Math. Mo. 60, 604 (1953). 856.

Hammond, H.K., III, Gloss measurement-past, present, and future. Am. Paint J. 41, No. 37, 94 (1957). 2143.

Hammond, H. K., III, Holford, W. L., Kuder M. L., Ratio-recording spectroradiometer. J 64C2, 151 (1960).

Hammond, H. K., III, Ingle, G. W., Measurement of color, gloss, and haze. Symposium on Plastics Testing, Am. Soc. Testing Materials Spec. Tech. Publ. No. 132 , p. 25 (1953). 760.

Hammond, H. K., III, Newman, S. B., Riddell, H. F., Becker value of manila rope by photoelectric reflectometry. ASTM Bull. No. 199, 84 (1954). 960.

Hammond, $H$. $K$., III, Nimeroff, $\dot{I}$., Minimizing anomalies in reflectance measurements with the Beckman quartz spectrophotometer. J. Opt. Soc. Am. 42, 367 (1952). 484.

Hammond, H. K., III, Nimeroff, I., Richmond, J, C., Crandall, J. R., Gloss meter comparisons. J. Am. Ceram. Soc. 39, No. 3, 103 (1956). 1724.

Specular-gloss measurement of ceramic materials. J. Am. Ceram. Soc. 39, No. 3, 103 (1956). 1886.

Hansen, $W$. C., Brauer, G. M., Davenport, $R$. $M$., Accelerating effect of aminos on polymerization of methyl methacrylate. Modern Plastics 34, No. 3,153 (1956). 1608.

Hansen, $W$. C., Margetis, $P$. M., Changes in agar-agar type duplicating material and agar-agar on heating and storage. J. Am. Dental Assoc. 54, 737 (1957). 2050 .

Hanson, W. B., Pellam, J.R., Second sound attenuation in liquid helium II. Phys. Rev. 95, 321 (1954). 1137 .

Thermal Rayleigh disk measurements in liquid helium II. Phys. Rev. 85, 216 (1952). 603.

Harding, J. E., Schwebel, A., Stockmann, L. L., Report on the determination of small amounts of radium in solution. J. Assoc. Official Agri. Chem. 41, No. 2, $311(1958) .2733$.

Harding, W. B., Koch, J. W., Jansen, R. J., Fading rate recorder for propagation research. Electronics 32, No. 51,78 (1959). 2949.

Hardy, $R$. C., Viscosity of $n$-hexadecane. J 61, 433 (1958) RP2912.

Hardy, R. C., Swindells, J. F., Snyder, C. F., Golden, P. E., Viscosities of sucrose solutions at various temperatures: tables of recalculated values. (1958) C440 Suppl.

Hardy, R. J., Martin, K. B., Jacobs, R. B., Operation of bearings and pumps at low temperatures. Proc. 1957 Cryogenic Engineering Conference, Paper D-6, p. 209, Edited by K. D. Timmerhaus, Chemical Engineering Dept., University of Colorado (Boulder, Colorado) (1958), 2676.

Performance of pumps with liquefied gases. Proc. 1956 Cryogenic Engineering Conference, Paper G-6, p. 295. Edited by K. D. Timmerhaus, Chemical Engineering Dept., University of Colorado (Boulder, Colorado) (1957). 2243.

Harman, A. W., Douglas, T. B., Enthalpy and heat capacity from $0^{\circ}$ to $900^{\circ} \mathrm{C}$ of three nickel-chromiumiron alloys of different carbon contents. J 60, 563 (1958) RP2870.

Heat content of sodium borohydride and of potassium borohydride from $0^{\circ}$ to $400^{\circ}$ C. J 60, 117 (1958) RP2828.

Harman, G. G., Crystal mount and techniques for measuring high frequency induced electroluminescence. Rev. Sci. Instr. 28, No. 2, 127 (1957). 2074.

Electrical properties of samarium doped barium titanate. Phys. Rev. 106, No. 6, 1358 (1957). 2105. Electroluminescence from the surface layer of $\mathrm{BaTiO}_{3}$, $\mathrm{SrTiO}_{3}$ and associated materials. Phys. Rev. 111, No. 1, 27 (1958). 2539.

Harman, G. G., Raybold, R. L., High frequency induced electroluminescence in zinc sulfide. Phys. Rev. 104, No. 6,1498 (1956). 1728.

Harned, H. S., Bates, R. G., Guggenheim, E. A., Ives, D. J., Janz, G. J., Monk, C. B., Robinson, R. A., Stokes, $R$. H., Wynne-Jones, W. F. J., Standard electrode potential of the silver, silver chloride electrode. J. Chem. Phys. 25, 361 (1956). 1895.

Harrington, $R$. D., Cavity techniques for permeability measurements in the VHF region. 6th Electronic Components Conf. Proc. May 27-29, 1955 (Los Angeles, Calif.) p. 27 (1955). 1283.

Characteristics and present requirements of ferrites. Proc. 13th Ann. Meeting Metal Powder Assoc. April 30-May 1, 1957 (Chicago, Illinois) p. 177 (1957). 2051.

Harrington, R. D., Grimes, D. M., Rasmussen, A. L., Magnetic properties of polycrystalline materials. J. Phys. Chem. Solids. 12, 28 (1959), 2980 A.

Harrington, R. D., Haas, P. H., Powell, R. C., Rasmussen, $A$. L., Instruments for magnetic high frequency measurements. Wright Air Development Center Tech. Report 54-238 (1954). 1043.

Harrington, R. D., Powell, R. C., High-frequency magnetic permeability measurements using toroidal coils. Proc. IRE 46, 784 (1958). 2584.

Harrington, R. D., Rasmussen, $A$. L., Initial and remanent permeability spectra of Yttrium iron garnet. Proc. IRE 47, 98 (1959). 2966.

Harrington, $R$. $F$., Effect of antenna size on gain, bandwidth, and efficiency. J 64D 1, 1 (1960).

Harris, $F$. $K$., Galvanometer efficiency as a design parameter. Commun. and Electronics, Am. Inst Elec, Engrs. 24, 176 (1956). 1717.

Harris, F. K., McGregor, M. C., Hersh, J. F., Cutkowsky, $R$. D., Kotter, $F, R$., New apparatus at the $\mathrm{Na}$ tional Bureau of Standards for absolute capacitance measurement. IRE Trans. Instrumentation I-7, No. 3-4, 253 (1958), 2647.

Harris, W. P., Cooter, I. L., A feedback amplifier with negative ouptut resistance for magnetic measurements. IRE Natl. Conv. Record, Pt. 5, p. 217 (1958). 2421.

Improved bridge method for the measurement of core losses in ferromagnetic materials at high flux densities. J 60, 509 (1958) RP2865.

The use of an AC bridge to measure core loss at high induction. Commun. and Electronics, Am. Inst. Elec. Engrs. No. 24, 252 (1956). 1954.

Harrison, G. R. Meggers, $W . F$., Zeeman effect in spark lines of ruthenium. J. Opt. Soc. Am. 43, 816 (1954). 1228.

Harrison, C. W., Jr., King, R. W. P., Response of a loaded electric dipole in an imperfectly conducting cylinder of finite length. J 64D 3, 289 (1960).

Harrison, W. N., Moore, D. G., Pitts, J. W., Role of nickel dip in the enameling of sheet steel. J. Am. Ceram. Soc. 37, No. 8, 363 (1954). 1133.

Harrison, W. N., Moore, D. G., Pitts, J. W., Richmond, $W . N$., The galvanic corrosion theory for adherence of porcelain enamel ground coats to steel. Inst. Vitreous Enamellers Ltd. Bull. (Ripley, England) 7, No. 4 (1957). 2358.

Harrison, W. N., Richmond, J. C., The evaluation of small color differences: I. Visual observations. Bull. Am. Ceram. Soc. 38, 292 (1959). 3078.

Harrison, W. N., Richmond, J. C., Crandall, J.R., Effects of acid treatment on acid and abrasion resistance 
of porcelain enamels. J. Am. Ceram. Soc. 33, 314 (1950). 51.

Harrison, W. N., Richmond, J. C., Lefort, H. G., Williams, $C$. $N$., Ceramic coatings for nuclear reactors (a progress report). J. Am. Ceram. Soc. 38, No. 2, 72 (1955). 1285

Harrison, W. N., Richmond, J. C., Moore, D. G., Kirkpatrick, $H$. B., Relation between roughness of interface and adherence of porcelain enamel to steel. Inst. Vitreous Enamellers Ltd. Bull. (Published by J. Am. Ceram. Soc.) 7, No. 5, 69 (1957). 2281.

Hart, E. J., Koch, H. W., Petree, B., Schulman, J. H., Taimuty, S. I., Wyckoff, H. O., Measurement systems for high-level dosimetry. 2d United Nations Intern. Conf. on the Peaceful Uses of Atomic Energy, Vol. 21, Health and Safety: Dosimetry and Standards, P/1927 USA, p. 188 (Pergamon Press, London, England) (1959). 2984.

Hart, V. E., A graphical method for single-point determination of intrinsic viscosity. J. Polymer Sci. 17, 215 (1955). 1235.

A semi-micro dilution viscometer. J. Polymer Sci. 17, 207 (1955). 1245.

Hart, I. E., Madorsky, S. L., Straus, S., Products volitalized from a channel and a furnace black on heating to $1,000^{\circ} \mathrm{C}$. Rubber Age 84, 238 (1953). 803. Thermal degradation of cellulosic materials. J 60, 343 (1958) RP2853.

Hart, I. E., Wall, L. A., Brown, D. W., Pyrolysis of $\alpha$ and $\beta$ deuterostyrene polymers. J. Polymer Sci. 15, No. 79,157 (1955). 1464

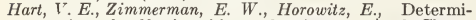
nation of sulfur in rubber vulcanizates. Ánal. Chem. 27, No. 10, 1606 (1955). 1311.

Hartley, J. L., Hudson, D. C., Moore, R. B., Sweeney, W. $T$., Some factors influencing the cutting characteristics of rotating dental cutting instruments. J. Am. Dental Assoc. 50, 377 (1955). 1494.

Hartley, J. L., Hudson, D. C., Sweeney, W. T., Dickson, G., Development of methods for evaluation of rotating dental diamond abrasive instruments. J. Am. Dental Assoc. 54, 637 (1957). 2085.

Hartley, J. L., Hudson, D. C., Sweeney, W. T., Richardson, $W$. P., Cutting characteristics of dental burs as shown by high speed photomicrography. U.S. Armed Forces Med. J. 8, 209 (1957). 2076.

Hartman, C. C., Shellac. Offic. Dig. Federation Paint Varnish Production Clubs 29, No. 393, 1028 (1957). 2298.

Hartman, W. J., Limit of spatial resolution of refractometer cavities. J 64D1, 65 (1960).

Hartman, $W . J$. Wilkerson, R. E., Path antenna gain in an exponential atmosphere. J 63D3, 273 (1959).

Hartsfield, $W$. L., Observations of distant meteor-trail echoes followed by ground scatter. J. Geophys. Research 60, $53(1955)$. 1421

Hartsfield, W. L., Silberstein, $R$., A comparison of CW field intensity and backscatter delay. Proc. IRE 40, 1700 (1952). 361.

Low-pass duplexing system for high-frequency pulse transmitters, Tele-Tech 13, 76 (1954). 1056.

Harvey, J. L., Pfeiffer, K. R., Brauer, G. M., Deterioration during storage of alginate hydrocolloidal dental impression material. U.S. Armed Forces Med. J. 5, No. 9, 315 (1954), 986.

Harvey, J.L., Wilson, $W . \dot{K}$, Effect of light on coated groundwood papers. Tappi 36, 459 (1953). 693. Effect of light on coated groundwood papers. Indian Pulp and Paper 9, 444 (1955). 1327.

Harvey, J. L., Wilson, W. K., Mandel, J., Worksman, $T$. L., Accelerated aging of record papers compared with normal aging. Tappi 38, No. 9, 543 (1955). 1251 .

Harvey, J. L., Wilson, W. K., Padgett, A. A., Effect of melamine resin on chemical tests of paper. Tappi 34,410 (1951). 227.

Harvey, $K$. B., Bass, $A . \dot{M}$., Infrared absorption of oxygen discharge products and ozone at $4^{\circ} \mathrm{K}$. J. Mol. Spectroscopy 2, 405 (1958). 2599.
Harvey, K. B., Brown, H. W., Etude aux infrarouges de certains solides condenses a partir de decharges en phase gazeuse. J. chim. phys. 56, No. 2392, 745 (1959). 2943.

Harvey, $K$. B., Giguere, $P$., An infrared study of hydrogen bonding in solid $\mathrm{H}_{2} \mathrm{O}$ and $\mathrm{H}_{2} \mathrm{O}-\mathrm{H}_{2} \mathrm{O}_{2}$ mixtures. $\mathrm{J}$. Mol. Spectroscopy 3, 36 (1959). 2894 .

Harvey, M. R., Bersch, C. F., Achhammer, B. G., Heat and ultraviolet aging of poly(vinyl chloride). J 60, 481 (1958) RP2863.

Harvey, M. R., Wall, L. A., Tryon, M., Oxidative degradation of styrene and a-deuterostyrene polymers. J. Phys. Chem. 60, 1306 (1956). 1802.

Harwell, R. A., Schaum, J. H., Versatile core boxes. Foundry 80, No. 5, 154 (1952). 619.

Harwell, R. H., Jr., Mordfin, L., Greene, G. E., Halsey, N., Bloss, R. L., Creep and static strengths of large bolted joints of forged aluminum alloys under various temperature conditions. Inst. of Aeronaut. Sci. Preprint No. 779 (1958). 2505.

Hasegawa, S., Garfinkel, S. B., Wexler, A., Simple humidity lag apparatus. Rev. Sci. Instr. 26, No. 12, 1196 (1955). 1487.

Hashman, J. S., Ruehrwein, R. A., Formation of ozone from atomic oxygen at low temperatures. J. Chem. Phys. 30, No. 3, 823 (1959). 2954.

Hasko, S., Roth, R. S., Beta-alumina-type structure in the system lanthana-alumina. J. Am. Ceram. Soc. 41, No. 4, 146 (1958). 2472.

Hatch, M. R., Jacobs, R. B., Richards, R. J., Boggs, R. N., Phelps, G. R., Prediction of pressure drop in twophase single-component fluid flow. Proc. 1958 Cryogenic Engineering Conference, Paper F-4, p. 357. Edited by K. D. Timmerhaus, Chemical Engineering Dept., University of Colorado (Boulder, Colorado) (1959). 3019.

Haueter, R. D., Greenwald, S., Alexander, S. N., SEAC. Proc., IRE 41, 1300 (1953). 820.

Haught, A. F., Mulligan, B. W., Correction for instrumental drift in flame photometry. J 61, 499 (1958) RP2918.

Haupt, G. W., An alkaline solution of potassium chromate as a transmittancy standard in the ultraviolet. Condensation of this paper appeared in J. Opt. Soc. Am. 42, 441 (1952). 388.

Haupt, G. W., Davis, R., Gibson, K. S., Spectral energy distribution of the International Commission on Illumination light sources $A, B$, and $C$. Condensation of this paper appeared in J. Opt. Soc. Am. 43, $172(1953)$. 834

Hawes, B. K., Selgin, P. J., Temperature prediction in electronic design. Elec. Mfg. 50, No. 4, 116 (1952). 564.

Hawkins, J. E., Armstrong, G. T., The physical properties of terpenes. III. The vapor pressures of alpha pinene and beta pinene. J. Am. Chem. Soc. 76, 3756 (1954). 1191A.

Haydon, G. $W$., Optimum frequencies for outer space communication. J 64D2, 105 (1960).

Hayes, W. D., Cuthill, J. R., Seebold, R. E., Nitriding phenomena in titanium and the $6 \mathrm{~A} 1-4 \mathrm{~V}$ titanium alloy. J 64A 1, 119 (1960).

Haynsworth, E. V., Applications of a theorem on partitioned matrices. J 63B2, 73 (1959).

Note on bounds for certain determinants. Duke Math. J. 24, No. 3, 313 (1957). 2218.

Hayward, E. V., Fuller, E. G., Elastic scattering of photons. Phys. Rev. 94, 732 (1954). 1006. Elastic scattering of photons by tantalum. Phys. Rev. Letters 1, No. 12, 465 (1958). 2535. Nuclear elastic scattering of photons. Phys. Rev. 95, 1106 (1954). 1086; Phys. Rev. 101, No. 2, 692 (1956). 1787

Parameter of the 15-Mev X-ray scattered by $\mathrm{C}^{12}$ Physica 22, 1138 (1956). 1803.

Photon scattering and self absorption in lead and bismuth at 7 Mev. Proc. Congress International de Physique Nucleaire, 1958, Nuclear Reaction in 
Nuclear Forces, Pt. 2, Chapter I, p. 62 (Paris, France) (1959). 3014.

Photon self-absorption and scattering by the 15.1Iev level in C12. Phys. Rev. 106, 991 (1957). 2248 .

Hayward, E. V., Fuller, E. G., Koch, H. W., Shape of the high-energy end of the electron bremsstrahlung spectrum. Phys. Rev. 109, 630 (1958). 2755.

Hayward, E. I., Hubbell, J. H., Backscattering of the cobalt-60 gamma rays from infinite media. J. Appl. Phys. 25, 506 (1954). 959.

The albedo of various materials for 1 Mev photons. Phys. Rev. 93, 955 (1954). 1170

Hayward, E. i., Hubbell, J. H., Titus, W. F., Energy and angular distribution of X-rays scattered in lead. Phys. Rev. 108, No. 6, 1361 (1957). 2111.

Hayward, $R$. W., Beta-ray spectrometers. Chapter in Vol. 5 of Advances in Electionics (Academic Press Inc., New York, N.Y.) (1953), 657.

NBS alpha standards coincidence counting, and radiative electron capture. Proc. Symp. on Measurements and Standards of Radioactivity, Natl, Acad. Sci., Natl. Research Council, Nuclear Science Series No. 24,12 (1958). 2644.

Nuclear electromagnetic radiations. Handbook of Physics, Edited by E. U. Condon and H. Odishaw, Chapter 6, Pt. 9, p. 9-106 (McGraw-Hill Book Co., Ine., New York, N.Y.) (1958). 2500.

The decay of the Cd115 isomers. Phys. Rev. 87, 202 (1952). 569.

Hayward, R. W., Ambler, E., Hoppes, D. D., Hudson, $R$. P., Absence of interference effects in the $\beta$-decay of polarized $\mathrm{CO}^{58}$ and $\mathrm{CO}^{58}$ nuclei. Phys. Rev. 108, 503 (1957). 2006.

Beta-gamma correlations from polarized manganese52. Phys. Rev. 110, No. 3, 787 (1958); Proc. Intern. Congr. on Nuclear Phys. (Paris, France, July 1958) and Proc. Kamerlingh Onnes Conf. on Low Temp. Phys. Leiden, The Netherlands, Supplement of Physica (June 1958). 2473.

Beta-gamma correlations from polarized nuclei. Proc. Congres Intern. de physique Nucleaire, 1958, Interactions Faibles, Chapter III, p. 831 (Paris, France) (1959). 2902.

Experiments on the beta-decay of polarized nuclei. Proc. Kamerlingh Onnes Conference, Leiden, Holland, 1958; Physica (Supplement) 24, S64 (1958). 2557.

Hayward, R. W., Ambler, E., Hoppes, D. D., Hudson, R. P. $W u, C$. S., Further experiments on $\beta$-decay of polarized nuclei. Phys. Rev. 106, No. 6, 1361 (1957). 2132.

Hayward, R. W., Hoppes, D. D., Disintegration of selenium-73. Bull. Am. Phys. Śoc. 30, No. 1, 33A (1955). 1316.

Disintegration of selenium-73. Phys. Rev. 101, No. 1, 93 (1956). 1680.

Gamma radiation from Ag105. Bull. Am. Phys. Soc. Series II, No. 1, 42 (1956). 1718

Inner bremsstrahlung from $V^{49}$. Bull. Am. Phys. Soc. 30, No. 3, 59A (1955). 1368.

Radiative electron capture in cesium-131. Phys. Rev. 104, No. 2, 368 (1956). 1834.

Radiative orbital electron capture in vanadium-49. Phys. Rev. 104, No. 1, 183 (1956). 1835.

Scintillation counting in experiments on parity conservation. Proc. 6th Scin. Counter Symp. IRF Trans. Nuclear Science NS-5, 161 (Tan. 1958). 2749.

Hayward, R. W. Hoppes, D. D., Ernst, H., The disintegration of $\mathrm{Ba}^{113}$. Phys. Rev. 93, 916 (1954). 1178. The disintegration of $\mathrm{Nb}^{91}$ and $\mathrm{Nb}^{92}$. Bull. Am. Phys. Soc. 29, No. 7, 16A (1954). 1179.

Hayward, R. W., Pessoa, E. F., Hoppes, D. D., van Lieshout, $R$., Gamma radiation from $\mathrm{Zn}^{63}$. Il Nuovo Cimento 11, 53 (1959). 2956.

Hayward, R. W., Wu, C. S., Ambler, E., Hoppes, D. D., Hudson, $R$. $P$., An experimental test of parity conservation in beta decay. Phys. Rev. 105, 1413 (1957). 2015.
Heath, D. F., Broida, H. P., Luminous reaction between carbon monoxide and atomic nitrogen. J. Chem. Phys. 26, 1352 (1957). 2183.

Spectroseopic survey of energy distributions of $\mathrm{OH}$ $\mathrm{C}_{2}$ and $\mathrm{CH}$ radicals in low-pressure acetylene-oxygen flames. J. Chem. Phys. 26, 223 (1957), 2316.

Heaton, $V$. E., Measuring time and frequency in Hawaii. Tele-Tech. 10, No. 3, 36 (1951). 257.

Hedberg, R. W., Kerns, D. M., Propagation constant in rectangular wave guide of finite conductivity. J. Appl. Phys. 25, No. 12, 1550 (1954). 1120.

Hefley, G., Doherty, R. H., Berger, E. L., An intermittent-action camera with absolute time calibration. J 64C2, 159 (1960).

Heindl, R. A., Foundry crucible service life. Am. Foundryman 22, 40 (1952). 454.

The refractories industry in some European countries. The Refractories Inst. Tech. Bull. No. 90, p. 1 (1952). 592 .

Heindl, R. A., Mackles, L., Mong, L. E., Chemical analyses, surface area, and thermal reactions of natural graphite, and refractoriness of the ashes. J. Am. Ceram. Soc. 36, 266 (1953). 665.

Heindl, R. A., Massengale, G. B., Mong, L. E., Permeability and some other properties of a variety of refractory materials. J. Am. Ceram. Soc. 36, 222 (1953). 794.

Heindl, R. A., Mohler, N. F., Oxidation studies of some natural graphites. J. Am. Ceram. Soc. 38, No. 3, 89 (1955). 1437

Heindl, R. A., Pendergast, W. L., Results of laboratory tests of high duty and super duty fireclay plastic refractories. Bull. Am. Ceram. Soc. 36, No. 1, 6 (1957). 2289

Heindl, R. A., Post, Z. A., Refractory castables, preparation and some properties. J. Am. Ceram. Soc. 33, 230 (1950). 125.

Helimian, $N . \dot{C}$., Chart speeds design of feedback amplifiers. Electronics 25, No. 9, 153 (1952). 410.

Constant-amplitude oscillator. Electronies 24, 164 (1951). 210.

Feedback amplifiers with stabilized output impedances. Tele-Tech 12, 103 (1953). 719.

Frequency-deviation meter plots drift. Electronics 25, No. 6, 134 (1952). 455; Radio and Television News (Radio-Electronic Engineering Section) 50, No. 4, 32 (1953). 723.

Helliwell, R. A., Gallet, R. M., A theory of the production of VLF noise (so-called drawn chorus) by traveling wave amplification in the exosphere of the earth. Symp. on the Prop. of VLF Radio Waves, Sponsored by the NBS Boulder Laboratories and IRE Prof. Group on Ant. Prop., January 23-25, 1957 (Boulder, Colorado), Paper No. 20, V 2 (1957). 2003.

Origin of "very-low-frequency emissions". J 63D1, 21 (1959).

Helliwell, R. A., Jean, A. G., Taylor, W. L., Some properties of lightning impulses which produce whistlers. Proc. IRE Letter 46, 1760 (1958). 2764.

Hellmann, M., Alexander, R. L., Jr., Coyle, C. F., Separation of isomeric polyphenyls by adsorption chromatography. Anal. Chem. 30, 1206 (1958). 2752.

Hellmann, M., Bilbo, A. J., The preparation of two fluorinated p-dihalobenzenes. J. Am. Chem. Soc. 75, 4590 (1953). 879 .

Hellmann, M., Bilbo, A. J., Pummer, W. J., Synthesis and properties of fluorinated polyphenyls. J. Am. Chem. Soc. 77, 3650 (1955). 1521.

Hellmann, M., Maienthal, M., Haber, C. P., Hymo, L. A. Carpenter, S., Carr, A.J., The preparation of some aryl silanes. 'J. Am. Chem. Soc. 76, 6392 (1954). 1192.

Hellmann, $M$., Mandlekern, L., Brown, D. W., Roberts, D. E., Quinn, F. A., Jr., The melting transition of polymethylene. J. Am. Chem. Soc. 75, 4093 (1953). 869.

Hellmann, M., Peters, E., Pummer, W. J., Wall, L. A., Hexafluorobenzene from the pyrolysis of tribromofluoromethane. J. Am. Chem. Soc. 79, 5654 (1957). 2151 . 
Hellmann, M., Stewart, J. E., Infrared spectra of crystalline polyphenyls. J 60, 125 (1958) RP2829.

Helson, H., Judd, D. B., Warren M. H., Object-color changes from daylight to incandescent filament illumination. Illum. Eng. 47, 221 (1952). 492.

Helson, H., Judd, D. B., Wilson, M., Color rendition in fluorescent sources of illumination. Illum. Eng. 51, $329(1956) .1651$.

Hendricks, S., Bramao, L., Cady, J. G., Swerdlow, M., Criteria for the characterization of kaolinite halloysite, and a related mineral in clays and soils. Soil Sci. 73, No. 4, 273 (1952). 420.

Henig, S., Determination of a general index of effort in sorting mail by conventional methods. (1960) TN54 (PB161555).

Henning, J.M., Glasgow, A. R., Jr., Ross, G. S., Horton, A. T. Enagonio, D., Dixon, H. D., Saylor, C. P., Furukawa, G. T., Reilly, M. L., Comparison of cryoscopic determinations of purity of benzene by therometric and calorimetric procedures. Anal. chim. acta 17, 54 (1957); Proc. of I.U.P.A. International Symposium on Purity Control by Thermal Analysis (Amsterdam, The Netherlands) (April 1957). 2062.

Henrici, $P$. A Neumann series for the product of two Whittaker functions. Proc. Am. Math. Soc. 4, 331 (1953). 630.

A subroutine for computations with rational numbers. J. Assoc. Computing Machinery 3, No. 1, 6 (1956). 1603.

Addition theorems for general Legendre and Gegenbauer functions. J. Rational Mech. Anal. 4, No.6, 983 (1955). 1253.

Application of two methods of numerical analysis to the computation of the reflected radiation of a point source. J. Wash. Acad. Sci. 45, 38 (1955). 1267.

Automatic computations with power series. J. Assoc. Computing Machinery 3, No. 1, 10 (1956). 1638.

Bergmans integraloperator erster art and Riemannsche funktion. Z. Angew. math. u phys. (Basel, Switzerland) 3, $229(1952) .401$.

Kleine Bemerkung zur asymptotischen enwicklung des fehlerintegrals. Z angew. math. $u$ phys. (Basel, Switzerland) 6, 145 (1955). 1383.

On certain series expansions involving Whittaker functions and Jacobi polynomials. Pacific J. Math. 5, Suppl. 1, 725 (1955). 1424.

On generating functions of the Jacobi polynomials. Pacific J. Math. 5, Suppl. 2, 923 (1955). 1426. On helical springs of finite thickness. Quart. Appl. Math. 13, No. 1, 106 (1955). 1427.

On rotational viscous flow through a tube. A. angew. math. phys. 5, 511 (1954). 1094.

On the representation of a certain integral involving Bessel functions by hypergeometric series. J. Math. and Phys. 36, No. 2, 151 (1957). 2236.

The quotient-difference algorithm. (1958) AMS49.

Ueber die funktionen von Gegenbauer. Arch. math. (Tübingen, Germany) 5, 92 (1954). 1221.

Henrici, $P$., Chaix, B., On the design of two-dimensional nozzles by the method of characteristics. J. Aeronaut. Sci. 22, 140 (1955). 1432.

Henry, R. L., Scal, R. $\dot{K}-F$, Shapiro, G., New techniques for electronic miniaturization. Proc. IRE (Sydney, Australia) 13, No. 3, 75 (1952). 489.

Herbold, R. J., Bates, D. M., Murcray, D. G., Shaw, C. C., Near infrared solar radiation measurements by balloon to an altitude of $100,000 \mathrm{ft}$. J. Opt. Soc. Am. 48, No. $12,1010(1958) .2645$.

Herbstreit, J. W., Signal generator system for low output levels. Electronics 26, 218 (1953), 826.

Herbstreit, J. W., Crysdale, J.H., Dickson, F. H., Egli, J.J., Wickizer, $\dot{G}$. S., Rebuttal to comments on "Large reduction of VHF transmission loss and fading by the presence of a mountain obstacle in bey ond-line-of-sight paths", by F. H. Dickson, J. J. Egli, J. W. Herbstreit, and G. S. Wickizer (Publ. in Proc. IRE 41, 967 (1953)). Proc. IRE 43, 627 (1955). 1384.

Herbstreit, J. W., Dickson, F. H., Egli, J. J., Wickizer, G. S., Large reductions of VHF transmission loss and fading by the presence of a mountain obstacle in beyond-lineof-sight paths. Proc. IRE 41, 967 (1953). 750.

Herbstreit, J. W., Kirby, R. S., Norton, K. A., Service range for air-to-ground and air-to-air communications at frequencies above 50 Mc. Proc. IRE 40, 525 (1952). 540

Herbstreit, J. W., Norton, K. A., Rice, P. L., Schafer, G. E., Radio wave scattering in tropospheric propagation. Conv. Record of the IRE, Pt. 2, Antennas and Communications, p. 85 (1953). 808 .

Herbstreit, J. W., Rice, P. L., Survey of Central Radio Propagation Laboratory research in tropospheric propagation 1948-1956. (1959) TN26 (PB151385).

Herbstreit, J. W., Rice, P. L., Mansfield, W. V., Groundto-air cochannel interference at $2900 \mathrm{Mc}$. IRE Trans Airborne Electronics AE-6, 1 (1952). 459.

Herbstreit, J. W., Thompson, $M$. C., Jr., Continuous phase difference measurements of earth satellites. Proc. IRE 46, No. 8, 1535 (1958). 2499.

Measurement of the phase of radio waves received over transmission paths with electrical lengths varying as a result of atmospheric turbulence. URSI Symp. on Electromagnetic Wave Theory, June 1955 (Ann Arbor, Mich.); Proc. IRE 43, 1391 (1955). 402 .

Measurements of the phase of signals received over transmission paths with electrical lengths as a result of atmospheric turbulence. IRE Trans. Ant. Prop. AP-4, 352 (1956). 1770.

Hermach, $F$. L., AC-DC transfer instruments for current and voltage measurements. IRE Trans. Instrumentation I-7, No. 3-4, 235 (1958). 2446.

Electrothermic instruments for the measurement of alternating current and voltage. Proc. Symp. Natl. Phys. Lab., Precisions of Electrical Measurement, Teddington, England. (H. M. Station Office, London, England) (1954). 1012.

Hazards from static electricity. Military Engr. 44, 287 (1952). 461.

Power supplies for 60-cycle tests of electrical instruments and meters. Instr. Soc. Am. Proc. 11, Paper No. 56-21-3 (1956). 1814.

The definition and measurement of the time constant and response time of thermal converters. Trans. Am. Inst. Elec. Engrs. 77, Pt. I, 277 (1958); Communications and Electronics No. 37, 277 (1958). 2809.

The testing of electrical instruments. Proc. Instr. Soc. Am. 8, 18 (1953). 886.

Hermach, F. L., Boone, T. H., MacArthur, E. H., McAuliff, R. C., Conductive flooring for hospital operating rooms. J 63C2, 113 (1959); (1960) Mono. 11.

Hermach, F. L., Peterson, C., Dunfee, B. L., Methods for measuring the " $\mathrm{Q}$ " of large reactors. Am. Inst. Elec. Engrs. Trans. Paper No. 56-87 (1956). 1776.

Hermach, F. L., Spinks, A. W., Portable potentiometer and thermostated containers for standard cells. Rev. Sci. Instr. 26, 770 (1955). 1450.

Hermach, F. L. Zapf, T. L., Lead resistance errors in watthour-meter phantom load test. Elec. World 141, 113 (1954). 1049.

Herman, R. C., Benedict, W. S., Silverman, S., R-branch heads of some $\mathrm{CO}_{2}$ infrared bands in the $\mathrm{CO}+\mathrm{O}_{2}$ flame spectrum. J. Chem. Phys. 19, 1325 (1951). 293.

Herman, R. C., Moore, G. E., Shuler, K. E., Silverman, S., The reactions of ammonia and hydrazine with oxygen atoms and hydrogen atoms in atomic flames. J. Phys. Chem. 60, 813 (1956). 1948.

Herman, R. C., Rubin, R. J., Vibrational energy exchange between diatomic molecules and a surface. J. Chem. Phys. 29, 591 (1958), 2862.

Herman, R. C., Shuler, K. E., Studies in nonequilibrium rate processes. IV. The rotating and vibrational relaxation of a system of rotating oscillators. J. Chem. Phys. 29, 366 (1958). 2787.

Herron, J. T., Franklin, J. L., Bradt, P., Mass spectrometric study of the reactions of some hydrocarbons 
with active nitrogen. Can. J. Chem. 37, 579 (1959). 2982.

Herron, J. T., Franklin. J, L., Bradt, P., Dibeler, V. H., Kinetics of nitrogen atom recombination. J. Chem. Phys. Letters 29, No. 1, 230 (1958); J. Chem. Phys. 30, 879 (1959). 2972.

Mass spectrometric study of the decomposition of hydrazoic acid by the electric discharge. J. Am. Chem. Soc. 80, 6188 (1958). 2623.

Hersh, J. F., McGregor, M. C., Cutkowsky, R. D., Harris, $F$. K., Kotter, F.R., New apparatus at the National Bureau of Standards for absolute capacitance measurement. IRE Trans. Instrumentation I-7, No. 3-4, 253 (1958). 2647.

Herzfeld, C. M., Incomplete equilibrium and temperature measurement. J. Wash. Acad. Sci 46, 269 (1956). 1735 .

On the dependence of the constants in the CurieWeiss law on exchange. J. Chem. Phys. 23, 398 (1955). 1431.

Theory of the forbidden transitions of nitrogen atoms trapped in solids. Phys. Rev. 10\%, 1239 (1957). 2392 .

Herzfeld, C. M., Bass, $A$. $M$., Frozen free radicals. Sci, American 3, 91 (1957). 2129.

Herzfeld, C. M., Broida, H. P., Interpretation of spectra of atoms and molecules in solid nitrogen condensed at $4.2^{\circ} \mathrm{K}$. Phys. Rev. 101, 606 (1956). 1747.

Herzfeld, C. M., Hutchison, C. A., Jr., Magnetic susceptibilities of some U(IV) compounds. J. Chem. Phys. 23, 1650 (1955). 1396.

Herzfeld, C. M., Levine, D. B., Effect of crystal field and spin-orbit coupling on magnetic susceptibility of systems with $f^{2}$ electron configuration. $\quad$ 61, 117 (1958) RP2893.

Hess, A. E., Powell, R. C., Jickling, R. M., High-frequency impedance standards at the National Bureau of Standards. IRE Trans. Instrumentation I-7, No. $3-4,270$ (1958). 2583.

Hess, A. E., Rasumussen, A. L., RF permeameter techniques for testing ferrite cores. Elec. Mfg. 61, 86 (1958). 2746.

Hestenes, $M$. R., An elementary introduction to the calculus of variations. Mathematics 23, No. 5, 249 (1950). 23.

Applications of the theory of quadratic forms in Hilbert space to the calculus of variations. Pacific J. Math. 1, No. 4, 525 (1951). 195.

Hetzer, H. B., Davis, M. M., Relative strengths of forty aromatic carboxylic acids in benzene. J 60, 569 (1958) RP2871.

The ionic dissociation of 2, 4-, 2, 6-, 3, 4-dichlorobenzoic acids in water. J. Phys. Chem. 61, 123 (1957). 2368.

The ionic dissociation of 2, 6-dimethylbenzoic acid in water. J. Phys. Chem, 61, 125 (1957). 2369.

The relative basicities of tribenzylamine and tribenzylamine oxide in benzene and water. Preparation and properties of tribenzylamine oxide. J. Am. Chem. Soc. 76, 4247 (1954). 1195.

Hewitt, C. A., Automatic cut-off valve for ion-exchange columns. Anal. Chem. 27, 865 (1955). 1273.

Hewitt, F. J., Crichlow, W. Q., Foldes, G., Horner, F., Shinkawa, H., Sullivan, A. W., Measurement of atmospheric noise. Recommendation No. 1 and Annex, Proc. XIIth Gen. Assembly Intern. Sci. Radio Union, Boulder, Colorado, August 22-September 5, 1957, XI, Pt. 4, 99, 1957; U.R.S.I. (Intern. Sci. Radio Union) Inform. Bull. No. 105, 10 (SeptemberOctober 1957). 2190.

Heydenburg, N. P., Temmer, G. M., Energy levels in F18 from alpha-particle reactions in nitrogen. Phys. Rev. 92, 89 (1953). 711.

Hidnert, $P$., Krider, H. S., Tables 142 to 144 , thermal expansion. 9th edition of the Smithsonian Phys. Tables (Smithsonian Institution, Washington D.C.) (1954). 1160.

Tables $4 \mathrm{f}-1$ through $4 \mathrm{f}-6$ on thermal expansion. Am.
Inst. Phys. Handbook (McGraw-Hill Book Co., Ine., New York, N.Y.) (1957). 2336.

Hilbert, G. E., Brode, W. R. Career opportunities in the Government. Chem. Eng. News 36, Pt. II, 70 (1958). 2484.

Hilkert, M., Ogburn, $F_{\text {., }}$ The nature, cause and effect of porosity in electrodeposits, II. Radiographic detection of porosity in electrodeposits. Ann. Proc. Am. Electroplaters' Soc. 43, 256 (1956), 1940.

Hill, J. P., Johnson, D. P., Cross, J, L., Bowman, H. A., Elastic distortion error in the dead weight piston gage. Ind. Eng. Chem. 49, 2046 (1957). 2103.

Hill, J. S., Waldo, G. V., Staras, H., Very high frequency tropospheric recording measurements of plane and circular polarized waves in the Great Lakes area. Abstract published in IRE Trans. Ant. Prop. AP-1, 42 (1952); entire paper published as FCC Memo. 62838. 6220 .

Hilsenrath, $J$., Sources of transport coefficients and correlations of thermodynamic and transport data. Second AGARD Combustion Colloquium, Liege, Belgium (Dec. 1955). 1501; Agard Selected Combustion Problems, II. Fundamentals and aeronautical applications. (Buttersworth Sci. Publ., London, England) p. $199,(1956)$. 1879 .

Hilsenrath, J., Green, M.S., Beckett, C. W., The internal energy of highly ionized gases. IXth Intern. Astronautical Congr. Proc. Amsterdam, Holland, 1958. Springer Verlag (Vienna, Austria), p. 120 (1959). 3081 .

Hilsenrath, J., Klein, M., Sumida, D. Y., Mechanized computation of thermodynamic tables at the National Bureau of Standards: the calculation of the equilibrium composition and thermodynamic properties of dissociation and ionized gaseous systems. Proc. Symp. Thermodyamic and Transport Properties of Gases, Liquids, and Solids, (Reprinted from Thermodynamic and Transport Properties of Gases, Liquids, and Solids), Am. Soc. Mech. Engrs. p. 416 (1959). 2988. Hilsenrath, J., Touloukian, Y. S., The viscosity, thermal conductivity, and Prandtl number for air, $\mathrm{O}_{2}, \mathrm{~N}_{2}$, $\mathrm{NO}, \mathrm{H}_{2}, \mathrm{CO}, \mathrm{CO}_{2}, \mathrm{H}_{2} \mathrm{O}, \mathrm{He}$ and A. Trans. Am. Soc. Mech. Engrs. 76, No. 6, 967 (1954). 1207.

Hilsenrath, J., Wegstein, J. H., Mechanized computation of thermodynamic tables at the National Bureau of Standards-The calculation of the equilibrium composition and thermodynamic properties of dissociated and ionized gaseous systems. Proc. Joint Conf. on Thermodynan ic and Transport Properties of Fluids, London, England, July 10-12, 1957 (Institution of Mechanical Engineers, London, England), p. 79 (1958). 2632.

Hilsenrath, $J$., White, D., Pressure-sensitive diaphragmtype null detector. Rev. Sci. Instr. 29, No. 7, 648 (1958). 2698.

Hipple, J. A., Dibeler, $V$. H., Mass spectrometry. Anal. Chem. 24, 27 (1952). 478.

Hipple, J. A., Gorman, J. G., Jones, E. J., Analysis of solids with the mass spectrometer. Anal. Chem. 23, 438; Phys. Rev. 82, 768 (1951). 194.

Hipple, J. A., Sommer, H., A combination crossed-field and time-of-flight mass spectrometer. Phys. Rev. 85,714 (1952). 360 .

A new method for the measurement of the isotopic abundance of solids. Phys. Rev. 83, 229 (1951). 180.

Hipple, J. A., Sommer, H., Craig, S. N., Hoffman, J. I., The effect of recent mass measurements on the Faraday Phys. Rey. 85, 740 (1952). 573.

Hiza, M. J., McClintock, R. M., Epoxy resins as cryogenic structural adhesives. Proc. 1957 Cryogenic Engineering Conference Paper F1-3, p. 305, Edited by K. D. Timmerhaus, Chemical Engineering Dept., University of Colorado (Boulder, Colorado) (1958); Modern Plastics 35, 172 (1958). 2547.

Hobbs., R. B., A suggested program for industry-sponsored shoe research. Leather and Shoes 123, 8 (1952). 379.

A technological challenge to the textile industry. Can. Textile J. 74, 53 (1957). 2002. 
Durability of boys' shoes. J. Home Economics 45, $32(1953)$. 691

Glass fiber papers. Sixth Annual Report of Tech. Assoc. Graphic Arts, p. 104 (1954). 1026.

New paper products in competition with textiles. Am. Dyestuff Reporter 45, 479 (1956). 1784.

Note on the indentation of heavy leather. J. Am. Leather Chemists Assoc. 68, 349 (1953). 776.

Present status of standards for shoe last sizes. J. Natl. Assoc. Chiropodists No. 10, $30(1950) .113$.

Service tests of boys' shoes. Am. Shoemaking Pt. 1, 222, No. 6,15 ; Pt. 2, 222, No. 7, 17 (1952). 541.

Hobbs, R. B., Jones, W. T., Annual review of pulp and paper industry. Ind. Eng. Chem. 48, 111 (1956). 1627.

Hobbs, R. B., Wilson, W. K. Annual review of pulp and paper industry. Ind. Eng. Chem. 49, 42A (1957). 2024

Paper production on the upswing. Chem. Eng. News 32, 40 (1954), 1103.

Pulp and paper. Chem. Eng. News 31, 40 (1953). 805. Pulp and Paper (Annual Review). Chem. Eng. News 33, 114 (1955). 1466.

Hobbs, R. B., Worthington, V., Measuring the stiffness of paper. Modern Packaging 26, 127 (1953). 762.

Hobbs, R. H., Stiehler, R. D., Steel, M. N., Richey, G. G., Mandel, $J .$, Power loss and operating temperature of tires. J 64C1, 1 (1960).

Hochstrasser, $U$., Numerical experiments in potential theory using the Nehari estimates. Math. Tables and Other Aids to Computation 12, 26 (1958). 2654.

Hochstrasser, U., Stoneley, R., The attenuation of Rayleigh waves with depth in a medium with two surface layers. Monthly Notice of Royal Astron. Soc. Geophys. (London, England) Supplement 7, 279 $(1957), 2344$.

Hockman, A., Carl, H. F., Jaffe, H.W., New techniques for evaluating natural corundum ores. Mining Eng. 6, No. 4, 402 (1954). 1081.

Hoffman, A.J., Generalization of a theorem of Konig. J. Wash. Acad. Sei 46, 211 (1956). 1719.

Geometry. Handbook of physics, Edited by E. U. Condon and $\mathrm{H}$. Odishaw, Chapter 8, Pt, 1, p, 1-97 (McGraw-Hill Book Co., Inc., New York, N.Y.) (1958). 2500 .

How to solve a linear programming problem. Proc. of Second Symp. Linear Programming, held jointly by NBS and the USAF, p. 397 (Dept. of Defense) (1955). 1360 .

Linear programming. Appl. Mech. Rev, 9, 185 (1956). 1757

Hoffman, A. J., Antosievicz, H. A., A remark on the smoothing problem. Management Sci. 1, No, 1, 92 (1954). 931.

Hoffman, A. J., Bellman, R., On a thenrem of Ostrowski and Taussky. Archiv. der mathematik 5, 123 (1954) 1091 .

Hoffman, A. J., Dantzig, G. B., Dilworth's theorem on partially ordered sets. Ann. Math. Studies No. 38, $207(1956), 1677$.

Hoffman, A. J., Fan, $K$., Some metric inequalities in the space of matrices. Proc. Am. Math. Soc. 6, No. 1, $111(1955), \quad 1497$

Hoffman, A. J., Fan, K., Glicksburg, I., Systems of inerualities involving convex funetions. Proc. Am. Math. Soc. 8, 617 (1957). 2335

Hoffman, A. J., Gaddum, J. W., Sokolowsky, D., On the solution of the caterer problem. Logistics Research Quart. 1, 223 (1954). 1099.

Hoffman, A.J., Jacobs, $W$., Smooth patterns of production. Management Sci. 1, 86 (1954). 1139.

Hoffman, A.J., Kruskal, J. B., Integral boundary points of convex polvhedra. Ann. Math. Studies No. 38, $223(1956), 1743$

Hoffman, A.J., Kuhn, H. W., On systems of distinct representatives. Ann. Math. Studies No. 38, 199 (1956). 1793

Systems of distinct representatives and linear programming. Am. Math. Mo. 63, 455 (1956). 1910.
Hoffman, A.J., Mannos, M., Sokolowsky, D., Wiegmann, N. A., Computational experience in solving linear programs. J. Soc. Ind. Appl. Math. 1, 17 (1953). 672 .

Hoffman, A. J., Newman, M., Straus, E. G., Taussky, O., The number of absolute points of a correlation. Pacific J. Math. 6, 83 (1956). 1943.

Hoffman, A. J., Wielandt, $H$., The variation of the spectrum of a normal matrix. Duke Math. J. 20, 37 (1953), 890 .

Hoffman, J. D., The thermodynamic driving force in nucleation and growth processes. Chem. Phys. Note 29, No. 5, 1192 (1958). 2834.

Theory of dielectric relaxation for a single-axis rotator in a crvstalline field, II. J. Chem. Phys. 23, No. 7, 1331 (1955). 1554 .

Hoffman, J. D., Lauritzen, $J . I ., J r$, Theory of formation of polymer ervstals with folded chains in dilute solution. J 64A 1, 73 (1960).

Hoffman, $J . D ., W e e k s, J, J$., Specific volume and degree of crvstallinity of semicrystalline poly (chlorotrifluoroethylene), and estimated specific volumes of the pure amorphous and crystalline phases. J 60, 465 (1958) RP2862.

The specific volume and degree of crystallinity of poly (chlorotrifluornethylene). J. Polymer Sci. 28, No. 117,472 (1958). 2831.

Hoffman, J. D., Weeks, J. J., Murphey, W. M., Experimental and theoretical study of kinetics of bulk crvstallization in poly(chlorotrifluoroethylene). J 63A1, $67(1959)$.

Hoffman, J. D., Zimm, B. H., Rate of thermal diffiusion of polvmer molecules in solution. J. Polymer Sci. 15, 405 (1955) 1472

Hoffman, J. I., The evolition of certified reference materials. Anal. Chem. 31, No. 12, 1934 (1959). 3078A.

Hoffman, J. I., Craig, D. N., A new method for determining the value of the Faraday. Phys. Rev. 80,487 (1950). 3.

Hoffman, J. I., Kushner, L. M., Synthetic detergents. Sci. Am. 185, No. 4, 26 (1951). 318.

Hoffman, J. I., Sommer, H., Craig, S. N., Hipple, J. A., The effeet of recent mass measurements on the Faraday. Phys. Rev. 85, 740 (1952). 573.

Hoge, $H$. $J$. A resistance-temperature relation for low temperature thermometry. Rev. Sci. Instr. 21, 815 (1950). 9.

Hoge, $E . W ., T s a i, D . H$. Reproducibility of a penand-chart type of recording instrument under dynamic conditions, ASME Paper 57A-209 (1957). 2286.

Holden, D. B., Moler, W. F., Tropospheric scatter propagation and atmospheric circulations. J 64D1, $81(1960)$.

Holford, W. L., Hammond, H. K., III, Kuder, M. L., Ratio-recording spectroradiometer, J 64C2, 151 (1960)

Holler, H. D., Studies on galvanic couples. I. Measurement of electromotive force and internal resistance of cells during current flow. J. Electrochem. Soc. 97, $271(1950) .141$.

Studies on galvanic couples, II. Some potentialcurrent relations in galvanic corrosion. J. Electrochem. Soc. 97, 277 (1950). 142.

Studies on galvanic couples. III. Polarization and cuthndie protection. J. Electrochem. Soc. 97, 453 (1950). 143.

Holleu, S. F., Kerper, M. J., Mong, L. E., Stiefel, M. B., Evaluation of tensil, compressive, torsional, transverse, and impact tests and correlation of results for brittle cermets. J 61, 149 (1958) RP2895.

Holleu, S. F., Walker, R. F., Zerfoss, S., Gross, L. J., Temperature of the inversion in cristobalite. $J$ 61, 251 (1958) RP2902.

Holshouser, W. L., and Bennett, J. A., Mechanical failures of metals in service. Eng. Materials Handbook 36, 2 (1958). 2629.

Holshouser, W. L., Frankel, H.E., Bennett, J. A., Effect of oleophobic films on metal fatigue. J $64 \mathbf{C} 2,147$ (1960). 
Holt, A. W., An experimental rapid access memory using diodes and capacitors. Proc. Assoc. Computing Machinery, p. 133 (Assoc, for Computing Machinery, New York, N.Y.) (1952). 392.

Holt, A. W., Davis, W. W., Computer memory uses conventional C-R tubes. Electronics 26, 178 (1953). 673 .

Holt, N. B., Isbell, H. S., Frush, H. L., Note on the prenaration of sodium amalgam in the form of pellets. J 64.11, 135 (1960).

Holt, N. B., Isbell, H. S., Frush, H. L., Moyer, J. D. Tritium-labeled compounds III. Aldoses-1-t. J 64.12, $177(1960)$

Holten, D. C., Richards, R.J., Robbins R. F., Jacobs, R. B., Heat transfer to boiling liquid nitrogen and hydrogen blowing axially through narrow annular passages. Proc. 1957 Cryogenic Engineering Conference, Paper $\mathrm{G}-3$, p. 375 , Edited by K. D. Timmerhaus, Chemical Engineering Department, University of Colorado (Boulder, Colorado) (1958). 2581.

Holzer, R. E., Saxon, D. S., Distribution of electrical conduction currents in the vicinity of thunderstorms. J. Geophys. Research 57, No, 2, 207 (1952), 432.

Honig, D. G., Gainen, L., Stanley, E. D., Linear programming in bid evaluation. Logistics Research Quart. 1, 48 (1954). 1051.

Hooper, E. C., Jr., Brown, B., Plastic phosphor matrix for fast neutron detection. Nucleonics 16, 96 (1958). 2693.

Hopper, $N$. J., Reinhart, H.W., Periodicals and serials received in the Library of the National Bureau of Standards, August 1958. (1958) C563, 1st Suppl.

Hoppes, D. D., Ambler, E., Hayward, R. W., Hudson, R. $P$., Absence on interference effects in the $\beta$-decay of polarized $\mathrm{CO}^{56}$ and $\mathrm{CO}^{58}$ nuclei. Phys, Rev, 108, 503 (1957). 2006.

Beta-gamma correlations from polarized manganese52. Phys, Rev. 110, No. 3, 787 (1958); Proc. Intern. Congr. on Nuclear Phys. (Paris, France, July 1958) and Proc. of Kamerlingh Onnes Conf. on Low Temp. Phys. Lieden. The Netherlands, Supplement of Physica. (June 1958). 2473.

Beta-gamma correlations from polarized nuclei. Proc. Congres Intern. de Physique Nucleaire, 1958, Interactions Faibles, Chapter III, p. 331 (Paris, France) (1959). 2902.

Experiments on the beta-decay of polarized nuclel. Proc. Kamerlingh Onnes Conference, Leiden, Holland, 1958, Physica (Supplement) 24, S64 (1958). 2557.

Hoppes, D. D., Ambler, E., Hayward, R. W., Hudson, R. P., $W u, C$. S., Further experiments on $\beta$-decay of polarized nuclei. Phys. Rev. 106, No. 6, 1361 (1957). 2132.

Hoppes, D. D., Hayward, R. W., Disintegration of selen. ium-73. Bull. Am. Phys. Soc. 30, No. 1, 33A (1955). 1316.

Disintegration of selenium-73. Phys. Rev. 101, No. 1, 93 (1956). 1680 .

Gamma radiation from Ag105. Bull. Am. Phys. Soc. Series II, No. 1, 42 (1956). 1718.

Inner bremsstrahlung from $V^{49}$. Bull. Am. Phys. Soc. 30, No. 3, 59A (1955). 1368.

Radiative electron capture in cesium-131. Phys. Rev. 104, No. 2, 368 (1956). 1834

Radiative orbital electron capture in vanadium-49. Phys. Rev. 104, No. 1, 30 (1956). 1835.

Scintillation counting in experiments on parity conservation. Proc. 6th Scin. Counter Symp. IRE Trans. Nuclear Science NS-5, 151 (Jan. 1958). 2749.

Hoppes, D. D., Hayward, R. W., Ernst, H., The disintegration of $\mathrm{Ba}^{113}$. Phys. Rev. 93, 916 (1954). 1178. The disintegration of $\mathrm{Nb}^{91}$ and $\mathrm{Nb}^{92}$. Bull. Am. Phys. Soc. 29, No. 7, 16A (1954). 1179.

Hoppes, D. D., Hayward, R. W., Pessoa, E. F., van Lieshout, $R$., Gamma radiation from $\mathrm{Zn}^{63}$. Il Nuovo Cimento 11, 53 (1959). 2956.

Hoppes, D. D., Wu, C. S., Ambler, E., Hayward, R. W. Hudson, $\vec{R}$. P., An experimental test of parity con- servation in beta decay. Phys. Rev. 105, 1413 (1957). 2015 .

Horl, E. M., Light emission from solid nitrogen during and after electron bombardment. J. Mol. Spectroscopy 3, No. 4, 425 (1959). 2975.

Horl, E. M., Marton, L., Cryostat for electron bombardment and electron diffraction work. Rev. Sei. Instr. 29, No. 10, 859 (1958), 2506.

Horl, E. M., Peyron, M., Brown, H. W., Broida, H. P. Etude spectroscopique des produits de la decharge electrique dan $\mathrm{L}^{\prime} \mathrm{A}$ zote condenses a l'etat solide a tres basse temperatures, prevues en faveur de l'existence d'Azote triatomique dan le solide. J. chim. phys. 56, No. 2392, 736 (1959). 2944.

Spectroscopic evidence for triatomic nitrogen in solids at very low temperatures. J. Chem. Phys. 30, 1304 (1959). 3054.

Horn, J. T., Bean, B. R., Radio refractive-index climate near the ground. J 63D3, 259 (1959).

Horn, J. T., Bean, B. R., Riggs, L. P., Synoptic study of the vertical distribution of the radio refractive index. J 63D2, 249 (1959).

Hornbeck, G. A., Spectroscopic determination of the dissociation energy of the $\mathrm{OH}$ radical. Fifth Symp. (International) on Combustion (Reinhold Publ. Corp., New York, N.Y.) p. 790 (1955). 1503.

Horner, F., Crichlow, W. Q., Foldes, G., Hewitt, F. J., Shinkawa, $H$., Sullivan, $A$. $W$., Measurement of atmospheric noise. Recommendation No. 1 and Annex, Proc. XIIth Gen. Assembly Intern. Sci. Radio Union, Boulder, Colorado, August 22-September 5, 1957, XI, Pt. 4, 99, 1957 . U.R.S.I. (Intern. Sci. Radio Union) Inform. Bull. No. 105, 10 (September-October 1957). 2190.

Horowitz, E., Connor, W. S., Variability of wool content in part wool blankets. ASTM Bull. No. 208, 42 (1955). 1571

Horowitz, E., Mandel, J. Comparison of sodium hydroxide and sulfuric acid methods for analysis of wool admixed with cotton and viscose rayon. Am. Dyestuff Reptr. 43, No. 19,613 (1954). 973.

Horowitz, E., Zimmerman, E. W., Hart, V. E., Determination of sulfur in rubber vulcanizates. Anal. Chem. 27, No. 10, 1606 (1955). 1311.

Horton, A. T., Glasgow, A. R., Jr., Ross, G. S., Enagonio, D., Dixon, H. D., Saylor, C. P., Furukawa, G. T., Reilly, M. L., Henning, J. M., Comparison of eryoscopic determinations of purity of benzene by thermometric and calorimetric procedures. Anal. chim. acta 17, 54 (1957); Proc. of I.U.P.A. Intern. Symp. on Purity Control by Thermal Analysis (Amsterdam, The Netherlands) (April 1957). 2062.

Horton, H. B., McPherson, J. L., Alexander, S. N. Glaser, E., Information processing in social and industrial research. Sci. Monthly 76, 100 (1953). 741.

Hosler, W. R., Blunt, R. F., Frederikse, H. P. R., Electrical and optical properties of intermetallic compounds, II. Gallium antimonide. Phys. Rev. 96, 576 (1954). 1007.

Electrical and optical properties of intermetallic compounds, IV. Magnesium stannide. Phys. Rev. 100, No. 2, 663 (1955). 1330.

Properties of $\mathrm{Mg}_{2} \mathrm{Sn}$. Phys. Rev. 98, 1533A (1955). 1460.

Hosler, W. R., Blunt, R. F., Frederikse H. P. R., Becker, $J . H$. Electrical and optical properties of intermetallic compounds, III. Aluminum antimonide. Phys. Rev. 96, 578 (1954). 1007

Hosler, W. R., Breckenridge, R. G., Electrical properties of titanium dioxide semiconductors. Phys. Rev. 91, 793 (1953). 704.

Hosler, W. R., Breckenridge, R. G., Blunt, R. E., Frederikse, H. P. R., Becker, J. H., Oshinsky, W., Electrical and optical properties of intermetallic compounds. I. Indium antimonide. Phys. Rev. 96, 571 (1954). 1007.

Hosler, $\dot{W}$. R., Breckenridge, R. G., Oshinsky, W. Electrical properties of indium antimonide. Phys. Rev. 91, 243 (1953). 703 . 
Hosler, $W$. R., Frederikse, $H . P . R$. Galvanomagnetic effects in InSb. Phys. Rev. 98, 1532A (1955). 1352. Galvanomagnetic effects in n-type InSb at $4.2^{\circ} \mathrm{K}$. Can. J. Phys. 34, 1377 (1956). 1716.

Galvanomagnetic effects in n-type indium antimonide. Phys. Rev. 108, 1136 (1957), 2135.

Galvanomagnetic effects in p-type indium antimonide. Phys. Rev. 108, 1146 (1957). 2136.

Oscillatory galvanomagnetic effects in n-type indium arsenide. Phys. Rev. 110, 880 (1958). 2678.

Hosler, W. R., Frederikse, H. P. R., Roberts, D. E. Electrical conduction in magnesium stannide at low temperatures. Phys. Rev. 101, No. 6, 1653 (1956). 1695.

Hosterman, E. R., Steiner, E. T., Aging of Karakul and seal fur skins. J. Am. Leather Chemists Assoc. 45, 579 (1950). 19.

Hotchkiss, R. N., Washer, F. E., Rosberry, F. W., Spurious resolution of photographic lenses. J. Opt. Soc. Am. 41, 600 (1951). 315 .

Hough, W. S., McCaulley, J. W., A relationship between the lower ionosphere and the [OI] 5577 nightglow emission. J. Geophys. Research 64, No. 12, 2307 (1959). 2880 .

Householder, J., Wait, J. R., Mixed-path ground-wave propagation: 2. Larger distances. J 59, 19 (1957) RP2770.

Pattern synthesis for slotted-cylinder antennas. J 63D3, 303 (1959).

Howard, D. M., Smith, F. C., Fatigue and static tents of flush riveted joints. Product Eng. Handbook No. 3, Section G., p. G2 (McGraw-Hill Book Co., Inc., New York, N.Y.) 1345.

Howard, D. M., Smith, I., Smith, F. C., Cumulative fatigue damage of axially loaded Alclad 75S-T6 and Alclad 24S-T3 aluminum-alloy sheet. Natl. Advisory Comm. Aeronaut. Tech. Note 3923 (1955), 1303.

Howard, F. L., Automotive research at the National Bureau of Standards. NADA Mag. 24, No. 3, 118 (1952), 399.

Howard, F. L., Broida, H. P., Levedahl, W. J., The emission spectra of first-stage combustion in an engine. J. Chem. Phys, 19, 797 (1951). 333.

Howard, F. L., Levedahl, W. J., Two-stage autoignition of some hydrocarbons. Ind. Eng. Chem. 43, 2805 (1951). 354

Howard, F. L., Mallonee, R. E., The determinations of freezing point of engine antifreezes. ASTM Bull. No. 172 , p. 43 (1951). 331 .

Howe, H. H., Note on the solution of Riceati's differential equation. J 64B2, 95 (1960).

Howe, H. H., Wait, J.R., Mode calculations for V.L.F. ionospheric propagation. Symp, on the Prop. of V.L.F. Radio Waves, Sponsored by the NBS Boulder Laboratories and IRE Prof. Group on Ant. and Prop., January 23-25, 1957 (Boulder, Colorado), Paper No.' 36, V. 3; Further comments, V. 4, 91. 2203.

The waveguide mode theory of VLF ionospheric propagation. Proc. IRE 45, 95 (1957), 2390.

Howland, W. H., Douglas, T. B., Epstein, L. F., Dever, $J$. L., Lithium: heat content from $0^{\circ}$ to $900^{\circ}$, triple point and heat of fusion, and thermodynamic properties of the solid and liquid. J. Am. Chem. Soc. 77, 2144 (1955). 1390.

Hoynes, D. S., Characteristies of metal-clad laminates. Elec. Mfg. 59, No. 4, 104 (1957). 2053.

Hubbard, D., Glaze, F. W., Blackburn, D. H., Osmalov, J. S., Black, $M$. H., Properties of arsenic sulfide glass. J 59, 83 (1957) RP2774.

Hubbard, D., Jenkins, L. B., Krumrine, E. M., Amelung glasses compared with some modern commercial glasses. Sci. Monthly LXXV, No. 6, 327 (1952). 387.

Hubbard, D., Krumrine, E. M., Stair, R., Australite (meteoric) glass. Trans. Am. Geophys. Union 37, No. 6, 767 (1956). 1636.

Hubbard, D., Pike, R. G., Increased chemical reactivity of the surface compared with that in the bulk volume of
Britton-Robinson universal buffers. J 59, 411 (1957) RP2811.

Miscellaneous observations on the alkali-aggregate reaction and the ionic charge on hydrated cement. Natl. Acad. Sci., Natl. Research Council. Publ. Highway Research Board Bull. 171, p. 16 (1958). 2639.

Physico-chemical studies of the destructive alkaliaggregate reaction in concrete. J 59, 127 (1957) RP2780.

Source of the non-migratable ionic charges developed by portland and high-alumina cements during hydration. Natl. Acad. Sci., Natl. Research Council. Proc. Highway Research Board 3\%, 256 (1958). 2768.

Hubbard, D., Pike, R. G., Insley, H., Mechanisms of alkali-aggregate reaction. J. Am. Concrete Inst. 27, No. 1,13 (1955). 1406.

Hubbard, R. W., Thompson, M. C., Jr., Phase generator for tropospheric research. Electronies 29, No. 10, $220(1956), \quad 1805$.

Hubbard, W. D., Kushner, L. M., Light scattering and micelle structure in the system sodium dodecyl sulfate-sodium chloride-water. J. Colloid. Sci. 10, No. 4, 428 (1955). 1386

On the determination of critical micelle concentration by a bubble pressure method. J. Phys. Chem. 57, 898 (1953). 787.

Viscometric and turbidimetric measurements on dilute aqueous solutions of a nonionic detergent. J. Phys. Chem. 58, 1163 (1954). 1225.

Hubbard, W. D., Kushner, L. M., Doan, A., Light scattering measurements of a fractionated nonionic detergent. J. Phys. Chem. 61, 371 (1957). 2176.

Hubbard, W. D., Kushner, L. M., Parker, R. A., Turbidity and viscosity measurements on some eationic detergents in water and in sodium chloride solutions. J 59, 113 (1957) RP2778.

Hubbell, J. H., Response of a large sodium iodide scintillation detector to high energy X-rays. Rev. Sci. Instr. 29, No. 1, 65 (1958). 2741.

Hubbell, J. H., Bach, R. L., Lamkin, J. C., Radiation field from a rectangular source. J 64C2, 121 (1960).

Hubbell, J. H., Berger, M. J., Reingold, I., Contribution of the annihilation radiation to the gamma-ray flux in lead. Phys. Rev. 113, No. 3, 857 (1959). 2917.

Hubbell, J. H., Fano, L., Beckett, C. W. Compressibility factor, density, specific heat, enthalpy, entropy, free energy function, viscosity, and thermal conductivity of steam. Natl. Advisory Comm. Aeronaut. Tech. Note 3273 (1956). 1657.

Hubbell, J.H., Hayward, E. V., Backscattering of the cobalt- 60 gamma rays from infinite media. J. Appl. Phys. 25, 506 (1954), 959.

The albedo of various materials for $1 \mathrm{Mev}$ photons. Phys. Rev. 93, 955 (1954). 1170.

Hubbell, J. H., Hayward, E. V., Titus, W. F., Energy and angular distribution of X-rays seattered in lead. Phys. Rev. 108, No. 6, 1361 (1957). 2111.

Hubbell, J. H., Schofield, N. E., Unserambling of gammaray scintillation spectrometer pulse-height distributions. IRE Trans. Nuclear Sci. NS-5, No. 3, 156 (1958), 2856.

Huber, C. E., Beatty, R. W., Lossy cable attenuator pads. Rev. Sci. Instr. 24, 1002 (1953). 754.

Hudson, D. C., Hartley, J. L., Moore, R. B., Sweeney, $W . T$., Some factors influencing the cutting characteristics of rotating dental cutting instruments. J. Am. Dental Assoc. 50, 377 (1955). 1494.

Hudson, D. C., Hartley, J. L., Sweeney, W. T., Dickson, $G$., Development of methods for evaluation of rotating dental diamond abrasive instruments. J. Am. Dental Assoc. 54, 637 (1957). 2085.

Hudson, D. C., Hartley, J. L., Sweeney, W. T., Richardson, $W$. P., Cutting eharacteristies of dental burs as shown by high speed photomicrography. U.S. Armed Forces Med. J. 8, 209 (1957). 2076.

Hudson, D. C., Kumpula, $J$. W. Ionization chambers for radiation data during dental X-ray exposure. U.S. Armed Forces Med. J. 6, No. 8, 1131 (1955). 1380. 
Hudson, D. C., Kumpula, J. W., Dickson, G., A panoramic X-ray dental machine. U.S. Armed Forces Med J. 8, No. 1, 46 (1957). 1993.

Hudson, D. C., Sweeney, W. T., Temperatures developed in rotating dental cutting instruments. J. Am. Dental Assoc. 48, 127 (1954). 1165.

Hudson, P. A., Allred, C. M., A dry, static calorimeter for $\mathrm{RF}$ power measurement. IRE Trans. Instrumentation I-7, No. 3-4, 292 (1958). 2419.

Hudson, R. P., Properties of the $\mathrm{Cr}^{+++}$ion in the paramagnetic alums at low temperatures. Phys. Rev. 88 , No. 3, 570 (1952). 522 .

The helium vapor-pressure scale of temperature. Temperature, Its Measurement and Control in Science and Industry. (Reinhold Publ. Corp., New York, N.Y.) 2, 185 (1955). 1535.

The magnetic threshold field curve of a superconductor. Phys. Rev. 85, No. 2, 382 (1952). 584.

Very low temperature data: properties of paramagnetic salts. Chapter 4-b, Am. Inst. Phys. Handbook (McGraw-Hill Book Co., New York, N.Y.) p. 14 (1957). 2408.

Hudson, $R$. P., Ambler, E., Absolute temperature below $1^{\circ} \mathrm{K}$ : chromic methylammonium alum as a thermometric substance. J. Chem. Phys. 27, 378 (1957). 2009.

Critical field phenomena in an isotropic paramagnetic crystal. Phys. Rev. 96, No. 4, 907 (1954). 981. Magnetic cooling. Published in Reports on Progress in Physics, Proc. British Phys. Soc. (London, England) 18, 251 (1955). 1395.

Magnitoye okhlazhdenye. Uzpekhi physicheskhikh nauk 47, 445 (1959). 2981.

Measurements of chromium potassium alum below $1^{\circ}$ K. Phys. Rev. 95, 1143 (1954), 1067.

Measurements on the saturation vapour pressure of liquid helium in the range $1.4^{\circ}-4.2^{\circ} \mathrm{K}$. Bull. Intern. Inst. Refrig. Commun. 147, 605 (1955). 1403.

New materials for magnetic cooling and thermometry below $1^{\circ} \mathrm{K}$. Phys. Rev. 102, 916 (1956). 1783.

Spin-spin absorption in chromic methylammonium alum and manganous chloride tetrahydrate at very low temperatures. Physica XXII, 866 (1956). 1887. Spin-spin absorption in manganous chloride tetrahydrate. Phys. Rev. 104, 1506 (1956). 1888. Spin-spin relaxation at very low temperatures in chromic methylammonium alum. Phys. Rev, 104, 1500 (1956). 1889.

Hudson, R. P., Ambler, E., Hayward, R. W., Hoppes, D. $D$., Absence of interference effects in the $\beta$-decay of polarized $\mathrm{CO}^{66}$ and $\mathrm{CO}^{68}$ nuclei. Phys. Rev. 108, 503 (1957). 2006.

Beta-gamma correlations from polarized manganese52. Phys. Rev. 110, No. 3, 787 (1958); Proc. Intern. Congr. on Nuclear Phys. (Paris, France, July 1958) and Proc. Kamerlingh Onnes Conf. on Low Temp. Phys. Leiden, The Netherlands, Supplement of Physica. (June 1958). 2473.

Beta-gamma correlations from polarized nuclei. Proc. Congres Intern. de Physique Nucleaire, 1958, Interactions Faibles, Chapter III, p. 831 (Paris, France) (1959). 2902.

Experiments on the beta-decay of polarized nuclei. Proc. Kamerlingh Onnes Conference, Leiden, Holland. 1958; Physica (Supplement) 24, S64 (1958). 2557.

Hudson, R. P. A mbler, E., Hayward, R. W., Hoppes, D. D., $W u, C$. S. Further experiments on $\beta$-decay of polarized nuclei. Phys. Rev. 106, No. 6, 1361 (1957). 2132.

Hudson, R. P., Ambler, E., Temmer, G. M. Alignment of cerium $^{141}$ and neodymium ${ }^{147}$. Phys. Rev. 97, 1212 (1955). 1255.

Alignment of three odd-A rare-earth nuclei. Phys. Rev. 101, No. 1, 196 (1956). 1611.

Alignment of three rare-earth isotopes. Bull. Intern. Inst. Refrig., Commun. 142, 255 (1955). 1256.

Hudson, R. P., Brickwedde, F. G., Ambler, E., Cryogenics. Ann. Rev. Phys. Chem. 6, 25 (1955).' 1301.
Hudson, R. P., de Klerk, D., Pellam, J.R., Normal fluid concentration in liquid helium II below $1^{\circ} \mathrm{K}$. Phys. Rev. 89, 662 (1953). 774.

Second sound velocity measurements below $1^{\circ} \mathrm{K}$. Phys. Rev. 89, 326 (1953). 822.

Hudson, R. P., McLane, C. $\dot{K}$., Magnetic and thermal properties of chromic methylamine alum below $1^{\circ} \mathrm{K}$. Phys. Rev. 95, No. 4, 932 (1954). 1057.

Hudson, R. P., Wu, C. S., Ambler, E., Hayward, R. W., Hoppes, D. D., An experimental test of parity conservation in beta decay. Phys. Rev. 105, 1413 (1957). 2015.

Huffman, E. W. D. Macurdy, L. B., Alber, H. K., BenedettiPichler, A. A., Carmichael, H., Corwin, A. H., Fowler, R. M., Kirk, P. L., Lashof, T. W., Terminology for describing the performance of analytical and other precise balances. Anal. Chem. 26, 1190 (1954). 1166.

Huffnagel, C. A., Rose, J., Broida, H. P., Gillespie, J. F., Rabile, P. J., Freis, E. D., A method for the study of the circulation in the dog using a mechanical left ventricle. J. Appl. Physiol. \%, 580 (1954). 920.

Hufford, G. A., A note on wave propagation through an inhomogeneous medium. J. Appl. Phys. 24, 268 (1953). 629.

An analysis of some anomalous properties of equiphase contours. Proc. IRE 38, 614 (1950). 22.

An integral equation approach to the problem of wave propagation over an irregular surface. Quart. Appl. Math. 9, No. 4, 391 (1952). 394.

Hughes, E. E., Diniak, A. W., Fujii, M., Determination of water content in Freon-12 circulating in a refrigeration system. Refrig. Eng. 62, No. 2, 56 (1954). 990.

Hughes, E. E., Gorden, R.,Jr., Determination of acetylene in air in concentrations from ten parts per billion to ten parts per million. Anal. Chem. 31, 94 (1959). 2928.

Hughes, E. E., Weaver, E. R., Diniak, A. W., Determination of water vapor from the change in electrical resistance of a hygroscopic film. J 60, 489 (1958) RP2864

Hughes, E. E., Weaver, E. R., Gunther, S. M., Schuhmann, S., Redfearn, $N$. T., Gorden, R., Jr., Interpretation of mass spectra of condensates from urban atmospheres. J 59, 383 (1957) RP2809.

Hughes, $J$. C., Testing of glass volumetric apparatus. (1958) C602.

Humphreys, C. J., Benedict, W. S., Plyler, E. K., The emission spectrum of OH from 1.4 to $1.7 \mu$. J.' Chem. Phys. 21, 398 (1953). 860.

Hunt, C. M., Discussion of paper, Properties of portland cement pastes cured at elevated temperatures and pressures. J. Am. Concrete Inst., 28, Pt. 2, No. 6, 1934 (1956). 1826.

Hunt, C. M., Dantzler, V., Tomes, L. A., Blaine, R. L., Reaction of portland cement with carbon dioxide. J 60, 441 (1958) R P2858.

Hunt, C. M., Tomes, L. A., Blaine, R. L., Some effects of aging on the surface area of portland cement paste. J 64A2, 163 (1960).

Some factors affecting the surface area of hydrated portland cement as determined by water-vapor and nitrogen absorption. J 59, 357 (1957) RP2806.

Hunt, G. A., Erdös, P., Changes of sign of sums of random variables. Pacific J. Math. 3, 673 (1953). 662.

Hunten, D. M., Roach, F. E., Chamberlain, J. W., A photometric unit for the airglow and aurora. J. Atmospheric and Terrest. Phys, 8, 345 (1956). 1958.

Hunter, J. S., Youden, W., J., Partially replicated latin squares." Biometrics 11, 399 (1955). 1442.

Huntoon, R. D., Basic standards for science and industry. Eléctronic Design 5, No. 16, 38 (1957); Proe. Conf. Instrumentation and Control in the Process Industries, February 6-7, 1957 (Armour Research Foundation, Illinois Institute of Technology, Chicago, Illinois) (1957). 2037.

Huntoon, R. D., Fano, U., Atomic definition of primary standards. Nature (London, England) 166, 167 (1950). 28. 
Huntoon, R. D., McNish, A. G., Importance of the Faraday to elemental constants and electricity standards. Nature (London, England) 181, 1194 (1958). 2593.

Present status of research on the physical constants at the (United States) National Bureau of Standards. N. 1 del Supplemento at Vol. 6, Serie X, Il Nuovo Cimento p. 146 (1957). 2263.

Hurlburt, E. H., Ellenwood, $R$. $\dot{C}$., The determination of impedance with a double-slug transformer. Proc. IRE 40, 1690 (1952). 570.

Huskey, H. D., Characteristics of the Institute for Numerical Analysis Computer. Math. Tables and Other Aids to Computation 4, No. 30, 103 (1950). 34.

Huskey H. D., Huskey, V. R., Electronic computers aiding management control. J. Accountancy 93, No. $1,69(1952)$. 441.

New frontiers in business management control are being established by electronic computers. J. Accountancy $93,69(1952) .488$.

Huskey, H. D., Thorensen, R., Ambrosio, B., F., Yowell, E. C., The SWAC-design features and operating experience. Proc. IRE 41, 1294 (1953). 885.

Huskey, V. R., Huskey, H. D., Electronic computers aiding management control. J. Accountancy 93, No. 1, 69 (1952). 441.

New frontiers in business management control are being established by electronic computers. J. Accountancy 93, 69 (1952). 488.

Hutchison, C. A., Jr., Herzfeld, C. $M_{\text {., }}$ Magnetic susceptibilities of snme U'(IV) compounds. J. Chem. Phys. 23, 1650 (1955). 1396.

Hutton, $U$. O., A tilting air-lubricated piston gage for pressures below one-half inch of mercury. J $63 \mathbf{C} 1$, 47 (1959).

Hutton, U. O., Lloyd, E. C., Johnson, D. P., Compact multi-anvil wedge type high-pressure" apparatus. J $63 \mathrm{C} 1,59$ (1959).

Hymo, L. A., Maienthal, M., Hellmann, M., Haber, C. P., Carpenter, S., Carr, A.J., The preparation of some aryl silanes. J. Am. Chem. Soc. 76, 6392 (1954). 1192.

Hyndman, J. R., Bender, C. P., Furukawa, G. T., Vapor pressure of benzene above $100^{\circ} \mathrm{C}$. Ind. Eng. Chem. 44, 387 (1952). 616.

\section{I}

Ikegami, F., A preliminary study of radiometeorological effects on beyond-horizon propagation. J 64D 3,239 (1960).

Ingle, G. W., Hammond, H. K., III, Measurement of color, gloss, and haze. Symposium Plastics Testing, Am. Soc. Testing Materials Spec. Tech. Publ. No. 132 , p. 25 (1953). 760.

Ingram, B. L., Bean, L., Removal of manganese prior to calcium and magnesium precipitations. Anal. Chem. 25, 1217 (1953). 814.

Inscoe, M. N., Gould, J. H., Corning, M. E., Brode, W. R., Relation between the absorption spectra and the chemical constitution of dyes: XXIX. Interaction of direct azo dyes in aqueous solution. J 60,65 (1958) RP2823.

Insley, H., Pike, R. G., Hubbard, D., Mechanisms of alkali-aggregate reaction. J. Am. Concrete Inst. 27, No. 1, 13 (1955). 1406.

Irish, C. R., Rosenberg, S. J., New ultra high strength steel. Materials and Methods 45, 145 (1957). 2214. Sigma phase in high-purity 18-10. Metal Progr. 61, No. 5, 92 (1952). 542 .

Irwin, L. K., Handmade bolts support the Capitol dome. Fasteners 13, No. 3, 5 (1958). 2577.

Irwin, L. K., Campbell, W. R., Duncan, R. C., Stress studies of bulkhead intersections for welded' tankers. Welding J. 31, No. 2, 68 (1952). 556.

Irwin, L. K., Chi, M. Elastic deformation in strips with holes loaded through pins. J 62, 147 (1959) RP2945.

Irwin, L. K., Ramberg, $W$., A pulse method for determining dynamic stress-strain relations. Trans. IXe, Congres Intern. de Mechanique Appliquee (Brussels, Belgium) 8, 480 (1957). 1997.
Longitudinal impact tests of long bars with a slingshot machine. Am. Soc. Testing Materials Spec. Tech. Publ. No. 176, "Symp. Impact Testing," June 1955 (1956). 1760 .

Isbell, H. S., Frush, H. L., Mutarotation, hydrolysis, and rearrangement reactions of glycosymalines. J. Organic Chem. 23, 1309 (1958), 2642.

Sodium borohydride reduction of aldonic lactones. J. Am. Chem. Soc. 78, No. 12, 2844 (1956). 1868. Synthesis of $\mathrm{C}^{14}$-labeled L-sorbose and L-ascorbic acid. J 59, 289 (1957) RP2800.

Isbell, H. S., Frush, H. L., Bruckner, B. H., Kowkabany, G. N., Wampler, G., Micromethylation of polysaccharides. Anal. Chem. 29, 1523 (1957). 2198.

Isbell, H. S., Frush, H. L., Holt, N. B., Note on the preparation of sodium amalgam in the form of pellets. J 64A 1,135 (1960).

Isbell, H. S., Frush, H. L., Holt, N. B., Moyer, J. D., Tritium-labeled compounds III. Aldoses-1-t. J 64A2, 177 (1960).

Isbell, H. S., Frush, H. L., Moyer, J. D., Use of carbon-14 and tritium for the study and characterization of cellulose and other polysaccharides. Tappi 40, No. 9, 739 (1957). 2403.

Isbell, H. S., Frush, H. L., Peterson, R. A., Tritiumlabeled compounds I. Radioassay of tritium-labeled compounds in "infinitely thick" films with a windowless, gas-flow, proportional counter. J 63A2, 171 (1959).

Isbell, H. S., Moyer, J. D., Carbon-14 earboxy-labeled polysaccharides. J 61, 71 (1958) RP2886.

Determination of reducing sugars and reducing end groups in polysaccarides by reaction with carbon-14labeled cyanide. Anal. Chem. 30, No. 12, 1975 (1958). 2517.

Preparation of carbon-14-labeled cyanide. Anal. Chem. 29, 393 (1957), 2262.

Structural analysis of clinical dextrans by periodate oxidation and isotope dilution techniques. Anal. Chem. 29, 1862 (1957). 2319.

Tritium-labeled compounds II. General-purpose apparatus, and procedures for the preparation, analysis, and use of tritium oxide and tritium-labeled lithium borohydride. J 63A2, 177 (1959).

Isbell, H. S., Schaffer, R., Branched-chain higher sugars. J. Am. Chem. Soc. 80, 756 (1958), 2476.

Branched-chain higher sugars. I. A 9-aldo-4-Cformyl-nonose derivative. J. Am. Chem. Soc. 81, 2178 (1959). 2903.

Branched-chain higher sugars. II. A diethylideneoxtose. J. Am. Chem. Soc. 81, 2838 (1959). 2904. Reaction of dimeric 5-aldo-1,2-O isopropylidene-Dxylo-pentofuranose with cyanide and the preparation of calcium 1,2-O-isopropylidene-L-idofururonate-6-C ${ }^{14}$ dihydrate. J. Am. Chem. Soc. 79, 3867 (1957). 2280 .

Structure of 5-aldo-1,2-O-isopropylidene-D-xylo-pentofuranose. J. Am. Chem. Soc. 79, 3864 (1957). 2321.

Synthesis of maltose-1-C 14 , maltobiono- $\delta$-lactone-1$\mathrm{C}^{14}$, and lithium maltobionate-1-C ${ }^{14}$ from 3-( $\alpha$-D-glucopyranosyl)-D-arabinose. J. Am. Chem. Soc. 78, No. 9,1887 (1956). 1909.

Isbell, H. S., Smith, F. A., Creitz, E. C., Frush, H. L., Moyer, J. D., Stewart, J. E., Infrared spectra of sugar acetates in solution. J 59, 41 (1957) RP2772.

Isbell, H.S., Tipson, R.S., Conformations of the pyranoid sugars. I. Classification of conformers. J 64A2, 171 (1960).

Conformations of the pyranoid sugars. II. Infrared absorption spectra of some aldopyranosides. J 64A3, 239 (1960).

Isbell, H. S., Tipson, R. S., Stewart, J. E., Infrared absorption spectra of some cyclic acetals of sugars. J 62, 257 (1959) RP2960.

Isaacs, T., Swanson, H. E., Cook, M. I., Evans, E. H., Standard X-ray diffraction powder patterns. (1960) C539, Vol. 9 . 
Ives, D. J., Bates, R. G., Guggenheim, E. A., Harned, H. S., Janz, G. J., Monk, C. B., Robinson, R. A., Stokes, R. H., Wynne-Jones, W. F. J., Standard electrode potential of the silver, silver chloride electrode. J. Chem. Phys. 25, 361 (1956). 1895.

\section{J}

Jackson, A., Clabaugh, W. S., Separation anddetermination of phosphate, silicate, and arsenate. J 62, 201 (1959) RP2954.

Jackson, J. L., Reactions en chaine de radicaux geles. J. chim. phys. 56, No. 2392, 771 (1959). 3030. Stabilized free radicals. J. Wash. Acad. Sci. 48, No. 6, 181 (1958). 2774.

Jackson, J. L., Griffing, V., Ransil, B. J., The magnetic interaction of $\mathrm{H}_{3}$. J.' Chem. Phys. 30, 1066 (1959). 3082.

Jackson, J. L., Montroll, E. W., Free radical statistics. J. Chem. Phys. 28, 1101 (1958). 2571.

Jacobs, R. B. Long distance transfer of liquefied gases. Proc. 1956 Cryogenic Engineering Conference, Paper G-7, p. 303, Edited by K. D. Timmerhaus, Chemical Engineering Dept., University of Colorado (Boulder, Colorado) (1957). 2177.

Recent advances in eryogenic engineering. ARS J. 29, No. 4, 245 (1959). 3031.

Single-phase transfer of liquefied gases. (1958) C596.

Jacobs, R. B., Hatch, M. R., Richards, R. J., Boggs, R. N., Phelps, G. R., Prediction of pressure drop in twophase single-component fluid flow. Proc. 1958 Cryogenic Engineering Conference, Paper F-4, p. 357. Edited by K. D. Timmerhaus, Chemical Engineering Dept., University of Colorado (Boulder, Colorado) (1959). 3019 .

Jacobs, R. B., Martin, K. B., Testing and operation of ball bearings submerged in liquefied gases. Proc. 1958 Cryogenic Engineering Conference, Paper $\mathrm{H}-2$, p. 476. Edited by K. D. Timmerhaus, Chemical Engineering Dept., University of Colorado (Boulder, Colorado) (1959); ASLE Trans. 2, No. 1, 101 (1959). 3063 .

Jacobs, R. B., Martin, K. B., Hardy, R. J., Operation of bearings and pumps at low temperatures. Proc. 1957 Cryogenic Engineering Conference, Paper D-6, p. 209, Edited by K. D. Timmerhaus, Chemical Engineering Dept., University of Colorado (Boulder, Colorado) (1958). 2676.

Performance of pumps with liquefied gases. Proc. 1956 Cryogenic Engineering Conference, Paper G-6, p. 295, Edited by K. D. Timmerhaus, Chemical Engineering Dept., University of Colorado (Boulder, Colorado) (1957). 2243.

Jacobs, R. B., Richards, R. J., Vacuum-insulated transfer tube. Rev. Sci. Inst. 28, 291 (1957). 2406.

Valve for cold fluids. Rev. Sci. Instr. 26, No. 7, 730 (1955). 1570.

Jacobs, R. B., Richards, R. J., Pestalozzi, W.J., Measurement of the flow of liquefied gases with sharp-edged orifices. Proc. 1958 Cryogenic Engineering Conference, Paper E-1, p. 272. Edited by K. D. Timmerhaus, Chemical Engineering Dept., University of Colorado (Boulder, Colorado) (1959). 2983.

Jacobs, R. B., Richards, R. J., Robbins, R. F., Holten, D. C., Heat transfer to boiling liquid nitrogen and hydrogen flowing axially through narrow annular passages. Proc. 1957 Cryogenic Engineering Conference, Paper G-3, p. 375 , Edited by K. D. Timmerhaus, Chemical Engineering Department, University of Colorado (Boulder, Colorado) (1958). 2581.

Jacobs, R. B., Van Gundy, D. A., Characteristics of some insulations for liquid oxygen transfer lines. Proc. 1956 Cryogenic Engineering Conference, Paper E-1, p. 156, Edited by K. D. Timmerhaus, Chemical Engineering Dept., University of Colorado (Boulder, Colorado) (1957). 2054.

Jacobs, W., Hoffman, A. J., Smooth patterns of production. Management Ścience 1, 86 (1954). 1139.
Jaffe, H. W., Carl, H. F., Hockman, A., New techniques for evaluating natural corundum ores. Mining Eng. 6, No. 4, 402 (1954). 1081 .

Jaffe, I., Prosen, E. J., Szwarc, M., Heats of combustion of some peroxides and the heats of formation of acetate, propionate, and butyrate radicals. J. Chem. Phys. 27, 416 (1957). 2148.

Jaffe, I., Seliger, H. H., Ziegler, C. A., Role of oxygen in the quenching of liquid scintillators. Phys. Rev. 101, No. 3, 998 (1956). 1858.

Jaffe, I., Ziegler, C. A., Seliger, H. H., Oxygen quencing and wavelength shifters in liquid scintillators. Phys. Rev. 99, No. 2, 663; Bull. Am. Phys. Soc. 30, No. 3, $63 \mathrm{~A}$ (1955). 1439.

Three ways to increase efficiency of liquid scintillators. Nucleonics 14, No. 5, 84 (1956). 1966.

Jameson, L. H., Factoren, die die Eigenschaften von Reyongeweben beeinflussen. Textil-Praxis (Stuttgart, Germany) 8, 769 (1953.) 718.

Jameson, L. H., Whittier, B. L., Schiefer, H. F., Factors affecting the properties of rayon fabrics. Textile Research J. 22, No. 9, 599 (1952). 446.

Jammick, P. M., Roach, F. E., The sky and eye. Sky and Telescope 17, 164 (1958). 2830.

Janes, H. B., Norton, K.A., Rice, P. L., Barsis, A. P., The rate of fading in propagation through a turbulent atmosphere. Proc. IRE 43, 1341 (1955). 1542.

Janes, H. B., Stroud, J. C., Decker, M. T., An analysis of propagation measurements made at 418 megacycles per second well beyond the radio horizon (a digest). J 64D3, 255 (1960).

An analysis of propagation measurements made at $418 \mathrm{Mc}$ well beyond the radio horizon. (1959) TN6 (PB151365).

Janes, H. B., Thompson, M.C., Jr., Measurements of phase stability over a low-level tropospheric path. J 63D 1, 45 (1959).

Janes, H. B., Wells, P. I., Some tropospheric scatter propagation measurements near the radio horizon. Proc. IRE 43, 1336 (1955). 1499.

Jansen, R. J., Koch, J. W., Harding, W. B., Fading rate recorder for propagation research. Electronics 32, No. 51, 78 (1959). 2949.

Janz, G. J., Bates, R. G., Guggenheim, E. A., Harned, H. S., Ives, D. J., Monk, C. B., Robinson, R. A., Stokes, R. H., Wynne-Jones, W. F. J., Standard electrode potential of the silver, silver chloride electrode. J. Chem. Phys. 25, 361 (1956). 1895.

Jean, A. G., Helliwell, R. A., Taylor, W. L., Some properties of lightning impluses which produce whistlers. Proc. IRE Letter 46, 1760 (1958). 2764.

Jean, A. G., Lange, L. J., Wait, J. R., Ionospheric reflection coefficients at VLF. Symp. on the Prop. of V. L. F. Radio Waves, Sponsored by the NBS Boulder Laboratories and IRE Prof. Group on Ant. and Prop., January 23-25, 1957 (Boulder, Colorado), Paper No. 31, V. 3, (1957). 2172.

Polarization of sferies. Symp. on the Prop. of V. L. F. Radio Waves, Sponsored by the NBS Boulder Laboratories and IRE Prof. Group on Ant. and Prop., January 23-25, 1957 (Boulder, Colorado) Abstracts, Paper No. 32, V. 3. 2257.

Jean, A. G., Taylor, W. L., Very-low-frequency radiation spectra of lightning discharges. J 63D2, 199 (1959).

Jefferies, J., Pottasch, S. R., The nova outburst. III. The ionization of hydrogen gas by an exciting star. Ann. Astrophys. J. 22, 318 (1959). 3085.

Jeffries, J. T., Thomas, $R$. N., Source function in a nonequilibrium atmosphere, II. Depth dependence of source function for resonance and strong subordinate lines. Astrophys. J. 127, No. 3, 667 (1958). 2767.

Jenkins, L. B., Hubbard, D., Krumrine, E. M., Amelung glasses compared with some modern commercial glasses. Sei. Monthly LXXV, No. 6, 327 (1952). 387

Jenkins, M. A., Crichlow, W. Q., Samson, C. A., Disney, R. T., Quarterly radio noise data March, April, May 1959. (1960) TN18-2 (PB151377-2).

Radio noise data for the International Geophysical 
Year July 1, 1957-December 31, $1958 . \quad$ (1959) TN18 (PB151377).

Jenkins, $W . D .$, Digges, T. G., Influence of strain rate and temperature on the creep of cold drawn ingot iron. Trans. Am. Soc. Metals 42, 1128 (1950). 73.

Jenkins, $W$. D., Johnson, C. R., Creep of annealed nickel, copper, and two nickel-copper alloys. J 60, 173 (1958) RP2836.

Creep of cold-drawn nickel. J 63C1, 1 (1959).

Jennings, C. W., Zentner, V., Brenner, A., Physical properties of electrodeposited metals, I: Nickel, 3: The effect of plating variables on the structure and properties of electrodeposited nickel. Plating 39, 865 (1952). 511 .

Jennings, W. H., Forziati, A. F., Losse, F. L., Pigman, W. $W$., Lawson, $M$. E., $J_{r}$., In vitro production of experimental enamel caries. J. Am. Dental Assoc. 53, 655 (1956). 1734.

Jennings, $W$. H., Losee, $F$. L., Lawson, M. E., Jr., Microstructure of the human tooth. A. The dentioenamel junction. J. Dental Research 36, No. 6, 911 (1957). 2190.

Jensen, $M$. $W$., Checking prepackaged commodities. (1959) H67.

Farm milk tank testing. Proc. 11th Annual Dairy Technology Conf. (Univ, of Md., College Park, Md.) p. 6 (Nov. 1955); Proc. Milk Industry Foundation Convention (Dairy Industries Foundation, Washington, D.C.) p. 41 (Oct. 1955). 1344; Milk Products J. p. 17 (1956). 1711.

Gaging and testing farm milk tanks. Proc. Natl. Conf. Bulk Milk Handling (Michigan State University, Lansing, Michigan) p. 62 (1957). 2134.

Paper milk containers. Scale J. 41, No. 12, 5 (1955). 1441.

The necessity for uniformity of test procedures. Scale J. 39, No. 3,7 (1952). 587 .

Weights and measures approval seals. Scale J. 41, No. 5, 2 (1955). 1580.

Jensen, $M$. $W$., Bussey, $W$. S., Weights and measures administration in the U.S. Supplement to "The Monthly Review" of the Inst. of Weights and Measures Admin. (April 1956). 1976.

Jerome, C. W., Judd, D. B., Specification of color rendering properties of fluorescent lamps. Preprint No. 4, Nat1. Tech. Conf. of the Illum. Eng. Soc., Pasadena, California (Aug. 21-24, 1950). 134.

Specification of color-rendering properties of fluorescent lamps. Illum. Eng. 48, 259 (1953). 833.

Jespersen, $J$. L., Lawrence, R. S., A preliminary analysis of amplitude scintillations of radio stars observed at Boulder, Colorado. (1959) TN20 (PB151379).

Jessup, R. S., Heat of mixing of polybutadiene and benzene. J 62, 1 (1959) RP2921.

Precise measurement of heat of combustion with a bomb calorimeter. (1960) Mono. 7.

Some thermodynamic properties of the systems polybutadiene-benzene and polyisobutene-benzene. J $\mathbf{6 0}$, 47 (1958) RP2820.

Jessup, R. S., Armstrong, G. T., Combustion calorimetry with fluorine: constant pressure flame calorimetry. J 64A 1, 49 (1960).

Jessup, R. S., Armstrong, G. T., Mears, T. W., Net heat of combustion of aviation gasoline and its correlation with other properties. Chem. \& Eng. Data Series 3, 20 (1958). 2646.

Jessup, R. S., Cogliano, J. A., Relation between net heat of combustion and aniline-gravity product of aireraft fuels. ASTM Bull. No. 201 (TP217), 55 (1954). 1128.

Jessup, R. S., McCoskey, R. E., Nelson, R. A., The heat of formation of tetrafluoromethane. J. Am. Chem. Soc. 77, 244 (1955). 1533.

Jessup, R. S., Rothberg, S., Net heat of combustion of AN-F-58 aircraft fuels. Ind. Eng. Chem. 43, 981 (1951). 265.

Jickling, R. M., Powell, R. C., Hess, A. E., High-frequency impedance standards at the National Bureau of Stand- ards. IRE Trans. Instrumentation I-7, No. 3-4, 270 (1958). 2583.

Joel, L. S., Zelen, $M$., The weighted compounding of two independent significance tests. Ann. Math. Stat. 30, No. 4, 885 (1959). 3098.

Johannesen, R. B., Lindberg, A. R., The temperatureindependent paramagnetism of ammonium hexabromoosmate (IV). J. Am. Chem. Soc. 76, 5349 (1954), 1201.

Johler, J. R., Propagation of the radiofrequency ground wave transient sinusoidal over a finitely conducting plane earth. Symp. on the Prop. of V.L.F. Radio Waves, sponsored by the NBS Boulder Laboratories and IRE Prof. Group on Ant. and Prop., January 23-25, 1957 (Boulder, Colorado), Paper No. 16, V. 2; Geofis. pura e appl., (Milano, Italy), 37, 116 (1957). 2267.

Transient radiofrequency ground waves over the surface of a finitely conducting plane earth. J 60, 281 (1958) RP2844.

Johler, J. R., Cottony, H. V., Cosmic radio noise intensities in the VHF band. Proc. IRE 40, 1053 (1952). 418.

Johler, J. R., Walters, L. C., Mean absolute value and standard deviation of the phase of a constant vector plus a Rayleigh-distributed vector. J 62, 183 (1959) RP2950.

On the theory of reflection of low- and very-low-radiofrequency waves from the ionosphere. J 64D3, 269 (1960).

Propagation of a ground wave pulse around a finitely conducting spherical earth from a damped sinusoidal source current. IRE Trans. Ant. Prop. AP-7, No. 1, 1 (1959). 3024.

Johler, J. R., Walters, L. C., Lilley, C. M., Low- and very low-radiofrequency tables of ground wave parameters for the spherical earth theory: The roots of Riccati's differential equation. (Supplementary numerical data for C573.) (1959) TN7 (PB151366).

$J o h n, F$., On integration of parabolic equations by difference methods. Comm. Pure and Appl. Math. 5, No. 2,155 (1952). 497 .

Johnson, C. R., Jenkins, $W . D$., Creep of annealed nickel, copper, and two nickel-copper alloys. J 60, 173 (1958) RP2836.

Creep of cold-drawn nickel. J 63C1, 1 (1959).

Johnson, D. P., Brombacher, W. G., Cross, J. L., Mercury barometers and manometers. (1960) Mono. 8.

Johnson, D. P., Cross, J. L., Hill, J. P., Bowman, H. A., Elastic distortion error in the dead weight piston gage. Ind. Eng. Chem. 49, 2046 (1957). 2103.

Johnson, D. P., Lloyd, E. C., Hutton, U. O., Compact multi-anvil wedge type high-pressure apparatus. J 63C 1, 59 (1959).

Johnson, D. P., Newhall, D. $H$., The piston gage as a precise measuring instrument. Trans. Am. Soc. Mech. Engrs. 75, 301 (1953). 877.

Johnson, F. S., Wilson, N. L., Tousey, R., Purcell, J. D., Moore, C. E., A revised analysis of the solar spectrum from 2990 to 2635 A. Astrophys. J. 119, No. 3, 590 (1954). 933.

Johnson, H., Rohrlich, F., Atomic electron affinities. Nature No. 4656, 183 (1959). 2900.

Johnson, H. D., Winter, J. C., Phillips, C. W., Grimes, J. $W$., Achenbach, $P$. R., Heat transfer measurements on refrigerated-food trailers. U.S. Dept. of Agri. Marketing Service (AMS-250), (June 1958), 2580.

Johnson, H. F., List of geomagnetic observatories and thesaurus of values. J. Geophys. Research 56, 431 (1951). 252 .

Johnson, P. V., McBurney, J. W., Durability of deaired brick. J.'Am. Ceram. Soc. 39, No. 5, 159 (1956). 1683.

Johnson, V. A., Frederiske, H. P. R., Scanlon, W. W., Methods of experimental physics. Chapter 7.6, Thermoelectric Effects. Edited by K. Lark-Horovitz and V. A. Johnson (Academic Press Inc., New York, N.Y.) p. 114 (1959). 2990. 
Johnson, $V$. J., A new arrangement for ortho-para conversion of liquid hydrogen in the large CEL-NBS liquefier. Proe. 1956 Cryogenic Engineering Conference, Paper A-4, p. 19, Edited by K. D. Timmerhaus, Chemical Engineering Dept., University of Colorado (Boulder, Colorado) (1957). 1988.

Removal of nitrogen from hydrogen with silica gel at low temperatures. Proc. 1957 Cryogenic Engineering Conference, Paper A-2, p. 11, Edited by K. D. Timmerhaus, Chemical Engineering Dept., University of Colorado (Boulder, Colorado) (1958). 2730.

Johnson, V. M., Bennett, L. H., Resistance diode bridge eircuit for temperature control. (1959) TN34 (PB151393).

Johnson, W.', Curtiss, L. F., Evans, R. D., Seaborg, G. T., Units of radioactivity. Rev. Sei. Instr. 21, 94 (1950). 163.

Johnson, W. H. Gilliland, A. A., Prosen, E. J., Heat of formation of titanium tetraiodide. J 63A2, 161 (1959).

Johnson, W. H., Miller, R. G., Prosen, E. J., Heat of formation of boron trichloride. J 62, 213 (1959) RP2956.

Johnson, W. H., Nelson, R. A., Prosen, E. J., Heat of formation of titanium tetrabromide. J 62, 67 (1959) RP2932.

Heat of formation of titanium tetrachloride. J 62, 49 (1959) RP2928.

Johnson, W. H., Prosen, E. J., Pergiel, F. Y., Heats of formation of diborane and pentaborane. J 61 , 247 (1958) RP2901.

Heat of reaction of diborane with water and the heat of formation of boric oxide. J 62, 43 (1959) RP2927.

Johnston, H. L., Friedman, A. S., Trzeciak, $M$., Pressurevolume temperature relations of liquid normal deuterium. J. Am. Chem. Soc. 76, 1552 (1954). 115.

Johnston, H. L., Keller, W. E., Friedman, A. S., The compressibility of liquid normal hydrogen from the boiling point to the central point at pressures up to 100 atmospheres. J. Am. Chem. Soc. 76, 1482 (1954). 1173 .

Johnston, R. G., Maki, A. G., Stair, R., Apparatus for the measurement of the normal spectral emissivity in the infrared. J 64C2, 99 (1960).

Johnston, R. G., Stair, R., Some studies of atmospheric transmittance on Mauna Loa. J 61, 419 (1958) RP2910.

Johnston, R. G., Stair, R., Bagg, T. C., Continuous measurement of atmospheric ozone by an automatic photoelectric method. Proc. Joint Army-Navy-Air Force Conf. on Elastomer Research and Devel., January 12-13, 1954 (Washington, D.C.), Natl. Acad. Sci.-Natl. Research Council Publ. 370, p. 75 (1954). 977.

Johnston, $R$. W., Koch, $H . W$., Editors, Multichannel pulse height analyzers. 'Natl. Acad. Sci.-Natl. Research Council Publ. 467 (1957). 2206.

Junes, E., Cemented piezoelectric accelerometers. Rev. Sci. Instr. 24, 1151 (1953). 660.

Jones, E., Edelman, S., Brooks, R., Saito, S., Smith, $E$. R., A stroboscopic vibration' analyzer. 'J $63 \mathrm{C} 2$, 97 (1959).

Jones, E., Edelman, S., Smith, E. R., Some developments in vibration measurement. J. Acoust. Soc. Am. 27, 728 (1955). 1492.

Jones, E., Smith, E. R., Edelman, S., Schmidt, V. A., Stroboscopic interferometer for vibration measurement. J. Acoust. Soc. Am. 30, 867 (1958). 2784.

Jones, E. J., Gorman, J. G., Hipple, J. A., Analysis of solids with the mass spectrometer. Anal. Chem. 23, 438; Phys. Rev. 82, 768 (1951). 194.

Jones, R. W., Leaderman, H., Smith, R. G., Rheology of polyisobutylene. II. Low molecular weight polymers. J. Polymer Sci. 14, 47 (1954). 1132.

Jones, $W$. T., Hobbs, R. B., Annual review of pulp and paper industry. Ind. Eng. Chem. 48, 111 (1956). 1627 .
Jordon, E. C., Cottony, H. V., Elliott, R. S., Rumsey, V. H., Siegel, K. M., Wait, J. R., Woodyard, O. C., Antennas and waveguides, and annotated bibliography. U.S.A. National Committee report, Intern. Sei. Radio Union Subcommission 6.3. IRE Trans. Ant. Prop. AP-y, $87(1959), 2897$.

U.S.A. National Committee report URSI Subcommission 6.3 , antennas and waveguides, and annotated bibliography. Proc. 12th Gen. Assembly, International Scientific Radio Union, August-September 1957 (Boulder, Colorado) 11, Pt. 6, 121 (1957). 2402.

Jorgensen, K. D., Posner, A. S., Study of the setting of plaster. J. Dental Research 38, 491 (1959), 3058.

Joseph, H. M., Kovasznay, L. S., Processing of two-dimensional patterns by scanning techniques. Science 118, 475 (1953). 802 .

Joseph, H. M., Newman, N., Eddy-current mutual-inductance transducers with high-conductivity reference plates. Commun. and Electronics (Publ. by Am. Inst. Elec. Engrs.) Paper 55-4, No. 17, p. 1 (1955). 1323.

Joseph, H. M., Stansbury, C., Automatic counting machine. Elec. Mfg. 49, No. 3, 100 (1952). 398.

$J u d d, D . B ., \quad$ A new point of view in the measurement of light and color. Anales real soc. espan. fis. y quim. (Madrid, Spain) 53A, No. 1-2, 43 (1957). 1989. Ceguera para el color y teorias de la vision cromatica. Anales real soc. espan. fis. y quim. (Madrid, Spain) 47, 35 (1951). 201.

Color vision and colorimetry. Handbook of Physics, Edited by E. U. Condon and H. Odishaw, Chapter 4, Pt. 6, p. 6-64 (McGraw-Hill Book Co., Inc., New York, N.Y.) (1958). 2500.

Description of color. Proc. Perkin Centennial (Ogden Printing Co., New York, N.Y.) (1957). 2080.

El sistema I. C. I. para la especificacion del color Anales de la real soc. Espan. fis, y quim. (Madrid, Spain) 46, 123 (1950). 52.

Entoptic color-perceptions of the macular pigment by observers of normal and color-defective vision according to the three-components theory. Proc. $1953 \mathrm{Col}-$ loquium on Optical Problems in Vision (Madrid, Spain) (1956). 1706.

La medida de la luz y el color. Anales real soc. Espan. fis. y quim. (Madrid, Spain) 53A, 43 (1958). 2609.

Measurement of light and color. Illum. Eng. 53, 61 (1958). 2627.

Medida del color en la industria. Boletin iberomericano de cultura tecnica (Madrid, Spain) 1, 13 (1957). 2197.

Progress report by Committee of Optical Society of America on uniform color scales. J. Opt. Soc. Am. 45, 673 (1955). 1459A.

Some color demonstrations I have shown. J. Opt. Soc. Am. 49, 322 (1959). 3046.

Summary of symposium on color of transparent and translucent products. ASTM Bull. No. 202, p. 72 (1954). 1155.

Judd, D. B., Helson, H., Warren, M. H., Object-color changes from daylight to incandescent filament illumination. Illum. Eng. 47, 221 (1952). 492.

$J$ udd, D. B., Helson, H., Wilson, M., Color rendition in fluorescent sources of illumination. Illum. Eng. 51, 329 (1956). 1651.

$J$ udd, D. B., Jerome, C. $W$., Specification of color rendering properties of fluorescent lamps. Preprint No. 4, Natl. Tech. Conf. of the Illum. Eng. Soc., Pasadena, California (August 21-24, 1950). 134; Illum. Eng. 48, 259 (1953). 833.

Judd, D. B., Keegan, H. J., Rheinboldt, W. C., Schleter, J. C., Menard, J. P., Digital reduction of spectrophotometric data to Munsell renotations. J. Opt. Soc. Am. 48, 863 (1958). 2522.

Judd, D. B., Plaza, L., Farnsworth, D., Tritanopia with abnormally heavy ocular pigmentation. J. Opt. Soc. Am. 40, 833 (1950). 161.

Judd, D. B., Schleter, J. C., Keegan, H. J., Extension of the Munsell renotation system. J. Opt. Soc. Am. 48, 863 (1958). 2559. 
$J u d d, D . B .$, Wyszecki, G., Extension of Munsell renotation svstem to very dark colors. J. Opt. Soc. Am. 46, 281 (1956). 1710.

Judson, L. V., Calibration of line standards of length and measuring tapes at the National Bureau of Standards. (1960) Mono. 15.

\section{K}

Kac, $M$., On some connections between probability theory and differential and integral equations. 2d Berkeley Symp. on Math. Stat, and Prob. held by the University of California from July 31 to Aug. 12, 1950 , p. 189 (1951). 278.

Kanagy, $J$. R., Heats of wetting of collagen, leather, and other organic and fibrous materials. J. Am. Leather Chemists Assoc. 49, 646 (1954). 1028.

Influence of temperature on the adsorption of chrome $\left(\mathrm{Cr}_{2} \mathrm{O}_{3}\right)$ by calf skin squares. J. Am. Leather Chemists Assoc. 52, 142 (1957). 2159.

Influence of temperature on the adsorption of water vapor by collagen and leather. J. Am. Leather Chemists Assn. 45. 12 (1950). 74.

Significance of the results of some physical tests on upper leather. J. Am. Leather Chemists Assoc. 50, No. 3, 112 (1955). 1485.

Specific heats of collagen and leather. J. Am. Leather Chemists Assoc. 50, No 10, 444 (1955). 1502.

Kanagy, J.R., Carter, T.J., A flex tension test for leather. J. Am. Leather Chemists Assoc. 49, 23 (1954). 917.

Kanagy, J. R., Cassel, J. M., Heats of wetting of modified collagen and other materials. J. Am. Leather Chemists Assoc. 52, 248 (1957). 2149.

Kanagy, J. R., Fouquet, B. H., Impregnation of sole leather with polymers. J. Am. Leather Chemists Assoc. 53, No, 6, 336 (1958), 2594 .

Kanagy, J. R., Kilduff, T. J., Cyclic strain testing machine for leather. J. Am. Leather Chemists Assoc. 50, No, 10,489 (1955), 1304

Kanagy, J. R., Leser, W., Randall, E. B., Carter, T. J. Mann, C., Influence of splitting on the strength of chrome-tanned steer hides. J. Am. Leather Chemists Assoc. 47, 329 (1952). 468

Kanagy, J. R., Mann, C., Mandel, J., Study of the variation of the physical and chemical properties of chrome-tanned leather and the selection of a sampling location. J. Soc. Leather Trades' Chemists (London, England) 36, 231 (1952). 558.

Kanagy, J. R., Mann, C., Mandel, J., Steel, M. N., Sampling of side upper leather II. J. Am. Leather Chemists Assoc. 47, 352 (1952). 535.

Kanagy J. R., Randall, E. B., Carter, T. J., Kilduff T. J. Mann, $C$., The variation of the physical and chemical properties of split and unsplit chrome-tanned leathers. J. Am. Leather Chemists Assoc. 47, 404 (1952), 601 .

Kanagy, J.R., Randall, E. B., Carter, T. J., Kinmonth, R. A., Mann, C., Variations of physical and chemical properties within and between vegetable retanned cow and steer sides. J. Am. Leather Chemists Assoc. 47, $726(1952) .618$

Kanagy, J. R., Randall, E. B., Mann, C., Mandel, J., Study of the burst test as applied to military upper leathers. J. Am. Leather Chemists Assoc. 48, 84 (1953), 842.

Kanagy, J. R., Robinson, M., Studies on leather by means of a sonic technique. J. Am. Leather Chemists Assoc. 51, 174 (1956). 1905.

Kanagy, J. R., Vickers, R. A., Factors affecting the water vapor permeability of leather. J. Am. Leather Chemists Assoc. 45, 211 (1950). 57

Kane, W. R., Broida, H. P., Polyhedral flames with hydrogen and hydrocarbons. J. Chem. Phys. 20, No. $6,1042(1952)$. 514

Rotational intensity distributions of $\mathrm{OH}$ and $\mathrm{OD}$ in electrodeless discharge through water vapor. Phys. Rev. 89, 1053 (1953). 817.

Rotational "temperature" of $\mathrm{OH}$ in diluted flames. J. Chem. Phys, 21, 347 (1953), 818.
Kanegis, J., Wolock, I., Plastics literature in Government reports. Am. Soc. Advances in Chem. Ser. No. $10,151(1954)$. 1109

Kaplan, A. M., Dahl, S., Fungicidal effectiveness of compounds applied to leather. J. Am. Leather Chemists. Assoc. 52, 536 (1957). 2131.

Laboratory and field exposure studies of leather fungicides. J. Am. Leather Chemists Assoc. 51, No. 3,119 (1956). 1754 .

Studies of leather fungicides. J. Am. Leather Chemists Assoc. 53, 103 (1958). 2788.

Kaplan, J., Shapley, A. H., The International Geophysical Year: a progress report. News Report Natl. Research Council 4, No, 6, 1 (1954). 1188.

Kaplan, L. D., Kostkowski, H. J., Absolute intensities of the 721 and $742 \mathrm{~cm}^{-1}$ bands of $\mathrm{CO}_{2}$. J. Chem. Phys. 26, 1252 (1957). 2007 .

Kaplansky, I., Completelv continuous normal operators with property L. Pacific J. Math. 3, 721 (1953). 671.

Karush, $W$. An iterative method for finding characteristic vectors of a symmetric matrix. Pacific J. Math. 1, No. 2, 233 (1951). 192.

Convergence of a method of solving linear problems. Proc. Am. Math. Soc. 3, No. 6, 839 (1952). 417. Determination of the extreme values of the spectrum of a bounded self-adjoint operator. Proc. Am. Math. Soc. 2, No. 6, 980 (1951). 220.

Kates, J., Wait, J. R., Radiation patterns of circumferential slots on moderately large conducting cvlinders. Inst. Elec. Engrs. Mono, No. 167R, 1 (1956) 1830 .

Kato, T., On the Hilbert matrix. Proc. Am. Math. Soc. 8,73 (1957). 2229.

Kato, T. Fujita, H., Nakata, Y., Newman, M., Estimation of the frequencies of thin elastic plates with free edges. J 59, 169 (1957) RP2784.

Kato, T., Taussky, O., Commutators of A and A*. J. Wash. Acad. Sci. 46, 38 (1956). 1653.

Katzin, J. C. Katzin, M., Pezzner, H., Koo, B. Y.-C., Larson, J. $V$., The trade-wind inversion as a transoceanic duct. J 64D3, 247 (1960).

Katzin, M., Koo, B. Y.-C., An exact earth-flattening procedure in propagation around a sphere. J 64D1, $61(1960)$

Katzin, M., Pezzner, H., Koo, B. Y.-C., Larson, J. V., Katzin, $J, C$., The trade-wind inversion as a transoceanic duct. J 64D $3,24 i$ (1960).

Kaufman, S. Wieder, H. H. Plastic electrets. J. Appl. Phys, 24, 156 (1953). 796.

Plastic electrets and their applications. Elec. Eng. 72, 511 (1953). 797.

Keegan, H. J., Colorimetry from precision spectrophotometry. J. Opt. Soc. Am. 48, 281 (1958). 2487. Computer defines color. Chem. Eng. News 37, No. $10,37(1959) .2913$.

New federal standard on colors. J. Opt. Soc. Am. 47, 330 (1957). 2212.

Safety color codes. Inter-Society Color Council News Letter No. 121, 10 (1955). 1481; Mag. of Standards 27, 21 (1956): J. Opt. Soc. Ám. 46, 74 (1956). 1861.

Keegan, H. J., Rheinboldt, W. C., Schleter, J. C., Menard, $J . P . J u d d, D . B$, Digital reduction of spectrophotometric data to Munsell renotations. J. Opt. Soc. Am. 48, 863 (1958), 2522.

Keegan, H. J., Schleter, J. C., Spectrophotometry and aerial photoreconnaissance. (Abstract) J. Opt. Soc. Am. 4\%, 1050 (1957). 2308.

Keegan, H. J., Schleter, J. C., Judd, D. B., Extension of the Munsell renotation system. J. Opt. Soc. Am. 48, 863 (1958). 2559 .

Keeler, R. N., Timmerhaus, $K$. D., Poisoning and reactivation of orthoparahydrogen conversion catalyst. Proc. 1958 Cryogenic Engineering Conference, Paper E-3, p. 296. Edited by K. D. Timmerhaus, Chemical Fngineering Dept., University of Colorado (Boulder, Colorado) (1959) 3017

Keery, W. J., Marsden, C. P., Moffit, J. K., Tabulation of data on receiving tubes. (1959) H68. 
Keinath, G., Recorder survey: recording surfaces and marking methods. (1959) C601.

The measurement of thickness. (1958) C585.

Keller, H. R., Marden, E., Pfeffer, H., The Haystaq system: past, present and future. Preprinted in Proc. Intern. Conf. on Scientific Infor. November 11-16, 1958 (American Documentation Inst., Washington D.C.) Area 5, p. 317 (1958). 2815.

Keller, W. E., Johnston, H. L., Friedman, A. S., The compressibility of liquid normal hydrogen from the boiling point to the central point at pressures up to 100 atmospheres. J. Am. Chem. Soc. 76, 1482 (1954). 1173.

Kelly, $K$. $L$. Central notations for the revised ISCCNBS color-name blocks. J 61, 427 (1958) RP2911.

Observer differences in color-mixture functions studied by means of a pair of metameric grays. J 60. 97 (1958) RP2825.

Kennedy, R. J., Kirn, F. S., Betatron X-ray: how much concrete for shielding? Nucleonics 12, 44 (1954). 961.

Kennedy, R. J., Kirn, F. S., Wyckoff, H. O., The attenuation of gamma rays at oblique incidence. Radiology 63, 94 (1954). 1171.

Kennedy, R.J., Miller, $W_{\text {., }}$ Attenuation of 86- and 176Mer synchrotron X-rays in concrete and lead. Radiol. Research 4, 360 (1956). $1635 \mathrm{~A}$.

Measurement of distortion produced in guarded electrostatic fields by grounded external plates. Rev. Sci. Instr. 25, 1031 (1954). 1066.

X-ray attenuation in lead, aluminum, and concrete in the range 275 to 525 kilovolts. Radiology 65 , No. 6, 920 (1955). 1582.

Kennedy, R. J., Smith, S. $\dot{W}$., Dose to walls in radiographic rooms. Radiology 64, 114A (1955). 1319.

Kerns, D. M., Half-round inductive obstacles in rectangular waveguide. J 64B2, 113 (1960). 2691.

WWV standard frequency transmissions. Proc. IRE Letter 46, 1881 (1958). "2872.

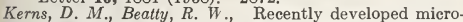
wave impedance standards and methods of measurement. IRE Trans. Instrumentation I- 7 , No. 3-4, 319 (1958). 2722.

Kerns, D. M., Dayhoff, E. S., Theory of diffraction in microwave interferometery. J 64B 1, 1 (1960).

Kerns, D. M., Hedberg, R. W., Propagation constant in rectangular wave guide of finite conductivity. J. Appl. Phys. 25, No. 12, 1550 (1954). 1120.

Kerns, D. M., MacPherson, A. C., A microwave micocalorimeter. Rev. Sci. Instr. 27 (1955). 1236. A new technique for the measurement of microwave standing wave ratios. Proc. IRE 44, 1024 (1956). 1594.

Matching loads on a magic tee. Electronics 23, No. 9, $190(1950)$. 83A.

Kerper, M. J., Mong, L. E., Stiefel, M. B., Holley, S. F., Evaluation of tensile, compressive, torsional, transverse, and impact tests and correlation of results for brittle cermets. J 61, 149 (1958) RP2895.

Kessler, $K . G_{.,}$Analysis of the first spectrum of ruthenium (Ru I). J 63A3, 213 (1959).

Forbidden lines in the spectra of impure mercury 198. Phys. Rev. 77, 559 (1950). 60.

Kessler, K. G., Barger, R. L., Schweitzer, W. G., Jr. Atomic beam sources and standard of length, IRE' Trans. Instrumentation 1-7, No. 3-4, 181 (1958). 2464 .

Kessler, K. G., McNally, J. R., Jr., Supplementary Zeeman data for the first spectrum of ruthenium (Ru I). J 63A3, 253 (1959).

Kessler, K. G., Prusch, S. B., Stegun, I. A., Analysis of a omic spectra with electronic digtail computers. J. Opt. Soc. Am. 46, No. 12, 1043 (1956). 1624.

Kessler, K. G., Schweitzer, W. G., Jr., Zeeman-split absorption lines as very narrow pass filters. J. Opt. Soc. Am. 49, 199 (1959). 3115.

Kessler, K. G., Trees, R. E., The nuclear moments of technetium-99. Phys. Rev. 92, 303 (1953). 875.
Kessler, M., Lyons, H., Rueger, L. J., Nuckolls, R. G. Microwave spectra of deutero-ammonias. Phys. Rev. 81, 630 (1951). 260.

Kettering, C. A., Montgomery, G. F., How the U.S. Weather Bureau uses Fax \& Telemetering: How FM expedites weather data distribution. FM-TV Radio Commun. 10, No. 10, 17 (1950). 69.

Keulegan, G. H., Determination of critical depth in spatially variable flow. 2nd Proc. Midwestern Conf. on Fluid Mechanies, March 17-19, 1952, Ohio State University, Engineering and Experiment Station Bull. 149, p. 67 (1952). 225.

Keulegan, G. H., Carpenter, L. H., Forces on cylinders and plates in an oscillating fluid. $J$ 60, 423 (1958) RP2857.

Khan, N. A., Marcus, $M$., Space of k-commutative matrices. J 64B1, 51 (1960).

Kiefer, J., Dvoretsky, A., Wolfowitz, J., Sequential decision problems for processes with continuous time parameter. Testing hypotheses. Ann. Math. Stat. 24, No. 2, 254 (1953). 824.

Kiess, C. C., Description and analysis of the second spectrum of molybdenum, Mo II. J 60, 375 (1958) RP2856.

Extension of the second spectrum of zirconium into the infrared. J. Opt. Soc. Am. 43, 1024 (1953). 717.

Kiess, C. C., Corliss, C. H., Deseription and analysis of the first spectrum of iodine. J 63A 1, 1 (1959).

Kiess, C. C., Corliss, C. H., Kuess, H. K., Corliss, L. R., High-dispersion spectra of mars. Astrophys. J. 126, No. 3, 579 (1957). 2152.

Kiess, C. C., Moore, C. E., Predicted lines of $\mathrm{Fe} I$ in the arc and in the sun. Astron. J. 55, 173 (1950). 111.

Kiess, H. K., Kiess, C. C., Corliss, C. H., Corliss, L. R., High-dispersion spectra of mars. Astrophys. J. 126, 3,579 (1957). 2152.

Kiess, N. H., Broida, H. P., Analysis of the (0.1) and (1.2) bands of the ${ }^{2} \Delta^{-2} \pi$ system of $\mathrm{CH}$. Astrophys. J. 123, No. 1, 166 (1956). 1625.

Emission spectra from mixtures of atomic nitrogen and organic substances. 7th Symp. on Combustion (Butterworths, London, England), p. 207 (1958). 2544 .

Spectrum of the $\mathrm{C}_{3}$ molecule between $3600 \AA$ and $4200 \AA$. Can. J. Phys. 34, 1471 (1956). 1885.

Spectrum of the $C_{3}$ molecule between $3600 \AA$ and $4200 \AA$. Mem. Soc. Roy. Sci. (Liege, Belgium) 18, 544 (1957). 2313.

Kilday, $M$., Shepherd, $M$., Schuhmann, $S$., Determination of carbon monoxide in studies of air pollution. Anal. Chem. 27, 380 (1955), 1309.

Kilduff, T. J., Kanagy, J. R., Cyclic strain testing machine for leather. J. Am. Leather Chemists Assoc. 50, No. 10, 489 (1955). 1304.

Kilduff, T. J., Oehler, R., Dahl, S., Treatment of leather with Castiloa and Hevea rubbers. J. Am. Leather Chemists Assoc. 45, 378 (1950). 160.

Treatment of leather with polyisobutylene. J. Am. Leather Chemists Assoc. 47, 642 (1952). 607.

Kilduff, T. J., Randall, E. B., Carter, T. J., Mann, C., Kanagy, J.R., The variation of the physical and chemical properties of split and unsplit chrome-tanned leathers. J. Am. Leather Chemists Assoc. 47, 404 (1952). 601.

Kilpatrick, E. L., A technique for sweep frequency polarization measurements at low frequencies. J. Geophys. Research 59, 345 (1954). 938.

Polarization measurements of low-frequency echoes. J. Geophys. Research 57, No. 2, 221 (1952). 513.

King, E. P., Estimating the standard deviation of a normal population. Ind. Quality Control 10, 30 (1953). 713.

On some procedures for the rejection of suspected data. J. Am. Stat. Assoc. 48, 531 (1953). 784.

Optimum grouping in one-criterion variance components analysis, J. Am. Stat. Assoc. 49, 637 (1954). 1102. 
Probability limits for the average chart when process standards are unspecified. Ind. Qual. Control 10, 62 (1954). 1116.

The operating characteristic of the average chart. Ind. Qual. Control 9, No. 3, 30 (1952). 588.

$K$ ing, E. P., Lukacs, $E$., A property of the normal distribution. Ann. Math. Stat. 25, No. 2, 389 (1954). 928.

King, R. W. P., Harrison, C. W., Response of a loaded electric dipole in an imperfectly conducting cylinder of finite length. J 64D3, 289 (1960).

King, T. A., Rodney, W. S., Malitson, I. H., Refractive index of arsenic trisulfide. J. Opt. Soc. Am. 48, No. 9, 633 (1958). 2726.

Kinmonth, R. A., Kanagy, J. R., Randall, E. B., Carter, T.J., Mann, C., Variations of physical and chemical properties within and between vegetable retanned cow and steer sides. J. Am. Leather Chemists Assoc. 47, $726(1952), 618$.

Kinmouth, R. A., Oehler, R., Davis, J. H., A pilot plant study of the process for treating heavy leather with polyisobutylene and other polymers. J. Am. Leather Chemists Assoc. 50, 16 (1955). 1240.

Kinter, E. B., Wintermyer, A. M., Swerdlow, $M$., Electron microscopy of soil clays and related materials. Public Roads J. Highway Research, 27, No. 5, 89 (1952). 439.

Kirby, R. C., Extreme useful range of VHF transmission by scattering from the lower ionosphere. IRE Conv. Record 6, Pt. 1, 112 (1958). 2560.

1958 eritique of $\mathrm{VHF}$ ionospheric scatter communication. (Survey Paper), Record of Natl. Symp. on Extended Range and Space Communications, October 6-7, 1958 (Washington, D.C.), p. 90 (1958). 2505A. $\mathrm{VHF}$ propagation by ionospheric scattering-survey of experimental results. IRE Trans. Commun. Systems CS-4, No. 1, 17 (1956). 1971.

Kirby, R. C., Bailey, D. K., Bateman, R., Radio transmission at VHF by scattering and other processes in the lower ionosphere. Proc. IRE 43, No. 10, 1181 (1955). 1469.

Kirby, R. C., Little, C. G., Conference on Arctic communication. J 64D 1, 73 (1960).

Kirby, R.S., Measurement of a service area for television broadcasting. Trans. IRE Trans. Broadeast Transmission Systems BTS-7, 23 (1957). 2189.

Kirby, R. S., Capps, F. M., Correlation in VHF propagation over irregular terrain. IRE Trans. Ant. Prop. AP-4, No. 1,77 (1956). 1663.

Kirby, R. S., Dougheriy, H. T., McQuate, P. L., VHF propagation measurements in the Rocky Mountain region. Trans. IRE Trans. Vehicular Commun. VC-6, 13 (1956). 1972 .

Kirby, R. S., Herbstreit, J. W., Norton, K. A., Service range for air-to-ground and air-to-air communications at frequencies above 50 Mc. Proc. IRE 40, 525 (1952). 540.

Kirby, R. S., Taff, J. M., Moore, H. S., Measurement of the effect of irregular terrain on VHF and UHF directive antenna patterns. IRE Trans. Ant. Prop. AP-3, 167 (1952). 481.

Kirk, P. L., Macurdy, L. B., Alber, H. K., BenedettiPichler, A. A., Carmichael, H., Corwin, A. H., Fowler, R. M., Huffman, E. W. D., Lashof, T. W., Terminology for describing the performance of analytical and other precise balances. Anal. Chem. 26, 1190 (1954). 1166.

Kirkpatrick, H. B., Richmond, J. C., A radioisotope study of the nickel dip. J. Am. Ceram. Soc. 39, No. 2, 39 (1956). 1599.

The nickel dip: a radioisotope study of metallic deposits in porcelain enameling. Natl. Advisory Comm. Aeronaut. Tech. Note 3577 (1956). 1941.

Kirkpatrick, H. B., Richmond, J. C., Moore, D. G., Harrison, $W . \quad N$., Relation between roughness of interface and adherence of porcelain enamel to steel. Inst. Vitreous Enamellers Ltd. Bull. (Published by J. Am. Ceram. Soc.) 7, No. 5, 69 (1957). 2281.
Kirn, F. S., Kennedy, R. J., Betatron X-rays: how much concrete for shielding? Nucleonics 12, 44 (1954). 961.

Kirn,F.S., Kennedy, R. J., Wyckoff, H. O., The attenuation of gamma rays at oblique incidence. Radiology 63, 94 (1954). 1171.

Kirn, F. S., Motz, J. W., Miller, W., Wyckoff, H. O., Gibson, H. F., Gamma-ray measurements by the magnetic analysis of Compton electrons. Rev. Sci. Instr. 24, 929 (1953). 725.

Kirsch, R. A., Cahn, L., Ray, L. C., Urban, G. H., Experiments in processing pictorial information with a digital computer. 1957 Eastern Joint Computer Conf. Proc. (Washington, D.C.) (1957). 2119.

Kirsch, R. A., Ray, L. C., Finding chemical records by digital computers. Science 126, No. 3278, 814 (1957). 2122 .

Kirstein, A. F., Chi, M., Flexural cracks in reinforced concrete beams. J.' Am. Concrete Inst. No. 54 (1958). 2566.

Kissinger, C. W., Perls, T. A., An integrating and differentiating bar-magnet velocity meter for use in ballistocardiography. Rev. Sci. Instr. 25, No. 10, 983 (1954). 951.

Jerkmeter for ballistocardiography. Rev. Sci. Instr. 27, No. 1, 51 (1956). 1752 .

Kissinger, H. E., McMurdie, H. F., Simpson, B., Thermal decomposition of manganous and ferrous carbonates. J. Am. Ceram. Soc. 39, No. 5, 168 (1956). 1960.

Kivelson, $D$., Lide, $D$. R., Jr., Internal rotation in methyltrifluoromethyl acetylene. J. Chem. Phys. 23, 2191 (1955). 1374.

Structure of the methylamine molecule. II. Theory of internal motions and application to $\mathrm{CD}_{3} \mathrm{NO}_{2}$. J. Chem. Phys. 27, 353 (1957). 2323.

Klebanoff, $P$. S., Characteristics of turbulence in a boundary layer with zero pressure gradient. Natl. Advisory Comm. Aeronaut. Tech. Note 3178 (1954). 968.

Klebanoff, P. S., Schubaver, G. B., Tidstrom, K. D., Measurement of the effect of two-dimensional and threedimensional roughness elements on boundary-layer transition. J. Aeronaut. Sci. 22, 11 (1955), 1400.

Klein, E. I., Ruthberg, S., On small precision bottles of quartz and low-loss glass. Rev. Sci. Instr. 28, No. 3,205 (1957). 2224.

Klein, M., Hilsenrath, J., Sumida, D. Y., Mechanized computation of thermodynamic tables at the National Bureau of Standards: the calculation of the eauilibrium composition and thermodynamic properties of dissociation and ionized gaseous systems. Proc. Symp. Thermodynamic and Transport Properties Gases, Liquids, and Solids (Reprinted from Thermodynamic and Transport Properties of Gases, Liquids, and Solids), Am. Soc. Mech. Engrs. p. 416 (1959). 2988.

Klein, R., Adsorption, diffusion, and evaporation of carbon monoxide on tungsten. J. Chem. Phys. 31, No. 5,1306 (1959). 2888.

Klein, R., McNesby, J. R., Scheer, M. D., Schoen, L. J., Kinetics of D atom reactions with $\mathrm{H}_{2}$. J. Chem. Phys. 30, No. 1, 58 (1959). 2971.

Klein, R., Pararas, J., An improved hydrogen atom beam furnace. (1960) TN47 (PB151406).

Klein, R., Scheer, M. G., The addition of hydrogen atoms to solid olefine at $-195^{\circ}$. J. Am. Chem. Soc. 80, 1007 (1958). 2803.

The reaction of hydrogen atoms with solid olefins at $-195^{\circ}$. J. Phys. Chem. 62, 1011 (1958). 2828.

Klein, R., Schoen, L. J., The energy dissociation of the $\mathrm{C}-\mathrm{H}$ bond in the formyl radical. J. Chem. Phys. Comments and Errata 29, 953 (1958). 2813.

Klein, R., Simpson, J. A., Production of a square temperature wave in a filament operating at low temperatures. Rev. Sci. Instr. 29, No. 9, 770 (1958). 2701.

Kleinschmidt, L. R., Influence of exposure conditions 
on the accelerated durability testing of asphalt. ASTM Bull. No. 213, p. 69 (1956). 1738.

Preparation of bituminous materials for spinning. ASTM. Bull. No. 193,53 (1953). 800.

Kleinschmidt, L. R., Snoke, H. R., Changes in the properties of an asphalt during the blowing operation. J 60, 169 (1958) RP2835.

Effect of light and water on the degradation of asphalt. J 63C 1,31 (1959).

Klier, E. P., The tensile properties of selected steels as a function of temperature. Welding Research Council Bull. 35 (1957). 2384.

Kline, G. $M$., Amerikanische kunststoffentwicklung im jahre 1949. Kunststoffe (Munchen, Germany) 40, No. $2,59(1950) .21$.

International plastic committee considers standard tests. Mag. of Standards 26, No. 10, 305 (1955). 1376 .

Plastics. J. Eng., Mech. Div., Proc. Am. Soc. Civil Engrs, 84, Paper 1071, EM 4 (1956); Grolier Encyclopedia (1956 ed.). 1811.

Preservation of the Declaration of Independence and the Constitution of the United States. ASTM. Bull. No. 178,32 (1951). 288.

Report on International Standards Organization Technical Committee 61. Mag. of Standards 26, No. 2, 47 (1955). 1477.

Research and standards for the plastics industry. Modern Plastics 31, No, 12, 127 (1954). 1130.

Ricerca e normalizzazione per I'industria delle materie plastische. Poliplasti (E.T.A.S. Editrice, Milano, Italy) 2, No. 2529, 62 (1956). 1857.

Standards for plastics. Chapter in Plastics in Building 95, Advances in Chemistry Series 10, 145 (1955). 1509.

Test methods, specifications and standards for plastics. Am. Chem. Soc. Advances in Chem. Ser. No. 10, 145 (1954). 1167

The year 1952 in review. Modern Plastics 30, 111 (1953). 894.

Kline, G. M., Achhammer, B. G., Tryon, M., Chemical structure and stability relationships in polymers. Kunststoffe combined with German Plastics Digest 49, No. 11, 600 (1959); Modern Plastics 37, No, 4, 131 (1959). 2910.

Kline, G. M., Reinhart, $F$. W., Plastics: organic. Medical Physics II, 728 (1950). 109.

Kline, G. M., Seymour, R. B., Plastics. Ind. Eng. Chem. 44, 2339 (1952). 512 .

Kline, G. M., Wolock, I., Axilrod, B. M., Sherman, M. A. George, D. A., Cohen. V., Development of craze and impact resistance in glazing plastics by multiaxial stretching. Natl. Advisory Comm. Aeronaut. Tech. Report 1290 (1957). 2084.

Kline, G. M., Wolock, I., Callomon, I. G., Plastics for aircraft. 'Part II. 'Transparent glazing materials. Army-Navy-Commerce Bull. ANC-17 (1957). 2255.

Klinkenberg, P. F. A., Meggers, W. F., Velasco, R., Catalan, $M . A$. Term analysis of the first spectrum of rhenium (Re I.) J 59, 319 (1957) RP2804.

Knecht, $R$. W., Relationships between aurora and sporadic-E echoes at Barrow, Alaska. J. Geophys. Research 61, 59 (1956). 1845.

Statistical results and their shortcomings concerning the ionosphere within the auroral zone. J. Atmospheric and Terrest. Phys. Special Suppl. 1957, Proc. of the Polar Atmosphere Symp. (Oslo, Norway) July 2-8, 1956, Pt. II, 109 (1957). 2317.

Knecht, R. W., Gautier, T. N., McNish, A. G., Lunar stratification of the F2 layer at Huancayo, Peru. Proc. General Assembly. Intern. Sci. Radio Union, Zurich, Switzerland, Sept. 11-22, 1950, VIII, Pt. 2, 216 (1950) 83.

Knecht, R. W. Smith, E. K., Some implications of slantEs. J. Atmospheric and Terrest. Phys Spec. Suppl. 1957, Proc. Polar Atmospheric Symp. July 1956 (Oslo, Norway), Pt. II, 195 (1957). 2304.

Knecht, R. W., Wright, J. W., Davies $K$., Ionospheric vertical soundings. Anals of the International Geo- physical Year 3, Pt. 1, 1 (Pergamon Press, New York, N.Y.) (IGY Instruction Manual: The Ionosphere, Pt. I) (1957). 2173.

Knudsen, F. P., Geller, R. F., Moreland, R. E., Physical characteristics of titaniun carbide type cermets at elevated temperatures. J. Am. Ceram. Soc. 38, No, 9, 312 (1955). 1445 .

Knudsen, F. P., Lang, S. M., Some physical properties of high-density thorium oxide. J. Am. Ceram. Soc. 39, No. 12, 415 (1956). 1877.

Knudsen, $H$. L., Earth currents near a top-loaded monopole antenna with special regard to electrically small L- and T-antennas. J 62, 283 (1959) RP2961.

Knudsen, $H$. L., Larsen, T., The electric field at the ground plane near a top-loaded monopole antenna with special regard to electrically small L- and Tantennas. J 64D2, 139 (1960).

$K o c h, H . W$., Direct observation of nuclear absorption and elastic scattering of X-rays. Proc. 1954 Glasgow Conference on Nuclear and Meson Physics (Pergamon Press, New York, N.Y.) (1955). 1312.

Gamma-ray spectroscopy. Chapter 15, Trace Analysis. Edited by Yoe \& Koch (John Wiley \& Sons, Inc., New York, N.Y.), p. 413 (1957). 2139.

Koch, H. W., Fano, U., Motz, J. W., Evaluation of bremsstrahlung cross sections at the high-frequency limit. Phys, Rev, 112, No, 5, 1679 (1958), 2551.

Koch, H. W., Foote, R. S., Scintillation spectrometers for measuring total energy of X-ray photons. Rev. Sci. Instr. 25, 746 (1954). 1136.

Total-absorption X-ray spectrometry, application to betatron experiments. Nucleonics 12, 51 (1954). 1213.

Koch, H. W., Fuller, E. G., Hayward, E., Shape of the high-energy end of the electron bremsstrahlung spectrum. Phys. Rev. 109, 630 (1958). 2755.

Koch H. W., Hart, E. J., Petree, B., Schulman, J. H., Taimuty, S. I., Wyckoff, H. O., Measurement systems for high-level dosimetry. 2d United Nations Intern. Conf. on the Peaceful Uses of Atomic Energy, Vol. 21, Health and Safety: Dosimetry and Standards, P/1927 USA, p. 188 (Pergamon Press, London, England) (1959). 2984.

Koch, H. W., Johnston, R. W., Editors, Multichannel pulse height analyzers. Natl. Acad. Sci.-Natl. Research Council Publ. 467 (1957). 2206.

$K o c h, H$. W., Starfelt, N., Differential cross-section measurements of thin-target bremsstrahlung produced by 2.7- to $9.7-\mathrm{Mev}$ electrons. Phys. Rev. 102, 1598 (1956). 1676.

Koch, H. W., Wyckoff, J. M., Response functions of total-absorption spectrometers. Reprint IRE Trans. Nuclear Sci. NS-5, No. 3, 127 (1958). 2740

$K o c h, H . W$. Zendle, $B$., Dose measurements in water using scintillation and ionization detectors. Radiation Research 1, No. 6, 568A (1954). 995.

Koch, H. W., Zendle, B., McElhinney, J., Boag, J. W., Studies of dose distributions in water for betatron X-rays to 37 Mev. Radiol. Research 5, No. 2, 107 (1956). 1904.

Koch, J. W. Modulation studies for VHF ionospheric scattering. Record of Natl. Symp. on Extended Range and Space Communications, October 6-7, 1958 (Washington, D.C.), p. 114 (1958). $2639 \mathrm{~A}$. Reception of space diversity transmitters. Wireless World (England) 65, No. 10, 512 (1959). 3033.

Koch, $J . W .$, Harding, $W . B .$, Jansen, R. J., Fading rate recorder for propagation research. Electronics 32, No. 51, 78 (1959). 2949.

Koenig, J. F., A relative damping criterion for linear systems. 'Applications and Industry, No. 9, 291 (1953); Trans. Am. Inst. Elec. Engr. 72, Pt. 2, 291 (1953), 633.

On the zeros of polynominals and the degree of linear systems. J. Appl. Phys. 24, 476 (1953). 790.

Koester, C. J., Phase shift effects in Fabry-Perot interferometry. J 64A3, 191 (1960). 
Koidan, $W$., An acoustic method for the measurement of vibration amplitudes. J. Acoust. Soc. Am. 26, 428 (1954). 946.

The condenser microphone as a displacement detector calibrator. J. Acoust. Soc. Am. 29, No. 7, 813 (1957). 2348 .

Koidan, W., Corliss, E. L. R., Mechancial impedance of the forehead and mastoid. J. Acoust. Soc. Am. 27, No. 6, 1164 (1955). 1404

Koller, H. R., Marden, E., Pfeffer, H., The Haystaq system: past, present and future. Preprinted in Proc. Intern. Conf. on Scientific Info. November 11-16, 1958 (American Documentation Inst., Washington, D.C.) Area 5, p. 317 (1958).

Konecnik, M., Weitzel, D. H., Blake, J. H., Flow conversion kinetics of ortho and parahydrogen. Proc. 1958 Cryogenic Engineering Conference, Paper E-2, p. 286. Edited by K. D. Timmerhaus, Chemical Engineering Dept., University of Colorado (Boulder, Colorado) (1959). 2953.

Koo, B. Y.-C., Katzin, $M$., An exact earth-flattening procedure in propagation around a sphere. J 64D 1 , $61(1960)$

Koo, B. Y.-C., Katzin, M., Pezzner, H., Larson, J. V., Katzin, $J$. C., The trade-wind inversion as a transoceanic duct. J 64D3, 247 (1960).

Kortman, C. M., Shaull, J. M., Comparing outputs from precision time standards. Electronies 24, No, 4, 102 (1951). 209.

Kosthowski, H. J., Rapport sur l'etalonnage de deux lampes etalons secondaires a ruban de tunstene par quatre laboratories nationaux. Proces-Verbaux Comite Consultatif Thermometrie, aupres du Comite International de Poids et Mesures (Paris, France) 5, 133 (1958), 2716.

Kostkowski, H. J., Bass, A. M., Slit function effects in the direct measurement of absorption line half-widths and intensities. J. Opt. Soc. Am. 46, No. 12, 1060 (1956). 1867.

Kostkowski, H.J., Broida, H. P., Experimental evidence for the existance of abnormal $\mathrm{OH}$ rotational "temperatures" in low pressure flames. J. Chem. Phys. 23, 754 (1955). 1339.

Experimental proof for the existence of nonthermal rotational distribution of $\mathrm{OH}\left({ }^{2} \Sigma^{+}\right)$in flames. J. Chem. Phys. 25, No. 4, 676 (1956). 1709. Spectral absorption method for determining population "temperatures" in hot gases. J. Opt. Soc. Am. 46, No. 4, 246 (1956). 1882 .

Kostkowski, H. J., Kaplan, L. D., Absolute intensities of the 721 and $742 \mathrm{~cm}^{-1}$ bands' of $\mathrm{CO}_{2}$. J. Chem. Phys. 26, 1252 (1957). 2007.

Kostkowski, H. J., Plyler, E. K., Optical systems for increasing the available intensity from flames. J. Opt. Soc. Am. 42, 360 (1952). 506.

Kotter, F. R., Electrical measurements-in the core curriculum. Proc. 3d Natl. Conf. on Analog and Digital Instrumentation, April 20-21, 1959, Philadelphia, Pennsylvania (Sponsored by Recording and Controlling Instrumentation Committee of AIEE). Am. Inst. Elec. Engrs. p. 15 (1959). 2935.

Kotter, F. R., McGregor, M. C., Hersh, J. F., Cutkowsky, R. D., Harris, F. K., New apparatus at the National Bureau of Standards for absolute capacitance measurement. IRE Trans. Instrumentation I-7, No. 3-4, 253 (1958). 2647.

Kovasznay, L. S., Development of turbulence-measuring equipment. Natl. Advisory Comm. Aeronaut. Tech. Note 2839 (1953). 686.

Kovasznay, L. S., Joseph, H. M., Processing of twodimensional patterns by scanning techniques. Science 118, 475 (1953). 802.

Kowkabany, G. N., Isbell, H. S., Frush, H. L., Bruckner, B. H., Wampler, G., Mieromethylation of polysaccharides. Anal. Chem. 29, 1523 (1957). 2198.

Kraichman, $M$. B., Basic experimental studies of the magnetic field from electromagnetic sources immersed in a semi-infinite conducting medium. J 64D 1, 21 (1960).
Krasny, J.F., Schiefer, H. F., Appel, W. D., Richey, G. G., Impact properties of yarns made from different fibers. Textile Research J. 23, 489 (1953). 734.

Kraus, O., Nuclear magnetic resonance in semiconductors. Phys. and Chem. of Solids (Pergamon Press, London, England) 8, 504 (1959). 3000.

Krauss, $M$,, Electronic structure of some diatomic hydrides. J. Chem. Phys. 28, 1021 (1958). 2543.

Krauss, M., Kropf, A., Vibrational structure in the ionization efficiency curves of hydrogen and deuterium molecules. J. Chem. Phys. 26, 1776 (1957). 2410.

Krauss, M., Reese, R. M., Dibeler, V. H., Multiple ionization of rare gases by electron impact. J 63A3, 201 (1959).

Krider, H. S., Hidnert, P., Tables 142 to 144, thermal expansion. 9th edition of the Smithsonian Phys. Tables (Smithsonian Institution, Washington, D.C.) (1954). 1160.

Tables $4 \mathrm{f}-1$ through $4 \mathrm{f}-6$ on thermal expansion. Am. Inst. Phys. Handbook (MeGraw-Hill Book Co., Inc., New York, N.Y.) (1957). 2336.

Krieger, L. A., Armstrong, G. T., Wong, P. K., Use of a direct current amplifier and recorder to balance a Mueller resistance bridge. Rev. Sci. Instr. 30, 339 (1959). 3107.

Kroll, $W . D$., Aerodynamic heating and fatigue. NASA Memorandum 6-4-59W (1959). 2889.

Effect of rib flexibility on the vibration modes of a delta-wing aircraft. J 60, 335 (1958) RP2852.

Kronenberg, $M$. L., Ogburn, F., Salmon, $H$. I., Electrolytic coatings on magnesium base alloys from alkaline chromate solutions. Plating 42, 271 (1955). 1332 .

Kropf, A., Krauss, $M$., Vibrational structure in the ionization efficiency curves of hydrogen and deuterium molecules. J. Chem. Phys. 26, 1776 (1957). 2410 .

Kropschot, R. H., McClintork, R. M., Van Gundy, D. A., Mechanical properties of some engineering materials. Proc. 1956 Cryogenic Engineering Conference, Paper C-2, p. 93 . Edited by K. D. Timmerhaus, Chemical Engineering Dept., University of Colorado (Boulder, Colorado) (1957). 2193.

Kropschot, R. H., Mikesell, R. P., An experimental study of the strength and fatigue of glass at very low temperatures. Proc. 1956 Cryogenic Engineering Conference, Paper D-5, p. 136. Edited by K. D. Timmerhaus, Chemical Engineering Dept. University of Colorado (Boulder, Colorado) (1957). 2014.

Strength and fatigue of glass at very low temperatures. J. Appl. Phys. 28, 610 (1957). 2318.

Krouskop, N. C., Glasgow, A. R., Rossini, F. D., Apparatus for determining treezing points at saturation pressure from time-temperature freezing and melting experiments. Anal. Chem. 22, 1521 (1950). 26.

Kruger, J., Corrosion research at the National Bureau of Standards. J. Ind. Eng. Chem. 50, 55A (1958). 2503.

Effect of illumination on the oxidation of copper single erystals in water. J. Appl. Phys. 28, No. 10, 1212 (1957). 2097.

Krumbein, W. C., Lieblein, J., Geological application of extreme-value methods to interpretation of cobbles and boulders in gravel deposits. Trans. Am. Geophys. Union 37, No. 3, 313 (1956). 1723.

Krumrine, E. M., Hubbard, D., Jenkins, L. B., Amelung glasses compared with some modern commercial glasses. Sei. Mo. LXXV, No. 6, 327 (1952). 387.

Krumrine, E. M., Hubbard, D., Stair, R., Australite (meteoric) glass. Trans. Am. Geophys. Union 37, No. 6, 767 (1956). 1636.

Kruskal, J. B., Aumann, R. J., The coefficient in an allocation problem. Naval Research Logistics Quart. 5, 111 (1958). 2808.

Kruskal, J. B., Hoffman, A. J., Integral boundary points of convex polyhedra. Ann. Math. Studies No. 38,223 (1956). 1743. 
Kryder, S. J., Birnbaum, G., Lyons, H., Microwave measurements of the dielectric properties of gases. J. Appl. Phys. 22, No. 1, 95 (1951). 259.

Kryder, S. J., Maryott, A. A., Dipole moment of perchloryl fluoride. J. Chem. Phys. 27, No. 5. 1221 (1957). 2089.

Krynitsky, A. I., Stern, H., Experimental production of nodular graphite in cast iron. Foundry 80, No. 3,$106 ;$ Pt. 2, 80, No.4, 98 (1952). 444.

Kuder, M. L.. A broadband, low-level, error-voltage detector. Rev. Sei. Instr. 25, No. 5, 464 (1954). 914.

Electron-tube curve generator. Electronics 25, No. $3,118(1952)$. 440.

Kuder, M. L., Hammond, H. K., III, Holford, W. L., Ratio-recording spectroradiometer. J 64C2, 151 (1960).

Kuchner, E. C., Leslie, R. T., Review of analytical distillation. Anal. Chem. 30, 629 (1958). 2744.

Kuentzel, L. E., Schoen, L. J., Broida, H. P., Glass dewars for optical studies at low temperatures. Rev. Sci. Instr. 29, No. 7, 633 (1958). 2575.

$K u h n, H$. W., Contractibility and convexity. Proc. Am. Math. Soc. 5, No. 5, 777 (1954). 978.

Solvability and consistency for linear equations and inequalities. Am. Math. Mo. 63, 217 (1956). 1869.

$K u h n, H$. W., Hoffman, A. J., On systems of distinct representatives. Ann. Math. Studies No. 38 (1956). 1793.

Systems of distinct representatives and linear programming. Am. Math. Mo. 63, 455 (1956). 1910.

Kumpula, $J . W .$, Hudson, $D$. C., Ionization chambers for radiation data during dental X-ray exposure. U.S. Armed Forces Med. J. 6, No. 8, 1131 (1955). 1380.

Kumpula, J. W., Hudson, D. C., Dickson, G., A panoramic X-ray dental machine. U.S. Armed Forces Med. J. 8, No. 1, 46 (1957) 1993.

Kumpula, J. W., Nelsen, R.J., Pelander, C. E., Hydraulic turbine contra-angle handpiece. J. Am. Dental Assoc. 47, 324 (1953). 732.

Kumpula, J. W., Stanford, J. W., Pafjenbarger, G. C., Sweeney, W.T., Determination of some compressive properties of human enamel and dentin. J. Am. Dental Assoc. 57, 487 (1958). 2518.

Kunkel, W. B., Analysis of ionic recombination including ion production during measurement. Phys. Rev. 84, 218 (1951). 193.

Kurzweg, U. H., Bass, A. M., Broida, H. P., Spectra of afterglows and discharges from nitrogen-oxygen mixtures. J. Mol. Spectroscopy 1, 184 (1957). 2307.

Kushner, L. M., Hoffman, J. I., Synthetic detergents. Sci. Am. 185, No. 4, 26 (1951). 318

Kushner, L. M., Hubbard, W. D., Light scattering and micelle structure in the system sodium dodecyl sulfate-sodium chloride-water. J. Colloid Sci. 10, No. 4, 428 (1955). 1386 .

On the determination of critical micelle concentrations by a bubble pressure method. J. Phys. Chem. 57, 898 (1953). 787.

Viscometric and turbidimetric measurements on dilute aqueous solutions of a nonionic detergent. J. Phys. Chem. 58, 1163 (1954). 1225.

Kushner, L. M., Hubbard, W. D., Doan, A., Light scattering measurements of a fractionated nonionic detergent. J. Phys. Chem. 61, 371 (1957). 2176.

Kushner, L. M., Hubbard, W. D., Parker, R. A., Turbidity and viscosity measurements on some cationic detergents in water and in sodium chloride solutions. J $\mathbf{5 9}$, 113 (1957) RP2778

Kushner, L. M., Parker, R. A., On the low rates of equilibration and dialysis experiments with ionic surfaceactive agents. J. Phys. Chem. 61, 822 (1957). 2232.

\section{$\mathbf{L}$}

Labrie, R. J., Senderoff, S., Electrolytic preparation of molybdenum from fused salts, IV. Preparation of reduced molybdenum chlorides from molybdenite concentrate. J. Electrochem. Soc. 102, 77 (1955). 1333.

Lagoda, H., Wiener, $M$., Background eradication in thicklayered emulsions. Rev. Sci. Instr. 21, No. 1, 39 (1950). 30 .

Laki, K., Mandelkern, L., Posner, A. S., Diorio, A. F., Mechanism of contraction in the muscle fibre - ATP system. Proc. Natl. Acad. Sci. 45, 814 (1959). 2986.

Lalos, G. T., Broida, H. P., Rotational temperature of $\mathrm{OH}$ in several flames. J. Chem. Phys. 20, No. 9, 1466 (1952). 534.

Lalos, G. T., Corruccini, R. J., Broida, H. $P_{\text {, }}$ Design and construction of a blackbody and its use in the calibration of a grating spectroradiometer. Rev. Sci. Instr. 29, 505 (1958). 2513.

Lam, D.G.,Jr., Wachtman, J.B., Jr. Young's modulus of various refractory materials as a function of temperature. J. Am. Ceram. Soc. 42, 254 (1959). 3114.

Lam, D. G., Jr., Wachtman, J. B., Jr., Tefft, W. E., Stinchfield, $R$. $P$., Elastic constants of synthetic single crystal corundum at room temperature. J 64A3, $213(1960)$.

Lamb, J. J., George, D. A., Baker, H. A., Sieffert, L. E., Impact strength of some thermosetting plastics at low temperatures. ASTM Bull. No. 181, 67 (1952). 464.

Lamb, J. J., Reinhart, F. W., Boor, L., Brown, C., Evaluation of the Boor-Quartermaster snag tester for coated fabrics and plastic films, ASTM Bull. No. 210,50 (1955). 1337.

Lamb, J. J., Slone, M. C., Reinhart, F. W., Properties of polymethyl alpha-chloroacrylate. Modern Plastics 29, No. 10, 109 (1952). 520.

Lamb, $V$. A., Metzger, W. H., Designing electroformed parts. Machine Design 25, 124 (1953). 683. Electroforming. Tool Engr. 33, 55 (1954). 1009.

Lamb, V. A., Young, J. P., Chromium plating of gun bores. Ann. Proc. Am. Electroplaters' Soc. 260 (1956). 1648.

Experimental plating of gun bores to retard erosion. (1960) TN46 (PB151405).

Lamkin, J. C., Berger, $M$. J., Sample calculations of gamma-ray penetration into shelters: contributions of sky shine and roof contamination. J 60, 109 (1958) RP2827.

Lamkin, J. C., Hubbell, J. H., Bach, R. L., Radiation field from a rectangular source. J 64C2, 121 (1960).

Lanczos, C., Chebyshev polynomials in the solution of large-scale linear systems. Proc. Assoc. Computing Machinery (Toronto, Canada), p. 124 (Assoc. for Computing Machinery, New York, N.Y.) (1952). 411.

Landsman, G. L., Skramstad, H. K., Control mechanisms. Handbook of Physics, Edited by E. U. Condon, and H. Odishaw, Chapter 9, Pt. 2, p. 2-69 (McGraw-Hill Book Co., Inc., New York, N.Y.) (1958). 2500.

Lang, S. M., Melting-point and phase-equilibrium determinations. High Temp. Technol. p. 409 (J. Wiley \& Sons, New York, N.Y., and Chapman and Hall, London, England) (1956). 1775.

Properties of high-temperature ceramics and cermets. Elasticity and density at room temperature. (1960) Mono. 6.

Lang, S. M., Geller, R. F., The construction and operation of thoria resistor-type furnaces. J. Am. Ceram. Soc. 24, 193 (1951). 328.

Lang, S. M., Knudsen, F. P., Some physical properties of high-density thorium oxide. J. Am. Ceram. Soc. 39, No. 12, 415 (1956). 1877.

Langdell, R. D., Rothman, S., Adelson, E., Schwebel, A., Adsorption of $\mathrm{C}^{14}$ dextran to human blood platelets and red blood cells in vitro. Vox Sanguinis 2, 104 (1957). 2011.

Lange, L. J., Jean, A. G., Wait, J. R., Ionospheric reflection coefficients at VLF. Symp. on the Prop. of V.L.F. Radio Waves, Sponsored by the NBS Boulder Laboratories and IRE Prof. Group on Ant. and Prop. 
January 23-25, 1957 (Boulder, Colorado), Paper No. 31 , V. 3 (1957). 2172 .

Polarization of sferies. Symp. on the Prop. of V.L.F. Radio Waves, Sponsored by the NBS Boulder Laboratories and IRE Prof. Group on Ant. and Prop., January 23-25, 1957 (Boulder, Colorado), Abstracts, Paper No. 32, V. 3.2257.

Lange, L. J., Taylor, W. L., Some characteristics of VLF propagation using atmospheric waveforms. In Recent Advances in Atmospheric Electricity; Proc. 2d Conf. on Atmospheric Electricity, Portsmouth, New Hampshire, May 20-23, 1958 (Pergamon Press, New York, N.Y.), p. 609 (1958). 2760.

Larsen, T., Knudsen, $H$. L., The electric field at the ground plane near a top-loaded monopole antenna with special regard to electrically small $L$ - and $T$ antennas. J 64D2, 139 (1960).

Larson, J. V., Katzin, M., Pezzner, H., Katzin, J. C., The trade-wind inversion as a transoceanic duct. J 64D3, 247 (1960).

Larson, R. R., Birnbaum, G., Bussey, H. E., The microwave measurement of variations in atmospheric refractive index. IRE Trans. Ant. Prop. AP-3, 74 (1952). 585.

Lashof, T. W., Macurdy, L. B., Testing a quick-weighing balance. Anal. Chem. 26, 707 (1954). 1168.

Lashof, T. W., Macurdy, L. B., Alber, H. K., BenedettiPichler, A. A., Carmichael, H., Corwin, A. H., Fowler, R. M., Huffman, E. W. D., Kirk, P. L., Terminology for describing the performance of analytical and other precise balances. Anal. Chem. 26, 1190 (1954). 1166 .

Lashof, T. W., Mandel, J., Worthington, $V$., Use of the sensitivity criterion for the comparison of the Bekk and Sheffield smoothness testers. Tappi 39, No. 7, 532 (1956). 1970.

Laufer, $J$., The structure of turbulence in fully developed pipe flow. Natl. Advisory Comm. Aeronaut. Tech. Note 2954 (1953), 884.

Laufer, J., Nimeroff, I., Spectral band-pass determinations by a dynamic approach. J. Opt. Soc. Am. 48, $864(1958), 2770$.

Laughlin, $R$. D., Decay constant in vibrating systems. Radio-Electronic Eng. Sect., Radio and Television News 49, 11 (1953). 678.

Launer, $H$. F., Wilson, $W . K$., Preparing cuprammonium solvent and cellulose solutions. Anal. Chem. 22, 375 (1950). 112.

Launer, H. F., Wilson, W. K., Flynn, J. H., Determination of glucose by means of sodium chlorite. Indian Pulp and Paper 9, 399 (1955). 1310.

Laurie, $V$. $W$., Comments on the structure of 1,1,1trifluoro-2-butyne. J. Chem. Phys. Letter 30, 1101 (1959). 2911.

Microwave spectrum of methyl germane. J. Chem. Phys. 30, 1210 (1959). 2992.

Note on the determination of molecular structure from spectroscopic data. J. Chem. Phys. 28, 704 (1958). 2651 .

Laurie, V. W., Schwendeman, R. H., Tables of line strengths for rotational transitions of asymmetric rotor molecules. (Pergamon Press, London, England) (1958). 2196.

Lauritzen, J.I., Jr., An analysis of dielectric loss in two urea addition compounds. J. Chem. Phys. 28, No. 1, 118 (1958). 2454 .

Lauritzen, $J . I ., J r ., H o f f m a n, J . D$., Theory of formation of polymer crystals with folded chains in dilute solution. J 64A1, 73 (1960).

Law, C. A., Craig, D. N., Hamer, W. J., Stability of silver and pyrex in perchloric acid-silver perchlorate solutions and in conductivity water. J 64A1, 127 (1960).

Lawrence, $R$. S., An investigation of the perturbations impassed upon radio waves penetrating the ionosphere. Proc. IRE 46, 1 (1958). 2458.
Continental maps of four ionospheric disturbances. J. Geophys. Research 58, 219 (1953). 675 .

Radio observations of interstellar neutral hydrogen clouds. Astrophys. J. 123, No. 1, 30 (1956). 1836. Lawrence, R. S., Bok, B. J., Menon, T. K., Radio observations (al-CM) of dense dark nebulae. Publ. Astron. Soc. Pacific 67, No. 395, 108 (1955). 1468.

Lawrence, $R$. S., Jesperson, $J$. L., A preliminary analysis of amplitude scintillations of radio stars observed at Boulder, Colorado. (1959) TN20 (PB151379).

Lawrence, R. S., Menzel, D. H., Warwick, J. W., A dynamic spectrum analyzer for Sacramento Peak. Harvard College Observatory (Cambridge, Mass.). Scientific Report 20 (1955). 1233.

Lawrence, $R$. S. Storey, L. R. O., A radio-astronomical test of the ballistic theory of light emission. Correspondence to Editor, Observatory 79, No. 911, 150 (1959). 2878.

Lawson, $D$. B., Increased rate of liquefaction of the National Bureau of Standards helium liquefier. Rev. Sci. Instr. 28, 204 (1957), 2157.

Lawson, M. E., Jr., Dickson, G., Forziati, A. F., Fluorescence of teeth reveals structure. N.Y. J. Dentistry 23, 299 (1953). 721.

Lawson, M. E., Jr., Dickson, G., Forziati, A. F., Schoonover, I. C., Fluorescence of teeth: a means of investigating their structure. J. Am. Dental Assoc. 45, 661 $(1952), 452$.

Lawson, M. E., Jr., Forziati, A. F., Losee, F. L., Pigman, $W$. W., Jennings, $W . H$. , In vitro production of experimental enamel caries. J. Am. Dental Assoc. 53, 655 (1956). 1734.

Lawson, M. E., Jr., Losee, F. L., Jennings, W. H., Microstructure of the human tooth. A. The dentioenamel junction. J. Dental Research 36, No. 6, 911 (1957). 2190.

Lawson, M. E., Jr., Rubb, N. W., Dickson, G., Sweeney, $W . T$., A method for measuring the mucosal surface contours of impressions, casts and dentures. J. Am. Dental Assoc. 54, 24 (1957). 1987.

Lazaro, E. J., Rose, J. C., Broida, H. P., Dynamies of complete right ventricular failure in dogs maintained with an extracorporeal left ventricle. J. Am. Heart Assoc. 4, No. 2, 173 (1956). 1685.

Leaderman, $H$., Approximations in linear viscoelasticity theory: delta-function approximations. J. Appl. Phys. 25, 294 (1954). 956.

Mechanical properties of high polymers. Ann. Rev. Phys. Chem, 9, 179 (1958). 2630.

Nonlinear viscoelastic behavior of rubber in shear. J. Polymer. Sci. 16, No. 82, 261 (1955). 1419.

Proposed nomenclature for linear viscoelastic behavior. Trans. Soc. Rheology I, 312 (1957), 2271. Rheology of polyisobutylene, I. Theory and experimental procedures. J. Polymer. Sci. 13, 371 (1954). 1132 .

Rheology of polyisobutylene, IV. Calculation of the retardation time function and dynamic response from creep data. Proc. Second Intern. Congress on Rheology, edited by V. G. W. Harrison, (Butterworth's Scientific Publications, London, England) (1954). 1132 .

Viscoelastic phenomena in amorphous high polymeric systems. Chapter 1, p. 1, Vol. II. Rheologytheory and application (Academic Press, Inc., New York, N.Y.) (1958). 2863.

Leaderman, $H_{\text {, }}$ Marvin, $R$. S., Dynamic compliance dynamic modulus, and equivalent Voight and Maxwell models for polyisobutylene. J. Appl. Phys. 24, 812 (1953). 692 .

Leaderman, H., Smith, R. G., Jones, R. W., Rheology of polyisobutylene. II. Low molecular weight polymers. J. Polymer Sci. 14, 47 (1954). 1132.

Leaderman, H., Smith, R. G., Williams, L. C., Rheology of polyisobutylene. III. Elastic recovery, nonNewtonian flow, and molecular weight distribution. J. Polymer Sci. 36, 233 (1959), 3037. 
Leder, L. B., Electron characteristic energy losses in metals and compounds. Phys. Rev. 103, No. 6, 1721 (1956). 1699.

Inelastic scattering of 20-kev electrons in metal vapors. Phys. Rev. 107, No. 6, 1569 (1957). 2158.

Leder, $\dot{L}$. B., Marton, L., Effect of chemical combination of the characteristic energy loss of electrons. Phys. Rev. 95, 1345 (1954). 997.

Energy loss of electrons in passage through thin films. Phys. Rev, 94, 203 (1954). 1014.

Temperature dependence of the characteristic energy loss of electons in aluminum. Phys. Rev. 112, No.2, 341 (1958), 2798.

Leder, L. B., Marton, L., Coleman, J. W., Schubert, D. C., Electron beam magnetometer. J 63C1, 69 (1959).

Leder, L. B., Marton, L., Mendlowitz, H., Characteristic energy losses of electrons in solids. Advances in Electronics and Electron Phys, 7, 183 (1955). 1287.

Leder, L. B., Marton, L., Mendlowitz, H., Simpson, J. A., Marton, $C$., Energy losses of electrons and their influence on resolving power. Proc. of the Toulouse Conf., Centre National de la Richerce Scientifique (Paris, France), p. 175 (1955). 1335.

Leder, L. B., Mendlowitz, H., Marton, L., Comparison of the characteristic energy losses of electrons with the fine structure of the X-ray absorption spectra, Phys. Rev. 101, No. 5, 1460 (1956). 1655.

Leder, L. B., Simpson, J. A., Improved electrical differentiation of retarding potential measurements. Rev. Sci. Instr. 29, No. 7, 571 (1958). 2596.

Ledley, $R$. S., Mathematical foundations and computational methods for a digital logic machine. J. Operations Research Soc. Am. 2, No. 3, 249 (1954). 1061.

The relation of occlusal surfaces to the stability of artificial dentures. J. Am. Dental Assoc. 48, 508 (1954). 1194.

Lee, A. A., Bouche, R. R., Ballard, C. R., On, F. J., Windinduced vibration of telephone and distribution conductors. Am. Inst. Elec. Engrs. Middle Eastern District Meeting Conf. Paper (April 1958), 2868.

Lee, T. G., McCamy, C. S., Shoub, H., Fire extinguishment by means of dry powder. Proc. 6th Symp. (International) on Combustion (Reinhold Publ. Corp., New York, N.Y.), p. 795 (1957). 2123.

Lee, W. M., Newman, S. B., Surface tension measurements with a strain-gauge-type testing machine. Rev. Sci. Instr. 29, 785 (19F8). 2794.

Lefert, H. G., Richmond, J. C., Williams, C. N., Harrison, $W$. N., Ceramic coatings for nuclear reactors (a progress report). J. Am. Ceram. Soc. 38, No. 2, 72 (1955). 1285.

Legate, A. C., Mordfin, L., Creep behavior of structural joints of aircraft materials under constant loads and temperatures. Natl. Advisory Comm. Aeronaut. Tech. Note 3842 (1957). 2071.

Lehmer, $D$. $H$., The sieve problem for all-purpose computers. Math. Tables and Other Aids to Computation 7, 6 (1953). 882

Leibfritz, W. A., Taylor, D. F., Adler, A. G., Physical properties of chromium cobalt alloys. J. Am. Dental Assoc. 56, 343 (1958). 2687.

Liebler, R. A., Forsythe, G. E., Matrix inversion by a Monte Carlo method. Math. Tables and Other Aids to Computation 4, No. 31, $127(1950), 84$.

Leighton, H. I., Field-strength variations recorded on a VHF ionospheric scatter circuit during the solar event of Feb. 23, 1956 . J. Geophys. Research Letter to Editor 62, 483 (1957). 2121.

Leighton, H. I., Billings, D. E., Solar $\mathrm{H}_{\alpha}$ filaments and geomagnetic disturbances. J. Atmospheric and Terrest. Phys. 7, 349 (1955). 1489.

Leighton, H. I., Ellyett, C. D., Solar cycle influence on the lower ionosphere and on VHF forward scatter. Proc. IRE 46, No. 10, 1711 (1958). 2759.

Leinbach, H., Litle, C. G., The riometer-a device for the continuous measurement of ionospheric absorption. Proc. IRE 47, No. 2, 315 (1959). 3091.
Leiner, A. L., Problems of logical design. Proc. Convention on Digital Techniques, Inst. Elec. Engrs., (London, England) (1956). 1818.

Leiner, A. L., Notz, W. A., Smith, J. L., Weinberger, A., Organizing a network of computers to meet deadlines. 1957 Eastern Joint Computer Conf. Proc. (Washington, D.C.), p. 115 (1957). 2240.

PILOT, a new multiple computer system. J. Assoc. Computing Mach. 6, No. 3, 313 (1959). 3016.

PILOT, the NBS multicomputer system. Proc. Eastern Joint Computer Conf. (Philadelphia, Pennsylvania), p. 164 (1958). 2692.

Leiss, J. E., Calculated behavior of a fast neutron spectrometer based on the total absorption principle. (1959) TN10 (PB151369).

Conference on photonuclear reactions. Phys. Today 11, No. 9,18 (1958). 2497.

Photoproduction of neutral mesons from carbon near threshold. Am. Phys. Soc. Ser. II, No. 2, 6 (1957). 2250 .

Leiss, J. E., Penfold, $A, S$, Analysis of photocross sections. Physics Dept. Rept. (University of Ill., Champaign, Ill.) (1958), 2460.

Leiss, J. E., Penner, S., Robinson, C. S., Range straggling of high-energy electrons in carbon. Phys. Rev, 107, 1544 (1957). 2279.

Leiss, J. E., Schrack, R. A., Angular distribution in neutral meson decay. Phys. Rev. 109, 1326 (1958). 2461.

Nuclear matter distributions from coherent neutral pion production. Rev. Modern Phys. 30, No. 2, 456 (1958). 2653.

LeLevier, $R$. E., Saxon, D. S. An optical model for nucleon-nuclei scattering. Phys. Rev. 87, 40 (1952). 395 .

Lengel, $J . H$. ., Dibeler, $V . H$. , Preparation of small quantities of germanium tetramethyl. J. Am. Chem. Soc. 74,2683 (1952). 517

Leser, W., Kanagy, J. R., Randall, E. B., Carter, T. J., Mann, $C$., Influence of splitting on the strength of chrome-tanned steer hides. J. Am. Leather Chemists Assoc. 47, 329 (1952). 468.

Leslie, R. T., Kuehner, E. C., Review of analytical distillation. Anal. Chem. 30, 629 (1958). 2744.

Levedahl, $W . J ., \quad$ Multistage autoignition of engine fuels. Fifth Symposium (International) on Combustion (Reinhold Publ. Corp., New York, N.Y.), p. 372 (1955). 1411.

Levedahl, W. J., Beckers, A., Mechanism of autoignition in benzene-air mixtures. Ind. Eng. Chem. 48, 411 (1956). 1773.

Levedahl, W. J., Broida, H. P., Emission spectra on autoignited heptane-air mixtures. Anal. Chem. 24, 1776 (1952). 442.

Levedahl, W. J., Broida, H. P., Howard, F. L., The emission spectra of first-stage combustion in an engine. J. Chem. Phys. 19, 797 (1951). 333.

Levedahl, W. J., Howard, F. L., Two-stage autoignition of some hydrocarbons. Ind. Eng. Chem. 43, 2805 (1951) 354 .

Levin, B. M., Newman, A. E., Distribution of incoming lettermail at the Baltimore, Maryland City Post Office. (1959) TN33 (PB151392).

Levin, E., Distribution of phase diagrams of interest to ceramists. Bull. Am. Ceram. Soc. 34, No. 12, 399 (1955). 1318.

Indentation pressure of a smooth circular punch. Quart. Appl. Math. 13, No. 2, 133 (1955). 1363.

Note on the circle theorem of hydrodynamics. Quart. Appl. Math. 12, No. 3, 315 (1954). 1084.

Levin, E. M., Block, S., Structural interpretation of immiscibility in oxide systems: 1. Analysis and calculation of immiscibility. J. Am. Ceram. Soc. 40, No. 3, 95 (1957). 2320 .

Structural interpretation of immiscibility in oxide systems: 2. Coordination principles applied to immiscibility. J. Am. Ceram. Soc. 40, No. 4, 113 (1957). $2320 \mathrm{~A}$. 
Structural interpretation of immiscibility in oxide systems: 3. Effect of alkalis and alumina in ternary systems. J. Am. Ceram. Soc. 41, No. 2, 49 (1958). 2785 .

Levin, E. M., Cleek, G. W., Shape of liquid immiscibility volume in the system barium oxide-boric oxide-silica. J. Am. Ceram. Soc. 41, No. 5, 175 (1958). 2754.

Levin, E. M., McMurdie, H. F., Hall, F. P., Compilation of phase diagrams of interest to ceramists. Book, Phase of Ceramists (Am. Ceram. Soc.) (1956). 1656.

Levin, E. M., Robbins, C. R., The system magnesium oxide-germanium dioxide. Am. J. Sci. 257, 63 (1959). 3094.

Levin, E. M., Roth, R. S., Phase equilibria in the subsystem barium disilicate dibarium trisilicate. J 62 , 193 (1959) RP2953.

Polymorphism in barium disilicate. Am. Mineralogist 44, No. 3-4, 452 (1959). 3018.

Levin, J., Construction and use of subroutines for the SEAC. Proc. Assoc. Computing Machinery, Jointly Sponsored by the Assoc. for Computing Machinery and the Mellon Institute, Pittsburgh, Pennsylvania, May 2-3, 1952, p. 173 (Assoc. for Computing Machinery, New York, N.X.) (1952). 416.

Levine, D. B., Her zfeld, C. M., Effect of crystal field and spin-orbit coupling on magnetic susceptibility of systems with $f^{2}$ electron configuration. J 61, 117 (1958) RP2893.

Levinos, S., Ehrlich, M., Stabilization film processing for $\mathrm{X}$ - and $\gamma$-ray dosimetry. Nucleonics 14, No. 7, 72 (1956) 1891 .

Levy, S., Determination of loads in the presence of thermal stresses. J. Aeronaut. Sci. 21, No. 10, 659 (1954). 988.

Influence coefficients of tapered cantilever beams computed on SEAC. Am. Soc. Mech. Engrs. Paper No. 52-A-25 (1952). 467; Trans. Am. Soc. Testing Materials 20, 1 (1953). 738.

Recent developments in the Ramberg vacuum tube acceleramoter. Proc. Soc. Exptl. Stress Anal. 9, No. 1,151 (1951). 297

Structural analysis and influence coefficients for delta wings. J. Aeronaut. Sci. 20, No. 7, 449 (1953). 840. Thermal stresses and deformation in beams. Aeronaut. Eng. Rev, 15, 10 (1956), 1962.

Levy, S., Cahill, W. F. Computation of vibration modes and frequencies on SEAC. J. Aeronaut. Sci. 22, No. 12,837 (1955). 1293.

Lewis, W. B., Astin, A. V., and Bullart, E. C., International comparisons of radioactivity standards. Science 123, 895 (1956). 1746.

Liberatore, L. C., Wilson, R. E., Aging changes in clinical thermometers. J. Am. Ceram. Soc. 35, 203 (1952). 385.

Lide, D. R., Jr. Effects of internal motion in the microwave spectrum of methylamine. J. Chem. Phys. 22, 1613 (1954). 1001.

Internal barrier in ethane. J. Chem. Phys. Note 29, 1426 (1958). 2603.

Microwave spectra of molecules exhibiting internal rotation. $V$. Barrier height in ethylchloride and ethyl bromide. J. Chem. Phys. 30, 37 (1959). 2991. Microwave spectrum of benzonitrils. J. Chem. Phys. 22, 1577 (1954). 1075.

Structure of the methylamine molecule. I. Microwave spectrum of $\mathrm{CD}_{3} \mathrm{ND}_{2}$. J. Chem. Phys. 27, 343 (1957). 2322.

Lide, D. R., Jr., Kivelson, D., Internal rotation in methyltrifluoromethyl acetylene. J. Chem. Phys. 23, 2191 (1955). 1374

Structure of the methylamine molecule, II. Theory of internal motions and application to $\mathrm{CD}_{3} \mathrm{NO}_{2}$. J. Chem. Phys. 27, 353 (1957). 2323.

Lide, D. R., Jr., Mann, D. E., Microwave spectra of molecules exhibiting internal rotation. I. Propylene. J. Chem. Phys. 27, 868 (1957); II. Methylallene. J. Chem. Phys. 27, 874 (1957). 2202.

Microwave spectra of molecules exhibiting internal ratation. III. Trimethylamine. J. Chem. Phys. 2y, 572 (1958). 2637.

Microwave spectra of molecules exhibiting internal ratation. IV. Isobutyane, tertiary butyl fluoride and trimethylphospine. J. Chem. Phys. 29, 914 (1958). 2638.

Search for the microwave spectrum of perchlorylfluoride. J. Chem. Phys. 25, 595 (1956). 1863.

The infrared spectrum and structure of perchlorylfluoride. J. Chem. Phys. 25, 1128 (1956). 1933.

Lide, D. R., Jr., Mann, D. E., Acquista, N., The infrared spectrum of tertiary butyl fluoride. J. Mol. Spectroscopy 2, 575 (1958), 2820.

Lide, D. R., Jr., Mann, D. E., Fristrom, R. M., The microwave spectrum and structure of sulfuryfluoride. J. Chem. Phys. 25, 734 (1957). 2372.

Lieblein, $J$., A new method of analyzing extreme-value data. Natl. Adv. Comm. Aero. Technical Note 3053 (1954). 922 .

On moments of order statistics from the Weibull distribution. Ann. Math. Stat. 26, No. 2, 330 (1955). 1428.

On the exact evaluation of the variances and covariances of order statistics in samples from the extremevalue distribution. Ann. Math. Stat. 24, No. 2 281 (1953). 788.

Two early papers on the relation between extreme values and tensile strength. Biometrika (London, England) 41, 559 (1954). 1219.

Lieblein, J., Gumbel, E. J., Some applications of extremevalue theory. Am. Stat. 8, 14 (1954). 1140.

Lieblein, J., Krumbein, W. C., Geological application of extreme-value methods to interpretation of cobbles and boulders in gravel deposits. Trans. Am. Geophys. Union 37 , No. 313 (1956). 1723.

Lieblein, J., Salzer, H. E., Table of the first moment of ranked extremes. J 59, 203 (1957) RP2787.

Liggett, $B$. W., Deitz, V.R., Color and turbidity of sugar products. Advances in Carbohydrate Chem. 9, 247 (1954). 970 .

Likins, R. C., Bavetta, L. A., Posner, A. S., Calcification in lysine deficiency. Arch, of Biochem, and Biophys. 70, 401 (1957). 2040.

Likins, R. C., Posner, A. S., Steere, A. C., Effect of calcium treatment on solubility and calcium uptake of synthetic hydroxyapatite and rat molar enamel. J. Am. Dental Assoc. 57, 335 (1958). 2528.

Lilley, C. M., Johler, J. R., Walters, L. C., Low- and very low-radiofrequency tables of ground wave parameters for the spherical earth theory: the roots of Riccati's differential equation. (Supplementary numerical data for C573). (1959) TN7 (PB151366).

Lincoln, J. V., McNish, A. G., Predictions of the present sunspot cycle. Trans. Am. Geophys. Union 35, 709 (1954). 112.

Lindberg, A. R., Johannesen, R. B., The temperatureindependent paramagnetism of ammonium hexabroosmate (IV). J. Am. Chem. Soc. 76, 5349 (1954). 1201.

Lindsay, R., Magnetic susceptibility of manganese solenide. Phys. Rev. 84, 569 (1951). 254.

Linnig, F. J., Mandel, J., Statistical methods in chemistry. Anal. Chem, 28, 770 (1956). 1896; Anal. Chem. 30, 739 (1958). 2779.

Study of accuracy in chemical analysis using linear calibration curves. Anal. Chem. 29, 743 (1957). 2328 .

Linnig, $F$. J., Mandel, J., Peterson, $J . M$., A plan for studying the accuracy and precision of an analytical procedure. Anal. Chem. 26, No. 7, 1102 (1954). 926.

Linnig, F. J., Milliken, L. T., Interference of antioxidant in the determination of low polymer in SBR synthetic rubber. J. Polymer Sci. XLI, No. 138, 544 (1959). 2967.

Linnig, F.J., Peterson, J. M., Edwards, D. M, Acherman, $W$. L., Chemical analysis of GR-S by complete solution procedures. Gross constituents in GR-S containing soap. Anal. Chem. 25, 1511 (1953). 666. 
Linnig, F. J., Schneider, A., Chemical analysis of GR-S by complete solution procedures. Titration of mineral and organic acids in toluene-ethanol solution. Anal. Chem. 25, 1515 (1953). 667.

Linnig, F. J., Stewart, J. E., A spectroscopic study of oils used in oil-extended rubber. J 59, 27 (1957) RP2771.

Infrared study of some structural changes in natural rubber during vulcanization. J 60, 9 (1958) RP2816.

Linnig, F. J., Tryon, M., Natural and synthetic rubbers: review of test methods. Anal. Chem. Appl. Revs. 31, Pt. II, No. 4, 767 (1959). 2997.

Lipkis, R. S., Use of subroutines on SWAC. Proc. Assoc. for Computing Machinery, p. 231 (1952). 614.

Lippincott, E. R., Van Valkenburg, A., Weir, C. E. Bunting, $E$. $\vec{N}$., Infrared studies on polymorphs of silicon dioxide and germanium dioxide. $\mathbf{J}$ 61, 61 (1958) RP2885.

Lippincott, E. R., Weir, C. E., Van Valkenburg, A., Bunting, $E$. N., Infrared studies in the 1- to 15-micron region to 30,000 atmospheres. J 63A 1, 55 (1959).

Lipson, H, G., Burstein, E., Davisson, J. W., Bell., E. E., Infrared photoconductivity due to neutral impurities in germanium. Phys. Rev. 93, 65 (1954). 1039.

Liss, R., Vacher, H. C., Mebs, R. W., Elastic and plastic behavior of the ferrite lattice in a low-alloy steel. Acta Met. 4, No. 5, 532 (1956). 1691.

Little, C. G., Kirby, R. C., Conference on Arctic communication. J 64D 1, 73 (1960).

Little, C. G., Leinbach, H., The riometer-a device for the continuous measurement of ionospheric absorption. Proc. IRE 47, No. 2, 315 (1959). 3091.

Little, R. E., McKinley, J. D., Jr., On-off temperature control for electrically heated filaments. Rev. Sci. Instr. 29, No. 12, 1143 (1958). 2660.

Livengood, J. C., Taylor, C. F., Tsai, D. H., Dynamics in the inlet system of a four-stroke single-cylinder engine. Trans. Am. Soc. Mech. Engrs. 77, No. 7, 1133 (1955), 1322

Lloyd, E. C., Hutton, U. C., Johnson, D. P., Compact Multi-anvil wedge type high-pressure apparatus. J 63C 1,59 (1959).

Lloyd, E. C., Wildhack, W. A., Dressler, R. F. Investigations of the properties of corrugated diaphragms. Am. Soc. Mech. Engrs. Preprint 55-A-181 (1955). 1378; Trans. Am. Soc. Mech. Engrs. 79, 65 (1957). 2166 .

Lloyd, $R$. L., The new safety code for portable metal ladders. Mag. of Standards 28, No. 4, 104 (1957). 2374.

Loberman, H., Weinberger, A., Formal procedures for connecting terminals with a minimum total wire length. J. Assoc. Computing Machinery 4, No. 4, 428 (1957). 2128.

Symbolic designations for electrical connections. J. Assoc. Computing Machinery 4, No. 4, 420 (1957). 2334.

Loebenstein, W. V., Direct nitrogen calibration of dead space in gas absorption systems. J. Chem. Phys. 29, No. 1, 236 (1958). 2524.

Precise evaluation of surface area with indirectly calculated dead space. J 60, 105 (1958) RP2826.

Loebenstein, W. V., Deitz, V. R., Oxygen chemisorption on carbon adsorbents. J. Phys. Chem. 59, 481 (1955). 1438.

Loebenstein, W. V., Weitzel, D. H., Draper, J. W., Park, $O$. $E$., Orthopara catalysis in liquid-hydrogen production. J 60, 221 (1958) RP2840.

Loftus, $J$., Gross, D., Flame spread properties of building finish materials. ASTM Bull. No. 230 (1958). 2565.

Loftus, J., Robertson, A. F., Gross, D., A method for measuring surface flammability of materials using a radiant energy source. Am. Soc. Testing Materials Proc. 56, 1437 (1956). 1593.

Logan, $H$. L., Mechanism of stress-corrosion cracking in the AZ31B magnesium alloy. J 61, 503 (1958) RP2919.

Studies of stress-corrosion cracking of Austenitic strainless steel. Research Suppl. Walting J. 37, 463S (1958). 2789 .

Logan, H. L., Sherman, R. J., $J_{r}$, Stress-corrosion cracking of type 304 austenitic stainless steel. Welding J. 35, No. 8, 389 (1956). 1898.

Logan, J. K., van Dijk, H., Durieux, M., Clement, J. R., The "1958 He $\mathrm{H}^{4}$ scale of temperatures", Part 2, Tables for the 1958 temperature scale. J 64A 1, 1 (1960); (1960) Mono. 10.

Lonberger, S. T., Roeser, W. F., Methods of testing thermocouples and thermocouple materials. (1958) C590.

London, $A_{\text {., }} \quad$ Determination of reverberant sound absorption coefficient from acoustic impedance measurement. J. Acoust. Soc. Am. 22, 263 (1950). 46.

Technical aspects of industrial and office quieting. Proc. Second Annual Natl. Noise Abatement Symp., Armour Research Foundation (Chicago, Illinois) 2, 75 (1951). 323.

Transmission of reverberant sound through double walls. J. Accoust. Soc. Am. 22, 270 (1950). 159.

Longley, A. G., Rice, P. L., Norton, K. A., Prediction of the cumulative distribution with time of ground wave and tropospheric wave transmission loss. Part 1. The prediction formula. (1959) TN15 (PB151374).

Longley, A. G., Williamson, $D$. A., Fuller, V. L., Rice, $P$. L., A summary of VHF and UHF tropospheric transmission loss data and their long-term variability. (1960) TN43 (PB151402).

Loos, B. D., Reinhart, F. W., DeLollis, N.J., Evaluation of adhesives for acoustical tile. ASTM Bull. No. $169,57(1950)$. 55.

Losee, F. L., Forziati, A. F., Pigman, W. W., Jennings, W. H., Lawson, M. E. Jr., In vitro production of experimental enamel caries. J. Am. Dental Assoc. 53,655 (1956). 1734 .

Losee, F. L., Jennings, W. H., Lawson, M.E., Jr., Microstructure of the human tooth. A. The dentioenamel junction. J. Dental Research 36, No. 6, 911 (1957). 2190.

Lowell, $P$. D., Hakkarinen, $W$. Allison, L. M., Mechanical stops for use with helical potentiometers. Rev. Sei. Instr. 25, No. 4, 398 (1954). 1070.

Lowrie, P. H., Jr., Rack for standard resistors. Rev. Sci. Instr. Letter to Editor 30, No. 4, 291 (1959). 3026 .

Lukacs, $E$., An essential property of the fourier transforms of distribution functions. Proc. Am. Math. Soc, 3, No. 3, 508 (1952). 391.

On strongly continuous stochastic processes. Sankhya 13, 219 (1954). 1095.

The stochastic independence of symmetric and homogeneous linear and quadratic statistics. Ann. Math. Stat. 23, No. 3, 442 (1952). 596.

Lukacs, E., King, E. P., A property of the normal distribution. Ann. Math. Stat. 25, No. 2, 389 (1954). 928.

Lukacs, E. Szasz, O., Certain fourier transforms of distributions. Can. J. Math. 3, No. 2, 140 (1951). 202.

Certain fourier transforms of distribution, II. Can. J. Math. 6, 186 (1954). 965.

On analytic characteristic functions. Pacific J. Math. 2, No. 4, 615 (1952). 496.

Lutes, O. S., Superconductivity of microscopic tin filaments. Phys. Rev. 105, No. 5, 1451 (1957). 2329.

Lutes, 0 . S., Broida, H. $P$., Abundance of free atoms in solid nitrogen condensed at $4.2^{\circ} \mathrm{K}$ from a gas discharge. J. Chem. Phys. 24, No. 2, 484 (1956). 1607.

Lutes, O. S., Maxwell, E., Superconducting transitions in tin whiskers. Phys. Rev. 97, No. 6, 1718 (1955). 1519 .

Threshold field properties of some superconductors. Phys. Rev. 95, 333 (1954). 1212.

Lutsky, M., Slawsky, M. M., Schneidlin, A. E., Pressure drop in pneumatic components and systems. Product Eng. Handbook No. 3, Section J, p. J2 (McGrawHill Book Co. Inc., New York, N.Y.) (1955). 1456. 
Lyon, H.W., Dickson, G., Schoonover, I. C., Effectiveness of vacuum investing in the elimination of surface defects in gold casting. J. Am. Dental Assoc. 46, 197 (1953). 697.

Mechanism of hygroscopic expansion of dental casting investments. J. Dental Research 34, 44 (1955). 1405.

Lyons, $H$., Microwave frequency dividers. J. Appl. Phys. 21, 59 (1950). 89.

Spectral lines as frequency standards. Ann. N.Y. Acad. Sci. 55, Art. 5, 831 (1952). 550.

The atomic clock, a universal standard of frequency and time. The American Scholar 19, 159 (1950). 148.

Lyons, H., Birnbaum, G., Kryder, S. J., Microwave measurements of the dielectric properties of gases. J. Appl. Phys. 22, No, 1, 95 (1951). 259.

Lyons, H., Nuckolls, R. G., Rueger, L. J., Microwave absorption spectrum of $\mathrm{ND}_{3}$. Phys. Rev. 89, 1101 (1953). 766 .

Lyons, H., Rueger, L. J., Nuckolls, R. G., A high temperature, broad-band Stark cell for microwave spectroscopy. Rev. Sci. Inst. 22, 428 (1951). 178.

Lyons, H., Rueger, L. J., Nuckolls, R. G., Kessler, M., Microwave spectra of deutero-ammonias. Phys. Rev. 81,630 (1951). 260.

\section{Mc}

McAuliff, R. C., Boone, T. H., Hermach, F. L., MacArthur' $E$. $H$., Conductive flooring for hospital operating rooms. J 63C2, 125 (1959); (1960) Mono. 11.

McBurney, $J$. W., American standard building code requirements for masonry. Mag. of Standards 25, No. 11, 358 (1954). 945 .

Cracking in masonry caused by expansion of mortar. Am. Soc. Testing Materials Proc. 52, 1228 (1952). 419.

Masonry cracking and damage caused by moisture expansion of clay tile. Am. Soc. Testing Materials Proc. 54, 1219 (1954). 1058.

Masonry requirements up to date. Standards $\mathbf{2 5}$, No. 11,358 (1954). 1059 .

The effects of weathering on certain mortars exposed in brick masonry with and without caps and flashings. Am. Soc. Testing Materials Proc. 56, 1273 (1956). 1927.

McBurney, J.W., Johnson, P. V., Durability of deaired brick. J. Am. Ceram. Soc. 39, No. 5, 159 (1956). 1683 .

McBurney, J. W., Richmond, J. C., Copeland, M. A., Relations among certain specification properties of building brick and effects of differences in raw materials and methods of forming. J. Am. Ceram. Soc. 35 , No. 12,309 (1952). 528 .

McCamy, $\dot{C}$. S., Survey of hazards of handling liquid oxygen. Ind. Eng. Chem. 49, 81A (1957). 2333.

McCamy, C. S., Shoub, H., Lee, T. G., Fire extinguishment by means of dry powder. Proc. 6th Symp. (International) on Combustion (Reinhold Publ, Corp., New York, N.Y.), p. 795 (1957). 2123.

McCann, F. R., Rothman, S., Improved Hepp-Scatchard osmometer. J. Polymer Sci. 18, 151 (1955). 1362.

McCann, F. R., Rothman, S., Mandel, J., Weissberg, S. G., Sorption of macromolecules to solid surfaces: sorption of dextran to cellulose nitrate membranes in the presence of serum albumin and surface-active agents. J. Colloid Sci. 10, No. 4, 338 (1955). 1500.

McCaulley, J. W., Hough, W. S., A relationship between the lower ionosphere and the [OI] 5577 nightglow emission. J. Geophys. Research 64, No. 12, 2307 (1959). 2880.

McCaulley, J. W., Roach, F. E., Marovich, E., Origin of [OI] 5577 in the airglow and the aurora. J 63D1, 15 (1959).

McCaulley, J. W., Roach, F. E., Purdy, C. M., Comparison of absolute intensities of [OI] 5577 in the auroral and subauroral zones. J 63D 1, 19 (1959).
McClennon, P. R., Conversion of payroll processes at the National Bureau of Standards from manual to punched cards. Federal Accountant 7, No. 2, 64 (1957). 2070.

Mc Clintock, $R$. M., Cryogenic characteristics of wire reisitance strain gages. 1956 Cryogenic Engineering Conference, Paper E-4, p. 172, Edited by K. D. Timmerhaus, Chemical Engineering Dept., University of Colorado (Boulder, Colorado) (1957). 2073. Low temperature properties of plastic foams. Soc. of Plastics Engrs. J. 14, No. 11, 36 (1958). 2620. Mechanical properties of insulating plastic foams at low temperatures. Proc. 1958 Cryogenic Engineering Conference, Paper B-6, p. 132. Edited by K. D. Timmerhaus, Chemical Engineering Department, University of Colorado (Boulder, Colorado) (1959). 2985 .

McClintock, R. M., Gibbons, H. P., Mechanical properties of structural materials at low temperatures. A compilation from the literature. (1960) Mono. 13.

McClintock, R. M., Hiza, M.J., Epoxy resins as cryogenic structural adhesives. Proc. 1957 Cryogenic Engineering Conference, Paper Fl-3, p. 305, Edited by K. D. Timmerhaus, Chemical Engineering Dept., University of Colorado (Boulder, Colorado) (1958), Modern Plastics 35, 172 (1958). 2547.

McClintock, R. M., Kropschot, R. H., Van Gundy, D. A., Mechanical properties of some engineering materials. Proc. 1956 Cryogenic Engineering Conference, Paper C-2, p. 93 . Edited by K. D. Timmerhaus, Chemical Engineering Dept., University of Colorado (Boulder, Colorado) (1957). 2193.

McCoskey, R. E., Jessup, R. S., Nelson, R. A., The heat of formation of tetrafluoromethane. J. Am. Chem. Soc. $7 \%, 244$ (1955). 1533.

McCrackin, F. L., Total collision cross sections of negative atomic iodine ions in nitrogen and argon. Phys. Rev. 86, No. 1, 135 (1952). 605.

McCrackin, F. L., Bersch, C. F., Creep behavior of transparent plastics at elevated temperatures. SPE. Tech. Paper 5, 94 (1959). 2921.

McCrackin, F. L., Bersch, C. F., Plitt, K. F., The effects of rates of straining on some tensile properties of polyester film. Modern Plastics 35, 171 (1957). 2354 .

McCrackin, F. L., Schiefer, H.F., Smith, J. C., Stone, W. K. Breaking velocities, strain energies, and theory neglecting wave propagation. Part II. Textile Research J. 25, 529 (1955). 1277.

Stress-strain relationships in yarns subjected to rapid impact loading. Am. Soc. Testing Materials Spec. Tech. Publ. 176, "Symposium on Impact Testing", 126 (1955). 1514.

Stress-strain relationships in yarns subjected to rapid impact loading. Am. Soc. Testing Materials Spec. Tech. Publ. 185, Symp. on Speed of Testing on NonMetallic Materials, p. 47 (1956). 1899.

McCrackin, F. L., Schiefer, H. F., Smith, J. C., Stone, $W . K$, Towne, $K$. M., Stress-strain relationship in yarns tested at rates of straining up to a million percent per minute. Physics of Wool and Other Viscoelastic Fibers, D, 148 (1956) (Proc. Intern. Wool Textiles Research Conf., August 20-25, 1955, Sydney, Australia) (1956). 1900.

McCrackin, F. L., Smith, J. C., Schiefer, H. F., Characterization of the high-speed impact behavior of textile yarns. (Conference Issue) J. Textile Inst. (Trans.) 50, No. 1, T55 (1959). 2909.

Stress-strain relationships in yarns subjected to rapid impact loading. III. Effect of wave propagation. Textile Research J. 25, No. 8, 701 (1955). 1516. Stress-strain relationships in yarns subjected to rapid impact loading: 5 . Wave propagation in long textile yarns impacted transversly. J 60, 517 (1958) RP2866; Textjle Research J. 28, No. 4, 288 (1958). 2783.

The impact absorbing capacity of textile yarns. ASTM Bull. No. 220, 52 (1957). 2363. 
McCraw, T. F., Marton, L., Simpson, J. A., Automatic instrument for electron scattering measurements. Rev. Sei. Instr. 26, No. 9, 855 (1955). 1274.

Distribution of electron scattering by gold. Phys. Rev. 99, No. 2, 495 (1955). 1317.

McCraw, T. F., Simpson, J. A., Marton, L., Distribution of electrons scattered by solids. Phys. Rev. 104, No. 1, 64 (1956). 1681.

McDonald, E. J., Quantitative chromatrographic procedure for determining dextrose in sugar mixtures. Anal. Chem. 29, 32 (1957). 2276.

Report on reducing sugars. J. Assoc. Official Agri. Chem. 41, No. 3, 623 (1958). 2732.

Report on reducing sugars. Effect of clarification with neutral lead acetate before determining reducing sugars in molasses. J. Assoc. Offic. Agr. Chemists $39,676(1956), \quad 1850$.

McDuffie, R. E., Tables of Median hourly values of the cosine of the solar zenith angle $(\chi)$ for thirty-five locations. (1959) TN5 (PB151364).

McElhinney, J., Zendle, B., Domen, S. R., Calorimetric determination of the power in a $1400 \mathrm{kev}$ X-ray beam. Radiol. Research 6, No. 1, 40 (1957). 2047.

McElhinney, J., Zendle, B., Koch, H. W., Boag, J.W., Studies of dose distributions in water for betatron X-rays to $37 \mathrm{Mev}$. Radiol. Research 5, No. 2, 107 (1956). 1904.

McGavin, R. E., Barsis, A. P., Report on comparative $100 \mathrm{Mc}$ measurements for three transmitting antenna heights. IRE Trans. Ant. Prop. A P-4, 168 (1956). 1848.

McGavin, R. E., Maloney, L. J., Study at 1,046 megacycles per second of the reflection coefficient of irregular terrain at grazing angles. J 63D2, 235 (1959).

McGinnies, $R$. T., Energy spectrum resulting from electron slowing down. (1958) C597.

X-ray attenuation coefficients from $10 \mathrm{kev}$ to 100 Mev. (1959) C583 Suppl.

McGinnis, C. L., Disintegration of $5.8^{\mathrm{d}} \mathrm{Sb}^{120}$. Bull. Am. Phys. Soc. 30, No. 1, 33A (1955). 1315.

Radioactivity of In $^{117}$ and Sbi17. Bull. Am. Phys. Soc. 29, No. 1, 43A (1954) 1124; Phys. Rev. 97, 93 (1955). 1471.

Radioactivity of $\mathrm{In}^{120} \mathrm{Sb}^{120}$. Phys. Rev, 109, 888 (1958). 2711.

McGregor, M. C., Hersh, J. F., Cutkowsky, R. D., Harris, $F . K ., K o t t e r, F . R$., New apparatus at the National Bureau of Standards for absolute capacitance measurement. IRE Trans. Instrumentation I-7, No. 3-4, 253 (1958). 2647.

McIntyre, D., Doderer, G. C., Absolute light-scattering photometer: I. Design and operation. J 62, 153 (1959) RP2946.

McIntyre, D., Doderer, G. C., O'Mara, J. H., Design and performance of a block-type osmometer. J 62, 63 (1959) RP2931.

McKean, W. J., Potter, $R$. F., An apparatus for measuring the piezoresistivity of semiconductors. J 59, 427 (1957) RP2814.

McKee, S. A., Oil flow in plain journal bearings. Trans. Am. Soc. Mech. Engrs. 74, 841 (1952). 494

McKee, S. A., Bair, E. N., Sr., White, H. S., Lubrication of plain journal bearings. The Virginia Tech. Eng. 23, 26 (1950). 82 .

McKee, S. A., Swindells, J. F., White, H. S., Mountjoy, W., For wear prevention. SAE J. 58, 47 (1950). 59.

McKenna, E., Cassel, $J$., Amide nitrogen of collagen and hide powder. J. Am. Leather Chemists Assoc. 48, 142 (1953). 645 .

Swelling of collagen and modified collagen. J. Am. Leather Chemists Assoc. 49, 553 (1954). 1159.

McKenna, E., Cassel, J., Glime, A., Studies on the polar amino acid content of collagen and related material. J. Am. Leather Chemists Assoc. 48, 277 (1953). 841.

McKinley, J. D., Jr., Little, R. E., On-off temperature control for electrically heated filments. Rev. Sci. Instr. 29, No. 12, 1143 (1958). 2660.
McKinley, J. D., Jr., Polanyi, J. C., Chemiluminescence in the system atomic sodium plus atomic hydrogen. Can. J. Chem. 36, 107 (1958). 2486.

McKinley, J. D., Jr., Shuler, K. E., Kinetics of the high temperature heterogeneous reaction of chlorine and nickel between 1200 and $1700^{\circ} \mathrm{K}$. J. Chem. Phys. 28, No. 6, 1207 (1958), 2608.

Kinetics of the reaction between nickel and chlorine above $1,100^{\circ} \mathrm{K}$. Proc. Intern. Meeting of Chemistry (Paris, France), Section de Chimie Minerale, p. 637 (1957). 2174.

McKinney, J. E., Edelman, S., Marvin, R. S., Apparatus for the direct determination of the dynamic bulk modulus. J. Appl. Phys. 27, 425 (1956). 1629.

McKinney, J. E., Roth, $F$. L., Carbon black differentation by electrical resistance of vulcanizates. Ind. Eng. Chem. 44, 159 (1952). 408.

McLane, C. K., Hudson, R. P., Magnetic and thermal properties of chromic methylamine alum below $1^{\circ} \mathrm{K}$. Phys. Rev. 95, No. 4, 932 (1954). 1057.

McLaughlin, W. L., Ehrlich, M., Film badge dosimetry: how much fading occurs? Nucleonics 12, No. 10, 34 (1954). 1021.

Limitations in the photographic dosimetry of Xand gamma-rays. Radiation Research 9, No. 1, 148 (1958). 2611.

Photographic dosimetry at total exposure levels below $20 \mathrm{mr}$. (1959) TN29 (PB151388).

Reciprocity law for X-rays, Part 1: validity for highintensity exposures in the negative region. J. Opt. Soc. Am. 46, No. 10, 797 (1956). 1838.

McLeish, C. W., Measurements of coastal deviation of high-frequency radio waves. J 64D 1, 57 (1960).

McLeod, G. L., Properties of some experimental map papers containing synthetic fibers. Tappi 41, No. 8, 430 (1958). 2703.

McLeod, G. L., Worksman, T. L., Improved dimensional stability in laminated map paper. Tech. Assoc. Pulp Paper Ind, 41, No. 9, 484 (1958). 2595.

McLernon $F$., Bay, $Z$., Cleland, $M$. R., Fast coincidences with Cerenkov counters. Phys. Rev. 87, 901 (1952). 447.

McMurdie, H. F., Kissinger, H. E., Simpson, B., Thermal decomposition of manganous and ferrous carbonates. J. Am, Ceram Soc. 39, No. 5, 168 (1956). 1960.

McMurdie, H. F., Levin, E. M., Hall, F. P., Compilation of phase diagrams of interest to ceramists. Book, Phase of Ceramists (Am. Ceram. Soc.) (1956). 1656.

McNally, J. R., Jr., Kessler, K. G., Supplementary Zeeman data for the first spectrum of ruthenium (Ru 1). J 63A3, 253 (1959).

McNesby,J.R., Photolysis of oxygen in solution in liquid nitrogen and in liquid carbon monoxide at $1849 \mathrm{~A}$. J. Chem. Phys. 31, No. 1, 283 (1959). 3013.

McNesby, J. R., Klein, R., Scheer, M. D., Schoen, L. J., Kinetics of D atom reactions with $\mathrm{H}_{2}$. J. Chem. Phys. 30, No. 1, 58 (1959). 2971.

McNish, A. G., Classification and nomenclature for standards of measurement. IRE Trans. Instrumentation I-7, No. 3-4, 371 (1958). 2486A.

Dimensions, units, and standards. Phys. Today 10, No. 4, 19 (1957). 2088.

Geomagnetic effects of high-altitude nuclear explosions, J. Geophys. Research 64, No. 12, 2253 (1959). 2959.

Study of radio reflections from meteor trails in research on the upper atmosphere. Air Force Cambridge Research Center: Geophysical Research Paper No. 7 (Proc. of the Conf. on Ionospheric Research, June 1949), p. 105 (1950). 144.

The basis of our measuring system. Proc. IRE 47, No. 5, 636 (1959). 3066.

McNish, A. G., Gautier, T. N., Knecht, R. W., Lunar stratification of the F2 layer at Huancayo, Peru. Proc. General Assembly, Intern. Sci. Radio Union, Zurich, Switzerland, Sept. 11-22, 1950, VIII, Pt. 2, 216 (1950). 83. 
McNish, A. G., Huntoon, R. D., Importance of the Faraday to elemental constants and electricity standards. Nature 181, 1194 (1958), 2593.

Present status of research on the physical constants at the (United States) National Bureau of Standards. N. 1 del Supplemento at Vol. 6, Serie X, Il Nuovo Cimento (Bologna, Italy) p. 146 (1957). 2263.

McNish, A. G., Lincoln, J. V., Predictions of the present sunspot cycle. Trans. Am. Geophys. Union 35, 709 (1954). 1112.

McPherson, A. E., A dual-amplitude axial-load fatigue machine. Proc. Soc. Exptl. Stress Anal. 10, 143 (1953). 626.

McPherson, A. T., A proposed standard for facilities for scientific conferences. Mag. of Standards 29, No. 7, 202 (1958). 2434.

Government and industry use of common standards. Proc. of the Eighth Natl. Conf. on Standards (Am. Std. Assoc.), p. 26 (1957). 2144.

Local standards laboratories. Instr. and Automation 32, 92 (1959), 2977.

Materials of construction: their properties and methods of test. J. Am. Inst. Arch. 29, No. 3, 143 (1958). 2625.

New standards for science and industry. Am. Soc. Quality Control, Proc. 4th Annual Quality Control Symp., Southern Methodist Univ., (Dallas, Texas), p. 103 (1959). 2998.

Science Fair. ATSM Bull. No. 237, 29 (1959). 3041.

Screw thread standardization. Standards Engrs. Soc. Proc. for 1956 (Proc. of the 5th Annual Natl. Meeting (Washington, D.C.)) (1956). 1862.

Standards-good and bad. Boletime de metrologia No, 4, 7 (1958), $2777 \mathrm{~A}$

Student research on ASTM problems. ASTM Bull. No. 217, 31 (1956), 1903.

The need for more knowledge of materials in civil engineering. Conf. Basic Research in Civil Engr. September 10-11, 1958, Am. Soc. Civil Engrs. Natl. Sci. Foundation, George Washington University (Washington, D.C.), p. 20 (1958). 2824

Unsolved problems in review. ASTM Bull. No. 210, p. $12(1955) .1568$.

McPherson, J. L., Alexander, S. N., Horton, H. B., Glaser, $E$., Information processing in social and industrial research. Sci. Monthly $\mathbf{7 6 ,} 100$ (1953). 741.

McQuate, P. L., Kirby, R. S., Dougherty, H. T., VHF propagation measurements in the Rocky Mountain region. IRE Trans. Vehicular Commun. VC-6, $13(1956) .1972$.

McReynolds, W. A., Schrodt, J. E., Fulk, M. M., Flanery, $W$. S., A comparison of insulating materials. Proc. 1957 Cryogenic Engineering Conference, Abstract, Paper G-7, p. 416, Edited by K. D. Timmerhaus, Chemical Engineering Department, University of Colorado, (Boulder, Colorado) (1958). 2416.

McVoy, $K$. W., Fano, $U$., Bremsstrahlung and the photoelectric effect as inverse processes. Phys. Rev. 116, No. 5, 1168 (1959). 2905.

McVoy, K. W., Fano, U., Albers, J.R., Interference of orbital and spin eurrents on bremsstrahlung and photoelectric effect. Phys. Rev. 116, No. 5, 1159 (1959). 2968.

Sauter theory of the photoelectric effect. Phys. Rev. 116, No. 5, 1147 (1959). 3040.

\section{M}

MacArthur, E. H., Boone, T. H., Hermach, F. L., McAuliff, R. C., Conductive flooring for hospital operating rooms. J 63C2, 125 (1959); (1960) Mono. 11.

Machlan, L. A., Hague, J. L., Determination of niobium and tantalum in titanium-base alloys. J 62, 53 (1959) RP2929.

Determination of titanium, zirconium, niobium, and tantalum in steels: separations by anion-exchange. J 62, 11 (1959) RP2923.

Photometric determination of tungsten in steel and titanium alloys with dithiol. J 59, 415 (1957) RP2812 .

Machlan, L. A., Hague, J. L., Meros, E. J., Determination of aluminum in precipitation hardening stainless steel and high temperature alloys. J 64A2, 181 (1960).

Macinko, J., Chelton, D. B., Dean, J., Hydrogen liquefaction by a dual pressure process. Refrig. Eng. 65, No. 8, 39 (1957). 2156.

Hydrogen liquefaction cycles. Proc. 1957 Crogenic Engineering Conference, Paper A-1, p. 1. Edited by K. D. Timmerhaus, Chemical Engineering Dept., University of Colorado (Boulder, Colorado) (1958). 2590.

Mackles, L., Heindl, R. A., Mong, L. E., Chemical analyses, surface area, and thermal reactions of natural graphite, and refractoriness of the ashes. J. Am. Ceram. Soc. 36, 266 (1953). 665.

Macmillan, R. S., Rusch, W. V. T., Golden, R. M., A very-low-frequency antenna for investigating the ionosphere with horizontally polarized radio waves. J 64D 1, 27 (1960).

MacPherson, A. C., Beatty, R. W., Mismatch errors in microwave power measurements. Proc. IRE 41, 1112 (1953). 769.

MacPherson, A. C., Kerns, D. M., A microwave microcalorimeter. Rev. Sci. Instr. 27 (1955). 1236.

A new technique for the measurement of microwave standing wave ratios. Proc. IRE 44, 1024 (1956). 1594.

Matching loads on a magic tee. Electronics 23, No. $9,190(1950)$. $83 \mathrm{~A}$.

Macurdy, L. B., Alber, H. K., Benedetti-Pichler, A. A., Carmichael, H., Corwin, A. H., Fowler, R. M., Huffman, E. W. D., Kirk, P. L., Lashof, T. W., Terminology for describing the performance of analytical and other precise balances. Anal. Chem. 26, 1190 (1954). 1166

Macurdy, L. B., Bowman, H. A., photoelectric followup and recording system, and its application to remote observations of the beam in high precision balances. J 63C2, 91 (1959).

Macurdy, L. B., Lashof, T. W., Testing a quick-weighing balance. Anal. Chem. 26, 707 (1954). 1168.

Madorsky, I., Wood, L. A., Paulson, R. A., Determination of copolymer composition by combustion analysis for carbon and hydrogen. J 64A2, 157 (1960).

Madorsky, S. L., Macromolecular chemistry. Science 121, 81 (1955). 1394 .

Pyrolysis of hydrocarbon polymers. Science 111, $360(1950) .116$.

Rates and activation energies of thermal degradation of styrene and acrylate polymers in a vacuum. J. Polymer Sci. 11, 491 (1953). 811.

Thermal degradation of polymers at low rates. $\mathbf{J} \mathbf{6 2}$, 219 (1959) RP2957.

Thermal degradation of polymers at temperatures up to about $800-900^{\circ} \mathrm{C}$. Proc. Joint Army-Navy-Air Force Conf. on Elastomer Research and Development, 2, 5th meeting (October 1958), p. 511 (Materials Laboratory, Wright Air Development Center, WrightPatterson Air Force Base, Ohio) (1958). 2843.

Rates of thermal degradation of polystyrene and polyethelene in a vacuum. J. Polymer Sci. 9, No. 2, 133 (1952). 526.

Thermal degradation of polystyrene and polyethylene. J. Polymer Sci. 13, 185 (1954), 1209.

Tungsten helical spring balance. Rev. Sci. Inst. 21, 393 (1950). 162.

Madorsky, S. L., Hart, V. E., Straus, S., Products volatilized from a channel and a furnace black on heating to $1,000^{\circ} \mathrm{C}$. Rubber Age 84, 238 (1953). 803 . Thermal degradation of cellulosic materials. $J \mathbf{6 0}$, 343 (1958) RP2853.

Madorsky, S. L., Straus, S., Thermal degradation of polyacrylonitrile, polybutadiene, and copolymers of butadiene with acrylonitrile and styrene. J 61, 77 (1958) RP2888.

Thermal degradation of polyethylene oxide and poly- 
propylene oxide. J. Polymer Sci. 36, 183 (1959). 3100 .

Thermal degradation of polymers as a function of molecular structure and branching. Consiglio $\mathrm{Na}-$ zionale Delle Richerche (Rome, Italy) 25, 3 (1955). 1557 .

Thermal degradation of polymers at high temperatures. J 63A3, 261 (1959).

Thermal degradation of unvulcanized and vulcanized rubber in a vacuum. Ind. Eng. Chem. 48, 1212 (1956). 1961

Madorsky, S. L., Wall, L. A., Brown, D. W., Straus, S. Simha, $R$., Depolymerization of polymethylene and polyethylene. J. Am. Chem. Soc. 76, 3430 (1954). 985.

Magat, M., Wall, L. A., Degradation des polymeres par les rayons et les neutrons. J. chim. phys. 50,308 (1953). 681.

Effects of atomic radiation on polymers. Modern Plastics 30, 111 (1953). 698.

Magill, A. A., Variation in distortion with magnification. J. Opt. Soc. Am. 45, No. 3, 148 (1955). 1572

Maienthal, M., Hellmann, M., Haber, C. P., Hymo, L. A., Carpenter, S., Carr, A. J., The preparation of some aryl silanes. J. Am. Chem. Soc. 76, 6392 (1954). 1192.

Mair, B. J., Petroleum hydrocarbons. Ind. \& Eng. Chem. 42, 1355 (1950). 107.

Mair, B. J., Westhaver, J. W., Rossini, F. D., Theoretical analysis of fractionating process of adsorption. Ind. \& Eng. Chem. 42, 1279 (1950). 156.

Maki, A. G., Stair, R., Johnston, R. G., Apparatus for the measurement of the normal spectral emissivity in the infrared. J 64C2, 99 (1960).

Malitson, I. H., Murphy, V. F., Jr., Rodney, W. S., Refractive index of synthetic sapphire. J. Opt. Soc. Am. 48, No. 1, 72 (1958). 2727.

Malitson, I. H., Rodney, W. S., Refraction and dispersion of thallium bromide iodide. J. Opt. Soc. Am. 46, No. 11, 956 (1956). 1842.

Malitson, I. H., Rodney, W. S., King, T. A., Refractive index of arsenic trisulfide. J. Opt. Soc. Am. 48, No. 9, 633 (1958). 2726.

Mallonee, R. E., Howard, F. L., The determination of freezing point of engine antifreezes. ASTM Bull. No. 172 , p. 43 (1951). 331.

Malloy, A., Soller W., Roberts, A. G., Evaluation of adhesion of organic coatings by ultracentrifugal and other methods. Paint, Oil, and Chem. Rev. 116, No. 18, $14 ; 116$, No. 19, 26 (1953). 715.

Malmberg, C. G., Dielectric constant of deuterium oxide. J 60, 609 (1958) RP2874.

Malmberg, M. S., Hamer, W. J., Rubin, B., Theoretical electromotive forces for cells containing a single solid or molten chloride electrolyte. J. Electrochem. Soc. 103, 8 (1956). 1957.

Maloney, L. J., Barghausen, A. F., Decker, M. T., Measurements of correlation, height gain, and path antenna gain at $1046 \mathrm{Mc}$ on spaced antennas far beyond the radio horizon. IRE Conv. Record 3, Pt. 1, 78 (1955). 1401.

Maloney, $\dot{L}$. J., McGavin, R. E., Study at 1,046 megacycles per second of the reflection coefficient of irregular terrain at grazing angles. J 63D 2, 235 (1959).

Malville, J. M., Artificial auroras resulting from the 1958 Johnston Island nuclear explosions. J. Geophys. Research 64, No. 12, 2267 (1959). 2899.

Mandel, $J ., \quad$ A note on confidence intervals in regression problems. Ann. Math. Stat. 49, No. 3, 903 (1958). 2429.

Chain block designs with two-way elimination of heterogeniety. Biometrics 10, 251 (1954). 966. Fitting a straight line to certain types of cumulative data. J. Am. Stat. Assoc. 52, 552 (1957). 2125.

Statistical principles of testing. Ind. Quality Control 12, 18 (1955). 1511.

Statistical treatment of analytical data. Proc. Conf on Chemical Control Problems, Natl. Plant Food Inst. p. 1 (October 1958). 2780.
The theory of extreme values. ASTM Bull. No. 236, 29 (1959); Tech. Eng. News 40, No. 7, 28 (1959). 3096.

The uncertainty of measurements. Chem. Corps Engineering Command, Proc. 2d Annual Statistical Engineering Symp. (Edgewood, Maryland), p. 57 (1956). 1953.

Mandel, J., Dahl, S., A collaborative study on the cinchonine method for determination of lignosulfonates in vegetable extract blends. J. Am. Leather Chemists Assoc. 52, 184 (1957). 1979.

Mandel, J., Decker, G. E., Mooney viscometer cure characteristics of Hevea rubber. ASTM Bull. No. 209, 47 (1955). 1409.

Mandel, J., Horowitz, E., Comparison of sodium hydroxide and sulfuric acid methods for analysis of wool admixed with cotton and viscose rayon. Am. Dyestuff Reptr. 43, No. 19, 613 (1954). 973.

Mandel, J., Kanagy, J. R., Mann, C., Study of the variation of the physical and chemical properties of chrome-tanned leather and the selection of a sampling location. J. Soc. Leather Trades' Chemists (London, England) 36, 231 (1952). 558.

Mandel, J., Lashof, T. W., Worthington, V., Use of the sensitivity criterion for the comparison of the Bekk and Sheffield smoothness testers. Tappi 39, No. 7, 532 (1956). 1970.

Mandel, J., Linnig, $F$. J., Statistical methods in chemistry. Anal. Chem. 28, 770 (1956). 1896.

Statistical methods in chemistry. Anal. Chem. 30, 739 (1958). 2779 .

Study of accuracy in chemical analysis using linear calibration curves. Anal. Chem. 29, 743 (1957). 2328 .

Mandel, J., Linnig, $F . J .$, Peterson, $J . M$. A plan for studying the accuracy and precision of an analytical procedure. Anal. Chem. 26, No. 7, 1102 (1954). 926.

Mandel, J., Mann, C., Sampling of leather. J. Sci. and Ind. Research 18A, No. 12, 575 (1959). 3039.

Mandel, J., Mann, C., Steel, M. N., Kanagy, J. R., Sampling of side upper leather II. J. Am. Leather Chemists Assoc. 47, 352 (1952). 535.

Mandel, J., Randall, E. B., Mann, C., Kanagy, J. R., Study of the burst test as applied to military upper leathers. J. Am. Leather Chemists Assoc. 48, 84 (1953). 842.

Mandel, J., Reinhart, $F$. W., Comparison of methods for measuring the tensile and tear properties of plastic films. ASTM Bull. No. 209, 50 (1955). 1290.

Mandel, J., Roth, F. L., Steel, $M$. N., Stiehler, R. D., Measurement of the aging of rubber vulcanizates. J 63C2, 141 (1959)

Mandel, J., Rothman, S., McCann, F. R., Weissberg, S. G., Sorption of macro-molecules to solid surfaces: sorption of dextran to cellulose nitrate membranes in the presence of serum albumin and surface-active agents. J. Colloid. Sci 10, No. 4, 338 (1955). 1500.

Mandel, J., Steel, M. N., Stiehler, R. D., Treadwear of tires. Influence of type of rubber and black. Ind. Eng. Chem. 43, 2901 (1951). 350.

Mandel, $J$. Stiehler, $R$. D., Evaluation of analytical methods by the sensitivity criterion. Anal. Chem. 29, 17 A (1957), 2112.

Statisties-A tool in testing rubber. ASTM Bull. No, 204, 44 (1955). 1512.

Mandel, J., Stiehler, R. D., Richey, G. G., Measurement of treadwear of commerical tires. Rubber Age 73, 201 (1953). 761

Mandel, J., Stiehler, R. D., Steel, M. N., Some factors influencing the road wear of tires. Trans. Inst. Rubber Ind. (Cambridge, England) 27, No. 6, 298 (1951). 308 .

Mandel, J., Stiehler, R. D., Steel, M. N., Richey, G. G., Hobbs, R. H., Power loss and operating temperature of tires. J 64C 1,1 (1960).

Mandel, J., Wilson, W. K. Harvey, J. L., Worksman, T. L., Accelerated aging of record papers compared with normal aging. Tappi 38, No. 9, 543 (1955). 1251. 
Mandelkern, L., Crystallization kinetics in polymeric systems. Chapter in book, Growth and Perfection of Crystals, p. 467 (John Wiley \& Sons, New York, N.Y.) (1958). 2507; SPE J. 15, 63 (1959). 2923. Kinetics of crystallization in polymers, II. Polymerdiluent mixtures. J. Appl. Phys. 26, 443 (1955). 1382.

The crystallization of flexible polymer molecules. Chem. Revs. 56, No. 5, 903 (1956). 1924.

The melting of crystalline polymers. Rubber Chem. and Technol. XXXII, No. 5, 1392 (1959). 3083.

Mandelkern, L., Flory, P. J., Melting of copolymers. J. Polymer Sci. 21, No. 98, 345 (1956). 1774.

Mandelkern, L., Fox, T. G., Dilute solution thermodynamic behavior of flexible chain molecules: polymethyl methacrylate. J. Chem. Phys. 21, 187 (1953). 687.

Mandelkern, L., Hellmann, M., Brown, D. W., Roberts, D. E., Quinn, F. A., Jr., The melting transition of polymethylene. J. Am. Chem. Soc. 75, 4093 (1953). 869.

Mandelkern, L., Martin, G. M., Glass formation in polymers: II. The system rubber-sulfur. J 62, 141 (1959). RP2944.

Mandelkern, L., Martin, G. M., Rogers, S. S., Volume temperature relations of amorphous polymers over an extended temperature range. J. Polymer Sci. 20, 579 (1956). 1975 .

Mandelkern, L., Newton, C. J., Roberts, D. E., Preferred orientation in stark rubber studied with an automatic integrating pole-figure goniometer. J. Appl. Phys. 26, No. 12, 1521 (1955). 1454.

Mandelkern, L., Posner, A. S., Diorio, A. F., Laki, K. Mechanism of contraction in the muscle fibre-ATP system. Proc. Natl. Acad. Sci. 45, 814 (1959). 2986.

Mandelkern, L., Quinn, F. A., Jr., Thermodynamies of crystallization in high polymers: poly(ethylene). J. Am. Chem. Soc. 80, 3178 (1958). 2846.

Mandelkern, L., Quinn, F. A., Jr., Flory, P. J., Kinetics of crystallization in polymers, I. Bulk polymers. J. Appl. Phys. 25, 830 (1954). 1047.

Mandelkern, L., Quinn, F. A., Jr., Roberts, D. E., Thermodynamics of crystallization in high polymers: gutta percha. J. Am. Chem. Soc. 78, 926 (1956). 1965.

Mandelkern, L., Roberts, D. E., Some properties of polymer networks formed from oriented chains of natural rubber. J. Am. Chem. Soc. 80, 1289 (1958). 2765 .

Thermodynamics of crystallization in high polymers: natural rubber. J. Am. Chem. Soc. 77, 781 (1955). 1558 .

Mandelkern, L., Roberts, D. E., Diorio, A. F., Reversible contractile processes in fibrous macromolecules. J. Am. Chem. Soc. 80, 500 (1958). 2743.

Mandelkern, L., Roberts, D. E., Flory, P. J., The isotropic length of polymer networks. J. Am. Chem. Soc. 79, 1515 (1959). 2370.

Mandelkern, L., Rogers, S. S., Glass formation in polymers: I. The glass transitions of the poly- $(n$-alkyl methacrylates), J. Phys. Chem. 61, 985 (1957). 2142.

Mandelkern, L., Scheraga, H. A., On the interpretation of hydrodynamic data for dilute protein solutions. J. Phys. Chem. 62, 370 (1958). 2668.

Mandelkern, L., Tryon, M., Quinn, F. A., Jr., The melting behavior of polybutadienes. J. Polymer Sci. 19, 77 (1956). 1938.

Mandelkern, L., Williams, L. C., Weissberg, S. G., Sedimentation equilibrium of flexible chain molecules. J. Phys. Chem. 61, 271 (1957). 2296.

Mandelkern, L., Worthington, C. R., Posner, A. S., Lowangle X-ray diffraction of fibrous polyethylene. Science 127, 1052 (1958). 2616.

Mann, C., Kanagy, J. R., Leser, W., Randall, E. B., Carter, T. J., Influence of splitting on the strength of chrome-tanned steer hides. J. Am. Leather Chemists Assoc. 47, 329 (1952). 468.
Mann, C., Kanagy, J. R., Mandel, J., Study of the variation of the physical and chemical properties of chrome-tanned leather and the selection of a sampling location. J. Soc. Leather Trades' Chemists (London, England) 36, 231 (1952). 558.

Mann, C., Kanagy, J. R., Randall, E. B., Carter, T. J., Kinmonth, R.A., Variations of physical and chemical properties within and between vegetable retanned cow and steer sides. J. Am. Leather Chemists Assoc. 47, 726 (1952). 618.

Mann, C., Mandel, J., Sampling of leather. J. Sci. and Ind. Research 18A, No. 12, 575 (1959). 3039.

Mann, C., Mandel, J. R., Steel, M. N., Kanagy, J. R., Sampling of side upper leather II. J. Am. Leather Chemists Assoc. 47, 352 (1952). 535.

Mann, C., Randall, E. B., Carter, T. J., Kilduff, T. J., Kanagy, J.R., The variation of the physical and chemical properties of split and unsplit chrometanned leathers. J. Am. Leather Chemists Assoc. 47, 404 (1952). 601 .

Mann, C., Randall, E. B., Kanagy, J. R., Mandel, J., Study of the burst test as applied to military upper leathers. J. Am. Leather Chemists Assoc. 48, 84 (1953). 842.

Mann, D. B., Birmingham, B. W., United States planning for the large scale liquefaction and transportation of helium. Physica 24, S144 (1958). 2855A.

Mann, D. B., Birmingham, B. W., Vander Arend, P. C., Technical aspects of large scale liquid helium liquefaction and transportation. Proc. 1957 Cryogenic Engineering Conference, Paper D-4, p. 191, Edited by K. D. Timmerhaus, Chemical Engineering Dept., University of Colorado (Boulder, Colorado) (1958). 2797.

Mann, D. B., Chelton, D. B., Byrns, R. A., A large liquid hydrogen bubble chamber. Proc. 1956 Cryogenic Engineering Conference, Paper H-2, p. 325, Edited by K. D. Timmerhaus, Chemical Engineering Dept., University of Colorado (Boulder, Colorado) (1957). 1984.

Mann, D. B., Chelton, D. B., Dean, J. W., Strobridge, T. R., Birmingham, $B$. W., Helium refrigeration and liquefaction using a liquid hydrogen refrigerator for precooling. (1960) TN39 (PB151398).

Mann, D. B., Scolt, L. E., Robbins, R. F., Birmingham, $B$. $W$., Temperature stratification in a nonventing liquid helium dewar. J 64C1, 19 (1960).

Mann, D. B., Steward, R. B., Thermodynamic properties of helium at low temperatures and high pressures. (1959) TN8 (PB151367).

Mann, $D$. $E$., The vibrational spectra of $n$-dimethylaminodiborane. J. Chem. Phys. 22, 70 (1954). 1205.

Mann, D. E., Acquista, N., Lide, D. R., Jr., The infrared spectrum of tertiary butyl fluoride. J. Mol. Spectroscopy 2, 575 (1958). 2820.

Mann, D. E., Acquista, N., Plyler, E. K., The vibrational spectrum of bromotrifluoroethylene. J. Chem. Phys. 22, 1199 (1954). 1206.

The vibrational spectrum of chlorotrifluoroethylene. J. Chem. Phys, 21, 1949 (1953). 893.

Vibrational spectra of trifluorethylene and trifluoroethylene-d. J. Chem. Phys. 22, 1586 (1954). 1224.

Mann, D. E., Broida, H. P., Squires, H. P., The decomposition of $\mathrm{CF}_{4}$ in flames. J. Chem. Phys. 22, 348 (1954). 1176.

Mann, D. E., Dibeler, V. H., Reese, R. M., Ionization and dissociation of perchlorylfluoride by electron impact. J. Chem. Phys. 27, 176 (1957). 2170.

Mann, $D . E$., Fano, $L$., Vibrational spectrum and force constants of diboron tetrachloride. J. Chem. Phys. 26, 1665 (1957). 2409 .

Mann, D. E., Lide, D. R., Jr., Microwave spectra of molecules exhibiting internal rotation. I. Propylene. J. Chem. Phys. 27, 868 (1957); II. Methylallene, J. Chem. Phys. 27, 874 (1957). 2202.

Microwave spectra of molecules exhibiting internal rotation, III. Trimethylamine. J. Chem. Phys. 27, 572 (1958). 2637. 
Microwave spectra of molecules exhibiting internal rotation. IV. Isobutyane, tertiary butyl fluoride and trimethylphospine. J. Chem. Phys. 29, 914 (1958). 2638 .

Search for the microwave spectrum of perchlorylfluoride. J. Chem. Phys. 25, 595 (1956). 1863.

The infrared spectrum and structure of perchlorylfluoride. J. Chem. Phys. 25, 1128 (1956). 1933.

Mann, D. E., Lide, D. R., Jr., Fristrom, R. M., The microwave spectrum and structure of sulfuryfluoride. J. Chem. Phys. 25, 734 (1957). 2372.

Mann, D. E., Meal, J. H., Plyler, E. K., Properties of fluorine compounds, the vibrational spectrum of tetrabromoethylene. J. Chem. Phys. 24, No. 5, 1018 (1956). 1825.

Vibrational spectrum of tetrabromoethylene. J. Chem. Phys. 24, 1018 (1956). 1973.

Mann, D. E., Plyler, E. K., Infrared and raman spectra of trans-difluorodichloroethylene. J. Chem. Phys. 26, 773 (1957). 2161.

The $\nu_{0}$ fundamental of $\mathrm{C}_{2} \mathrm{~F}_{8}$. J. Chem. Phys. 21, 1116 (1953). 888.

Vibrational spectrum of fluorotrichloroethylene. J. Chem. Phys. 23, No. 11, 1989 (1955). 1577.

Mann, D. E., Plyler, E. K., Acquista, N., Vibrational spectrum of 1-fluoro-1-chloroethylene. J. Chem. Phys. 23, 2122 (1955). 1576.

Mann, $H$. B., The estimation of parameters in certain stochastic processes. Sankhya (Calcutta, India.) 11, Pt. 2, 97 (1951). 334.

Mann, $W$. B., Preparation and maintenance of radioactive standards by the National Bureau of Standards. Acta Radiol. Suppl. 117, 50 (1955). 1455.

Radium standards, internal gas counting and the United States radioactivity standards program. Proc. Symp. on Measurements and Standards of Radioactivity, Natl. Research Council, Nuclear Science Series, No. 24, 17 (1958). 2714.

The preparation and maintenance of standards of radioactivity. J. Appl. Radiation and Isotopes 1, $3(1956) .1944$.

Mann, W. B., Bay, Z., Seliger, H. H., Wyckoff, H. O., Measurement of $W_{\text {sir }}$ for sulfur-35 betarays. Radiol. Research Abstract 5, No. 4, 9 (1956). 1769.

Mann, W. B., Seliger, H. H., Preparation, maintenance, and application of standards of radioactivity. (1958) C594.

Radioactivity standardization in the United States. Proc. 2d Intern. Conf. on the Peaceful Uses of Atomic Energy, United Nations (Geneva, Switzerland) 21, 90 (1958). 2712 .

Mann, W. B., Seliger, H. H., Cavallo, L. M., Average energy of sulfur-35 beta decay. J 60, 447 (1958) RP2859.

Mann, W. B., Stockmann, L. L., Youden, W. J., Schwebel, A., Mullen, P. A., Garfinkel, S. B., Preparation of new solution standards of radium. $\mathbf{J} \mathbf{6 2}, 21$ (1959) RP2924.

Manning, J.R., Correlation correction to the activation energy for diffusion in crystalline solids. Phys. Rev. Letters 1, No. 10,501 (1958). 2502.

Correlation effect in impurity diffusion. Phys. Rev. 116, No. 4, 819 (1959). 2919.

Manning, R., Newkirk, G. A., Curtis, G. W., Watson, D. K. Shelby, $J$., The inner solar corona during June 1959. Nature 184, 1308 (1959). 3080.

Mannos, M., Hoffman, A. J., Sokolowsky, D., Wiegmann, N. A., Computational experience in solving linear programs. J. Soc. Ind. Appl. Math. 1, 17 (1953). 672 .

Mansfield, W. V., Norton, K. A., Vogler, L. E., Short, P. J., The probability distribution of the amplitude of a constant vector plus a Rayleigh-distributed vector. Proc. IRE 43, 1354 (1955). 1541.

Mansfield, W. V., Rice, P. L., Herbstreit, J. W. Groundto-air cochannel interference at 2900 Mc. IRE Trans. Airborne Electronics AE-6, 1 (1952). 459.
Marcus, $M$., A determinantal ineouality of H. P. Robertson. J. Wash. Acad. Sci. 47, 264 (1957). 1980.

Mathematics - a note on values of a quadratic form. J. Wash. Acad. Sci. 47, No. 4, 97 (1957). 2186.

Basic theorems in matrix theory. (1959) AMS57.

On a determinantal inequality. Am. Math. Monthly (Math. Notes) 65, 266 (1958). 2657.

On doubly stochastic transforms of a vector. Oxford Quart. J, Math. 9, 74 (1958). 2658.

On subdeterminants of doubly stochastic matrices. Illinois J. Math. 1, 583 (1957). 2227.

Marcus, M., Goldman, A. J., Convexity of the field of a linear transformation. Can. Math. Bull. 2, 15 (1959). 2918.

Marcus, $M$., Khan, $N$. A., Space of $k$-commutative matrices. J 64B 1, 51 (1960).

Marcus, M., Moyles, B. N., Westwick, R., Some extreme value results for indefinite Hermitian matrices. Illinois J. Math. 1, 449 (1957). 2303.

Marcus, M., Newman, M., On the minimum of the permanent of a doubly stochastic matrix. Duke Math. J. 26, 61 (1959). 3008.

Marcus, $M$., Thompson, $R$., A note on symmetric functions of eigenvalues. Duke Math. J. 24, 43 (1957). 1990.

Marden, E., Koller, H. R., Pfeffer, H., The Haystaq system: past, present and future. Preprinted in Proc. Intern. Conf. on Scientific Infor. November 11-16, 1958 (American Documentation Inst., Washington D.C.) Area 5, p. 317 (1958). 2815.

Margetis, $P$. M., Hansen, $W$. C., Changes in agar-agar type duplicating material and agar-agar on heating and storage. J. Am. Dental Assoc. 54, 737 (1957). 2050 .

Margoshes, J., Scribner, B. F., The plasma jet as a spectroscopic source. 'Spectrochem. Acta XIV, No. 2, 138 (1959). 3088.

Marimont, $R$. B., A new method of checking the consistency of precedence matrices. J. Assoc. Computing Mach. 6, No. 2, 164 (1959). 2876.

Marinelli, L., Taylor, L. S., The measurement of ionizing radiations for biological purposes. Biological Effects of Radiation (McGraw-Hill Book Co., Inc., New York, N.Y.) (1954). 1189.

Marlow, $W$. $F$., Medlock, $R$. $W$., A carbon-14 beta-ray standard, benzoic acid-7-C in toluene, for liquid scintillation counters. J 64A2, 143 (1960).

Marovich, E., Roach, F. E., A monochromatic low-latitude aurora. J 63D3, 297 (1959).

Aurora of October 22/23, 1958, at Rapid City, South Dakota. J 64D2, 205 (1960).

Marovich, E., Roach, F. E., McCaulley, J. W., Origin of [OI] 5577 in the airglow and the aurora. J $63 \mathrm{D} 1,15$ (1959).

Marovich, E., Roach, F. E., Megill, L. R., Rees, M. H., The height of nightglow, 5577. J. Atmospheric and Terrest. Phys. 12, 171 (1958), 2817.

Marsden, C. P., A survey of the application of ceramics as vacuum tube envelopes. Third Annual Meeting Tube Techniques Conf. (Advisory Group on Electron Tubes, New York, N.Y.) (1956). 1604.

Marsden, C. P., Keery, W. J., Moffitt, J.K., Tabulation of data on receiving tubes. (1959) $\mathrm{H} 68$.

Marshall, P. A., Wales, M., Rothman, S., Weissberg, S. G., Application of viscosity methods to the study of branching in polysaccharides. Ann. N.Y. Acad. Sci. $\mathbf{5 7}, 353$ (1953). 654 .

Marshall, P. A., Wales, M., Weissberg, S. G., Intrinsic viscosity-molecular weight relationships for dextran. J. Polymer Sci, 10, 229 (1953). 745.

Martin, G. M., Mandelkern, L., Glass formation in polymers: II. The system rubber-sulfur. J 62, 141 (1959) RP2944.

Martin, G. M., Rogers, S. S., Mandelkern, L., Volume temperature relations of amorphous polymers over an extended temperature range. J. Polymer Sci. 20, 579 (1956). 1975. 
Martin, G. M., Roth, F. L., Stiehler, R. D., Behavior of "pure gum" rubber vulcanizates in tension. Trans. Inst. Rubber Ind. 32, No. 6, 189 (1956). 1640.

Martin, K. B., Jacobs, R. B., Testing and operation of ball bearings submerged in liquid gases. Proc. 1958 Cryogenie Engineering Conference, Paper $\mathrm{H}-2$, p. 476. Edited by K. D. Timmerhaus, Chemical Engineering Dept., University of Colorado (Boulder, Colorado) (1959); ASLE Trans. 2, No. 1, 101 (1959). 3063

Martin, K. B., Jacobs, R. B., Hardy, R. J., Operation of bearings and pumps at low temperatures. Proc. 1957 Cryogenic Engineering Conference, Paper D-6, p. 209, Edited by K. D. Timmerhaus, Chemical Engineering Dept., University of Colorado (Boulder, Colorado) (1958), 2676.

Performance of pumps with liquefied gases. Proc. 1956 Cryogenic Engineering Conference, Paper G-6, p. 295, Edited by K. D. Timmerhaus, Chemical Engineering Dept., University of Colorado (Boulder, Colorado) (1957). 2243.

Martin, $W$. C., Energy levels and spectrum of neutral helium ( $\mathrm{He} \mathrm{I})$. J 64A 1, 19 (1960)

Value of the Rydberg constant. Phys. Rev. 116, No. 3, 654 (1959). 3109.

Marton, C., Marton, L., Leder, L. B., Mendlowitz, H., Simpson, J. A., Energy losses of electrons and their influence on resolving power. Proc. of the Toulouse Conference, Centre National de la Richerche Scientifique (Paris, France), p. 175 (1955). 1335.

Marton, L., Conference on quantum interactions of the free electron. Rev. Mod. Phys. 28, No. 3, 172 (1956). 1659.

Electron interference and phase effects. Electron interference phenomena. Proc. Intern. Conf. Electron Mieroscopy, July 1954 (Royal Microscopical Society, London, England), p. 272 (1956). 1701.

Electron interferometer. Phys. Rev. 85, No. 6, 1057 (1952). 438.

Electron interferometery. Seience 118, 470 (1953). 707.

Electron scattering phenomena. J. Sci. Ind. Research 16a, No. 6, 221 (1957). 2109.

Electrons vs, photons: a comparison of microseopes. J. Opt. Soc. Am. 40, 269 (1950). 54.

Exploration of electrostatic and magnetic fields. Sei. Monthly 71, 3 (1950). 56.

"Introduction" to July 1956 issue, Rev. Mod. Phys. eontaining all papers of the Intern. Conf. "Quantum Interaetions of the Free Electron" 28, No. 3, 171 (1956). 1748 .

Limiting resolution of electron spectrometers. Rev. Sci. Instr. 29, No. 5, 438 (1958). 2612.

Measuring with the electron. J. Sci. Ind. Research 16A, No. 10, 429 (1957). 2192.

Men and electrons. J. Wash. Acad. Sci. 49, No. 4, 97 (1959). 2989.

Sur les limites du pouvoir de resolution des spectrometres corpusculaires. Bull. acad. roy. de Belg., p. 159 (March 1958). 2791.

Marton, L., Horl, E. M., Cryostat for electron bombardment and electron diffraction work. Rev. Sei. Instr. 29, No. 10,859 (1958). 2506 .

Marton, L., Leder, L. B., Effect of chemical combination of the characteristic energy loss of electrons. Phys. Rev, 95, 1345 (1954). 997.

Energy loss of electrons in passage through thin films. Phys. Rev. 94, 203 (1954). 1014.

Temperature dependence of the characteristic energy loss of electrons in aluminum. Phys. Rev. 112, No. 2, 341 (1958). 2798 .

Marton, L., Leder, L. B., Coleman, J W., Schubert, D. C., Electron beam magnetometer. J 63C1, 69 (1959).

Marton, L., Leder, L. B., Mendlowitz, H., Characteristic energy losses of electrons in solids. Advances in Electronies and Electron Phys. 7, 183 (1955). 1287 Comparison of the characteristie energy losses of electrons with the fine structure of the X-ray absorp- tion spectra. Phys. Rev. 101, No. 5, 1460 (1956). 1655.

Marton, L., Leder, L. B., Mendlowitz, H., Simpson, J. A., Marton, $C$., Energy losses of electrons and their influence on resolving power. Proc. of the Toulouse Conference, Centre National de la Richerche Scientific (Paris, France), p. 175 (1955). 1335

Marton, L., Reverdin, D. L., Electron optical properties of space-charged clouds. J. Appl. Phys, 21, 842 (1950). 53.

Stroboscopic mapping of time-variable fields. J. Appl. Phys. 21, 617 (1950). 140.

Marton, L., Schrack, R. A., Placious, R. C., A field emitter point projection X-ray microscope. X-ray Mieroscopy and Microradiography (Academic Press Inc., New York, N.Y.), p. 287 (1957). 1982.

Marton, L., Schubert, D. C., Mielczarek, S. R., Electron optical image of an atomic beam. J. Appl. Phys. 27, No. 4, 419 (1956). 1704 .

Marton, L., Simpson, J. A., Electron absorption spectrometer using an improved velocity analyzer. Rev. Sci. Instr. 29, No. 7, 567 (1958). 2541.

Marton, L., Simpson, J. A., McCraw, T. F., Automativ instrument for electron scattering measurements. Rev. Sci. Instr. 26, No. 9, 855 (1955). 1274.

Distribution of electron scattering by gold. Phys. Rev. 99, No. 2, 495 (1955). 1317.

Distribution of electrons scattered by solids. Phys. Rev. 104, No. 1, 64 (1956). 1681.

Marton, L., Simpson, J. A., Suddeth, J. A., An electron interferometer. Rev. Sci. Instr. 25, No. 11, 1099 (1954). 947.

Electron beam interferometer. Phys. Rev. 90, 490 (1953). 706.

Marton, L., Simpson, J. A., Suddeth, J. A., Wagner, $M$. D., Watanabe, $H$., Effect of degree in orientation and crystal size on the scattering of 20 -ker. electrons by aluminum. Phys. Rev. 110, No. 5, $1057(1958)$. 2529.

Marton, L., Suddeth, J. A., High-vacuum temperature gradient furnace. Rev. Sci. Instr. 29, No. 5, 440 (1958). 2588.

Marvin, R. S., Measurement of dynamic properties of rubber. Ind. Eng. Chem. 44, 696 (1952). 480. The dynamic mechanical properties of polyisobutylene. Proc. Second Intern. Congr. Rheol. Edited by V. G. W. Harrison (Butterworth's Scientific Publications, London, England) (1954). 1183.

Marvin, R. S., Aldrich, R., Sack, H. S., The dynamic bulk viscosity of polyisobutylene. J. Appl. Phys. 25, No. $10,1213(1954), 1182$.

Marvin, R. S., Edelman, S., McKinney, J. E., Apparatus for the direct determination of the dynamic bulk modulus. J. Appl. Phys. 27, 425 (1956). 1629.

Marvin, R. S., Leaderman, H., Dynamic compliance, dynamic modulus, and equivalent Voight and Maxwell models for polyisobutylene. J. Appl. Phys. 24, 812 (1953). 692

Maryott, A. A., Birnbaum, G., Absorption in the lowfrequency wing of the $\mathrm{NH}_{3}$ inversion spectrum. $J$. Chem. Phys. 21, 1774 (1953). 639.

Change in the inversion spectrum of $\mathrm{ND}_{3}$ from resonant to nonresonant absorption. Phys. Rev. 92, 270 (1953). 661.

Microwave absorption in compressed gases; saturated hydrocarbons. J. Chem. Phys. 24, 1022 (1956). 1778 .

Microwave absorption in compressed oxygen. Phys. Rev. 99, 1886 (1955). 1408.

Nonresonant absorption of symmetric top molecules. Collision cross section. J. Chem. Phys. 29, 1422 (1958). 2648

Temperature dependence of the microwave absorption of $\mathrm{NH}_{3}$. J. Chem. Phys. 22, No. 8, 1457 (1954). 1162.

Maryott, A. A., Birnbaum, G., Wacker, P. F., Microwave absorption by the nonpolar gas $\mathrm{CO}_{2}$. J. Chem. Phys. 22, 1782 (1954). 1074. 
Maryott, A. A., Buckley, F., Tables of dielectric dispersion data for pure liquids and dilute solutions. (1958) C589.

Maryott, A. A., Kryder, S. J., Dipole moment of perchloryl fluoride. J. Chem. Phys. 27, No. 5, 1221 (1957). 2089.

Marzetta, $L$. A., High-power pulser aids cathode studies. Electronics 27, 178 (1954), 1029.

Marzetta, L. A., Gilford, S. R., Gregg, D. E., Shadle, O. W., Ferguson, T. B., An improved cuvette densitometer for cardiac output determination by the dvedilution method. Rev. Sci. Instr. 24, 696 (1953). 650 .

Marzullo, S., Bunting, E. N., Dielectric properties of titania or tin oxide containing varying proportions of rare-earth oxides. J. Am. Ceram. Soc. 41, No. 1, 40 (1958). 2520.

Note on the dielectric properties of magnesiumstrontium titanates. J. Am. Ceram. Soc. 40, No. 8, 285 (1957). 2220.

Masi, J.F., Survey of experimental heat capacities of ten technically important gases. Trans. Am. Soc. Mech. Engrs, p. 1067 (1953). 843.

Thermodynamic properties of gaseous difluorodichloromethane (freon-12). J. Am. Chem. Soc. 74, 4738 (1952). 604 .

Mason, H. L., Sensitivity and life data on Bourdon tubes. Trans. Am. Soc. Mech. Engrs. 30, No. 1, 65 (1956). 1864.

The integration of concepts in the terminology of measurement and control. Trans. Am. Soc. Mech. Engrs. No. 1, 1 (1956). 1935.

Mason, R. D., Jr., Block, S., Burley, G., Perloff, A., Refinement of the crystal structure of triclinic magnesium pyroborate. J 62, 95 (1959) RP2936.

Massengale, G. B., Mong, L. E., Heindl, R. A., Permeability and some other properties of a variety of refractory materials. J. Am. Ceram. Soc. 36, 222 (1953). 794.

Mathey, R. G., Watstein, D., Evaluation of width of cracks in concrete at the surface of reinforcing steel by means of tensile bond specimens. Proc. RILEM Symp. on Bond and Crack Formation of Reinforced Concrete (Stockholm, Sweden) (1957). 2113.

Strains in beams having diagonal cracks. J. Am. Concrete Inst. 30, No. 6, Title No. 55-46 (1958); Proc. Am. Concrete Inst. 55, 717 (1958-59), 2782.

Matsushita, S., A study of the morphology of ionospheric storms. J. Geophys. Research 64, 305 (1959). 2884. Some studies of the upper atmosphere in the auroral zone. Ann. Geophys. 14, 483 (1958). 2766.

Mauer, F. A., Black, I. A., Bolz, L. H., Brooks, F. P. Peiser, $H$. S., A liquid-helium cold cell for use with an X-ray diffractometer. J 61, 367 (1958) RP2907.

Mauer, F. A., Bolz, L. H., Boyd, M. E., Peiser, H. S., A re-examination of the crystal structure of $\alpha$ and $\beta$ nitrogen. Acta Cryst. 12, 237 (1959). 2879.

Mauer, F. A., Bolz, L. H., Peiser, H. S., Low-temperature X-ray studies on Rice's blue material. J. Chem. Phys. 30, No. 1, 349 (1959). 2979.

Maxwell, E., Isotype effect in the superconductivity of mercury. Phys. Rev. 78, 477 (1950). 81.

Similarity properties of the two-fluid model of superconductivity. Phys. Rev. 87, No. 6, 1126 (1952). 543.

Superconductivity of the isotopes of tin. Phys. Rev. 86, No. 2, 235 (1952). 560.

The isotope effect in superconductivity. Phys. Today 5, No. 12, 14 (1952); Phys. Rev. 5, 13 (1952). 581.

Maxwell, E., Lutes, O. S., Superconducting transitions in tin whiskers. Phys. Rev. 97, No. 6, 1718 (1955). 1519.

Threshold field properties of some superconductors. Phys. Rev. 95, 333 (1954). 1212.

Maxwell, E., Schmidt, F. A., Liquid helium cryostat with an integral super-conducting resonator. Suppl. au Bull. de l'Institut Intern. du Froid, Commission 1, Delft, Holland, Annexe 1958-1, p. 95 (1958). $2610 \mathrm{~A}$.
Maxwell, E., Vacher, H. C., Bechtoldt, C. J., Structure of some iridium-osmium alloys. J. Metals 6, No. 1, 80 (1954); Trans. Am. Inst. Mining Engrs. 200, 80 (1954). 1152.

Maxwell, E., Williams, W. E., Jr., Liquid level indicator for condensed gases at low temperature. Rev. Sei. Instr, 25, No. 2, 111 (1954). 1052.

Maxwell, E. L., Watt, A. D., Characteristics of atmospheric noise from 1 to $100 \mathrm{Kc} / \mathrm{s}$. Symp. on the Prop. of V. L. F. Radio Waves, Sponsored by the NBS Boulder Laboratories and IRE Prof. Group on Ant. and Prop., January 23-25, 1957 (Boulder, Colorado), Paper No. 35, V. 3; Proc. IRE 45, 787 (1957). 2052. Low-frequency propagation paths in arctic areas. $J$ 63D1, 99 (1959).

Measured statistical characteristics of VLF atmospheric radio noise. Proc. IRE 45, 55 (1957). 2188.

Maxwell, L. H., Wachtman, J. B., Jr., A bend-test method of determining the stress required to cause ereep in tension. ASTM Bull. No. 211, 38 (1956). 1584A.

Plastic deformation of ceramic-oxide single crystals. J. Am. Ceram. Soc. 37, 291 (1954). 1108.

Plastic deformation of ceramic-oxide single crystals, II. J. Am. Ceram. Soc. 40, No. 11, 377 (1957). 2253.

Mazur, J., Rubin, R. J., Quantum-mechanical calculation of the probability of an exchange reaction for constrained linear encounters. J. Chem. Phys. 31, No. 5,1395 (1959). 3025.

Mazur, P., Oppenheim, I., Density expansions of distribution functions. I. Virial expansion for finite closed systems; canonical ensemble. Physica 23, 197 (1957). 2077.

Density expansions of distribution functions. II. Density expansions in the grand canonical ensemble. Physica 23, 216 (1957). 2078.

Meal, J. H., Mann, D. E., Plyler, E. K., Properties of fluorine compounds, the vibrational spectrum of tetrabromoethylene. J. Chem. Phys. 24, No. 5, 1018 (1956). 1825.

Vibrational spectrum of tetrabromoethylene. J. Chem. Phys. 24, 1018 (1956). 1973.

Meaney, F. M., Bean, B. R., The monthly refractive gradient for the United States and its application to predicting the geographical and annual trend of VHF transmission loss. Proc. Conf. Radio Meteorol. (The University of Texas, Austin, Texas) Supplement III-2 (1953). 870.

Mears, T. W., Armstrong, G. T., Jessup, R. S., Net heat of combustion of aviation gasoline and its correlation with other propeities. Chem. \& Eng. Data Series 3, 20 (1958). 2646.

Mears, T. W., Florin, R. E., High-temperature liquids. (Oct. 1954) Conf. on Reactor Heat Transfer, Report ENL 2446, 89, Brookhaven National Laboratories (Brookhaven, Long Island, N.Y.) (1954). 1032.

Mebs, R. W., Ballard, D. B., Transmission of ultrasonic vibrations in metals. Wright Air Devel. Ctr. (Ohio) Tech. Rept. 56-457 (1957). 2398.

Mebs, R. W., Vacher, H. C., Liss, R., Elastic and plastic behavior of the ferrite lattice in a low-alloy steel. Acta Met. 4, No. 5, 532 (1956). 1691.

Medlock, $R$. W., Marlow, $W . F$., A carbon-14 beta-ray standard, benzoic acid-7-Cit in toluene, for liquid scintillation counters. J 64A2, 143 (1960).

Meggers, E. R., Building construction and maintenance. (1959) BMS140, 3d ed.

Meggers, W.F., Emission spectroscopy. Anal Chem. 24, No. 1, 23' (1952). 443; Anal. Chem. 26, 54 (1954). 1013; Anal. Chem. 28, 616 (1956). 1705.

Present status and future prospects of emission spectroscopy. Rev. Universelles des Mines (Liege, Belgique) No. 5 , p. 7 (1959). 3021 .

Spectroscopy. Encyclopedia Brittanica 21, 180 (1955). 1504 .

Stark effect. Encyclopedia Brittanica 21, 334 (1955). 1510 .

The present experimental status of rare-earth spectra. Opt. i Spekt. 6, 429 (1959). 3090. 
The Zeeman effect in the first spectrum of hafnium. J. Opt. Soc. Am. \&4, 348 (1954), 1208.

What use is spectroscopy? Appl. Spectroscopy 6, No. 4,6 (1952). 622 .

Zeeman effect. Encyclopedia Brittanica 23, 940 (1955). 1584.

Meggers, $W . F$. Catalan, $M$. A., Sales, $M$., Term analysis of the second spectrum of rhenium (Re II). J 61, 441 (1958) RP2914.

Meggers, W. F., Coleman, C. D., Bozman, W. R., Table of wavenumbers, $2000 \mathrm{~A}$ to $7000 \mathrm{~A}$. (1960) Mono. 3 , Vol. I.

Table of wavenumbers, $7000 \mathrm{~A}$ to $1000 \mu$. (1960) Mono. 3, Vol. II

Meggers, $\dot{W}$. F., Corliss, C. H., Improved description of hafnium spectra. J 61, 269 (1958) RP2904.

Meggers, $W$. F., Harrison, $G$. R., Zeeman effect in spark lines of ruthenium. J. Opt. Soc. Am. 43, 816 (1954). 1228 .

Meggers, W. F., Klinkenberg, P.F.A., Velasco, R, Catalán, $M$. $A$., Term analysis of the first spectrum of rhenium (Re I). J 59, 319 (1957) RP2804.

Meggers, W. F., Moore, C. E., Data concerning atomic spectra. J. Opt. Soc. Am. 43, 415 (1953). 677.

Notation for atomic spectra. J. Opt. Soc. Am. 43, 422 (1953). 775.

Meggers, $W . F$., Scribner, $B . F$., Index to the literature on spectrochemical analysis. Part III-1946-1950 (226 pgs), ASTM (1954). 1036.

Index to the literature on spectrochemical analysis, Part IV, 1950-1955. Am. Soc. for Testing Materials Publ. No. 41D (1959). 2965.

Meggers, $W . F$, Shenstone, A. G., The second spectrum of ruthenium ( $\mathrm{Ru}$ II). J 61, 373 (1958) RP2908.

Meggers, $W . F$., Stanley, R. W., Wavelengths from thorium-halide lamps. J 61, 95 (1958) RP2891.

Megill, L. R., Roach, $F$, E., Rees, M. H., Marovich, E., The height of nightglow 5577. J. Atmospheric and Terrest. Phys. 12, 171 (1958). 2817.

Megill, L. R., Roach, F. E., Tandberg-Hanssen, E., Movements of airglow cells. J. Atmospheric and Terrest. Phys. 13, 122 (1958). 2640.

Possible movements of 5577 ainglow. J. Atmospheric and Terrest. Phys. 13, 122 (1958). 2695.

The characteristic size of airglow cells. J. Atmospheric and Terrest. Phys. 13, 113 (1958). 2807.

Meinel, A. B., Roach, $F$. E., Nightglow heights: a reinterpretation of old data. Astrophys. J. 3, 554 (1955). 1416.

The height of the nightglow by the van Rhijn method. Astrophys. J. 122, No. 3, 530 (1955). 1534

Meissner, $P$., Sequential flow cooling of electronic equipment. Elec. Mfg. 58, No. 6, 120 (1956). 1865.

Meissner, P., Stone, R. O., Schwarz, $K$. $M$., Expendable modules as bases for disposal-at-failure maintenance. (1960) TN41 (PB151400)

Menard, J. P., Keegan, H. J., Rheinboldt, W. C., Schleter, $J$. C., Judd, D. B., Digital reduction of spectrophotometric data to Munsell renotations. J. Opt. Soc. Am. 48, 863 (1958). 2522.

Menard, J. P., Rheinboldt, $W$. $C$., Mechanized conversion of colorimetric data to M̈unsell renotations. J. Opt. Soc. Am. 48, 864 (1958). 2633.

Mendlowitz, $H$., A high energy electron-nuclear scattering. Phys. Rev. 102, No. 2, 527 (1956). 1591.

Note on accelerating components in particle spectrometers. Rev. Sci. Instr. 29, No. 8, 701 (1958). 2649. Quantum interactions of the free electron. Phys. Today 9, No. 8, 27 (1956). 1829.

Simplified approach to spin in Dirac theory. Am. J. Phys. 26, No. 1, 17 (1958). 2757.

Mendlowitz, $H$., Case, $K$. $M$., Double scattering of electrons with magnetic interaction. Phys. Rev. 97, $33(1955)$. 1320

Mendlowitz, H., Leder, L. B., Marton, L., Comparison of the characteristic energy losses of electrons with the fine structure of the X-ray absorption spectra. Phys. Rev. 101, No. 5, 1460 (1956). 1655.
Mendlouitz, H., Marton, L., Leaer, L. B., Characteristic energy losses of electrons in solids. Advances in Electronics and Electron Phys. 7, 183 (1955). 1287.

Mendlowitz, H., Marton, L., Leder, L. B., Simpson, J. A., Marton, C., Energy losses of electrons and their influence on resolving power. Proc. of the Toulouse Conference, Centre National de la Richerche Scientique (Paris, France), p. 175 (1955). 1335.

Menon, T. K., Bok, B. J., Lawrence, R. S., Radio observations (al-CM) of dense dark nebulae. Publ. Astron. Soc. Pacific 67, No. 395, 108 (1955). 1468.

Menzel, D. H., Warwick, J. W., Lawrence, R. S., A dynamic spectrum analyzer for Sacramento Peak. Harvard College Observatory (Cambridge, Massachusetts) Scientific Report 20 (1955). 1233.

Meros, E. J., Machlan, L. A., Hague, J. L., Determination of aluminum in precipitation hardening stainless steel and high temperature alloys. J 64A2, 181 (1960).

Merrill, $P . W$., Lines of the chemical elements in astronomical spectra; Appendix A-C. E. Moore and P. W. Merrill, Partial Grotrian Diagrams of Astrophysical Interest. p. 103 Carnegie Inst. Washington, D.C., Publ. 610 (1956). 1758.

Merrill, $\dot{P}$. $W$., Moore, $C$. E., Partial Grotrian diagrams of astrophysical interest. Appendix A, Carnegie Inst. Washington, D.C., Publ. 610, p. 103 (1956). 1758.

Merrill, R. G., Optimum antenna height for ionospheric scatter propagation. IRE Natl. Conv. Record, Pt. I, p. $10(1959)$. 3011.

Metzger, $W$. H., Jr., Characteristics of deposits. Am. Soc. Testing Materials Symp. Electroless Nickel Plating, 265, 13 (1959). 2908.

Metzger, W. H., Lamb, V. A., Designing electroformed parts. Machine Design 25, 124 (1953). 683. Electroforming. Tool Engr. 33, 55 (1954). 1009.

Meyerson, M. R., Rosenberg, S. J., A method of controlled slack quenching of impact bars. Rev. de Metallurgie 55, No. 4, 317 (1958). 2426.

Impact properties of slack-quenched alloy steels. J 59, 273 (1957) RP2799.

The influence of heat treating variables on the martensite transformation in SAE 1050 steel. Trans. Am. Soc. Metals 45, 1225 (1954). 1186.

Michaelis, $R$. E. Investigation of homogeneity of six proposed NBS-GMC zinc-base spectrographic standards. General Motors Spectrographic Committee Spec. Publ. (1955). 1377.

Recent developments in NBS spectrographic standard samples of iron-base alloys. Appl. Spectroscopy 12, 114 (1958). 2719.

Michels, A., Botzen, A., Friedman, A. S., Sengers, V. J., The thermal conductivity of argon for temperatures between $0^{\circ} \mathrm{C}$ and $75^{\circ} \mathrm{C}$ and at pressures up to 2500 atmospheres. Physica 22, 121 (1956). 1952.

Michels, A., Cox, J. A. M., Botzen, A., Friedman, A. S., Contribution to the study of transport phenomena in gases at high densities. J. Appl. Phys. 26, 843 (1955). 1296.

Middleton, $H$. K., Hydraulic research in the United States. 1957. M221; (1958) C., M224; (1959) M227.

Mielczarek, E. V., Frederikse, H. P. R., Thermoelectric power of indium antimonide. Phys. Rev. 99, 1889 (1955). 1559.

Mielczarek, S. R., Marton, L., Schubert. D. C., Electron optical image of an atomic beam. J. Appl. Phys. 27, No. 4, 419 (1956). 1704.

Mielenz, K. D., Use of Chebychev polynomials in thin film computations. J 63A3, 297 (1959).

Mikesell, R. P., Kropschot, R. H., An experimental study of the strength and fatigue of glass at very low temperatures. Proc. 1956 Cryogenic Engineering Conference, Paper D-5, p. 136 , Edited by K. D. Timmerhaus, Chemical Engineering Dept., University of Colorado (Boulder, Colorado) (1957). 2014.

Strength and fatigue of glass at very low temperatures. J. Appl. Phys. 28, 610 (1957). 2318. 
Mikesell, R. P., Reed, R. P., Some mechanical properties of Mylar and Dacron polyester strands at low temperatures. Rev. Sci. Instr. 29, No. 8, 734 (1958). 2761.

The impact testing of various alleys at low temperatures. Proc. 1957 Cryogenic Engineering Conference, Paper F-4, p. 316, Edited by K. D. Timmerhaus, Chemical Engineering Dept., University of Colorado (Boulder, Colorado)(1958). 2819.

The stability of austenitic stainless steels at low temperatures as determined by magnetic measurements. Proc. 1958 Cryogenic Engineering Conference, Paper B-2, p. 84, Edited by K. D. Timmerhaus, Chemical Engineering Dept., University of Colorado (Boulder, Colorado) (1959). 3093.

The tensile and impact strength of annealed and welded 5086 aluminum down to $20^{\circ} \mathrm{K}$. Proc. 1958 Crvogenic Engineering Conference, Paper B-3, p. 101. Edited by K. D. Timmerhaus, Chemical Engineering Dept., University of Colorado (Boulder, Colorado) (1959). 3095.

Miller, J.A., Improved photogrid techniques for determination of strain over short gage lengths. Proc. Soc. Exptl. Stress Anal. Semiann. Rept. 10, 29 (1952). 465 .

Stress-strain and elongation graphs for alclad aluminum-alloy $24 \mathrm{~S}-\mathrm{T} 86$ sheet. Natl. Advisory Comm. for Aeronaut., Washington, D.C. TN 2094 (1950). 139.

Miller, J. A., Albert, A. L., Mechanical tests on specimens from large aluminum-alloy forgings. Natl. Advisory Comm. Aeronaut. Tech. Note 3729 (1956). 1772 .

Miller, J. C. P., A method for the determination of converging factors, applied to the asymptotic expansions for the parabolic cylinder functions. Proc. Cambridge Phil. Soc. (London, England) 48, Pt. 2, 243 (1952). 366.

Miller, J. C. P., Fox, L., Notes on numerical analysis. 5. Table-making for large arguments. Math. Tables and Other Aids to Computation 5, 163 (1951). 272.

Miller, R. G., Johnson, W. H., Prosen, E. J., Heat of formation of boron trichloride. J 62, 213 (1959) RP2956.

Miller, $W$., Effective earth's radius for radiowave propagation beyond the horizon. J. Appl. Phys. 22, No. 1, 55 (1951). 228.

Relative back-scattering of electrons and positrons. Phys. Rev. 82, No. 3, 452 (1951). 300.

Miller, $W$., Kennedy, $R$. J., Attenuation of $86-$ and $176-\mathrm{Mev}$ synchrotron X-rays in concrete and lead. Radiol. Research 4, 360 (1956). 1635A.

Measurement of distortion produced in guarded electrostatic fields by grounded external plates. Rev. Sci. Instr. 25, 1031 (1954). 1066.

$\mathrm{X}$-ray attenuation in lead, aluminum, and concrete in the range 275 to 525 kilovolts. Radiology 65 , No. 6,920 (1955). 1582 .

Miller, W., Motz, J. W., Differential cross-section measurement for 1-Mev bremsstrahlung. Phys. Rev. 96, $544 \mathrm{~L}$ (1954). 993.

Miller, W., Motz, J.W., Cialella, C., Thick target bremsstrahlung specta for $1.00-, 1.25-$, and $1.40-\mathrm{Mev}$ electrons. Phys. Rev. 96, No. 5, 1344 (1954). 1211.

Miller, $W .$, Motz, J. W., Wyclooff, H. O., Eleven-Mev thick target bremsstrahlung. Phys. Rev. 89, 968 (1953). 709.

Miller, W., Motz, J. W., Wyckoff, H. O., Gibson, H. F., Kirn, F. S., Gamma-ray measurements by the magnetic analysis of Compton electrons. Rev. Sci. Instr. 24, 929 (1953). 725.

Milliken, L. T., Frozen free radicals: the problem and the program at the National Bureau of Standards. Capital Chemist $\boldsymbol{\gamma}$, No. 8, 244 (1957). 2130. Quantitative determination of copper in natural rubber. Rubber Age $\boldsymbol{7 1}$, No. 1, 64 (1952). 523.

The Government's bookstore. Capital Chemist 7 , No. 1,10 (1957). 2361.
Milliken, L. T., Linnig, F. J., Interference of antioxidant in the determination of low polymer in SBR synthetic rubber. J. Polymer Sci. XLI, No. 138, 544 (1959), 2967.

Milne, W. E., Numerical methods associated with Laplace's equation. Proc. 2d Symp. on Large-Scale Digital Cal. Mach., Annals of the Harvard Computation Laboratory, Cambridge, Massachusetts XXVI, 152 (1951). 274.

Minkoff, G. J., Everett, A. J., Broida, H. P., Ultraviolet spectrophotometry of low pressure explosions. Fifth Intern. Symp. on Combustion (Reinhold Publ. Corp., New York, N.Y.), p. 779 (1955). 1565.

Minkoff, G.J., Scherber, F. I., Energy release from discharged monatomic gases trapped at $4^{\circ} \mathrm{K}$. J. Chem. Phys. 28, No. 5, 992 (1958). 2546.

Suppression of bubbling in boiling refrigerants as an aid in optical measurements. J. Opt. Soc. Am. 48, 358 (1958). 2790.

Minkoff, G. J., Scherber, F. I., Gallagher, J. S., Energetic species trapped at $4.2^{\circ} \mathrm{K}$ from gaseous discharges. J. Chem. Phys. 30, 753 (1959). 2942.

Minkoff, G.J., Scherber, F. I., Stober, A. K., Suppression of bubbling in boiling refrigerants." Nature 180, 1413 (1957). 2330.

Missimer, J. K., O'Leary, M. J., Scribner, B. W., Resin bonding of offset papers containing mineral fillers. Tappi 34, 506 (1951). 303.

Missimer, J. K., O'Leary, M.J., Scribner, B. W., Erving, $J$. $J$., Manufacture of paper from glass fibers. Tappi 35, No. 7, 289 (1952). 477.

Missimer, J.K., Scribner, B. W., Shaw, M. B., O'Leary, $M, J$., Resin bonding of hardwood fibers in offset papers. Tappi 34, 481 (1951). 302.

Mitchell, J. A., Schoonover, I. C., Dickson, G., Vacher, $H$. C., Some factors affecting the dimensional stability of the Ag-Sn (Cu-Zn) amalgams. J. Dental Research 34, No. 2, 273 (1955). 1493.

Mitchell, N. D., New light on self-ignition. Natl. Fire Prot. Quart. 45, No. 2, 165 (1951). 266.

Mizushima, M., Theory of the stark effect of the NO molecule. Phys. Rev. 109, No. 5, 1557 (1958). 2840.

Mockler, $R$. $C$., Etalons de temps atomiques et moléculaires au National Bureau of Standards. ProcèsVerbaux des Séances, Rapport et Annexes, IRE Session (1957), Comité Consultatif pour la Définition de la Seconde, p. 38, (Comité International des Poids et Mesures. 2. serie, tome 26-B) Paris, France, Gauthier-Villars (1958). 2549.

Mockler, R. C., Barnes, J., Beehler, R., Salazar, H., Fey, L., The ammonia maser as an atomic frequency and time standard. IRE Trans. Instrumentation I-7, No, 3-4, 201 (1958). 2804.

Moffit, J. K., Marsden, C. P., Keery, W. J., Tabulation of data on receiving tubes. (1959) H68.

Mohler, F. L., Isotopic abundance ratios reported for reference samples stocked by the National Bureau of Standards. (1960) TN51 (PB161552).

Reference samples of isotopic abundance. Science 122, No. 3164,334 (1955). 1475.

Mohler, F. L., Bradt, P., Analysis of fluorinated polyphenyls by mass spectrometer. Anal. Chem. 2z, 875 (1955). 1263.

Mass spectra and relative sensitivities of some polyphenyls. J 60, 143 (1958) RP2831.

Mohler, F. L., Bradt, P., Dibeler, V. H., Mass spectra of aromatic hydrocarbons filtered from smoky air. J 60, 615 (1958) RP2876.

Mohler, F. L., Dibeler, V. H., Quinn, E., Redetermination of mass spectra of deuteromethanes. J 61, 171 (1958) RP2896.

Mohler, F. L., Dibeler, V. H., Reese, R. M., Ionization and dissociation of hexafluorobenzene by electron impact. J. Chem. Phys. 26, No. 2, 304 (1957). 2168. Ionization and dissociation of methyl siloxanes by electron impact. J. Chem. Phys. 21, 180 (1953). 747. 
Some ions of high kinetic energy in mass spectra of polyatomic molecules. J. Chem. Phys. 22, 394 (1954). 1144.

The ionization potential of the $\mathrm{CF}_{3}$ radical and some fluorocarbon bond energies obtained by electron impact. J. Chem. Phys, 20, 761 (1952). 580.

Mohler, F. L., Florin, R. E., Wall, L. A., Quinn, E., Dimethylphosphinoborine trimer: mass spectra and thermal decomposition. J. Am. Chem. Soc. $\boldsymbol{\gamma} 6,3344$ (1954). 994

Mohler, F. L, Quinn, E. I. Mass spectra of some deuterostyrenes. J 62, 39 (1959) RP2926.

Mohler, F. L., Wall, L. A., Straus, S., Florin, R. E., Bradt, P., Phosphinoborine compounds: mass spectra and pyrolysis. J $\mathbf{6 3 A} 1,63$ (1959).

Mohler, $N$. F., Heindl, R. A., Oxidation studies of some natural graphites. J. Am. Ceram. Soc. 38, No. 3, $89(1955) 1437$.

Mohler, W. F., Holden, D. B., Tropospheric scatter propagation and atmospheric circulations. J 64D 1, 81 (1960).

Mong, L. $\dot{E}$., Adelman, D. M., Control of factors affecting the reproducibility of mechanical properties of refractory semi-dry press specimens. J. Am. Ceram. Soc. 41, No. 7, 267 (1958). 2501.

Mong, L. E., Donoghue, J. J., Zirconia and alumina shapes. Am. Ceramic Soc. Bull. 29, 405 (1950). 173 .

Mong, L. E., Kerper, M. J., Stiefel, M. B., Holley, S. F., Evaluation of tensile compressive, torsional, transverse, and impact tests and correlation of results for brittle cermets. J 61, 149 (1958) R P2895.

Mong, L. E., Mackles, L., Heindl, R. A., Chemical analyses, surface area, and thermal fractions of natural graphite, and refractoriness of the ashes. J. Am. Ceram. Soc. 36, 266 (1953). 665.

Mong, L. E., Massengale, G. B., Heindl, R. A, Permeability and some other properties of a variety of refractory materials. J. Am. Ceram. Soc. 36, 222 (1953). 794

Mong, L. E., Pendergast, W. L., Dynamic and static tests for mechanical properties of fired plastic refractories and other more resilient materials, J. Am. Ceram. Soc. 39, No, 9, 293 (1956) 1684.

Mong, L. E., Schneider, S. J., Elasticity, strength, and other related properties of some refractory castables. J. Am. Ceram. Soc, 41, No. 1, 27 (1958). 2536. Thermal length changes of some refractory castables. J 59, 1 (1957) RP2768.

Monk, C. B., Bates, R. G., Guggenheim, E. A., Harned, H. S. Ives, D. J. Janz, G. J. Robinson, R. A., Stokes, $R$. H., Wynne-Jones, W. F. J., Standard electrode potential of the silver, silver chloride electrode. J. Chem. Phys. 25, 361 (1956). 1895.

Montgomergy, G. F., A comparison of amplitude and angle modulation for narrow-band communication of binary-coded messages in fluctuation noise. Proc. IRE 42, 447 (1954), 915.

An incremental delay pulse generator. Electronics 42, No, 2, 218 (1951), 191

Communication via meteor bursts. Radio-Electronics 29, 88 (1958). 2491 .

High input impedance transistor amplifier. Electronic Design 6, No. 16, 48 (1958). 2586.

Intermediate-frequency gain stabilization with inverse feedback. Proc. IRE 38, 662 (1950). 78.

Intermittent communications with a fluctuating signal. Proc. IRE 45, No. 12, 1684 (1957). 2163. Message error in diversity frequency-shift reception. Proc. IRE 42, No. 7, 1184 (1954). 1071.

Monostable multivibrator design. Tele-Tech. 9, No. 10, $31(1950), 93$.

On the transmission error function for meteor-burst communication. Proc. IRE 46, $1423(1958), 2674$. The cascade-binary counter with feedback. J. Appl. Phys. 22, 780 (1951). 326.

The diode reactance modulator. Flec. Eng. 77, 615 (1958); AIEE Trans. Commun. and Electronics 78, No. $40,980(1959) .3070$.
Transistor beta tester. Electronics 30, No. 5, 198. (1957). 2396.

Ultrasonic switch aids diversity reception. Electronics 28, No. 11, 1169 (1955). 1564.

Wireless microphone uses F-M modulation. Electronics 31. No. 1, 54 (1958). 2869.

Montgomery, G. F., Bailey, D. K., Bateman, R., Berkner, L. V., Booker, H. G., Purcell, E. M., Salisbury, M. W.', Wiesner, J. B., A new kind of radio propagation at very high frequencies observable over long distances. Phys. Rev. 86, No. 2, 141 (1952). 369.

Montgomery, G. F., Kettering, C. A., How the U. S. Weather Bureau uses Fax \& Telemetering: how FM expedites weather data distribution. FM-TV Radio Comm. 10, No. 10, 17 (1950). 69.

Montgomery, G. F., Stansbury, C., Clamp-on microammeter measures a-c current. Electronics 30, No. 12 152 (1957). 2056.

The clamp-type alternating-current microammeter. Am. Inst. Elec. Engrs. Paper No. 57114 (1957). 2347.

Montgomery, G. F., Sugar, G.R., The utility of meteor burst for intermittent radio communication, Proc. IRE 45, No. 12,1684 (1957). 2389.

Montgomery, G. F., Sulzer, P. G., Wide-range resonators for VHF and UHF. Electronics 42, No. 5, 200 (1951). 357 .

Montgomery, G. F., Sulzer, P. G., Gerks, I. H., An U-H-F moon relay. Proc. IRE 40, 36 (1952). 396

Montroll, E. W., Bazley, N.W., Rubin, R.J., Shuler, K. E., Studies in nonequilibrium rate processes, III. The vibrational relaxation of a system of anharmonic oscillators. J. Chem. Phys. 28, 700 (1958). 2786.

Montroll, E. W., Jackson, J. L., Free radical statistics. J. Chem. Phys. 28, 1101 (1958). 2571.

Montroll, E. W., Shuler, K. F., Application of the theory of stochastic processes to chemical kinetics. Chapter in book, Advances in Chemical Physics (Interscience Publ., New York, N.Y.) 1, p. 361 (1959). 2898 .

Studies in nonequilibrium rate processes. I. The relaxation of a system of harmonic oscillators. J. Chem. Phys. 26, 454 (1957). 2324.

The application of the theory of stochastic processes to chemical kinetics. Chapter in book, Advances in Chemical Physics (Interscience Publ. Inc., New York, N.Y.) 1, 361 (1958). 2805.

Mooney, R. C. L., Crystal structure of anhydrous indium phosphate and thallic phosphate by X-ray diffraction. Acta Cryst. 9, No. 95-204 Pt. 2, 113 (1956). 1667. The crystal structure of aluminum phosphate and gallium phosphate low cristobalite type. Acta. Cryst. 9, 728 (1956). 1923.

The structure of anhydrous scandium phosphate. Acta Gryst. 9, 677 (1956). 1950.

Moore, C. E., A multiplet table of astrophysical interest Part I. Table of multiplets and Part II. Finding list of all lines in the table of multiplets. (1959) TN36 (PB151395)

Atomic energy levels (as derived from the analyses of optical spectra). (1958) C467, Vol. III.

Atomic spectra. Chapter 19 of Fundamental Formulas of Physics, Editor D. H. Menzel. p. 451. (PrenticeHall, Ine., New York, N.Y.) (1955). 1269.

Atomic spectra for the astrophysicist. Science 113, No. 2946, 669 (1951) 197.

Atoms and ions in the sun. Science 119, No. 3093, 499 (1954). 958.

Henry Norris Russell. Observatory 77, No. 897, 67 (1957). 2150.

Infrared multiplets and the solar spectrum. J. Opt. Soc. Am. 43, No. 11, 1014 (1953). 743.

Repcrt on Joint Commission for Spectroseopy meeting. J. Opt. Soc. Am, 46, 1004 (1956). 1849.

Table 664 "Elements in the Sun's Atmosphere"; Forsythe, W. E., Smithsonian Physical Tables, Vol. 120, Publ. 4169 (Smithsonian Institution, Washington, D.C.) (1954) 1138

Tables of spectra. Trans. Intern. Astron. Union 9, 212 (1957). 2337. 
Technetium in the sun. Science 114, 59 (1951). 322, The identification of solar lines, Chapter 4 of The Sun. The identification of solar lines, ch. 4 of The Sun, vol. I of the Solar System. p. 186 (Edited by G. P. Kuiper, The University of Chicago Press, Chicago, Illinois) (1953). 864 .

Vistas in astronomy, Chapter 13, Vol. 2, Atomic Spectra-Their role in astrophysies. p. 1209 (Pergamon Press, New York, N.Y.) (1956). 1974.

Moore, C. E., Broida, H. P., $\mathrm{CH}$ in the solar spectrum. J 63A 1, 19 (1959).

Molecules in the solar spectrum. 7th Intern. Astrophys. Symp. Proc. Memoirs of Royal Soc. (Liege, Belgium) Series 4, Vol. 18, 217 (1957). 2205. $\mathrm{OH}$ in the solar spectrum. J 63A3, 279 (1959).

Rotational lines of $\mathrm{CH}, \mathrm{OH}$ and $\mathrm{CN}$ in the solar spectrum. 7th Intern. Astrophys. Symp. Proc. Memoirs of Royal Soc. (Liege, Belgium) Series 4, Vol. 18, 252 (1957). 2292.

Moore, C. E., Kiess, C. C., Predicted lines of Fe $\mathbf{I}$ in the arc and in the sun. Astron. J. 55, 173 (1950). 111.

Moore, C. E., Meggers, W. F., Data concerning atomic spectra. J. Opt. Soc. Am. 43, 415 (1953). 677.

Notation for atomic spectra. J. Opt. Soc. Am. 43, 422 (1953). 775.

Moore, C. E., Merrill, P. W., Partial Grotrian diagrams of astrophysical interest. Appendix A, Carnegie Inst. Washington, D.C., Publ. 610, p. 103 (1956). 1758.

Moore, C. E., Wilson, N. L., Tousey, R., Purcell, J. D., A revised analysis of the solar spectrum from 2990 to 2635A. Astrophys. J. 119, No. 3, 590 (1954). 933.

Moore, D. G., Bonding agents for high-temperature strain gages. Am. Soc. Testing Materials, Spec. Tech. Publ. No. 230, 106 (1958). 2474.

Weatherability of porcelain enamels. Proc. of Conf. on Architectural Porcelain Enamel, Auspices of Architectural Division-Porcelain Enamel Institute, p. 39 (1955). 1578 .

Weathering of porcelain enamel. Proc. Porcelain Enamel Institute 17, 136 (1955). 1579.

Moore, D. G., Cuthill, J. R., Protection of low-strategic alloys with a chromium boride-nickel cermet coating. Am. Ceram. Soc. Bull. 34, 11 (1955). 1463.

Moore, D. G., Eubanks, A. G., Effeet of the oxygen content of the furnace atmosphere of the adherence of vitreous coatings to iron. J. Am. Ceram. Soc. 38, No. 7, 226 (1955). 1329.

Influence of copper ions on the adherence of vitreous coatings to stainless steel. J. Am. Ceram. Soc. 39, No. 10,357 (1956). 1737.

Moore, D. G., Eubanks, A. G., Richmond, J. C., Studies of oxidation behavior of enameling iron during firing. Finish 11, No. 5, 50 (1954). 1153.

Moore, D. G., Pitts, J. W., Buzzard, E., Resistance measurement of ceramic-type strain-gage cements. Am. Soc. Testing Materials, Spec. Tech. Publ. No. 230, 67 (1958). 2739 .

Moore, D. G., Pitts, J. W., Harrison, W. N., Role of nickel dip in the enameling of sheet steel. J. Am. Ceram. Soc. 37, No. 8, 363 (1954). 1133.

Moore, D. G., Pitts, J. W., Richmond, J. C., Harrison, $W$. N., The galvanic corrosion theory for adherence of porcelain enamel ground coats to steel. Inst. Vitreous Enamellers Ltd. Bull. (Ripley, England) 7, No. 4 (1957). 2358.

Moore, D. G., Richmond, J. C., Kirkpatrick, H. B., Harrison, $W . \quad N$., Relation 'between roughness of interface and adherence of porcelain enamel to steel. Inst. Vitreous Enamellers Ltd. Bull. (Publ. by J. Am. Ceram. Soc.) 7, No. 5, 69 (1957). 2281.

Moore, $D$. G., Thornton, $H$. R., Effect of oxygen on the bonding of gold to fused silica. J 62, 127 (1959) RP2942.

Moore, G. A., Preparation of metallic iron of high purity. J. Metals 5, No. 11, 1443 (1953); Trans. Am. Inst. Mining \& Metallurgical Engrs. (Mining Branch) 197, 1443 (1953). 801.
Moore, G. E., Shuler, K. E., Silverman, S., Herman, R. C., The reactions of ammonia and hydrazine with oxygen atoms and hydrogen atoms in atomic flames. J. Phys. Chem. 60, 813 (1956). 1948.

Moore, H. S., Kirby, R. S., Taff, J. M., Measurement of the effect of irregular terrain on VHF and UHF directive antenna patterns. IRE Trans. Ant. Prop. AP-3, 167 (1952). 481.

Moore, H. S., Phillips, M. L., Dispersion of F2-layer critical frequencies. Proc. IRE 39, 717 (1951). 225.

Moore, H. S., Stein, M., Comparison of variously derived solar indexes. J. Geophys. Research 55, 423 (1950). 40.

Moore, R. B., Hudson, D. C., Hartley, J. L., Sweeney, W. T., Some factors influencing the cutting characteristies of rotating dental cutting instruments. J. Am. Dental Assoc. 50, 377 (1955). 1494.

Moore, R. C., Forecasting of disturbed high frequency communication conditions. J. Atmospheric and Terrest. Phys. Special Suppl. 1957, Proc. of the Polar Atmosphere Symp., July 2-8 1956 (Oslo, Norway), Pt. II, 147 (1957). 2127.

Moore, W. J., Wall, L. A., Pyrolysis of mixtures of ethane and ethane-ds. J. Am. Chem. Soc. 73, 2840 (1951). 291.

Mordfin, L., Creep and creep rupture characteristics of some riveted and spot-welded lap joints of aircraft materials. Natl. Advisory Comm. Aeronaut. Tech. Note 3412 (1955). 1299.

Mordfin, L., Greene, G. E., Halsey, N., Harwell, R. H., $J$ r., Bloss, R. L., Creep and static strengths of large bolted joints of forged aluminum alloys under various temperature conditions. Inst. of Aeronaut. Sci. Preprint No. 779 (1958). 2505.

Mordfin, L., Halsey, N., Granum, P. J., Investigation of bearing creep of two forged aluminum alloys. (1960) TN55 (PB161556).

Mordfin, L., Legate, A. C., Creep behavior of structural joints of aircraft materials under constant loads and temperatures. Natl. Advisory Comm. Aeronaut. Tech. Note 3842 (1957). 2071.

Moreland, R. E., Knudsen, F. P., Geller, R. F., Physical characteristics of titanium carbide type cermets at elevated temperatures. J. Am. Ceram. Soc. 38, No. 9,312 (1955). 1445.

Morey, F. C., Bean, H. S., Correction factors for the balancing effect of a gas in one leg of a manometer. Instruments 24, 528; Oil and Gas J. 50, No. 11, 107 (1951). 212.

Morgan, A. H., Precise time synchronization of widely separated clocks. (1959) TN22 (PB151381).

Morgan, A. H., Phelps, F. P., Research at NBS Boulder Laboratories on quartz crystal resonator and oscillators at low temperatures. Proc. 12th Annual Symp. on Frequency Control, May 6-8, 1958, U.S. Ármy Signal Research and Development Laboratory (Ft. Monmouth, N.J.), p. 162 (1958). 2734.

Morgan, G. H., Broida, H. P., Optical spectrophotometrie analysis of hydrogen-deuterium mixtures in presence of air. Anal. Chem. 24, 799 (1952). 505.

Morowitz, H. J., Broida, H. P., Flames. Sci. Monthly 80, No. 1, 3 (1955). 1347.

Morris, P. P., Jr, Dibeler, V. H., Franklin, J. L., Mass spectrometric study of the rate of thermal decomposition of hydrazoic acid. J 61, 41 (1958) RP2881.

Morrow, W. L., Aldehyde, carboxyl, pentosan, and degree of polymerization data on the ICCA "standard" pulps. Tech. Assoc. Pulp Paper Ind. 42, No. 3, 169 (1959). 2890.

Morrow, W. L., Flynn, J. H., Wilson, W. K., Degradation of cellulose in a vacuum with ultraviolet light. J 60, 229 (1958) RP2841.

Morse, P. M., Pellam, J. R., Thermal Rayleigh disk in liquid helium II. Phys. Rev. 78, 474 (1950). 157.

Mosburg, E. R., Jr., Seintillation counter method of intercomparing neutron source strengths by means of a manganous sulfate bath. J 62, 189 (1959) RP2952. 
Moshanos, M. G., Brauer, E. M., White, E. E., Jr., The reaction of metal oxides with $o$-ethyoxybenzoic acid and other chelating agents. J. Dental Research 37, 547 (1958). 2829.

Standards for neutron flux measurement and neutron dosimetry. 2d United Nations Internat. Conf. on the Peaceful Uses of Atomic Energy, Vol. 21, Health and Safety; Dosimetry and Standards, P/752, USA, p. 92 (Pergamon Press, London, England) (1959). 3055 .

Motz, $J, W$., Bremsstrahlung differential cross-section measurements for 0.5 - and $1.0-\mathrm{Mev}$ electrons. Phys. Rev. 100, No. 6, 1560 (1955). 1278.

Bremsstrahlung polarization measurements for 1.0 Mev electrons. Phys. Rev. 104, No. 3, 557 (1956). 1643.

Motz, J. W., Fano, U., Koch, H. W. Evaluation of bremsstrahlung cross sections at the high-frequency lin it. Phys. Rev. 112, No. 5, 1679 (1958). 2551A.

Motz, J. W., Miller, W., Differential cross-section measurement for 1-Mev bremsstrahlung. Phys. Rev. 96, 544L (1954). 993.

Motz, J. W., Miller, W., Cialella, C., Thick target bremsstrahlung spectra for 1.00-, 1.25-, and 1.40Mev electrons. Phys. Rev. 96, No. 5, 1344 (1954). 1211.

Motz, J. W., Miller, W., Wyckoff, H. O., Eleven-Mev thick target bremsstrahlung. Phys. Rev. 89, 968 (1953). 709.

Motz, J. W., Miller, W., Wyckoff, H. O, Gibson, H. F., Kirn, F. S., Gamma-ray measurements by the magnetic analysis of Compton electrons. Rev. Sći. Instr. 24, 929 (1953). 725

Motz, J. W., Placious, R. C., Bremsstrahlung crosssection measurement for 50-kev electrons. Phys. Rev. 109, 235 (1958), 2477.

Polarization near the high-frequency limit of $500-\mathrm{kev}$ bremsstrahlung. Phys. Rev. 112, No. 4, 1039 (1958) 2694.

Motzkin, T.S., The assignment problem. Proc. 6th Symp. Appl. Math. of Am. Math. Soc. (Santa Monica, Calif., 1953), p. 109 (1956), 1921.

Two consequences of the transposition theorem on linear inequalities. Econometrica 19, No. 2, 184 (1951). 353.

Motzkin, T. S., Forsythe, G. E., An extension of Gauss' transformation for improving the condition of systems of linear equations. Math Tables and Other Aids to Computation 6, No. 37, 9 (1952). 393.

Motzkin, T. S., Raiffa, H., Thompson, G. L., Thrall, R. M., The double description method. Contributions to the theory of games II. Edited by H. W. Kuhn and A. W. Tucker. Annals of Math. Study 28, Chapter 3 (Princeton, N.J.) (1953). 857.

Motzkin, T. S., Schoenberg, I. J., On lineal entire functions of $n$ complex variables. Proc. Am. Math. Soc. 3, No. 4,517 (1952). 498 .

The relaxation method for linear inequalities. Can. J. Math. 6, 393 (1954). 1197.

Motzkin, T. S., Straus, E. G., Valentine, $F$. A., The number of farthest points. Pacific J. Math. 3, 221 (1953). 876 .

Motzkin, T. S., Taussky, O., On representations of finite groups. Koninkl, Nederl. Akademie Van Wetenschappen Proc. (Amsterdam, Holland), Series A, 55. No. 5; Indag. Math (Amsterdam, Holland) 14, No. $5,511(1952) .499$.

Pairs of matrices with property L. Trans. Am. Math. Soc. 73, No. 1, 108 (1952). 507; Proc. Natl. Acad. Sci. 39, 961 (1953). 791.

Pairs of matrices with property L. II. Trans. Am. Math. Soc. 80, No. 2, 387 (1955). 1440.

Motzkin, T. S., Walsh, J. L., Least pth power polynomials on a real finite point set. Trans. Am. Math. Soc. 78, No. 1, 67 (1955). 1385.

On the derivative of a polynomial and Chebyshev approximation. Proc. Am. Math. Soc. 4, 76 (1953). 786.
Motzkin, T. S., Wasow, W., On the approximation of linear elliptic differential equations by difference equations with positive coefficients. J. Math. and Phys. 31, 253 (1953). 785.

Mountjoy, W., McKee, S. A., Swindells, J. F., White, H. S., For wear prevention. SAE J. 58, 47 (1950). $\quad 59$.

Mowery, W. E., Burns, C. L., Dickson, G., Sweeney, W. T., Dimensional stability of denture base resins. J. Am. Dental Assoc. 57, 345 (1958.). 2523.

Moyer, J. D., Isbell, H. S., Carbon-14 carboxy-labeled polysaccharides. J 61, 71 (1958) RP2886.

Determination of reducing sugars and reducing end groups in polysaccharides by reaction with carbon-14labeled cyanide. Anal. Chem. 30, No. 12, 1975 (1958). 2517.

Preparation of carbon-14-labeled cyanide. Anal. Chem. 29, 393 (1957). 2262.

Structural analysis of clinical dextrans by periodate oxidation and isotope dilution techniques. Anal. Chem. 29, 1862 (1957). 2319.

Tritium-labeled compounds II. General-purpose apparatus, and procedures for the preparation, analysis, and use of tritium oxide and tritium-labeled borohydride. J 63A2, 177 (1959).

Moyer, J. D., Isbell, H. S., Frush, H. L., Use of carbon14 and tritium for the study and characterization of cellulose and other polysaccharides. Tappi 40, No. 9,739 (1957). 2403.

Moyer, J. D., Isbell, H. S., Frush, H. L., Holt, N. B., Tritium-labeled compounds III. Áldoses-l-t. J 64A 2, 177 (1960).

Moyer, J. D., Isbell, H. S., Smith, F. A., Creitz, E. C. Frush, H. L., Stewart, J. E., Infrared spectra of sugar acetates in solution. J 59, 41 (1957) RP2772.

Moyer, $J . W_{1}, \quad$ Free radicals research symposium. Phys. Today 11, 14 (1958). 2572.

Moyer, $J . W .$, Broida, $H$. P., Spectroscopic analysis of deuterium in hydrogen-deuterium mixtures. J. Opt. Soc. Am. 42, 37 (1952). 551.

Moyles, B. N., Marcus, M., Westwick, R., Some extreme value results for indefinite Hermitian matrices. Illinois J. Math. 1, 449 (1957). 2303.

Mrose, M. E., Clark, J.R., Perloff, A., Burley, G., Studies of borate minerals. VI: Veatchite. Am. Mineralogist 44, No. 11-12, 1141 (1959). 3057.

Muehthause C. O., Neutron physics. Handbook of Physics. Edited by E. U. Condon and H. Odishaw, Chapter 7, Pt. 9, p. 9-125 (MeGraw-Hill Book Co., Inc., New York, N.Y.) (1958). 2500.

Pile oscillator measurements of eta. Nuclear Sci. and Engr. 5, No. 4, 225 (1959). 3015.

Mullen, L. O., Arnett, R. W., Warren, K. A., Some methods for reducing heat lead through support members in liquefied gas storage vessels. Proc. 1958 Cryogenic Engineering Conference, Paper G-1, p. 410. Edited by K. D. Timmerhaus, Chemical Engineering Dept., University of Colorado (Boulder, Colorado) (1959). 3049.

Mullen, P. A., Mann, W. B., Stockmann, L. L., Youden, W. J., Schwebel, A., Garfinkel, S. B., Preparation of new solution standards of radium. J 62, 21 (1959) RP2924.

Mulligan, B. W., Blau, E. J., Raman spectrum of triethylborane.' J. Chein. Phys. 26, 1085 (1957). 2278.

Mulligan, $B$. W., Haught, $A$. $F$., Correction for instrumental drift in flame photometry. J 61, 503 (1958) RP2918.

Mulliken, R. S., Plyler, E. K., Molecular complexes and their spectra. IX. Infrared absorption by iodine in its pyridine complexes and in benzene. J. Am. Chem. Soc. 81, 823 (1959), 2994.

Muncy, J. H., National Bureau of Standards preferred circuits program. Elec. Eng. 74, No. 12, 1088 (1955). 1412.

Preferred circuits manual with engineering notes. Bu. Aeronaut Report 3492 (1954). 1113.

Reliability starts with circuit design. Aviation Age 27, 1 (1957), 2283. 
Mundy, R. E., Cooter, I. L., Cunife wire magnets of small size. J 59, 379 (1957) RP2808.

Munson, I. K., Mierowave power stabilizer. Rev. Sci, Instr. 21, 622 (1950). 90.

Murcray, D. G., Gates, D. M., Shaw, C. C., Herbold, R. J., Near infrared solar radiation measurements by balloon to an altitude of $100,000 \mathrm{ft}$. J. Opt. Soc. Am. 48, No. $12,1010(1958) .2645$.

Murphey, W. M., Hoffman, J. D., Weeks, J. J., Experimental and theoretical study of kinetics of bulk crystallization in poly(chlorotrifluoroethylene). J 63A 1,67 (1959).

Murphy, A., Wait, $J . R$. ., Further studies of the influence of a ridge on the low-frequency ground wave. J 61, 57 (1958) RP2884.

Multiple reflections between the earth and the ionosphere in VLF propagation. Ceofisica pura e appl. 35, $61(1956)$, 1781 .

The geometrical optics of VLF sky wave propagation. Symp. on the Prop. of V. L. F. Radio Waves, Sponsored by the NBS Boulder Laboratories and IRE Prof. Group on Ant. and Prop., January 23-25, 1957 (Boulder, Colorado), Paper No. 5, V. 1; Proc. IRE 45, $754(1957) .2360$.

Murphy, V. F., Jr., Malitson, I. H., Rodney, W. S., Refractive index of synthetic sapphire. J. Opt. Soc. Am. 48, No. 1, 72 (1958). 2727.

Mutcher, $M, K .$, Reinhart, $F$.W., Fluorescent sunlamps in laboratory aging tests for plastics. ASTM Bull. No. 212,45 (1956). 1713.

Myerson, R. L., Sweeney, W. T., Rose, E. E., Semmelman, J. O., Proposed specification for plastic teeth. J. Prosthetic Dentistry 7, 420 (1957). 2272.

\section{$\mathrm{N}$}

Nakata, Y., Kato, T., Fujita, H., Newman, M., Estimation of the frequencies of thin elastic plates with free edges. J 59, 169 (1957) RP2784.

Nall, J. R., Haliday, W. G., Drain casting of a highgrade magnesium oxide support used in an experimental vacuum tube. Rev. Sei. Instr. 25, No. 12, 1225 (1954). 996.

Napolitano, A., Waxler, $R$. M., Relative stress-optical coefficients of some National Bureau of Standards optical glasses. J 59, 121 (1957) RP2779.

Natrella, $M, G$., A note on the computation of $X^{2}$. Am. Stat. 13, No. 1, 20 (1959). 2877.

Nelms, A. T., Energy loss and range of electrons and positrons. (1958) C577 Suppl.

Nelms, A. T., Cooper, J., $\mathrm{U}_{235}$ fission product decay spectra at various times after fission. Health Physies 1, 427 (1959). 3105.

Nelms, A. T., Fano, U., An approximate expression for gamma-ray degradation spectra. J 59, 207 (1957) RP2788.

Nelson, R. A., Band, W., The vapor pressure of $\mathrm{He}^{3}-\mathrm{He}^{4}$ mixtures. Phys. Rev. 88, No. 6, 1431 (1952). 599.

Nelson, R. A., Jessup, R. S., McCoskey, R. E., The heat of formation of tetrafluoromethane. J. Am. Chem. Soc. 77, 244 (1955). 1533.

Nelson, R. A., Johnson, W. H., Prosen, E. J., Heat of formation of titanium tetrachloride. J 62, 49 (1959) RP2928.

Heat of formation of titanium tetrabromide. J $\mathbf{6 2}$, 67 (1959) RP2932.

Nelsen, R.J., Relationships of dental materials to elinical subjects. J. Dental Education 19, 62 (1955). 1476.

Nelsen, R. J., Paffenbarger, G. C., Sweeney, W. T., Direct and indirect filling resins: a review of some physical and chemical properties. J. Am. Dental Assoc. 47, $516(1953) .688$.

Nelsen, R.J., Pelander, C. E., Kumpula, J.W., Hydraulic turbine contra-angle handpiece. J. Am. Dental Assoc. 47, 324 (1953). 732.

Nelsen, R. J., Smith, S. W., Dangers of radiation. J. Am. Dental Assoc. 50, No. 5, 581 (1955). 1305.

Nelsen, R. J., Wolcott, R. B., Paffenbarger, G. C., Fluid exchange at the margins of dental restorations. J. Am. Dental Assoc. 44, 288 (1952). 451.
Nettleton, R. E., Goldberg, K., Green, M. S., Dense subgraphs and connectivity. Can. J. Math. 11, 262 (1959). 2925.

Nettleton, R. E., Green, M. S., Moebius function on the lattice of dense subgraphs. J 64B 1, 41 (1960).

Expression in terms of molecular distribution functions for the entropy density in an infinite system. J. Chem. Phys. 29, 1365 (1958). 2558.

Newhall, D. H., Johnson, D. P., The piston gage as a precise measuring instrument. Trans. Am. Soc Mech. Engrs, 75, 301 (1953). 877.

Newkirk, G., Jr., A model of the electron corona with reference to radio observations. Paris Symp. Radio Astronomy (IAU Symp. No. 9 and URSI Symp. No. $1)$, paper 27 , p. $149(1959) .2875$.

Newkirk, G. A., Curtis, G. W., Watson, D. K., Manning, $R$., Shelby, J., The inner solar corona during June 1959. Nature 184, 1308 (1959). 3080.

Newhirk, T. F., Differential thermal analysis above $1200^{\circ} \mathrm{C}$. J. Am. Ceram. Soc. 41, 409 (1958), 2521.

Newkirk, T. F., Thwaite, R. D., Pseudoternary system calcium oxide-monocalcium aluminate $\left(\mathrm{CaO} \cdot \mathrm{Al}_{2} \mathrm{O}_{3}\right)-$ dicalcium ferrite $\left(2 \mathrm{CaO} \cdot \mathrm{Fe}_{2} \mathrm{O}_{3}\right)$. J 61, 233 (1958) RP2900.

Newman, A. E., Levin, B. M., Distribution of incoming letter mail at the Baltimore, Maryland City Post Office. (1959) TN33 (PB151392).

Newman, A. E., Severo, N. E., A statistical chain-ratio method for estimating relative volumes of mail to given destinations. J 64C1, 37 (1960).

Distribution of mail by destination at the San Francisco, Los Angeles, and Baltimore post offices. (1959) TN27 (PB151386).

Newman, A. E., Severo, N. C., Young, S. M., Zelen, M., Some applications of statistical sampling methods to outgoing letter mail characteristics. (1959) TN16 (PB151375).

Newman, E. S., A thermochemical study of the reaction of calcium hydroxide, silica gel, and water. J 59, 187 (1957) RP2785.

Heat of formation of potassium calcium silicate. J 62, 207 (1959) RP2955.

Heat of formation of sodium calcium aluminate. J 61, 75 (1958) RP2887.

An alternative proof of a theorem on unimodular groups. Proc. Am. Math. Soc, 6, 998 (1955). 1260. An identity for the coefficients of certain modular forms. J. London Math. Soc. 30, 488 (1955). 1262.

Newman, $M$., A table of the coefficients of the powers of $\eta(\gamma)$. Konikl. Ned. Akad. Wetenschap. Proc. 59, 2 (1956); Indag. Math. 18, 2 (1956). 1605.

An inclusion theorem for modular groups. Proc. Am. Math. Soc. 8, 125 (1957). 2016.

Congruences for the coefficents of modular forms and for the coefficients of $j(\tau)$. Proc. Am. Math. Soc. 9, 609 (1958). 2498.

Congruences for the coefficients of modular forms and some new congruences for the partition function. Can. J. Math. 9, 549 (1957), 2068.

Construction and application of a class of modular functions. Proc. London Math. Soc. 7, 334 (1957). 2069.

Further identities and congruences for the coefficients of modular forms. Can. J. Math. 10, 577 (1958). 2573 .

Generalizations of identities for the coefficients of certain modular forms. J. London Math. Soc. 31, 205 (1956). 1720.

Kantorovich's inequality. J 64B1, 33 (1960).

On the existence of identities for the coefficients of certain modular forms. J. London Math. Soc. 31, 350 (1956). 1797.

Remarks on some modular identities. Trans. Am. Math. Soc. 73, No. 2, 313 (1952). 529.

Some theorems about pr $(\eta)$. Can. J. Math. 9, 68 $(1957), 2305$.

Structure theorems for modular subgroups. Duke Math. J. 22, No. 1, 25, (1955). 1517. 
The coefficients of certain infinite products. Proc. Am. Math. Soc. 4, 435 (1953). 853.

The normalizer of certain modular subgroups. Can. J. Math. 8, No. 1, 29 (1956). 1942.

Weighted restricted partitions. Acta arith. V, 371 (1959). 3112.

Newman, $M$., Goldberg, $K$., Pairs of matrices of order two which generate free groups. Illinois J. Math. 1, No. 3, 446 (1957). 2241.

Newman, M., Goldberg, K., Straus, E. G., Swift, J. D., The representation of integers by binary cuadratic rational forms. Arch. math. (Tübinger, Germany) $5,12(1954) .1198$

Newman, M., Hoffman, A. J., Straus, E, G., Taussky, O., The number of absolute points of a correlation. Pacific J. Math. 6, 83 (1956). 1943.

Newman, $M_{\text {, }}$ Kato, T., Fujita, H., Nakata, $Y$., Estimation of the frequencies of thin elastic plates with free edges. J 59, 169 (1957) RP2784.

Newman, $M$., Marcus, $M$., On the minimum of the permanent of a doubly stochastic matrix. Duke Math. J. 26, 61 (1959). 3008 .

Newman, M., Taussky, O., Classes of positive definite unimodular eireulants. Can. J. Math. 9, 71 (1957). 2057.

On a generalization of the normal basis in abelian algebraic number fields. Commun. on Pure and Appl. Math. (New York University, New York, N.Y.) 9, 85 (1956). 1791

Newman, M., Todd, J., The evaluation of matrix inversion programs. J. Soc. Indust. Appl. Math. 6, 466 (1958). 2814.

Newman, N., Joseph, H. M., Eddy-current mutualinductance transducers with high-conductivity reference plates. Commun. and Electronics (Published by Am. Inst. Elee. Engrs.), Paper 55-4, No. 17, p. 1 (1955). 1323

Newman, P. A., Bay, Z., Comparison of the ionization produced in air by alpha particles near $5-\mathrm{Mev}$ and by beta particles. Bull. Am. Phys. Soc. II :4, No. 4, 217 (1959). 2912.

Newman, S. B., A new technique for cutting very thin sections and its application to the electron microscopy of fibers. ASTM Bull. No, 163, 57 (1950). 4. Some problems in ultramicrotomy. Bull. Intern. Assoc. Med. Museums 32, 100 (195i). 309.

Newman, S. B., Hammond, H. K., III, Riddell, H. F., Becker value of manila rope by photoelastic reflectometry. ASTM Bull. No. 199, 84 (1954). 960.

Newman, S. B., Lee, $W . M_{\text {., }}$ Surface tension measurements with a strain-gauge-type testing machine. Rev. Sci. Instr. 29, 785 (1958), 2794.

Newman, S. B., Reinhart, F. W., Plastic springs. Product. Eng. 27, 183 (1956).' 1810.

Reinforced plastic springs. SPE J. 12, 8 (1956). 1843.

Newman, S. B., Riddell, H. F., The drying twist in plant fibers. Textile Research J. 24, 113 (1954). 1181.

Newman, S. B., Toner, S. D., Achhammer, B. G., Surface erosion of filled plastics. Modern plasties 36, 135 (1958). 2792.

Newman, S. B., Wolock, I., Fracture phenomena and molecular weight in polymethyl methacrylate. J. Appl. Phys. 29, No. 1, 49 (1958). 2569.

Newton, $C$. $J$., False negative permanent strains observed with resistance wire strain gages. ASTM Bull. No, 235, 42 (1959). 2950.

Newton, C. J., Burnett, H. C., Crystallography of cold drawn music wire. Wire and Wire Products 33, No. 1, 66 (1958). 2509.

Variations in preferred orientation and modulus of elasticity in cold drawn music spring wire. Wire and Wire Products 30, No. 11, 1375 (1955). 1573.

Newton, C. J., Mandelkern, L., Roberts, D. E., Preferred ol ientation in stark rubber studied with an automatic integrating pole-figure goniometer. J. Appl. Phys. 26, No. 12, $1521(1955), 1454$.

Newton, C. J., Vacher, H. C., An X-ray study of textural stresses in two-phase alloys. J 59, 239 (1957) RP2793.
Residual lattice strains in sectioned bars of plastically deformed iron. J. Metals 7, No. 11, 1193 (1955). 1479 .

Nicastro, L. J., Caswell, R. S., A double-pulse totalabsorption fast neutron spectrometer. (1959) TN1 (PB151360).

Nigro, J. P., Skramstad, H. K., Ernst, A. A., An analogdigital simulation for the design and improvement of man-machine systems. 1957 Eastern Joint Computer Conf. Proc. (Inst. Radio Engrs., New York, N.Y.) (1958). 2453.

Nimeroff, $I$., Analysis of goniophotometric reflection eurves. Condensation, J. Opt. Soc. Am. 42, 579 (1952). 397.

Propagation of errors in spectrophotometric colorimetry. J. Opt. Soc. Am. 43, 531 (1953). 804. Propagation of errors in tristimulus colorimetry. J. Opt. Soc. Am. 47, 697 (1957). 2266.

Status of ASTM methods and standards for appearance evaluation. Am. Soc. Testing Materials Spec. Tech. Publ. 258, 3 (1959). 3055A.

Nimeroff, I., Hammond, H. K., III, Minimizing anomalies in reflectance measurements with the Beckman quartz spectrophotometer. J. Opt. Soc. Am. 42, 367 (1952). 484

Nimeroff, I., Hammond, H. K. III, Richmond, J. C., Crandell, J. R., Specular-gloss measurement of ceramic materials. J. Am. Ceram. Soc. 39, No. 3, 103 (1956). 1886.

Gloss meter comparisons. J. Am. Ceram. Soc. 39, No. 3, 103 (1956). 1724 .

Nimeroff, I., Laufer, $J$., Spectral band-pass determinations by a dymanic approach. J. Opt. Soc. Am. 48, 864 (1958). 2770.

Nivert, J.M., Jr., Hamilton, E. H., Waxler, R. M., Properties of zinc borosilicate glasses. J 62, 59 (1959) RP2930.

Nobel, L. D. C., Building technology at the National Bureau of Standards. Informes construc. (Madrid, Spain) 4, No, 36, 1 (1951). 200.

Noble, C. E., Goodman, S., Russell, S. B., Effect of internal radiant beat transfer on temperature distribution, thermal stress, and deflection in box beams. J 62, 175 (1959) RP2949.

Norrish, R. G. W., Edgecombe, S. H. C., Thrush, B. A., The flash photolysis of chlorine monoxide. Proc. Roy. Soc. (London, England) 243, 24 (1957). 2357.

Norton, K. A., Carrier frequency dependence of the basic transmission loss in tropospheric forward scatter propagation. (1960) TN53 (PB161554). Low and medium frequency radio propagation (abstract). Congres Intern. sur la Propagation des Ondes Radio-Êlectriques, October 6-11, 1958 (Liége, Belgique) Résumé des commun. p. 20 (1958). 2615. Point-to-point relaying via the scatter mode of tropospheric propagation. IRE Trans. Commun. Systems CS-4, No. 1, 39 (1956). 1812.

Recent experimental evidence favouring the $p \mathrm{~K}_{1}(\rho)$ correlation function for describing the turbulence of refractivity in the troposphere and stratosphere. J. Atmospheric and Terrest. Phys. 15, 206 (1959). 3032 .

System loss in radio wave propagation. J 63D 1, 53 (1959).

Technical considerations leading to an optimum allocation of radio frequencies in the band 25 to 60 Mc. (1959) TN13 (PB151372).

Transmission loss in radio propagation. Proc. IRE 41, 146 (1953). 901 .

Transmission loss in radio propagation, II. (1959) TN12 (PB151371).

Transmission loss of space waves propagated ove1 irregular terrain. IRE Trans. Ant. Prop. AP-3, (1952). 606

Norton, K. A., Allen, E. W., The variation with frequency of the signal range of FM and television broadcasting stations. J. Brit. Inst. Radio Engr. 11, 93 (1951). 347 . 
Norton, K. A., Herhstreit, J. W., Rice, P. L., Schafer, $G$. E., Radio wave scattening in tropospheric propagation. Conv. Record of the IRE, Pt. 2, Antennas and communications, p. 85 (1953). 808.

Norton, K. A.. Kirby, R. S., Herbstreit, J. W., Service range for air-to-ground and air-to-air communications at frequencies above 50 Mc. Proc. IRE. 40, 525 (1952). 540 .

Norton, $K$. A., Rice, $P$. L., Gapless coverage in air-toground communications at frequencies above $50 \mathrm{Mc}$. Proc. IRE. 40, 470 (1952). 457.

Norton, K. A., Rice, P. L., Longley, A. G., Prediction of the cumulative distribution with time of ground wave and tropospheric wave transmission loss. Part 1. The prediction formula. (1959) TN15 (PB151374).

Nortcn, K. A., Rice, P. L., Vogler, L. E., The use of angular distance in estimating transmission loss and fading range for propagation through a turbulent atmosphere over irregular terrain. Proc. IRE 43, 1488 (1955). 1550.

Norton, K. A., Schultz, E. L., Yarbrough, H., The probability distıibution of the phase of the resultant vector sum of a constant vector plus a Rayleigh distributed vector. J. Appl. Phys. 23, No. 1, 137 (1952). 591.

Norton, K. A., Staras, H., Blum, M., A statistical approach to the problem of multiple radio interference to FM and television service. IRE Trans. Ant. Prop. AP-1, 13 (1952). 378

Norton, K. A., Vogler, L. E., Mansfield, W. V., Short, $P$. $J$., The probability distribution of the amplitude of a constant vector plus a Rayleigh-distributed vector. Proc. IRE 43, 1354 (1955). 1541.

Norton, K. A., Wiesner, J. B., The scatter propagation issue. Introduction to special issue of Proc. IRE. Proc. IRE. 43, 1181 (1955). 1544.

Norton, R. B., Wright, J. W., Analysis of ionospheric vertical soundings for electron density profile data. 1. Facilities for convenient manual reduction of ionograms. (1959) TN14 (PB151373).

Notz, W. A., Leiner, A. L., Smith, J. L., Weinberger, A., Organizing a network of computers to meet deadlines. 1957 Eastern Joint Computer Conf. Proc. (Washington, D.C.), p. 115 (1957). 2240.

PILOT, a new multiple computer system. J. Assoc. Computing Mach. 6, No. 3, 313 (1959). 3016. PILOT, the NBS multicomputer system. Proc. Eastern Joint Computer Conf. (Philadelphia, Pa.), p. 164 (1958). 2692.

Nuckolls, R. G., Lyons, H., Rueger, L. J., Kessler, M., Microwave spectra of deutero-ammonias. Phys. Rev. 81, 630 (1951). 260.

Nuckolls, R. G., Rueger, L. J., A broad-band coaxial stark cell for microwave spectroscopy. Rev. Sci. Instr. 23, 635 (1952), 358.

Nuckolls, R. G., Rueger, L. J., Lyons, H., A high temperature, broad-band stark cell for microwave spectroscopy. Rev. Sci. Instr. 22, 428 (1951). 178. Microwave absorption spectrum of $\mathrm{ND}_{3}$. Phys. Rev. 89,1101 (1953). 766 .

Obayashi, T., Measured frequency spectra of very-lowfrequency atmospherics. J 64D 1, 41 (1960).

Oberhettinger, $F$., On a modification of Watson's lemma. J 63B 1,15 (1959).

On asymptotic series for functions occurring in the theory of diffraction of waves by wedges. J. Math. and Phys. 34, No. 4, 245 (1956). 1792.

On some expansions for Bessel integral functions. J 59, 197 (1957) RP2786.

On the derivative of Bessel functions with respect to the order. J. Math. and Phys. 37, 75 (1958). 2666. On the diffraction and reflection of waves and pulses by wedges and corners. J 61, 343 (1958) RP2906. On the Lerch zeta functions. Pacific J. Research 6, No. 1, 117 (1956). 1798
On the propagation of electromagnetic and acoustic pulses, Part I: Diffraction of pulses by wedges. Z. Phys. 146, 423 (1956). 1799.

Ocks, R. G., Carpenter, R. J., Experimental equipment for communication utilizing meteor bursts. IRE Wescon Conv. Session 46: Ionospheric Prop. August 20-23, 1957, San Francisco, Calif. IRE Wescon Conv. Record, Pt. 1, 283 (1957). 2117.

Odishaw, H., Scientific measurement and technological progress. Ind. Lab. 3, No. 9, 8 (1952), 537.

Oeller, R., Dahl, S., The determination of nitrogen in leather by K jeldahl method. J. Am. Leather Chemists Assoe. 46, 317 (195'). 332 .

Oehler, R., Dahl, S., Kilduff, T. J., Treatment of leather with polyisobutylene. J. Am. Leather Chemists Assoc. 47, 642 (1952). 607.

Oekler, R., Davis, J.H., Method for restoring original appearance of impregnated leather. J. Am. Leather Chemists Assoc. 50, 38 (1955). 1407.

Oeiler, R., Davis, J. H., Kinmouth, R. A., A pilot plant study of the process for treating heavy leather with polyisobutylene and other polymers. J. Am. Leather Chemists Assoc. 50, 16 (1955). 1240.

Oehler, R., Kilduff, $T$. J., Dahl, S., Treatment of leather with Castiloa and Hevea rubbers. J. Am. Leather Chemists Assoc. 45, 378 (1950). 160.

Ogburn, $F$., Plating standards and specifications. Electroplating Eng. Handbook, Chapter 7 (Reinhold Publ. Corp., New York, N.Y.) (1955). 1447.

Specifications for electrodeposited coatings. Elec. Mfg. 48, 119 (1951). 313.

Ogburn, F., Cameron, J. M., Acceptance sampling of electroplated articles. Plating 41, No. 1, 43 (1954). 940.

Ogburn, F., Hilkert, M., The nature, cause and effect of porosity in electrodeposits, II. Radiographic detection of porosity in electrodeposits. Ann. Proc. Am. Electroplaters' Soc. 43, 256 (1956). 1940.

Ogburn, $F$. Salmon, H. I. Commutator for periodically changing the direction and magnitude of direct currents. Plating 43, 343 (1956). 1652.

Notes on post-treatment for HAE coatings. Plating 43, 1251. (1956). 1786.

Ogburn, F., Salmon, H. I., Kronenberg, M. L., Electrolytic coatings on magnesium base alloys from alkaline chromate solutions. Plating 42, 271 (1955). 1332.

Ogburn, F., Strang, A. G., Measurement of surface roughness of electrodeposited and electropolished surfaces by means of the microinterferometer. 37th Annual Proc. Am. Electroplaters' Soc. 125 (1950). 86.

O'Grady, M., Wait, J.R., Surface currents excited by an infinite slot on half-planes and ribbons. IRE. Trans. Ant. Prop. AP-4, No. 1, 47 (1956). 1907

Olcott, R. N., Chatterjee, S. D., Absence of spontaneous emission of neutrons from samarium. Phys. Rev. $85,147(1952) .383$.

Oldenberg, O., Broida, H.P., Application of photoelectric multiplier tubes to the sensitive measurement of absorption or of changes of relative light intensities. J. Opt. Soc. Am. 40, 381 (1950). 27.

The thermal hydrogen-oxygen reaction at relatively low temperatures. J. Chem. Phys. 19, 196 (1951). 345 .

O'Leary, M. J., Scribner, B. W., Missimer, J. K., Resin bonding of offset papers containing mineral fillers. Tappi 34, 506 (1951). 303. ary, M. J., Scribner, B. W., Missimer, J. K., Erving, $J$. $J$. Manufacture of paper from glass fibers. Tappi 35, No. 7, 289 (1952). 477.

O'Leary, M. J., Scribner, B. W. Shaw, M. B., Missimer, $J$. K., Resin bonding of hardwood fibers in offset papers. Tappi 34, 481 (1951). 302.

Oliver, $D . W$., Lindeck potentiometer. Rev. Sci. Instr. 26, No. 11, 1078 (1955). 1389.

Oliver, D. W., Spiegel, Y., Jr., Caswell, R. S., Age to indium resonance for 'D-D neutrons in water. Nuclear Sci. and Eng. 4, No. 4, 546 (1958). 2450. 
Olsen, H. L., Berl, W. G., Gayhart, E. L., Broida, H. P., and Shuler, K. E., Spectroscopic observations on pentaborane-air flames and explosions. J. Chem. Phys. 25, No. 4, 797 (1956). 1883.

Olson, $C$. A., Spencer, L. $V$., Energy spectra of cascade electrons and photons. J 60, 85 (1958) RP2824.

Olver, $F . W . J .$, Uniform asymptotic expansions for Weber parabolic cylinder functions of large orders. J 63B2, 131 (1959).

O'Mara, J. H., Doderer, G. C., McIntyre, D., Design and performance of a block-type osmometer. J 62, 63 (1959) RP2931.

On, F. J., Bouche, R. R., Lee, A. A., Ballard, C. R., Windinduced vibration of telephone and distribution conductors. Am. Inst. Elec. Engrs. Middle Eastern District Meeting Conf. Paper (April 1958). 2868.

Oppenheim, I., Quantum mechanical effects on the surface tension of simple lixuids. Il Nuovo Cimento Suppl. IX, No. 1, 180 (1958). 2708.

Statistical mechanical theory of transport processes. Proc. Midwestern Conf. on Theoretical Phys., Washington University (St, Louis, Mo.), p. 142.2778.

Oppenheim, I., Mazur, P., Density expansions of distribution functions. I. Virial expansion for finite closed systems; canonical ensemble. Physica 23, 197 (1957). 2077

Density expansions of distribution functions. II. Density expansions in the grand canonical ensemble. Physica 23, 216 (1957). 2078.

Oppenheim, I., Ross, J., Temperature dependence of distribution functions in cuantum statistical mechanics. Phys. Rev, 107, No. 1, 28 (1957), 2340.

Ordway, $F$., Crystal chemistry symposium. Ceramic Age 58, No. 5, 28 (1951). 215.

Orem, T. H., Growth of preferentially oriented aluminum single crystals. J 60, 547 (1958) RP2868.

Twinned epitaxy of copper on copper. J 60, 597 (1958) RP2873

Oshinsky, W., Breckenridge, R. G., Blunt, R. F., Hosler, $W . R .$, Frederikse, H.P. R., Becker, J.H., Electrical and optical properties of intermetallic compounds. I. Indium antimonide. Phys. Rev. 96, 571 (1954). 1007

Oshinsky, W., Breckenridge, R. G., Hosler, W. R., Electrical properties of indium antimonide. Phys. Rev. 91, 243 (1953). 703 .

Osmalov, J. S., Glaze, F. W., Blackburn, D. H., Hubbard, D., Black, M. H., Properties of arsenic sulfide glass. J 59, 83 (1957) RP2774.

Ostrow, S. M., Maximum usable frenuencies and lowest usable frequencies for the path Washington to Resolute Bay. J. Geophys. Research Letter to Editor 59, 3 (1954), 1062

Ostrow, S. M., Pokempner, $M$., The differences in the relationship between ionospheric critical frequencies and sunspot number for different sunspot cycles. J. Geophys. Research 57, No, 4, 473 (1952), 571.

Ostrowski, A. M., A method of speeding up iterations with superlinear convergence. J. Math. Mech. 7, 117 (1958), 2427

Bounds for the greatest latent root of a positive matrix. J. London Math. Soc. (London, England) 27, Pt. 2, No, 106, 253 (1952). 405.

Determinanten mit ueberwiegender Hauptdiagonale und die absolute Konvergenz von linearen iterationsprozessen. Comment, Math. helv. 30, 175 (1956). 1671 .

Generalization of a theorem of Osgood to the case of continuous approximation. Proc. Am. Math. Soc. 1, No. 5, $648(1950), 64$.

Note on an infinite integral. Duke Math. J. 18, No. 2,355 (1951). 270 .

Note on Vincent's theorem. Annals of math. 52, $702(1950) .96$.

On Gauss' speeding up device in the theory of single step iteration. Math. Tables and Other Aides to Computation 12, No, 62, 116 (1958), 2659.

On and over and under relaxation in the theory of the cyclic single step iteration. Math. Tables and Other Aids to Computation $\mathbf{7}, 152$ (1953). 781.

On the bounds of a one-parametric family of matrices. J. reine angew, math. (Berlin, Germany) 200, 190 (1958), 2663.

On the convergence of the Rayleigh quotient iteration for the computation of the characteristic roots and vectors, II. Arch. Ratl. Mech. Anal. 2, No. 5, 423 (1959). 3003.

On the convergence of the Rayleigh quotient iteration for the computation of characteristic roots and vectors, VI. (Usual Rayleigh quotient for nonlinear elementary divisors). Arch. Ratl. Mech. Anal. 4, No. 2, 153 (1959), 3004 .

On the linear iteration procedures for symmetric matrices. Rend. Mat. App. [V] VIII, No. 3-4, 140 (1954). 1097.

On the rounding off of difference tables for linear interpolation. Math. Tables and Other Aids to Computation 6, No. 40, 212 (1952). 504.

On the spectrum of a one parametric family of matrices, J. reine angew, math. (Berlin, Germany) 193,143 (1954). 1100.

On two problems in abstract algebra connected with Horner's rule. Studies in Math. and Mechanies, p. 40 (Academic Press Inc., New York, N.Y.) (1954). 1101.

Two explicit formulae for the distribution function of the sums of $n$ uniformly distributed independent variables. Archiv der mathematik (Basel, Switzerland) 3, No. 6, 3 (1952). 609.

Un nouveau critère d'univalence des transformations dans un $\mathrm{R}^{\mathrm{n}}$. Comp. rend. acad. sci. 248, 348 (1959). 3106.

Ostrowski, A. M., Taussky $O$., On the variation of the determinant of a positive definite matrix. Koninkl. Nederl. Akademie Van Wetenschappen (Amsterdam, Holland). Proc. Series A, 54, No .5; Indag. Math. 13, No. 5 (1951). 284.

Oswald, V. A., Jr., Fletcher, S. L., Jr., Proposals for the mechanical resolution of German syntax patterns. Modern Language Forum 36, No. 3-4, 1 (1951). 290.

Otto, E. M., Eicke, W. G., Jr., The gassing of dry cells. J. Electrochem. Soc. 104, 199 (1957). 2359.

Padgett, A. A., Wilson, W. K., Reaction of sodium chlorite with some adloses and modified celluloses. Tappi 38, 292 (1955). 1473.

Padgett, A. A., Wilson, W. K., Harvey, J. L., Effect of melamine resin on chemical tests of paper. Tappi 34, 410 (1951). 227.

Padgett, D. W., Caswell, R. S., Gabbard, R. F., Doering, $W$. P., Attenuation of 14.1 Mevs neutrons in water. Nuclear Sci. and Engr. 2, 143 (1957). 2032.

Paffenbarger, G. C., Dental materials and instruments 1951-55. Intern. Dental J. 6, No. 3, 299 (1956). 1669.

Dental research. Book, Developments in Dentistry During the Last 25 Years. Med. Digest of India, p. 351 (Bombay, India, 1957). 2079.

Résumé of dental research. J. Am. Coll. Dentists, 22, 21 (1955), 1480 .

Silicate cements: how to Select and Use Them. Trans. Am. Dental Soc. of Europe (John Wright \& Sons Ltd., Bristol, England) (1957). 2299.

Paffenbarger, G. C., Bowen, R. L., Scientific developments and methods in practice (Dentistry in 1967). J. Am. Dental Assoc. 56, 785 (1958). 2748.

Paffenbarger, G. C., Caul, H. J., Sweeney, W. T., Relationships between residual monomer and some properties of self-curing dental resins. J. Am. Dental Assoc. 53, 60 (1956). 1846.

Paffenbarger, G. C., Grunewald, A. H., Dickson, G., The effect of molding processes on some properties of denture resins. J. Am. Dental Assoc. 44, 269 (1952). 572 . 
Paffenbarger, G. C., Grunewald, A. H., Dickson, G., Schoonover, 1. C., Silicate cement: method of mixing in a closed container to prevent effects of exposure to atmosphere. J. Am. Dental Assoc. 46, 184 (1953), 827.

Paffenbarger, G. C., Nelsen, R. J., Sweeney, IV. T., Direet and indireet filling resins: a review of some physical and chemical properties. J. Am. Dental Assoc. 47, $516(1953)$. 688.

Paffenbarger, G. C., Nelsen, R. J., Wolcott, R. B., Fluid exchange at the margins of dental restorations. J. Am. Dental Assoc. 44, 288 (1952). 451.

Paffenbarger, G. C., Sacchi, H., A simple technique for making porcelain jacket crowns. J. Am. Dental Assoc. 54, 366 (1957). 2000.

Paffenbarger, G. C., Schoubee, P.J., Sweeney, W. T., Resin cements and posterior-type direct-filling resins. J. Am. Dental Assoc, 52, 584 (1956). 1854.

Paffenbarger, G. C., Souder, W., American Dental Assocition contribution to dental science: physical research. J. Am. Dental Assoc. 58, 97 (1959), 2891.

Paffenbarger, G. C., Stanford, J. W., Processing denture base resins: heat-curing type. J. Am. Dental Assoc. 53, 72 (1956). 1819.

Review of 1956 dental research: dental materials and instrumentation. J. Am. Dental Assoc. 54, 465 (1957), 2290.

Silicate cements: how to select and use them. Dental Practioneer 8, No, 12, 387 (1958), 2756.

Zinc phosphate and silicate cements. Chapter in Dental Clin. North American, p. 561 (W. B. Saunders Co., Philadelphia Pa., 1958). 2873.

Paffenbarger, G. C., Stanford, J. W., Burns, C. L., American Dental Association specification No. 13 for selfcuring repair resins. J. Am. Dental Assoc. 51, 425 $(1955) .1257$.

Paffenbarger, G. C., Stanford, J. W., Kumbula, J. W., Sweeney, W. T., Determination of some compressive properties of human enamel and dentin. J. Am. Dental Assoc. 57, 487 (1958). 2518.

Paffenbarger, G. C., Stanford, J.W., Sweeney, W. T., A revision of American Dental Association specification No. 3 for dental impression compound. J. Am. Dental Assoc. 51, 56 (1955). 1244.

American Dental Association specifications for dental materials (third ed.) (1958). 2451.

Paffenbarger, G. C., Swaney, A. C., Caul, H. J., Sweeney, W. T., American Dental Association specification No. 12 for denture base resin (second revision). J. Am. Dental Assoc. 46, 54 (1953). 644.

Paffenbarger, G. C., Swaney A. C., Schoonover, I. C., Dickson, G., Ảmerican Dental Ássociation Specification No. 9 for dental silicate cement, First revision, effective July 1, 1950. J. Am. Dental Assoc. 40, $186(1950) .20$.

Paffenbarger, $\dot{C}$. G., Sweeney, $W . T$., American Dental Association specifications for dental materials. J. Am. Dental Assoc. 2d ed. (1956). 1614.

Dental materials specifications and certification annual for 1954. Am. Dental Assoc. Publication (1955). 1307.

Paffenbarger, G. C., Sweeney, W. T., Schoubee, P.J., Dental cements. 'Intern. Dental J. 5, 584 (1955).' 1306.

Paffenbarger, G. C., Wolcott, $R$. B., Schoonover, $I . C$, Direct resinous filling materials: temperature rise during polymerization. J. Am. Dental Assoc. 42, 253 (1951). 223.

Page, B. L., The graduation of precise circles. Surveying and Mapping 13, 149 (1953). 862.

Page, C. H., Contributions to Dictionary of Physics and Electronics. (D. Van Nostrand Co., Inc., New York, N.Y.) (1955). 1660A.

Electronic circuits. Handbook of Physics. Ed. by E. U. Condon and $\mathrm{H}$. Odishaw, Chapter 4, Pt. 4, p. 4-47 (McGraw-Hill Book Co., Inc., New York, N.Y.) (1958). 2500.

Harmonic generation with ideal rectifiers. Proc. IRE 46, No. 10, 1738 (1958). 2578.

Information theory. J. Wash. Acad. Sci. 41, 245 (1951). 250A.
Instantaneous power spectra. J. Appl. Phys. 23, 103 (1952). 469

Physical mathematics. (D. Van Nostrand Co. Inc., New York, N.Y.) (1955). $1445 \mathrm{~A}$.

The algebra of electronics. (D. Van Nostrand Co. Inc., New York, N.Y.) (1958), 2803A.

Units and dimensions in physics. Am. J. Phys. 20, 1 (1952). 611.

Pagel, B. E. F., Branscomb, L. M., Atomic and molecular negative ions in stellar atmospheres. Monthly notices roy. astron. soc. 118, No. 3,258 (1958). 2463.

Pall, G., Taussky, O., The number of representations of a quadratic form as a sum of four squares. Proc. roy. Irish acad. Sect. A., No. 3, 23 (1957). 2375.

Pangborn, E. F., Zimmerman, E. W., Determination of grease in leather. J. Am. Leather Chemists Assoc. 46, 342 (1951). 219.

Pararas, J., Klein, $R$., An improved hydrogen atom beam furnace. (1960) TN47 (PB151406).

Park, J. H., Cones, H. N., Sphere-gap volt-time curvesstandards for steep front surge measurements. Am. Inst. Elec. Engrs. Conf. Paper No. CP 57-215 (1957). 2314.

Park, J. J., Fickle, D. P., Uranium-platinum system. J 64A 1, 107 (1960).

Park, O. E., Weitzel, D. H., Iron catalyst for production of liquid para-hydrogen. Rev. Sci. Instr. 27, No. 1, 57 (1956), 1751 .

Park, O. E., Weitzel, D. H., Draper, J. W., Timmerhaus, K. D., Van Valin, C. C., Catalysis of the ortho-parahydrogen conversion. Proc. 1956 Cryogenic Engineering Conference, Paper A-4, p. 12. Edited by K. D. Timmerhaus, Chem. Eng. Dept., Univ. of Colorado (Boulder, Colo.) (1957). 2048.

Park, O. E., Weitzel, D. H., Loebenstein, W. V., Draper, $J$. $W$., Ortho-para catalysis in liquid-hydrogen production. J 60,221 (1958) RP2840.

Parker, H. S., Burdick, M. D., The effect of particle size on the bulk density and strength properties of uranium dioxide specimens. J. Am. Ceram. Soc. 39, No. 5 (1956). 1926

Parker, M. S., Stromberg, R. R., Quasius, A. R., Toner, S. $D$., Adsorption of polyesters on glass, silica, and alumina. J 62, 71 (1959) RP2933.

Parker, R. A., Kushner, L. M., On the low rates of equilibration in dialysis experiments with ionic surfaceactive agents. J. Phys. Chem. 61, 822 (1957). 2232.

Parker, R. A., Kushner, L. M., Hubbard, W. D., Turbidity and viscosity measurements on some cationic detergents in water and in sodium chloride solutions. J 59, 113 (1957) RP2778.

Parker, R. A., Wasik, S. P., On the electroviscous effect in dilute aqueous solutions on ionic detergents. J. Phys. Chem. 62, 967 (1958). 2667.

Parks, P. C., Swanson, H. E., Gilfrich, N. T., Cook, M. I., Stinchfield, R., Standard X-ray diffraction powder patterns. (1959) C539, Vol. 8.

Parsons, D. E., Building research in the U.S. before 1947. Proc. Conference on Building Research, Ottawa, October 21-23, 1953, Bull. 1 of Div. of Bldg. Research, Natl. Research Council, Ottawa, Canada, p. 1 (1955). $\quad 1279$.

Patridge, F. M., Report on the methods used in the 1949 AGA-ASME orifice meter tests. Instruments $\mathbf{2 3}$, 189 (1950). 128.

Paulson, R. A., Wood, L. A., Madorsky, I., Determination of copolymer composition by combustion analysis for carbon and hydrogen. J 64A2, 157 (1960).

Payne, L. E., Upper and lower bounds for the center of flexure. J 64B2, 105 (1960).

Payne, L. E., Pell, W. H., The Stokes flow problem for a class of axially symmetric bodies. Abstract Notices Am. Math. Soc. 5, 468 (1958). 2832.

Pearl, M., On a theorem of M. Riesz. J 62, 89 (1959) RP2935.

Peck, R. C., Pineo, V. C., A circuit for simultaneously recording the range, amplitude, and duration of radartype reflections. Rev. Sci. Instr. 22, 112 (1951). 176. 
Peiser, H. S., Black, I. A., Bolz, L. H., Brooks, F. P. Mauer, F. A., A liquid-helium cold cell for use with an X-ray diffractometer. J 61, 367 (1958) RP2907.

Peiser, H. S., Bolz, L. H., Boyd, M. E., Mauer, F. A., A re-examination of the crystal structure of the $\alpha$ and $\beta$ nitrogen. Acta Cryst. 12, 237 (1959). 2879.

Peiser, H. S., Bolz, L. H., Mauer, F. A., Low-temperature X-ray studies on Rice's blue material. J. Chem. Phys. 30, No. 1, 349 (1959). 2979.

Pelander, C. E., Brown, F. P., Tough problems solved with tricky tooling. Machinery 64, No. 1, 149 (1957). 2395 .

Pelander, C. E., Nelsen, R.J., Kumpula, J.W., Hydraulic turbine contra-angle handpiece. J. Am. Dental Assoc. 47, 324 (1953). 732 .

Pell, $W . H$. Graphical solution of the single degree of freedom vibration problem with arbitrary damping and restoring forces. J. Appl. Mech. 24, No. 2, 311 (1957). 2145.

The elastic problem for a ring of uniform force in an infinite body. J 60, 365 (1958) RP2855.

Pell, W. H., Payne, L. E., The Stokes flow problem for a class of axially symmetric bodies. Abstract Notices Am. Math. Soc. 5, 468 (1958). 2832.

Pellam, J. R., Observations of second sound radiation by the thermal Pitot tube. Phys. Rev. 78, 818 (1950). 101 .

Second sound propagation in liquid helium II. Phys. Today 6, $4(1953)$. 821 .

Pellam, $J$. R., Broida, $H . P$. A note on the preparation of solid ozone and atomic oxy gen. J. Chem. Phys. 23, $409(1955) . \quad 1239$.

Phosphorescence of atoms and molecules of solid nitrogen at $4.2^{\circ} \mathrm{K}$. Phys. Rev. 95, No. 3, 845 (1954). 1104.

Pellam, J. R., deKlerk, D., Hudson, R. P., Normal fluid concentration in liquid helium II below $1^{\circ} \mathrm{K}$. Phys. Rev. 89, 662 (1953). 774.

Second sound velocity measurements below $1^{\circ} \mathrm{K}$. Phys. Rev. 89, 326 (1953). 822.

Pellam, J. R., Hanson, W. B., Second sound attenuation in liquid helium II. Phys. Rev. 95, 321 (1954) 1137.

Thermal Rayleigh disk measurements in liquid helium II. Phys. Rev. 85, 216 (1952). 603.

Pellam, J. R., Morse, P. M., Thermal Rayleigh disk in liquid helium II. Phys. Rev. 78, 474 (1950). 157.

Pellam, J. R., Weinstock, B., Thermal Rayleigh disk measurements in $\mathrm{He}^{3}-\mathrm{He}^{4}$ mixtures. Phys. Rev. 89, 521 (1953). 895 .

Pendergast, W. L., Heindl, R. A., Results of laboratory tests of high duty and super duty fireclay plastic refractories. Bull. Am. Ceram. Soc. 36, No. 1, 6 (1957). 2289 .

Pendergast, $W$. L., Mong, L. E., Dynamic and static tests for mechanical properties of fired plastic refractories and other more resilient materials. J. Am Ceram. Soc. 39, No. 9, 293 (1956). 1684.

Pendleton, T. A., Underearth crystal resonators, Proc. IRE 41, 1616 (1953). 903.

Penfold, A. S., Leiss, J. E., Analysis of photocross sections. Physics Dept. Rept. (University of Ill., Champaign, Ill.) (1958). 2460.

Penner, S., Leiss, J. E., Robinson, C. S., Range straggling of high-energy electrons in carbon. Phys. Rev. 107, 1544 (1957), 2279.

Pennington, W. A., Frankel, H. E., Bennett, J.A., Fatigue properties of high strength steels. Trans. Am. Soc. Metals 52 (1959). 2951.

Pergiel, F, $Y$., Johnson, $W, H$, Prosen, E. J., Heat of reaction of diborane with water and the heat of formation of boric oxide. J 62, 43 (1959) RP2927.

Pergiel, F. Y., Prosen, E. J., Johnson, W. H., Heats of formation of diborane and pentaborane. J 61, 247 (1958) RP2901

Perloff, A., Temperature inversions of anhydrous gallium orthophosphate. J. Am. Ceram. Soc. 39, No. 3, 83 $(1956), 1916$.
Perloff, A., Block, S., Burley, G., Mason, R. D., Jr., Refinement of the crystal structure of triclinic magnesium pyroborate. J 62, 95 (1959) R P2936.

Perloff, A., Clark, J. R., Mrose, M. E., Burley, G., Studies of borate minerals. VI: Veatchite. Am. Mineralogist 44, No. 11-12, 1141 (1959). 3057.

Perloff, A., Posner, A. S., Preparation of pure hydroxyapatite crystals. Science 124, No. 3222, 583 (1956). 1816.

Perloff, A., Posner, A. S., Diorio, A. F., Refinement of the hydroxyapatite structure. Acta Cryst. 11, 308 (1958). 2724.

Perls, $T$. A., Electrical noise from instrument cables subjected to shock and vibration. J. Appl. Phys. 23, No. 6, 674 (1952). 437 .

Perls, T. A., Buchmann, E., A bar magnet velocity meter. Rev. Sci. Instr. 22, 475 (1951). 174

Perls, $T$. A., Kissinger, $C$. $W$., An integrating and differentiating bar-magnet volocity meter for use in ballistocardiography. Rev. Sci. Instr. 25, No. 10, 98 ? (1954). 951

Jerkmeter for ballistocardiography. Rev. Sci. Instr. 27, No. 1, 51 (1956). 1752.

Perls, T. A., Wildhack, W. A., A simple type $\mathrm{X}(t)-Y$ recorder. Rev. Sci. Instr. 22, No. 7, 541 (1951). 184.

Perry, L. B., Wait, J. R., Calculations of ionospheric reflection coefficients at very low radio frequencies. J. Geophys. Research 62, No. 1, 43 (1957). 2044.

Pessoa, E. F., Hayward, R. W., Hoppes, D. D., van Lieshout, $R$., Gamma radiation from $\mathrm{Zn}^{63}$. Il Nuovo Cimento II, 53 (1959). 2956.

Pestalozzi, W.J., Richards, R. J., Jacobs, R. B., Measurement of the flow of liquefied gases with sharpedged orifices. Proc. 1958 Cryogenic Eng., Conf. Paper E-1, p. 272. Edited by K. D. Timmerhaus, Chem Eng. Dept., Univ. of Colorado (Boulder, Colo.) (1959). 2983

Peters, E., Hellmann, M., Pummer, W. J., Wall, L. A., Hexafluorobenzene from the pyrolysis of tribromofluoromethane. J, Am. Chem. Soc. 79, 5654 (1957). 2151 .

Peterson, C., Dunfee, B. L., Hermach, F. L., Methods for measuring the "Q" of large reactors. Am. Inst. Elec. Engrs. Trans. Paper No. 56-57 (1956). 1776.

Peterson, J. M., Linnig, F. J., Edwards, D. M., Acherman, $W$. L., Chemical analysis of GR-S by complete solution procedures. Gross constituents in GR-S containing soap. Anal. Chem, 25, 1511 (1953). 666.

Peterson, J. M., Linnig, F. J., Mandel, J., A plan for studying the accuracy and precision of an analytical procedure. Anal. Chem. 26, No. 7, 1102 (1954) 926.

Peterson, R. A., Isbell, H. S., Frush, H. L., Tritiumlabeled compounds I. Radioassay of tritium-labeled compounds in "infinitely thick" films with a windowless, gas-flow, proportional counter. J 63A2, 171 (1959).

Petree, B., Foote, R. S., A pulsed magnetic extractor for removing the electron beam from a betatron. Rev. Sci. Instr. 25, 694 (1954). 929.

Petree, B., Fuller, E. G., Weiss, M. S., Photoneutron vields in the rare-earth region. Phys. Rev. 112, No. 2, 554 (1958), 2686.

Petree, B., Hart, E. J., Koch, H. W., Schulman, J. H. Taimuty, S. I., Wyckoff, H. O., Measurement systems for high-level dosimetry. 2d United Nationals Intern. Conf. on the Peaceful Uses of Atomic Energy, Vol. 21, Health and Safety: Dosimetry and Standards, P/1927 USA, p. 188 (Pergamon Press, London, England, 1959). 2984.

Petree, $B$, Weiss, M.S., Fuller, E. G. Photoneutron yields in the rare earth region. Bull. Am. Phys. Soc. Series II, No. 2, 16 (1957). 2249.

Peyron, $M$., Broida, $H$. P., Evaporation of active species trapped in a solid condensed from discharged nitrogen. J. Chem. Phys. 28, 725 (1958). 2551A. Luminescence of solid nitrogen $\left(4.2^{\circ} \mathrm{K}\right)$ containing atoms of free radicals. Effect of traces of oxygen 
hydrogen and water vapor. J. Phys. Radium 19, No. 4, 480 (1958). 2622.

Spectra emitted from solid nitrogen condensed at very low temperature from a gas discharge. J. Chem. Phys. 30, No. 1, 139 (1959). 3052.

Peyron, M., Horl, E. M., Brown, H. W., Broida, H. P. Etude spectroscopique des produits dé la décharge électrique dans L'Azote condensés a l'état solide à trés bassé temperatures, prévues en faveur de l'existence d'Azote triatomique dans le solide. J. chim. phys. 56, No. 2392, 736 (1959). 2944.

Spectroscopic evidence for triatomic nitrogen in solids at very low temperatures. J. Chem. Phys. 30, 1304 (1959). 3054.

Pezzner, H., Katzin, M., Koo, B. Y.-C., Larson, J. V., Katzin, J. C., The trade-wind inversion as a transoceanic duct. J 64D 3,247 (1960).

Pfeffer, H., Koller, H. R., Marden, E., The Haystaq system: past, present and future. Preprinted in Proc. Intern. Conf. on Scientific Infor. November 11-15, 1958 (American Documentation Inst., Washington, D.C.) Area 5, p. 317 (1958). 2815.

Pfeiffer, K. R., Harvey, J. L., Brauer, G. M., Deterioration during storage of alginate hydrocolloidal dental impression material. U.S. Armed Forces Med. J. 5, No. 9, 315 (1954). 986.

Phelps, F. P., Detwinning quartz crystals. (1959) TN3 (PB151362).

Stability of quartz resonators at very low temperatures. Proc. 11th Ann. Symp. on Frequency Control May 7-9, 1957 (Ft. Monmouth, N.J.), p. 256 (1957). 2315

Phelps, F. P., Morgan, A. H., Research at NBS Boulder Laboratories on quartz crystal resonator and oscillators at low te mperatures. Proc. 12th Annual Symp. on Frequency Control, May 6-8, 1958, U.S. Army Signal Research and Development Laboratory (Ft. Monmouth, N.J.), p. 162 (1958). 2734.

Phelps, F. P., Plyler, E. K., Growth and infrared transmission of cesium iodide crystals. J. Opt. Soc. Am. 42, 432 (1952). 460

Transmittance of cesium bromide crystals. J. Opt. Soc. Am. 41, 209 (1951). 349.

Phelps, G. R., Hatch, M. R., Jacobs, R. B., Richards, R. J., Boggs, R. N., Prediction of pressure drop in two: phase single-component fluid flow. Proc. 1958 Cryogenic Engineering Conference, Paper F-4, p. 357. Edited by K. D. Timmerhaus, Chem. Eng. Dept., Univ. of Colorado (Boulder, Colo.) (1959). 3019 .

Phillips, C. W., Trucks and trailers. Vol. 1, Chapter 32, Am. Soc. Refrig. Engrs. Data Book, p. 32 (1959). 3013.

Phillips, C. W., Achenbach, P. R., Water demand rates for electric drinking-water coolers of the bubbler type. Refrig. Eng., 62, No. 12, 37 (1954). 1226.

Phillips, C. W., Johnson, H. D., Winter, J. C., Grimes, J. W., Achenbach, P. R., Heat transfer measurements on refrigerated-food trailers U.S. Dept. of Agri. Marketing Service (AMS-250), (June 1958). 2580.

Phillips, M. L., Moore, H. S., Dispersion of F2-layer critical frequencies. Proc. IRE 39, 717 (1951). 225.

Pigman, W. W., Forziati, A. F., Losee, F. L., Jennings, $W$. H., Lawson, $M$. E., Jr., In vitro production of experimental and enamel caries. J. Am. Dental Assoc. 53, 655 (1956). 1734.

Pike, R. G., Pressures developed in cement pastes and mortars by the alkali-aggregate reaction. Natl. Acad. Sci., Natl. Research Council, Highway Research Board Bull. 171, p. 34 (1958). 2699.

Pike, R. G., Hubbard, D., Increased chemical reactivity of the surface compared with that in the bulk volume of Britton-Robinson universal buffers. $J$ 59, 411 (1957) RP2811.

Miscellaneous observations on the alkali-aggregate reaction and the ionic charge on hydrated cement. Natl. Acad. Sci., Natl. Research Council. Publ. Highway Research Board Bull. 171, p. 16 (1958). 2639.
Physio-chemical studies of the destructive alkaliaggregate reaction in concrete. $J \mathbf{5 9}, 127$ (1957) RP 2780 .

Source of the non-migratable ionic charges developed by portland and high-alumina cements during hydration. Natl. Acad. Sei., Natl. Research Council. Proc. Highway Research Board 37, 256 (1958). 2768.

Pike, R. G., Hubbard, D., Insley, H., Mechanisms of alkali-aggregate reaction. J. Am. Concrete Inst. 27, No. 1, 13 (1955). 1406.

Pinching, G. D., Bates, R. G., Dissociation constant of aqueous ammonia at $0^{\circ}$ to $50^{\circ}$ from EMF studies of the ammonium salt of a weak acid. J. Am. Chem. Soc. 72, $1393(1950) .49$.

Note on the strength of picric acid. Experientia 10, 482 (1954). 1085.

Triathonolamin als puffer-substanz. Helv, chim. acta (Basil, Switzerland) $\mathbf{3 7}, 1439$ (1954). 1217.

Uber pH-Werte nichtwässeriger Losungen. Helv. chim. acta (Basil, Switzerland) 38, 699 (1955). 1563.

Pineo, $V . C$., A comparison of meteor activity with occurrence of sporadic $E$ reflections. Science 112, 50 (1950). 2.

Oblique-incidence measurements of the heights at which ionospheric scattering of the $\mathrm{VHF}$ radio waves occurs. J. Geophys. Research 61,165 (1956). 1790. Off-path propagation at VHF. Proc. IRE 46, 922 (1958). 2656

Pineo, $V . C$., Gautier, $T$. $N$., The wave-frequency dependence of the duration of radar-type echoes from meteor trails. Science 114, 460 (1951). 348.

Pineo, V. C., Peck, R. C., A circuit for simultaneously recording the range, amplitude, and duration of radartype reflections. Rev. Sci. Instr. 22, 112 (1951). 176.

Pitls, J. W., Buzzard, E., Moore, D. G., Resistance measurement of ceramic-type strain gage cements. Am. Soc. Testing Materials, Spec. Tech. Publ. No. 230, 67 (1958). 2739 .

Pitts, J. W., Moore, D. G., Harrison, W. N., Role of nickel dip in the enameling of sheet steel. J. Am. Ceram. Soc. 37,No. 8, 363 (1954). 1133.

Pitts, J. W., Moore, D. G., Richmond, J. C., Harrison, $W . N$., The galvanic corrosion theory for adherence of porcelain enamel ground coats to steel. Inst. Vitreous Enamellers Ltd. Bull. (Ripley, England) 7, No. 4 (1957). 2358.

Pizer, R. S., Roberts, A. G., Improved dip coater. Anal. Chem. 26, 790 (1954). 1033.

Protective coating adhesion measurement using an electronic averaging device for the adherometer. ASTM Bull. No. 221, 53 (1957). 2273.

Pizer, R. S., Roberts, A. G., Crouse, W. A., Abrasive jet method for measuring abrasion resistance of organic coatings. ASTM Bull. No. 208, 36 (1955). 1249.

Placious, R. C. Marton, L., Schrack, R. A., A field emitter point projection X-ray microscope. X-ray Microscopy and Microradiography (Academic Press Inc., New York, N.Y.), p. 287 (1957). 1982.

Placious, R. C., Motz, J. $W$., Bremsstrahlung crosssection measurement for $50-\mathrm{kev}$ electrons. Phys. Rev. 109, 235 (1958). 2477.

Polarization near the high-frequency limit of $500-\mathrm{kev}$ bremsstrahlung. Phys. Rev. 112, No. 4, 1039 (1958). 2694.

Placious, R. C., Schrack, R. A., Apparatus for etching fine points under controlled conditions. Rev. Sci. Instr. 27, No. 6, 412 (1956). 1628 .

Plaza, L., Judd, D. B., Farnsworth, D., Tritanopia with abnormally heavy ocular pigmentation. J. Opt. Soc. Am. 40, 833 (1950). 161.

Plitt, K. F., Bersch, C. F., McCrackin, F. L., The effects of rates of straining on some tensile properties of polyester film. Modern Plastics 35, 171 (1957). 2354 .

Plumb, H. H., Edlow, M. H., Constant temperature liquid helium bath and reproductibility of resistance thermometers. Rev. Sei. Instr. 30, 376 (1959). 2916. 
Plush, R. W., Florman, E. F., Measured statistical characteristics and narrow-band teletype message errors on a single-sideband 600-mile-long ultrahighfrequency tropospheric radio link. J 64D2, 125 (1960)

Plush, R. W., Watt, A. D., Power requirements and choice of an optimum frequency for a worldwide standard-frequency broadcasting station. J 63D 1 , 35 (1959).

Plyler, E. K., Combination bands of ethylene. J. Chem. Phys. 19, 658 (1951). 206.

Further infrared measurements with a cesum iodide prism molecular spectroscopy. The Inst. Petrol. (London, England) (1955). 1351.

Infrared absorption spectra of twelve substituted benzene derivatives from 15 to 40 mierons. Faraday Society Discussion No. 9 (Univ. Press, Aberdeen, Scotland) (1950). 75 .

Infrared flame spectra. Mikrochim. acta 2-3, 421 (1955). 1366.

Infrared measurements, past and present. J. Franklin Inst. 259, No. 1, 17 (1955). 1367.

Mésures dans l'infrarouge avec un prisme en iodure de cesium. Extrait du J. de Physique et le Radium (Paris, France). 15, 519. (1954). 1072.

Plyler, E. K., Acquista, N., Infrared absorption of liquid water from 2 to 42 microns. J. Opt. Soc. Am. 44, 505 (1954). 1038.

Infrared measurements with a cesium iodide prism. J. Opt. Soc. Am, 43, 977 (1953). 742.

Infrared spectrometry with a cesium iodide prism. J. Opt. Soc. Am. 43, 212 (1953). 744.

Small grating spectrometer for the far infrared region. J. Chem. Phys. 23, 752 (1955). 1488.

The use of cesium iodide prisms to 50 microns. N. 3 del Suppl. al Vol. 2, Ser. X, Il Nuovo Cimento, p. 629 (Bologna, Italy) (1955). 1551.

Transmittance and reflectance of cesium iodide in the far infrared region. J. Opt. Soc. Am. 48, No. 9, 668 (1958). 2852.

Plyler, E. K., Allen, H. C., Jr., Infrared spectrum of hydrogen sulfide, II. The $5100 \mathrm{~cm}^{-1}$ region. J. Chem. Phys. 22, 1104 (1954). 1040.

Infrared spectrum of hydrogen sulfide. J. Chem. Phys. 25, No. 6, 1132 (1956). 1740.

$\nu_{3}$ band of methane. J. Chem. Phys. 26, No. 4, 972 (1957). 2221.

Some vibrational-rotational bands of deuterated methanes. J 63A27, 145 (1959).

The infrared spectrum of hydrogen sulfide in the $5100 \mathrm{~cm}^{-1}$ region. J. Chem. Phys. 22, 1104 (1954). 1187 .

The structure of ethylene from infrared spectra. J. Am. Chem. Soc. 80, 2673 (1958). 2833.

Plyler, E. K., Allen, H. C., Jr., Blaine L. R., Infrared spectrum of carbonyl sulfide. J. Chem. Phys. 26, No. 2, 400 (1957). 2162 .

Some infrared bands of deuterium sulfide. J 59, 211 (1957) RP2789.

The emission spectrum of $\mathrm{OH}$ from 2.8 to $4.1 \mu$. Spectrochem. acta 9, 126 (1957). 2356.

The infrared spectrum and rotational constants of carbon disulfide. J. Am. Chem. Soc. 78, 4843 (1956). 1932.

Plyler, E. K., Allen, H. C. Jr., Cross, P. C., Blaine, L. R., Infrared spectrum of hydrogen sulfide from $2200-$ $2800 \mathrm{~cm}^{-1}$. J. Chem. Phys. 24, No. 1, 35 (1956). 1741 .

Plyler, E. K., Allen, H. C., Jr., Tidwell, E. D., Emission spectrum of carbon monoxide from 2.3 to 2.5 microns. J 61, 53 (1958) RP2883.

Infrared spectra of hydrogen cyanide and deuterium cyanide. J. Chem. Phys. 25, No. 2, 302 (1956). 1739.

The infrared spectrum of acetylene-d . J. Am. Chem. Soc. 78, 3034 (1956). 1934.

Vibrational energy of deuterium cyanide. J. Chem. Phys. 23, No. 7, 1356 (1955). 1575.
Plyler, E. K., Ball, J. J., Filters for the infrared region. J. Opt. Soc. Am. 42, 269 (1952). 450. The infrared emission spectra of $\mathrm{OH}, \mathrm{CO}$, and $\mathrm{CO}_{2}$ from $3 \mu$ to $5.5 \mu$. J. Chem. Phys. 20, 1178 (1952). 578.

Plyler, E. K., Benedict, W. S., Energy transfer in hot gases. Reprint of "High-resolution spectra of hydrocarbon flames in the infrared" from Symp. at Washington, D.C. (1954). 1016.

Interaction of stretching vibrations and inversion in ammonia. J. Chem. Phys. 24, No. 4, 904 (1956). 1744.

Vibration-rotation bands of ammonia. II. 'The molecular dimensions and harmonic frequencies of ammonia and deuterated ammonia. Can. J. Phys. 35,1235 (1957). 2411.

Rotation-vibration spectra of deuterated water vapor. J. Chem. Phys. 24, 1139 (1956). 1859.

The rotation-vibration spectra of deuterated water vapor. J. Chem. Phys. 24, No. 6, 1139 (1956). 1949.

The vibration-rotation spectrum of $\mathrm{D}_{2} \mathrm{O}$. J. Chem. Phys. 21, 1301 (1953). 891.

The vibration-rotation spectrum of HDO. J. Chem. Phys. 21, 1302 (1953). 892.

Plyler, E. K., Benedict, W. S., Humphreys, C. J., The emission spectrum of $\mathrm{OH}$ from 1.4 to $1.7 \mu$. J."Chem. Phys. 21, 398 (1953). 860.

Plyler, E. K., Benedict, W. S., Silverman, J., Precise measurements in the infrared spectrum of carbon monoxide. J. Chem. Phys. 20, 775 (1952). 516.

Plyler, E. K., Benedict, W. S., Tidwell, E. D., Vibrationrotation bands of ammonia: 1. The combination bands $\nu_{2-}\left(\nu_{1}, \nu_{3}\right)$. J 61, 123 (1958) RP2894.

Vibration-rotation bands of ammonia, III. The region 3.2-4.3 microns. J. Chem. Phys. 29, 829 (1958). 2861.

Plyler, E. K., Blaine, L. R., Infrared high-resolution grating spectrometer. J 62, 7 (1959) RP2922.

Infrared emission spectrum of methane at 3.3 microns. J 59, 317 (1957) RP2803.

Infrared measurements with a small grating from 100 to 300 microns. J 60, 55 (1958) RP2812.

Transmittance of materials in the far infrared. J 64C1, 55 (1960).

Plyler, E. K., Blaine, L. R., Connor, W. S., Velocity of light from the molecular constants of carbon monoxide. J. Opt. Soc. Am. 45, No. 2, 102 (1955). 1574.

Plyler, E. K., Danti, A., Blaine, L. R., Tidwell, E. D., Vibration-rotation structure in absorption bands for the calibration of spectrometers from 2 to 16 microns. J 64A 1, 29 (1960); (1960) Mono. 16.

Optical systems for increasing the available intensity from flames. J. Opt. Soc. Am. 42, 360 (1952). 506.

Plyler, E. K., Mann, D. E., Infrared and raman spectra of trans-difluorodichloroethylene. J. Chem. Phys. 26, 773 (1957). 2161.

The $\nu_{9}$ fundamental of $\mathrm{C}_{2} \mathrm{~F}_{6}$. J. Chem. Phys. 21, 1116 (1953) 888 .

Vibrational spectrum of fluorotrichloroethylene. J. Chem. Phys. 23, No. 11, 1989 (1955). 1577.

Plyler, E. K., Mann, D. E., Acquista, N., The vibrational spectrum of bromotrifuoroethylene. J. Chem. Phys. 22, 1199 (1954), 1206.

The vibrational spectrum of chlorotrifluoroethylene. J. Chem. Phys. 21, 1949 (1953). 893.

Vibrational spectrum of 1-fluoro-1-chloroethylene. J. Chem. Phys. 23, 2122 (1955). 1576.

Vibrational spectra of trifluoroethylene and trifluoroethylene-d. J. Chem. Phys. 22, 1586 (1954). 1224.

Plyler, E. K., Mann, D. E., and Meal, J. H., Properties of fluorine compounds, the vibrational spectrum of tetrabromoethylene. J. Chem. Phys. 24, No. 5, 1018 (1956). 1825.

Vibrational spectrum of tetrabromoethylene. J. Chem. Phys. 24, 1018 (1956). 1973.

Plyler, E. K., Mulliken, R. S., Molecular complexes and their spectra. IX. Infrared absorption by iodine in 
its pyridine complexes and in benzene. J. Am. Chem. Soc. 81, 823 (1959). 2994.

Plyler, E. K., Phelps, F. P., Growth and infrared transmission of cesium iodide crystals. J. Opt. Soc. Am. 42, 432 (1952). 460 .

Transmittance of cesium bromide crystals. J. Opt. Soc. Am. 41, 209 (1951). 349.

Plyler, E. K., Stephens, R. E., Rodney, W. S., Spindler, R.J., Refractive index of potassium bromide for infrared radiant energy. J. Opt. Soc. Am. 43, 110 (1953). 812.

Plyler, E. K., Tidwell, E. D., Infrared emission spectra of flames under high resolution. J 61, 263 (1958) RP2903.

The precise measurement of the infrared spectra of molecules of the atmosphere. Mémoires Roy. Sei. Soc. Liége, quatrième ser., Inst. Astrophys. (Liége, Belgium) 28, 426 (1957). 2377.

Plyler, E. K. Tidwell, E. D., Allen, H. C., Jr., Near infrared spectrum of nitrous oxide. J. Chem. Phys. 24, No. 1,95 (1956). 1782 .

Plyler, E. K., Tidwell, E. D., Blaine, L. R., Infrared absorption spectrum of methane from 2470 to 3200 $\mathrm{cm}^{-1}$. J 64A3, 201 (1960).

Plyler, E. K., Tilton, L. W., Stephens, R. E., Refractive index of silver chloride for visable and infrared radiant energy. J. Opt. Soc. Am. 40, 540 (1950). 124.

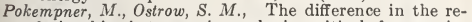
lationship between ionospheric critical frequencies and sunspot number for different sunspot cycles. J. Geophys. Research 5\%, No. 4, 473 (1952). 571 .

Polanyi, J.C., McKinley, J. D., Chemiluminescence in the system atomic sodium plus atomic hydrogen. Can. J. Chem. 36, 107 (1958). 2486.

Pollack, H., Davis, P., Complex biorthogonality for certain sets of polynomials. Duke Math. J. 21, No. 4, 653 (1954). 975.

Pomerantz, P., Fookson, A., Rich, E. H., The preparation of high-purity hydrogen deuteride. Science 112, 748 (1950). 153.

Pons, D. C., Wier, J. E., Axilrod, B. M., Effects of humidity during fabrication of polyester laminates. SPE J. 8, No. 9, 8 (1952). 435.

Effects of resin coating methods and other variables on physical properties of glass-fabric reinforced polyesters. Natl. Advisory Comm. Aeronaut. Research Memo. RM54G26 (1954). 1004.

Pope, C.I., A note on the estimation of sodium carbonate in photographic developers. J. Soc. Motion Picture Television Engrs. 64, 420 (1955). 1238.

Formation of silver sulfide in the photographic image during fixation. J 64C1, 65 (1960).

Posner, A.S., Fluoridation. Encyclopedia Chem. Supp., p. 128 (Reinhold Publ. Corp., New York, N.Y.) (1958). 2567 .

The structure of bone mineral. Clinical Orthopedics No. 9 , p. 5 (1957). 2383.

$\mathrm{X}$-ray diffraction studies of tooth structures. Norelco Reptr. 2, 26 (1955). 1583.

Posner, A. S., Bienenstock, A., Block, S., Radial distribution study of vitreous barium borate. J 64A3, $229(1960)$.

Posner, A. S., Jorgensen, K. D., Study of the setting of plaster. J. Dental Research 38, 491 (1959). 3058.

Posner, A. S., Likins, R. C., Bavetta, L. A., Calcification in lysine deficiency. Arch. of Biochem. and Biophys. 70, 401 (1957). 2040.

Posner, A. S., Likins, R. C., Steere, A. C., Effect of calcium treatment on solubility and calcium uptake of synthetic hydroxyapatite and rat molar enamel. J. Am. Dental Assoc. 57, 335 (1958). 2528.

Posner, A. S., Mandelkern, L., Diorio, A. F., Laki, K., Mechanism of contraction in the muscle fibre-ATP system. Proc. Natl. Acad. Sci. 45, 814 (1959). 2986.

Posner, A. S., Mandelkern, L., Worthington, C. R., Lowangle X-ray diffraction of fibrous polyethylene. Science 127, 1052 (1958). 2616.
Posner, A. S., Perloff, A., Preparation of pure hydroxyapatite crystals. Science 124, No. 3222,583 (1956). 1816.

Posner, A.S., Perloff, A., Diorio, A. F., Refinement of the hydroxyapatite structure. Acta eryst. 11, 308 (1958). 2724.

Posner, A. S., Stephenson, S. R., Crystallographic investigation of tricalcium phosphate hydrate. J. Dental Research 31, No. 3, 371 (1952). 421.

Isomorphous substitution in enamel apatite. J. Am. Dental Assoc. 46, 257 (1953). 748.

Post, Z. A., Heindl, R. A., Refractory castables, preparation and some properties. J. Am. Ceram. Soc. 33, $230(1950) .125$.

Pottasch, S. R., Bright rims in diffuse nebulae. Rev. Mod. Phys. 30, No. 3, 1053 (1958). 2479.

Conditions at the ionization and shock fronts in collisions of gas clouds - bright rims in diffuse nebulae, Part IV. Rev. Modern Phys. 30, No. 3, 1053 (1958). 2496A.

Departures of hydrogen from L.T.E. in a stellar atmosphere and the consequent structure of the solar chromosphere. Comm. de l'Observatoire Royal de Belgique No. 157, entitled The empirical determination of the stellar photospheric structure, Paper 11, 67 (1959). 2927.

The nova outburst. I. The nova outburst; II. The radiative cooling of an expanding shell; IV. The intensity of $\mathrm{H}_{\alpha}$; and $\mathrm{V}$. The temperature and radius of the central exciting star and observation of elements other than hydrogen. Ann. Astrophys. J. 22, 297, 310, 318,394 , and 412 (1959). 3085.

Variations of gamma cassiopeiae. (1959) TN21 (PB151380).

Pottasch, S. R., Jefferies, J., The nova outburst. III. The ionization of hydrogen gas by an exciting star. Ann. Astrophys. J. 22, 318 (1959). 3085.

Pottasch, S. R., Thomas, R. N., Departures from the Saha equation under varying conditions of Lyman continuous opacity. Astrophys. J. 130, No. 3, 941 (1959). 2926.

Potter, A., Using an electronic computer to reduce weathering test data. Proc. Porcelain Enamel Inst. 20, 73 (1958). 2858.

Potter, R. F., Cross sections for charge transfer collisions of low energy ions in $\mathrm{N}_{2}$ and $\mathrm{O}_{2}$. J. Chem. Phys. 22, 974 (1954). 982.

Elastic moduli of indium antimonide. Phys. Rev. 103, No. 1,47 (1956). 1693.

Indirect transitions in indium antimonide. Phys. Rev, 103, 861 (1956). 1736.

Ion-atom interchange in air. J. Chem. Phys. 23, No. 12, $2462(1955)$. 1379.

Piezoresistance of indium antimonide. Phys. Rev. 108, No. 3, 652 (1957). 2252.

The ionic character and elastic moduli of zinc blend lattices. Phys. Chem. Solids, 3, 223 (1957). 2367.

Potter, $R . F$., McKean, $W . J$., An apparatus for measuring the piezoresistivity of semiconductors. J 59, 427 (1957 RP2814.

Powell, F.J., Robinson, H. E., Cosgrove, L. A., Thermal resistance of airspaces and fibrous insulations bounded by reflective surfaces. (1957) BMS151.

Powell, $R$. C., A simplified method for measuring the attenuation of balanced transmission lines. Proc. Natl. Electronics Conf. 1951 (Natl. Electronics Conf., Inc., Chicago, Ill.) 7, 287 (1952). 376.

American dielectric standards. Elec. J. 153, 1779 (1954). 944.

Powell, R. C., Dalke, J. L., Advancements in dielectrics, 1952. Proc. IRE 41, 452 (1953). 642.

Advancements in dielectrics, 1953. Proc. IRE 4.2, 705 (1954). 943 .

Measuring power of low-loss dielectrics. Electronics 24, No. 8, 224 (1959). 256.

Powell, R. C., Haas, P. H., Harrington, R. D., Rasmussen, $A$. L., Instruments for magnetic high frequency measurements. Wright Air Development Center (Ohio) Tech. Rept. 54-238 (1954). 1043. 
Powell, R. C., Harrington, R. D., High-frequency magnetic permability measurements using toroidal coils. Proc. IRE 46, 784 (1958). 2584 .

Powell, R. C., Jickling, R. M., Hess, A. E., High-frequency impedance standards at the National Bureau of Standards. IRE Trans. Instrumentation I- $\boldsymbol{\gamma}$, No. 3-4, 270 (1958), 2583.

Powell, R. L., Aboud, A. A., Electrical contact resistance of copper, copper functions at low temperatures. Rev. Sci. Instr. 29, 248 (1958). 2537.

Powell, R. L., Bunch, M. D., Calibration of thermocouples at low temperatures. Proc. 1957 Cryogenic Engineering Conference, Paper E-6, p. 269, Edited by K. D. Timmerhaus, Chem. Eng. Dept., Univ. of Colorado (Boulder, Colo. ) (1958). 2483.

The thermal emf of several thermometric alloys. Suppl. au Bull. Inst. Intern. du Froid (Delft, Holland), Comm. 1, 129 (1958). 2833A.

Powell, R. L., Bunch, M. D., Corruccini, R.J., Thermal emf of some thermometric alloys. In Low-Temperature Physics and Chemistry; 1957 Proc. 5th Intern. Conf., Univ. of Wisconsin (Madison, Wis.), p. $484(1958), 2844 \mathrm{~A}$

Powell, R. L., Coffin, D. O., Low-temperature thermal conductivity of a free-machining copper. Rev. Sci. Instr. 26, 516 (1955). 1393.

Powell, R. L., Hall, W. J., Roder, H. M., Thermal and electrical conductivity of aluminum and aluminum alloys. In Low-Temperature Physics and Chemistry; 1957 Proc. 5 th Intern. Conf., p. 389, Univ, of Wisconsin (Madison, Wis., 1958). 2841A.

Thermal conductivities of common commercial aluminum alloys. Proc. 1957 Cryogenic Eng. Conf., Paper G-6, p. 408, Edited by K. D. Timmerhaus, Chem. Eng. Dept., Univ. of Colorado (Boulder, Colo.) (1958) 2842 .

Transport properties of dilute alloys of copper. Physica 24, S176 (1958), 2852A.

Thermal and electrical conductivity of pure copper. In Low-Temperature Physies and Chemistry; 1957 5 th Intern. Conf., Univ. of Wisconsin, p. 364 (Madison, Wis., 1958). 2841B

Powell, R. L., Roder, H. M., Rogers, W. M., Low-temperature thermal conductivity of some commercial coppers. J. Appl. Phys, 28, 1282 (1957). 2181.

Powell, R. L., Rogers, $W$. M., Tables of transport integrals $J_{n}(x) \equiv \int_{0}^{x} \frac{e^{z} z^{n} d z}{\left(e^{z}-1\right)^{2}} \cdot(1958)$ C595.

Powell, R. L., Rogers, W. M., Coffin, D. O., An apparatus for measurement of thermal conductivity of solids at low temperatures. J 59, 349 (1957) RP2805.

Powell, R. L., Rogers, $W$. M., Hall, $W$. J., Tables of transport integrals: a supplement. J G3B1, 23 (1959)

Powell R. L., Rogers, W. M., Roder, H. M., Thermal conductivities of copper and copper alloys. Proc. 1956 Cryogenic Eng. Conf., Paper E-3, p. 166, Edited by K. D. Timmerhaus, Chem. Eng. Dept., Univ. of Colorado (Boulder, Colo.) (1957). 2393.

Powlitch, F. J., Robinson, H. E., The thermal insulatum value of airspaces. Housing and Home Finance Agency (Washington, D.C.) Housing Research Paper No. $32(1954)$. 1203.

Projector, T. $H_{\text {., }}$ Effective intensity of flashing lights. Illum. Eng. 52, No. 12, 630 (1957). 2101.

The use of zonal constants in the calculation of beam flux. Illum. Eng. 48, 189 (1953). 887.

Versatile goniometer for projection photometry. Illum. Eng. 48, 192 (1953). 904.

Proschan, $F$., Confidence and tolerance intervals for the normal distribution. J. Am. Stat. Assoc. 48, 550 (1953) 674

Control charts may be all right, but-. Ind. Qual. Control 9, 56 (1953). 676.

Rejection of outlying observations. Am. J. Phys. 21, 520 (1953). 813 .

Use of random numbers. Ind. Qual. Control 9, No. $1,32(1952)$. 613 .
Prosen, E. J., Calorimetric combustion in a bomb of compounds containing carbon, hydrogen, oxygen and nitrogen. Chapter 6 in the book Experimental Thermochemistry, p. 129 (Interscience Publ. Inc., New York, N.Y., 1956). 1645.

Prosen, E.J., Deitz, V.R., Reaction of carbon monoxide with graphite carbon at $450^{\circ} \mathrm{C}$. Nature 118, 109 (1958). 2717.

Prosen, E. J., Jaffe, I., Szwarc, $M$., Heats of combustion of some peroxides and the heats of formation of acetate propionate, and butyrate radicals. J. Chem. Phys. 27, 416 (1957). 2148 .

Prosen, E. J., Johnson, W. H., Gilliland, A. A., Heat of formation of titanium tetraiodide. $J$ 63A2, 161 (1959).

Prosen, E. J., Johnson, W. H. Miller, R. G., Heat of formation of boron trichloride. J 62, 213 (1959) RP2956.

Prosen, E. J., Johnson, W. H., Nelson, R. A., Heat of formation of titanium tetrachloride. J 62, 49 (1959) RP2928.

Heat of formation of titanium tetrabromide. J 62, 67 (1959) RP2932.

Prosen, E. J., Johnson, W. H., Pergiel, F. Y., Heats of formation of diborane and pentaborane. $J$ 61, 247 (1958) RP2901.

Heat of reaction of diborane with water and the heat of formation of boric oxide. J 62, 43 (1959) RP2927.

Pruitt, J. S., Scintillation crystal-TV device X-rays objects in motion. Nucleonics 13, No. 8, 26 (1955). 1484 .

Prusch, S. B., Kessler, K. G., Stegun, I. A., Analysis of atomic spectra with electronic digital computers. J. Opt. Soc. Am. 46, No. 12, 1043 (1956). 1624.

Pryce, M. H. L., Eisenstein, J. C., The electronic structure and magnetic properties of uranyl-like ions, II. Plytonyl. Proc. Roy. Soc. (London, England) 238, 31 (1957), 2355.

Pummer, $\dot{W}$. J., Florin, R. E., Wall, L. A., Fluorination of haloaromatic compounds. J 62, 107 (1959) RP2938 .

Reactions of aromatic fluorocarbons with hydrogen. J 62, 119 (1959) RP2940.

Synthesis of some disubstituted 3,4,5,6-tetrafluorobenzenes. J 62, 113 (1959) RP2939.

Pummer, W. J., Hellmann, M., Bilbo, A. J., Synthesis and properties of fluorinated polyphenyls. J. Am. Chem. Soc. 77, 3650 (1955), 1521.

Pummer, W. J., Hellmann, M., Peters, E., Wall, L. A. Hexafluorobenzene from the pyrolysis of tribromofluoromethane. J. Am. Chem. Soc. 79, 5654 (1957). 2151.

Pummer, W. J., Wall, L. A., Reactions of hexafluorobenzene. Science 127, 643 (1958). 2718. Reactions of pentafluorohalobenzenes. J 63A2, 167 (1959).

Purcell, E. M., Bailey, D. K., Bateman, R., Berkner, L. V. Booker, H. G., Montgomery, G. F., Salisbury, M. W.', Wiesner, $\dot{J}$. $\dot{B}$., A new kind of radio propagation at very high frequencies observable over long distances. Phys. Rev. 86, No. 2, 141 (1952). 369.

Purcell, J. D., Wilson, N. L., Tousey, R., Johnson, F. S., Moore, $C$.'E., A revised analysis of the solar spectrum from 2990 to 2635 A. Astrophys. J. 119, No. 3, 590 (1954). 933 .

Purcell, J. R., Draper, J. W., Weitzel, D. H., A unique thermal conductivity gas analyzer. Proc. 1957 Cryogenic Eng. Conf., Paper D-4, p. 191, Edited by K. D. Timmerhaus, Chem. Eng. Dept., Univ, of Colorado (Boulder, Colo.) (1958). 2443.

Purcell, J. R., Goodwin, R. D., Direct-coupled power amplifier for cryostat heating control. Rev. Sci. Instr. 26, 581 (1957). 2090.

Purdy, C. M., Roach, F. E., McCaulley, J. W., Comparison of absolute intensities of [OI] 5577 in the auroral and subauroral zones. J 63D1, 19 (1959). 
Quasius, A. R., Stromberg, R. R., Toner, S. D., Parker, M. $S$., Adsorption of polyesters on glass, silica, and alumina, J 62, 71 (1959) RP2933.

Duinn, E., Florin, R. E., Wall, L. A., Mohler, F. L., Dimethylphosphinoborine trimer: mass spectra and thermal decomposition. J. Am. Chem. Soc. 76, 3344 (1954). 994.

Duinn, E., Mohler, F. L., Mass spectra of some deuterostyrenes. J 62, 39 (1959) RP2926.

Quinn, E., Mohler, F. L., Dibeler, V. H., Redetermination of mass spectra of deuteromethanes. J 61, 171 (1958) RP2896.

Duinn,F.A.,Jr., Bestul, A.B., Belcher, H.V., Bryant, C.B., Viscosities of 0.25 to 90 percent GR-S rubber solutions. J. Phys. Chem. 56, 432 (1952) 621.

Ouinn, F. A., JT., Mandelkern, L., Thermodynamics of crystallization in high polymers: poly(ethylene). J. Am. Chem. Soc. 80, 3178 (1958). 2846.

Quinn, $\dot{F}$. A., $J_{T}$., Mandelkern, L., Flory, P. $J_{\text {., }}$ Kinetics of crystallization in polymers. I. Bulk polymers. J. Appl. Phys. 25, 830 (1954). 1047.

Quinn, F. A., Jr., Mandelkern, L., Hellmann, M., Brown, D. W., Roberts, D. E., The melting transition of polymethylene. J. Am. Chem. Soc. 75, 4093 (1953). 869.

Duinn, F. A., Jr., Mandelkern, L., Roberts, D.E., Thermodynamics of crystallization in high polymers: gutta percha. J. Am. Chem. Soc. 78, 926 (1956). 1965.

Duinn, F. A., Jr., Mandelkern, L., Tryon, M., The melting behavior of polybutadienes. J. Polymer Sci. 19, 77 (1956). 1938.

Duinn, F. A., Jr., Roberts, D. E., Work, R. N., Volumetemperature relationships for the room temperature transition in Teflon. J. Appl. Phys. 22, 1084 (1951). 356.

\section{$\mathbf{R}$}

Rabile, P. J., Rose, J. C., Broida, H. P., Hufnagel, C. A., Gillespie, J.F., Freis, E. D., A method for the study of the circulation in the dog using a mechanical left ventricle. J. Appl. Physiol. 7, 580 (1954). 920.

Rabinow, $J$., Developing seals for abrasive-fluid mixtures. Machine Design 23, 128 (1951). 221.

Rabinowitz, $P$., The use of sub-routines on SEAC for numerical integrations of differential equations and for Gaussian quadrature. Proc. Assoc. for Computing Machinery, Toronto, Canada, p. 88 (Assoc. for Computing Machinery, New York, N.Y.) (1952). 598.

Rabinowitz, P., Abramowitz, $M$., Evaluation of coulomb wave functions along the transition line. Phys. Rev. 96, No. 1, 77 (1954). 1018.

Rabinowitz, P., Cole, K. S., Antosiewicz, H. A., Automatic computation of nerve excitation. J. Soc. Indust. Appl. Math. 3, 153 (1955). 1272.

Rabinowitz, P., Davis, P., A multiple-purpose orthonormalizing code and its uses. J. Assoc. Computing Mach. 1, No. 4, 183 (1954). 921 .

Additional abscissas and weights for Gaussian quadratures of high order: values for $n=64,80$, and 96 . J 60, 613 (1958) RP2875.

Numerical experiments in potential theory using orthonormal functions. J. Wash. Acad. Sci. 46, 12 (1956). 1789.

On the estimation of quadrature errors for analytic functions. Math. Tables and Other Aids to Computation 8, No. 48, 193 (1954). 1096.

Some Monte Carlo experiments in computing multiple integrals. Math. Tables and Other Aids to Computation 10, 1 (1956). 1875.

Racah, G., Fano, U., Irreducible tensorial sets. Academic Press Inc., New York, N.Y. (1959). 2970.

Raiffa, H., Motzkin, T. S., Thompson, G. L., Thrall, R. M., The double description method. Contributions to the theory of games II. Edited by H.W. Kuhn and A. W. Tucker, Annals of Math. Study 28, Chapter 3 (Princeton, N.J.) (1953). 857.
Ramberg, $W_{\text {., }} \quad$ Calibration of shock and vibration pickups. Noise Control 3, No. 5, 23 (1957). 2046.

Looking ahead in mechanics. J. Wash. Acad. Sci. 43, 241 (1953). 752.

Mechanical testing of solid materials. Appl, Mech. Rev. 5, 241 (1952). 482.

Ramberg, $W$., Irwin, $L$. K., A pulse method for determining dynamic stress-strain relations. Trans. IX ${ }^{\circ}$ Congrés intern. de méchanique appliquée (Brussels, Belgium) 8, 480 (1957). 1997.

Longitudinal impact tests of long bars with a slingshot machine. Am. Soc. Testing Materials, Spec. Tech. Publ. No. 176, "Symp. Impact Testing," June 1955 (1956). 1760.

Randall, D. L., A study of some of the meteorological effects on radio propagation at $96.3 \mathrm{me}$ between Richmond, Va., and Washington, D.C. Bull. Am. Meteorol. Soc. 35, 56 (1953). 635.

Randall, E. B., Carter, T. J., Kilduff, T. J., Mann, C., Kanagy, $J . R$., The variation of the physical and chemical properties of split and unsplit chrometanned leathers. J. Am, Leather Chemists Assoc. 47, 404 (1952). 601 .

Randall, E. B., Kanagy, J. R., Carter, T. J., Kinmonth, R. A., Mann, C., Variations of physical and chemical properties within and between vegetable retanned cow and steer sides. J. Am. Leather Chemists Assoc. 47, 726 (1952). 618.

Randall, E. B., Kanagy, J. R., Leser, W., Carter, T., Mann, C., Influence of splitting on the strength of chrome-tanned steer hides. J. Am. Leather Chemists Assoc. 47, 329 (1952). 468.

Randall, E. B., Mann, C., Kanagy, J. R., Mandel, J., Study of the burst test as applied to military upper leathers. J. Am. Leather Chemists Assoc. 48, 84 (1953). 842.

Ransil, B. $J$., Application of configuration interaction to the $\mathrm{H}_{3}$ complex. J. Chem. Phys. 26, No. 4, 97 (1957). 2027.

Ransil, B. J., Griffing, V., Jackson, J. L., The magnetic interaction of $\mathrm{H}_{3}$. J. Chem. Phys. 30, 1066 (1959). 3082 .

Ransom, $F$. A., A time-proportional electronic thermostat. Cont. Eng. 1, 85 (1954). 939.

Raskin, $W$. H., Robertson, A. F., Adiabatic apparatus for the study of self-heating of poorly conducting materials. Rev. Sci. Instr. 25, 541 (1954). 942.

Rasmussen, A. L., Grimes, D. M., Harrington, R. D., Magnetic properties of polycrystalline materials. J. Phys. Chem. Solids 12, 28 (1959). 2980A.

Rasmussen, A. L., Haas, P. H., Harrington, R. D., Powell, $R$. C., Instruments for magnetic high frequency measurements. Wright Air Development Center (Ohio) Tech. Rept. 54-238 (1954). 1043.

Rasmussen, A. L., Harrington, R. D., Initial and remanent permeability spectra of Yttrium iron garnet. Proc. IRE 47, 98 (1959). 2966.

Rasmussen, A. L., Hess, A. E., RF permeameter techniques for testing ferrite cores. Elec. Mfg. 61, 86 (1958). 2746.

Ray, L. C., Kirsch, R. A., Finding chemical records by digital computers. Science 126, No. 3278, 814 (1957). 2122 .

Ray, L. C., Kirsch, R. A., Cahn, L., Urban, G. H., Experiments in processing pictorial information with a digital computer. 1957 Eastern Joint Computer Conf. Proc., Washington, D.C. (1957). 2119.

Raybold, R. L., Harman, G. G., High frequency induced electroluminescence in zinc sulfide. Phys. Rev. 104, No. 6, 1498 (1956). 1728.

Reaves, J., Bias supplied for direct-coupled circuits. Electronics 27, No. 8, 172 (1954). 962. Low-capacitance power supply. Radio-Electron. Eng. 24, No. 6, 16 (1955). 1391.

Rebbert, R., Schoen, L. J., Electrical discharge induced luminescence of solids at low temperatures. J. Mol. Spectroseopy 3, No, 4, 417 (1959). 2934. 
Reber, G., Galactic radio waves. Leaflet Astron. Soc. Pacific No. 295 (1950). 63.

Motion in the solar atmosphere as deduced from radio measurements. Science 113, No, 2934, 312 (1951). 263.

Reber, G., Hagen, J., Haddock, $F . T$., NRL Aleutian radio eclipse expedition. Sky and Telescope 10, No. 5,111 (1951). 273.

Rechtin, E., Bateman, R., Florman, E. F., Tait, A., A source of error in radio phase measuring systems. (Discussion), Proc. IRE 39, 436 (1951). 186.

Redfearn, N. T., Weaver, E. R., Hughes, E. E., Gunther, S. M., Schuhmann, S., Gorden, R., Jr., Interpretation of mass spectra of condensates from urban atmospheres. J 59, 383 (1957) RP2809.

Reed, R. P., Mikesell, R. P., Some mechanical properties of Mylar and Dacron polyester strands at low temperatures. Rev. Sci. Instr. 29, No. 8, 734 (1958). 2761

The impact testing of various alloys at low temperatures. Proc. 1957 Cryogenic Eng. Conf., Paper F-4, p. 316, Edited by K. D. Timmerhaus, Chem. Eng. Dept., Univ. of Colorado (Boulder, Colo.) (1958). 2819. The stability of austenitic stainless steels at low temperatures as determined by magnetic measurements. Proc. 1958 Cryogenic Eng. Conf., Paper B-2, p. 84, Edited by K. D. Timmerhaus, Chem. Eng. Dept., Univ. of Colorado (Boulder, Colo.) (1959). 3093.

The tensile and impact strength of annealed and welded 5086 aluminum down to $20^{\circ} \mathrm{K}$. Proc. 1958 Cryogenic Eng. Conf., Paper B-3, p. 101. Edited by K. D. Timmerhaus, Chem. Eng. Dept., Univ. of Colorado (Boulder, Colo.) (1959). 3095.

Rees, M. H., Roach, F. E., The absolute photometry of the Gegenschein. Symp. on the Airglow and the Aurora, September 1955, Belfast (Pergamon Press, London, England), p. 142 (1956). 1919.

Rees, $M$. H., Roach, F. E., Megill, L. R., Marovich, $E$., The height of nightglow 5577. J. Atmospheric and Terrest. Phys. 12, 171 (1958). 2817

Reese, $R$. M., Dibeler, $V$. H., Ionization and dissociation of $\mathrm{NF}_{3}$ by electron impact. J. Chem. Phys, 24, 1175 (1956). 1749

Mass spectrometry. Anal. Chem. 30, 604 (1958). 2624 .

Multiple ionization of sodium vapor by electron impact. J. Chem. Phys. 31, 282 (1959). 2996.

Reese, R. M., Dibeler, $V$. H., Franklin, J. L., Electron impact studies of hydrazine and the methyl-substituted hydrazines. J. Am. Chem. Soc. 81, 68 (1959). 2939

Electron impact studies of sulfur dioxide and sulfuryl fluoride. J. Chem. Phys. 29, No. 4, 880 (1958). 2542 .

Ionization and dissociation of oxygen difluoride by electron impact. J. Chem. Phys. 27, 1296 (1957). 2169 .

Reese, R. M., Dibeler, V. H., Mann, D. E., Ionization and dissociation of perchlorylfluoride by electron impact. J. Chem. Phys. 27, 176 (1957). 2170.

Reese, R. M., Dibeler, V. H., Mohler, F. L., Ionization and dissociation of hexafluorobenzene by electron impact. J. Chem. Phys, 26, No. 2, 304 (1957). 2168. Ionization and dissociation of methyl siloxanes by electron impact. J. Chem. Phys. 21, 180 (1953). 747. The ionization potential of the $\mathrm{CF}_{3}$ radical and some fluorocarbon bond energies obtained by electron impact. J. Chem. Phys. 20, 761 (1952). 580.

Reese, R. M., Franklin, J. L., Dibeler, V. H., Krauss, $M$. Ionization and dissociation of hydrazoic acid and methyl azide by electron impact. J. Am. Chem. Soc. 80, 298 (1958). 2604.

Reese, $R$. M., Krauss, M., Dibeler, $V$. H., Multiple ionization of rare gases by electron impact. J. 63A3, 201 (1959).

Reese, R. M., Mohler, F. L., Dibeler, V. H., Some ions of high kinetic energy in mass spectra of polyatomic molecules. J. Chem. Phys. 22, 394 (1954). 1144.
Reeves, R. E., Rowen, J. W., Forziati, F. H., Spectrophotometric evidence for the absence of free aldehyde groups in periodate-oxidized cellulose. J. Am. Chem. Soc. 73,4484 (1951). 314.

Reggia, $F$., Design details of a linear magnetic UHF oscillator attenuator. FM-TV Radio Commun. 11, No. 10,16 (1951). 216.

Ultra high frequency magnetic attenuator. RadioElectronic Eng. Sect. Radio and TV News 49, No. 4, 12 (1953). 902.

Reggia, F., Beatty, $R$. $W$., Characteristics of the magnetic attenuator at UHF. Proc. IRE 41, 93 (1953). 663.

Reichard, T. W., Spinner, S., Tefft, W. E., A comparison of experimental and theoretical relations between Young's modulus and the flexural and longitudinal resonance frequencies of uniform bars. J 64A2, 147 (1960).

Reid, J. G., Engineering management in a government laboratory. Proc. Natl. Elec. Conf. (Am. Inst. Elec. Engrs.) 8, 734 (1953). 712.

Reid, W. E., Jr., Bish, J. M., Brenner, A., Electrodeposition of metals from organic solutions. III. Preparation and electrolysis of titanium and zirconium compounds in nonaqueous media. J. Electrochem. Soc. 104, No. 1, 21 (1957). 2106.

Reid, W. E., Jr., Connor, J. H., Wood, G. B., Electrodeposition of metals from organic solutions. V. Electrodeposition of magnesium and magnesium alloys. J. Electrochem. Soc. 104, 38 (1957). 2108.

Reilly, M. L., Glasgow, A. R., Jr., Ross, G. S., Horton, A. T., Enagonio, D., Dixon, 'H. D., Saylor, C. P., Furukawa, G. T., Henning, J. M., Comparison of cryoscopic determinations of purity of benzene by thermometric and calorimetric procedures. Anal. chim. acta 17, 54 (1957); Proc. of I.U.P.A. Intern. Symp. on Purity Control by Thermal Anal. (Amsterdam, The Netherlands) (April 1957). 2062

Reiney, M. J., Achhammer, B. G., Wall, L. A., Reinhart, $F$. W., Study of degradation of polysty rene by means of mass spectrometry. J. Polymer Sci. 8, No. 5, $555(1952) .557$

Reingold, I., Berger, $M$. J., Hubbell, J. H., Contribution of the annihilation radiation to the gamma-ray flux in lead. Phys. Rev. 113, No. 3, 857 (1959). 2917.

Reinhart, $F . M$., Corrosion factors in design. Product Eng. 22, No. 7, 101 (1951). 213.

Corrosion of aluminum alloys by exhaust gases. Corrosion 10, No. 12, 421 (1954). 979.

Stress corrosion cracking of a gold wedding ring. Corrosion 11, 503t (1955). 1513.

The effect of heat treatment of the susceptibility of sand cast aluminum alloy 220 to stress corrosion cracking. Corrosion 13, 17 (1957). 2349.

Reinhart, $F$. M., Ellinger, G. A., Effect of 20 -year marine exposure on some aluminum alloys. Am. Soc. Testing Materials Spec. Tech. Publ. No. 175, 47 (1956). 1689.

Reinhart, $F$. W., Accuracy. Juniata College Bull. 55, No. 4,2 (1958). 2448.

Nature of adhesion. J. Chem. Educ. 31, 128 (1954). 1080.

Plastics. The Encyclopedia of Chemistry (Reinhold Publ. Corp., New York, N.Y.), p. 756 (1957). 2254.

Relation between actual and artificial weathering. Am. Soc. Testing Materials Spec. Tech. Publ. 236, 57 (1958). 2729 .

Resistencia a la tension de los plasticos. Revista de plasticos \%, No. 41, 236 (1956). 1855.

Selecting engineering adhesives. Product Eng. 22, 123 (1951). 306.

Survey of adhesion and types of bonds involved. Chapter in book "Adhesion and adhesives, fundamentals and practice," p. 9 (John Wiley \& Sons, Inc., New York, N.Y., 1954). 1157.

Tension testing of plastics. Am. Soc. Testing Materials Symp. on Tension Testing of Nonmetallic Materials No. 194 (1957). 2341. 
Reinhart, F. W., Achhammer, B. G., Reiney, M. J., Wall, $L$. A., Study of degradation of polystrene by means of mass spectrometry. J. Polymer Sci. 8, No. 5, 555 (1952). 557 .

Reinhart, F. W., Boor, L., Brown, C., Lamb, J. J., Evaluation of the Boor-Quartermaster snag tester for coated fabries and plastic films. ASTM Bull. No. 210, 50 $(\div 955), 1337$.

Reinhart, F. W., Good, C. L., Turner, P. S., Wolock, I., Comparison of mechanical properties of flat sheets, molded shapes and postforined shapes of cotton fabric-phenolic laminates. Natl. Advisory Comm. Aeronaut. Tech. Note 3825 (1957). 2063.

Reinhart, F. W., Kline, G. M., Plastics: organic. Medical Physics, II, 728 (1950). 109.

Reinhart, F. W., Loos, B. D., DeLollis, N.J., Evaluation of adhesives for acoustical tile. ASTM Bull. No. $169,57(1950)$. 55 .

Reintart, F. W., Mandel, J., Comparison of methods for measuring the tensile and tear properties of plastic films. ASTM Bull. No. 209, 50 (1955). 1290.

Reinhart, F. W., Mutchler, M. K., Fluorescent sunlamps in laboratory aging tests for plastics. ASTM Bull. No. 212,45 (1956). 1713 .

Reinhart, F. W., Newman, S. B., Plastic springs. Product Eng. 27, 183 (1956). 1810.

Reinforced plastic springs. SPE J. 12, 8 (1956). 1843.

Reinhart, F. W., Slone, M. C., Measurement of creep of acrylic glazing plasties exposed to temperature gradients. SPE J. 10, 29 (1954). 1065.

Properties of plastic films. Modern Plastics 31, 203 (1954). 1122 .

Reinhart, F. W., Slone, M. C., Lamb, J. J., Properties of polymethyl alpha-chloroacrylate. Modern Plastics 29. No. 10, $109(1952) .520$.

Reinhart, F. W., Toner, S. D., Wolock, I., Eifects of molding pressure on strength properties of glass-fiber reinforced plastics. SPE J. 14, 40 (1958). 2533.

Reinhart, H. W., Hopper, N. J., Periodicals and serials received in the Library of the National Bureau of Standards, August 1958. (1958) C563, 1st Suppl.

Reverdin, D. L., Electron-optical exploration of space charge in a cut-off magnetron. J. Appl. Phys. 22, 257 (1951). 230.

Reverdin, D. L., Marton, L., Electron optical properties of space-charged clouds. J. Appl. Phys. 21, 842 (1950). 53.

Stroboscopic mapping of time-variable fields. J Appl. Phys. 21, 617 (1950), 140.

Reynolds, $M . M$., Fulk, $M . M$., Emissivities of metallic surfaces at $76^{\circ} \mathrm{K}$. J. Appl. Phys. 28, 1464 (1957). 2110 .

Rheinboldt, W. C., Greub, W., Non-self-adjoint boundary value problems in ordinary differential equations. J 64B2, 83 (1960).

Rheinboldt, W. C., Keegan, H. J., Schleter, J. C., Menard, $J$. P., Judd, D. B., Digital reduction of spectrophotometric data to Munsell renotations. J. Opt. Soc. Am. 48, 863 (1958). 2522.

Rheinboldt, $W . C$., Menard, J. P. Mechanized conversion of colorimetric data to Munsell renotations. J. Opt. Soc. Am. 48, 864 (1958). 2633.

Rhodes, I., Blanch, G., Table of characteristic values of Mathieu's equation for large values of the parameter. J. Wash. Acad. Sci. 45, 166 (1955). 1523.

Rice, $J$. R., Criteria for the existence and equioscillation of best Tchebycheff approximations. J 64B2, 91 (1960)

Rice, $P$. L., A comparison of methods for evaluating trends in time selies of tropospheric radio field strength data. IRE Trans. Ant. Prop. AP-3, 144 (1952). 362.

Some methods for evaluating trends in time series of tropospheric radio field strength data. IRE Trans. Ant. Prop. AP-3, 144 (1952), 546.

Rice, $P$. L., Daniel, $F$. T., Radio transmission loss versus distance and antenna height at $100 \mathrm{Mc}$. IRE Trans. Ant. Prop. AP-3, 59 (1955). 1470.
Rice, P. L., Herbstreit, J.W., Survey of Central Radio Propagation Laboratory research in tropospheric propagation 1948-1956. (1959) TN26 (PB151385).

Rice, P. L., Herbstreit, J. W., Norton, K. A, Schafer, $G$. E., Radio wave scattering in tropospheric propagation. Conv. Record on the IRE, Pt. 2, Antennas and communications, p. 85 (1953). 808.

Rice, P. L., Longley, A. G., Norton, K. A., Prediction of the cumulative distribution with time of ground wave and tropospheric wave transmission loss. Part 1. The prediction formula. (1959) TN15 (PB 151374)

Rice, P. L., Mansfield, W. V., Herbstreit, J. W. Groundto-air cochannel interference at $2900 \mathrm{Mc}$. IRE Trans. Airbourne Electronics AE-6, 1 (1952). 459.

Rice, $P$. L., Norton, $K$. A., Gapless coverage in air-toground communications at frequencies above $50 \mathrm{Mc}$. Proc. IRE 40, 470 (1952). 457

Rice, P. L., Norton, K. A., Janes, H. B., Barsis, A. P. The rate of fading in propagation through a turbulent atmosphere. Proc. IRE 43, 1341 (1955). 1542.

Rice, P. L., Norton, K. A., Vogler, L. E., The use of angular distance in estimating transmission loss and fading range for propagation through a turbulent atmosphere over irregular terrain. Proc. IRE 43 , 1488 (1955). 1550.

Rice, P. L., Williamson, D. A., Fuller, V. L., Longley, A, G., A summary of VHF and UHF tropospheric transmission loss data and their long-term variability. (1960) TN43 (PB151402).

Rich, E. H., Fookson, A., Pomerantz, P., The preparation of high-purity hydrogen deuteride. Science 112, $748(1950) .153$.

Richards, R. J., A high vacuum seal-off valve. Rev. Sci. Instr. 25, 520 (1954). 919.

Richards, R. J., Hatch, M. R., Jacobs, R. B., Boggs, R. N., Phelps, G. R., Prediction of pressure drop in twophase single-component fluid flow. Proc. 1958 Cryogenic Engineering Conference, Paper F-4, p. 357. Edited by K. D. Timmerhaus, Chem. Eng. Dept., Univ. of Colorado (Boulder, Colo.) (1959). 3019.

Richards, R. J., Jacobs, R. B., Vacuum-insulated transfe tube. Rev. Sci. Instr. 28, 291 (1957). 2406.

Valve for cold fluids. Rev. Sci. Instr. 26, No. 7, 730 (1955). 1570.

Richards, R. J., Jacobs, R. B., Pestalozzi, W.J., Measurement of the flow of liquefied gases with sharp-edged orifices. Proc. 1958 Cryogenic Eng. Conf., Paper E-1, p. 272. Edited by K. D. Timmerhaus, Chem. Eng. Dept., Univ. of Colorado (Boulder, Colo.) (1959). 2983.

Richards, R. J., Robbins, R. F., Jacobs, R. B., Holten, D. C., Heat transfer to boiling liquid nitrogen and hydrogen flowing axially through narrow annular passages. Proc, 1957 Cryogenic Eng. Conf., Paper G-3, p. 375. Edited by K. D. Timmerhaus, Chem. Eng, Dept., Univ, of Golorado (Boulder, Colo.) (1958). 2581.

Richardson, J.F., Scott, A. H., Ehrlich, P., Dielectric measurements up to $500^{\circ} \mathrm{C}$, symposium on temperature stability of electrical insulating materials. Proc. Am. Soc. Testing Materials Spec. Tech. Publ. No. 161 (1954). 992.

Richardson, J.M., Experimental evaluation of the oxygen microwave absorption as a possible atomic frequency standard. J. Appl. Phys. 29, No. 2, 137 (1958). 2555.

Richardson, J. M., Faris, J. J., Excess noise in microwave crystal diodes used as rectifiers and harmonic generators. IRE Trans. Microwave Theory and Tech. MT'T-5, No. 3, 208 (1957). 2114.

Richardson, J. M., Riley, R. B., Performance of threemillimeter harmonic generators and crystal detectors. IRE Trans. Microwave Theory and Tech. MTT-5, 131 (1957). 2244.

Richardson, W. P., Hartley, J. L., Hudson, D. C., Sweeney, W. T., Cutting characteristics of dental burs as shown by high speed photomicrography. U.S. Armed Forces Med. J. 8, 209 (1957). 2076. 
Richey, G. G., Hobbs, R. H., Stiehler, R. D., Temperature studies of air in a truck tire. Rubber Age 79, 273 (1956). 1917.

Richey, G. G., Schiefer, H. F., Appel, W. D., Krasny, J. F., Impact properties of yarns made from different fibers. Textile Research J. 23, 489 (1953). 734

Richey, G. G., Stiehler, R. D., Mandel, J., Measurement of treadwear of commercial tires. Rubber Age 73, 201 (1953). 761.

Richey, G. G., Stiehler, R. D., Steel, M. N., Mandel, J., Hobbs, R. H., Power loss and operating temperature of tires. J 64C1, 1 (1960).

Richmond, J. C., Coatings for space vehicles. Proc. 1st Symp. Surface Effects on Space And Craft Materials, Sponsored by USAF Air Research and Development Command and Lockheed Aireraft Corp., May 12-13, 1959 (Palo Alto, Calif.), p. 182 (John Wiley and Sons, Inc., New York, N.Y., 1959). $2910 \mathrm{~A}$.

Discussion of the paper "Heat treatment affects properties of enamel-steel composities," by J. H. Healey and L. K. Breeze. J. Am. Ceram. Soc. 41, 386 (1958). 2525.

Revision of Commercial Standard CS115-44 for porcelain enameled hot water tanks. Proc. Porcelain Enamel Inst. 20, 51 (1958). 2745.

Some methods used at the National Bureau of Standards for measuring thermal emittance at high temperatures. Proc. 1st Symp. Surface Effects on Space and Craft Materials, Sponsored by USAF Air Research and Development Command and Lockheed Aircraft Corp., May 12-13, 1959 (Palo Alto, Calif.), p. 182 (John Wiley and Sons, Inc., New York, N.Y., 1959). 3049 A.

Richmond, J. C., Cuthill, J. R., Tighe, N. J., The effect of a ceramic coating on the creep behavior of some high temperature alloys. Bull. Am. Ceram. Soc. 39, 4 (1959). 3072.

Richmond, J. C., Eubanks, A. G., Crandall, J. R., Flexibility of thin porcelain enameled sheet steel. Bull. Am. Ceram. Soc. 36, No. 2, 59, (1957). 2126.

Richmond, J. C., Eubanks, A. G., Moore, D. G., Studies of oxidation behavior of enameling iron during firing. Finish 11, No. 5, 50 (1954). 1153.

Richmond, J. C., Gordon, C. C., A thickness gage for ceramic coatings. J. Am. Ceram. Soc. 33, 295 (1950). 13.

Richmond, J. C., Harrison, W. N., The evaluation of small color differences: I. Visual observations. Bull. Am. Ceram. Soc. 38, 292 (1959). 3078.

Richmond, J. C., Harrison, W. N., Crandall, J.R., Effects of acid treatment on acid and abrasion resistance of porcelain enamels. J. Am. Ceram. Soc. 33, 314 (1950). 51 .

Richmond, J. C., Kirkpatrick, H. B., A radiosotope study of the nickel dip. J. Am. Ceram. Soc. 39, No. 2, 39 (1956). 1599.

The nickel dip: a radioisotope study of metallic deposits in porcelain enameling. Natl. Advisory Comm. Aeronaut. Tech. Note 3577 (1956). 1941.

Richmond, J. C., Lefort, H. G., Williams, C. N., Harrison, $W$. N. Ceramic coatings for nuclear reactors (a progress report). J Am. Ceram, Soc. 38, No. 2, 72 (1955). 1285 .

Richmond, J. C., McBurney, J. W., Copeland, M. A., Relations among certain specification properties of building brick and effects of differences in raw materials and methods of forming. J. Am. Ceram. Soc. 35, No. 12, 309 (1952). 528.

Richmond, J. C., Moore, D. G., Kirkpatrick, H. B., Harrison, $W$. N., Relation between roughness of interface and adherence of porcelain enamel to steel. Inst. Vitreous Enamellers Ltd. Bull. (Published by J. Am. Ceram. Soc.) 7, No. 5, 69 (1957). 2281.

Richmond, J. C., Moore, D. G., Pitts, J. W., Harrison, $W . N$., The galvanic corrosion theory for adherence of porcelain enamel ground coats to steel. Inst. Vitreous Enamellers Ltd. Bull. (Published by J. Am. Ceram. Soc.) 7, No. 4 (1957). 2358.
Richmond, J. C., Nimeroff, I., Hammond, H. K. III, Crandall, J. R., Gloss meter comparisons. J. Am. Ceram. Soc. 39, No. 3, 103 (1956). 1724.

Specular-gloss measurement of ceramic materials. J. Am. Ceram. Soc. 39, No. 3, 103 (1956). 1886.

Richmond, J. C., Stewart, J. E., Infrared emission spectrum of silicon carbide heating elements. J. 59, 405 (1957) RP2810.

Spectral emittance of ceramic-coated and uncoated specimens of Inconel and stainless steel. J. Am. Ceram. Soc. 42, No. 12, 633 (1959). 3053.

The spectral emittance of uncoated and ceramic-coated Inconel and type 321 stainless steel. NASA Memo 4-9-59W (1959). 3092.

Riddell, H. F., Newman, S. B., The drying twist in plant fibers. Textile Research J. 24, 113 (1954). 1181.

Riddell, H. F., Newman, S. B., Hammond, H. K. III, Becker value of manila rope by photoelectric reflectometry. ASTM Bull. No. 199, 84 (1954). 960.

Riddle,J.L., Temperature du zine et comparison de deux determinations du point du soufre dans un appareil ferme. Proc.-Verbaux Comité Consultatif de Thermometric, auxprés du Comité Intern. des Poids et Mésures (Paris, France) 5, 111 (1958). 2799.

Rieger, C. J., Carpenter, F. G., Light scattering by commercial sugar solutions. J 63A3, 205 (1959).

Ries, $F$. X. Selby, M. C., Behrent, L. F. RF voltmeter calibrating consoles. IRE Natl. Conv. Record 6, Pt. 5, 251 (1958). 2747.

Riggs, L. P., Bean, B. R., Synoptic variation of the radio refractive index. J 63D 1,91 (1959).

Riggs, L. P., Bean, B. R., Horn, J. D., Synoptic study of the vertical distribution of the radio refractive index. J 63D2, 249 (1959).

Riley, R. B., Convenient microwave harmonic generator. Rev. Sei. Instr. 27, 174 (1956). 1662.

Riley, R. B., Richardson, J. M., Performance of threemillimeter harmonic generators and crystal detectors. IRE Trans. Microwave Theory and Tech. MTT-5. 131 (1957). 2244

Ritz, $V$. H., Broad and narrow beam attenuation of $\operatorname{Ir}^{192}$ gamma rays in concrete, steel, and lead. Nondestructive Testing 16, No. 3, 269 (1958). 2480. Standard free-air chamber for the measurement of low energy $\mathrm{X}$-rays (20 to 100 kilovolts-constant-potential). J 64C1, 49 (1960).

Ritz, V. H., Attix, F. H., A determination of the gammaray emission or radium. J 59, 293 (1957) RP2801.

Ritz, $V . H .$, DeLaVergne, L., Attia, F. H., Cavity ionization as a function of wall material. J 60, 235 (1958) RP2842.

Roach, F. E., A review of observational results in airglow photometry. Ann. geophys. (Paris, France) II, No. 2, 214 (1955). 1242.

Photometric observations of the airglow. Ann. Intern. Geophys. Year 4, Pt. 1, 115 (1957). 2247.

The night airglow. Proc. IRE 47, 267 (1959). 3084. The nightglow: a review of observational results. Proc. Intern. Union Geodesy and Geophys. (Rome, Italy) (1954). 1191.

The night airglow. American Geophys. Union, Geophys, and the IGY, Geophys. Monograph No. 2, 97 (1958), 2825.

Roach, F. E., Elvey C. T., Aurora and airglow. Sci. Am. 193, No. 3, 140 (1955). 1271.

Roach, F. E., Hunten, D. M., Chamberlain, J. W., A photometric unit for the airglow and aurora. J. Atmospheric and Terrest. Phys. 8, 345 (1956). 1598.

Roach, F. E., Jammick, P. M., The sky and eye. Sky and Telescope 17, 164 (1958). 2830.

Roach, F. E., Marovich, E., A monochromatic low-latitude aurora. J 63D3, 297 (1959). Aurora of October $22 / 23,1958$, at Rapid City, South Dakota. J 64D2, 205 (1960).

Roach, F. E., McCaulley, J. W., Marovich, E., Origin of [OI] 5577 in the airglow and the aurora. J 63D1, 15 (1959). 
Roach, F. E., McCaulley, J. W., Purdy C. M., Comparison of absolute intensities of [OI] 5577 in the auroral and subauroral zones. J 63D1, 19 (1959).

Roach, F. E., Megill, L. R., Rees, M. H., Marovich, E. The height of nightglow 5577. J. Atmospheric and Terrest. Phys. 12, 171 (1958). 2817.

Roach, F. E., Meinel, A. B., Nightglow heights: a reinterpretation of old data. Astrophys. J. 3, 554 (1955). 1416

The height of the nightglow by the van Rhijn method. Astrophys. J. 122, No. 3, 530 (1955). 1534.

Roach, F. E., Rees, M. H., The absolute photometry of the Gegenschein. Symp. on the Airglow and the Aurora, September 1955, Belfast (Pergamon Press, London, England), p. 142 (1956). 1919.

Roach, F. E., Tandberg-Hanssen, E., Excitation mechanisms of the oxygen 5577 emission in the upper atmosphere. J 63D 3, 319 (1959).

Roach, F. E., Tandberg-Hanssen, E., Megill, L. R., Movements of airglow cells. J. Atmospheric and Terrest. Phys. 13, 122 (1958). 2640.

Possible movements of 5577 airglow. J. Atmospheric and Terrest. Phys. 13, 122 (1958). 2695.

The characteristic size of airglow cells. J. Atmospheric and Terrest. Phys. 13, 113 (1958). 2807.

Robbins, C. R., Levin, E. $M$., The system magnesium oxide-germanium dioxide. Am. J. Sci. 257, 63 (1959). 3094 .

Robbins, R. F., Richards, R. J., Jacobs, R. B., Holten, $D$. C., Heat transfer to boiling liquid nitrogen and hydrogen flowing axially through narrow annular passages. Proc. 1957 Cryogenic Eng. Conf., Paper $\mathrm{G}-3$, p. 375 , Edited by K. D. Timmerhaus, Chem. Eng. Dept., Univ, of Colorado (Boulder, Colo.) (1958). 2581.

Robbins, R. F., Scott, L. E., Mann, D. B., Birmingham, $B$. $W$., Temperature stratification in a nonventing liquid helium dewar. J 64C1, 19 (1960).

Roberts, A. G., Crouse, W. A., Pizer, R. S., Abrasive jet method for measuring abrasion resistance of organic coatings. ASTM Bull. No. 208, 36 (1955). 1249.

Roberts, A. G., Malloy, A., Soller, W., Evaluation of adhesion of organic coatings by ultracentrifugal and other methods. Paint, oil, and Chem. Rev, 116, No. 18, 14; 116, No. 19, 26 (1953). 715.

Roberts, A. G., Pizer, R. S., Improved dip coater. Anal. Chem. 26, 790 (1954), 1033.

Roberts, A. G., Pizer, R. S., Progective coating adhesion measurement using and electronic averaging device for the adherometer. ASTM Bull. No. 221, 53 (1957). 2273.

Roberts, A. G. Weissberg, S. G., Film-forming properties of cellulose acetate propionates. Effect of solvents, diluents, and plasticizers. Ind. Eng. Chem. 43, 2088 (1951). 237.

Roberts, D. E., Frederikse, H. P. R., Hosler, W. R., Electrical conduction in magnesium stannide at low temperatures. Phys. Rev, 101, No. 6, 1653 (1956). 1695.

Roberts, D. E., Mandelkern. L., Some properties of polymer networks formed from oriented chains of natural rubber. J. Am. Chem. Soc. 80, 1289 (1958). 2765. Thermodynamics of crystallization in high polymers: natural rubber. J. Am. Chem. Soc. 77, 781 (1955). 1558.

Roberts, D. E., Mandelkern, L., Diorio, A. F., Reversible contractile processes in fibrous macromolecules. J. Am. Chem. Soc. 80, 500 (1958), 2743.

Roberts, D. E., Mandelkern, L., Flory, P. J., The isotropic length of polymer networks. J. Am. Chem. Soc. 79, 1515 (1957). 2370.

Roberts, D. E., Mandelkern, L., Hellmann, M., Brown, D. W., Quinn, F. A., Jr., The melting transition of polymethylene, J. Am. Chem. Soc. 75, 4093 (1953). 869.

Roberts, D. E., Mandelkern, L., Quinn, F. A. Jr., Thermodynamics of crystallization in high polymers: gutta percha. J. Ám. Chem. Soc. 78, 926 (1956). 1965.
Roberts, D. E., Newton, C. J., Mandelkern L.,, Preferred orientation in stark rubber studied with an automatic integrating pole-figure goniometer. J. Appl. Phys. 26, No. 12, 1521 (1955), 1454.

Roberts, D. E., Quinn, F. A., Jr., Work, R. N., Volumetemperature relationships for the room temperature transition in Teflon. J. Appl. Phys. 22, 1084 (1951) 356.

Robertson, A. F., Fire research at the National Bureau of Standards. Proc. Fire Research, 1st Correlation Conf. 1956, Natl. Academy of Sci. Publ. No. 475, p. 40 (1957). 2124.

Fire research in the United States. Fire Research and Fire Prevention Conf., 1958 Bulletin No. 2, Division Building Research, Natl. Research Council Publ. NRC 5011, p. 51 (Ottawa, Canada) (1958). 2563.

Some activities of the NBS in the field of fire research. Natl. Acad. Sci.-Natl. Research Council Publ. No. 475 (1956). 1870.

Robertson, A. F., Gross, D., An electrical-analog method for transient heat-flow analysis. J 61, 105 (1958) RP2892.

Self-ignition temperatures of materials from kineticreaction data. J 61, 413 (1958) RP2909.

Robertson, A. F., Gross, D., Loftus, J., A method for measuring surface flammability of materials using a radiant energy source. Am. Soc. Testing Materials Proc. 56, 1437 (1956). 1593.

Robertson, A. F., Raskin, W. H., Adiabatic apparatus for the study of self-heating of poorly conducting materials. Rev. Sci. Instr. 25, 541 (1954). 942.

Robertson, A. F., Ryan, J. V., Proposed criteria for defining load failure of beams, floors, and roof constructions during fire tests. J 63C2, 121 (1959).

Robinson, C.S., Leiss, J.E., Penner, S., Range straggling of high-energy electrons in carbon. Phys. Rev. 107, 1544 (1957). 2279.

Robinson, H. E. Cosgrove, L. A. Powell, F. J., Thermal resistance of airspaces and fibrous insulations bounded by reflective surfaces. (1957) BMS151.

Robinson, H. E., Powlitch, F. J., The thermal insulating value of airspaces. Housing and Home Finance Agency (Washington, D.C.) Housing Research Paper No. 32 (1954). 1203.

Robinson, H. E., Watson, T. W., Interlaboratory comparison of thermal conductivity determinations with guarded hot plates. ASTM. Spec. Tech. Publ. No. 119 (1951). 251 .

Robinson, M., Kanagy, J.R., Studies on leather by means of a sonic technique. J. Am. Leather Chemists Assoc. 51, 174 (1956). 1905.

Robinson, R. A., Bates, R. G., Guggenheim, E. A., Harned, H. S., Ives, D. J., Janz, G. J., Monk, C. B., Stokes, R. H., Wynne-Jones, W. F. J., Standard electrode potential of the silver, silver chloride electrode. J. Chem. Phys. 25, 361 (1956). 1895.

Roder, H. M., Hall, W. J., Powell, R. L., Thermal and electrical conduetivity of aluminum and aluminum alloys. In Low-Temperature physies and chemistry; 1957 Proc. 5th Intern. Conf., Univ. of Wisconsin (Madison, Wis.), p. 389 (1958). 2841A.

Roder, H. M., Powell, R. L., Thermal conductivities of copper and copper alloys. Proc. 1956 Cryogenic Eng. Conf., Paper E-3, p. 166, Edited by K. D. Timmerhaus, Chem, Eng. Dept., Univ, of Colorado (Boulder, Colorado) (1957). 2393.

Roder, H. M., Powell, R. L., Hall, W. J., Thermal and electrical conductivity of pure copper. In Low-Temperature physics and chemistry; 1957 Proc. 5th Intern. Conf., University of Wisconsin (Madison, Wisconsin), p. 364 (1958). $2841 \mathrm{~B}$.

Thermal conductivities of common commercial aluminum alloys. Proc. 1957 Cryogenic Eng. Conf. Paper G-6, p. 408 , Edited by K. D. Timmerhaus, Chem. Eng. Dept., Univ. of Colorado (Boulder, Colo.) (1958). 2842 .

Transport properties of dilute alloys of copper. Physica 24, S176 (1958). 2852A. 
Roder, H. M., Powell, R. L., Rogers, W. M., Low temperature thermal conductivity of some commercial coppers. J. Appl. Phys. 28, 1282 (1957). 2181.

Rodney, W. S., Optical properties of cesium iodide. J. Opt. Soc. Am. 45, No. 11, 987 (1.955). 1436.

Rodney, $W$. S., Djurle, E., Infrared phase contrast refractometer. J. Opt. Soc. Am. 48, No. 6, 388 (1958). 2600 .

Rodney, W. S., Malitson, I. H., Refraction and dispersion of thallium bromide iodide. J. Opt. Soc. Am. 46, No. 11,956 (1956). 1842.

Rodney, W. S., Malitson, I. H., King, T. A., Refractive index of arsenic trisulfide. J. Opt. Soc. Am. 48, No. 9, 633 (1958). 2726.

Rodney, W. S., Malitson, I. H., Murphy, V. F., Jr., Refractive index of synthetic sapphire. J. Opt. Soc. Am. 48, No. 1, 72 (1958). 2727

Rodney, W. S., Spindler, R. J., Index of refraction of fused-quartz glass for ultraviolet, visible, and infrared wavelengths. J. Opt. Soc. Am. 44, No. 9, 677 (1954). 1035

Refractive indices of cesium bromide. J. Opt. Soc. Am. 4\%, 431 (1952). 527.

Rodney, W.S., Stephens, R. E., Plyler, E.K., Spindler, R.J., Refractive index of potassium bromide for infrared radiant energy. J. Opt. Soc. Am. 43, 110 (1953). 812.

Roeser, W. F., Lonberger, S. T., Methods of testing thermocouples and thermocouple materials. (1958) C590.

Rogers, S. S., Mandelkern, L., Glass formation in polymers: I. The glass transitions of the poly-(n-alkyl methacrylates). J. Phys. Chem. 61, 985 (1957). 2142 .

Rogers, S. S., Martin, G. M., Mandelkern, L., Volume temperature relations of amorphous polymers over an extended temperature range. J. Polymer Sci. 20, 579 (1956). 1975 .

Rogers, W. M., Hall, W. J., Powell, R. L., Tables of transport integrals: a supplement. $J$ 63B $1, \quad 23$ (1959).

Rogers, W. M., Powell, R. L., Tables of transport integrals $J_{n}(z) \equiv \int_{0}^{x} \frac{e^{z} z^{n} d z}{\left(e^{z}-1\right)^{2}} \cdot \quad$ (1958) C595.

Rogers, W. M., Powell, R. L., Coffin, D. O., An apparatus for measurement of thermal conductivity of solids at low temperatures. J 59, 349 (1957) RP2805.

Rogers, W. M., Powell, R. L., Roder, H. M., Low-temperature thermal conductivity of some commercial coppers. J. Appl. Phys. 28, 1282 (1957). 2181. Thermal conductivities of copper and copper alloys. Proc. 1956 Cryogenic Eng. Conf., Paper E-3, p. 166, Edited by K. D. Timmerhaus, Chem. Eng. Dept., Univ. of Colorado (Boulder, Colo., 1957). 2393.

Rohrlich, $F$., Johnson, H., Atomic electron affinities. Nature No. 4656, 183 (1959). 2900.

Romanoff, $M$., Results of Bureau of Standards 45-year corrosion study. Petroleum Eng. 29, No. 3 and 4 615 (1957). 2287.

Romanoff, M., Denison, I. A., Effect of exposure to soils on the properties of asbestos-cement pipes. Corrosion 10, 169 (1954). 998.

Rosberry, F. W., Equipment and method for photoelectric determination of image contrast suitable for using square-wave targets. J 64C1, 57 (1960).

Rosberry, F. W. Hotchkiss, R. N., Washer, F. E. Spurious resolution of photographic lenses. J. Opt. Soc. Am. 41, 600 (1951). 315 .

Rosberry, F. W., Washer, F. E., New resolving power test chart. J. Opt. Soc. Am. 41, 597 (1951). 268.

Rose, E. E., Sweeney, W. T., Myerson, R. L., Semmelman, J. O., Proposed specification for plastic teeth. J. Prosthetic Dentistry 7,420 (1957). 2272.

Rose, J. C., Broida, H. P., Effects of plastic and steel surfaces on clotting time of human blood. Proc. Soc. Exp. Biol. Med. J. 86, 384 (1954). 1003.

Rose, J. C., Broida, H. P., Freis, E. D., A variable heart pump permitting independent control of rate, output and ejection velocity. Science 115, No. 2996, 603 (1952). 381.

Rose, J. C., Broida, H. P., Hufnagel, C. A., Gillespie, J. F., Rabile, P. J., Freis, E. D., A method for the study of the circulation in the dog using a mechanical left ventricle. J, Appl. Physiol. 7, 580 (1954). 920.

Rose, J. C., Lazaro, E. J., Broida, H. P., Dynamics of complete right ventricular failure in dogs maintained with an extracorporeal left ventricle. J. Am. Heart Assoc. 4, No. 2, 173 (1956). 1685.

Rosenberg, S.J., Note on thermal stabilization of austenite iron-carbon-nickel alloys. Acta Met. 376 (1953). 777.

Temper brittleness of boron-treated steel. Wright Air Devel. Ctr. (Ohio) Tech. Rept. 56-396 (1956). 1913.

Rosenberg, S. J., Carwile, N. L., A study of 17-7 PH stainless steel. WADC Tech. Rept. 58-653 (1959). 2883.

Rosenberg, S. J., Grimsley, J. D., A study of the impact properties of boron-treated steels. J. Iron and Steel Inst. 182, 278 (1956). 1601.

Rosenberg, S. J., Irish, C. R., New ultra high strength steel. Materials and Methods 45, 145 (1957). 2214. Sigma phase in high-purity 18-10. Metal Progr. 61, No. 5, 92 (1952). 542.

Rosenberg, S. J., Meyerson, M. R., A method of controlled slack quenching of impact bars. Rev. de met. 55, No. 4, 317 (1958). 2426.

Impact properties of slack-quenched alloy steels. J 59, 273 (1957) RP2799.

The influence of heat treating variables on the martensite transformation in SAE 1050 steel. Trans. Am. Soc. Metals 45, 1225 (1954). 1186.

Rosenblatt, J. R. On prediction of system behavior. Proc. of the New York University-Industry Conf. on Reliability Theory (New York, N.Y.), p. 39 (June 1958). $2660 \mathrm{~A}$.

On prediction of system performance from information on component performance. Proc. IRE Western Joint Computer Conf. (Los Angeles, Calif.), p. 85, (1957), 2223.

On some aspects of prediction in the study of complex systems. Proc. NYU-RCA Working Conf. on Theory of Reliability (Ardsley-on-Hudson, N.Y.), p. 24 (1957). 2225.

Rosenwasser, H., De Juren, J. A., Pulse-height measurements of recoils from $\mathrm{B}^{10}(n, \alpha) \mathrm{Li}^{7}$. Phys. Rev. 93, 831 (1954). 1123.

Ross, G. S., Frolen, L. J., Simple rotating molecular still. J 62, 187 (1959) RP2951.

Ross, G. S., Glasgow, A. R., Jr., Improved stainless steel needle valve. Anal. Chem. 26, 2003 (1954). 1034.

Ross, G. S., Glasgow, A. R., Jr., Horton, A. T., Enagonio, D., Dixon, H. D., Saylor, C. P., Furukawa, G. T., Reilly, M. L., Henning, J. M., Comparison of cryoscopic determinations of purity of benzene by thermometric and calorimetric procedures. Anal. Chim. Acta 1\%, 54 (1957); Proc. of I.U.P.A. Intern. Symp. on Purity Control by Thermal Anal. (Amsterdam, The Netherlands) (April 1957). 2062.

Ross, J., Amdur, I., On the calculation of properties of gases at elevated temperatures. Combustion and Flames 2, 412 (1958). 2664.

Ross, J., Oppenheim, F., Temperature dependence of distribution functions in quantum statistical mechanics. Phys. Rev. 10\%, No. 1, 28, (1957). 2340.

Rossini, F, D., Glasgow, A. R., Krouskop, N. C., Apparatus for determining freezing points at saturation pressure from time-temperature freezing and melting experiments. Anal. Chem. 22, 1521 (1950). 26.

Rossini, F. D., Mair, B. J., Westhaver, J. W., Theoretical analysis of fractionating process of adsorption. Ind. \& Eng. Chem. 42, 1279 (1950). 156.

Roth, F. L., Time-temperature behavior of rubber from measurements of indentation hardness. Proc. Joint Army-Navy-Air Force Conf, on Elastomer Research and Development 2, 5th meeting (Oetober 1958), p. 399 (Materials Laboratory, WADC, Wright-Patterson Air Force Base, Ohio) (1958). 2848. 
$R o t h, F . L$., Decker, $G, E$., Influence of variations in rotors, dies, and rate of shear on Mooney viscosity. India Rubber World 128, 339 (1953). 740.

Roth, F. L., Decker, G. E., Stiehler, R. D., Temperature control during mixing of rubber compounds. Rubber World 132, $482(1955), 1526$.

Roth, F. L., Mandel, J., Steel, M. N., Stiehler, R. D., Measurement of the aging of rubber vulcanizates. J 63C2, 141 (1959).

Roth, F. L., Martin, G. M., Stiehler, R. D., Behavior of "pure gum" rubber vulcanizates in tension. Trans. Inst. Rubber Ind. 32, No. 6, 189 (1956). 1640.

Roth, F. L., McKinney, J. E., Carbon black differentiation by electrical resistance of vulcanizates. Ind. Eng. Chem. 44, 159 (1952). 408.

Roth, $F$, $L_{*}$, Stiehler, $R$. D., Standard natural rubber. Rubber Age 78, 77 (1955). 1505.

Tension testing of rubber rings. ASTM Bull. No. 233, 38 (1958). 2801.

Vulcanization characteristics of natural rubber. Symp. on Recent Developments in the Evaluation of Natural Rubber, 1952; Am. Soc. Testing Materials Spec. Tech. Publ. No. 136, p. 50 (1953); India Rubber World 127, 783 (1953); Rubber Chem, Tech. 26, 593 (1953). 909 .

Roth, R.S., Phase ecuilibrium relations in the binary system lead oxide-niobium pentoxide. J 62, 27 (1959) RP2925.

Revision of the phase equilibrium diagram of the binary system calcia-titania, showing the compound $\mathrm{Ca}_{4} \mathrm{Ti}_{3} \mathrm{O}_{10}$. J 61, 437 (1958) RP2913.

Unit-cell data of the lead niobate $\mathrm{PbNb}_{2} \mathrm{O}_{6}$. Acta cryst. 10, Pt. 6, 437 (1957). 2401.

Zirconia reactions in binary oxide systems. J. Am. Ceram. Soc. 39, No. 5, 196 (1956). 1978.

Roth, R. S., Hasko, S., Beta-alumina-type structure in the system lanthana-alumina. J. Am. Ceram. Soc. 41, No. 4, 146 (1958). 2472.

Roth, R. S., Levin, E. M., Phase equilibria in the subsystem barium disilicate-dibarium trisilicate. J 62, 193 (1959) RP2953.

Polymorphism in barium disilicate. Am. Mineralogist 44, No. 3-4, 452 (1959). 3018.

Roth, R. S., Walker, R. F., Schneider, S. J., Some evidence for structural anomalies in pure cristobalite. J. Am. Ceram. Soc. 42, No. 12, 642 (1959). 3048.

Rothberg, S., Jessup, R. S., Net heat of combustion of $\mathrm{AN}-\mathrm{F}-58$ aircraft fuels. Ind. Eng. Chem. 43, 981 (1951). 265

Rothman, $\dot{S}$., Device for filtering and precise dispensing of solutions in closed systems. Anal. Chem. 22, 367 (1950). 47.

Rothman, S., Adelson, E., Schwebel, A., Langdell, R. D., Adsorption of $\mathrm{C}^{14}$ dextran to human blood platelets and red blood cells in vitro. Vox Sanguinis 2, 104 (1957). 2011.

Rothman, S., Mandel, J., McCann, F. R., Weissberg, S. G., Sorption of macromolecules to solid surfaces; sorption of dextran to cellulose nitrate membranes in the presence of serum albumin and surface-active agents. J. Colloid Sci. 10, No. 4, 338 (1955), 1500 .

Rothman, S., McCann, F. R., Improved Hepp-Seatehard osmometer. J. Polymer Sci. 18, 151 (1955). 1362.

Rothman, S., Schwebel, A., Weissberg, S. G., Sorption of dextran to collodion membranes. J. Polymer Sci. 11, 381 (1953). 832.

Rothman, S., Simha, R., Weissberg, S. G., Viscosities of very dilute polymer solutions. J. Polymer Sci. 5, 141 (1950). 166.

Rothman, S., Wales, M., Marshall, P. A., Weissbera, S. G. Application of viscosity methods to the study of branching in polysaccharides. Ann. N.Y. Acad. Sci. $\mathbf{5 7}, 353(1953) .654$

Roubique, C. J., Crichlow, W. Q., Spaulding, A. D., Beery, W. $M$., Determination of the amplitude-probability distribution of atmospheric radio noise from statistical moments. J 64D1, 49 (1950).

Rowen, J.W., Forziati, F. H., Reeves, R. E., Spectrophotometric evidence for the absence of free aldehyde groups in periodate-oxidized cellulose. J Am. Chem. soc. 73, 4484 (1951). 314

Rubin, B., Hamer, W. J., Malmberg, M. S., Theoretical electromotive forces for cells containing a single solid or molten chloride electrolyte. J. Electrochem. Soc. 103, 8 (1956). 1957.

Rubin, R.J., Bazely, N.W., Montroll, E. W., Shuler, K. E., Studies in nonequilibrium rate processes, III. The vibrational relaxation of a system of anharmonic oscillators. Errata in J. Chem. Phys. 28, 700 (1958). 2786.

Rubin, R. J., Herman, R. C., Vibrational energy exchange between diatomic molecules and a surface. J. Chem. Phys. 29, 591 (1958). 2862.

Rubin, R. J., Mazur, J., Quantum-mechanical calculation of the probability of an exchange reaction for constrained linear encounters. J. Chem. Phys. 31, No. $5,1395(1959), 3025$.

Rubin, $R$. J., Shuler, $K$. E., On the relaxation of vibrational nonequilibrium distributions, III: The effect of radiative transitions on the relaxation behavior. J. Chem. Phys. 26, No. 1, 137 (1957). 2235.

On the relaxation of vibrational nonequilibrium distributions: I. Collisional relaxation of a system of harmonic oscillators. J. Chem. Phys. 25, 59 (1956); II. The effect of the collisional transition probabilities on the relaxation behavior, J. Chem. Phys. 25, 68 (1956). 1847.

Rubin, S., A low voltage regulator. Television Eng. 3, No. 3,8 (1952). 364

Application of low $\mathrm{Mu}$ triodes to series regulator circuits. Trans. of RETMA Symp. (1956). 1630. Stable-output oscillator. Electronics 25, No. 10, 154 (1952). 552.

Rubin, S., Boggs, G. E., Gated-beam mixer. Electronics 24, 196 (1951). 243.

Rubin, S., Scott, D., Gas tube stabilized power supplies. Tele-Tech 14, No. 7,70 (1955). 1353.

Rucker, N., DeLollis, N. J., Weir, J. E., Comparative strengths of some adhesive-adherend systems. Trans. Am. Soc. Mech. Engrs. 73, 183 (1951). 208.

Rueger, L. J., Teflon insert makes needle vacuum tight. Rev. Sci. Inst. 24, 551 (1953). 848.

Rueger, L. J., Lyons, H., Nuckolls, R. G., A high temperature, broad-band Stark cell for microwave spectroscopy. Rev. Sci. Inst. 22, 428 (1951). 178.

Rueger, L. J., Lyons, H., Nuckolls, R. G., Kessler, M., Microwave spectra of deutero-ammonias. Phys. Rev. 81, 630 (1951). 260.

Rueger, L. J., Nuckolls., R. G., A broad-band coaxial Stark cell for microwave spectroscopy. Rev. Sci. Instr. 23, 635 (1952). 358 .

Rueger, L. J., Nuckolls, R. G., Lyons, H., Microwave absorption spectrum of $\mathrm{ND}_{3}$. Phys. Rev. 89, 1101 (1953). 766 .

Ruegg, F. W., Halpern, C., A study of sampling of flame gases. J 60, 29 (1958) RP2818.

Ruegg, $F . W$., Shafer, $M$. R., Liquid-flowmeter calibration techniques. Trans. Am. Soc. Mech. Engrs. 80; No. 7, 1369 (1958). 2613.

Ruehrwein, R. A., Hashman, J. S., Formation of ozone from atomic oxygen at low temperatures. J. Chem. Phys. 30, No. 3, 823 (1959). 2954.

Rumfelt, A. Y., Schafer, G. E., Mismatch errors in cascade-connected variable attenuators. IRE Trans. Microwave Theory and Tech. MTT-7, No. 4, 447 (1959). 2993.

Rumsey, V. H., Cottony, H. V., Elliott, R. S., Jordan, E. C., Siegel, K.'M., Wait, J. R., Woodyard, O. C., Antennas and waveguides, and annotated bibliography. U.S.A. National Committee Report, Intern. Sci. Radio Union Subcomm. 6.3. IRE Trans. Ant. Prop. AP-7, 87 (1959), 2897

U.S.A. National Committee report URSI Subcommission 6.3, antennas and waveguides, and annotated bibliography. Proc. 12th Gen. Assembly, Intern. Sci. Radio Union, August-September 1957 (Boulder, Colorado), on 11, Pt. 6, 121 (1957). 2402. 
Rupp, N.W.,Dickson, G., Lawson, M.E.,Jr., Sweeney, W. T., A method for measuring the mucosal surface contours of impressions, casts and dentures. J. Am. Dental Assoc. 54, 24 (1957). 1987.

Rusch, W. V. T., Macmillan, R. S., Golden, R. M., A very-low-frequency antenna for investigating the ionosphere with horizontally polarized radio waves. J 64D 1, 27 (1960).

Russell, H. H., The National Bureau of Standards 1000lb balance. The Scale J. 41, No. 11 (1955). 1539.

Russell, S. B. Goodman, S., Poisson's ratio of aireraft sheet materials for large strains. Proc. First Midwestern Conf. on Solid Mechanies (Univ. of III., Urbana, Illinois), p. 1 (1953). 798.

Russell, S. B., Goodman, S., Noble, C. E., Effect of internal radiant heat transfer on temperature distribution, thermal stress, and deflection in box beams. J 62, 175 (1959) RP2949.

Ruthberg, S., Microwave double-sweep method for analysis of time-dependent cavity characteristics. Rev. Sci. Instr. 29, No. 11, 999 (1958). 2636.

Ruthberg, S., Klein, E. I., On small precision bottles of quartz and low-loss glass. Rev. Sci. Instr. 28, No. 3, 205 (1957), 2224 .

Rutishauser, $H$., Solution of eigenvalue problems with the LR-transformation. (1958) AMS49.

Ryan, J. V., Robertson, $A . F$, Proposed eriteria for defining load failure of beams, floors, and roof construction during fire tests. J $63 \mathrm{C} 2,121$ (1959).

Ryan, W. E., Ellenwood, R. C., A VHF and microwave matching termination. Proc. IRE 41, 104 (1953). 638.

Ryan, W. E., Sorrows, H. E., Ellenwood, R. C., Evaluation of coaxial slotted-line impedance measurements. Proc. IRE 39, 162 (1951). 234.

Ryge, G., Dickson, G., Schoonover, I. C., Dental amalgam: the effect of mechanical condensation of some physical properties. J. Am. Dental Assoc. 45, 269 (1952). 423.

Rynders, G. F., Van Valkenberg, A., Synthetic cuspidine. Am. Mineralogist 43, No, 11 and 12, 1195 (1958). 2795 .

Sacchi, H., Paffenbarger, G. C., A simple technique for making porcelain jacket crowns. J. Am. Dental Assoc. 54, 366 (1957). 2000.

Sack, H. S., Marvin, R. S., Aldrich, R., The dynamic bulk viscosity of polyisobutylene. J. Appl. Phys. 25, No. 10,1213 (1954). 1182 .

Sager, E. E., Byers, F. C., Dissociation of 4-chloro-4'aminodiphenylsulfone. J 59, 245 (1957). RP2794.

Saito, S., Edelman, S., Brooks, R., Jones, E., Smith, E. R., A stroboscopic vibration analyzer. J 63C2, 97 (1959).

Salaman, R., Agy, V., Davies, $K$. An atlas of obliqueincidence ionograms. (1959) TN31 (PB151390).

Salazar, H., Mockler, R. C., Barnes, J., Beehler, R., Fey, $L ., \quad$ The ammonia maser as an atomic frequency and time standard. IRE Trans. Instrumentation I-7, No, 3-4, $201(1958), 2804$

Sales, M., Meggers, W.F., Catalán, M.A., Term analysis of the second spectrum of rhenium ( $R e$ II). J 61. 441 (1958) RP2914.

Salisbury, M. W., Bailey, D. K., Bateman, R., Berkner, L. V., Booker, H. G., Montgomery, G. F., Purcell, E. M., Wiesner, J. B., A new kind of radio propagation at very high frequencies observable over long distances. Phys. Rev. 86, No. 2, 141 (1952). 369.

Salmon, H. I., Ogburn, F., Commutator for periodically changing the direction and magnitude of direct currents. Plating 43, 343 (1956). 1652.

Notes on post-treatment for HAE coatings. Plating 43, 1251 (1956). 1786

Salmon, H. I., Ogburn, F., Kronenberg, M. L., Electrolytic coatings on magnesium base alloys from alkaline chromate solutions. Plating 42, 271 (1955). 1332

Salzberg, C. D., Greenstone, R., Systematic ionospheric winds. J. Geophys. Research 56, No. 4, 521 (1951). 319 .
Salzer, $H, E$., An elementary note on powers of quaternions. Am. Math. Mo. 59, No. 5, 298 (1952). 390. Coefficients for polar complex interpolation. $\mathrm{J}$. Math. and Phys. 24, No. 2, 96 (1950). 36.

Formulas for calculating the error function of a complex variable. Math. Tables and Other Aids to Computation 5, 67 (1951). 241.

Formulas for finding the arguments for which a function has a given derivative. Math. Tables and Other Aids to Computation 5, No. 36, 213 (1951). 242.

Formulas for inverse osculatory interpolation in the complex plane. J 59, 233 (1957) RP2792.

Formulas for numerical differentiation in the complex plane, J. Math. and Phys, 31, No. 3, 155 (1952). 453.

Formulas for numerical integration of first and second order differential equations in the complex plane. J. Math. and Phys. 29, No. 3, 207 (1950). 61. Radix table for obtaining hyperbolic and inverse hyperbolic functions to many places. J. Math. and Phys, 32, 197 (1953). 810.

Radix table for trigonometric functions and their inverses to high accuracy. Math. Tables and Other Aids to Computation 5, 9 (1951). 296.

Tables of osculatory interpolation coefficients. (1959) AMS56.

Zeros of the derivative of Bessel functions of fractional order. Math. Tables and Other Aids to Computation VII, 69 (1953). 912.

Salzer, H. E., Lieblein, J., Table of the first moment of ranked extremes. J 59, 203 (1957) RP2787.

Samson, C. A., Effects of high-altitude nuclear explosions on radio noise. J 64D 1, 27 (1960).

Samson, C. A., Crichlow, W. Q., Disney, R. T., Jenkins, M. A., Quarterly radio noise data-March, April, May 1959. (1960) TN18-2 (PB151377-2).

Radio noise data for the International Geophysical Year July 1, 1957-December 31, $1958 . \quad$ (1959) TN18 (PB151377).

Samson, C. A., Disney, R. T., Operating instructions for ARN -2 auxiliary log-linear noise recorder. (1960) TN45 (PB151404).

Sandholzer, M.W., Some properties of flameproof fabrics. Am. Dyestuff' Reptr. 48, No. 2, 37 (1959). 3050.

Sanford, J.W., Caul, H. J., Serio, A. F., Properties of self-curing denture base resins. J. Am. Dental Assoc. 44, $295(1952) .521$.

Saunders, J. B., An improved optical test for spherical aberration. Letter to Editor, J. Opt. Soc. Am. 44, No. 8,664 (1954). 950 .

Parallel testing interferometer. J 61, 491 (1958) RP2917.

The Kosters double-image prism. Appendix C of "Concepts of Classical Opties", p. 393 (W. H. Freeman and Co., San Francisco, California) (1958). 2822 .

Saunders, J. B., Gross, F. L., Interferometer for large surfaces. J 62, 137 (1959) RP2943.

Savage, $I . R$., Contributions to the theory of rank order statistics - the two sample case. Ann. Math Stat. 27, 3 (1956), 1661.

On the independence of tests of randomness and other hypotheses. J. Am. Stat. Assoc. 52, 53 (1957). 2231

Savage, I. R., Grab, E., Tables of expected values of 1/X for positive Bernoulli and Poisson variables. J. Am. Stat. Assoc. 49, 169 (1954). 1161.

Savitt, J., Recombination and attachment in the Fregion during the eclipse of May 20, 1947. J. Geophys. Research 55, 385 (1950). 121.

Sawada, F. H., Carter, C. J., Freeze, P. D., Blackburn, G. F., Development and evaluation of ceramic coatings for thermocouples. Wright Air Devel. Ctr. (Ohio) Tech. Rept. 56-135 (1956). 1674.

Saxon, D. S., Cahn, A. S., Jr., Modes of vibration of a suspended chain. Quart. J. Mech. and Appl. Math, 6, Pt. 3, 273 (1953). 770. 
Saxon, D. S., Gerjuoy, E., Application of variational methods to intermediate and high energy scattering. Phys. Rev. 94, 478 (1954). 955.

Fariational calculation of scattering cross sections. Phys. Rev. 85, No. 5, 939 (1952). 617.

Variational principles for the acoustic field. Phys. Rev. 94, No. 6, 1445 (1954). 1223.

Saxon, D. S., Holzer, R. E., Distribution of electrical conduction currents in the vicinity of thunderstorms. J. Geophys. Research 57, No. 2, 207 (1952). 432.

Saxon, D. S., LeLevier, R. E., An optical model for nucleon-nuclei scattering. Phys. Rev. 87, 40 (1952). 395.

Saylor, C. P., Calculation of cryoseopic data. Anal. chim. acta 17, 36 (1957). 2042.

Saylor, C. P., Brice, A. T., Zernike, F., Color phase contrast microscopy: requirements and applications. J. Opt. Soc. Am. 40, 329 (1950). 37.

Saylor, C. P., Glasgow, A. R., Jr., Ross, G. S., Horton, A. T., Enagonio, D., Dixon, H. D., Furukawa, G. T. Reilly, M. L., Henning, J. M., Comparison of cryoscopic determinations of purity of benzene by thermometric and calorimetric procedures. Anal. chim. acta 17, 54 (1957); Proc. of I.U.P.A. International Symposium on Purity Control by Thermal Analysis (Amsterdam, The Netherlands) (April 1957). 2062.

Sayre, W. R., Furey, J. J., Hague, J. L., Determination of nitrogen and sulfur in steel. Anal. Chem. 24, 199 (1952). 426.

Scal, $R$. $K-F$., New techniques for fabrication of airborne electronic equipment. Proc. IRE 43, No. 1, 4 (1955). 1415.

Scal, R. K-F., Henry, R. L., Shapiro, G., New techniques for electronic miniaturization. Proc. IRE (Sydney, Australia) 13, No. 3, 75 (1952). 489.

Scanlon, $W$. W., Frederiske, H.P.R., Johnson, V. A. Methods of experimental physies. Chapter 7.6, Thermoelectric Effects. Edited by Lark-Horovitz and V. A. Johnson (Academic Press Inc., New York, N.Y.), p. 114 (1959). 2990.

Schach, $A$., Two forms of mathematical induction. Math. Mag. 32, No. 2, 83 (i958). 2855.

Schafer, G. E., A modulator for microwave mixers. IRE Trans Microwave Theory and Tech. MTT-6, 333 (1958). 2428.

Schafer, G. E., Beatty, R. W., A method for measuring the directivity of directional couplers. IRE Trans. Microwave Theory and Tech. MTT-6, No. 4, 419 (1958). 2425

Schafer, G. E., Herbstreit, J. W., Norton, K. A., Rice, P. L., Radio wave scattering in tropospheric propagation. Conv. Record of IRE, Pt. 2, Antennas and communications, p. 85 (1953). 808.

Schafer, G. E., Rumfell, A. Y., Mismatch errors in cascade-connected variable attenuators. IRE Trans. Microwave Theory and Tech. MTT-7, No. 4, 447 (1959). 2993.

Schaffer, R., Isbell, H. S., Bंranched-chain higher sugars. J. Am. Chem. Soc. 80, 756 (1958). 2476.

Branched-chain higher sugars. I. A 9-aldo-4-Cformylnonose derivative. J. Am. Chem. Soc. 81, 2178 (1959). 2903.

Branched-chain higher sugars. II. A diethylideneoxtose. J. Am. Chem. Soc. 81, 2838 (1959). 2904 Reaction of dimeric 5-aldo-1,2-O isopropylidene-Dxylo-pentofuranose with cyanide and the preparation of calcium 1, 2-O-isopropylidene-L-idofururonate-6C14 dihydrate. J. Am. Chem. Soc. 79, 3867 (1957). 2280 .

Structure of 5-aldo-1,2-O-isopropylidene-D-xylo-pentofuranose. J. Am. Chem. Soc. 79, 3864 (1957). 2321.

Synthesis of maltose-1-C14, maltobiono- $\delta$-lactone1-C ${ }^{14}$, and lithium maltobionate-1-C ${ }^{14}$ from $3-(\alpha-\mathrm{D}-$ glucopyranosyl)-D-arabinose. J. Am. Chem. Soc. 78, No. 9, 1887 (1956). 1909.

Schaum, J. H., Harwell, R. A., Versatile core boxes. Foundry 80, No. 5, 154 (1952). 619.
Scheer, M. D., Klein, R., The addition of bydrogen atoms to solid olefine at $-195^{\circ}$. J. Am. Chem. Soc. 80, 1007 (1958). 2803.

The reaction of hydrogen atoms with solid olefins at $-195^{\circ}$. J. Phys. Chem. 62, 1011 (1958). 2828.

Scheer, M. D., Klein, R., McNesby, J. R., Schoen, L. J., Kinetics of D atom reactions with $\mathrm{H}_{2}$. J. Chem. Phys. 30, No, 1,58 (1959), 2971.

Scheid, $F_{\text {., }}$ Radial distribution of the center of gravity of random points on a unit circle. J 60, 307 (1958). $\mathrm{RP} 2847$

Scheraga, H. A., Mandelkern, L., On the interpretation of hydrodynamic data for dilute protein solutions. J. Phys. Chem. 62, 370 (1958). 2668.

Scherber, F. I., Minkoff, G. J., Energy release from discharged monatomic gases trapped at $4^{\circ} \mathrm{K}$. J. Chem. Phys. 28, No. 5, 992 (1958). 2546.

Suppression of bubbling in boiling refrigerants as an aid in optical measurements. J. Opt. Soc. Am. 48, 358 (1958). 2790.

Scherber, F. I., Minkoff, G. J., Gallagher, J. S., Energetic species trapped at $4.2^{\circ} \mathrm{K}$ from gaseous discharges. J. Chem. Phys. 30, 753 (1959). 2942.

Scherber, F. I., Minkoff, G. J., Stober, A. K., Suppression of bubbling in boiling refrigerants. Nature 180 , 1413 (1957). 2330.

Schiefer, $H . F$., Abrasion resistance measurements on cotton fabrics made with the blade type abradent and the Schiefer abrasion testing machine. Proc. Spring Meeting, Textile Qual. Control Assoc. p. 65 (1952). 382.

Textile Forschung and Prufung am National Bureau of Standards. Sonderdruck aus melliand textilberichte (Heidelberg, Germany), 32, No. 125, 188 (1951). 325 .

Thickness and compressibility of fabrics. ASTM Bull. No. 192 (TP 138), 48 (1953). 898.

Schiefer, H. F., Appel, W. D., Krasny, J. F., Richey, $G$. G., Impact properties of yarns made from different fibers. Textile Research J. 23, 489 (1953). 734.

Schiefer, H. F., Jameson, L. H., Whittier, B. L., Factors affecting the properties of rayon fabrics. Textile Research J. 22, No. 9, 599 (1952). 446.

Schiefer, H. F., McCrackin, F. L., Smith, J. C., Stone, $W$. $K$., Breaking velocities, strain energies, and theory neglecting wave propagation. Pt. II. Textile Research J. 25, 529 (1955). 1277.

Schiefer, H. F., Smith, J. C., The design of impactresisting fabric structures. Chapter 13 on Textile Engineering, Encyclopedia of Man-Made Textiles (Textile Book Publ., Division of Interscience Publ., New York, N.Y.) ) (1959). 3069.

Schiefer, H. F., Smith, J. C., McCrackin, F. L., Characterization of the high-speed impact behavior of textile yarns. (Conf. Issue) J. Textile Inst. (Trans.) 50, No. 1, T55 (1959). 2909.

Stress-strain relationships in yarns subjected to rapid impact loading. III. Effect of wave propagation. Textile Research J. 25, No. 8, 701 (1955). 1516. Stress-strain relationships in yarns subjected to rapid impact loading: 5. Wave propagation in long textile yarns impacted transversely. J 60,517 (1958) RP2866; Textile Research J. 28, No. 4, 288 (1958). 2783. The impact absorbing capacity of textile yarns. ASTM Bull. No. 220, 52 (1957). 2363.

Schiefer, H. F., Smith, J. C., McCrackin, F. L., Stone, $W$. $K$., Stress-strain relationships in yarns subjected to rapid impact loading. Am. Soc. Testing Materials Spec. Tech. Pub. 176, "Symposium on Impact Testing", 126 (1955). 1514.

Stress-strain relationships in yarns subjected to rapid impact loading. Am. Soc. Testing Materials Spec. Tech. Publ 185, Symp. on Speed of Testing on NonMetallic Materials, p. 47 (1956). 1899.

Schiefer, H. F., Smith, J. C., McCrackin, F. L., Stone, $W$. $K$., Towne, $K$. $M$., Stress-strain relationship in yarns tested at rates of straining up to a million percent per minute. Physics of Wool and Other Viseoelastic Fibers, D, 148 (1956) (Proc. Intern. Wool 
Textiles Research Conf. August 20-25, 1955, Sydney, Australia) (1956). 1900.

Schiefer, H. F., Stone, W. K., Fox, G., Stress-strain relationships in yarns subjected to rapid impact loading. Part I. Equipment, testing procedure, and typical results. Textile Research J. 25, 520 (1955). 1515.

Schiefer, H. F., Werntz, C. W., Interpretation of tests for resistance to abrasion of textiles. Textile Research J. 22, 1 (1952). 473.

Ueber den Einfluss de1 Gewebekonstruktoon auf den Scheuerwiderstand. Textil-Praxis (Stuttgart, Germany) 57, ] (1952). 610.

Schleter, J. C., Judd, D. B., Keegan, H. J., Extonsion of the Munsell renotation system. J. Opt. Soc. Am. 48, $863(1958) .2559$.

Schleter, J. C., Keegan, H. J., Spectrophotometry and aerial photoreconnaissance. (Abstract) J. Opt. Soc. Am. 47, 1050 (1957). 2308.

Schleter, J. C., Keegan, H. J., Rheinboldt, W. C., Menard, J. P., Judd, D. B., Digital 1eduction of spectrophotometric datar to Munsell renotations. J. Opt. Soc. Am. 49, 863 (1958). 2522.

Schmidt, F. A., Maxwell, E., Liquid helium cryostat with an integral super-condueting resonator. Suppl. au Bull. de l'Institut Intern. du Froid, Commission 1, Delft, Holland, Annexe 1958-1, p. 95 (1958). 2610A.

Schmidt, P. S., Fixed capacitors of electronic circuits. Elec. Mfg. 47, No. 5, 100 (1951). 238.

Schmidt, V. A., Smith, E. R., Edelman S., Jones, E., Stroboscopic interferometer for vibration measurement. J. Acoust. Soc. Am. 30, 867 (1958). 2784.

Schneider, A., Linnig, F. J., Chemical analysis of GR-S by complete solution procedures. Titration of mineral and organic acids in toluene-ethanol solution. Anal. Chem. 25, 1515 (1953). 667.

Schneider, S. J., Mong, L. E., Elasticity, strength, and other related properties of some refractory castables. J. Am. Ceram. Soc. 41, No. 1, 27 (1958). 2536 .

Thermal length changes of some refractory castables. J 59, 1 (1957) RP2768.

Schneider, S. J., Walker, R. F., Roth, R. S., Some evidence for structural anomalies in pure cristobalite. J. Am. Ceram. Soc. 42, No. 12, 642 (1959). 3048.

Schneidlin, A. E., Slawsky, M. M., Lutsky, M., Pressure drop in pneumatic components and systems. Product Eng. Handbook No. 3, Section J, p. J2 (McGrawHill Book Co., Inc. New York, N.Y.). 1456.

Schoen, L. J., Klein, R., The energy dissociation of the $\mathrm{C}-\mathrm{H}$ bond in the formyl radical. J. Chem. Phys. Comments and Errata 29, 953 (1958). 2813.

Schoen, L. J., Klein, R., McNesby, J. R., Scheer, M. D., Kineties of $\mathrm{D}$ atom reactions with $\mathrm{H}_{2}$. J. Chem. Phys. 30, No. 1, 58 (1959). 2971.

Schoen, L. J., Kuentzel, L. E., Broida, H. P., Glass dewars for optical studies at low temperatures. Rev. Sci. Instr. 29, No, 7, 633 (1958). 2575.

Schoen, L. J., Rebbert, $R$., Electrical discharge induced luminescence of solids at low temperatures. J. Mol. Spectroscopy 3, No. 4, 417 (1959). 2934.

Schoenberg, I. J., A remark on M. M. Day's characterization of inner-product spaces and a conjecture of L. M. Blumenthal. Proc. Am. Math. Soc. 3, 961 (1952), 373.

An isoperimetric inequality for closed curves convex in even-dimensional Euclidean spaces. Acta math. (Uppsala, Sweden) 91, 143 (1954). 952.

On smoothing operations and their generating functions. Bull. Am. Math. Soc. 59, 199 (1953). 783.

Schoenberg, I. J., Motzkin, T. S., On lineal entire functions of $n$ complex variables. Proc. Am. Math. Soc. 3, No, 4, $517(1952) 498$.

The relaxation method for linear inequalities. Can. J. Math. 6, 393 (1954). 1197.

Schoenberg, I. J., Whitney, A., On polya frequency functions. III. The positivity of translation determinants with an application to the interpolation problem by spline curves. Trans. Am. Math. Soc. 74, 246 (1953). 782 .
Schofield, N. E., Hubbell, J. H., Unscrambling of gammaray scintillation spectrometer pulse-height distributions. IRE Trans. Nuclear Sci. NS-5, No. 3, 156 (1958). 2856.

Schoonover, I. C., Caul, H. J., The color stability of direct filling resins. J. Am. Dental Assoc. 47, 448 (1953). 854.

Schoonover, I. C., Dickson, G., Forziati, A. F., Lawson, M. $E$. $J r$. Fluorescence of teeth: a means of investigating their structure. J. Am. Dental Assoc. 45, $661(1952) .452$.

Schoonover, I. C., Fischer, T, E., Deterioration of silicate cements in the tropics. U.S. Armed Forces Med. J. 2, 907 (1951). 217.

Schoonover, I. C., Fischer, T. E., Serio, A. F., Sweeney, $W$. T., Bonding of plastic teeth to heat cured denture base resins. J. Am. Dental Assoc. 44, 285 (1952). 402 .

Schoonover, I. C., Glasson, G. F., Sweeney, W. T., The effect of preheating on thermal expansion of silicagypsum investments. J. Am. Dental Assoc. 48, 433 (1954). 1184.

Schoonover, I. C., Grunewald, A. H., Dickson, G., Paffenbarger, G. C., Silicate cement: method of mixing in a closed container to prevent effects of exposure to atmosphere. J. Am. Dental Assoc. 46, 184 (1953). 827.

Schoonover, I. C., Lyon, H. W., Dickson, G., Effectiveness of vacuum investigating in elimination of surface defects in gold castings. J. Am. Dental Assoc. 46, 197 (1953). 697.

Mechanism of hygroscopic expansion of dental casting investments. J. Dental Research 34, 44 (1955). 1405 .

Schoonover, I. C., Mitchell, J. A., Dickson, G., Vacher, $H$. C., Some factors affecting the dimensional stability of the Ag-Sn (Cu-Zn) amalgams. J. Dental Research 34, No. 2, 273 (1955). 1493.

Schoonover, I. C., Paffenbarger, G. C., Swaney, A. C., Dickson, G., American Dental Association Specification No. 9 for dental silicate cement, First revision, effective July 1, 1950. J. Am. Dental Assoc. 40, 186 (1950). 20.

Schoonover, I. C., Ryge, G., Dickson, G., Dental amalgam: effect of mechanical condensation of some physical properties. J. Am. Dental Assoc. 45, 269 (1952). 423.

Schoonover, I. C., Smith, D. L., Direct filling resins: dimensional changes resulting from polymerization shrinkage and water sorption. J. Am. Dental Assoc. 46, 540 (1953). 689 .

Schoonover, I. C., Smith, D. L., Ferguson, G. W., Microstructure of dental amalgam. J. Am. Dental Assoc. 47, 305 (1953). 765.

Schoonover, I. C., Sweeney, W. T., Brauer, G. M., Crazing of acrylic resins. J. Am. Dental Research 34, 306 (1955). 1298.

Schoonover, I. C., Wolcott, R. B., Paffenbarger, G. C., Direct resinous filling materials: temperature rise during polymerization. J. Am. Dental Assoc. 42, 253 (1951). 223.

Schoubee, P. J., Paffenbarger, G. C., Sweeney, W. T., Dental cements.. Intern.' Dental J. 5, 584 (1955).' 1306.

Resin cements and posterior-type direct-filling resins. J. Am. Dental Assoc. 52, 584 (1956). 1854.

Schrack, $R$. A. High vacuum gimbals. Rev. Sci. Instr. 27, No. 3, 172 (1956). 1731.

Physics - The electrostatic field and the symmetry of snowflakes. J. Wash. Acad. Sci. 48, No. 9, 273 (1958). 2689 .

Schrack, $R$. A., Leiss, $J$. E., Angular distribution in neutral meson decay Phys. Rev. 109, 1326 (1958). 2461 .

Nuclear matter distributions from coherent neutral pion production. Rev. Modern Phys. 30, No. 2, $456(1958) .2653$.

Schrack, R. A., Marton, L., Placious, R. C., A field emitter point projection X-ray microscope. X'-ray Microscopy 
and Microradiography (Academic Press Inc. New York, N. Y.), p. 287 (1957). 1982.

Schrack, R. A., Placious, R. C., Apparatus for etching fine points under controlled conditions. Rev. Sei. Instr. 27, No. 6, 412 (1956). 1628.

Schriesheim, A., Greenfeld, S. H., Water-soluble degradation products of asphalt. ASTM Bull. No. 220, 43 (1957). 2413.

Schrodt, J. E., Fulk, M. M., Devereaux, R. J., Heat transfer through foams and powders. Proc. 1956 Cryogenic Engineering Conference, Paper E-3, p. 166, Edited by K. D. Timmerhaus, Chemical Engineering Dept., University of Colorado (Boulder, Colorado) (1957). 2147.

Schrodt, J. E., Fulk, M. M., McReynolds, W. A., Flanery, $W$. S. A comparison of insulating materials. Proc. 1957 Cryogenic Engineering Conference, Abstract, Paper G-7, p. 416 , Edited by K. D. Timmerhaus, Chemical Engineering Department, University of Colorado, (Boulder, Colorado) (1958). 2416.

Schrodt, J. P., Hamer, W. J., Dry cell standards broadened-now includes mercury-, air-, and flat-type cells. Mag. of Standards 26, No. 5, $132(1955) .1321$.

Schubauer, G. B., Mechanism of transition at subsonic speeds. Proc. Symp. on Boundry Layer Research, Intern. Union for Theo. and Appl. Research, August 26-29, 1957 (Freiburg, Germany), p. 85 (1957). 2195. Turbulent processes as observed in boundary layer and and pipe. J. Appl. Phys. 25, 188 (1954). 1218.

Schubauer, G. B., Klebanof, P. S., Tidstrom, K. D., Measurement of the effect of two-dimensional and threedimensional roughness elements on boundary-layer transition. J. Aeronaut. Sci. 22, 11 (1955). 1400.

Schubauer, G. B., Tchen, C. M., Turbulent motion. Seetion B, Vol. V, Princeton Series, High Speed Aerodynamics and Jet Propulsion, "Turbulent Flows and Heat Transfer", p. 75 (Princeton University Press, Princeton, N. J.) (1959). 3104.

Schubert, D. C., Coleman, J. W., Leder, L. B., Marton, L., Electron beam magnetometer. J 63C1, 69 (1959).

Sehubert, D. C., Marton, L., Mielczarek, S. R., Electron optical image of an atomic beam. J. Appl. Phys. 27, No. 4,419 (1956). 1704 .

Schuhmann, S., Shepherd, M., Kilday, M., Determination of carbon monoxide in studies of air pollution. Anal. Chem. 27, 380 (1955). 1309.

Schuhmann, S., Weaver, E. R., Hughes, E. E., Gunther, S. M., Redfearn, N. T., Gorden, R., Jr., Interpretation of mass spectra of condensates from urban atmospheres. J 59, 383 (1957) RP2809.

Schulkin, M., Average radio-ray refraction in the lower atmosphere. Proc. IRE 40,554 (1952). 400.

Schulman, J. H., Hart, E. J., Koch, H. W., Petree, B., Taimuty, S. I., Wyckoff, H. O., M̉easurement systems for high-level dosimetry. 2d United Nations Intern. Conf. on the Peaceful Uses of Atomic Energy, Vol. 21, Health and Safety: Dosimetry and Standards, P/1927 USA, p. 188 (Pergamon Press, London, England) (1959). 2984.

Schulman, J. H., Shurcliff, W., Ginther, R. J., Attix, F. H., Radiophotoluminescence dosimetry system of the U.S. Navy. Nucleonies 11, 52 (1953). 809.

Schultz, E. L., Norton, K. A., Yarbrough, H., The probability distribution of the phase of the resultant vector sum of a constant vector plus a Rayleigh distributed vector. J. Appl. Phys. 23, No. 1, 137 (1952). 591.

Schwarz, K. M., Stone, R. O., Expendable modules as bases for disposal-at-failure maintenance. (1960) TN41 (PB151400).

Schwarz, L., Errata in "Tables relating to Hankel integrals of order zero." Published in Luftfahrtforschung 20, No. 12, 341 (1943), and translated by J. Lotsof, Cornell Aeronautics Laboratory, May, 1946. Math Tables and Other Aids to Computation IV, No. $30,100(1950) .147$.
Schwarzenbach, G., Bates, R. G., Note on the strength of picric acid. Experientia 10, 482 (1954). 1085.

Triathanolamin als puffersubstanz. Helv, chim. acta 37, 1439 (1954). 1217.

Uber pH-werte nichtwässeriger Lösunger. Helv. chim. acta (Basil, Switzerland) 38, 699 (1955). 1563 .

Schwebel, $A$., Clamp for handling radioactive liquids. Nucleonics 14, No. 9, 112 (1956). 1649.

Schwebel, A., Harding, J. E., Stockmann, L. L., Report on the determination of small amounts of radium in solution. J. Assoc. Official Agri. Chem. 41, No. 2, 311 (1958). 2733.

Schwebel, A., Mann, W. B., Stockmann, L. L., Youden, W. J., Mullen, P. A., Garfinkel, S. B., Preparation of new solution standards of radium. $J 62,21$ (1959) RP2924.

Schwebel, A., Rothman, S., Adelson, E., Langdell, R. D., Adsorption of $\mathrm{C}^{14}$ dextran to human blood platelets and red blood cells in vitro. Vox Sanguinis 2, 104 (1957). 2011.

Schwebel, A., Rothman, S., Weissberg, S. G., Sorption of dextran to collodion membranes. J.'Polymer Sei. 11, 381 (1953). 832 .

Schwebel, A., Seliger, H. H., The standardization of beta-emitting nuclides. Nucleonies 12, No. 7, 54 (1954). 1200.

Schwebel, A., Ziegler, C. A., Technique for monitoring tritiated-water vapor in air. Nucleonics 15, No. 1, 64 (1957). 2338

Schweitzer, W. G., Jr., Kessler, K. G., Zeeman-split absorption lines as very narrow pass filters. J. Opt. Soc. Am. 49, 199 (1959). 3115.

Schweitzer, W. G., Jr., Kessler, K. G., Barger, R. L., Atomic beam sources and standard of length. IRE Trans. Instrumentation I-7, No. 3-4, 181 (1958). 2464 .

Schweitzer, $W . G ., J r ., Z a h n, C$. T., Excitation and separation of pure high-order modes in large high-Q eavities. J. Appl. Phys. 27, No. 8, 929 (1956). 1708.

Schwendeman, R. H., Laurie, V. W., Tables of line strengths for rotational transitions of asymmetric rotor molecules. (Pergamon Press, London, England) (1958). 2796.

Schwerdtfeger, $W . J ., \quad$ Current and potential relations for the cathodic protection of steel in a high resistivity environment. J 63C1, 37 (1959).

Current and potential relations for the cathodic protection of steel in salt water. J 60, 153 (1958) RP2833.

Electrical measurements in the selection of bolt materials for service underground. Corrosion 10, 355 (1954). 1008.

Schwerdtfeger, $W$. J., Denison, I. A., Geometric factors in electrical measurements relating to corrosion and its prevention. Corrosion 2, 25 (1955). 1354.

Scott, A. H., An instrument for mechanically differentiating curves. Rev. Sci. Instr. 21, 397 (1950). 24.

Scott, A. H., Ehrlich, P., Richardson, J. F., Dielectric measurements up to $500^{\circ} \mathrm{C}$, symposium on temperature stability of electrical insulating materials. Proc. Am. Soc. Testing Materials Spec. Tech. Publ. No. 161 (1954). 992.

Scott, D., Rubin, S., Gas tube stabilized power supplies. Tele-Tech 14, No. 7, (1955). 1353.

Scott, L. E., Robbins, R. F., Mann, D. B., Birmingham, $B$. $W$., Temperature stratification in a nonventing liquid helium dewar. J 64C1, 19 (1960).

Scott, R. B. Activities of the National Bureau of Standards Cryogenic Engineering Laboratory. Conf. de physique des basses temperatures, September 2-8, 1955 , p. 368 (Union Intern. de Physique pure et applique Institut Intern. du Froid) (Paris, France) (1955). 1252.

Cryogenic Engineering. (D. Van Nostrand Co., New York, N.Y.) (1959). 2922.

Low temperature scales from $90^{\circ}$ to $5^{\circ} \mathrm{K}$. Am. Inst. 
Phys. Temperatures, its measurement and control in Seience and Industry 2, 179 (Reinbold Publ. Corp., New York, N.Y.) (1955). 1392.

Scott, R. B., Armstrong, G. T., Brickwedde, F. G., The vapor pressures of the deuteromethanes. J. Chem. Phys. 21, 1297 (1953). 889.

Seribner, B. F., Automation in the laboratory. Capital Chemist 8, 38 (1958). 2468.

Emission spectroscopy. Anal. Chem. 30, 596 (1958). 2545.

Scribner, B. F., Corless, C. H., Emission spectrographic standards. Anal. Chem. 23, 1548 (1951). 232.

Scribner, B. F., Margoshes, J., The plasma jet as a spectroscopic source. Spectrochem. acta XIV, No. 2, 138 (1959). 3088.

Scribner, B. F., Meggers, $W$. $F$., Index to the literature on spectrochemieal analysis. Part III. 1946-1950 (226 pgs.), Am. So. Testing Materials Spec. Tech. Publ. 41-C (1954). 1036.

Index to the literature on spectrochemical analysis Pt. IV, 1950-1955. Am. Soc. for Testing Materials Publ. No. 41D (1959). 2965.

Scribner, B. W., O'Leary, M. J., Missimer, J. K., Resin bonding of offset papers containing mineral fillers. Tappi 34, 506 (1951). 303.

Scribner, B. W., O'Leary, M.J., Missimer, J. K., Erving, $J$. ' $J$., Manufacture of paper from glass fibers. Tappi 35, No. 7, 289 (1952). 477.

Scribner, B. W., Shaw, M. B., O'Leary, M. J., Missimer, $J$. $K$., Resin bonding of hardwood fibers in offset papers. Tappi 34, 481 (1951). 302.

Scuderi, T. S., Cleek, G. W., Effect of fluorides on infrared transmittance of certain silicate glasses. J. Am. Ceram. Soc. 42, No. 12, 599 (1959). 2933.

Seaborg, G. T., Curtiss, L. F., Evans, R. D., Johnson, W. Units of radioactivity. Rev. Sci. Instr. 21, 94 (1950). 163.

Seaman, R. G., Bekkedahl, N., The ASTM erude natural rubber subcommittee. Rubber World 189, 693 (1959). 3065.

Seebold, R. E., Cuthill, J. R., Hayes, W. D., Nitriding phenomena in titanium and the $6 \mathrm{~A} 1-4 \mathrm{~V}$ titanium alloy. J 64A 1, 119 (1960).

Seidel, W., Beckenbach, E. F., Szasz, O., Recurrent determinants of Legendre and of ultraspherical polynomials. Duke Math. J. 18, 1 (1951). 298.

Seidel, $W$., Szasz, O., On positive harmonic functions of ultraspherical polynomials. J. London Math. Soc. (London, England) 26, 36 (1951). 276.

Selby, $M$. C., Accurate radio-frequency microvoltages. Elec. Eng. 72, 515; Am. Inst. Elee. Engrs. Trans. p. 1 (May issue); Electronics 6, 158 (1953). 640. Conical coaxial capacitors and their advantages. J 63C2, 87 (1959).

High-frequency standards of the Electronic Calibration Center, National Bureau of Standards, Boulder Laboratories, Boulder, Colorado. IRE Trans. Instrumentation I-7, No. 3-4, 262 (1958). 2585 .

R. F. micropotentiometers. (Radio-Electronic Eng. Sect.) Radio \& TV News 50, No. 4, 5 (1953). 807. VTM circuits. Electronies 23, No. 5, 110 (1950). 168.

Selby, M. C., Behrent, L. F., Ries, F. X., RF voltmeter ealibrating consoles. IRE Natl. Conv. Record $\mathbf{6}, \mathrm{Pt}$. 5,251 (1958). 2747

Seldin, I. L., Brode, W. R., Spoerri, P. E., Wyman, G. M., The relation between the absorption spectra and the chemical constitution of dyes. XXVIII. The hydration of azo dyes in organic solvents. J. Am. Chem. Soc. 77, 2762 (1955). 1543.

Selgin, P. J., Solderless electronic assembly with cellular units. Elec. Mfg. 52, 139 (1953). 830.

Selgin, P. J., Hawes, B. K., Temperature prediction in electronic design. Elec. Mfg. 50, No. 4, 116 (1952). 564.

Seliger, H.H., Applications of standards of radioactiv- ity. Intern J. Appl. Radiation and Isotopes 1, 215 (1956). 1631.

Improvements in liquid scintillators. Proc. Northwestern Technical Institute Symp. on Liquid Scintillation Counting, p. 115 (Pergamon Press, New York, N.Y.) (1958). 2597.

Liquid scintillation counting. Proc. Symp. on Measurements, and Standards of Radioactivity, Natl. Acad. Sci., Natl. Research Council, Nuclear Science Series No. 24, 47 (1958). 2614.

Saturation backscattering of positive electrons. Phys. Rev. 78, 491 (1950). 131.

Scintillation counting of beta emitters. Proc. Intern. Conf. on Radioisotopes in Scientific Research UNESCO (1958). 2750.

The backseattering of positrons and electrons. Phys. Rev. 88, 408 (1952). 567.

The transmission of monoenergetic positrons and electrons. Phys. Rev. 95, 610A (1954). 1204.

Transmission of positrons and electrons. Phys. Rev. 100, No. 4, 1029 (1955). 1560.

Seliger, H. H., Agranoff, B., Improvements in liquid scintillators. Anal. Chem. 31, No. 9, 1607 (1959). 2964.

Seliger, H. H., Bay, Z., Absolute measurement of $\mathrm{W}_{\mathrm{sir}}$ for polonium-210 alpha particles. Radiation Research 9, No. 1, 90 (1958). 2444.

Collection of ions produced by alpha particles in air. Bull. Am. Phys. Soc. II, 3, No. 3, Session Y-9 (1958). 2488.

Seliger, H. H., Bay, Z., Mann, W. B., Wyckoff, H. O., Measurement of $\mathrm{W}_{\mathrm{air}}$ for sulfur- 35 beta rays. Radiol. Research Abstract 5, No. 4, 9 (1956). 1769.

Seliger, H. H., Bay, Z., Wyckoff, H. O., Absolute measurement of $W_{\text {air }}$ for sulfur-35 beta rays. Radiation Research $\boldsymbol{\gamma}$, No. 6, 558 (1957). 2080.

Seliger, H. H., Cavallo, L., Culpepper, S. V., The halflife of $\mathrm{I}^{131}$.' Phys. Rev, 90, 443 (1953). 863.

Selzger, H. H., Mann, W. B., Preparation, maintenance, and application of standards of radioactivity. (1958). C594.

Radioactivity standardization in the United States. Proc. 2d Intern. Conf. on the Peaceful Uses of Atomic Energy, United Nations (Geneva, Switzerland) 21, 90 (1958). 2712.

Setiger, H. H., Mann, W. B., Cavallo, L. M., Average energy of sulfur-35 beta decay. J 60, 447 (1958) RP2859.

Seliger, H. H., Schwebel, A., The standardization of betaemitting nuclides. Nucleonies 12, No. 7, 54 (1954). 1200.

Seliger, $\dot{H}$. H., Smith, C. C., Improvement in response

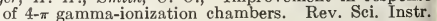
24, 474 (1953). 735.

Seliger, H. H., Ziegler, C. A., Hysteresis effect in multiplier phototube noise. J. Appl. Phys. 26, No. 10, 1225 (1955). 1361.

Liquid-scintillator temperature effects. Nucleonies 14, 4, No. 49 (1956). 1759.

Temperature effects in gas-free liquid scintillators. IRE Trans. Nuclear Science NS-3, 4 (1956). 1915.

Seliger, H.H., Ziegler, C. A., Jaffe, I., Oxygen quenching and wavelength shifters in liquid scintillators. Phys. Rev. 99, No. 2, 663; Bull. Am. Phys. Soc. 30, No. 3, $63 \mathrm{~A}(1955)$. 1439.

Role of oxygen in the quenching of liquid scintillators. Phys. Rev. 101, No. 3, 998 (1956). 1858.

Three ways to increase efficiency of liquid scintillators. Nucleonies 14, No. 5, 84 (1956). 1966.

Semmelman, J. O., Sweeney, W. T., Myerson, R. L., Rose, E. E., Proposed specification for plastic teeth. J. Prosthetic Dentistry 7, 420 (1957). 2272.

Sen, $H . K$. ., An estimate of the density and motion of solar material from observed characteristics of solar radio outbursts. Australian J. Phys. 6, 67 (1953). 649.

Nonlinear theory of space-charge wave in moving, interacting electron beams with application to solar radio noise. Phys. Rev. 97, 849 (1955). 1418. 
Solar "enhanced radiation" and plasma oscillations. Phys. Rev. 88, No. 4, 816 (1952). 544.

Space charge wave amplification in a shock front and the fine structure of solar radio noise. Australian J. Phys, 7, 30 (1954). 1149.

Structure of a magnetohydrodynamic shock wave in a plasma of infinite conductivity. Phys. Rev. 102, 5 (1956). 1902.

The non-Maxwellian distribution in a shock front and the anomaly of the chromospheric temperature. Phys. Rev. 92, 861 (1953). 874.

Sen, H. K., Feinstein, J., Radio wave generation by multi-stream charge interaction. Phys. Rev. 83, No. 2,405 (1951). 295.

Sen, $H . K$. White, $M . L$, Thermal and gravitational excitation of atmospheric oscillations. J. Geophys. Research 60, 483 (1955). 1555.

Senderoff, S., Couch, D. E., The electrolytic preparation of molybdenum from fused salts. V. Electrorefining studies in the presence of tin, iron, copper, silicon, and nickel. Trans. Metallurgical Soc. of Am. Inst. Metallurgical Engrs. p. 320 (1958), 2811.

Senderoff, S., Labrie, R. J., Electrolytic preparation of molybdenum from fused salts. IV. Preparation of reduced molybdenum chlorides from molybdenite concentrate. J. Electrochem. Soc. 102, 77 (1955). 1333.

Sengers, V. J., Michels, A., Botzen, A., Friedman, A. S., The thermal conductivity of argon for temperatures between $0^{\circ} \mathrm{C}$ and $75^{\circ} \mathrm{C}$ and at pressures up to 2500 atmospheres. Physica 22, 121 (1956). 1952

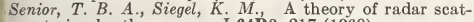
tering by the moon. J 64D3, 217 (1960).

Serio, A. F., Caul, H. W., Sanford, $J$. W., Properties of self-curing denture base resins. J.'Am. Dental Assoc. 44, 295 (1952). 521.

Serio, A. F., Schoonover, I. C., Fischer, T. E., Sweeney, $W . T$." Bonding of plastic teeth to heat cured denture base resins. J. Am. Dental Assoc. 44, 285 (1952). 402.

Severo, N. C., A statistician and the post office: a case history in operations research. Trans. 1959 Conf. Admin. Appl. Div. of Am, Soc. Quality Control (Milwaukee, Wis.) (1959). 2881.

Asymptotic behavior of tests on the mean of a logarithmic-normal distribution with known variances. Ann. Math. Stat. 28, 1044 (1957). 2030.

Severo, $N$. C., Newman, A. E., A statistical chain-ratio method for estimating relative volumes of mail to given destinations. J 64C 1,37 (1960).

Distribution of mail by destination at the San Francisco, Los Angeles, and Baltimore post offices. (1959) TN27 (PB151386).

Severo, N. C., Newman, A. E., Young, S. M., Zelen, M., Some applications of statistical sampling methods to outgoing letter mail characteristics. (1959) TN16 (PB151375).

Seymour, R. B., Kline, G. M., Plastics. Ind. Eng. Chem. 44, 2339 (1952). 512.

Shaaf, J. H., Shaull, J. M., Precision quartz resonator frequency standards. Proc. IRE 42, No. 8, 1300 (1954). 1111.

Shack, $R$. V., Outline of characteristics of an image-forming system. J. Opt. Soc. Am. 9, 755 (1956). 1801.

Shack, R. V., Washer, F. E., Feder, D. P., Panel discussion-Report on the NBS optical image evaluation symposium, reprinted from Photogrammetric Eng. 18, 575 (1952). 532.

Shadle, O. W., Ferguson, T. B., Gregg, D. E., Gilford, S. R., Evaluation of a new cuvette densitometer for determination of cardiac output. Circulation Research 1, No. 3, 200 (1953). 714.

Shadle, O.W., Gilford, S. R., Gregg, D. E., Ferguson, T. B., Marzetta, $L$. A., An improved cuvette densitometer for cardiac output determination by the dye-dilution method. Rev. Sci. Instr. 24, 696 (1953). 650.

Shafer, $M . R$. Ruegg, $F$. $W$., Liquid-flowmeter calibration techniques. Trans. Am. Soc. Mech. Engrs. 80, No. 7, 1369 (1958). 2613.
Shalek, R. J., Bonner, T. W., Branscomb, L. M., The emission spectra of nitrogen bombarded by high energy protons. Phys. Rev. 85, 739 (1952). 576.

Shalek, R. J., Branscomb, L. M., Bonner, T. W., Intensity distributions in nitrogen bands exeited in auroras and by high-energy protons and hydrogen atoms. Trans. Am. Geophys. Union 35, 107 (1954). 1044.

Shapiro, G., Henry, R. L., Scal, $R . K-F$, New techniques for electronic miniaturization. Proc. IRE (Sydney, Australia) 13, No, 3, 75 (1952). 489.

Shapiro, H., Couch, D. E., Brenner, A., The use of nickelaluminum alloy coatings for the protection of molybdenum from oxidation. J. Electrochem. Soc. 105, No. 8, 485 (1958), 2837.

Shapiro, H., Couch, D. E., Taylor, J. K., Brenner, A., Protection of molybdenum from oxidation at elevated temperatures. J. Electrochem. Soc. 105, No. 8, 450 (1958). 2705.

Shapley, A.H., Chairman, Commission III on ionospheric radio, World-wide soundings committee: Status report, Intern. Sci. Radio Union (U.S.R.I.), Inform. Bull., No. 108, 13 (1958). 2490.

Clues to ionospheric conditions in the southern auroral zone. In Antarctica in the Intern. Geophys. Year; based on a Symp. on the Antarctic, Cosponsored by the U.S. Natl. Comm. for the IGY of the Natl. Acad. Sci., Natl. Sci. Foundation and Am. Geophys. Union (Washington, D.C.), p. 86 (1956). 1650.

First report of the special committee on world-wide ionospheric soundings. Intern. Sei. Radio Union (URSI) Inform. Bull. No. 99, 48 (1956). 1712. Geographic distribution of geophysical stations on the polar cap. J. Atmospheric and Terrest. Phys. Special Supp. 1957, Proc. of the Polar Atmosphere Symp., July 2-8, 1956, (Oslo, Norway) Pt. II, 108 (1957). 2141.

IGY instruction manual, Pt. I: World days and communications. Ann. Intern. Geophys. Year Vol. $\gamma$, Pt. 1, p. 1 (Pergamon Press, New York, N.Y.) (1959). 2962 .

Proposed recommendation on ionospheric vertical soundings after the I.G.Y. (Memorandum No. 18), Intern. Sci. Radio Union (U.R.S.I.) Inform. Bull. No. 111,42 (1958). 2704.

Second report of the Special Committee on world-wide ionospheric soundings of the URSI/AGI Committee, May 1957, Boulder, Colorado, National Bureau of Standards, Central Radio Propagation Laboratory (1957). 2294.

The day-to-day coordination of IGY observations. Proc. IRE 47, 323 (1959). 3068.

The ionosphere IGY program in the Arctic. Proc. Arctic Cong. (Stockholm, Sweden May 22-25, 1956). Int. Sci. Radio Union Info. Bull. 98, 29 (1956). 1936. To the edge of space. Sci. Teacher 25, No. 2, 69 (1958). 2849 .

World-wide soundings committee: Memorandum 17Future work. U.R.S.I. (Intern. Sci. Radio Union) Inform. Bull. No. 110, 55 (July-August 1958). 2870.

Shapely, A. H., Kaplan, J., The International Geophysical Year: a progress report. News Report Natl. Research Council 4, No. 6, 1 (1954). 1188.

Shapely, A. H., Slutz, R. J., Probing the ionosphere. In American Geophysical Union, Geophysies and the IGY. Geophysical Monograph No. 2, 45 (1958). 2700 .

Shartsis, L., Bestul, A. B., Shermer, H. F., Immiscibility and surface tension of some simple borates. J. Am. Ceram. Soc. 41, 507 (1958). 2592.

Surface tension of alkaline-earth borates containing 3 mole percent of potassium oxide. J. Am. Ceram. Soc. 42, 242 (1959). 3059.

Shartsis, L., Capps, W., Spinner, S., Viscosity and electrical resistivity of molten alkali borates. J. Am. Ceram. Soc. 36, 319 (1953). 907.

Shartsis, L., Coughanour, L. W., Shermer, H. F., Viscosity density, and electrical resistivity of molten alkalineearth borate glasses with three mole percent of po- 
tassium oxide. J. Am. Ceram. Soc. 41, 324 (1958). 2864.

Shartsis, L., Shermer, H. F., Surface tension, density, viscosity, and eleetrical resistivity of molten binary alkaline-earth borates. J. Am. Ceram. Soc. 37, No. 11, 544 (1954). 1156.

Shartsis, L., Spinner, S., Capps, W., Density, expansivity and viscosity of molten alkali silicates. J. Am. Ceram. Soc. 35, No. 6, 155 (1952). 422.

Shartsis, L., Weir, C. E., Compressibility of binary alkali borate and silicate glasses at high pressures. J. Am. Ceram. Soc. 38, No. 9, 299 (1955). 1292.

Compressibility of binary alkaline-earth borate glasses at high pressures. J. Am. Ceram. Soc. 39, No. 9, 319 (1956). 1658.

Shaull, J.M., Frequency multipliers and converters for measurement and control. Tele-Tech 14, No. 4, Section 1, 86 (1955). 1349 .

High precision automatic frequency comparator and recorder. Tele-Tech 14, No. 1, 58 (1955). 1356.

Wide range decade frequency generator. Tele-Tech 9, No. 11,36 (1950). 170.

Adjustment of high-precision frequency and time standards. Proc. IRE 38, 6 (1950). 18.

Shaull, J. M., Kortman, C. M., Comparing outputs from precision time standards. Electronies 24, No. 4, 102 (1951). 209.

Shaull, J. M., Shaaf, J. H., Precision quartz resonator frequency standards. Proc. IRE 42, No. 8, 1300 (1954). 1111.

Shaw, C. C., Gates, D. M., Murcray, D. C., Herbold, R. J., Near infrared solar radiation measurements by balloon to an altitude of $100,000 \mathrm{ft}$. J. Opt. Soc. Am. 48, No. 12, 1010 (1958). 2645.

Shaw, M. B., Scribner, B. W., O'Leary, M. J., Missimer, $J$. $K$., Resin bonding of hardwood fibers in offset papers. Tappi 34, 481 (1951). 302.

Sheehan, W. D., Sweeney, W. T., Yost, E. L., Mechanical properties of direct filling resins. J. Am. Dental Assoc. 49, 513 (1954). 1069.

Shelby, J., Newkirk, G. A., Curtis, G. W., Watson, D., Manning, $R$., The inner solar corona during June 1959. Nature 184, 1308 (1959). 3080.

Shenstone, A. G., Meggers, W. F., The second spectrum of ruthenium (Ru II). J 61, 373 (1958) RP2908.

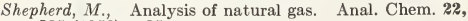
885 (1950). 25.

Shepherd, M., Schuhmann, S., Kilday, M., Determination of carbon monoxide in studies of air pollution. Anal. Chem. 27, 380 (1955). 1309.

Shereshefsky, J. L., Weir, C. E., Adsorption of gases and vapors on glass spheres. II. Two dimensional condensation of oxygen. J. Phys. Chem. 60, 1162 (1956). 1609.

Sherfey, J. M., Brenner, A., Electrochemical calorimetry. J. Electrochem. Soc. 105, 665 (1958). 2538.

Sherman, $M$. A., Axilrod, B. $M$., Stress and strain at onset of crazing of polymethyl methacrylate at various temperatures. Natl. Advisory Comm. Aeronaut. Tech. Note 2778 (1952). 555.

Stress and strain at onset of crazing of polymethyl methacrylate at various temperatures. ASTM Bull. No. 191,65 (1953). 838.

Stress-solvent crazing of acrylics. Modern Plastics 30, 130 (1953). 839.

Sherman, M. A., Axilrod, B. M., Cohen, V., Wolock, I., Effects of biaxial stretch-forming. Modern Plastics 30, 117 (1952). 434.

Effects of moderate biaxial stretch-forming on tensile and crazing properties of acrylic plastic glazing. Natl. Advisory Comm. Aeronaut. Tech. Note 2779 (1959). 436.

Sherman, M. A., Kline, G. M., Wolock, I., Axilrod, B. M., George, D. A., Cohen, $V$., Development of craze and impact resistance in glazing plasties by multiaxial stretching. Natl. Advisory Comm. Aeronaut. Tech. Report 1290 (1957). 2084.
Sherman, M. A., Wolock, I., Axilrod, B. M., Biaxial stretch-forming of acrylics. Modern Plastics 31, 128 (1953). 658.

Effect of molecular weight on the crazing and tensile properties of polymethyl methacrylate. Natl. Advisory Comm. Aeronaut. Research Memo. 54AO4 (1954). 999.

Effects of high degrees of biaxial stretch-forming on crazing and other properties of acrylic plastic glazing. Natl. Advisory Comm. Aeronaut. Research Memo. 53D14 (1953). 699.

Sherman, R. J., Logan, H. L., Stress-corrosion cracking of type 304 austenitic stainless steel. Welding J. Research Suppl. 35, No. 8, 389 (1956). 1898.

Shermer, H. F., Bestul, A. B., Shartsis, L., Immiscibility and surface tension of some simple borates. J. Am. Ceram. Soc. 41, 507 (1958). 2592.

Shermer, H. F., Coughanour, L. W., Shartsis, L., Viscosity density, and electrical resistivity of molten alkaline-earth borate glasses with three mole percent of potassium oxide. J. Am. Ceram. Soc. 41, 324 (1958). 2864.

Shermer, $\dot{H}$. F., Shartsis, L., Surface tension, density, viscosity, and electrical resistivity of molten binary alkaline-earth borates. J. Am. Ceram. Soc. 37, No. 11,544 (1954). 1156

Shermer, H., F., Shartis, L., Bestul, A. B., Surface tension of alkaline-earth borates containing 3 mole-percent of potassium oxide. J. Am. Ceram. Soc. 42, 242 (1959), 3059 .

Sherrard, E. J., Simulator for use in development of jet engine controls. (1957) C584.

Sherwood, J. E., A remote-control method of opening ampoules of active materials. Rev. Sci. Instr. 21, 570 (1950). 8

Shiffman, C. A., Thermoconductivity of tin-indium alloys in the normal state. Proc. Phys. Soc. (London, England) 71, No. 460, 597 (1958).

Shinkawa, H., Crichlow, W. O., Foldes, G., Hewitt, $F$. Horner, H., Sullivan, $A$. W. Measurement of atmospheric noise. Recommendation No. 1 and Annex, Proc. XIIth Gen. Assembly Intern. Sci. Radio Union, Bouider, Colorado, August 22-September 5, 1957, XI, Pt. 4, 99, 1957. U.R.S.I. (Intern. Sci. Radio Union) Inform. Bull. No. 105, 10 (September-October 1957). 2190 .

Shoaf, J. $H$., The NBS primary frequency standard. Radio and TV News 49, No. 110 (1953). 872.

Short, P. J., Norton, K. A., Vogler, L. E., Mansfield, $W . V$. , The probability distribution of the amplitude of a constant vector plus a Rayleigh-distributed vector. Proc. IRE 43, 1354 (1955). 1541.

Shoub, H., McCamy, C. S., Lee, T. G., Fire extinguishment by means of diy powder. Proc 6th Symp. (International) on Combustion (Reinhold Publ. Corp., New York, N.Y.), p. 795 (1957). 2123.

Shuler, $K$. $E$., Analysis of vibrational relaxation data in shock wave experiments. J. Chem. Phys. Note 30, 1631 (1959). 2896.

On the kinetics of elementary gas phase reactions at high temperatures. Fifth Symposium (International) on Combustion (Reinhold Publ. Corp., New York, N.Y.), p. 56 (1955). 1433.

On the perturbation of the vibrational equilibrium distribution reactant molecules by chemical reactions. 7 th Symp. on Combustion (Butterworth, London, England), p. 87 (1958). 2671.

On the specification of transient nonequilibrium systems. 6th Intern. Symp. on Combustion, (Reinhold Publ. Coip., New York, N.Y.), p. 371 (1957). 2237. Studies in nonequilibrium rate processes, II. The relaxation of vibrational nonequilibrium in distributions in chemical reactions and shock waves. J. Phys. Chem 61, 849 (1957). 2325.

Shuler, K. E., Bazley, N. W., Montroll, E. W., Rubin, R. J., Studies in nonequilibrium rate processes, III. The vibrational relaxation of a system of anharmonic oscillators. Errata in J. Chem. Phys. 28, 700 (1958). 2786 . 
Shuler, K. E., Berl, W. G., Gayhart, E. L., Olsen, H. L., Broida, H. P., Spectroscopic observations on pentaborane-air flames and explosions. J. Chem. Phys. 25, No. 4, 797 (1956). 1883.

Shuler, K. E., Broida, $H$. P., Kinetics of $\mathrm{OH}$ radicals from flame emission spectra. IV. A study of the hydrogenoxygen flame. J. Chem. Phys. 20, 168 (1952). 475. Kinetics of $\mathrm{OH}$ radicals from flame emission spectra. V. A study of acetylene-oxygen flame. J. Chem. Phys, 20, 1383 (1952). 476.

Spectroscopic study of electronic flame temperatures and energy distributions. J. Chem. Phys. 27, No. 4, 933 (1957), 2310.

Spectroscopic study of electronic flame temperatures and energy distributuions. Rev. French Instr. Petro. and Ann. Combus. Liquid 13, 620 (1958). 2771.

Shuler, $K$. E., Herman, $R$. $C$., Studies in nonequilibrium rate processes. IV. The rotating and vibrational relaxation of a system of rotating oscillators. J. Chem. Phys. 29, 366 (1958), 2787.

Shuler, K. E., McKinley, J. D., Jr. Kineties of the high temperature heterogeneous reaction of chlorine and nickel between 1200 and $1700^{\circ} \mathrm{K}$. J. Chem. Phys. 28, No. 6, 1207 (1958). 2608.

Kinetics of the reaction between nickel and chlorine above $1,100^{\circ} \mathrm{K}$. Intern. Meeting of Chemistry (Paris, France), Section de Chimie Minerale, p. 637 (1957). 2174.

Shuler, K. E., Montroll, E. W., Application of the theory of stochastic processes to chemical kineties. Chapter in book, Advances in Chemical Physics (Interscience Publ. Inc., New York, N.Y.) 1 p. 361 (1959). 2898. Studies in nonequilibrium rate processes. I. The relaxation of a system of harmonic oscillators. J. Chem. Phys, 26, 454 (1957). 2324.

The application of the theory of stochastic processes to chemical kinetics. Advances in Chemical Physics (Interscience Publ. Inc,, New York, N.Y.) 1361 (1958). 2805.

Shuler, K. E., Moore, G. E., Silverman, S., Herman, R. C., The reactions of ammonia and hydrazine with oxygen atoms and hydrogen atoms in atomic flames. J. Phys. Chem. 60, 813 (1956). 1948.

Shuler, K. E., Rubin, R. J., On the relaxation of vibrational nonequilibrium distributions: I. Collisional relaxation of a system of harmonic oscillators. J. Chem. Phys. 25, 59 (1956); II. The effect of the collisional transition probabilities on the relaxation behavior. J. Chem. Phys. 25, 68 (1956). 1847.

On the relaxation of vibrational nonequilibrium distributions, III: The effect of radiative transitions on the relaxation behavior. J. Chem. Phys. 26, No, 1 , 137 (1957). 2235.

Shurcliff, W., Schulman, J. H., Ginther, R. J., Attix, F. H., Radiophotoluminescence dosimetry system of the U.S. Navy. Nucleonics 11, 52 (1953). 809.

Shutts, R. E., Albrite, J. P., Whitlock, M. B., Jr., Cook, R. K., Corliss, E. L. R., Burkhard, M. D., Research in normal threshold of hearing. A.M.A., Arch. Otolaryngol. 68, 194 (1958). 2735 .

Siddiqui, $M$. M., Covariances of least-squares estimates when residuals are correlated. Ann. Math. Stat. 29, 1251 (1958). 2504.

Distribution of a serial correlation coefficient near the ends of the range. Ann. Math. Stat. 29, 852 (1958). 2527

Sieffert, L. E., Lamb, J. J., George, D. A., Baker, H. A. Impact strength of some thermosetting plasties at low temperatures. ASTM Bull. No. 181, 67 (1952). 464.

Siegel, K. M., Cottony, H. V., Elliott, R. S., Jordan, E. C., Rumsey, V. H., Wait, J. R., Woodyard, O. C., Antennas and waveguides, and annotated bibliography. U.S.A. National Committee report, Intern. Sci. Radio Union Subcommission 6.3. IRE Trans, Ant. Prop. AP-7, 87 (1959). 2897

U.S.A. National Committee report URSI Subcommission 6.3 , antennas and waveguides, and annotated bibliography, Proc. 12th Gen. Assembly, Intern'
Scientific Radio Union, August-September 1957 (Boulder, Colorado), 11, Pt. 6, 121 (1957). 2402.

Siegel, K. M., Senior, T. B. A., A theory of radar scattering by the moon. J 64D3, 217 (1960).

Sigler, $P$. A., Safe floors and floor finishes. Soap Sanit. Chemicals 26, No. 9, 121 (1950). 130.

Sigler, P. A., Boone, T. H., Materiales para pisos, Ingenieria Internacional Construccion. (McGraw-Hill Book Co., Inc., New York, N.Y.) 40, No. 7, 50 (1952). 479 .

Silberstein, R., A long-distance pulse-propagation experiment on 20.1 megacycles. J. Geophys. Research 63, No. 3, 445 (1958). 2423.

A note on sweep-frequency backscatter observations. J. Geophys. Research 59, 138 (1954). 924.

Comments on studies of transequatorial ionospheric propagation, by Villard-Stein-Yeh. J. Geophys. Research 62, 645 (1957), 2326.

High-frequency scatter sounding experiments at the National Bureau of Standards. Science 118, 759 (1953). 730

Interpretation of high-frequency $\mathrm{C}-\mathrm{W}$ field-intensity records with the aid of simultaneous pulse data. Proc. IRE 40, 974 (1952). 472.

Sweep frequency backscatter-some observations and deductions. IRE Trans. Ant. Prop. AP-2, No. 2, 56 (1954). 1158.

The long distance horizontal radiation pattern of a high-frequency antenna. IRE Trans, Ant. Prop. $\mathbf{A P}-\mathbf{5}, 397(1957) .2371$.

The origin of the current nomenclature for the ionospheric layers. J. Atmospheric and Terrest. Phys. 13, 382 (1959). 3086.

The use of sweep-frequency backscatter data for determining oblique-incidence ionospheric characteristics. Geophys. Research 63, 335 (1958). 2838.

Tropospheric effects on 6-MC pulses. Proc. IRE Letter 46, No. 12, 1968 (1958). 2854.

Silberstein, R., Hartsfield, W. L., A comparison of CW field intensity and backscatter delay, Proc. IRE 40, 1700 (1952). 361

Low-pass duplexing system for high-frequency pulse transmitters. Tele-Tech 13, 76 (1954). 1056.

Silsbee, F. B., Does rationalization change units? Elec. Eng. 76, 4 (1957). 2094.

Physics. Measure for measure: some problems and paradoxes of precision. J. Wash. Acad. Sci. 41, 213 (1951), 287.

Standards for electrical measurements. Elec. Eng. \% 0 , No, 3, 202 (1951). 316.

The ampere. Proc. IRE 47, No. 5, 643 (1959). 3064. Silverman, J., Plyler, E. K., Benedict, W. S., Precise measurements in the infrared spectrum of carbon monoxide. J. Chem. Phys. 20, 175 (1952). 516.

Silverman, S., Benedict, W. S., Herman, R. C., R-branch heads of some $\mathrm{CO}_{2}$ infrared bands in the $\mathrm{CO}+\mathrm{O}_{2}$ flame spectrum. J. Chem. Phys. 19, 1325 (1951). 293.

Silverman, S. Moore, G, E. Shuler, $K, E$., Herman, R. C., The reactions of ammonia and hydrazine with oxygen atoms and hydrogen atoms in atomic flames. J. Phys. Chem. 60, 813 (1956). 1948.

Silverstein, $A_{\text {, }}$ Building and using dielectric amplifiers. Electronics 27, 150 (1954). 963.

Simha, R., Degradation of high polymers. J. Polymer Sci. Letter to Editor 5, 515 (1950). 43.

Simha, R., Rothman, S., Weissberg, S. G., Viscosities of very dilute polymer solutions. J. Polymer Sci. 5, $141(1950)$. 166.

Simha, R., Wall, L. A., Kinetics of chain depolymerization.' J. Phys. Chem. 56, No. 6, 707 (1952). 474. Mechanism of high energy radiation effects in polymers. J. Chem. Phys, 61, 425 (1957). 2194.

Mechanisms of polymer formation and decomposition. Catalysis Ser. 6, Chapter 3, p. 191, edited by P. H Emmett (Reinhold Pub. Corp., New York, N.Y.) (1958). 2631.

Some aspects of depolymerization kinetics. J. Polymer Sci. 6, No, 1, 39 (1951). 307. 
Simha, R., Wall, L. A., Blatz, P.J., Depolymerization as a chain reaction. J. Polymer Sci. 5, 615 (1950). 44.

Simha, R., Wall, L. A., Bram, J., High-speed computations in the kinetics of free-radical degradation, I. Random initiation. J. Chem. Phys. 29, No. 4, 894 (1958). 2587

Simha, R., Wall, L. A., Madorsky, S. L., Brown, D. W., Straus, S., Depolymerization of polymethylene and polyethylene. J. Am. Chem. Soc. 76, 3430 (1954). 985 .

Simmons, B., Biagi, F., Pressure calibration of condenser microphones above $10,000 \mathrm{cps}$. J. Acoust. Soc. Am. 26, 693 (1954). 1114.

Simpson, B., Kissinger, $H, E$. McMurdie, $H . F$., Thermal decomposition of manganous and ferrous carbonates. J. Am. Ceram. Soc. 39, No. 5, 168 (1956). 1960.

Simpson, J. A., Electron interference experiments. Rev. Mod. Phys. 28, No. 3, 254 (1956). 1702.

The theory of the three-crystal electron interferometer. Rev. Sei. Instr. 25, No. 11, 1105 (1954). 1202 .

Simpson, J.A., Klein, R., Production of a square temperature wave in a filament operating at low temperatures. Rev. Sci. Instr. 29, No, 9, 770 (1958). 2701.

Simpson, J. A., Leder, L. B., Improved electrical differentiation of retarding potential measurements. Rev. Sci. Instr. 29, No. 7, 571 (1958) 2596.

Simpson, J. A., Marton, L., Electron absorption spectrometer using an improved velocity analyzer. Rev. Sci. Instr. 29, No. 7, 567 (1958), 2541

Simpson, J. A., Marton, L., Leder, L. B., Mendlowitz, $H$., Marton, C., Energy losses of electrons and their influence on resolving power. Proc. of the Toulouse Conf., Centre Natl. de la Richerche Scientifique (Paris, France), p. 175 (1955). 1335

Simpson, J.A., Marton, L., McCraw, T. F., Automatic instrument for electron scattering measurements. Rev. Sci. Instr. 26, No. 9, 855 (1955). 1274.

Distribution of electron scattering by gold. Phys. Rev. 99, No. 2, 495 (1955). 1317.

Simpson, J. A., Marton, L., Suddeth, J. A., An electron interferometer. Rev. Sci. Instr. 24, No. 11, 1099 (1954). 947

Electron beam interferometer. Phys, Rev, 90, 490 (1953). 706

Simpson, J. A., Marton, L., Suddeth, J. A., Wagner, M. D., Watanabe, $H$., Effect of degree in orientation and crystal size on the scattering of 20-kev electrons by aluminum. Phys. Rev. 110, No. 5, 1057 (1958). 2529 .

Simpson, J. A., McCraw, T. F., Marton, L., Distribution of electrons scattered by solids. Phys. Rev, 104, No. 1, 64 (1956). 1681

Simpson, J. A., Van Bronkhorst, A., Modification of electron microscope for electron optical shadow method. Rev. Sci. Instr. 21, 669 (1950) 91.

Sisler, F. D., Cloud, P. E., Jr., Friedman, I., Dibeler, V. H., Microbiologieal fractionation of the hydrogen isotopes. Science 127, 13 (1958). 2635.

Skramstad, H. K., Ernst, A. A., Nigro, J. P., An analogdigital simulation for the design and improvement of man-machine systems, 1957 Eastern Joint Computer Conf. Proc. (Inst. Radio Engrs., New York, N.Y.) (1958). 2453.

Skramstad, H. K., Landsman, G. L., Control mechanisms, Handbook of Physies, Edited by E. U. Condon and H. Odishaw, Chapter 9, Pt. 2, p. 2-69 (MeGraw-Hill Book Co., Inc., New York, N.Y.) (1958). 2500.

Skramstad, H. K., Wright, J. H., An analog computer for radioactive fallout prediction. Proc. Natl. Simulation Council (1956). 1616.

Slawsky, M. M., Lutsky, M., Schneidlin, A. E., Pressure drop in pneumatic components and systems. Product Eng. Handbook No. 3, Section J, p. J2 (MeGrawHill Book Co., Inc., New York, N.Y.) (1955). 1456.
Slawsky, M. M., Tsai, D. H., Determination and correlation of flow capacities of pneumatic components. (1957) C588.

Slone, M. C., Lamb, J. J., Reinhart, F. W., Properties of polymethyl alpha-chloroacrylate. Modern Plastics 29, No. 10, 109 (1952). 520.

Slone, M. C., Reinhart, $F$. W., Measurement of creep of acrylic glazing plastics exposed to temperature gradients. SPE J. 10, 29 (1954). 1065.

Properties of plastic films. Modern Plastics 31, 203 (1954). 1122

Slutz, R. J., Atmospheric research, International Geophysical Year. Americana Annual p. 60. (An Encyclopedia of the Events of 1956, Americana Corp., New York, N.Y.) (1957), 2031.

Slutz, R. J., Shapley, A. H., Probing the ionosphere. In American Geophysical Union, Geophysics and the IGY, Geophysical Monograph No. 2, 45 (1958). 2700 .

Smith, A. N., Devaney, T. E., Fields in electrically short ground systems. J 63D2, 175 (1959).

Smith, C. C. Seliger, $H$. $H$., Improvement in response of 4- $\pi$ gamma-ionization chambers. Rev. Sci. Instr. 24, 474 (1953). 735 .

Smith, D. K., Bibliography on molecular and crystal structure models. (1960) Mono. 14

Smith, D. L., Caul, H.J., Alloys of gallium with powered metals as possible replacement for dental amalgam. J. Am. Dental Assoc. 53, 315 (1956). 1613.

Smith, D. L., Caul, H.J., Sweeney, W. T., Some physical properties of gallium copper-tin alloys. J. Am. Dental Assoc. 53, 677 (1956). 1876.

Smith, D. L., Ferguson, G. W., Schoonover, I. C., Microstructure of dental amalgam. J. Am. Dental Assoc. 47,305 (1953). 765 .

Smith, D. L. Schoonover, I. C., Direct filling resins: dimensional changes resulting from polymerization shrinkage and water sorption. J. Am. Dental Assoc. 46, 540 (1953). 689 .

Smith, E. E., Wyckoff, H. O., Aston, G. H., A comparison of X-ray standards. British J. Radiol. 27, No. 318, 325 (1954). 916.

Smith, E. K. Bureau of Standards comments on Loran communications. IRE Trans. Aeronaut. Navigational Elec. ANE-4, 84, (1957). 2039.

Sporadic E observed on VHF oblique incidence circuits, North Atlantic Treaty Organization. Sporadic E ionization; Ionospheric Research Meeting, AGARD Avionics Panel, September 1958, (Cambridge, England), AGARDograph 34, p. 129 (1958). 2773 .

The effect of sporadic-E on television reception. IRE Trans. Ant. Prop. AP-2, 54 (1952). 574; J. Radio-Electronics 24, No. 6, 54 (1953). 859

Smith, E. K., Bateman, R., Finney, J. W., Tveten, L. $H$., Watts, $J . M$., IGY observations of F-layer scatter in the far east. J. Geophys. Research 64, 403 (1959). 2963.

Smith, E. K., Davis, R. M., Ellyett, C. D., The effect of sporadic $\mathrm{E}$ on VHF transmission in North America. IRE Spec. Govt. Issue 47, No. 5, 762 (1959). 3074.

Smith, E. K. Finney, J. W., Report on the IGY obliqueincidence sporadic- $E$ and $F$-scatter program. (1960) TN48 (PB151407)

Smith, E. K., Knecht, R. W., Some implications of slantEs. J. Atmospheric and Terrest. Phys. Spec. Suppl. 1957, Proc. Polar Atmospheric Symp. July 1956 (Oslo, Norway), Pt. II, 195 (1957). 2304.

Smith, E. K., Thomas, J.A., A survey of the present knowledge of sporadic-E ionization. J. Atmospheric and Terrest. Phys. 13, 295 (1959). 2885.

Smith, E. K., Weintraub, S., The constants in the equation for atmospheric refractive index at radio frequencies. Proc. IRE 41, 1035 (1953). 855.

Smith, E. R., Edelman, S., Brooks, R., Saito, S., Jones, E., A stroboscopic vibration analyzer. J 63C2, 97 (1959). 
Smith, E. R., Edelman, S., Jones, E., Some developments in vibration measurement. J. Acoust. Soc. Am. 27, 728 (1955). 1492 .

Smith, E. R., Edelman, S., Jones, E., Schmidt, V. A., Stroboscopic interferometer for vibration measurement. J. Acoust. Soc. Am. 30, 867 (1958). 2784.

Smith, E. R., Sulzer, P. G., Edelman, S., High-speed stroboscope for accelerometer calibration. Rev. Sci. Instr. 25, No. 8, 837 (1954). 1031.

Smith, F. A., Isbell, H. S., Creitz, E. C., Frush, H. L., Moyer, J. D., Stewart, J. E., Infrared spectra of sugar acetates in solution. J 59, 41 (1957) RP2772.

Smith, F. C., Howard, D. M., Fatigue and static tents of flush riveted joints. Product Eng. Handbook No. 3, Section G. p. G2 (McGraw-Hill Book Co., Inc., New York, N.Y.) (1955). 1345.

Smith, F. C., Smith, I., Howard, D. M., Cumulative fatigue damage of axially loaded Alclad $75 \mathrm{~S}-\mathrm{T} 6$ and Alclad 24S-T3 aluminum-alloy sheet. Natl. Advisoly Comm. Aeronaut. Tech. Note 3923 (1955). 1303.

Smith, I., Howard, D. M., Smith, F. C., Cumulative fatigue damage of axially loaded Alclad 75S-T6 and Alclad 24S-T3 aluminum-alloy sheet. Natl. Advisory Comm. Aeronaut. Tech. Note 3932 (1955). 1303.

Smith, J., Wildhack, W. A., Stern, J., Documentation in instrumentation. Studies in Coordinate Indexing Vol. 111, Documentation, Inc. (1956). 1682.

Smith, J. C., Rapid impact loading of textile yarns. Proc. Symp. on High-Speed Testing, Plas-Tech. Equipment Corp. (Interscience Publ. Inc., New York, N.Y.) (1958). 2715.

Smith, J. C., McCrackin, F. L., Schiefer, H. F., Characterization of the high-speed impact behavior of textile yarns. (Conf. Issue) J. Textile Inst. (Trans.) 50, No. 1, T55 (1959). 2909.

Stress-strain relationships in yarns subjected to rapid impact loading. III. Effect of wave propazation. Textile Research J. 25, No. 8, 701 (1955). 1516.

Stress-strain relationships in yarns subjected to rapid impact loading: 5. Wave propagation in long textile yarns impacted transversely. J 60, 517 (1958) RP2866; Textile Research J. 28, No. 4, 288 (1958). 2783.

The impact absorbing capacity of textile yarns. ASTM Bull. No. 220, 52 (1957). 2363.

Smith, J. C., McCrackin, F. L., Schiefer, H. F., Stone, $W$. $\dot{K}$., 'Breaking velocities, strain energies, and theory neglecting wave propagation. Pt. II. Textile Research J. 25, 529 (1955). 1277.

Smith, J. C., Schiefer, H. F., The design of impactresisting fabric structures. Chapter 13 on Textile Engineering, Encyclopedia of Man-Made Textiles (Textile Book Publs., Division of Interseience Publs., New York, N.Y.) (1959). 3069.

Smith, J. C., Schiefer, H. F., McCrackin, F. L., Stone, $\dot{W} \cdot \dot{K} ., \quad$ Stress-strain relationships in yarns subjected to rapid impact loading. Am. Soc. Testing Materials Spec. Tech. Publ. 176, "Symposium on Impact Testing", 126 (1955). 1514.

Stress-strain relationships in yarns subjected to rapid impact loading. Am. Soc. Testlng Materials Spec. Tech. Publ. 185, Symp. on Testing on Non-Metallic Materials, p. 47 (1956) 1899.

Smith, J. C., Schiefer, H. F., McCrackin, F. L., Stone, $W . K$., Towne, $K$. M., Stress-strain relationship in yarns tested at rates of straining up to a million percent per minute. Physics of Wool and Other Viscoelastic Fibers, D, 148 (1956) (Proc. Intern. Wool Textiles Research Conf., August 20-25, 1955, Sydney, Australia) (1956). 1900.

Smith, J. L., Leiner, A. L., Notz, W. A., Weinberger, A., Organizing a network of computers to meet deadlines. 1957 Eastern Joint Computer Conf. Proc. (Washington, D.C.) p. 115 (1957). 2240.

PILOT, a new multiple computer system. J. Assoc. Computiny Mach. 6, No. 3, 313 (1959). 3016.

PILOT, the NBS multicomputer system. Proc. Eastern Joint Computer Conf. (Philadelphia, Pa.) p.
164 (1958). 2692.

Smilh, J. L., Weinberger, A., A logic for high-speed addition. (1958) C591, Sect. 1.

A one-microsecond adder using one-megacycle circuitry. IRE Trans. Electronic Computers EC-5, No. 2, 65 (1956). 1596.

Shortcut multiplication for binary digital computers. (1958) C591, Sect. 1.

The logical design of a one-microsecond parallel adder using one-megacycle circuitry. Am. Inst. Elec. Engrs. Proc. Western Joint Computer Conf. T-85 (1956). 1937.

Smith, N., Color television. Sci. Am. 183, No. 6, 13 (1950). 38.

Influence of the sun upon the ionosphere. (Part of a Conference on the sun in the service of man.) Proc. Am. Acad. Arts 79, 181 (1951). 250.

Smith, N., Condon, E. U., Bailey, S. L., Everitt, W. L., Fink, $D . G$., The present status of color television; a report by the Senate Advisory Committee on Color Television. Proc. IRE 38, 980 (1950). 154.

Smith, R. G., Leaderman, H., Jones, R. W., Rheology of polyisobutylene. II. Low molecular weight polymers. J. Polymer Sci. 14, 47 (1954). 1132.

Smith, R. G., Leaderman, H., Williams, L. C., Rheology of polyisobutylene. III. Elastic recovery, non-Newtonian flow, and molecular weight distribution. J. Polymer Sci. 36, 233 (1959). 3037.

Smith, R. O., Wildhack, W. A., A basic method of determining the dynamic characteristics of accelerometers by rotation. J. Instr. Soc. Am. 1, No. 12, Paper No. 54 (1954). 913.

Smith, $R$. W. The federal basis for weights and measures. (1958) Ċ593.

Smith, S. J., Branscomb, L. M., Electron affinity of atomic oxygen. Phys. Rev. 98, No. 4, 1127 (1955). 1334.

Electron affinity of atomic sulfur and empirical affinities of the light elements. J. Chem. Phys. 25, No. 3, 598 (1956). 1698.

Experimental cross section for photodetachment of electrons from H-and D-. Phys. Rev. 98, No. 4, 1028 (1955). 1338.

Negative oxygen ions in the upper atmosphere: the affinity and radiative attachment coefficient of atomic oxygen. Trans. Am. Geophys. Union 36, 5, 755 (1955). 1413.

Smith, S. J., Branscomb, L. M., Burch, D. S., Geltman, S., Photodetachment cross section and the electron affinity of atomic oxygen. Phys. Rev. 111, No. 2, 504 (1958). 2683.

Photodetachment studies of negative ions. Third Intern. Conf. on Ionization Phenomena in Gases, Societa Italiana di Fiscia. (Venice, Italy) (1957). 2246.

Smith, S. J., Burch, D. S., Photodetachment cross section of the negative hydrogen ion. Phys. Rev. Letters 2, No. 4, 1645 (1959). 3012.

Relative measurement of the photodetachment cross section for $\mathrm{H}^{-}$. Phys. Rev. 116, No. 5, 1125 (1959). 3035 .

Smith, S. J., Burch, D. S., Branscomb, L. M., Experimental photodetachment cross section and the ionospheric detachment rate for $0_{2}$. Ann. Geophys. Tome 14, No. 2, 225 (1958), 2556.

Photodetachment of $0_{2-}$. Phys. Rev. 112, 171 (1958). 2684.

Smith, S. W., Protection in the use of dental roentgenographic equipment. J. Am. Dental Assoc. 51, 12 (1955). 1462.

Smith, S. W., Amory, H. I., Military field X-ray equipment. Radiology 59, N̉o. 6, 879 (1952). 483.

Smith, S. W., Kennedy, R. J., Dose to walls in radiographic rooms. Radiology 64, 114A (1955). 1319.

Smith, S. W., Nelsen, R. J., Dangers of radiation. J. Am. Dental Assoc. 50, No. 5, 581 (1955). 1305. 
Smith, S. W., Taylor, J. K., A coulometric-titration coulometer. J 63C1, 65 (1959).

Polarographic determination of trace quantities of iodide. Anal. Chem. 29, 301 (1957). 2258.

Precise coulometric titrations. Science 124, 3228 (1956). 1815.

Precise coulometric titration of acids and bases. J 63A2, 153 (1959).

Smith, W. H., Multipurpose standard for microchemical analysis. Anal. Chem. 30, 149 (1958). 2641.

Snoke, $H, R$., Asphalt research at the National Bureau of Standards. ASTM Bull. No. 220, p. 41 (1957). 2029.

Research on roofings. Building Research Advisory Board Conf. Report, Research Corporation Conf. (Building Research Advisory Board, Div, of Engr. and Ind. Natl. Research Council, Washington, D.C.) (1950). 129 .

Snoke, H. R., Kleinschmidt, $L$. R., Changes in the properties of an asphalt during the blowing operation. J 60, 169 (1958) RP2835.

Effect of light and water on the degradation of asphalt. J 63C1, 31 (1959).

Snyder, C. F., Report on sugars and sugar products. J. Assoc. Offic. Agri. Chemists 39, 676 (1956). 1852.

Snyder, C. F., Swindells, J. F., Hardy, R. C., Golden, P. E., $V$ iscosities of sucrose solutions at various temperatures: tables of recalculated values. (1958) C440 Suppl.

Snyder, W. F., Corliss, E. L. R., Calibration of audiometers. J. Acoust. Soc. Am. 22, 837 (1950). 31.

Sokolowsky, D., Gaddum, J. W., Hoffman, A. J., On the solution of the caterer problem. Logistics Research Quart. 1, 223 (1954). 1099.

Sokolowsky, D., Hoffman, A. J., Mannos, M., Wiegmann, $N$. A., Computational experience in solving linear programs. J. Soc. Ind. Appl. Math. 1, 17 (1953). 672.

Solow, $M$., Greene, $F$. M., Development of VHF fieldintensity standards. Proc. IRE 40, No. 5, 573 (1952). 427

Soller, W., Malloy, A., Roberts, A. G., Evaluation of adhesion of organic coatings by ultracentrifugal and other methods. Paint, Oil, and Chem. Rev. 116, No. 18,$14 ; 116$, No. 19,26 (1953), 715.

Sommer, H., Craig, S. N., Hoffman, J. I., Hipple, J. A., The effect of recent mass measurements on the Faraday. Phys. Rev. 85, 740 (1952). 573.

Sommer, H., Hipple, J. A., A combination crossedfield and time-of-flight mass spectrometer. Phys. Rev. 85, 714 (1952). 360.

A new method for the measurement of the isotopic abundance of solids. Phys. Rev. 83, 229 (1951). 180.

Sorrows, H. E., Ryan, W.E., Ellenwood, R. C., Evaluation of coaxial slotted-line impedance measurements. Proc. IRE 39, $162(1951), 234$.

Soudsr, W., Paffenbarger, G. C., American Dental Association contribution to dental science: physical research. J. Am. Dental Assoc. 58, 97 (1959), 2891.

Southard, T. H., Yowell, E. C. An alternative "preditorcorrector" process. Math. Tables and Other Aids to Computation, VI, No. 40, 253 (1952). 389.

Spaulding, A. D., Crichlow, W. Q., Roubique, C. J., Beery, $W . M$., Determination of the amplitude-probability distribution of atmospheric radio noise from statistical moments. J 64D 1, 49 (1960).

Spencer, $L . V$., Calculation of peaked angular distributions from Legendre polynomial expansions and an application to the multiple scattering of charged particles. Phys. Rev. 90, 146 (1953). 659. A theory of cavity ionization. Cancer Yearbook (Yearbook Publishers, Chicago, Ill.), p. 499 (1956) 1606.

Energy dissipation by fast electrons, (1959) Mono. 1. Penetration and diffusion of X-rays: calculation of spatial distributions by semi-asymptotic methods. Phys. Rev. 88, No. 4, 793 (1952). 509. The theory of electron penetration. Phys. Rev. 98, 1597 (1955). 1548.
Spencer, L. $V$., Attix, F. H., A cavity ionization theory including the effects of energetic secondary electron. Radiology 64, No. 1, 113 (1955). 1229.

A theory of cavity ionization. Radiol. Research 3, 239 (1955). 1247.

Spencer, L. V., Berger, $M$. J., General relations between fluxes from collimated point and plane sources of radiation. Phys. Rev. 113, No. 2, 408 (1959). 2957. Penetration of gamma rays from isotropic sources through aluminum and concrete. (1959) TN11 (PB151370).

Some radiological applications of gamma-ray transport theory. Radiation Research 10, No. 5, 552 (1959). 3051.

Spencer, L. V., Blanchard, C. H., Multiple scattering of relativistic electrons. Phys. Rev, 93, 114 (1954). 1079.

Spencer, $L . \quad V$., Doggett, J. A., Elastic scattering of electrons and positrons by point nuclei. Phys. Rev. 103, No. 6, 1597 (1956). 1694.

Spencer, L. V., Fano, $U$., Energy spectrum resulting from electron slowing down. Phys. Rev. 93, 1172 (1954). 1015.

Spencer, L. V., Goldstein, H., Wilkins, J., Systematic calculations of gamma-ray penetration. Phys. Rev. $89,1150(1953)$. 847.

Spencer, L. V., Olson, C. A., Energy spectra of cascade electrons and photons. J 60, 85 (1958) RP2824.

Spencer, $L$. V., Wolff, C., Penetration and diffusion of hard X-rays: polarization effects. Phys. Rev. 90, 510 (1953). 793.

Spiegel, $V ., J r .$, Oliver, D. W., Caswell, R. S., Age to indium resonance for D-D neutrons in water. Nuclear Sci. and Eng. 4, No. 4, 546 (1958). 2450.

Spindler, R. J., Rodney, $W$. S., Index of refraction of fused-quartz glass for ultraviolet, visible, and infrared wavelengths. J. Opt. Soc. Am. 44, No. 9, 677 (1954). 1035 .

Refractive indices of cesium bromide. J. Opt. Soc. Am. 42, 431 (1952). 527.

Spinảler, R. J., Stephens, R. E., Plyler, E. K., Rodney, W. S., Refractive index of potassium bromide for infrared radiant energy. J. Opt. Soc. Am. 43, 110 (1953). 812 .

Spinks, A. W., Hermach, F. L., Portable potentiometer and thermostated containers for standard cells. Rev, Sci. Instr. 26, 770 (1955). 1450.

Spinner, S., Elastic moduli of glasses at elevated temperatures by a dynamic method. J. Am. Ceram. Soc. 39, No. 3, 113 (1956). 1692.

Relation between refractive index and elastic moduli of a borosilicate glass after heat treatment. J. Am. Ceram. Soc 39, No. 11, 390 (1956) 1844.

Spinner, S., Cleek, G. W., Hamilton, E. H., Determination and use of the sag point as a reference point in the heating of glasses. J 59, 227 (1957) RP2791.

Spinner, S., Reichard, T. W., Tefft, W. E., A comparison of experimental and theoretical relations between Young's modulus and the flexural and longitudinal resonance frequencies of uniform bars. $J$ 64A2, 147 (1960).

Spinner, S., Shartsis, L., Capps, W., Density expansivity and viscosity of molton alkali silicates. J. Am. Ceram. Soc. 35, No. 6, $155(1952), 422$.

Viscosity and electrical resistivity of molten alkali borates. J. Am. Ceram. Soc. 36, 319 (1953). 907.

Spinner, S., Valore, R. C., Jr., Comparison of theoretical and empirical relations between the shear modulus and torsional resonance frequencies for bars of rectangular cross section. J 60, 459 (1958) RP2861.

Spoerri, P. E., Brode, W. R., Seldin, I. L., Wyman, G. M., The relation between the absorption spectra and the chemical constitution of dyes. XXVIII. The hydration of Azo dyes in organic solvents. J. Am. Chem. Soc. 7\%, 2762 (1955). 1543.

Squires, H. P., Mann, D. E., Broida, H. P., The decomposition of $\mathrm{CF}^{4}$ in flames. J. Chem. Phys, 22, 348 (1954). 1176. 
Stair, R., Filter radiometry and some of its applications. J. Opt. Soc. Am. 43, 971 (1953). 720.

Les Tektites-Ces objets de verre tombes du ciel proviennent-ils d'une planete perdue? Sciences et Avenir (Paris, France), No. 116, 441 (1956). 1756. Tektites and the lost planet. Smithsonian Institution Annual Report No. 4194, 217 (Smithsonian Institution, Washington, D.C.) (1955). 1525 .

Tektites and the lost planet. Sci. Monthly 83, 1 (1956). 1912.

Tektites, meteoric glass. Discovery (Norwich, England) 17, No. 10, 408 (1956), 1912A.

The spectral radiant energy from the sun through varving degrees of smog at Los Angeles. Proc. of 3d Natl. Air Pollution Symp. (Los Angeles, Calif.) (1955). 1545.

The spectral-transmissive properties of plastics for use in eye protection. Am. Standards Assoc. Special Issue (New York, N.Y.) (1955). 1456.

The spectral-transmissive properties of some of the tektites. Geochim. et cosmochim. acta (Pergamon Press, New York, N.Y.) 7, 43 (1955). 1547.

Stair, R., Bagg, T. C., Johnston, R. G., Continuous measurement of atmospheric ozone by an automatic photoelectric method. Proc. Joint Army-Navy-Air Force Conf. on Elastomer Research and Devel., January 12-13, 1954 (Washington, D.C.), Natl. Acad. Sci. Natl. Research Council Publ. 370, p. 75 (1954). 977

Stair, R., Hubbard, D., Krumrine, E. M., Australite (meteoric) glass. Trans. Am. Geophys. Union 37, No. 6, 767 (1956). 1636

Stair, R., Johnston, R. G., Some studies of atmospheric transmittance on Mauna Loa. J 61, 419 (1958) RP2910

Stair, R., Maki, A. G., Johnston, R. G., Apparatus for the measurement of the normal spectral emissivity in the infrared. J 64C2, 99 (1960).

Stanford, J.W., Burns, C. L., Paffenbarger, G. C., American Dental Association specification No. 13 for selfcuring repair resins. J. Am. Dental Assoc. 51, 425 (1955). 1257.

Stanford, $\dot{J} . W_{.}$, Paffenbarger, G. C., Processing denture base resins: heat-curing type. J. Am. Dental Assoc. 53, 72 (1956). 1819.

Review of 1956 dental research: dental materials and instrumentation. J. Am. Dental Assoc. 54, 465 (1957). 2290.

Silicate cement: how to select and use them. Dental Practitioner 8, No. 12, 387 (1958). 2756.

Zinc phosphate and silicate cements. Chapter in Dental Clin. North American, p. 561 (W. B. Saunders Co., Philadelphia, Pa.) (1958). 2873.

Stanford, $J, W$, Paffenbarger, G, C. Kumpula, J. W. Sweeney, $W . T$., Determination of some compressive properties of human enamel and dentin. J. Am. Dental Assoc. 5\%, 487 (1958). 2518.

Stanford, J. W., Paffenbarger, G. C., Sweeney, W. T., A revision of American Dental Association specification No. 3 for dental impression compound. J. Am. Dental Assoc. 51, 56 (1955). 1244 .

American Dental Association specifications for dental materials (third ed.) (1958). 2451.

Stang, A. H. The tensile forces in tightened bolts. Product Éng. 22, No. 2, 118 (1951). 344.

Stanley, C., Brenner, A., The adhesion of electroplated coatings to titanium. Ann. Proc. Am. Electroplaters' Soc, p. 123 (1956), 1920.

Stanley, E. D., Gainen, L., Honig, D. J., Linear programming in bid evaluation. Logistics Research Quart. 1, 48 (1954). 1051.

Stanley, G. $M_{\text {., }} \quad$ Layered earth propagation in the vicinity of Point Barrow, Alaska. J 64.D1, 95 (1960).

Stanley, $R$. $W$., Meggers, $W$. $F$., Wavelengths from thorium-halide lamps. J $\mathbf{6 1}, 95$ (1958) RP2891.

Stansbury, C., Joseph, H. M., Automatic counting machine. Elec. Mfg. 49, No. 3, 100 (1952). 398.
Stansbury, C., Montgomery, G. $F$., Clamp-on microammeter measures a-c current. Electronics 30, No. 12, 152 (1957). 2056.

The elamp-type alternating-current microammeter. Am. Inst. Elec. Engrs. Paper No. 57114 (1957). 2347.

Staras, H., Scattering of electromagnetic energy in a randomly inhomogeneous atmosphere. J. Appl. Phys. 23, No. 10, 1152 (1952). 536.

Staras, H., Hill, J. S., Waldo, G. V., VHF tropospheric recording measurements of plane and circular polarized waves in the Great Lakes area. Abstract Publ. in IRE Trans. Amt. Prop. AP-1, 42 (1952); entire paper published as FCC memo 62838. 620

Staras, H., Norton, K. A., Blum, M., A statistical approach to the problem of multiple radio interference to FN and television service. IRE Trans. Ant. Prop. AP-1, 43 (1952). 378.

Starfelt, N., The use of scintillation spectrometers in the study of continuous gamma- and X-ray spectra between 0.01 and 20 Mev. Kungl. Fysiografiska Sallskapets I. Lund Forhandlinger $\mathrm{Bd} \mathrm{26,} \mathrm{Nr} 5$ (University of Lund, Lund, Sweden) (1956). 1955.

Starfelt, N., Koch, $H$. W., Differential cross-section measurements of thin-target bremsstrahlung produced by 2.7 - to $9.7-\mathrm{Mev}$ electrons. Phys. Rev. 102, 1598 (1956). 1676.

Staugaitis, C. L., Burnett, H. C., Endurance of helical springs related to properties of the wire. Metal Progr. 64, 77 (1953). 710.

The behavior of long helical springs under fluctuating load. ASTM Bull. No. 55, 918 (1955). 1530.

Stavroudis, $O . N$., Lens design: a new approach. J 63 B 1 , $31(1959)$

Steel, M. N., Mandel, J., Roth, F. L., Stiehler, R. D., Measurement of the aging of rubber vulcanizates. J 63C2, 141 (1959).

Steel, M. N., Mandel, J., Stiehler, R. D., Treadwear of tires. Influence of type of rubber and black. Ind. Eng. Chem. 43, 2901 (1951). 350.

Steel, M. N., Mann, C., Mandel, J., Kanagy, J. R., Sampling of side upper leather II. J. Am. Leather Chemists Assoc. 47, 352 (1952). 535.

Steel, M. N., Stiehler, R. D., Mandel, J., Some factors influencing the road wear of tires. Trans. Inst. Rubber Ind. (Cambridge, England) 27, No. 6, 298 (1951). 308.

Steel, M. N. Stiehler, R. D., Richey, G. G., Mandel, J., Hobbs, R. H., Power loss and operating temperature of tires. J 64C1, 1 (1960).

Stegun, I. A., Abramowitz, M., Generation of Bessel functions on high speed computers. Math. Tables and Other Aids to Computation 11, 255 (1957). 2140 .

Pitfalls in computation. J. Soc. Indust. Appl. Math. 4, 207 (1956). 1809 .

The generation of Coulomb wave functions by means of recurrence relations. Phys. Rev. 98, 1851 (1955). 1532.

Stegun, I. A., Kessler, K. G., Prusch, S. B., Analysis of atomic spectra with electronic digital computers. J. Opt. Soc. Am. 46, No. 12, 1043 (1956). 1624.

Steifel, $M . B$. Application of photoelasticity to the design of tensile specimens for ceramic metal materials. Bull. Am. Ceram. Soc. 34, No. 5, 133 (1955). 1266

Stein, $M$., Moore, $H$. S., Comparison of variously derived solar indexes. J. Geophys. Research 55, 423 (1950). 40.

Stein, M. L., Sufficient conditions for the convergence of Newton's method in complex Banach spaces. Proc. Am. Math. Soc. 3, No. 6, 858 (1952). 559.

Stein, $P_{\text {., }}$ The convergence of Seidel iterants of nearly symmetric matrices. Math. Tables and Other Aids to Computation V, 236 (1951). 329.

Steiner, E. T., Hosterman, E. R., Aging of Karakul and seal fur skins. J. Am. Leather Chemists Assoc. 45, 579 (1950). 19. 
Steinert, L. A., Bussey, H. E., An exact solution for a eylindrical cavity containing a gyromagnetic material. Proc. IRE 45, Pt. 1, 693 (1957). 2013.

Exact solution for a gyromagnetic sample and measurements on a ferrite. IRE Trans. Microwave Theory and Tech. MT'T-6, 72 (1958). 2552.

Stephens, R. E., Cross-field error in unsymmetrical range finders. J. Opt. Soc. Am. 45, No. 3, 146 (1955). 1300 .

Note on measurement of sinewave response of lenses. J. Opt. Soc. Am. 49, No. 4, 413 (1959). 2999.

Range finders, using projected images. J 60, 327 (1958) RP2851.

Secondary chromatic aberration. J. Opt. Soc. Am. 47, No. 12, 1135 (1957). 2295.

Selection of glasses for three color achromats. J. Opt. Soc. Am. 49, No. 4, 398 (1959). 3043.

The effect of illuminance of a lens interposed between the source and the illuminated surface. J. Opt. Soc. Am. 43, 704 (1953). 858.

Stephens, R. E., Plyler, E. K., Rodney, W. S., Spindler, R. J. Refractive index of potassium bromide for infrared radiant energy. J. Opt. Soc. Am. 43, 110 (1953). 812.

Stephens, R. E., Plyler, E. K., Tilton, L. W., Refractive index of silver chloride for visible and infrared radiant energy. J. Opt. Soc. Am. 40, 540 (1950). 124.

Stephenson, S. R., Posner, A. S., Crystallographic investigation of tricaleium phosphate hydrate. J. Dental Research 31, No. 3, 371 (1952). 421.

Isomorphous substitution in enamel apatite. J. Am. Dental Assoc. 46, 257 (1953). 748.

Stern, H., Krynitsky, A. I., Experimental production of nodular graphite in cast iron. Foundry 80, No. 3, 106 ; Pt. 2, 80, No. 4, 98 (1952). 444.

Stern, $J$ A system of names for binary numbers. Science 128, 594 (1958). 2441.

Stern, J., Wildhack, $W$. A., The peek-a-boo system. Chapter 6, Punched Cards, p. 125 (Reinhold Publ. Corp. New York, N.Y.) (1959). 3087.

Stern, J., Wildhack, W. A., Smith, J., Documentation in instrumentation. Studies in Coordinate Indexing Vol. 111. Documentation, Inc. (1956). 1682.

Stevens, $M$. E., Interdisciplinary approach to science personnel. Personnel Administration 12, No. 3, 27 (1950). 77.

The interconnection of two digital computers. Am. Inst. Elec. Engrs. Fall General Meeting, CP 55-736 (Chicago, Ill.) (1955). 1536.

Stevens, M.E., Alexander, S. N., Comparative characteristics of medium-priced fully automatic computers for statistical applications. Proc. of First Conf. on the Design of Experiments in Army Research, Development and Testing. Office of Ordnance Research (Durham, N.C.) OORR 57-1 (June 1957). 2061.

Stewart, J. E., Infrared absorption spectra of urea, thiourea, and some thiourea-alkali halide complexes. J. Chem. Phys. 26, 248 (1957). 2160.

Infrared absorption spectrum of $n$-dimethyl-aminodiborane. J. Chem. Phys. 23, No. 11, 2204 (1955). 1365.

Low-temperature microcell for infrared study of condensed gases. Anal. Chem. 20, 2073 (1958). 2619. Temperature microcell for infrared study of condensed gases. Anal. Chem, 30, 2073 (1958). 2800.

Use of partially oriented solid samples and unpolarized radiant energy in the analysis of infrared absorption spectra. J. Chem. Phys. 23, No. 5, 986 (1955). 1569 .

Stewart, J. E., Hellmann, $M$., Infrared spectra of crystalline polyphenyls. J 60, 125 (1958) RP2829.

Stewart, J. E., Isbell, H. S., Smith, F. A., Creitz, E. C., Frush, H. L., Moyer, J. D., Infrared spectra of sugar acetates in solution. J 59, 41 (1957) RP2772.

Stewart, J. E., Linnig, $F$. J., A spectroseopic study of oils used in oil-extended rubber. J 59, 27 (1957) RP2771.

Infrared study of some structural changes in natural rubber during vulcanization. $\mathbf{J}$ 60, 9 (1958) RP2816.
Stewart, R. B., Mann, D. B., Thermodynamic properties of helium at low temperatures and high pressures. (1959) TN8 (PB151367).

Stewart, J. E., Richmond, J. C., Infrared emission spectrum of silicon carbide heating elements. J 59, 405 (1957) RP2810.

Spectral emittance of ceramic-coated and uncoated specimens of Inconel and stainless steel. J. Am. Ceram. Soc. 42, No. 12, 633 (1959). 3053.

The spectral emittance of uncoated and ceramiccoated Inconel and type 321 stainless steel. NASA Memo 4-9-59W (1959). 3092.

Stewart, J. E., Tipson, R. S., Isbell, H. S., Infrared absorption spectra of some cyclic acetals of sugars. J 62, 257 (1959) RP2960.

Stiefel, E. L., Kernel polynomials in linear algebra and their numerical applications. (1958) A MS49.

Stiefel, M. B., Kerper, M. J., Mong, L. E., Holley, S. F., Evaluation of tensile, compressive, torsional, transverse, and impact tests and correlation of results for brittle cermets. J 61, 149 (1958) RP2895.

Stiehler, R. D., Abrasion-resistance. Encyclopedia of Chemistry (Reinhold Publ. Corp., New York, N.Y.), p. 1 (1957). 2005.

Proof testing rubber tires. Ordnance 36, No. 192 , 1018 (1952). 519.

The National Bureau of Standards and its services to industry. Industries et Sciences. (L'Association des Ingenieurs Techniciens de Charleroi. Rhode-StaintGenese-lez-Bruxelles, Belgium) 30, No. 4, 17 (1956). 1939.

Stiehler, R. D., Decker, G. E., Temperature measurements in the Mooney viscoineter. ASTM Bull. No. 195, (TP7) (1954). 1164.

Stiehler, R. D. Mandel, J. Evaluation of analytical methods by the sensitivity criterion. Anal. Chem. 29, 17 A (1957). 2112 .

Statisties-A tool in testing rubber. ASTM Bull. No. 204, 44 (1955). 1512 .

Stiehler, R. D., Mandel, J., Roth, F. L., Steel, M. N., Measurement of the aging of rubber vulcanizates. J 63C2, 141 (1959).

Stiehler, R. D., Mandel, J., Steel, M. N., Treadwear of tires. Influence of type of rubber and black. Ind. Eng. Chem. 43, 2901 (1951). 350.

Stichler, R. D., Martin, G. M., Roth, F. L., Behavior of "pure gum" rubber vulcanizates in tension. Trans. Inst. Rubber Ind. 32, No, 6, 189 (1956). 1640.

Stiehler, R. D., Richey, G. G., Mandel, J., Measurement of treadwear of commercial tires. Rubber Age 73, 201 (1953). 761.

Stiehler, R. D., Roth, F. L., Standard natural rubber. Rubber Age 78, 77 (1955). 1505.

Tension testing of rubber rings. ASTM Bull. No. 233,38 (1958). 2801.

Vulcanization characteristics of natural rubber. Symp. on Recent Developments in the Evaluation of Natural Rubber, 1952; Am. Soc. Testing Materials Spec. Tech. Publ. No. 136, p. 50 (1953); India Rubber World 127, 783 (1953); Rubber Chem. Tech. 26, 593 (1953). 909.

Stichler, R. D., Roth, F. L., Decker, G. E., Temperature control during mixing of rubber compounds. Rubber World 132, 482 (1955). 1526.

Stiehler, $R, D$, Steel, $M, N$, Mandel, $J$, Some factors influencing the road wear of tires. Trans. Inst. Rubber Ind. (Cambridge, England) 27, No. 6, 298 (1951). 308.

Stiehler, R. D., Steel, M. N., Richey, G. G., Mandel, J., Hobbs, $R$. H., Power loss and operating temperature of tires. J $\mathbf{6 4 C} 1,1$ (1960).

Stimson, $H$. F., Heat units and temperature scales for calorimetry. Am. J. Phys, 23, 614 (1955). 1355. The present status of temperature scales. Science 116, 339 (1952). 590 .

Stinchfield, R., Swanson, H. E., Gilfrich, N. T., Cook, M. I., Parks, $P$. C., Standard X-ray diffraction powder patterns. (1959) C539, Vol. 8.

Stinchfield, R. P., Wachtman, J. B., Jr., Tefft, W. E., Lam, 
D. G., Jr., Elastic constants of synthetic single crystal corundum at room temperature. J 64A3, $213(1960)$

Stober, A.K., Minkoff, G. J., Scherber, F. I., Suppression of bubbling in boiling refrigerants. Nature 180, 1413 (1957). 2330.

Stockmann, L. L., Harding, J. E., Schwebel, A., Report on the determination of small amounts of radium in solution. J. Assoc. Official Agri. Chem. 41, No. 2, 311 (1958). 2733.

Stockmann, L. L., Mann, W. B., Youden, W. J., Schwebel A., Mullen, P. A., Garfinkel, S. B., Preparation of new solution standards of radium. J 62, 21 (1959) RP2924.

Stokes, R. H., Bates, R. G., Guggenheim, E. A., Harned, H. S., Ives, D. J., Janz, G.J., Monk, C. B., Robinson, R. A., Wynne-Jones, W. F. J., Standard electrode potential of the silver, silver chloride electrode. J. Chem. Phys. 25, 361 (1956). 1895.

Stone, J. J., Goldman, A. J., A symmetric continuous poker model. J 64B1, 35 (1960).

Stone, R. O., Meissner, P., Schwarz, K. M., Expendable modules as bases for disposal-at-failure maintenance. (1960) TN41 (PB151400).

Stone, W. K., McCrackin, F. L., Schiefer, H. F., Smith, J.C., Breaking velocities, strain energies, and theory neglecting wave propagation, Pt. II. Textile Research J. 25, 529 (1955). 1277.

Stone, W. K., Schiefer, H. F., Fox, G., Stress-strain relationships in yarns subjected to rapid impact loading. Part I. Equipment, testing procedure, and typical results. Textile Research J. 25, 520 (1955). 1515.

Stone, W. K., Schiefer, H. F., Smith, J. C., McCrackin, $F$. L., Stress-strain relationships in yarns subjected to rapid impact loading. Am. Soc. Testing Materials Spec. Tech. Publ. 176, "Symp. on Impact Testing", 126 (1955). 1514.

Stress-strain relationships in yarns subjected to rapid impact loading. Am. Soc. Testing Materials Spec. Tech. Publ. 185, Symp. on Speed of Testing on NonMetallic Materials, p. 47 (1956). 1899.

Stone, W. K., Schiefer, H. F., Smith, J. C., McCrackin, $F$. L., Towne, K. M., Stress-strain relationship in yarns tested at rates of straining up to a million percent per minute. Physics of Wool and Other Viscoelastic Fibers, D, 148 (1956) (Proc. Intern. Wool Textiles Research Conf., August 20-25, 1955, Sydney, Australia). (1956). 1900.

Stoneley, R., The transmission of Rayleigh waves across an ocean floor with two surface layers. Bull. Seismological Soc. Am. 47, 7 (1957). 2387.

Stoneley, R., Hochstrasser, U., The attenuation of Rayleigh waves with depth in a medium with two surface layers. Monthly Notices of Royal Astron. Soc. Geophys. (London, England) Supplement $\gamma, 279$ (1957). 2344.

Storey, L. R. O., Correlation of solar noise fluctuations in harmonically related bands. J 63D3, 293 (1959).

Method for measuring local electron density from an artificial satellite. J 63D3, 325 (1959).

The joint use of the ordinary and extraordinary virtual height curves in determining ionospheric layer profiles. J 64D2, 111 (1960).

Storey, L. R. O., Lawrence, R. S., A radio-astronomical test of the ballistic theory of light emission. Correspondence to Editor, Observatory $\boldsymbol{7 9}$, No. 911, 150 (1959). 2878.

Strang, A. G., Ogburn, F., Measurement of surface roughness of electrodeposited and electropolished surfaces by means of the microinterferometer. 37th Annual Proc. Am. Electroplaters' Soc. 125 (1950). 86.

Straus, E. G., Goldberg, K., Newman, M., Swift, J. D., The representation of integers by binary quadratic rational forms. Arch. math. (Tübinger, Germany) 5,12 (1954). 1198 .

Straus, E. G., Hoffman, A. J., Newman, M., Taussky, O., The number of absolute points of a correlation. Pacific Math. 6, 83 (1956). 1943.

Straus, E. G., Motzkin, T. S., Valentine, F. A., The number of farthest points. Pacific J. Math. 3, 221 (1953). 876.

Straus, E. G., Taussky, O., Algebraic equations satisfied by roots of natural numbers. Pacific J. Math 6,97 (1956). 1610 .

Straus, S., Madorsky, S. L., Thermal degradation of polyacrylonitrile, polybutadiene, and in copolymers of butadiene with acrylonitrile and styrene. J 61, 77 (1958) RP2888.

Thermal degradation of polyethylene oxide and polypropylene oxide. J. Polymer Sci. 36, 183 (1959). 3100 .

Thermal degradation of polymers as a function of molecular structure and branching. Consiglio Nazionale Delle Richerche (Rome, Italy) 25, 3 (1955). 1557.

Thermal degradation of polymers at high temperatures. J 63A3, 261 (1959).

Thermal degradation of unvuleanized and vuleanized rubber in a vacuum. Ind. Eng. Chem. 48, 1212 (1956). 1961.

Straus, S., Madorsky, S. L., Hart, V. E., Products volitalized froin a channel and a furnace black on heating to $1,000^{\circ} \mathrm{C}$. Rubber Age 84, 238 (1953). 803. Thermal degradation of cellulosic materials. J $\mathbf{6 0}$, 343 (1958) RP2853.

Straus, S., Stromberg, R. R., Achhammer, B. G., Infrared spectra of thermally degraded poly (vinyl chloride). J 60, 147 (1958) RP 2832.

Thermal decomposition of poly (vinyl chloride). J Polymer, Sci. 35, No. 129, 355 (1959). 3099.

Straus, S., Wall, L. A., Influence of impurities on the pyrolysis of polyamides. J 63A 3, 269 (1959). Pyrolysis of polyamides. J 60, 39 (1958) RP2819.

Straus, S., Wall, L. A., Florin, R. E., Mohler, F. L., Bradt, P., Phosphinoborine compounds: mass spectra and pyrolysis. J 63A 1, 63 (1959).

Straus, S., Wall, L. A., Madorsky, S. L., Brown D., Simha, $R$., Depolymerization of polymethylene and polyethylene. J. Am. Chem. Soc. 76, 3430 (1954). 985.

Strobridge, T. R., Chelton, D. B., Dean, J. W., Birmingham, B. W., Mann, D. B., Helium refrigeration and liquefaction using a liquid hydrogen refrigerator for precooling. (1960) TN39 (PB151398).

Stromberg, R. R., Bersch, C. F., Achhammer, B. G., Structural changes in irradiated plasties films. SPE Tech. Paper 5, 5 (1959). 3056.

Stromberg, R. R., Quasius, A. R., Toner, S. D., Parker, $M$. S., Adsorption of polyesters on glass, silica, and alumina. J 62, 71 (1959) RP2933.

Stromberg, R. R., Straus, S., Achhammer, B. G., Infrared spectra of thermally degraded poly(vinyl chloride). J 60, 147 (1958) RP2832.

Thermal decomposition of poly(vinyl chloride). J. Polymer Sci. 35, No. 129, 355 (1959). 3099.

Stromberg, R. R., Swerdlow, M., Pores in collagen and leather. J. Am. Leather Chemists Assoc. 50, No. 7, $336(1955), \quad 1449$.

Stroud, J. C., Janes, H. B., Decker, M. T., An analysis of propagation measurements made at 418 megacy cles per second well beyond the radio horizon (a digest), J 64D 3, 255 (1960).

An analysis of propagation measurements made at $418 \mathrm{Me}$ well beyond the radio horizon. (1959) TN6 (PB151365).

Sturrock, P. A., Note on the focusing of electron beams in certain magnetic fields. Proc. Phys. Soc. (London. England) 63, 954 (1950). 95.

Perturbation characteristic functions and their application to electron optics. Proc. Roy. Soc. (London, England) [A] 210, 269 (1951). 286.

Suddeth, J. A., Marton, L., High-vacuum temperature gradient furnace. Rev. Sci. Instr. 29, No. 4, 894 (1958). 2588.

Suddeth, J. A., Marton, L., Simpson, J. A., An electron interferometer. Rev. Sci. Instr. 25, No. 11, 1099 (1954). 947.

Electron beam interferometer. Phys. Rev. 90, 490 (1953). 706. 
Suddeth, J. A., Marton, L., Simpson, J. A., Wagner, M. D. Watanabe, $H$., Effect of degree in orientation and crystal size on the scattering of 20 -kev electrons by aluminum, Phys. Rev. 110, No. 5, 1057 (1958). 2529.

Sugar, G. R., Estimation of correlation coefficients from scatter diagrams. J. Appl. Phys. 25, 354 (1954). 1017.

Some fading characteristics of regular VHF ionospheric propagation. Proc. IRE 43, No. 10, 1432 (1955). 1495 .

Sugar, G. R., Montgomery, G. F., The utility of meteor burst for intermittent radio communication. Proc, IRE 45, No. 12, 1684 (1957). 2389.

Suguira, M., Chapman, S., Arc-lengths along the lines of force of a magnetic dipole. J. Geophys. Research 61, 485 (1956). 1633.

Sullivan, A. W., Crichlow, W. O., Foldes, G., Hewitt, F. J., Horner, $F$., Shinkawa, $H$., Measurement of atmospheric noise. Recommendation No. 1 and Annex, Proc. XIIth Gen. Assembly Intern. Sci. Radio Union, Boulder, Colo. August 22-September 5, 1957, XI, Pt. 4, 99, 1957. U.R.S.I. (Intern. Sci. Radio Union) Inform. Bull. No. 105, 10 (September-October 1957). 2190.

Sulzer, $P$. G., A note on a bridged-T network. Proc. IRE 39, 819 (1951). 181 .

A note on a selective R-C bridge. Proc. IRE 40, 338 (1952). 370.

A reliable locked-oscillator pulse timer. Tele-Tech 12, No. 4, 68 (1953). 634.

A survey of audio-frequency power amplifier circuits. Audio Eng. 35, No. 5, 15 (1959). 187.

A VHF match meter. Television Eng. 1, No. 7, 4 (1950). 15.

Coupling chart for solenoid coils. Television Eng. 1, No. 6, 20 (1950). 41.

Frequency and amplitude stability of the cathodecoupled oscillator. Proc. IRE 38, 540 (1950). 62. High-stability bridge-balancing oscillator. Proc. IRE 43, 701 (1955). 1358.

High-stability frequency standards. Tele-Tech 14, No. 6, 108 (1955). 1359.

Junction transactor cireuit applications. Electronics 26, No. 8, 170 (1953). 749.

Low-distortion transistor audio oscillator. Electronies 26, 171 (1953), 755 .

Modified locked-oscillator frequency dividers. Proc. IRE 39, 1535 (1951), 261.

Modified resonant circuits match impedances. TeleTech 9, No. 11, 41 (1950). 92 .

Precision transistor oscillator. Radio and TV News (Radio-Elec. Eng. Sect.) 49, No. 5, 18 (1953), 799. R-F solenoid design chart. Tele-Tech 10, No. 5, 45 (1951). 294.

Series-resonant high-voltage supply. Electronics $\mathbf{2 5}$, No. 9, 157 (1952). 539.

Stable electronic voltage regulator. Electronics 23, No. $12,162(1950)$. 135.

Sweep-frequency pulse-transmission measurements over a 2400-KM path. J. Geophys. Research 60, 483 (1955). 1520.

Transistor band spotter. Radio and Television News, (Radio-Electronic Eng. Sect.) 49, No. 6, 52 (1953). 899.

Transistor frequency standard. Electronics 36, No. 5, 206 (1953). 900.

Tuning systems employing feedback amplifiers.

Electronics 24, 252 (1951). 352.

Wide-band $\mathrm{FM}$ adapter reduces interference. RadioElectronies 21, 24 (1950). 169.

Wide-range R-C oscillator. Electronics 23, No. 9, $88(1950) .171$.

Sulzer, P. G., Ferguson, E. E., Sweep-frequency obliqueincidence ionospheric measurements over a $1,150 \mathrm{~km}$ path. Proc. IRE 40, 1124 (1952). 561.

Sulzer, $P$. G., Montgomery, G. F., Wide-range resonators for VHF and UHF, Electronics 42, No. 5, 200 (1951). 357.
Sulzer, P. G., Montgomery, G. F., Gerks, I. H., An UHF moon relay. Proc. IRE 40, 36 (1952). 396.

Sulzer, P. G., Smith, E. R., Edelman, S., High-speed stroboscope for accelerometer calibration. Rev. Sci. Instr. 25, No. 8, 837 (1954). 1031.

Sumida, D. Y., Hilsenrath, J., Klein, $M$., Mechanized computation of thermodynamic tables at the National Bureau of Standards: the calculation of the equilibrium composition and thermodynamic properties of dissociation and ionized gaseous systems. Proc. Symp. Thermodynamic and Transport Properties of Gases, Liquids, and Solids (Reprinted from Thermodynamic and Transport Properties of Gases, Liquids, and Solids, p. 416, Am. Soc. Mech. Engrs., 1959). 2988.

Swaney, A. C., Paffenbarger, G. C., Caul, H. J., Sweeney, $W . T$. American Dental Association specification No. 12 for denture base resin (second revision). J. Am. Dental Assoc. 46, 54 (1953). 644.

Swaney, A. C., Paffenbarger, G. C., Schoonover, I. C., Dickson, G., American Dental Association Specification No. 9 for dental silicate cement, First revision, effective July 1, 1950. J. Am. Dental Assoc. 40, 186 (1950). 20 .

Swanson, H. E., Cook, M. I., Isaacs, T., Evans, E. H., Standard X-ray diffraction powder patterns. 1960 C539, Vol. 9.

Swanson, H. E., Gilfrich, N. T., Cook, M. I., Standard X-ray diffraction powder patterns. (1957) C539, Vol. 7.

Swanson, H. E., Gilfrich, N. T., Cook, M. I., Stinchfield, R., Parks, P. C., Standard X-ray diffraction powder patterns. (1959) C539, Vol. 8.

Swarzenbach, G., Bates, R. G., Die bestimmung thermodynamischer aciditatskonstanten. Helv́. chim. acta (Basil, Switzerland) 37, 1069 (1954). 991.

Sweeney, $W . T$., Acrylic resins in prosthetic dentistry. Dental Clin. of North America 593 (Nov. 1958). 2449.

Characteristics of ultrasonic vibrations. J. Am. Dental Assoc. 55, 819 (1957). 2055.

Developments in instruments. Intern. Dental J. 8, No. 2, 238 (1958). 2519.

Resins in prosthetic dentistry. Chapter in the Dental Clin. of North America. p. 593 (W. B. Saunders Co., Philadelphia, P\&., 1958). 2738.

The program of training and graduate instruction in dental materials at the National Bureau of Standards. J. Dental Educ. 22, 217 (1958). 2827.

Effect of temperature and degree of polymerization on the sorption of water by polymethyl methacrylate. Modern Plastics 32, No. 7, 138 (1955). 1328.

Sweeney, W. T., Brauer, G. M., Schoonover, I. C., Crazing of acrylic resins. J. Am. Dental Research 34, 306 (1955). 1298.

Sweeney, $\dot{W}$. T., Caul, H. J., Gneug, W., Transverse strength testing machine for denture resins. J. Am. Dental Assoc. 49, 174 (1954). 1216.

Sweeney, W. T., Caul, H. J., Paffenbarger, G. C., Relationship between residual monomer and some properties of self-curing dental resins. J. Am. Dental Assoc. 53, 60 (1956). 1846.

Sweeney, W. T., Glasson, G. F., Schoonover, I. C., The effect of preheating on thermal expansion of silicagypsum investments. J. Am. Dental Assoc. 48, 433 (1954). 1184.

Sweeney, W. T., Hartley, J. L., Hudson, D. C., Dickson, G., Development of methods for evaluation of rotating dental dia mond abrasive instruments. J. Am. Dental Assoc. 54, 637 (1957). 2085.

Sweeney, W. T., Hartley, J. L., Hudson, D. C., Richardson, $W$. P., Cutting characteristics of dental burs as shown by high speed photomicrography. U.S. Armed Forces Med. J. 8, 209 (1957). 2076.

Sweeney, W. T., Hudson, D. C. Temperatures developed in rotating dental cutting instruments. J. Am. Dental Assoc. 48, 127 (1954). 1165.

Sweeney, $W$. T., Hudson, D. C., Hartley, J. L., Moore, $R$. B., Some factors influencing the cutting charac- 
teristics of rotating dental cutting instruments. J Am. Dental Assoc. 50, 377 (1955). 1494.

Sweeney, W. T., Mowery, W. E., Burns, C. L., Dichson, G., Dimensional stability of denture base resins. J. Am. Dental Assoc. 57, 345 (1958). 2523.

Sweeney, W. T., Myerson, R. L., Rose, E. E., Semmelman, J.O. Proposed specification for plastic teeth. J. Prosthetic Dentistry 7, 420 (1957), 2272.

Sweeney, W. T., Paffenbarger, G. C., American Dental Association specifications for dental materials. J. Am. Dental Assoc. 2d Ed. (1956). 1614.

Dental materials specifications and certification annual for 1954. Am. Dental Assoc. Publ. (1955) 1307.

Sweeney, W. T., Paffenbarger, G. C., Nelsen, R. J., Direct and indirect filling resins: a review of some physical and chemical properties. J. Am. Dental Assoc. 47, 516 (1953). 688.

Sweeney, W. T., Paffenbarger, G. C., Schoubee, P. J., Dental cements. Intern, Dental J. 5, 584 (1955), 1306.

Sweeney, W. T., Paffenbarger, G. C., Stanford, J.W. American Dental Association specifications for dental materials (third ed.) (1958), 2451.

Sweeney, W. T., Rupp, N. W., Dickson, G., Lawson, M. $E$, Jr., A method for measuring the mucosal surface contours of impressions, casts and dentures. J. Am. Dental Assoc. 54, 24 (1957), 1987.

Sweeney, W. T., Schoonover, I. C., Fischer, T. E., Serio, $A . F$., Bonding of plastic teeth to heat cured denture base resins. J. Am. Dental Assoc, 44, 285 (1952). 402 .

Sweeney, W. T., Schoubee, P. J., Paffenbarger, G. C., Resin cements and posterior-type direct-filling resins. J. Am. Dental Assoc. 52, 584 (1956). 1854.

Sweeney, W. T., Sheehan, W. D., Yost, E. L., Mechanical properties of direct filling resins. J. Am. Dental Assoc. 49, 513 (1954). 1069.

Sweeney, W. T., Smith, D. L., Caul, H.J., Some physical properties of gallium copper-tin alloys. J. Am. Dental Assoc. 53, 677 (1956). 1876.

Sweeney, W. T., Stanford, J. W., Paffenbarger, G. C., A revision of American Dental Association specification No. 3 for dental impression compound. J. Am. Dental Assoc. 51, 56 (1955). 1244.

Sweeney, $W . T$, Stanford, $J, W$. Paffenbarger, $G, C$, $K u m p u l a, J . W .$, Determination of some compressive properties of human enamel and dentin. J. Am. Dental Assoc. 57, 487 (1958). 2518.

Sweeney, W. T., Swaney, A. C., Paffenbarger, G. C., Caul, $H . J$. American Dental Association specification No. 12 for denture base resin (second revision). J. Am. Dental Assoc. 46, 54 (1953). 644.

Sweeney, $W . T$, and Taylor, D. F., A proposed specification for dental chromium-cobalt casting alloys. J. Am. Dental Assoc. 54, 44 (1957). 1996.

Sweeney, W. T., Yost, E. L. Fee, J. G., Physical properties of plastic teeth. J. Am. Dental Assoc. 56, 883 (1958), 2688

Suerdlow, $M$., Electron microscopy. Anal, Chem. 26, 178 (1954). 1011.

Swerdlow, M., Bramao, L., Cady, J. G., Hendricks, S., Criteria for the characterization of kaolinite halloysite, and a related mineral in clays and soils. Soil Sci. 73 , No. 4, 273 (1952). 420.

Swerdlow, M., Dalton, A. J.,Birks, L. S., Electron microscopy. Anal. Chem. 28, Pt. II, No, 4, 597 (1956). 1703.

Swerdlow, M., Kinter, E, B, Wintermyer, A. $M$, Electron microscopy of soil clays and related materials. Public Roads Highway Research J. 27, No. 5, 89 (1952). 439.

Swerdlow, $M$., Stromberg, $R . R$. Pores in collagen and leather. J. Am. Leather Chemists Assoc. 50, No. 7, 336 (1955), 1449.

Swift, J. D., Goldberg, K., Newman, M., Straus, E. G., The representation of integers by binary quadratic rational forms. Arch. math. (Tübinger, Germany) 5, $12(1954)$. 1198 .
Swindells, J. $F_{\text {., }}$ Calibration of liquid-in-glass thermometers. (1959) C600.

Swindells, J.F., McKee, S. A., White, H. S., Mountjoy, W. For wear prevention. SAE J. 58, 47 (1950). 59.

Swindells, J. F., Snyder, C. F., Hardy, R. C., Golden, $P$. E., Viscosities of sucrose solutions at various tempreatures: tables of recalculated values. (1958) C440 Suppl.

Swindells, J. F., White, H. S., Belcher, H. V., Oil-soaked felt-pad lubrication of ball bearings at high speed and high temperature. Lubrication Eng. 11, 182 (1955). 1422 .

Synge, $J, L .$, Elastic waves in anisotropic media. J. Math. and Phys. 35, 323 (1957). 2104.

Synge, J.L., Cahill, $W . F$, The torsion of a hollow square. Quart. Appl. Math. 15, No. 3, 217 (1957). 2385.

Szasz, O., Gibbs' phenomenon for Hausdorff means. Trans. Am. Math. Soc. 69, 440 (1950). 65.

Indentities and inequalities concerning orthogonal polynomials and Bessel functions. J. D'anal. math. (Jerusalem, Israel) 1, 116 (1951). 249.

Inequalities concerning ultraspherical polynomials and Bessel functions. Proc. Am. Math. Soc. 1, No. 2,256 (1950). 72 .

On a summation of O. Perron. Math. Z. (Berlin, Germany) 52, 631 (1950), 102.

On a Tauberian theorem for Abel summability. Pacific J. Math 1, 117 (1951). 275.

On some trigonometric transforms. Pacific J. Math. 1, No. 2, 291 (1951). 279.

On the Gibb's phenomenon for a class of linear transforms. Acad. Serve. Sci. Publ. Inst. Math. 4, 135 (1952). 500.

On the Gibbs' phenomenon for Euler means. Acta Scientiarum Mathematicarum (Szeged, Hungary) 12, Pt. B, 107 (1950). 103.

On the relative extrema of Bessel function. Bollettino della Unione Mat. Ital. (Bologna, Italy) (Firenze) III, 225 (Dec, 1950). 104.

On the relative extrema of the Hermite orthogonal functions. J. Indian Math. Soc. (Madras, India) 15, Pt. B, 129 (1951). 283.

On the relative extrema of ultraspherical polynomials. Bollettino della Unione Mat. Ital. (Bologna, Italy) Ser, 3, Anno 5, No, 2, 125 (1950). 106.

Summation of slowly convergent series. J. Math. and Phys, 28, No. 4, $272(1950) .145$.

Tauberian theorems for summability $\left(R_{1}\right)$. Am. J. Math. 73, No. 4, 779 (1951). 321.

Szasz, O., Beckenbach, E. F., Seidel, W., Recurrent determinants of Legendre and of ultraspherical polynomials. Duke Math. J. 18, 1 (1951). 298.

Szasz, O. Lukacs. E., Certain fourier transforms of distributions. Can. J. Math. 3, No. 2, 140 (1951). 202.

Certain Fourier transforms of distribution: II. Can. J. Math 6, 186 (1954). 965.

On analytic characteristic functions, Pacific J. Math. 2, No. 4, 615 (1952). 496.

Szasz, O. Seidel, $W$ On positive harmonic functions of ultraspherical polynomials. J. London Math. Soc. (London, England) 26, 36 (1951). 276.

Sze, $W . C$. Measurement of voltage ratio at audio frequencies. Commun. Electronic 32, 444 (1957). 2191.

Szwarc, M., Jaffe, I., Prosen, E. J., Heats of combustion of some peroxides and the heats of formation of acetate, propionate, and butyrate radicals. J. Chem. Phys. 27, 416 (1957). 2148.

\section{T}

Taback, L., Analog-digital conversion equipment for electrocardiographic data. (1960) TN42 (PB151401). A function generator for two independent variables. Control Eng. 3, No. 11, 129 (1956). 1590.

Taff, J. M., Kirby, R. S., Moore, H. S., Measurement of the effect of irregular terrain on VHF and UHF directive antenna patterns. IRE Trans. Ant. Prop. AP-3, 167 (1952). 481. 
Taimuty, S. I., Hart, E. J., Koch, H. W., Petree, B., Schulman, J. H., Wyckoff, H. O., Measurement systems for high-level dosimetry, 2d United Nations Intern. Conf. on the Peaceful Uses of Atomic Energy, Vol. 21, Health and Safety: Dosimetry and Standards, P/1927 USA, p. 188 (Pergamon Press, London, England) (1959). 2984.

Tait, A., Bateman, R., Florman, E. F., A source of error in radio phase measuring systems. Proc. IRE 38, 612 (1950). 10.

Tait, A., Bateman, R., Florman, E. F., Rechtin, E., A source of error in radio phase measuring systems. (Discussion), Proc. IRE 39, 436 (1951). 186.

Tandberg-Hanssen, E., Curtis, W., Watson, $K$., The emission of HeI, $\lambda$ 10830, during the great flare of August 26, 1958. Astrophys. J. 129, No. 1, 238 (1959). 3077.

Tandberg-Hanssen, E., Roach, F. E., Excitation mechanisms of the oxygen 5577 emission in the upper atmosphere. J 63D3, 319 (1959).

Tandberg-Hanssen, E., Roach, F. E., Megill, L. R., Movements of airglow cells. J. Atmospheric and Terrest. Phys. 13, 122 (1958). 2640.

Possible movements of 5577 airglow. J. Atmospheric and Terrest. Phys. 13, 122 (1958), 2695.

The characteristic size of airglow cells. J. Atmospheric and Terrest. Phys. 13, 113 (1958). 2807.

Tate, D. R., Bouche, R. R., Autographic stress-strain recorders. ASTM Bull. No. 228, 33 (1958). 2465.

Taussky, O., A note on group matrices. Proc. Am. Math. Soc. 6, 984 (1955). 1237.

Classes of matrices and quadratic fields. Pacific J. Math. 1, 127 (1951). 205.

Characteristic roots of quaternion matrices. Archiv der math. (Wurzburg, Germany) 5, 99 (1954). 967. Classes of matrices and quadratic fields (II). J. London Math. Soc. (London, England) 27, Pt. 2, No. 106, 237 (1952). 412.

Commutativity in finite matrices. Am. Math. Mo. 64, 229 (1957). 2060.

Generalized commutators of matrices and permutations of factors in a product of three matrices. Studies in Math. and Mech., p. 67, (Academic Press Inc., New York, N.Y.) (1954). 1023.

1. Notes on numerical analysis, 2. Note on the condition of matrices. Math. Tables and Other Aids to Computation 4, No. 30, 111 (1950). 98.

On matrix classes corresponding to an ideal and its inverse. Illinois J. Math. 1, 108 (1957). 2222.

Some computational problems in algebraic number theory. Proc. 6th Symp. Appl. Math. of Am. Math. Soc. (Santa Monica, Calif., 1953) p. 187 (1956). 1873. Unimodular integral circulants. Math. z. (Berlin, Germany) 63, 286 (1955). 1567.

Taussky, O., Fan, K., Todd, J., An algebraic proof of the isoperimetric inequality for polygons. J. Wash. Acad. Sei. 45, No. 11, 334 (1955). 1259.

Discrete analogs of inequalities of Wirtinger. Monatsh mathematic (Vienna, Austria) Bd. $59 / 2$ (1955). 1313.

Taussky, O., Hoffman, A. J., Newman, M., Straus, E. G., The number of absolute points of a correlation. Pacific J. Math. 6, 83 (1956). 1943.

Taussky, O., Kato, T., Commutators of A and A*, J. Wash. Acad. Sei. 46, 38 (1956). 1653.

Taussky, O., Motzkin, T. S., On representations of finite groups. Koninkl, Nederl. Akademie Van Wetenschappen Proc. (Amsterdam, Holland), Series A, 55, No. 5; Indag. math. (Amsterdam, Holland) 14, No. 5, 511 (1952). 499.

Pairs of matrices with property L. Trans. Am. Math. Soc. 73, No. 1, 108 (1952). 507: Proc. Natl. Acad. Sei. 39, 961 (1953). 791.

Pairs of matrices with property L, II. Trans. Am. Math Soc. 80, No. 2, 387 (1955). 1440.

Taussky, $O$., Newman, $M$., Classes of positive definite unimodular circulants. Can. J. Math. 9, 71 (1957). 2057.

On a generalization of the normal basis in abelian algebraic number fields, Commun, on Pure and Appl. Math. (N. Y. Univ. New York, N.Y.) 9, 85 (1956). 1791 .

Taussky. O., Ostrowski, A. M., On the variation of the determinant of a positive definite matrix. Koninkl. Nederl. Akademie Van Wetenschappen (Amsterdam, Holland). Proc. Series. A, 54, No. 5; Indag. Math. 13, No. 5 (1951). 284.

Taussky, O., Pall, G., The number of representations of a quadratic form as a sum of four squares. Proc. roy. Irish acad. Sect. A., No. 3, 23 (1957). 2375.

Taussky, O., Straus, E. G., Algebraic equations satisfied by roots of natural numbers. Pacific J. Math. 6, 97 (1956). 1610 .

Taussky, $\dot{O}_{\text {., }}$ Todd. J., Commuting bilinear transformations and matrices. J. Wash. Acad. Sci. 46, No. 12, 373 (1956). 1654.

Generation and testing of pseudo-random numbers. Proc. Symp. Monte Carlo Methods (Gainesville, Fla., $1954)$, p. 15 (1956). 1722.

Systems of equations, matrices and determinants. Mathematics Mag. 26, No. 1, Sept. p. 9 and 26, No. 2, Dec. p. 71 (1952). 562 .

Taylor, C. F., Livengood, J.C., Tsai, D. H., Dynamies in the inlet system of a four-stroke single-cylinder engine. Trans. Am. Soc. Mech. Engrs. 77, No. 7, 1133 (1955). 1322.

Taylor, D. F., Leibfritz, W. A., Adler, A. G., Physical properties of chromium cobalt alloys. J. Am. Dental Assoc. 56, 343 (1958). 2687.

Taylor, D. F., Sweeney, W.T., A proposed specification for dental chromium-cobalt casting alloys. J. Am. Dental Assoc. 54, 44 (1957). 1996.

Taylor, $J$. K., The Washington area scientists-forteachers program. J. Chem. Educ. 33, 461 (1956). 1956.

Taylor, J. K., Couch, D. E., Shapiro, H., Brenner, A., Protection of molybdenum from oxidation at elevated temperatures. J. Electrochem. Soc. 105, No. 8, 450 (1958). 2705.

Taylor, J. K., Smith, S. W., A coulometric-tritration coulometer. J 63C1, 65 (1959).

Polarographic determination of trace quantities of iodide. Anal. Chem. 29, 301 (1957). 2258.

Precise coulometric titrations. Science 124, No. 3228,940 (1956). 1815.

Precise coulometric titration of acids and bases. J 63A2, 153 (1959).

Taylor, L. S., Basis for standards for radiation protection. Proc. Meeting Society for Nondestructive Testing, J. Wash. Acad. Sci. 46, No. 3, 69 (1956). 1639. Brief history of the National Committee on radiation protection and measurements. Health Phys. J. 1, 3 (1958). 2478.

Can we legislate ourselves into radiation safety? Nucleonics 13, No. 3, 17 (1955). 1280.

Current situation with regard to permissible radiation exposure levels. Radioloyg 69, No. 1,6 (1957). 2075. Editorial on Roentgen ray protection design. Am. J. Roentgenol. Radium Therapy Nuclear Med. 68, 972 (1952). 433

Education in radiation protection. Janeway Lecture, 1954, Am. J. Roentgenol. Radium Therapy Nuclear Med. 73, 193 (1955). 1324.

Effect of new lower permissible exposure levels on use of radioactive isotopes. Proc. United Nations Educational, Scientific and Cultural Organization Conf. Paris, France (Sept. 1957); Impact 8, 209 (1957). 2099 .

Exposure standards and radiation protection regulations. Sections 3, p. 2, Radiation Hygiene Handbook, edited by Hansen Blatz (MeGraw-Hill Book Co., Inc. New York, N.Y.) (1959). 2947.

History of the International Commission on Radiological Protection. Health Phys. J. 1, 2 (1958). 2589 .

Influence of lower permissible levels on atomic operations in the United States. Proc. 2d Intern. Conf. on 
the Peaceful Uses of Atomic Energy, United Nations (Geneva, Switzerland) 23, 211 (1958). 2598.

Legislative control of radiation. Radiology 66, No. 2,246 (1956). 1755 .

Maximum permissible radiation exposures to man. Radiation Research 6, No. 4, 513 (1957); Radiology 68, No. 2, 260 (1957). 2187.

National Bureau of Standards Semi-Centennial 19011951. Am. J. Roentgenol. Radium Therapy Nuclear Med. 67, 112 (1952). 486.

Permissible exposure to ionizing radiation. Proc, of Intern. Conf. on Peaceful Uses of Atomic Energy (Geneva, Switzerland) (1955). 1443.

Practical suggestions for reducing radiation exposure in diagnostic examinations. Am. J. Roentgenol. Radium Therapy Nuclear Med. 78, No. 6, 983 (1957). 2259 .

Radiation exposure and the use of radioisotopes. Impact of Sci, on Soc. (Paris, France) 8, No. 4 (1957). 2277 .

Radiation exposure as a reasonable calculated risk. Proc. UNESCO Conf. (Paris, France, Sept. 1957); Impact 8, 209 (1957); Health Phys. J. 1, 62 (1958). 2709.

Radiation protection for the general practitioner. Southern Med. J. of the Southern Medical Assoc. 49, 8 (1956). 1831.

Radiation regulations. Hospitals 30, No. 13, 23 (1956). 1832.

Science in radiology. Radiology 64, 429 (1955). 1483. State control of protection against ionizing radiation. Am. J. Roentgenol., Radium Therapy Nuclear Med. 71, 691 (1954); Acta radiol. (Stockholm, Sweden) Suppl. 116, 21 (1954). 1151.

Teaching pay in the Soviet Union. Letter to Editor Science Teacher 22, No. 6, 264 (1955). 1524.

The philosophy underlying radiation protection. Am. J. Roentgenol. 77, No. 5, 914 (1957). 2376. The International Commission of Radiological Units and Measurements. Intern. Assoc. (Pergamon Press, London, England) 9, 594 (1957). 2366.

Taylor, L. S., Gray, L. H., Wyckoff, H. O., Program of the International Commission on Radiological Units and Measurements. 2d United Nations Intern. Conf. on the Peaceful Uses of Atomic Energy, Vol. 21, Health and Safety: Dosimetry and Standards, P/2243 WHO, p. 81 (Pergamon Press, London, England) (1959), 3022.

Taylor, L. S., Marinelli, L., The measurement of ionizing radiations for biological purposes. Biological Effects of Radiation (McGraw-Hill Book Co., Inc., New York, N.Y.) (1954). 1189.

Taylor, W. C., Bogue, R. H., A sulfate susceptibility test for portland cements. Portland Cement Assoc. Fellowship 58, 223 (1950). 11.

Taylor, W. L., Spectrum analysis of sferics. Symp. on the Prop., of V. L. F. Radio Waves, Sponsored by the NBS Boulder Laboratories and IRE Prof. Group on Ant. Prop., January 23-25, 1957 (Boulder, Colo.), Paper No. 33, V. 3 (1957), 2312.

Taylor, W. L., Helliwell, R. A., Jean, A. G., Some properties of lightning impulses which produce whistlers. Proc. IRE Letter 46, 1760 (1958). 2764.

Taylor, W. L., Jean, A. G., Very-low-frequency radiation spectra of lightning discharges. J 63D2, 199 (1959).

Taylor, W. L., Lange, L. J., Some characteristics of VLF progagation using atmospheric waveforms. In Recent Advances in Atmospheric Electricity; Proc. 2d Conf. on Atmospheric Electricity, Portsmouth, New Hampshire, May 20-23, 1958 (Pergamon Press, New York, N.Y.), p. 609 (1958). 2760.

Tayman, W. P., Washer, F. E., Darling, W. R., Evaluation of lens distortion and photographic method, J 61, 509 (1958) RP2920.
Tchen, C. M., Approximate theory on the stability of interfacial waves between two streams. J. Appl. Phys. 27, No. 12, 1533 (1956). 1632.

Diffusion of particles in turbulent flow. Advances in Geophys. 6: "Atmospheric Diffusion and Air Pollution". p. 165, Proc. Symp. Oxford, England, August 1958 (Academic Press Inc., New York, N.Y.) (1959). 2929.

Heat delivery in a compressible flow and applications to hot-wire anemometry. Natl. Advisory Comm. Aeronaut, Tech. Note 2436 (1951), 247.

Interfacial waves in viscoelastic media. J. Appl. Phys. 27, No. 5, 431 (1956). 1745.

Motion of small particles in skew shape suspended in a viscous liquid. J. Appl. Phys. 25, 463 (1954). 1077. Random flight with multiple partial correlations. J. Chem. Phys. 20, No. 2, 214 (1952). 525.

Stability of oscillations of superposed fluids. J. Appl. Phys. 27, No. 7, 760 (1956). 1890.

Transport processes as functions of the Heisenberg and Obukoff theories of turbulence. Phys. Rev. 93, 4 (1954). 1215.

Tchen, C. M., Schubauer, G. B., Turbulent motion. Section B, Vol. V, Princeton Ser., High Speed Aerodynamics and Jet Propulsion, "Turbulent Flows and Heat Transfer", p. 75 (Princeton University Press, Princeton, N.J.) (1959). 3104.

Teele, R. P., Measuring circuit for radiometers. J. Opt. Soc. Am. 44, No. 11, 860 (1954). 1068.

Tefft, W. E., Spinner, S., Reichard, T. W., A comparison of experimental and theoretical relations between Young's modulus and the flexural and longitudinal resonance frequencies of uniform bars. J 64A2, 147 (1960).

Tefft, W. E., Wachtman, J. B., Jr., Effect of suspension position on apparent values of internal friction determined by Forster's method. Rev. Sci. Instr. 29, No. 6, 517 (1958). 2530.

Tefft, W. E., Wachtman, J. B., Jr., Lam, D. B., Jr., Stinchfield, $R$. P., Elastic constants of synthetic single crystal corundum at room temperature. J 64A3, 213 (1960).

Teichroew, D., Use of continued fractions in high-speed computing. Math Tables and Other Aids to Computation 6, No. 39, 127 (1952). 612.

Temmer, G. M., Ambler, E., Hudson, R. P., Alignment of cerium $^{141}$ and neodynium ${ }^{147}$. Phys. Rev. 97, 1212 (1955). 1256.

Alignment of three odd-A rare-earth nuclei. Phys. Rev. 101, No. 1, 196 (1956). 1611.

Alignment of three rare-earth isotopes. Bull. Intern. Inst. Refrig. Commun. 142, 255 (1955). 1256.

Temmer, G. M., Bloembergen, N., Nuclear magnetic resonance of aligned radioactive nuclei. Phys. Rev. 89, 883 (1953). 778.

Temmer, G. M., Heydenburg, N. P., Energy levels in F18 from alpha-particle reactions in nitrogen. Phys. Rev. 92, 89 (1953). 711.

Temmer, G. M., Wyckoff, J. M., Alpha-gamma angular correlation in ionium $\left(\mathrm{TH}^{230}\right)$. Phys. Rev. 92, 913 (1953). 643.

Temperley, $H . N . V$., Treatment of liquid $\mathrm{He}^{3}$ based on the "cell" model, Phys. Rev. 97, 835 (1955). 1562.

Tenebaum, M., Glasgow, A. R., Jr., Gryoscopic determination of purity of highly reactive substances. Anal. Chem. 28, 1907 (1956). 1666.

Thayer, G. D., Bean, B. R., Central Radio Propagation Laboratory exponential reference atmosphere. J 63D3, 315 (1959).

CRPL exponential reference atmosphere. (1959). Mono. 4.

On models of the atmospheric radio refractive index. Proc. IRE 47, No. 5, 740 (1959). 3002.

Thayer, G. D., Cahoon, B. A., Bean, B. R., Tables for the statistical prediction of radio ray bending and elevation angle error using surface values of the refractive index. (1960) TN44 (PB151403). 
Theron, J. J., An automatic controller for radiofrequency induction-heating unit. Rev. Sci. Instr. 24, 281 (1953). 647.

Thomas, H. A., Microwave power measurements employing electron beam techniques. Proc. IRE 45, No, 2 , 205 (1957). 2201.

Nuclear-resonance magnetic field control. Electronics 25, No. 1, 114 (1952). 491.

Thomas, J. A., Smith, E. K., A survey of the present knowledge of sporadic-E ionization. J. Atmospheric and Terrest. Phys, 13, 295 (1959). 2885.

Thomas, J. L., Driscoll, R. L., Cutkosky, R. D., Status of absolute measurements at the National Bureau of Standards. Procès-verbaux, C.I.P.M., p. 36 (1958). 2781 .

Thomas, R. N., Athay, R. G., On the use of the early Balmer lines to extend the photospheric model. Astrophys. J. 127, No. 1, 96 (1958). 2675.

Thomas, R. N., Burgers, J. M., Preface to Proc. 3d Symp. on Cosmical Gas Dynamics. Smithsonian Astrophysical Observatory, June 1957 (Cambridge, Massachusetts), Rev. Modern Phys. 30, 908 (1958). 2697.

Thomas, $R$. N., Jeffries, J.T., Source function in a nonequilibrium atmosphere. II. Depth dependence of source function for resonance and strong subordinate lines. Astrophys. J. 127, No. 3, 667 (1958), 2767.

Thomas, R. N., Pottasch, S. R., Departures from the Saha equation under varying conditions of Lyman continuous opacity. Astrophys. J. 130, No. 3, 941 (1959). 2926.

Thompson, G. L., Motzkin, T. S., Raiffa, H., Thrall, R. M., The double description method. Contributions to the theory of games II, Edited by H. W. Kuhn and A. W. Tucker, Annals of Math. Study 28, Chapter 3 (Princeton, N.J.) (1953). 857.

Thompson, J. G., Corrosion observing $3 \mathrm{~d}$ year anniversary of "Corrosion Technology". Corrosion Tech. 3, No. 4,105 (1956). 1664.

Corrosion research at the National Bureau of Standards. Corrosion Tech. (London, England) 2, 102 (1955), 1297.

Nickel and its alloys. (1958) C592

Thompson, J. P., Early history and development of building codes, BOCA News (Publ, by the Building Officials Conference of America, Inc.) 6, No, 1, 2 (1957). 2095.

How the National Bureau of Standards contributes to building codes. BOCA News (Publ. by the Building Officials Conference of America, Inc.) 4, No. 12, 1 (1956). 1733 .

New standards wind load requirements. Eng. News-Record 158, No, 6, 33 (1957), 2213.

Thompson, M. C., Jr., Crystal-stabilized pulse-pair generator. Rev. Sci. Instr. 26, 617 (1955), 1302

Thompson, M. C., Jr., Cateora, J. V., High-order harmonics for X-band oscillator stabilization. Rev. Sci. Instr. 28, 656 1957). 2153.

Thompson, M. C., Jr., Cook, R. K., Waterhouse, R. V., Berendt, R. D., Edelman, S., Measurement of correlation coefficients in reverberant sound fields. J. Acoust. Soc. Am. 27, No. 6, 1072 (1955). 1399.

Thompson, M. C., Jr., Freethey, F. E., Waters, D. M., Fabrication techniques for ceramic X-band cavity resonators. Rev. Sci. Instr. 29, No, 10, 865 (1958). 2561 .

Thompson, M. C., Jr., Greenspan, M., A logarithmic slidewire for a self-balancing potentiometer. Rev. Sci. Instr. 22, 799 (1951). 179.

An eleven megacycle interferometer for low pressure gases. J. Acoust. Soc. Am. 25, 92 (1953). 648.

Thompson, M. C., Jr., Herbstreit, J. W., Continuous phase difference measurements of earth satellites. Proc. IRE 46, No. 8, 1535 (1958). 2499.

Measurement of the phase of radio waves received over transmission paths with electrical lengths varying as a result of atmospheric turbulence. USRI
Symp, on Electromagnetic Wave Theory, June 1955 (Ann Arbor, Mich.); Proc. IRE 43, 1391 (1955). 1402 .

Measurements of the phase of signals received over transmission paths with electrical lengths as a result of atmospheric turbulence. IRE Trans. Ant. Prop. AP-4, 352 (1956). 1770.

Thompson, M. C., Jr., Hubbard, R. W., Phase generator for tropospheric research. Electronics 29, No. 10, $220(1956)$. 1805 .

Thompson, M. C.,Jr., Janes, H.B., Measurements of phase stability over a low-level tropospheric path. J 63D1, 45 (1959).

Thompson, M. C., Jr., Tschiegg, C. E., Greenspan, M., Subharmonic crystal oscillators, Rev. Sci. Instr. 25, 8 (1954). 1154.

Thompson, $M . \dot{C}_{\text {, }} J_{r .}$ Vetter, $M . J .$, A compact microwave refractometer for use in small aircraft. Rev. Sci. Instr. 29, No, 12, 1093 (1958). 2415.

Single path phase measuring system for three-centimeter radio waves. Rev. Sci. Instr. 29, 148 (1958). 2758 .

Thompson, M. C. Jr., Waters, D. M., Comparison of phase difference and Doppler shift measurements for studying ionospheric fine structure using earth satellites. Proc. IRE Letter 46, 1960 (1958). 2493. Simple technique for diplexing $10,000 \mathrm{Mc}$ and video signals on coaxial cables. Rev. Sci. Instr. 28, 206 (1957). 2300.

Thompson, R., Marcus, $M$., A note on symmetric functions of eigenvalues. Duke Math. J. 24, 43 (1957). 1990.

Thoraeus, R., Wyckoff, H.O., Calibration of the portable Swedish free-air chamber equipment at the U.S. National Bureau of Standards. Acta radiol. $\mathbf{4 6}$, 741 (1956). 1644.

Thorensen, $R$., Design features of a magnetic drum memory for the National Bureau of Standards Western Automatic Computer (SWAC). Proc. Symp. on Electronic Computers held by the Western Section of the Elec. Computers Prof, Group of the IRE, April 30, May 1-2, 1952 (Univ, of Calif. Los Angeles Calif.) II, 1-II:9 (1952). 424.

Thorensen, R., Huskey, H. D., Ambrosio, B. F., Yowell, $E, C$. The SWAC-design features and operating experience. Proc. IRE 41, 1294 (1953). 885.

Thornton, H. R., Moore, D. G., Effect of oxygen on the bonding of gold fused silica. J 62, 127 (1959) RP2942

Thrall, R. M., Motzkin, T. S., Raiffa, H., Thompson, G. L., The double description method. Contributions to the theory of games II, Edited by H. W. Kuhn and A. W. Tucker, Annals of Math. Study 28, Chapter 3 (Princeton, N.J.) (1953). 857.

Thrush, B. A., Kinetic spectroscopy in the far ultraviolet: the flash photolvsis of ethyl compounds. Proc. Roy Soc. 243, 555 (1)58). 2607.

Thrush, B. A., Edgecombe, S. H. C., Norrish, R. G. W., The flash photolysis of chlorine monoxide. Proc. Roy. Soc. (London, England) 243, 24 (1957). 2357.

Thwaite, R. D., Newkirk, T. $F$., Pseudoternary system calcium oxide-monocalcium aluminate $\left(\mathrm{CaO} \cdot \mathrm{Al}_{2} \mathrm{O}_{3}\right)$ dicalcium ferrite $\left(2 \mathrm{CaO} \cdot \mathrm{Fe}_{2} \mathrm{O}_{3}\right)$. J 61, 233 (1958) RP2900.

Tidstrom, K. D., Klebanoff, P. S., Schubauer, G. B., Measurement of the effect of two-dimensional and threedimensional roughness elements on boundary-layer transition. J. Aeronaut. Sci. 22, 11 (1955). 1400.

Tidwell, E. D., Allen, H. C., Jr, Plyler, E. K., Infrared spectra of hydrogen cyanide and deuterium cyanide. J. Chem. Phys. 25, No. 2, 302 (1956). 1739.

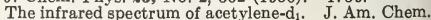
Soc. 78, 3034 (1956). 1934.

Vibrational energy of deuterium cyanide. J. Chem. Phys. 23, No. 7, 1356 (1955). 1575.

Tidwell, E. D. Benedict, W. S., Plyler, E. K., Vibrationrotation bands of ammonia: 1. The combination bands $\nu_{2}-\left(\nu_{1}, \nu_{3}\right)$. J 61, 123 (1958) RP2894. 
Tidwell, E. D., Plyler, E. K., Infrared emission spectra of flames under high resolution. J 61, 263 (1958) RP2903.

The precise measurement of the infrared spectra of molecules of the atmosphere. Mémoires royal sei. soc. Liége, quatrième ser., Inst. Astrophys. (Liége, Belgium) 28, 426 (1957). 2377.

Tidwell, E. D., Plyler, E. K., Allen, H. C., Emission spectrum of carbon monoxide from 2.3 to 2.5 microns. J 61, 53 (1958) RP2883.

Near infrared spectrum of nitrous oxide. J. Chem. Phys. 24, No. 1, 95 (1956). 1782.

Tidwell, E. D., Plyler, E. K., Benedict, W. S., Vibrationrotation bands of ammonia, III. The region 3.2-4.3 microns. J. Chem. Phys, 29, 829 (1958). 2861.

Tidwell, E. D., Plyler, E. K., Blaine, L. R., Infrared absorption spectrum of methane from 2470 to 3200 $\mathrm{cm}^{-1}$. J 64A3, 201 (1960).

Tidwell, E. D., Plyler, E. K., Danti, A., Blaine, L. R. Vibration-rotation structure in absorption bands for the calibration of spectrometers from 2 to 16 microns. J 64A 1, 29 (1960); (1960) Mono. 16

Tighe, N. J., Cuthill, J. R., Richmond, J. C., The effect of a ceramic coating on the creep behavior of some high temperature alloys. Bull. Am. Ceram. Soc. 39, 4 (1959). 3072 .

Tilton, $L . W$., Density formula for alkali silicate glasses from annealing to glass-processing temperatures. J 61, 463 (1958) RP2915.

Nonerystal ionic model for silica glass. J 59, 139 (1957) RP2782.

Role of vitrons in alkali silicate binary glasses. J 60 , 351 (1958) RP2854.

Structural rearrangements in silicate glasses during annealing. J. soc. glass technol. (British) 40, T338 (1956). 1901.

Tilton, L. W., Plyler, E. K., Stephens, R. E., Refractive index of silver chloride for visible and infrared radiant energy. J. Opt. Soc. Am. 40, 540 (1950). 124.

Timmerhaus, K. D., Flynn, T. M., Weitzel, D. H., Distillation of hydrogen isotopes. Colorado Eng. 55 , 12 (1958). 2526.

Pilot plant studies of the low temperature distillation of hydrogen isotopes. Proc. 1958 Cryogenic Eng. Conf., Paper $\mathrm{H}-1$, p. 464 , Edited by K. D. Timmerhaus, Chemical Engineer Dept., Univ. of Colorado (Boulder, Colo.) (1958); Chem. Eng. Progr. 54, No. 6, 35 (1958). 2691.

Timmerhaus, K. D., Flynn, T. M., Weitzel, D. H., Draper $J$. W., Separation of hydrogen isotopes by multicomponent distillation. Proc. 1957 Cryogenic Eng. Conf., Paper A-6, p. 58, Edited by K. D. Timmerhaus, Chemical Engineering Dept., Univ, of Colorado (Boulder, Colo.) (1958). 2751.

Timmerhaus, K. D., Flynn, T. M., Weitzel, D. H., Vander Arend, P. C., Draper, J. W., Distillation of hydrogendeuterium mixtures. Paper A-6, p. 39, Edited by K. D. Timmerhaus, Chem. Eng. Dept. Univ. of Colorado (Boulder, Colo.) (1957). 2093.

Timmerhaus, K. D., Keeler, R. N., Poisoning and reactivation of orthoparahydrogen conversion catalyst. Proc. 1958 Cryogenic Eng. Conf., Paper E-3, p. 296. Edited by K. D. Timmerhaus, Chem. Eng. Dept., Univ. of Colorado (Boulder, Colo.) (1959). 3017.

Timmerhaus, K. D., Weitzel, D. H., Draper, J. W., Park, O. E., Van Valin, C. C., Catalysis of the orthoparahydrogen conversion. Proc. 1956 Cryogenic Eng. Conf., Paper A-4, p. 12, Edited by $K$. D Timmerhaus, Chem. Eng. Dept. Univ. of Colorado (Boulder, Colo.) (1957). 2048.

Timmerhaus, K. D., Weitzel, D. H., Flynn, T. M., Low temperature distillation of hydrogen isotopes. Chem. Eng. Progr. 54, No. 6, 35 (1958). 2618.

Tipson, R. S., Isbell, H. S., Conformations of the pyranoid sugars. I. Classification of conformers, J 64A2, 171 (1960).

Conformations of the pyranoid sugars. II. Infrared absorption spectra of some aldopyranosides. 64A3, 239 (1960).
Tipson, R. S., Isbell, H. S., Stewart, J. E., Infrared absorption spectra of some cyclic acetals of sugars. J 62, 257 (1959) RP296̧.

Titus, W. F., Measurement of the gamma-ray dose near the interface between two media. Nuclear Sci. and Eng. 3, No. 5, 609 (1958). 2628.

Total photelectric cross sections of copper, molybdenum, silver, tantalum, and gold at $662 \mathrm{kev}$. Phys. Rev. 115, No. 2, $351(1959)$. 3101.

Titus, W. F., Hubbell, J. H., Hayward, E. V., Energy and angular distribution of X-rays scattered in lead. Phys. Rev. 108 No. 6, 1361 (1957). 2111.

Todd, $J_{\text {., }}$ A direct approach to the problem of stability in the numerical solution of partial differential equations. Comm. Pure Appl. Math. 9, No. 3, 597 (19.56). 1588.

Begrundung fur die Beschaftigung mit numerisher analysis, Yahres. Bericht de DMV (Tübingen, Germany) 58, No, 1, 11 (1955), (Translation of "Motivation for working in numerical analysis"). 1275. Experiments in the solution of differential equations by Monte Carlo methods. J. Wash. Acad. Sci. 44, No. 12,377 (1954). 1019.

Motivation for working in numerical analysis. Commun. on Pure and Appl. Math. 8, No. 1, 97 (1955). 1410.

Notes on modern numerical analysis. I. Solution of diferential equations by recurrence relations. Math. Tables and Other Aids to Computation 4, No. 29, 39 (1950). 97.

Numerical analysis. Review of Proc. Sym. Applied Mathematics Vol. VI, Science 124, No. 3233, 1211 (1956). 1788.

Obituary notice on L. F, Richardson. Math. Tables and Other Aids to Computation 8, 242 (1954). 1088. On the relative extrema of the Laguerre orthogonal functions. Bollettino della unione mat. Ital. (Bologna, Italy) Ser. 3, Anno 5, No. 2, 122 (1950). 105. The condition of certain matrices, III. J 60, 1 (1958) RP2815.

The condition of a certain matrix. Cambridge Phil. Sic. 46, Pt. 1, $116(1950) .149$.

The condition of certain matrices, II. Arch. math. (Tübinger, Germany) V, 249 (1954). 1175.

Todd, J., Fan, K., A determinantal inequality. J. London Math. S’oc. 30, 58 (1955). 1232.

Todd, J., Fan, K., Taussky, $O$., An algebraic proof of the isoperimetric inequality for polygens. J. Wash. Acad. Sci. 45, No. 11, 334 (1955). 1259.

Discrete analogs of inequalities of Wirtinger. Monatsh. math. (Vienna, Austria) Bd. 59/2 (1955). 1313.

Todd, J., Newman, M., The evaluation of matrix inversion programs.' J. Soc. Indust. Appl. Math. 6, 466 (1958). 2814.

Todd, J., Taussky, O., Commuting bilinear transformations and matrices. J. Wash. Acad. Sci. 46, 373 (1956). 1654.

Generation and testing of pseudo-random numbers. Proc. Symp. Monte Carlo Methods (Gainesville, Fla., 1954), p. 15 (1956). 1722.

Systems of equations, matrices and determinants. Math. Mag. 26, No. 1, Sept. p. 9 and 26, No. 2, Dec. p. $71(1952) .562$.

Toman, $K$., Focusing, defocusing, and refraction in a circularly stratified atmosphere. J 64D3, 287 (1960).

Tomes, L. A., Hunt, C. M., Blaine, R. L., Some effects of aging on the surface area of portland cement paste. J 64A2, 163 (1960).

Some factors affecting the surface area of hydrated portland cement as determined by water-vapor and nitrogen adsorption. J 59, 357 (1957) RP2806.

Tomes, L. A., Hunt, C. M., Dantzler, V., Blaine, R. L., Reaction of portland cement with carbon dioxide. J 60, 441 (1958) RP2858.

Tompkins, C. B., Machine attacks on problems whose variables are permutations. Proc. Am. Math. Soc. 6th Symp. Appl. Math. of Am. Math. Soc. (Santa Monica, Calif. 1953) (1956). 1762. 
Toner, S. D., An evaluation of various thermoplastic materials for use as iodine vapor barriers. SPE J. 15, 48 (1959). 2892 .

Determination of resin content of glass fiber polyester laminates containing a calcium carbonate filler. Anal. Chem. 28, 1109 (1956). 1672.

Toner, S. D., Newman, S. B., Achhammer, B. G., Surface erosion of filled plasties. Modern Plastics 36, 135 (1958). 2792

Toner, S. D., Stromberg, R. R., Duasius, A. R., Parker, $M$. S., Adsorption of polyesters on glass, silica, and alumina. J 62, 71 (1959) RP2933.

Toner, S. D., Wolock, I., Reinhart, $F$. W., Effects on molding pressure on strength properties of glass-fiber reinforced plastics. SPE J. 14, 40 (1958). 2533.

Torgesen, J. L., Douglas, T. B., Ball, A. F., Heat capacity of crystalline dextrose between $25^{\circ}$ and $95^{\circ} \mathrm{C}$. J. Am. Chem. Soc. 73, 1360 (1951). 246.

Tosini, P. C., Magnetic drum directory and programming system for codesorting letter mail. (1960) TN50 (PB151409).

Touloukian, Y. S., Hilsenrath, $J$., The viscosity, thermal conductivity, and Prandtl number for air, $\mathrm{O}_{2}, \mathrm{~N}_{2}, \mathrm{NO}$, $\mathrm{H}_{2}, \mathrm{CO}, \mathrm{CO}_{2}, \mathrm{H}_{2} \mathrm{O}, \mathrm{He}$ and $\mathrm{A}$. Trans. Am. Soc. Mech. Engrs, 76, No. 6, 967 (1954), 1207.

Tousey, R., Wilson, N. L., Purcell, J. D., Johnson, F. S., Moore, $C . E$. A revised analysis of the solar spectrum from 2990 to 2635 A. Astrophys. J. 119, No. 3, 590 (1954). 933.

Towne, K. M., Schiefer, H. F., Smith, J. C., McCrackin, $F$. L., Stone, $W$. $K$., Stress-strain relationship in yarns tested at rates of straining up to a million percent per minute. Physics of Wool and Other Viscoelastic Fibers, D, 148 (1956) (Proc. Intern. Wool Textiles Research Conf., August 20-25, 1955, Sydney, Australia) (1956). 1900 .

Townes, C. H., Atomic clock and frequency stabilization on microwave spectral lines. J. Appl. Phys. 22, 1365 (1951). 196

Trees, $R$. E., Comparison of first, second, and third approximations in Bacher and Goudsmit's theory of atomic spectra. J. Opt. Soc. Am. 48, 293 (1958). 2492 .

Hyperfine structure formulas for LS coupling. Phys. Rev. 92, 308 (1953). 733.

Low even configurations in the first spectrum of ruthenium (Ru I), part 2. J 63A3, 255 (1959).

Polarization energy for $3 \mathrm{~d}$ and $4 \mathrm{p}$ electrons. Phys. Rev. 97, 686 (1955), 1448.

Trees, R. E., Coleman, C. D., Tables for diagonalizing second-order matrices. J 60, 201 (1958) RP2838.

Trees, $R$. E., Kessler, $K$. G., The nuclear moments of technetium-99. Phys. Rev, 92, 303 (1953). 875.

Tregoning, L. J., Frush, H. L., Isotope effect in oxidation of D-mannitol-2-C14 by acetobacter suboxydans. Science 128, No. 3324, 597 (1958), 2606.

Tryon, $M$., Analytical procedures: natural and synthetic rubbers. Anal. Chem. 29, 714 (1957). 2020.

Tryon, M., Achhammer, B. G., Kline, G. M., Chemical structure and stability relationships in polymers. Kunststoffe combined with German Plastics Digest 49, No. 11, 600 (1959); Modern Plastics 37, No. 4, 131 (1959). 2910.

Tryon, M., Bekkedahl, N., Natural and synthetic rubbers. Biennial review of physical and chemical test methods. Anal. Chem. 27, 589 (1955). 1412A.

Tryon, M., Linnig, F. J., Natural and synthetic rubbers: review of test methods. Anal. Chem. Appl. Revs. 31, Pt. II, No. 4, 767 (1959). 2997.

Tryon, M., Mandelkern, L., Quinn, F. A., Jr., The melting behavior of polybutadienes. J. Polymer Sci. 19, 77 (1956). 1938 .

Tryon, M., Wall, L. A., Oxidative degradation of a series of deuterated styrene polymers. J. Phys. Chem. 62, No. 6, 697 (1958). 2680.

Thermal and photochemical processes in polystyrene in the glassy state. Nature 178, 101 (1956). 1958.
Tryon, M., Wall, L. A., Harvey, M. R., Oxidative degradation of styrene and $\alpha$-deuterostyrene polymers. J. Phys. Chem. 60, 1306 (1956). 1802.

Trzeciak, M., Friedman, A. S., Johnston, H. L., Pressurevolume temperature relations of liquid normal deuterium. J. Am. Chem. Soc. 76, 1552 (1954). 1115.

$T$ sai, D. H., Discussion of paper "Pressure-flow characteristies of pneumatic valves", by F. D. Ezekiel and J. L. Shearer. ASME Trans. 79, 1589 (1957) 2092. Discussion of the paper "Wave action in diffusers for exhaust-pipe systems, with special reference to the scavenging of two-stroke engines", by F. J. Wallace and G. Boxer. Proc. Inst. Mech. Engrs, 170, 1152 (1956). 1679.

Effect of size on the inlet-system dynamics in fourstroke, single-cylinder engines. Trans. Am. Soc. Mech. Engrs. 78, No. 1, 197 (1956). 1687.

Tsai, D. H., Hogue, E. W. Reproducibility of a pen-andchart type of recording instrument under dynamic conditions. ASME Paper 57A-209 (1957). 2286.

Tsai, D. H., Slawsky, M. M., Determination and correlation of flow capacities of pneumatic components. (1957) C588.

Tsai, D. H., Taylor, C. F., Livengood, J.C., Dynamies in the inlet system of a four-stroke single-cylinder engine. Trans. Am. Soc. Mech. Engrs. 77, No. 7, 1133 (1955). 1322.

Tschiegg, C. E., Greenspan, $M$., An accurate time-modulated pulse eircuit. Rev. Sci. Instr. 24, 183 (1953). 646.

Effect of dissolved air on the speed of sound in water. J. Acoust. Soc. Am. 28, 501 (1956). 1686.

Sing-around ultrasonic velocimeter for liquids. Rev. Sci. Instr. 28, No. 11, 897 (1957). 2301.

Speed of sound in water by a direct method. J 59, 249 (1957) RP2795.

Tables of the speed of sound in water. J. Acoust. Soc. Am. 31, No. 1. 75 (1959). 3061.

Tschiegg, C. E., Greenspan, M., Breckenridge, F., Temperature coefficient of the speed of sound in water near the turning point. J. Acoust. Soc. Am. 28, 500 (1956). 1914

Tschiegg, C. E., Thompson, M. C., Jr., Greenspan, M., Subharmonic crystal oscillators. Rev. Sci. Instr. 25, 8 (1954). 1154.

Tucker, N. J., Bean, L., A note on the oxyquinolate determination of magnesium oxide in cement. ASTM Bull. No. 201, (TP224), 62 (1954). 925.

Turner, P. S., Reinhart, F. W., Good, C. L., Wolock, I., Comparison of mechanical properties of flat sheets, molded shapes and postformed shapes of cotton fabric-phenolic laminates. Natl. Advisory Comm. Aeronaut. Tech. Note 3825 (1957). 2063.

Turner, $W$. J., Photoemission from silver into sodium chloride, and thallium bromide. Phys. Rev. 101, No. 6, 1653 (1956). 1807.

Turner, W. J., Burstein, E., Davisson, J. W., Bell, E. E., Lipson, H. G., Infrared photoconductivity due to neutral impurities in germanium. Phys. Rev. 93, 65 (1954). 1039.

Tveten, L. H., Bateman, R., Finney, J. W., Smith, E. K., Watts, $\dot{J}$. $M$., IGY observations of ' $F$-layer scatter in the Far East. J. Geophys. Research 64, 403 (1959). 2963.

\section{$\mathrm{U}$}

Udelson, $B . J$. Effect of microwave signals incident upon different regions of a de hydrogen glow. J. Appl. Phys. Letter to Editor 28, No. 3, 380 (1957). 2098 .

Udelson, B. J., Creedon, J. E., French, J. C., Microwave measurements of the properties of a de hydrogen discharge. J. Appl. Phys, 28, No. 6, 717 (1957). 2200.

Underhill, A. B., Waddell, J. H., Stark broadening functions for the hydrogen lines. (1959) C603.

Urban, G. H., Kirsch, R. A., Cahn, L., Ray, L. C., Experiments in processing pictorial information with 
a digital computer. 1957 Eastern Joint Computer Conf. Proc. Washington, D.C. (1957). 2119.

Utlaut, $W . F .$, Factors affecting radio propagation in the TV and FM bands. Tele-Tech 14, No. 6, 98 (1955). 1342

\section{$\mathbf{v}$}

Vacher, H. C., Bechtoldt, C. J., Maxwell, E., Structure of some iridium-osmium alloys. J. Metals 6, No. 1, 80 (1954); Trans. Am. Inst. Mining Engrs. 200, 80 (1954). 1152 .

Vacher, H. C., Liss, R., Mebs, R. W., Elastic and plastic behavior of the ferrite lattice in a low-alloy steel. Acta met. 4, No. 5, 532 (1956). 1691.

Vacher, H. C., Mitchell, J. A., Schoonover, I. C., Dickson, $G$., Some factors affecting the dimensional stability of the Ag-Sn (Cu-Zn) amalgams. J. Dental Research 34, No. 2, 273 (1955). 1493.

Vacher, H. C., Newton, C.J., An X-ray study of textural stresses in two-phase alloys. J 59, 239 (1957) RP2793.

Residual lattice strains in sectioned bars of plastically deformed iron. J. Metals 7, No. 11, 1193 (1955). 1479 .

Valentine, F. A., Motzkin, T. S., Straus, E. G., The number of farthest points. Pacific J. Math. 3, 221 (1953). 876.

Valore, R. C., Jr., Cellular concretes, Part 1. Composition and methods of preparation. J. Am. Concrete Inst. 25, 773 (1954); Part 2. Physical properties. J. Am. Concrete Inst. 25, 817 (1954). 964.

Insulating concretes. J. Am. Concrete Inst. 28, 5 (1956). 1742 .

Volume changes in small concrete cylinders during freezing and thawing. Title No. 46-26, J. Am. Concrete Inst. 21, 417 (1950). 167.

Valore, $R$. C., Jr., Green, W. C., Air replaces sand in "no-fines" concrete. J. Am. Concrete Inst. 22, No. 10, 833 (1951). 190.

Valore, R. C., Jr., Spinner, S., Comparison of theoretical and empirical relations between the shear modulus and torsional resonance frenuencies for bars of rectangular cross section. J 60, 459 (1958) RP2861.

Van Bronkhorst, A., Simpson, J. A., Modification of electron microscope for electron optical shadow method. Rev. Sci. Instr. 21, 669 (1950). 91.

van Dantzia, $D$., Chaines de Markoff dans les ensembles abstraits et applications aux processus avec regions absorbantes et au probleme des boucles (formerly, Time-discrete stochastic processes in arbitrary sets, with applications to processes with absorbing regions and to the problems of loops in Markoff chains) Ann. Inst. H. Poincare (Paris, France) 14, 145 (1955). 1286.

van Dijk, H., Durieux, M., Clement, J. R., Logan, J. K. The "1958 $\mathrm{He}^{4}$ scale of temperatures", Part 2. Tables for the 1958 temperature scale. J 64A1, 1 (1960); (1960) Mono. 10.

Van Gundy, D. A., Jacobs, R. B., Characteristics of some insulations for liquid oxygen transfer lines. Proc. 1956 Cryogenic Eng. Conf., Paper E-1, p. 156, Edited by K. D. Timmerhaus, Chem. Eng. Dept., Univ. of Colorado (Boulder, Colo.) (1957). 2054.

Van, Gundy, D. A., Kropschot, R. H., McClintock, R. M. Mechanical properties of some engineering materials. Proc. 1956 Cryogenic Eng. Conf., Paper C-2, p. 93, Edited by K. D. Timmerhaus, Chem. Eng. Dept., Univ, of Colorado (Boulder, Colo.) (1957). 2193.

Van Holde, K. E., Wales, $M$., The concentration dependence of the sedimentation constants of flexible macromolecules. J. Polymer Sci. 14, 81 (1954). 1174.

Van Lieshout, R., Hayward, R. W., Pessoa, E. F., Hoppes, D. D., Gamma radiation from $\mathrm{Zn}^{63}$. Il Nuovo Cimento 11, 53 (1959). 2956.

Van Valin, C. C., Weitzel, D. H., Draper, J. W., Design data for ortho-parahydrogen converters. Proc. 1957 Cryogenic Eng. Conf., Paper B-2, p. 73, Edited by
K. D. Timmerhaus, Chem. Eng. Dept., Univ. of Colorado (Boulder, Colo.) (1958), 2514.

Van Valin, C. C., Weitzel, D. H., Draper, J. W., Park, $O$. E., Timmerhaus, K. D., Catalysis of the orthoparahydrogen conversion. Proc. 1956 Cryogenic Eng. Conf., Paper A-4, p. 12, Edited by K. D. Timmerhaus, Chem. Eng. Dept. Univ. of Colorado (Boulder, Colo.) (1957). 2048.

Van Valkenburg, A., Bunting, E. N., Some properties of diamond. Am. Mineralogist 43, 102 (1958), 2762.

Van Valkenburg, A., Lippincott, E. R., Weir, C. E., Bunting, E. N., Infrared studies on polymorphs of silicon dioxide and germanium dioxide. $J$ 61, 61 (1958) RP2885.

Van Valkenburg, A., Rynders, G.F., Synthetic cuspidine. Am. Mineralogist 43, No. 11 and 12, 1195 (1958). 2795.

Van Valkenburg, A., Weir, C. E., Studies of beryllium chromite and other beryllia compounds with $\mathrm{R}_{2} \mathrm{O}_{3}$ oxides. J 64A 1, 103 (1960).

Van Valkenburg, A., Weir, C. E., Lippincott, E. R., Bunting, $E$. N., Infrared studies in the 1- to 15micron region to 30,000 atmospheres. J $\mathbf{6 3 A} 1,55$ (1959)

Vander Arend, P. C., Flynn, T. M., Weitzel, D. H., Timmerhaus, $K$. D., Draper, $J$. $W$., Distillation of hydrogen-deuterium mixtures. Proc. 1956 Cryogenic Eng. Conf., Paper A-6, p. 39, Edited by K. D. Timmerhaus, Chem. Eng. Dept., Univ. of Colorado (Boulder, Colo.) (1957). 2093.

Vander Arend, P. C., Mann, D. B., Birmingham, B. W. Technical aspects of large scale liquid helium liquefaction and transportation. Proc. 1957 Cryogenic Eng. Conf., Paper D-4, p. 191, Edited by K. D. Timmerhaus, Chem. Eng. Dept., Univ. of Colorado (Boulder, Colo.) (1958). 2797.

Velasco, R., Klinkenberg, P. F. A., Meggers, W. F., Catalan. $M$. A. Term analysis of the first spectrum of rhenium (Re I). J 59, 319 (1957) RP2804.

Vetter, $M$. J., Thompson, $M$. C., Jr., A compact microwave refractometer for use in small aircraft. Rev. Sci. Instr. 29, No. 12, 1093 (1958). 2415.

Single path phase measuring system for three-centimeter radio waves. Rev. Sci. Instr. 29, 148 (1958) 2758 .

Vickers, R. A., Kanagy, J. R., Factors affecting the water vapor permeability of leather. J. Am. Leather Chemists Assoc. 45, 211 (1950). 57.

Victor, A. C., Douglas, T. B., Heat content of zirconium and of five compositions of zirconium hydride from $0^{\circ}$ to $900^{\circ} \mathrm{C}$ J 61, 13 (1958) RP2878.

Vinti, $J$. $P$. New method of solution for unretarded satellite orbits. J 63B 2, 105 (1959).

Theory of the effect of drag on the orbital inclination of an earth satellite. J 62, 79 (1959) RP2934.

Vinti, J. P., Dressler, R. F., The functional synthesis of linear plots. J 64C2, 115 (1960).

Vogler, L. E., Norton, K. A., Mansfield, W. V., Short, P. J., The probability distribution of the amplitude of constant vector plus a Rayleigh-distributed vector. Proc. IRE 43, 1354 (1955). 1541.

Vogler, L. E., Norton, K. A., Rice, P. L., The use of angular distance in estimating transmission loss and fading range for propagation through a turbulent atmosphere over irregular terrain. Proc. IRE 43, 1488 (1955). 1550

\section{W}

Wachtman, J. B., Jr., Creep of crystalline nonmetals. Am. Soc. Metals Symp., Oct. 6, 1956, Creep and Recovery p. 344 (1957). 2072

Wachtman, J. B., Jr., Lam, D. G., Jr., Young's modulus of various refractory materials as a function of temperature. J. Am. Ceram. Soc. 42, 254 (1959). 3114.

Wachtman, J. B., Jr., Maxwell, L. H., A bend-test method of determining the stress required to cause creep in tension. ASTM Bull. No. 211, 38 (1956). 1584A. 
Plastic deformation of ceramic-oxide single crystals. J. Am. Ceram. Soc. 37, 291 (1954). 1108.

Plastic deformation of ceramic-oxide single crystals, II. J. Am. Ceram. Soc. 40, No. 11, 377 (1957). 2253.

Wachtman, J. B., Jr., Tefft, W. E., Effect of suspension position on apparent values of internal friction determined by Forster's method. Rev. Sci. Instr. 29, No. 6,517 (1958). 2530.

Wachtman, J. B., Jr., Tefft, W. E., Lam, D. G., Jr., Stinchfield, $R$. P., Elastic constants of synthetic single crystal corundum at room temperature. $J$ 64A3, 213 (1960).

Wacker, P. F., Birnbaum, G., Maryott, A. A., Microwave ahsorption by the nonpolar gas $\mathrm{CO}^{2}$. J. Chem. Phys. 22, 1782 (1954). 1074 .

Wacks, M. E., Dibeler, V. H., Electron impact studies of aromatic hydrocarbons. I. Benzene, napthalene, anthracene, and penanthrene. J. Chem. Phys. 31, No. 6, 1557 (1959). 2938.

Waddell, J. H., Underhill, A. B., Stark broadening functions for the hydrogen lines. (1959) C603.

Wade, C., Jr., Abramowitz, M., Cahill, W. F., Heat transfer in laminar flow through a tube. J $\mathbf{6 2}, 101$ (1959) RP2937.

Wagner, M. D., Marton, L., Simpson, J. A., Suddeth, J. A. Watanabe, $H$. . Effect of degree in orientation and crystal size on the scattering of 20 -kev electrons by aluminum. Phys. Rev, 110, No, 5, 1057 (1958). 2529.

Wait, J.R., A low-frequency annular-slot antenna. J 60, 59 (1958) RP2822.

A note on the propagation of the transient ground wave. Symp. on the Prop. of V.L.F. Radio Waves, Sponsored by the NBS Boulder Laboratories and IRE Prof. Group on Ant. and Prop., January 23-25, 1957 (Boulder, Colo.), Appendix D, V. 4, 67; Can. J. Phys. 35, 1146 (1957). 1992

A phenomenological theory of induced electrical polarization. Can. J. Phys. 36, 1634 (1958). 2431. A study of earth currents near a VLF monopole antenna with a radial wire ground system. Proc. IRE 46, No. 8, 1 (1958). 2437.

A study of VLF field strength data: both old and new. Geofisica pura e applicata (Milano, Italy) 41, 73 (1958). 2438.

A survey and bibliography of recent research in the propagation of VLF radio waves. (1960) TN58 (PB161559).

An extension to the mode theory of VLF ionospheric propagation. J. Geophys. Research 63, No. 1, 125 (1958), 2456.

Comments on the attenuation versus frequency characteristics. Symp. on the Prop. of V.L.F. Radio Waves, Sponsored by the NBS Boulder Laboratories and IRE Prof. Group on Ant. and Prop., January 23-25, 1957 (Boulder, Colo.), Appendix A, V. 4, 43; Proc. IRE 45, 768 (1957). 2059.

Currents excited on a conducting surface of large radius of curvature. IRE Trans. Microwave Theory and Tech. MTT-4, 143 (1956). 1668.

Diffraction of a spherical wave pulse by a half plane screen. Can. J. Phys. 35, No. 5, 693 (1957). 2086.

Diurnal change of ionospheric heights deduced from phase velocity. Proc. IRE 47, No. 5, 98 (1959). 2931.

Downcoming radio waves, measurement of characteristics. Elec. and Radio Engr. 36, No. 3, 106 (1959) 2932.

Earth currents near a monopole antenna with symmetrical top loading. J 62, 247 (1959) RP2959.

Effect of the ground screen on the field radiated from a monopole. IRE Trans. Ant. Prop. AP-4, 179 (1956). 1688.

Excitation of surface waves on conducting, stratified dielectric-clad and corrugated surfaces. $J \mathbf{5 9}, 365$ (1957) RP2807.

Excitation of surface waves on plane and curved impedance boundaries. Congrès intern. sur la propagation des ondes radio-electriques, October 6-11, 1958 (Liége, Belgium). Resumé des commun., p. 26 (1958), 2553.

Insulated loop antenna immersed in a conducting medium. J 59, 133 (1957) RP2781.

Introduction to the VLF papers. Proc. IRE 45, 739 (1957). 2165.

Low frequency radiation from a horizontal antenns over a spherical earth. Can. J. Phys. 34, 586 (1956). 1761 .

Note on L.F. portable antennas operating over ice and snow covered terrain. Symp. on the Prop. of V.L.F. Radio Waves, Sponsored by the NBS Boulder Laboratories and IRE Prof. Group on Ant. and Prop. January 23-25, 1957 (Boulder, Colo.), Appendix C, V. $4,62.2219$.

On the calculations of transverse current loss in buried wire ground systems. Appl. Sci. Research 7, Sec. B, 81 (1958). 2665.

On the conductance of slots. IRE Trans. Ant. Prop. A P-4, 124 (1956). 1796.

On the electromagnetic response of an imperfectly conducting thin dyke. Geophysics XXIV, No. 1, 167 (1959). 3006.

On the measurement of the conductivity of a fluid contained in a cylindrical vessel. Can. J. Technol. 34,410 (1957), 2233

On the mode theory of VLF ionospheric propagation. Geofis. pura e appl. (Milan, Italy) 37, 103 (1957). 2234.

On the theory of propagation of electromagnetic waves along a curved surface. Can. J. Phys. 36, 9 (1958). 2673 .

On the theory of reflection from a wire grid parallel to an interface between homogeneous media. Appl. Sci. Research Section B, 6, 259 (1957). 2238.

On the theory of reflection from a wire grid parallel to an interface between homogeneous media (II). Appl. Sci. Research Section B, 7, No. 5, 355 (1959). 3010.

On the waveform of a radio atmosphere at short ranges. Proc. IRE 44, 1052 (1956). 1800.

Pattern of a flush-mounted microwave antenna. J 59, 255 (1957) RP2796.

Propagation of a pulse across a coast line. Proc. IRE 45, 1550 (1957). 2265.

Propagation of very-low-frequency pulses to great distances. J 61, 187 (1958) RP2898.

Radiation from a small loop immersed in a semiinfinite conducting medium. Can. J. Phys. 37, 672 (1959). 3028.

Radiation resistance of dipoles in an interface between two dielectrics. Can. J. Phys. 34, 24 (1956). 1833.

Radio wave propagation in an inhomogeneous atmosphere. (1959) TN24 (PB151383).

Recent theoretical studies of the propagation of long waves (abstract). Congrès Intern. sur la Propagation des Ondes Radio-Electriques, October 6-11, 1958 (Liége, Belgium). Resumé des commun,, p. 27 (1958). 2721.

Shielding of a transient electromagnetic dipole field by a conductive sheet. Can. J. Phys. 34, 890 (1956). 1866 .

Some solutions for electromagnetic problems involving spheroidal, spherical, and cylindrical bodies. 64B 1, 15 (1960).

Terrestrial propagation of very-low-frequency radio waves. A theoretical investigation. J 64D2, 153 (1960).

The attenuation versus frequency characteristics of VLF radio waves. Proc. IRE 45, 768 (1957). 2345. The calculation of the field in a homogeneous conductor with a wavy interface. Proc. IRE 47, No. 6, 1155 (1959). 3067.

The effective electrical constants of soil at low frequencies. Proc. IRE 45, 1411 (1957). 2352.

The impedance of a wire grid parallel to a dielectric 
interface, IRE Trans, Microwave Theory and Tech. MTT-5, 99 (1957), 2364.

The mode theory of V.L.F. ionospheric propagation for finite ground conductivity. Symp. on the Prop. of V.L.F. Radio Waves, Sponsored by the NBS Boulder Laboratories and IRE Prof. Group on Ant. and Prop., January 23-25, 1957 (Boulder, Colo.), Paper No. 6, V. 1; Proc. IRE 45, 760 (1957), 2373. The radiation pattern of an antenna mounted on a surface of large radius of curvature. Proc. IRE 44, 694 (1956). 1946.

The transient behavior of the electromagnetic ground wave on a spherical earth. IRE Trans. Ant. Prop. AP-5, 2, 198 (1957). 2386.

Transient fields of a vertical dipole over a homogeneous curved ground. Can. J. Phys. 34, 27 (1956). 1967.

Transmission and reflection of electromagnetic waves in the presence of stratified media. J 61, 205 (1958) RP2899.

Transmission loss curves for propagation at very low radio frequencies. IRE Trans. Commun. Systems CS-6, No, 2, 58 (1958), 2851.

Tramsmission of power in radio propagation. Elec. and Radio Engr. 36, No. 4, 146 (1959). 3102.

Wait, J. R., Carter, N. F., Field strength calculations for E.L.F. radio waves.' (1960) TN52 (PB161553).

Wait, J. R., Conda, A. M., Diffraction of electromagnetic waves by smooth olustacles for grazing angles. J 63D2, 181 (1959).

On the diffraction of electromagnetic pulses by curved conducting surfaces. Can. J. Phys, 37, 1384 (1959). 3005 .

On the measurement of ground conductivity at VLF. IRE Tran. Ant. Prop. AP-6, No. 3, 273 (1958). 2670.

Pattern of an antenna on a curved lossy surface. IRE Trans. Ant. Prop. AP-6, No. 4, 348 (1958). 2682 .

Radiation from slots on dielectric clad and corrugated cylinders. J 59, 307 (1957) RP2802.

The patterns of a slot-array antenna on a finite and imperfect ground plane. L'onde electrique 38, No. 376 bis (Proc. Intern. Congress Ultra High Frequency Circuits and Antennas, October 21-26, 1957, Paris, France), p. 7 (1958). 2826.

Wait, J. R., Cottony, H. V., Elliott, R. S., Jordan, E. C., Rumsey, V. H., Siegel, K. M., Woodyard, O. C., Antennas and waveguides, and annotated bibliography, U.S.A. National Committee report, Intern. Sci. Radio Union Subcomm. 6.3. IRE Trans. Ant. Prop. AP-7, 87 (1959). 2897.

U.S.A. National Committee report URSI Subcommission 6.3, antennas and waveguides, and annotated bibliography. Proc. 12th Gen. Assembly, International Scientific Radio Union, AugustSeptember 1957 (Boulder, Colo.), 11, Pt. 6, 121 (1957). 2402.

Wait, J. R., Frood, D. G., An investigation of slot radiators in rectangular metal plates. Proc. IRE (London, England) 103, Pt. B, No. 7, 103 (1956). 1622.

The radiation pattern and conductances of slots cut on rectangular metal plates. Proc. IRE 44, 1469 (1956). 1947.

Wait, J. R., Householder, J., Mixed-path ground-wave propagation: 2. Larger distances. J 59, 19 (1957) RP2770.

Pattern synthesis for slotted-cylinder antennas. J 63D3, 303 (1959).

Wait, J. R., Howe, H. H., Mode calculations for V.L.F. ionospheric propagation. Symp. on the Prop. of V.L.F. Radio Waves, Sponsored by the NBS Boulder Laboratories and IRE Prof. Group on Ant. and Prop., January 23-25, 1957 (Boulder, Colorado), Paper No. 36, V. on 3; Further comments, V. 4, 91. 2203. The waveguide mode theory of VLF ionospheric propagation. Proc. IRE 45, 95 (1957), 2390.
Wait, J. R., Jean, A. G., Lange, L. J., Ionospheric reflection coefficients at VLF. Symp, on the Prop. of V.L.F. Radio Waves, Sponsored by the NBS Boulder Laboratories and IRE Prof. Group on Ant. and Prop., January 23-25, 1957 (Boulder, Colorado), Paper No. 31, V. 3 (1957). 2172.

Polarization of sferics. Symp, on the Prop. of V.L.F. Radio Waves, Sponsored by the NBS Boulder Laboratories and IRE Prof. Group on Ant. and Prop., January 23-25, 1957 (Boulder, Colo.), Abstracts, Paper No. 32, V. 3. 2257.

Wait, J. R., Kates, J., Radiation patterns of circumferential spots on moderately large conducting cylinders. Inst. Elec. Engrs. Mono. No. 167R, 1 (1956). 1830.

Wait, J.R., Murphy, A., Further studies of the influence of a ridge on the low-frequency ground wave. J 61, 57 (1958) RP2884.

Multiple reflections between the earth and the ionosphere in VLF propagation. Geofisica pura e applicata 35, 61 (1956). 1781

The geometrical optics of VLF sky wave propagation. Symp. on the Prop. of V.L.F, Radio Waves, Sponsored by the NBS Boulder Laboratories and IRE Prof. Group on Ant. and Prop., January 23-25, 1957 (Boulder, Colo.), Paper No, 5, V. 1; Proc. IRE 45, 754 (1957). 2360.

Wait, J. R., O'Grady, M., Surface currents excited by an infinite slot on half-planes and ribbons. IRE Trans. Ant. Prop. AP-4, No. 1, 47 (1956). 1907.

Wait, J. R., Perry, L. B., Calculations of ionoshperic reflection coefficients at very low radio frequencies. J. Geophys. Research 62, No. 1, 43 (1957). 2044.

Wald, A., Dvoretzky, A., Wolfowitz, J., Elimination of randomization in certain statistical decision procedures and zero-sum two-person games, Ann. Math. Stat. 22, 1 (1951). 231.

Relations among certain ranges of vector measures. Pacific J. Math. 1, 59 (1951). 299.

Waldo, G. V., Hill, J. S., Staras, H., VHF tropospheric recording measurements of plane and circular polarized waves in the Great Lakes area. Abstract published in IRE Trans. Ant. Prop. AP-1, 42 (1952); entire paper published as FCC Memo. 62838. 620.

Wales, M., Marshall, P. A., Rothman, S., Weissberg, S. G., Application of viscosity methods to the study of branching in polysaccharides. Ann. N.Y. Acad. Sci. 57, 353 (1953). 654 .

Wales, M., Marshall, P. A., Weissberg, S. G., Intrinsic viscosity-molecular weight relationships for dextran. J. Polymer Sci. 10, 229 (1953), 745.

Wales, M., Van Holde, K. E., The concentration dependence of the sedimentation constants of flexible macromolecules. J. Polymer Sci. 14, 81 (1954). 1174.

Walker, H. H., Surface resistivity of cotton, wool, and nylon fabrics at different temperatures and relative humidities. Textile Research J. 28, No. 12, 1047 (1958), 2793.

Walker, $R . F_{\text {., }}$ The mechanisms of material transport during sintering. J. Am. Ceram. Soc. 38, No. 6, 187 (1955). 1538.

Two resistance-type hot-pressing furnaces for laboratory use. Rev. Sci. Instr. 28, No. 7, 563 (1957). 2399.

Walker, R. F., Schneider, S. J., Roth, R. S., Some evidence for structural anomalies in pure cristobalite. J. Am. Ceram. Soc. 42, No. 12, 642 (1959). 3048.

Walker, R. F., Zerfoss, S., Holley, S. F., Gross, L. J., Temperature of the inversion in cristobalite. J 61, 251 (1958) RP2902.

Wall, L. A., Atomic radiation and polymers. J. Soc. Plastics Engr. 12, No. 3, 17 (1956). 1634.

Factors influencing the behavior of polymers exposed to high-energy radiation. J. Polymer Sci. 17, 141 (1955). 1343 .

Thermal decomposition of polymers. Chem. Corps Rept. of Symp. VII, Incendiary gels and their instrumentation, p. 103 (June 1955). 1556. 
Wall, L. A., Achhammer, B. G., Reiney, M. J., Reinhart, F. W., Study of degradation of polystrene by means of mass spectrometry. J. Polymer Sci. 8, No. 5, 555 (1952), 557.

Wall, L. A., Brown, D. W., Gamma irradiation of polymethyl methacrylate and polystrene. J. Phys. Chem. 61, 129 (1957). 2138.

Pyrolysis of poly-alpha-methylstyrene. J. Phys. Chem. 62, 848 (1958). 2707.

Use of deuterium to investigate extent of branching in polystyrene. J. Polymer Sci, 14, No. 78, 513 (1954). 1220.

Wall, L. A., Brown, D. W., Florin, R. E., Electron spin resonance spectra from gamma-irradiated solid nitrogen. J. Chem. Phys. 30, No. 2, 602 (1959). 2940.

Wall, L. A., Brown, D. W., Hart, V. E., Pyrolysis of $\alpha$ and $\beta$ deuterostyrene polymers. J. Polymer Sci. 15. No. 79,157 (1955). 1464.

Wall, L. A., Caul, H. J., Acquista, N., Determination of the monomer content of polymethyl methacrylate. J. Am. Dental Assoc. 53, 56 (1956). 1673.

Wall, $L$. A., Florin, R. E., Effect of structure on the thermal decomposition of polymers. J 60, 451 (1958) RP2860.

Wall, L. A., Florin, R. E., Mohler, F. L., Duinn, E., Dimethylphosphinoborine trimer: mass spectra and thermal decomposition. J. Am. Chem. Soc. 76, 3344 (1954). 994.

Wall, L. A., Florin, R. E., Pummer, W. J., Fluorination of haloraomatic compounds, J 62, 107 (1959) RP2938.

Reactions of aromatic fluorocarbons with hydrogen. J 62, 119 (1959) RP2940.

Synthesis of some disubstituted 3,4,5,6-tetrafluorobenzenes. J 62, 113 (1959) RP2939.

Wall, L. A., Harvey, $M$. R., Tryon, $M$., Oxidative degradation of styrene and $\alpha$-deuterostyrene polymers. J. Phys. Chem. 60, 1306 (1956). 1802.

Wall, L. A., Hellmann, M., Peters, E., Pummer, W. J., Hexafluorobenzene from the pyrolysis of tribromofluoromethane. J. Am. Chem. Soc. 79, 5654 (1957). 2151.

Wall, L. A., Madorsky, S. L., Brown, D. W., Straus, S., Simha, R., Depolymerization of polymethylene and polyethylene. J. Am. Chem. Soc. 76, 3430 (1954). 985 .

Wall, L. A., Magat, M., Degradation des polymeres par les rayons et les neutrons. J. chim. phys. 50, 308 (1953). 681 .

Effects of atomic radiation on polymers. Modern Plastics 30, 111 (1953). 698.

Wall, L. A., Moore, W. J., Pyrolysis of mixtures of ethane and ethane-d $d_{6}$. J. Am. Chem. Soc. 73, 2840 (1951). 291.

Wall, L. A., Pummer, $W$. J., Reactions of hexafluorobenzene. Science 127, 643 (1958). 2718.

Reactions of pentafluorohalobenzenes. J 63A2, 167 (1959)

Wall, $L, A$., Simha, $R$., Kinetics of chain depolymerization. J. Phys. Chem. 56, No. 6, 707 (1952). 474. Mechanism of high energy radiation effects in polymers. J. Chem. Phys, 61, 425 (1957). 2194.

Mechanisms of polymer formation and decomposition. Catalysis Ser. 6, Chapter 3, p. 191, ed. by P. H Emmett (Reinhold Publ. Corp., New York, N.Y.) (1958), 2631.

Some aspects of depolymerization kinetics. J. Polymer Sci. 6, No. 1, 39 (1951). 307

Wall, L. A., Simha, R., Blatz, P. J., Depolymerization as a chain reaction. J. Polymer Sci. 5, 615 (1950). 44.

Wall, L. A., Simha, R., Bram, J., High-speed computations in the kinetics of free-radical degradation. I Random initiation. J. Chem. Phys. 29, No. 4, 894 (1958). 2587.

Wall, L. A., Straus, S., Influence of impurities on the pyrolysis of polyamides. J 63A3, 269 (1959). Pyrolysis of polyamides. J 60,39 (1958) RP2819.
Wall, L. A., Straus, S., Florin, R. E., Mohler, F. L., Bradt $P$., Phosphinoborine compounds: mass spectra and pyrolysis. J 63A 1, 63 (1959).

Wall, L. A., Tryon, $M$., Oxidative degradation of a series of deuterated styrene polymers. J. Phys. Chem. 62, No. 6, 697 (1958), 2680.

Thermal and photochemical processes in polystyrene in the glassy state. Nature 178, 101 (1956). 1958.

Walsh, J. L., Best approximation polynomials of given degree. Proc. 6th Symp. Appl. Math. (Am Math. Soc. (Santa Monica, Calif., 1953), p. 213 (1956). 1641.

Walsh, J. L., Davis, P., On representations and extension of bounded linear functionals defined on classes of analytic functions. Trans. Am. Math. Soc. 76 No. 2, 190 (1954). 1093.

Walsh, J. L., Motzkin, T. S., Least pth power polynomials on a real finite point set. Trans. Am. Math. Soc. 78, No. 1, 67 (1955). 1385

On the derivative of a polynomial and Chebyshev approximation. Proc. Am. Math. Soc. 4, 76 (1953). 786.

Walters, L. C., Johler, J. R., Mean absolute value and standard deviation of the phase of a constant vector plus a Rayleigh-distributed vector. J 62, 183 (1959) RP2950.

On the theory of reflection of low- and very-lowradiofrequency waves from the ionosphere. J 64D3, 269 (1960).

Propagation of a ground wave pulse around a finitely conducting spherical earth from a damped sinusodal source current. IRE Trans. Ant. Prop. AP-7, No. 1, 1 (1959). 3024

Walters, L. C., Johler, J. R., Lilley C. M. Low- and verylow-radiofrequency tables of ground wave parameters for the spherical earth theory: the roots of Riccati's differential equation. (Supplementary Numerical data for C573). (1959) TN7 (PB151366).

Walz, $A$., Compressible turbulent boundary layers with heat transfer and pressure gradient in flow direction. J 63B 1, 53 (1959).

Wampler, G., Isbell, H. S., Frush, H. L., Bruckner, B. H., Kowkabany, G. N., Micromethylation of polysaccharides, Anal, Chem. 29, 1523 (1957). 2198.

Warren, G., Some statistical designs for experiments. Proc. 12th Ann, Porcelain Enamel Inst. Forum (Univ. of Illinois, Urbana, Illinois) p. 76 (1950). 133.

Warren, K. A., Arnett, R. W., Mullen, L. O., Some methods for reducing heat leak through support members in liquefied gas storage vessels. Proc. 1958 Cryorenic Eng. Conf., Paper G-1, p, 410. Edited by K. D. Timmerhaus, Chem. Eng. Dept., Univ. of Colorado (Boulder, Ćolo.) (1959). 3049.

Warren, M. H., Helson, H., Judd, D. B., Object-color changes from daylight to incandescent filament illumination. Illum. Eng. 47, 221 (1952). 492.

Warwick, C. S., Green coronal line intensity and geomagnetism. J. Geophys. Research 64, No. 5, 527 (1959), 2960.

Warwick, $\dot{C} . S_{.}$Wood, $M . B$., A study of limb flares and associated events. Astrophys. J. 128, No. 3, 801 (1959). 2882.

Geomagnetic disturbance and velocity of slow-drift solar radio bursts. Nature 184, 1471 (1959). 2958.

Warwick, J. V. Menzel, D. H., Lawrence, R. S., A dynamic spectrum analyzer for Sacramento Peak. Harvard College Observatory, Cambridge, Massachusetts Scientific Rept. 20 (1955). 1233.

Washer, F. E., A simplified method of locating the point of symmetry. Photogrammetric Eng. XXIII, No. 1, 75 (1957). 2001.

Calibration of airplane cameras. Photogrammetric Eng. 23, No. 5, 890 (1957). 2045.

Instrument for measuring the marginal power of spectacle lenses. J. Opt. Soc. Am. 45, No. 9, 719 (1955). 1369.

Optical T-bench method of measuring longitudinal spherical aberration. J 61, 31 (1958) RP2880.

Prism effect, camera tipping, and tangential distor- 
tion. Photogrammetric Engr. 23, No. 4, 721 (1957). 2264.

Report of the Committee on the American Standards Association. Photogrammetric Engr. XXXV, No. 2, 309 (1959). 3036.

Sources of error in various methods of airplane camera calibration. Photogrammetric Eng. 22, No. 4, 727 (1956). 1878.

Testing of photographic lenses at the National Bureau of Standards. Photographic Eng. 5, No. 1, 37 (1954). 1169.

The effect of prism on the location of the principal point. Photogrammetric Eng. 23, No. 13, 520 (1957). 2350.

Washer, F. E., Case, F. A., Calibration of precision airplane mapping cameras. Photogrammetric Eng. 16, $502(1950)$. 32

Washer, F. E., Darling, W. R., Evaluation of lens distortion by the inverse nodal slide. J 63C2, 105 (1959).

Evaluation of lens distortion by the modified goniometric method. J 63C2, 113 (1959).

Marginal performance of corrected opthalmic lenses. J 60, 551 (1958) RP2869.

Factors affecting the accuracy of distortion measurements made on the nodal slide optical bench. J. Opt. Soc, Am. 49, No. 6, 517 (1959). 2948.

Washer, F. E., Feder, D. P. Shack, R. V., Panel discussion-Report on the NBS optical image evaluation symposium, reprinted from Photogrammetric Eng. 18, 575 (1952). 532 .

Washer, F. E., Hotchkiss, R. N., Rosberry, F. W., Spurious resolution of photographic lenses. J. Opt. Soc. Am. 41, 600 (1951). 315 .

Washer, F. E., Rosberry, J., New resolving power test chart. J. Opt. Soc. Am, 41, 597 (1951). 268.

Washer, F. E., Tayman, W. P., Darling, W. R., Evaluation of lens distortion by visual and photographic methods. J 61, 509 (1958) RP2920.

Wasik, S. P., Parker, R. A., On the electroviscous effect in dilute aqueous solutions on ionic detergents. J. Phys. Chem. 62, 967 (1958). 2667.

Wasilik, $J . H$., Anisotropic relaxation peak in the internal friction of crystalline quartz. Phys. Rev. 105, 1174 (1957). 2022.

An elasticity and dielectric loss of quartz. J. Appl. Phys. 27, No. 7, 836 (1956). 1620.

Wasilik, J. H., Flippen, R. B., Piezoelectric effect in indium antimonide. Phys. Rev. Letters 1, No. 7, 233 (1958), 2690.

Wasow, W., Asymptotic solution of the differential equation of hydrodynamic stability in a domain containing a transition point. Ann. Math. 58, 222 (1953). 656.

Discrete approximations to elliptic differential equations. Zeit. angew math. phys. (Basel, Switzerland) 6, Pt. 2, 81 (1955). 1314.

Metodi probabilisticl per la soluzione numerica di alcuni problemi di analisi. Rend. mat. app. V, XI, 336-346 (Roma 1952); issued separately as Publicazioni dell' Istituto per le Applicazioni del Calcolo $\mathrm{N}$. 354 (Roma, Italy) (1953). 763.

On singular perturbation problems in theory of nonlinear vibrations. Actes du Colloque International des Vibrations Non Lineaires, Ile de Porquerolles, France. Publ. sci, et techn. du ministère de l'air No. 281 (1951). 277.

On the asymptotic transformation of certain distributions into the normal distribution. Proc. 6th Symp. Appl. Math., of Am. Math. Soc. (Santa Monica, Calif., 1953), p. 251 (1956). 1795.

On the duration of random walks. Ann. Math. Stat. 22, No. 2, 199 (1951). 281.

On the inversion of matrices by random walks. Math. Tables and Other Aids to Computation VI, No. 38, 78 (1951). 282.

Singular perturbation methods for nonlinear oscillations. Proc. Symp. on Nonlinear Circuit Analysis, held by the Polytech. Inst. of Brooklyn, N.Y. (1953). 828.

Wasow, W., Motzkin, T. S., On the approximation of linear elliptic differential equations by difference equations with positive coefficients. J. Math, and Phys. 31, 253 (1953). 785.

Watanabe, H., Marton, L., Simpson, J. A., Suddeth, J. A., Wagner, M. D., Effect of degree of oricntation and crystal size on the seattering of 20 -kev electrons by aluminum, Phys. Rev, 110, No. 5, 1057 (1958). 2529.

Waterhouse, $R$. V., Interference patterns in reverberant sound fields.' J. Acoust. Soc. Am. 27, 247 (1955). 1373.

Noise control requirements in building codes. Chapter 40, Handbook of Noise Control (McGraw-Hill Book Co., New York, N.Y.) (1957). 2215.

On standard methods of measurement in architectural acoustics. J. Acoust. Soc. Am. 29, 544 (1957), 2226. Output of a sound source in a reverberation chamber and other reflecting environments. J. Acoust. Soc. Am 30, 4 (1958). 2679.

The power output of a sound source in a reflecting environment. Dissertation (Catholic University of America, Washington, D.C.) (1959). 3089.

Transmission of reverberant sound through walls. Acustica 4, 290 (1954). 1214.

Waterhouse, R. V., Berendt, R. D., Reverberation chamber study of the sound power output of subsonic air jets. J. Acoust. Soc. Am. 30, 114 (1958). 2742.

Waterhouse, R. V., Cook, R. K., New method of recording the sound transmission loss of walls as a continuous function of frequency. J. Acoust. Soc. Am. 27, No. 5,967 (1955). 1414.

Waterhouse, R. V., Cook, R. K., Berendt, R. D., Measurements on sound absorbers for jet-engine test cells. J. Acoust. Soc. Am. 28, No. 4, 688 (1956). 1771.

Waterhouse, R. V., Berendt, R. D., Cook, R. K., Sound insulation of wall, floor, and door constructions. (1958) BMS144, 2d Suppl.

Waterhouse, R. V., Cook, R. K., Berendt, R. D., Edelman, S., Thompson, M. C., Jr., Measurement of correlation coefficients in reverberant sound fields. J. Acoust. Soc. Am. 27, No. 6, 1072 (1955). 1399.

Waters, D. M., Thompson, M. C., Jr., Comparison of phase difference and Doppler shift measurements for studying ionospheric fine structure using earth satellites. Proc. IRE, Letter 46, 1960 (1958), 2493.

Simple technique for diplexing 10,000 Me and video signals on coaxial cables. Rev. Sci. Instr. 28, 206 (1957). 2300.

Waters, D. M., Thompson, M. C., Jr., Freethey, F. E., Fabrication techniques for ceramic X-band cavity resonators. Rev. Sci. Instr. 29, No. 10, 865 (1958). 2561.

Watson, D., Newkirk, G. A., Curtis, G. W., Manning, R., Shelby, J., The inner solar corona during June 1959. Nature 184, 1308 (1959). 3080.

Watson, K., Tandberg-Hanssen, E., Curtis, W., The emission of $\mathrm{HeI}, \lambda 10830$, during the great flare of August 26, 1958. Astrophys. J. 129, No. 1, 238 (1959). 3077 .

Watson, $\dot{T} .$, ., Robinson, $H . E$., Interlaboratory comparison of thermal conductivity determinations with guarded hot plates. Am. Soc. Testing Materials Spec. Tech. Publ. No, 119 (1951). 251.

Watstein, $D$. Effect of straining rate on the compressive strength and elastic properties of concrete. J. Am. Concrete Inst. 24, No. 49-52, 729 (1953). 694. Properties of concrete at high rates of loading. Am. Soc. Testing Materials Spec. Tech. Publ. 176, "Symp. Impact Testing," June 1955 (1956). 1824.

Watstein, D., Mathey, R. G., Evaluation of width of eracks in concrete at the surface of reinforcing steel by means of tensile bond specimens. Proc. RILEM Symp. on Bond and Crack Formation of Reinforeed Concrete (Stockholm, Sweden) (1957). 2113.

Strains in beams having diagonal cracks. J. Am. 
Concrete Inst. 30, No. 6, Title No. 55-46 (1958); Proc. Am. Concrete Inst. 55, 717 (1958-59). 2782.

Watt, A. D., Spectrum of frequency-shift radio phototransmissions. IRE Trans. Commun. Systems CS4, 27 (1956). 1884 .

Watt, A. D., Coon, R. M., Zurich, V. J., Reduction of adjacent-channel interference from on-off keyed carriers. IRE Trans. Commun. Systems CS $=4$, 41 (1956). 1841.

Wrtt, A. D., Maxwell, E. L., Characteristics of atmospheric noise from 1 to $100 \mathrm{Kc} / \mathrm{s}$. Symp. on the Prop. of V. L. F. Radio Waves, Sponsored by the NBS Boulder Laboratories and IRE Prof. Group on Ant. and Prop., January 23-25, 1957 (Boulder, Colo.), Paper No.'35, V. 3; Proc. IRE 45, 787 (1957). 2052. Low-frequency propagation paths in arctic areas. $\mathrm{J}$ 63D 1, 99 (1959).

Measured statistical characteristics of VLF atmospheric radio noise. Proc. IRE 45, 55 (1957). 2188.

Watt, $A . D .$, Plush, $R . W$., Power requirements and choice of an optimum frequency for a worldwide standardfrequency broadcasting station. J 63D1, 35 (1959).

Watt, A. D., Zurick, V. J., Coon, R. M., Reduction of adjacent-channel interference components from frequency-shift-keyed carriers. IRE Trans. Commun. Systems CS-6, 39 (1958). 2723.

Watts, J. M., A note on the polarization of low frequency ionosphere echoes. J. Geophys. Research 57, No. 2, 287 (1952). 371.

An observation of audio-frequency electromagnetic noise during a period of solar disturbance. J. Geophys. Research 62, 199 (1957). 2019.

Audio-frequency electromagnetic hiss recorded at Boulder in 1956. Symp. on the Prop of V. L. F. Radio Waves, Sponsored by the NBS Boulder Laboratories and IRE Prof. Group on Ant. and Prop., January 23-25, 1957 (Boulder, Colo.), Paper No. 17, V. 2; Geofis. pura e appl., (Milano, Italy) 37, 169 (1957). 2033.

Complete night of vertical-incidence ionosphere soundings covering frequency range from $50 \mathrm{Kc} / \mathrm{s}$ to 25 Mc/s. J. Geophys. Research 62, 484 (1957). 2064. Oblique incidence propagation at $300 \mathrm{kc}$ using the pulse technique. J. Geophys. Research 57, 487 (1952). 493 .

The interpretation of night-time low-frequency ionograms. J. Geophys. Research 63, 717 (1958). 2821.

Watts, J. M. Bateman, R., Finney, J. W., Smith, E. K., Tveten, $L$. $H$., IGY observations of $F$-layer scatter in the far east. J. Geophys. Research 64, 403 (1959). 2963.

Watts, J. M., Blair, J. C., Brown, J. N., An ionosphere recorder for low frequencies. J. Geophys. Research 58,99 (1953). 651 .

Watts, J. M., Brown, J. N., Effects of ionosphere disturbances on low frequency propagation. J. Geophys. Research 56, No. 3, 403 (1951). 229.

Ionosphere observations at $50 \mathrm{kc}$. J. Geophys. Research 55, $179(1950) .80$.

Some results of sweep-frequency investigation in the low frequency band. J. Geophys. Research 59, 71 (1954). 1146 .

Watts, J. M., Ellyett, C., Stratification in the lower ionosphere. J 63D2, 117 (1959).

Waxler, R. M., Adams, L. H., Temperature-induced stresses in solids of elementary shape. (1960) Mono. 2.

Waxler, R. M., Hamilton, E. H., Nivert, J. M., Jr., Properties of zinc borosilicate glasses. J 62, 59 (1959) RP2930.

Waxler, $R_{\text {. }}$ M., Napolitano, A., Relative stress-optical coefficients of some National Bureau of Standards optical glasses. J 59, 121 (1957) RP2779.

Weaver, E. R., Hughes, E. E., Diniak, A. W., Determination of water vapor from the change in electrical resistance of a hygroscopic film. J 60, 489 (1958) RP2864.

Weaver, E. R., Hughes, E. E., Gunther, S. M., Schuhmann, S., Redfearn, N. T., Gorden, R., Jr., Interpretation of mass spectra of condensates from urban atmospheres. J 59, 383 (1957) RP2809.

Weaver, F. D., Notes on the care and use of electrical instruments. Instruments 23, 1236 (1950). 99. Scale and reading errors of electrical indicators. Instr. and Automation 2\%, No. 11, 1812 (1954). 1134.

Weeks, J. J., Hoffman, J. D., Specific volume and degree of crystallinity of semicrystalline poly(chlorotrifluoroethylene), and estimated specific volumes of the pure amorphous and crystalline phases. J 60, 465 (1958) RP2862.

The specific volume and degree of crystallinity of poly(chlorotrifluoroethylene). J. Polymer Sci. 28, No. 117, 472 (1958). 2831.

Weeks, J.J., Hoffman, J. D., Murphey, W. M., Experimental and theoretical study of kinetics of bulk erystallization in poly (chlorotrifiuoroethylene). J 63A1, 67 (1959).

Wegstein, J.H., A numerical solution of the helium wave equation with the SEAC. Proc. Assoc. Computing Machinery, Toronto, Canada, p. 34 (Assoc. for Computing Machinery, New York, N.Y.) (1952). 372. Accelerating convergence of iterative processes. Communications, Assoc. Comp. Mach. 1, No. 6, 9 (1958). 2447.

Automatic coding principles. Proc. Symp. on Advanced Programming Methods for Digital Computers, Washington, D.C., June 1956 (Office of Naval Research, Department of the Navy, Washington, D.C.), p. 3 (1958), 2466 .

Wegstein, J.H., Alexander, S. N., Programming of scientific computers. Control Eng. 3, No. 5, 87 (1956). 1820.

Wegstein, J. H., Hilsenrath, J., Mechanized computation of thermodynamic tables at the National Bureau of Standards-the calculation of the equilibrium composition and thermodynamic properties of dissociated ionized gaseous systems. Proc. Joint Conf. on Thermodynamic and Transport Properties of Fluids, London, England, July 10-12, 1957 (Inst. of Mech. Eng. London, England) p. 79 (1958). 2632.

Weinberg, J. G., Bennett, J. A., A toric bending specimen for investigation of geometrical factors in fatigue. ASTM Bull. No. 234, p. 53 (1958). 2442.

Effect of crystal orientation of fatigue-crack initiation in polycrystalline alumium alloys. Natl. Advisory Comm. Aeronaut. Tech. Note 3990 (1957). 2096.

Weinberger, A., Leiner, A. L., Notz, W. A., Smith, J. L., Organizing a network of computers to meet deadlines. 1957 Eastern Joint Computer Conf. Proc. (Washington, D.C.), p. 115 (1957). 2240.

PILOT, a new multiple computer system. J. Assoc. Computing Mach. 6, No. 3, 313 (1959). 3016.

PILOT, the NBS multicomputer system. Proc. Eastern Joint Computer Conf. (Philadelphia, Pa.), p. 164 (1958), 2692.

Weinberger, A., Loberman, $H$., Formal procedures for connecting terminals with a minimum total wire length. J. Assoc. Computing Machinery 4, No. 4, 428 (1957). 2128.

Symbolic designations for electrical connections. J. Assoc. Computing Machinery 4, No. 4, 420 (1957). 2334 .

Weinberger, A., Smith, J. L., A logic for high-speed addition. (1958) C591, Sect. 1.

A one-microsecond adder using one-megacycle circuitry. IRE Trans. Electronic Computers EC-5, No. 2,65 (1956). 1596 .

Shortcut multiplication for binary digital computers. (1958) C591, Sect. 1.

The logical design of a one-microsecond parallel adder using one-megacycle circuitry. Am. Inst. Elec. Engrs. Proc. Western Joint Computer Conf. T-85, (1956). 1937.

Weinstock, B., Pellam, J. R., Thermal Rayleigh disk measurements in $\mathrm{He}^{3}-\mathrm{He}^{4}$ mixtures. Phys. Rev. 89, 521 (1953). 895.

Weintraub, S., Slide rule computes radio refractive index of air. Electronics 26, No. 1, 182 (1953). 829. 
Weintraub, S., Smith, E. K., The constants in the equation for atmospheric refractive index at radio frequencies. Proc. IRE 41, 1035 (1953). 855.

Weir, C. E., Lattice energies of ionic cubic crystals. Phys. Rev. 108, No. 1, 19 (1957). 2175.

Physical and physico-chemical constants of leather and collagen. J. Soc. Leather Trades' Chemists 36, 155 (1952). 510 .

Resistivity dielectric constant, power factor of leather. J. Am. Leather Chemists' Assoc. 47, 711 (1952). 533.

Weir, C. E., Carter, T. J., Rate of shrinkage of tendon collagen: further effects of tannage and liquid enviroment on the activation constants of shrinkage. J. Am. Leather Chemists Assoc. 45, 421 (1950). 120.

Weir, C. E., Lippincott, E. R., Van Valkenburg, A. Bunting, $E$. $N$., Infrared studies in the 1- to 15-micron region to 30,000 atmospheres. J $\mathbf{6 3 A} 1,55$ (1959). Infrared studies on polymorphs of silicon dioxide and germanium dioxide. J 61, 61 (1958) RP2885.

Weir, C. E., Shartsis, L., Compressibility of binary alkali borate and silicate glasses at high pressures. J. Am. Ceram. Soc. 38, No. 9, 299 (1955). 1292. Compressibility of binary alkaline-earth borate glasses at high pressures. J. Am. Ceram. Soc. 39, No. 9, 319 (1956). 1658.

Weir, C. E., Shereshefsky, J. L., Adsorption of gases and vapors on glass spheres, II. Two dimensional condensation of oxygen. J. Phys. Chem. 60, 1162 (1956). 1609 .

Weir, C. E., Van Valkenburg, A., Studies of beryllium chromite and other beryllia compounds with $\mathrm{R}_{2} \mathrm{O}_{3}$ oxides. J 64A1, 103 (1960).

Weir, C. E., Yoder, H. S., Change of free energy with pressure of the reaction nepheline + albite $=2$ jadeite. Am. J. Sci. 249, 683 (1951). 203.

Weir, J. E., DeLollis, N. J., Rucker, N., Comparative strengths of some adhesive-adherend systems. Trans. Am. Soc. Mech. Engrs. 73, 183 (1951), 208.

Weiss, M. S., Fuller, E. G., Splitting of the giant resonance for deformed nuclei. Phys. Rev. 11\%, No. 2, 560 (1958), 2772.

Weiss, M. S., Fuller, E. G., Petree, B., Photoneutron yields in the rare-earth region. Phys. Rev. 112, No. 2,554 (1958). 2686.

Weiss, M. S., Petree, B., Fuller, E. G., Photoneutron yields in the rare earth region. Bull. Am. Phys. Soc. Series II, No. 2, 16 (1957). 2249.

Weissberg, S. G., Mandelkern, L., Williams, L. C., Sedimentation equilibrium of flexible chain molecules. J. Phys. Chem. 61, 271 (1957), 2296.

Weissberg, S. G., Roberts, A. G., Film-forming properties of cellulose acetate propionates. Effect of solvents, diluents, and plasticizers. Ind. Eng. Chem. 43, 2088 (1951). 237.

Weissberg, S. G., Rothman, S., Mandel, J., McCann, F. R., Sorption of macromolecules to solid surfaces: sorption of dextran to cellulose nitrate membranes in the presence of serum albumin and surface-active agents. J. Colloid Sci. 10, No. 4, 338 (1955). 1500.

Weissberg, S. G., Rothman, S., Schwebel, A., Sorption of dextran to collodion membranes. J. Polymer Sci. 11, 381 (1953). 832.

Weissberg, S. G., Rothman, S., Simha, R., Viscosities of very dilute polymer solutions. J. Polymer Sci. 5, 141 (1950). 166.

Weissberg, S. G., Wales, M., Marshall, P. A., Intrinsic viscosity-molecular weight relationships for dextran. J. Polymer Sci. 10, 229 (1953). 745.

Weissberg, S. G., Wales, M., Marshall, P. A., Rothman, S., Application of viscosity methods to the study of branching in polysaccharides. Ann. N.Y. Acad. Sci. 5\%, 353 (1953). 654 .

Weissler, $P$. G., Cook, $R$. $K$., Piezoelectric constants of alpha- and beta-quartz at various temperatures. Phys. Rev. 80, 712 (1950). 108.

Weitzel, D. H., Blake, J. H., Konecnik, M., Flow conversion kinetics of ortho and para-hydrogen. Proc. 1958 Cryogenic Eng. Conf., Paper E-2, p. 286 . Edited by K. D. Timmerhaus, Chem. Eng. Dept., Univ. of Colorado (Boulder, Colo.) (1959). 2953.

Weitzel, D. H., Draper, J. W., Park, O. E., Timmerhaus, $K$. D., Van Valin, C. C., Catalysis of the orthoparahydrogen conversion. Proc. 1956 Cryogenic Eng. Conf., Paper A-4, p. 12 . Edited by K. D. Tin.merhaus, Chem. Eng. Dept., Univ. of Colorado (Boulder, Colo.) (1957). 2048.

Weitzel, D. H., Flynn, T. M., Timmerhaus, K. D., Distillation of hydrogen isotopes. Colorado Engr. 55, 12 (1958). 2526.

Pilot plant studies of the low temperature distillation of hydrogen isotopes. Proc. 1958 Cryogenic Eng. Conf., Paper H-1, p. 464. Edited by K. D. Timmerhaus, Cherrical Engineering Dept., University of Colorado (Boulder, Colo.) (1958); Chem. Eng. Progr. 54, No. 6, 35 (1958). 2691.

Weitzel, D. H., Flynn, T. M., Timmerhaus, K. D., Draper, $J$. $W$., Separation of hydrogen isotopes by multicomponent distillation. Proc. 1957 Cryogenic Eng. Conf., Paper A-6, p. 58, Edited by K. D. Timmerhaus, Chem. Eng. Dept., Univ. of Colorado (Boulder, Colo.) (1958). 2751 .

Weitzel, D. H., Flynn, T. M., Timmerhaus, K. D., Vander Arend, P. C., Draper, J. W., Distillation of hydrogen-deuterium mixtures. Proc. 1956 Cryogenic Eng. Conf., Paper A-6, p. 39, Edited by K. D. Timmerhaus, Chem. Eng. Dept., Univ. of Colorado (Boulder, Colo.) (1957). 2093.

Weitzel, D. H., Loebenstein, W. V., Draper, J. W., Park, $O$. E., Orthopara catalysis in liquid-hydrogen production. J 60, 221 (1958) RP2840.

Weitzel, D. H., Park, O. E., Iron catalyst for production of liquid para-hydrogen. Rev. Sci. Instr. 27, No. 1, 57 (1956). 1751.

Weitzel, D. H., Purcell, J. R., Draper, J.W., A unique thermal conductivity gas analyzer. 'Proc. 1957 Cryogenic Eng. Conf. Paper D-4, p. 191. Edited by K. D. Timmerhaus, Chem. Eng. Dept., Univ, of Colorado (Boulder, Colo.) (1958). 2443.

Weitzel, D. H., Timmerhaus, K. D., Flynn, T. M., Low temperature distillation of hydrogen isotopes. Chem. Eng. Progr. 54, No. 6, 35 (1958). 2618.

Weitzel, D. H., Van Valin, C. C., Draper, J. W., Design data for ortho-parahydrogen converters. Proc. 1957 Cryogenic Eng. Conf., Paper B-2, p. 73. Edited by K. D. Timmerhaus, Chem. Eng. Dept., University of Colorado (Boulder, Colo.) (1958). 2514.

Weitzel, $D . H$. White. L. E., Continuous analysis of ortho-parahydrogen mixtures. Rev. Sci. Instr. 26 , 290 (1955). 1294.

Weitzel, D. H., Wilson, W. A., Vapor phase ortho-para conversion in the large CEL-NBS hydrogen liquefier. Proc. 1957 Cryogenic Eng. Conf., Paper B-3, p. 85. Edited by K. D. Timmerhaus, Chem. Eng. Dept., Univ, of Colorado (Boulder, Colo.) (1958). 2859.

Wells, P. I., Janes, H. B., Some tropospheric scatter propagation measurements near the radio horizon. Proc. IRE 43, 1336 (1955). 1499.

Wells, T. E., Measurement of the resistance-strain relation and Poisson's ration for copper wires. Paper No. 56-8-3, Proc. Instr. Soc. Am. 11, No. 9, 377 (1956). 1768 .

Werntz, C. W., Schiefer, H. F. Interpretation of tests for resistance to abrasion of textiles. Textile Research J. 22, 1 (1952). 473.

Ueber den einfluss der Gewebekonstruktoon auf den Scheuerwiderstand. Textil-praxis (Stuttgart, Germany) 57, 1 (1952). 610.

West, $E . D$., The heat capacity of sulfur from $25^{\circ}$ to $250^{\circ}$, the heats and temperatures of transitions and fusion. J. Am. Chem. Soc. 81, 29 (1959). 3079.

West, E. D., Ginnings, D. C., An adiabatic calorimeter for the range $30^{\circ}$ to $500^{\circ} \mathrm{C}$. J 60,309 (1958) RP2848.

Automatic temperature regulation and recording in precision adiabatic calorimetry. Rev. Sci. Instr. 28, 1070 (1957). 2035.

The heat capacity of aluminum oxide in the range 
$300^{\circ}$ to $700^{\circ}$ K. J. Phys. Chem. 61, 1573 (1957). 2362 .

Westfall, F. O., Corliss, C. H., Bozman, W. R., Electrodeless metalhalide lamps. J. Opt. Soc. Am. 43, 398 (1953). 705.

Westhaver, J. W., Mair, B. J., Rossini, F. D., Theoretical analysis of fractionating process of adsorption. Ind. \& Eng. Chem. 42, 1279 (1950). 156.

Westmoreland, J.C., A meter for timing the flow of very small volumes of a gas. Natl Advisory Comm. Aeronaut. RM 52109 (1952). 365.

Determination of viscosity of exhaust-gas mixtures at elevated temperatures. Natl. Advisory Comm. Aeronaut, Tech. Note 3180 (1954). 989.

Westwick, R., Marcus, M., Moyles, B. N., Some extreme value results for indefinite Hermitian matrices. Illinois J. Math. 1, 449 (1957). 2303.

Wexler, A., Electric hygrometers. (1957) C586.

Wexler, A., Hasegawa, S., Garfinkel, S. B., Simple humidity lag apparatus. Rev. Sci. Instr. 26, No. 12, 1196 (1955). 1487.

Wheelon, A. D., Radio-wave scattering by trospheric irregularities. J 63D2, 205 (1959).

White, D., Hilsenrath, J., Pressure sensitive diaphragmtype null detector. Rev. Sci. Instr. 29, No. 7, 648 (1958). 2698.

White, E. E., Jr., Brauer, E. M., Moshanos, M. G., The reaction of metal oxides with o-ethyoxybenzoic acid and other chelating agents. J. Dental Research 37, 547 (1958). 2829.

White, H.S., Friction and endurance of prelubricated and unlubricated ball bearings at high speeds and extreme temperatures. J 63C1, 19 (1959).

Laboratory test with turboprop lubricants. Am. Snc. Lubrication Engrs. Trans. 1, 51 (1958). 2610. Materials for small, oil-free bearings. Materials and Methods 45, No. 4, 135 (1957). 2184.

White, H. S., Bair, E. N., Sr., McKee, S. A., Luhrication of plain journal kearings. The Virginia Tech. Eng. 23, 26 (1950). 82.

White, H. S., Belcher, H. V., Effects of capillary shape on flow characteristics and degradation of polymer solutions. J 60, 215 (1958) RP2839.

White, H. S., McKee, S. A., Swindells, J. F., Mountjoy, W., For wear prevention. SAE J. 58, 47 (1950). 59.

White, H. S., Swindells, J. F., Belcher, H. V., Oil-soaked felt-pad lubrication of ball bearings at high speed and high temperature. Lubrication Eng. 11, 182 (1955). 1422 .

White, H. S., Zei, D., Special lubricants tests. Mech. Eng. 73, 657 (1951). 311.

White, H. W., Grease consistometer is modified to operate at constant shear rates, higher viscosities. Petroleum Processing 6, 285 (1951). 245.

White, L. E., Weitzel, D. H., Continuous analysis of ortho-parahydrogen mixtures. Rev. Sci. Instr. 26, 290 (1955). 1294.

White, M. L., Extension of the Sen-White paper on atmospheric oscillations. J. Geophys. Research 60, 531 (1955). 1341.

Gravitational and thermal oscillations in the earth's upper atmosphere. J. Geophys. Research 61, 489 (1956). 1726.

On the atmospheric dynamo theory. J. Geophys. Research 62, 329 (1957). 2228.

White, M. L., Sen, H. K., Thermal and gravitational excitation of atmospheric oscillations. J. Geophys. Research 60, 483 (1955). 1555.

Whitlock, M. B., Jr., Albrite, J. P., Shutts, R. E., Cook, R. K., Corliss, E. L. R., Burkhart, M. D. Research in normal threshold of hearing. A.M.A. Arch. Otolaryngol. 68, 194 (1958). 2735 .

Whitney, A., Schoenberg, I. J., On polya frequency functions. III. The positivity of translation determinants with an application to the interpolation problem by spline curves. Trans. Am. Math. Soc. $74,246(1953)$. 782 .

Whitney, J. E., Brode, W. R., Gould, J. H., Wyman, G. M., A comparative survey of spectrophotometers in the
210-760 $\mathrm{m} \mu$ region. J. Opt. Soc. Am. 43, 862 (1953). 625 .

Whittier, B. L., Jameson, L. H., Schiefer, H. F., Factors affecting the properties of rayon fakrics. Textile Research J. 22, No. 9, 599 (1952). 446.

Wichers, E., Can the scales of atomic weights and nuclidic masses be unified? Phys. Today 12, No. 1, 28 (1959). 2906.

Reagent chemicals, American Chemical Society Specifications, 1955. Prepared by the Comm. on Analytical Reagents, Publ. by Am. Chem. Soc. (1955). 1474. Report of the committee on atomic weights of the American Chemical Society. J. Am. Chem. Soc. 72, 1431 (1950). 127; J. Am. Chem. Soc. 74, 2447 (1952). 531 ; J. Am. Chem. Soc. 76, 2033 (1954). 1129. Report on atomic weights for 1956-57. J. Am. Chem. Soc. 80, 4121 (1958). 2731.

Standard samples. Science 117, 7 (1953). 835.

Wichers, E., Bates, R. G., Precise intercomparison of acids by differential potentiometric titration with hydrogen electrodes. J 59, 9 (1957) RP2769.

Precise intercomparison of acids by differential potentiometric titration with the hydrogen electrode. Actas do congress (Lisboa, Portugal) 1, 3 (1957). 2260 .

Wichers, E., Gordon, C. L., Purification of mercury and its physical properties. Ann. N.Y. Acad. Sci. 65, No. 5, 369 (1957). 2275.

Wickizer, G. S., Crysdale, J. H., Herbstreit, J. W., Dickson, F. H., Egli, J.J., Rebuttal to comments on "Large reduction of VHF transmission loss and fading by the presence of a mountain obstacle in beyond-lineof-sight paths", by F. H. Dickson, J. J. Egli, J. W. Herbstreit, and G. S. Wickizer. (Publ. in Proc. IRE 41, 967, (1953)). Proc. IRE 43, 627 (1955). 1384.

Wickizer, G. S., Dickson, F. H., Egli, J.J., Herbstreit, J. $W$., Large reductions of VHF transmission loss and fading by the presence of a mountain obstacle in bevond-line-of-sight paths. Proc. IRE 41, 967 (1953). 750 .

Wieder, B., Some results of a sweep-frequency propagation experiment over an $1150-\mathrm{KM}$ east-west path. J. Geophys. Research 60, 395 (1955). 1498.

Wieder, H. H., Kaufman, S., Plastic electrets. J. Appl. Phys. 24, 156 (1953). 796.

Plastic electrets and their applications. Elec. Eng. 72, 511 (1953). 797.

Wiegmann, N. A., Pairs of normal matrices with property L. Proc. Am. Math. Soc. 4, 35 (1953). 792.

Wiegmann, N. A., Hoffman, A. J., Mannos, M., Sokolowsky, D., Computational experience in solving linear programs. J. Soc. Ind. Appl. Math. 1, 17 (1953). 672 .

Wielandt, H., An extremum property of sums of eigenvalues. Proc. Am. Math. Soc. 6, No. 1, 106 (1955). 1234 .

Einschliessung von Eigenwarten hermitescher Matrizen nach dem Abschnittsverfahren. Arch. math. (Tübenger, Germany) 5, 108 (1954). 1005.

Error bounds for the eigenvalues of summetric integral equations. Proc. 6th Symp. Appl. Math., Am. Math. Soc. (Santa Monica, Calif., 1953), p. 261 (1956). 1707.

On eigenvalues of sums of normal matrices. Pacific J. Math. 5, 633 (1955). 1425.

Wielandt, H., Hoffman, A. J., The variation of the spectrum of a normal matrix. Duke Math. J. 20, 37 (1953). 890.

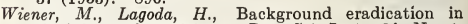
thick-layered emulsions. Rev. Sci. Instr. 21, No. 1, 39 (1950). 30.

Wier, J. E., Pons, D. C., Axilrod, B. M., Effects of humidity during fabrication of polyester laminates. SPE J. 8, No. 9, 8 (1952). 435.

Effects of resin coating methods and other variables on physical properties of glass-fabric reinforced polyesters. Natl. Advisory Comm. Aeronaut. Research Memo. RM54G26 (1954). 1004. 
Wiesner, J. B., Bailey, D. K., Bateman, R., Berkner, L. V., Booker, H. G., Montgomery, $G, F$. Purcell, $E$. $M$.'

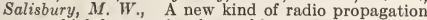
at very high frequencies observable over long distances. Phys, Rev. 86, No. 2, 141 (1952). 369.

Wiesner, J. B., Norton, $K$. A., The scatter propagation issue, Introduction to special issue of Proc. of the IRE. Proc. IRE 43, 1181 (1955). 1544.

Wildhack, W. A., A versatile pneumatic instrument based on critical flow. Rev. Sci. Instr. 21, No. 1, $25(1950) .14$.

Effect of transverse atmospheric drag on satellite orbits. Science 128, 309 (1958), 2532.

Instrumentation-Revolution in industry, science, and warfare. Science 120, 15A (1954). 1042.

Instrumentation and automation. Science 122, 1146 (1955). 1370.

Instrumentation and the future of mankind. Instr. Soc. Am. J. 2, No. 9, 430 (1955). 1371.

Instrumentation in perspective. Science 112, 515 (1950). 76.

Instrumentation research at the National Bureau of Standards. Ann. N.Y. Acad. Sci. 60, 809 (1955). 1372.

New tools for research. Science 114, No. 2962, 3 (1951). 269 .

Review of some methods of flow measurement. Science 120, No, 3110, 191 (1954). 1131.

Wildhack, W. A., Dressler, $R . F$., Lloyd, $E$. C., Investigations of the properties of corrugated diaphragms. Am. Snc. Mech. Engrs. Preprint 55-A-181 (1955). 1378; Trans. Am. Soc. Mech. Engrs. 79, 65 (1957). 2166 .

Wildhack, W. A., Perls, T. A., A simple type X $(t)-\mathrm{Y}$ recorder. Rev. Sci. Instr. 22, No. 7, 541 (1951). 184.

Wildhack, W. A., Smith, R. O., A basic method of determining the dynamic characteristics of accelerometers by rotation. J. Instr: Soc. Am. 1, No. 12, Paper No. 54-40-3, (1954). 913.

Wildhack, W. A., Stern, J., The peek-a-boo system. Chapter 6, Punched Cards, p. 125 (Reinhold Publ. Co., New York, N.Y.) (1959). 3087.

Wildhack, W. A., Stern, J., Smith, J., Documentation in instrumentation. Studies in Coordinate Indexing Vol. 111, Documentation, Inc. (1956). 1682.

Wilkerson, R. E., Hartman, W. J., Path antenna gain in an exponential atmosphere. J 63D2, 273 (1959).

Wilkins, J.,Jr., Goldstein, H., Spencer, L.V., Systematic calculations of gamma-ray penetration. Phys. Rev. 89,1150 (1953). 847.

Williams, C. N., Richmond, J. C., Lefort, H. G., Harrison, $W$. N., Ceramic coatings for nuclear reactors (a progress report). J. Am. Ceram. Soc. 38, No. 2, 72 (1955). 1285.

Williams, L. C., Leaderman, H., Smith, R. G., Rheology of polyisobutylene. III. Elastic recovery, non-Newtonian flow, and molecular weight distribution. J. Polymer Sci. 36, 233 (1959). 3037.

Williams, L. C., Mandelkern, L., Weissberg, S. G., Sedimentation equilibrium of flexible chain molecules. J. Phys, Chem. 61, 271 (1957). 2296.

Williams, M. L., Analysis of brittle behavior in ship plates. Am. Soc. Testing Materials Spec. Tech. Publ. No. 158, 11 (1954). 954.

Williams, $M$. L., Ellinger, G. A., Investigation of structural failures of welded ships. Welding J. 32, 498-S (1953), 746 .

Williams, $W . E ., J r$. A high-power pulse generator. Radio-Electronic Eng. Section, Radio and TV News 49, 32 (1953). 627.

High-power square-pulse generator. Electronics 25, No. 10, 144 (1952). 463.

Voltage breakdown tester. Rev. Sci. Instr. 24, 458 (1953). 908.

Williams, W. E., Jr., Maxuell, E., Liquid level indicator for condensed gases at low temperature. Rev. Sci. Instr. 25, No. 2, 111 (1954), 1052.
Williamson, D. A., Fuller, V. L., Longley, A. G., Rice, $P$. L., A summary of VHF and UHF tropospheric transmission loss data and their long-term variability. (1960) TN43 (PB151402).

Wilson, A. C., Impedance of a corner-reflector antenna as a function of the diameter and length of the driven element. J 64D2, 135 (1960).

Wilson, A. C., Cottony, H. V., Gains of finite-size cornerreflector antennas. IRE Trans. Ant. Prop. AP-6, No. 4, 366 (1958). 2574.

Wilson, M., Helson, H., Judd, D. B., Color rendition in fluorescent sources of illumination. Illum. Eng. 51, 329 (1956). 1651.

Wilson, N. L., Tousey, R., Purcell, J. D., Johnson, F. S., Moore, C. $E$., A revised analysis of the solar spectrum from 2990 to $2635 \mathrm{~A}$. Astrophys, J. 119, No. 3,590 (1954). 933 .

Wilson, $R$. E., Standards of temperature. Phys. Today 6, $10(1953)$. 836.

Wilson, R. E., Arnold, R. D., Thermometry and pyrometry. Handbook of Physics, Edited by E. U. Condon and $\mathrm{H}$. Odishaw, Chapter $3, \mathrm{Pt} .4$, p. 5-30 (McGraw-Hill Book Co., Inc., New York, N.Y), (1958). 2500 .

Wilson, R. E., Liberatore, L. C., Aging changes in clinical thermometers. J. Am. Ceram. Soc. 35, 203 (1952). 385 .

Wilson, S. W., Barbrow, L. E., Vertical distribution of light from gas-filled candle-power standards. Illum. Eng. 53, 645 (1958). 2860.

Wilson, $W$. A., Breathing oxygen storage dewars, Proc. 1956 Cryogenic Eng. Conf., Paper B-1, p. 54. Edited by K. D. Timmerhaus, Chemical Engineering Dept., Univ. of Colorado (Boulder, Colo.) (1957). 2038.

Wilson, W. A., Weitzel, D. H., Vapor phase ortho-para conversion in the large CEL-NBS hydrogen liquefier. Proc, 1957 Cryogenic Eng. Conf., Paper B-3, p. 85, Edited by K. D. Timmerhaus, Chem. Eng. Dept., Univ. of Colorado (Boulder, Colo.) (1958). 2859.

Wilson, $W . K$., Determination of aldehyde in cellulose: a review of methods. Tappi 38, 274 (1955). 1308. Record papers and their preservation. Capital Chemist 6, 46 (1956). 1840.

Degradation of cellulose in a vacuum with ultraviolet light. J 60, 229 (1958) RP2841.

Wilson, $W . K$., Forshee, B. W., Degradation of cellulose acetate films. SPE J. 15, No. 2, 146 (1959). 2924. Preservation of documents by lamination. (1959) Mono. 5.

Wilson, $W . K$., Harvey, J. L., Effect of light on coated groundwood papers. Tappi 36, 459 (1953). 693. Effect of light on coated groundwood papers. Indian Pulp and Paper 9, 444 (1955). 1327.

Wilson, $W . K$., Harvey, J. L., Mandel, J., Worksman, T. L., Accelerated aging of record papers compared with normal aging. Tappi 38, No. 9, 543 (1955). 1251.

Wilson, $\dot{W} . K$., Harvey, J. L., Padgett, A. A., Effect of melamine resin on chemical tests of paper. Tappi 34, 410 (1951), 227.

Wilson, W. K., Hobbs, R. B., Annual review of pulp and paper industry. Ind. Eng. Chem, 49, 42A (1957). 2024 .

Paper production on the upswing. Chem. Eng. News 32, 40 (1954). 1103.

Pulp and paper. Chem. Eng. News 31, 40 (1953). 805 .

Pulp and Paper (Annual Review). Chem. Eng. News 33,114 (1955). 1466.

Wilson, W. K., Launer, H. F., Flynr, J. H., Determination of glucose by means of sodium chlorite. Indian Pulp and Paper 9, 399 (1955). 1310.

Preparing cuprammonium solvent and cellulose solutions. Anal, Chem. 22, 375 (1950), 112.

Wilson W. K., Padgett, A. A., Reaction of sodium chlorite with some adloses and modified celluloses. Tappi 38, 292 (1955). 1473.

Windsor, $M$., Volcanoes on the moon. Nature 184, 1556 (1959). 3111. 
Winter, J. C., Johnson, H. D., Phillips, C. W., Grimes$J$. W., Achenbach, P. R., Heat transfer measuref ments on refrigerated-food trailers. U.S. Dept. of Agri. Marketing Service (AMS-250), (June 1958). 2580.

Wintermyer, A. M., Kinter, E. B., Swerdlow, M., Electron microscopy of soil clays and related materials. Public Roads Highway Research J. 27, No. 5, 89 (1952). 439.

Wolcott, R. B., Nelsen, R. J., Paffenbarger, G. C., Fluid exchange at the margins of dental restorations. J. Am. Dental Assoc. 44, 288 (1952). 451.

Wolcott, R. B., Pafjenbarger, G. C., Schoonover, I. C., Direct resinous filling materials: temperature rise during polymerization. J. Am. Dental Assoc. 42, 253 (1951). 223.

Wolfowitz, J., Dvoretsky, A., Kiefer, J., Sequential decision problems for processes with continuous time parameter. Testing hypotheses. Ann. Math. Stat. 24, No. 2, 254 (1953).

Wolfowitz, J., Dvoretzky, A., Wald, A., Elimination of randomization in certain statistical decision procedures and zero-sum two-person games. Ann. Math. Stat. 22, 1 (1951). 231.

Relations among certain ranges of vector measures. Pacific J. Math. 1, 59, (1951). 299.

Wolock, I., Axilrod, B. M., Sherman, M. A., Biaxial stretch-forming of acrylics. Modern Plastics 31, 128 (1953). 658.

Effects of high degrees of biaxial stretch-forming on crazing and other properties of acrylic plastic glazing. Natl. Advisory Comm. Aeronaut. Research Memo. RM53D14 (1953). 699.

Wolock, I., Axilrod, B. M., Sherman, M. A., Cohen, V., Effects of biaxial stretch-forming. Modern Plastics 30, $117(1952), 434$.

Effects of moderate biaxial stretch-forming on tensile and crazing properties of acrylic plastic glazing. Natl. Advisory Comm. Aeronaut. Tech. Note, 2779 (1952). 436.

Wolock, I., George, D. A., Effects of multiaxial stretching on crazing and other properties of transparent plastics. Natl. Advisory Comm. Aeronaut. Research Memo 54F22 (1954). 1002.

Effects of multiaxial stretching on crazing and other properties of transparent plastics. SPE J. 12, 20 (1956), 1690.

Wolock, I., Kanegis, $J$., Plasties literature in Government reports. Am. Chem. Soc. Advances in Chem. Ser. No, 10, 151 (1954). 1109.

Wolock, I., Kline, G. M., Axilrod, B. M., Sherman, M. A., George, $D$, A, Cohen, $V$, Development of craze and impact resistance in glazing plastics by multiaxial stretching. Natl. Advisory Comm. Aeronaut. Tech. Report 1290 (1957). 2084.

Wolock, I., Kline, G., M., Callomon, I. G., Plastics for aircraft. Part II. Transparent glazing materials. Army-Navy-Commerce Bull. ANC-17 (1957). 2255.

Wolock, I., Newman, S. B., Fracture phenomena and molecular weight in polymethyl methacrylate. J. Appl. Phys. 29, No. 1, 49 (1958). 2569

Wolock, I., Reinhart, F. W., Good, C. L., Turner, P. S., Comparison of mechanical properties of flat sheets, molded shapes and postformed shapes of cotton fabric-phenolic laminates. Natl. Advisory Comm. Aeronaut. Tech. Note. 3825 (1957). 2063.

Wolock, I., Sherman, $M$. A., Aailrod, B. $M$., Effect of molecular weight on the crazing and tensile properties of polymethyl methacrylate. Natl. Advisory Comm. Aeronaut. Research Memo. 54A04 (1954). 999.

Wolock, I., Toner, S. D., Reinhart, F. W., Effects of molding pressure on strength properties of glassfiber reinforced plastics. SPE J. 14, 40 (1958). 2533.

Wolff, $C$., Spencer, $L$. V., Penetration and diffusion of hard X-rays: polarization effects. Phys. Rev. 90, 510 (1953). 793.

Wolfowitz, J., Dvoretsky, A., Kiefer, J., Sequential decision problems for processes with continuous time parameter, testing hypotheses, Ann, Math. Stat. 24, No. 2, 254 (1953). 824.

Wong, P. K., Armstrong, G. T., Krieger, L. A., Use of a direct current amplifier and recorder to balance a Mueller resistance bridge. Rev. Sci. Instr. 30, 339 (1959), 3107.

Wood, G. B., Brenner, A., Electrodeposition of metals from organic solutions. IV. Electrodeposition of beryllium and beryllium alloys. J. Electrochem. Soc. 104, No. 1, 29 (1957). 2107.

Wood, G. B., Connor, J. H., Reid, W. E., Jr., Electtrodeposition of metals from organic solutions. V. Electrodeposition of magnesium and magnesium alloys. J. Electrochem. Soc. 104, 38 (1957). 2108.

Wood, J. W., Fanning, R. J., Rapid extraction of resins from chilte and guayule rubbers. Rubber Age 68 , No. 2, 195 (1950), 119.

Wood, L. A., Crystallization in butadiene-styrene copolymers. J. Appl. Phys. 25, No. 7, 851 (1954). 983. Elastic properties of pure-gum rubber vulcanizates. Proc. Joint Army-Navy-Air Force Conf. on Elastomer Research and Development 2, 5th meeting (October 1958), p. 363 (Materials Lab. Wright Air Development Ctr. Wright-Patterson Air Force Base, Ohio) (1958), 2534.

Glass transition temperatures of copolymers. J. Polymer Sci. 28, No. 117, 319 (1958). 2576.

Physical chemistry of synthetic rubbers. Chapter $\mathrm{X}$ in the book of "Synthetic Rubber", p. 316 (John Wiley \& Sons, Inc., New York, N.Y.) (1954). 1107. Physics - the elasticity of rubber. J. Wash. Acad. Sci. 47, No. 9, 281 (1957). 2251.

Stress-strain relation of pure-gum rubber vulcanizates in compression and tension. J 60, 193 (1958) RP2837. The elasticity of rubber. Rubber Chem. and Technol. 31. No. 5, 959 (1958), 2810A.

Values of physical constants of different rubbers. Section of Smithsonian Physical Tables, 9th Ed. Edited by W. E. Forsyth (Smithsonian Institution, Washington, D.C.) (1954). 1222.

Wood, L. A., Madorsky, I., Pawlson, R. A., Determination of copolymer composition by combustion analysis for carbon and hydrogen. J 64A2, 157 (1960).

Wood, $M$. B., A study of magnetic maps of the sun. Ástrophys. J. 124, 447 (1956), 1600.

Wood, $M$. B., Warwick, C. S., A study of limb flares and associated events. Astrophys. J. 128, No. 3, 801 (1959), 2882.

Geomagnetic disturbance and velocity of slow-drift solar radio bursts. Nature 184, 1471 (1959). 2958.

Wood, R. E., Experiments with the cell $\mathrm{Bi}, \mathrm{Bi}_{2} \mathrm{O}_{3}, \mathrm{ZnC}_{2} \mathrm{l} /$ $\mathrm{ZnCl}_{2} / \mathrm{ZnCl}_{2}, \mathrm{Zn}$ between 450 and $510^{\circ}$. J. Phys. Chem. 63, 525 (1959), 2946.

Punched-cell batteries with polyethylene glycol electrolytes. J. Electrochem. Soc. 103, 417 (1956). 1828.

Wax batteries. Proc. 12th Annual Battery Research and Develop. Conf. Sponsored by Power Sources Div. U.S. Army Signal Corp. Research \& Devel. Lab. (Ft. Monmouth, New Jersey), p. 62 (1958). 2866.

Wood, R. E., Hamer, W. J., Electrolytic conductivity and electrode processes. Handbook of Physics Edited by E. U. Condon and H. Odishaw, Chapter 9 , Pt. 4, p. 4-138. 2500.

Wood, W. H., Low-cost microvolt potentiometer. Rev. Sci. Instr. 28, No. 3, 202 (1957). 2178.

Woodyard, O. C., Cottony, H. V., Elliott, R. S., Jordan, E. C., Rumsey, V. H., Siegel, K. M., Wait, J. R., Antennas and waveguides, and annotated bibliography. U.S.A. National Comm. Rept. Intern. Sci. Radio Union Subcomm. 6.3. IRE Ant. Prop. AP-7, 87 (1959). 2897.

U.S.A. National Committee report URSI Subcommission 6.3, antennas and waveguides, and annotated bibliography. Proc. 12th Gen. Assembly, International Scientific Radio Union, August-September 1957 (Boulder, Colorado) 11, Pt. 6, 121 (1957). 2402. 
Woolley, H. W., Effect of dissociation on thermodynamic properties of pure diatomic gases. Natl. Advisory Comm. Aeronaut. Tech. Note 3270 (1955). 1326.

Representation of gas properties in terms of molecular clusters. J. Chem. Phys. 21, 236 (1953). 815.

Thermodynamic properties of gaseous nitrogen. Natl. Advisory Comm. Aeronaut. Tech. Note 3271 (1956). 1964 .

Thermodynamic properties of gases at high temperature: 1 . Chemical equilibrium among molecules, atoms, and atomic ions considered as clusters. J 61, 469 (1958) RP2916.

Woolley, H. W., Beckett, C. W., Green, M. S., Thermochemistry and thermodynamics of substance. Ann. Rev. Phys. Chem. 7, 287 (1956). 1963.

Woolley, W. H., Benedict, W. S., Generalized tables of corrections to thermodynamic properties for nonpolar gases. Natl. Advisory Comm. Aeronaut. Tech. Note 3272 (1956). 1721.

Work, R. N., Quinn, F. A., Jr., Roberts, D. E., Volumetemperature relationships for the room temperature transition in Teflon. J. Appl. Phys. 22, 1084 (1951). 356.

Worksman, T. L., McLeod, G. L., Improved dimensional stability in laminated map paper, Tech. Assoc. Pulp Paper Ind. 41, No. 9, 484 (1958). 2595.

Worksman, T. L., Wilson, W. K., Harvey, J. L., Mandel. $J$., Accelerated aging of record papers compared with normal aging. Tappi 38, No. 9, 543 (1955). 1251.

Worthington, C. R., Mandelkern, L., Posner, A. S., Lowangle X-ray diffraction of fibrous polyethylene. Science 127, 1052 (1958). 2616.

Worthington, V., Extending the range of the NBS stiffness tester. Tappi $\mathbf{3 7}, 384$ (1954), 1020.

Worthington, V., Carson, F. T., Diaphragm error and testing rate in the paper bursting test. Tappi 35, No. 12,539 (1952). 428.

The stiffness of paper. Tappi 36, 36 (1953). 883.

Worthington, $V$., Hobbs, $R$. B., Measuring the stiffness of paper. Modern Packaging 26, 127 (1953). 762.

Worthington, V., Lashof, T. W., Mandel, J., Use of the sensitivity criterion for the comparison of the Bekk and Sheffield smoothness testers. Tappi 39, No. 7, 532 (1956). 1970.

Wranglen, G., Brenner, A., Cathode film studies by the drainage method. Sartrych ur svensk kemish tidskrift (Sweden) 67, 81 (1955). 1282.

Wright, J. H., Skamstad, H. K., An analog computer for radioactive fallout prediction. Proc. Natl. Simulation Council (1956). 1616.

Wright, J. W., Analysis of ionospheric vertical soundings for electron density profile data. (1959) TN19 (PB151378).

Wright, J. W., Fine, L. A., Mean electron density variations of the quiet ionosphere. (1960) TN40-1 (PB151399-1); (1960) TN40-2 (PB151399-2).

Wright, J. W., Knecht, R. W., Davies, K., Ionospheric vertical soundings. Annals of the International Geographical Year, 3, Pt. 1. 1 (Pergamon Press, New York, N.Y.) (IGY Instruction Manual: The Ionosphere Part 1) (1957). 2173.

Wright, J. W., Norton, R. B., Analysis of ionospheric vertical soundings for electron density profile data. 1. Facilities for convenient manual reduction of ionograms. (1959) TN14 (PB151373).

$W u, C$. S., Ambler, E., Hayward, R. W., Hoppes, D. D., Hudson, R. P., An experimental test of parity conservation in beta decay. Phys, Rev, 105, 1413 (1957). 2015.

Further experiments on $\beta$-decay of polarized nuclei. Phys. Rev. 106, No. 6, 1361 (1957). 2132.

Wyckoff, H. O., Measurement of cobalt-60 and cesium137 gamma rays with a free-air chamber. J 64C2, $87(1960)$.

Radiation attenuation data. Section B, p. 1, Radiation Hygiene Handbook, Edited by Hanson Blatz (McGraw-Hill Book Co., Inc., New York, N.Y., 1959). $\quad 3027$.
Radiation protection in therapeutic radiology. Am J. Roentgenol. Radium Therapy Nuclear Med. 73, 655 (1955), 1467 .

Wyckoff, H. O., Allisy, A., DeLaVergne, L., An intercomparison of the French and U.S. roentgen ray standards. Acta Radiol. 48, No. 6, 486 (1957) 2017.

Wyckoff, H. O., Aston, G. H., A comparison of X-ray standards. Acta radiol. Suppl. 117, 17 (1955). 1230 .

Wyckoff, H. O., Aston, G. H., Smith, E. E., A comparison of X-ray standards. British J. Radiol. 27, No. 318, 325 (1954) 916 .

Wyckoff, H. O., Attix, F. H., Design of fresh-air ionization chambers. (1957)' H64.

Wyckoff, H. O., Bay, Z., Mann, W. B., Seliger, H. H. Measurement of $\mathrm{W}_{\mathrm{Bir}}$ for sulfur-35 beta-rays. Radiol. Research Abstract 5, No. 4, 9 (1959). 1769.

Absolute measurement of $W_{\mathrm{Bir}}$ for sulfur-35 beta rays. Radiation Research 7, No. 6, 558 (1957). 2008 .

Wyckoff, H. O., Goldstein, N., Fano, U., Directional distribution of 1040 -kev radiation from a high volt ge X-ray tube. J. Appl. Phys, 22, 417 (1951). 224.

Wyckoff, H. O., Hart, E. J., Koch, H. W., Petree, B., Schulman, J. H., Taimuty, S. I., Measurement systems for high-level dosimetry. 2d United Nations Intern. Conf. on the Peaceful Uses of Atomic Energy, Vol. 21, Health and Safety: Dosimetry and Standards, P/1927 USA, p. 188 (Pergamon Press, London, England) (1959). 2984.

Wyckoff, H. O., Kirn, F. S., Kennedy, R. J., The attenuation of gamma rays at oblique incidence. Radiology 63, 94 (1954). 1171.

Wyckoff, H. O., Motz, J. W., Miller W., Eleven-Mev thick target bremsstrahlung. Phys. Rev. 89, 968 (1953). 709.

Wyckoff, H. O., Motz, J. W., Miller, W., Gibson, H. F., Kirn, F. S., Gamma-ray measurements by the magnetic analysis of Compton electrons. Rev. Sci. Instr. 24, 929 (1953). 725.

Wyckoff, H. O., Taylor, L. S., Gray, L. H., Program of the International Commission on Radiological Units and Measurements, 2d United Nations Intern. Conf. on the Peaceful Uses of Atomic Energy, Vol. 21, Health and Safety: Dosimetry and Standards, $\mathrm{P} / 2243$ WHO, p. 81 (Pergamon Press, London, England) (1959). 3022 .

Wyckoff, H. O., Thoraeus, R., Calibration of the portable Swedish free-air chamber equipment at the U.S. National Bureau of Standards. Acta radiol. 46, 741 (1956). 1644.

Wyckoff, $J . M$., The construction and operation of a high pressure diffusion cloud chamber. Phys. Rev. 87, 185 (1952). 568.

Wyckoff, J. M., Koch, $H$. W., Response functions of total-absorption spectrometers. Reprint IRE Trans. Nuclear Sci. NS-5, No. 3, 127 (1958), 2740.

Wyckoff, J. M., Temmer, G. $M$., Alpha-gamma angular correlation in ionium (TH ${ }^{230}$ ). Phys. Rev. 92, 913 (1953). 643.

Wyly, R. S., Comments on capacities of plumbing drains. Yearbook of the Am. Soc. Sanit. Engr. 36, 243 (1958). 2489. Problems of drainage and venting. Master Plumber and Heating Contractor 21, No. 3, 22 (1951). 289.

Wyly, R. S., Bain, K, Faegre, M. L., Behavior of young children under conditions simulating entrapment in refrigerators. Pediatrics 22, No. 4, Pt. I, 628 (1958). 2471.

Wyly, R. S., Eaton, H. N., Capacity of plumbing stacks. The Plumber and J. of Heating (London, England) 76, No. 912, 26 (1955). 1281.

Wyman, G. M., Bilbo, A. J., Steric hindrance to coplanarity in o-fluorobenzidines. J. Am. Chem. Soc. 75, 5312 (1953). 837.

Wyman, G. $M$., Brode, W. R., The relation between the absorption spectra and the chemical constitution of 
dyes. XXII. Cis-trans isomerism in thioindigo dyes. J. Am. Chem. Soc. y3, 1487 (1951). 341. The relation between the absorption spectra and the chemical constitution of dyes. XXIV. Absorption spectra of some thioindigo dyes in sulfuric acid. J. Am. Chem. Soc. \%3, 4267 (1951). 342.

Wyman, G. M., Brode, W. R., Gould, J. H., The relation between the absorption specta and the chemical constitution of dyes. XXV. Phototropism and cis-trans isomerism in aromatic Azo compounds. J. Am. Chem. Soc. 74, No. 18, 4641 (1952). 593.

The relation between the absorption spectra and the chemical constitution of dyes. XXVI. Effect of solvent and of temperature on the cis-trans isomerization of Azo dyes. J. Am. Chem. Soc. 75, 1856 (1953). 881.

Wyman, G. M., Brode, W. R., Gould, J. H., Whitney, J.E., A comparative survey of spectrophotometers in the $210-760 \mathrm{~m} \mu$ region. J. Opt. Soc. Am. 43, 862 (1953). 625.

Wyman, G. M., Brode, W. R., Seldin, I. L., Sperri, P. E., The relation between the absorption spectra and the chemical constitution of dyes. XXVIII. The hydration of Azo dyes in organic solvents. J. Am. Chem. Soc. $7 \gamma, 2762$ (1955). 1543.

Wynne-Jones, W. F. J., Bates, R. G., Guggenheim, E. A., Harned, H. S., Ives, D. J., Janz, G. J., Monk, C. B., Robinson, R. A., Stokes, R. H., Standard electrode potential of the silver, silver chloride electrode. J. Chem. Phys. 25, 361 (1956). 1895.

Wyszecki, G., A graphical interpretation of a threecomponents theory of chromatic adaptation in terms of the CIE chromaticity diagram. J. Opt. Soc. Am. 44, 787 (1954). 918 .

A regular rhombohedral lattice sampling of Munsell renotation space. J. Opt. Soc. Am. 44, 725 (1954). 930.

Invariance of insidedness in projective transformations of the Maxwell traingle. J. Opt. Soc. Am. 44, 524 (1954). 1046.

Wyszecki, G., Judd, D. B., Extension of Munsell renotation system to very dark colors. J. Opt. Soc. Am. 46, $281(1956)$. 1710.

\section{$\mathbf{Y}$}

Yannakopoulos, T., Brenner, A., Isolation of the diffusion layer at an electrode and the determination of concentration polarization. J, Electrochem. Soc. 105, No. 9, 521 (1958). 2605.

Yarbrough, $H$., Norton, $K$. A., Schultz, E. L., The probability distribution of the phase of the resultant vector sum of a constant vector plus a Rayleigh distributed vector. J. Appl. Phys. 23, No. 1, 137 (1952). 591.

Yates, $W$. A., Davidson, $M$., Wide-range calibrator for vibration pickups. Electronics 26, 183 (1953). 911.

Yevdjevich, $V$. M., Analytical integration of the differential equation for water storage. J 63B 1, 43 (1959). Effect of sudden water release on the reservoir free outflow hydrograph. J 63B2, 117 (1959).

Yoder, H. S., Weir, C. E., Change of free energy with pressure of the reaction nepheline + albite $=2$ jadeite. Am. J. Sci. 249, 683 (1951), 203.

Yokley, C. R., Ferguson, R. E., Products of cool flame oxidation of propane-2-C ${ }^{13}$. 7 th Symp. on Combustion (Butterworths, London, England), p. 113 (1958). 2702 .

Separation of the products of cool flame oxidation of propane. Combustion and Flame 2 , No. 2, 117 (1958). 2753.

Yost, E. L., Sweeney, W. T., Fee, J. G., Physical properties of plastic teeth. J. Am. Dental Assoc. 56, 883 (1958). 2688.

Yost, E. L., Sweeney, W. T., Sheehan, W. D., Mechanical properties of direct filling resins. J. Am. Dental Assoc. 49, 513 (1954), 1069.

Youden, $W$. $J$., A note on the four by four Latin squares. Biometrics 6, 289 (1950). 5.

Comparative tests in a single laboratory. ASTM Bull. No. 166, 48 (1950). 39 .
Design of experiments in operations research. Operations Research for Management II, 282 (1956). 1670. Design of experiments in research and development. Proc. of Conference on Design of Experiments in Army Research, Development, and Testing, Office of Ordnance Research Report 57-1, (Durham, N.C.), p. $9(1957) .2083$.

How statistics improves physical, chemical, and engineering measurements. Revised text of a lecture, presented earlier by Dr. Youden, has been issued in mimeographed form by (and copies are available from) the Committee on Experimental Design, Agricultural Research Administration U. S. Department of Agriculture, Washington, D.C. 68.

Index for rating diagnostic tests. Cancer 3, No. 1, 32 (1950). 71.

Instrumental drift. Science 120, 627 (1954). 1041. Locating sources of variability in a process. Ind. Eng. Chem. 43, 2059 (1951). 253.

National physical standards and design of experiment. Bull. International Statistical Inst. 35, 191 (1957). 2209.

Problems arising in engineering investigations. Proc. 1st Annual Statistical Engineering Symposium, held by the Chemical Corps Engineering Agency at Army Chemical Center, Md., 60 (1955). 1457.

Sets of three measurements. Sci. Monthly 77, 143 (1953). 825.

Statistical aspects of analytical determinations. The Analyst 7\%, No. 921, 874 (1952). 553.

Statistical design of experiments. Handbook of Physics, Edited by E. U. Condon and H. Odishaw, Chapter 13, Pt. 1, p. 1-165 (McGraw-Hill Book Co., Inc., New York, N.Y.) (1958). 2500.

Statistics and planning tests at elevated temperatures. Proceedin $\sigma_{s}$ of the Society for Experimental Stress Analysis XI, No. 2, 219 (1950). 137.

Statistics in analytical chemistry. Ann. New York Acad. of Sci. 52, Art. 6, 815 (1950). 138.

Technique for locating source of variability in a process. Ind. Eng. Chem. 45, No. 9, 2059 (1951). 324 .

The fisherian revolution in methods of an experimentation. J. Am. Stat. Assoc. 46, 47 (1951). 335.

The interpretation of chemical data. Conf. Papers of the 5th Annual Convention of the Am. Soc. for Quality Control, May 23, 1951 (Rochester Institute of Technology, Rochester, N.Y.), 8, No. 6, 90 (1952). 579.

The principles of experimental design. Appeared as a chapter in "Research Operations in Industry," (Papers delivered at the Third Annual Conference on Industrial Research, June 1953), p. 317 (Kings Crown Press, Columbia University, New York, N.Y.) (1953), 880 .

Youden, W. J., Cameron, J. M., The selection of a limited number from many possible conditioning treatments for alloys to achieve best coverage and statistical evaluation. Am. Soc. Testing Materials Proc. 50, 951 (1950), 155.

Use of statistics to determine precision of test methods. Am. Soc. Testing Materials Spec. Tech. Publ. No. 103, 27 (1950). 165.

Youden, $W$. J., Conner, $W$. S., Making one measurement do the work of two. Chem. Engr. Progr. 49, 549 (1953). 757 .

The chain block design. Biometrics 9, 127 (1953). 852.

Youden, $W$. J., Hader, R. J., Experimental statistics. Anal. Chem, 24, 120 (1952). 445.

Youden, W. J., Hunter, J.S., Partially replicated latin squares. Biometrics 11, 399 (1955). 1442.

Youden, W. J., Mann, W. B., Stockmann, L. L., Schwebel, A., Mullen, P. A., Garfinkel, S. B., Preparation of new solution standards of radium. J 62, 21 (1959) RP2924.

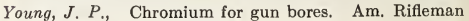
104, 39 (1956). 1647. 
Young, J. P., Lamb, V. A., Chromium plating of gun bores. Ánn. Proc. Am. Electroplaters' Soc. $\mathbf{2 6 0}$ (1956). 1648.

Experimental plating of gun bores to retard erosion. (1960) TN46 (PB151405).

Young, S. M., Severo, N. C., Newman, A. E., Zelen, M., Some applications of statistical sampling methods to outgoing letter mail characteristics. (1959) TN16 (PB151375).

Yowell, E. C., Some general precepts for programmers. Proc. of the Symp. on Elec. Computers held by the Western Section of the Elec. Computers Prof. Group at the IRE, April 30, May 1-2, 1952 (University of California, Los Angeles, California) X:1-X:6 (1952), 545.

The determination of eigenvectors and eigenvalues of symmetrio matrices. Proc. Computation Seminar, IBM, p. 112 (1951). 330.

Yowell, E. C., Blanch, G., A guide to tables on punched cards. Math. Tables and Other Aids to Computation 5, No. 36, 185 (1951). 177.

Addendum to a guide to tables on punched cards. Math. Tables and Other Aids to Computation VII, 1 (Jan. 1953). 641.

Yowell, E. C., Huskey, H. D., Thorensen, R., Ambrosio, $B, F$., The SWAC-design features and operating experience, Proc, IRE 41, 1294 (1953). 885.

Yowell, E. C., Southard, T. H., An alternative "preditorcorrector" process. Math. Tables and Other Aids to Computation, VI, No. 40, 253 (1952). 389.

\section{$\mathbf{Z}$}

Zacharisen, D. H., World maps of $\mathrm{f} 2$ critical frequencies and maximum usable frequency factors. (1959) TN2 (PB151361).

Zahn, C. T., Schweitzer, W. G., Jr., Excitation and separation of pure high-order modes in large high-Q cavities. J. Appl. Phys. 27, No. 8, 929 (1956). 1708.

Zalubas, $R$., A preliminary list of levels and $g$-values for the first spectrum of Thorim (Th I). J 63A3, 275 (1959).

New description of thorium spectra. (1960) Mono. 17.

Zapf, T. L., Capacitor calibration by step-up methods. J 64C1, 75 (1960).

Variable capacitor calibration with an inductive voltage divider bridge. (1960) TN57 (PB161558).

Zapf, T. L., Hermach, F. L., Lead resistance errors in watthour-meter phantom load test. Elec. World 141, 113 (1954). 1049.

Zei, D., White, H. S., Special lubricants tests. Mech. Eng. 73, 657 (1951). 311.

Zelen, $M$., A note on partially balanced designs. Ann. Math. Stat. 25, No. 3, 599 (1954). 923.

Analysis for some partially balanced incomplete block designs having a missing block. Biometrics 10, No. 2, 273 (1954). 953.

Experiments with many factors, Proc. 3d Annual Statistical Engineering Symp. (Chemical Center, Md.), p. 1 (1957). 2120.

Multi-factor experiments for evaluating reliability. Proc, of Joint Military Industry Guided Missile Reliability Symp. (Point Mugu, Calif.), p. 2-17. (1957). 2207.

The analysis of convariance for incomplete block design. Biometrics 13, No. 3, 309 (1957). 2342.

The analysis of incomplete block designs, J. Am. Stat. Assoc. 52, 204 (1957). 2343.

The use of group divisible designs for asymmetrical factorial arrangements. Ann. Math. Stat. 29, 22 (1958). 2836.

Zelen, M., Connor, W. S., Fractional factorial experiment designs for factors at three levels. (1959). AMS54 Multi-factor experiments. Ind. Quality Control 15, No. 9, 1 (March 1959). 2995.
Zelen, M., Eisenhart, C., Elements of probability. Handbook of Physies, Edited by E. U. Condon and H. Odishaw, Chapter 12, Pt. 1, p. 1-134 (McGraw-Hill Book Co., Ine., New York, N.Y.) (1958). 2500.

Zelen, M., Joel, L. S., The weighted compounding of two independent significance tests. Ann. Math. Stat. 30, No. 4, 885 (1959), 3098.

Zelen, M., Severo, N. C., Newman, A. E., Young, S. M., Some applications of statistical sampling methods to outgoing letter mail characteristics. (1959) TN16 (PB151375).

Zemantowsky, $M$. T., Synthetically bonded molding sand. Am. Foundryman 23, 57 (1953). 846.

Zendle, B., Boag, J. W., Scattered radiation contribution to depth dose data for 35-Mev and 90-Mev synchrotron X-rays. Pbys. Rev. 95, 608A (1954). 1135 .

Zendle, B., Koch, H. W., Dose measurements in water using scintillation and ionization detectors. Radiation Research 1, No. 6, 568A (1954). 995.

Zendle, B., Koch, H. W., McElhinney, J., Boag, J. W., Studies of dose distributions in water for betatron X-rays to $37 \mathrm{Mev}$. Radiol. Research 5, No. 2, 107 (1956). 1904.

Zendle, B., McElhinney, J., Domen, S. R., Calorimetrio determination of the power in a $1400 \mathrm{kev}$ X-ray beam. Radiol. Research 6, No, 1, 40 (1957). 2047.

Zentner, V., Brenner, A., Jennings, $C$. W., Physical properties of electrodeposited metals, I: Nickel, 3: The effect of plating variables on the structure and properties of electrodeposited nickel. Plating $\mathbf{3 9}$, 865 (1952). 511.

Zerfoss, S., Walker, R. F., Holley, S. F., Gross, L. J., Temperature of the inversion in cristobalite. J 61, 251 (1958) RP2902.

Zernicke, F., Saylor, C. P., Brice, A. T., Color phase contrast microscopy: requirements and applications. J. Opt. Soc. Am. 40, 329 (1950). 37.

Ziegler, C. A., Schwebel, A., Technique for monitoring tritiated-water vapor in air. Nucleonios 15, No. 1, 64 (1957). 2338.

Ziegler, C. A., Seliger, H. H., Hysteresis effect in multiplier phototube noise. J. Appl. Phys, 26, No. 10, 1225 (1955). 1361.

Liquid-scintillator temperature effects. Nucleonics 14, No. 4, 49 (1956). 1759.

Temperature effects in gas-free liquid scintillators. IRE Trans. Nuclear Science NS-3, 4 (1956). 1915.

Ziegler, C. A., Seliger, H. H., Jaffe, I., Oxygen quenching and wavelength shifters in liquid scintillators. Phys. Rev. 99, No. 2, 663; Bull. Am. Phys. Soc. 30, No. 3, $64 \mathrm{~A}$ (1955). 1439.

Role of oxygen in the quenching of liquid scintillators. Phys. Rev. 101, No. 3, 998 (1956). 1858.

Three ways to inorease efficienoy of liquid scintillators. Nucleonics 14, No. 5, 84 (1956). 1966.

Ziegler, $W . T$., The vapor pressures of some hydrocarbons in the liquid and solid state at low temperatures. (1959) TN4 (PB151363).

Zimm, B. H., Hoffman, J. D., Rate of thermal diffusion of polymer molecules in solution. J. Polymer Sci. 15,405 (1955). 1472.

Zimmerman, E. W., Hart, V. E., Horowitz, E., Determination of sulfur in rubber vulcanizates. Anal. Chem. 27, No. 10, 1606 (1955). 1311.

Zimmerman, E. W., Pangborn, E. F., Determination of grease in leather. J. Am. Leather Chemists Assoc. 46, 342 (1951). 219.

Zurich, V. J., Watt, A. D., Coon, R. M., Reduction of adjacent-channel interference components from frequency-shift-eyed carriers. IRE Trans. Commun. Systems CS-6, 39 (1958). 2723.

Reduction of adjacent-channel interference from onoff keyed carriers. IRE Trans. Commun. Systems CS-4, 41 (1956), 1841. 


\section{SUBJECT INDEX}

\section{A}

$\mathrm{A}$ and $\mathrm{A}^{*}$, commutators, 1653

Abelian, algebraic number fields, generalization of the normal basis, 1791

groups of unimodular matrices, 2886.

Aberration, chromatic, secondary, 2295.

longitudinal spherical, optical T-bench method of measuring, RP2880.

Abrasion, textiles, interpretation of tests for resistance, 473. resistance, 2005 .

resistance measurements on cotton fabrics made with the blade type abradant and the Schiefer abrasion testing machine, 382 .

testing, 1089

testing machine, Schiefer, 382.

Abrasive jet method for measuring abrasion resistance of organic coatings, 1249.

Abrasive-fluid mixtures, 221.

Abscissas and weights for Gaussian quadratures of high order, additional: values for $n=64,80$, and 96 , RP2875.

Absence, interference effects in the $\beta$-decay of polarized $\mathrm{CO}^{56}$ and $\mathrm{CO}^{58}$ nuclei, 2006.

spontaneous emission of neutrons from samarium, 383 .

Absolute, intensities of the 721 and $742 \mathrm{~cm}^{-1}$ bands of $\mathrm{CO}_{2}$, 2007.

light-scattering photometer: I. Design and operation, RP2946.

measurement, 780 .

measurement of $W_{\text {sir }}$ for polonium-210 alpha particles, 2444.

measurement of $\mathrm{W}_{\text {air }}$ for sulfur-35 beta rays, 2008. measurements at the NBS, status, 2781 .

photometry of the Gegenschein, 1919.

points of a correlation, number of, 1943 .

temperature below $1^{\circ} \mathrm{K}$ : chromic methylammonium alum as a thermometric substance, 2009.

Absorbent materials, absorption of sound patches, 2010. Absorption, bands for the calibration of spectrometers from 2 to 16 microns, J 64A1, 29; Mono.16.

concrete of X-rays and gamma-rays, 1250.

change in the inversion spectrum of $\mathrm{ND}_{3}$ from resonant to nonresonant, 661 .

infrared, of liquid water from 2 to 42 microns, 1038 .

low-frequency wing of the $\mathrm{NH}_{3}$ inversion spectrum, 639.

line half-widths and intensities, slit function effects in the direct measurement, 1867 .

lines as very narrow pass filters, Zeeman-split, 3115 . sound by patches of absorbent materials, 2010 .

spectra and the chemical constitution of dyes, 1543.

spectra and the chemical constitution of dyes, relation. XXII. Cis-trans isomerism in thioindigo dyes, 341.

spectra and the chemical constitution of dyes. XXIV. Absorption spectra of some thioindigo dyes in sulfuric acid, 342 .

spectra and the chemical constitution of dyes, relation between, RP2823.

spectra, infrared, $75 ; 1569$.

spectra infrared, of some cyclic acetals of sugars, RP2960.

spectra of solids condensed at low temperatures from electric discharges, 2445.

spectra of some aldopyranosides, conformations of the pyranoid sugars, $\mathrm{J} 64 \mathrm{~A} 3,239$. spectral, method for determining population "temperatures" in hot gases, 1882.

spectrum of $\mathrm{ND}_{3}$, microwave, 766 .

spectrum of vibrationally excited $\mathrm{N}_{2}$ in active nitrogen, 2887.

Abstract algebra, two problems connected with Horner's rule, 1101.

Abundance of free atoms in solid nitrogen condensed at $4.2^{\circ} \mathrm{K}$ from a gas discharge, 1607 .

$\mathrm{Ac}^{227}$, nuclear moments, 1420 .

$\mathrm{A}-\mathrm{C}$, bridge for the audio-frequency range, 1615 .

bridge to measure core loss at high induction, use of, 1954 .

current, clamp-on-microammeter measures, 2056.

zero locator, 2414.

AC-DC transfer instruments for current and voltage measurements, 2446.

Accelerated aging of record papers compared with normal aging, 1251 .

Accelerating, components in particle spectrometers, 2649. convergence of iterative processes, 2447 .

effect of aminos on polymerization of methyl methacrylate, 1608.

Accelerators, pulsed, charge-storage pulse-height analyzers for use with, 2485 .

sulfinic acid derivatives, in the polymerization of methyl methacrylate, 1906.

Accelerometer, calibration, high-speed stroboscope for, 1031.

ceramic, 175 .

Ramberg vacuum tube, recent developments, 297.

Accelerometers, cemented piezoelectric, 660 .

dynamic characteristies, by rotation, 913 .

Acceptance sampling, electroplated articles, 940 .

variables with special reference to the ease in which quality is measured by average of dispersion, 16 .

Accomplishments at the International Conference in Bournemouth, England, Part III, 188.

Accuracy, 2448.

Accurate, radio-frequency microvoltages, 640 .

time-modulated pulse circuit, 646 .

Acetabacter suboxydans, isotope effect in oxidation of D-mannitol-2-C14, 2606.

Acetals, cyclic, of sugars, infrared absorption spectra, RP2960.

Acetate, cellulose, films, degradation, 2924.

cellulose, propionates, 237.

propionate, and butyrate radicals, heats of combustion of some peroxides and the heats of formation, 2148.

Acetylene, air, 2928.

methyltrifluoromethyl, internal rotation, 1374.

Acetylene-d $d_{1}$ infrared spectrum, 1934.

Acetylene-oxygen, fiame, kinetics of $\mathrm{OH}$ radicals, 476. flames, 2311.

Achromats, three color, selection of glasses, 3043 .

Acid, o-ethyoxybenzoic and other ehelating agents, 2829. picric, 1085.

sulfinic, derivatives as accelerators in the polymerization of methyl methacrylate, 1906.

treatment, effects of, on acid and abrasion resistance of porcelain enamels, 51 .

weak, ammonium salt, 49 .

Acids, bases, precise coulometric titration, J 63A2, 153 .

benzene, aromatic carboxylic, relative strengths, RP2871.

intercomparison of, RP2769. 
precise intercomparison of, by differential potentiometric titration with the hydrogen electrode, 2260.

Acoustic, field, 1223.

impedance measurement, 46.

impedance of right circular cylindrical enclosure, 941.

method for the measurement of vibration amplitudes, 946.

pulses, electromagnetic and, propagation, 1799.

wave field, diffraction and reflection, RP2906.

Acoustical tile, evaluation of adhesives, 55 .

Acoustics, architectural, standard methods of measurement, 2226.

Acrylate and styrene polymers in a vacuum, 811 .

Acrylic, plastic glazing, 699 .

plastic, glazing, properties of, 436 .

resins, crazing, 1298.

resins in prosthetic dentistry, 2449.

Acrylics, biaxial stretch-forming of, 658 .

stress-solvent crazing of, 839 .

Action of boron in exhancing the hardenability of steel, 851 .

Activities, NBS in the field of fire research, 1870.

National Bureau of Standards Cryogenic Engineering Laboratory, 1252.

Addendum to a guide to tables on punched cards, 641 .

Addition, multiplication, high-speed methods, application of digital computers to, C591, Sect. 1.

hydrogen atoms to solid olefins at $-195^{\circ}, 2803$.

theorems for general Legendre and Gegenbauer functions, 1253 .

Adherence of vitreous coatings to iron, effect of oxygen content of the furnare atmosphere, 1329.

Adherometer, electronic averaging device for, protective measurement, coating adhesion, 2273 .

Adhesion, electroplated coatings to titanium, 1920.

nature of, 1080 .

organic coatings by ultracentrifugal and other methods, evaluation of, 715 .

survey, 1157.

Adhesive-adherent systems, 208.

Adhesives, acoustical tile, 55 .

cryogenic structural, epoxy resins, 2547.

engineering, selecting, 306 .

Adiabatic, apparatus for the study of self-heating of poorly conducting materials, 942 .

calorimeter for the range $30^{\circ}$ to $500^{\circ} \mathrm{C}$, RP2848.

calorimetry, automatic temperature regulation and recording, 2035.

demagnetization and the temperature scale below $1^{\circ} \mathrm{K}, 384$.

Adjustable sliding termination for rectangular waveguide, 2012.

Admittance of a diode with a retarding field, 566 .

Adsorption, $\mathrm{C}^{14}$ dextran to human blood platelets and red blood cells in vitro, 2011.

diffusion, and evaporation of carbon monoxide on tungsten, 2888.

fractionating process, 156.

gases and vapors on glass spheres. II. Two dimensional condensation of oxygen, 1609 .

heats of, from charge-transfer complex theory, 2582.

polyesters on glass, silica and alumina, RP2933.

Advancements in dielectrics, $642 ; 943$.

Advances (developments) in synthetic materials in America in $1949,21$.

Aerial photoreconnaissance, spectrophotometry, 2308.

Aerodynamic, heating and fatigue, 2889.

phenomena in stella atmospheres, TN30 (PB151389).

Aerodynamical mechanisms producing electronic density fluctuations in turbulent ionized layers, 1254 .

Agar-agar type duplicating material and agar-agar on heating and storage, changes, 2050.

Age to indium resonance for D-D neutrons in water, 2450.

Agenda for commission on symbols, units, and nomenclature of the International Union of Pure and Applied Physics, 189.

Aging, changes in clinical thermometers, 385 .

heat, and ultraviolet, of poly (vinyl chloride), RP2863.
Karakul and seal fur skins, 19.

rubber vulcanizates, measurement, J 63C2, 141.

some effects of, on the surface of portland cement paste, J 64A2, 163.

Air, alpha particles in, collection of ions produced, 2488. dissolved on the speed of sound in water, effect, 1686 . interchange in, ion-atom, 1379.

lubricated piston gage for pressures below one-half inch of mercury, tilting, $\mathrm{J} 63 \mathrm{C} 1,47$.

mercury-, and flat-type cells, dry cell standards broadened-now includes, 1321 .

nitrogen, and oxygen, rotational relaxation, 3038 .

pollution, determination of carbon monoxide in studies, 1309.

replaces sand in "no-fines" concrete, 190.

shock, fragmentation of waterdrops in the zone behind an, RP2843.

slide rule computes radio refractive index of, 829 .

technique for monitoring tritiated-water vapor, 2338 .

truck tire, temperature studies, 1917.

Airborne, electronic equipment, new techniques for fabrication, 1415.

television station, TN35 (PB151394).

Aircraft, delta-wing, effect of rib flexibility on the vibration modes of, RP2852.

lighting, research, NBS development and testing, 771.

materials, creep and creep rupture characteristics of some riveted and spot-welded lap joints, 1299 .

materials, creep behavior of structural joints of, under constant load and temperatures, 2071.

plastics, 2255.

sheet materials for large strains, Poisson's ratio, 798.

Airglow, cells, 2640.

cells, characteristic size, 2807.

5577, possible movements, 2695.

night, 2825.

oxygen red lines, 2681.

photometric observations, 2247.

photometry, review of observational results, 1242.

Airglow and aurnra, 1271 .

origin of [OI] $5577, \mathrm{~J} 63 \mathrm{D} 1,15$.

photometric unit, 1598.

Airplane, camera calibration, 1878.

cameras, calibration, 2045.

mapping cameras, 32 .

Airspaces, fibrous insulations bounded by reflective surfaces, thermal resistance, BMS151.

thermal insulating value, 1203.

Airy functions, integrals of, AMS52.

Albedo of various materials for $1 \mathrm{Mev}$ photons, 1170 .

Aldehyde, carboxyl, pentosan, and degree of polymerization data on the ICCA "standard" pulps, 2890.

cellulose, determination, 1308.

Aldonic lactones, sodium borohydride reduction, 1868 .

Aldopyranosides, infrared absorption spectra of, conformations of the pyranoid sugars, J $64 \mathrm{~A} 3,239$.

Aldoses-1- $t$, J 64A2, 177.

modified celluloses, reaction of sodium chlorite, 1473.

Aleutian, NRL, radio eclipse expedition, 273.

Algebra, abstract, two problems connected with Horner's rule, 1101 .

electronics, $2803 \mathrm{~A}$.

Algebraic, equations satisfied by roots of natural numbers. 1610.

number theory: computation of cyclic cubic units, RP2783.

number theory estimates based on the Dedekind etafunction, 1871 .

proof of the isoperimetric inequality for polygons, 1259.

Alignate hydrocolloidal dental impression material, 986.

Alignment, cerium ${ }^{141}$ and neodynium ${ }^{147}, 1255$.

three odd-A rare-earth nuclei, 1611.

three rare-earth isotopes, 1256.

Alkali, borates, molten, viscosity and electrical resistivity of, 907 .

silicate binary glasses, role of vitrons, RP2854. 
silicate glasses, density formula for, from annealing to glass-processing temperatures, RP2915.

Alkali-aggregate, reaction and the ionic charge on hydrated cement, 2639.

reaction in concrete, physical-chemical studies, RP2780.

reactions, mechanism, 1406 .

Alkaline, chromate solutions, electrolytic coatings on magnesium base alloys, 1332 .

solution of potassium chromate as a transmittancy standard in the ultraviolet, 388 .

solutions for $\mathrm{pH}$ control, 1612 .

Alkaline-earth borates, 3059 .

Alkalis and alumina in ternary systems, 2785.

Allocation problem, coefficients, 2808.

Alloy, aluminum, alclad, 24S-T 86 sheet, 139.

aluminum, sheet, 1303.

magnesium, specimens, fatigue strength, 1531 .

nickel, copper, and nickel-copper, annealed, creep of, RP2836.

steels, slack-quenched impact properties, RP2799.

Alloys, aluminum, 2841A.

aluminum, effect of 20-year marine exposure, 1689 .

aluminum, two forged, investigation of bearing creep, TN55 (PB161556).

aluminum under various temperature conditions, 2505.

austenite iron-carbon-nickel, note on thermal stabilization, 777 .

chromium-cobsilt casting, for dental, proposed specification, 1996.

chromium cobalt, physical properties, 2687.

common commercial aluminum, thermal conductivities, 2842.

copper, thermal conductivities, 2393.

enthalpy and heat capacity from $0^{\circ}$ to $900^{\circ} \mathrm{C}$. of three nickel-chromium-iron of different carbon contents, RP2870.

gallium copper-tin, some physical properties, 1876 .

gallium with powdered metals as possible replacement for dental amalgam, 1613.

iridium-osmium, 1152 .

low-strategic, with a ehromium boride-nickel cermet coating, protection, 1463.

low temperatures, impact testing, 2819.

thermometric, $2833 \mathrm{~A}$.

thermometric, thermal emf, 2844A.

tin-indium in the normal state, thermoconductivity, 2845.

two-phase textural stresses in. RP2793.

Alpha, particles in air, collection of ions produced, 2488.

particles near 5-Mev and by beta particles, 2912 .

standards, NBS, 2644.

Alpha-chloro-acrylate, polymethyl, properties, 520 .

Alpha-gamma angular correlation in ionium ( $\left.\mathrm{TH}^{230}\right), 643$.

Alpha-particle, energy levels in $\mathrm{F}^{18}$, i aactions in nitrogen, 711.

Alternative, derivations of Fox's escalator formulae for latent roots, 386.

"predictor-corrector" process, 389.

proof of a theorem on unimodular groups, 1260.

Alum, chromic methylammonium, as a thermometric substance, absolute temperature below $1^{\circ} \mathrm{K}$, 2009.

Alumina, alkalis in ternary systems, 2785.

lanthana, svstem, 2472 .

shapes, zirconis, 173 .

Aluminum, alloys, thermal and electrical conductivity, $2841 \mathrm{~A}$.

alloys, two forged, investigation of bearing creep, TN55 (PB161556).

antimonide, 1007.

concrete, isotropic sources through, penetration of gamma rays, TN11 (PB151370).

degree in orientation and crystal size on the scattering of 20 -kev eleetrons, 2529 .

determination in precipitation hardening stainless steel and high temperature alloys, J 64A2, 181.

electrodeposition of, hydride bath, 363 . electrons in, temperature dependence of the characteristic energy loss, 2798.

foil, mineral-wool blanket, and mineral-wool board, thermal insulating properties, BMS151.

gallium phosphate, low cristobalite type, erystal structure, 1923.

lead, and concrete in the range 275 to 525 kilovolts, X-ray attenuation, 1582 .

oxide, heat capacity, RP2848

oxide in the range $300^{\circ}$ to $700^{\circ} \mathrm{K}$, heat capacity, 2362 .

single crystals, growth of, RP2868.

steel, separation and determination of small quantities of, $\mathrm{J} 64 \mathrm{~A} 3 ; 235$

welded $5086 ; 3095$.

Aluminum-alloy, forgings, large, mechanical tests on specimens, 1772 .

sheet, 1303.

Aluminum alloy, alclad, 24S-T86 sheet, 139 .

Aluminum alloys, common commercial, thermal conductivities, 2842.

corrosion of, by exhaust gases, 979 .

effect of 20-year marine exposure, 1689.

under various temperature conditions, 2505 .

Amalgam, dental, 1613.

dental, microstructure, 765 .

dental: the effect of mechanical condensation of some physical properties, 423 .

sodium, in the form of pellets, preparation of, J $64 \mathrm{~A} 1$, 135 .

Amalgams, Ag-Sn (Cu-Zn), stability of, 1493.

Amelung glasses compared with some modern commercial glasses, 387.

American Chemical Society specifications, reagent chemicals, 1474

American Denta, 1 Association, contribution to dental science, 2891.

specification No. 3 for dental impression compound, revision, 1244

specification No. 9 for dental silicate cement, 20 .

specification No.12 for denture base resin, 644 .

specification No, 13 for self-curing repair resins, 1257.

specifications for dental materials, $1614 ; 2451$.

American dielectric standards, 944.

standard building code requirements for masonry, 945 .

American Standards Association, Report of the Committee, 3036.

Amerikanische Kunststoffentwicklung im Jahre 1949, 21. Amide nitrogen of collagen and hide powder, 645 .

Amino acid, polar, content of collagen and related material, 841 .

Aminos on polymerization of methyl methacrylate, accelerating effect, 1608 .

Ammonia, deuterated, 2411.

hydrazine with oxygen atoms and hydrogen atoms in atomic flames, reactions, 1948 .

inversion in, interaction of stretching vibrations, 1744.

maser as an atomic frequency and time standard, 2804 .

nitrogen, oxygen, hydrogen, and water, electric discharge, 2309

vibration-rotation bands, $2411 ; 2862 ; \mathrm{RP} 2894$.

Ammonias, deutero, microwave spectra, 260.

Ammonium, hexabromoosmate, 1201.

salt of a weak acid, 49 .

Ampere, 3064.

Amplifier, audio-frequency power, circuits, a survey, 187. cryostat heating control, 2090.

direct current, and recorder to balance a Müeller resistance bridge, 3107 .

feedback, with negative output resistance for magnetic measurements, 2421.

impedance transistor, 2586 .

Amplifiers, gain stable mixers with current feedback, 1022 . dielectric, 963 .

feedback, chart speeds design, 410 .

feedback, tuning systems, 352 .

feedback, with stabilized output impedance, 719. 
Amplitude, constant vector plus a Rayleigh-distributed vector, 1541 .

scale, and spectrum of refractive index inhomogeneities in the first 125 meters of the atmosphere, 1258.

scintillations of radio stars observed at Boulder, Colorado, a preliminary analysis, TN20 (PB151379).

stability of the cathode-coupled oscillator, frequency, 62.

stabilization of a microwave signal source, 2452.

Amplitude-probability distribution of atmospheric radio noise from statistical moments, determination, J 64D1, 49.

Amplitudes, vibration, acoustic method for the measurement of, 946 .

Amorphous, high polymeric systems, viscoelastic phenomena, 2863 .

polymers over an extended temperature range, volume temperature relations, 1975.

Ampoules of active materials, 8 .

Analog, computer for radioactive fallout prediction, 1616 .

equipment, representation of nonlinear functions of two input variables, 2285 .

Analog-digital, conversion equipment for electrocardiographic data, TN42 (PB151401).

simulation for the design and improvement of manmachine systems, 2453.

Analogs, discrete, of inequalities of Wirtlinger Monatsh 1313.

Analysis, anomalous properties of equiphase contours, 22 .

atomic spectra with electronic digital computere 1624

brittle behavior in ship plates, 954

calculation of immiscibility, 2320 .

collagen and collagen derivatives, paper chromatographic, 1597 .

continuous of ortho-parahydrogen mixtures, 1294.

convariance for incomplete block designs, 2342 .

dielectric loss in two urea addition compounds, 2454 .

films in, of tritium-labeled compounds, J 63A2, 171.

first spectrum of ruthenium (Ru I), J 63A3, 213.

fluorinated polyphenyls by mass spectrometer, 1263 .

goniophotometric reflection curves, 397.

hydrogen-deuterium mixtures in presence of air, optical spectrophotometric, 505 .

incomplete block designs, 2343.

ionic recombination including ion production during messurements, 193

ionospheric vertical soundings for electron density profile data. TN14 (PB151373); TN19 (PB151378).

millimicrosecond pulses using a charge-storage type pulse-height analyzer, 2895.

natural gas, 25.

numerical, modern, solution of. I. Differential equations by recurrence relations, 97 .

numerical, motivation for working, 1410.

numerical. 2. Note on the condition of matrices, 98.

numerical, notes, $271 ; 272$.

partially balanced incomplete block designs having a missing block, 953 .

photocross, 2460 .

propagation measurements made at 418 megacycles per second well beyond the radio horizon (a digest), J 64D3, 255.

propagation measurements made at $418 \mathrm{Mc}$ well beyond the radio horizon, TN6 (PB151365).

solar spectrum from 2990 to 2635 A, revised, 933 .

solids with the mass spectrometer, 194.

spectrochemical, index to the literature, 1036; 2965.

vibrational relaxation data in shock wave experiments. 2896.

(0.1) and (1.2) bands of the ${ }^{2} \Delta-{ }^{2} \pi$ system of $\mathrm{CH}$, 1625 .

Analyst, modern, and $\mathrm{pH}, 2242 \mathrm{~A}$.

Analytic, characteristic functions, 496 .

comparison of suggested configurations for automatic mail sorting equipment, J 63B2, 83 . functions, $1093 ; 1096$

functions, periodic, numerical integration, 3009 .

study of war games, 1264.

systems for linear differential equations, 932.

Analytical, chemistry, statistics, 138 .

data, statistical treatment, 2780 .

determinations, statistical aspects, 553 .

distillation, 2744.

integration of the differential equation for water storage, J 63B1, 43.

mass spectrometry, 1626 .

methods by the sensitivity criterion, evaluation, 2112 .

procedure, a plan for studying the accuracy and precision of, 926 .

procedures: natural and synthetic rubbers, 2020.

Analyzer, stroboscopic vibration, $\mathrm{J} 63 \mathrm{C} 2,97$.

Analyzing, extreme-value data, 922.

straight-line data, 652 .

Anelasticity of quartz, 653 .

Anemometry, hot-wire, 247.

Anesthesia, physological monitor, 1446.

Angular, correlation of radiations with parallel angular mcments, 2021.

correlations, geometrical characterization of nuclear states and the theory of, 726 .

Angular distribution, multiple-scattered gamma radiation from a plane isotropic source, 1265 .

neutral meson decry, 2461.

Anhydrous, gallium orthophosphate, temperature inversions, 1916 .

indium phosphate and thallic phosphate by X-ray diffraction, crystal structure, 1667.

scandium phosphate, structure, 1950.

Anisotropic, inhomogeneous medium, wave propagation in, 910 .

media, elastic waves, 2104.

relaxation peak in the internal friction of crystalline quartz, 2022.

Annealed nickel, copper, and two nickel-copper alloys, creep of, RP2836.

Annealing, silicate glasses during, structural rearrangements, 1901 .

Annual, report of the Director of the National Bureau of Standards for fiscal years ending June 30 .

1957: M223

1958: M226

1959: M229

review of pulp and paper industry, 1627; 2024.

Annular-slot antenna, low frequency, RP2822.

Anodic (HAE) corting on the fatigue strength of magnesium alloy specimens, $1325 ; 1531$.

Another milestone in screw thread standardization, 2025.

Antenna, annular-slot, low frequency, RP2822.

curved lossy surface, 2682.

function of the diameter and length of the driven element, impedance of a corner-reflector, J $64 \mathrm{D} 2,135$.

gain, path, in an exponential atmosphere, $J$ 63D3, 273.

gain, techniques for accurate measurement, C598.

height at $100 \mathrm{Mc}$, radio transmission loss vs distance, 1470 .

heights, three transmitting, report on comparative 100 Mc measurements, 1848.

high-frequency, long distance horizontal radiation pattern, 2371 .

horizontal, over a spherical earth, low frequency radiation, 1761 .

insulated loop, immersed in a conducting medium, RP2781.

microwave, pattern of a flush mounted, RP2796.

monopole with symmetrical top loading, earth currents near, RP2959.

optimum, height for ionospheric scatter propagation, 3011 .

patterns, 481 .

radiation pattern of, mounted on a surface of large radium of curvature, 1946. 
sizé, effect of, on gain, bandwidth, and efficiency, $\mathrm{J} 64 \mathrm{D} 1,1$.

slot-array, on a finite and imperfect ground plane, patterns, 2826.

top-loaded monopole, earth currents near with special regard to electrically small $L$ - and $T$-antennas, RP2961.

top-loaded monopole, the electric field at the ground plane with special regard to electrically small L-and T-antennas, J 64D2, 139.

VHF monopole, 2437.

very-low-frequency, for investigating the ionosphere with horizontally polarized radio waves, J 64D1, 27.

Antennas, waveguides, 2402.

annotated bibliography, 2897.

corner-reflector, gains of finite-size, 2574.

high-gain, for VHF scatter propagation, 1729.

low frequency, portable, operating over ice and snow covered terrain, 2220 .

slotted-cylinder, pattern synthesis, J 63D3, 303.

spaced, far beyond the radio horizon, 1401 .

Antifreezes, engine, determination of freezing point, 331.

Antimonide, indium, elastic moduli, 1693.

electrical properties, 703 .

indirect transitions, 1736.

piezoelectric effects, 2690 .

piezoresistance, 2252.

thermoelectric power, 1559 .

Antimonide, n-type indium, galvanomagnetic effects, 2135 p-type indium, galvanomagnetic effects, 2136 .

Antioxidants on asphalt durability, effects of, J $64 \mathrm{C} 1,13$. Apatite, enamel, isomorphous substitution, 748.

Apparatus, determining freezing points at saturation pressure from time-temperature freezing and melting experiments, 26.

direct determination of the dynamic bulk modulus, 1629 .

etching fine points under controlled conditions, 1628.

measurement of the normal spectral emissivity in the infrared, $\mathrm{J} 64 \mathrm{C} 2,99$.

measurement of thermal conductivity of solids at lon temperatures, RP2805.

measuring the piezoresistivity of semiconductors, RP2814.

simple humidity, 1487.

Application, configuration interaction to the $\mathrm{H}_{3}$ complex, 2027.

low $\mathrm{M}_{\mu}$ triodes to series regulator circuits, 1630 .

Monte Carlo method to a problem in gamma-ray diffusion, 1618 .

photoelasticity to the design of tensile specimens for ceramic metal materials, 1266.

photoelectric multiplier tubes to the sensitive measurement of absorption or of changes of relative light intensities, 27.

preparation, and maintenance, of standards of radioactivity, C594.

RF micropotentiometers for calibration of signal generators to $1000 \mathrm{Mc}$, TN37 (PB151396).

theory of stochastic processes to chemical kinetics $2805 ; 2898$.

two methods of numerical analysis to the computation of the reflected radiation of a point source, 1267 .

variational methods to intermediate and high energy scattering, 955 .

viscosity methods to the study of branching in polysaccharides, 654 .

Applications, extreme-value theory, 1140.

standards of radioactivity, 1631 .

statistical sampling methods to outgoing letter mail characteristics, TN16 (PB151375).

theorem on partitioned matrices, J $63 \mathrm{~B} 2,73$.

theory of quadratic forms in Hilbert space to the calculus of variations, 195 .

Applied optics at NBS, 655 .

Approximate, daytime constancy of the absorption of radio waves in the lower ionosphere, 2661. expression for gamma-ray degradation spectra, RP2788.

theory on the stability of interfacial waves between two streams, 1632.

Approximation of linear elliptic differential equations by difference equations with positive coefficients, 785.

Approximations, discrete, to elliptic differential equations, 1314.

linear viscoelasticity theory: delta-function approximations, 956.

Are-discharge tubes in regulated low-voltage supplies, 615 Arc-lengths along the lines of force of a magnetic dipole, 1633.

Architectural acoustics, standard methods of measurement, 2226.

Arctic, communication, conference on, J 64D1, 73.

ionosphere IGY program, 1936.

Argon, nitrogen, negative atomic iodine ions, 605.

temperatures between $0^{\circ}$ and $75^{\circ} \mathrm{C}$ and at pressures up to 2500 atmospheres, thermal conductivity, 1952.

Arithmetic, 2500

Aromatic, carboxylic acids in benzene at $25^{\circ} \mathrm{C}$, relative strengths, RP2871.

hydrocarbons, electron impact studies, 2938.

hydrocarbons filtered from smoky air, mass spectra RP2876.

Arsenate, silicate, and phosphate, separation and determination, RP2954.

Arsenic sulfide glass, properties, RP2774.

trisulfide, refractive index, 2726.

Arsenide, $n$-type indium, oscillatory galvanomagnetic effects, 2678.

Artificial, auroras resulting from the 1958 Johnston Island nuclear explosions, 2899.

dentures, 1194.

Aryl silanes, 1192

Asbestos-cement pipes, 998.

L-ascorbic acid-C ${ }^{14}$, synthesis of, RP2800.

Ashes, refractoriness, chemical analyses, surface area, and thermal reactions of natural graphite, and, 665 .

Aspects, charge and discharge processes in lead-acid storage batteries, 1141 .

depolymerization kinetics, 307.

gamma ray penetration theory relevant to shielding problems, 2028.

large scale Jiquefaction of hydrogen, 1491.

prediction in the study of complex systems, 2225.

tropospheric radio wave propagation, 1872 .

Asphalt, changes in the properties, during the blowing operations, RP2835.

durability, effects of antioxidants, J 64C1, 13.

effect of light and water on the degradation of, J $63 \mathrm{C} 1$, 31.

influence of exposure conditions on the accelerated durability testing, 1738 .

research at the National Bureau of Standards, 2029.

water-soluble degradation products, 2413.

Asphalts, infrared spectra of, J 63A2, 189.

Assignment problem, 1921.

ASTM, crude natural rubber subcommittee, 3065 .

methods and standards for appearance evaluation, status of, $3055 \mathrm{~A}$.

problems, student reasearch, 1903.

Astronomical spectra, lines of the chemical elements, 1758.

Astronomy, new method of solution for unretarded satellite orbits, J 63B2, 105.

solar radio, at the NBS, 1490.

Astrophysical, a multiplet table, of interest, TN36 (PB 151395).

interest, partial Grotrian diagrams, 1758.

Astrophysics, TN36 (PB151395).

atomic spectra-their role, 1974 .

Asymmetric rotor molecules, tables of line strengths for rotational transitions, 2796 .

Asymmetrical factorial arrangements, the use of group divisible designs, 2836 . 
Asymptotic, behavior of tests on the mean of a logarithmico-normal distribution with known variances. 2030.

expansion of error integrals, brief note, 1383.

expansions for the parabolic cylinder functions, 366 .

expansions in general regions, uniqueness theory, 2400.

lower bounds for the frequencies of certain polygonal membranes, 957.

series for functions occurring in the theory of diffraction of waves by wedges, 1792 .

solution of the differential equation of hydrodynamic stability in a domain containing a transition point, 656 .

transformation of certain distributions into normal distribution, 1795.

Atlas of oblique-incidence ionograms, TN31 (PB151390).

Atmosphere, circularly stratified, focusing, defocusing, and refraction, J $64 \mathrm{D} 3,287$.

CRPL exponential reference, Mono. 4.

earth's, OH emission, 3001.

earth's outermost, interplanetary space, 2969.

earth's upper, gravitational and thermal oscillations, 1726 .

exponential reference, Central Radio Propagation Laboratory, J 63D3, 315.

first 125 meters, amplitude, scale, and spectrum of refractive index inhomogeneities, 1258.

furnace, on the adherence of vitreous coatings to iron, effect of oxygen content, 1329.

infrared spectra of molecules, 2377.

inhomogeneous, radio wave propagation, TN24 (PB151383).

lower, average radio-ray refraction, 400.

non-equilibrium, source function, 2767.

path antenna gain in an exponential, J 63D3, 273.

radio, at short ranges, waveform, 1800 .

radio waves in, oxygen and water vapor absorption, 2241.

solar, motion in, as deduced from radio measurements, 263.

sun, elements in, 1138.

turbulent, over irregular terrain, propagation, 1550.

turbulent, rate of fading in propagation, 1542 .

upper, excitation mechanisms of the oxygen 5577 emission, J 63D3, 319.

upper, in the auroral zone, 2766.

upper, negative oxygen ions, 1413.

upper, study of radio reflections from meteor trains in research, 144.

Atmospheres, stellar, 2463.

stella, aerodynamic phenomena, TN30 (PB151389).

Atmospheric, bending of radio waves, 2806 .

circulations and tropospheric scatter propagation, $J$ $64 \mathrm{D} 1,81$

dynamo theory, 2228.

horizontal inhomogeneity, effect of upon ray tracing J 63D3, 287.

negative ions, photodetachment, 2245 .

noise from 1 to $100 \mathrm{Kc} / \mathrm{s}$, characteristics, 2052.

noise, measurement, 2189.

noise, VLF, 2351.

oscillations, extension of the Sen-White paper, 1341. oscillations, thermal and gravitational excitation, 1555.

ozone, continuous measurement, by an automatic photoelectric method, 977.

pressure, rotational and vibrational "temperatures" of $\mathrm{CH}$ in flames, 816 .

radio noise data, report, 1851 .

radio noise from statistical moments, determination of the amplitude-probability distribution, J $64 \mathrm{D} 1,49$.

radio noise, VLF, 2188.

radio refractive index, 3002 .

refractive index at radio frequencies, 855 .

refractive index, microwave measurement of variations, 585 .

research, $2031 ; 2462$. tides in barometric pressure, solar and lunar, 1586.

total, bending of radio rays at small elevation angles, TN44 (PB151403).

transmittance of Mauna Loa, studies, RP2910.

transverse, drag on satellite orbits, 2532 .

turbulence, 1402 .

turbulence, messurement of the phase of signals receited over transmission paths with electrical lengths, 1770 .

waveforms, some characteristics of VLF propagation, 2760 .

Atmospherics, measured frequency spectra of very-lowfrequency, J 64D $1,41$.

Atom, improved hydrogen, beam furnace, TN47 (PB151406).

nitrogen, recombination, kinetics of, 2972.

Atomic, berm, electron optical image, 1704.

besm sources and standird of length, 2464 .

clock, a universal standard of frequency and time, 148.

clock and frequency stabilization on microwave spectral lines, 196.

definition of primarv standards, 28.

electron affinities, 2900.

energv levels, C467, Vol. III.

explosions, temperature, 1528.

flames, reactions of ammonia and hydrazine with oxygen atoms and hydrogen atoms, 1948.

flames: spectra "temperatures" and products, 1268.

frequency and time standard, 2804.

frequency standard, 2555 .

hydrogen plus atomic sodium, chemiluminescence in the system, 2486.

molecular negative ions in stellar atmospheres, 2463.

molecular time standards at the NBS, 2549.

negative, iodine ions in nitrogen and argon, total collision cross section, 605 .

nitrogen and carbon monoxide, luminous reaction, 2183.

nitrogen and organic substances, 2544.

operations in the United States, influence of lower permissibles levels on, 2598.

oxygen and carbon monoxide, luminous reaction between, 866 .

oxygen and solid ozone, note on the preparation 1239.

oxygen at low temperatures, 2954.

oxygen, electron affinity, $1334 ; 2812$.

oxygen, photodetachment cross section and the electron affinity, 2683.

oxygen, the affinity and radiative attachment coefficient, 1413.

radiation and polymers, 1634 .

radiation on polymers, effects of, 698 .

sodium plus atomic hydrogen, chemiluminescence in th , system, 2486.

spectra, 1269 .

spectra, Bacher and Goudsmit's theory of, comparison of first, second, and third approximations, 2492 . spectra, data concerning, 677 .

spectra for the astrophysicist, 197.

spectra, notation for, 775 .

spectra with electronic digital computers, analysis, 1624.

spectra-their role in astrophysics, 1974.

sulfur and empirical affinities of the light elements, electron affinity, 1698.

theory of electromagnetic interactions in dense materials, 1635 .

weights, 127.

weights and nuclidic masses, 2906.

weights for 1956-57, report, 2731 .

weights, report of the committee of the American Chemical Society, 531; 1129.

Atoms, free, in solid nitrogen condensed at $4.2^{\circ} \mathrm{K}$ from a gas discharge, abundance, 1607.

free radicals, 2622 . 
free radicals, luminescence of solid nitrogen $\left(4.2^{\circ} \mathrm{K}\right)$, 2182 .

hydrogen, intensity distributions in nitrogen bands excited in auroras and by high-energy protons, 1044.

hydrogen, the addition, to solid olefins at $-195^{\circ}, 2803$.

hydrogen, with solid olefins at $-195^{\circ}$, reaction, 2828 .

ions in the sun, 958 .

molecules, basic physics, 2901

molecules of solid nitrogen, 1104

nitrogen, trapped in solids, 2392.

oxygen and hydrogen in atomic flames, reactions of ammonia and hydrazine, 1948.

spectra, and molecules in solid nitrogen condensed at $4.2^{\circ} \mathrm{K}$, interpretation, 1747.

Attenuation, coefficients for X-rays from $10 \mathrm{kev}$ to 100 Mev, C583 Suppl.

data, radiation, 3027 .

due to rainfall, 29.

86- and 176-Mev synchrotron X-rays in concrete and lead, $1635 \mathrm{~A}$.

14,1-Mev neutrons in water, 2032.

gamma-ray, 724.

gamma rays at oblique incidence, 1171.

Rayleigh waves with depth in a medium with two surface layers, 2344.

versus frequency characteristics, comments, 2059.

versus frequency characteristics of VLF radio waves, 2345 .

Attenuator, magnetic, at UHF, characteristics, 663 .

magnetic, ultra-high frequency, 902.

pads, lossy cable, 754 .

piston, chart for $\mathrm{TE}_{11}, 664$.

UHF oscillator, 216 .

Attenuators, cascade-connected, 33.

Audio oscillator, low-distortion transistor, 755 .

Audio-frequencies, measurement of voltage ratio, 2191.

Audio-frequency, electromagnetic hiss recorded at Boulder in $1956,2033$.

electromagnetic noise during a period of solar disturbance, 2019.

power amplifier circuits, a survey, 187.

range, A-C bridge, 1615 .

Audiometer earphones, 632 .

Audiometers, calibration, 31 .

Aurora, a monochromatic low latitude, J 63D3, 297. airglow, 1271 .

Aurora and airglow, photometric unit, 1598.

middle and low latitudes, 2346.

October 22/23, 1958, at Rapid City, South Dakota J 64D2, 205.

sporadic-E echoes at Barrow, Alaska, relationship, 1845.

Auroral, absorption zone, location of, 1054.

observations, visual, 2412 .

subauroral zones, [OI] 5577 in, comparison of absolute intensities, J 63D 1, 19.

zone, ionosphere, 2317.

zone, some studies of the upper atmosphere, 2766

zone, southern, clues to ionospheric conditions, 1650 .

Aurorars, artificial, 2899.

Austenite iron-carbon-nickel alloys, note on thermal stabilization, 777 .

Austenitic, stainless steel, 2789.

stainless steel, stress corrosion cracking of type 304 , 1898.

stainless steels at low temperatures as determined by magnetic measurements, 3093.

Australite (meteoric) glass, 1636.

Autographic stress-strain recorders, 2465.

Autoignited heptane-air mixtures, emission spectra, 442.

Autoignition, benzene-air mixtures, mechanism, 1773. engine fuels, multistage, 1411.

two-stage, of some hydrocarbons, 354.

Automatic, coding principles, 2466.

computation of nerve excitation, 1272.

computations with power series, 1638 .

computers, development, 1675.

computing machines, 233. controller for radiofrequency induction-heating unit, 647.

counting machine, 398.

cut-off valve for lon-exchange columns, 1273

data processing in Government (1956), progress report, 1822 .

frequency comparator and recorder, high precision, 1356.

instrument for electron scattering measurements, 1274 .

lens design methods, 2034.

temperature regulation and recording in precision adiabatic calorimetry, 2035.

timer simplifies small-current measurements, 2036.

Automation, brief look at the history, 1585.

future management information systems, 2467.

future office and factory, 1715.

instrumentation, 1370 .

laboratory, in the, 2468 .

Automotive research at the NBS, 399.

Average, energy of sulfur-35 beta decay, RP2859.

radio-ray refraction in the lower atmosphere, 400 .

Axial line source, surface-wave resonance effect in a reactive cylindrical structure excited by an, J 64D1, 13 .

Axial-load fatigue machine, dual amplitude, 626 .

Azide, methyl and hydrazoic acid by electron impact ionization and dissociation, 2604.

Azo dyes, 881 .

direct interaction in aqueous solution RP2823.

indigo dyes steric effects 1897 .

organic solvents hydration. 1543 .

Bacher and Goudsmit's theory of atomic spectra comparison of first second and third approximations. 2492 .

Background eradication in thick-layered emulsions, 30.

Backscatter, delay and $\mathrm{CW}$ field intensity, comparison, 361 .

frequency 1158.

Backscattering of, gamma-rays, 934.

gamma rays, cobalt-60, from infinite media, 959.

positive electrons, 131.

positrons and electrons, 567.

Balance, NBS 1000-lb, 1539.

quick-weighing, 1168.

Balances, a photoelectric followup and recording system and its application to remote observations of the beam in high precision, $\mathrm{J} 63 \mathrm{C} 2,91$.

analytical and other precise, 1166.

Ball bearings, at high speed and high temperature, oilsoaked felt-pad lubrication, 1422 .

high speeds and extreme temperatures, friction and endurance of prelubricated and unlubricated, $\mathrm{J}$ $63 \mathrm{C} 1,19$

submerged in liquefied gases, 3063.

Ballistocardiography, 951 .

jerkmeter, 1752.

Balmer lines, early, to extend the photospheric model, 2675 .

Banach, complex, spaces, Newton's method, 559.

Bandwidth, television, conservation, communication theory aspects, TN25 (PB151384).

Bar magnet velocity meter, 174 .

Bar-magnetic velocity meter for use in ballistocardiography, 951.

Barium, borate, vitreous, radial distribution study, J $64 \mathrm{~A} 3,229$.

disilicate-dibarium trisilicate, subsystem, phase equilibria, RP2953.

disilicate, polymorphism, 3018 .

hydrogen orthophosphate, crystal structure of, RP2817.

oxide-boric oxide-silica, shape of liquid immisicibility volume, 2754

oxide-boric oxide-silicate, some properties of glasses in the system, 2763 . 
titanate, 3062 .

titanate, sammarium doped, electricalproperties, 2105.

Barometers, mercury, and manometers, Mono. 8.

Bars, long, with a sling-shot machine, longitudinal impact tests, 1760 .

rectangular, shear modulus and torsional resonance frequencies, RP2861.

uniform, comparison of experimental and theoretical relations between Young's modulus and the flexural and longitudial resonance frequencies, $\mathrm{J} 64 \mathrm{~A} 2,147$.

Bases and acids, precise coulometric titration, J 63A2, 153

Basic, experimental studies of the magnetic field from electromagnetic sources immersed in a semiinfinite conducting medium, J 64D 1, 21.

method of determining the dynamic characteristics of accelerometers by rotation, 913 .

physics of atoms and molecules, 2901.

research in Europe, 2470.

standardization, 2469.

standards for science and industry, 2037.

standards in science, 198.

theorems in matrix theory, AMS57.

Basis, for standards for radiation protection, 1639. measuring system, of our, 3066 .

$\mathrm{Ba}^{113}$, disintegration, 1178 .

$\mathrm{BaTiO}_{3}, \mathrm{SrTiO}_{3}$, and associated materials, surface layer, electroluminescence, 2539

Batteries, and dry cells, specification, H71.

lead, storage, 751 .

modern, 2204.

punched-cell, with polyethylene glycol electrolytes, 1828.

storage, lead-acid, some aspects of the charge and discharge processes in, 1141.

thermal, 602 .

wax, 2866 .

Battery, electrochemical, system, 2436.

Beam flux, use of zonal constants in the calculation of, 887.

Beams, strains in, having diagonal cracks, 2782.

thermal stresses and deformation, 1962.

Bearing creep of two forged aluminum alloys, TN55 (PB161556).

Bearings, and pumps at low temperatures, operation, 2676.

lubrication, 82.

oil-free, small, materials, 2184.

plain journal, oil flow, 494 .

Becker, value of manila rope by photoelectric reflectometry, 960 .

Beckman, quartz spectrophotometer, minimizing anomalies in reflectance measurements, 484 .

Begrundung fur die Beschaftigung mit numerischer analysis, 1275 .

Behavior, in the large of the integral curves of a nonlinear differential equation, 2662.

long helical springs under fluctuating load, 1530.

"pure gum" rubber vulcanizates in tension, 1640.

young children under conditions simulating entrapment in refrigerators, 2471.

Bekk and Sheffield smoothness testers, use of the sensitivity criterion for the comparison, 1970.

Bend-test method of determining the stress required to cause creep in tension, $1584 \mathrm{~A}$.

Benzene, derivatives from 15 to 40 microns, infrared absorption spectra of twelve substituted, 75 .

purity of, thermometric and calorimetric procedures, comparison of cryoscopic determinations, 2062.

relative strengths of forty aromatic carboxylic acids in, at $25^{\circ} \mathrm{C}, \mathrm{RP} 2871$.

vapor pressure of, above $100^{\circ} \mathrm{C}, 616$.

Benzene-air mixtures, mechanism of autoignition, 1773.

Benzenes, pentafluorohalo-, reactions, J 63A2, 167.

tetrafluoro, 1,2,4-, preparation of from 2-chloroheptafiuorotoluene, RP2939.
Benzoic acid-7- $\mathrm{C}^{14}$ in toluene, for liquid scintillation counters a carbon-14 beta-ray standard, J 64A2, 143.

Benzonitrils, microwave spectrum of, 1075.

Bergman's, integraloperator erster art und Riemannsche Funktion, 401.

integral operator of the first kind and the Riemannian function, 401 .

Berkeley symposium on mathematical statistics and probability, 114.

Bernoulli and Poisson positive, variables, tables of expected values of $1 / \mathrm{X}, 1161$

Beryllium, and beryllium alloys, 2107.

between $0^{\circ}$ and $900^{\circ} \mathrm{C}$, specific heat, 312 .

chromite and other beryllia compounds with $\mathrm{R}_{2} \mathrm{O}_{3}$ oxides, studies of, J $64 \mathrm{~A} 1,103$.

oxide, thermal conductivity, from $40^{\circ}$ to $750^{\circ} \mathrm{C}$, RP2775.

Bessel function, relative extrema, 104.

Bessel functions, 249.

fraction order, zeros of the derivative, 912.

generation of, on high speed computers, 2140.

hypergometric series, representation, 2236.

polynomials, 72 .

respect to the order, on the derivative, 2666 .

Bessel integral functions, expansions, RP2786.

Bessel-Clifford, and Bessel functions, 214.

functions, terms of, regular and irregular Coulomb wave functions expressed, 1127.

Best approximation polynomials of given degree, 1641.

Beta decay, average energy of sulfur-35, RP2859. experimental test of parity conservation, 2015 . polarized nuclei, 2557.

polarized nuclei, further experiments, 2132

Beta emitters, scintillation counting, 2750.

rays, absolute measurement of $W_{\mathrm{s} \text { ir }}$ for sulfur-35, 2008.

tesier, transistor, 2396.

Beta-alumina-type structure in the system lanthanaalumina, 2472

Beta-emitting nuclides, 1200.

Beta-gamma correlations from polarized manganese-52, 2473.

correlations from polarized nuclei, 2902.

Beta-ray, sources, industrial, safe design and use of, H66. spectrometers, 657 .

standard, carbon-14, benzoic acid-7-C $\mathrm{C}^{14}$ in toluene, for liquid scintillation counters, J 64A2, 143.

Betatron, experiments, 1213.

pulsed magnetic extractor for removing the electron beam from, 929 .

X-rays: how much concrete for shielding, 961.

$\mathrm{X}$-rays to $37 \mathrm{Mev}$, studies of dose distributions in water, 1904.

Betatrons, injection in, electron, 1700.

Bias supplied for direct-coupled circuits, 962.

Biaxial, stretch-forming effects, 434 .

stretch-forming of acrylics, 658 .

Bibliography, and survey of recent research in the propagation of VLF radio waves, TN58 (PB161559).

molecular and crystal structure models, Mono. 14.

nickel and its alloys, C592.

physical equilibria and related properties of some cryogenic systems, TN56 (PB161557).

selected, of statistical literature, 1930 to 1957: I. Correlation and regression theory, J 64B1, 55 .

selected, of statistical literature, 1930 to 1957: II. Time series, J 64B1, 69.

selected, on building construction and maintenance, BMS140,3d ed.

Bilinear transformations and matrices, commuting, 1654. Binary, alkali borate and silicate glasses at high pressures, compressibility, 1292.

alkaline-earth borate glasses at high pressures, compressibility, 1658.

alkaline-earth borates, 1156.

numbers, system of names, 2441.

oxide systems, zirconia reactions, 1978. 
quadratic rational forms, representation of integers, 1198

Biology, radiology, 1125 .

Biorthogonality, complex, for certain sets of polynomials, 975.

Bismuth and lead at $7 \mathrm{Mev}, 3014$.

Bisulfate ion, ionization, 3076.

Bituminous, materials for spinning, preparation, 800 .

materials, uniform films, method of preparing, 628 .

Bivariate normal distribution function and related functions, AMS50.

Blackbody, design and construction, and its use in the calibration of a grating spectroradiometer, 2513 .

Blade type abradant and the Schiefer abrasion testing machine, 382 .

Blankets, part wool, variability of wool content, 1571 .

Block designs, having a missing block, analysis for some partially balanced, 953 .

incomplete, 2116.

incomplete, analysis, 2343.

incomplete, analysis of convariance, 2342.

Block-type osmometer, design and performance, RP2931.

Blocks of symmetrical group divisible designs, 548 .

Blood, human, effects of plastic and steel surfaces on clotting time of, 1003 .

human, platelets and red blood cells in vitro, adsorption of $\mathrm{C}^{14}, 2011$.

Blumenthal, conjecture of, Day's characterization of inner-product spaces, remark, 373.

Bodies containing radioactive isotopes, safe handling of, $\mathrm{H} 65$.

Body burdens and maximum permissible concentrations of radionuclides in air and in water for occupational exposure, H69.

Bolometric power measurements, a self-balancing directcurrent bridge for, RP2776.

Bolt materials for service undergound, electrical measurements in the selection of, 1008 .

Bolts, handmade, support the Capitol dome, 2577.

tightened, tensile forces, 344 .

Boltzmann equation from the statistical mechanical point of view, 1922 .

Bomb, calorimeter, precise measurement of heat of combustion, Mono.7.

calorimetric combustion, of compounds containing carbon, hydrogen, oxygen, and nitrogen, 1645 .

Bombardment, electron cryostat for, and electron diffration work, 2506.

Bonding agents for high-temperature strain gages, 2474.

Bonding of plastic teeth to heat cured denture base resins, 402.

Bone char, eurrent problems in refining, 984 .

process in sugar refining, 132.

review of pressure drop across columns, 1243.

Bone mineral, structure, 2383.

Boolean geometry L., 403.

Boor-Quartermaster snag tester for coated fabrics and plastic films, evaluation, 1337.

Borate minerals, 3057 .

vitreous barium, radial distribution study, J 64A3, 229 .

Borates, alkali, molten, viscosity and electrical resistivity of, 907 .

alkaline-earth, 3059.

binary alkaline-earth, 1156.

simple, immiscibility and surface tension, 2592.

Boric oxide, the heat of formation, heat of reaction of diborane with water, RP2927.

Borohydride, sodium, reduction of aldonic lactones, 1868. Borohydrides, sodium and potassium, heat content of, from $0^{\circ}$ to $400^{\circ} \mathrm{C}, \mathrm{RP} 2828$.

Boron enhancing the hardenability of steel, action of, 851 . needs well-made steel plus intelligent heat treatment, 199.

steels, 1642 .

trichloride, heat of formation, RP2956.

Boron-treated, steel, temper brittleness, 1913.

steels, study of the impact properties, 1601.
Bose-Einstein condensation and the lambda transition in liquid helium, 2475 .

Boulders and cobbles in gravel deposits, geological application of extreme-value methods to interpretation, 1723 .

Boundary layer, and pipe, 1218.

transition, 1400.

with zero pressure gradient, characteristics of turbulence, 968 .

Boundary layers, compressible turbulent, with heat transfer and pressure gradient in flow direction, $J$ $63 \mathrm{~B} 1,53$.

Boundary value problems for multiply connected domains, 404.

Bounds, for eigenvalues of Hermitian matrices by the segment method, 1005 .

greatest latent root of a positive matrix, 405 .

one-parametric family of matrices, 2663 .

Bourdon tubes, sensitivity and life data, 1864.

Bragg-Gray cavity principle for measuring energy dissipation, 1083.

Brakelights and taillights, configuration, 1587

Branched-chain, higher sugars, $2476 ; 2903 ; 2904$.

Breaking velocities, strain energies, and theory neglecting wave propagation, 1277 .

Breathing oxygen storage dewars, 2038.

Bremsstrahlung, and the photoelectric effect, 2968.

cross-section measurement for 50-kev electrons, 2477.

cross sections at the high-frequency limit, evaluation, 2551.

differential cross-section measurements for 0.5- and 1.0-Mev electrons, 1278 .

eleven-Mev thick target, 709.

500-kev, 2694.

inner, from $\mathrm{V}^{49}, 1368$.

1-Mev, 993.

photoelectric effect as inverse processes, 2905.

polarization measurements for 1.0 -Mev electrons, 1643.

Sauter approximation, high-frequency limit, 2961.

spectra, 1211.

spectrum, electron, shape of high energy, 2755.

thin-target, differential cross-section measurement, 1676.

Brick, de-aired, durability, 1683.

masonry with and without caps and flashings, effects of weathering on certain mortars exposed in, 1927.

Bridge, $\mathrm{R}-\mathrm{C}$, selective, note, 370 .

Bridge-balancing oscillator, high-stability, 1358

Bridged-T network, 181.

Brief history of the National Committee on Radiation Protection and Measurements, 2478.

Brief, look at the history of automation, 1585 .

note on the asymptotic expansion of error integrals, 1383.

Bright rims in diffuse nebulae, 2479

Brittle cermets, evaluation of tensile, compressive, torsional, transverse, and impact tests and correlation of results, RP2895.

Britton-Robinson universal buffers, increased chemical reactivity of the surface compared with that in the bulk volume of, RP2811.

Broad and narrow beam attenuation of $\operatorname{Ir}^{192}$ gamma rays in concrete, steel, and lead, 2480 .

Broad-band, coaxial stark cell for microwave spectroscopy, 358.

low-level, error-voltage detector, 914

Broadcast reception, worldwide standard frequency, 623.

Broadcasting, television, measurement of a service area, 2190.

station, worldwide standard-frequency, power requirements and choice of an optimum frequency, J $63 \mathrm{D} 1,35$.

stations, FM and television, variation with frequency of the signal range, 347 .

Bromide, cesium, crystals, transmittance, 349 .

cesium, refractive indices, 527 . 
potassium, for infrared radiant energy, 812

thallium, iodide, refraction and dispersion, 1842 .

Bromotrifluoroethylene, vibrational spectrum, 1206.

Bubble chamber, a large liquid hydrogen, 1984.

Buffer, solutions, surface reactivity, RP2811. substance, triathanolamine, 1217.

Buffers for $\mathrm{pH}$ standardization at $60^{\circ}$ to $95^{\circ} \mathrm{C}, \mathrm{RP} 2797$.

Building, and using dielectric amplifiers, 963 .

brick, properties of, and effects of differences in raw materials and methods of forming, relations among certain specifications, $\mathbf{5 2 8}$

code requirements for masonry, 945.

Building codes, early history and development, 2095.

how the National Bureau of Standards contributes, 1733.

noise control requirements, 2216.

Building, construction and maintenance, selected bibliography, BMS140, 3d ed.

finish materials, flame spread properties, 2565.

Materials and Structure Reports (see list p. 63. Indexed under specific subjects).

research in the U.S. before 1947,1279 .

technology at the NBS, 200.

Bulk crystallization in poly(chlorotrifluorethylene), experimental and theoretical study of kinetics, J $63 \mathrm{~A} 1,67$.

Bunsen flames, 207.

Bureau of Standards comments on Loran communications, 2039 .

Butadiene-styrene copolymers, crystallization, 983.

Butyrate, acetate, propionate radicals, heats of combustion of some peroxides and the heat of formation, 2148 .

\section{C}

$\mathrm{C}_{2}$ radical in hydrocarbon flames, 1434 .

$\mathrm{C}_{3}$ molecule between $3600 \AA$ and $4200 \AA$, spectrum, 1885 .

$\mathrm{C}_{\mathrm{s}}{ }^{139}$ narrow hyperfine absorption lines of, 2643 .

Cable, lossy, attenuator pads, 754 .

Cables, coaxial, video signals, 2300.

Calcia-titania, revision of the phase equilibrium diagram of the binary system showing the compound $\mathrm{Ca}_{4} \mathrm{Ti}_{3} \mathrm{O}_{10}, \mathrm{RP} 2913$.

Calcification in lysine deficiency, 2040.

Calcium, aluminate hydrate mono, observations on, RP2777.

aluminate hydrates, a study, RP2877.

hydroxide, a thermochemical study of the reaction of, RP2785.

magnesium precipitations, removal of manganese prior to, 814 .

phosphates, precipitated basic, pyrophosphate formation upon ignition, 1465.

silicate hydrates, 1172

treatment on solubility and calcium uptake of synthetic hydroxyapatite and rat molar enamel, 2528 .

Calculated calibration points for negative ion appearance potentials, 2481.

Calculating error function of a complex variable, formulas, 241 .

number of chain scissions in the photochemical degradation of solid polymers, equation, 2455 .

Calculation of, an optical merit function and its derivatives with respect to the system parameters, 2041.

beam flux, use of zonal constants, 887 .

cryoscopic data, 2042.

energy dissipation by gamma radiation near the interface between two media, 2043.

gaseous heat conduction in dewars, 2482.

peaked angular distributions from legendre polynominal expansions and an application to the multiple scattering of charged particles, 659

Calculation of departures of radio wave bending from normal, J 64D3, 259.

equilibrium composition and thermodynamic properties of dissociation and ionized gaseous systems, $2632 ; 2988$. field in a homogeneous conductor with a wavy interface, 3067 .

properties of gases at elevated temperatures 2664 .

scattering cross sections, variational 617 .

spatial distributions by semi-asymptotic methods: penetration and diffusion of X-rays, 509.

Calculations, of ionospheric reflection coefficients at very low radio frequencies, 2044.

systematic, of gamma-ray penetration, 847 .

transverse current loss in buried wire ground systems, 2665.

Calculus, of variations, applications of the theory of quadratic forms in Hilbert space, 195.

variations, elementary introduction, 23.

Calf skin squares, influence of temperature on the adsorption of chrome $\left(\mathrm{Cr}_{2} \mathrm{O}_{3}\right), 2159$

Calibration, absolute time, intermittent-action camera, $J$ $64 \mathrm{C} 2,159$.

accelerometer, high-speed stroboscope for, 1031.

airplane camera, source of error in various methods, 1878.

airplane cameras, 2045.

audiometers, 31 .

calculated, points for negative ion appearance potentials, 2481.

capacitor, by step-up methods, $\mathrm{J} 64 \mathrm{C} 1,75$.

commercial field-strength meters, 4070 .

condenser microphones, 1114.

grating spectroradiometer, design and construction of a blackbody and its use, 2513 .

line standards of length and measuring tapes at the NBS, Mono, 15.

liquid-flowmeter, techniques, 2613.

liquid-in-glass thermometers, $\mathrm{C} 600$.

portable Swedish free-air chamber equipment at the U.S. National Bureau of Standards, 1644

precision airplane mapping cameras, 32 .

shock and vibration pickups, 2046.

spectrometers from 2 to 16 microns, vibrationrotation structure in absorption bands for, $J$ $64 \mathrm{A1}, 29$; Mono.16.

thermocouples at low temperatures, 2483.

VHF field-intensity meters, 151.

with an inductive voltage divider bride, TN57 (PB161558).

Calibrator, detector, microphone condenser, 2348.

wide-range, for vibration pickups, 911.

Calorimeter, adiabatic, for the range $30^{\circ}$ to $500^{\circ} \mathrm{C}, \mathrm{RP}$ 2848.

bomb, precise measurement of heat of combustion, Mono. 7.

dry, static, for rf power measurement, 2419.

microwave, 1236 .

Calorimetric, and thermometric procedures, comparison of cryoscopic determinations of purity of benzene, 2062 .

combustion in a bomb of compounds containing carbon, hvdrogen, oxygen, and nitrogen, 1645 .

determination of the power in a $1400 \mathrm{kev}$ X-ray beam, 2047.

Calorimetry adiabatic, automatic temperature regulation and recording, 2035.

combustion with fluorine: constant pressure flame calorimetry, J 64A1, 49 .

electrochemical, 2538.

heat units and temperature scales, 1355.

Camera, calibration, airplane, sources of error in various methods, 1878

intermittent-action, with absolute time calibration, J $64 \mathrm{C} 2,159$.

tipping, prism effect, and tangential distortion, 2264, Cameras, airplane, calibration, 2045. airplane mapping, 32 .

Can the scales of atomic weights and nuclidic masses be unified? 2906.

Can we legislate ourselves into radiation safety? 1280. Candlepower standards, gas-filled, 2860.

Canonical ensemble, virial expansion for finite closed systems, $2077 ; 2078$. 
Cantilever beams, tapered, computed on SEAC, 738.

Capacitance, low, power supply, 1391.

measurement, absolute, 2647.

Capacities, flow, of pneumatic components, determination and correlation of, C588.

plumbing drains, 2489 .

Capacitor calibration by step-up methods, J 64C1, 75 . variable, calibration with an inductive voltage divider bridge, TN57 (PB161558)

Capacitors and diodes, experimental rapid access memory, 392.

conical coaxial, and their advantages, J $63 \mathrm{C} 2,87$.

Capacity, plumbing stacks, 1281.

requirement of a mail sorting device, RP2948.

requirement of a mail sorting device: II, J 63B2, 79 .

Capillary, shape, effects on flow characteristics and degradation of polymer solutions, R P2839.

viscometry, $2047 \mathrm{~A}$.

Carbide, titanium, type cermets at elevated temperatures, physical characteristics, 1445 .

Carbohydrate derivatives, infrared spectra, RP2772.

Carbohydrates, carbon-14-labeled, RP2800.

general-purpose apparatus for preparation of tritiumlabeled, J 63A2, 177.

tritium labeled, assay of, J 63A2, 171.

Carbon, adsorbents, oxygen chemisorption, 1438.

black differentiation by electrical resistance of vulcanizates, 408 .

dioxide, reaction of portland cement with, RP2858.

disulfide, infrared spectrum and rotational constants, 1932 .

electrons in, range straggling of high-energy, 2279.

formation in hydrocarbon flames, isotopic tracer study, 2018 .

graphite at $450^{\circ} \mathrm{C}$, reaction of carbon monoxide, 2717.

hydrogen, oxygen, and nitrogen, calorimetric combustion in a bomb of compounds containing, 1645 .

monoxide and atomic nitrogen, luminous reaction, 2183.

monoxide and atomic oxygen, luminous reaction between, 866.

monoxide from 2.3 to 2.5 microns, emission spectrum, R P2883.

monoxide, infrared spectrum, 516.

monoxide in studies of air pollution, 1309.

monoxide, liquid, 3013 .

monoxide on tungsten, 2888.

monoxide, velocity of light from the molecular constants, 1574 .

monoxide with graphite carbon at $450^{\circ} \mathrm{C}$, reactio 3 . 2717.

near threshold, photoproduction of neutral mesons, 2250 .

stable, isotope analysis of optical spectroscopy, 1892.

Carbon and hydrogen, determination of copolymer composition by combustion analysis, J $64 A 2,157$.

Carbon-14, beta-ray standard, benzoic acid-7-C ${ }^{14}$ in toluene, for liquid scintillation counters, J 64A2, 143.

carboxy-labeled polysaccharides, RP2886.

labeled cyanide, determination of reducing sugars and reducing end groups in polysaccharides by reaction, 2517.

labeled cyanide, preparation, 2262.

Carbonate, sodium, in photographic developers, note on estimation, 1238.

Carbonates, manganous and ferrous, thermal decomposition, 1960.

Carbonyl sulfide, inf rared spectrum, 2162.

Carboxyl, aldehyde, pentosan, and degree of polymerization data on the ICCA "standard" pulps, 2890 .

Cardiac, output determination by the dye-dilution method, an improved cuvette densitometer for, 650 .

output, evaluation of a new cuvette densitometer for determination of, 714

Career opportunities in the Government, 2484.

Carrier frequency dependence of the basic transmission loss in tropospheric forward scatter propagation, TN53 (PB161554).
Cascade electrons and photons, energy spectra, RP2824.

Cascade-binary counter with feedback, 326 .

Cascade-connected, attenuators, 33 .

variable attenuators, mismateh errors, 2993.

Cassiopeiae, gamma, variations, T N21 (PB151380).

Cast iron, nodular graphite in, experimental production, 444

Castables, refractory, preparation and some properties, 125 .

refractory, strength, elasticity, and other related properties, 2536 .

Castiloa and Hevea rubbers, 160.

Castings, gold, effectiveness of vacuum investing in the elimination of surface defects, 697 .

Catalysis of the ortho-parahvdrogen conversion, 2048.

Caterer problem, solution, 1099

Cathode, film studies by the drainage method, 1282 . studies, high-power pulser aids, 1029.

Cathode-coupled oscillator, 62 .

Cathode-rav tubes, electrostatic deflection plates, J 64C2, 103.

Cathodic, protection, polarization, 143.

steel in a high resistivity environment, current and potential relations for, $\mathrm{J} 63 \mathrm{C} 1,37$.

steel in salt water, current and potential relations, RP2833.

Cationic detergents in water, turbidity and viscosity measurements on, RP2778.

Cavities, large high-Q, excitation and separation of pure high-order modes, 1708.

limit of spatial resolution of refractometer, J 64D1, 65

Cavity, characteristics, time-dependent, 2636.

ionization as a function of wall material, RP2842.

ionization theory of, $1247 ; 1606$.

ionization, theory including the effects of energetic secondary electron, 1229 .

resonator dielectric measurements on rod samples, 2907.

techniques for permeability measurements in the VHF region, 1283.

$\mathrm{Cd}^{115}$ isomers, decay of, 569 .

Ceguera para el color y teorias de la vision cromatica, 201.

Cell, dry, standards broadened-now includes mercury-, air-, and flat-type cells, 1321.

Cells, airglow, 2640.

dry, and batteries, specification, H71.

dry, gassing, 2359 .

photoflash, standard test, 1506.

resistance, during current flow, 141.

standard, portable potentiometer and thermostated containers, 1450 .

Cellular, concretes. Part 1. Composition and methods of preparation; Part 2. Physical properties, 964. units, solderless electronic assembly, 830 .

Cellulose, acetate films, degradation, 2924

acetate propionates, 237 .

dezradation in a vacuum with ultraviolet light, RP2841.

determination of aldehyde, 1308.

periodate-oxidized, 314 .

water evaporation, 547 .

Cellulose and, polysaccharides, 2403. solvent solutions, 112.

Celluloses and aldoses, reaction of sodium chlorite, 1473.

Cellulosic materials, thermal degradation, RP2853.

Cement, 1284.

analysis, 937 .

asbestos, pipes, 998 .

hydrated, 2639 .

manganese in, flame photometric determination, 1930. note on the oxyquinolate determination of magnesium oxide in, 925 .

paste, portland, some effects of aging on the surface area, J 64A2, 163.

pastes and mortars by the alkali-aggregate reaction, 2699.

portland, chemistry, 327 .

portland, flame photometric determination of strontium, 1346. 
portland, hydrated, some factors, affecting the surface area, RP2806.

portland, improvements in flame photometric determination of sodium in, 736 .

portland, magnesium oxide in, 2893 .

portland, pastes cured at elevated temperatures and pressures, properties, 1826.

portland, reaction with carbon dioxide, RP2858.

silicate, dental, 20.

silicate: how to select and use them, 2299; 2756 .

silicate, method of mixing in a closed container to prevent effects of exposure to atmosphere 827 .

Cemented piezoelectric accelerometers, 660 .

Cements, ceramic-type strain gage, 2739.

dental, 1306.

portland, a sulfate susceptibility test, 11.

portland and high-alumina, during hydration, 2768 .

portland, strontium oxide content, 2832 .

resin and posterior-type direct-filling resins, 1854.

silicate, and zinc phosphate, 2873.

silicate, deterioration in the tropics, 217.

Central notations for the revised ISCC-NBS color-name blocks, RP2911.

Radio Propagation Laboratory, 2049.

Radio Propagation Laboratory exponential reference atmosphere, J 63D3, 315.

Ceramic accelerometer of wide frequency range, 175.

coated and uncoated specimens of Inconel and stainless steel, 3053.

coated Inconel and type 321 stainless steel, 3092.

coating on the creep behavior of some high temperature alloys, 3072 .

coatings for nuclear reactors, 1285 .

coatings for thermocouples, development and evaluation, 1674 .

coatings, thickness gage, 13 .

materials, specular-gloss measurement, 1886.

metal materials, 1266.

oxide single crystals, plastic deformation, $1108 ; 2253$.

type strain-gage cements, 2739.

$\mathrm{X}$-band cavity resonators, fabrication techniques, 2561

Ceramics, a survey for nuclear reactors, 12.

cermets, properties of high-temperature, Mono.6.

vacuum tube envelopes, survey of the application, 1604.

Ceramists, compilation of phase diagrams of interest, 1656.

distribution of phase diagrams of interest, 1318 .

Cerenkov counters, fast coincidences, 447.

Cerium $^{141}$ and neodynium ${ }^{147}$, alignment, 1255.

Cermet, chromium boride-nickel, coating, protection of low-strategic alloys, 1463.

Cermets and ceramies, properties of high-temperature, Mono.6.

titanium carbide type, at elevated temperatures, physical characteristics, 1445 .

Certain Fourier transforms of distribution, 202; 965.

series expansions involving Whittaker functions and Jacobi polynomials, 1424 .

Certification and specifications, dental materials, annual for $1943,1307$.

Certified reference materials, 3078A.

Cesium bromide crystals, transmittance, 349 .

bromide, refractive indices, 527 .

iodide crystals, growth and infrared transmission, 460.

iodide in the far infrared region, transmittance and reflectance, 2852 .

iodide, optical properties, 1436.

iodide prism, 1072.

iodide prism, infrared measurements, 742 .

iodide prism, infrared spectrometry, 744 .

iodide prisms to 50 microns, 1551 .

Cesium-131, radiative electron capture, 1834

Cesium-137 and cobalt-60 gamma rays with a free-air chamber, measurement of, J $64 \mathrm{C} 2,87$.
$\mathrm{CH}$ flames at atmospheric pressure, rotational and vibrational "temperatures" of, 816 .

$\mathrm{OH}$, and $\mathrm{HCO}$ in flame spectra, using deuterium as tracer, mechanism of formation, 868 .

solar spectrum, J $63 \mathrm{~A} 1,19$.

system of, 1625 .

Chain block design, 852 .

block designs with two-way elimination of heterogeneity, 966 .

depolymerization, kinetics. 474 .

molecules, flexibility, sedimentation equilibrium, 2296 .

ratio, statistical, method for estimating relative volumes of mail to given destinations, J $64 \mathrm{C} 1,37$. reactions of frozen radicals, 3030 .

suspended, modes of viration, 770 .

Chaines de Markoff dans les ensembles abstraits et applications aux processus avec regions absorbantes et au probleme des boucles, 1286

Chamber, a large liquid hydrogen bubble, 1984 .

Change in the inversion spectrum of $\mathrm{ND}_{3}$ from resonant to nonresonant absorption, 661 .

free energy with pressure of the reaction nephelene + albite $=2$ jadeite, 203 .

Changes, in agar-agar type duplicating material and agar-agar on heating and storage, 2050.

sign of sums of random variables, 662 .

Char, bone, review of pressure drop across columns, 1243.

Characteristic energy loss of electrons in aluminum, temperature dependence, 2798.

energy losses of electrons in solids, 1278.

functions, analytic, 496

roots of quaternion matrices, 967.

size of airglow cells, 2807 .

Characteristics, and atmospheric noise from 1 to $100 \mathrm{Kc} / \mathrm{s}$, 2052 .

cryogenic of wire resistance strain gages, 2073.

cutting, of dental burs as shown by high speed photomicrography, 2076 .

deposits, 2908.

Institute for Numerical Analysis computer, 34.

magnetic attenuator at $\mathrm{UHF}, 663 ; 902$.

metal-clad laminates, 2053.

outline of, an image-forming system, 1801.

some insulations or liquid oxygen transfer lines, 2054.

turbulence in a boundary layer with zero pressure gradient, 968 .

ultrasonic vibrations, 2055

Characteristics of VLF propagation using atmospheric waveforms, 2760 .

Characterization of kaolinite, halloysite, and a related mineral in clays and soils, criteria, 420 .

high-speed impact behavior of textile yarns, 2909.

Charge-storage pulse-height analyzers for use with pulsed accelerators, 2485 .

Chart, average, operating characteristic, 588 .

coupling, for solenoid coils, 41 .

speeds design of feedback amplifiers, 410 .

$\mathrm{TE}_{11}$ model piston attenuator, 664 .

test, power, 268 .

Charts, control, may be all right, but-, 676 .

Chebyshev and polynomial approximation, derivative, 786 .

polynomials in the solution of large-scale linear systems, 411.

polynomials in thin film computations, use, J $63 \mathrm{~A} 3$, 297.

Checking money-value graduations on a computing scale, 204.

prepackaged commodities, H67.

Chemical analyses, surface area, and thermal reactions of natural graphite, and refractoriness of the ashes, 665 .

color constitution, 1289.

combination of the characteristic energy loss of electrons, effect of, 997.

compounds, optical rotation of polarized light, 285 .

constitution of dyes, absorption spectra, relation. XXVIII. The hydration of Azo dyes in organic solvents, 1543 . 
constitution of dyes, absorption spectra, relation. XXII. Cis-trans isomerism in thioindigo dyes, 341.

constitution of dyes, absorption spectra, relation. XXIV. Absorption spectra of some thioindigo dyes in sulfuric acid, 342 .

data, interpretation, 579 .

education in Switzerland, 969.

elements in astronomical spectra, lines, 1758.

equilibrium among molecules, atoms, and atomic ions considered as clusters, RP2916.

kinetics, stochastic processes, 2898.

kinetics, the application of the theory of stochastic processes, 2805 .

methods for separating and determining metals contained in platiniferous materials, 668 .

physical properties of chrome-tanned leather and the election of sampling location, study of the variation, 558 .

physical properties of split and unsplit chrome-tanned leathers, variation, 601.

physical properties, review, direct and indirect filling resins, 688 .

physics, and engineering measurements, how statistics improve, 68 .

reactions and shock waves, 2325

reactivity at the surface compared with that in the bulk volume of buffer solutions, RP2811.

research, autoclaves in, high pressure gas handling equipment, 2154 .

spectroscopy, 35 .

structure and stability relationships in polymers, 2910 .

Chemical analysis, GR-S by complete solution procedures, 666.

GR-S by complete solution procedures. Gross constituents in GR-S containing soap, 667 .

using linear calibration curves, 2328.

Chemicals, reagent, American Chemical Society specifications, 1474 .

Chemiluminescence as a tool in the study of liquid flow boundaries, 1646 .

in the system atomic sodium plus atomic hydrogen, 2486.

Chemisorption, oxygen, on carbon adsorbents, 1438.

Chemistry, analytical, 138.

crystal, symposium, 215.

macromolecular, 1394.

organic phosphorus, 2494

physical of synthetic rubbers, 1107

portland cement, 327 .

statistical methods, $1896 ; 2779$.

Chilte and guayule rubbers, 119.

Chloride, manganous, tetrahydrate at very low temperatures, spin-spin absorption in chronic methylammonium alum, 1887 .

manganous, tetrahydrate, spin-spin, 1888.

molten, electrolyte, theoretical electromotive forces for cells containing a single solid, 1957 .

silver, silver, electrode, standard electrode potential, 1895 .

Chlorine and nickel, above $1,100^{\circ} \mathrm{K}$, kinetics of the reaction between, 2174 .

between 1,200 and $1,700^{\circ} \mathrm{K}$, kinetics of the high temperature heterogeneous reaction, 2608 .

Chlorine monoxide, flash photolysis, 2357.

Chlorite, sodium, determination of glucose, 1310 .

sodium with some aldoses and modified celluloses, reaction, 1473 .

4-Chloro-4'-aminodiphenylsulfone, dissociation of, RP2794

Chlorotrifluoroethylene, vibrational spectrum of, 893 .

Chromatic aberration, secondary, 2295.

Chromaticity diagram, 918.

Chromatographic, paper, analysis for collagen and collagen derivatives, 1597.

Chromatography, adsorption, separation of isomeric polyphenyls, 2752 .
Chrome-tanned leathers, split and unsplit, properties of, 601.

steer hides, influence of splitting on the strength, 468 . variation of the physical and chemical properties, 558.

Chromic, methylamine alum, 1057.

methylammonium alum and manganous chloride tetrahydrate at very low temperatures, spin-spin absorption, 1887 .

methylammonium alum as a thermometric substance, absolute temperature below $1^{\circ} \mathrm{K}, 2009$.

methylammonium alum, spin-spin relaxation in very low temperatures, 1889

Chromium, boride-nickel cermet coating, protection of low-strategic alloys, 1463.

cobalt alloys, physical properties, 2687.

gun bores, 1647 .

plating of gun bores, 1648

potassium alum, 1067 .

Chromosphere, solar, 2927.

Chromotographic, quantitative, procedure for determining dextrose in sugar mixtures, 2276.

Cinchonine method for determination of lignosulfonates in vegetable extract blends, 1979 .

Circle theorem of hydrodynamics, 1084

Circles, precise, graduation of, 862 .

Circuit, design, 2283.

high-speed memory, pulse generator, 1827.

junction transactor, 749 .

parameters, effect of variation of, on the excitation of spectra by capacitor discharges, 696 .

pulse, accurate time-modulated, 646 .

resistance diode bridge, for temperature control, TN34 (PB151393).

simultaneously recording the range, amplitude, and duration of radar-type reflections, 176 .

Circuitry, one-megacycle, one-microsecond adder, 1596.

Circuits, audio-frequency power amplifier, a survey, 187. coincidence, millimicrosecond, 1779 .

communication, effect of echo on the operation of high frequency, 2810 .

direct-coupled, bias supplied, 962.

electronic, $238 ; 2500$.

incidence, VHF oblique, 2773.

modified resonant, match impedances, 92

preferred, manual with engineering notes, 1113

preferred, NBS, program, 1412 .

VTM, 168.

Circulants, integral, unimodular, 1567

unimodular, classes of positive definite, 2057 .

Circular, cylindrical enclosure, right acoustic impedance of, 941.

disks containing the eigenvalues of a matrix, note, 2650 . punch, smooth, indentation pressure, 1363 .

Circulation in the dog using a mechanical left ventricle, a method for the study of, 920 .

Civil enineering, 2824 .

Clamp for handling radioactive liquids, 1649.

Clamp-on-microammeter measures a-c current, 2056.

Clamp-type alternating-current microammeter, 2347.

Class of mean value functions, 1 .

Classes of matrices and quadratic fields, $205 ; 412$.

positive definite unimodular circulants, 2057.

Classification, and nomenclature for standards of measurement, $2486 \mathrm{~A}$.

conformers: conformations of the pyranoid sugars, J $64 \mathrm{~A} 2,171$.

Clay tile, moisture expansion of, 1058.

Clays, soil, and related materials, electron microscopy, 439.

Climate, radio-refractive-index, near the ground, J 63D3, 259.

Climatic variation of absolute humidity, note, 1991.

Climatology, of ground-based radio ducts, J 63D1, 29. radio, meeting on, 2564 .

Clinical, dextrans by periodiate oxidation and isotope dilution techniques, structural analysis, 2319 . thermometers, aging changes, 385 . 
Clock, atomic, 148; 196.

Clocks, widely separated, precise time synchronization, TN22 (PB151381).

Closure to discussion by Prof. Maxwell of Stress and strain at the onset of crazing of polymethyl methacrylate at various temperatures, 669 .

Cloud chamber, high pressure diffusion, construction and operation, 568 .

Clouds, hydrogen, interstellar neutral, radio observations, 1836 .

space-charged, electron optical properties, 53.

Clues to ionospheric conditions in the southern auroral zone, 1650

Clusters, chemical equilibrium among molecules, atoms, and atomic ions, RP2916.

$\mathrm{CO}_{2}$, nonpolar gas, 1074 .

Coastal deviation of high-frequency radio waves, measurements, J 64D1, 57.

Coater, dip, improved, 1033.

Coatings, for space vehicles, $2910 \mathrm{~A}$.

organic, abrasive jet method for measuring abrasion resistance, 1249 .

Coaxial, cables, video signals, 2300. capacitors, conical, and their advantages, J $63 \mathrm{C} 2,87$. stark cell, broad-band for microwave spectroscopy, 358.

Cobalt alloys, chromium, physical properties, 2687.

Cobalt- 60 , backscattering of, 959 .

cesium-137 gamma rays with a free-air chamber, measurement of, J $64 \mathrm{C} 2,87$.

Cobbles and boulders in gravel deposits, geological application of extreme-value methods to interpretation, 1723.

Code, checking, computer aids, 413.

orthonormalizing, multiple-purpose, its uses, 921.

safety, new, for portable metal ladders, 2374.

Codes, and standards, governmental aspects, specifications, 1880.

building, early history and development, 2095 .

building, how the National Bureau of Standards contributes, 1733.

building, noise control requirements, 2216 .

color, safety, $1481 ; 1861$.

Coding, automatic, principles, $1637 ; 2466$.

Coefficients, for allocation problem, 2808.

certain infinite products, 853 .

certain modular forms, existence of identities, 1797. certain modular forms, generalizations of identities, 1720 .

polar complex interpolation, 36 .

powers of $\eta(\lambda)$ table, 1605 .

reverberant sound fields, measurement of correlation, 1399.

Coils, solenoid, coupling chart, 41 .

toroidal, high-frequency magnetic permeability measurements, 2584

Cold, cell, liquid helium, for use with an X-ray diffractometer, RP2907.

fluids, valve for, 1570 .

Collaborative study on the cinchonine method for determination of lignosulfonates in vegetable extract blends, 1979 .

Collagen, leather, and other organic and fibrous materials, heats of wetting, 1028 .

derivatives, paper chromatographic analysis, 1597.

heat, degradation, 2511.

hide powder, amide nitrogen of, 645 .

modified collagen, 1159 .

modified, and other materials, heats of wetting, 2149 . reaction of nitrous acid, 1837 .

studies of the polar amino acid content, 841

tendon shrinkage, 120

Collagen and leather, influence of temperature on the absorption of water vapor, 74

physical and physico-chemical constants, 510 .

pores, 1449.

specific heats, 1502 .

Collection of ions produced by alpha particles in air, 2488 .
Collision, path from the earth to the moon in the restricted problem of three bodies, 359 .

liquid drops and rigid steel spheres, pits in metals, J $64 \mathrm{A1} 1,61$.

Collisional relaxation of a system of harmonic oscillators, $1847 ; 2235$.

Collodion membranes, sorption of dextran to, 832

Color, blindness and theories of color vision, 201.

chemical constitution, 1289 .

codes, safety, $1481 ; 1861$.

computer defines, 2913.

demonstrations, 3046 .

description, 2080.

differences, 3078 .

gloss and haze, measurement of, 760 .

light, a new point of view in the measurement, 1989.

light, measurement, $2609 ; 2627$.

measurement in industry, 2197.

mixture functions studied by means of a pair of metameric grays, observer difference, RP2825.

name blocks, revised ISCC-NBS, central notations, RP2911.

perceptions, entroptic, of the macular pigment by observers of normal and color-defective vision according to the three-components theory, 1706.

phase contrast microscopy: requirements and applications, 37.

rendering properties of fluorescent lamps, specifications of, $134 ; 833$.

rendition in fluorescent sources of illumination, 1651.

scales, uniform, progress report by Committee of Optical Society of America, 1459A.

specification, 52 .

stability of direct filling resins, 854 .

television, 38 .

television, present status, 154.

three, achromats, glasses for, 3043 .

turbidity of sugar products, 970 .

vision and colorimetry, 2500 .

vision color blindness and theories, 201.

Colorfastness of textiles, international methods for testing, 1375 .

textiles tests for, 850 .

Colorimetrie data to Munsell renotations, mechanized conversion, 2633.

determination of tetrachlorohydroquinone, 670 .

Colorimetry and color vision, 2500.

precision spectrophotometry, 2487.

spectrophotometric, propagation of errors in, 804 .

tristimulus, propagation of errors, 2266 .

Colors, dark, extension of Munsell renotation system, 1710 .

new federal standard, 2213.

Combination, bands of ethylene, 206.

crossed-field and time-of-flight mass spectrometer, 360 .

Combinatorial analysis of a mail sorting device, J 63B2, 79.

Combined translational and relaxational dispersion of sound in gases, 971 .

Combustion, analysis for carbon and hydrogen, determination of copolymer composition, J 64A2, 157.

Bunsen flames, 207.

calorimetry with fluorine: constant pressure flame calorimetry, J 64A1, 49.

heat of, precise measurement, with a bomb calorimeter, Mono.7.

net heat, of aviation gasoline and its correlation with other properties, 2646.

Comments on a, recent paper by Deresiewicz on plane waves in a thermoelastic solid, 2058.

attenuation versus frequency characteristics, 2059.

capacites of plumbing drains, 2489.

structure of 1,1,1-trifluoro-2-butyne, 2911.

Commercial, field-strength meters, calibration, 407.

sugar solutions, light seattering, J 63A3, 205.

tires, measurement of treadwear, 761 .

Commission III on ionospheric radio, world-wide soundings committee, 2490. 
Commodities, checking prepackaged, H67.

Communication, arctic, conference on, J 64D 1, 73.

circuits, effect of echo on the operation of high frequency, 2810

circuits, high-frequency, 3073 .

conditions, high-frequency, 2127

experimental equipment for, utilizing meteor bursts, 2117.

line wires, pretied tie, 2432.

meteor-burst, transmission error function, 2674.

narrow-band, of binary coded messages in fluctuation noise, 915 .

optimum frequeneies for outer space, J 64D2, 105 .

theory aspects of television bandwidth conservation, TN25 (PB151384).

via meteor bursts, 2491 .

Communications, air, at frequencies above $50 \mathrm{Mc}, 540$. air-to-ground, at frequencies above $50 \mathrm{Mc}$, gapless coverage, 457 .

intermittent, with a fluctuating signal, 2163.

Loran, Bureau of Standards comments, 2039.

marine radio, propagation characteristics of the frequency band 152-162 Mc, 1945.

$k$-commutative matrices, space of, $\mathrm{J} 64 \mathrm{~B} 1,51$

Commutativity in finite matrices, 2060.

Commutator for periodically changing the direction and magnitude of direct currents, 1652 .

Commutators of A and A*, 1653.

matrices, some remarks, 1145 .

Commuting bilinear transformations and matrices, 1654

Compact, microwave refractometer for use in small aircraft, 2415.

multi-anvil wedge type high-pressure apparatus, J $63 \mathrm{C} 1,59$.

Comparative, characteristics of medium-priced fully automatic computers for statistical applications, 2061.

$100 \mathrm{mc}$ measurements at distances far beyond the radio horizon, 972

strengths of some adhesive-adherend systems, 208.

survey of spectrophotometers in the $210-760 \mathrm{~m} \mu$ region, 625 .

tests in a single laboratory, 39.

Comparator, and recorder, high precision automatic frequency, 1356.

noise, for microwaves, 773 .

Comparing outputs from precision time standards, 209.

Comparison, of absolute intensities of [O1] 5577 in the auroral and subauroral zones, J 63D1, 19.

amplitude and angle modulation for narrow-band communication of binary-coded messages in fluctuation noise, 915 .

annual mean solar and lunar atmospheric tides in barometric pressure, as regards to their worldwide distribution of amplitude and phase, 1586.

characteristic energy losses of electrons with the fine structure of the X-ray absorption spectra, 1655.

cryoscopic determinations of purity of benzene by thermometric and calorimetric procedures, 2062. CW field intensity and backscatter delay, 361 .

experimental and theoretical relations between Young's modulus and the flexural and longitudinal resonance frequencies of uniform, bars, J $64 \mathrm{~A} 2,147$

first, second, and third approximations in Bacher and Goudsmit's theory of atomic spectra, 2492.

ionization produced in air by alpha particles near 5-Mev and by beta particles, 2912.

insulating materials, 2416.

mechanical properties of flat sheets, molded shapes, and postformed shapes of cotton fabric-phenolic laminates, 2063.

meteor activity with occurrence of sporadic E reflections, 2.

methods for evaluating trends in time series of tropospheric radio field strength data, 362 .

methods for measuring the tensile and tear properties of plastic films, 1290 . phuse difference and Doppler shift measurements for studying ionosplierle fine structure using enrth satellites, 2493.

sodium hydroxide and sulfuric acid methods for mulysis of wool admixed with cotton and viscore ruyon, 973 .

theories and experiments for the hydraulic dam-brenk wave, 974 .

variously derived solar indexes, 40 .

X-ray standards, $916 ; 1230$.

Comparison theorem for eigenvalues of normal matrices, 1231.

Compatibility equations, of strain rate and strain, RP2813.

Compilation of phase diagrams of interest to ceramists, 1656.

Complementary use of nuclear magnetic resonance and infrared absorption studies in organic phosphorus chemistry, 2494.

Complete night of vertical-incidence ionosphere soundings covering frequency range from $50 \mathrm{Kc} / \mathrm{s}$ to 25 $\mathrm{Mc} / \mathrm{s}, 2064$.

Complete sequences and approximations in normed linear spaces, 2065

Completely continuous normal operators with property $\mathbf{L}$, 671.

Complex, arguments, tables of the exponential integral AMS51.

biorthogonality for certain sets of polynomials, 975 .

plane, formulas for inverse osculatory interpolation in the complex plane, RP2792.

plane, formulas for numerical differentiation, 453 .

systems, some aspects of prediction in the study, 2225.

Components, for mechanized production, 1291.

microwave, 258.

pneumatic and systems, pressure drop, 1456.

waveguide, electroforming of, for the millimeterwave-length range, C587.

Composition, of apparent shearing forces during shear degradation of polymers, 976 .

resistors, noise, $1416 \mathrm{~A}$.

resistors, proposed index of current noise, 1241.

Compound semiconductors, 2495.

Compounds, and metals, energy losses, electron characteristic, 1699.

intermetallic, electrical and optical properties, 1007; 1330.

intermetallic, energy loss, 2937.

intermetallic, photoeffects, 1444 .

semiconducting intermetallic, 823.

two urea addition, analysis of dielectric loss, 2454.

Compressibility, binary alkali borate and silicate glasses at high pressures, 1292.

binary alkaline-earth borate glasses at high pressures, 1658 .

factor, density, specific heat, enthalpy, entropy, free energy function, viscosity, and thermal conductivity of steam, 1657.

liquid normal hydrogen from the boiling point to the central point at pressures up to 100 atmospheres, 1173 .

Compressible turbulent boundary layers with heat transfer and pressure gradient in flow direction, $\mathrm{J} 63 \mathrm{~B} 1$, 53.

Computation, capacity of a cube, 779 .

cyclic cubic units, RP2783.

effective intensity of flashing lights, 2066.

high-speed, of ideal gas thermodynamic functions, 1357.

ideal gas thermodynamic functions, high-speed machine, 1030.

Laboratory, scientific teamwork, 819.

pitfalls 1809 .

vibration modes and frequencies on SEAC, 1293.

$x^{2}, 2877$.

Computational, experience in solving linear programs, 672. methods for a digital logic machine, 1061 .

problems in algebraic number theory, 1873. 
Computations, automatic, with power series, 1683 .

high-speed, in the kinetics of free-radical degradation, 2587.

thin film, Chebychev polynomials in use, J 63A3, 297. rational numbers, subroutine, 1603 .

Computer aids to code checking, 413.

analog for radioactive fallout prediction, 1616 .

defines color, 2913.

development and applications, survey, 2332.

digital, experiments in processing pictorial information, 2119

electronic, to reduce weathering test data, 2858.

Institute for Numerical Analysis, characteristics, 34.

memory uses conventional C-R tubes, 673.

NBS Western automatic (SWAC), design features of a magnetic drum memory, 424 .

new multiple, system, PILOT, 3016.

Computers, 2496.

allpurpose, sieve problem for, 885 .

automatic, development, 1675 .

digital, finding chemical records, 2122.

digital, system design of, at-the NBS: methods for high-speed addition and multiplication, C591, Sect. 1 .

electronic, aiding management control, 441 .

electronic digital, analysis of atomic spectra, 1624.

electronic, European experience, 1336.

electronic, in air traffic control, role, 2380.

electronic, new frontiers in business management, 488 .

high speed, generation of Bessel functions, 2140.

high-speed, in statistics, some examples, 2302.

medium-priced fully automatic, 2061.

network of, to meet deadlines, organizing, 2240 .

scientific, programming, 1820 .

statistics, use, 2404.

two digital, interconnection, 1536.

Computing, high-speed, use of continued fractions, 612. hypergeometric series, programs, 1118.

multiple integrals, some Monte Carlo experiments, 1875 .

operating characteristics of single sample plans, 563 .

Concentration dependence of the sedimentation constants of flexible macromolecules, 1174 .

Concrete, alkali-aggregate reaction in, physical-chemical studies, RP2780.

aluminum, and lead in the range 275 to 525 kilovolts, $\mathrm{X}$-ray attenuation, 1582 .

aluminum, isotropic sources through, penetration of gamma rays, TN11 (PB151370)

beams, reinforced, diagonal tension 222 .

beams, reinforced, flexural cracks, 2566 .

cellular, preparation, 964

cylinders during freezing and thawing, 167.

evaluation of width of cracks, at the surface of reinforcing steel by means of tensile bond specimens, 2113 .

heavy steel-aggregate, 1678 .

high rates of loading, properties, 1824 .

lead, attenuation of $86-$ and $176-\mathrm{Mev}$ synchrotron $\mathrm{X}$-rays, $1635 \mathrm{~A}$.

"no-fines", 190.

properties of, 694 .

reinforced, cracking of, flexural members, 1665

shielding, betatron X-rays, 961 .

steel, and lead, broad and narrow beam attenuation of $\mathrm{Ir}^{192}$ gamma rays, 2480 .

$\mathrm{X}$-rays and gamma-rays, absorption, 1250.

Concretes, insulating, 1742 .

Condensation interfaces in two-phase flows, 2067.

Condenser microphone as a displacement detector calibrator, 2348 .

Condition, for radiation from a solar plasma, 414 .

certain matrix, 149.

certain matrices, II, 1175 .

Conditions at the ionization and shock fronts in collisions of gas clouds-bright rims in diffuse nebulae, 2496 A.

Conductance of slots, 1796.
Conductive flooring, or hospital operating rooms, J $63 \mathrm{C} 2$, 125; Mono. 11

Conductivity, electrical; tables of transport integrals, C595.

finite, propagation constant in rectangular wave guide, 1120 .

fluid contained in a cylindrical vessel, measurement, 2233.

plasmas to microwaves, 2914 .

thermal, of solids at low temperatures, RP2805.

thermal; tables of transport integrals, C595.

Conductors, distribution, and telephone, wind-induced vibration, 2868.

pore, properties, 1461 .

Conference, arctic communication, J 64D1, 73.

National, on Weights and Measures. See National Conference on Weights and Measures.

photonuclear reactions, 2497.

quantum interactions of the free electron, 1659 .

scientific, proposed standard for facilities, 2434 .

Confidence, and tolerance intervals for the normal distribution, 674 .

intervals for a proportion, 1660.

intervals for the expectation of a Poisson variable, 2915.

Configuration, interaction to the $\mathrm{H}_{3}$ complex, application, 2027.

taillights and brakelights, Reports of Night Visibility Committee, Highway Research Board, 1587.

Configurations for automatic mail sorting equipment, analytic comparison, J 63B2, 83 .

Conformations, of the pyranoid sugars: classification of conformers, J 64A2, 171.

pyranoid sugars. II. Infrared absorption spectra of some aldopyranosides, J 64 A3, 239.

Congruences, for the coefficients of modular forms and for the coefficients of $j(\tau), 2498$.

coefficients of modular forms and some new congruences for the partition function, 2068 .

coefficients of modular forms, further identities, 2573.

new, for the partition function, modular forms, 2068 .

Conical coaxial capacitors and their advantages, J $63 \mathrm{C} 2$, 87.

Connections between probability theory and differential and integral equations, 278.

Conservation, parity, scintillation counting in experiments, 2749 .

Consistometer, grease, constant shear rates, higher viscosities, 245.

Constant temperature liquid helium bath and reproducibility of resistance thermometers, 2916.

Constant-amplitude oscillator, 210.

Constant-level device for liquids, 415 .

Constants, elastic, of synthetic single crystal corundum at room temperature, J $64 \mathrm{~A} 3,213$.

equation for atmospheric refractive index at radio frequencies, 855 .

Constitution, and Declaration of Independence of the U.S., preservation, 288.

color and chemical, 1289.

Construction, and application of a class of modular functions, 2069 .

design of a blackbody and its use in the calibration of a grating spectroradiometer, 2513 .

design of a liquid hydrogen temperature refrigeration system, TN38 (PB151397).

floor, wall, and door, sound insulation, BMS144, 2d Suppl.

maintenance, building, selected bibliography, BMS140 $3 \mathrm{~d}$ ed.

materials of: their properties and methods of test, 2625.

operation of a high pressure diffusion cloud chamber. 568.

operation of thoria resistor-type furnaces, 328 .

use of subroutines for the SEAC, 416 .

Containers, milk, paper, 1441.

Continental maps of four ionospheric disturbances, 675 . 
Continuous, analysis of ortho-parahydrogen mixtures, 1294 .

measurement of atmospheric ozone by an automatic photoelectric method, 977.

phase difference measurements of earth satellites, 2499 .

Contractibility and convexity, 978 .

Contractile, reversible processes in fibrous macromolecules, $27+3$.

Contribution, of the annihilation radiation to the gamma-ray flux in lead, 2917.

determination of eigenvalues, AMS49.

dictionary of physics and electronics, 1660A.

Handbook of Physics: 2500.

Arithmetic.

Color vision and colorimetry.

Control mechanisms.

Electrolytic conductivity and electrode processes.

Electronic circuits.

Elements of probability

Geometry.

Integral equations.

Neutron physics.

Nuclear electromagnetic radiations.

Statistical design of experiments.

Thermometry and pyrometry.

study of transport phenomena in gases at high densities, 1296.

theory of rank order statistics-the two sample case, 1661 .

Control, and measurement, integration of concepts in the terminology, 1935.

charts may be all right, but-, 676 .

factors affecting the reproducibility of mechanical properties of refractory semi-dry press specimens, 2501 .

mechanisms, 2500 .

Controls, jet engine, simulator for use in development, C584.

Convenient microwave harmonic generator, 1662.

Convergence, of a method of solving linear problems, 417.

Newton's method in complex Banach spaces, 559 .

numerical iteration, 856 .

Rayleigh quotient iteration for the computation of characteristic roots and vectors, $3003 ; 3004$.

Seidel iterants of nearly symmetric matrices, 329 .

series, $\mathbf{1 4 5}$.

Converging factors, method for the determination of, applied to the asymptotic expansions for the parabolic cylinder functions, 366 .

Conversion of payroll processes at the National Bureau of Standards, 2070.

Converters and frequency multipliers for measurement control, 1349

Convex, cones, theorem on, with applications to linear inequalities, 380 .

functions or linear transforms, 2115.

normed linear spaces, 1350.

polyhedra, intergral boundary points, 1743.

Convexity, and contactibility, 978 .

field of a linear transformation, 2918.

Cooling, magnetic, 1395.

Copolymer composition by combustion analysis for carbon and hydrogen, determination, J 64A2, 157.

Copolymers, butadiene-styrene, crystallization, 983.

melting, 1774 .

temperatures of, glass transition, 2576 .

Copper, and copper alloys, thermal conductivities, 2393. coalesced, thermal conductivity, RP2805.

dilute alloys, $2852 \mathrm{~A}$.

free-machining, low-temperature thermal conductivity, 1393.

functions at low temperatures, 2537.

ions, influence of, on the adherence of vitreous coatings to stainless steel, 1737 .

molybdenum, silver, tantalum, and gold at $662 \mathrm{kev}$, total photoelectric cross sections, 3101.

natural rubber, 523 . nickel, and nickel-copper alloy, amnealed, creep of RP2836.

pure, thermal and electrical conductivity, 2841B. tin, iron, silicon, and niekel, electrorefining studics,
2811.

twinned epitaxy of, RP2873.

wires, measurement of the resistance-strain relation and Poisson's ratio, 1768 .

Copper-tin alloys, gallium, some physical properties, 1876.

Coppers, commercial, low-temperature thermal cenductivity, 2181

Core, a spherical, impedance characteristies of a uniform current loop, J 64D3, 295.

bozes, versatile, 619 .

losses in ferromagnetic materials, RP2865.

Corpuscular spectrometers, 2791.

Correction, factors for the balancing effect in one leg of a U-type manometer, 211.

factors for the balancing effect of a gas in one leg of a manometer, 212.

instrumental drift in flame photometry, RP2918.

Corrections, generalized tables of, to thermodynamic properties for nonpolar gases, 1721 .

Correlation, correction to the activation energy for diffusion in crystalline solids, 2502.

effects in impurity diffusion, 2919.

number of absolute points of, 1943 .

solar noise fluctuations in harmonically related bands, J 63D3, 293.

VHF propagation over irregular terrain, 1663.

Corrosion, and its prevention, geometric factors in electrical measurements, 1354 .

aluminum alloys by exhaust gases, 979 .

factors in design, 213 .

galvanic, potential-current relations, 142.

galvanic theory for adherence of porcelain enamel ground coats to steel, 2358 .

observing $3 \mathrm{~d}$ year anniversary of "Corrosion Technology." 1664.

research at the National Bureau of Standards, 1297; 2503 .

stress, cracking of a gold wedding ring, 1513.

stress, cracking of austenitic stainless steel, 2789 .

stress, cracking of type 304 austenitic stainless steel, 1898.

stress, cracking, sand cast aluminum alloy $220 ; 2349$.

study, 45-year, results of Bureau of Standards, 2287.

Corrugated, diaphragms, investigations of the properties, 1378.

diaphragms, properties, of, investigations, 2166.

Corundum, ores, 1081 .

synthetic single erystal, elastic constants of, at room temperature, J $64 \mathrm{~A} 3,213$.

Cosine of the solar zenith angle $(x)$ for thirty-five locations, tables of median hourly values, TN5 (PB151364).

Cosmic, examples of heat conduction in very rare rotating or expanding gases, 2920 .

radio noise intensities in the VHF band, 418 .

Cosmic-ray, fast, neutrons, sea-level latitude variation, 343.

Cosmical gas dynamics, 2697.

Cotton, and viscose rayon, 973 .

fabrics, abrasion resistance on, made with the blade type abradant and the Schiefer abrasion testing machine, 382 .

wool, and nylon fabrics at different temperatures and relative humidities, 2793 .

Coulomb, wave function by means of recurrence relations, generation, 1532.

wave functions along the transition line, evaluation of, 1018.

wave functions expressed in terms of Bessel-Clifford and Bessel functions, 214.

wave functions expressed in terms of Bessel-Clifford functions, regular and irregular, 1127

wave functions in the transition region, 980 .

Coulometer, coulometric-titration. J $63 \mathrm{C1}, 65$. 
Coulometric titration of acids and bases, precise, J63A2, 153. tritrations, precise, 1815 .

Coulometric-titration coulometer, J $63 \mathrm{C} 1,65$.

Counter sources, $4 \pi$, self-absorption in, RP2859.

Counters, Cerenkov, fast coincidences, 447.

Counting machine, automatic, 398.

Couplers, direction, directivity of, method for measuring, 2425.

Coupling chart for soleniod coils, 41 .

Covariances of least-squares estimates when residuals are correlated, 2504.

Coverage of standard frequency station WWVH, 42.

$\mathrm{Cr}^{+++}$ion in the paramagnetic alums, properties of low temperatures, 522 .

Cracking, in masonry eaused by expansion of mortar, 419. reinforced concrete flexural members, 1665.

Crazing, of acrylic resins, 1298.

polymethyl methacrylate at various temperatures, 555 .

Creep, and acrylic glazing plastics, 1065.

annealed nickel, copper, and two nickel-copper alloys, RP2836.

behavior of structural joints of aircraft materials under constant load and temperatures, 2071.

behavior of transparent plastics at elevated temperatures, 2921 .

cold-drawn nickel, J $63 \mathrm{C} 1,1$.

creep rupture characteristics of some riveted and spot-welded lap joints of aircraft materials, 1299.

crystalline nonmetals, 2072.

static strengths of large bolted joints of forged aluminum alloys under various temperature conditions, 2505 .

Cristobalite, inversion temperature, RP2902.

low, type, crystal structure of aluminum phosphate and gallium phosphate, 1923.

pure, structural anomalies, 3048 .

Criteria, for the characterization of kaolinite, halloysite, and a related mineral in clays and soils, 420 .

existence and equioseillation of best Tchebycheff approximations, J 64B2, 91.

Critical field phenomena in an isotropic paramagnetic crystal, 981.

Critique, 1958, of VHF ionospheric seatter communication, 2505 A.

Cross, section for photodetachment of electrons from $\mathrm{H}$ and D-, experimental, 1338.

section, photodetachment, and the electron affinity of atomic oxygen, 2683.

sections for charge transfer collisions of low energy ions in $\mathrm{N}_{2}$ and $\mathrm{O}_{2}, 982$.

Cross-field error in unsymmetrical range finders, 1300.

CRPL exponential reference atmosphere, Mono.4.

Cryogenic, characteristics of wire resistance strain gages, 2073.

engineering, 2922.

Engineering Laboratories of the NBS, activities, 1252.

engineering, recent advances, 3031 .

struetural adhesives, epoxy, 2547.

systems, bibliography of the physical equilibria and related properties, TN56 (PB161557).

Cryogenics, 1301.

Cryoseopic, determination of purity of highly reactive substances, 1666 .

determinations of purity of benzene by thermometric and calorimetric procedures, comparison, 2062.

Cryostat, for electron bombardment and electron diffraction work, 2506 .

heating control, amplifier, 2090.

liquid helium, with an integral super-conducting resonator, $2610 \mathrm{~A}$.

temperature control, wide-range, 3113.

Crystal, and molecular structure models, bibliography, Mono.14.

chemistry symposium, 215.

detectors and three-millimeter harmonic generators, performance, 2244 . growth, and electrical and optical properties of gray tin, studies, 1518.

mount and techniques for measuring high frequency induced electroluminescence, 2074.

orientation on fatigue-crack initiation in polycrystalline aluminum alloys, effect, 2096.

oscillators, subharmonic, 1154.

paramagnetic, isotropic, 981 .

resonators, underearth, 903.

structure of $\alpha$ and $\beta$ nitrogen, 2879.

structure of aluminum phosphate and gallium phosphate, low cristobalite type, 1923.

structure of anhydrous indium phosphate and thallic phosphate by X-ray diffraction, 1667.

structure of barium hydrogen orthophosphate, RP2817.

structure of triclinic magnesium pyroborate, refinement, RP2936.

Crystal-stabilized pulse-pair generator, 1302.

Crystal-TV, scintillation, device X-rays objects in motion, 1484.

Crystalline dextrose between $25^{\circ}$ and $95^{\circ} \mathrm{C}$, heat capacity, 246.

field, single-axis rotator in, theory of dielectric relaxation, 1554.

nonmetals, creep, 2072.

polymers, melting, 3083.

polyphenyls, infrared spectra of, RP2829.

quartz, internal friction, 2022.

solids, diffusion in, correlation correction to the activation energy, 2502 .

Crystallinity of poly(chlorotrifluoroethylene), specific volume and degree, 2831.

Crystallization, butadiene-styrene copolymers, 983.

flexible polymer molecules, 1924.

high polymers: natural rubber, thermodynamics, 1558.

high polymers, thermodynamics, 2846.

kinetics in polymeric systems, $2507 ; 2923$.

polymers, kinetics, $1047 ; 1382$.

thermodynamies of, in high-polymers; gutta percha, 1965.

Crystallographic, cold drawn music wire, 2509.

data for yohimbine hydrochloride, 2508 .

investigation of tricalcium phosphate hydrate, 421.

Crystals, cesium bromide, transmittance, 349 .

copper single, in water, oxidation of, effect of illumination, 2097

folded chains in dilute solution, theory of formation of polymer J $64 \mathrm{~A} 1,73$.

germanium single, thermoelectric power in, 896 .

gray tin, 2672.

ionic cubic, lattice energies, 2175.

pure hydroxyapatite, preparation, 1816.

quartz, detwinning, TN3, (PB151362).

single aluminum, growth of, RP2868.

single, ceramic oxide, plastic deformation, 2253

single, plastic deformation of ceramic-oxide, 1108

Cube, eapacity of, computation, 779 .

Cumulative, data, fitting a straight line to certain types, 2125 .

fatigue damage of axially loaded Alelad $75 \mathrm{~S}-\mathrm{T} 6$ and Alclad 24S-T3 aluminum-alloy sheet, 1303.

Cunife wire magnets of small size, RP2808.

Cuprammonium solvent and cellulose solutions, 112.

Curie-Weiss law on exchange, dependence of the constants, 1431.

Current, and potential relations for the cathodic protection of steel in a high resistivity environment, $\mathrm{J} 63 \mathrm{C} 1,37$.

potential relations for the cathodic protection of steel in salt water, RP2833.

measured with a Pellat-type electrodynamometer, RP2845.

measurement with the National Bureau of Standards current balance, RP2846.

problems in refining with bone char, 984.

situation with regard to permissible radiation exposure levels, 2075. 
Currents, earth, near a top-loaded monopole antenna, with special regard to electricity small L- and T- antennas, RP2961.

excited on a conducting surface of large radius of curvature, 1668.

Cuspidine, synthetic, 2795.

Cutting characteristies of dental burs as shown by high speed photomicrography, 2076.

Cuvette, densitometer for cardiac output determination by the dye-dilution method, 650 .

densitometer, new, for determination of cardiac output, evaluation of, 714 .

CW field intensity, and backscatter delay, comparison, 361.

high-frequency, records with the aid of simultaneous pulse data, interpretation, 472 .

Cyanide, carbon-14-labeled, preparation, 2262.

deuterium, vibrational energy, 1575 .

hydrogen and deuterium cyanide, infrared spectra, 1739.

Cyclic, acetals of sugars, infrared absorption spectra, RP2960.

cubic units, a computation of, RP2783.

single step iteration, over and under relaxation in the theory of, 781 .

strain testing machine for leather, 1304.

Cylinder functions, parabolic, 366 .

parabolic, asymptotic expansions, 366 .

Cylinder of finite length, response of a loaded electric dipole, J 64D3, 289.

Cylinders, and plates, forces on, in an oscillating fluid, RP2857.

circumferential slots on moderately large conducting, radiation patterns, 1830 .

Cylindrical, cavity containing a gyromagnetic material, 2013.

circular, enclosure, right, acoustic impedance of, 941 . spheroidal, and spherical bodies, J $64 \mathrm{~B} 1,15$.

structure excited by an axial line source, surfacewave resonance effect in a reactive, J 64D1, 13 .

vessel, conductivity of a fluid contained in, measurement, 2233.

Cyroscopic data, calculation, 2042.

\section{D}

Dacron and Mylar polyester strands at low temperatures, some mechanical properties, 2761.

Dampproofing and waterproofing materials, 164.

Dangers of radiation, 1305 .

Data, concerning atomic spectra, 677.

cyroscopic, calculation, 2042.

extreme-value, new method of analyzing, 922 .

processing, automatic, in the Government (1956). progress report, 1822.

receiving tubes, $\mathrm{H} 68$.

straight-line, analyzing, 652 .

suspected, some procedures for the rejection of, 784 .

Day-to-day coordination of IGY observations, 3068 .

Day's, M. M., characterization of inner-product spaces and a conjecture of L. M. Blumenthal, remark, 373 .

Daytime constancy of the absorption of radio waves in the lower ionosphere, 2661.

Dead space, indirectly calculated, RP2826.

Deaired brick, durability, 1683 .

Decay, $\mathrm{Cd}^{115}$ isomers, 569 .

constant in vibrating systems, 678 .

Declaration of Independence and the Constitution of the US, preservation, 288.

Decomposition, CF 4 in flames, 1176.

hydrazoic acid by the electric discharge, mass spectrometric study, 2623.

thermal of polymers, 1556 .

thermal, of poly (vinyl chloride), 3099.

Dedekind, eta-function, some algebraic number theory estimates, 1871 .

number formula, cubic class, a numerical study of, RP2798.
Definition and measurement of the time constant and response time of thermal converters, 2809 .

Defocusing, focusing, and refraction in a circularly stratified atmosphere, $\mathrm{J} 64 \mathrm{D} 3,287$.

Deformation, elastic, in strips with holes loaded through pins, RP2945.

twinning in charpy $\mathrm{V}$-noteh specimens of ingot iron, 679.

Deformed nuclei, 2772 .

Degradation, asphalt, effect of light and water on the, $\mathrm{J} 63 \mathrm{C} 1,31$.

cellulose acetate films, 2924 .

cellulose in a vacuum with ultraviolet light, RP2841. collagen by heat, 2511 .

des polymeres par les rayons et les neutrons, 681 .

flow characteristics of polymer solutions, effect of capillary shape, RP2839.

high poly mers, 43 .

mechanical shear, temperature dependence, 1527.

polyisobutylenes on shearing in solution, 682 . polymer studies by absorption and mass spectrom-
etry, 515 .

polymers, high temperatures, thermal, J 63A3, 261.

polymers, shearing forces, 976 .

polymers, thermal as a function of molecular structure and branching, 1557.

polystyrene by means of mass spectrometry, study, 557.

products of asphalt, watersoluble, 2413.

range straggling of high-energy radiations, 680 .

solid polymers, photochemical, 2455.

thermal, of cellulosic materials, RP2853.

thermal, of polyethylene oxide and polypropylene oxides, 3100 .

thermal, of polymers, 2843.

thermal of polymers at low rates, RP2957.

thermal, of unvulcanized and vulcanized rubber in a vacuum, 1961.

Degree in orientation and crystal size on the scattering of 20-kev electrons by aluminum, 2529 .

Delegate reports on small tools, 2417.

Delta wings, structural analysis and influence coefficients for, 840 .

Delta-funetion approximations, 956.

Delta-wing aircraft, effect of rib flexibility on the vibration modes of, RP2852.

Demagnetization, adiabatic, and the temperature scale below $1^{\circ} \mathrm{K}, 384$.

Demands on teachers in a technological society, 2512.

Dense subgraphs and connectivity, 2925.

Densitometer, cuvette, for cardiac output determination by the dye-dilution method, improvea, 650 .

cuvette, new, for determination of cardiac output, evaluation of, 714 .

Density, entropy, in an infinite system, 2558.

expansions of distribution functions. I. Virial expansion for finite closed systems; canonical ensemble, 2077.

expansions of distribution functions. II. Density expansions in the grand canonical ensemble, 2078.

expansivity, and viscosity of molten alkali silicates, 422.

formula for alkali silicate glasses from annealing to glass-processing temperatures, RP2915.

local clectron, method for measuring from an artificial satellite, J 63D3, 325 .

Dental, amalgam, 1613.

amalgam, microstructure, 765 .

ainalgam: the effect of mechanical condensation of some physical properties, 423.

burs, cutting characteristics, 2076.

casting investments, mechanism of hygroscopic expansion, 1405.

cements, 1306.

cements, silicate, 20 .

chromium-cobalt easting alloys, proposed specification, 1996.

cutting instruments, rotating, $1165 ; 1494$. 
impression compound, revision of American Dental Association specification No. 3, 1244.

impression material, alignate hydrocolloidal, 986.

materials, American Dental Association specifications, $1614 ; 2451$.

materials and instrumentation, review of 1956 dental research, 2290.

materials and instruments 1951-55, 1669.

materials at the NBS, the program of training and graduate instruction, 2827.

materials, relationships of, to clinical subjects, 1476 . materials specifications and certification annual for $1943,1307$.

research, 2079 .

research, résume, 1480 .

research, reviews of 1956: dental materials and instrumentation, 2290.

resins, self-curing, relationship between residual monomer and some properties, 1846.

restorations, margins of, fluid exchange, 451.

roentgenographic equipment, protection, in the use, 1462 .

rotating, diamond abrasive instruments, 2085.

science, physical research, 2891.

$\mathrm{X}$-ray exposure, radiation data during, ionization chambers, 1380 .

X-ray film, 2091.

X-ray machine, panoramic, 1993.

Dentin and enamel, human, determination of some compressive properties, 2518 .

Dentionoenamel junction, 2199.

Dentistry, prosthetic, acrylic resins, 2449.

prosthetic, resins, 2738 .

Denture resins, 1216 .

American Dental Association specification No. 12, 644.

dimensional stability, 2523 .

heat cured, bonding of plastic teeth, 402 .

processing: heat-curing type, 1819.

self-curing, properties, 521 .

some properties, effect of molding processes, 572 .

Departures, from the Saha equation under varying conditions of Lyman continuous opacity, 2926.

hydrogen from L.T.E. in a stellar atmosphere and the consequent structure of the solar chromosphere, 2927.

Dependence of the constants in the Curie-Weiss law on exchange, 1431 .

Depolymerization, chain kinetics, 444.

chain, reactions, 44.

kinetics, some aspects, 307.

polymethylene and polyethylene, 985 .

Deposits, characteristics, 2908.

Derivative, Bessel functions with respect to the order, 2666 .

polynomial and Chebyshev approximation, 786 .

Description, analysis of the first spectrum of iodine, $\mathrm{J} 63 \mathrm{~A} 1,1$.

color, 2080.

method, double, 857 .

new, thorium spectra, Mono.17.

states in quantum mechanics by density matrix and operator techniques, 2081.

Design, construction of a blackbody and its use in the calibration of grating spectroradiometer, 2513 .

construction of a liquid hydrogen temperature refrigeration system, TN38 (PB151397).

data for ortho-parahydrogen converters, 2514.

details of a linear magnetic UFH oscillator attenuator, 216.

experiments in operations research, 1670.

experiments in research and development, 2083.

features of a magnetic drum memory for the NBS Western automatic computer (SWAC), 424.

impact-resisting fabric structures, 3069 .

improvement of man-machine systems, analog-digital simulation, 2453.

performance of a block-type osmometer, R P2931.

performance of a portable infiltration meter, 2082. simple DC resistance thermometer bridges for widerange temperature control, 2515 .

simple resistance thermometer bridges for wide-range control of low temperatures, 2516.

single frequency filters, TN23 (PB151382).

two-dimentional nozzles by the method of characteristics, 1432 .

using mathematics, 355 .

Designing electroformed parts, 683.

Designs, balanced, note on, 923 .

Detector, null, pressure-sensitive diaphragm-type, 2698.

Detergent, fractionated nonionic, light scattering measurements, 2176.

Detergents, ionic, 2667.

synthetic, 318 .

Deterioration, during storage of alginate hydrocolloidal dental impression material, 986.

microbiological, of leather, prevention, 1817.

silicate cements in the tropics, 217.

Determinantal inequality, $1232 ; 2657$.

H. P. Robertson, 1980 .

Determinanten mit ueberwiegender Hauptdiagonale und die absolute Konvergenz von linearen Iterationsprozessen, 1671.

Determinants, of Legendre polynomials, second order, 305 . predominant principal diagonals and the absolute convergence of linear iteration processes, 1671.

Determination of, acetylene in air in concentrations from ten parts per billion to ten parts per million, 2928.

aldehyde in cellulose: a review of methods, 1308.

aluminum in precipitation hardening stainless steel and high temperature alloys, J $64 \mathrm{~A} 2,181$.

amplitude-probability distribution of atmospheric radio noise from statistical moments, J 64D1, 49. attenuation from impedance measurements, 45 .

carbon monoxide in studies of air pollution, 1309.

copolymer composition by combustion analysis for carbon and hydrogen, J 64A2, 157.

correlation of flow capacities of pneumatic components, C588.

critical depth in spatially variable flow, 425 .

critical micelle concentrations by a bubble pressure method, 787.

dissociation constants in a mixture of two weak acids by electromotive force measurements, 218 .

dynamic stress-strain curves from stain waves in long bars, 684 .

eigenvectors and eigenvalues of symmetric matrices, 330.

extreme values of the spectrum of a bounded selfadjoint operator, 220 .

freezing point of engine antifreezes, 331.

gamma-ray emission of radium, RP2801.

general index of effort in sorting mail by conventional methods. TN54 (PB161555).

glucose by means of sodium chlorite, 1310.

grease in leather, 219.

impedance with a double-slug transformer, 570 .

inorganic constituents in sucrose solutions, 987.

loads in the presence of thermal stresses, 988.

monomer content of polymethyl methacrylate, 1673

niobium and tantalum in titanium-base alloys, RP2929.

nitrogen and sulfur in steel, 427.

nitrogen in leather by Kjeldahl method, 332 .

phosphate by differential spectrophometry, 685 .

reducing sugars and reducing end groups in polysaccharides by reaction with carbon-14-labeled eyanide, 2517.

resin content of glass fiber polyester laminates containing a calcium carbonate filler, 1672 .

reverberant sound absorption coefficient from acoustic impedance measurement, 46.

some compressive properties of human enamel and dentin, 2518.

sulfur in rubber vulcanizates, 1311.

thermodynamic acidity constants, 991. 
titanium, zirconium, niobium, and tantalum in steels: separations by anion-exchange, RP2923.

use of the sag point as a reference point in the heating of glasses, RP2791.

viscosity of exhaust-gas mixtures at elevated temperatures, 989 .

water content in Freon-12 circulating in a refrigeration system, 990.

Detwinning quartz erystals, TN3 (PB151362).

Deuterated, ammonia, 2411.

styrene polymers, 2680 .

Deuteride, hydrogen, 153.

Deuterium, as a tracer, mechanism of formation of $\mathrm{OH}$, $\mathrm{CH}$, and $\mathrm{HCO}$ in flame spectra, 868 .

cyanide and hydrogen cyanide, infrared spectra, 1739 . cyanide, vibrational energy, 1575 .

hydrogen, mixtures, distillation, 2093.

hydrogen molecules, 2410 .

hydrogen-deuterium mixtures, spectroscopic analysis, 551 .

liquid normal, pressure-volume temperature relations of, 1115 .

oxide, dielectric constant of, RP2874.

sulfide, some infrared bands of, RP2789.

use of, to investigate extent of branching in polystyrene, 1220.

Deutero-ammonias, microwave spectra, 260.

Deuteromethanes, redetermination of mass spectra, RP2896.

vapor pressures of, 889

Deuterostyrene $\alpha$ and $\beta$ pryolysis, polymers, 1464 .

Deuterostyrenes, mass spectra of some, RP2926.

Developing seals for abrasive-fluid mixtures, 221.

Development of, automatic computers, 1675 .

craze and impact resistance in glazing plastics by multiaxial stretching, 2084

evaluation of ceramic coatings for thermocouples, 1674

methods for evaulation of rotating dental diamond abrasive instruments, 2085.

some infrared transmitting glasses, 1177

turbulence-measuring equipment, 686 .

VHF field-intensity standards, 427.

Developments in, instruments, 2519.

recent, the field of microwave power measurements at the NBS, 2720.

synthetic materials in America in 1959, 21.

vibration measurement, 1492

Deviation, standard, and mean absolute value of the phase of a constant vector plus a Rayleigh-distributed vector, RP2950.

Device for filtering and precise dispensing of solutions in closed systems, 47.

Dewar, nonventing liquid helium, temperature stratification, J $64 \mathrm{C} 1,19$

Dewars, calculation of gaseous heat conduction, 2482.

Dextran, intrinsic viscosity-molecular weight relationships, 745 .

sorption of, collodion membranes, 832 .

sorption of serum albumin and surface-active agents, cellulose nitrate membranes in the presence of, 1500 .

Dextrans, clinical, by periodate oxidation and isotope dilution techniques, structural analysis, 2319.

Dextrose in sugar mixtures, quantitative chromotographic procedure for determining, 2276.

Diagnostic tests, index for rating, 71 .

Diagonal tension in reinforced concrete beams, 222.

Diagonalizing second-order matrices, tables for, RP2838.

Diagrams, phase, of interest to ceramists, compilation, 1656.

Dialysis experiments with ionic surface-active agents; low rates of equilibration, 2232

Diameter and length of the driven element, impedance of a corner-reflector antenna as a function of the, J $64 \mathrm{D} 2,135$.

Diamond, some properties, 2762.

Diaphragm, error and testing rate in the paper bursting test, 428 .

theory, Haringx, invited discussion, 2167.
Diaphragms, corrugated, design of, 1981.

corruggted, investigations of the properties, 1378; 2166.

Diaphragm-type null detector, pressure-sensitive, 2698.

Diathermy, generators, 429 .

rules and the physician, 430 .

Diatomic, free radicals of the isotopic hydrides of oxygen and sulphur, 1357.

gases, pure, thermodynamic properties, effect of dissociation, 1326.

hydrides, electronic structure, 2543.

molecular negative ions, 2841 .

molecules, oxygen, nitrogen, nitricocide, and their molecule ions, 2394.

molecules, vibrational energy exchange between, 2862 .

Diborane, heat of reaction of, with water and the heat of formation of boric oxide, RP2927.

pentaborane, heats of formation, RP2901.

Diboron tetrachloride, vibrational spectrum and force constants, 2409.

Dielectric, amplifiers, 963.

cavity resonator, measurements on rod samples, 2907.

constant of deuterium oxide, RP2874.

constant of hydrogen-bonded liquids, RP2905.

constant of water vapor, 431.

constant, resistivity, and power factor of leather, 533 .

dispersion data for pure liquids and dilute solutions, tables, C589.

elastic, and piezoelectric constants of quartz, method for dynamic determination, Mono. 9 .

elasticity loss of quartz, 1620.

interface, impedance of a wire grid parallel, 2364 .

loss in two urea addition compounds, analysis, 2454.

measurements from 100 to $400 \mathrm{Mc}$, variable length reentrant cavity, 638 .

measurements up to $500^{\circ} \mathrm{C}$, symposium on temperature stability of electrical insulating materials, 992.

properties of gases, 259 .

properties of magnesium-strontium titanates, note, 2221 .

properties of titania or tin oxide containing varying proportions or rare-earth oxides, 2520 .

properties, polymer physics and their relation to, 2440 .

relaxation for a single-axis rotator in a crystalline field, theory, 1554

standards, American, 944.

Dielectrics, advancements in, 642; 943.

dipoles in an interface between two, radiation resistance, 1833 .

low-loss, measuring power factor, 256 .

Dies, rotors, and rate of shear on Mooney viscosity, 740 . Differences in the relationship between ionospheric critical frequencies and sunspot number for different sunspot cycles, 571 .

Differential, cross-section measurements for 1-Mev bremsstrahlung, 993 .

cross-section measurements of thin-target bremsstrahlung produced by 2.7- to 9.7-Mev electrons, 1676.

integral geometry, metric methods in, 764 .

thermal analysis above $1200^{\circ} \mathrm{C}, 2521$.

Differential equations, elliptic, discrete approximations, 1314.

experiments in the solution, by Monte Carlo methods, 1019.

hydrodynamic stability in a domain containing a transition point, asymptotic solution of, 656 .

integrable forcing term, stable systems, 1893 .

integral, some connections between probability theory and differential, 278.

linear, analytic systems, solutions, 932 .

non-linear, behavior in the large of the integral curves, 2662 .

non-linear, of the second order with integrable forcing term, 1429 .

numerical solution, 1588.

ordinary, non-self-adjoint boundary value problems, J $64 \mathrm{~B} 2,83$

recurrent relations, solution of, 97 .

Riccati's, note on the solution, J 64B2, 95. 
roots of Riccati's: low- and very low-radiofrequency tables of ground wave parameters for the spherical earth theory, TN7, (PB151366).

Differentiation, numerical, in the complex plane, formulas, 453.

Diffraction, electromagnetic pulses by curved conducting surfaces, 3005 .

electromagnetic waves by smooth obstacles for grazing angles, $J 63 \mathrm{D} 2,181$.

powder patterns, standard X-ray, C539, Vols. 7, 8, 9.

reflection of waves and pulses by wedges and corners, RP2906.

spherical wave pulse by a half plane screen, 2086 .

waves by wedges, 1792 .

Diffuse nebulae, bright rims, 2479 .

Diffusion, impurity, 2919.

particles in turbulent flow, 2929.

particles with memory, 2087.

penetration of hard X-rays: polarization effects, 793 .

Difluoride, oxygen, by electron impact, 2169.

Difluorodichloromethane (freon-12), gaseous, thermodynamic properties, 604

Digital, analog, simulation for the design and improvement of man-machine systems, 2453.

computers, system design at the NBS: methods for high-speed addition and multiplication. C591, Sect. 1 .

computers, two, interconnection, 1536 .

logic machine, computational methods, 1061.

reduction of spectrophotometric data to Munsell renotations, 2522 .

Dihedral and trihedral angles, sums of, in a tetrahedron 597

Dilute, alloys of copper, $2852 \mathrm{~A}$.

aqueous solutions on ionic detergents, 2667 .

protein solutions, interpretation of hydrodynamic data, 2668.

solution, thermodynamic behavior of flexible chain molecules: polymethyl methacrylate, 687 .

Dilworth's theorem on partially ordered sets, 1677 .

Dimensional, stability of denture base resins, 2523 .

two, patterns by scanning techniques, processing of, 802.

Dimensions, units, and standards, 2088.

units in physics, 611 .

2,6-Dimethylbenzoic acid in water, 2369.

Dimethylmercury, isotope analysis, 1381.

Dimethylphosphinoborine trimer: mass spectra and thermal decomposition, 994 .

Diode, reactance modulator, 3070 .

resistance, bridge circuit for temperature control, TN34 (PB151393)

retarding field, 566 .

Diodes and capacitors, experimental rapid access memorv. 392.

Dioxide, magnesium oxide-germanium, 3094

titanium, semiconductors, electrical properties, 704 .

uranium, specimens, effect of particle size on the bulk density and strength properties, 1926.

Dip coater, improved, $949 ; 1033$.

Dipole, in an imperfectly conducing eylinder of finite length, J 64D3, 289.

magnetic, arc-lengths along the lines of force, 1633.

moment of perchloryl fluoride, 2089

Dipoles in an interface between two dielectrics, radiation resistance, 1833 .

Dirac theory, simplified approach to spin, 2757.

Direct, approach to the problem of stability in the numerical solution of partial differential equations, 1588 .

filling resins: dimensional changes resulting from polymerization shrinkage and water absorption, 689 .

filling resins, mechanical properties, 1069.

indirect filling resins: a review of some physical and chemical properties, 688.

nitrogen calibration of dead space in gas absorption systems, 2524 .

observation of nuclear absorption and elastic scattering of X-rays, 1312. resinous filling materials: temperature rise during polymerization, 223 .

Direct-coupled, circuits, 962 .

power amplifier for cryostat heating control, 2090.

Direct-current bridge for accurate bolometric power measurements, RP2776.

Direct-indicating recording instruments, 690.

Directional, couplers, directivity of, method for measuring, 2425.

distribution of 1040-kev radiation from a high voltage $\mathrm{X}$-ray tube, 224.

Disaster monitoring with amateur photographic film and with dental X-ray film, 2091.

Diserete, analogs of inequalities of Wirtinger Monatsh, 1313.

approximations to elliptic differential equations, 1314.

Discussion of, design of corrugated diaphragms, 1981 .

paper "Heat treatment affects properties of enamelsteel composites," 2525.

paper "Heavy Steel-Aggregate Concrete," 1678.

paper "Pressure-flow characteristics of pneumatic valves," 2092.

paper "Wave action in diffusers for exhaust-pipe systems, with special reference to the scavenging of two-stroke engines," 1679.

Disintegration, $\mathrm{Ba}^{113}, 1178$.

$5.8^{\mathrm{d}} \mathrm{Sb}^{120}, 1315$.

$\mathrm{Nb}^{21}$ and $\mathrm{Nb}^{92}, 1179$

scheme of $\mathrm{I}^{131}, 150$.

selenium-73, i316; 1680 .

Dispersion, dielectric, data for pure liquids and dilute solutions, tables, C589.

F2-layer critical frequencies, 225.

function of paramagnetic relaxation, 2418.

$\mathrm{NH}_{3}$ in the microwave region, 48 .

sound in gases, 971 .

Dissociation, 4-chloro-4'-aminodiphenylsulfone, RP2794.

ionization of hydrazoic acid and methyl azide by electron impact, 2604.

ionization of oxygen difluoride by electron impact, 2169.

ionization of perchlorylfuoride by electron impact, 2170.

Dissociation constant, aqueous ammonia at $0^{\circ}$ to $50^{\circ}$ from EMF studies of the ammonium salt of a weak acid, 49.

d, $l$-malic acid, RP2947.

Distillation, analytical, 2744 .

hydrogen-deuterium mixtures, 2093.

hydrogen isotopes, 2526.

multicomponent, separation of hydrogen isotopes, 2751 .

Distinct representatives, on systems, 1793.

Distinction between initiation and propagation of a fatigue crack, 1925.

Distortion, inverse nodal slide, evaluation of, $\mathrm{J} 63 \mathrm{C} 2$, 105.

low, transistor audio oscillator, 755 .

magnification, variation, 1572 .

modified goniometric method, evaluation of lens, J $63 \mathrm{C} 2,113$

Distribution, electrical conduction currents in the vicinity of thunderstorms, 432 .

electron scattering by gold, 1317.

electrons scattered by solids, 1681 .

functions, essential property of the Fourier transforms, 391 .

incoming lettermail at the Baltimore, Maryland City Post Office, TN33 (PB151392).

linear energy transfer, or "ion density" for fast neutrons in water, 1180

mail by destination at the San Francisco, Los Angeles, and Baltimore post offices, TN27 (PB151386).

matter with temperature in the emission corona, 2930.

normal, note, 1595 .

normal, property of, 928 .

phase diagrams of interest to ceramists, 1318. 
serial correlation coefficient near the ends of the range, 2527.

Diurnal change of ionospheric heights deduced from phase velocity, 2931.

Divided differences, modified, 1092.

Documentation in instrumentation, 1682.

Documents, preservation, by lamination, Mono.5

Does rationalization change units, 2094.

Door, wall, and floor constructions, sound insulation, BMS144, 2d Suppl.

Doppler shift measurements for studying ionospheric fine structure using earth satellites, comparison of phase differences, 2493.

Dose, distributions in water for betatron X-rays to 37 Mev, studies, 1904.

measurements in water using scintillation and ionization detectors, 995 .

walls in radiographic rooms, 1319.

Dosimeter, gamma-ray, neutron-insensitive, 2211.

photographic personnel, for $\mathrm{X}$-radiation in the range from $30 \mathrm{kev}$ to beyond $1 \mathrm{Mev}, 1994$.

Dosimeters, radiation survey meters, pocket chambers, X-ray calibration, 172 .

Dosimetry, film badge, 1021.

high-level, measurement systems, 2984.

ionizing radiations, introductory remarks on, 1045 .

photographic, at total exposure levels below $20 \mathrm{mr}$, TN29 (PB151388).

photographic, of X-and gamma-rays, 2611.

radiophotoluminescence, system of the U.S. Navy, 809

Double, description method, 857.

scattering of electrons with magnetic interaction, 1320

Double-pulse total-absorption fast neutron spectrometer, TN1 (PB151360).

Double-slug transformer, determination of impedance, 570 .

Doubly stochastic transforms of a vector, 2658 .

Downcoming radio waves, measurement of characteristics, 2932.

Drain casting of a high-grade magnesium oxide support used in an experimental vacuum tube, 996.

Drainage, method, cathode film studies, 1282.

venting, problems, 289.

Drift, instrumental, correction for in flame photometry, RP2918.

Dry cell standards broadened-now includes mercury-, air-, and flat-type cells, 1321

Dry cells, batteries, specification, H71. gassing, 2359 .

Dry, static calorimeter for RF power measurement, 2419.

Drying twist in plant fibers, 1181 .

Dual-amplitude axial-load fatigue machine, 626 .

Durability, boys' shoes, 691. deaired brick, 1683 .

Duration of random walks, 281.

Dyes, azo, 881 .

azo, and indigo, steric effects, 1897 .

azo, in organic solvents, hydration, 1543 .

chemical constitution, relation between the absorption spectra. XXV. Phototropism and cis-trans isomerism in aromatic azo compounds, 593.

chemical constitution, relation between the absorption spectra. XXII. Cis-trans isomerism in thioindigo dyes, 341 .

chemical constitution, relation between the absorption spectra. XXIV. Cis-trans isomerism in thioindigo dyes in sulfuric acid, 342 .

chemical constitution, absorption spectra, relation. XXVIII. The hydration of azo dyes in organic solvents, 1543 .

direct azo, interaction in aqueous solution, RP2823.

phototropic, shutter-illumination device for the study, 374.

Dynamic, bulk viscosity of polyisobutylene, 1182

characteristics of accelerometers by rotation, 913.

compliance, dynamic modulus, and equivalent Voight and Maxwell models for polvisobutylene, 692 .

determination of the elastic, dielectric, and piezoelectrie constants of quartz, Mono. 9 . measurements of the magnetoclastic properties of ferrites, TN49 (PB151408).

mechanieal properties of polyisobutylene, 1183.

spectrum for Sacremento Peak, 1233.

static tests for mechanical properties of fired plastic refractories and other more resilient materials, 1684

Dynamics, complete right ventricular failure in dogs maintained with an extracorporeal left ventricle, 1685 cosmieal gas, 2697.

inlet system of a four-stroke single-cylinder enginc, 1322

Dynamo, atmospheric, theory, 2228.

E

Early, history and development of building codes, 2095

work in applied optics at the NBS, 226.

Earphones, audiometer, probe tube method for the transfer of threshold standards, 632 .

ears and couplers, 1199

Earth, electrical resistance, 1928.

environment, 3071 .

ionosphere, multiple reflections between, in VLF propagation, 1781 .

propagation in the vicinity of Point Barrow, Alaska, J 64D1, 95.

rare, region, photoneutron yields, 2249

Earth currents, near a monopole antenna with symmetrical top loading, RP2959.

near a top-loaded monopole antenna with a special regard to electrically small $\mathrm{L}$ - and $\mathrm{T}$-antennas, RP2961.

near a VLF monopole antenna with a radial wire ground system, 2437 .

Earth satellite, comparison of phase difference and Doppler shift measurements for studying ionospheric fine structure, using, 2493.

continuous phase difference measurements, 2499.

theory of the effect of drag on the orbital inclination of, RP2934.

Earth-flattening procedure in propagation around a sphere, J $64 \mathrm{D} 1,61$.

Earth's exosphere, very low-frequency emissions, 3097.

upper atmosphere, gravitation and thermal oscillations, 1726 .

Echo on the operation of high frequency communication circuits, 2810 .

Echoes, low frequency ionosphere, note on the polarization, 371 .

Eclipse, geomagnetic, phenomena, survey of, 1908.

May 20, 1957, 121.

Economic aspects of the bone char process in sugar refining, 132.

Eddy-current mutual-inductance transducers with highconductıvity reference plates, 1323.

Edge of space, 2849.

Editorial on Roentgen ray protection design, 433 .

Education in radiation protection, 1324.

Effect, Zeeman, 1584.

Effect of, anodic (HAE) coating on the fatigue strength of magnesium alloy specimens, $1325 ; 1531$.

antenna size on gain, bandwidth, and efficiency, $J$ 64D1, 1.

atmospheric horizontal inhomogeneity upon ray tracing, J 63D3, 287.

calcium treatment on solubility and calcium uptake of synthetic hydroxyapatite and rat molar enamel, 2528.

ceramic coating on the creep behavior of som " high temperature alloys, 3072 .

chemical combination of the characteristic energy loss of electrons, 997.

crystal field and spin-orbit coupling on magnetic susceptibility of systems with $f^{2}$ electron configuration, RP2893.

crystal orientation on fatigue-crack initiation in polycrystalline aluminum alloys, 2096. 
degree in orientation and crystal size on the scattering of 20 -kev electrons by aluminum, 2529 .

dissociation on thermodynamic properties of pure diatomic gases, 1326 .

dissolved air on the speed of sound in water, 1686 .

earth's magnetic field on m.u.f. calculations, 3075 .

echo on the operation of high frequency communication circuits, 2810 .

exposure to soils on the properties of asbestos-cement pipes, 998.

fluorides on infrared transmittance of certain silicate glasses, 2933.

fuel-immersion on laminated plastics, 50 .

ground screen on the field radiated from a monopole, 1688.

heat treatment of the susceptibility of sand cast aluminum alloy 220 to stress corrosion eracking, 2349 .

illuminance of a lens interposed between the source and the illuminated surface, 858 .

illumination on the oxidation of copper single crystals in water, 2097.

internal radiant heat transfer on temperature distribution, thermal stress, and deflection in box beams, RP2949.

light and water on the degradation of asphalt, $\mathrm{J} 63 \mathrm{C} 1$, 31.

light on coated groundwood papers, $693 ; 1327$

melamine resin on chemical tests of paper, 227 .

microwave signals incident upon different regions of a de hydrogen glow, 2098.

molding processes on some properties of denture resins, 572 .

molecular weight on the crazing and tensile properties of polymethyl methacrylate, 999 .

multipath distortion on the choice of operating frequencies for high-frequency communication circuits, 3073 .

new lower permissible exposure levels on use of radioactive isotopes, 2099.

oxygen content of the furnace atmosphere on the adherence of vitreous coatings to iron, 1329

oxygen on the bonding of gold to fused silica, RP2942

particle size on the bulk density and strength properties of uranium dioxide specimens, 1926.

preheating on thermal expansion of silica-gypsum investiments, 1184.

prism on the location of the principal point, 2350 .

receiver bandwidth on amplitude distribution of VLF atmospheric noise, 2351.

recent mass-measurements on the faraday, 573 .

sample preparation on precision in the hide substance determination, 1000 .

size on the inlet-system dynamics in four-stroke, single-cylinder engines, 1687.

small irregularities on the constitutive relations for the ionosphere, J 63D2, 135.

sporadic $\mathrm{E}$ on television reception, $574 ; 859$.

sporadic $\mathrm{E}$ on VHF transmission in North America, 3074.

straining rate on the compressive strength and elastic properties of concrete, 694 .

strain-temperature history on the tensile behavior of titanium and a titanium alloy, RP2897.

sudden water release on the reservoir free outflow hydrograph, J 63B2, 117.

super-refractive layers on tropospheric signal characteristics in the Pacific coast region, 2100.

suspension position on apparent values of internal friction determined by Forster's method, 2530 .

temperature and degree of polymerization on the sorption of water by polymethyl methacrylate, 1328.

tensile strain at low temperatures on deformation twinning in ingot iron, 695 .

textile fiber structure on wear resistance, 610 .

transverse atmospheric drag on satellite orbits, 2532.

20-year marine exposure on some aluminum alloys, 1689 . variation of circuit parameters on the excitation of spectra by capacitor discharges, 696 .

Effective, earth's radius for radiowave propagation beyond the horizon, 228 .

electrical constants of solid at low frequencies, 2352.

intensity of flashing lights, 2101.

Effectiveness of vacuum investing in the climination of surface defects in gold castings, 697 .

Effects of, acid treatment on acid and abrasion resistance of porcelain enamels, 51 .

aging on the surface area of portland cement paste, J $64 \mathrm{~A} 2,163$

antioxidants on asphalt durability, J 64C1, 13.

atomic radiation on polymers, 698 .

biaxial stretch-forming, 434 .

boundary and inhomogeneities on the penetration of gamma radiations, 2101

fungicides on deterioration of leather, 2353.

high degrees of biaxial stretch-forming on crazing and other properties of acrylic plastic glazing, 699 .

high-altitude nuclear explosions on radio noise, $J$ $64 \mathrm{D} 1,37$

humidity during fabrication of polyester laminates, 435.

internal motion in the microwave spectrum of methylamine, 1001.

ionosphere disturbances on low frequency propagation, 229 .

low temperatures and notch depth on the mechanical behavior of an annealed commercially pure titanium, RP2790.

moderate biaxial stretch-forming on tensile and crazing properties of acrylic plastic glazing, 436 .

molding pressure on strength properties of glass-fiber reinforeed plastics, 2533.

multiaxial stretching on crazing and other properties of transparent plastics, $1002 ; 1690$.

plastic and steel surfaces on clotting time of human blood, 1003.

rates of straining on some tensile properties of polyester film, 2354.

resin coating methods and other variables on physical properties of glass-fabric reinforced polyesters, 1004.

self-absorption of rotational "temperatures" of $\mathrm{OH}$ in flames, 700 .

vibrational state on rotational intensity distribution in $\mathrm{OH}, 701$.

weathering on certain mortars exposed in brick masonry with and without caps and flashings, 1927.

Eigenvalues, bounds for, of Hermitian matrices by the segment method, 1005.

eigenvectors of symmetric matrices, determination, 330 .

extremum property of sums, 1234.

free plate, RP2784.

further contributions to the solution of simultaneous linear equations and the detemination of, AMS49.

matrix, circular disks containing, note, 2650 .

normal matrices, comparison theorem, 1231.

sums of normal matrices, 1425 .

symmetric functions, note, 1990.

symmetric integral equations, error bounds, 1767.

Eigenvectors and eigenvalues of symmetric matrices, determination, 330 .

Eight decade logarithmic ohmmeter, 1619.

Einschliessung von eigenwerten hermitescher Matrizen nach dem abschnittsverfahren, 1005.

El sistema I. C. I. para la especificacion del color, 52.

Elastic, constants and static strains with hydrostatic pressure, variation, 2407.

constants of synthetic single crystal corundum at room temperature, J 64A3, 213.

deformation in strips with holes loaded through pins, RP2945.

dielectric, and piezoelectric constants of quartz, method for dynamic determination, Mono.9.

distortion error in the dead weight piston gage, 2103. 
moduli of a borosilicate glass after heat treatment, 1844 .

moduli of glasses at elevated temperatures by a dvnamic method, 1692 .

moduli of indium antimonide, 1693.

moduli of zinc blend lattices, ionic character, 2367.

nuclear, scattering of photons, 1787 .

plastic behavior of the ferrite lattice in a low-alloy steel, 1691 .

problem for a ring of uniform force in an infinite body, RP2855.

properties of pure-gum rubber vulcanizates, 2534

recovery, non-Newtonian flow, and molecular weight distribution, 3037

scattering, and absorption of X-rays, direct observation, 1312

scattering of electrons and positrons by point nuclei, 1694 .

scattering of photons, 1006.

scattering of photons by tantalum, 2535.

waves in anisotropic media, 2104.

Elasticity, dielectric loss of quartz, 1620.

rubber, $2810 \mathrm{~A}$

rubber, physics, 2251.

strength, and other related properties of some refractory castables, 2536 .

Electrets, plastic, 796 .

plastic, and their applications, 797 .

Electric, discharge, mass spectrometric study of the decomposition of hydrazoic acid, 2623.

discharge through nitrogen, oxygen, hydrogen, water, and ammonia, 2309 .

discharges, absorption spectra of solids condensed at low temperatures, 2445.

field at the ground plane near a top-loaded monopole antenna with special regard to electrically small Land T-antennas, J 64D2, 139.

hygrometers, C586.

Electrical, conduction currents in the vicinity of thunderstorms, distribution, 432 .

conduction in magnesium stannide at low temperatures, 1695.

conductivity and Hall effect in oxide cathodes, 1063 conductivity, tables of transport integrals, C595.

connections, symbolic designations, 2334.

constants of solid at low frequencies, 2352.

contact resistance of copper, copper functions at low temperatures, 2537.

differentiation of retarding potential measurements, 2596.

discharge in Azote condensed to a solid state at very low temperature, 2944.

discharge induced luminescence of solids at low temperatures, 2934.

indicating instruments used in early Edison central stations, 702 .

indicators, 1134.

induced, polarization, 2431

insulating materials, 992.

noise from instrument cables subjected to shock and vibration, 437.

optical properties of gray tin, studies of crystal growth, 1518 .

optical properties of intermetallic compounds, 1330.

optical properties of intermetallic compounds. I. Indium antimonide, II. Gallium antimonide, and III. Aluminum antimonide, 1007.

properties of indium antimonide, 703 .

properties of sammarium doped barium titanate, 2105 .

properties of titanium dioxide semiconductors, 704

resistance of vulcanizates, carbon black differentiation, 408.

resistance to the earth of a live tree, 1928.

resistivity of molten alkaline-earth borate glasses with three mole percent of potassium oxide, 2864.

resistivity of molten binary alkaline-earth borates, 1156. thermal conductivity of aluminum and aluminum alloys, $2841 \mathrm{~A}$.

thermal conductivity of pure copper, 2841B.

viscosity resistivity of molten alkali borates, 907

Electrical instruments, 99.

meters, 1814

testing of, 886 .

Electrical measurements, core curriculum, 2935.

geometric factors, relating to corrosion and its prevention, 1354

selection of bolt materials for service underground, 1008 . standards, 316 .

Electrical-analog method for transient heat-flow analysis, RP2892.

Electrically, heated filaments, on-off temperature control, 2660

short ground systems, fields, J 63D2, 175.

Electricity, standards, 2593.

static, hazards from, 461

Electrocardiographic data, T N42 (PB151401).

Electrochemical, battery system, 2436.

calorimetry, 2538.

theory, fifty years, 448 .

Electrode, determination of concentration polarization, 2605 .

processes and electrolytic conductivity, 2500.

silver, 1486.

silver-silver oxide, 1998

standard, potential of the silver, silver chloride electrode, 1895.

Electrodeless metal-halide lamps, 705 .

Electrodeposited, coatings, specifications, 313 .

electropolished surfaces by means of the microinterferometer, measurement of surface roughness, 86 . metals, and nickel, 511 .

Electrodeposition, aluminum, hydride bath, 363 .

fifty years, 449

metals, 2936.

some of the less common metals from nonaqueous media, 1331.

Electrodeposition of metals from organic solutions, Part I, General survey, 1696.

Part II: Further studies on the electrodeposition of aluminum from a hydride bath, 1697.

Part III. Preparation and electrolysis of titanium and zirconium compounds in nonaqueous media, 2106.

Part IV. Electrodeposition of beryllium and beryllium alloys, 2107.

Part V: Electrodeposition of magnesium and magnesium alloys, 2108 .

Electrodeposits, porosity in, nature, cause and effect, 1940.

Electrodes, hydrogen, titration of acids with, RP2769.

Electrodynamometer, Pellat-type measurement of current with, RP2845.

Electroformed parts, designing, 683.

Electroforming, 1009.

precision waveguide components in the millimeter wavelengths, technique, 2339.

waveguide components for the millimeter-wavelength range, $\mathrm{C} 587$.

Electroless, nickel plating, 2327

plating comes of age, 1010 .

Electroluminescence, high frequency induced, in zine sulfide, 1728 .

measuring high frequency, crystal mount and techniques, 2074

surface layer of $\mathrm{BaTiO}_{3}, \mathrm{SrTiO}_{3}$, and associated materials, 2539.

Electrolysis of organic solvents with reference to the electrodeposition of metals, 2936.

Electrolyte, single solid or molten chloride, theoretical electromotive forces for cells containing, 1957.

Electrolytes, polyethylene glycol, punched-cell batteries, 1828.

Electrolytic, coatings on magnesium base alloys from alkaline chromate solutions, 1332 .

conductivity and electrode processes, 2500. 
preparation of molybdenum from fused salts, 1333; 2811.

Electrolyzed gas film, 865 .

Electromagnetic, acoustic pulses, propagation, 1799 .

audio-frequency, noise during a period of solar disturbance, 2019.

energy in a randomly inhomogeneous atmosphere, seattering, 536.

field of rotating uniformly magnetized sphere, $\mathbf{5 7 5}$.

ground wave on a spherical earth, transient behavior, 2386.

hiss, audio-frequency, recorded in Boulder in 1956, 2033.

interactions in dense materials, atomic theory, 1635. nuclear, radiations, 2500 .

problems involving spheroidal, spherical, and cylindrical bodies, J 64B1, 15 .

pulses by curved conducting surfaces, 3005 .

response of an imperfectly conducting thin dyke, 3006. sources immersed in a semi-infinite conducting medium, basic experimental studies of the magnetic field, J $64 \mathrm{D} 1,21$.

transient, dipole field by a conductive sheet, 1866 . wave field, diffraction and reflection, RP2906.

Electromagnetic waves, along a curved surface, theory of propagation, 2673.

diffraction of, by smooth obstacles for grazing angles, J $63 \mathrm{D} 2,181$

plasma, 3023.

presence of stratified media, transmission and reflection, RP2899.

Electromotive, theoretical forces for cells containing a single solid or molten chloride electrolyte, 1957.

Electromotive force, measurement, 141.

measurements, 218 .

method of determining ionization constants: ionization of bisulfate ion, 3076 .

thermal, tables of transport integrals, C595.

Electron, absorption spectrometer using an improved velocity analyzer, 2541 .

affinities, atomic, 2900 .

bombardment and electron diffraction work, 2506 .

bombardment, light emission from solid nitrogen, 2975.

bremsstrahlung spectrum, shape of high-energy, 2755 .

characteristic energy losses in metals and compounds, 1699.

characteristic energy losses in some intermetallie compounds, 2937.

corona with reference to radio observations, model, 2875 .

data processing equipment, evaluation, 1261.

energetic secondary, cavity ionization theory including the effects, 1229

free, conference on quantum interactions, 1659.

free, quantium interactions, 1829.

injection in betatrons, 1700 .

interference and phase effects. Electron interference phenomena, 1701.

interference experiments, 1702.

interferometer, $438 ; 947 ; 1202$.

interferometry, 707 .

measuring with, 2191

microscope, modification, for electron optical shadow method, 91

optical image of an atomic beam, 1704.

optical properties of space-charged clouds, 53 .

optics, 286.

orbital, capture in vanadium, radiative, 1835 .

penetration, theory, 1548 .

radiative, capture in cesium-131, 1834 .

slowing down, energy spectrum resulting from, 1015.

spectra, C597.

spectrometers, 2612

spin resonance spectra from gamma-irradiated solid nitrogen, 2940.

Electron affinity, atomic oxygen, $1334 ; 2812$.

atomic sulfur and empirical affinities of the light elements, 1698 photodetachment eross section, of atomic oxygen, 2683.

Electron beam, 929.

interferometer, 706 .

magnetometer, J $63 \mathrm{C} 1,69$.

techniques, microwave power measurements, 2201.

Electron beams, certain magnetic fields, note on the focusing, 95 .

interacting, with application to solar radio noise, 1418 .

Electron density, fluctuations in the ionosphere, 2381.

local, method for measuring from an artificial satellite, J $63 \mathrm{D} 3,325$.

profile data, analysis of ionospheric vertical soundings, TN14 (PB151373); TN19 (PB151378).

variations of the quiet ionosphere, TN40-1 (PB151399-1); TN40-2 (PB151399-2).

Electron impact, 580 .

dissociation and ionization of perchlorylfuoride, 2170 .

ionization and dissociation of hexafluorobenzene, 2168 .

ionization and dissociation of hydrazoic acid and methyl azide, 2604.

ionization and dissociation of methyl siloxanes, 747 . ionization and dissociation of $\mathrm{NF}_{3}, 1749$.

multiple ionization of rare gases by, J 63A3, 201.

multiple ionization of sodium vapor, 2996.

oxygen diffuoride, 2169.

Electron impact studies, aromatic hydrocarbons, 2938. hydrazine and the substituted hydrazines, 2939. sulfur dioxide and sulfuryl fluoride, 2542.

Electron microscopy, $1011 ; 1703$.

fibers, 4 .

soil clays and related materials, 439 .

Electron scattering, by gold, distribution, 1317. measurements, automatic instrument, 1274. phenomena, 2109

Electron-nuclear scattering, high energy, 1591.

Electron-optical exploration of space charge in a cut-off magnetron, 230.

Electron-tube curve generator, 440.

Electron, averaging device for the adherometer, protective coating adhesion measurement, 2273.

circuits, 238; 2500.

computer to reduce weathering test data, 2858 .

data processing systems, future developments, 2955.

density fluetuations in turbulent ionized layers, 1254.

design, temperature prediction, $\mathbf{5 6 4}$.

digital computers, analysis of atomic spectra, 1624.

flame temperatures and energy distributions, spectroscopic study, 2310; 2771 .

miniaturization, new techniques, 489.

quenching of $\mathrm{OH}\left({ }^{2} \Sigma^{+}\right)$in flames and its significance in the interpretation of rotational relaxation, 2941.

streams, certain, relation between the conductance and the noise power spectrum, 503 .

structure and magnetic properties of uranyl-like ions, 2355.

structure of some diatomic hydrides, 2543.

thermostat, time-proportional, 939.

thickness gage: circuitry, operating characteristics, and calibration, further studies, 2133.

voltage regulator, 135 .

Electronic computers, 488.

aiding management control, 441.

air traffic control, role, 2380 .

European experience, 1336.

Electronic equipment, airborne, new techniques for fabrication, 1415 .

maintenance, TN41 (PB151400).

sequential flow cooling, 1865.

Electronics, algebra of, 2803A.

industry, physical standards, 1808.

measurements and standards, 2776.

physics, dietionary of, contributions, 1660A.

Electrons, 2989.

aluminum, temperature dependence of the characteristic energy loss, 2798.

carbon, range straggling of high-energy, 2279.

cascade, and photons, energy spectra, RP2824. 
Compton, gamma-ray measurements by the magnetic analysis of, 725 .

energy loss, characteristic, 997.

energy loss of, in passage through thin films, 1014.

fast, energy dissipation, Mono.1.

50-kev, bremsstrahlung cross-section measurements, 2477.

from $\mathrm{H}$ - and D-, experimental cross section for photodetachment, 1338

1.0-Mev, bremsstrahlung polarization measurements, 1643.

positive, 131.

relativistic, multiple scattering, 1079 .

scattered by solids, distribution, 1681.

secondary: average energy loss per ionization, 538.

solids, characteristic energy losses, 1287.

standards, 1508.

their influence on resolving power, energy losses. 1335.

$3 \mathrm{~d}$ and $4 \mathrm{p}$, polarization energy, 1448 .

20 -kev, by aluminum, 2529.

$20-\mathrm{kev}$, in metal vapors, inelastic scattering, 2158.

2.7- to 9.7-Mev, 1676 .

vs photons: a comparison of microscopes, 54 .

with fine structure of the X-ray absorption spectra, comparison of the characteristic energy losses, 1655 .

with magnetic interaction, double scattering, 1320 .

0.5 - and $1.0-\mathrm{Mev}$, bremsstrahlung differential crosssection measurements, 1278

Electrons and positrons, 1204.

backscattering, 567 .

elastic scattering by point nuclei, 1694 .

relative backscattering, 300 .

transmission, 1560

Electroplated articles, 940.

coatings to titanium, adhesion, 1920

Electrorefining studies in the presence of tin, iron, copper, silicon, and nickel, 2811.

Electrostated fields by grounded external plates, 1066.

Electrostatic, deflection plates for cathode-ray tubes, $\mathrm{J} 64 \mathrm{C} 2,103$.

field and type symmetry of snowflakes, 2689.

magnetic fields, 56 .

Electrothermic instruments for the measurement of alternating current and voltage, 1012 .

Electroviscous effect in dilute aqueous solutions on ionic detergents, 2667.

Elementary, inequalities relating to a gamma and incomplete gamma function, 3047 .

introduction to the calculus of variations, 23 .

note on powers of quaternions, 390 .

Elements, atomic energy levels, C467, Vol. III.

mathematical theory of probability, 708 .

non-negative, topological proofs for certain theorems on matrices, 2850 .

probability, 2500 .

sun's atmosphere, 1138

Elevated temperatures, evaluation of resistance strain gages, 2420 .

statistics and planning tests, 137.

Eleven megacycle interferometer for low pressure gases, 648.

Eleven-Mev thick target bremsstrahlung, 709 .

E.L.F. radio waves, TN52 (PB161553).

Elimination of randomization in certain statistical decision procedures and zero-sum two-person games, 231.

Elliptic, differential equations, discrete approximations, 1314.

linear, differential equations by difference equations with positive coefficients, 785 .

Embedding theorem for balanced incomplete block designs, 948 .

Emission, $\mathrm{He} \mathrm{I}, \lambda \mathrm{10803}$, during the great flare of August 26, 1958,3077

$\mathrm{OH}$, in the earth's atmosphere, 3001

spectrographic standards, 232 .

spontaneous, of neutrons from samarium, 383 .
Emission spectra, autoignited heptane-air mixturcs, 442. first-stage combustion in an engine, 333.

mixtures of atomic nitrogen aud organic substances, 2544

nitrogen bombarded by high energy protons, 576

Emission spectroscopy, 443; $1013 ; 1705 ; 2545 ; 3021$.

Emission spectrum, carbon monoxide from 2.3 to 2.5 microns, RP2883.

OH from 1.4 to $1.7 \mu, 860$.

$\mathrm{OH}$ from 2.8 to $4.1 \mu, 2356$.

Emissivities of metallic surfaces at $76^{\circ} \mathrm{K}, 2110$.

Emulsions, thick-layered, background eradication, 30 .

Enamel, apatite, isomorphous substitution in, 748 .

caries, experimental vitro production, 1734 .

human, and dentin, determination of some compressive properties, 2518 .

porcelain, ground coats to steel, 2358 .

rat molar, 2528.

Enameled, porcelain, hot water tanks, 2745. ranges, 1090 .

Enameled-steel composites, heat treatment affects properties, discussion of the paper, 2525.

Enameling, iron during firing, 1153.

porcelain, a radioisotope study of metallic deposits, 1941.

sheet steel, 1133.

Enamels, procelain, effects of acid treatment on acid and abrasion resistance, 51 .

porcelain, weatherability, $1578 ; 1579$.

Endurance of helical springs related to properties of the wire, 710 .

Energetic species trapped at $4.2^{\circ} \mathrm{K}$ from gaseous discharges, 2942.

Energy, angular distribution of X-rays scattered in lead, 2111.

characteristic, loss of electrons in aluminum, temperature dependence, 2798.

dissociation of the $\mathrm{C}-\mathrm{H}$ bond in the formyl radical, 2813.

dissociation, of the $\mathrm{OH}$ radical, 1503.

distribution, spectroscopic study of electronic flame temperatures, 2310.

high electron-nuclear scattering, 1591.

infrared radient, $124 ; 812$.

release from discharged monatomic gases trapped at $4^{\circ} \mathrm{K}, 2546$,

spectra of eascade electrons and photons, RP2824.

spectral, distribution to the International Commission on Illumination Light sources A, B, and C, 834.

spectrum resulting from electron slowing down, C597; 1015.

transfer in hot gases, 1016.

vibration, of deuterium eyanide, 1575 .

Energy dissipation, fast electrons, Mono.1.

gamma radiation near the interface between two media, calculation, 2043.

high-energy X-rays, 1150.

Energy levels, in $F^{18}$ from alpha-particle reactions in nitrogen, 711

spectrum of neutral helium $\left({ }^{4} \mathrm{He} \mathrm{I}\right), \mathrm{J} 64 \mathrm{Al}, 19$.

Energy loss, of electrons in passage through thin films, 1014.

range of electrons and positrons, C577 Suppl.

Energy losses, of electrons and their influence on resolving power, 1335.

electrons in solids, characteristic, 1287.

metals and compounds, electron characteristic, 1699 .

Engine antifreezes, determination of freezing point, 331.

emission spectra of first-stage combustion, 333 .

four-stroke, single-cylinder, effect of size on the inletsystem dynamics, $1322 ; 1687$.

fuels, multistage autoignition, 1411

jet, controls, simulator for use in development, C584.

Engineer at the National Bureau of Standards. 1929.

Engineering, adhesives, selecting, 306.

civil, 2824.

cryogenic, 2922. 
cryogenic, recent advances, 3031 .

investigations, problems arising, 1457.

management in a government laboratory, 712 .

materials, mechanical properties, 2193.

physics, and chemical measurements, how statistics improve, 68 .

Engines, expansion, for hydrogen liquefiers, J $64 \mathrm{C} 1,25$.

jet-type, high velocity exhaust gases, 1602.

two-stroke, scavenging of, wave action in diffusers for exhaustpipe systems, 1679 .

Enthalpy, heat, specific entropy, free energy function entropy, free energy function, viscosity, and thermal conductivity of steam, compressibility factor, density, 1657 .

Entoptic color-perceptions of the macular pigment by observers of normal and color-defective vision according to the three-components theory. 1706.

Entropy, density in an infinite system, 2558.

specific heat, enthalpy, free energy function, viscosity, and thermal conductivity of steam, compressibility factor, density, 1657 .

Envelopes, vacuum tube, survey of the application of ceramics, 1604.

Epitaxy, in metal-rock salt pairs, 367 .

twinned, of copper on copper, RP2873.

Epoxy resins as cryogenic structural adhesives, 2547.

Equation, atmospheric refractive index at radio frequencies, 855 .

calculating the number of chain scissions in the photochemical degradation of solid polymers, 2455 .

differential, analytical integration for water storage, J $63 \mathrm{~B} 1,43$

differential, occurring in the problem of heat convection in laminar flow through a tube, 789 .

differential of hydrodynamic stability in a domain containing a transition point, asymptotic solution, 656

differential, Riccati's, note on the solution, J 64B2, 95.

differential, roots of Riccati's: low- and very lowradiofrequency tables of ground wave parameters for the spherical earth theory, TN7 (PB151366).

helium wave, with the SEAC, numerical solution, 372 .

Equations, differential, experiments in the solution, by Monte Carlo methods, 1019.

differential, in the complex plane, 61 .

differential, ordinary, non-self-adjoint boundary value problems, J $64 \mathrm{~B} 2,83$.

differential, solution of, by recurrence relations, 97 .

interpolation series, linear functional, 1050.

linear elliptic differential, 785 .

linear, solving, 831.

positive coefficients, 785 .

Equator, magnetic, $50 \mathrm{Mc}$ oblique transmission experiment near the, 2626 .

Equilibration in dialysis experiments with ionic surfaceactive agents, low rates, 2232 .

Equilibrium, flexibility chain molecules, sedimentation, 2296.

incomplete, and temperature measurement, 1735 .

phase, and melting-point, determinations, 1775.

Equipment and method for photoelectric determination of image contrast suitable for using square wave targets, J 64C1, 57.

Erosion, damage to solids caused by high-speed collision with rain, $\mathrm{RP} 2882$.

experimental plating of gun bores to retard, TN46 (PB151405)

surface, of filled plastics, 2792.

Errata in tables relating to Hankel integrals of order zero, 147

Error, bounds for the eigenvalues of symmetric integral equations, 1707 .

Error function, 241.

integrals, asymptotic expansion, brief note, 1383 .

radio phase measuring systems, 186.

various methods of airplane camera calibration, 1878 .

Errors, propagation of, in spectrophotometric colorimetry, 804

Es, slant, some implications, 2304.
Essential, property of the Fourier transforms of distribution functions, 391 .

similarity: a counter-example, 2548.

Estimate for the density and motion of solar material from observed characteristics of solar radio outbursts, 649 .

Estimating the standard deviation of a normal polulation, 713.

Estimation of, correlation coefficients from scatter diagrams, 1017

frequencies of thin elastic plates with free edges, RP2784.

parameters in certain stochastic processes, 334 .

quadrature errors for analytic functions, 1096.

Etalons, et méasures de fréquence et d'intervalle de temps aux Etats-Unis d'Amérique de 1954 a $1957,2550$.

temps atomique et moléculaires au National Bureau of Standards, 2549.

Etching fine points under controlled conditions, apparatus, 1628.

Ethane, ethane- $\mathrm{d}_{6}$, pyrolysis of mixtures, 291. internal barrier, 2603.

Ethylene, combination bands, 206.

infrared spectra, structure, 2833.

Etude, infrarouges de certains solides condenses a partir de decharges en phase gazeuse, 2943.

spectroscopique des produits de la decharge electrique dan L'Azote condenses a l'etat solide a tres basse temperature, prevues en faveur de l'existence d'Azote triatomique dan le solide, 2944.

Euclidean spaces, isoperimetric inequality for closed curves convex in even-dimensional, 952.

Euler means, Gibbs' phenomenon, 103.

European experience with electronic computers, 1336.

Evaluation, adhesion of organic coatings by ultracentrifugal and other methods, 715 .

adhesives for acoustical tile, $\mathbf{5 5}$.

analytical methods by the sensitivity criterion, 2112 .

appearance, status of ASTM methods and standards for, $3055 \mathrm{~A}$.

automatic computing machines, 233.

Boor-Quartermaster snag tester for coated fabrics and plastic films, 1337.

bremsstrahlung cross sections at the high-frequency limit, 2551.

coaxial slotted-line impedance measurements, 234.

Coulomb wave functions along the transition line, 1018.

electronic data processing equipment, 1261.

integral $\int_{0}^{\infty} e^{-u^{2}-(x / u)} d u, 716$.

matrix inversion programs, 2814.

new cuvette desitometer for determination of cardiac output, 714 .

resistance strain gages at elevated temperatures, 2420.

small color differences: I. Visual observations, 3078.

tensile, compressive, torsional, transverse, and impact tests and correlation of results for brittle cermets, RP2895.

various thermoplastic materials for use as iodine vapor barriers, 2892 .

width of cracks in concrete at the surface of reinforcing steel by means of tensile bond specimens, 2113.

Evaluation, active species trapped in a solid condensed from discharged nitrogen, $2551 \mathrm{~A}$.

water from cellulose, 547 .

Evaluation of lens distortion, inverse nodal slide, J $63 \mathrm{C} 2$, 105.

modified goniometric method, J 63C2, 113.

visual and photographic methods, RP2920.

Evidence for structural anomalies in pure cristobalite, 3048 .

Evolution of certified reference materials, 3078.

Exact, earth-flattening procedure in propagation around a sphere, J 64 D1, 61.

evaluation of the variances and covariances of order statistics in samples from the extreme-value distribution, 788 . 
solution for a cylindrical cavity containing a gyromagnetic material, 2013.

solution for a gyromagnetic sample and measurements on a ferrite, 2552 .

Examples of the use of high-speed computers in statistics, 2302 .

Excess noise in microwave crystal diodes used as rectifiers and harmonic generators, 2114.

Excitation, mechanisms of the oxygen 5577 emission in the upper atmosphere, J 63D 3, 319.

oxygen red lines in twilight, 2554.

separation of pure high-order modes in large high-Q cavities, 1708 .

surface waves on conducting, stratified, dielectricclad, and corrugated surfaces, RP2807.

surface waves on plane and curved impedance boundaries, 2553

Exhaust-gas mixtures at elevated temperatures, determination of viscosity, 989 .

Exhaust-pipe systems, wave action in diffusers, with special reference to the scavenging of two-stroke engines, 1679.

Existence, identities for the coefficients of certain modular forms, 1797.

Existence theorems and extreme solutions for inequalities concerning convex functions or linear transforms, 2115.

Expansion, engines for hydrogen liquefiers, J 64C1, 25.

theorem for pseudo-analytic functions, 577 .

Expansions, uniform asymptotic, for Weber parabolic cylinder functions of large orders, J 63B2, 131

Expansivity, viscosity, and density, molten alkali silicates, 422 .

Expendable modules as bases for disposal-at-failure maintenance, TN41 (PB151400).

Experiences with incomplete block designs: examples, 2116.

Experiment, designs: fractional factorial experiment designs for factors at three levels, AMS54.

national physical standards and design, 2210 .

Experimental, cross section for photodetachment of electrons from $\mathrm{H}$ - and $\mathrm{D}-$-, 1338.

design, principles, 880 .

equipment for communication utilizing meteor bursts, 2117.

evaluation of the oxygen microwave absorption as a possible atomic frequency standard, 2555 .

evidence for the existence of abnormal $\mathrm{OH}$ rotational "temperatures" in low pressure flames, 1339.

photodetachment cross section and the ionospheric detachment rate for $\mathrm{O}_{2}^{-}, 2556$.

plating of gun bores to retard erosion, TN46 (PB151405).

production of nodular graphite in cast iron, 444.

proof for the existence of nonthermal rotational distributions of $\mathrm{OH}\left({ }^{2} \Sigma^{+}\right)$in flames. 1709.

rapid access memory using diodes and capacitors, 392 .

statistics, 445 .

study of the strength and fatigue of glass at very low temperatures, 2014.

temperature measurements in flames and hot gases, 1340.

test of parity conservation in beta decay, 2015 .

theoretical study of kinetics of bulk crystallization in poly (chlorotrifluoroethylene), J $63 \mathrm{~A} 1,67$.

Experimentation, Fisherian revolution in methods of, 335. Experiments, beta-decay of polarized nuclei, 2557.

cell $\mathrm{Bi}, \mathrm{Bi}_{2} \mathrm{O}_{3}, \mathrm{ZnCl}_{2} / \mathrm{ZnCl}_{2} / \mathrm{ZnCl}_{2}, \mathrm{Zn}$ between 450 and $510^{\circ}, 2946$.

electron interference, 1702.

fast coincidence, techniques and theory, 1911.

high-frequency, at the NBS scatter sounding, 730 .

many factors, 2120 .

multi-factor, 2995 .

operations research, design, 1670 .

parity conservation, scintillation counting, 2749.

processing pictorial information with a digital computer, 2119.

statistical designs, 133. solution of differential equations by Monte Carlo methods, 1019.

Exploration of electrostatic and magnetic fields, 50.

Explosions, atomic, temperatures, 1528.

low pressure, ultraviolet spectrophotometry, 1565.

pentaborane-air flames, spectroscopic observations, 1883.

Exponential, atınosphere, path antenna gain, J 63D3, 273.

CRPL, reference atmosphere, Mono.4.

integral $\int_{1}^{\infty} e^{-x t} t^{-n} d t$ for large values of $n, \mathrm{RP} 2941$

integral for complex arguments, tables, AMS51.

reference atmosphere, Central Radio Propagation Laboratory, J 63D3, 315 .

Exposure standards and radiation protection regulations, 2947

Expression in terms of molecular distribution functions for the entropy density in an infinite system, 2558

Extending the range of the NBS stiffness tester, 1020.

Extension of Gauss' transformation for improving the condition of systems of linear equations, 393 .

mode theory of VLF ionospheric propagation, 2456.

Munsell renotation system, 2559.

Munsell renotation system to very dark colors, 1710 .

second spectrum of zirconium into the infrared, 717 .

Sen-White paper on atmospheric oscillations, 1341 .

Extrema, Bessel function, relative, 104.

relative, of the Hermite orthogonal functions, 283.

Extreme useful range of VHF transmission by seattering from the lower ionosphere, 2560 .

Extreme values, tensile strength, two early papers on the relation between, 1219

theory, 3096.

Extreme-value, distribution: table of the first moment of ranked extremes, RP2787.

results for indefinite Hermitian matrices, 2303.

theory, some applications, 1140 .

Extremum property of sums of eigenvalues, 1234.

Eye, and sky, 2830.

protection, plastics for, 1547 .

\section{F}

F-region, eclipse of May 20, 1957, 121 .

F-scatter and sporadic-E program, TN48 (PB151407).

F2 layer, at Hyancayo, Peru, lunar stratification, 83.

critical frequencies, 225 .

Fabric structures, design of impact-resisting, 3069.

Fabric-phenolic laminates, cotton, 2063.

Fabrication, of airborne electronic equipment, new techniques, 1415.

polyester laminates, effects of humidity, 435 .

techniques for ceramic X-band cavity resonators, 2561 .

Fabrics, coated, and plastic films, 1337.

flameproof, 3050 .

rayon, properties, factors affecting, 446 .

rayon, properties of, 718 .

thickness and compressibility of, 898 .

woven in high temperature laundering, test for dimensional changes, 2802.

Fabry-Perot interferometry, phase shift effects, J 64A3, 191.

Facility for the evaluation of resistance strain gages at elevated temperatures, 2420.

Facilities for convenient manual reduction of ionograms, TN14 (PB151373).

Factoren, die die Eigenschaften von Reyongeweben beeinflussen, 718 .

Factorial, asymmetrical, arrangements, use of group divisible designs, 2836 .

fractional, experiment designs for factors at three levels, AMS54.

fractional experiments of $2^{m} 3^{n}$ series, 2568 .

Factors, affecting radio propagation in the TV and FM bands, 1342 .

affecting the accuracy of distortion measurements made on the nodal slide optical bench, 2948 . 
affecting the dimensional stability of the Ag-Sn (Cu-Zn) amalgams, 1493.

affecting the properties of rayon fabrics, 446 .

affecting the water vapor permeability of leather, 57.

converging, method for the determination of, applied to the asymptotic expansions for the parabolic cylinder functions, 366 .

influencing the behavior of polymers exposed to highenergy radiation, 1343 .

influencing the cutting characteristics of rotating dental cutting instruments, 1494.

influencing the road wear of tires, 308 .

properties of rayon fabrics affect, 718 .

Fading, characteristics of regular VHF ionospheric propagation, 1495 .

properties of a signal imposed on a constant signal, C599.

rate recorder for propagation research, 2949.

False negative permanent strains observed with resistance wire strain gages, 2950 .

Faraday, mass measurements, 573 .

elemental constants and electricity standards, 2593.

Farm, milk tank testing, 1344; 1711 .

Fast coincidences with Cerenkov counters, 447.

Fatigue and, static tests of flush riveted joints, 1345.

crack, distinction between initiation and propagation, 1925.

properties of high strength steels, 2951.

strength of glass at very low temperatures, an experimental study, 2014 .

stressing, damaging effect, 1566 .

Fax and telemetering: how FM expedites weather data distribution, 69 .

Federal basis for weights and measures, C593.

Feedback, amplifier with negative output resistance for magnetic measurements, 2421.

amplifiers, tuning systems, 352 .

amplifiers with stabilized output impedances, 719 . $\mathrm{FeO}$, new infrared band system, 368 .

Fermi beta-distribution function, numerical evaluation, 100.

Ferrite, cores, RF permeameter techniques for testing, 2746 .

exact solution for a gyromagnetic sample and measurements, 2552.

lattice in low-alloy steel, elastic and plastic behavior, 1691.

Ferrites, characteristics and present requirements, 2051. magnetoelastic properties, TN49 (PB151408).

Ferromagnetic materials, measurement of core losses, RP2865.

Ferrous and manganous carbonates, thermal decomposition, 1960.

Fiber, papers, glass, 1026.

structure, textile, on wear resistance, effect, 610 .

Fibers, different, impact properties of yarns made from, 734.

electron microscopy of, 4 .

hardwood, resin bonding in offset papers, 302 .

properties and behavior of, when impacted at high velocities, RP2866.

properties of, 1121 .

synthetic, properties of some experimental map papers containing, 2703

Fibre, muscle-ATP'system, 2986.

Fibrous, insulations bounded by reflective surfaces, thermal resistance, BMS151.

macromolecules, reversible contractile processes, 2743 .

polyethylene, low-angle X-ray diffraction, 2616 .

Field, emitter point projection X-ray microscope, 1982. ground screen, radiated from a monopole, 1688 .

strength calculations for E.L.F. radio waves, TN52 (PB161553).

Field-strength, meters, commercial, calibration, 407.

variations recorded on a VHF ionospheric scatter circuit during the solar event of February 23, 1956, 2121.

Fields in electrically short ground systems, J 63D2, 175.
Fifth-order aberration in an optical system, 235.

Fifty, oblique transmission experiment near magnetic equator, 2626 .

years of electrochemical theory, 448.

years of electrodeposition, 449 .

years of scientific standardization, 236.

years of signal lighting, $2562 ; 2952$.

Filaments, electrically heated, on-off temperature control, 2660

Filling resins, direct, color stability, 854 .

Film, badge dosimetry: how much fading occurs? 1021. cathode, studies by the drainage method, 1282 .

dental X-ray, 2091.

for $\mathrm{X}$ - and $\gamma$-ray dosimetry, stabilization processing, 1891.

polyester, tensile properties, 2354 .

Film-forming properties of cellulose acetate propionates, 237.

Films, cellulose acetate, degradation, 2924.

bituminous materials, uniform, method of preparing, 628.

oleophobic, on metal fatigue, effect of, J $64 \mathrm{C} 2,147$. plastic, and coated fabries, 1337 .

plastics, comparison of methods for measuring the tensile and tear properties, 1290.

plastic, properties of, 1122 .

plastics, irradiated, 3056.

thin, energy loss of electrons in passage through, 1014.

Filter radiometry and some of its applications, 720 .

Filtering, device, 47.

Filters, for the infrared region, 450 .

pass, Zeeman-split absorption lines, 3115 . single frequency, design, TN23 (PB151382).

Finding, chemical records by digital computers, 2122.

list, a multiplet table of astrophysical interest, TN36 (PB151395).

Finite, groups, representations, 499. matrices, communtativity, 2060.

Fire, extinguishment by means of dry powder, 2123 .

research at the National Bureau of Standards, 2124. research, field of, some activities of the National $\mathrm{Bu}$ reau of Standards, 1870 .

research in the United States, 2563.

tests, proposed criteria for defining load failure of beams, floors, and roof constructions during, J $63 \mathrm{C} 2,121$.

First, meeting on radio climatology, 2564.

report of the special committec on world-wide ionospheric soundings, 1712 .

Fisherian revolution in methods of experimentation, 335 .

Fitting a straight line to certain types of cumulative data, 2125.

Fixed capacitors of electronic circuits, 238.

Fixture unit ratings as used in plumbing system design, 239.

Flame, calorimetry, constant pressure: combustion calorimetry with fluorine, J $64 \mathrm{~A} 1,49$.

cool, oxidation of propane-2-C $\mathrm{C}^{13}$, products, 2702.

emission spectra, kinetics of $\mathrm{OH}$ radicals, 475.

gases, sampling, RP2818.

photometric determination of manganese in cement, 1930.

photometric determination of strontium in portland cement, 1346 .

spectra, infrared, 1366.

speeds, measurement of, by a nozzle burner method, RP2867.

spread properties of building finish materials, 2565 .

Flameproof fabrics, 3050 .

Flames, 1347.

acetylene-oxygen, 2311.

atomic: spectra "temperatures" and products, 1268. atmospheric pressure, rotational and vibrational "temperatures" of $\mathrm{CH}$ in, 816 .

bunsen, 207.

effects of self-absorption of rotational "temperatures" of $\mathrm{OH}$ in, 700 .

hot gases, experimental temperature measurements, 1340.

low pressure, 1339. 
methane-air, rotation temperatures of $\mathrm{OH}, 304$.

pentaborane-air and explosions, spectroscopic observations, 1883 .

several, rotational temperatures of $\mathrm{OH}, 534$.

Flammability of materials using a radiant energy source, method for measuring, 1593 .

Flares, limb, 2882.

Flash photolysis of chlorine monoxide, 2357.

Flatness of large surfaces, testing of, by interferometer, RP2943.

Flex tension test for leather, 917 .

Flexible polymer molecules, crystallization, 1924 .

Flexibility of thin porcelain enameled sheet steel, 2126.

Flexural cracks in reinforced concrete beams, 2566.

Flexure, center of, upper and lower bounds, J 64B2, 105.

Floor, wall, and door constructions, sound insulation of, B.IS144, 2d Suppl.

Flooring, conductive, for hospital operating rooms, $\mathrm{J} 63 \mathrm{C} 2$, 125; Mono. 11.

materials, 479 .

Floors and floor finishes, 130.

walls, transmission of airborne noise, 2397.

Flow, behavior of concentrated GR-S rubber solutions, 58 . capacities of pneumatic components, determination and correlation of, C588.

characteristics and degradation of polymer solutions, effects of capillary shape, RP2839.

conversion kinetics of ortho and parahydrogen, 2953. measurements, review of some methods, 1131 .

Fluctuating signal imposed on a constant signal, on the theory of fading properties, C599.

Fluctuations, in harmonically related bands, correlation of solar noise, J 63D3, 293.

refractive index of the atmosphere at microwave frequencies, 240.

Fluid, conductivity of, contained in a cylindrical vessel, measurement, 2233.

exchange at the margins of dental resotrations, 451 . heat flow in, with eddying flow, 1727 .

normal, concentration in liquid helium II below $1^{\circ}$ $\mathrm{K}, 774$.

Fluids, cold, valve for, 1570 .

superposed, stability of oscillations, 1890.

Fluorescence, and average lifetime of excited $\mathrm{OH}\left({ }^{2} \Sigma^{+}\right)$in flames, 1348 .

teeth: a means of investigating their structure, 452 . teeth reveals structure, 721 .

Fluorescent, lamps, 134.

color-rendering properties, specifications of, 833 . sources of illumination, color rendition, 1651 .

sunlamps in laboratory aging tests for plastics, 1713 .

Fluoridation, 2567.

Fluoride, lithium: heat content from $0^{\circ}$ to $900^{\circ}$, the melting point and heat of fusion, 1053 .

perchloryl, dipole moment, 2089.

tertiary butyl, 2820 .

Fluorides on infrared transmittance of certain silicate glasses, 2933.

Fluorinated, polyphenyls by mass spectrometer, analysis, 1263.

polyphenyls, synthesis and properties, 1521 .

Fluorination of haloaromatic compounds, RP2938.

Fluorine, combustion calorimetry: constant pressure flame calorimetry, J 64A1, 49.

compounds, properties of, the vibrational spectrum of tetrabromoethylene, 1825

1-Fluoro-1-chloroethylene, vibrational spectrum, 1576.

Fluorocarbon bond energies obtained by electron impact, 580 .

Fluorocarbons, aromatic, reactions with hydrogen, RP2940.

Fluorotrichloroethylene, vibrational spectrum, 1577.

Flush riveted joints, fatigue and static tests, 1345.

FM, adapter, wide-band reducted interference, 169.

data reduction from magnetic tape recorders, 722 .

modulation, wireless microphone uses, 2869.

television broadcasting stations, variation with frequency of the signal range, 347 . television service, statistical approach to the problem of multiple radio interference, 378 .

TV bands, factors affecting radio propagation, 1342. Foams and powders, heat transfer, 2147.

Focusing, defocusing, and refraction in a circularly stratified atmosphere, J 64D3, 287.

Forbidden lines in the spectra of impure mereury 198, 60.

Forecasting of disturbed high frequency communication conditions, 2127.

Forces on cylinders and plates in an oscillating fluid, RP2857.

Forehead and mastoid, mechanical impedance, 1404

Formal, power series for $\log e^{x} e^{y}, \quad 1931$.

procedures for connecting terminals with a minimum total wire length, 2128 .

Formation, of ozone from atomic oxygen at low temperatures, 2954

polymer crystals with folded chains in dilute solution, theory of, $J 64 \mathrm{~A} 1,73$.

silver sulfide in the photographic image during fixation, J $64 \mathrm{Cl}, 65$.

titanium tetrachloride, heat, RP2928.

Formula, Fox's escalator, for latent roots, 386 .

Formulas, for calculating the error function of a complex variable, 241.

finding the arguments for which a function has a given derivative, 242.

hyperfine structure, for LS coupling, 733 .

inverse osculatory interpolation in the complex plane, $\mathrm{RP} 2792$

numerical differentiation in the complex plane, 453 .

numerical integration of first and second order differential equations in the complex plane, 61 .

Formyl radical, the energy dissociation of the $\mathrm{C}-\mathrm{H}$ bond, 2813.

Forster's method, effect of suspension position on apparent values of internal friction determined by, 2530 .

Foundry crucible service life, 454.

Fourier, transforms, certain, of distribution: II. 965.

transforms of distribution, 202.

transforms of distribution functions, essential property, 391 .

Fox's escalator formulae for latent roots, alternative derivations, 386 .

Fractional, factorial experiment designs for factors at three levels, AMS54.

factorial experiments of $2^{m} 3^{n}$ series, 2568 .

Fractionated, microbiological of the hydrogen isotopes, 2635 .

Fractionating process of adsorption, 156.

Fracture phenomena and molecular weight in polymethyl methacrylate, 2569.

Fragmentation of waterdrops in the zone behind an air shock, RP2843.

Free, energy function, specific heat, enthalpy, entropy, viscosity, and thermal conductivity of steam, compressibility factor, density, 1657 .

machining copper, low-temperature thermal conductivity, 1393.

precession determination of the proton gyromagnetic ratio, 2422 .

radical stabilization in condensed phases, 2570 .

radical statistics, 2571 .

radicals at low temperatures, stabilization, Mono.12; 2316.

radicals, luminescence of solid nitrogen $\left(4.2^{\circ} \mathrm{k}\right)$ coning atoms, 2622 .

radicals, frozen, 2129 .

radicals, frozen: the problem and the program at the National Bureau of Standards, 2130.

radicals research at the National Bureau of Standards, 2973.

radicals research symposium, 2572 .

radicals, stabilized, 2774 .

Free-air ionization chambers, design of, H64.

Free-radical degradation, high-speed computations in the kinetics, 2587.

Freezing, of water in pipes and vessels, 861 .

point of engine antifreezes, determination, 331 . 
points, apparatus for determining, at saturation pressure from time-temperature freezing and melting experiments, 26.

Freon-12, determination of water content in, circulating in a refrigeration system, 990 .

Frequencies, above $50 \mathrm{Mc}$, gapless coverage in air-toground communications, 457.

audio, measurement of voltage ratio, 2191.

high, observable over long distances, new kind of radio propagation, 369 .

ionospheric critical, and sunspot number for different sunspot cycles, 571 .

low, effective electrical constants of solid, 2352.

low radio, calculations of ionspheric reflection coefficients, 2044.

low radio, transmission loss curves for propagation, 2851.

low, sweep, polarization measurements, 938 .

lowest usable, and maximum usable, for the path Washington to Resolute Bay, 1062.

microwave, atmosphere, 240.

optimum, for outer space communication, J 64D2, 105.

Frequency, and amplitude stability of the cathode-coupled oscillator, 62 .

audio, power amplifier circuits, a survey, 187.

backscatter, sweep, 1158.

band $152-162 \mathrm{Mc}$ which is available for marine radio communications, propagation characteristics, 1945.

carrier, dependence of the basic transmission loss in tropospheric forward scatter propagation, TN53 (PB161554).

characteristics, comments on the attenuation, 2059.

characteristics of VLF radio waves, 2345.

characteristics versus attenuation, 205.

continuous function, 1414 .

dependence of VHF ionospheric scattering, TN9 (PB151368).

dividers, microwave, 89 .

dividers, modified locked-oscillator, 261.

factors, world maps of $\mathrm{f} 2$ critical frequencies and maximum usable, TN2 (PB151361).

generator, wide range, 170 .

high, impedance standards at the NBS, 2583.

high, induced electroluminescence in zinc sulfide, 1728

high, limit of $500-\mathrm{kev}$ bremsstrahlung, polarization, 2694.

high, measurements, instruments for, 1043.

high precision automatic, comparator and recorder, 1356.

high, standards of the Electronic Calibration Center, NBSBL, 2585.

high, very, field-intensity meters, 739 .

high, very, transmission loss, 750 .

intermediate, gain stabilization with inverse feedback, 78 .

low, band, some results of sweep-frequency investigation, 1146.

low, ionosphere echoes, note on the polarization, 371 .

low, propagation, 229 .

low, radiation from a horizontal antenna over a spherical earth, 1761.

low, standard transmissions, 2179.

multipliers and converters for measurement and control, 1349.

polya functions, 782 .

power requirements for a worldwide standard-frequency broadeasting station, J $63 \mathrm{D} 1,35$.

primary standard of, 737 .

radio, mass spectrometer, 117.

range, 175 .

range from $50 \mathrm{Kc} / \mathrm{s}$ to $25 \mathrm{Mc} / \mathrm{s}, 2064$.

signal range of FM and television broadeasting stations, variation, 347 .

single, filters, design, TN23 (PB151382).

spectra of very-low-frequency atmospherics, measured, J 64D $1,41$.

standard at low temperature, 1589.

standard, atomic, 2555. standard, broadeast at very low frequency, 2270 .

standard, NBS primary, 872 .

standard, station WWVH, 42.

standard, transistor, 90D.

standard, transmission and time signals, 3054A.

standards, 456.

standards, high stability, 1359.

standards, precision quartz resonator, 1111.

standards, spectral lines, 550 .

sweep, backscatter observations, note on, 924 .

time in Hawaii, measuring, 257.

time, standard, 1190.

time, standard, atomic clock, 148.

time standards, half-precision, 18.

time standards in the U.S.A. from 1954 to $1957,2550$.

transmissions, WWV standard, $2871 ; 2872$.

ultra-high, characteristics of the magnetic attenuator, 663 .

ultra-high, magnetic attenuator, 902.

very high, ionospheric scatter communication, 1958 critique, $2505 \mathrm{~A}$.

very high, ionospheric scattering, modulation studies, 2639 A.

very high, propagation by ionospheric scatteringsurvey of experimental results, 1971.

very high propagation measurements in the Rocky Mountain region, 1972.

very-high transmission loss, 870 .

very low, emissions generated in the earth's exosphere, 3097 .

very low, ionospheric propagation, 2203.

very low, noise, 2003.

very low, propagation in the presence of a transverse magnetic field, J 64D3, 265.

Frequency-deviation, meter plots drift, 455; 723 .

Frequency-high, pulse transmitters, 1056.

Frequency-shift, keyed carriers, 2723.

radio photo-transmissions, spectrum, 1884.

reception, 1071.

Friction and endurance of prelubricated and unlubricated ball bearings at high speeds and extreme temperatures, J $63 \mathrm{C} 1,19$.

Frozen, free radicals, 2129.

free radicals: the problem and the program at the National Bureau of Standards, 2130.

products from the nitrogen microwave discharge, thermometric study, 2847.

Fuel-immersion, laminated plastics, 50.

Fuels, aircraft, 1128.

aircraft, AN-F-58, net heat of combustion, 265.

engine, multistage autoignition, 1411.

Fully convex normed linear spaces, 1350.

Function, generator for two independent variables, 1590 . aid in the fitting of kinetic data to a rate equation, 1983.

Funetions, generating, smoothing operations, 783 .

Hermite orthogonal, 283.

positive harmonic, of ultraspherical polynomials, 276. struve, properties, 2269.

value, class of, 1.

Fungicides on deterioration of leather, 2353.

Fungicidal effectiveness of compounds applied to leather, 2131.

Fungicides, leather, laboratory and field exposure studies, 1754.

leather, studies, 2788.

Fur skins, aging of karakul and seal, 19.

Furnace, gradient, high-vacuum temperature, 2588.

improved hydrogen atom beam, TN47 (PB151406).

Furnaces, thoria and resistor-type, construction and operation, 328 .

two resistance-type hot-pressing, for laboratory use, 2399.

Further contributions to the solution of simultaneous linear equations and the determination of eigen values, AMS49.

experiments on $\beta$-decay of polarized nuclei, 2132. 
identities and congruences for the coefficients of modular forms, 2573 .

infrared measurements with a cesium iodide prism molecular spectroseopy, 1351.

studies of an electronic thickness gage: circuitry operating characteristies, and calibration, 2133.

studies of the influence of a ridge on the low-frequency ground wave, RP2884.

Future developments of electronic data processing systems, 2955 .

Fused salts, electrolytic preparation of molybdenum, 2811

Fused-quartz glass for ultraviolet, visible, and infrared wavelengths, index of refraction of, 1035 .

Future office and factory automation, 1715 .

\section{G}

Gage, area, a reduction of, for use at low temperatures, 7.

electronic thickness: circuitry, operating characteristies, and ealibration, further studies, 2133. piston, 2103.

thickness, ceramic coatings, 13 .

Gages, strain, bonding agents for high-temperature, 2474 wire resistance strain, eryogenic characteristics, 2073. wire strain, 2950.

Gaging and testing farm milk tanks, 2134

Gain stable mixers and amplifiers with current feedback, 1022.

Gains of finite-size corner-reflector antennas, 2574.

Galactic radio waves, 63 ,

Gallium, alloys of, with powdered metals as possible replacement for dental amalgam, 1613.

aluminum phosphate, low cristobalite type, erystal structure, 1923.

antimonide, 1007.

copper-tin alloys, some physical properties, 1876.

orthophosphate, anhydrous gallium, temperature inversion, 1916.

vapor, mass spectrum of, 758 .

Galvanic, corrosion theory for adherence of porcelain enamel ground coats to steel, 2358. couples, $138 ; 141 ; 142 ; 143$.

Galvanomagnetic, effects in InSb, 1352.

effects in $n$-type indium antimonide, 2135

effects in n-type InSb at $4.2^{\circ} \mathrm{K}, 1716$.

effects in p-type indium antimonide, 2136.

oscillatory effects in n-type indium arsenide, 2678.

Galvanometer efficiency as a design parameter, 1717.

Games with random payoff matrices, 2137.

Gamma, and incomplete gamma function, 3047.

cassiopeiae, variations, TN21 (PB151380).

function, 2974.

irradiation of polymethyl methacrylate and polystyrene 2138

radiation from $\mathrm{Ag}^{105}, 1718$.

Gamma radiation from $\mathrm{Z}_{\mathrm{n}}^{63}, 2956$.

radiation, multiple-scattered from a plane isotropic source, angular distribution, 1265 .

radiation near the interface between two media, calculation of energy dissipation, 2043.

radiations, penetration of, 2102 .

Gamma rays, and X-, photographic dosimetry, 2611.

backscattering of the cobalt-60, from infinite media, 959.

cobalt-60 and cesium-137, with a free air chamber, measurement of, $\mathrm{J} 64 \mathrm{C} 2,87$.

isotropic sources through aluminum and concrete, penetration, TN11 (PB151370).

oblique incidence, 1171

similarity principle in the backseattering of, 934

$\mathrm{X}$-rays, absorption by concrete, 1250 .

Gamma-ionization chambers, improvement in response of $4-\pi, 735$.

Gamma-ray, attenuation, 724

backscattering and transmission, Monte Carlo calculations of, 1076 .

degradation spectra, an approximate expression for, RP2788.

diffusion, application of the Monte-Carlo method to a problem, 1618 . doses near the interface between two media, measurement, 2628.

dosimeter, neutron-insensitive, 2211.

dosimetry and $\mathrm{X}$-, stabilization film, processing, 1891 .

emission of radium, determination of, RP2801.

measurements by the magnetic analysis of Compton electrons, 725 .

penetration into shelters, RP2827.

penetration theory relevant to shielding problems, aspects, 2028 .

penetrations, 847 .

scintillation spectrometer pulse-height distributions, 2856.

spectroscopy, 2139 .

transport theory, 3051 .

X-ray spectra between 0.01 and $20 \mathrm{Mev}, 1955$.

Gapless coverage in air-to-ground communications at frequencies above $50 \mathrm{Mc}, 457$.

Gas, absorption systems, 2524.

analyzer, unique thermal conductivity, 2443 .

clouds, ionization and shock fronts in collisions, $2496 \mathrm{~A}$.

$\mathrm{CO}_{2}$, nonpolar, 1074 .

cosmical, dynamics, 2697.

discharge, low temperatures, 3052.

discharge, spectra emitted from a solid nitrogen condensed at $4.2^{\circ} \mathrm{K}, 1881$.

elementary phase reactions at high temperatures, 1433.

film, electrolyzed, 865 .

high pressure, handling equipment for autoclaves in chemical research, 2154.

ideal, thermodynamic functions, high-speed computation, 1357.

internal, counting, 2714 .

liquefied, storage vessels, 3049 .

natural, analysis of, 25 .

pound of, 927 .

properties in terms of molecular clusters, representation of, 815 .

streams, measurement of high temperature, 85 .

temperatures, measurement of, by immersion-type instrument, 867.

thermodynamic functions, high-speed machine computation of, 1030 .

tube stabilized power supplies, 1353.

volumes of, meter for timing the flow, 365 .

Gas-exhaust mixtures at elevated temperatures, determination of viscosity, 989 .

Gas-filled candlepower standards, 2860.

Gas-free liquid scintillators, temperature effects, 1915.

Gaseous, discharges, energetic species trapped at $4.2^{\circ} \mathrm{K}$, 2942.

heat conduction in dewars, ealculation, 2482.

nitrogen, thermodynamic properties, 1964

state, infrareds of certain solids, 2943.

systems, ionized, $2632 ; 2988$.

Gases, and vapors on glass spheres, adsorption, 1609.

calculation of properties, 2664.

compressed, microwave absorption; saturated hydrocarbons, 1778 .

condensed, liquid level indicator, at low temperatures, 1052.

condensed, low-temperature microcell for infrared study, 2619.

condensed, temperature microcell for infrared study, 2800 .

exhaust, corrosion of aluminum alloys by, 979 .

five monoatomic, propagation of sound, 1823 .

flame, sampling, RP2818.

high-velocity exhaust of jet-type engines, 1602 .

hot, and flames, experimental temperature measurements, 1340 .

hot, energy transfer in, 1016.

hot, temperatures, 1882.

high densities, contribution in the study of transport phenomena, 1296.

ionized, internal energy, 3081. 
ionized, thermal diffusion, 2844.

liquefied, ball bearings submerged in, 3063 .

liquefied, long distance transfer, 2177

liquefied, performance of pumps, 2243

liquefied, single-phase transfer, C596.

liquefied, with sharp-edged orifices 2983.

low pressure, an eleven megacycle interferometer for, 648.

monatomic, trapped at $4^{\circ} \mathrm{K}, 2546$

nonpolar, generalized tables of corrections to thermodynamic properties, 1721 .

properties of, 259.

pure diatomic, 1326.

rare rotating or expanding, cosmic examples of heat conduction, 2920.

sound in, combined translational and relaxational dispersion of, 971 .

survey of experimental heat capacities, 843 .

thermodynamic properties of, at high temperature, RP2916.

various buffer, narrow hyperfine absorption lines of $\mathrm{C}_{\mathrm{n}}{ }^{133}, 2643$.

water, measurement, RP2864.

Gasoline, aviation, and its correlation with other properties, net heat of combustion, 2646.

Gassing of dry cells, 2359

Gated-beam mixer, 243.

Gauss', speeding up device in the theory of single step iteration, 2659 .

Gerling on relaxation, 244

transformation for improving the condition of systems of liner equations, extension, 393.

Gaussian quadratures of high order, abscissas and weights for: values for $n=64,80$, and 96 , RP2875.

functions, 1221.

Gegenbauer and Legendre functions, general, addition theorems, 1253 .

Gegenschein, absolute photometry, 1919.

General, precepts for programers, 545 .

relations between fluxes from collimated point and plane sources of radiation, 2957.

system, signal, 826 .

Generalization, normal basis in abelian algebraic number field, 1791.

theorem of Konig. 1719.

theorem of Osgood to the case of continuous approximation, 64

Generalizations of identities for the coefficients of certain modular forms, 1720 .

Generalized, commutators of matrices and permutations of factors in a product of three matrices, 1023

tables of corrections to thermodynamic properties for nonpolar gases, 1721 .

Generating functions of the Jacobi polynomials, 1426 .

Generation, and testing of pseudo-random numbers, 1722.

Bessel functions on high speed computers, 2140.

Coulomb wave function by means of recurrence relations, 1532 .

Generator, electron-tube curve, 440 .

frequency, wide range, 170 .

high-power square-pulse, 463.

microwave harmonic, 1662.

phase, for tropospheric research, 1805.

pulse, and high-speed memore circuit, 1827.

pulse, delay ineremental, 191.

pulse, high-power, 627

pulse-pair, crystal-stabilized, 1302

Generators, diathermy, 429 .

Genuine cooperation between manufacturers and weights and measures officials, 458.

Geographic, and temporal distribution of polar blackouts, 1024 .

distribution of geophysical stations on the polar eap, 2141.

Geographical and height distribution of the gradient of refractive index, 727 .

Geological application of extreme-value methods to interpretation of cobbles and boulders in gravel deposits, 1723
Geomagnetic, disturbance and velocity of slow-drift solar radio bursts, 2958 .

disturbances, solar $\mathrm{H} \boldsymbol{\alpha}$ filaments, 1489

eclipse phonomena, survey, 1908 .

effects of high-altitude nuclear explosions, 2959.

observatories and thesaurus of values, 252 .

Geometric factors in electrical measurements relating to corrosion and its prevention, 1354.

Geometrical, characterization of nuclear states and the theory of angular correlations, 726 .

factors in fatigue, toric bending specimen for investigation, 2442.

optics at V.I.F. sky wave propagation, 2360.

Geometry, 2500.

Boolean, 403.

integral and differential, metric methods in, 764 .

metric differential, 87.

Geophysical, stations on the polar cap, geographic distribution, 2141 .

year, International, July 1, 1957-December 31, 1958, radio noise data, TN18 (PB151377).

German syntax patterns, proposals for the mechanical resolution, 290 .

Germane, methyl, microwave spectrum, 2992.

Germanium below room temperature, thermoelectric power of, 897.

infrared photoconductivity due to neutral impurities in, 1039 .

single crystals, thermoelectric power in, 896 .

tetramethyl, small quantities, preparation, 517 .

Giauque-Kelving proposal, 1025.

Gibbs, phenomenon for a class of linear transforms, 500 . phenomenon for Euler means, 103.

phenomenon for Hausdorff means, 65.

Gimbals; high vacuum, 1731.

Glass, analysis, recent trends, 1126.

arsenic sulfide, properties, RP2774.

australite (meteoric), 1636.

boroscilicate, after heat treatment, 1844.

dewars for optical studies at low temperatures, 2575 .

fatigue and strength at very low temperatures, an experimental study, 2014.

fiber papers, 1026.

fiber polyester laminates containing a calcium carbonate filler, determination of resin content, 1672 .

fibers, paper from, manufacture, 477.

formation in polymers: I. The glass transitions of the poly-( $n$-alkyl methacrylates), 2142.

formation in polymers. II. The system rubbersulfur, RP2944.

formation, stable, shape of the liquidus surface as a criterion of, RP2872.

low-loss and quartz, small precision bottles, 2224.

optical, production in the United States, history of, 731.

radiant, heating panels, 807 .

silica, noncrystal ionic model for, RP2782.

spheres, adsorption of gases and vapors, 1609.

strength and fatigue of, at very low temperatures, 2318.

transition temperatures of copolymers, 2576 .

volumetric apparatus, testing, C602.

Glass-fabric reinforced polysters, 1004.

Glass-fiber reinforced plastics, effects of molding pressure on strength properties, 2533.

Glasses, alkali silicate, density formula for, RP2915.

amelung, compared with some modern commercial glasses, 387 .

binary alkali borate and silicate at high pressures, compressibility, 1292.

binary alkaline-earth borate, at high pressures, compressibility, 1658.

elastic moduli, at elevated temperatures by a dynamic method, 1692

infrared energy, transmittance, 1561.

molten alkaline-earth borate, with three mole percent of potassium oxide, 2864.

optical, relative stress-optical coefficients, RP2779. 
sag point as a reference point in heating of, R P2791. silicate, 2933.

silicate, during annealing, structural rearrangements, 1901.

sodium titanium silicate, properties, RP2890.

structure of alkali silicate binary, RP2854.

system barium oxide-boric oxide-silicate, some properties, 2763 .

three color achromats, 3043 .

transmitting, infrared, 1177.

zinc borosilicate, properties, RP2930.

Glassware, railroad-signal, an evaluation of the luminoustrasmittance requirements, RP2849.

Glassy state, polystyrene in, thermal and photochemical processes in, 1958.

Gloss, color, and haze, measurement of, 760 .

measurements - past, present, and future, 2143.

meter comparisons, 1724 .

Glucose means of sodium chlorite, 1310.

Glycol, polythylene, electrolytes, punched-cell batteries, 1828 .

Glycosylamines, mutarotation, hydrolysis, and rearrangement reactions, 2642 .

Gold, bonding of to fused silica, effect of oxygen, RP2942.

Gold, castings, effectiveness of vacuum investing in the elimination of surface defects, 697 .

copper, molybdenum, silver, tantalum, and gold at $662 \mathrm{kev}$, total photoelectric cross sections, 3101. electron scattering by, distributoin, 1317.

Goniometer, automatic integrating pole-figure, preferred orientation in stark rubber, 1454

versatile, for projection photometry, 904.

Goniometric method, evaluation of lens distortion by the modified, J $63 \mathrm{C} 2,113$.

Goniophotometric reflection curves, analysis, 397.

Goudsmit and Bacher's theory of atomic spectra, eomparison of first, second, and third approximations, 2492 .

Government, and industry use of common standards, 2144 .

researchers, 158.

specifications, 1725 .

specifications, sources and identifying symbols, 549 .

Government's bookstore, 2361.

Gradient reflections, nature, 586 .

Graduation of precise circles, 862 .

Graphical, interpretation of a three-components theory of chromatic adaption in terms of the CIE chromaticity diagram, 918.

method for single-point determination of intrinsic viscosity, 1235 .

solution of the single-degree-of-freedom vibration problem with arbitrary damping and restoring forces, 2145 .

Graphite, carbon at $450^{\circ} \mathrm{C}$, reaction of carbon monoxide, 2717.

natural, and refractoriness of the ashes, chemical analyses, surface area, and thermal reactions of, 665 .

Graphites, natural, oxidation studies, 1437.

Gravitational, and thermal excitation of atmospheric oscillations, 1555 .

thermal oscillations in the earth's upper atmosphere, 1726.

Gray tin crystals and their rate of growth, on the quality, 2672.

Grazing, angles, diffraction of electromagnetic waves by smooth obstacles, J 63D2, 181.

angles, study at 1,046 megacycles per second of the reflection coefficient of irregular terrain, $J 63 \mathrm{D} 2$, 235.

Grease consistometer is modified to operate at constant shear rates, higher viscosities, 245 .

Green coronal line intensity and geomagnetism, 2960.

Grid, parallel wire, transmission and reflection, J 63D1, 87.

Grotrian diagrams, partial, of astrophysical interest, 1758.
Ground, homogeneous curved, transient field of vertical dipole, 1967.

systems, electrically short, fields, J $63 \mathrm{D} 2,175$.

wave pulse around a finitely conducting spherical earth from a damped sinusoidal source current, 3024 .

waves, transient radiofrequency, over the surface of a finitely conducting plane earth, RP2844.

Groundwood papers, effect of light, 693 .

Ground-based radio duets, climatology, J 63D1, 29.

Ground-to-air cochannel interference at $2900 \mathrm{Mc}, 459$.

Ground-wave propagation, R P2770.

Growth, and infrared transmission of cesium iodide crystals, 460 .

preferentially oriented aluminum single erystals, RP2868.

Guayule and chilte rubbers, 119.

Guide to tables on punched cards, 177.

Gun bores, ehromium, 1647.

ehromium plating, 1648 .

retard erosion, experimental plating, TN46 (PB151405).

Gyromagnetic, materials, cavity containing, 2013.

proton, ratio, $2422 ; 2706$.

sample and measurements on a ferrite, exact solution, 2552

\section{H}

$\mathrm{H}^{3}$ complex, applieation of configuration interaction, 2027.

HAE coatings, notes on post-treatment, 1786 .

Hafnium, spectra, improved deseription, RP2904.

spectrum of, 1208 .

Half-life of $\mathrm{I}^{131}, 863$.

Half-planes and ribbons, surface currents exicited by an infinite slot, 1907.

Halloysite, kaolinite, and a related mineral in clays and soils, criteria for the characterization, 420 .

Haloaromatic compounds, fluorination, RP2938.

Handbook of physics, contributions: 2500 .

Arithmetic.

Color vision and colorimetry.

Control mechanisms.

Electrolytic conductivity and electrode processes. Electronic circuits.

Elements of probability.

Geometry.

Integral equations.

Neutron physies.

Nuclear electromagnetic radiations.

Statistical design of experiments.

Thermometry and pyrometry.

Handmade bolts support the Capitol dome, 2577.

Hankel, determinants, AMS49.

integrals of order zero, tables relating, errata, 147

Hardwood fibers in offset papers, resin bonding, 302 .

Haringx diaphragm theory, invited discussion, 2167.

Harmonic, generation with ideal rectifiers, 2578.

oscillators, 1847.

subharmonic, and linear functions of two variables, 280.

Harmonics, radiated, measurement of, source of error, 377. Hausdorff means, Gibbs' phenomenon, 65 .

Haystaq system: past, present and future, 2815.

Hazards from static electricity, 461.

Haze, color, and gloss, measurement of, 760 .

$\mathrm{HCO}, \mathrm{OH}$, and $\mathrm{CH}$ in flame spectra, using deuterium as tracer, mechanism of formation, 868.

$\mathrm{He}^{4}$ scale of temperatures, 1958, J 64A1, 1; Mono. 10.

Head regulator for cutoff valve, 2146 .

Hearing, mechanistic aspects, 2987.

Hearing, normal threshold, research, 2735.

Heart pump, variable, permitting independent control of rate, output, and ejection velocity, 381 .

Heat, and ultraviolet aging of poly(vinyl chloride), RP2863.

capacities of ten technically important gases, 843 .

eapacity and enthalpy from $0^{\circ}$ to $900^{\circ} \mathrm{C}$ of three nickel-chromium-iron alloys of different carbon contents, RP2870. 
capacity of aluminum oxide, RP2848.

capacity of aluminum oxide in the range $300^{\circ}$ to $700^{\circ} \mathrm{K}, 2362$.

capacity of erystalline dextrose between $25^{\circ}$ and $95^{\circ}$ C, 246.

capacity of potassium and three potassium-sodium alloys between $0^{\circ}$ and $800^{\circ}$, the triple point and heat of fusion of potassium, 462 .

capacity of sulfur from $25^{\circ}$ to $250^{\circ}$, the heats and temperatures of transition and fusion, 3079 .

capacity standards for the range $14^{\circ}$ to $122^{\circ} \mathrm{K}, 729$.

combustion (net) and aniline-gravity product of aircraft fuels, relation between, 1128 .

combustion with a bomb calorimeter, precise measurement, Mono.7.

conduction, cosmic examples, in very rare rotating or expanding gases, 2920 .

degradation of collagen by, 2511.

delivery in a compressible flow and applications to hot-wire anemometry, 247.

flow in a fluid with eddying flow, 1727 .

fusion, and heat content of lead, 1027.

fusion and the melting point, heat content from $0^{\circ}$ to $900^{\circ}$, lithium fluoride, 1053.

gaseous conduction in dewars, calculation, 2482

mixing of polybutadiene and benzene, RP2921.

net of combustion of AN-F-58 aircraft fuels, 265 .

net of combustion of aviation gasoline and its correlation with other properties, 2646.

pump, 1227.

reaction of diborane with water and the heat of formation of boric oxide, RP2927.

reducing, leak through support members in liquefied gas storage vessels, 3049 .

specific, enthalpy, entropy, free energy function, viscosity, and thermal conductivity of steam, compressibility factor, density, 1657 .

specific, of beryllium between $0^{\circ}$ and $900^{\circ} \mathrm{C}, 312$.

treating variables on the martensite transformations in SAE 1050 steel, 1186.

treatment, 199.

treatment affects properties of enamel-steel composites, discussion of the paper, 2525 .

treatment of the susceptibility of sand cast aluminum alloy 220 to stress corrosion cracking, 2349.

units and temperature scales for calorimetry, 1355

Heat content, of zirconium and of five compositions of zirconium hydride from $0^{\circ}$ to $900^{\circ} \mathrm{C}$, RP2878.

lead from $0^{\circ}$ to $900^{\circ}$, and the heat of fusion, 1027 .

potassium borohydride, RP2828.

sodium borohydride and of potassium borohydride from $0^{\circ}$ to $400^{\circ} \mathrm{C}, \mathrm{RP} 2828$.

study, some thermodynamic properties, 2839 .

Heat of formation, of boron trichloride, RP2956.

potassium calcium silicate, RP2955.

sodium calcium aliminate, RP2887.

tetrafluoromethane, 1533 .

titanium tetrabromide, RP2932.

titanium tetrachloride, RP2928.

titanium tetraiodide, J 63A2, 161.

Heat transfer, boiling liquid nitrogen and hydrogen flowing axially through narrow annular passages, 2581 .

effect of internal radiant, on temperature distribution, thermal stress, and deflection in box beams, RP2949.

foams and powders, 2147.

laminar flow, 2579 .

laminar flow through a tube, RP2937.

measurements on refrigerated-food trailers, 2580 .

Heat-flow analysis, transient, electrical-analog method for, RP2892.

Heated, electrically filaments, on-off temperature control, 2660 .

Heating, aerodynamic, 2889.

control, cryostate, amplifier, 2090.

elements, silicon carbide, spectral emittance of, RP2810.

glasses, sag point as a reference point in, RP2791. panels, radiant glass, 807 .
Heats, adsorption from charge-transfer complex theory, 2582.

combustion of some peroxides and the heats of formation of acetate, propionate, and butyrate radicals, 2148 .

formation of diborane and pentaborane, RP2901.

liquid metals and liquid salts, specific, 2306.

specific, of collagen and leather, 1502 .

temperatures of transition and fusion, 3079 .

wetting of collagen, leather, and other organic and fibrous materials, 1028.

wetting of modified collagen and other materials, 2149.

Height, curves, virtual, joint use of the ordinary and extraordinary in determining ionospheric layer profiles, J $64 \mathrm{D} 2,111$.

gain and path antenna gain, measurements of correlation, 1401 .

nightglow 5577, 2817 .

nightglow by the van Rhijn method, 1534.

pulse, analyzers, multichannel, 2207.

Heights, nightglow: a reinterpretation of old data, 1416. Heisenberg and Obukoff theories of turbulence, 1215. Helical, potentiometers, 1070.

springs, long, under fluctuating load, behavior, 1530 .

springs of finite thickness, 1427.

tungsten, spring balance, 162.

Helium, at low temperatures and high pressures, TN8, (PB151367).

Joule-Thomson process in the liquefaction of, RP2834.

large scale, helium liquefaction and transportation, 2797.

liquefaction and transportation, $2855 \mathrm{~A}$.

liquefier, National Bureau of Standards, increased rate of liquefaction, 2157.

liquid, attenuation, 1137.

liquid, bath and reproducibility of resistance thermometers, constant temperatures, 2916.

liquid, below $1^{\circ} \mathrm{K}$, normal fluid concentration in 774.

liquid, cryostat with an integral super-conducting resonator, $2610 \mathrm{~A}$.

liquid, lambda transition, 2475 .

II, liquid, rayleigh disk, 157 .

II, liquid, second sound propagation in, 821 .

('He I), neutral, energy levels and spectrum of, J $64 \mathrm{~A} 1,19$.

nonventing, liquid dewar, temperature stratification, J $64 \mathrm{Cl}, 19$.

4, photodisintegration, 1106.

rarefied, propagation of sound, 115 .

refrigeration and liquefaction using a liquid hydrogen refrigerator for precooling, TN39 (PB151398).

vapor-pressure scale of temperature, 1535.

vapor-pressure scale of temperatures, 2818.

wave equation with the SEAC, numerical solution, 372.

Hepp-Scatchard osmometer, improved, 1362.

Heptane-air, autoignited, mixtures, emission spectra, 442 Hermite orthogonal functions, 283.

Hermitian matrices, by the segment method, bounds for eigenvalues, 1005 .

indefinite, 2303.

inequalities concerning positive-definite, 1496

Herzberg bands of $0^{2}$ in an oxygen afterglow and in the night sky spectrum, 1185 .

Heterogeneity, chain block designs with two-way elimination of, 966 .

Heterogeneous reaction of chlorine and nickel between $1200^{\circ}$ and $1700^{\circ} \mathrm{K}$, kinetics of high temperature, 2608.

Hevea and Castiloa rubbers, 160.

rubber, Mooney viscometer cure characteristics, 1409.

Hexabromoosmate, ammonium, 1201.

$\mathrm{n}-\mathrm{H}$ exadecane, viscosity, RP2912.

Hexafluorobenzene, by electron impact, ionization and dissociation, 2168.

pyrolysis of tribromofluoromethane, 2151.

reactions, 2718 . 
Hide substance determination, effect of sample preparation on precision in, 1000.

Hides, chrome-tanned steer, influence of splitting on the strength, 468 .

High energy, electron-nuclear scattering, 1591. radiation effects in polymers, mechanism, 2194.

High, frequencies observable over long distances, new kind of radio propagation, 369 .

input impedance transistor amplifier, 2586.

polymers, thermodynamics of erystallization, 2846. precision automatic frequency comparator and recorder, 1356.

pressure apparatus, compact multianvil wedge type, $\mathrm{J} 63 \mathrm{C} 1,59$.

pressure gas handling equipment for autoclaves in chemical research, 2154.

pressures and low temperatures, thermodynamic properties of helium, TN8 (PB151367).

temperature alloys, determination of aluminum in precipitation hardening stainless steel, J 64A2, 181.

temperature, broad-band stark cell for microwave spectroscopy, 178 .

temperatures, precision thermostat, 110.

vacuum gimbals, 1731 .

vacuum seal-off valve, 919 .

very, frequency propagation by ionospheric scatteringsurvey of experimental results, 1971.

very, frequency propagation measurements in the Rocky Mountain region, 1972.

voltage $\mathrm{X}$-ray tube, 224.

High-altitude nuclear explosions on radio noise, effects, $\mathrm{J} 64 \mathrm{D} 1,37$.

High-density theorium oxide, some physical properties, 1877.

High-dispersion spectra of Mars, 2152.

High-energy, radiation, behavior of polymers exposed, factors influencing the behavior, 1343 .

radiations, degradation and range straggling of, 680 . $\mathrm{X}$-rays, 1150.

High-frequency, antenna, long distance horizontal radiation pattern, 2371 .

communication circuits, effect of echo on the operation, 2810.

communication circuits, multipath distortion, 3073 .

communication conditions, 2127 .

$\mathrm{C}-\mathrm{W}$ field-intensity records with the aid of simultaneous pulse data, interpretation, 472 .

impedance standards at the National Bureau of Standards, 2583.

induced electroluminescence in zine sulfide, 1728.

limit, evaluation of bremsstrahlung cross sections, 2551 .

limit of bremsstrahlung in the Sauter approximation, 2961.

limit of 500-kev bremsstrahlung, polarization, 2694.

magnetic permeability measurements using toroidal coils, 2584 .

measuring, induced electroluminescence, erystal mount and technique, 2074.

radiowaves, measurements of coastal deviation, $\mathrm{J} 64 \mathrm{Di}, 57$.

scatter sounding experiments at the NBS, 730 .

standards for the Electronic Calibration Center, NBSBL, 2585.

High-gain antennas for VHF scatter propagation, 1729.

High-order harmonics for X-band oscillator stabilization, 2153.

High-power, pulse generator, 627.

pulser aids cathode studies, 1029.

square-pulse generator, 463 .

High-precision frequency and time standards, 18.

High-purity $18-10$, sigma phase, 542 .

High-repetition-rate mercury pulser, 2155.

High-resolution grating spectrometer, infrared, RP2922.

High-speed, computation of ideal gas thermodynamic functions. II. The diatomic free radicals of the isotopic hydrides of oxygen and sulphur, 1357 .

computations in the kinetics of free-radical degradation. I. Random initiation, 2587. flip-flops for the millimicrosecond region, 1730 .

machine computation of ideal gas thermodyuamic functions, 1030.

motion pictures in textile researeh, 66 .

stroboscope for accelerometer calibration, 1031.

High-stability, bridge-balaneing oseillator, 1358 .

frequeney standards, 1359.

High-temperature, liquids, 1032.

strain gages, bonding agents, 247 .

High-vacuum temperature gradient furnace, 2588.

High-velocity streams of hot gas, 1163 .

High-voltage supply, series-resonant, 539.

Higher-order approximations in ionospheric wave-propagation, 67.

Hilbert, matrix, 2229 .

space to the calculus of variations, applications of the theory of quadratic forms, 195 .

Hindered rotation in symmetric-asymmetric molecules, 1732 .

History, of automation, brief look, 1585 .

glass, optical, production in the United States, 731.

International Commission on Radiological Protection, 2589.

vertical-incidence ionosphere sounding at the National Bureau of Stantards, TN28 (PB 151387).

Hoist, novel rocket, 2430 .

Hollow square, torsion, 2385.

Homogeneity of six proposed NBS-GMC zine-base spectrographic standards, investigation, 1377.

Homogeneous, conductor with a wavy interface, calculation of the field, 3067 .

curved ground, transient fields of vertical dipole, 1967.

media, theory of reflection from a wire grid parallel to an interface, 2238.

Horizon, tropospheric propagation, 351.

Horizontally polarized radio waves, a very-low-frequeney antenna for investigating the ionosphere with, J 64D1, 27.

Horner's rule, two problems in abstract algebra connected with, 1101.

Hospital, operating rooms, conductive flooring, J $63 \mathrm{C} 2$, 125; Mono.11.

Hot gases, temperatures, 1882.

How the National Bureau of Standards contributes to building codes, 1733 .

How to solve a linear programming problem, 1360.

How statisties improve physies, chemical, and engineering measurements, 68 .

Human enamel and dentin, determination of some compressive properties, 2518 .

Humidity, absolute, note on the climatic variation, 1991. during fabrication of polyester laminates, effects, 435.

lag apparatus, simple, 1487.

Hydrated cement, 2639.

Hydrates, calcium silicate, 1172.

Hydraulic, dam-break wave, 974.

research in the United States:

1957: M221.

1958: M224.

1959: M227.

turbine contra-angle handpiece, 732 .

Hydrazine, and ammonia with oxygen atoms and hydrogen atoms in atomic flames, reactions, 1948.

substitute hydrazines, electron impact studies, 2939.

Hydrazoic, acid and methyl azide by electron impact, ionization and dissociation, 2604.

acid, mass spectrometric study of the decomposition of, by the electric discharge, 2623.

acid, mass spectrometric study of the rate of thermal decomposition, RP2881.

Hydride bath for the electrodeposition of aluminum, 363 .

Hydrides, diatomic, electronic structure, 2543.

Hydrocarbon, flames, $\mathrm{C}_{2}$ radical, 1434.

flames, isotopic tracer study of carbon formation, 2018. polymers, pyrolysis, 116.

Hydrocarbons, and hydrogen, polyhedral flames, 514.

aromatic, electron impact studies, 2938.

aromatic, filtered from smoky air, mass spectra, RP2876. 
liquid and solid state at low temperatures, vapor pressures of, TN4 (PB151363).

nitrogen, active, 2982 .

petroleum, 107.

saturated, microwave absorption in compressed gases, 1778.

some, two-stage autoignition, 354

Hydrochloride, yohimbine, erystallographic data, 2508.

Hydrocolloidal, alignate, dental impression material, 986.

Hydrodynamic, data for dilute protein solutions, interpretation, 2668 .

stability in a domain containing a transition point, asymptotic solution of the differential equation, 656 .

Hydrodynamics, note on the circle theorem, 1084.

Hydrogen, and boiling liquid nitrogen flowing axially through narrow annular passages, heat transfer, 2581.

atoms, the addition, to solid olefins at $-195^{\circ}, 2803$. atoms with solid olefins at $-195^{\circ}$, reaction, 2828 .

bonding in solid $\mathrm{H}_{2} \mathrm{O}$ and $\mathrm{H}_{2} \mathrm{O}-\mathrm{H}_{2} \mathrm{O}_{2}$ mixtures, 2894.

carbon, determination of copolymer composition by combustion analysis, $\mathrm{J} 64 \AA 2,157$.

carbon, oxygen, and nitrogen calorimetric combustion in a bomb of compounds containing, 1645 .

clouds, interstellar neutral, radio observations, 1836.

cyanide and deuterium cyanide, infrared spectra, 1739

departures of, from L.T.E. in a stellar atmosphere and the consequent structure of the solar chromosphere, 2927.

dc, discharge, microwave measurements of the properties, 2200 .

deuteride, 153 .

deuterium molecules, 2410 .

electrode, 2260 .

electrodes, titration of acids with, RP2769.

hydrocarbons, polyhedral flames, 514 .

hydrogen evolution reactions, 3007 .

glow, dc, effect of microwave signals, 2098.

improved, atom beam furnace, TN47 (PB151406).

ion, 3012 .

isotopes, distillation, 2526.

isotopes, low temperature distillation, $2618 ; 2691$.

isotopes, microbiological fractionation, 2635 .

isotopes, separation, by multicomponent distillation, 2751

large liquid, bubble chamber, 1984 .

large scale liquefaction, some aspects, 1491.

lines, stark broadening function for, $\mathrm{C} 603$.

liquefaction by a dual pressure process, 2156 .

liquefaction cycles, 2590 .

liquefier, large $\mathrm{CEL}-\mathrm{NBS}$, vapor phase ortho-para conversion, 2859.

liquefiers, expansion engines for, $\mathrm{J} 64 \mathrm{C} 1,25$.

liquid, in the large CEL-NBS liquefier, new arrangement for ortho-para conversion, 1988 .

liquid, no-loss storage, mechanical refrigeration process, 1986.

liquid, ortho-para catalysis in production, RP2840.

liquid, refrigerator for precooling, TN39 (PB151398)

liquid temperature refrigeration system, design and construction, TN38 (PB151397).

negative ion, photodetachment of, 1105.

nitrogen, oxygen, water, and ammonia, electric discharge, 2309.

normal, liquid, 1173

sulfide, infrared spectrum, $1040 ; 1187 ; 1740$.

sulfide, infrared spectrum of, from $2200-2800 \mathrm{~cm}^{-1}$, 1741 .

Hydrogen-oxygen reaction at the second explosion limit, 248.

thermal, reaction at relatively low temperatures, 345 .

Hydrogen-deuterium, mixtures, deterium in, spectroscopic analysis, 551 .

mixtures, distillation, 2093.

Hydrogen-bonded liquids, dielectric constant, RP2905.

Hydrograph, sudden water release, J 63B2, 117.

Hydrographs, river flow, J 63B1, 43.
Hydrolysis, mutarotation, and rearrangement reactions of glycosylamines, 2642.

Hydroxyapatite, pure, crystals, preparation, 1816 . synthetic, and rat molar enamel, 2528.

structure, refinement, 2724 .

Hygrometers, electric, C586.

Hygroscopic expansion of dental casting investments, mechanism, 1405.

Hyperbolic and inverse hyperbolic functions to many places, radix table for obtaining, 810

Hyperfine structure formulas for LS coupling, 733.

Hypergeometric, series, programs for computing, 1118.

series, representation of a certain integral involving Bessel functions, 2236.

Hypotheses, other, and randomness, independence of tests, 2231.

Hysteresis effect in multiplier phototube noise, 1361.

I

$\mathrm{I}^{131}$, disintegration, 150 .

half-life, 863 .

Identification, microdetermination of nickel, 70 . solar lines, 864

Identities and inequalities concerning orthogonal polynomials and Bessel functions, 249 .

Identity, for the coefficients of certain modular forms, 1262.

relationship for fractional replicates of the $2^{n}$ series, 2230 .

Ignition, Kel-F and teflon, 2591.

precipitated basic calcium phosphates, pyrophosphate formation, 1465 .

self, new light, 266.

IGY instruction manual, Pt. 1: World days and communications, 2962.

ionosphere, program in the Arctic, 1936.

NBS equatorial region V.H.F. scatter research program, 2209 .

oblique-incidence sporadic-E and F-scatter program, TN48 (PB151407).

observations, day to day, 3068 .

observations of F-layer scatter in the far east, 2963.

Illuminance of a lens interposed between the source and the illuminated surface, 858 .

Illumination, color rendition of fluorescent sources, 1651. daylight to incandescent filament, object-color changes from, 492 .

shutter, device for the study of phototropic dyes, 374 .

Image constrast suitable for using square waves targets, J $64 \mathrm{C} 1,57$.

photographic, during fixation, J $64 \mathrm{C} 1,65$.

Image-forming system, outline of characteristics, 1801.

Immiscibility, oxide systems, 2320; $2320 \mathrm{~A}$.

surface tension of some simple borates, 2592 .

Impact, absorbing capacity of textile yarns, 2363.

bars, method of controlled slack quenching, 2426 .

ionization and dissociation of methyl siloxanes by electron, 747

properties of slack-quenched alloy steels, RP2799.

properties of yarns made from different fibers, 734 .

strength of some thermosetting plastics at low temperatures, 464 .

test for leather based on an alternating punching force, 2457 .

testing of various alloys at low temperatures, 2819 . tests of textile yarns, RP2866.

Impedance boundaries, plane and curved, excitation of surface waves, 2553 .

characteristies of a uniform current loop having a spherical core, J 64D3, 295.

corner-reflector antenna as a function of the diameter and length of the driven element, J 64D2, 135 .

mierowave standards and methods of measurement, 2722 .

standards, high-frequency, 2583.

wire grid parallel to a dielectric interface, 2364.

Implications of Liapunov's conditions for stability, 1142. slant-Es, 2304. 
Importance of the Faraday to elemental constants and electricity standards, 2593.

Impregnation of sole leather with polymers, 2594 .

Improved cuvette densitometer for cardiac output determination by the dye-dilution method, 650 .

description of hafnium spectra, RP2904.

dimensional stability in laminated map paper, 2595.

dip coater, 1033.

8-hydroxyquinoline method for the determination of magnesium oxide in portland cement, 2893.

electrical differentiation of retarding potential measurements, 2596.

Hepp-Scatchard osmometer, 1362.

hydrogen atom beam furnace, TN47 (PB151406).

optical test for spherical abberration, 950 .

photogrid techniques for determination of strain over short gage lengths, 465 .

stainless steel needle valve, 1034 .

Improvement, gain stability of the superheterodyne mixer through the application of negative feedback, 466 .

response of 4- $\pi$ gamma-ionization chambers, 735 .

Improvements in flame photometric determination of sodium in portland cement, 736 .

liquid scintillators, $2597 ; 2964$

$\mathrm{In}^{117}$ and $\mathrm{Sb}^{117}$ radioactivity, $1124 ; 1471 ; 2711$.

Inclusion theorum for modular groups, 2016.

Incomplete block designs, 948 .

equilibrium and temperature measurement, 1735.

Inconel and stainless steel, 3053.

ceramic coated, and type 321 stainless steel, 3092.

Increased rate of liquefaction of the National Bureau of Standards helium liquefier, 2157.

Incremental delay pulse generator, 191.

Indentation pressure of a smooth circular punch, 1363.

Independence of tests of randomness and other hypotheses, 2213.

Index, diagnostic tests, rating, 71.

notation, generalization of, for absolute tensors of arbitrary order, J $64 \mathrm{~B} 2,99$.

proposed, of current noise for composition resistors, 1241 .

radio refractive, synoptic study of the vertical distribution, J $63 \mathrm{D} 2,249$.

refraction, Mono. 3, Vol. I and II.

refraction of fused-quartz glass for ultraviolet, visible, and infrared wavelengths, 1035 .

refractive, gradient, geographical and height distribution of the, 727 .

spectrochemical analysis, literature on, 1036; 2965.

Indigo and azo-dyes, steric effects, 1897.

Indirect transitions in indium antimonide, 1736.

Indium, anhydrous, phosphate and thallic phosphate by X-ray diffraction, crystal structure, 1667 .

$n$-type, arsenide, oscillatory galvanomagnetic effects, 2678.

resonance for D-D neutrons in water, 2450 .

Indium antimonide, electrical and optical properties of intermetallic compounds, 1007.

elastic moduli, 1693.

electrical properties, 703 .

indirect transitions, 1736 .

$n$-type, galvanomagnetic effects, 2135 .

piezoelectric effect, 2690 .

piezoresistance, 2252.

$p$-type, galvanomagnetic effects, 2136

thermoelectric power, 1559.

Industrial, and office quieting, technical aspects, 324 .

beta-ray sources, safe design and use of, H66.

social research, information processing in, 741 .

Industry, and Government, use of common standards, 2144.

NBS and its service to, 1939.

textile, technological challenge, 2002.

Inelastic collisions the Moliere theory of multiple scattering, 1037.

scattering of 20 -kev electrons in metal vapors, 2158.
Inequalities concerning positive-definite IIermitian matrices, 1496

clementary, relating to a gamma and incomplete gamma function, 3047

ultraspherical polynomials and Bessel functions, 72

Inequality, determinantal, $1232 ; 2657$.

Kiantorovich's, J 64B1, 33.

Robertson, II. P., determinantal, 1980

Infiltration meter, portable, design and performance, 2082.

Infinite integral, note, 270 .

products, eoefficients of, 853 .

slot on half-planes and ribbons, surface currents excited by, 1907.

Influence coefficients of tapered cantilever beams computed on SEAC, $467 ; 738$.

copper ions on the adherence of vitreous coatings to stainless steel, 1737.

exposure conditions on the accelerated durability testing of asphalt, 1738 .

ground on the calibration and use of VHF fieldintensity meters, $151 ; 739$.

heat treating variables on the martensite transformations in SAE 1050 steel, 1186.

impurities on the pyrolysis of polyamides, J 63A3, 269.

lower permissible levels on atomic operations in the United States, 2598.

splitting on the strength of chrome-tanned steer hides, 468.

strain rate and temperature on the creep of cold drawn ingot iron, 73.

sun upon the ionosphere, 250.

temperature on the adsorption of chrome $\left(\mathrm{Cr}_{2} \mathrm{O}_{3}\right)$ by calf skin squares, 2159 .

temperature on the adsorption of water vapor by collagen and leather, 74 .

variations in rotors, dies, and rate of shear on Mooney viscosity, 740 .

Information processing in social and industrial research, 741 .

systems, automation and future management, 2467 .

theory, $250 \mathrm{~A}$.

theory aspects of propagation through time-varying media, $1143 ; 1364$.

Infrared, bands of deuterium sulfide, RP2789.

emission spectra of flames under high resolution, RP2903.

emission spectra of $\mathrm{OH}, \mathrm{CO}$, and $\mathrm{CO}_{2}$ from $3 \mu$ to $5.5 \mu, 578$.

emission spectrum of methane at 3.3 microns, RP2803.

emission spectrum of silicon carbide heating elements, RP2810.

energy by glasses, transmittance, 1561 .

extension of the second spectrum of zirconium, 717 .

far, region, transmittance and reflectance of cesium iodide, 2852.

far, transmittance of materials, $\mathrm{J} 64 \mathrm{C} 1,55$.

flame spectra, 1366.

flame spectra, emission of, under high resolution, RP2903.

growth transmission of cesium iodide crystals, 460 . high-resolution grating spectrometer, RP2922.

measurements, 1072 .

measurements, past and present, 1367.

measurements with a cesium iodide prism, 742 .

measurements with a cesium iodide prism molecular spectroscopy, 1351.

measurements with a small grating from 100 to 300 microns, RP2821.

multiplets and the solar spectrum, 743 .

near, solar radiation measurements by balloon to an altitude of $100,000 \mathrm{ft}$., 2645 .

new, band system of $\mathrm{FeO}, 368$.

normal spectral emissivity, apparatus for the measurement, J 64C2, 99. 
phase contrast refractometer, 2600.

photoconductivity due to neutral impurities in germanium, 1039 .

radiant energy, $124 ; 812$.

raman spectra of trans-difluorodichloroethylene, 2161.

region, far, small grating spectrometer, 1488.

region, filters for, 450 .

spectrometry with a cesium iodide prism, 744 .

studies in the 1 - to 15 -micron region to 30,000 atmospheres, J 63A1, 55 .

studies on polymorphs of silicon dioxide and germanium dioxide, RP2885.

study of condensed gases, low-temperature microcell, 2619.

study of condensed gases, temperature microcell, 2800.

study of hydrogen bonding in solid $\mathrm{H}_{2} \mathrm{O}$ and $\mathrm{H}_{2} \mathrm{O}$ $\mathrm{H}_{2} \mathrm{O}_{2}$ mixtures, 2894.

study of some structural changes in natural rubber during vulcanization, RP2816.

synthesis absorption spectra of some $m$-polyphenyls, 1951 .

transmittance of certain silicate glasses, effect of fluorides, 2933 .

transmitting glasses, 1177.

wavelengths, ultraviolet, and visible, 1035.

Infrared absorption, iodine in its pyridine complexes and in benzene. 2994 .

liquid water from 2 to 42 microns, 1038.

oxygen discharge products and ozone at $4^{\circ} \mathrm{K}, 2599$. spectra, analysis, 1569 .

spectra of some aldopyranosides, conformations of the pyranoid sugars, J $64 \mathrm{~A} 3,239$.

spectra of some cyclic acetals of sugars, RP2960.

spectra of twelve substituted benzene derivatives from 15 to 40 microns, 75 .

spectra of urea, thiourea, and some thiourea-alkali halide complexes, 2160 .

spectrum of methane from 2,470 to $3,200 \mathrm{~cm}^{-1}, \mathrm{~J} 64 \mathrm{~A} 3$, 201.

spectrum of $n$-dimethyl-amino-diborane, 1365.

studies in organic phosphorus chemistry, 2494.

Infrared spectra, asphalts, J $63 \mathrm{~A} 2,189$.

crystalline polyphenyls, RP2829.

hydrogen cyanide and deuterium cyanide, 1739 .

molecules of the atmosphere, 2377.

structure of ethylene, 2833.

sugar acetates in solution, RP2772.

thermally degraded poly(vinyl chloride), RP2832.

Infrared spectrum, acetylene-d, 1934.

carbon monoxide, 516; RP2883.

carbonyl sulfide, 2162 .

hydrogen sulfide, $1040 ; 1740$.

hydrogen sulfide from $2200-2800 \mathrm{~cm}^{-1}, 1741$.

hydrogen sulfide in the $5100 \mathrm{~cm}^{-1}$ region, 1187 .

nitrous oxide, 1782 .

rotational constants of carbon disulfide, 1932.

structure of perchlorylfluoride, 1933.

tertiary butyl fluoride, 2820 .

Infrareds of certain solids condensed to leave discharges in a gaseous state, 2943 .

Ingot iron, deformation twinning in charpy $V$-noteh specimens of, 679 .

effect of strain rate and temperature, 73 .

effect of tensile strain at low temperatures on deformation twinning in, 695 .

Initial and remanent permeability spectra of Yttrium iron garnet, 2966.

Inlet system of a four-stroke single-cylinder engine, dynamics, 1322.

Inner bremsstrahlung from $\mathrm{V}^{40}, 1368$.

solar corona during June 1959, 3080.

Inorganic constitutents in sucrose solutions, determination, 987.

Input, high, impedance transistor amplifier, 2586.

Input-output, key or bottleneck, 2601.

InSb, galvanomagnetic effects, 1352.

Instantaneous power spectra, 469 .

Institute for Numerical Analysis, 152; 337.
Instructions, operating, for ARN-2 auxiliary log-linear noise recorder, $\mathrm{TN} 45$ (PB151404).

Instrument, automatic, for electron scattering measurements, 1274 .

cables subjected to shock and vibration, electricall noise, 437.

measuring marginal power of spectacle lenses, 1369. measuring, piston gage, 877 .

mechanically differentiating curves, 24 .

pneumatic, based on critical flow, 14.

recording, under dynamic conditions, 2286.

Instrumental drift, 1041 .

Instrumentation, automation, 1370 .

dental materials, review of 1956 dental research, 2290 . documentation, 1682 .

future of mankind, 1371.

meteorological, at the NBS, 1073.

perspective, 76 .

research at the NBS, 1372.

revolution in industry, science, and warfare, 1042. where we stand, 2602 .

Instruments, AC-DC transfer, for current and voltage measurements, 2446.

dental, cutting, 1165 .

dental materials, 1951-55, 1669.

developments, 2519.

electrical, 99.

electrical indicating, used in early Edison central stations, 702 .

electrical, testing of, 886 .

electrothermic, 1012 .

immersion-type, measurement of gas temperatures, 867.

magnetic high frequency measurements, 1043.

meters, electrical, 1814 .

recording, direct-indicating, 690 .

rotating dental liamond abrasive, 2085.

Insulated loop antenna immersed in a conducting medium, RP2781.

Insulating, concretes, 1742.

materials, comparison, 2416.

materials, electrical, 992.

properties, thermal, of airspaces and fibrous insulations bounded by reflective surfaces, BMS151. thermal, 1203.

Insulation, sound, of wall, floor, and door constructions, BMS144, 2d Suppl.

Insulations, characteristics of some, for liquid oxygen transfer lines, 2054 .

Integrability of strain rate and strain, conditions of, RP2813.

Integral, $\int_{0}^{2} e^{-\mu 3} d u$, table, 320 .

boundary points of convex polyhedra, 1743.

circulants, unimodular, 1567.

differential equations, 278.

differential geometry, metric methods in, 764 .

equation approach to the problem of wave propagation over an irregular surface, 394.

equations, 2500 .

equations, symmetric, error bounds for the eigenvalues, 1707 .

infinite, note, 270 .

large, curves of a non-linear differential equation, behavior, 2662

transformations, relations between, and summation methods, J 63B1, 1.

Integrals, airy functions, AMS52.

function space, 185.

multiple, some Monte Carlo experiments in computing, 1875 .

practical evaluation, 1098.

tables of transport: a supplement, J 63B1, 23.

Integrating and differentiating bar-magnetic velocity meter for use in ballistocardiography, 951 .

Integration, concepts in the terminology of measurement and control, 1935.

parabolic equations by difference methods, 497 . 
Intensities, absolute, of [OI] 5577 in the auroral and subauroral zones, comparison, J 63D 1, 19.

Intensity distributions in nitrogen bands excited in auroras and by high-energy protons and hydrogen atoms, 1044 .

Interacting electron beams with application to solar radio noise, 1418 .

Interaction of stretching vibrations and inversion in ammonia, 1744 .

Intercomparing neutron source strengths by means of a manganous sulfate bath, scintillation counter method, RP2952.

Intercomparison, French and U.S. roentgen ray standards, 2017.

roentgen standards of Great Britain and USA, 1621.

Interconnection of two digital computers, 1536.

Interdisciplinary approach to science personnel, 77 .

Interfacial waves in viscoelastic media, 1745 .

Interference, antioxidant in the determination of low polymer in SBR synthetic rubber, 2967.

orbital and spin currents on bremsstrahlung and photoelectric effect, 2968.

patterns in reverberant sound fields, 1373.

wide-band FM adapter reduces, 169.

Interferometer, electron, $438 ; 706 ; 947$

large surfaces, RP2943.

low pressure gases, 648 .

microwave Fabry-Perot, reflectors for, 3034.

parallel testing, RP2917.

stroboscopic, for vibration measurement, 2784 .

Interferometry, electron, 707.

Fabry-Perot, phase shift effects, J 64A3, 191.

microwave, J $64 \mathrm{~B} 1,1$.

Interlaboratory comparison of thermal conductivity determinations with guarded hot plates, 251.

Intermediate and high energy scattering, application of variation methods, 955 .

Intermetallic compounds, electrical and optical properties, 1330.

optical and electrical properties of, 1007.

photoconductivity and photovoltaic, 1806.

photoeffects, 1444.

semiconducting, 823 .

Intermittent-action camera with absolute time calibration, J 64C2, 159.

Intermittent communications with a fluctuating signal, 2163.

Internal barrier in ethane, 2603.

energy of highly ionized gases, 3081 .

gas counting, 2714 .

reflection in the troposphere and propagation beyond the horizon, 470 .

rotation in methyltrifluoromethyl acetylene, 1374 .

International relations, National Bureau of Standards and its, 339 .

Commission on Radiological Units and Measurements, $2366 ; 3022$.

comparisons of radioaetivity standards, 1746 .

co-operation on standardization of textile testing methods, 471 .

exchange of scientific information, 79.

Geophysical Year July 1, 1957-December 31, 1958, radio noise data, $\mathrm{T} N 18$ (PB151377).

Geophysical Year, radio and ionospheric observations, J $63 \mathrm{D} 1,1$

Geophysical Year: progress report, 1188.

methods for testing colorfastness of textiles, 1375 .

plastic committee considers standard tests, 1376.

Standards Organization Technical Committee 61 report, 1477 .

textile work shows fast progress, 2164 .

Interplanetary space and the earth's outermost atmosphere, 2969.

Interpolation, coefficients for polar complex, 36 .

coefficients, tables of osculatory, AMS56.

problem, 782 .

series, linear functional equations, 1050 .
Interpretation, ehemical data, 579.

high-frequeney $\mathrm{C}$ - IV field-intensity reeords with the aid of simultaneous pulse data, 172 .

hydrodynamie data for dilute protein solutions, 2668.

mass spectra of eondensates from urban atmospheres, RP'2809.

night-time low-frequency ionograms, 2821

radar waves from metcor trails, 338 .

spectra of atoms and molecules in solid nitrogen condensed at $4.2^{\circ} \mathrm{K}, 17 \pm 7$.

tests for resistance to abrasion of textiles, 473 .

Intervals for a proportion, confidence, 1660 .

Intrinsic viscosity, graphical method for singlc-point determination, 1235.

viscosity-molecular weight relationships for dextran, 745 .

Introduction, July 1956 issue, Revs. Mod. Phys, containing all papers of the Intern. Conferenec "Quantum Interactions of the Free Electron," 1748.

VLF papers, 2165

Introductory remarks on the dosimetry of ionizing radiations, 1045 .

Invariance of insidedness in projective transformations of the Maxwell triangle, 1046.

Inverse feedback, intermediate-frequency gain stabilization, 78 .

nodal slide, evaluation of lens distortion by, $\mathrm{J} 63 \mathrm{C} 2$, 105.

Inversion of matrices by random walks, 282 .

Investigation, bearing creep of two forged aluminum alloys, TN55 (PB161556).

homogeneity of six proposed NBS-GMC zinc-base spectrographic standards, 1377.

perturbations impassed upon radio waves penetrating the ionosphere, 2458.

slot radiators in rectangular metal plates, 1622 .

structural failures of welded ships, 746 .

Investigations of the properties of corrugated diaphragms, $1378 ; 2166$.

Invited diseussion on the Haringx diaphragm theory, 2167.

Iodide, potassium, fast neutron detector, 1451.

sodium, scintillation detector to high energy X-rays, 2741

thallium bromide, refraction and dispersion, 1842.

titanium tetra-, heat of formation, J $63 \mathrm{~A} 2,161$.

trace quantities, polarographic determination, 2258.

Iodide-cesium, far infrared region, transmittance and reflectance, 2852.

optical properties, 1436.

prism, infrared measurements, 742 .

prism, infrared spectrometry, 744 .

prism molecular spectroscopy, further infrared measurements, 1351

prisms, to 50 microns, 1551 .

Iodine, analysis of first spectrum, J 63A1, 1.

vapor barriers, 2892.

Ion, atom interchange in air, 1379

bisulfate, ionization, 3076 .

exchange columns, automatic cut-off valve, 1273.

hydrogen negative, photodetachment of, $1105 ; 3012$.

negative, appearance potentials, 2481.

production during measurements, 193.

Ionic, character and elastic moduli of zinc blend lattices, 2367.

charge and alkali-aggregate reaction on hydrated cement, 2639

eubic crystals, lattice energies, 2175.

detergents, electroviscous effect, in dilute aqueous solutions, 2667.

dissociation of 2,4-, 2,6-, 3,4-dichlorobenzoie acids in water, 2368.

dissociation of 2,6-dimethylbenzoic acid in water, 2369 .

model, nonerystal, for silica glass, RP2782.

non-migratable, charges developed by portland and high-alumina cements during hydration, source, 2768 .

recombination including ion production during measurements, 193. 
Ionium $\left(\mathrm{TH}^{230}\right)$, alpha-gamma angular correlation, 643 . Ionization, average energy loss, secondary electrons, 538. bisulfate ion, 3076 .

cavity, as a function of wall materials, RP2842.

cavity, theory, $1229 ; 1247 ; 1606$.

chambers for radiation data during dental X-ray exposure, 1380 .

chambers, free air, design of, H64.

chambers, standard free-air, plate separation requirements, 1110.

constants, 3076

detectors, dose measurement in water using scintillation and, 995.

dissociation of hexafluorobenzene by electron impact, 2168.

dissociation of hydrazoic acid and methyl azide by electron impact, 2604.

dissociation of methyl siloxanes by electron impact, 747.

dissociation of $\mathrm{NF}_{3}$ by electron impact, 1749

dissociation of oxygen difluoride by electron impact, 2169.

dissociation of perchlorylfluoride by electron impact, 2170 .

efficiency curves of hydrogen and deuterium molecules, 2410 .

gamma, chambers, improvement in response of $4 \pi$, 735 .

multiple, of sodium vapor by electron impact, 2996.

potential of the $\mathrm{CF}_{3}$ radical and some fluorocarbon bond energies obtained by electron impact, 580 .

produced in air by alpha particles near 5-Mev and by beta particles, 2912 .

rare gases by electron impact, multiple, J 63A3, 201.

shock fronts in collisions of gas clouds, $2496 \mathrm{~A}$.

sporadic-E, 2885.

Ionized, gases, $2632 ; 2988 ; 3081$.

gases, thermal diffusion, 2844.

layers, turbulent, aerodynamical mechanisms producing electronic density fuctuations, 1254.

Ionizing radiation, 1151 .

biological purposes, 1189 .

permissible exposure, 1443.

Ionograms, facilities for convenient manual reduction, TN14 (PB151373).

night-time low-frequency, interpretation, 2821.

oblique-incidence, TN31 (PB151390).

Ionosphere, $1537 ; 2700$.

auroral zone, 2317 .

disturbances on low frequency propagation, 229.

echoes, low frequency, note on the polarization, 371 .

effect of small irregularities on the constitutive relations, J 63D2, 135.

electron density fluctuations, 2381.

$41 \mathrm{Mc} / \mathrm{sec}$., observation on vertical-incidence scatter, 2655.

horizontally polarized radio waves, a very-low-frequency antenna for investigating the, $\mathrm{J} 64 \mathrm{D} 1,27$.

IGY program in the Aretic, 1936.

influence of the sun, 250 .

lower, and on VHF forward seatter, 2759.

lower, and the [OI] $557 \%$ nightglow emission, 2880 .

lower, extreme useful range of VHF transmission by scattering, 2560.

lower, radio transmission at VHF by scattering and other processes, 1469

lower, radio waves, 2661.

lower, stratification in, J 63D2, 117.

measurements over a $1,150 \mathrm{~km}$ path, 561 .

multiple reflections between earth and, in VLF propagation, 1781

observations at $50 \mathrm{kc}, 80$.

quiet, mean electron density variations, TN40-1 (PB151399-1); TN40-2 (PB151399-2).

radio waves penetrating, 2458 .

recorder for low frequencies, 651 .

soundings covering frequency range from $50 \mathrm{Kc} / \mathrm{s}$ to $25 \mathrm{Mc} / \mathrm{s}, 2064$. theory of reflection of low- and very-low-radiofrequency waves, J 64D3, 269.

vertical-incidence, sounding at the National Bureau of Standards, TN28 (PB151387).

Ionospheric absorption, measurement, 3091.

characteristics, use of sweep-frequency backscatter data for determining oblique-incidence, 2838 .

conditions in the southern auroral zone, 1650 .

critical frequencies and sunspot number for different sunspot cycles, 571 .

data, 1117

detachment rate for $0_{\overline{2}}$, experimental photodetachment cross section, 2556 .

disturbances, continental maps of, 675 .

drift measurements in the United States, results, 2288.

effects produced by solar flare radiation, 1750 .

fine structure using earth satellites, comparison of phase difference and Doppler shift measurements, 2493.

forward scatter, 2171.

heights deduced from phase velocity, 2931.

investigations using the sweep-frequency pulse technique at oblique incidence, J $63 \mathrm{D} 2,151$.

irregularities, scattering at oblique incidence, 1482.

Ionospheric layer profiles, joint use of the ordinary and extraordinary virtual height eurves in determining, J 64D2, 111.

layers, current nomenclature, 3086.

origin, radio noise, 118 .

propagation by the scatter-sound method, 2326 . propagation, very low frequency, 2203.

propagation, V.L.F., for finite ground conductivity, mode theory, 2373.

propagation, VLF, mode theory, 2234.

propagation, VLF, waveguide mode theory, 2390.

radio, 2490 .

reffection coefficients at very low radio frequencies, calculations, 2044.

reflection coefficients at VLF from sferies measurements, 2172

scatter communication, 1958 eritique of VHF, 2505A. scatter propagation, 3011 .

scattering, modulation studies for VHF, 2639A.

seattering of the VHF radio waves, 1790 .

scattering-survey of experimental results, very high frequency propagation, 1971.

soundings, world-wide, first report of the special committee, 1712 .

storms, 2884.

vertical soundings, 2173 .

vertical soundings for electron density profile data TN14 (PB151373); TN19 (PB151378).

vertical sounds after the I.G.Y. proposed recommendation, 2704.

VHF propagation, regular, 1495.

VHF, scatter circuit during the solar event of February 23, 1956, field-strength variations recorded, 2121 .

VHF, scattering, frequency dependence, TN9 (PB151368).

VLF, propagation, mode theory, 2456.

wave-propagation, 67 .

winds, systematic, 319 .

world-wide soundings of the URSI/AGI Committee, second report of the special committee, 2294 .

Ions, atmospheric negative, photodetachment, 2245. atoms in the sun, 958

collection of produced by alpha particles in air, 2488.

copper, influence, on the adherence of vitieous coatings to stainless steel, 1737 .

ions high kinetic energy in mass spectra of polyatomic molecules, 1144 .

low energy, in $\mathrm{N}_{2}$ and $\mathrm{O}_{2}, 982$.

molecule, ideal gas thermal functions to $25,000 \mathrm{deg}$. $\mathrm{K}$ for diatomic molecules, oxygen, nitrogen nitricocide, 2394 .

negative, 2210 . 
negative atomic iodine, nitrogen and argon, 605 . negative, diatomic molecular, 2841 .

negative oxygen, in the upper atmosphere, 1413.

negative, photodetachment studies, 2246 .

uranyl-like, 2355.

$\mathrm{Ir}^{102}$ gamma rays in concrete, steel, and lead, broad and narrow bean attenuation, 2480 .

Iridium-osmium alloys, 1152 .

Iron catalyst for production of liquid para-hydrogen, 1751 .

enameling, during firing, 1153.

ingot, deformation twinning in charpy $V$-notch specimens of, 679

ingot, effect of strain rate and temperature, 73.

ingot, effect of tensile strain at low temperatures on deformation twinning in, 695 .

metallic, high purity, preparation, 801 .

plastically deformed, residual lattice strains in sectioned bars, 1479 .

tin, copper, silicon, and nickel, electrorefining studies, 2811.

Iron-base alloys, NBS spectrographic standard samples, 2719.

Iron-carbon-nickel alloys, austenite, note on thermal stabilization, 777 .

Iron, cast, nodular graphite in, experimental production, 444.

Irreducible tensorial sets, 2970 .

Irregularities, small effect of, on the constitutive relations for the ionosphere, J $63 \mathrm{D} 2,135$.

tropospheric, radio-wave scattering, J 63D2, 205.

ISCC-NBS color-name blocks, central notations for the revised, RP2911.

Isobutyane, tertiary butyl fluoride and trimethlphospine, 2638.

Isolation of the diffusion layer at an electrode and the determination of concentration polarization, 2605.

Isomeric polyphenyls by adsorption chromatography, separation, 2752.

Isomers, $\mathrm{Cd}^{115}$, decay of, 569 .

Isomorphous substitution in enamel apatite, 748 .

Isoperimetric inequality, closed curves convex in evendimensional euclidean spaces, 952 .

polygons, algebraic proof, 1259 .

Isotope analysis using dimethylmercury, 1381

dilution techniques, structural analysis of clinical dextrans by periodate oxidation, 2319.

effect in oxidation of D-mannitol-2-C 14 by acetabacter suboxydans, 2606.

effect in superconductivity, 581 .

effect in the superconductivity or mercury, 81 .

hydrogen, distillation, 2526.

hydrogen, low temperature distillation, $2618 ; 2691$.

hydrogen, microbiological fractionation, 2635.

hydrogen, separation, by multicomponent distillation, 2751 .

radioactive, lower permissible levels, 2099.

radioactive, safe handling of bodies containing, $\mathrm{H} 65$. stable carbon, analysis of optical spectroscopy, 1892

three rare-earth, alignment, 1256 .

tin, superconductivity, 560 .

Isotopic abundance of solids, 180.

abundance ratios reported for reference samples stocked by the National Bureau of Standards, TN51 (PB161552).

abundance, reference samples, 1475 .

hydrides of oxygen and sulphur, diatomic free radicals, 1357 .

tracer study of earbon formation in hydrocarbon flames, 2018.

Isotropic length of polymer networks, 2370.

paramagnetic crystal, 981.

source, plane, angular distribution of multiple-scattered gamma radiation, 1265 .

sources through aluminum and conerete, gamma rays, TN11 (PB151370)

Iteration, cyclic single step, 781 .
Iterations with superlinear convergenec, method of speeding up, 2427.

Iterative inethod for finding characteristic vectors of a symmetric matrix, 192.

proccsses, acceleratilig convergence, 2447.

Jacobi, polynomials and Whittaker functions, certain series expansions involving, 1424.

polynomials, generating functions, 1426

Jerkmeter for ballistocardiography, 1752 .

Jet abrasive method for measuring abrasion resistance of organic coatings, 1249 .

engine controls, simulator for use in development, C584.

engine test cells, measurements on sound absorbers, 1771

Jets, subsonic air, reverberation chamber study of the sound power output, 2742 .

Joint use of the ordinary and extraordinary virtual height curves in determining ionospheric layer profiles, J $64 \mathrm{D} 2,111$.

Joints, flush riveted, fatigue and static tests, 1345 .

riveted and spot-welded lap, of aircraft materials, creep and creep rupture characteristics, 1299.

Joule-Thomson process in the liquefaction of helium, RP2834.

Junction transactor eireuit applications, 749 .

Kantorovich's inequality, J 64B1, 33 .

Kaolinite, halloysite, and a related mineral in clays and soils, criteria for the characterization, 420 .

Kel-F and teflon, ignition, 2591.

Kinetic data to rate equation, function to the aid in the fitting, 1983.

spectroscopy in the far ultraviolet: the flash photolysis of ethyl compounds, 2607.

Kinetics, eapillary shear degradation in concentrated polymer solutions, 1723 .

chain depolymerization, 474 .

chemical, stochastic processes, 2898 .

chemical, the applieation of the theory of stochastic processes, 2805 .

crystallization in polymers. I. Bulk Polymers, 1047.

crystallization in polymers. II. Polymer-diluent mixtures, 1382 .

D atom reactions with $\mathrm{H}_{2}, 2971$.

depolymerization, some aspects, 307 .

elementary gas phase reactions at high temperatures, 1433.

free-radical degradation, high-speed computations, 2587.

high temperature heterogeneous reaction of chlorine and nickel between 1200 and $1700^{\circ} \mathrm{K}, 2608$.

nitrogen atom recombination, 2972.

OH radicals, acetylene-oxygen flame, 476 .

$\mathrm{OH}$ radicals from flame emission spectra, 475 .

ortho and parahydrogen, 2953.

polymeric systems, crystallization, $2507 ; 2923$.

reaction between nickel and chlorine above $1,100^{\circ} \mathrm{K}_{1}$ 2174 .

Kinorama as an evaluator in aviation lighting, 582 .

Kjeldhal method, determination of nitrogen in leather, 332.

Kleine Bermerkung zur asymptotischen Entwicklung des fehlerintegrals, 1383.

Konig, theorem of, 1719 .

Kosters double-image prism, 2822.

\section{L}

La recherche sur les radicaux libres au National Bureau of Standards, 2973.

Laboratory aging tests for plastics, fluorescent sunlamps, 1713.

automation, 2468 . 
field exposure studies of leather fungicides, 1754

government, engineering management in, 712 .

standard microphones, 1048.

tests with turboprop lubricants, 2610.

Lactones, aldonic, sodium borohydride reduction, 1868.

Ladders, portable metal, new safety code, 2374.

Laguerre orthogonal functions, relative extrema, 105.

Lambda transition in liquid helium, 2475.

La medida de la luz y el color, 2609.

Laminar flow, heat transfer, 2579.

through a tube, heat transfer, RP2937.

through a tube, solution of the differential equation occurring in the problem of heat convection in, 789.

Laminated map paper, improved dimensional stability, 2595 .

Laminates, metal-clad, characteristics, 2053.

Lamination, preservation of documents, Mono.5.

Lamp, two secondary tungsten filament, standards, 2716 .

Lamps, fluorescent, 134.

metal-halide, electrodeless, 705 .

Lanthana-alumina, system, 2472 .

Laplace's equation, numerical methods associated with, 274

Large liquid hydrogen bubble chamber, 1984.

reduction of VHF transmission loss and fading by the presence of a mountain obstacle in beyondline-of-sight paths, 1384 .

reductions of VHF transmission loss and fading by the presence of a mountain obstacle in beyondline-of-sight paths, 750 .

surfaces, testing of by interferometer, RP2943.

Latent roots, Fox's escalator formulae, 386.

positive matrix, 405.

Latin squares, a note on the four by four, 5 .

partially replicated, 1442 .

Lattice energies of ionic cubic crystals, 2175.

regular rhombohedral, sampling, of Munsell renotation space, 930 .

residual, strains in sectioned bars of plastically deformed iron, 1479 .

Lattices, zine blend, ionic character and elastic moduli, 2367.

Layered earth propagation in the vicinity of Point Barrow, Alaska, J 64D1, 95.

Layers, compressible turbulent boundary, with heat transfer and pressure gradient in flow direction, J $63 \mathrm{~B} 1,53$.

Lead, aluminum, and concrete in the range 275 to 525 kilovolts, X-ray attenuation, 1582 .

bismuth at $7 \mathrm{Mev}, 3014$.

concrete, and steel, broad and narrow beam attenuation of $\mathrm{Ir}^{192}$ gamma rays, 2480 .

concrete, attenuation of $86-$ and $176-\mathrm{Mev}$ synchrotron X-rays, $1635 \mathrm{~A}$.

gamma-ray flux, 2917.

heat content, from $0^{\circ}$ to $900^{\circ}$, and the heat of fusion, 1027.

niobate $\mathrm{PbNb}_{2} \mathrm{O}_{6}$, unit-cell data, 2401

oxide-niobium pentoxide, phase equilibrium relations in the binary system, RP2925.

resistance errors in watthour-meter tests, 1049.

storage batteries-essential to modern transportation, 751 .

$\mathrm{X}$-rays scattered in, energy and angular distribution, 2111.

Lead-acid storage batteries, 1141.

Least pth power polynomials on a real finite point set, 1385.

Leather, Castiloa and Hevea rubbers, treatment, 160.

chrome-tanned, 558 .

collagen, and other organic and fibrous materials, heat of wetting, 1028 .

collagen, influence of temperature on the adsorption of water vapor, 74 .

collagen, physical and physico-chemical constants, 510 .

collagen, pores, 1449

collagen, specific heats, 1502 . compounds applied to, fungieidal effectiveness, 2131. cyclic strain testing machine, 1304.

flex tension test for, 917 .

fungicides, deterioration, on, 2353.

fungicides, laboratory and field exposure studies, 1754.

fungicides, studies, 2788.

grease in, 219.

heavy, note on the indentation of, 776 .

heavy, with polyisobutylene and other polymers, pilot plant study of the process for treating, 1240 .

impact test for, based on an alternating punching force, 2457 .

impregnated, method for restoring original appearance, 1407.

Kjeldhal method, determination of nitrogen, 332.

polyisobutylene, treatment, 607.

power factor, resistivity, dielectric constant, 533 .

prevention of microbiological deterioration, 1817.

sampling of, 3039 .

side upper, sampling, 535.

sole, with polymers, impregnation, 2594 .

studies on, by means of a sonic technique, 1905.

upper, significance of the results of some physical tests, 1485 .

water vapor permeability, 57 .

Leathers, ehrome-tanned, properties of split and unsplit, 601.

mulitary upper, 842.

Legendre, Gegenbauer functions, general, addition theorems, 1253.

polynomials, second order determinants, 305 .

ultraspherical polynomials, recurrent determinants, 298.

Legislative control of radiation, 1755 .

Lemma, Watson's modification, J 63B1, 15.

Length, changes, thermal, of refractory castables, RP2768. response of a loaded electric dipole in an imperfectly conducting cylinder, J 64D3, 289.

standard of and atomic beam sources, 2464 .

standards and light waves, 1387 .

standards, calibration at NBS, C572.

Lengths, short gage, improved photogrid techniques for determination, 465 .

Lens, automatic, design methods, 2034.

design: a new approach, J 63B1, 31 .

distortion by the inverse nodal slide, evaluation of, $\mathrm{J} 63 \mathrm{C} 2,105$.

distortion by the modified goniometric method, evaluation of, $\mathrm{J} 63 \mathrm{C} 2,113$.

distortion, evaluation of, by visual and photographic methods, RP2920.

effect of illuminance, interposed between the source and the illuminated surface, 858 .

Lenses, corrected ophthalmic, marginal performance of, RP2869.

photographic, spurious resolution, 315 .

photographic, testing, 1169.

sine-wave response, 2999.

spectacle, instrument for measuring the marginal power, 1369.

Leonard Euler's integral: a historieal profile of the gamma function, 2974 .

Lerch zeta functions, 1798 .

Les Tektites-Ces objets de verre tombes du ciel proviennent-ils d'une planete perdue? 1756.

Letter, outgoing, mail eharacteristics, some applications of statistical sampling methods, TN16 (PB151375).

Lettermail, incoming, at the Baltimore, Maryland City Post Office, distribution, TN33 (PB151392).

Liapunov's conditions for stability, implications, 1142.

Light, coated groundwood papers, effect, 1327.

color, a new point of view in the measurement, 1989. color, measurement, 2609; 2627.

emission from solid nitrogen during and after electron bombardment, 2975 .

emission, radio-astronomical test of the ballistic theory, 2878 . 
intensities, application of photoelectric multiplier tubes to the sensitive measurement of absorption or of changes, 27.

molecular constants of carbon monoxide, velocisty, 1574 .

new, on self-ignition, 266.

polarized, optical rotation, by chemical compounds, 285.

scattering and miscelle structure in the system sodium dodecyl sulfate-sodium chloride-water, 1386.

scattering by coinmercial sugar solutions, J $63 \mathrm{~A} 3,205$.

scattering measurements of a fractionated non-ionic detergent, 2176 .

sources, A, B, and C, spectral energy distribution, 834 .

vertical distribution of, from gas-filled candlepower standards, 2860.

waves and length standards, 1387.

Lighting, aircraft, NBS research, development and testing, 771 .

aviation, kinorama as an evaluator, 582 .

signal, $2562 ; 2952$.

Lightning discharges, VLF radiation spectra of, J $63 \mathrm{D} 2$, 199.

some properties of, impulses which produce whistlers, 2764.

tree from the viewpoint, 1248 .

Lights, condenser-discharge, photometer for measurement of the effective intensity, 2685 .

flashing, effective intensity, 2066;2101.

Lignolsulfonates in vegetable extract blends, cinchonine method for determination, 1979.

Lime-alumina-water, a study of the system at $1^{\circ} \mathrm{C}$, RP2877.

Limit of spatial resolution of refractometer cavities, J $64 \mathrm{D} 1,65$.

Limitations, photographic dosimetry of $\mathrm{X}$ - and gammarays, 2611 .

rapid signal analysis, 1388 .

Limiting polarization of magneto-ionic waves, 583.

resolution of electron spectrometers, 2612 .

thickness of an electrolyzed gas film capable of sustaining a given negative pressure, 865 .

Limits of the power resolution of corpuscular spectrometers, 2791.

Lindeck potentiometer, 1389.

Line, shape and $\mathrm{f}$ value in the $\mathrm{OH}^{2} \Sigma^{+}-{ }^{2} \pi$ transition, 2976 . standards of length and measuring tapes, calibration at NBS, Mono.15.

strengths for rotational transitions of asymmetric

rotor molecules, tables, 2796 .
Linear calibration curves, study of accuracy in chemical analysis, 2328 .

differential equations, 932.

elliptic differential equations by difference equations with positive coefficients, 785 .

entire functions of $n$ complex variables, 498 .

equalities, on systems, 1794 .

form, certain, minimum, J $64 \mathrm{~B} 1,49$.

functional equations and interpolation series, 1050.

functionals defined on classes of analytic functions, 1093.

functions of two variables, subharmonic and harmonic 280.

interpolation, rounding off of difference tables, 504. iteration procedures for symmetric matrices, 1097.

iteration processes, determinants with predominant principal diagonals and the absolute convergence, 1671 .

normed, spaces, 2065 .

Linear equations, determination of eigenvalues, AMS49. extension of Gauss' transformation for improving the condition of systems, 393 .

iterative solutions, A.MS49.

solvability and consistency, 1869 .

solving, 831 .

Linear inequalities, $1196 ; 1197$.

solvability and consistency, 1869 .

two consequences of the transposition theorem, 353 .

two existence theorems for systems, 608 .
Lincar plots, functional synthesis, J $64 \mathrm{C} 2,115$.

Linear problems, convergence of a method, 417. programs, couputational experience in solving, 672. spaces, full convex normed, 1350 .

transformation, 2918.

transforms, class of, Gibbs phenomenon, 500.

transforms or convex functions, 2115.

viscoclastic behavior, proposed nomenclature, 2271 .

viscoelasticity theory: delta-function approximations, 956.

Linear programming, 1757.

bid evaluation, 1051

large-scale, Chebyshev polynomials in the solution, 411.

problems, how to solve, 1360 .

proceedings of the Second Symposium, 1458.

relative damping criterion for, 633 .

systems, degree of, zeros of polynomials, 790 .

systems of distinct representatives, 1910 .

Lines of the chemical elements in astronomical spectra, 1758.

Liquefaction, helium, RP2834.

helium refrigeration, using a liquid hydrogen refrigerator for precooling, TN39 (PB151398)

hydrogen, by a dual pressure process, 2156 .

hydrogen, cycles, 2590 .

increased rate, of the National Bureau of Standards liquefier, 2157.

large scale, of hydrogen, some aspects, 1491

transportation of helium, $2855 \mathrm{~A}$.

transportation, technical aspects of large scale liquid helium, 2797.

Liquefied gas storage vessels, 3049.

Liquefied gases, 3063.

long distance transfer, 2177.

performance of pumps, 2243 .

single-phase transfer, C596.

with sharp-edged orifices, 2983.

Liquefier, helium, National Bureau of Standards increased rate of liquefaction, 2157 .

hydrogen, expansion engines for, $\mathrm{J} 64 \mathrm{C1}, 25$.

Liquid and solid state at low temperatures, TN4 (PB151363).

drops and rigid steel spheres, pits in metals caused by collision, $J 64 \mathrm{A1}, 61$.

flow boundaries, chemiluminescence as a tool in the study, 1646.

hydrogen, large, bubble chamber, 1984.

immiscibility volume in the system barium oxideboric oxide-silica, 2754 .

large scale, helium liquefaction and transportation, technical aspects, 2797.

level indicator for condensed gases at low temperatures, 1052 .

metals and liquid salts, specific heats, 2306.

nitrogen, photolysis of oxygen in solution, 3013 .

nonventing, helium dewar, temperature stratification, $\mathrm{J} 64 \mathrm{C} 1,19$.

normal hydrogen, 1173.

oxygen, survey of hazards of handling, 2333.

oxygen transfer lines, characteristics of some insulations, 2054.

para-hydrogen, iron catalyst, 1751 .

viscous, 1077.

Liquid helium, 1137.

based on the "cell" model, treatment, 1562.

below $1^{\circ} \mathrm{K}$, normal fluid concentration in, 774 .

cryostat with an integral super-conducting resonator, $2610 \mathrm{~A}$.

range $1.4^{\circ}-4.2^{\circ} \mathrm{K}$, measurements on the saturation vapor pressure, 1403.

second sound propagation in, 821 .

thermal Rayleigh disk measurements, 603.

Liquid hydrogen in the large CEL-NBS liquefier, new arrangement for orthopara conversion, 1988.

no-loss storage, mechanical refrigeration process, 1986.

refrigerator for precooling, TN39 (PB151398). 
temperature refrigeration system, design and construction, TN38 (PB151397).

Liquid scintillation, counters, carbon-14 beta-ray standard, benzoic acid-7- $\mathrm{C}^{14}$ in toluene, J 64A2, 143. counting, 2614.

Liquid scintillators, 2597; 2964.

gas-free, temperature effects, 1915.

oxygen quenching and wavelength shifters, 1439 .

quenching of, role of oxygen, 1858 .

three ways to increase efficiency, 1966.

Liquid-flowmeter calibration techniques, 2613.

Liquid-helium cold cell for use with an X-ray diffractometer, RP2907.

Liquid-hydrogen production, ortho-para catalysis in, RP2840.

Liquid-in-glass thermometers, calibration, C600.

Liquid-scintillator temperature effects, 1759 .

Liquids, constant-level device, 415.

high-temperature, 1032.

pure, and dilute solutions, dielectric dispersion data, tables, C589.

radioactive, 1649 .

simple, quantum mechanical effects on the surface tension, 2708.

sing-around ultrasonic velocimeter, 2301.

solids for, note on the displacement pressure method for measuring the affinity, 490 .

Liquidus surface, shape of, as a criterion of stable glass formation, RP2872.

Literature on spectrochemical analysis, index to, 1036.

statistical, selected bibliography, J $64 \mathrm{~B} 1,55$; J $64 \mathrm{~B} 1$, 69.

Lithium borohydride, tritium-labeled, preparation and use of, $J 63 \mathrm{~A} 2,177$.

fluoride: heat content from $0^{\circ}$ to $900^{\circ}$, melting point and heat of fusion, 1053

heat content from $0^{\circ}$ to $900^{\circ}$, triple point and heat of fusion, and thermodynamic properties of the solid and liquid, 1390.

Load failure of beams, floors, and roof constructions during fire tests, proposed criteria for defining, J $63 \mathrm{C} 2,121$.

Local standards laboratories, 2977.

Locating sources of variability in a process, 253.

Location of the auroral absorption zone, 1054 .

Logarithmic attenuator, 935 .

ohmmeter, 1619.

slidewire for a self-balancing potentiometer, 179 .

Logarithmic-normal distribution with known variances, 2030.

Logarithms, table of natural, for arguments between five and ten to sixteen decimal places, AMS53.

$\log e^{z} e^{y}$, formal power series, 1931.

Logical design of a one-microsecond parallel adder using one-megacycle circuitry, 1937

design, problems, 1818.

Long distance horizontal radiation pattern of a highfrequency antenna, 2371.

distance pulse-propagation experiment on 20.1 megacycles, 2423.

transfer of liquefied gases, 2177.

Long-range correlation model of the photonuclear effect, 2669 .

Longitudinal impact tests of long bars with a slingshot machine, 1760.

spherical aberration, optical T-bench method of measuring, RP2880.

Looking ahead in mechanics, 752 .

nucleus, 753 .

Loop, of a uniform current, having a spherical core, impedance characteristics, J 64D3, 295.

Loran communications, Bureau of Standards comments, 2039.

Lossy cable attenuator pads, 754 .

Lototsky method for summation, J 63B1, 1.

Low and medium frequency radio propagation, 2615.

electrical conduction in magnesium stannide, 1695.

even configurations in the first spectrum of ruthenium (Ru I), part 2, J 63A3, 225. frequencies, technique for sweep frequency polarization measurements, 938.

glass dewars for optical studies, 2575 .

high pressures, thermodynamic properties, TN8 (PB151367).

impact strength of some thermosetting plastics, 464 . notch depth, effects of, on the mechanical behavior of an annealed commercially pure titarium, RP2790.

pressure explosions, ultraviolet spectrophotometry, 1565 .

properties of materials, 2268.

properties of the $\mathrm{Cr}^{+++}$ion in the paramagnetic alums, 522 .

quartz crystal resonators and oscillators, 2734.

radio frequencies, 2044.

rates of equilibration in dialysis experiments with ionic surface-active agents, 2232.

reduction of area gage, 7 .

removal of nitrogen from hydrogen with silica gel, 2730 .

some mechanical properties of Mylar and Dacron polyester strands, 2761 .

stability of quartz resonators, 2315 .

stabilization of free radicals, Mono.12; 2316.

strength and fatigue of glass, 2318.

temperatures, TN4 (PB151363).

voltage regulator, 364 .

Low, very, frequency, ionospheric propagation, 2203.

frequency, noise, 2003.

temperature, an experimental study of the strength and fatigue of glass, 2014.

Low- and very low-radiofrequency tables of ground wave parameters for the spherical earth theory: the roots of Riccati's differential equation, TN7 (PB151366).

Low-angle X-ray diffraction of fibrous polyethylene, 2616.

Low-capacitance power supply, 1391.

Low-cost microvolt potentiometer, 2178.

Low-distortion transistor audio oscillator, 755 .

Low-energy ions in $\mathrm{N}_{2}$ and $\mathrm{O}_{2}, 982$.

Low-energy limit of the photonuclear pseudodeuteron effect, 2978.

Low-frequency band, some results of sweep-frequency investigation, 1146.

echoes, polarization measurements, 513.

ground wave, further studies of the influence of a ridge, $\mathrm{RP} 2884$.

ionosphere echoes, note on the polarization, 371 .

night-time, ionograms, interpretation, 2821.

portable antennas operating over ice and snow covered terrain, 2220.

propagation, ionospheric disturbances, 229.

propagation paths in arctic areas, J 63D1, 99.

radiation from a horizontal antenna over a spherical earth, 1761 .

standard transmissions, 2179.

very, standard frequency broadcast, 2270.

wing of the $\mathrm{NH}_{3}$ inversion spectrum, absorption, 639 .

Low-latitude aurora, a monochromatic, J 63D3, 297.

Low-pass duplexing system for high-frequency pulse transmitters, 1056 .

Low-temperature distillation of hydrogen isotopes, 2618; 2691.

frequency standard, 1589.

microcell for infrared study of condensed gases, 2619. physics, $1055 ; 2180$.

properties of plastic foams, 2620.

scales from $90^{\circ}$ to $5^{\circ} \mathrm{K}, 1392$.

thermal corductivity of free-machining copper, 1393.

thermal conductivity of some commercial coppers, 2181.

thermometry, resistance-temperature relation, 9 .

X-ray studies on Rice's blue material, 2979 .

Lower and upper bounds for the center of flexure, J 64B2, 105 .

LR-transformation, solution of eigenvalue problem with, AMS49. 
LS coupling, hyperfine structure formulas for, 733 .

L.T.E., departures of hydrogen from, 2927.

Lubricants tests, special, 311 .

turboprop, laboratory tests, 2610.

Lubrication of plain journal bearings, 82 .

oil-soaked felt-pad, of ball bearings at high speed and high temperature, 1422

Luminescence de L'azote solide $\left(4,2^{\circ} \mathbf{K}\right)$ contenant des atomes ou radicaux libres, 2182 .

Luminescence of solid nitrogen $\left(4.2^{\circ} \mathrm{K}\right)$ containing atoms or free radicals, 2182 .

containing atoms of free radicals. Effect of traces of oxygen, hydrogen and water vapor. 2622.

solids at low temperatures, 2934.

Luminous reaction between carbon monoxide and atomic nitrogen, 2183.

reaction between carbon monoxide and atomic oxygen, 866.

Luminous-transmittance requirements for railroad-signal glassware in terms of standard source A of the International Commission on Illumination, an evaluation of, RP2849.

Lunar and solar atmospheric tides in barometric pressure, 1586.

stratification of the F2 layer at Hyancayo, Peru, 83.

Lyanpunov's second method, survey, 2439.

Lysine deficiency, calcification, 2040.

\section{M}

Machine attacks on problems whose variables are permutations, 1762 .

automatic counting, 398 .

cyclic strain testing, for leather, 1304.

Machines, computing, 233 .

retrieving and correlating recorded information, 1763.

Macromolecular chemistry, 1394.

Macromolecules, fibrous, reversible contractile processes, 2743.

flexible, 1174 .

Macro-molecules to solid surfaces, sorption, 1500.

Magnesium, alloy specimens, effect of an anodic (HAE) coating on the fatigue strength, 1325 .

alloy specimens, fatigue strength, 1531 .

alloys and magnesium, 2108.

base alloys from alkaline chromate solutions, electrolytic, 1332.

calcium precipitations, removal of Manganese prior to, 814 .

oxide-germanium dioxide, 3094.

oxide, high-grade, drain casting of, 996.

oxide in cement, 925 .

oxide in portland cement, 2893.

stannide at low temperatures, electrical conduction, 1695.

Magnesium-strontium titanates, note on the dielectric properties, 2221.

Magnetic, attenuator, ultra-high frequency, $663 ; 902$

Compton electrons, gamma-ray measurements, 725 .

cooling, $1395 ; 2981$.

cooling and thermometry below $1^{\circ} \mathrm{K}$, new materials, 1783.

dipole, arc-lengths along the lines of force, 1633 .

drum directory and programming system for codesorting letter mail, TN50 (PB151409).

drum memory for the NBS Western automatic computer (SWAC), design features, 424.

electrostatic fields, 56 .

equator, $50 \mathrm{Mc}$ oblique transmission experiment, 2626. extractor, pulsed, 929 .

field control, nuclear-resonance, 491

field, earth's, on m.u.f. calculations, 3075 .

field from electromagnetic sources immersed in a semiinfinite conducting medium, basic experimental studies, J $64 \mathrm{D} 1,21$.

field, transverse, on the mode theory of very-low-frequency propagation, J 64D3, 265.

fields, note on the focusing of electron beams, 95 . high-frequency measurcments, instruments for, 1043.

high-frequency, permeability measurements using toroidal coils, 2584 .

interaction, electrons with, double seattering, 1320 . interaction of $\mathrm{H}_{3}, 3082$.

losses at low temperatures, 2980.

maps of the sun, study, 1600 .

measurements, amplifier fecdback, 2421

measurements, core losses in ferromagnetic material at high flux densities, RP2865.

measurements, stability of austenitic stainless stcels at low temperatures, 3093.

nuclear, resonance and infrared absorption studies in organic phosphorus chemistry, 2494.

nuclear, resonance of aligned radioactive nuclei, 778 nuclear, resonance in semiconductors, 3000 .

properties below $1^{\circ} \mathrm{K}, 756$.

properties of polyerystalline materials, $2980 \mathrm{~A}$.

properties of uranyl-like ions, 2355.

specimen mount for fractography, 2424.

susceptibilities of some U(IV) compounds, 1396.

susceptibility of manganese solenide, 254 .

susceptibility of systems with $f^{2}$ electron configuration, effect of crystal field and spin-orbit coupling, RP2893.

tape recorders, FM data reduction from, 722 .

thermal properties of chromic methylamine alum below $1^{\circ} \mathrm{K}, 1057$.

threshold field curve of superconductor, 584 .

Magnetized sphere, 575 .

Magnetoelastic properties of ferrites, TN49 (PB151408).

Magneto-ionic waves, limiting polarization, 583 .

Magnetohydrodynamic shock wave in a plasma of infinite conductivity, structure, 1902

Magnets, Cunife wires, RP2808.

Magnetometer, electron beam, J 63C1, 69.

Magnification, variation in distortion, 1572.

Magnitoye okblazhdenye, 2981.

Magnitude and direction of direct currents, commutator for periodically changing, 1652 .

Mail, distribution of, by destination at the San Francisco, Los Angeles, and Baltimore post offices, TN27 (PB151386).

letter, magnetic drum directory and programming system for codesorting, TN50 (PB151409).

sorting, by conventional methods, TN54 (PB161555).

sorting device, capacity requirement, RP2948; J 63B2, 79.

sorting equipment, analytic comparison of suggested configurations, J $63 \mathrm{~B} 2,83$.

to given destinations, statistical chain-ratio method for estimating relative volumes, $\mathrm{J} 64 \mathrm{C} 1,37$.

Maintenance and preparation of standards of radioactivity, 1944.

electronic equipment, TN41 (PB151400).

preparation, and application of standards of radioactivity, C594.

Making one measurement do the work of two, 757 .

Malic acid, dissociation constant, RP2947.

Maltose-1-C ${ }^{14}$, maltobionodeltalactone-1-C ${ }^{14}$, and lithium maltobionate-1-C ${ }^{14}$ from 3-( $\alpha$-D-glucopyranosyl)D-a rabinose, synthesis, 1909 .

Man-machine systems, analog-digital simulation for the design and improvement, 2453.

Managanese-52, polarized, beta-gamma correlation, 2473.

Management, control, electronic computers aiding, 441

future, information systems, automation, 2467 .

Manganese, flame photometric determination, in cement, 1930.

removal of, prior to calcium and magnesium percipitations, 814

solenide, magnetic susceptibility, 254.

thermal neutron activation cross section, 1397.

Manganous, chloride tetrahydrate at very low temperatures, spin-spin absorption in chronic methylammonium alum, 1887.

chloride tetrahydrate, spin-spin absorption, 1888.

ferrous carbonates, thermal decomposition, 1960. 
Manila rope, Becker value, by photoelectric reflectometry, 960.

D-mannitol-2-C ${ }^{14}$, isotope effect in oxidation, by acetabacter suboxydans, 2606.

Manometer, 212.

U-typ , 211.

Manometers and mercury barometers, Mono.8.

Manufacture of paper from glass fibers, 477 .

Map, laminated paper improved dimensional stability, 2595 .

papers, experimental, containing synthetic fibers, 2703.

Mapping, stroboscopic, of time-variable fields, 140.

Maps, magnetic of the sun, study, 1600.

world, of $f 2$ critical frequencies and maximum usable frequency factors, TN2, (PB151361).

Marginal performance of corrected ophthalmic lenses, RP2869.

Marine, exposure on some aluminum alloys, effect of 20 year, 1689.

radio communications, propagation characteristics of the frequency band 152-162 MC, 1945.

Marking methods and recording surfaces, recorder survey, C601.

Mars, high-dispersion spectra, 2152.

Martensite transformations in SAE 1050 steel, 1186.

Masonry, building code requirements for, 945 .

cracking and damage caused by moisture expansion of clay tile, 1058 .

cracking in, caused by expansion of mortar, 419 . requirements up to date, 1059 .

Mass, measurements on the faraday, effect, 573. spectrometric study of the decomposition of hydrazoic acid by the electric discharge, 2623.

spectrometric study of the rate of thermal decomposition of hydrazoic acid, RP2881.

spectrometric study of the reactions of some hydrocarbons with active nitrogen, 2982.

spectrum of gallium vapor, 738 .

Mass spectra, aromatic hydrocarbons filtered from smoky air, RP2876.

condensates from urban atmospheres, interpretation of, RP2809.

polyatomic molecules, 1144 .

pyrolysis: phosphinoborine compounds, J 63A1, 63 .

relative sensitivities of some polyphenyls, RP2831.

some deuterostyrenes, RP2926.

thermal decomposition, dimethylphosphinoborine trimer, 994.

Mass spectrometer, 194.

analysis of fluorinated polyphenyls, 1263.

combination crossed-field and time-of-flight, 360 .

radio frequency, 117 .

Mass spectrometry, $478 ; 1060 ; 1764 ; 2624$.

absorption, polymer degradation studies, 515 .

analytical, 1626 .

study of degradation of polystyrene, 557 .

Mastoid and forehead, mechanical impedance, 1404.

Matching loads on a magic tee, $83 \mathrm{~A}$.

Material transport during sintering, mechanisms, 1538.

Materials, 255.

aircraft sheet, for large strains, Poisson's ratio, 798. bituminous materials for spinning, preparation, 800 . construction: their properties and methods of test, 2625 .

dense, atomic theory of electromagnetic interactions, 1635 .

flooring, 479 .

low temperatures, properties, 2268.

refractory, permeability and some other properties of a variety of, 794 .

research on effects of prestraining and notch sharpness on the notch strength, 1853.

self-ignition temperatures of, from kinetic-reaction data, RP2909.

small, oil-free bearings, 2184.

standard, issued by the NBS, C552, 3d ed. structural, at low temperatures, mechanical properties, Mono.13.

synthetic, in America in 1959, developments, 21.

Mathematical foundations and computational methods for a digital logic machine, 1061

induction, two forms, 2855.

services useful in industry, 759 .

statistics and probability, proceedings of the Berkeley symposium, review, 114 .

theory of probability, elements, 708 .

Mathematical tables, addendum to a guide to tables on punched cards, 641 .

characteristic values of Mathieu's equation for large values of the parameter, 1523 .

Coulomb wave functions expressed in terms of Bessel-Clifford functions and Bessel functions, 214.

Coulomb wave functions in the transition region, 980 .

evaluation of Coulomb wave functions along the transition line, 1018.

exponential integral for complex arguments, AMS51.

generation of Bessel functions on high speed computers, 2140.

generation of Coulomb wave functions by means of recurrence relations, 1532 .

guide to tables on punched cards, $177 ; 641$.

integrals of airy functions, AMS52.

integrals of Struve functions, 146.

natural logarithms for arguments between five and ten to sixteen decimal places, AMS53.

osculatory inter-polation coefficients, AMS56.

radix table for trigonometric functions and their inverses to high accuracy, 296.

relating to Hankel integrals of order zero, 147.

table of the integral, $\int_{0}^{z} e^{-\mu^{3}} \mathrm{du}, 320$.

tablemaking for large arguments, 272.

Mathematics for design, 355.

determinantal inequality of H. P. Robertson, 1980 note on values of a quadratic form, 2186.

physical, 1445A.

Mathieu's differential equation and the spheroidal wave equation, programming for finding the characteristic values, 518 .

equation for large values of the parameter, 1523 .

Matric, plastic phosphor, for fast-neutron detection, 2093. Matrices, AMS $49 ; 2876 ; 2886$.

Matrices, bounds of a one-parametric family, 2663.

$k$-commutative, space of, J 64B1, 51 .

commutators of, some remarks, 1145.

condition of, RP2815; 1175 .

doubly stochastic, subdeterminants, 2227.

finite commutativity, 2060 .

group, 1237.

Hermitian, indefinite, 2303.

Hermitian, inequalities concerning positive-definite, 1496.

normal, comparison theorem for eigenvalues, 1231 .

normal, eigenvalues of sums, 1425 .

normal, pairs of, with property L, 792 .

one parametric family, spectrum, 1100.

pairs of, order two which generate free groups, 2242 .

pairs of, with property L, 507; 791; 1440 .

partitioned, applications of a theorem on, J 63B2, 73.

permutations of factors in a product of three matrices, generalized commutations of, 1023.

quadratic fields, $205 ; 412$.

quaternion, 967 .

random notes on, RP2850.

random payoff, games, 2137 .

random walks, inversion, 282.

second-order, tables for diagonalizing, RP2838.

space of, metric inequalities, 1497.

symmetric, convergence of Seidel iterants, 329 .

symmetric, determination of eigenvectors and eigenvalues, 330 .

symmetric, linear iteration procedures, 1097. 
theorem on alternatives for pairs, 1246 .

transformations, commuting bilinear, 1654 .

unimodular, of order two which commute, 1969.

with non-negative elements, 2850.

Matrix, classes corresponding to an ideal and its inverse, 2222 .

condition, 149 .

doubly stochastic, 3008 .

eigenvalues of, circular disks containing, note, 2650.

Hilbert, 2229.

inversion by a Monte Carlo method, 84

inversion programs, 2814

minimization problem, 1985 .

new, transformations for obtaining characteristic vectors, 267.

normal, spectrum of, variation, 890 .

positive, bounds for the greatest latent root, 405 .

positive definite, 284 .

principal submatrices of a full-rowed non-negative, J $63 \mathrm{~B} 1,19$.

symmetric, 192 .

theory, basic theorems, AMS57.

Maximum, permissible body burdens and maximum permissible concentrations of radionuclides in air and in water for occupational exposure, H69.

permissible radiation exposures to man, 2187.

usable frequencies and lowest usable frequencies for the path Washington to Resolute Bay, 1062.

Maxwell triangle, invariance of insidedness in projective transformations of, 1046 .

Mc, 50 , oblique transmission experiment near the magnetic equator, 2626.

Mean absolute value and standard deviation of the phase of a constant vector plus a Rayleigh-distributed vector, RP2950.

deviation of the Poisson distribution, 2823.

electron density variations of the quiet ionosphere, TN40-1 (PB151399-1); TN40-2 (PB151499-2).

Meaning and standardization of $\mathrm{pH}$ measurements, 1767.

Measured frequency spectra of very-low-frequency atmospherics, J 64D1, 41.

statistical characteristics and narrow-band teletype message errors on a single-sideband 600-milelong ultrahigh-frequency tropospheric radio line, J $64 \mathrm{D} 2,125$.

statistical characteristics of VLF atmospheric radio noise, 2188.

values, reliability. Part I. Fundamental concepts, 594

Measurement, absolute, 780 .

absolute capacitance, 2647.

absolute, of $W_{\text {air }}$ for polonium-210 alpha particles, 2444.

accurate, of antenna gain, techniques, C598.

aging of rubber vulcanizates, $\mathrm{J} 63 \mathrm{C} 2,141$.

architectural acoustics, standard methods, 2226 .

atmospheric noise, 2189.

burning velocities, 1064

ceramic materials, specular-gloss, 1886.

characteristies, downcoming radio waves, 2932 .

conductivity of a fluid contained in a cylindrical vessel, 2233.

control, integration of concepts in the terminology, 1935 .

definition of the time constant and response time of thermal converters, 2809 .

cobalt- 60 and cesium- 137 gamma rays with a freeair chamber, J $64 \mathrm{C} 2,87$.

color, gloss, and haze, 760 .

color, in industry, 2197.

continuous, of atmospheric ozone by an automatic photoelectric method, 977

control, frequency multipliers and converters, 1349.

correlation coefficients in reverberant sound fields, 1399.

creep of acrylic glazing plastics exposed to temperature gradients, 1065.

current with the NBS current balance, RP2846. direct, of absorption line half-widths and intensitics, slit funetion effects, 1867 .

distortion produced in guarded elcetrostatic fields by grounded external plates, 1066

dynamic properties of rubber, 480 .

effect of irregular terrain on VIIF and UII F directive antenua patterns, 481 .

effect of two-dimensional and three-dimensional roughness elements on boundary-layer transition, 1400 .

effective intensity of condenser-discharge lights, photometer, 2685.

electromotive force, 141

50 -kev electrons, bremsstrahlung cross-section, 2477.

flame speeds by a nozzle burner method. RP2867.

flow of liquefied gases with sharp-edged orifices, 2983 .

gamma-ray dose near the interface between two media, 2628.

gas temperatures by immersion-type instruments, 867 . ground conductivity at VLF, 2670.

high temperature in gas streams, 85 .

infrared, past and present, 1367 .

ionizing radiations for biological purposes, 1189 .

ionospheric adsorption, 3091 .

isotopic abundance of solids, 180 .

light and color, $1989 ; 2609 ; 2627$.

low energy X-rays, standard, J 64C1, 49.

magnetoelastic properties of ferrites, TN49 (PB151408).

making one do the work of two, 757 .

microwave, of variations in atmospheric refractive index, 585 .

microwave standing-wave ratios, new technique, 1594.

neutron flux, and neutron dosimetry, 3055 .

normal spectral emissivity in the infrared, apparatus for, J $64 \mathrm{C} 2,99$

photodetachment cross section for $\mathrm{H}^{-}, 3035$.

physical, and medicine, 795 .

precise and race for technological supremacy, 2696.

precise, of heat of combustion with a bomb calorimeter, Mono. 7.

precise, of the infrared spectra of molecules of the atmosphere, 2377.

precision, 2261 .

protective coating adhesion, using an electronic averaging device for the adherometer, 2273.

radiated harmonics, source of error, 377.

radio, of winds aloft, 524 .

RF power, 2419.

resistance, of ceramic-type strain-gage cements, 2739 .

resistance-strain relation and Poisson's ratio for copper wires, 1768 .

service area for television broadcasting, 2190.

sine-wave response of lenses, 2999.

standards of, classification and nomenclature, $2486 \mathrm{~A}$

surface roughness of electrodeposited and electropolished surfaces by means of the microinterferometer, 86 .

systems for high-level dosimetry, 2984 .

systems, power spectra and the minimum detectable signal, 1430.

technological progress, scientific, 537 .

temperature, and incomplete equilibrium, 1735.

theoretical study of electrical conductivity and Hall effect in oxide cathodes, 1063.

thickness, C585.

treadwear of commercial tires, 761 .

vibration amplitudes, acoustic method, 946.

vibration, developments, 1492.

vibration, stroboscopic interferometer, 2784 .

voltage ratio at audio frequencies, 2191.

$\mathrm{W}_{\text {nir }}$ for sulfur-35 beta-rays, $1769 ; 2008$.

Measurements, absolute, at the NBS, status, 2781.

balloon to an altitude of $100,000 \mathrm{ft}$., near infrared solar radiation, 2645 .

bremsstrahlung polarization, 1643 .

chromium potassium alum below $i^{\circ} \mathrm{K}, 1067$.

coastal deviation of high-frequency radio waves, $J$ $64 \mathrm{D} 1,57$. 
comparative $100 \mathrm{Mc}$, at distances far beyond the radio horizon, 972 .

correlation, height gain, and path antenna gain at 1046 $\mathrm{Mc}$ on spaced antennas far beyond the radio horizon, 1401.

cross-section for 0.5 - and 1.0 -Mev electrons, bremsstrahlung differential, 1278.

current and voltage, AC-DC transfer instruments, 2446.

dielectric, from 100 to $400 \mathrm{Mc}$, variable length reentrant cavity, 638.

dielectric, up to $500^{\circ} \mathrm{C}, 992$.

Doppler shift, for studying ionospheric fine structure using earth satellites, comparison of phase difference, 2493.

dose, in water using scintillation and ionization detectors, 995.

earth satellites, continuous phase difference, 2499.

electrical, $1008 ; 2935$.

electrical, geometric factors, relating to corrosion and its prevention, 1354 .

electrical, standards, 316 .

electromotive force, 218 .

electron scattering, a utomatic instrument, 1274.

factors affecting the accuracy of distortion, made on the nodal slide optical bench, 2948.

ferrite, gyromagnetic sample, exact solution, 2552.

flow, review of some methods, 1131.

fractionated nonionic detergent, light scattering, 2176.

gamma-ray, by the magnetic analysis of Compton electrons, 725 .

gloss-past, present, and future, 2143.

heat transfer on refrigerated-food trailers, 2580.

impedance coaxial slotted-lime, 234 .

impedance, determination of attenuation, 45 .

indentation hardness, time-temperature behavior of rubber, 2848.

infrared with a cesium iodide prism, $742 ; 1072 ; 1351$.

instruments for magnetic high frequency, 1043.

ion production, 193 .

ionospheric drift in the United States, results, 2288 .

ionosphere over a $1,150 \mathrm{~km}$ path, 561 .

low-frequency echoes, polarization, 513 .

made at 418 megacycles per second well beyond the radio horizon, $\mathrm{J} 64 \mathrm{D} 3,255$.

magnetic, feedback amplifier, 2421.

magnetic, stability of austenitic stainless steels at low temperatures, 3093.

mass, on the faraday, effect, 573 .

methods of, recently developed microwave impedance standards, 2722

mierowave attenuation, with accuracies from 0.0001 to 0.06 decibel over a range of 0.01 to 50 decibels, J $64 \mathrm{C} 2,139$.

microwave, of the dielectric properties of gases, 259.

microwave, of the properties of a de hydrogen discharge, 2200.

microwave power, employing electron beam techniques, 2201.

microwave power, field of, 2720 .

mismatch errors in microwave power, 769 .

near the radio horizon, tropospheric scatter propagation, 1499.

noise, of composition resistors, 1417

oblique-incidence, of the heights at which ionospheric scattering of the VHF radio waves occurs, 1790.

1-Mev bremsstrahlung, 993.

optical, 2790.

over a $2400-\mathrm{KM}$ path, sweep-frequency pulse-transmission, 1520 .

permeability in the VHF region, eavity techniques, 1283.

permeability, using toroidal coils, high-frequency magnetic, 2854 .

$\mathrm{pH}$, meaning and standarization, 1767 .

phase of radio waves received over transmission paths with electrical lengths varying as a result of atmospheric turbulence, 1402. phase of signals received over transmission paths with electrical lengths as a result of atmospheric turbulence, 1770 .

phase stability over a low-level tropospheric path, $\mathrm{J} 63 \mathrm{D} 1,45$.

physies, chemical, and engineering, how statistics improve, 68 .

pile oscillator, of eta, 3015.

plane and circular polarized waves in the Great Lakes area, VHF tropospheric recording, 620 .

propagation, made at $418 \mathrm{Mc}$ well beyond the radio horizon, analysis of, TN6 (PB151365).

pulse-height, of recoils from $\mathrm{B}^{10}(n, \alpha) \mathrm{Li}^{7}, 1123$.

radiation protection, brief history of the National Committee, 2478.

radio, 263.

reflectance, with the Beckman quartz spectrophotometer, minimizing anomalies, 484 .

refractive index, quantitative determination of natural rubber, 292.

report on comparative $100 \mathrm{Me}$, for three transmitting antenna heights, 1848 .

retarding potential, electrical differentiation, 2596.

rod samples, cavity resonator dielectric, 2907.

saturation vapor pressure of liquid helium in the range $1.4^{\circ}-4.2^{\circ} \mathrm{K}, 1403$.

sets of three, 825 .

sferics, ionsopheric reflection coefficients at VLF, 2172.

small-current, automatic timer, 2036.

sound absorbers for jet-engine test cells, 1771 .

standards for electronics, 2776.

standards for steep front surge, sphere-gap volt-time curves, 2314

standards of frequencies and time intervals in the U.S.A. from 1954 to $1957,2550$.

surface tension, with a strain-gauge-type testing maehine, 2794 .

temperature, in flames and hot gases, experimental, 1340.

temperature, in high-velocity streams of hot gas, 1163. temperature, in the Mooney viscometer, 1164.

thermal Rayleigh disk, in $\mathrm{He}^{3}-\mathrm{He}^{4}$ mixtures, 895 .

thermal Rayleigh disk, in liquid helium II, 603.

thin-target bremsstrahlung, 1676.

uncertainty, 1953.

units, radiological, International Commission, 2366; 3022 .

velocity, below $1^{\circ} \mathrm{K}, 822$.

very high frequency propagation, in the Rocky Mountain region, 1972 .

viscometric and turbidimetric, on a dilute aqueous solution of a nonionic detergent, 1225 .

Measures, vector, relations among certain ranges, 299.

Measures and weights, administration in the U. S, 1976. administration, NBS and state, 485 .

approval scale, 1580 .

federal basis for, C593.

officials and manufacturers, genuine cooperation, 458 .

training program, 182.

Measuring abrasion resistance of organic coatings, 1249.

affinity of liquids for solids, 490 .

attenuation of balanced transmission lines, 376 .

cireuit for radiometers, 1068.

directivity of directional couplers, method, 2425.

energy dissipation, 1083.

equipment, development, 686 .

high frequency induced electroluminescence, crystal mount and technique, 2074.

marginal power of spectacle lenses, instrument for, 1369 .

mucosal surface contours of impressions, easts, and dentures, method, 1987.

power factor of low-loss dielectries, 256.

stiffness of paper, 762 .

surface flammability of materials using a radiant energy source, method, 1593.

system, basis of, 3066 . 
system for three-centimeter radio waves, single path phase, 2758 .

systems, a source of error in radio phase, 10 .

systems, radio phase, 186.

tapes, calibration of line standards at NBS, C572.

tersile and tear properties of plastic films, comparison of methods, 1290.

thermal emittance at high temperatures, $3049 \mathrm{~A}$.

time and frequency in Hawaii, 257.

with the electron, 2192 .

Mechanical, failures of metals in service, 2629.

impedance of the forehead and mastoid, 1404.

properties of direct filling resins, 1069.

properties of high polymers, 2630.

properties of insulating plastic foams at low temperatures, 2985.

properties of Mylar and Dacron ployester strands at low temperatures, 2761.

properties of refractory semi-dry press specimens, 2501.

properties of some engineering materials, 2193.

properties of structural materials at low temperatures, Mono.13.

quadratures, problems in the theory, 1423.

quantum, calculation of the probability of an exchange reaction for constrained linear encounters, 3025 .

quantum, effects on the surface tension of simple liquids, 2708.

refrigeration process for the no-loss storage of liquid hydrogen, 1986.

resolution of German syntax patterns, proposals, 290.

shear degradation, temperature dependence, 1527.

stops for use with helical potentiometers, 1070 .

testing of solid materials, 482 .

tests on specimens from large aluminum-alloy forgings, 1772 .

theory of transport processes, statistical, 2778.

Mechanically differentiating curves, 24 .

Mechanics, looking ahead, 752.

quantum, by density matrix and operator techniques, description of states, 2081.

quantum, polarization, 936 .

statistical, quantum, 2340.

Mechanism, alkali-aggregate reactions, 1406.

autoignition in benzene-air mixtures, 1773.

contraction in the muscle fibre - ATP system, 2986.

formation of $\mathrm{OH}, \mathrm{CH}$, and $\mathrm{HCO}$ in flame spectra, using deuterium as tracer, 868 .

high energy radiation effects in polymers, 2194.

hydroscopic expansion of dental casting investments, 1405.

stress-corrosion cracking in the AZ31B magnesium alloy, RP2919.

transition at subsonic speeds, 2195.

Mechanisms, aerodynamical, producing electronic density fluctuations in turbulent ionized layers, 1254

control, 2500.

material transport during sintering, 1538.

polymer formation and decomposition, 2631.

Mechanistic aspects of hearing, 2987.

Mechanized computation of thermodynamic tables at the National Bureau of Standards: The calculation of the equilibrium composition and thermodynamic properties of dissociation and ionized gaseous systems, $2632 ; 2988$.

production, components, 1291 .

Media, time-varying, propagation through, information theory aspects, 1364 .

Median hourly values of the cosine of the solar zenith angle $(X)$ for thirty-five locations, tables of, TN5 (PB151364).

Medicine, and physical measurement, 795 .

Medida del color en la industria, 2197.

Megacycle, eleven, interferometer for low pressure gases, 648.
418 per second well beyond the radio horizon, J $64 \mathrm{D} 3$, 255.

$20.1,2423$.

Melamine resin on chemical tests of paper, effect of, 227 .

Melting behavior of polybutadienes, 1938.

copolymers, 1774 .

erystalline polymers, 3083 .

transition of polymethylene, 869 .

Melting-point and phase-equilibrium determinations, 1775.

Membranes, collodion, sorption of dextran to, 832 .

Men and electrons, 2989.

Mercury barometers and manometers, Mono, 8 .

198, impure, spectra, 60.

isotope effeet, 81 .

pulser, ligh-repetition-rate, 2155.

purification of, and its physical properties, 2275 .

Mercury-, air-, and flat-type cells, dry cell standards broadened-now includes, 1321 .

Meson, neutral, decay, angular distribution, 2461

Mesons, neutral, from carbon near threshold, photoproduction, 2250 .

Message error in diversity frequency-shift reception, 1071

Metal, fatigue, effect of oleophobic films, J $64 \mathrm{C} 2,147$.

materials, ceramic, 1266.

oxides with $o$-ethyoxybenzoic acid and other chelating agents, 2829.

plates, rectangular, investigation of slot radiators, 1622 .

plates, rectangular, radiation patterns and conductances of slots cut on, 1947.

progress, cleaning and finishing, 1459 .

vapors, inelastic scattering of 20 -kev, 2158 .

Metal-clad laminates, characteristics, 2053.

Metal-halide lamps, electrodeless, 705.

Metal-rock salt pairs, epitaxy, 367 .

Metallic, iron of high purity, preparation of, 801

porcelain enameling, radioisotope study, 1941.

surfaces at $76^{\circ} \mathrm{K}, 2110$.

Metals contained in platiniferous materials, chemical methods for separating and determining, 668 .

electrodeposited, $511 ; 2936$.

electrodeposition of, from organic solutions, 1696; 1697.

energy losses, electron characteristic, 1699.

less common, from nonaqeous media, electrodeposition, 1331.

liquid and liquid salts, specific heats, 2306.

mechanical failures in service, 2629 .

nickel and its alloys, C592.

pits in, by collision with liquid drops and soft metal spheres, RP2958.

pits in, caused by collision with liquid drops and rigid steel spheres, J $64 \mathrm{~A} 1,61$.

powdered, alloys of gallium with, as possible replacements for dental amalgam, 1613.

ultrasonic vibrations in, transmission, 2398.

Meteor activity with occurrence of sporadic-E reflections, 2.

burst for intermittent radio communication, 2389 .

bursts, communication, 2491 .

bursts, experimental equipment for communication utilizing, 2117.

trail, nature of the decay, 501 .

trails, radar waves from, interpretation, 338 .

trails, wave-frequency dependence of the duration of radar-type echoes, 348.

trains, study of radio reflections from, in research on the upper atmosphere, 144.

Meteor-burst communication, transmission error function, 2674.

Meteor-train cchoes followed by ground scatter, observations of distance, 1421 .

Meteoric glass, Tektites, 1912A.

Meter, bar-magnetic velocity, 951.

gloss, comparisons, 1724 .

match, VHF, 15.

Meter plots drift, frequency-deviation, 455 .

portable infiltration, design and performance, 2082.

timing the flow of very small volumes of a gas, 365 . 
Meterological effects on radio propagation at $96.3 \mathrm{Mc}$ between Richmond, Va., and Washington, D.C., study, 635 .

effects on scattered radio waves, 1874 .

instrumentation at the NBS, 1073.

observations in radio wave propagation, 2331.

theory of wavelength dependence in ultra-high frequency transhorizon propagation, J 64D3, 231.

Meters electrical, and instruments, 1814.

field-strength, commercial, calibration, 407.

VFH field-intensity meters, 739.

water-current, rating, 340 .

wholesale, testing, 565 .

Methacrylate, methyl, accelerating effect of aminos on polymerization, 1608 .

methyl, sulfinic acid derivatives as accelerators in the polymerization, 1906 .

polymethyl, 838; $999 ; 1328$.

polymethyl, and polystyrene, gamma irradiation, 2138.

polymethyl, at various temperatures, stress and strain at onset of crazing, $555 ; 669$.

polymethyl, determination of the monomer content, 1673.

polymethyl, dilute solution thermodynamic behavior of flexible chain molecules, 687.

polymethyl, fracture phenomena and molecular weight, 2569.

Methane from 2,470 to $3,200 \mathrm{~cm}^{-1}$, infrared absorption spectrum, J $64 \mathrm{~A} 3,201$.

infrared emission spectrum, at 3.3 microns, RP2803.

$\nu_{3}$ band, 2206.

Methane-air flames, rotation temperatures of $\mathrm{OH}, 304$.

Methanes, deuterated, some vibrational-rotational bands, $\mathrm{J} 63 \mathrm{~A} 2,145$.

Method for determination of converging factors, applied to the asymptotic expansions for the parabolic cylinder functions, 366 .

determining rate equation for reactions in which the concentration of the reactant is unknown, 1592 .

dynamic determination of the elastic, dielectric, and piezoelectric constants of quartz, Mono.9.

measurement of the isotopic abundance of solids, 180 .

measuring local electron density from an artificial satellite, J $63 \mathrm{D} 3,325$.

measuring surface flammability of materials using a radiant energy source, 1593.

measuring the directivity of directional couplers, 2425.

measuring the mucosal surface contours of impressions, casts, and dentures, 1987.

restoring original appearance of impregnated leather, 1407 .

study of the circulation in the dog using a mechanical left ventricle, 920 .

Method of controlled slack quenching of impact bars, 2426.

preparing uniform films of bituminous materials, 628 .

speeding up iterations with superlinear convergence, 2427 .

Methods, evaluating trends in time series of tropospheric radio field strength data, 546 .

experimental physics, 2990.

measuring the " $Q$ " of large reactors, 1776.

Methods reducing heat leak through support members in liquefied gas storage vessels, 3049 .

Methyl, azide and hydrazoic acid by electron impact, ionization and dissociation, 2604.

germane, microwave spectrum, 2992.

methacrylate, accelerating effect of aminos on polymerization, 1608 .

methacrylate, sulfinic acid derivatives as accelerators in the polymerization, 1906.

siloxanes by electron impact, 747 .

Methylamine, alum, ehromic, 1057.

microwave spectrum of, 1001 .

molecule, structure, $2322 ; 2323$.
Methylammonium alum, chromic, as a thermometric substance, absolute temperature below $1^{\circ} \mathrm{K}$, 2009.

alum, chromic, spin-spin relaxation at very low temperatures, 1889 .

chromic, alum and manganous chloride tetrahydrate at very low temperatures, spin-spin absorption, 1887.

Methyltrifluoromethyl acetylene, internal rotation, 1374.

Metodi probabilistici per la soluzione numerica di alcur.i problemi di analisi, 763 .

Metric, differential geometry, 87 .

inequalities in the space of matrices, 1497.

methods in integral and differential geometry, 764 .

Metrology and production, 1777.

$\mathrm{Mg}_{2}-\mathrm{Sn}$, properties, 1460 .

Micelle, critical, concentrations by a bubble pressure method, 787 .

Microammeter, clamp-on, measures a-c current, 2056. clamp-type alternating-current, 2347.

Microbiological deterioration of leather, prevention, 1817.

fractionation of the hydrogen isotopes, 2635.

Microcalorimeter, a refined X-band microwave, $\mathrm{J} 63 \mathrm{C} 1$, 77.

microwave, 1236.

Microcell, infrared study of condensed gases, temperature, 2800 .

low-temperature, for infrared study of condensed gases, 2619.

Microchemical analysis, multipurpose standard, 2641,

Microdetermination and identification of nickel, 70 .

Microinterferometer, measurement of surface roughness of electrodeposited and electropolished surfaces, 86.

Micromethylation of polysaccharides, 2198.

Microns, 15 to 40 , infrared absorption spectra of twelve substituted benzene derivatives, 75 .

2 to 42,1038 .

Microphone, condenser, as a displacement detector calibrator, 2348.

wireless, uses F-M modulation, 2869.

Microphones, condenser, 1114.

standard, laboratory, 1048.

Micropotentiometers, RF, 806.

calibration of signal generators to $1000 \mathrm{Mc}, \mathrm{TN}-37$ (PB151396).

Microscopic tin filaments, superconductivity, 2329.

Microscopy, color phase contrast: requirements and applications, 37 .

electron, of fibers, $4 ; 1011 ; 1703$.

electron, of soil clays and related materials, 439 .

Microstructure, dental amalgam, 765 .

human tooth. A. The dentioenamel junction, 2199.

Microvolt, low-cost, potentiometer, 2178 .

Microvoltages, accurate radio-frequency, 640 .

Microwave absorption, 1162.

nonpolar gas $\mathrm{CO}_{2}, 1074$.

compressed gases; saturated hydrocarbons, 1778 .

compressed oxygen, 1408.

spectrum of $\mathrm{ND}_{3}, 766$.

Microwave, antenna, pattern of a flush-mounted, RP2796. attenuation measurements with accuracies from 0.0001 to 0.06 decibel over a range of 0.01 to 50 decibels, J $64 \mathrm{C} 2,139$.

attenuation statistics estimated from rainfall and water vapor statistics, 88 .

compact, refractometer for use in small aircraft, 2415 . components: precision casting vs. electroforming, 258.

crystal diodes used as rectifiers and harmonic generators, excess noise, 2114.

double-sweep method for analysis of time-dependent cavity characteristies, 2636 .

Fabry-Perot interferometer, reflectors for, 3034 .

frequencies, atmosphere, 240 .

frequency dividers, 89.

harmonic generator, 1662. 
impedance standards and methods of measurement, 2722 .

interferometry, J 64B1, 1.

measurement of variations in atmospheric refractive index, 585 .

measurements of the dielectric properties of gases, 259

measurements of the properties of a de hydrogen discharge, 2200.

microcalorimeter, 1236 .

microcalorimeter, a refined $\mathrm{X}$-band, $\mathrm{J} 63 \mathrm{C} 1,77$.

mixers, modulator, 2428.

oxygen, absorption as a possible atomic frequency standard, experimental evaluation, 2555.

power measurements employing electron beam techniques, 2201.

power measurements mismatch errors in, 769 .

power measurements, rceent developments, 2720 .

power stabilizer, 90 .

refractometer, recording, $6 ; 122$.

region, dispersion in $\mathrm{NH}_{3}, 48$.

signal source, amplitude stabilization, 2452 .

signals incident upon different regions of a de hydrogen glow, 2098.

spectra of deutero-ammonias, 260.

spectra of molecules exhibiting internal rotation, $2202 ; 2637 ; 2638 ; 2991$.

spectroscopy, broad-band coaxial stark cell, 178; 358.

spectrum and structure of sulfurylfluoride, 2372 .

spectrum of benzonitrils, 1075 .

spectrum of $\mathrm{CD}_{3} \mathrm{ND}_{2}, 2322$.

spectrum of methyl germane, 2992.

spectrum of methylamine, 1001.

spectrum of perchlorylfluoride, search for, 1863 .

standing-wave ratios, new technique for measurement, 1594.

VHF matching termination, 637.

Microwaves, conductivity of plasmas, 2914.

noise comparator, 773 .

suppressing, by zonal screens, 317 .

Military field X-ray equipment, 483.

Milk containers, paper, 1441.

farm, tank testing, $1344 ; 1711$.

farm, tanks, gaging and testing, 2134.

Millimeter, wavelength dispersion of water vapor, 767 . wavelengths, waveguide components, 2339.

Millimicrosecond, coincidence circuits, 1779.

pulses using a charge-storage type pulse-height analyzer, 2895.

region, high-speed flipflops, 1730 .

Mineral, bone, structure, 2383 .

fillers, resin bonding of offset papers containing, 303 .

Minerals, borate, 3057 .

Miniaturization, electronic, new techniques, 489.

Minimax theorems, 768 .

Minimization, matrix, problem, 1985.

Minimizing anomalies in reflectance measurements with the Beckman quartz spectrophotometer, 484 .

Minimum of, certain linear form, J $64 \mathrm{~B} 1,49$.

permanent of a doubly stochastic matrix, 3008 .

Miscellaneous observations on the alkali-aggregate reaction and the ionic charge on hydrated cement, 2639.

Miscelle structure and light scattering in the system sodium dodecyl sulfate-sodium chloride-water, 1386.

Mismatch errors, cascade-connected variable attenuators, 2993.

microwave power measurements, 769 .

Mixed-path ground-wave propagation: 2. Larger distances, RP2770.

Mode, expansion in the low-frequency range for propagation through a curved stratified atmosphere, J $63 \mathrm{D} 1,75$.

theory of VLF ionospheric propagation, 2234.

theory of VLF ionospheric propagation for finite ground conductivity, 2373.

theory of very-low-frequency propagation in the presence of a transverse magnetic field, J 64D3, 265. V.L.F. ionospheric propagation, 2203.
Model, chromosphere from radio aud optical data, 2874. electron corona with reference to radio observations, 2875 .

epitaxy in metal-rock salt pairs, 367.

Models of the atmospheric radio refractive index, 3002.

Modern, batteries, 2204

numerical analysis. I. Solution of differential equations by recurrence relations, 97 .

Modes of vibration of a suspended chain, 770 .

Modification, electron microscope for electron optical shadow method, 91.

Watson's lemma, J 63B1, 15.

Modified, differences, divided, 1092

locked-oscillator frequency dividers, 261.

resonance circuits match impedances, 92 .

Modular, certain, subgroups, normalizer, 1942.

forms and some new congruences for the partition function, 2068 .

forms, certain, generalizations of identities for the coefficients, 1720 .

forms, certain, identity for the coefficients, 1262 .

forms, coefficients of, further identities and congruences, 2573.

functions, class, 2069.

groups, inclusion theorem, 2016.

identities, remarks on, 529 .

subgroups, structure theorems, 1517.

Modulator, diode reactance, 3070.

microwave mixers, 2428.

Modules, expendable, as bases for disposal-at-failure maintenance, TN41 (PB151400).

Moduli, elastic of glasses at elevated temperatures by a dynamic method, 1692.

elastic of indium antimonide, 1693.

elastic of zine blend lattices, ionic character, 2367.

Modulus, dynamic bulk, apparatus tor the direct determination, 1629 .

variations in preferred, and orientation of elasticity in cold drawn music spring wire, 1573.

Young's, of various refractory materials as a function of temperature, 3114 .

Moebius function on the lattice of dense subgraphs, J 64B1, 41.

Mold growth and the $\mathrm{pH}$ of tartrate buffer solutions, 262 .

Molecular, atomic negative ions in stellar atmospheres, 2463.

atomic time standards at the NBS, 2549.

clusters, representation of gas properties in terms of, 815 .

complexes and their spectra, IX. Infrared absorption by iodine in its pyridine complexes and in benzene, 2994.

constants of carbon monoxide, velocity of light, 1574 .

crystal structure models, bibliography, Mono.14.

diatomic, negative ions, 2841.

dimensions and harmonic frequencies of ammonia and deuterated ammonia, 2411.

distribution functions for the entropy density in an infinite system, 2558 .

spectroscopy, iodide prism, infrared measurements with a cesium iodide prism, 1351.

still, simple rotating, RP2951.

structure and branching, thermal degration of polymers as a function, 1557

structure from spectroscopic data, 2651.

weight distribution, 3037 .

weight in polymethyl methacrylate, fracture phenomena, 2569

weight on the crazing and tensile properties of polymethyl methacrylate, 999 .

Molecule, ions, ideal gas thermal functions to $25,000 \mathrm{deg}$. $\mathrm{K}$ for diatomic molecules, oxygen, nitrogen, nitricocide, 2394.

methylamine, structure, $2322 ; 2323$.

NO, stark effect, 2840.

spectrum of the $\mathrm{C}_{3}$, between $3600 \mathrm{~A}$ and $4200 \mathrm{~A}, 2313$. 
Molecules atmosphere, infrared spectra, 2377.

chain, flexibility, sedimentation equilibrium, 2296.

deuterium and hydrogen, 2410.

diatomic, 2861 .

exhibiting internal rotation, microwave spectra, 2202 ; $2638 ; 2991$

flexible chain, dilute solution thermodynamic behavior of: polymethyl methacrylate, 687 .

flexible polymer, crystallization, 1924 .

microwave spectra of, exhibiting internal rotation, 2637.

polymer in solution, rate of thermal diffusion, 1472 .

reactant, by chemical reactions, 2671

solar spectrum, 2205 .

symmetric top, nonresonant absorption, 2218; 2648.

symmetric-asymmetric, hindered rotation, 1732 .

Molecules, atoms, basic physics, 2901.

atoms of solid nitrogen, 1104.

atoms spectra in solid nitrogen condensed at $4.2^{\circ} \mathrm{K}$, interpretation, 1747.

rotor, asymmetric, tables of line strength for rotational transitions, 2796.

Moliere theory of multiple scattering, 1037.

Molten alkali, borates, viscosity and electrical resistivity of, 907

alkali silicates, density, expansivity, and viscosity, 422 .

alkaline-earth borate glasses with three mole percent of potassium oxide, 2864 .

Molybdenum, copper, silver, tantalum, and gold at 662 kev, total photoelectric cross sections, 3101.

fused salts, electrolytic preparation, $1333 ; 2811$.

oxidation at elevated temperatures, 2705 .

oxidation, protection of, 2837.

preparation of trichloride and tetrachloride, J 63A2, 185

second spectrum, description and analysis, RP2856.

Moments of order statisties from the Weibull distribution, 1428 .

Monatomic gases trapped at $4^{\circ} \mathrm{K}, 2546$.

Monev-value graduations on a computing scale, 204.

Monitoring, radiation, 2710.

Monochromatic low-latitude aurora, J 63D3, 207.

Monoenergetic positrons and electrons, 1204.

Monomer, polymethyl methacrylate, determination, 1673.

residual and some properties of self-curing dental resins, relationship, 1846

Monopole, antenna, top-loaded, the electric field at the ground plane with special regard to electrically small $L$-and $T$-antennas, $J 64 \mathrm{D} 2,139$.

antenna with symmetrical top loading, earth currents near, RP2959.

effect of the ground sereen on the field radiated, 1688 .

Monostable multivibrator design, 93.

Monoxide, carbon, and atomic nitrogen, luminous reaction, 2183.

carbon, in studies of air pollution, 1309

carbon, on tungsten, 2888.

carbon, velocity of light from the molecular constants, 1574 .

carbon, with graphite carbon at $450^{\circ} \mathrm{C}$, reaction, 2717 .

chlorine, flash photolysis, 2357.

Monte Carlo, calculations of $\gamma$-ray backscattering and transmission, 1076.

experiments in computing multiple integrals, 1875.

method, matrix inversion, 84.

method to a problem in gamma-ray diffusion, application, 1618 .

methods, experiments in the solution of differential equations, 1019

Monthly refractive gradient for the United States and its application to predicting the geographical and annual trend of VHF transmission loss, 870 .

Moon, theory of radar scattering, J 64D3, 217

UHF, relay, 396.

volcanoes on, 3111 .

Mooney viscometer, cure characteristics of Hevea rubber, 1409.

die closure, 1082.

temperature measurements, 1164.
Morphology of ionospheric storms, 2884.

Mortar, expansion in, cracking in masonry, 419.

Mortars, cement pastes by the alkali-aggregate reaction, 2699.

certain, effects of weathering on, exposed in brick masonry with and without caps and flashings, 1927.

Motion, small particles in skew shape suspended in a viscous liquid, 1077.

two cylinders in an ideal fluid, RP2889.

Motion, solar atmosphere as deduced from radio measurements, 263.

storm-D region, 1078 .

turbulent, 3104 .

Motivation for working in numerical analysis, 1410.

Movements of airglow cells, 2640.

$\mathrm{M}_{\mu}$, low, triodes to series regulator circuits, 1630 .

Mucosal surface contours of impressions, casts, and dentures, method for measuring, 1987.

M.U.F. calculations, effect of the earth's magnetic field, 3075 .

Multi-anvil wedge type high-pressure apparatus, J $63 \mathrm{C} 1$, 59.

Multiaxial stretching on crazing and other properties of transparent plastics, 1002.

Multichannel pulse height analy zers, 2206.

Multicomponent distillation, separation of hydrogen isotopes, 2751 .

Multicomputer system, 2692.

Multi-factor experiments, 2995.

evaluating reliability, 2207 .

Multiple, integrals, some Monte Carlo experiments in computing, 1875 .

ionization of rare gases by electron impact, J 63A3, 201.

ionization of sodium vapor by electron impact, 2996. reflections between the earth and the ionosphere in VLF propagation, 1781 .

scattering, inelastic collisions and the Moliere theory, 1037 .

seattering of relativistic electrons, 1079.

Multiple-purpose orthonormalizing code and its uses, 921.

Multiplet table of astrophysical interest, TN36 (PB151395).

Multiplets, infrared, solar spectrum, 743 .

Multiplication and addition, methods for high-speed, application of digital computers to, C591, Sect. 1 .

Multiplier phototube noise, hysteresis effect, 1361.

Multipliers, frequency, and converters for measurements control, 1349.

Multipurpose standard for microchemical analysis, 2641.

Multistage autoignition of engine fuels, 1411.

Multi-stream charge interaction, radio wave generation, 295 .

Multivibration, monostable, design, 93.

Munsell, renotation space, regular rhombohedral lattice sampling, 930 .

renotation system, extension, 2559.

renotation system to very dark colors extension, 1710 .

renotations, mechanized conversion of colorimetric data, 2633.

renotations, spectrophotometric data, 2522.

Muscle fibre-ATP system, 2986.

Music spring wire, cold drawn, 1573.

Music wire, cold drawn, crystallography, 2509.

Mutarotation, hydrolysis, and rearrangement reactions of glycosylamines, 2642.

Mylar and Dacron polyester strands at low temperatures, some mechanical properties, 2761.

\section{$\mathrm{N}$}

n-dimethylaminodiborane, 1205.

n-dimethyl-amino-diborane, infrared absorption specrum, 1365.

$n$-type, indium arsenide, oscillatory galvanomagnetic effects, 2678.

InSb at $4.2^{\circ} \mathrm{K}$, galvanomagnetic effects, 1716 .

$\mathrm{N}_{2}$ and $\mathrm{O}_{2}$ low energy ions in, 982 .

$\mathrm{NaI}$ (TI) scintillation counters, response function, 1856. 
Narrow hyperfine absorption lines of $\mathrm{C}_{8}{ }^{133}$ in various buffer gases, 2643 .

Narrow-band teletype message errors on a single-sideband 600 -mile-long ultrahigh-frequency tropospheric radio link, measured statistical characteristics, $\mathrm{J} 64 \mathrm{D} 2,125$.

National Bureau of Standards, international relations, 339.

services to industry, 1939.

National Committee on Radiation Protection and Measurements, brief history, 2478 .

National Conference on Weights and Measures:

Forty-second, 1957, M222.

Forty-third, 1958, M225.

Forty-fourth, 1959, M228.

National physical standards and design of experiment, 2209.

Natural and synthetic rubbers, $94 ; 264 ; 487 ; 772 ; 1412 \mathrm{~A}$. review of test methods, 2997.

Natural gas, analysis of, 25 .

Nature, adhesion, 1080 .

cause and effect of porosity in electrodeposits, 1940. decay of a meteor trail, 501 . gradient reflections, 586 .

$\mathrm{Nb}^{91}$ and $\mathrm{Nb}^{92}$, disintegration, 1179 .

NBS, aircraft lighting, research, development and testing, 771.

alpha standards, coincidence counting, and radiative electron capture, 2644.

equatorial region V.H.F. scatter research program for the I.G.Y., 2208.

1000 -lb balance, 1539 .

preferred circuits program, 1412.

primary frequency standard, 872 .

semi-centennial 1901-1951, 486 .

state weights and measures administration, 485 .

Near infrared, solar radiation measurements by balloon to an altitude of $100,000 \mathrm{ft}$., 2645 .

spectrum of nitrous oxide, 1782 .

Nebulae, dense dark, radio observations (al-CM), 1468.

diffuse, bright rims, $2496 \mathrm{~A} ; 2479$.

Necessity for uniformity of test procedures, 587.

Need for more knowledge of materials in civil engineering, 2824.

Needle vacuum, 848 .

Negative, feedback, improvement in gain stability of the superheterodyne mixer through the application, 466 .

hydrogen ion, 3012 .

ion appearance potentials, 2481.

ion, hydrogen, photodetachment of, 1105

oxygen ions in the upper atmosphere: The affinity and radiative attachment coefficient of atomic oxygen, 1413

Negative ions, 2210.

atmospheric, photodetachment, 2245.

atomic and molecular, in stellar atmospheres, 2463. photodetachment studies, 2246.

Nehari, estimates, numerical experiments in potential theory, 2654.

Neodynium ${ }^{147}$ and cerium ${ }^{141}$, alignment, 1255.

Nephelene + albite $=2$ jadeite, 203.

Nerve excitation, automatic computation, 1272.

Net heat of combustion, AN-F-58 aircraft fuels, 265.

aviation gasoline and its correlation with other properties, 2646.

Neumann series for the product of two Whittaker functions, 630

Neutral meson decay, angular distribution, 2461.

Neutron, activation cross section, manganese thermal, 1397.

dosimetry and neutron flux measurements, standards, $2777 ; 3055$.

fast, detection, plastic phosphor matrix, 2693.

fast, potassium iodide, detector, 1451 .

fast, spectrometer based on the total absorption principle, TN10 (PB151369).

fast, spectrometer, double-pulse total-absorption, TN1 (PB151360). flux measurement and neutron dosimetry, 3055 .

physics, 2500

radiation up to 30 million electron volts, protection against, $\mathrm{H} 63$.

source strengths, scintillation counter method of intercomparing by means of a manganous sulfate bath, RP2952.

Neutron-insensitive gamma-ray dosimeter, 2211.

Neutrons, D-D, in water, age to indium resonance, 2450.

degradation des polymeres par les rayons, 681 .

fast cosmic-ray, sea-level latitude variation, 343 .

fast cosmic-ray, with altitudc, variation of intensity, 600 .

fast, in water, 1180

fast, reflection of from water, $\mathrm{J} 63 \mathrm{~A} 2,101$.

14,1-Mev in water, attenuation, 2032.

samarium, emission of, 383 .

New, apparatus at the NBS for absolute capacitance measurement, 2647.

arrangement for ortho-para conversion of liquid hydrogen in the large CEL-NBS liquefier, 1988.

criterion of univalence of transformations in $R^{n}, 3106$.

federal standard on colors, 2212.

frontiers in business management control are being established by electronic computers, 488 .

infrared band system of $\mathrm{FeO}, 368$.

kind of radio propagation at very high frequencies observable over long distances, 369 .

light on self-ignition, 266.

materials for magnetic cooling and thermometry below $1^{\circ} \mathrm{K}, 1783$.

matrix transformations for obtaining characteristic vectors, 267.

method analyzing extreme-value data, 922 .

method for determining the value of the Faraday, 3 .

method of checking the consistency of precedence matrices, 2876 .

method of recording the sound transmission loss of walls as a continuous function of frequency, 1414.

method of solution for unretarded satellite orbits, J $63 \mathrm{~B} 2,105$.

paper products in competition with textiles, 1784 .

point of view in the measurement of light and color, 1989.

resolving power test chart, 268 .

safety code for portable metal ladders, 2374

standard wind load requirements, 2213.

standards for science and industry, 2998.

technique for the measurement of microwave standingwave ratios, 1594

techniques for electronic miniaturization, 489 .

techniques for evaluating natural corundum ores, 1081.

techniques for fabrication of airborne electronic equipment, 1415 .

tools for research, 269

ultra high strength steel, 2214

Newton's method in complex Banach spaces, 559 .

$\mathrm{NF}^{3}$ by electron impact, ionization and dissociation, 1749.

Nickel, chlorine, above $1,100^{\circ} \mathbf{K}$, kinetics of the reaction between, 2174

chlorine, between 1200 and $1700^{\circ} \mathrm{K}$, kinetics of the high temperature heterogeneous reaction, 2608.

cold-drawn, ereep of, $\mathrm{J} 63 \mathrm{C} 1,1$.

copper, and two nickel-copper alloys, annealed, creep of, RP2836.

electrodeposited, 511.

identification and microdetermination, 70 .

plating, electroless, 2327 .

tin, iron, copper, and silicon, electrorefining studies, 2811 .

Nickel dip, a radioisotope study of metallic deposits in porcelain enameling, 1941 .

enameling of sheet steel, 1133 .

radioisotope study, 1599 .

Nickel-aluminum alloy coatings for the protection of molybdenum from oxidation, 2837.

Nickel-chromium-iron alloys, heat capacity and enthalpy, RP2870. 
Night afterglow, 3084 . airglow, 2825 .

Nightglow, 5577, height, 2817.

heights: a reinterpretation of old data, 1416.

review of observational results, 1191.

van Rhijn method, height, 1534 .

Night-time low-frequency ionograms, interpretation, 2821.

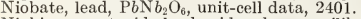

Niobium, pentoxide-lead oxide, phase equilibrium relations in the binary system, RP2925.

tantalum in titanium-base alloys, determination of, RP2929.

titanium, zirconium, and tantalum in steels, determination of, RP2923.

Nitracocide, diatomic molecules, oxygen, nitrogen, and their molecule ions, 2394.

Nitriding phenomena in titanium and the $6 \mathrm{Al}-4 \mathrm{~V}$ titanium alloy, J $64 \mathrm{~A} 1,119$.

Nitrogen, active, absorption spectrum of vibrationally excited $\mathrm{N}_{2}, 2887$.

active, hydrocarbons, 2982.

$\alpha$ and $\beta, 2879$.

argon, negative atomic iodine ions, 605 .

atom recombination, kinetics of, 2972 .

atomic, and carbon monoxide, luminous reaction, 2183.

atomic and organic substances, 2544

atoms trapped in solids, 2392

bands excited in auroras and by high-energy protons and hydrogen atoms, intensity distributions, 1044.

boiling liquid and hydrogen flowing axially through narrow annular passages, 2581.

calibration of dead space in gas absorption systems, 2524 .

carbon, hydrogen, and oxygen, calorimetric combustion in a bomb of compounds containing, 1645.

diatomic molecules, oxygen, nitricocide, and their molecule ions, 2394 .

discharged, 2551A.

emission spectra of, bombarded by high energy protons, 576.

energy levels in $\mathrm{F}^{18}$ from alpha-particle reactions, 711 . gamma-irradiates solid, electron spin resonance spectra, 2940 .

gaseous, thermodynamic properties, 1964.

isotope analysis by optical spectroscopy, 2775 .

leather by the Kjeldhal method, determination, 332 . liquid, 3013 .

microwave discharge, thermometric study of the frozen products from, 2847.

oxygen, and air, rotational relaxation, 3038 .

oxygen, hydrogen, water, and ammonia, electric discharge, 2309.

removal, from hydrogen with silica gel at low temperatures, 2730 .

solid, condensed at $4.2^{\circ} \mathrm{K}$ from a gas discharge, abundance of free atoms, 1607 .

solid, condensed at $4.2^{\circ} \mathrm{K}$ from a gas discharge, spectra emitted, 1881.

solid, condensed at $4.2^{\circ} \mathrm{K}$, interpretation of spectra of atoms and molecules, 1747 .

solid, at $4.2^{\circ} \mathrm{K}$, phosphorescence of atoms and molecules, 1104

solid $\left(4.2^{\circ} \mathrm{K}\right)$ containing atoms of free radicals, 2622.

solid $\left(4.2^{\circ} \mathrm{K}\right)$, luminescence of, containing atoms or free radicals, 2182 .

solid, gas discharge, 3052

solid, light emission from, 2975 .

sulfur in steel, determination, 426

triatomic, in solids at very low temperatures, 3054 .

Nitrogen-oxygen mixtures, spectra of after glows and discharges, 2307.

Nitrous, acid with collagen, reaction, 1837 .

oxide, near infrared spectrum, 1782.

NO molecule, stark effect, 2840 .

Nodal slide, evaluation of lens distortion by the inverse, $\mathrm{J} 63 \mathrm{C} 2,105$.

optical bench, 2948 .
Noise, airborne, transmission of, through walls and floors, 2397.

atmospheric, measurement, 2189.

comparator for microwaves, 773 .

composition resistors, $1416 \mathrm{~A}$.

control requirements in building codes, 2215.

data, radio, TN18-2 (PB151377-2).

investigation at VLF by the NBS, 2216.

measurements of composition resistors. I. The method and equipment. II. Characteristics and comparison of resistors, 1417.

multiplier phototube, hysteresis effect, 1361 .

radio, data for the International Geophysical Year July 1, 1957-December 31, 1958, TN18 (PB151377).

radio, of ionospheric origin, 118.

recorder, ARN-2 auxiliary log-linear, TN45 (PB151404).

solar radio, 1418 .

Noncrystal ionic model for silica glass, RP2782.

Nonequilibrium rate processes, studies, $2324 ; 2325 ; 2786$; 2787

systems, transient, specification, 2237.

Nonlinear, oscillations, singular perturbation methods, 828.

theory of space-charge wave in moving, interacting electron beams with application to solar radio noise, 1418.

viscoelastic behavior of rubber in shear, 1419 .

Non-linear differential equations of the second order with integrable forcing term, 1429 .

Non-Maxwellian distribution in a shock front and the anomaly of the chromospheric temperature, 874 .

Non-self-adjoint boundary value problems in ordinary differential equations, $\mathbf{J} 64 \mathrm{~B} 2,83$.

Nonmetals, creep of crystalline, 2072.

Nonresonant absorption of symmetric top molecules: shape of the nonresonant spectra, 2217;2648.

Normal, distribution, note, 1595 .

distribution, property of, 928 .

fluid concentration in liquid helium II below $1^{\circ} \mathrm{K}, 774$.

Normalizer of certain modular subgroups, 1942.

Notation for atomic spectra, 775 .

Notch depth and low temperatures, effects of, on the mechanical behavior of an annealed commercially pure titanium, RP2790.

Note, accelerating components in particle spectrometers, 2649.

adjustment of Mooney viscometer die closure, 1082. bounds for certain determinants, 2218 .

Bragg-Gray cavity principle for measuring energy dissipation, 1083.

circle theorem of hydrodynamics, 1084.

circular disks containing the eigenvalues of a matrix, 2650 .

climatic variation of absolute humidity, 1991.

computation of $x^{2}, 2877$.

confidence intervals in regression problems, 2429 .

determination of molecular structure from spectroscopic data, 2651.

dielectric properties of magnesium-strontium titanates, 2220 .

displacement pressure method for measuring the affinity of liquids for solids, 490 .

estimation of sodium carbonate in photographic developers, 1238 .

focusing of electron beams in certain magnetic fields, 95 .

four by four Latin squares, 5 .

group matrices, 1237 .

indentation of heavy leather, 776 .

infinite integral, 270 .

L.F. portable antennas operating over ice and snow covered terrain, 2219.

measurement of sine-wave response of lenses, 2999.

normal distribution, 1595 .

oxyquinolate determination of magnesium oxide in cement, 925 . 
partially balanced designs, 923 .

polarization of low frequency ionosphere echoes, $37 \mathrm{I}$.

preparation of sodium amalgain in the form of pellets, J $64 \mathrm{~A} 1,135$.

preparation of solid ozone and atomic oxygen, 1239. propagation of the transient ground wave, 1992.

selective R-C bridge, 370 .

strength of pieric acid, 1085 .

sweep-frequency backscatter observations, 924 .

symmetric functions of eigenvalues, 1990.

thermal stabilization of austenite iron-carbon-nickel alloys, 777 .

Vincent's theorem, 96.

wave propagation through an inhomogeneous medium, 629.

Notes, care and use of electrical instruments, 99.

numerical analysis, $97 ; 98 ; 271 ; 272$.

post-treatment for HAE coatings, 1786 .

Nouveaucritére d'univalence des transformations dans un $\mathrm{R}^{n}, 3106$.

Nova outburst, 3085 .

Novel rocket hoist, 2430.

Nozzle burner method, measurement of flame speeds, RP2867.

Nozzles, two-dimensional, by the method of characteristics, design, 1432 .

NRL Aleutian radio eclipse expedition, 273.

$\nu_{3}$ band of methane, 2221 .

Nuclear, absorption and elastic scattering of X-rays, direct observation, 1312 .

elastic scattering of photons, $1086 ; 1787$.

electromagnetic radiations, 2500 .

explosions, geomagnetic effects of high-altitude, 2959.

high-altitude, explosions on radio noise, J 64D1, 37.

magnetic resonance and infrared absorption studies in organic phosphorus chemistry, 2494 .

magnetic resonance in semiconductors, 3000 .

magnetic resonance of aligned radioactive nuclei, 778 .

matter distributions from eoherent neutral pion production, 2653.

moments of $\mathrm{Ac}^{227}, 1420$.

moments of technetium-99, 875 .

reactors, a survey of ceramics, 12.

reactors, ceramic coatings, 1285 .

states and the theory of angular correlations, geometrical characterization of, 726 .

Nuclear-resonance magnetic field control, 491.

Nucleation and growth processes, 2834.

Nuclei, deformed, 2772 .

point, elastic scattering of electrons and positrons, 1694.

polarized, 2902 .

polarized, beta-decay, 2557 .

polarized, $\beta$-decay of, further experiments, 2132.

radioactive, aligned, nuclear magnetic resonance of, 778.

three odd-A rare-earth, alignment, 1611.

Nucleon-nuclei scattering, an optical model, 395.

Nucleonics, 1087.

Nucleus, looking into, 753.

Nuclides, beta-emitting, 1200 .

Nuclidic masses and atomic weigints, 2906.

Null detector, pressure-sensitive diaphragm-type, 2698.

Number, absolute points of a correlation, 1943.

farthest points, 876 .

representations of a quadratic form as a sum of four squares, 2375 .

theory, algebraic, some computational problems, 1873.

Numbers, natural, roots of, algebraic equations, 1610 . psuedo-random, generation and testing, 1722 . rational, subroutine for computations, 1603 .

Numerical analysis:

accelerating convergence of iterative processes, 2447. alternative derivations of Fox's esculator formulae for latent roots, 386 .

application of the Monte Carlo method to a problem in gamma-ray diffusion, 1618 . application of two methods of numerical analysis to the computation of the reflected radiation of a point source, 1267 .

applications of a theorem in partitioned matrices $\mathrm{J} 63 \mathrm{~B} 2,73$

applications of the theory of quadratic forms in Hilbert space to the calculus of variations, 195.

approximation of linear elliptic differential equations by difference equations with positive coefficients, 785

asymptotic lower bounds for the frequencies of certain polygonal membranes, 957 .

asymptotic solution of the differential equation of hydrodynamic stability in a domain containing a transition point, 656 .

automatic computation of nerve excitation, 1272.

automatic eomputations with power series, 1638.

basic theorems in matrix theory, AMS57.

Begrundung für die Beschaftigung mit numerischer Analysis, 1275 .

best approximation polynomials of given degree, 1641.

bounds for certain determinants, 2218 .

bounds for the greatest latent root of a positive matrix, 405 .

bounds of a one-parametric family of matrices, 2663. certain series expansions involving Whittaker functions and Jacobi polynomials, 1424 .

changes of sign of sums of random variables, 662 .

characteristic roots of quaternion matrices, 967 .

Chebyshev polynomials in the solution of largescale linear systems, 411.

circular disks containing the eigenvalues of a matrix, 2650 .

elasses of matrices and quadratic fields, 205 .

classes of positive definite unimodular circulants, 2057.

commutativity in finite matrices, 2060.

comparison theorem for eigenvalues of normal matrices, 1231.

complete sequences and approximations in normal linear spaces, 2065.

eomplex biorthogonality for certain sets of poly nomials, 975 .

computation of cyclic cubic units, RP2783.

computation of the capacity of a cube, 779 .

computation of vibration modes and frequencies on SEAC, 1293.

computational experience in solving linear programs, 672 .

computational problems in algebraic number theory, 1873.

condition of a certain matrix, 149.

condition of certain matrices, III, RP2815.

condition of matrices, 98 .

congruences for the coefficients of modular forms and some new congruences for the partition function, 2068.

construction and application of a elass of modular - functions, 2069

continued fractions in high-speed computing, 612 . convergence of a method of solving linear problems, 417.

convergence of numerical iteration, 856 .

convergence of Seidel iterants of nearly symmetric matrices, 329 .

convergence of the Rayleigh quotient iteration for the computation of characteristic roots and vectors, $3003 ; 3004$.

dense subgraphs and connectivity, 2925.

derivative of a polynomial and Chebyshev approximation, 786

determinantal inequality, $1232 ; 2657$.

determinantal inequality of H. P. Robertson, 1980. determinanten mit ueberwiegender Hauptdiagonale und die absolute Konvergenz von linearen Iterationsprozessen, 1671.

determination of eigenvectors and cigenvalues of symmetric matrices, 330 . 
determination of the extreme values of the spectrum of a bounded self-ajoint operator, 220 .

direct approach to the problem of stability in the numerical solution of partial differential equations, 1588 .

discrete analogs of inequalities of Wirtinger, 1313. discrete approximations to elliptic differential equations, 1314

eigenvalues of sums of normal matrices, 1425 .

elementary inequalities relating to a gamma and incomplete gamma function, 3047 .

error bounds for the eigenvalues of symmetric integral equations, 1707.

estimation of quadrature errors for analytic functions, 1096.

European experience with electronic computers, 1336. evaluation of matrix inversion programs, 2814.

evaluation of the integral $\int_{0}^{\infty} e^{-u 2-(x / u)} d u, 716$.

existence theorems and extreme solutions for inequalities concerning convex functions for linear transforms, 2115.

experiments in the solution of differential equations, 1019.

extension of Gauss' transformation for improving the condition of systems of linear equations, 393 .

extreme value results for indefinite Hermitian matrices, 2303.

formal power series for $\log e^{x} e^{y}, 1931$.

formulas for calculating the error function of a complex variable, 241 .

formulas for finding the arguments for which a function has a given derivative, 242 .

formulas for numerical differentiation in the complex plane, 453 .

formulas for numerical integration of first and second order differential equations in the complex plane, 61.

foundations for applications, 1275 .

further contributions to the solution of simultaneous linear equations and the determination of Eigenvalues, AMS49.

Gauss' speeding up device in the theory of single step iteration, 2659.

Gauss to Gerling on relaxation, 244.

generalization of a theorem of Osgood to the case of continuous approximation, 64 .

generation and testing of pseudo-random numbers, 1722 .

Gibbs' phenomenon for Euler means, 103.

Gibbs' phenomenon for Hausdorff means, 65.

Hilbert matrix, 2229.

how to solve a linear programming problem, 1360.

identities and inequalities concerning orthogonal polynomials and Bessel functions, 249.

inequalities concerning ultraspherical polynomials and Bessel functions, 72 .

infinite integral, 270.

Institute for Numerical Analysis of the National Bureau of Standards, $152 ; 337$

integration of parabolic equations by difference methods, 497 .

inversion of matrices by random walks, 282 .

kleine Bemerkung zur asymptotischen Entwicklung des Fehlerintegrals, 1383.

Leonard Euler's integral: a historical profile on the gamma function, 2974 .

linear functional equations and interpolation series, 1050.

linear iteration procedures for symmetric matrices, 1097.

machine attacks on problems whose variables are permutations, 1762 .

mathematical services useful in industry, 759 .

matrix inversion, 84

matrix minimization problem, 1985 .

matrix, principal submatrices of a fullrowed non-negative, J $63 \mathrm{~B} 1,19$. method for the determination of converging factors, applied to the asymptotic expansions for the parabolic cylinder functions, 366 .

method of speeding up iterations with superlinear convergence, 2427.

methods for evaluating trends in time series of tropospheric radio field strength data, 546 .

metodi probabilistici per la soluzione numerica di alcuni problemi di analisi, 763 .

minimum of the permanent of a doubly stochastic matrix, 3008 .

modified divided differences, 1092 .

Monte Carlo calculations of $\gamma$-ray backscattering and transmission, 1076.

Monte Carlo experiments in computing multiple integrals, 1875 .

motivation for working in numerical analysis, 1410 .

multiple-purpose orthonormalizing code and its uses, 921.

Neumann series for the product of two Whittaker functions, 630 .

new matrix transformations for obtaining characteristic vectors, 267

number of absolute points of a correlation, 1943.

number of representations of a quadratic form as a sum of four squares, 2375 .

numerical analyst's fifteen-foot shelf, 631 .

numerical experiments in potential theory using or thonormal functions, 1789 .

numerical experiments in potential theory using the Nehari estimates, 2654.

numerical integration of periodic analytic functions, 3009 .

numerical methods associated with Laplace's equation, 274.

numerical solution of equations involving differential operators with constant coefficients, 502 .

numerical solution of the helium wave equation with SEAC, 372 .

numerical study of Dedekind's cubic class number formula, RP2798.

over and under relaxation in the theory of the cyclic single step iteration, 781 .

pairs of matrices of order two which generate free groups, 2242 .

pitfalls in computation, 1809

polya frequency functions. III, The positivity of translation determinants with an application to the interpolation problem of spline curves, 782 . practical evaluation of integrals, 1098 .

probability of a saddle point, 2379 .

problem in the theory of mechanical quadratures, 1423. proceedings of the Second Symposium in Linear Programming, held jointly by the National Bureau of Standards and the United States Air Force, 1458 .

radix table for obtaining hyperbolic and inverse hyperbolic functions to many places, 810 .

relative extrema of Bessel function, 104.

relative extrema of the Hermite orthogonal functions, 283.

relative extrema of the Laguerre orthogonal functions, 105.

relative extrema of ultraspherical polynomials, 106. relaxation and step-by-step methods, 301 .

relaxation method for linear inequalities, 1196; 1197. remark on the smoothing problem, 931.

representation for solutions of analytic systems of linear differential equations, 932.

representation of a eertain integral involving Bessel functions by hypergeometric series, 2236 .

rounding off of difference tables for linear interpolation, 504.

smoothing operations and their generating functions, 783.

solution of differential equations, by recurrence relations, 97.

solving linear algebraic equations can be interesting, 831 
some inequalities concerning positive-definite Hermitian matrices, 1496.

stable systems of differential equations with integrable forcing term, 1893.

subdeterminants of doubly stochastic matrices, 2227.

subroutine for computations with rational numbers, 1603.

sufficient conditions for the convergence of Newton's method in complex Banach spaces, 559 .

summation method of $\mathrm{O}$. Perron, 102.

summation of slowly convergent series, 145 .

symmetric functions of eigenvalues, 1990.

systems of equations, matrices, and determinants, 562 .

systems of inequalities involving convex functions, 2335 .

Tauberian theorem for Abel summability, 275.

Tauberian theorems for summability, 321 .

theorem of Ostrowski and Taussky, 1091.

theorem of M. Riesz, RP2935.

topological proofs for certain theorems on matrices with non-negative elements, 2850.

two consequences of the transposition theorem on linnear inequalities, 353 .

two explicit formulae for the distribution function of the sum of $n$ uniformly distributed independent variables, 609 .

two problems in abstract algebra connected with Horner's rule, 1101.

uniqueness theory for asymptotic expansions in general regions, 2400 .

values of a quadratic form, 2186.

variation of the determinant of a positive definite matrix, 284.

vibration of a square clamped plate, 1435 .

Vincent's theorem, 96.

use of random numbers, 613 .

weighted restricted partitions, 3112 .

zero of $\mathrm{I}_{n+1}(x) \mathrm{J}_{n}(x) \mathrm{I}_{n+1}(x), 624$.

zeros of polynomials and the degree of linear systems, 790 .

Numerical, computation, exponential integral, RP2941.

differentiation in the complex plane, formulas, 453 .

evaluation of the Fermi beta-distribution function, 100.

evaluation, Simpson's rule, Weiner's integrals in function space, 185 .

experiments in potential theory using orthonormal functions, 1789

experiments in potential theory using the Nehari estimates, 2654.

integration, formulas for, 61 .

integration of periodic analytic functions, 3009 .

integrations of differential equations and for Gaussian quadrature, use of sub-routines SEAC, 598.

iteration, convergence of, 856 .

methods associated with Laplace's equation 274.

solution of certain problems in analysis, probability methods, 763 .

solution of equations involving differential operators with constant coefficients, 502

solution of partial differential equations, 1588 .

solution of the helium wave equation with the SEAC, 372 .

study of Dedekind's eubic class number formula, RP2798.

values of the intensities of the Stark components of the hydrogen lines and the Stark broadening functions, C603.

Nylon, cotton, and wool fabrics at different temperatures and relative humidities, 2793 .

o

$\mathrm{O}_{2}$, and $\mathrm{N}_{2}$, low energy ions in, 982 .

photodetachment, 2684

Obituary notices on L. F. Richardson, 1088.

Object-color changes from daylight to incandescent filament illumination, 492 .

Oblique incidence propagation at $300 \mathrm{ke}$ using the pulse technique, 493.

Oblique-incidence, ionograms, TN31 (PB151390). measurements of the heights at which ionospheric scattering of the $\mathrm{VHF}^{3}$ radio waves oecurs, 1790 .

Observation, audio-frequency electromagnetic noise during a period of solar disturbauce, 2019 .

vertical-ineidence seatter from the ionosphere at 41 $\mathrm{Me} / \mathrm{sec} ., 2655$.

Observations, abrasion testing, 1089.

distance meteor-trail cchocs followed by ground scatter, 1421.

evaporation of water from cellulose, 547

field exposures of enameled ranges, 1090 .

hydrated monocalcium aluminate and monostrontium aluminate, RP2777.

miscellaneous, on the alkali-aggregate reaction and the ionic charge on hydrated cement, 2639

outlying, rejection of, 813 .

second sound radiation by the thermal Pitot tube, 101.

sweep-frequency backseatter, note on, 924 .

Observatories, geomagnetic and thesaurus of values, 252 .

Observer differences in color-mixture functions studied by means of a pair of metameric grays, RP2825.

Obukoff and Heisenberg theories of turbulence, 1215.

Ocular pigmentation, tritanopia, 161 .

$\mathrm{OD}$ and $\mathrm{OH}$ in electrodelen discharge through water vapor, rotational intensity distributions of, 817 .

o-fluoro-benzidines, 837 .

Off-path propagation at VHF, 2656.

$\mathrm{OH}$, OD in electrodelen discharge through water vapor, rotational intensity distributions of, 817 .

$\mathrm{CH}$, and $\mathrm{HCO}$ in flame spectra, using deuterium as tracer, mechanism of formation, 868 .

effects of vibrational state on rotational intensity distribution, 701

emission in the earth's atmosphere, 3001.

emission spectrum, from 1.4 to $1.7 \mu, 860$.

flames, effects of self-absorption of rotational "temperatures", 700 .

methane-air flames, rotation temperatures, 304 .

several flames, rotational temperatures, 534.

radical, dissociation energy, 1503 .

rotational "temperature", in diluted flames, 818 .

rotational "temperatures" in low pressure flames, experimental evidence for existence of abnormal, 1339.

solar spectrum, J 63A3, 279 .

$\left(2 \Sigma^{+}\right)$in flames, $1348 ; 2941$.

${ }_{2}^{+}$, wall effects on rotational population, 2865 .

Ohmmeter, logarithmic, 1619.

Oil flow in plain journal bearings, 494.

Oil-soaked felt-pad lubrication of ball bearings at high speed and high temperature, 1422

Oils used in oil-extended rubber, RP2771.

Olefins, solid, at $-195^{\circ}$, reaction of hydrogen atoms, 2828 .

solids at $-195^{\circ}$, the addition of hydrogen atoms, 2803 .

Oleophobic films on metal fatigue, effect of, J $64 \mathrm{C} 2,147$.

On-off temperature control for electrieally heated filaments, $2459 ; 2660$.

One-microsecond, adder using one-megacycle circuitry, 1596.

parallel adder using one-megacycle circuitry, logical design, 1937.

Operating, characteristic of the average chart, 588 .

instructions for ARN-2 auxiliary log-linear noise recorder, TN45 (PB151404).

rooms, conductive flooring for hospital, J $63 \mathrm{C} 2,125$

Operation, bearings and pumps at low temperatures, 2676.

construction of a high pressure diffusion cloud chamber, 568.

construction of thoria resistor-type furnaces, 328 .

smoothing, and their generating functions, 783 .

Operations research, design of experiments, 1670.

Optical, bench, 2948.

detection of narrow $\mathrm{Rb}^{87}$ hyperfine absorption lines, 2677.

electrical properties of gray tin, studies of erystal growth, 1518.

electrical properties of intermetallic compounds, 1007 ; 1330 .

electron, exploration of space, 230 . 
electron, properties of space charged clouds, 53 .

glass production in the United States, history of, 731. glasses, relative stress-optical coefficients of, RP2779. image, electron, of an atomic beam, 1704.

image evaluation, report on the NBS, symposium, 532. measurements, 2790 .

merit function and its derivatives with respect to the system parameters, calculation, 2041.

model for nucleon-nuclei scattering, 395 .

properties of cesium iodide, 1436 .

radio data, 2874 .

rotation of polarized light by chemical compounds, 285 .

shadow method, electron, modification of electron microscope, 91.

spectrophotometric analysis of hydrogen-deuterium mixtures in presence of air, 505 .

spectroseopy, stable carbon isotope analysis, 1892.

spectroseopy, stable nitrogen isotope analysis, 2775 .

studies at low temperatures, 2575 .

system, 235.

systems for increasing the available intensity from flames, 506.

T-bench method of measuring longitudinal spherical aberration, RP2880.

test for spherical aberration, 950 .

Optics, applied, at NBS, $226 ; 655$.

electron, 286.

geometrical at V.L.F. sky wave propagation, 2360 .

Optimum, antenna height for ionospheric seatter propagation, 3011 .

frequencies for outer space communication, J 64D2, 105.

grouping on one-criterion variance components analysis, 1102.

Orbits, satellite, effect of transverse atmospheric drag, 2532.

satellite, unretarded, new method of solution, J $63 \mathrm{~B} 2$, 105 .

Ores, corundum, 1081

Organic, coatings, abrasive jet method for measuring abrasion resistance, 1249

evaluation of adhesion of, by ultracentrifugal and other methods, 715 .

Organic, phosphorus chemistry, 2494

plasties, 109 .

solutions, electrodeposition of metals, $1696 ; 1697$.

solvents, electrolysis of, with reference to the electrodeposition of metals, 2936.

substances and atomic nitrogen, 2544 .

Organizing a network of computers to meet deadlines, 2240.

Orientation, variations in preferred, and modulus of elasticity in cold drawn music spring wire, 1573.

Orifice meter tests, 128 .

Origin, current nomenclature for the ionospheric layers, 3086.

electronically excited $\mathrm{C}_{2}$ radical in hydrocarbon flames, 1434.

[OI] 5577 in the airglow and the aurora, J 63D1, 15.

"very-low-frequency emissions", J 63D1, 21

Ortho and parahydrogen, flow conversion kinetics, 2953.

Orthogonal, Hermite, functions, 283.

Laguerre, functions, relative extrema, 105.

polynomials, AMS49.

polynomials and Bessel functions, 249.

Orthonormalizing code, multiple-purpose, its uses, 921.

Orthoparahydrogen conversion catalyst, 3017 .

Ortho-para, catalysis in liquid-hydrogen production, RP2840.

conversion of liquid hydrogen in the large CEL-NBS liquefier, new arrangement, 1988.

Ortho-parahydrogen, conversion, catalysis, 2048.

eonverters, design data, 2514 .

mixtures, continuous analysis, 1294.

Orthophosphate, anhydrous gallium, temperature inversion, 1916.
Oscillations, nonlinear, singular perturbation methods, 828.

superposed fluids, stability, 1890 .

Oscillator, audio, low-distortion transistor, 755 .

eathode-coupled, 62.

constant-amplitude, 210 .

high-stability bridge-balancing, 1358 .

locked, modified, frequency dividers, 261.

locked, pulse timer, 634 .

$\mathrm{R}-\mathrm{C}$, wide-range, 171 .

stable-output, 552 .

transistor, precision, 799.

X-band, stabilization, high-order harmonics, 2153.

Oscillators, anharmonic, the vibrational relaxation of a system, 2786 .

harmonic, $1847 ; 2235$.

harmonic, the relaxation of a system, 2324 .

rotating, 2787.

subharmonic crystal, 1154.

Oscillatory, galvanomagnetic effects in $n$-type indium arsenide, 2678.

interpolation coefficients, tables, AMS56.

interpolation in the complex plane, formulas for, RP2792.

Osgood, theorem of, to the case of continuous approximation, generalization, 64 .

Osmometer, block-type, design and performance, RP2931.

Hepp-Scatchard, improved, 1362.

Outgoing letter mail characteristies, some applications of statistical sampling methods, TN16 (PB151375).

Outline of characteristics of an image-forming system, 1801.

Output of a sound source in a reverberation chamber and other reflecting environments, 2679.

Output-input, key or bottleneck, 2601.

Over and under relaxation in the theory of the cyclic single step iteration, 781 .

Oxidation, behavior of enameling iron during firing, 1153. cellulose by ozone in small concentrations, 589 .

cool flame, of propane-2-C ${ }^{13}$, products, 2702.

cool flame, of propane, separation, 2753.

D-mannitol-2-C14 by acetabacter suboxydans, isotope effect, 2606.

elevated temperatures, protection of molybdenum from, 2705.

periodate, and isotope dilution techniques, 2319 .

studies of some natural graphites, 1437.

Oxidative degradation, series of deuterated styrene polymers, 2680 .

styrene and $\alpha$-deuterostyrene polymers, 1802.

Oxide, binary, systems, zirconia reactions, 1978.

cathodes, measurement and theoretical study of electrical conductivity and Hall effect, 1063.

ceramic, single crystals, plastic deformation, 2253.

electrode, silver-silver, 1998.

high-density thorium, some physical properties, 1877. magnesium, in cement, 925 .

nitrous, near infrared spectrum, 1782.

potassium, 3059 .

strontium, content of portland cements, 2382.

tin or titania containing varying proportions or rareearth oxides, dielectric properties, 2520.

tribenzylamine, in benzene and water, 1195 .

Oxide systems, immiscibility in, structural interpretation, $2320 ; 2320 \mathrm{~A}$.

structural interpretation of immisicibility, $2320 ; 2785$.

Oxide-germanium, magnesium dioxide, 3094.

Oxides, metal, with o-ethyoxybenzoic acid and other chelating agents, 2829 .

$\mathrm{R}_{2} \mathrm{O}_{3}$, studies of beryllium chromite and other beryllia compounds, J 64A1, 103.

Oxyacetylene flame spectra, emission of, under high resolution, RP2903.

Oxygen, carbon, hydrogen, and nitrogen, calorimetric combustion in a bomb of compounds containing, 1645.

chemisorption on carbon adsorbents, 1438 .

compressed, microwave absorption, 1408 . 
content of the furnace atmosphere on the adherence of vitreous coatings to iron, effect, 1329 .

diatomic molecules, nitrogen, nitricocide, and their molecule ions, 2394.

difluoride by electron impact, 2169

discharge products and ozone at $4^{\circ} \mathrm{K}$, infrared absorption, 2599 .

effect on the bonding of gold to fused silica, RP2942.

5577 emission in the upper atmospherc, excitation mechanisms, J $63 \mathrm{D} 3,319$.

Herzberg band of $\mathrm{O}^{2}, 1185$.

hydrogen atoms in atomic flames, reactions of ammonia and hydrazine, 1948.

hydrogen, reaction at the second explosion limit, 248.

ions, negative, in the upper atmosphere, 1413.

liquid, survey of hazards of handling, 2333.

liquid, transfer lines, characteristics of some insulations, 2054

microwave absorption as a possible atomic frequency standard, experimental evaluation, 2555.

nitrogen, and air, rotational relaxation, 3038.

nitrogen, hydrogen, water, and ammonia, electric discharge, 2309.

photolysis, in solution in liquid nitrogen and in liquid carbon monoxide at $1849 \mathrm{~A}, 3013$.

quenching and wavelength shifters in liquid scintillators, 1439.

red lines in the airglow, 2681

red lines in twilight, excitation, 2554.

role of, in the quenching of liquid scintillators, 1858 .

storage dewars, breathing, 2038.

sulphur, diatomic free radicals of the isotopic hydrides, 1357.

water vapor absorption of radio waves in the atmosphere, 2241.

Oxygen, atomic, and carbon monoxide, 866 .

electron affinity, $1334 ; 2812$.

low temperatures, 2954.

photodetachment cross section and the electron affinity, 2683.

solid ozone, note on the preparation, 1239.

Oxyquinolate determination of magnesium oxide in cement, 925 .

Ozone, atmospheric, 977.

atomic oxygen at low temperatures, 2954.

$4^{\circ} \mathrm{K}$, infrared absorption of oxygen discharge products, 2599 .

oxidation of cellulose, in small concentrations, 589.

solid and atomic oxygen, note on the preparation, 1239.

p-dihalobenzenes, preparation of two fluorinated, 879 .

Packages, checking, H67.

Pads, attenuator, lossy cable, 754 .

Pairs, of matrices of order two which generate free groups, 2242.

matrices with property L, 507; 791; 1440 .

normal matrices with property L, 792 .

Panoramic X-ray dental machine, 1993.

Paper and pulp, 805.

annual review, $1466 ; 2024$.

industry, annual review, 1627.

Paper, bursting test, diaphragm error and testing rate, 428 . chemical tests, 227.

chromatographic analysis for collagen and collagen derivatives, 1597 .

glass fibers, manufacture, 477.

laminated map, improved dimensional stability, 2595. measuring the stiffness, 762 .

milk containers, 1441.

new, products in competition with textiles, 1784 .

production on the upswing, 1103.

stiffness of, 883 .

Papers, coated groundwood, effect of light, 1327 cylinder functions, asymptotic expansions, 366 . equations by difference methods, integration, 497 experimental map, containing synthetic fibers, 2703. glass fiber, 1026. groundwood, effect of light, 693 .

offset, contain mincral fillers, resin bonding, 303 .

offset, resin bonding of hardwood fibers, 302 .

record, accelcrated aging of, compared with normal aging, 1251.

record, and their prescrvation, 1840 .

Parahydrogen and ortho, flow conversion kinetics, 2953.

Parallel testing interferometer, R P2917.

Paramagnetic, relaxation, dispersion function, 2418.

salts, properties of, 2408 .

Parameter, continuous time, sequential decision problems for processes, 824 .

design, galvanometer efficiency, 1717.

large values of, Mathieu's equation, 1523.

Parameters, certain stochastic processes, estimation, 334 .

eircuit, effect of variation, on the excitation of spectra by capacitor discharges, 696 .

15-Mev X-rays scattered by $\mathrm{C}^{12}, 1803$.

Parity conservation, scintillation counting in experiments, 2749.

Partial, Grotrian diagrams of astrophysical interest, 1758. reflections in tropospheric propagation, 508 .

Partially, balanced designs, 1148.

replicated latin squares, 1442 .

Path antenna gain in an exponential atmosphere, $\mathrm{J} 63 \mathrm{D} 3$, 273.

Pattern, of a flush-mounted microwave antenna, RP2796. antenna on a curved lossy surface, 2682.

synthesis for slotted-cylinder antennas, J 63D3, 303.

Patterns, of a slot-array antenna on a finite and imperfect ground plane, 2826.

smooth, of production, 1139 .

Payroll processes at the National Bureau of Standards, conversion, 2070 .

Peek-a-boo system, 3087.

Pellat-type electrodynamometer, measurement of current, RP2845.

Pellets, preparation of sodium amalgam in the form of, $\mathrm{J} 64 \mathrm{~A} 1,135$.

Penetration, and diffusion of hard X-rays: polarization effects, 793 .

diffusion of X-rays: calculation of spatial distributions by semi-asymptotic methods, 509 .

electron, theory, 1548.

gamma rays from isotropic sources through aluminum and concrete, TN11 (PB151370).

Pentaborane-air flames and explosions, spectroseopic observations, 1883.

Pentafluorohalobenzenes, reactions, J 63A2, 167 .

Pentosan, aldehyde, carboxyl, and degree of polymerization data on the ICCA "standard" pulps, 2890.

Perchloric acid-silver perchlorate solutions and conductivity water, stability of silver and Pyres, J 64A1, 127.

Perchlorylfuoride by electron impact, dissociation and ionization, 2170.

dipole moment, 2089 .

infrared spectrum and structure, 1933.

search for the microwave spectrum, 1863.

Performance, of pumps with liquefied gases, 2243.

three-millimeter harmonic generators and crystal detectors, 2244.

Periodicals and serials received in the NBS Library, C563, 1st Suppl.

Permeability and some other properties of a variety of refractory materials, 794 .

Permissible, body burdens and maximum permissible concentrations to radionuclides in air and in water for occupational exposure, $\mathrm{H} 69$.

exposure to ionizing radiation, 1443 .

lower, levels of atomic operations in the United States, influence, 2598.

Perron, O., summation method, 102.

Perturbation, characteristic functions and their application to electron optics, 286.

petroleum, hydrocarbons, 107.

vibrational equilibrium distribution reactant molecules by chemical reactions, 2671 .

$\mathrm{pH}$, and the modern analyst, $2242 \mathrm{~A}$. 
control, alkaline solutions, 1612 .

measurements, meaning and standardization, 1767.

standards at $60^{\circ}$ to $95^{\circ} \mathrm{C}, \mathrm{RP} 2797$.

tartrate buffer solutions, 262 .

values in nonaqueous solutions, 1563 .

Phase, constant vector plus a Rayleigh-distributed vector, mean absolute value and standard deviation RP2950.

diagrams distribution, of interest to ceramists, 1318 .

diagrams of interest to ceramists, compilation, 1656 .

equilibria in the subsystem barium disilicate-dibarium trisilicate, RP2953.

equilibrium diagram of the binary system calciatitania, showing the compound $\mathrm{C} a_{4} \mathrm{Ti}_{3} \mathrm{O}_{10}$, revision, RP2913.

equilibrium relations in the binary system lead oxide-niobium pentoxide. RP2925.

generator for tropospheric research, 1805 .

shift effects in Fabry-Perot interferimetry, J 64A3, 191.

stability, measurements of, over a low-level tropospheric path, J 63D1, 45.

Phenomena, electron scattering, 2'09.

Phenomenological theory of induced electrical polarization, 2431

Philosophy underlying radiation protection, 2376.

Phosphate, aluminum and gallium, low cristobalite type, crystal structure, 1923.

anhydrous indium and thallic phosphate by X-ray diffraction, crystal structure, 1667 .

anhydrous scandium, structure, 1950.

determination, by differential spectrophotometry, 685.

hydrate, tricalcium, crystallographic investigation, 421.

silicate, and arsenate, separation and determination, RP2954.

zinc, and silicate cements, 2873.

Phosphates, calcium, precipitated basic, pyrophosphate formation upon iginition, 1465 .

Phosphinoborine compounds: mass spectra and $\mathrm{J} 63 \mathrm{Al}$, 63.

Phosphor matric, plastic, for fast-neutron detection, 2693.

Phosphorescence of atoms and molecules of solid nitrogen at $4.2^{\circ} \mathrm{K}, 1104$.

Photochemical, and thermal processes in polystyrene in the glassy state, 1958.

degradation of solid polymers, 2455 .

Photoconductivity, and photovoltaic effect in intermetallic compounds, 1806 .

infrared, due to neutral impurities in germanium, 1039.

Photocross, analysis, 2460.

Photodetachment, atmospheric negative ions, 2245.

cross section and the electron affinity of atomic oxygen, 2683.

cross section and the ionospheric detachment rate for $0_{2}^{-}, 2556$.

cross section for $\mathrm{H}^{-}$, relative measurement, 3035 . cross section of the negative hydrogen ion, 3012 . diatomic molecular negative ions, 2841 .

electrons from $\mathrm{H}$ - and D-, experimental cross section, 1338.

$0 \overline{2}, 2684$

hydrogen negative ion, 1105 .

studies of negative ions, 2246 .

Photodisintegration of helium 4, 1106.

Photoeffects in intermetallic compounds, 1444.

Photoelastic constants of some optical glasses, RP2779.

Photoelasticity to the design of tensile specimens for ceramic metal materials, application, 1266.

Photoelectric, and bremsstrahlung, effect as inverse processes, 2905 .

cross sections of copper, molybdenum, silver, tantalum, and gold at $662 \mathrm{kev}, 3101$. determination of image contrast suitable for using square wave targets, equipment and method, $\mathrm{J} 64 \mathrm{C} 1,57$.

effect, Sauter theory, 3040 .

followup and recording system, and its application to remote observations of the beam in high precision balances. J $63 \mathrm{C} 2,91$.

method, automatic, continuous measurement of atmospheric ozone, 977 .

multiplier tubes, 27.

reflectometry, Becker value of manila rope by, 960 . Photoemission from silver into sodium chloride, thallium chloride, and thallium bromide, 1807.

Photoflash cells, standard test, 1506.

Photographic, developers, sodium carbonate in, note on the estimation, 1238 .

dosimetry at total exposure levels below $20 \mathrm{mr}$, TN29 (PB151388).

dosimetry of X-and gamma-rays, 2611.

film and dental X-ray film, 2091.

image, formation of silver sulfide in, during fixation, $\mathrm{J} 64 \mathrm{C} 1,65$.

lenses, spurious resolution, 315 .

lenses, testing, 1169.

personnel dosimeter for X-radiation in the range from $30 \mathrm{kev}$ to beyond $1 \mathrm{Mev}, 1994$.

Photolysis, absolute light-scattering: I. Design and operation, RP2946.

determination of tungsten in steel and titanium alloys with dithiol, RP2812.

flame, determination of manganese in cement, 1930.

flame, determination of strontium in portland cement, 1346.

flash, of chlorine monoxide, 2357.

measurement of the effective intensity of condenserdischarge lights, 2685.

observations of the airglow, 2247.

oxygen in solution in liquid nitrogen and in liquid carbon monoxide at $1849 \mathrm{~A}, 30: 3$.

unit for the airglow and aurora, 1598 .

Photometry, absolute, of the Gegenschein, 1919.

airglow, review of observational results, 1242 .

projection, versatile goniometer for, 904 .

Photomicrography, high speed, cutting characteristics of dental burs, 2076.

Photon, scattering and self absorption in lead and bismuth at $7 \mathrm{Mev}, 3014$.

self-absorption and scattering by the 15.1-Mev level in $\mathrm{C}^{12}, 2248$.

Photoneutron, yields in the rare earth region, $2249 ; 2686$.

Photons, cascade, and electrons, energy spectra, RP2824.

elastic scattering of, 1006 .

elastic scattering of, by tantalum, 2535 .

$1 \mathrm{Mev}, 1170$.

nuclear elastic scattering, $1086 ; 1787$.

vs electrons: a comparison of microscopes, 54 .

$\mathrm{X}$-rays, 1136 .

Photonuclear, effect, long-range correlation model, 2669.

pseudodeuteron effect, 2978 .

reactions, 2497 .

Photoproduction of neutral mesons from carbon near threshold, 2250 .

Photoreconnaissance, aerial, and spectrophotometry, 2308.

Phototropic dyes, shutter-illumination device for the study, 374.

Phototroprism and cis-trans isomerism in aromatic azo compounds, 593.

Phototube noise, multiplier, hysteresis effect, 1361 .

Photovoltaic and photoconductivity effect in intermetallic compounds, 1806.

Physical, and chemical properties of chrome-tanned leather and the election of sampling location, study of the variation, 558 .

chemical properties of split and unsplit chrome-tanned leathers, variation, 601 .

chemical properties, review, direct and indirect filling resins, 688 . 
chemical properties within and between vegetable retanned cow and steer sides, variations, 618 .

chemistry of synthetic rubbers, 1107.

constants at the (United States) NBS, present status of research, 2263 .

equilibria and related properties of some cryogenic systems, a bibliography, TN56 (PB161557).

mathematics, $1445 \mathrm{~A}$.

measurement and medicine, 795 .

physico-chemical constants of leather and collagen, 510.

properties of chromium cobalt alloys, 2687.

properties of electrodeposited metals, I: Nickel, 3: The effect of plating variables on the structure and properties of electrodeposited nickel, 511.

properties of gallium copper-tin alloys, 1876 .

properties of glass-fabric reinforced polyesters, 1004. properties of high-density thorium oxide, 1877.

properties of plastic teeth, 2688.

properties of terpenes. III. The vapor pressures of alpha pinene and beta pinene, $1191 \mathrm{~A}$.

standards for the electronics industry, 1808.

standards, national, and design of experiment, 2210.

titanium carbide type cermets at elevated temperatures, characteristics, 1445.

Physical-chemical studies of the destructive alkali-aggregate reaction in concrete, RP2780.

Physics, and electronics, dictionary of, contributions, $1660 \mathrm{~A}$.

elasticity of rubber, 2251.

electrostatic field and the symmetry of snowflakes, 2689.

chemical and engineering measurements, how statistics improve, 68 .

experimental, 2990.

low temperature, $1055 ; 2180$.

measure for measure: some problems and paradoxes of precision, 287.

neutron, 2500.

polymer, and their relation to dielectric properties, 2440 .

pure and applied, 189.

units and dimensions, 611 .

Physiological monitor for anesthesia, 1446.

Picric acid, 1085.

Piezoelectric, accelerometers, cemented, 660.

constants of alpha- and beta-quartz at various temperatures, 108.

effect in indium antimonide, 2690.

elastic, dielectric constants of quartz, method for dynamic determination, Mono. 9 .

Piezoresistance of indium antimonide, 2252.

Piezoresistivity of semiconductors, apparatus for measuring, RP2814.

Pigmentation, ocular, tritanopia, 161.

Pile oscillator measurements of eta, 3015 .

Pillowcases and sheets, standards help in the selection, 136.

PILOT, a new multiple computer system, 3016.

NBS multicomputer system, 2692.

plant studies of the low temperature distillation of hydrogen isotopes, 2691.

plant study of the process for treating heavy leather with polyisobutylene and other polymers, 1240 .

Pion, coherent neutral, production, nuclear matter distributions, 2653 .

Pipe flow, fully developed, structure of turbulence, 884 .

Pipes, asbestos-cement, 998.

Piston, attenuator, chart for $\mathrm{TE}_{11}$ model, 664 .

gage as a precise measuring instrument, 877 .

gage, dead weight, 2103.

gage for pressures below one-half inch of mercury, tilting air-lubricated, $\mathrm{J} 63 \mathrm{C} 1,47$.

Pitfalls in computation, 1809 .

Pits, in metals caused by collision with liquid drops and rigid steel spheres, J 64A1, 61 .

metals caused by collision with liquid drops and soft metal spheres, RP2958.
Plan for studying the accuracy and precision of an analytical procedure, 926 .

Planet, lost, and Tektites, 1525; 1912.

Plant fibers, 1181.

Plasma, electromagnetic waves, 3023.

infinite conductivity, structure of a magnetohydrodynamic shock wave, 1902.

jet as a spectroscopic source, 3088 .

oscillations, solar "enhanced radiation", 544 .

solar, condition for radiation, 414.

Plasmas, conductivity of, to microwaves, 2914.

Plaster, setting of, 3058.

Plastic, acrylic, glazing, 699.

elastic behavior of the ferrite lattice in low-alloy steel, 1691.

Committee, International, considers standard tests, 1376.

deformation of ceramic-oxide single crystals, $1108 ; 2253$.

electrets and their applications, 797 .

electrets, transparent, 796 .

films, and coated fabrics, 1337.

films, comparison of methods of measuring the tensile and tear properties, 1290.

films, properties of, 1122 .

fired, refractories and other more resilient materials, dynamic and static tests for mechanical properties, 1684.

foams, low temperature properties, 2620.

insulating, foams at low temperatures, 2985.

phosphor matrix for fast-neutron detection, 2693.

refractories, 2289 .

springs, 1810.

springs, reinforced, 1843.

steel surfaces on clotting time of human blood, effect of, 1003.

teeth, bonding of, to heat cured denture base resin, 402.

teeth, physical properties, 2688.

teeth, proposed specification, 2272 .

Plastics, 512; $1811 ; 2254$.

aircraft, 2255 .

eye protection, spectratransmissive properties, 1547.

filled, surface erosion, 2792.

glass-fiber reinforced, effects of molding pressure on strength properties, 2533.

glazing, acrylic, 1065 .

glazing, by multiaxial stretching, 2084.

industry, standardization research, 1857.

industry, standards and research for, 1130.

irradiated, films, 3056.

laboratory aging tests for, fluorescent sunlamps, 1713.

laminated, fuel-immersion, 50.

literature in Government reports, 1109.

organic, 109.

resistance to tension, 1855.

standards, 1509.

tension testing, 2341.

test, methods, specifications, and standards, 1167.

thermosetting, at low temperatures, 464.

transparent, creep behavior, 2921.

transparent, effects of multiaxial stretching on crazing, 1002 .

transparent, effects of multiaxial stretching on crazing and other properties, 1690.

year 1952 in review, 894 .

Plate separation requirements for standard free-air ionization chambers, 1110 .

Plates, electrostatic deflection for cathode-ray tubes, $J$ $64 \mathrm{C} 2,103$.

external, grounded, electrostated fields, 1066.

ship, analysis of brittle behavior, 954 .

square clamped, vibration, 1435 .

Plating at NBS, research, 2736.

electroless, 1010.

electroless nickel, 2327.

experimental, of gun bores to retard erosion, TN46 (PB151405).

standards and specifications, 1447. 
Platiniferous materials, chemical methods for separating and determining metals contained in, 668 .

Platinum-uranium system, J 64A1, 107.

Plumbing, drains, capacities, 2489.

stacks, capacity, 1281.

system design, 239.

Pneumatic, components and systems, pressure drop, 1456. components, determination and correlation of flow capacities of, C588.

valves, characteristics, pressure flow, discussion of paper, 2092.

Point Barrow, Alaska, layered earth propagation in the vicinity of, $\mathrm{J} 64 \mathrm{D} 1,95$.

Point-to-point radio relaying via the scatter mode of tropospheric propagation, 1812.

Poisoning and reactivation of orthoparahydrogen conversion catalyst, 3017 .

Poisson, and Bernoulli, positive, variables, tables of expected values of $1 / \mathrm{X}, 1161$.

distribution, mean deviation, 2823.

Poisson's ratio for copper wires, measurement of the resistance-strain relation, 1768 .

ratio of aircraft sheet materials for large strains, 798

Poker model, continuous, symmetric, J 64B1, 35 .

Polar, blackout occurence patterns, 2256.

blackouts, geographic and temporal distribution of, 1024 .

Polarization, bremsstrahlung, measurements, 1643.

concentration, 2605.

effects: penetration and diffusion of hard X-rays, 793.

energy for $3 d$ and $4 p$ electrons, 1448.

high-frequency limit of 500 bremsstrahlung, 2694.

induced electrical, 2431.

limiting, of magneto-ionic waves, 583 .

low frequency ionosphere echoes, 371.

measurements of low-frequency echoes, 513.

quantum mechanics, 936.

sferics, 2257 .

sweep frequency, measiurements at low frequencies, technique for, 938 .

Polarized $\mathrm{CO}^{56}$ and $\mathrm{CO}^{58}$, absence of interference effects in the $\beta$-decay, 2006.

light, optical rotation, by chemical compounds, 285. manganese- 52 , beta-gamma correlations, 2473 .

nuclei, beta-decay, 2557.

nuclei, $\beta$-decay of, further experiments, 2132 .

nuclei, beta gamma correlation, 2902 .

waves, VHF tropospheric recording measurements of plane and circular, in the Great Lakes area, 620 .

Polarographic determination of trace quantities of iodide, 2258 .

Pollution, air, determination of carbon monoxide in studies, 1309.

Polya freauency functions, III, The positivity of translation determinants with an application to the ir terpolation problem by spline curves, 782 .

Polyacrylonitrile, polybutadiene, and copolymers of butadiene with acrylonitrile and styrene, thermal degradation, RP2888.

Poly-alpha-methylstyrene, pyrolysis, 2707.

Polyamides, influence of impurities on the pyrolysis, $\mathrm{J} 63 \mathrm{~A} 3,269$

pyrolysis of, RP2819.

Polyatomic molecules, 1144.

Polybutadiene, and benzene, heat of mixing, RP2921.

Polybutadiene-benzene, some thermodynamic properties of the system, RP2820.

Polybutadienes, melting behavior, 1938 .

Poly (chlorotrifluoroethylene), semicrystalline, specific volume and degree of crystallinity, RP2862.

specific volume and degree of erystallinity, 2831.

Polycrystalline aluminum alloys, crystal orientation on fatique-crack initiation, 2096 .

materials, magnetic properties, $2980 \mathrm{~A}$.

Polyester, film, tensile properties, 2354.

laminates, effect of humidity during fabrication, 435 .
Polyesters, absorption on glass, silica, and alumina, RP2933.

glass-fabric reinforced, 1004

Polyethylene, and fibrous, low-angle X-ray diffraction, 2616 .

glycol electrolytes, punched-cell batteries, 1828 .

oxide and polypropylene oxides, thermal degradation, 3100 .

polymethylene, depolymerization, 985 .

polystyrene in a vacuum, rates of thermal degradation, 526 .

polystyrene, thermal degradation, 1209

Polygonal membranes, asymptotic lower bounds for the frequencies of, 957 .

Polygons, algebraic proof of the isoperimetric inequality, 1259 .

Polyhedra, convex, integral boundry points, 1743 .

flames with hydrogen and hydrocarbons, 514.

Polyisobutene-benzene, some thermodynamic properties of the systems, RP2820.

Polyisobutylene, and other polymers, pilot plant study of the process for treating heavy leather, 1240.

dynamic compliance, dynamic modulus, and equivalent Voight and Maxwell models, 692.

dynamic mechanical properties, 1183 .

rheology, 1132; 3037 .

treatment of leather, 607 .

viscosity, 1182 .

Polyisobutylenes on shearing in solution, degradation, 682.

Polymer, crystals with folded chains in dilute solution, theory of formation of, J $64 \mathrm{~A} 1,73$.

degradation studies by absorption and mass spectrometry, 515 .

dilute, solutions, $141 ; 166$.

formation and decomposition, 2631.

molecules, flexible, crystallization, 1924.

molecules in solution, rate of thermal diffusion, 1472

networks formed from oriented chains of natural rubber, some properties, 2765 .

networks, isotropic length, 2370.

physics, and their relation to dielectric properties, 2440 .

solutions, concentrated, kinetics of capillary shear degradation, 1753 .

solutions, effects of capillary shape of flow characteristics and degradation, RP2839.

structure research at the National Bureau of Standards. 1813 .

Polymer-diluent mixtures, 1382.

Polymeric, amorphous high, systems, viscoelastic phenomena, 2863.

systems, crystallization kinetics, 2507; 2923.

Polymerization, data on the ICCA "standard" pulps, 2890.

methyl methacrylate, accelerating effect of aminos, 1608 .

methyl methacrylate, sulfinic acid derivatives as accelerators, 1906 .

shrinkage and water sorption, dimensional changes: direct filling resins, 689 .

sorption of water by polymethyl methacrylate, effect of temperature and degree, 1328.

temperature rise, direct resinous filling materials, 223 .

Polymers, $\alpha$ and $\beta$ deuterostyrene, pyrolysis, 1464 .

amorphous, over an extended temperature range, volume temperature, 1975 .

atomic radiation, 1634 .

chemical structure and stability relationships. 2910 .

crystalline, 3083 .

degradation of, 976 .

deuterated styrene, 2680.

effect of structure on the thermal decomposition of, RP2860.

effects in, mechanism of high energy radiation, 2194. effects of atomic radiation on, 698 .

exposed to high-energy radiation, factors influencing the behavior, 1343 .

glass formation in: the glass transitions of the poly(n-alkyl methacrylates), 2142. 
glass formation. II. Rubber-sulfur system, RP2944. high, degradation, 43 .

high, mechanical properties, 2630.

high temperatures, thermal degradation, $\mathrm{J} 63 \mathrm{~A} 3,261$.

high, thermodynamics of crystallization; gutta percha, 1965

high, thermodynamics of erystallization: natural rubber, 1558 .

high, thermodynamies of erystallization, poly(ethylene), $28 \pm 6$.

hydrocarbon, pyrolysis, 116.

kinetics of crystallization in, $1047 ; 1382$.

les rayons et les neutrons, degradation, 681 .

sole leather, impregnation, 2594.

solid, photochemical degradation, 2455 .

styrene and acrylate, in a vacuum, 811 .

styrene and $\alpha$-deuterostyrene, oxidative degradation, 1802 .

thermal decomposition, 1556 .

thermal degradation at low rates, RP2957.

thermal degradation of, as a function of molecular structure and branching, 1557.

thermal degradation, temperatures up to about $800-900^{\circ} \mathrm{C}, 2843$

Polymethyl, alpha-chloro-acrylate, properties, 520 .

melting transition of, 869 .

methacrylate, $838 ; 999 ; 1328$.

methacrylate and polystyrene, gamma irradiation, 2138 .

methacrylate at various temperatures, stress and strain at onset of crazing, 555;669.

methacrylate, determination of monomer content, 1673 .

methacrylate: dilute solution thermodynamic behavior of flexible chain molecules, 687.

methacrylate, fracture phenomena and molecular weight, 2569.

polyethylene, depolymerization, 985 .

Polymorphism in barium disilicate, 3018 .

Polymorphs of silicon dioxide and germanium dioxide, infrared studies, RP2885.

Polynomial Chebyshev approximation, derivative, 786 legendre, 659 .

Polynomials, Chebyshev, in the solution of large-scale linear systems, 411.

Chebychev, use of, in thin film computations, J 63A3, 297.

complex biorthogonality for certain sets of, 975 .

given degree, best approximation, 1641 .

least pth power, on a real finite point set, 1385 .

Legendre, second order determinants, 305.

orthogonal, and Bessel functions, 249.

ultraspherical, $276 ; 298$.

ultraspherical, and Bessel functions, inequalities, 72 .

ultraspherical, relative extrema, 106 .

zeros, J 63B1, 21.

zeros of, and the degree of linear systems, 790 .

Polyphenyls, crystalline, infrared spectra, RP2829.

fluorinated, by mass spectrometer, analysis, 1263 .

fluorinated, synthesis and properties, 1521 .

isomeric, by adsorption chromatography, separation, 2752 .

mass spectra and relative sensitivities, RP2831.

$m$-polyphenyls, synthesis and infrared absorption spectra, 1951.

Polypropylene oxides and polyethylene oxide, thermal degradation, 3100 .

Polysaccharides, and cellulose, 2403.

application of viscosity methods to the study of branching in, 654 .

carbon-14, carboxy-labeled, RP2880.

micromethylation, 2198.

reaction with carbon-14-labeled cyanide, determination of reducing sugars and reducing end groups, 2517.

Polystyrene, 1220 .

glassy state, thermal and photochemical processes, 1958.

polyethylene, degradation, thermal, 1209. polyethelene in a vacuun, rates of thermal degradation, 526 .

polymethyl methacrylate, gamma irradiation, 2138. study of degradation, by means of mass spectrometry,

Polytetrafluoroethylene, thermal conductivity, RP2805.

Poly (vinyl chloride), heat and ultraviolet aging, RP2863. infrared spectra of thermally degraded, R P 2832 . thermal decomposition, 3099 .

Population, normal, estimating the standard deviation of 713.

Porcelain, enamel ground coats to steel, 2358.

enamel to steel, adherence of, relation between roughness of interface and adherence, 2281 .

enameled hot water tanks, $27+5$.

enameling, a radioisotope study of metallic deposits in, 1941

enameling industry, practical test methods, 1452 .

enamels, weatherability, $1578 ; 1579$

jacket erowns, 2000 .

thin, enameled sheet steel, flexibility, 2126.

Pore conductors, properties, 1461 .

Pores in collagen and leather, 1449.

Porosity in electrodeposits, nature, cause and effect, 1940.

Portable potentiometer and thermostated containers for standard cells, 1450 .

Portland and high-alumina cements during hydration, 2768.

Portland eement, 736 .

chemistry, 327 .

flame photometric determination of strontium, 1346 .

hydrated, some factors affecting the surface area, $\mathrm{RP} 2806$.

past, some effects of aging on the surface area, J $64 \mathrm{~A} 2$, 163.

reaction with carbon dioxide, RP2858.

Positive harmonic functions of ultra-spherical polynomials, 276.

Positrons, and electrons, 1204.

electrons, backscattering, 567 .

electrons, elastic scattering by point nuclei, 1694

electrons, energy loss and range of, C577 Suppl.

electrons, relative back-scattering, 300 .

electrons, transmission, 1560

energy loss and range of, C577 Suppl.

Possible movements of 5577 airglow, 2695.

Potassium, alum, chromium, 1067.

borohydride, heat content of, from $0^{\circ}$ to $400^{\circ} \mathrm{C}$, RP2 28 .

bromide for infrared radiant energy, 812 .

calcium silicate, heat of formation, RP2955.

chromate as a transmittance standard in the ultraviolet, alkaline solution, 388 .

iodide fast neutron detector, 1451 .

oxide, $2364 ; 3059$.

three potassium-sodium alloys between $0^{\circ}$ and $800^{\circ}$, the triple point and heat of fusion of potassium, heat capacity, 462 .

Potential, retarding, measurements, electrical differentiation, 2596.

theory using the Nehari estimates, numerical experiments, 2654 .

Potentiometer, and thermostated containers for standard cells, portable, 1450 .

Lindeck, 1389.

low-cost microvolt, 2178.

self-balancing, 179 .

Potentiometers, helical, 1070.

Pound of gas, 927 .

Powder, dry, fire extinguishment, 2123.

Powder patterns, diffraction, standard X-ray, C539, Vols. $7,8,9$.

Powders and foams, heat transfer, 2147

Power, loss and operating temperature of tires, J 64C1, 1. microwave, measurements, 2720 .

microwave, measurements, mismateh errors in, 769 . 
Power output of a sound source in a reflecting environment, 3089.

requirements and choice of an optimum frequency for a worldwide standard-frequency broadcasting station, J 63D1, 35 .

resolution of corpuscular spectrometers, 2791.

spectra and the minimum detectable signal in measurement systems, 1430 .

spectra, instantaneous, 469 .

stabilizer, microwave, 90 .

supplies for 60-cycle tests of electrical instruments and meters, 1814.

supply, low-capacitance, 1391

test chart, new, resolving, 268.

$\operatorname{pr}(n)$, some theorems, 2305.

Practical, evaluation of integrals, 1098

suggestions for reducing radiation exposure in diagnostic examinations, 2259 .

test methods for the Porcelain Enameling Industry, 1452 .

Prandtl number, 1207.

Precise, coulometric titrations, 1815.

coulometric titration of acids and bases, J 63A2, 153 .

evaluation of surface area with indirectly calculated dead space, RP2826.

intercomparison of acids by differential potentiometric titration with hydrogen electrodes, RP2769.

intercomparison of acids by differential potentiometric titration with the hydrogen electrode, 2260 .

measurement and race for technological supremacy, 2696.

measurement of heat of combustion with a bomb calorimeter, Mono.7.

measurement of the infrared spectra of molecules of the atmosphere, 2377.

measurements in the infrared spectrum of carbon monoxide, 516 .

time synchronization of widely separated clocks, TN22 (PB151381)

Precision, 5-10 kv high-voltage supply, 1453.

measurement, 2261 .

problems and paradoxes, 287.

quartz resonator frequency standards, 1111

thermostat for high temperatures, 110 .

transistor oscillator, 799 .

Predicted lines of $\mathrm{Fe} \mathrm{I}$ in the arc and in the sun, 111.

Prediction, cumulative distribution with time ground wave and tropospheric wave transmission loss. Part 1. The prediction formula, TN15 (PB151374).

pressure drop in two-phase single-component fluid flow, 3019 .

study of complex systems, some aspects, 2225 .

sunspot numbers for cycle 20,3020 .

system behavior, $2660 \mathrm{~A}$.

system performance from information on component performance, 2223.

Predictions of the present sunspot cycle, 1112.

Predictor-corrector, alternative, process, 389.

Preface to Proc. 3d Symp. on cosmical gas dynamics, 2697.

Preferred, circuits manual with engineering notes, 1113.

circuits program, NBS, 1412.

orientation in stark rubber studied with an automatic integrating pole-figure goniometer, 1454 .

Preliminary, analysis of amplitude scintillations of radio stars observed at Boulder, Colorado, TN20 (PB151379).

list of levels and $g$-values for the first spectrum of thorium ( $\mathrm{Th} \mathrm{I}$ ), J $63 \mathrm{~A} 3,275$.

results of the National Bureau of Standards radio and ionospheric observations during the International Geophysical Year, J 63D1, 1.

study of radiometeorological effects on beyondhorizon propagation, J $64 \mathrm{D} 3,239$.

Prelubricated and unlubricated ball bearings at high speeds and extreme temperatures, friction and endurance of, J $63 \mathrm{C} 1,19$.

Prepackaged commodities, checking, H67.
Preparation, and maintenance of radioactive standards by the NBS, 1455 .

bituminous materials for spinning, 800 .

carbon-14-labeled cyanide, 2262.

high-purity hydrogen deuteride, 153.

maintenance, and application of standards of radioactivity, C594.

maintenance of standards of radioactivity, 1944.

metallic iron of high purity, 801 .

new solution standards of radium, RP2924.

pure hydroxyapatite crystals, 1816.

small quantities of germanium tetramethyl, 517 .

some aryl silanes, 1192 .

trichloride and tetrachloride of molybdenum, J $63 \mathrm{~A} 2,185$.

two fluorinated $p$-dihalobenzenes, 879 .

Preparing euprammonium solvent and cellulose solutions, 112 .

Present, experimental status of rare-earth spectra, 3090

status and future prospects of emission spectroscopy, 3021.

status of color television, 154.

status of research on the physical constants at the (United States) NBS, 2263.

status of standards for shoe last sizes, 113.

status of temperature scales, 590 .

Preservation, of documents by lamination, Mono.5.

Declaration of Independence and the Constitution of the U.S., 288.

record papers, 1840

Press specimens, semi-dry, 2501.

Pressure calibration of condenser microphones above $10,000 \mathrm{cps}, 1114$.

drop in pneumatic components and systems, 1456.

drop in two-phase single-component fluid flow, 3019.

Pressure-flow characteristics of pneumatic valves, discussion of the paper, 2092.

Pressure-sensitive diaphragm-type null detector, 2698.

Pressure-volume temperature relations of liquid normal deuterium, 1115 .

Pressures, developed in cement pastes and mortars by the alkali-aggregate reaction, 2699.

wind, in various areas of the United States, BMS152.

Pretied tie for communication line wires, 2432.

Prevention of microbiological deterioration of leather, 1817.

Primary, standard of frequency, 737.

standards, atomic definition, 28.

Principal submatrices of a full-rowed nonegative matrix, $\mathrm{J} 63 \mathrm{~B} 1,19$.

Principle of randomization in the design of experiments, 2378 .

Principles of experimental design, 880 .

Prism, cesium iodide, infrared measurements, 742 .

Prism, cesium iodide, infrared spectrometry, 744 .

Prism, effect, camera tipping, and tangential distortion, 2264 .

Kosters double-image, 2822.

location of the principal point, effect, 2350 .

Probability, distribution of the amplitude of a constant vector plus a Rayleigh-distributed vector, 1541 .

distribution of the phase of the resultant vector sum of a constant vector plus a Rayleigh distributed vector, 591 .

elements, 2500 .

elements of a mathematical theory, 708 .

limits for the average chart when process standards are unspecified, 1116.

methods for the numerical solution of certain problems in analysis, 763 .

saddle point, 2379.

theory and differential and integral equations, 278 .

Probe tube method for the transfer of threshold standards between audiometer earphones, 632 .

Probing the ionosphere, 2700.

Problem, in matrix minimization, 1985.

self-heating of a spherical body, RP2773. 
stability in the numerical solution of partial differential equations, 1588 .

theory of mechanical quadratures, 1423 .

Problem, smoothing, remark on, 931.

Problems, algebraic number theory, some computational, 1873.

arising in engineering investigations, 1457 .

drainage and venting, 289.

linear programming, how to solve, 1360 .

logical design, 1818.

multiply connected domains, boundary value, 404 .

random walks in space, 310 .

theory of non-linear vibrations, 277.

ultramicrotomy, 309.

unsolved, in review, 1568 .

Procedure, analytical, 926.

improve the quality of ionospheric data, 1117.

Procedures for the rejection of suspected data, 784 .

Proceedings, of the Berkeley symposium on mathematical statistics and probability, 114.

Processing, denture base resins: heat-curing type, 1819. mechanized, components, 1291.

production, and metrology, 1777.

products, of cool flame oxidation of propane-2-C 13, 2702 .

second symposium in Linear Programming, 1458.

smooth patterns, 1139 .

square temperature wave in a filament operating at low temperatures, 2701 .

two-dimensional patterns by scanning techniques, 802 .

volatilized from a channel and a furnace black on heating to $1,000^{\circ} \mathrm{C}, 803$.

Profiles, ionospheric laver, joint use of the ordinary and extra-ordinary virtual height curves in determining, $\mathrm{J} 64 \mathrm{D} 2$, 111.

Program, of the International Commission on Radiological Units and Measurements, 3022.

training and graduate instruction in dental materials at the NBS, 2827 .

training, weights and measures, 182.

Programmers, some general precepts, 545.

Programming, for finding the characteristic values of Mathieu's differential equation and the spheroidal wave equation, 518 .

linear, $1458 ; 1757$.

linear, in bid evaluation, 1051.

linear, systems of distinct representatives, 1910 .

problems, linear, how to solve, 1360 .

scientific computers, 1820 .

system for codesorting letter mail, TN50 (PB151409).

Programs for computing hypergeometric series, 1118.

Progress, in metal cleaning and finishing, 1459.

report by Committee of Optical Society of America on Uniform Color Scales, 1459A.

report on aut?matic data processing in Government (1956), 1822.

report on SEAC, 1995.

Projected images used with range finders, 'RP2851.

Prolonged space-wave fadeouts at 1,046 Mc observed in Cheyenne Mountain propagation program, 1119.

Proof testing rubber tires, 519.

Propagation, and initiation of fatigue crack, distinction between, 1925.

around a sphere, an exact earth-flattening procedure, $\mathrm{J} 64 \mathrm{D} 1,61$.

beyond-horizon, preliminary study of radiometeorological effects on, J 64D3, 239.

characteristics of the frequency band 152-162 Mc which is available for marine radio communications, 1945 .

constant in rectangular wave guide of finite conductivity, 1120 .

curved stratified atmosphere, mode expansion in the low-frequency range, $\mathrm{J} 63 \mathrm{D} 1,75$.

electromagnetic and acoustic pulses, Part I: diffraction of pulses by wedges, 1799 .

electromagnetic waves along a curved surface, 2673. errors in spectrophotometric colorimetry, 804. errors in tristimulus colorimetry, 2266.

ground wave pulse around a finitely conducting spherical earth from a damped sinusoidal source current, 3024 .

ionospheric, by the scatter-sound method, 2326 .

ionospheric scatter, 3011.

ionospheric, very low frequency, 2203.

long waves, 2721.

low frequency, ionosphere disturbances, 229 .

low-frequency, in arctic areas, J 63D1, 99.

measurements, an analysis of, made at 418 megacycles per second well beyond the radio horizon (a digest), J 64D3, 255.

measurements made at $418 \mathrm{Mc}$ well beyond the radio horizon, analysis of, TN6 (PB151365).

measurements near the radio horizon, tropospheric scatter, 1499.

oblique incidence, at $300 \mathrm{kc}$ using the pulse technique, 493.

off-path, at VHF, 2656.

production of electromagnetic waves in a plasma, 3023.

pulse across a coast line, 2265 .

radio, at 96.3 mc between Richmond, Va., and Washington, D.C., study of some of the meteorological effects, 635 .

radio electrical, 2405 .

radio frequency ground wave transient sinusoidal over a finitely conducting plane earth, 2267 .

radio ground waves, $\mathrm{RP} 2770$.

radio, in the TV and FM bands, factors affecting, 1342.

radio, low and medium frequency, 2615 .

radio, new kind, at very high frequencies observable over long distances, 369

radio research at the NBS, 2713.

radio, transmission loss in, 90 .

radio, transmission loss, II, TN12 (PB151371).

radio, transmission power, 3102 .

radio wave, 2331 .

radio wave, beyond the horizon, 228 .

radio wave, in an inhomogeneous atmosphere, TN24 (PB151383)

research, fading rate recorder, 2949.

scatter, 1544

second sound, in liquid helium, II, 821.

sound in five monoatomic gases, 1823 .

sound in rarefied helium, 115.

sweep-frequency, 1498.

terrestrial, of very-low-frequency radio waves - a theoretical investigation, J 64D2, 153.

time-varying media, information theory, aspects, 1143; 1364 .

transient ground wave, note, 1992.

tropospheric, beyond the horizon, $351 ; 470$.

tropospheric, beyond the horizon, role of partial reflections, 595 .

tropospheric forward scatter, TN53 (PB161554)

tropospheric 1948-1956, TN26 (PB151385).

tropospheric, partial reflections, 508 .

tropospheric, point-to-point radio relaying via the scatter mode, 1812

tropospheric, radio wave scattering in, 808 .

tropospheric radio wave, some aspects, 1872.

tropospheric scatter and atmospheric circulations, J $64 \mathrm{D} 1,81$.

turbulent atmosphere over irregular terrain, 1550.

turbulent atmosphere, rate of fading, 1542.

very high frequency by ionospheric scattering-survey of experimental results, 1971

very high frequency measurements in the Rocky Mountain region, 1972 .

VHF ionospheric, regular, 1495.

VHF over irregular terrain, 1663

VHF scatter, high-gain antennas, 1729.

very-low-frequency, in the presence of a transverse magnetic field, J 64D3, 265.

VLF ionospheric for finite ground conductivity, 2373.

VLF ionospheric, mode theory, 2234; 2456. 
VLF ionospheric, waveguide mode theory, 2390.

VLF multiple reflections between the earth and the ionosphere, 1781.

very-low-frequency pulses to great distances, RP2898.

VLF radio waves, TN58 (PB161559).

VLF skywave geometrical optics, 2360.

VLF using atmospheric waveforms, 2760.

very low radio frequencies, transmission loss curves, 2851.

vicinity of Point Barrow, Alaska, layered earth, J $64 \mathrm{D} 1,95$.

Propagation, wave, 1147.

anisotropic, inhomogeneous medium, 910.

breaking velocities, strain energies, and theory neglecting, 1277 .

ionospheric, 67 .

long textile yarns impacted transversly, 2783.

over an irregular surface, integral equation approach to the problem, 394 .

through an inhomogeneous medium, 629 .

Propane-2-C $\mathrm{C}^{13}$, products of cool flame oxidation, 2702. separation of the products of cool flame oxidation, 2753.

Properties of arsenic sulfide glass, RP2774.

concrete at high rates of loading, 1824

corrugated diaphragms, investigations, $1378 ; 2166$.

$\mathrm{Cr}^{+++}$ion in the paramagnetic alums at low temperatures, 522 .

diamond, 2762 .

fibers, 1121.

fluorine compounds, the vibrational spectrum of tetrabromoethylene, 1825 .

gases at elevated temperatures, 2664.

glasses in the system barium oxide-boric oxide-silica, 2763.

impact, boron-treated steels, study, 1601 .

intermetallic compounds, electrical and optical, 1007.

lightning impulses which produce whistlers, 2764 .

magnetic, below $1^{\circ} \mathrm{K} ; 756$.

materials at low temperatures, 2268 .

mechanical, of some engineering materials, 2193.

mechanical, of structural materials at low temperatures, Mono.13.

$\mathrm{Mg}_{2}-\mathrm{S} n, 1460$.

physical, chromium cobalt alloys, 2687.

plastic films, 1122.

properties of flameproof fabrics, 3050 .

similarity, of the two-fluid model of superconductivity, 543 .

struve functions, 2269 .

thermodynamic, of helium at low temperatures and high pressures, TN8 (PB151367).

transport, of dilute alloys of copper, $2852 \mathrm{~A}$.

Property, of additively closed families of distributions, 2433.

experimental map papers containing synthetic fibers, 2703.

normal distribution, 928 .

polymer networks formed from oriented chains of natural rubber, 2765 .

polymethyl alpha-chloro-acrylate, 520 .

pore conductors, 1461 .

portland cement pastes cured at elevated temperatures and pressures, 1826.

rayon fabries, factors affecting, 446 .

self-curing denture base resins, 521 .

sodium titanium silieate glasses, RP2890.

synthesis of fluorinated polyphenyls, 1521 .

zinc borosilicate glasses, RP2930.

Propionate, acetate, and butyrate radicals, heats of combustion of some peroxides and the heats of formation, 2148.

Proposal for standard frequeney broadeast at very low frequency, 2270 .

Proposals for the mechanical resolution of German syntax patterns, 290.

Proposed, criteria for defining load failure of beams, floors, and roof constructions during fire tests, J $63 \mathrm{C} 2$, 121 . index of current noise for composition resistors, 1241. nomenclature for linear viscoelastic behavior, 2271 . recommendation on ionospheric vertical soundings after the I.G.Y., 2704.

specification for dental chromium-cobalt casting alloys, 1996.

specification for plastic teeth, 2272 .

standard for facilities for scientific conferences, 2434.

Prosthetic, dentistry, acrylic resins, 2449.

dentistry, resins, 2738.

Protection, design, Roentgen ray, editorial, 433.

ionizing radiation, 1151 .

low-strategic alloys with a chromium boride-nickel cermet coating, 1463 .

molyhdenum from oxidation at elevated temperatures, 2705 .

neutron radiation up to 30 million electron volts, H63.

use of dental roentgenographic equipment, 1462.

Protective coating adhesion measurement using an electronic averaging device for the adherometer, 2273.

Protein, dilute, solutions, interpretation of hydrodynamic data, 2668.

Proton, gyromagnetic ratio, $2422 ; 2706$.

Proton, high energy, emission spectra of nitrogen bombarded, 576 .

Protons, high-energy, and hydrogen atoms, intensity distributions in nitrogen bands excited in auroras, 1044.

Pyrolysis of poly-alpha methylstyrene, 2707.

Pseudo-analytic functions, expansion theorem, 577.

Pseudo-random numbers, generation and testing, 1722.

Pseudoternary system calcium oxide-monocalcium aluminate $\left(\mathrm{CaO} \cdot \mathrm{Al}_{2} \mathrm{O}_{2}\right)$-dicalcium ferrite $\left(2 \mathrm{CaO} \cdot \mathrm{Fe}_{2} \mathrm{O}_{3}\right)$, RP2900.

pth power polynomials on a real finite point set, 1385.

Pulp and paper, 805 .

annual review, 1466.

Pulp and paper industry, annual review, 1627; 2024.

Pulps, ICCA "standard", 2890.

Pulse, across a coast line, propagation, 2265.

circuit, accurate time-modulated, 646 .

generator and high-speed mémoire circuit, 1827.

generator, incremental delay, 191.

height analyzers, multichannel, 2207.

height measurements of recoils from $\mathrm{B}^{10}(n, x) \mathrm{Li}^{7}$, 1123.

method for determining dynamic stress-strain relations, 1997.

sky wave phenomena observed at $100 \mathrm{Kc}, 2274$.

Pulsed magnetic extractor for removing the electron beam from a betatron, 929 .

Pumps, and bearings at low temperatures, operation, 2676. performance of, with liquefied gases, 2243.

Punched, cards, tables, 177.

cards, tables on, addendum to guide, 641 .

cell batteries with polyethylene glycol electrolytes, 1828.

Purification of mercury and its physical properties, 2275.

Pyranoid sugars, conformations of. II. Infrared absorption spectra of some aldopyranosides, J 64A3, 239.

Pyrex and silver, stability in perchloric acid-silver percholrate solutions in conductivity water, J $64 \mathrm{~A} 1$, 127.

Pyrolysis, of $\alpha$ and $\beta$ deuterostyrene polymers, 1464. hydrocarbon polymers, 116.

mixtures of ethane and ethane- $d_{6}, 291$.

polyamides, RP2819.

polyamides, influence of impurities, J 63A3, 269.

tribromofluoromethane, hexafluorobenzene, 2151.

Py rometry and thermometry, 2500.

Pyrophosphate formation upon ignition of precipitated basic calcium phosphates, 1465 .

\section{Q}

Quadratic fields, and matrices, classes, 412.

form, a note on values, mathematics, 2186 . 
Quadrature errors for analytic functions, 1096.

Quality of gray tin erystals and their rate of growth, 2672.

Quantitative, chromotographic procedure for determining dextrose in sugar mixtures 2276 .

determination of copper in natural rubber, 523 . determination of natural rubber hydrocarbon by refractive index measurements, 292.

Quantum, interactions of the free electron, $1659 ; 1829$.

mechanical calculation of the probability of an exchange reaction for constrained linear encounters, 3025 .

mechanical effects on the surface tension of simple liquids, 2708 .

mechanies by density matrix and operator techniques, description of states, 2081.

Quarterly radio noise data-March, April, May 1959 , TN18-2 (PB151377-2).

Quartz, alpha- and beta-, piezoelectric constants at various temperatures, 108

anelasticity of, 653 .

crystal resonators and oscillators at low temperatures, 2734.

crystalline, 2022.

crystals, detwinning, TN3 (PB151362).

elasticity and dielectric loss, 1620 .

method for the dynamic determination of the elastic, dielectric, and piezoelectric constants of, Mono.9. precision, resonator frequency standards, 1111 .

resonators at very low temperatures, stability, 2315. small precision bottles, and low-loss glass, 2224 .

Quaternion matrices, 967.

Quaternions, powers of, elementary note, 390.

$\mathbf{R}$

$\mathrm{R}^{\mathrm{n}}$, new criterion of univalence of transformations, 3106 .

Rack for standard resistors, 3026 .

Radar, scattering by the moon, theory, J $64 \mathrm{D} 3,217$. waves from meteor trails, interpretation, 338 .

Radar-type, echoes from meteor trails, wave-frequency dependence of the duration, 348 . reflections, 176 .

Radial distribution of the eenter of gravity of random points on a unit circle, RP2847.

Radiant, energy source, method for measuring surface flammability of materials, 1593 .

energy, unpolarized, in the analysis of infrared absorption spectra, 1569

glass heating panels, 807 .

Radiated harmonics, measurement of, source of error, 377 .

Radiation, atomic, and polymers, 1634 .

atomic, on polymers, effects of, 698 .

attenuation data, 3027 .

contribution of the annihilation, to the gamma-ray flux in lead, 2917.

dangers, 1305 .

data during dental $\mathrm{X}$-ray exposure, ionization chambers, 1380 .

enhanced, solar, and plasma oscillations, 544 .

exposure and the use of radioisotopes, 2277 .

exposure as a reasonable calculated risk, 2709.

exposures to man, maximum permissible, 2187.

field from a rectangular source, J $64 \mathrm{C} 2,121$.

gamma, from $\mathrm{Ag}^{105}, 1718$.

gamma, from $\mathrm{Z} n^{63}, 2956$.

gamma, multipled-scattered, from a plane isotropic source, angular distribution, 1265.

gamma, near the interface between two media, calculation of energy dissipation, 2043.

general relations between fluxes from collimated point and plane sources, 2957.

high-energy, behavior of polymers exposed, factors influencing the behavior, 1343 .

high energy, effects in polymers, mechanism, 2194.

ionizing, permissible exposure, 1443.

legislative control, 1755 .

low frequency, from a horizontal antenna over a spherical earth, 1761 monitoring over long-distance telephone lines and direet field lines, 2710 .

near infrared solar, measurements by balloon to an altitude of $100,000 \mathrm{ft} ., 2645$.

neutron, up to 30 million elcetron volts, protection against, $\mathrm{H} 63$.

pattern, horizontal, of a high-frequency antenna, long distance, 2371 .

pattern of an antenna mounted on a surface of large radium of curvature, 1946 .

patterns and conductances of slots eut on rectangular metal plates, 1947

patterns of eircumferential slots on moderately large conducting cylinders, 1830.

permissible, exposure levels, 2075.

point source, 1267 .

reducing, exposure in diagnostic examinations, practical suggestion, 2259 .

regulations, 1832 .

resistance of dipoles in an interface between two dielectrics, 1833.

safety, 1280 .

scattcred, 1135 .

slots on dielectric-clad and corrugated cylinders, RP2802.

small loop immersed in a semi-infinite conducting medium, 3028.

solar flare, ionospheric effects, 1750 .

solar plasma, condition, 414,

spectra, VLF, of lightning discharges, J 63D2, 199.

survey meters, pocket chambers and dosimeters, $\mathrm{X}$-ray ealibration, 172 .

thermal Pitot tube, 101.

Radiation protection, $\mathrm{H} 65$.

basis for standards, 1639 .

education, 1324.

general practitioner, 1831.

measurements, brief history of the National Committee, 2478

philosophy, 2376.

regulations, exposure standards, 2947.

therapeutic radiology, 1467.

Radiations, dosimetry of ionizing, introductory remarks on, 1045 .

gamma, penetration of, 2102.

high-energy, degradation and range straggling of, 680 . ionizing, for biological purposes, 1189.

nuclear electromagnetic, 2500.

parallel angular moments, angular correlations, 2021.

Radiative, electron capture in cesium-131, 1834.

electron capture, NBS alpha standards, coincidence counting, 2644.

orbital electron capture in vanadium, 1835.

transitions on the relaxation behavior, the effect of, 2235 .

Radiators, slot in rectangular metal plates, investigation, 1622 .

Radical, $\mathrm{C}_{2}$, in hydrocarbon flames, 1434.

distribution study of vitreous barium borate, J $64 \mathrm{~A} 3$, 229 .

free, degradation, high-speed computations in the kinetics, 2587

free, stabilization in condensed phases, 2570.

$\mathrm{OH}$, dissociation energy, 1503 .

Radicals, acetate, propionate, and butyrate, heats of combustion of some peroxides and the heat of formation, 2148 .

diatomic free, of the isotopic hydrides of oxygen and sulphur, 1357

frozen, chain reactions, 3030 .

low-pressure acetylene-oxygen flames, 2311.

trapped, 2853.

Radicals free, 2622.

atoms, luminescence of solid nitrogen $\left(4.2^{\circ} \mathrm{K}\right), 2182$.

frozen, 2129.

frozen: the problem and the program at the National Bureau of Standards, 2130.

low temperatures, stabilization of, Mono.12; 2316.

research at the National Bureau of Standards, 2973. 
research symposium, 2572. stabilized, 2774 .

Radio, atmosphere at short ranges, waveform, 1800 . atmospheric, refractive index, 3002 .

climatology, 2564.

communication, intermittent, utility of meteor burst, 2389.

ducts, ground-based, climatology, J 63D1, 29.

eclipse, expedition, NRL, Aleutian, 273.

electrical propagation, 2405.

field strength data, tropospheric, 362 .

frequencies, constants in the equation for atmospheric refractive index, 855 .

frequencies in the band 25 to $60 \mathrm{Mc}, \mathrm{TN} 13$ (PB151372).

frequencv ground wave transient sinusoidal over a finitely conducting plane earth, propagation, 2267.

frequency mass spectrometer, 117.

ionospheric observations during the International Geophvsical Year, J 63D1, 1.

ionospheric world-wide soundings Committee, 2490.

low, frequencies, calculations of ionospheric reflections, 2044.

low, frequencies, transmission loss curves for propagation, 2851.

marine, communications, propagation characteristics of the frequency band 152-162 Mc which is available for, 1945 .

measurement of winds aloft, 524 .

measurements, 263.

multiple, interference to FM and television service, statistical approach to the problem, 378 .

observations, model of the electron corona, 2875 .

observations (al-CM) of dense dark nebulae, 1468.

observations of interstellar neutral hydrogen clouds, 1836 .

optical data, 2874 .

outbursts, solar, an estimate of the density and motion of solar material from observed characteristics 649 .

phase measuring systems, a source of error, 10; 186.

photo-transmissions, spectrum of frequency-shift, 1884.

point-to-point, relaving via the scatter mode of tropospheric propagation, 1812 .

ray bending, TN44 (PB151403).

ravs at small elevation angles, 2388 .

reflections from meteor trails in research on the upper atmosphere, study, 144 .

solar, astronomy at the NBS, 1490.

solar, bursts, 2958.

stars observed at Boulder, Colorado, amplitude scintillations, preliminary analvsis, TN20 (PB151379).

surveying systems, phase measurement type, 22 .

transmission at VHF by scattering and other processes in the lower ionosphere, 1469 .

transmission loss vs distance and antenna height at $100 \mathrm{Mc}, 1470$.

tropospheric, field strength data, 546 .

tropospheric, wave propagation, some aspects, 1872

VLF, waves, propagation, TN58 (PB161559).

waveguide components, electroforming, C587.

Radio horizon, an analysis of propagation measurements made at 418 megacycles per second, J 64D3, 255 .

analysis of propagation measurements, TN6 (PB151365).

comparative $100 \mathrm{mc}$ measurements at distances far beyond the, 972 .

spaced antennas far beyond, 1401.

tropospheric scatter propagation measurements, 1499.

Radio noise, atmospheric, from statistical moments, determination of the amplitude-probability distribution J 64D1, 49 .

atmospheric, report, 1851 .

cosmic, intentities in the VHF band, 418.

data, quarterly, TN18-2 (PB151377-2).

effects of high-altitude nuclear explosions, J 64D1, 37 .

International Geophysical Year July 1, 1957-December 31,1958 , TN18 (PB151377).

ionospheric origin, 118. solar, $1149 ; 1418$.

VLF atmospheric, 2188.

Radio propagation, excitation of surface waves on conducting, stratified, dielectric-clad, and corrugated surfaces, RP2807.

fields of an insulated loop immersed in a conducting medium, RP2781.

low and medium frequency, 2615.

mixed-path ground-wave propagation, RP2770.

new kind, at very high frequencies observable over long distances, 369 .

96.3 mc between Richmond, Va., and Washington, D.C., study of some of the meteorological effects, 635 .

pattern of a flush-mounted microwave antenna, RP2796.

research at the National Bureau of Standards, 2713. transmission loss, 901; TN12 (PB151371).

transmission power, 3102 .

TV and FM bands, factors affecting, 1342.

Radio wave, bending from normal, calculation of the departures, J 64D3, 259.

generation by multi-stream charge interaction, 295 .

propagation, 2331.

propagation in an inhomogeneous atmosphere, TN24 (PB151383).

propagation, system loss, J 63D1, 53.

scattering by tropospheric irregularities, J 63D2, 205.

scattering in tropospheric propagation, 808 .

Radio waves, TN52 (PB161553).

atmosphere, oxygen and water vapor absorption, 2241 .

atmospheric bending, 2806.

downcoming, 2932.

galactic, 63 .

lower ionosphere, 2661.

measurement of the phase, received over transmission paths with electrical lengths varying as a result of atmospheric turbulence, 1402 .

measurements of coastal deviation of high-frequency, J 64D1, 57.

penetrating the ionosphere, 2458 .

scattered, some meteorological effects, 1874.

three-centimeter, single path phase measuring system, 2758.

VLF frequency characteristics, 2345.

VLF waveguide mode theory, 2391.

Radio-astronomical test of the ballistic theory of light emission, 2878 .

Radio-frequency microvoltages, accurate, 640 .

Radio-ray, average, refraction in the lower atmosphere, 400 .

Radio-refractive-index, climate near the ground, J 63D3, 259.

air, slide rule computes, 829 .

synoptic study of the vertical distribution, J 63D3, 249. synoptic variation, J $63 \mathrm{D} 1,91$.

Radioactive, fallout prediction, analog computer, 1616.

isotopes, bodies containing, safe handling of, $\mathrm{H} 65$.

isotopes, new lower permissible exposure levels, 2099. liquids, 1649 .

nucei, aligned, nuclear magnetic resonance of, 778 . standards, preparation and maintenance by the NBS, 1455 .

Radioactivity, assay, of tritium compounds, J 63A2, 177 . In ${ }^{117}$ and $\mathrm{Sb}^{117}, 1124 ; 1471$.

$\mathrm{In}^{120} \mathrm{Sb}^{120}, 2711$.

preparation and maintenance of standards, 1944.

standardization in the United States, 2712.

standards, international comparisons, 1746.

standards of, applications, 1631 .

standards, preparation, maintenance, and application, C594.

standards program, 2714.

units, 163.

Radioassay of tritium-labeled compounds in "infinitely thick" films with a windowless, gas-flow, proportional counter, tritium-labeled compounds I., J $63 \mathrm{~A} 2,171$. 
Radiofrequency, ground waves, transient, over the surface of a finitely conducting plane earth, RP2844.

induction-heating unit, automatic controller for, 647

low, tables of ground wave parameters for the spherical earth theory: the roots of Riccati's differential equation, TN7 (PB151366).

waves, low- and very-low, on the reflection from the ionosphere, J 64D3, 269.

Radiographic rooms, dose to walls, 1319.

Radioisotope study, of metallic deposits in porcelain enameling, nickel dip, 1941.

nickel dip, 1599 .

Radioisotopes, radiation exposure and the use, 2277.

Radiological, applications of gamma-ray transport theory, 3051 .

physics, 1125 .

units and measurements, International Commission, $2366 ; 3022$.

Radiological protection, International Commission, history, 2589 .

Radiology, biology, 1125 .

science, 1483 .

therapeutic, radiation protection, 1467 .

Radiometeorological effects on beyond-horizon propagation, preliminary study of, J $64 \mathrm{D} 3,239$.

Radiometers, measuring circuit, 1068 .

Radiometry, filter, some of its applications, 720 .

Radionuclides in air and in water for occupational exposure, maximum permissible body burdens and maximum permissible concentrations, $\mathrm{H} 69$.

Radiophotoluminescence dosimetry system of the U.S, Navy, 809.

Radiowave propagation beyond the horizon, 228 .

Radium, determination of the gamma-ray emission of, RP2801.

large, of curvature, radiation pattern of an antenna mounted on a surface, 1946 .

preparation of new solution standards, RP2924.

small amounts in solution, report on the determination, 2733.

standards, internal gas counting and the United States radioactivity standards program, 2714 .

Radius, large, of curvature, currents excited on a conducting surface, 1668.

Radix table, for obtaining hyperbolic and inverse hyperbolic functions to many places, 810 .

trigonometric functions and their inverses to high accuracy, 296.

Railroad-signal glassware in terms of a standard source A of the International Commission on Illumination, an evaluation of the luminous-transmittance requirements for, RP2849.

Rain, erosion damage to solids caused by high-speed collision with, RP2882.

Rainfall, attenuation, 29 .

Raman, spectra, infrared, of trans-difluorodichloroethylene, 2161 .

spectrum of triethylborane, 2278.

Ramberg vacuum tube accelerometer, recent developments, 297.

Random, flight with multiple partial correlations, 525 .

notes on matrices, RP2850.

numbers, use, 613 .

variables, changes of sign of sums, 662 .

Random walks, duration, 281.

inversion of matrices, 282.

space, problems, 310 .

Randomization, certain statistical decision procedures and zero-sum two-person games, 231.

design of experiments, principle, 2378 .

Randomly inhomogeneous atmosphere, scattering of electromagnetic energy, 536 .

Randomness and other hypotheses, independence of tests, 2231.

Range finders, unsymmetrical, cross-field errors, 1300 .

using projected images, RP2851.

Range straggling of high-energy electrons in carbon, 2279.

Rapid City, South Dakota, the aurora of October 22/23, 1958, J 64D2, 205.
Rapid extraction of resins from chilte and guayule rubbers, 119.

impact loading of textile yarns, 2715 .

impact loading, yarns subjected to, stress-strain relationships, 1899.

Rapport sur l'etalonnage de deux lampes etalons secondaires a ruban de tungstene par quatre laboratories nationaux, 2716.

Rare earth region, photoneutron yields, 2249; 2686.

Rate, nonequilibrium processes, studies, 2786.

fading in propagation through a turbulent atmosphere, 1542 .

shrinkage of tendon collagen: further effects of tannage and liquid environment on the activation constants of shrinkage, 120.

thermal diffusion of polymer molecules in solution, 1472 .

Rates, activation energies of thermal degradation of styrene and acrylate polymers in a vacuum, 811 .

thermal degradation of polystyrene and polyethelene in a vacuum, 526 .

Rating of water-current meters, 340 .

Ratio, Poisson's, of aircraft sheet materials for large strains, 798 .

proton gyromagnetic, $2422 ; 2706$.

Ratio-recording spectroradiometer, J $64 \mathrm{C} 2,151$.

Rational numbers, subroutine for computations, 1603.

Ray, reflected suppression, 123.

roentgen, standards, intercomparison of French and U.S., 2017.

tracing, effect of atmospheric horizontal inhomogeneity, J 63D3, 287.

Rayleigh, disk in liquid helium II, 157.

disk measurements in liquid helium II, thermal, 603 .

distributed vector, $591 ; 1541$.

quotient iteration for the computation of characteristic roots and vectors, $3003 ; 3004$.

waves across an ocean floor with two surface layers, transmission, 2387.

waves with depth in a medium with two surface layers, attenuation, 2344.

Rayon fabrics, properties, factors affecting, 446 .

properties of, 718 .

Rayons et les neutrons, degradation des polymeres, 681 .

$\mathrm{Rb}^{87}$ narrow hyperfine absorption lines, 2677 .

R-branch heads of some $\mathrm{CO}_{2}, 293$.

$\mathrm{R}-\mathrm{C}$ bridge, selective, note, 370 .

oscillator, wide-range, 171.

Reaction, carbon monoxide with graphite carbon at $450^{\circ} \mathrm{C}$, 2717.

dimeric 5 -aldo-1,2-0-isopropylidene-D-xylo-pentofuranose with cyanide and the preparation of calcium 1,2-0-isotropylidene- L-idof ururonate-6$\mathrm{C}^{14}$ dihydrate, 2280.

hydrogen atoms with solid olefins at $-195^{\circ}, 2828$.

metal oxides with o-ethyoxybenzoic acid and other chelating agents, 2829.

nitrous acid with collagen, 1837 .

sodium chorite with some aldoses and modified celluloses, 1473 .

Reactions ammonia and hydrazine with oxygen atoms and hydrogen atoms in atomic flames, 1948.

aromatic fluorocarbons with hydrogen, RP2940.

frozen radicals, chain, 3030 .

hexafluorobenze, 2718 .

Reactions en chaine de radicaux geles, 3030 .

Reactive, cylindrical structure excited by an axial line source, surface-wave resonance effect in a, $J$ $64 \mathrm{D} 1,13$.

substances, highly, cryoscopic determination of purity, 1666.

Reactors, large, methods for measuring the " $Q$ ", 1776. nuclear, a survey of ceramics, 12.

nuclear, ceramic coatings, 1285.

Reagent chemicals, American Chemical Society specications, 1474 .

Receiving tubes, tabulation of data, H68. 
Recent, advances in cryogenic engineering, 3031.

developments in NBS spectrographic standard samples of iron-base alloys, 2719.

developments in the field of microwave power measurements at the National Bureau of Standards, 2720

developments in the Ramberg vacuum tube accelerometer, 297.

experimental evidence favouring the $p \mathrm{~K}_{1}(p)$ correlation function for describing the turbulence of refractivity in the troposphere and stratosphere, 3032

theoretical studies of the propagation of long waves, 2721.

trends in glass analysis, 1126.

Recently developed microwave impedance standards and methods of measurement, 2722 .

Reception of space diversity transmitters, 3033.

Reciprocity law for X-rays, $1838 ; 1839$.

Recombination and attachment in the F-region during the eclipse of May $20,1957,121$

Record papers and their preservation, 1840.

Recorded information, retrieving and correlating, machines, 1763 .

Recorder, simple type X $(t)-\mathrm{Y}, 184$.

survey: recording surfaces and marking methods, C601.

Recorders, autographic stress-strain, 2465.

magnetic tape, FM data reduction from, 722 .

Recording, instruments, direct-indicating, 690 .

instruments under dynamic conditions, 2286.

microwave refractometer, $6 ; 122$.

sound transmission loss of walls as a continuous function of frequency, new method, 1414 .

surfaces and marking methods, recorder survey, C601.

system, a photoelectric followup and its application to remote observations of the beam in high precision balances, $\mathrm{J} 63 \mathrm{C} 2,91$.

torsion testing machine for wire 2435 .

Rectangular waveguide, 2012.

half-round inductive obstacles, J 64B2, 113.

Rectifiers, ideal, harmonic generation, 2578.

Recurrent determinants of Legendre and of ultraspherical polynomials, 298 .

Red lines in the airglow, oxygen, 2681.

Redetermination of mass spectra of deuteromethanes, RP2896.

Reduction, adjacent-channel interference components from frequency-shift-keyed carriers, 2723.

adjacent-channel interference from on-off keyed, carriers, 1841.

area gage for use at low temperatures, 7.

Reexamination of the crystal structure of $\alpha$ and $\beta$ nitrogen, 2879.

Reference, materials, certified, 3078 .

samples of isotopic abundance, 1475 .

samples stocked by the National Bureau of Standards, isotopic abundance ratios, TN51 (PB161552)

Refined X-band microwave microcalorimeter, J $63 \mathrm{C1}, 77$

Refinement, crystal structure of triclinic magnesium pyroborate, RP2936.

hydrox yapatite structure, 2724

Reflected ray suppression, 123 .

Reflection, coefficient of irregular terrain at grazing angles, study at 1,046 megacycles per second, J $63 \mathrm{D} 2$, 235 .

curves, goniophotometric, analysis, 397.

fast neutrons from water, J 63A2, 101.

low- and very-low-radiofrequency waves from the ionosphere, J 64D3, 269.

transmission by a parallel rvire grid, J 63D 1, 87.

Reflectionless waveguide termination, 183.

Reflections, gradient, nature, 586 .

Reflective surfaces, thermal resistance of airspaces and fibrous insulations bounded by, BMS151.

Reflectors for a microwave Fabry-Perot interferometer, 3034 .
Refraction, dispersion of thallium bromide iodide, 1842.

focusing, and defocusing in a circularly stratified atmosphere, J 64D3, 287.

Refractive index, arsenic trisulfide, 2726.

atmosphere at microwave frequencies, 240.

elastic moduli of a borosilicate glass after heat treatment, 1844 .

equation for atmospheric, at radio frequencies, 855 .

geographical and height distribution of the gradient of, 727

indices of cesium bromide, 527 .

measurements, quantitative determination of natural rubber, 292.

potassium bromide for infrared radiant energy, 812 . radio, synoptic variation, $63 \mathrm{D} 1,91$.

silver chloride for visible and infrared radiant energy, 124.

synthetic sapphire, 2727

Refractivity in troposphere and stratosphere, 3032.

Refractometer, cavities, limit of spatial resolution, J 64D1, 65.

compact microwave, for use in small aircraft, 2415 .

infrared phase contrast, 2600 .

microwave, recording, $6 ; 122$.

Refractories industry in some European countries, 592.

Refractory castables, elasticity, strength, and other related properties, 2536.

preparation and some properties, 125 .

thermal length changes of, RP2768.

Refractory materials, permeability and some other properties of a variety of, 794

Refrigerants, bubbling in boiling, suppression, 2330.

Refrigerated-food trailers, heat transfer measurements. 2580 .

Refrigeration, helium, and liquefaction using a liquid hydrogen refrigerator for precooling, TN39 (PB151398)

mechanical, process for the no-loss storage of liquid hydrogen, 1986.

system, determination of water content in Freon-12 circulation in, 990 .

system, liquid hydrogen temperature, design and construction, TN38 (PB151397).

Refrigerators, entrapment in, behavior of young children under conditions simulating, 2471.

Regression problems, a note on confidence intervals, 2429.

Regular, Coulomb wave functions, irregular, expressed in terms of Bessel-Clifford functions, 1127.

rhombohedral lattice sampling of Munsell renotation space, 930.

Regulated supply offsets line changes, 2728.

Regulations, radiation, 1832.

Regulator, low voltage, 364 .

Reinforced, concrete, cracking of, flexural members, 1665 . plastic springs, 1843.

Rejection of outlying observations, 813 .

Relation, among the blocks of symmetrical group divisible designs, 548 .

occlusal surfaces to the stability of artificial dentures, 1194.

Relation between, absorption spectra and the chemical constitution of dyes. XXIV. Absorption spectra of some thioindigo dyes in sulfuric acid, 342 .

absorption spectra and the chemical constitution of dyes. XXII. Cis-trans isomerism in thioindigo dyes, 341.

absorption spectra and the chemical constitution of dyes. XXVI. Effect of solvent and of temperature on the cis-trans isomerization of azo dyes, 881 .

absorption spectra and the chemical constitution of dyes. XXV. Phototroprism and cis-trans isomerism in aromatic azo compounds, 593.

absorption spectra and the chemical constitution of dyes. XXVIII. The hydration of azo dyes in organic solvents, 1543 .

actual and artificial weathering, 2729 .

conductance and the noise power spectrum of certain electronic streams, 503 . 
net heat of combustion and aniline-gravity produet of aircraft fuels, 1128 .

refractive index and elastic moduli of a borosilieate glass after heat treatment, 1844 .

roughness of interface and adherence of porcelain enamel to steel, 2281 .

Relations, among certain ranges of vector measures, 299. among certain specification properties of building brick and effects of differences in raw materials and methods of forming, 528 .

between summation methods and integral transformations, J 63B1, 1.

Relationship between residual monomer and some properties of self-curing dental resins, 1846.

between the lower ionosphere and the [OI] 5577 nightglow emission, 2880 .

between sporadic-E and aurora echoes at Barrow, Alaska, 1845.

dental materials to elinical subjects, 1476 .

Relative, back-seattering of electrons and positrons, 300 . basicities of tribenzylamine and tribensylamine oxide in benzene and water, 1195 .

damping eriterion for linear systems, 633.

measurement of the photodetachment cross section for $\mathrm{H}^{-}, 3035$.

stress-optical coefficients of some National Bureau of Standards optical glasses, RP2779.

Relative extrema, Bessel function, 104.

Hermite orthogonal functions, 283.

Laguerre orthogonal functions, 105.

ultraspherical polynomials, 106.

Relaxation, method for linear inequalities, 1196; 1197 reliability, measured values. Part I. Fundamenta concepts, 594 .

starts with circuit design, 283.

vibrational nonequilibrium distributions, 1847 ; 2235 ; $2324 ; 2786$

Reliable locked-oscillator pulse timer, 634 .

Remark, M. M. Day's characterization of inner-product spaces and a conjecture of L. M. Blumenthal, 373. smoothing problem, 931 .

Remarks, commutators of matrices, 1145.

some modular identities, 529 .

Remote-control method of opening ampoules of active materials, 8 .

Removal, manganese prior to calcium and magnesium precipitations, 814 .

nitrogen from hydrogen with silica gel at low temperatures, 2730 .

Report of the, Committee on atomic weights of the American Chemical Society, $127 ; 531 ; 1129$.

committee on the American Standards Association, 3036.

subcommission on the question: What are the most readily measured characteristics of terrestrial radio noise from which the interference to different types of communications systems can be determined? 2284.

Report on, atomic weights for $1956-57,2731$.

comparative $100 \mathrm{Mc}$ measurements for three transmitting antenna heights, 1848.

International Standards Organization Technical Committee 61,1477

Joint Commission for spectroscopy meeting, June 1956,1849

reducing sugars, 2732 .

reducing sugars, effect of clarification with neutral lead acetate before determining reducing sugar in molasses, 1850 .

revision of atmospheric radio noise data, CCIR Report No. 65, VIII Plenary Assembly, 1851.

sugars and sugar products, 1852.

Report on the, determination of small amounts of radium in solution, 2733 .

methods used in the 1949 AGA-ASME orifice meter tests, 128 .
NIBS optical image evaluntion symposium, 532.

second revision of Rowland's table of solar wavelengths, 1478

Reports submitted for four national laboratories on the standardization (ealibration) of two secondary tumgsten filament lamp standards, 2716.

Representation, certain integral involving bessel functions by hypergeometrie series, 2236 .

gas properties in terms of molecular clusters, 815 .

integers by binary quadratic rational forms, 1198.

nonlinear functions of two input variables on analog equipment, 2285

solutions of analytic systems of linear differential equations, 932 .

Representations, extension of bounded linear functionals defined on classes of analytic functions, 1093 .

finite groups, 499.

Reproducibility of a pen-and-chart type of recording instrument under dynamie conditions, 2286.

Reproducible and stable silver-silver oxide electrode, 1998.

Research, atmospheric, 2031; 2462.

automotive at the NBS, 399.

basic in Europe, 2470

building in the U.S. before 1947, 1279 .

corrosion at the NBS, $1297 ; 2503$.

dental, 2079.

dental, résumé, 1480 .

development and testing, NBS, aireraft lighting, 771 .

effects of prestraining and notch sharpness on the notch strength of materials, 1853.

everybody's business, 2737 .

fire, in the United States, 2563.

highlights at the National Bureau of Standards. Annual Report, 1958: M226; 1959: M229.

NBS Boulder Laboratories on quartz erystal resonators and oscillators at low temperatures, 2734 .

new tools for, 269 .

normal threshold of hearing, 2735 .

operations, design of experiments, 1670.

physical constants at the (United States) NBS, present status, 2263.

plating at NBS, 2736

radio propagation at the NBS, 2713.

recent, in the propagation of VLF radio waves, bibliography and survey, TN58 (PB161559).

roofings, 129.

social and industrial, information processing in, 741 .

standards for the plasties industry, 1130.

sterochemical, 554 .

symposium, free radicals, 2572 .

systematic, 3060 .

Researchers, Government 158.

Residual lattice strains in sectioned bars of plastically deformed iron, 1479.

Resin, cements and posterior-type direct-filling resins, 1854.

coating methods and other variables on physical properties of glass-fabric reinforced polyesters, effects of, 1004 .

bonding of hardwood fibers in offset papers, 302.

bonding of offset papers containing mineral fillers, 303.

Resinous filling materials, 223 .

Resins, acrylic, crazing, 1298.

aerylic, in prosthetic dentistry, 2449.

denture base, dimensional stability, 2523.

denture, some properties, effect of molding processes, 572 .

direct filling, 1069

epoxy, as cryogenic structural adhesives, 2547

filling, direct and indirect: a review of some physical and chemical properties, 688 .

filling, direct, color stability of, 854 .

filling, direct: dimensional ehanges resulting from polymerization shrinkage and water sorption, 689.

prosthetic dentistry, 2738 .

rapid extraction, from chilte and guayule rubbers, 119. 
repair, self-curing, American Dental Association specification No. 13, 1257.

Resistance, abrasion, 2005.

diode bridge circuit for temperature control, TN34 (PB151393)

measurement of ceramic-type strain-gage cements, 2739 .

tension in plastics, 1855 .

Resistance-temperature relation for low temperature thermometry, 9 .

Resistencia a la tension de los plasticos, 1855 .

Resistivity, dielectric constant, and power factor of leather, 533.

Resistors, composition, proposed index of current noise, 1241.

noise in composition, $1416 \mathrm{~A}$.

standara, rack for, 3026 .

Resolution of the dissociation constants d,l-malic acid from $0^{\circ}$ to $50^{\circ} \mathrm{C}$, RP2947.

Resolving, new, power test chart, 268.

Resonance, effect in a reactive cylindrical structure excited by an axial line source, surface-wave, J 64D1, 13.

frequencies, flexural and longitudinal, of uniform bars, comparision of experimental and theoretical relations between Young's modulus, J 64A2, 147.

Resonator, integral super-conducting, liquid helium cryostat, $2610 \mathrm{~A}$.

Resonators, crystal, underearth, 903.

quartz, at very low temperatures, stability, 2315 . wide-range for $\mathrm{VHF}$ and $\mathrm{UHF}, 357$.

Response, earphones in ears and couplers, 1199.

function of NaI (TI) scintillation counters, 1856.

functions of total-absorption spectrometers, 2740 .

large sodium iodide scintillation detector to high energy X-rays, 2741

loaded electric dipole in an imperfectly conducting cylinder of finite length, J 64D3, 289

Results of Bureau of Standards 45-year corrosion study, 2287.

ionospheric drift measurements in the United States, 2288 .

laboratory tests of high duty and super duty fireclay plastic refractories, 2289 .

sweep-frequency investigation in the low frequency band, 1146.

sweep-frequency propagation experiment over an $1150-K M$ east-west path, 1498.

Résumé of dental research, 1480.

Retarding potential measurements, electrical differentiation 2596.

Reverberant, sound fields, interference patterns, 1373. sound fields, measurement of correlation coefficients, 1399.

sound through double walls, 159 .

sound through walls, 1214.

Reverberation chamber, other reflecting environments, output of a sound source, 2679.

study of the sound power output of subsonic air jets, 2742 .

Reversible contractile processes in fibrous macromolecules, 2743 .

Review, analytical distillation, 2744 .

dental research, 1956: dental materials and instrumentation, 2290.

methods of flow measurements, 1131

observational results in airglow photometry, 1242.

plastics, year 1952,894 .

pressure drop across columns of bone char, 1243.

proceedings of the Berkeley symposium on mathematical statistics and probability, 114 .

state of the art and future trends in electrochemical battery system. I. The more common systems, 2436 .

Revised analysis of the solar spectrum from 2990 to $2635 \mathrm{~A}$, 933.
Revision of, American Dental Association specification No. 3 for dental impression compound, 1244.

Commercial Standard CS115-44 porcelain enameled hot water tanks, 2745 .

phase equilibrium diagram of the binary system calcium-titania, showing the compound $\mathrm{Ca}_{4} \mathrm{Ti}_{3} \mathrm{O}_{10}$, RP2913.

RF, micropotentiometer, 806.

micropotentiometers for calibration of signal generators to $1000 \mathrm{Mc}$, TN37 (PB151396).

permeameter techniques for testing ferrite cores, 2746 . power measurement, 2419.

solenoid design chart, 294.

voltmeter calibrating consoles, 2747 .

Rhenium (Re II), term analysis of the second spectrum, RP2914.

Rhenium, term analysis of the first spectrum, RP2804.

Rheology of polyisobutylene, $1132 ; 3037$.

Rhombohedral, regular, lattice sampling of Munsell renotation space, 930 .

Rib flexibility, effect on the vibration modes of a deltawing aircraft, RP2852.

Ribbons and half-planes, surface currents excited by an infinite slot, 1907 .

Ricctai's differential equation, J 64B2, 95 .

Ricerca e normalizzazione per I'Industria delle materie plastische, 1857.

Richardson, L. F., obiturary notice, 1088.

Ridge, further studies of the influence of a, on the lowfrequency ground wave, RP2884.

Riesz, M., theorem of, RP2935.

Ring conformation of sugar acetates, RP2772.

Rings; rubber, tension testing, 2801.

Riometer $-a$ device for the continuous measurement of ionospheric absorption, 3091.

Riveted and spot-welded lap joints of aircraft materials, creep and creep rupture characteristics, 1299.

Robertson, H. P., a determinantal inequality, 1980.

Rocket hoist, 2430.

Roentgen, ray protection design, editorial, 433.

ray standards, intercomparison of French and U.S., 2017.

standards of Great Britain and USA, intercomparison, 1621.

Roentgenographic dental equipment, protection in the use, 1462.

Role of, electronic computers in air traffic control, 2380. nickel dip in the enameling of sheet steel, 1133.

oxygen in the quenching of liquid scintillators, 1858.

partial reflections in tropospheric propagation beyond the horizon, 595 .

Roofings, research, 129 .

Roots of natural numbers, algebraic equations, 1610 .

Rotating, dental cutting instruments, 1165.

dental diamond abrasive instruments, 2085.

molecular still, simple, RP2951.

Rotation, dynamic characteristics of, 913.

internal, in methyltrifluoromethyl acetylene, 1374.

temperatures of $\mathrm{OH}$ in methane-air flames, 304.

Rotation-vibration spectra of deuterated water vapor, $1859 ; 1949$.

Rotational, distributions, nonthermal, of $\mathrm{OH}\left({ }^{2} \Sigma^{+}\right)$in flames, 1709 .

intensity distributions of $\mathrm{OH}$ and $\mathrm{OD}$ in electrodelen discharge through water vapor, 817 .

lines of $\mathrm{CH}, \mathrm{OH}$ and $\mathrm{CN}$ in the solar spectrum, 2292.

relaxation in nitrogen, oxygen, and air, 3038 .

temperature of $\mathrm{OH}$ in diluted flames, 818.

temperatures of $\mathrm{OH}$ in several flames, 534 .

vibrational relaxation of a system of rotating oscillators, 2787.

vibrational "temperatures" of $\mathrm{CH}$ in flames at atmospheric pressure, 816 .

viscous flow through a tube, 1094 .

Rotator, single-axis in a crystalline field, theory of dielectric relaxation, 1554

Rotors, dies, and rate of shear on Mooney viscosity, 740 .

Rounding off of difference tables for linear interpolation, 504. 
Rowland's table, second revision, of solar wave-lengths, report, 1478 .

Rubber, compounds, temperature control, 1526

crude natural, AST II subcommittee, 3065 .

dynamic properties of, measurement, 480 .

elasticity, $2810 \mathrm{~A}$.

elasticity, physies, 2251.

GR-S, solution, flow behavior, 58 .

GR-S, solutions, viscosities of 0.25 to 90 percent, 621 .

Hevea, Mooney, viscometer cure characteristics, 1409. natural, 909.

natural, hydrocarbon by refractive index measurements, quantitative determination, 292.

natural, infrared study of some structural changes in during vulcanization, RP2816.

natural, quantitative determination of copper, 523.

natural, some properties of polymer networks formed from oriented, chains, 2765 .

natural, standard, 1505.

natural, symposium on recent developments in the evaluation of, 844 .

natural, thermodynamies of crystallization in high polymers, 1558 .

oil-extended, oils used in, RP2771.

pure-gum, vulcanizates, elastic properties, 2534.

pure gum, vulcanizates in tension, behavior, 1640 .

rings, tension testing, 2801.

shear, nonlinear viscoelastic behavior, 1419 .

stark, 1454.

synthetic, 2967.

time-temperature behavior, from measurements of indentation hardness, 2848.

tires, proof testing, 519 .

tool in testing, statistics, 1512.

unvulcanized and vulcanized in a vacuum, thermal degradation, 1961.

vulcanizates, determination of sulfur, 1311.

vulcanizates, measurement of the aging, J $63 \mathrm{C} 2,141$.

vulcanizates, pure-gum, stress-strain relation in compression and tension, RP2837.

Rubbers, Castiloa and Hevea, treatment of leather, 160. chilte and guayule, 119.

different, values of physical constants, 1222.

natural and synthetic, $94 ; 264 ; 487 ; 772 ; 1412 \mathrm{~A} ; 2997$.

natural and synthetic, analytical procedures, 2020.

synthetic, physical chemistry of, 1107.

Russell, Henry Norris, 2150.

Ruthenium, 1228.

$(\mathrm{Ru} \mathrm{I})$, analysis of the first spectrum, J 63A3, 213.

$(\mathrm{Ru} \mathrm{I})$, low even configurations in the first spectrum, part 2, J $63 \mathrm{~A} 3,255$.

(Ru II), second spectrum, RP2908.

(Ru I), supplementary Zeeman data for the first spectrum, J 63A3, 253.

Rydberg constant, value of, 3109 .

\section{$\mathbf{S}$}

Saddle point, probability, 2379.

Safe, design and use of industrial beta-ray sources, H66. floors and floor finishes, 130 .

handling of, cadovers containing radioactive isotopes, $\mathrm{H} 65$.

Safety, code, new, for portable metal ladders, 2374.

color codes, $1481 ; 1861$.

radiation, 1280 .

Sag point as a reference point in the heating of glasses, RP2791.

Salt pairs, metal-rock, epitaxy, 367 .

Salts, fused, electrolytic preparation of moldybdenum, $1330 ; 2811$.

liquid and liquid metals, specific heats, 2306

paramagnetic, properties of, 2408 .

Samarium, emission, spontaneous, of neutrons, 383.

Samples, reference, of isotopic abundance, 1475 . standard, 835 .

standard, and reference materials issued by the NBS, C552, 3d ed.
Sampling flame gases, RP2818.

leather, 3039 .

side upper leather II, 535 .

statistical, methods to outgoing letter mail characteristics, some applications, TN16 (PB151375).

variables, 16.

Sand, cast aluminum alloy 220 to stress corrosion cracking, 2349 .

in "no-fines" concrete, 190.

molding, 846 .

Sapphire, synthetic, refractive index, 2727.

Satellite, artificial, method for measuring local electron density, $J 63 \mathrm{D} 3,325$.

orbits, effect of trensverse atmospheric drag, 2532.

orbits, unretarded, new method of solution for, J 63 B2, 105.

Satellites, earth, comparison of phase difference and Doppler shift measurements for studying ionospheric fine structure using, 2493.

continuous phase difference measurements, 2499.

Saturation backscattering of positive electrons, 131.

Sauter theory of the photoelectric effect, 3040 .

$\mathrm{Sb}^{117}$ and $\mathrm{In}^{117}$, radioactivity, $1124 ; 1471$.

Scale, amplitude, and spectrum of refractive index inhomogeneities in the first 125 meters of the ionosphere, 1258.

$\mathrm{He}^{4}$, of temperatures, 1958, J 64A1, 1; Mono.10.

reading errors of electrical indicators, 1134.

temperature, helium vapor-pressure, 1535 .

weights and measures, 1580 .

Scales, atomic weights and nuclidic masses, 2906.

low temperature, from $90^{\circ}$ to $5^{\circ} \mathrm{K}, 1392$.

temperature, present status, 590 .

Scandium, anhydrous, phosphate, structure, 1950.

Scatter propagation issue, 1544 .

Scatter-sound method, ionospheric propagation, 2326.

Scattered radiation contribution to depth dose data for 35-Mev and 90-Mev synchrotron X-rays, 1135.

Scattering, cross sections, variational calculation, 617 .

double, of electrons with magnetic interaction, 1320.

electromagnetic energy in a randomly inhomogeneous atmosphere, 536 .

oblique incidence from ionospheric irregularities, 1482. radar, by the moon, J 64D3, 217.

Schiefer abrasion testing machine, 382.

Science, basic standards, 198.

fairs, 3041 .

personnel, interdisciplinary approach, 77 .

radiology, 1483 .

Scientific, conferences, proposed standard for facilities, 2434.

developments and methods in practice (Dentristry in 1967), 2748.

information, international exchange, 79 .

measurement and technological progress, 537.

standardization, fifty years, 236.

teamwork in a Computation Laboratory, 819.

Scientists' and public's responsibility, 2293.

Scintillation, and ionization detectors, dose measurements in water using, 995 .

counter method of intercomparing neutron source strengths by means of a manganous sulfate bath, RP2952.

counters, $\mathrm{NaI}$ (TI) response function, 1856.

counting in experiments on parity conservation, 2749. counting of beta emitters, 2750 .

crystal-TV device X-rays objects in motion, 1484.

detector, large sodium iodide, to high energy $\mathrm{X}$-rays, 2741 .

gamma-ray, spectrometer pulse-height distributions, 2856.

liquid, counting, 2614.

spectrometers for measuring total energy of X-ray photons, 1136.

Scintillator, liquid, temperature effects, 1759

Scintillators, liquid, improved 2597.

gas-free liquid, temperature effects, 1915.

liquid, oxygen quenching and wavelength shifters, 1439. 
liquid, quenching of, role of oxygen, 1858.

liquid, three ways to increase efficiency, 1966.

Screw thread, standardization, 1862.

standardization, another milestone, 2025.

standards for Federal Services, H28 (1957) Part II. why, must be everybody's business, 1977.

SEAC, 820 . world unification, 1581 .

computation of vibration modes and frequencies, 1293.

construction and use of subroutines for, 416 .

influence coefficients of tapered cantilever beams computed, $467 ; 738$.

numerical solution of the helium wave equation, 372 . progress report, 1995.

use of sub-routines, for numerical integrations of differential equations and for Gaussian quadrature, 598.

Sea-level latitude variation of fast cosmic-ray neutrons, 343.

Search for the microwave spectrum of perchlorylfluoride, 1863.

Second, order determinants of Legendre polynomials, 305.

report of the special committee on world-wide ionospheric soundings of the URSI/AGI Committee, 2294

sound attenuation in liquid helium II, 1137.

sound propagation in liquid helium II, 821 .

sound velocity measurements below $1^{\circ} \mathrm{K}, 822$.

spectrum of ruthenium (Ru I1), RP2908.

Secondary, chromatic aberration, 2295.

electrons: average energy loss per ionization, 538

Sedimentation equilibrium of flexible chain molecules, 2296.

Segment method, bounds for eigenvalues of Hermitian matrices, 1005 .

Seidel iterants of nearly symmetric matrices, convergence, 329

Selected bibliography on building construction and maintenance, BMS140, 3d ed

Selecting engineering adhesives, 306 .

Selection, glasses for three color achromats, 3043 .

limited number from many possible conditioning treatments for alloys to achieve best coverage and statistical evaluation, 155

Selenium-73, disintegration, $1316 ; 1680$.

Self-absorption in $4 \pi$-counter sources, RP2859.

Self-balancing direct-current bridge for accurate bolometric power measurements, RP2776.

Self-curing denture base resins, properties, 521.

Self-heating of a spherical body, RP2773.

Self-ignition temperatures of materials from kineticreaction data, RP2909.

Semiautomatic Townsend balance system, 3044.

Semiconducting intermetallic compounds, 823 .

Semiconductors, apparatus for measuring the piezoresistivity of, RP2814.

compound, 2495 .

electrical properties of titanium dioxide, 704 .

nuclear magnetic resonance, 3000 .

Semicrystalline poly(chlorotrifluoroethylene), specific volume and degree of crystallinity of, and estimated specific volumes of the pure amorphous and crystalline phases, RP2862.

Semi-infinite conducting medium, basic experimental studies of the magnetic field from electromagnetic sources immersed, J 64D1, 21.

Semi-micro dilution viscometer, 1245 .

Sensitivity and life data on Bourdon tubes, 1864.

Sen-White paper on atmospheric oscillations, extension of, 1341

Separation, and determination of phosphate, silicate, and arsenate, RP2954.

determination of small quantities of aluminum in steel, J 64A3, 235.

hydrogen isotopes by multicomponent distillation, 2751 . isomeric polyphenyls by adsorption chromatography, 2752 .

products of cool flame oxidation of propane, 2753 .

Sequential, decision problems for processes with continuous time parameter, 824 .

flow cooling of electronic equipment, 1865.

Series-resonant high-voltage supply, 539 .

Service, area of an airborne television station, TN35 (PB151394).

Nation's technology, 1999.

range for air-to-ground and air-to-air communications at frequencies above $50 \mathrm{Mc}, 540$.

tests of boys' shoes, 541 .

Sets of three measurements, 825 .

Sferics, measurements, ionospheric reflection coefficients at VLF, 2172 .

polarization, 2257.

spectrum analysis, 2312.

Shape of, high-energy end of the electron bremsstrahlung spectrum, 2755 .

liquid immiscibility in the system barium oxideboric oxide-silica, 2754.

liquidus surface as a criterion of stable glass formation, RP2872.

Sharp line absorption in silica near $3400 \mathrm{~cm}^{-1}, 2297$.

Shear, degradation of polymers, 976 .

modulus and torsional resonance frequencies of bars of rectangular cross section, RP2861.

rubber in, nonlinear viscoelastic behavior, 1419 .

Sheet steel, 1133.

Sheets and pillowcases, standards help in the selection, 136.

Sheffield smoothness testers, use of the sensitivity criterion for comparison, 1970 .

Shellac, 2298.

Shelters, gamma-ray penetration, RP2827.

Shielding, problems, aspect of gamma-ray penetration theory, 2028.

transient electromagnetic dipole field by a conductive sheet, 1866.

Ships, welded, investigation of structural failures, 746 .

Shock wave experiments, 2896.

Shoe, last sizes, 113 .

research, suggested problem for industry-sponsored, 379 .

Shoes, boys', durability, 691 .

service tests, 541 .

Shutter-illumination device for the study of phototropic dyes, 374.

Sieve problems for all-purpose computers, 882 .

Sigma phase in high-purity 18-10, 542 .

Signal, analysis, transients, 1968.

fluctuating, intermittent communications, 2163.

generator system for low output levels, 826 .

generators to $1000 \mathrm{Mc}$, calibration of, TN37 (PB151396)

glassware, railroad, an evaluation of the luminoustransmittance requirements, RP2849.

lighting, $2562 ; 2952$.

minimum detectable, measurement systems, power spectra, 1430 .

range of FM and television broadcasting stations, variation with frequency, 347 .

rapid, analysis, limitations, 1388.

source, microwave, amplitude stabilization, 2452.

Significance of the results of some physical tests on upper leather, 1485

Silanes, aryl, 1192.

Silica gel, a thermochemical study of the reaction of, RP2785.

low temperatures, 2730.

Silica, glass, noncrystal ionic model for, RP2782.

near $3400 \mathrm{~cm}^{-1}$, sharp line absorption, 2297.

Silica-gypsum investiments, 1184 .

Silicate, and binary alkali borate glasses at high pressures, compressibility, 1292.

calcium, hydrates, 1172 .

cement, dental, 20 . 
cement: how to select and use them, 2299; 2756 .

cement: method of mixing in a closed container to prevent effects of exposure to atmosphere, 827 cenlents and zinc phosphate, 2873.

cements, deterioration in the tropies, 217.

glasses, alkali density formula, RP2915.

glasses during annealing, structural rearrangements. 1901.

glasses, infrared transmissions, 2933.

glasses, role of vitrons in alkali, RP2854.

phosphate, and arsenate, separation and determina tion, RP2954.

Silicates, molten alkali, density, expansivity, and viscosity, 422 .

Silicon, carbide heating elements, infrared emission spectrum of, RP2810.

tin, iron, copper, and nickel, electrorefining studies, 2811.

Siloxanes, methyl, by electron impact, 747 .

Silver, and Pyrex, stability in perchloric acid-silver perchlorate solutions and in conductivity water, J $64 \mathrm{~A} 1,127$.

Silver, chloride for visible and infrared radient energy, refractive index, 124.

copper, molybdenum, tantalum, and gold at $662 \mathrm{kev}$, total photoelectric eross sections, 3101 .

electrode, 1486.

silver chloride electrode, standard electrode potential, 1895.

sodium chloride, thallium chloride, and thallium bromide, photoemission, 1807.

sulfide in the photographic image during fixation, formation of, $\mathrm{J} 64 \mathrm{C1}, 65$.

Similarity, principle in the backscattering of $\gamma$-rays, 934 properties of the two-fluid model of superconductivity, 543.

Simple, humidity lag apparatus, 1487.

rotating molecular still, RP2951.

technic for making porcelain jacket crowns, 2000 .

technique for diplexing $10,000 \mathrm{Mc}$ and video signals on coaxial cables, 2300 .

type $\mathrm{X}(t)-\mathrm{Y}$ recorder, 184.

Simplified, apprach to spin in Dirac theory, 2757.

method for measuring the attenuation of balanced transmission lines, 376.

method of locating the point of symmetry, 2001.

Simpon's rule for the numerical evaluation of Weiner's integrals in function space, 185 .

Simulator for use in development of jet engine controls, $\mathrm{C} 584$.

Simultaneous linear equations, determination eigenvalues, AMS49.

Sing-around ultrasonic velocimeter for liquids, 2301.

Single frequency filters, design, TN23 (PB151382).

path phase measuring system for three-centimeter radio waves, 2758 .

Single-phase transfer of liquefied gases, C596.

Singular, perturbation methods for nonlinear oscillations, 828.

perturbation problems in theory of non-linear vibrations, 277 .

Sinusoidal, damped, source current, 3024.

Skew shap suspended in a viscous liquid, motion of small particles in, 1077.

Skin, calf, squares, influence of temperature on the adsorption of chrome $\left(\mathrm{Cr}_{2} \mathrm{O}_{3}\right), 2159$.

Skins, fur, aging of karakul and seal, 19.

Sky, and eye, 2830 .

wave, pulse, phenomena observed at $100 \mathrm{Kc}, 2274$.

Slack-quenched alloy steels, impact properties, RP2799.

Slant-Es, some implications, 2304.

Slide rule computes radio refractive index of air, 829 .

Slit function effects in the direct measurement of absorption line half-widths and intensities, 1867.

Slots, conductance, 1796.

Slotted-cylinder antennas, pattern synthesis, J 63D3, 303.

Small, current-measurements, 2036.

grating spectrometer for the far infrared region, 1488 . precision bottles of quartz and low-loss glass, 2224 .
Smog at Los Angeles, spectral radiant energy from the sun through varying degrees, 1545 .

Smooth patterns of production, 1139.

Smooth-obstacle diffraction of electromagnetic waves for grazing angles, J 631 2, 181.

Smoothing, operations and their generating functions, 783 . problem, remark on, 931.

Social and industrial research, information processing in, 741 .

Sodium, amalgam in the form of pellets, preparation of, J 64 A1, 135.

borohydride, heat content of, from $0^{\circ}$ to $400^{\circ} \mathrm{C}$, RP2828.

borohydride reduction of aldonic lactones, 1868 .

calcium aluminate, heat of formation, RP2887.

carbonate in photographic developers, note on estimation, 1238 .

chloride solutions, turbidity and viseosity measurements in, RP2778.

chloride, thallium chloride, and thallium bromidc, photoemission from silver into, 1807.

chlorite, determination of glucose, 1310.

chlorite with some aldoses and modified celluloses, reaction, 1473 .

dodecyl sulfate-sodium chloride-water, light scattering and miscelle structure, 1386 .

flame photometric determination, in portland cement, 736.

hydroxide and sulfuric acid methods for analysis of wool admixed with cotton and viscose rayon, 973 .

iodide scintillation detector to high energy X-rays, 2741.

vapor by electron impact, multiple ionization, 2996.

Soil clays and related materials, electron microscopy, 439.

Solar, atmosphere, motion in, as deduced from radio measurements, 263.

chromosphere, 2927

eycle influence on the lower and on VHF forward scatter, ionosphere, 2759.

disturbance, observation of audiofrequency electromagnetic noise, 2019.

"enhanced radiation" and plasma oscillations, 544.

flare radiation, ionospheric effects, 1750 .

$\mathrm{H}_{\alpha}$ filaments and geomagnetic disturbance3, 1489.

indexes, variously derived, comparison, 40.

inner, corona during June 1959,3080.

lines, identification of, 864 .

lunar atmospheric tides in barometric pressure, as regards to their worldwide distribution of amplitude and phase, 1586.

plasma, condition for radiation, 414 .

radiation, near infrared, measurements by balloon to an altitude of $100,000 \mathrm{ft}$., 2645 .

radio astronomy at the NBS, 1490 .

radio bursts, 2958 .

radio noise, $1149 ; 1418$.

radio outbursts, an estimate of the density and motion of solar material from observed characteristics, 649.

spectrum, analysis, revised, 933 .

spectrum, CH, J 63A1, 19.

spectrum, infrared multiplets, 743.

spectrum, molecules, 2205.

spectrum, OH, J 63A $3,279$.

spectrum, rotational lines of $\mathrm{CH}, \mathrm{OH}$, and $\mathrm{CN}, 2292$.

wave-lengths, Rowland's report on the second revision, 1478 .

zenith angle $(x)$ for thirty-five locations, tables of median hourly values of the cosine of, TN 5 (PB151364).

Solderless electronic assembly with cellular units, 830 .

Sole leather with polymers, impregnation, 2594.

Solenide, manganese, magnetic susceptibility, 254.

Solenoid, coils, coupling chart, 41 .

design chart, $\mathrm{R}-\mathrm{F}, 294$.

Solid, materials, mechanical testing, 482.

nitrogen condensed at $4.2^{\circ} \mathrm{K}$ from a gas discharge, spectra emitted, 1881. 
nitrogen $\left(4.2^{\circ} \mathrm{K}\right)$ containing atoms of free radicals, 2622.

state, liquid, at low temperatures, TN4 (PB151363).

Solids, at low temperatures, 2934.

elementary shape, temperature-induced, Mono.2.

Solution, of the caterer problem, 1099.

differential equation occurring in the problem of heat convection in laminar flow through a tube, 789 .

Solutions, cuprammonium and cellulose, 112.

dilute polymer, 166 .

Solvability and consistency for linear equations and inequalities, 1869 . Solving linear algebraic equations can be interesting,

Sonic technique, studies on leather by means of, 1905 .

L-Sorbose-C ${ }^{14}$, synthesis of, RP2800.

Sorption, of dextran to collodion membranes, 832 .

macro-molecules to solid surfaces: sorption of dextran to cellulose nitrate membranes in the presence of serum albumin and surface-active agents, 1500.

Sorting, device (mail), capacity requirement of, J $63 \mathrm{~B} 2$, 79.

equipment (mail), analytic comparison of suggested configurations for, J $63 \mathrm{~B} 2,83$.

Sound, absorbers for jet-engine test cells, measurements, 1771 .

absorption coefficient from acoustic measurement, 46.

fields, reverberant, interference patterns, 1373.

insulation of wall, floor, and door constructions, BMS144, 2d Suppl.

patches of absorbent materials, 2010 .

power output of subsonic air jets, reverberation chamber study, 2742 .

propagation of, in five monoatomic gases, 1823.

propagation, second, in liquid helium II, 821 .

rarefied helium, propagation, 115 .

reverberant, fields, measurement of correlationcoefficients, 1399 .

reverberant through double walls, 159 .

reverberant, through walls, 1214 .

source in a reverberation chamber and other reflecting environments, qutput, 2679.

speed of, in water, by a direct method, RP2795.

speed of, in water, effect of dissolved air, 1686 .

speed of, in water near the turning point, temperature coefficient, 1914

transmission loss of walls as a continuous function of frequency, new method of recording, 1414.

velocity measurements below $1^{\circ} \mathrm{K}$, second, 822 .

Sounding, vertical-incidence ionosphere, at the National Bureau of Standards, TN28 (PB151387).

Source of error in the measurement of radiated harmonics, 377.

error in radio phase measuring systems, 10.

function in a non-equilibrium atmoshpere. II. Depth dependence of source function for resonance and strong subordinate lines, 2767.

non-migratable ionic charges developed by portland and high-alumina cements during hydration, 2768 .

Sources of error in various methods of airplane camera calibration, 1878 .

identifying symbols of Government specifications, 549.

transport coefficients and correlations of thermodynamic and transport data, 1501; 1879.

Space, communication, outer, optimum frequencies, J $64 \mathrm{D} 2,105$.

diversity transmitters, reception, 3033 .

edge of, 2849 .

$k$-commutative matrices, J 64B1, 51 .

problems on random walks, 310 .

vehicles, coatings for, $2910 \mathrm{~A}$.

waves propagated over irregular terrain, transmission loss, 606 .
Space-charge, wave amplification in a shock front and the fine structure of solar radio noise, 1149 .

wave in moving, interacting electron beams with application to solar radio noise, nonlinear theory, 1418.

Space-charged clouds, electron optical properties, 53.

Space-wave fadeouts at $1,046 \mathrm{Mc}, 1119$.

Spatial, distribution of energy dissipation by high-energy $\mathrm{X}$-rays, 1150 .

resolution of refractometer cavities, J $64 \mathrm{D} 1,65$.

Spatially variable flow, determination of critical depth, 425 .

Special lubricants tests, 311 .

Specific, dental silicate cement, No. 9, American Dental Association, 20.

heat of beryllium between $0^{\circ}$ and $900^{\circ} \mathrm{C}, 312$.

heats of collagen and leather, 1502 .

heats of liquid metals and liquid salts, 2306.

volume and degree of crystallinity of poly(chlorotrifluoroethylene), 2831 .

volume and degree of crystallinity of semicrystalline poly(chlorotrifluoroethylene), and estimated specific volume of the pure amorphous and crystalline phases, RP2862.

Specification, for cells (dry) and batteries, H71.

color rendering properties of fluorescent lamps, 134.

proposed, for dental chromium-cobalt casting alloys, 1996.

transient nonequilibrium systems, 2237.

Specifications, American Chemical Society, reagent chemical, 1474 .

American Dental Association, for dental materials, 2451.

certain, properties of building brick and effects of differences in raw materials and methods for forming, relations, 528 .

certification, dental materials, annual for 1943, 1307. color, 52 .

color-rendering properties of fluorescent lamps, 833 .

dental materials, American Dental Association, 1614. electrodeposited coatings, 313 .

government, 1725 .

government, sources and identifying symbols, 549

standards and codes, governmental aspects, 1880 .

standards for plating, 1447.

Spectacle lenses, instrument for measuring the marginal power, 1369.

Spectra, absorption, and chemical constitution of dyes, relation between, RP2823.

absorption, of solids condensed at low temperatures from electric discharges, 2445 .

afterglows and discharges from nitrogen-oxygen mixtures, 2307 .

astronomical, lines of the chemical elements, 1758 .

atomic, 1269 .

atomic, data concerning, 677 .

atomic, for the astrophysicist, 197.

atomic, notation for, 775 .

atomic - their role in astrophysics, 1974.

atomic, with electronic digital computers, analysis, 1624.

bremsstrahlung, 1211.

electron spin resonance, from gamma-irradiated solid nitrogen, 2940.

emission, autoignited heptane-air mixtures, 442 .

emission, of first-stage combustion in an engine, 333 .

emitted from solid nitrogen condensed at very low temperatures from a gas discharge, 3052 .

emitted from solid nitrogen condensed at $4.2^{\circ} \mathrm{K}$ from a gas dischargee, 1881 .

energy, of cascade electrons and photons, RP2824.

flame emission, kineties of $\mathrm{OH}$ radicals, 475 .

gamma-ray degradation, an approximate expression for, RP2788.

impure mercury 198,60 .

infrared absorption, $75 ; 1569$.

infrared absorption, of some cyclic acetals of sugars, RP2960. 
infrared absorption, of urea, thiourea, and some thiourea-alkali halide complexes, 2160.

infrared and raman of trans-difluorodichloroethylene, 2161.

infrared flame, 1366.

infrared, of asphalts, J63A2, 189.

infrared, of crystalline polyphenyls, RP2829.

infrared, of hydrogen cyanide and deuterium cyanide, 1739 .

infrared, of molecules of the atmosphere, 2377.

infrared, of sugar acetates in solution, RP2772.

infrared, of thermally degraded poly(vinyl chloride), RP2832.

mars, high-dispersion, 2152 .

mass, and relative sensitivities of some polyphenyls, RP2831.

mass, of aromatic hydrocarbons filtered from smoky air, RP2876.

microwave, of deutero-ammonias, 260.

microwave of molecules exhibiting internal rotation, 2202; 2637; 2638; 2991.

molecular complexes, 2994.

power, and the minimum detectable signal in measurement systems, 1430 .

power, instantantaneous, 469.

rare-earth, 3090 .

rotation-vibration, of deuterated water vapor, 1859 ; 1949.

shape of the nonresonant, 2218 .

synthesis and infrared absorption, of some $m$-polyphenyls, 1951 .

tables, 2337.

thorium, new description, Mono. 17.

trifluoroethylene, 1224 .

various times after fission, 3105 .

VLF radiation, of lightning discharges, J 63D2, 199.

Yttrium iron garnet, 2966.

Spectral, absorbency of 4 -chloro- $4^{\prime}$-aminodiphenylsulfone, RP2794.

absorption method for determining population "temperatures" in hot gases, 1882.

band-pass determinations by a dynamic approach, 2770 .

emittance of a silicon carbide heating element, RP2810.

emittance of ceramic-coated and uncoated specimens of Inconel and stainless steel, 3053 .

Spectral emittance of uncoated and ceramic coated Inconel an type 321 stainless steel, 3092 .

energy distribution to the International Commission on Illumination light sources A, B, and C, 834 .

lines as frequeney standards, 550 .

radiant energy from the sun through varying degrees of smog at Los Angeles, 1545.

Spectral-transmissive, properties of plastics for use in eye protection, 1547 .

properties of some of the tektites, 1546.

Spectrochemical analysis, index to the literature, $1036 ; 2965$.

Spectrographic, emission, standards, 232.

standard samples of iron-base alloys, 2719.

standards, investigation of homogeneity of six proposed NBS-GMC zinc-base, 1377.

Spectrometer, electron absorption, 2541.

fast neutron, TN1 (PB151360); TN10 (PB151369).

infrared high-resolution grating, RP2922.

mass, analysis of fluorinated polyphenyls, 1263.

mass, analysis of solids, 194.

mass, combination crossed-field and time-of-flight, 360 .

mass, radio frequency, 117 .

pulse-height distributions, 2856.

small grating, for the far infrared region, 1488.

Spectrometers, beta-ray, 657 .

calibration of, from 2 to 16 microns, Mono.16.

corpuscular, 2791 .

electron, 2612.

particle, note on accelerating components, 2649.

response functions of total-absorption, 2740 .

scintillation, for measuring, 1136 . scintillation, in the study of continuous gamma- and X-ray spectra between 0.01 and 20 Mev, use, 1955 .

Spectronetric, mass, study of the decomposition of hydrazoic acid by the electric discharge, 2623.

mass, study of the reactions of some hydocarbons with active nitrogen, 2982.

Spectrometry, infrared, with a cesium iodide prism, 744 . mass, $478 ; 1060 ; 1764 ; 2624$.

mass, analy tical, 1626 .

mass, and absorption, polymer degradation, 515 .

mass, study of degradation of polystyrene, 557 .

Spectrophotometer, Beckman quartz, 484.

Spectrophotometers in the $210-760 \mathrm{~m} \mu$ rcgion, 625 .

Spectrophotometric colorimetry, propagation of errors in, 804.

data to Munsell renotations, 2522.

evidence for the absence of free aldehyde groups in periodate-oxidized cellulose, 314 .

optical, analysis of hydrogen-deuterium mixtures in presence of air, 505.

Spectrophotometry and aerial photoreconnaissance, 2308. determination of phosphate, 685 .

low pressure explosions, ultraviolet, 1565.

precision, colorimetry, 2487.

Spectroradiometer, grading, design and construction of a blackbody and its use in the calibration, 2513 . ratio-recording, $\mathrm{J} 64 \mathrm{C} 2,151$.

Spectroscopic, analysis of deuterium in hydrogen-deuterium mixtures, 551 .

data, determination of molecular structure, 2651.

determination of the dissociation energy of the $\mathrm{OH}$ radical, 1503.

evidence for triatomic nitrogen in solids at very low temperatures, 3054 .

observations on pentaborane-air flames and explosions, 1883.

source, plasma jet, 3088 .

studies of solids condensed at $4.2^{\circ} \mathrm{K}$ from electric discharge through nitrogen, oxygen, hydrogen, water, and ammonia, 2309.

study of electronic flame temperatures and energy distributions, $2771 ; 2310$.

study of oils used in oil-extended rubber, RP2771.

survey of energy distributions of $\mathrm{OH}, \mathrm{C}_{2}$, and $\mathrm{CH}$ radicals in low-pressure acetylene-oxygen flames, 2311.

Spectroscopy, 1504 .

cesium iodide prism molecular, further infrared measurements, 1351.

chemical, 35 .

emission, $443 ; 1013 ; 1705 ; 2545 ; 3021$.

gamma-ray, 2139.

kinetic, in the far ultraviolet, 2607.

meeting, June 1956, Report on Joint Commission, 1849.

microwave, broad-band coaxial stark cell, $178 ; 358$.

optical, stable carbon isotope analysis, 1892.

optical, stable nitrogen isotope analysis, 2775 .

what use is it, 622 .

Spectrum, absorption, of vibrationally excited $\mathrm{N}_{2}$ in active nitrogen, 2887.

amplitude, and scale of refractive index in homogeneities in the first 125 meters of the atmosphere, 1258 .

analysis of sferics, 2312 .

bounded self-adjoint operator, 220 .

bromotrifluoroethylene, 1206.

$\mathrm{C}_{3}$ molecule between $3600 \AA$ and $4200 \AA, 1885 ; 2313$

electron density fluctuations in the ionosphere, 2381 .

energy levels of neutral helium ( $\left.{ }^{\mathrm{He}} \mathrm{I}\right), \mathrm{J} 64 \mathrm{~A} 1,19$.

bremsstrahlung, electron, 2755.

$\mathrm{D}_{2} \mathrm{O}$, vibration-rotation, 891 .

dynamic, for Sacremonto Peak, 1233.

emission, of $\mathrm{OH}$ from 1.4 to $1.7 \mu, 860$.

emission of $\mathrm{OH}$ from 218 to $4.1 \mu, 2356$.

energy, resulting from electron slowing down, 1015 
first, of iodine, description and analysis of, J $63 \mathrm{~A} 1,1$. frequency-shift radio phototransmissions, 1884 .

hafnium, 1208 .

HDO, vibration-rotation, 892.

infrared absorption, of $n$-dimethyl-amino-diborane, 1365 .

infrared, and rotational constants of carbon disulfide, 1932 .

infrared, and structure of perchlorylfluoride, 193.

infrared, hydrogen sulfide, 1187.

infrared emission, of methane at 3.3 microns, RP2803.

infrared multiplets, 743 .

infrared, of acetylene- $\mathrm{d}_{1}, 1934$

infrared, of carbonyl sulfide, 2162.

infrared, of hydrogen sulfide, $1040 ; 1740$.

infrared, of hydrogen sulfide from $2200-2800 \mathrm{~cm}^{-1}$, 1741 .

infrared, of nitrous oxide, 1782 .

infrared, of perchlorylfluoride, 1933.

infrared, of tertiary butyl fluoride, 2820 .

inversion, absorption in the low-frequency wing of the $\mathrm{NH}_{3}, 639$.

mass, of gallium vapor, 758 .

methane from 2,470 to $3,200 \mathrm{~cm}^{-1}$, infrared absorption, J 64A3, 201.

microwave, and structure of sulfurylfluoride, 2372 .

microwave, benzonitrils, 1075 .

microwave, of methyl germane, 2992.

microwave, of perchlorylfluoride, search for, 1863 .

$\mathrm{ND}_{3}$, microwave absorption, 766 .

normal matrix, variation, 890 .

molecules, 2205 .

$\mathrm{OH}, \mathrm{J} 63 \mathrm{~A} 3,279$.

one parametric family of matrices, 1100.

raman of triethylborane, 2278.

rhenium, first, term analysis of, RP2804.

rotational lines of $\mathrm{CH}, \mathrm{OH}$, and $\mathrm{CN}, 2292$.

second, of molybdenum, description and analysis, RP2856.

second, of zirconium into the infrared, extension of, 717

solar, CH, J $63 \mathrm{~A} 1,19$.

solar, rotational, of $\mathrm{CH}, \mathrm{OH}$, and $\mathrm{CN}, 2292$.

Spectrum, vibrational, of chlorotrifluoroethylene, 893 .

1-fluoro-1-chloroethylene, 1576.

fluorotrichloroethylene, 1577 .

tetrabromoethylene, 1973.

Specular-gloss measurement of ceramic materials, 1886.

Speed, of sound in water by a direct method, RP2795. sound in water near the turning point, temperature coefficient, 1914.

Sphere, an exact earth-flattening procedure in propagation, J 64D1, 61

Sphere-gap volt-time curves-standards for steep front surge measurements, 2314.

Spherical, aberration, improved optical test, 950 .

body, a problem in self-heating, RP2773

core, impedance characteristies of a uniform current loop, J 64D3, 295.

earth from a sinusoidal source current, 3024 .

spheroidal, and cylindrical bodies, J $64 \mathrm{~B} 1,15$.

wave pulse by a half plane screen, diffraction, 2086 .

Spheroidal, spherical, and cylindrical bodies, J 64B1, 15. wave equation, 518 .

Spin-spin, absorption in chronic methylammonium alum and manganous chloride tetrahydrate at very low temperatures, 1887.

absorption in manganous chloride tetrahydrate, 1888 .

relaxation at very low temperatures in chromic methylammonium alum, 1889.

Spinning, preparation of bituminous materials for, 800 .

Splitting of the giant resonance for deformed nuclei, 2772 .

Sporadic E, and aurora, echoes at Barrow, Alaska, relationship, 1845 .

F-scatter program, TN48 (PB151407).

ionization, 2885 .

reflections, 2 .

television reception, effect on, $574 ; 859$.
VHF oblique incidence circuits, 2773.

VHF transmission in North America, 3074.

Springs, plastic, 1810.

plastic, reinforced, 1843.

Spurious resolution of photographic lenses, 315 . Square, clamped plates, vibration, 1435 .

hollow, torsion, 2385.

Squares, Latin, a note on the four by four, 5 .

Stability, of austenitic stainless steels at low temperatures as determined by magnetic measurements, 3093 .

interfacial waves between two streams, approximate theory, 1632.

oscillations of superposed fluids, 1890.

phase, measurements of over a low-level tropospheric path, J 63D1, 45.

quartz resonators at very low temperatures, 2315 .

silver and Pyrex in perchloric acid-silver perchlorate solutions and in conduetivity water, J $64 \mathrm{~A} 1,127$.

Stabilization, amplitude of a microwave signal source, 2452 .

film processing for $\mathrm{X}$ - and $\gamma$-ray dosimetry, 1891.

free radical, in condensed phases, 257 .

free radicals at low temperatures, Mono. 12; 2316.

Stabilized free radicals, 2774 .

Stabilizer, power, microwave, 90.

Stable, carbon isotope analysis of optical spectroscopy, 1892.

electronic voltage regulator, 135 .

nitrogen isotope analysis by optical spectroscopy, 2775 .

systems of differential equations with integrable forcing term, 1893.

voltage controlled logarithmic attenuator, 935 .

Stable-output oscillator, 552 .

Stacks, plumbing, capacity, 1281.

Stainless steel, austenitic, stress corrosion cracking of type 304,1898

determination of aluminum in high temperature alloys, J $64 \mathrm{~A} 2,181$.

$17-7 \mathrm{PH}, 2883$.

Standard, American, building code requirements for masonry, 945 .

chamber for the measurement of low energy X-rays, J $64 \mathrm{C} 1,49$

cells, portable potentiometer and thermostated containers, 1450.

deviation of a normal population, estimating, 713 .

electrode potential of the silver, silver chloride electrode, 1895.

federal, new, on colors, 2213.

free-air ionization chambers, plate separation requirements, 1110

frequency and time, atomic clock, 148.

materials, issued by the NBS, C552, 3d ed.

methods of measurement in architectural acoustics, 2226 .

microphones, laboratory, 1048.

multipurpose for microchemical analysis, 2641.

natural rubber, 1505 .

primary, of frequency, 737.

proposed, for facilities for scientific conferences, 2434. resistors, rack for, 3026 .

samples, 835 .

samples and reference materials, issued by the NBS, C552, 3d ed.

test for photoflash cells, 1506.

transistor frequency, 900.

transmissions, low frequency, 2179.

transmittance, in the ultraviolet, 388 .

wind load requirements, new, 2214.

WWV, frequency transmissions, $2871 ; 2872$.

X-ray diffraction powder patterns, C539, Vols. 7, 8, 9. Standard, frequency, at low temperatures, 1589.

broadcast at very low frequency, 2270 .

broadcast reception, world-wide, 623 .

broadcasting station, world-wide, the power requirements and choice of an optimum frequency, J $63 \mathrm{D} 1,35$.

NBS primary, 872 . 
station WWVH, 42.

transmission and time signals, $3054 \mathrm{~A}$.

Standardization, of beta-emitting nuelides, 1200.

meaning of $\mathrm{pH}$ measurements, 1767

radioaetivity, in the United States, 2712.

research in the plasties industry, 1857.

serew thread, 1862.

screw thread, another milestone, 2025.

sulfur- 35 by $4 \pi$-eounting, RP2859.

textile testing methods, international eooperation, 471,

Standards, and eodes, governmental aspeets, speeifieations, 1880 .

Assoeiation, Ameriean, report of the eommittee, 3036. basic, for scienee and industry, 2037.

common, government and industry use, 2144

development of VHF field-intensity, 427.

dielectrie, American, 944.

dimensions, and units, 2088.

eleetrieal measurements, 316 .

electronics, 1508.

emission speetrographic, 232.

frequency, 456 .

frequency, precision quartz resonator, 1111

frequeney, spectral lines, 550 .

good and bad, $2777 \mathrm{~A}$.

help in the seleetion of sheets and pilloweases, 136.

high-frequeney impedanee, 2583.

laboratories, local, 2977.

length and light waves, 1387.

measurement, elassifieation and nomenelature, $2486 \mathrm{~A}$.

measurements for electronies, 2776 .

measurements of frequencies and time intervals in the U.S.A. from 1954 to $1957,2550$.

NBS, 1507.

national physieal, and design of experiment, 2210.

neutron flux measurement and neutron dosimetry, 3055 .

new, for science and industry, 2998.

$\mathrm{pH}$ measurements from $60^{\circ}$ to $95^{\circ} \mathrm{C}, \mathrm{RP} 2797$.

physical for the eletronics industry, 1808.

plastics, 1509.

primary, atomic definition, 28.

radiation proteetion, 1639 .

radiation proteetion regulations, exposure, 2947.

radioaetive, preparation and maintenanee by the NBS, 1455 .

radioactivity, applieations, 1631.

radioaetivity, international eomparisons, 1746.

radioaetivity, preparation and maintenanee, 1944.

radioaetivity, preparation, maintenanee, and application, C594.

radioaetivity, program, 2714 .

range $14^{\circ}$ to $122^{\circ} \mathrm{K}$, heat capacity, 729 .

research for the plastics industry, 1130 .

roentgen of Great Britain and USA, intercomparison, 1621.

roentgen ray, intercomparison of French and U.S., 2017.

seience, 198.

scientific, fifty years, 236.

serew-thread for Federal Services, H28 (1957) Part II.

shoe last sizes, 113.

specifieations for plating, 1447.

temperature, 836 .

time, at the NBS, molecular and atomie, 2549.

time, precision, 209.

$\mathrm{X}$-ray, comparison, $916 ; 1230$.

Stannide, magnesium at low temperatures, eleetrieal conduetion, 1695 .

Stark, broadening funetion for the hydrogen lines, C603. effeet, 1510 .

effect of NO molecule, 2840.

Stars, radio, observed at Boulder, Colorado, amplitude scintillations, preliminary analysis, TN20 (PB151379).

State, eontrol of proteetion against ionizing radiation, 1151 .

NBS weights and measures administration, 485 .
Static ealorimeter, applieations, eomparative eharneteristies of mediun-prieed fully automatio computers, 2061.

approach to the problem of multiple radio interferenee to FM and television service, 378 .

aspeets of analytieal determinations, 553.

chain-ratio method for estimating relative volumes of mail to given destination, $\mathrm{J} 64 \mathrm{C} 1,37$.

characteristics and narrow-band teletype message errors on a single-sideband 600-mile-long ultrahigh-frequeney tropospheric radio link, measured, J 64D2, 125.

characteristies of VLF atmospherie radio noise, measured, 2188.

decision proeedures and zero-sum two-person games, 231.

design of experiments, 2500.

designs for experiments, 133 .

dry, for rf power measurement, 2419.

dynamie tests for meehanieal properties of fired plastie refractories and other more resilient matcrials, 1684 .

electrieity, hazards from, 461

fatigue tests of flush riveted joints, 1345 .

methods in ehemistry, $1896 ; 2779$.

predietion of radio ray bending, TN44 (PB151403)

prineiples of testing, 1511 .

results and their shorteomings eoneerning the ionosphere within the auroral zone, 2317.

sampling methods to outgoing letter mail charaeteristies, some applieations, TN16 (PB151375).

treatment of analytieal data, 2780 .

Statistieal engineering:

aeeeptanee sampling by variables, 16 .

aeeeptance sampling of eleetroplated articles, 940.

analysis for some partially balaneed incomplete bloek designs having a missing block, 953 .

analysis of eovariance for ineomplete bloek design, 2342 .

analysis of ineomplete bloek designs, 2343.

analyzing straight-line data, 652 .

aspeets of predietion in the study of complex systems, 2225 .

asymptotie behavior of tests on the mean of a logarithmie-normal distribution with known varianees, 2030.

asymptotie transformation of certain distributions into the normal distribution, 1795 .

eertain Fourier transforms of distributions, 202. eertain fourier transforms of distribution: II, 965. ehain block design, 852 .

ehain block designs with two-way elimination of heterogeneity, 966.

ehanges of sign of sums of random variables, 662 . eomparative tests in a single laboratory, 39

components of varianee in preparing sehedules for sampling of baled wool, 346 .

computation of $x^{2}, 2877$.

eomputers in statisties, 2404.

confidenee and toleranee intervals for the normal distribution, 674 .

confidenee intervals for the expectation of a Poisson varible, 2915.

confidenee intervals in regression problems, 2429 . eonnections between probability theory and differentail and integral equations, 278 .

contributions to Dietionary of Physics and Electronics, 1660 .

eontributions to Handbook of Physies, 2500.

eontributions to the theory of rank order statistiesthe two sample case, 1661 .

control eharts may be all right but-, 676 .

design of experiments in operations researeh, 1670.

design of experiments in research and development, 2083.

deviation of the Poisson distribution, 2823.

distribution of a serial correlation coefficicnt near the ends of the range, 2527 .

duration of random walks, 281 . 
elements of a mathematical theory of probability, 708.

elimination of randomization in certain statistical decision procedures and zero-sum two-person games, 231 .

embedding theorem for balanced incomplete block designs, 948.

essential property of the Fourier transforms of distribution function, 391.

estimating the standard deviation of a normal population, 713 .

estimation of parameters in certain stochastic processes, 334 .

evaluation, 155 .

evaluation of analytical methods by the sensitivity criterion, 2112.

exact evaluation of the variances and covariances of order statistics in samples from the extreme-value distribution, 788 .

examples of the use of high-speed computers in statisties, 2302.

experimental statistics, 445 .

experiments with many factors, 2120 .

Fisherian revolution in methods of an experimentation, 335.

fitting a straight line to certain types of cumulative data, 2125.

four by four Latin squares, 5 .

fractional factorial experiment designs for factors at three levels, AMS54.

fractional factorial experiments of $2^{\mathrm{m}} 3^{\mathrm{n}}$ series, 2568 .

function to aid in the fitting of kinetic data to a rate equation, 1983.

further contributions to the solution of simultaneous linear equations and the determination of Eigenvalues, A MS49.

geological application of extreme-value methods to interpretation of cobbles and boulders in gravel deposits, 1723 .

index for rating diagnostic tests, 71 .

information theory aspects of propagation through time varying media, 1143 .

instrumental drift, 1041 .

interpretation of chemical data, 579 .

literature, 1930 to 1957 , selected bibliography, J 64B1 $55 ; \mathrm{J} 64 \mathrm{~B} 1,69$.

locating sources of variability in a process, 253 .

making one measurement do the work of two, 757 .

mathematical services useful in industry, 759 .

mechanical point of view, Boltzmann equation, 1922.

mechanical theory of transport processes, 2778 .

mechanies, quantum, 2340.

multi-factor experiments, 2995.

multi-factor experiments for evaluating reliability, 2207.

National physical standards and design of experiment, 2209 .

new method of analyzing extreme-value data, 922 .

normal distribution, 1595 .

operating characteristic of the average chart, 588 .

optimum grouping in one-criterior variance components analysis, 1102 .

partially balanced designs, 923 .

Physics. Measure for measure: some problems and paradoxes of precision, 287.

prediction of system performance from information on component performance, 2223.

principle of randomization in the design of experiments, 2378.

principles of experimental design, 880 .

probability distribution of the amplitude of a constant vector plus a Rayleigh-distributed vector, 1541 .

probability distribution of the phase of the resultant vector sum of a constant vector plus a Rayleigh distributed vector, 591 .

probability limits for the average chart when process standards are unspecified, 1116.

problems on random walks in space, 310 . procedures for the rejection of suspected data, 784 . property of additively closed families of distributions, 2433.

property of the normal distribution, 928 .

random flight with multiple partial correlations, 525 . rejection of outlying observations, 813 .

relations among certain ranges of vector measures, 299.

relations among the blocks of symmetrical group divisible designs, 548 .

reliability of measured values, 594 .

sampling of leather, 3039.

selection of a limited number from many possible conditioning treatments for alloys to achieve best coverage and statistical evaluation, 155.

sequential decision problems for processes with continuous time parameter, 824 .

sets of three measurements, 825 .

smooth patterns of production, 1139 .

statistical approach to the problem of multiple radio interference to FM and television service, 378 .

statistical aspects of analytical determinations, 553 .

statistical designs for experiments, 133.

statistical methods in chemistry, 1896; 2779.

statistical principles of testing, 1511 .

statistical results and their shortcomings concerning the ionosphere within the auroral zone, 2317 .

statistical treatment of analytical data, 2780 .

statistician and the post office: a case history in operations research, 2881 .

statistics - a tool in testing rubber, 1512.

statistics and planning tests at elevated temperatures, 137.

statisties in analytical chemistry, 138.

stochastic independence of symmetric and homogeneous linear and quadratic statistics, 596.

strongly continuous stochastic processes, 1095 .

study of accuracy in chemical analysis using linear calibration curves, 2328.

studying the accuracy and precision of an analytical procedure, 926 .

suppression of bubbling in boiling refrigerants, 2330 . survey of adhesion and types of bonds involved, 1157 . tables for constructing and for computing the operating characteristics of single sample plans, 563 .

tables of the bivariate normal distribution function and related functions, AMS50.

technique for locating sources of variability in a process, 324 .

theory of extreme values, 3096 .

uncertainty of measurements, 1953.

uniqueness of the triangular association scheme, 2835.

use of statistics to determine precision of test methods, 165.

weighted compounding of two independent significance tests, 3098 .

Statistician and the post office: a case history in operations research, 2881 .

Statistics-a tool in testing rubber, 1512.

analytical chemistry, 138.

computers in, 2404 .

determine precision of test methods, use, 165 .

experimental, 445 .

free radical, 2571.

high-speed computers in, some examples, 2302.

improve physies, chemical, and engineering measurements, 68 .

order, in samples from the extreme-value distribution, 788.

planning tests at elevated temperatures, 137.

symmetric and homogeneous linear and quadratic, stochastic independence, 596 .

two sample case, contributions to the theory of rank order, 1661 .

Weibull distribution, moments of order, 1428.

Status, of absolute measurements at the National Bureau of Standards, 2781.

ASTM methods and standards for appearance evaluation, $3055 \mathrm{~A}$.

Steam, thermal conductivity, 1657. 
Steam-driven water jets for propulsion purposes, RP2830. Steel, action of boron in enhancing the hardenability of, 851.

Austenitic stainless, 2789.

Austenitic stainless, stress corrosion cracking of type 304,1898 .

boron-treated, temper brittleness, 1913.

cathodic protection in salt water, RP2833.

concrete, and lead, broad and narrow beam attenuation of $\operatorname{Ir}^{182}$ gamma ravs, 2480.

determination of nitrogen and sulfur, 426 .

high resistivity environment, current and potential relations for the cathodic protection of, $\mathrm{J} 63 \mathrm{C} 1$, 37.

low-alloy, ferrite lattice in, elastic and plastic behavior, 1691 .

photometric determination of tungsten in, RP2812.

plastic surfaces on clotting time of human blood, effect of, 1003 .

porcelain enamel ground coats, 2358 .

reinforcing, by means of tensile bond specimens, 2113 .

SAE 1050, 1186.

separation and determination of small quantities of aluminum in, $\mathrm{J} 64 \mathrm{~A} 3,235$.

17-7 PH stainless, 2883.

stainless, Inconel, 3053.

stainless, influence of copper ions on the adherence of vitreous coatings, 1737 .

stainless, needle value, improved, 1034.

stainless, type 321 and Inconel, ceramic coated, 3092.

strength, new ultra high, 2215 .

thin porcelain enameled sheet, flexibility, 2126.

Steels, boron, 1642.

boron-treated, study of the impact properties, 1601.

determination of titanium, zirconium, niobium, and tantalum, RP2923.

high strength fatigue properties, 2951.

selected, tensile properties of, as a function of temperature, 2384 .

slack-quenched alloy, impact properties, RP2799.

stainless, austenitic, at low temperatures as determined by magnetic measurements, 3093 .

Stellar atmospheres, 2463.

atmospheres, aerodynamic phenomena, TN30 (PB151389).

Steric, effects in azo- and indigo dyes, 1897.

hindrance to coplanarity in o-fluoro-benzidines, 837 .

Sterochemical research, 554 .

Stiffness, of paper, 883 .

paper, measuring, 762 .

tester, NBS, extending the range of, 1020.

Stochastic, doubly, transforms of a vector, 2658.

independence of symmetric and homogeneous linear and quadratic statistics, 596 .

matrices, doubly, subdeterminants, 2227.

matrix, doubly, 3008 .

problems in wave propagation, 1147.

processes, 1095.

processes, certain, estimation of parameters, 334.

processes in arbitrary sets, with applications to processes with absorbing regions and to the problems of loops in Markoff chains, time discrete, 1286.

processes to chemical kinetics, the application of the theory, 2805; 2898 .

Stokes flow problem for a class of axially symmetric bodies, 2832.

Stokes-parameter technique for the treatment of polarization in quantum mechanics, 936.

Storage, Dewars, breathing oxygen, 2038.

lead, batteries, 751 .

Storm-D region, motion of, 1078.

Strain, gages at elevated temperatures, 2420 .

gages, bonding agents for high-temperature, 2474 .

rate and strain, compatibility equations and conditions of integrability, RP2813.

Strain-gage-type testing machine, surface tension measurements, 2794 .

Strains in beams having diagonal cracks, 2782 .
Stratification, in the lower ionosphere, J 63D2, 117.

lunar, of the F2 layer at Hyancayo, Peru, 83.

Stratosphere and troposphere, refractivity, 3032.

Strength, and fatigue, of glass at very low temperatures, 2318.

experimental study, 2014.

Stress and strain at onset of crazing of polymethyl methacrylate at various temperatures, $555 ; 669 ; 838$.

Stress-corrosion cracking, of Austenitic stainless steel, 2789 .

AZ31B magnesium alloy, mechanism of, RP2919. gold wedding ring, 1513.

type 304 austenitic stainless steel, 1898.

Stress-solvent crazing of acrylics, 839 .

Stress-strain, curves from strain waves in long bars, determination of dynamic, 684 .

elongation graphs for alclad aluminum alloy $24 \mathrm{~S}-$ T86 sheet, 139

recorders, autographic, 2465.

relation of pure-gum rubber vulcanizates in compression and tension, RP2837.

relations, dynamic pulse method for determining, 1997.

relationships in yarns subjected to rapid impact loading, RP2866;1514;1515; 1516;1899; 2783.

relationship in yarns tested at rates of straining up to a million percent per minute, 1900 .

Stress studies of bulkhead intersections for welded tankers, 556 .

Stresses (residual) in two-phase alloys, RP2793.

thermal, determination of loads in the presence of, 988.

Stressing fatigue, damaging effect, 1566.

Strips with holes loaded through pins, elastic deformation, RP2945.

Stroboscope, high-speed, for accelerometer calibration, 1031 .

Stroboscopic interferometer for vibration measurement, 2784.

mapping of time-variable fields, 140.

vibration analyzer, $\mathrm{J} 63 \mathrm{C} 2,97$.

Strongly continuous stochastic processes, 1095.

Strontium, aluminate hydrate, observations on, RP2777. portland cement, flame photometric determination, 1346.

oxide content of portland cements, 2382.

Structural, analysis and influence coefficients for delta wings, 840 .

analysis of clinical dextrans by periodate oxidation and isotope dilution techniques, 2319 .

anomalies in pure cristobailite, 3048 .

changes in irradiated plastics films, 3056 .

changes in natural rubber during vulcanization, infrared study, RP2816.

failures of welded ships, investigation of, 746 .

interpretation of immiscibility in oxide systems, 2320; $2320 \mathrm{~A} ; 2785$.

materials at low temperatures, mechanical properties, Mono.13.

rearrangements in silicate glasses during annealing, 1901.

Structure, in alkali silicate binary glasses, RP2854.

anhydrous scandium phosphate, 1950.

bone mineral, 2383.

ethylene from infrared spectra, 2833.

5-aldo-1,2-0-isopropylidene-D-xylo-pentofuranose 2321 .

iridium-osmium alloys, 1152 .

magnetohydrodynamic shock wave in a plasma of infinite conductivity, 1902.

methylamine molecule. I. Microwave spectrum of $\mathrm{CD}_{3} \mathrm{ND}_{2}, 2322$.

methylamine molecule. II. Theory of internal motions and application to $\mathrm{CD}_{3} \mathrm{NO}_{2}, 2323$.

polymer research at the National Bureau of Standards, 1813.

theorems for modular subgroups, 1517.

turbulence in fully developed pipe flow, 884 . 
vibration-rotation, in absorption bands for the calibration of spectrometers from 2 to 16 microns, Mono.16.

Struve functions, properties, 2269.

tables of intervals, 146 .

Student research on ASTM problems, 1903.

Studies, of atmospheric transmittance on Mauna Loa, RP2910.

beryllium chromite and other beryllia compounds with $\mathrm{R}_{2} \mathrm{O}_{3}$ oxides, $\mathrm{J} 64 \mathrm{Al}, 103$.

borate minerals. VI: Veatchite, 3057 .

crystal growth, and electrical and optical properties of gray tin, 1518 .

dose distributions in water for betatron $\mathrm{X}$-rays to 37 Mev, 1904.

electroless nickel plating, 2327.

galvanic couples. I. Measurement of electromotive force and internal resistance of cells during current flow, 141.

galvanic couples. II, Some potential-current relations in galvanic corrosion, 142 .

galvanic couples. III. Polarization and cathodic protection 143 .

leather by means of a sonic technique, 1905 .

leather fungicides, 2788.

nonequilibrium rate processes. I, The relaxation of a system of harmonic oscillators, 2324 .

nonequilibrium rate processes. II. The relaxation of vibrational nonequilibrium in distributions in chemical reactions and shock waves, 2325 .

nonequilibrium rate processes. III. The vibrational relaxation of a system of anharmonic oscillators, 2786.

nonequilbrium rate processes. IV. The rotational and vibrational relaxation of a system of rotating oscillators, 2787.

oxidation behavior of enameling iron during firing, 1153

polar amino acid content of collagen and related material, 841 .

stress corrosion cracking of Austenitic stainless steel, 2789 .

transequatorial ionospheric propagation by the scatter-sound method, 2326.

Study, of accuracy in chemical analysis using linear calibration curves, 2328 .

burst test as applied to military upper leathers, 842 .

degradation of polystyrene by means of mass spectrometry, 557.

earth currents near a VLF monopole antenna with a radial wire ground system, 2437 .

experimental, of the strength and fatigue of glass at very low temperatures, 2014.

impact properties of boron-treated steels, 1601 .

limb flares and associated events, 2882.

magnetic maps of the sun, 1600

1,046 megacycles per second of the reflection coefficient of irregular terrain at grazing angles, J 63D2, 235.

meteorological effects on radio propagation at 96.3 Mc between Richmond, Va., and Washington, D.C., 635 .

morphology of ionospheric storms, 2884.

operations involved in cement analysis, 937 .

radio reflections from meteor trails in research on the upper atmosphere, 144.

setting of plaster, 3058 .

17-7 PH stainless steel, 2883.

thermistor materials for use as temperature-sensing elements in the high-velocity exhaust gases of jet-type engines, 1602 .

upper atmosphere in the auroral zone, 2766.

variation of the physical and chemical properties of chrome-tanned leather and the selection of sampling location, 558 .

VLF field strength data: both old and new, 2438 .

Styrene, acrylate polymers in a vacuum, 811 .

$\alpha$-deuterostyrene polymers, oxidative degradation, 1802.
Subdeterminants of doubly stochastic matrices, 2227.

Subgraphs, dense, moebius function on, $\mathbf{J} 64 \mathbf{B} 1,41$.

Subharmonic crystal oscillators, 1154.

harmonic and linear functions of two variables, 280 .

Sub matrices of a full-rowed non-negative matrix, principal, J $63 \mathrm{~B} 1,19$.

Subroutine for computations with rational numbers, 1603 .

Subsonic air jets, reverberation chamber study of the sound power output, 2742 .

speeds, mechanism of transition, 2195 .

Sucrose solutions, determination of inorganic constituents in, 987 .

viscosities of, at various temperatures: tables of recalculated values, C440 Suppl.

Sufficient conditions for the convergence of Newton's method in complex Banach spaces, 559.

Sugar, acetates, infrared spectra, RP2772.

mixtures, determining dextrose in, quantitative chromotographic procedure for determining, 2276.

products, color and tubidity of, 970 .

refining, bone char process, 132 .

solutions, commercial, light scattering, J 63A3, 205.

Sugars, conformations of the pyranoid, 'J 64A2, 171; J 64A3, 239.

higher, branched-chain, $2476 ; 2903 ; 2904$.

infrared absorption spectra of some cyclic acetals, RP2960.

reducing and reducing end groups in polysaccharides by reaction with carbon-14-labeled cyanide, determination, 2517.

reducing, report, $1850 ; 2732$.

sugar products, report, 1852 .

tritium-labeled, $\mathrm{J} 64 \mathrm{~A} 2,177$.

Suggested problem for industry-sponsored shoe research, 379.

Sulfate susceptibility test for portland cements, 11 .

Sulfide, carbonyl, infrared spectrum, 2162.

hydrogen, infrared spectrum of, from 2200-2800 $\mathrm{cm}^{-1}, 1741$.

silver, in the photographic image during fixation, formation of, $\mathrm{J} 64 \mathrm{C} 1,65$.

Sulfinic acid derivatives as accelerators in the polymerization of methyl methacrylate, 1906.

Sulfur, atomic and empirical affinities of light elenents, electron affinity, 1698.

dioxide and sulfuryl fluoride, electron impact studies, 2542 .

nitrogen in steel, determination, 426.

oxygen, diatomic free radicals of the isotopic hydrides, 1357.

rubber vulcanizates, 1311 .

Sulfur-35 beta decay, average energy, RP2859.

Sulfuric acid, absorption spectra of some thioindigo dyes, 342 .

methods for analysis of wool admixed with cotton and viscose rayon, 973 .

Sulfurylfluoride, microwave spectrum and structure, 2372.

Summary of, symposium on color of transparent and translucent products, 1155 .

VHF and UHF tropospheric transmission loss data, TN43 (PB151402).

Summation method of 0 . Perron, 102.

Summation methods and integral transformations, J 63B1, 1.

Summation of slowly convergent series, 145 .

Sums of dihedral and trihedral angles in a tetrahedron, 597

Sun, and the arc, predicted lines of Fe I, 111.

atoms and ions in, 958 .

study of magnetic maps, 1600 .

technetium in, 322 .

upon the ionosphere, 250.

Sunlamps, fluorescent, in laboratory aging tests for plastics, 1713.

Sun's atmosphere, elements in, 1138.

Sunspot, cycle, 1112 .

numbers for cycle $20 ; 571 ; 3020$.

Superconducting transitions in tin whiskers, 1519.

Superconductivity, isotope effect, 581

isotopes of tin, 560 .

microscopic tin filaments, 2329. 
Superconductor, field curve, magnetic threshold, 584 .

Superconductors, 1212.

Superposed fluids, stability of oscillations, 1890.

Supplementary Zeeman data for the first spectrum of ruthenium (Ru I), J 63A3, 253.

Suppressing microwaves by zonal sereens, 317 .

Suppression of bubbling in boiling refrigerants, 2330.

optical measurements, aid in, 2790 .

Sur les limites du pouvoir de resolution des spectrometres corpusculaires, 2791.

Sur l'utilization des observations météorologiques courantes en propagation radió́lectrique, 2331.

Surface, currents excited by an infinite slot on halfplanes and ribbons, 1907.

erosion of filled plasties, 2792.

Surface area, hydrated portland cement, determination by water-vapor and nitrogen adsorption, RP2806.

precise evaluation of, with indirectly calculated dead

space, $\mathrm{RP} 2826$.
Surface reactivity, buffer solutions, RP2811.

cotton, wool, and nylon fabrics at different temperatures and relative humidities, 2793.

Surface tension, alkaline-earth borates containing 3 mole percent of potassium oxide, 3059 .

density, viscosity, and electrical resistivity of molten binary alkaline-earth borates, 1156 .

measurements with a strain-gauge-type testing machine, 2794.

some simple borates, 2592.

with body and surface concentrations, variations, 3110 .

Surface waves, excitation, on conducting, stratified, dielectric-clad, and corrugated surfaces, RP2807.

Surface-wave resonance effect in a reactive cylindrical structure excited by an axial line source, J 64D1, 13.

Survey, recorder, C601.

Survey of, adhesion and types of bonds involved, 1157.

application of ceramies as vacuum tube envelopes, 1604 .

audio-frequency power amplifier circuits, 187.

bibliography of recent research in the propagation of VLF radio waves, TN58 (PB161559).

Central Radio Propagation Laboratory research in tropospheric propagation 1948-1956, TN26 (PB151385).

ceramics for nuclear reactors, 12

computer development and applications, 2332.

experimental heat capacities of ten technically important gases, 843.

geomagnetic eclipse phenomena, 1908.

hazards of handling liquid oxygen, 2333.

Lyapunov's second method, 2439.

present knowledge of sporadic- $\mathrm{E}$ ionization, 2885.

recent advances in polymer physics, and their relation to dielectric properties, 2440 .

Suspended chain, modes of vibration of, $\mathbf{7 7 0}$.

SWAC, NBS Western Automatic computer, design features of a magnetic drum memory, 424 .

use of subroutines, 614 .

SWAC-design features and operating experience, 885 .

Swedish free-air chamber, portable, equipment at the NBS, ealibration, 1644 .

Sweep-frequency, backscatter data for determining oblique-incidence ionospheric characteristics, use, 2838.

backscatter observations, note on, 924 .

backscatter-some observations and deductions, 1158.

oblique-incidence ionosphere measurements over a $1,150 \mathrm{KM}$ path, 561 .

polarization measurements at low frequencies, 938.

pulse technique at oblique incidence, ionospheric investigation using, J $63 \mathrm{D} 2,151$.

pulse-transmission measurements over a $2400-\mathrm{KM}$ path, 1520.

Swelling of collagen and modified collagen, 1159.

Switeh aids, ultrasonic, diversity reception, 1564 .

Symbolic designations for electrical connections, 2334.

Symbols, units and nomenclature, pure, and applied physics, 189.
Symmetric, continuous poker model, J 64B1, 35 .

functions of eigenvalues, note, 1990 .

integral equations, error bounds for the eigenvalues, 1707.

matrix, 192.

top molecules, nonresonant absorption, 2648 .

Symmetric matrices, determination of eigenvectors and eigenvalues, 330 .

linear iteration procedures, 1097.

Symmetric-asymmetric molecules, hindered rotation, 1732.

Symmetrical group divisible designs, blocks, 548 .

Symmetry, point, simplified method of locating, 2001.

Symposium, color of transparent and transulcent products, 1155 .

erystal chemistry, 215.

NBS optical image evaluation, report, 532 .

recent developments in the evaluation of natural rubber, 844 .

research, free radicals, 2571.

second, in Linear Programming, proceedings, 1458.

temperature stability of electrical insulating materials, 992.

Synchronization, precise time, of widely separated clocks, TN22 (PB151381).

Synoptic, study of the vertical distribution of the radio refractive index, J 63D21, 249.

variation of the radio refractive index, J 63D1, 91.

Syntax, German, patterns, proposals for the mechanical resolution, 290 .

Synthesis, and infrared absorption spectra of some $m$-polyphenyls, 1951.

$C^{14}$-labeled $L$-sorbose and L-ascorbic acid, RP2800.

functional, of linear plots, J $64 \mathrm{C} 2,115$.

maltose-1-C $\mathrm{C}^{14}$, maltobionodeltalactone-1- $\mathrm{C}^{14}$, and lithium maltobionate-1-C ${ }^{14}$ from 3-( $\alpha$-D-glucopyranosyl)-D-arabinose, 1909.

properties of fluorinated polyphenyls, 1521.

slotted-cylinder antennas, $63 \mathrm{D} 3,303$.

some disubstituted 3,4,5,6-tetrafluorobenzes, R P2939.

Synthetic, and natural rubbers, 264; $487 ; 772 ; 1412 \mathrm{~A} ; 2997$.

cuspidine, 2795.

detergents, 318 .

fibers, properties of some experimental map papers containing, 2703.

materials in America in 1949, developments, 21.

rubbers, physical chemistry of, 1107.

sapphire, refractive index, 2727 .

single crystal corundum, elastic constants of, at room temperatures, J 64A3, 213.

textiles, $845 ; 1522$.

Synthetically bonded molding sand, 846 .

System, behavior, prediction of, $2660 \mathrm{~A}$.

design of digital computers at the NBS: methods for high-speed addition and multiplication, C591, Sect. 1.

lime-alumina-water at $1^{\circ} \mathrm{C}, \mathrm{RP2877}$.

loss in radio wave propagation, $\mathrm{J} 63 \mathrm{D} 1,53$.

magnesium oxide-germanium dioxide, 3094 .

names for binary numbers, 2441.

Systematic, calculations of gamma-ray penetration, 847 . ionospheric winds, 319 .

research, 3060 .

Systems, distinct representatives and linear programming, 1910.

equations, matrices, and determinants, 562 .

inequalities involving convex functions, 2335 .

linear, zeros of polynomials and the degree of, 790 .

tuning, amplifiers, feedback, 352 .

vibrating, decay constant in, 678 .

Systems of, distinct representatives, 1793.

linear inequalities, 1794.

$\mathbf{T}$

Table, coefficients of the powers of $\eta(\gamma), 1605$.

first moment of ranked extremes, RP2787.

radix, for obtaining hyperbolic and inverse hyperbolic functions to many places, 810 .

wavenumbers, Mono.3, Vol. I and Vol. II.

Wilson quotients and the third Wilson prime, 636. 
Tables, bivariate normal distribution function and related functions, AMS50.

characteristic values of Mathieu's equation for large values of the parameter, 1523 .

constructing and for computing the operating characteristics of single sample plans, 563 .

diagonalizing second-order matrices, RP2838.

dielectric dispersion data for pure liquids and dilute solutions, C589.

difference, for linear interpolation, rounding off, 504 . expected values of $1 / \mathrm{X}$ for positive Bernoulli and Poisson variables, 1161 .

exponential integral for complex arguments, AMS51.

4f-1 through $4 f-6$ on thermal expansion, 2336.

generalized, of corrections to thermodynamic properties for nonpolar gases, 1721.

integral, $\int_{0}^{2} e^{-\mu 3} \mathrm{du}, 320$.

intervals of Struve functions, 146.

line strengths for rotational transitions of asymmetric rotor molecules, 2796 .

median hourly values of the cosine of the solar zenith angle $(x)$ for thirty-five locations, TN5 (PB151364).

natural logarithms for arguments between five and ten to sixteen decimal places, AMS53.

$1958 \mathrm{He}^{4}$ temperature scale, J 64A 1-25; Mono. 10.

142 to 144 , thermal expansion, 1160.

osculatory interpolation coefficients, A MS56.

physical, 1138.

punched cards, a guide, 177

punched cards, a guide, addendum, 641.

radix, for trigonometric functions and their inverses to high accuracy, 296.

recalculated values: viscosities of sucrose solutions at various temperatures, C440 Suppl.

relating to Hankel integrals of order zero, errata, 147 spectra, 2337.

speed of sound in water, 3061 .

statistical prediction of radio ray bending, TN44 (PB151403).

thermodynamic, mechanized computation at the NBS, $2632 ; 2988$.

transport integrals, C595.

transport integrals: a supplement, J 63B1, 23.

viscosities of sucrose solutions at various temperatures: tables of recalculated values, C440 Suppl.

Tabulation of data on receiving tubes, H68.

Tacan coverage and channel requirements, $2337 \mathrm{~A}$.

Taillights and brakelights, configuration, 1587 .

Tangential distortion, prism, effect, and camera tipping, 2264.

Tank, farm milk, testing, 1344.

Tankers, welded, stress studies of bulkhead intersections, 556.

Tantalum, copper, molybdenum, and gold at $662 \mathrm{kev}$, total photoelectric cross sections, 3101 .

elastic scattering of photons, 2535 .

niobium, titanium, and zirconium in steels, determination, RP2923.

titanium-base alloys, determination of niobium, RP2929.

Tape recorders, magnetic, FM data reduction from, 722 .

Tapered cantilever beams computed on SEAC, influence coefficients, 467 .

Tapes, testing of measuring, C572.

Targets, square wave, $\mathrm{J} 64 \mathrm{C} 1,57$.

Tartrate buffer solutions, 262 .

Tauberian, theorem for Abel summability, 275.

theorems for summability $\left(R_{1}\right), 321$.

Tchebycheff approximations, criteria for the existence and equioscillation, $\mathrm{J} 64 \mathrm{~B} 2,91$.

Teaching pay in the Soviet Union, 1524 .

Technetium in the sun, 322 .

Technetium-99, nuclear moments, 875 .

Technical aspects, industrial and office quieting, 324.

large scale liquid helium liquefaction and transportation, 2797.
Technical considerations leading to an optimum allocation of radio frequencies in the band 25 to 60 Mc, TN13 (PB151372).

Technique for, eutting very thin sections and its application to the electron microscopy of fibers, 4. locating sources of variability in a process, 323 .

monitoring tritiated-water vapor in air, 2338 .

sweep frequency polarization measurements at low frequencies, 938 .

Techniques, accurate measurement of antenna gain, C598. electroforming of precision waveguide components in the millimeter wavelengths, 2339 .

scanning, processing of two-dimensional patterns, 802.

theory of fast coincidence experiments, 1911.

Technological, challenge to the textile industry, 2002. measurement progress, scientific, 537.

society, demands on teachers, 2512.

supremacy, precise measurement and race for, 2696 .

Technology, Nation's, service for, 1999.

Tee, magic, matching loads, $83 \mathrm{~A}$.

Teeth, fluorescence of, 721 .

fluorescence of: a means of investigating their structure, 452 .

plastic, bonding of, to heat cured denture base resin, 402.

plastic, physical properties, 2688 .

plastic, proposed specification, 2272 .

Teflon, insert makes needle vacuum tight, 848 .

Kel-F, ignition, 2591.

transition in, volume-temperature relationships for the room temperature, 356 .

Tektites, lost planet, $1525 ; 1912$.

meteoric glass, $1912 \mathrm{~A}$.

spectral-transmissive properties, 1546 .

these glass objects fallen from the sky; do they come from a lost planet? 1756 .

Telemetering and fax: how FM expedites weather data distribution, 69 .

Television, airborne, station, TN35 (PB151394).

bandwidth conservation, communication theory aspects, TN25 (PB151384).

broadcasting, measurement of a service area, 2190.

color, 38 .

color, present status, 154.

FM broadcasting stations, variation with frequency of the signal range, 347 .

FM service, statistical approach to the problem of multiple radio interference, 378 .

reception, effect of sporadic $\mathrm{E}, 574 ; 859$.

Temper brittleness of boron-treated steel, 1913 .

Temperature, absolute, below $1^{\circ} \mathrm{K}$ : chromic methylammonium alum as a thermometric substance, 2009 .

adsorption of water vapor by collagen and leather, 74 . aluminum alloys under various conditions, 2505 .

between $0^{\circ}$ and $75^{\circ} \mathrm{C}$ and at pressures up to 2500 atmospheres, thermal conductivity of argon, 1952.

chromospheric, 874 .

coefficient of the speed of sound in water near the turning point, 1914.

coefficients of non-Newtonian viscosity at fixed shearing stress and at fixed rate of shear, 849 .

constant, liquid helium bath and reproducibility of resistance thermometers, 2916.

degree of polymerization on the sorption of water by polymethyl methacrylate, effect, 1328 .

du point du zinc et comparison de deux déterminations du point du soufre dans un appareil fermé, 2799.

effects in gas-free liquid scintillators, 1915.

effects, liquid-scintillator, 1759 .

emission corona, 2930.

function, tensile properties of selected steels, 2384 .

function of, Young's modulus of various refractory materials, 3114 .

helium vapor-pressure scale, 1535.

high, alloys, 3072 .

high, ceramics and cermets, Mono.6. 
high, heterogeneous reaction of chlorine and nickel between 1200 and $1700^{\circ} \mathrm{K}$, kineties, 2608 .

high, laundering, test for dimensional changes in woven fabries, 2802.

high, liquids, 1032 .

high, strain gages, bonding agents, 2474.

high-vacuum gradient furnace, 2588.

influence of, on the adsorption of chrome $\left(\mathrm{Cr}_{2} \mathrm{O}_{3}\right)$ by calf skin squares, 2159 .

influence of strain rate, on the creep of cold drawn ingot iron, 73.

inversion in cristobalite, RP2902.

inversions of anhydrous gallium orthophosphate, 1916.

low, data: properties of paramagnetic salts, 2408

low, distillation of hydrogen isotopes, 2618; 2691.

low, microcell for infrared study of condensed gases, 2619

low, physics, $1055 ; 2180$.

low, properties of plastic foams, 2620.

low, reduction of area gage, 7 .

low, scales from $90^{\circ}$ to $5^{\circ} \mathrm{K}, 1392$.

low, thermal conductivity of free-machining copper, 1393.

measurement and control, C590.

measurement and incomplete equilibrium, 1735.

microcell for infrared study of condensed gases, 2800 . on-off, control for electrically heated filaments, 2660.

power loss and operating, of tires, J $64 \mathrm{C} 1,1$.

prediction in electronic design, 564 .

pressure-volume, relations of liquid normal deuterium, 1115 .

range, extended, volume temperature relations of amorphous polymers, 1975.

resistance, relation for low temperature thermometry, 9.

room, thermoelectric power of germanium, 897 .

rotational, of $\mathrm{OH}$ in diluted flames, 818 .

scale below $1^{\circ} \mathrm{K}$, adiabatic demagnetization, 384 .

square, wave in a filament operating at low temperatures, 2701.

stability of electrical insulating materials, 992.

standards of, 836 .

stratification in a nonventing liquid helium Dewar, J $64 \mathrm{C} 1,19$.

studies of air in a truck tire, 1917.

transition in teflon, 356 .

very low, an experimental study of the strength and fatigue of glass, 2014.

volume, relations of amorphous polymers over an extended temperature range, 1975 .

zine point and the comparison of two kinds of determinations of the sulfur point in a closed apparatus, 2799.

Temperature control, cryostat, wide-range, 3113.

during mixing of rubber compounds, 1526.

resistance diode bridge circuit, TN34 (PB151393).

wide-range, design of simple DC resistance thermometer bridges, 2515.

Temperature dependence, breakdown field of barium titanate, 3062.

characteristic energy loss of electrons in aluminum, 2798.

distribution functions in quantum statistical mechanics, 2340.

mechanical shear degradation, 1527.

microwave absorption of $\mathrm{NH}_{3}, 1162$.

Temperature measurements, flames and hot gases, experimental, 1340.

high-velocity streams of hot gas, 1163.

Mooney viscometer, 1164 .

Temperature scales, heat units for calorimetry, 1355

present status, 590 .

Temperature-independent paramagnetism of ammonium hexabromoosmate, 1201.

Temperature-induced stresses in solids of elementary shape, Mono.2.

Temperature-sensing elements in the high-velocity exhaust gases of jet-type engines, 1602 .
Temperatures in atomic explosions, 1528.

copolymers, glass transition, 2576.

developed in rotating dental cutting instruments, 1165 .

electronic flame, and energy distributions, spectroscopic study, 2310.

elevated, calculation of properties of gases, 2664

elevated, creep behavior of transparent plastics, 2921.

elevated, determination of viscosity of exhaust-gas mixtures, 989.

elevated, elastic moduli of glasses, by a dynamic method, 1692.

elevated, evaluation of resistance strain gages, 2420 .

elevated, physical characteristics of titanium carbide type cermets, 1445 .

elevated, protection of molybdenum from oxidation, 2705.

elevated, statistics and planning, 137.

gas, measurement of, by immersion type instruments, 867.

heats of transition and fusion, 3079 .

helium-vapor pressure scale, 2818.

high, kinetics of elementary gas phase reactions, 1433.

high, measurement in gas streams, 85 .

high, measuring thermal emittance, 3049A.

high, precision thermostat, 110 .

high, thermodynamic properties, 2394.

hot gases, 1882 .

low, TN4 (PB151363); 2730.

low, calibration of thermocouples, 2483.

low, copper functions, 2537.

low, effect of tensile strain, on deformation twinning in ingot iron, 695 .

low, electrical conduction in magnesium stannide, 1695 .

low, form electric discharges, absorption spectra of solids condensed, 2445.

low, formation of ozone from atomic oxygen, 2954.

low, frequency standards, $\mathbf{1 5 8 9}$.

low, from a gas discharge, 3052 .

low, glass dewars for optical studies, 2575 .

low, impact strength of some thermosetting plastics, 464.

low, impact testing of various alloys, 2819

low, liauid level indicator for condensed gases at, 1052 .

low, luminescence of solids, 2934.

low, magnetic losses, 2980.

low, mechanical properties of insulating plastic foams, 2985.

low, mechanical properties of structural materials, Mono.13.

low, operation of bearings and pumps, 2676.

low, properties of materials, 2268 .

low, properties of the $\mathrm{Cr}^{+++}$ion in the paramagnetic alums, 522 .

low, quartz erystal resonators and oseillators, 2734.

low, some mechanical properties of Mylar and Dacron polyester strands, 2761

low, stability of austenitic stainless steels, as determined by magnetic measurements, 3093 .

low, stability of quartz resonators, 2315 .

low, stabilization of free radicals, 2316; Mono.12.

low, strength and fatigue of glass, 2318 .

low, thermal conductivity of some commercial coppers, 2181 .

low, triatomic nitrogen, 3054 .

low, wide-range control, design of simple resistance thermometer bridges, 2516.

$1958 \mathrm{He}^{4}$ seale, J 64A1, 1; Mono.10.

piezoelectric constants of alpha- and beta-quartz, 108.

relatively low, thermal hydrogen-oxygen reaction, 345 .

rotational and vibrational, of $\mathrm{CH}$ in flames at atmospheric pressure, 816 .

rotational, of $\mathrm{OH}$ in methane-air flames, 304.

rotational, of $\mathrm{OH}$ in several flames, 534 . 
stress and strain at onset of crazing of polymethyl methacrylate, 838 .

up to about $800-900^{\circ} \mathrm{C}$, thermal degradation of polymers, 2843 .

various, stress and strain at onset of crazing of polymethyl methacrylate, 555 .

various, viscosities of sucrose solutions: tables of recalculated values, C440 Suppl.

very low, in chromic methylammonium alum, spinspin relaxation, 1889 .

very low, spin-spin absorption in chromic methylammonium alum and manganous chloride tetrahydrate, 1887 .

Tendon collagen, shrinkage, 120 .

Tensile, behavior of titanium and a titanium alloy, effect of strain-temperature, RP2897.

crazing properties of acrylic plastic glazing, effects of moderate biaxial stretch-forming, 436.

forces in tightened bolts, 344 .

impact strength of annealed and welded 5086 aluminum down to $20^{\circ} \mathrm{K}, 3095$.

properties of polyester film, 2354 .

properties of selected steels as a function of temperature, 2384 .

strength and extreme values, two early papers on the relation between, 1219 .

Tension, creep in, bend-test method of determining the stress required, $1584 \mathrm{~A}$.

surface, measurements with a strain-gauge-type testing machine, 2794.

testing of plastics, 2341 .

testing of rubber rings, 2801 .

Tensorial sets, 2970.

Tensors, absolute, of arbitrary order, generalization of the index notation, J 64B2, 99.

Term analysis of the first spectrum of rhenium, (Re I), RP2804.

(Re II), RP2914.

Terminology, describing the performance of analytical and other precise balances, 1166.

measurement and control, integration of concepts, 1935.

Terpenes, physical properties, 1191A.

Terrain, irregular, study at 1,046 megacycles per second of the reflection coefficient of, at grazing angles, $\mathrm{J}$ 63D2, 235.

Terrestrial, propagation of very-low-frequency radio waves-a theoretical investigation, J $64 \mathrm{D} 2,153$.

radio noise, 2284 .

Tertiary butyl fluoride, 2820 .

trimethylphospine, isobutyane, 2638.

Test, chart, power, 268 .

dimensional changes in woven fabrics in high temperature laundering, 2802 .

flex tension, for leather, 917.

procedure-error evaluation, 39.

procedures, necessity for uniformity, 587.

Test methods, practical, for the Porcelain Enameling Industry, 1452.

specifications, and standards for plastics, 1167.

Tester, voltage breakdown, 908 .

Testing, colorfastness of textiles, international methods, 1375.

electrical instruments, 886.

farm milk tank, 1344 .

generation of pseudo-random numbers, 1722 .

glass volumetric apparatus, C602.

impact, of various alloys at low temperatures, 2819

machine for wire, recording torsion, 2435.

machine, strain-gauge-type, surface tension measurements, 2794 .

mechanical, of solid materials, 482 .

operation of ball bearings submerged in liquefied gases, 3063.

photographic lenses at the NBS, 1169.

quick-weighing balance, 1168 .

research, development, NBS, aircraft lighting, 771. statistical principles, 1511 . thermocouples and thermocouple materials, methods of, C590.

wholesale meters, 565 .

Tests, colorfastness of textiles under consideration in the International Organization for Standardization, 850.

diagnosis, index for rating, 71 .

independence, of randomness and other hypotheses, 2231.

laboratory, with turboprop lubricants, 2610.

lubricants, special, 311 .

Tetrabromoethylene, vibrational spectrum, 1973.

vibrational spectrum, properties of fluorine compounds, 1825 .

Tetrachloride, diboron, vibrational spectrum and force constants, 2409.

trichloride of molybdenum, preparation, J 63A2, 185.

Tetrachlorohydroquinone, colorimetric determination, 670 . Tetrafluorobenzenes, disubstituted 3,4,5,6-synthesis, RP2939.

Tetrafluoromethane, heat of formation, 1533.

Tetrahedron, sums of dihedral and trihedral angles, 597.

Tetraiodide, titanium, heat of formation $J 63 \mathrm{~A} 2,161$.

Tetramethyl, germanium, small quantities, preparation, 517.

Textile fiber structure on wear resistance effect, 610 . Forschung and Prufung am NBS, 325.

industry, technological challenge, 2002.

research and testing at NBS, 325 .

research, high-speed motion pictures, 66 .

testing methods, standardization of, international co-operation, 471.

work, international, shows fast progress, 2164.

Textile yarns, characterization of the high-speed impact behavior, 2909.

impact absorbing capacity, 2363.

long, impacted transversely, wave propagation, 2783. rapid impact loading, 2715.

rapid impact testing, RP2866.

Textiles, 188.

abrasion of, interpretation of tests for resistance, 473.

at the NBS, 1529.

international methods for testing colorfastness, 1375.

new paper products in competition, 1784.

synthetic, $845 ; 1522$.

tests for colorfastness, 850

Textural stresses in two-phase alloys, RP2793.

Thallium, bromide iodide, refraction and dispersion, 1842.

bromide, sodium chloride, and thallium chloride, photoemission from silver into, 1807.

chloride, sodium chloride, and thallium bromide, photoemission from silver into, 1807.

Theorem, alternatives for pairs of matrices, 1246 .

convex cones, with applications to linear inequalities, 380.

embedding, for balanced incomplete block designs, 948 .

expansion, for pseudo-analytic functions, 577 .

modular groups, 2016

Ostrowski and Taussky, 1091.

Riesz, M., RP2935.

Tauberian, for Abel summability, 275.

transposition, on linear inequalities, two consequences, 353.

Theorems, about pr(n), 2305.

basic, in matrix theory, AMS57.

minimax, 768 .

partially balanced designs, 1148 .

structure, for modular subgroups, 1517.

Tauberian, for summability $\left(\mathrm{R}_{1}\right), 321$.

Theoretical, analysis of fractionating process of adsorption, 156 .

electromotive forces for cells containing a single solid or molten chloride electrolyte, 1957.

investigation, terrestrial propagation of very-lowfrequency radio waves, J 64D2, 153 . 
Theory, cavity ionization, $1247 ; 1606$.

cyclic single step iteration, over and under relaxation in, 781 .

dielectric relaxation for a single-axis rotator in a crystalline field, 1554 .

diffraction in microwave interferometry, J 64B1, 1 . effect of drag on the orbital inclination of an earth satellite, RP2934.

electrochemical, fifty years, 448 .

electron penetration, 1548 .

extreme values, 3096 .

fading properties of a fluctuating signal imposed on a constant signal, C599.

fast coincidence experiments, techniques, 1911.

forbidden transitions of nitrogen atoms trapped in solids, 2392.

formation of polymer crystals with folded chains in dilute solution, J $64 \mathrm{~A} 1,73$.

information, $250 \mathrm{~A}$.

non-linear vibrations, singular perturbation problems, 277.

production of VLF noise (so-called drawn chorus) by traveling wave amplification in the exosphere of the earth, 2003.

propagation of electromagnetic waves along a curved surface, 2673.

radar scattering by the moon, J 64D3, 217.

reflection from a wire grid parallel to an interface between homogeneous media, 2238; 3010 .

reflection of low- and very-low-radiofrequency waves from the ionosphere, J $64 \mathrm{D} 3,269$.

stark effect of the NO molecule, 2840.

three-crystal electron interferometer, 1202.

threshold energy dependence of photodetachment of diatomic molecular negative ions, 2841.

wavelength dependence on ultrahigh frequency transhorizon propagation based on meteorological considerations, J $64 \mathrm{D} 3,231$.

Thermal, analysis above $1200^{\circ} \mathrm{C}$, differential, 2521 .

batteries, 602 .

converters, 2809.

diffusion in ionized gases, 2844 .

electrical conductivity of aluminum and aluminum alloys, $2841 \mathrm{~A}$.

electrical conductivity of pure copper, 2841B.

electromotive force, tables of transport integrals, C595.

E.M.F. of several thermometric alloys, 2833A.

emf of some thermometric alloys, $2844 \mathrm{~A}$.

emittance at high temperatures, measuring, 3049A

gravitational excitation of atmospheric oscillations, 1555 .

gravitational oscillations in the earth's upper atmosphere, 1726 .

hydrogen-oxygen reaction at relatively low temperatures, 345 .

insulating value of airspace, 1203.

length changes of some refractory castables, RP2768. photochemical processes in polystyrene in the glassy state, 1958.

Rayleigh disk in liquid helium II, 157.

Rayleigh disk measurements in liquid helium II, 603.

Rayleigh disk measurements in $\mathrm{He}^{3}-\mathrm{He}^{4}$ mixtures, 895.

resistance of airspaces and fibrous insulations bounded by reflective surfaces, BMS151.

stress in box beams, effect of internal radiant heat transfer, RP2949.

Thermal conductivities, common commercial aluminum alloys, 2842.

copper and copper alloys, 2393.

Thermal conductivity, 1207.

argon for temperatures between $0^{\circ}$ and $75^{\circ} \mathrm{C}$ and at pressures up to 2500 atmospheres, 1952 .

beryllium oxide from $40^{\circ}$ to $750^{\circ} \mathrm{C}$, RP2775.

commercial coppers, low-temperature, 2181.

determinations with guarded hot plates, 251 .

free-machining copper, low temperature, 1393

gas analyzer, unique, 2443. solids at low temperatures, RP2805.

steam, 1657 .

tables of transport integrals, C595.

Thermal decomposition, manganous and ferrous carbonates, 1960 .

mass spectra, dimethylphosphinoborine trimer, 994.

polymers, 1556 .

polymers, effect of structure, RP2860.

poly (vinyl chloride), 3099 .

rate of hydrazoic acid, mass spectrometric study, RP2881.

Thermal degradation, cellulosic materials, RP2853.

polyacrylonitrile, polybutadiene and copolymers of butadiene with acrylonitrile and styrene, RP2888. polyethylene oxide and polypropylene oxides, 3100 .

polymers as a function of molecular structure and branching, 1557 .

polymers at high temperatures, J 63A3, 261.

polymers at low rates, RP2957.

polymers at temperatures up to about $800-900^{\circ} \mathrm{C}$, 2843.

polystrene and polyethylene, 1209.

polystyrene and polyethelene in a vacuum, rates, 526 .

styrene and acrylate polymers in a vacuum, 811 .

unvulcanized and vulcanized rubber in a vacuum, 1961.

Thermal expansion, 1160 .

silica-gypsum investiments, 1184 .

tables $4 \mathrm{f}-1$ through $4 \mathrm{f}-6,2336$.

Thermal stresses, deformation in beams, 1962 .

determination of loads in the presence of, 988 .

Thermally degraded poly(vinyl chloride) infrared spectra, RP2832.

Thermistor materials for use as temperature-sensing elements in the high-velocity exhaust gases of jettype engines, 1602 .

Thermochemical study of the reaction of calcium hydroxide, silica gel, and water, RP2785.

Thermochemistry and thermodynamics of substances, 1963.

Thermoconductivity of tin-indium alloys in the normal state, 2845 .

Thermocouple testing and materials, C590.

Thermocouples, development and evaluation of ceramic coatings, 1674 .

low temperatures, calibration, 2483

Thermodynamic, acidity constants, 991 .

dilute solution, behavior of flexible chain molecules: polymethyl methacrylate, 687 .

driving force in nucleation and growth processes, 2834 .

gas, functions, high-speed machine computation of, 1030.

transport data, sources of transport coefficients and correlations, $1501 ; 1879$.

Thermodynamic properties, from a heat content study, 2839.

gaseous difluorodichloromethane (freon-12), 604.

gaseous nitrogen, 1964 .

gases at high temperature: 1. Chemical equilibrium among molecules, atoms, and atomic ions considered as clusters, RP2916.

helium at low temperatures and high pressures, TN8, (PB151367).

high temperatures: ideal gas thermal functions to 25,000 deg. $\mathrm{K}$ for diatomic molecules, oxygen, nitrogen, nitrococide, and their molecule ions, 2394 .

nonpolar gases, generalized tables of corrections, 1721. polybutadiene-benzene, RP2820.

pure diatomic gases, effect of dissociation, 1326.

solid and liquid, triple point and heat of fusion, 1390.

Thermodynamic tables, at the National Bureau of Standards, mechanized computation, $2632 ; 2988$.

Thermodynamics and thermochemistry of substances, 1963.

Thermodynamics of crystallization in high polymers; gutta percha, 1965.

natural rubber, 1558 .

poly(ethylene), 2846 . 
Thermoelastic solid, plane waves in, comments on a recent paper by Deresiewicz, 2058.

Thermoelectric power, germanium below room temperature, 897.

germanium single crystals, 896 .

indium antimonide, 1559.

tables of transport integrals, C595.

Thermometer, bridges design of simple DC resistance, for wide-range temperature control, 2515.

design of simple resistance, bridges for wide-range control of low temperatures, 2516.

Thermometers, clinical, aging changes, 385 .

liquid-in-glass, calibration, $\mathrm{C} 600$.

resistance, 2916.

Thermometric, calorimetric procedures, comparison of cryoscopic determinations of purity of benzene, 2062 .

study of the frozen products from the nitrogen microwave discharge, 2847.

substance, chromic methylammonium alum, absolute temperature below $1^{\circ} \mathrm{K}, 2009$.

Thermometric alloys, $2833 \mathrm{~A}$.

thermal emf, 2844A.

Thermometry, below $1^{\circ} \mathrm{K}$, new materials for magnetic cooling, 1783.

low-temperature, resistance-temperature relation, 9. pyrometry, 2500 .

Thermoplastic materials for use as iodine vapor barriers, 2892 .

Thermostat, electronic, time-proportional, 939.

precision, for high temperatures, 110 .

Thermostated containers for standard cells, portable potentiometer, 1450

Thick target bremsstrahlung spectra for 1.00-, 1.25-, and 1.40- Mev electrons, 1211

Thickness, compressibility of fabrics, 898 . gage for ceramic coatings, 13. measurement of, C585.

Thioindigo dyes, cis-trans isomerism, 341. sulfuric acid, absorption spectra, 342 .

Thiourea, urea, and some thiourea-alkali halide complexes, infrared absorption spectra, 2160 .

Thoria resistor-type furnaces, construction and operation, 328.

Thorium, oxide, high-density, some physical properties, 1877.

spectra, new description, Mono.17.

(Th I), a preliminary list of levels and $g$-values for the first spectrum of, $\mathrm{J} 63 \mathrm{~A} 3,275$.

Thorium-halide lamps, wavelengths from, RP2891.

Thread, screw, standardization, 1862 .

Threads, screw, must be everybody's business, 1977. screw, world unification, 1581 .

Three ways to increase efficiency of liquid scintillators, 1966.

Three-crystal electron interferometer, 1202.

Threshold field properties of some superconductors, 1212.

Thunderstorms, distribution of electrical conduction currents in the vicinity, 432 .

Tile, acoustical, evaluation of adhesives, 55 .

clay, moisture expansion of, 1058.

Tilting air-lubricated piston gage for pressures below onehalf inch of mercury, $\mathrm{J} 63 \mathrm{C} 1,47$.

Time, calibration, absolute, intermittent-action camera, J $64 \mathrm{C} 2,159$

constant and response time of thermal converters, 2809 .

discrete stochastic processes in arbitrary sets, with applications to processes with absorbing regions and to the problems of loops in Markoff chains, 1286.

series of tropospheric radio field strength data, 362 . signals, standard frequency transmission, 3054A.

standard and atomic frequency, 2804 .

synchronization of widely separated clocks, TN22 (PB151381).

Time and frequency, Hawaii, measuring, 257.

standard, atomic clock, 148. standard, new type, need for, 1190.

standards in the U.S.A. from 1954 to $1957,2550$.

Time standards, adjustment of high-precision frequency, 18.

NBS, atomic and molecular, 2549.

precision, 209.

Time-dependent cavity characteristics, 2636.

Time-proportional electronic thermostat, 939 .

Time-temperature behavior of rubber from measurements of indentation hardness, 2848.

Time-variable fields, stroboscopic mapping, 140.

Timer, automatic, simplified small-current measurements, 2036 .

Tin, crystals, gray, 2672 .

iron, copper, silicon, and nickel, electrorefining studies, 2811.

isotopes of, superconductivity, 560 .

oxide or titania containing varying proportions or rare-earth oxides, dielectric properties, 2520 .

whiskers, superconducting transitions, 1519 .

Tin-indium alloys in the normal state, thermoconductivity, 2845 .

Tire, truck, temperature studies of air in, 1917.

Tires, commercial, measurement of treadwear, 761 .

power loss and temperature, J $64 \mathrm{Cl}, 1$.

road wear, some factors influencing, 308

rubber, proof testing, 519 .

treadwear of, influence of type of rubber and black, 350 .

Titanate, barium, 3062 .

barium, sammarium doped, electrical properties, 2105 .

Titanates, magnesium-strontium, note on the dielectric properties, 2221 .

Titania or tin oxide containing varying proportions or rareearth oxides, dielectric properties, 2520.

Titanium, adhesion of electroplated coatings, 1920 .

alloys with dithiol, RP2812.

carbide type cermets at elevated temperatures, physical characteristics, 1445.

dioxide simiconductors, electrical properties, 704 .

effects of low temperatures and notch depth on the mechanical behavior of, RP2790.

$6 \mathrm{Al}-4 \mathrm{~V}$ titanium alloy, nitriding phenomena, $\mathrm{J} 64 \mathrm{Al}$, 119

tetrabromide, heat of formation, RP2928; RP2932.

tetraiodide, heat of formation $\mathrm{J} 63 \mathrm{~A} 2,161$.

titanium alloy, effect of strain-temperature history on the tensile behavior, RP2897.

zirconium compounds in nonaqueous media, 2106.

zirconium, niobium, and tantalum in steels, determination, RP2923.

Titanium-base alloys, determination of niobium and tantalum, RP2929.

Titration, acids, differential, RP2769.

predise coulometric, of acids and bases, J 63A2, 153

Tooling, trickery, tough problems solved, 2395.

Tools, new, for research, 269.

small, a delegate reports, 2417.

Tooth, human, microstructure. A. The dentioenamel junction, 2199.

structures, X-ray diffraction studies, 1583.

Top-loaded monopole antenna, earth currents near, RP2959.

Topological proofs for certain theorems on matrices with non-negative elements, 2850 .

Toric bending specimen for investigation of geometrical factors in fatigue, 2442 .

Toroidal coils, high-frequency magnetic permeability measurements, 2584 .

Torsion, of a hollow square, 2385 .

testing machine for wire, recording, 2435.

Total-absorption X-ray spectrometry, application to betatron experiments, 1213.

Total, collision cross sections of negative atomic iodine ions in nitrogen and argon, 605 .

photoelectric cross sections of copper, molybdenum, silver, tantalum, and gold at $662 \mathrm{kev}, 3101$.

Tough problems solved with tricky tooling, 2395. 
Townsend balance system, semiautomatic, 3044 .

Trade wind inversion as a transoceanic duct, J 64D3, 247.

Trailers and trucks, 3103.

Transactor circuit, junction, 749

Trans-difluorodichloroethylene, infrared and raman spectra, 2161.

Transfer of liquefied gases, long distance, 2177.

Transfluorodichloroethylene, infrared and raman spectra, 2161.

Transformations, integral, relations between and summation methods, J 63B1, 1.

matrices, commuting and bilinear, 1654 .

projective, of the Maxwell triangle, invariance of insidedness in, 1046.

Transformer, double-slug, determination of impedance, 570 .

Transforms, trigonometric, 279.

Transient, electromagnetic ground wave on a spherical earth, behavior, 2386 .

error, uniform, RP2879.

fields of a vertical dipole over a homogeneous curved ground, 1967.

radio waves, RP2844.

Transients in signal analysis, 1968.

Transistor, amplifier, 2586.

band spotter, 899 .

beta tester, 2396.

frequency standard, 900

low-distortion, audio oscillator, 755 .

oscillator, precision, 799 .

Transition region, coulomb wave functions in, 980 .

Transitions, indirect in indium antimonide, 1736.

rotational of asymmetric rotor molecules, tables of line strengths, 2796.

tin whiskers, superconducting, 1519.

Transmission, air-borne noise through walls and floors, 2397.

cesium iodide crystals, growth and infrared, 460 . error function for meteor-burst communication, 2674 .

$50 \mathrm{Mc}$ oblique, experiment near the magnetic equator, 2626.

lines, balanced, measuring the attenuation of, 376 . monoenergetic positrons and electrons, 1204

positrons and electrons, 1560.

power in radio propagation, 3102 .

radio at VHF by scattering and other processes in the lower ionosphere, 1469.

radio, loss vs distance and antenna height at $100 \mathrm{Mc}$, 1470 .

Rayleigh waves across an ocean floor with two surface layers, 2387.

reflection by a parallel wire grid, J $63 \mathrm{D} 1,87$.

reflection of electromagnetic waves in the presence of stratified media, RP2899.

reverberant sound through double walls, 159 .

reverberant sound through walls, 1214 .

ultrasonic vibrations in metals, 2398.

Transmission loss, curves for propagation at very low radio frequencies, 2851 .

fading range for propagation through a turbulent atmosphere over irregular terrain, 1550.

radio propagation, 901 .

radio propagation. II, TN12 (PB151371).

space waves propagated over irregular terrain, 606 .

tropospheric forward scatter propagation, TN53 (PB161554).

Transmissions, standard, low frequency, 2179.

WWV standard frequency, $2871 ; 2872$

Transmittance cesium bromide crystals, 349 .

infrared energy by glasses, 1561 .

materials in the far infrared, J $64 \mathrm{C} 1,55$.

reflectance of cesium iodide in the far infrared region, 2852

Transmitters, high-frequency pulse, 1056.

space diversity, reception, 3033 .

Transparent, plastics, 1002.

translucent products, 1155.
Transport, coefficients and correlations of thermodynamic and transport data, 1879.

data, thermodynamic, 1501.

integrals, tables, C595.

integrals, tables of: a supplement, J 63B1, 23.

phenomena in gases at high densities, contribution to the study, 1296.

processes as functions of the Heisenberg and Obukoff theories of turbulence, 1215 .

processes, statistical mechanical theory, 2778 .

properties of dilute alloys of copper, $2852 \mathrm{~A}$.

Transportation, liquefaction of helium, $2855 \mathrm{~A}$.

liquefaction, technical aspects of large scale liquid helium, 2797.

Transposition theorem on linear inequalities, two consequences, 353

Transulcent and transparent products, 1155

Transverse strength testing machine for denture resins, 1216.

Trapped radicals, 2853.

Treadwear of, commercial tires, measurement of, 761 .

tires, influence of type of rubber and black, 350 .

Treatment of, leather with Castiloa and Hevea rubbers, 1600 .

leather with polyisobutylene, 607 .

liquid $\mathrm{He}^{3}$ based on the "cell" model, 1562.

Tree from the viewpoint of lightning, 1248 .

Triangular association scheme, uniqueness, 2835.

Triathanolamin als puffersubstanz, 1217.

Triathanolamine as a buffer substance, 1217.

Triatomic nitrogen in solids at very low temperatures, 3054 .

Tribenzylamine oxide in benzene and water, 1195 .

Tribromofluoromethane, hexafluorobenzene from the pyrolysis, 2151.

Tricalcium phosphate hydrate, crystallographic investigation, 421.

Trichloride and tetrachloride of molybdenum, preparation, $\mathrm{J} 63 \mathrm{~A} 2,185$.

Triethylborane, raman spectrum, 2278.

Trifluoroethylene, 1224

Trigonometric, functions and their inverse to high accuracy, radix tables, 296.

transforms, 279 .

Trihedral and dihedral angles, sums of, in a tetrahedron, 597.

Trimethylphospine, isobutyane, and tertiary butyl fluoride, 2638 .

Tristimulus colorimetry, propagation of errors, 2266 .

Trisulfide, arsenic, refractive index, 2726.

Tritanopia with abnormally heavy ocular pigmentation, 161.

Tritiated-water vapor in air, technique for monitoring, 2338.

Tritium, carbon-14 for the study and charaoterization of cellulose and other polysaccharides, 2403.

oxide and tritium-labeled lithium borohydride, general-purpose apparatus for preparation, analysis and use of, J 63A2, 177.

Tritium-labeled compounds I. Radioassay of tritiumlabeled compounds in "infinitely thick" films with a windowless, gasflow, proportional counter, J $63 \mathrm{~A} 2,171$.

II. General-purpose apparatus, and procedures for the preparation, analysis, and use of tritium oxide and tritium-labeled lithium borohydride, J 63A2, 177 .

III. Aldoses-1- $t, \mathrm{~J} 64 \mathrm{~A} 2,177$.

Tritrations, precise coulometric, 1815 .

Troposphere, propagation beyond the horizon, 470 .

stratosphere, refractivity, 3032 .

Tropospheric, effects on 6-ḾC pulses, 2854

forward scatter propagation, TN53 (PB161554).

irregularities, radio-wave scattering by, J 63D2, 205.

path, low-level, measurements of phase stability over, J $63 \mathrm{D} 1,45$.

radio field strength data, $362 ; 546$

radio wave propagation, some aspects, 1872 . 
research, phase generator, 1805 .

scatter propagation and atmospheric circulations, J $64 \mathrm{D} 1,81$.

scatter propagation measurements near the radio horizon, 1499.

signal characteristics in the Pacific coast region, effect of super-refractive layers, 2100.

transmission loss data, TN43 (PB151402).

ultrahigh-frequency, radio link, measured statistical characteristics and narrow-band teletype message errors on a single-sideband 600 -mile long, J 64D2, 125.

$\mathrm{VHF}$, recording measurements of plane and circular polarized waves in the Great Lakes area, 620 .

wave transmission loss, TN15 (PB151374).

Tropospheric propagation, beyond the horizon, 351 .

beyond the horizon, role of partial reflections, 595 .

1948-1956, TN26 (PB151385).

partial reflections, 508 .

point-to-point radio relaying via the scatter mode. 1812 .

radio wave scattering in, 808 .

Trucks and trailers, 3103 .

Tubes, gas, stabilized power supplies, 1353.

rotational viscous flow, 1094 .

transfer, vacuum-insulated, 2406.

Tubes, Bourdon, sensitivity and life data, 1864.

cathode-ray, electrostatic deflection plates, J $64 \mathrm{C} 2$, 103.

photoelectric multiplier, 27.

receiving, tabulation of data, $\mathrm{H} 68$.

Tungsten, carbon monoxide, 2888 .

helical spring balance, 162 .

steels, photometric determination, RP2812.

Tuning systems employing feedback amplifiers, 352 .

Turbidity and viseosity measurements on some cationic detergents in water and in sodium chloride solutions, RP2778.

Turbine, hydraulic, contra-angle handpiece, 732 .

Turbulence, 1215.

fully developed pipe flow, structure of, 884 .

Turbulence-measuring equipment, development, 686 .

Turbulent, boundary layers with heat transfer and pressure gradient in flow direction, J 63B1, 53 .

flow, difussion of particles, 2929 .

motion, 3104 .

processes as observed in boundary layer and pipe, 1218.

TV and FM bands, factors affecting radio propagation, 1342.

Twilight, oxygen red lines, excitation, 2554.

Twinned epitaxy of copper on copper, RP2873.

Two, consequences of the transposition theorem on linear inequalities, 353 .

cylinders in an ideal fluid, on the motion, RP2889.

early papers on the relation between extreme values and tensile strenght, 1219.

existence theorems for systems of linear inequalities, 608.

explicit formulae for the distribution function of the sums of $n$ uniformly distributed independent variables, 609 .

forms of mathematical induction, 2855.

independent variables, function generator, 1590 .

problems in abstract algebra connected with Horner's rule, 1101

resistance-type hot-pressing furnaces for laboratory use, 2399

variables, subharmonic, harmonic, and linear, 280.

Two-dimensional, nozzles by method of characteristics, design, 1432.

patterns by scanning techniques, processing of, 802 .

Two-stage autoignition of some hydrocarbons, 354 .

\section{$\mathbf{u}$}

$\mathrm{U}_{235}$ fission product decay spectra at various times after fission, 3105 .

U(IV) compounds, magnetic susceptibilities, 1396.

U-type manometer, 211.
Uber pH-Werte nichtwasseriger losungen, 1563.

den Einfluss der Gewebekonstrukcon auf den Scheuerwiderstand, 610 .

die Funktionen von Gegenbauer, 1221.

Ultra-high frequency, characteristics of the magnetic attenuator, 663 .

directive antenna patterns, measurement of the effect of irregular terrain, 481 .

magnetic attenuator, 902 .

moon relay, 396 .

oscillator attenuator, 216.

transhorizon propagation based on meteorological considerations, J 64D3, 231.

tropospheric transmission loss data, TN43 (PB151402)

wide-range resonators, 357 .

Ultra high strength steel, new, 2215 .

Ultracentrifugal, and other methods, evaluation of adhesion of organic coatings by, 715 .

Ultramicrotomy, problems, 309.

Ultrasonic, measurements, a method for calculation, 2407. sing-around, velocimeter for liquids, 2301.

switch aids diversity reception, 1564 .

vibrations, characteristics, 2055 .

vibrations in metals, transmission, 2398.

Ultraspherical polynomials, 298.

relative extrema, 106 .

Ultraviolet, alkaline solution of potassium chromate as a transmittance standard, 388 .

far, kinetic spectroscopy, 2607.

heat aging of poly (vinyl chloride), RP2863.

spectrophotometry of low pressure explosions, 1565. visible, and infrared wave-lengths, 1035 .

Uncertainty of measurements, 1953.

Underearth crystal resonators, 903.

Underground, service, electrical measurements in the selection of bolt material, 1008.

Understressing as a means of eliminating the damaging effect of fatigue stressing, 1566.

Uniform asymptotic expansions for Weber parabolic cylinder functions of large orders, J 63B2, 131.

color scales, progress report by Committee of Optical Society of America, $1459 \mathrm{~A}$.

transient error, RP2879.

Unimodular circulants, 2057.

groups, alternative proof of a theorem, 1260.

integral circulants, 1567.

matrices of order two which commute, 1969.

Unique thermal conductivity gas analyzer, 2443 .

Uniqueness theory for asymptotic expansions in general regions, 2400.

triangular association scheme, 2835.

Unit-cell data of the lead niobate $\mathrm{PbNb}_{2} \mathrm{O}_{3}, 2401$.

United States planning for the large scale liquefaction and transportation of helium, $2855 \mathrm{~A}$.

Units, cellular, 830 .

dimensions, and standards, 2088.

dimensions in physics, 611 .

does rationalization change, 2094.

heat, and temperature scale for colorimetry, 1355.

measurements, radiological, 3022 .

measurements, radiological, International Commission, 2366.

radioactivity, 163 .

Unpolarized radiant energy in the analysis of infrared absorption spectra, 1569 .

Unscrambling of gamma-ray scintillation spectrometer pulse-height distributions, 2856.

Unsolved problems in review, 1568.

Unsteady nonlinear waves in sloping channels, 2857.

Unsymmetrical range finders, cross-field error, 1300.

Uranium dioxide specimens, effect of particle size on the bulk density and strength properties, 1926.

Uranium-platinum system, J $64 \mathrm{~A} 1,107$.

Urban atmospheres, interpretation of condensates from, RP2809.

Urea, thiourea, and some thiourea-alkali halide complexes, infrared absorption spectra, 2160 .

two, addition compounds, analysis of dielectric loss, 2454 . 
U.S.A. National Committee Report, URSI subcommission 6.3 , antennas and waveguides and annotated bibliography, $3108 ; 2402$.

Use of, AC bridge to measure core loss at high induction, 1954.

admixtures as integral waterproofing and dampproofing materials, 164.

angular distance in estimating transmission loss and fading range for propagation through a turbulent atmosphere over irregular terrain, 1550.

carbor-14 and tritium for the study and characterization of cellulose and other polysaccharides, 2403. cesium iodide prisms to 50 microns, 1551 .

Chebychev polynomials in thin film computations, J $63 \mathrm{~A} 3,297$.

components of variance in preparing schedules for sampling of baled wool, 346 .

computers in statistics, 2404.

continued fractions in high-speed computing, 612 .

current meteorological observations in radio wave. propagation, 2331.

deuterium to investigate extent of branching in polystyrene, 1220 .

direct current amplifier and recorder to balance a Mueller resistance bridge, 3107 .

early Balmer lines to extend the photospheric model, 2675.

group divisible designs for asymmetrical factorial arrangements, 2836.

nickel-aluminum alloy coatings for the protection of molybdenum from oxidation, 2837.

partially oriented solid samples and unpolarized radiant energy in the analysis of infrared absorption spectra, 1569.

random numbers, 613.

scintillation spectrometers in the study of continuous gamma- and X-ray spectra between 0.01 and $20 \mathrm{Mev}, 1955$.

sensitivity criterion for the comparison of the Bekk and Sheffield smoothness testers, 1970.

statistics to determine precision of test methods, 165 . subroutines on SWAC, 614 .

sub-routines on SEAC for numerical integrations of differential equations and for Gaussian quadrature, 598 .

surface weather observation to predict the total atmospheric bending of radio rays at small elevation angles, 2388.

sweep-frequency backscatter data for determining oblique-incidence ionospheric characteristics, 2838.

zonal constants in the calculated of beam flux, 887 .

Using, arc-discharge tubes in regulated low-voltage supplies, 615 .

electronic computer to reduce weathering test data, 2858.

mathematics for design, 355 .

Utility of meteor burst for intermittent radio communication, 2389.

Utilization of observations of meterological occurrence in radio electrical propagation, 2405 .

Utilizzazione delle osservazioni meterologiche correnti nella propagation radio-electrica, 2405 .

\section{v}

$\mathrm{V}_{9}$ fundamental of $\mathrm{C}_{2} \mathrm{~F}_{6}, 888$.

Vacuum, high, gimbals, 1731 .

needle, 848 .

tube, accelerometer, recent developments, 297.

tube, experimental, drain casting of a high-grade magnesium oxide support used in, 996 .

Vacuum-insulated transfer tube, 2406.

Value, class of mean, functions, 1.

Faraday, 3.

Rydberg constant, 3109.

$g$-values for the first spectrum of thorium (Th I), a preliminary list of levels, $\mathrm{J} 63 \mathrm{~A} 3,275$.

Values of physical constants of different bubbers, 1222.
Valve, cold fluids, 1570

cutoff, heat regulator, 2146.

needle, improved stainless steel, 1034.

seal-off, high vacuum, 919.

Van Rhijn method, height of the nightglow, 1534.

Vanadium, radiative orbital electron capture, 1835 .

Vapor, gallium, mass spectrum of, 758 .

phase ortho-para conversion in the large CEL-NBS hydrogen liquefier, 2859.

water, millimeter wavelength dispersion of, 767 . water, permeability of leather, 57 .

water, rotational intensity distributions of $\mathrm{OH}$ and $O D$ in electrodelen discharge through, 817 .

Vapor pressure, benzene above $100^{\circ} \mathrm{C}, 616$.

$\mathrm{He}^{3}-\mathrm{He}^{4}$ mixtures, 599 .

helium, scale of temperature, 1535 .

saturation, of liquid helium in the range $1.4^{\circ}-4.2^{\circ} \mathrm{K}$, measurements, 1403.

Vapor pressures, deuteromethanes, 889 .

some hydrocarbons in the liquid and solid state at low temperatures, TN4 (PB151363).

Vapors and gases on glass spheres, adsorption, 1609.

Variability, in a process, technique for locating source, 323. wool content in part wool blankets, 1571 .

Variable capacitor calibration with an inductive voltage divider bridge, TN57 (PB161558).

Variable, heart pump permitting independent control of rate, output, and ejection velocity, 381

lenght re-entrant cavity for dielectric measurements from 100 to $400 \mathrm{Mc}, 638$.

Variables, two independent, function generator, 1590.

Variation, determinant of a positive definite matrix, 284 . distortion with magnification, 1572 .

elastic constants and static strains with hydrostatic pressure: a method for calculation from ultrasonic measurements, 2407.

frequency of the signal range of FM and television broadcasting stations, 347 .

intensity of fast cosmic-ray neutrons with altitude, 600 .

physical and chemical properties of split and unsplit chrome-tanned leather, 601 .

spectrum of a normal matrix, 890 .

synoptic, of the radio refractive index, J $63 \mathrm{D} 1,91$

Variational, calculation of scattering cross sections, 617 . principles for the acoustic field, 1223.

Variations, calculus of, elementary introduction, 23. gamma cassiopeiae, TN21 (PB151380).

physical and chemical properties within and between vegetable retanned cow and steer sides, 618 .

preferred orientation and modulus of elasticity in cold drawn music spring wire, 1573.

surface tension with body and surface concentrations, 3110 .

Veatchite, 3057.

Vector, double stochastic transforms, 2658.

measures, relations among certain ranges, 299

Vectors, constant, plus a Rayleigh-distributed, mean absolute value and standard deviation of the phase, RP2950.

symmetric matrix, 192.

Vegetable extract blends, lignolsulfonates 1979 .

Vehicles, space, coatings for, 2910A.

Velocimeter, sing-around ultrasonic for liquids, 2301.

Velocities, burning, measurement of, 1064 .

Velocity, light from the molecular constants of carbon monoxide, 1574 .

measurements below $1^{\circ} \mathrm{K}, 822$.

meter, bar magnet, 174 .

Venting and drainage, problems, 289.

Ventricle, mechanical left, method for the study of the circulation in the $\mathrm{dog}, 920$

Ventricular, right, failure in dogs maintained with an extracorporeal left ventricle, 1685 .

Versatile, core bozes, 619 .

goniometer for projection photometry, 904.

pneumatic instrument based on critical flow, 14 .

Vertical distribution of light from gas-filled candlepower standards, 2860 . 
Vertical-incidence, ionosphere sounding at the National Bureau of Standards, TN28 (PB151387).

scatter from the ionosphere at $41 \mathrm{Mc} /$ sec., observation, 2655.

VHF band, cosmic radio noise intensities, 418.

field-intensity meters, 739 .

field-intensity standards, development, 427.

forward scatter and the lower oinosphere, 2759 .

intensity meters, 151 .

ionospheric propagation, regular, 1495.

ionospheric scatter circuit during the solar event of February 23, 1956, field-strength variations recorded, 2121.

ionospheric seatter communication, 1958 critique, $2505 \mathrm{~A}$.

ionospheric scattering, frequency dependence, TN9 (PB151368).

ionospheric scattering, modulation studies, $2639 \mathrm{~A}$.

match meter, 15 .

microwave matching termination, 637.

oblique incidence circuits, 2773.

off-path propagation, 2656.

propagation by ionospheric scattering-survey of experimental results, 1971 .

propagation measurements in the Rocky Mountain region, 1972.

propagation over irregular terrain, 1663.

radio transmission at, by scattering and other processes in the lower ionosphere, 1469.

radio waves, oblique-incidence measurements of the heights at which ionospheric scattering occurs, 1790 .

region, permeability measurements, cavity techniques, 1283.

scatter propagation, high-gain antennas, 1729 .

scatter research program for the I.G.Y., NBS equatorial region, 2209.

transmission by scattering from the lower ionosphere, extreme useful range, 2560.

transmission in North America, effect of Sporadic E, 3074 .

transmission loss, $750 ; 870 ; 1384$.

tropospheric recording measurements of plane and circular polarized waves in the Great Lakes area, 620 .

UHF directive antenna patterns, measurement of the effect of irregular terrain, 481.

UHF tropospheric transmission loss data, TN43 (PB151402).

UHF wide-range resonators, 357.

VLF, antenna for investigating the ionosphere with horizontally polarized radio waves, J 64D1, 27. atmospheric noise, 2351 .

atmospheric radio noise, 2188.

emissions generated in the earth's exosphere, 3097.

emissions, origin of, J 63D1, 21.

field strength data, study, 2438 .

ionospheric propagation, 2203; 2234; $2456 ; 2390$.

ionospheric propagation for finite ground conductivity, mode theory, 2373.

ionospheric reflection coefficients at, from sferics measurements, 2172 .

measurement of ground conductivity, 2670 .

monopole antenna with a radial wire ground system, 2437.

noise investigation, by the NBS, 2217.

noise, theory of production, 2003 .

papers, introduction, 2165.

propagation, multiple reflections between the earth and ionosphere, 1781.

propagation using atmospheric waveforms, some characteristics, 2760 .

pulses, propagation of, to great distances, RP2898. radiation spectra of lightning discharges, J 63D2, 199. radio waves, frequency characteristies, 2345.

radio waves, propagation, TN58 (PB161559).

radio waves, waveguide mode theory, 2391 .

sky wave propagation, geometrical optics, 2360 .
Very low temperature, an experimental study of the strength and fatigue of glass, 2014.

data: properties of paramagnetic salts, 2408.

Vessel, cylindrical, conductivity of a fluid contained in, measurement, 2233.

Vibrating systems, decay constant in, 678.

Vibration, measurement, developments, 1492.

measurement, stroboscopic interferometer, 2784.

problem with arbitrary damping and restoring forces, 2145.

square clamped plates, 1435 .

stroboscopic analyzer, J $63 \mathrm{C} 2,97$.

suspended chain, modes of, 770 .

thin elastic plates with free edges, RP2784.

wind-induced, of telephone and distribution conductors, 2868.

Vibration-rotation, bands of ammonia, 2411; 2862.

bands of ammonia: 1 . The combination bands $\nu_{2}-\left(\nu_{1}, \nu_{3}\right)$, RP2894.

spectrum of D O, 891 .

spectrum of HDO, 892.

structure in absorption bands for the calibration of spectrometers from 2 to 16 microns, J $64 \mathrm{~A} 1,29$; Mono.16.

Vibrational, energy exchange between diatomic molecules and a surface, 2861 .

energy of deuterium cyanide, 1575.

equilibrium distribution reactant molecules by chemical reactions, 2671.

nonequilibrium distributions, relaxation, $1847 ; 2235$; $2324 ; 2786$.

relaxation data in shock wave experiments, 2896.

relaxation of a system of anharmonic oscillators, 2786 .

Vibrational spectra, $n$-dimenthylaminodiborane, 1205 .

trifluorethylene and trifluorethylene-d, 1224.

Vibrational spectrum, bromotrifluoroethylene, 1206.

chlorotrifluoroethylene, 893.

fluorotrichloroeth ylene, 1577.

1-fluoro-1 chloroethylene, 1576.

force constants of diboron tetrachloride, 2409.

structure in the ionization efficiency curves of hydrogen and deuterium molecules, 2410.

tetrabromoethylene, $1825 ; 1973$.

Vibrational-rotational bands of deuterated methanes, $J$ $63 \mathrm{~A} 2,145$.

Vibrations, stretching and inversion in ammonia, interaction, 1744.

ultrasonic, characteristics, 2055.

ultrasonic in metals, transmission, 2398.

Video signals on coaxial cables, 2300 .

Vincent's theorem, note, 96 .

Viscoelastic, linear, behavior, proposed nomenclature, 2271 .

media, interfacial waves, 1745 .

phenomena in amorphous high polymeric systems, 2863.

rubber in shear, nonlinear, 1419.

Viscoelasticity, linear, theory: delta-function approximations, 956.

Viscometer, Mooney, 1164; 1409.

semi-micro dilution, 1245.

Viscometric and turbidimetric measurements on dilute aqueous solutions of a nonionic detergent, 1225.

Viscometry, capillary, 2047A.

Viscose and cotton rayon, 973 .

Viscosities, higher, grease consistometer is modified to operate at constant shear rates, 245 .

sucrose solutions at various temperatures: tables of recalculated values, C440 Suppl.

very dilute polymer solutions, 166 .

0.25 to 90 percent GR-S rubber solutions, 621 .

Viscosity, density and electrical resistivity of molten alkaline-earth borate glasses with three mole percent of potassium oxide, 2864 .

electrical resistivity of molten alkali borates, 907 .

exhaust-gas mixtures at elevated temperatures, determination of 989 .

expansivity and density, molten alkali silicates, 422 . 
intrinsic, graphical method for single-point determination, 1235 .

methods to the study of branching in polsaccharides, application of, 654 .

Mooney, 740.

$n$-hexadecane, RP2912.

non-Newtonian, 849.

specific heat, enthalpy, entropy, free energy function, and thermal conductivity of steam, compressibility factor, density, 1657 .

thermal conductivity, and Prandtl number for air, $\mathrm{O}, \mathrm{N}_{2}, \mathrm{NO}, \mathrm{H}_{2}, \mathrm{CO}, \mathrm{CO}_{2}, \mathrm{H}_{6} \mathrm{O}, \mathrm{He}$ and $\mathrm{A}, 1207$.

turbidity measurements of some cationic detergents in water and sodium chloride solutions, RP2778.

Viscosity-molecular, intrinsic, weight relationships for dextran, 745 .

Visible, ultraviolet, and infrared wavelengths, 1035.

Vision, color and colorimetry, 2500.

color-defective and normal, according to the threecomponents theory, entroptic color-perceptions of the macular pigment by observers, 1706 .

Voltage, breakdown tester, 908 .

divider bridge, inductive, TN57 (PB161558).

electronic, regulator, 135.

error, detector, broadband, 914 .

low, regulator, 364 .

ratio at audio frequencies, measurement, 2191.

Visual auroral observations, 2412 .

Vitreous coatings to stainless steel, adherence of, influence of copper ions, 1737.

Vitro production of experimental enamel caries, 1734.

Vitron, noncrystal ionic model for silica glass, RP2782.

Vitrons in alkali silicate binary glasses, RP2854.

Volcanoes on the moon, 3111 .

Voltmeter, RF, calibrating consoles, 2747.

Volume, changes in small concrete cylinders during freezing and thawing, 167 .

temperature relations of amorphous polymers over an extended temperature range, 1975 .

Volume-temperature relationships for the room temperature transition in teflon, 356.

Volumetric apparatus, glass, testing, C602.

VTM circuits, 168

Vulcanizates, electrica! resistance, 408.

pure-gum rubber, 2534 .

rubber, determination of sulfur, 1311.

Vulcanization, characteristics of natural rubber, 909.

natural rubber, infrared study of structural changes, RP2816.

\section{w}

$\mathrm{W}_{\text {air }}$ for plonium-210 alpha particles, absolute measurement, 2444.

sulfur-35 beta-rays, measurement, 1769 .

Wall, effects on rotational population of $\mathrm{OH}^{2} \Sigma^{+}, 2865$.

floor, and door constructions, sound insulation, BMS144, 2d Suppl.

material, cavity ionization as a function of, RP2842.

Walls, double, reverberant sound, 158 .

floors, transmission of airborne noise, 2397.

radiographic rooms, dose, 1319 .

War games, analytic study, 1264 .

Washington area scientists-for-teachers program, 1956.

Water, a thermochemical study of the reaction of, RP2785. content in Freon-12, circulating in a refrigeration system, determination of, 990 .

current meters, rating, 340 .

demand rates for electric drinking-water coolers of the bubbler type, 1226.

deuterated, vapor, rotation-vibration spectra, 1949 .

dose distributions in, for betatron X-rays to $37 \mathrm{Mev}$, studies, 1904.

dose measurements, using scintillation and ionization detectors, 995 .

effect of dissolved air on the speed of sound, 1686 .

evaporation, from cellulose, 547 .

freezing of, in pipes and vessels, 861 .

jets, steam driven, for propulsion purposes, RP2830.

liquid, infrared absorption, from 2 to 42 microns, 1038 . nitrogen, oxygen, and ammonia, electric discharge, 2309.

porcelain enameled hot, tanks, 2745 .

release, effect on the free outflow hydrometer, J $63 \mathrm{~B} 2$, 117.

speed of sound in, near the turning point, temperature coefficient, 1914.

speed of sound, tables, 3061 .

storage, analytical integration of the differential equation, J $63 \mathrm{~B} 1,43$.

tritiated, vapor in air, technique for monitoring, 2338.

Water vapor, determination from the change in electrical resistance of a hygroscopic film, RP2864.

deuterated, rotation-vibration spectra, 1859.

dielectric constant, 431.

influence of temperature on the absorption, by collagen and leather, 74 .

millimeter wavelength dispersion of, 767 .

oxygen absorption of radio waves in the atmosphere, 2241.

permeability of leather, 57 .

rainfall, statistics, microwave attenuation, 88 .

rotational intensity distributions of $O H$ and $O D$ in electrodelen discharge through, 817 .

Water-soluble degradation products of asphalts, 2413.

Water-vapor and nitrogen adsorption, determination of surface area of hydrated portland cement by, RP2806.

Waterdrops, fragmentation in the zone behind an air shock, RP2843.

Waterproofing and dampproofing materials, 164.

Watson's lemma, modification, J 63B1, 15.

Watthour-meter test, 1049.

Wave action in diffusers for exhaust-pipe systems, with special reference to the scavenging of two-stroke engines, 1679 .

Wave-frequency dependence of the duration of radar-type echoes from meteor trails, 348 .

Wave, function, Coulomb, by means of recurrence relations, generation, 1532 .

pulse, spherical, by a half plane screen, diffraction, 2086.

radio, bending from normal, calculation of the departures, J 64D3, 259.

transient ground, note on the propagation, 1992.

transmission loss, tropospheric, TN15 (PB151374).

Wave propagation, 1147.

anisotropic, inhomogeneous medium, 910.

breaking velocities, strain energies, and theory neglecting, 1277 .

inhomogeneous medium, 629.

iosopheric, 67 .

irregular surface, integral equation approach to the problem, 394 .

long textile yarns impacted transversely, RP2866.

tropospheric radio, some aspects, 1872.

Waveform of a radio atmosphere at short ranges, 1800.

Waveguide components, millimeter wavelengths, precision, 2339.

millimeter-wavelength range, electroforming of, C587.

mode theory of the propagation of VLF radio waves, 2391.

mode theory of VLF ionospheric propagation, 2390.

rectangular, adjustable sliding termination, 2012.

rectangular, half-round inductive obstacles, J 64B2, 113.

termination, reflectionless, 183 .

Waveguides and antennas, 2402; 2897.

Wavelength, dependence in ultrahigh frequency transhorizon propagation based on meteorological considerations, J 64D3, 231.

dispersion of water vapor millimeter, 767 .

shifters and oxygen quenehing in liquid scintillators, 1439.

Wavelengths from thorium-halide lamps, RP2891.

Wavenumbers, tables of, Mono. 3, Vol. I and Vol. II.

Waves, electromagnetic, along a eurved surface, theory of propagation, 2673.

interfacial between two streams, 1632 . 
interfacial in viscoelastic media, 1745.

light and length standsrds, 1387.

long, propagation, 2721.

nonlinear, in sloping channels, unsteady, 2857.

pulses, on the diffraction and reflection of, by wedges and corners, RP2906.

radio, atmospheric bending, 2806.

radio, penetrating the ionosphere, 2458 .

scattered radio, some meteorological effects, 1874 .

surface, on plane and curved impedance boundaries, excitation, 2553.

three-centimeter radio, single path phase measuring system, 2758.

Wax batteries, 2866 .

Wear prevention, 59

Weather data distribution, how FM expedites: fax and telemetering, 69 .

Weatherability of porcelain enamels, 1578 .

Weathering, certain mortars exposed in brick masonry with and without caps and flashings, effects, 1927

porcelain enamel, 157.

relation between actual and artificial, 2729 .

test data, using an electronic computer to reduce, 2858 .

Weber parabolic cylinder functions of large orders, uniform asymptotic expansions. J $63 \mathrm{~B} 2,131$.

Wedges and corners on the diffraction and reflection of waves and pulses by, RP2906.

Weibull distribution, moments of order statistics, 1428.

Weight, intrinsic viscosity-molecular, 745 .

molecular in polymer methacrylate, fracture phenomena, 2569

molecular, on the crazing and tensile properties of polymethyl methacrylate, effect of, 999 .

piston gage, 2103.

Weighted, compounding of two independent significance tests, 3098 .

restricted partitions, 3112 .

Weights, atomic, 127.

1956-57, report, 2731.

report of the committee of the American Chemical Society, 531; 1129 .

Weights and measures, administration in the U.S., 1976. administration, NBS and state, 485 .

approval scale, 1580 .

checking prepackaged commodities, H67.

federal basis for, C593.

National Conferences:

Forty-second, 1957, M222

Forty-third, 1958, M225

Forty-fourth, 1959, M228

National Conference Reports, (See National Conference on Weights and Measures).

officials and manufacturers, genuine cooperation, 458. training program, 182.

Weiner's integrals in function space, 185 .

Welded ships, investigation of structural failures, 746 .

What is the heat pump? 1227

use is spectroscopy? 622 .

Whistlers, some properties of lightning impulses which produce, 2764.

Whittaker functions, Jacobi polynomials, certain series expansions involving, 1424.

product of two, Neumann series, 630.

Why screw threads must be everybody's business, 1977.

Wide-band FM adapter reduces interference, 169.

Wide-range, calibrator for vibration pickups, 911 .

cryostat temperature control, 3113.

decade frequency generator, 170.

$\mathrm{R}-\mathrm{C}$ oscillator, 171 .

resonators for VHF and UHF, 357.

Wilson quotients and the third Wilson prime, table, 636.

Wind, load requirements, new standard, 2214 .

pressures in various areas of the United States, BMS152.

trade, inversion as a transoceanic duct, J 64D3, 247.

Wind-induced vibration of telephone and distribution conductors, 2868.
Winds, aloft, radio measurement, 524 .

ionospheric, systematic, 319 .

Wings, delta, structural analysis and influence coefficients for, 840 .

Wire, buried, ground systems, calculations of transverse current loss, 2665.

cold drawn music, crystallography, 2509.

endurance of helical springs related to properties of, 710.

grid parallel to a dielectric interface, impedance, 2364 .

grid parallel to an interface between homogeneous media, theory of reflection, 2238 .

length, minimum total, formal procedures for connecting terminals, 2128 .

magnets, Cunife, RP2808.

music spring, cold drawn, 1573.

recording torsion testing machine, 2435 .

resistance strain gages, cryogenic characteristics, 2073.

strain gages, 2950 .

Wireless microphone uses F-M modulation, 2869.

Wires, copper, measurement of the resistance-strain relation and Poisson's ratio, 1768.

line, pretied tie for communication, 2432.

Wood, baled, use of components of variance in preparing schedules for sampling, 346 .

cotton, and nylon fabrics at different temperatures and relative humidities, 2793 .

Wool content in part wool blankets, variability, 1571.

World maps of $\mathrm{f} 2$ critical frequencies and maximum usable frequency factors, TN2 (PB151361).

unification of screw threads, 1581.

World-wide, ionospheric soundings, first report of the special committee, 1712.

soundings committee: Memorandum 17-Future work, 2870 .

standard frequency broadcast reception, 623.

standard frequency broadcasting station, the power requirements and choice of an optimum frequency for, J $63 \mathrm{D} 1,35$.

WWV, standard frequency transmissions, $2871 ; 2872$. standard frequency station, 42.

\section{$\mathbf{x}$}

$\mathrm{X}$ - and $\gamma$-ray dosimetry, stabilization film processing, 1891 . gamma-rays, photographic dosimetry, 2611.

$\mathrm{X}$-band, ceramic, cavity resonators, 2561 .

oscillator stabilization, high-order harmonics, 2153.

$\mathrm{X}$-radiation in the range from $30 \mathrm{kev}$ to beyond $1 \mathrm{Mev}$, photographic personnel dosimeter, 1994.

$\mathrm{X}$-ray, absorption spectra, 1655 .

attenuation coefficients from $10 \mathrm{kev}$ to $100 \mathrm{Mev}$, C583 Suppl.

attenuation in lead, aluminum, and concrete in the range 275 to 525 kilovolts, 1582 .

beam, $1400 \mathrm{kv}$, calorimetric determination of the power, 2047 .

calibration of radiation survey meters, pocket chambers and dosimeters, 172 .

dental, film, 2091 .

dental machine, panoramic, 1993.

diffraction, crystal structure of anhydrous indium phosphate and thallic phosphate, 1667.

diffraction powder patterns, standard, C539, Vols. 7, 8,9 .

diffraction studies of tooth structures, 1583.

equipment, military field, 483 .

exposure, dental, radiation data during, ionization chambers, 1380.

gamma-spectra between 0.0 ; and $20 \mathrm{Mev}, 1955$.

low-angle, diffraction of fibrous polyethylene, 2616. low-temperature, studies on Rice's blue material, 2979 .

microscope, field emitter point projection, 1982.

photons, 1136.

spectrometry, 1213 .

standards, comparison, $916 ; 1230$.

study of textural stresses in two-phase alloys, RP2793. tube, high voltage, 224 . 
X-rays betatron, 961

betatron, to $37 \mathrm{Mev}$, studies of dose distributions in water, 1904.

concrete and lead, attenuation of $86-$ and $176-\mathrm{Mev}$ synchrotron, $1635 \mathrm{~A}$.

gamma-rays, absorption by concrete, 1250 .

hard, penetration and diffusion of: polarization effects, 793.

high-energy, $1150 ; 2741$.

low energy, standard free-air chamber for measurement, J $64 \mathrm{C} 1,49$.

nuclear absorption and elastic scattering, direct observations, 1312 .

penetration and diffusion, 509 .

reciprocity law, $1838 ; 1839$.

scattered by $\mathrm{C}^{12}$, parameters of the 15 -Mev, 1803.

scattered in lead, energy and angular distribution, 2111.

scintillation crystal-TV, 1484.

synchrotron, 1135 .

\section{$\mathbf{Y}$}

Yarns, from different fibers, impact properties, 734. stress-strain relationships in, RP2866.

stress-strain relationships, subjected to rapid impact loading, $1899 ; 2783$.

subjected to rapid impact loading, stress-strain, 1514; $1515 ; 1516$.

tested at rates of straining up to a million percent per minute, stress-strain relationship, 1900 .

textile, high-speed impact behavior, 2909 .

textile, impact absorbing capacity, 2363.

textile, rapid impact loading, 2715 .

Yohimbine hydrochloride, crystallographic data, 2508.

Young's modulus, flexural and longitudinal resonance frequencies of uniform bars, comparison of experimental and theoretical relations, J 64A2, 147. various refractory materials as a function of temperature, 3114.
Yttrium iron garnet, spectra, 2966.

$\mathbf{z}$

Zeeman data, supplementary, for the first spectrum of ruthenium (Ru I), J 63A3, 253.

Zeeman effect, 1584 .

first spectrum of hafnium, 1208.

spark lines of ruthenium, 1228.

Zeeman-split absorption lines as very narrow pass filters, 3115 .

Zero pressure gradient, characteristics of turbulence in a boundary layer, 968 .

Zeros, certain polynomials, J 63B1, 21 . certain polynomials, $\mathrm{J} 63 \mathrm{~B} 1,21$.

derivative of Bessel functions of fractional order, 912. $\mathrm{I}_{n+1}(x) \mathrm{J}_{n}(x)+\mathrm{J}_{n+1}(x) \mathrm{I}_{n}(x), 624$.

polynomials and the degree of linear systems, 790 .

Zeta functions, Lerch, 1798.

Zinc, blend lattices, ionic character and elastic moduli, 2367.

boroxilicate glasses, properties, RP2930.

phosphate and silicate cements, 2873.

point, temperature, 2799 .

sulfide, high frequency induced electroluminescence, 1728 .

Zirconia, alumina shapes, 173 .

reactions in binary oxide systems, 1978.

Zirconium, extension of the second spectrum, into the infrared, 717.

five compositions of zirconium hydride, heat content of from $0^{\circ}$ to $900^{\circ} \mathrm{C}, \mathrm{RP} 2878$.

titanium compounds in nonaqueous media, 2106.

niobium, titanium, and tantalum in steels, determination, RP2923.

Zirconium-hydrogen system: some thermodynamic properties from a heat content study, 2839.

Zone, auroral absorption, location of, 1054 . 



\section{U.S. DEPARTMENT OF COMMERCE}

Luther It. Hodges, Secretary

NATIONAL BUREAU OF STANDARDS

A. V. Astin, Director

\section{THE NATIONAL BUREAU OF STANDARDS}

The scope of activities of the National Bureau of Standards at its major laboratories in Washington, D.C., and Boulder, Colo., is suggested in the following listing of the divisions and sections engaged in technical work. In general, each section carries out epecialized research, development, and engineering in the field indicated by its title. A brief description of the activities, and of the resultant publications, appears on the inside of the front cover.

WASHINGTON, D.C.

ELECTRICITY. Resistance and Reactance. Electrochemistry. Electrical Instruments. Magnetic Measurements. Dielectrics.

METROLOGY. Photometry and Colorimetry. Refractometry. Photographic Research. Length. Engineering Metrology. Mass and Scale. Volumetry and Densimetry.

HEAT. Temperature Physics. Heat Measurements. Cryogenic Physics. Equation of State. Statistical Physics.

RADIATION PHYSICS. X-Ray. Radioactivity. Radiation Theory. High Energy Radiation. Radiological Equipment. Nucleonic Instrumentation. Neutron Physics.

ANALYTICAL AND INORGANIC CHEMISTRY. Pure Substances. Spectrochemistry. Solution Chemistry. Analytical Chemistry. Inorganic Cbemistry.

MECHANICS. Sound. Pressure and Vacuum. Fluid Mechanics. Engineering Mechanics. Rheology. Combustion Controls.

ORGANIC AND FIBRoUS MATERIALS. Rubber. Textiles. Paper. Leather. Testing and Specifications. Polymer Structure. Plastics. Dental Research.

METALLURGY. Thermal Metallurgy. Chemical Metallurgy. Mechanical Metallurgy. Corrosion. Metal Physics. Electrodeposition.

MINERAL PRODUCTS. Engineering Ceramics. Glass. Refractories. Enameled Metals. Crystal Growth. Constitution and Microstructure.

BUILDING RESEARCH. Structural Engineering. Fire Research. Mechanical Systems. Organic Building Materials. Codes and Safety Standards. Heat Transfer. Inorganic Building Materials.

APPLIED MATHEMATICS. Numerical Analysis. Computation. Statistical Engineering. Mathematical Pbysics.

DATA PROCESSING SYSTEMS. Components and Techniques. Digital Circnitry. Digital Systems. Analog Systems. Applications Engineering.

ATOMIC PHYSICS. Spectroscopy. Radiometry. Solid State Physics. Electron Physics. Atomic Physics.

INSTRUMENTATION. Engineering Electronics. Electron Devices. Electronic Instrumentation. Mechanical Instruments. Basic Instrumentation.

PHYSICAL CHEMISTRY. Thermochemistry. Surface Chemistry. Organic Chemistry. Molecular Spectroscopy. Molecular Kinetics. Mass Spectrometry. Molecular Structure and Radiation Chemistry.

- Office of Weights and Measures.

BOULDER, COLO.

C.RYOGENIC ENGINEERING. Cryogenic Equipment. Cryogenic Processes. Properties of Materials. Gas Liquefaction. IONOSPHERE RESEARCH AND PROPAGATION. Low Frequency and Very Low Frequeney Research. Ionosphere Research. Prediction Services. Sun-Earth Relationships. Field Engineering. Radio Warning Services.

RADIO PROPAGATION ENGINEERING. Data Reduction Instrumentation. Radio Noise. Tropospheric Measurements. Tropospheric Analysis. Propagation-Terrain Effects. Radio-Meteorology. Lower Atmosphere Physics.

RADIO STANDARDS. High Frequency Electrical Standards. Radio Broadcast Service. Radio and Microwave Materials. Atomic Frequency and Time Interval Standards. Electronic Calibration Center. Millimeter-Wave Research. Microwave Uircuit Standards.

a ADIO SYSTEMS. High Frequency and Very High Frequency Research. Modulation Research. Antenna Research. Yavigation Systems. Space Telecommunications.

UPPER ATMOSPHERE AND SPACE PHYSICS. Upper Atmosphere and Plasma Physics. Ionosphere and Exosphere fcatter. Airglow and Aurora. Ionospheric Radio Astronomy. 




